Fall 2009

\title{
2009 Miracle Yearbook
}

Cedarville University

Follow this and additional works at: https://digitalcommons.cedarville.edu/yearbooks

Part of the Higher Education Commons, Organizational Communication Commons, and the Public Relations and Advertising Commons

\section{Recommended Citation}

Cedarville University, "2009 Miracle Yearbook" (2009). Yearbooks. 14.

https://digitalcommons.cedarville.edu/yearbooks/14

This Book is brought to you for free and open access by DigitalCommons@Cedarville, a service of the Centennial Library. It has been accepted for inclusion in Yearbooks by an authorized administrator of DigitalCommons@Cedarville. For more information, please contact digitalcommons@cedarville.edu. 

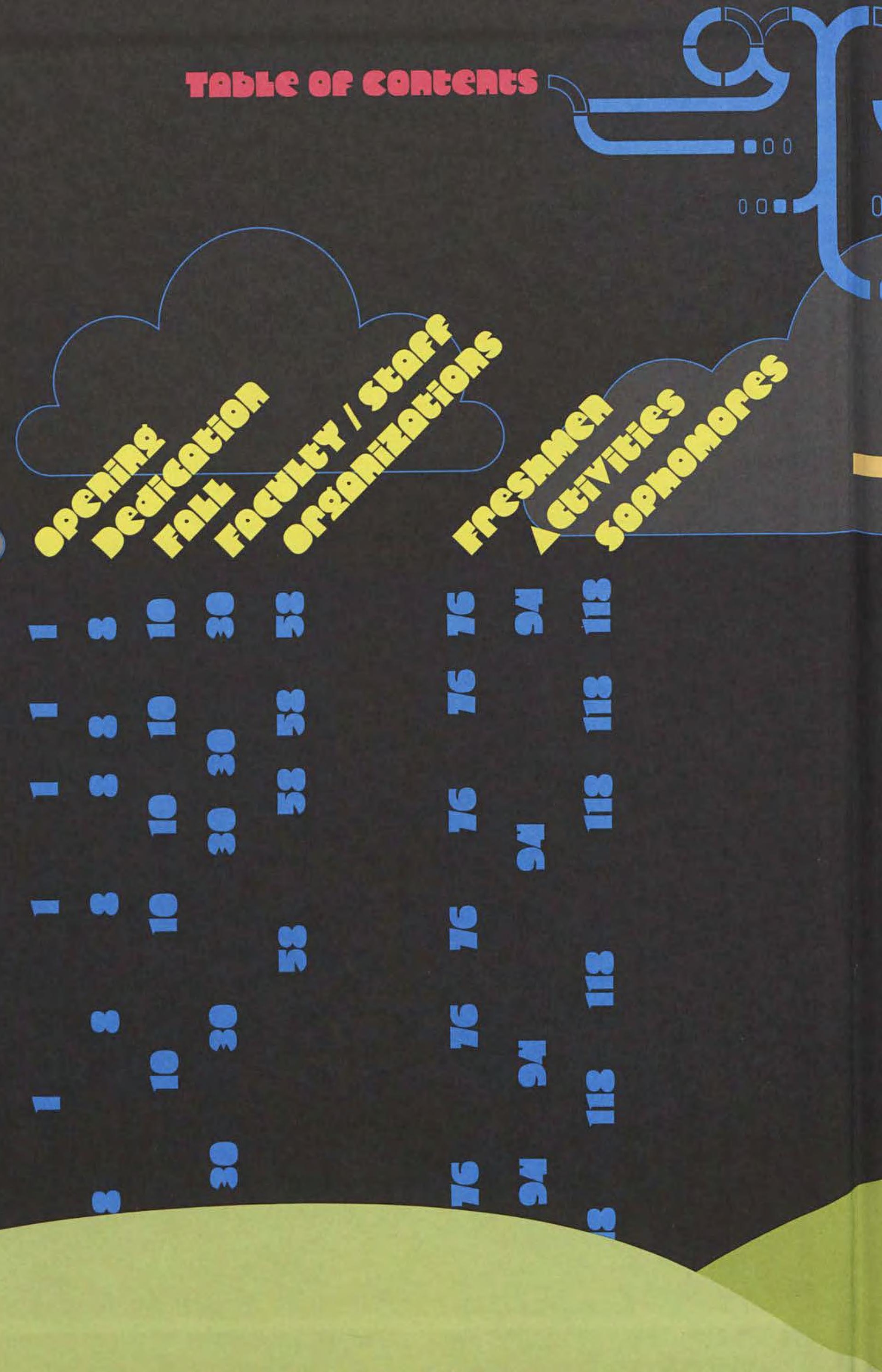


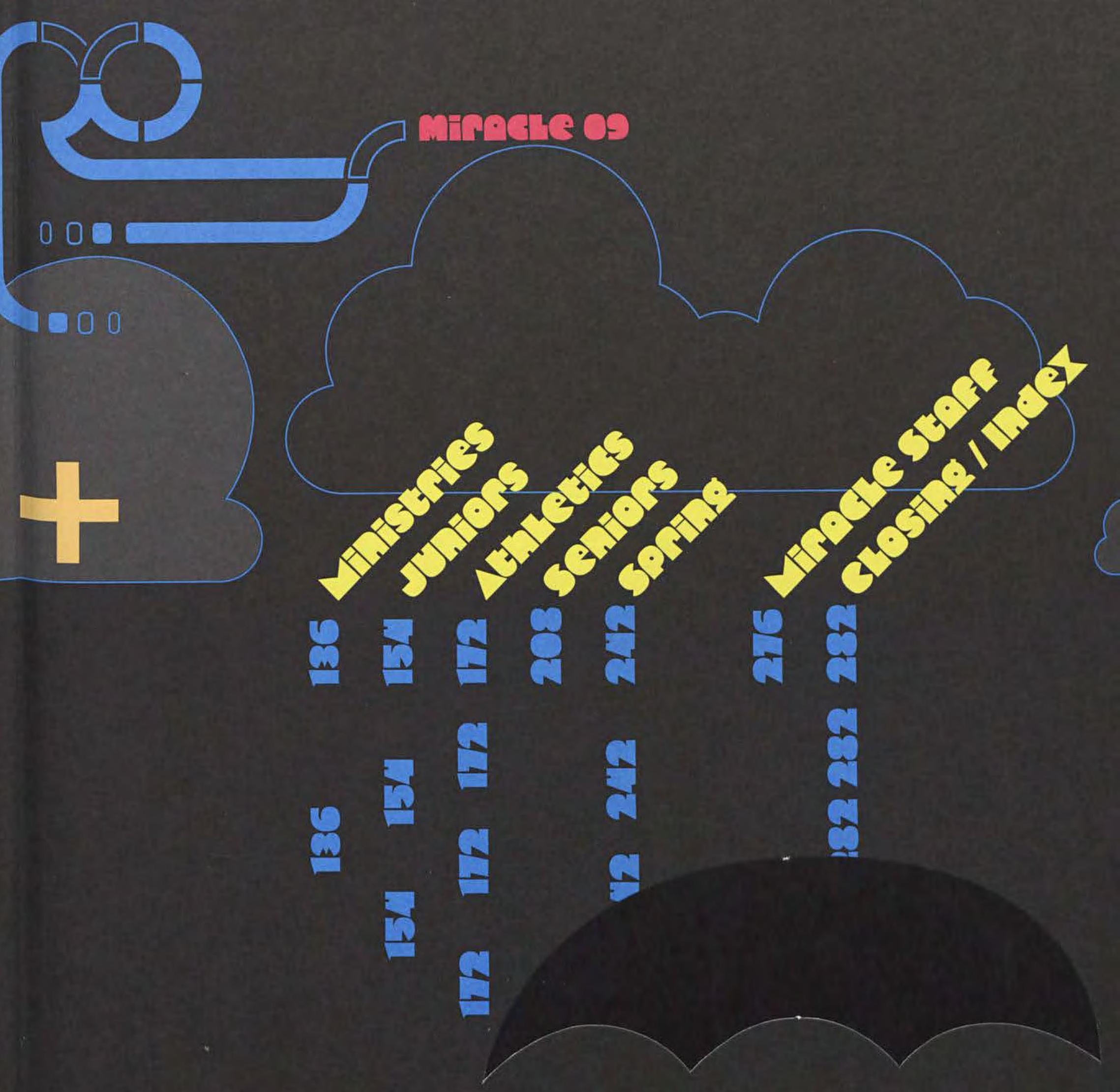




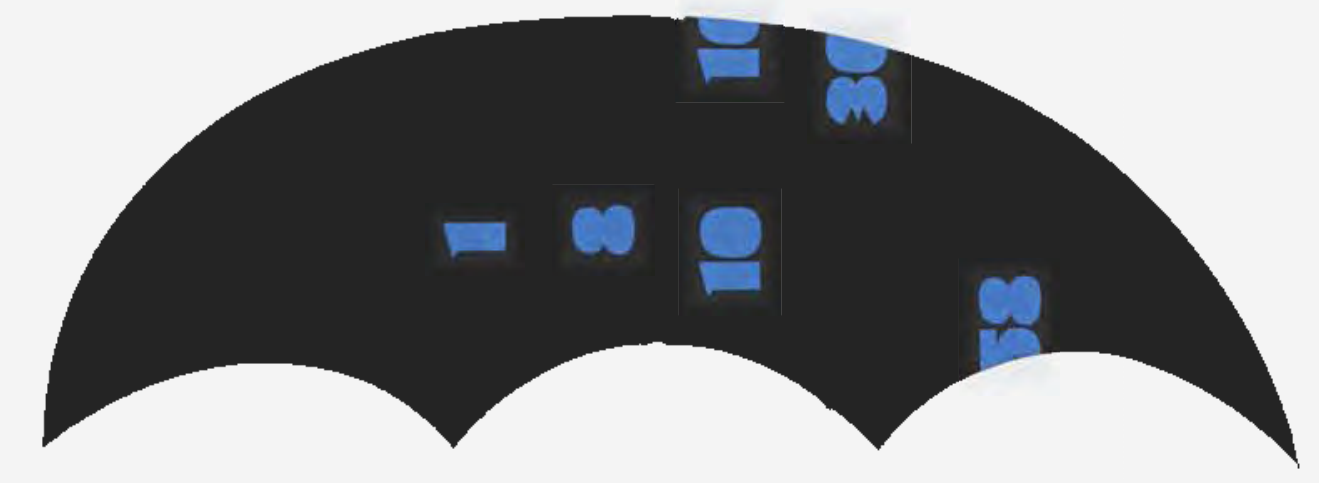




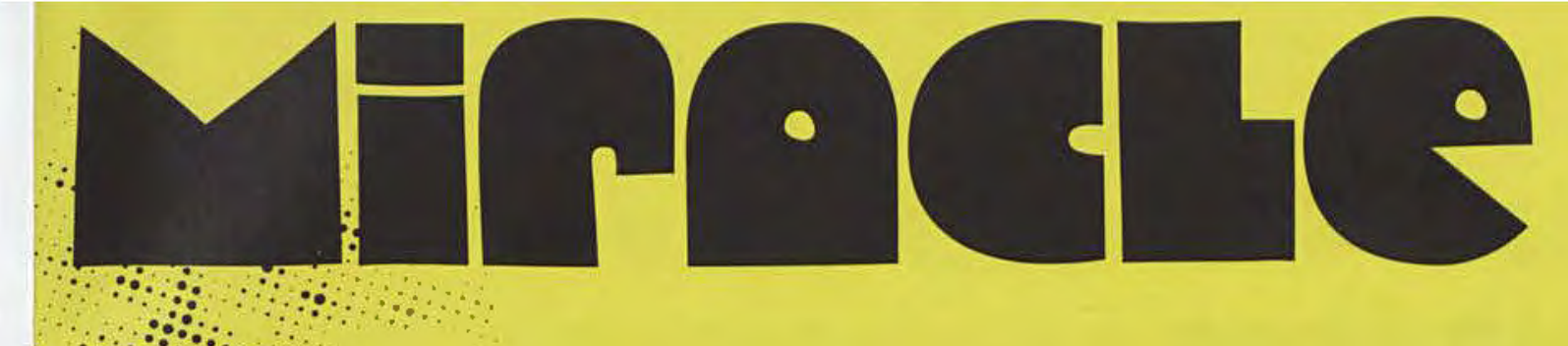




\section{s new th}

500

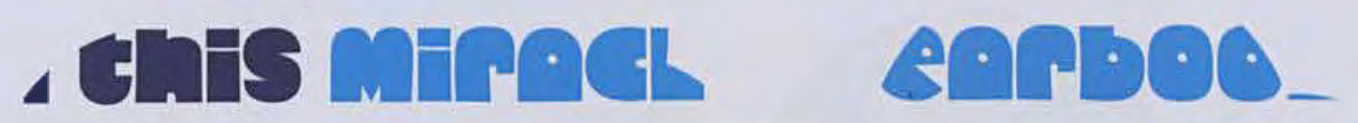

CS 50 a

semeshane new ther you

at the !

ו

שמ.
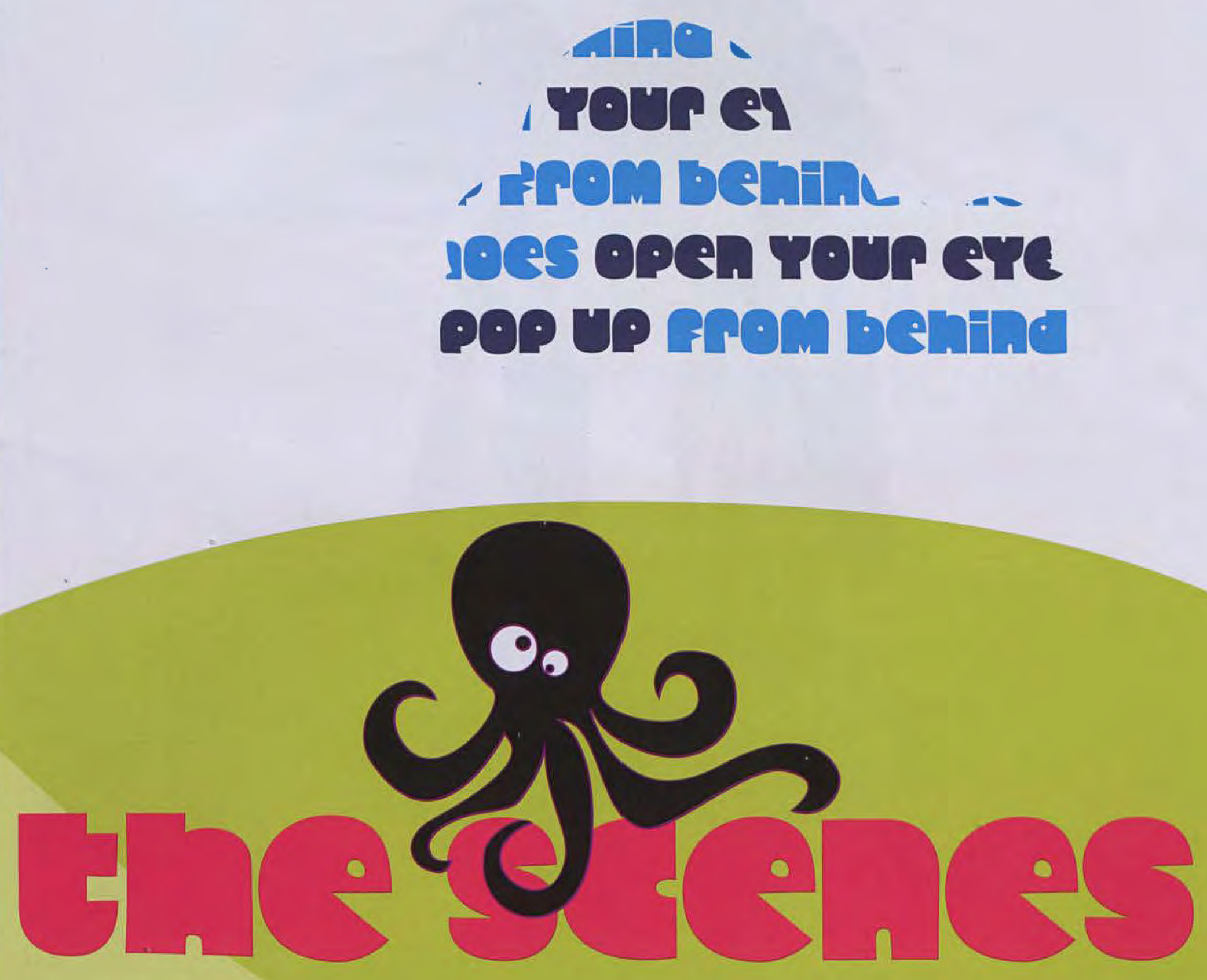

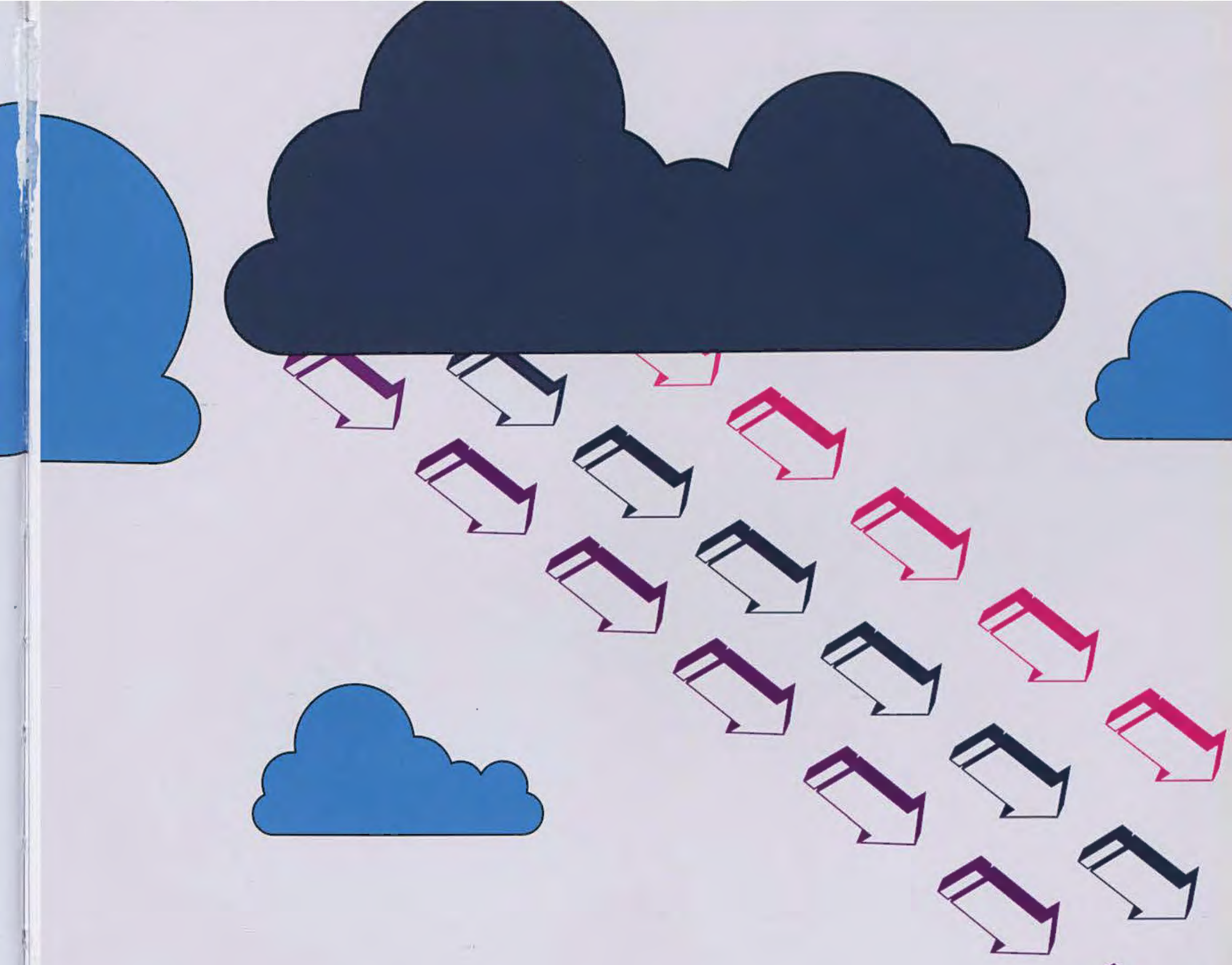


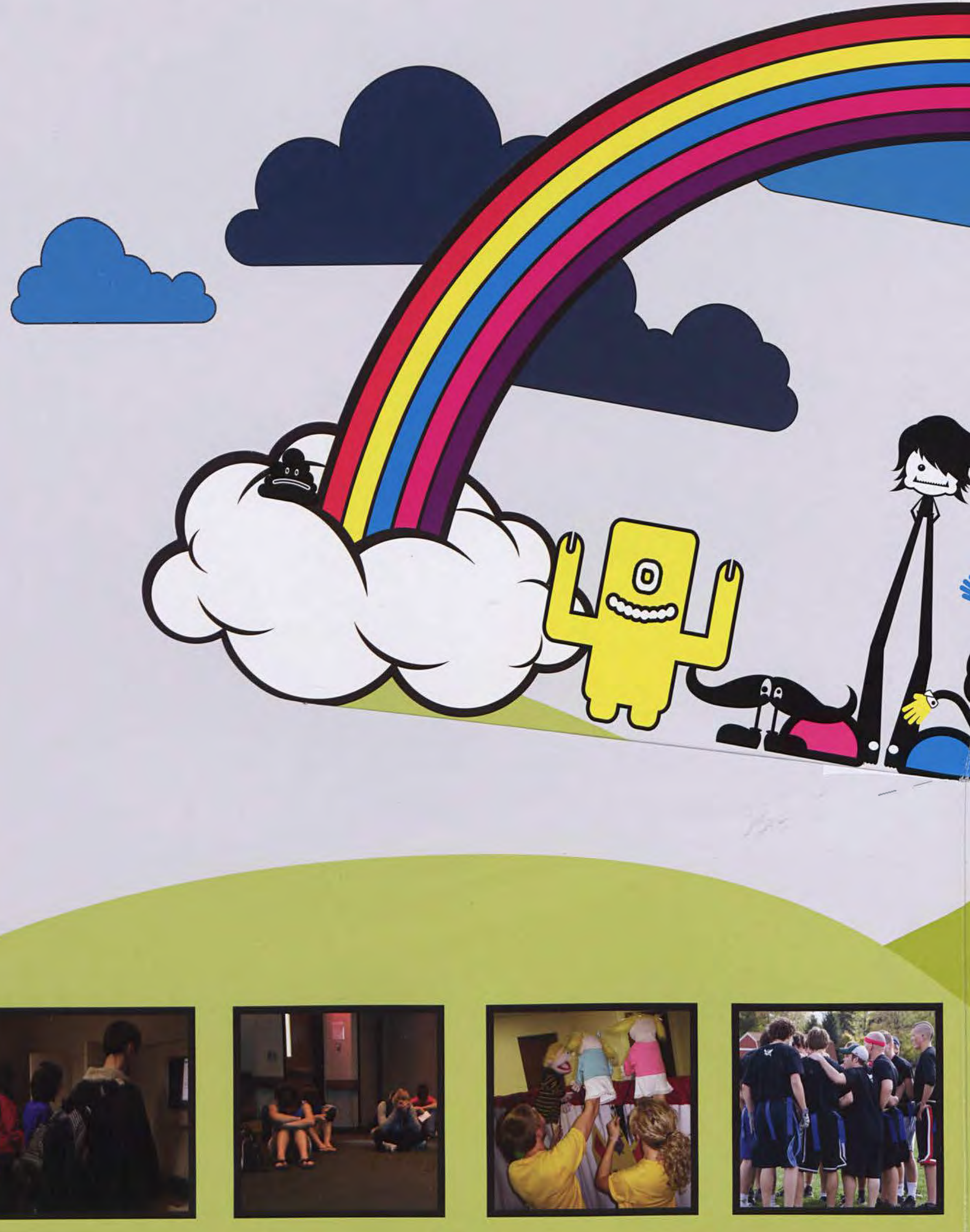




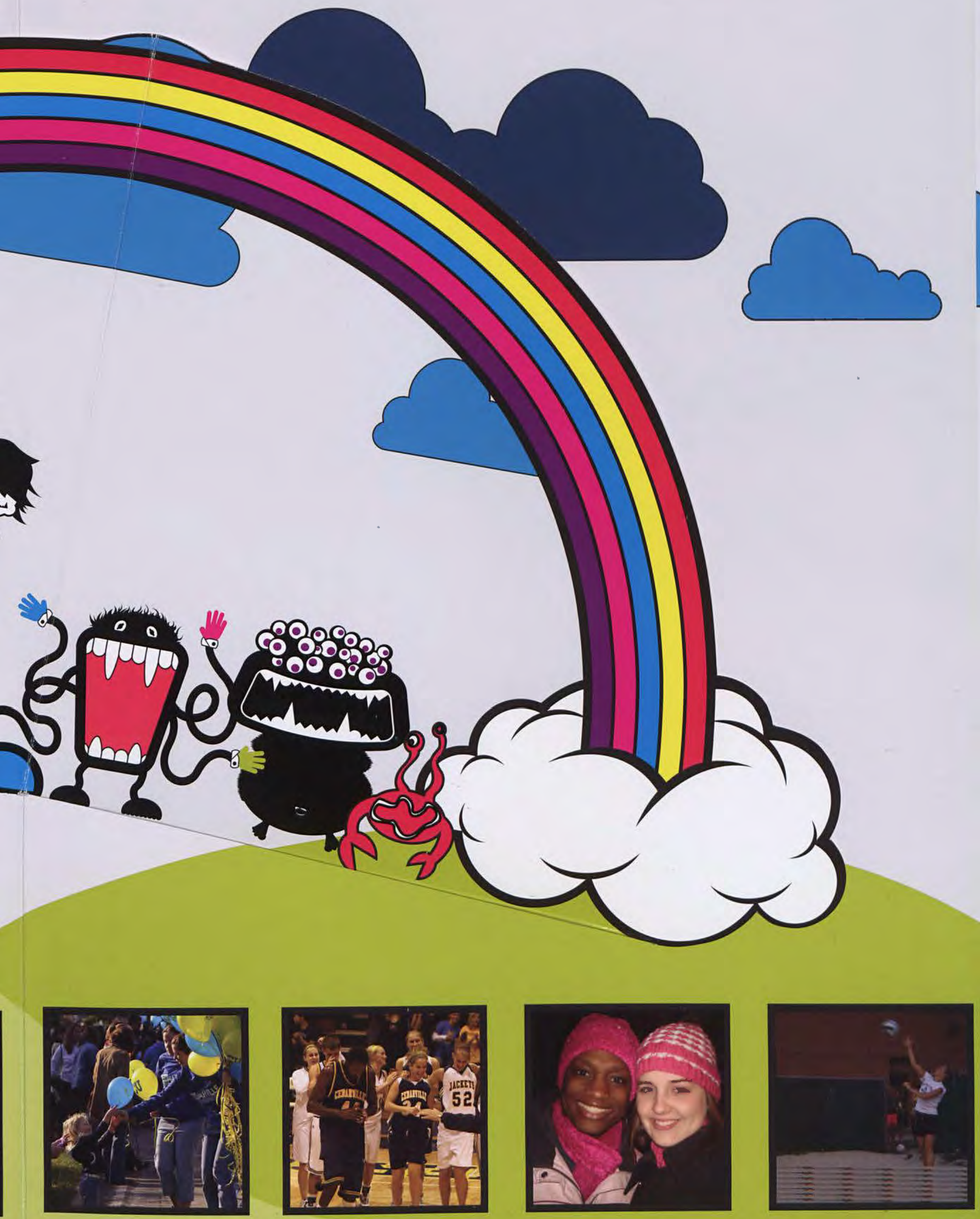




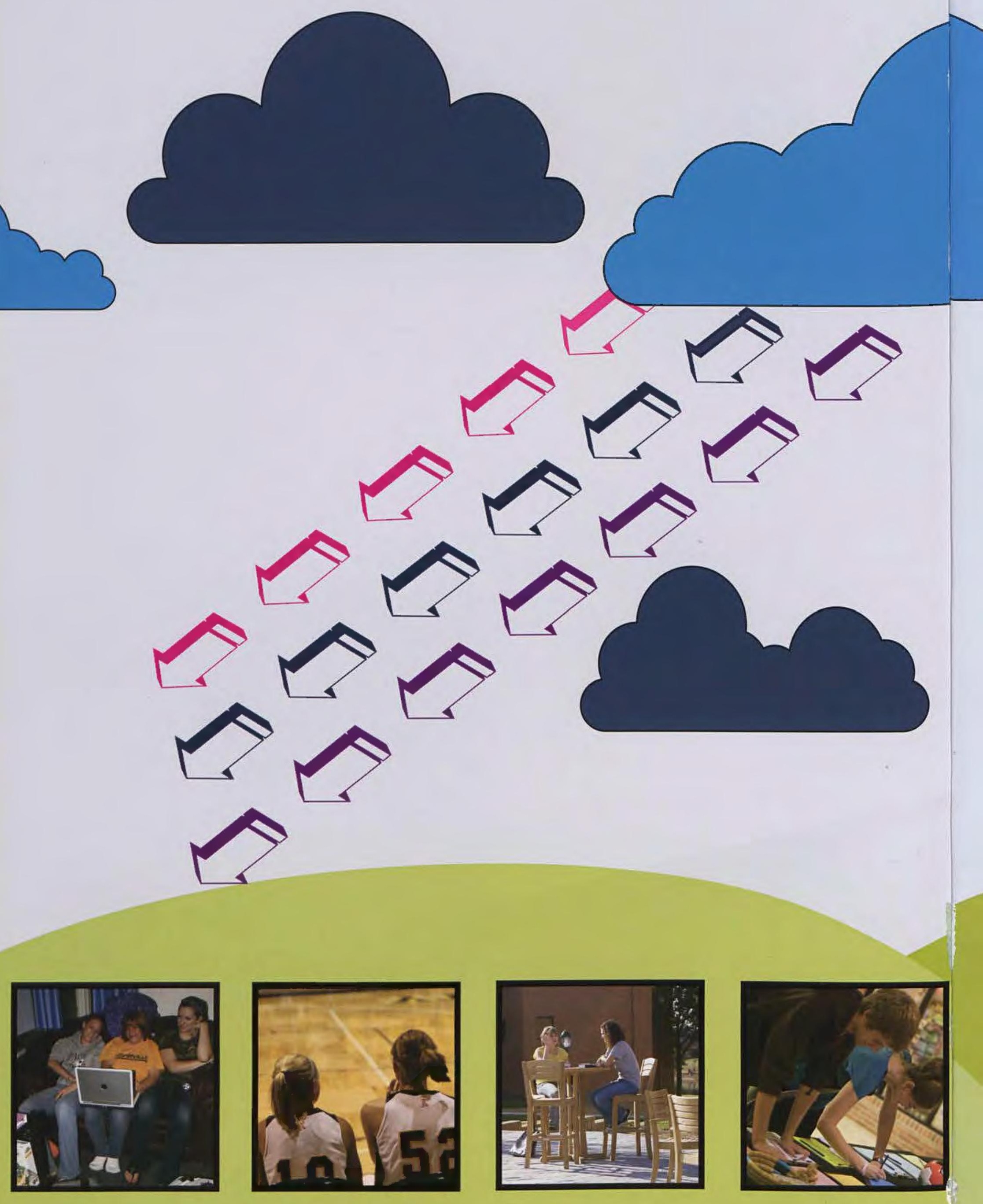




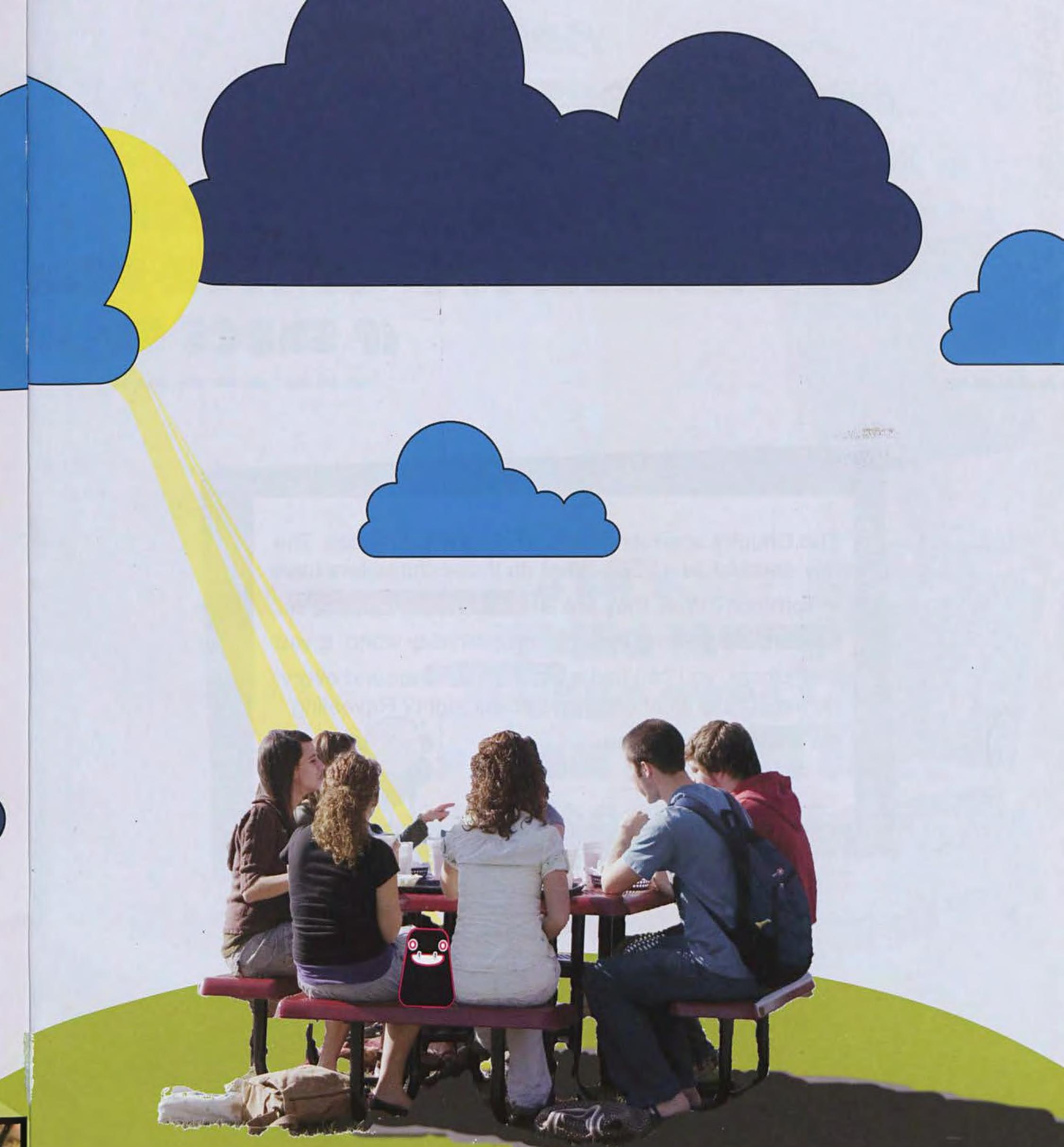




\section{PSOO \\ anbECPS PCo

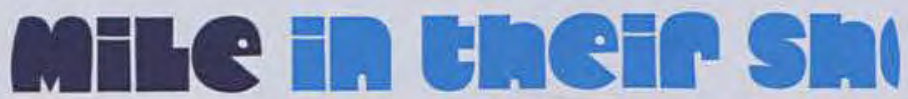 cere mensters oo}

CI ROU CTAE

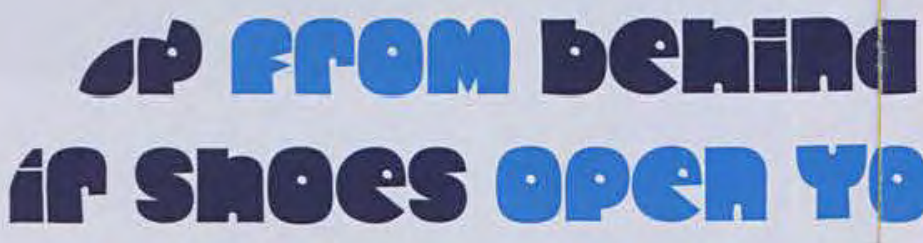

The Chuck's scanner. The sophomore from Brazil. The boy dressed as a $\mathrm{WO}$. What do these characters have in common? Well, they are all बे SURBOS walking through our everyday world. If you look closer, you can find a EURPurSO around every corner. That's what college is about, right? Ręvealing the $\Omega$ in the routine.

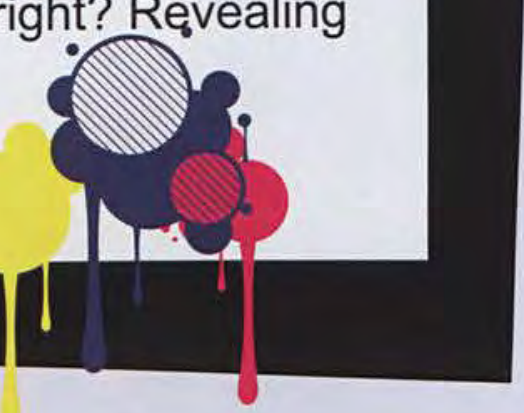



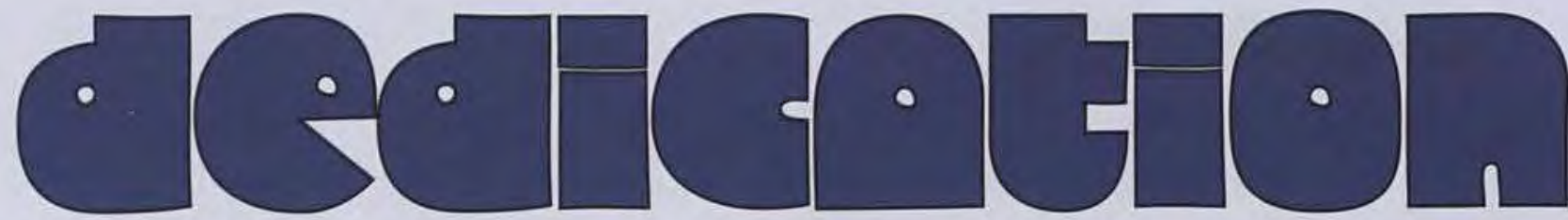

As Vice President of Student Life, Dr. Carl Ruby is quick to point out that $92 \%$ of a student's college career is spent outside of the classroom. Because of this, Dr. Ruby is especially intentional about meeting the academic and non-academic needs of the student body. Along with the rest of the Student Life staff, Dr. Ruby works to provide a campus environment that promotes open and honest dialogue, authentic, Christ-like living, and cultural diversity.

Dr. Ruby and his beautiful wife, Suzy, have been married for twenty-three years and have four daughters: Meagan, a sophomore education major at CU; Kelsey, a junior at Southeastern High School; Erin, a sophomore at Southeastern; and Leah, a 5th grader at Miami View Elementary. Often helping his girls with various activities, Dr. Ruby plays an active role in each of their lives.

Known for his sense of humor and fun-loving personality, Dr. Ruby enjoys reading, skiing, and other outdoor activities. He is also an active member of Midtown Community Church. Dr. Ruby has worked on several church missions trips and has lent his baking and sales skills at the church cinnamon-roll booth at the fair.

Dr. Ruby has been a part of the Cedarville family since he graduated from Cedarville University in 1983. He then went on to Wheaton College to pursue his Masters and finally to receive his Doctorate at Ohio University in 1996. Aside from his two years at Wheaton, Dr. Ruby has worked in a variety of positions at Cedarville since his graduation.

Through his commitment to "making our campus look more like God's kingdom," Dr. Ruby has left an undeniable mark on Cedarville University and its student body. Dr. Ruby has challenged students to think critically and in a Christ-like way about many important issues facing the world today. Through his redemptive discipline approach, Dr. Ruby not only works to make Cedarville's campus a well-ordered place to live, he ultimately points each student towards the love of Jesus Christ.
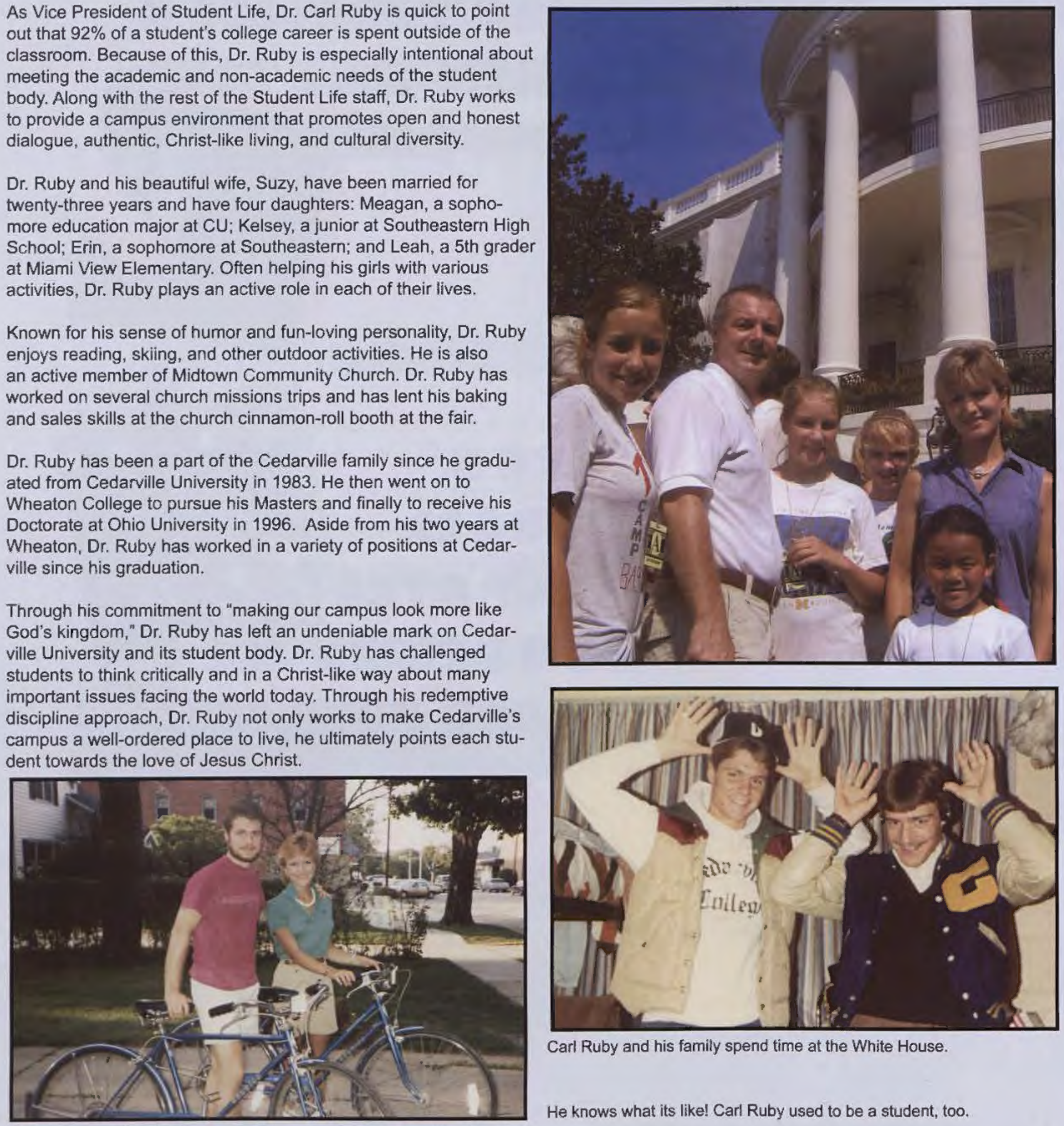

Carl Ruby and his family spend time at the White House.

He knows what its like! Carl Ruby used to be a student, too.

Carl Ruby and his wife, Suzy, spend time together outside riding bicycles.
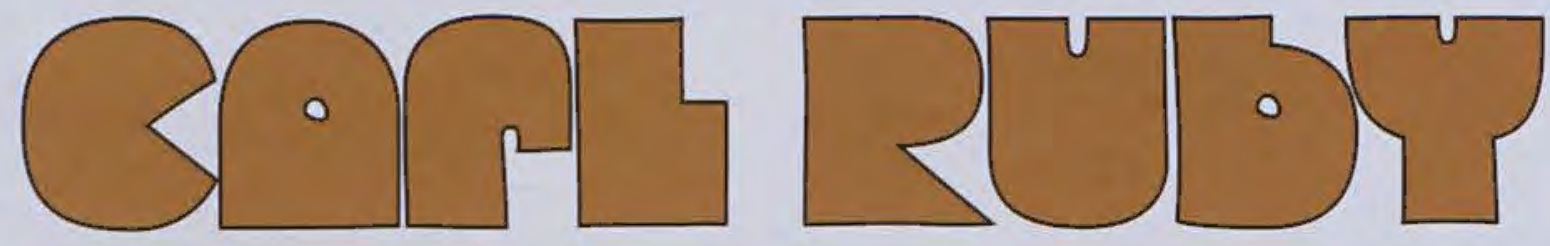

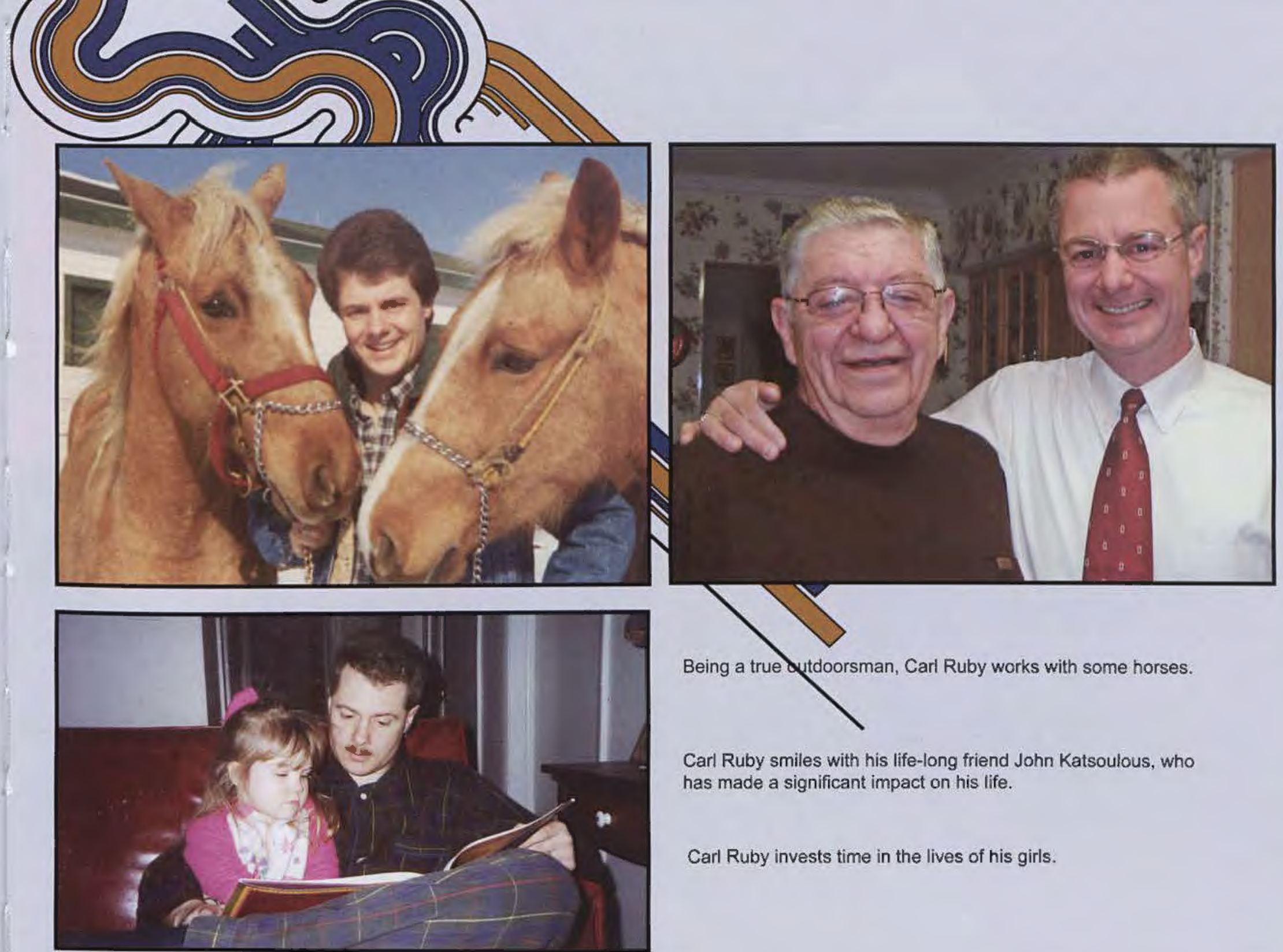

Carl Ruby smiles with his life-long friend John Katsoulous, who has made a significant impact on his life.

Carl Ruby invests time in the lives of his girls.

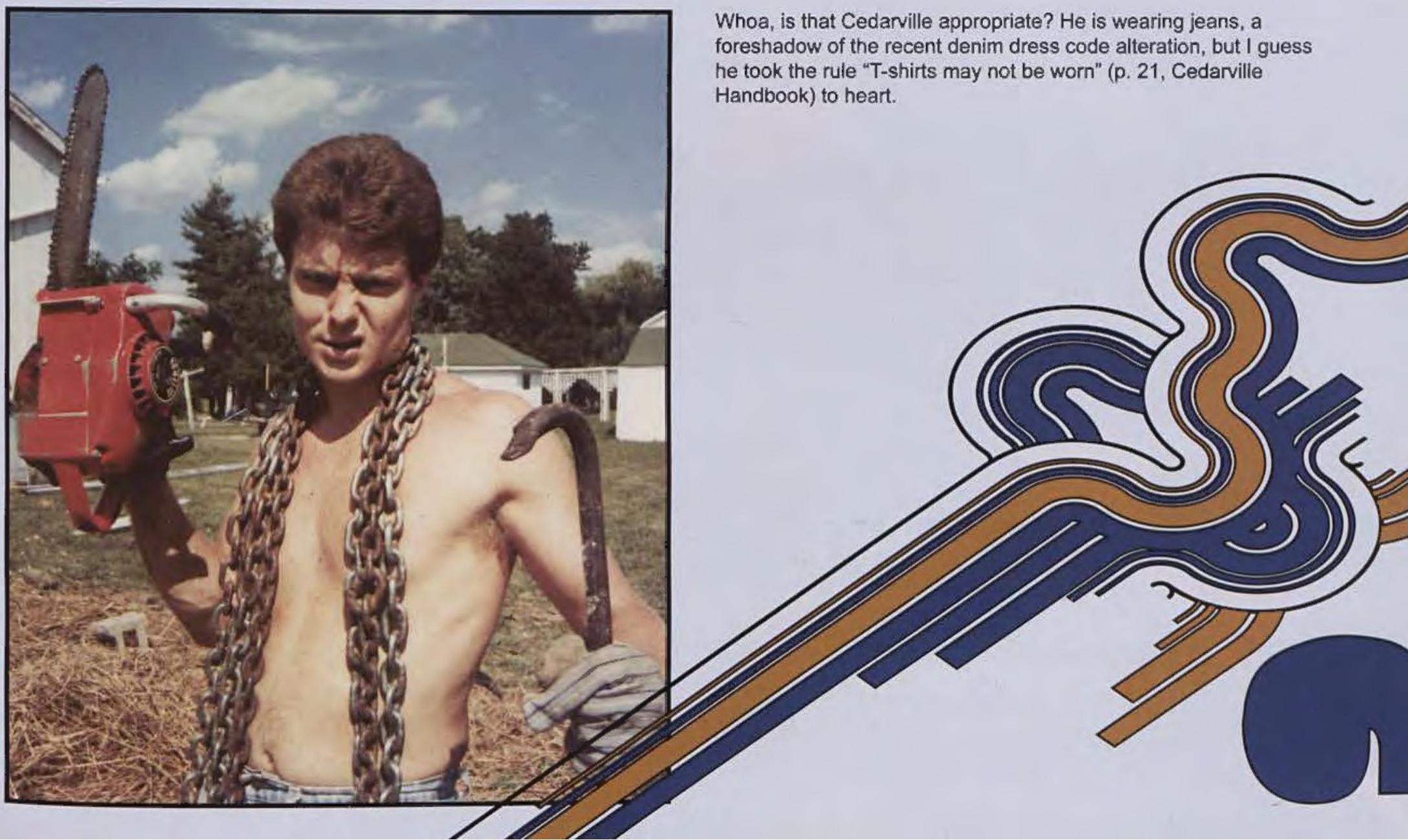




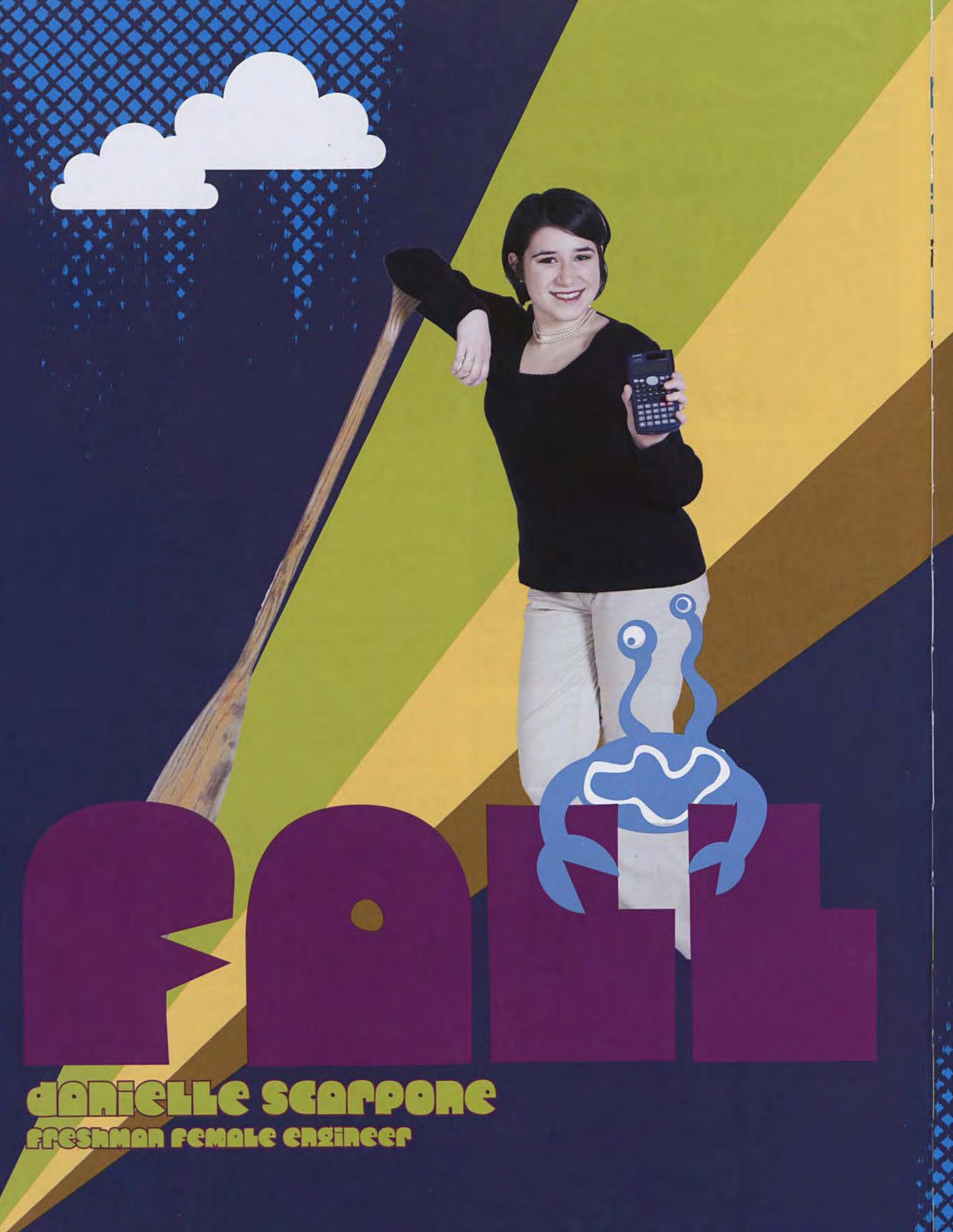


My heart was pounding as the canoe left the shore. My team's endless hours of hard work peaked in this moment - our cardboard canoe holding two of our members was gliding across Cedar Lake. The day was bright but chilly as the fall air crept into the early October afternoon. I stood at the rock, screaming loudly in support of our boat, staring at its cardboard edges as though I could keep them afloat with my eyes. It just had to reach the other side of the lake.

The truth is I knew the canoe would float. In my Engineering Profession class, my professor taught us how to calculate the dimensions of the boat to ensure its success. Still, with my grade on the line and my teammates inches away from the freezing water, a girl has her doubts. Once the boat was in the water, my teammates couldn't move the wrong way or the boat would tear, only adding to the pressure of the voyage. The cardboard was wobbling -1 thought our construction would be more stable. The anxiety was building as I watched them glide a little awkwardly across the lake. They just needed to paddle faster, why were they rowing so slowly?

I've always wanted to study science. When I was a kid, my favorite shows were Bill Nye the Science Guy and The Magic School Bus. I would borrow books from the library to try the experiments inside, absorbing the information and searching for ways to learn more. Because I was homeschooled, I had the opportunity to fully explore my interests and study math and science in depth. By junior year of high school I realized I wanted to become an engineer - I like the challenge that the projects hold, the balance of careful precision, and creativity engineering requires. I wanted to study engineering, and this is why I came to Cedarville.

After spending time here at Cedarville, I realize that the University has so much more to offer me than just a strong engineering program. I chose Cedarville because of the outstanding engineering department, but I am getting so much more than a good academic education. I love the genuine spiritual atmosphere and my unit mates in Printy. My engineering classes are interesting in both the academics and the social dynamics. Since there are so few female engineers, each of us girls must work to develop her identity within the male-dominated major. I find that the girls either can't stop talking about the guys or become one of the guys - I don't think I fit in either category. I definitely find that we girls stick together, but it's great to explore new friendships growing within the classroom.

In a one-credit hour intro class for engineers, we were split into teams of four and given the assignment to build a canoe out of cardboard that would successfully carry two of our team members across Cedar Lake. My project team was the only co-ed team to participate, and the four of us made a dynamic group. We labored for hours in the Maddox lounge to construct our cardboard canoe, preparing for the fateful Friday of Homecoming weekend where our beautiful final product would be judged. The moment of truth.

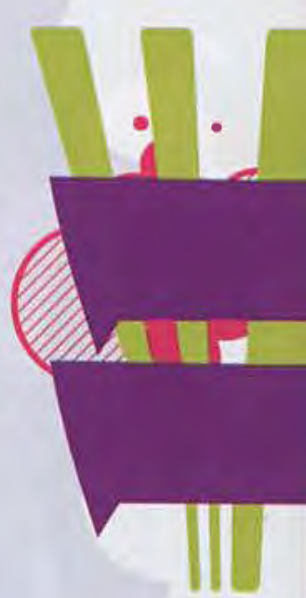



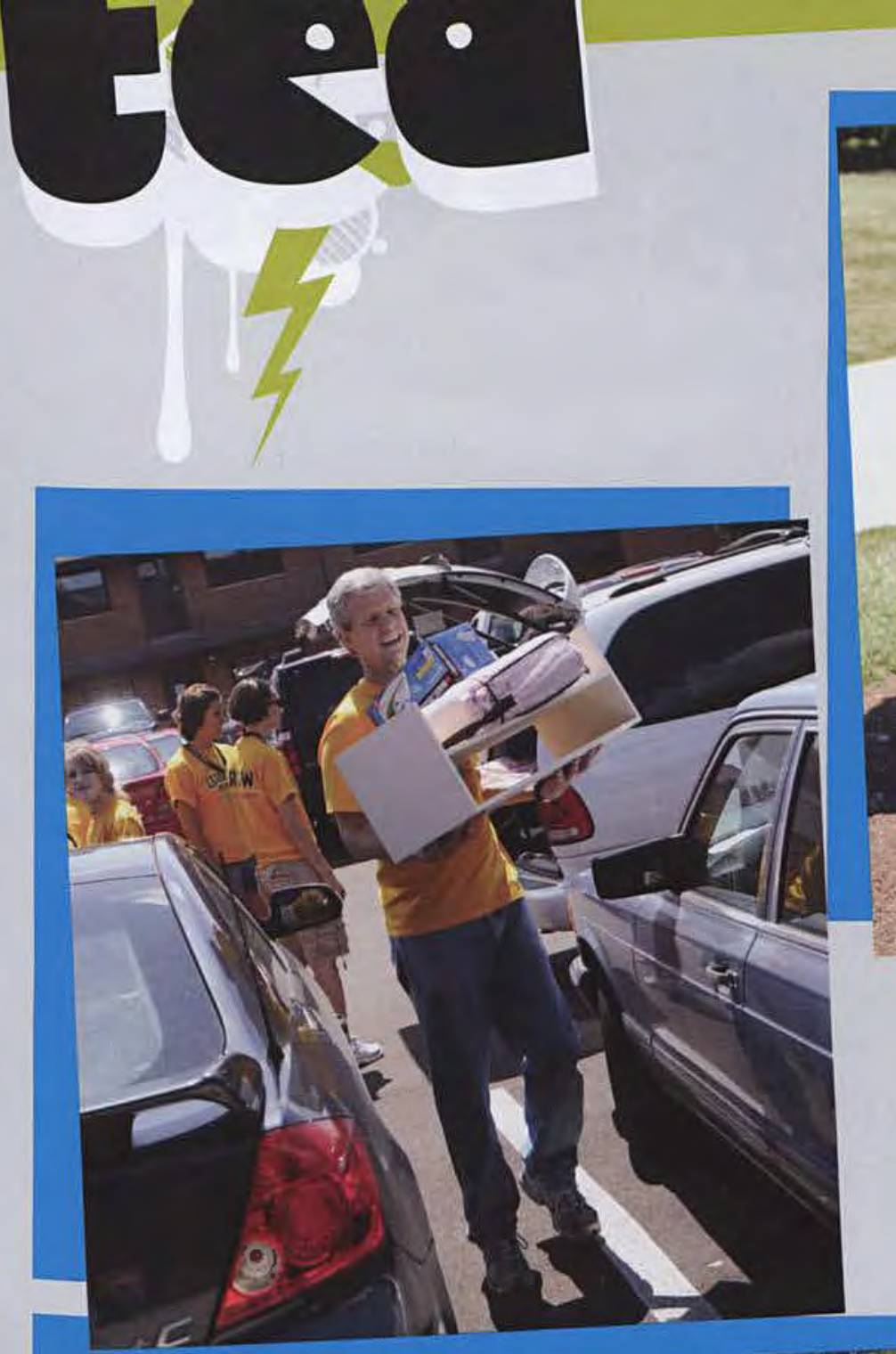

The Rock, a signature of life at the "Ville, welcomes students back to campus.

Always a familiar face during Getting Started, Dr. Brown lends a Always a familiar face during Geth many trips from car to dorm room hand as he

and back.

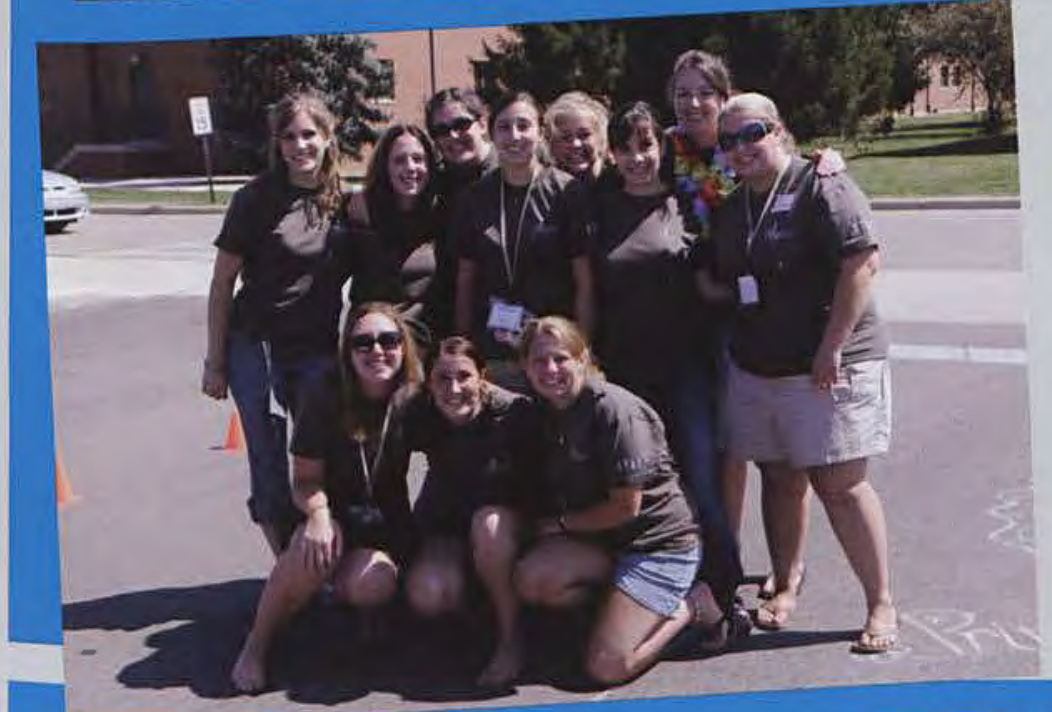

"We as Printy RAs spent two whole days cheering in the Printy girls! What God had in store for us in the coming year!" says Bri Mottashed.

anticipates a lot of time spent at his desk Obviously someone anticipates a lot com chies in this com 


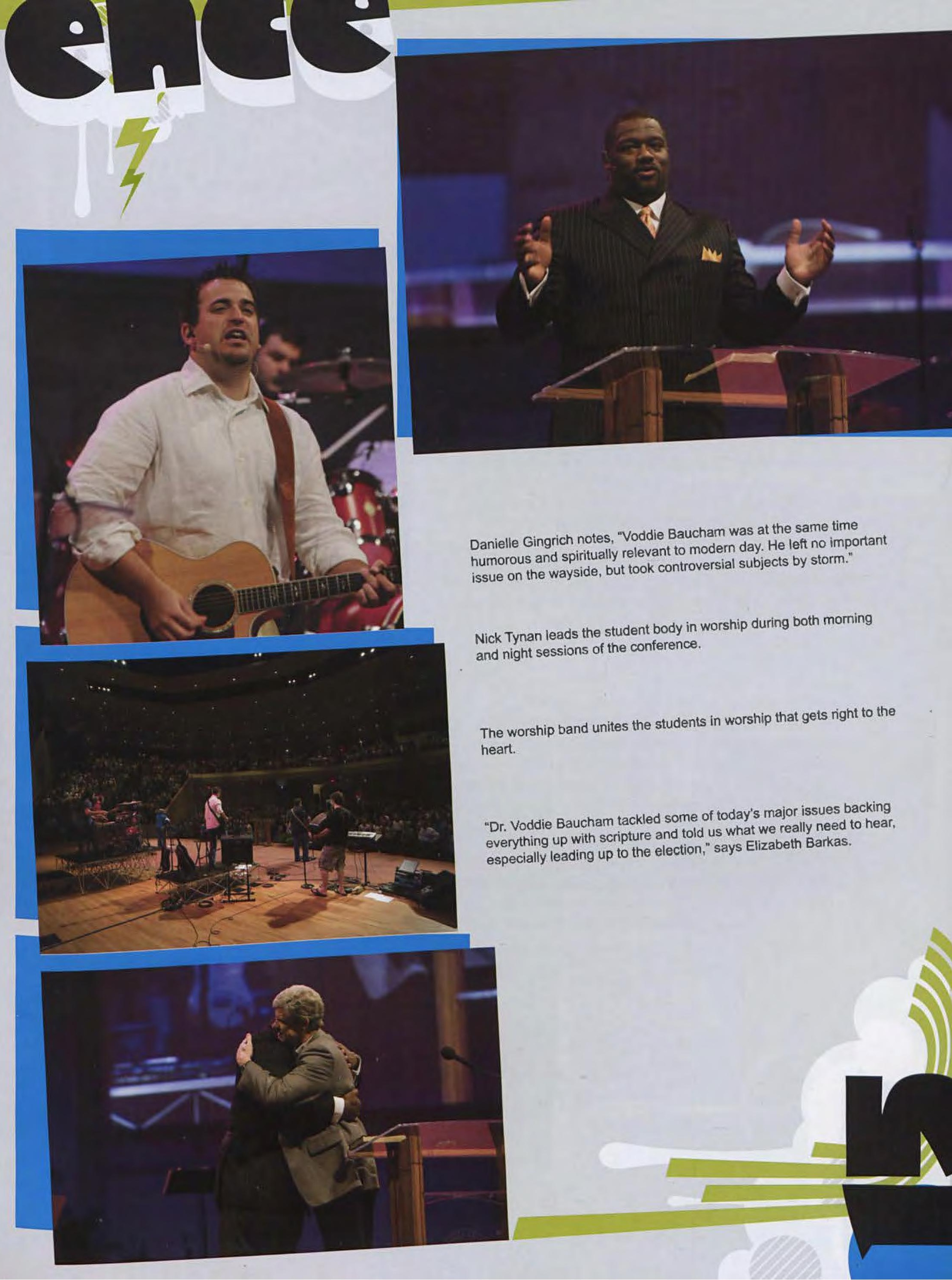




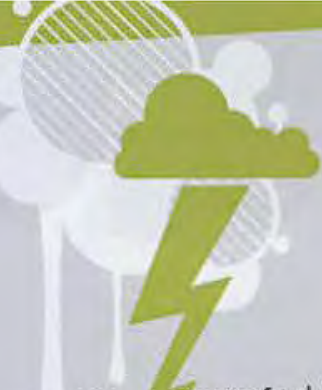

Many Cedarville students feel like kids again as they excitedly infamous blue and yellow CU balloons.
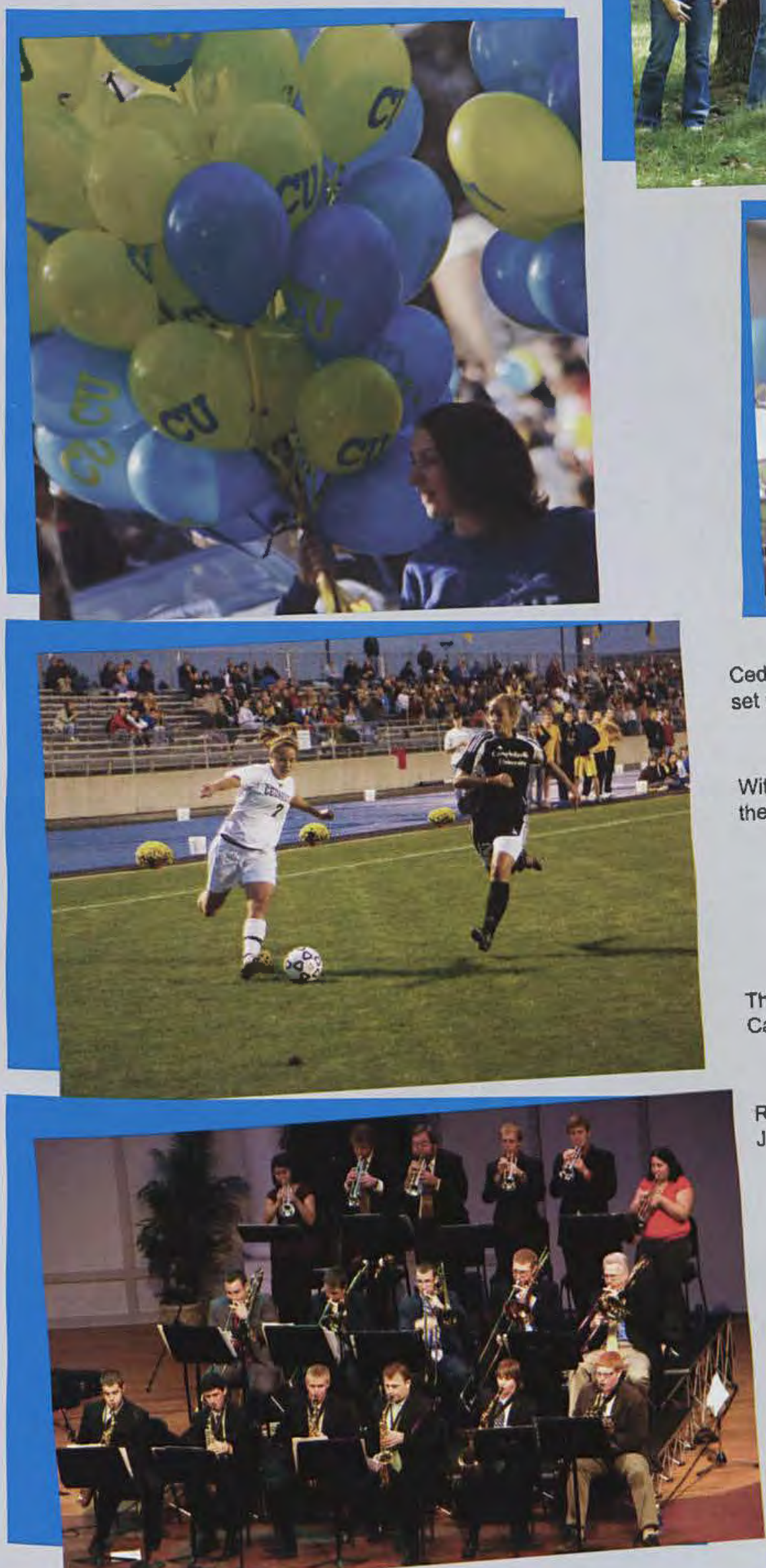
(I)

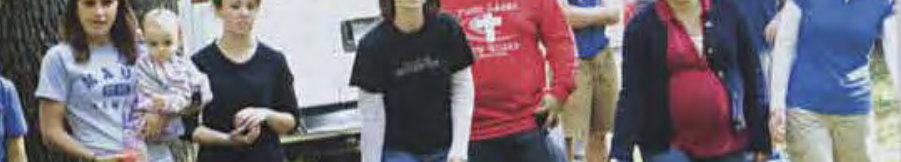

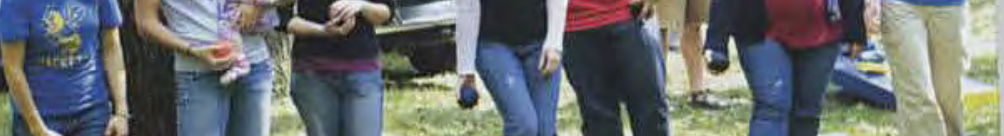

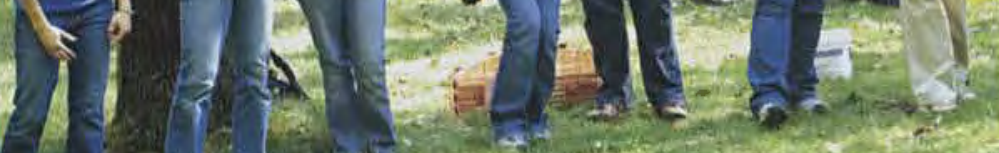

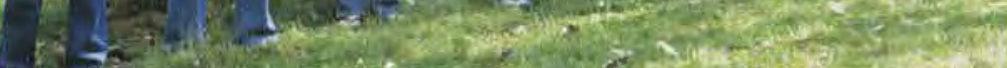

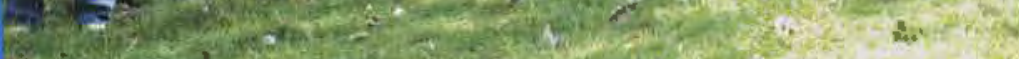

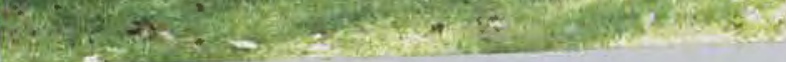

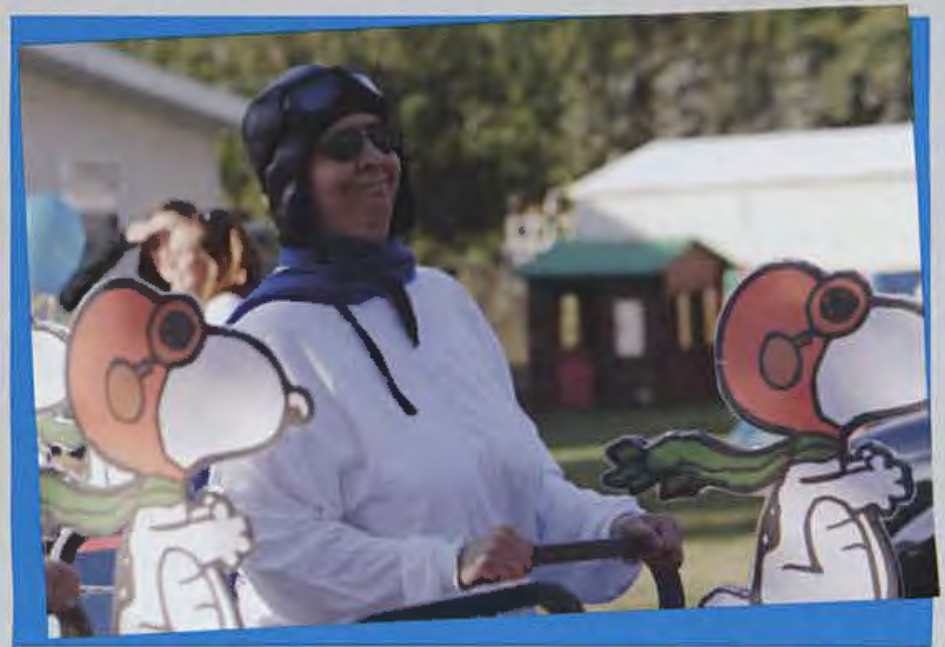

and alumi participate in one of the many games set up for Homecoming.

theme, the library staff turn the flight.

The always persistent Kelly Wise goes in for a goal against Campbellsville University. Ready to showcase their awesome for the occasion. Band holds a special performanct 


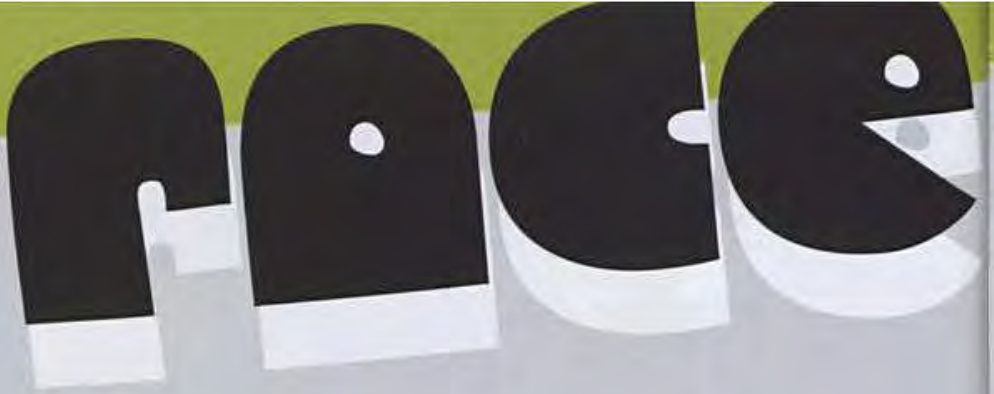

The $15^{\text {th }}$ annual Cardboard Canoe Race took place this year over Homecoming weekend. Hundreds of people crowded around Cedar Lake to watch the freshman engineers paddle themselves across the lake with only forty feet of cardboard and fifty-five yards of tape separating them from the water. Even some of the engineers were surprised that they could get across. Dan Lewis exclaimed, "I couldn't believe that so many people floated on cardboard!" One hundred and two freshmen participated in this year's event, one of the largest turnouts in Cedarville history.

The boat builders worked in teams of three or four and had two weeks to complete their boats. Some teams worked through the night during the final days of preparation, and their hard work paid off. Fred Anderson declared, "It was the adrenaline rush of a lifetime!"

The crowd looked on as twenty-six teams raced their way to the finish line. "I liked that some of the boats sank; it was more fun than watching them all get across," noted Maggie Casillo. The race was judged based on speed, but there was also a creative design competition sponsored by Caterpillar. Five academic department boats also raced against each other. The library once again beat out the other teams to reclaim the coveted department trophy.

After the races were over, the surviving boats and their passengers headed back into the water to battle each other in the demolition derby. One by one, teams plunged into the lake, laughing as they went down. Even though all the participants ended up soaking in the algae-infested waters, they all had a good time.

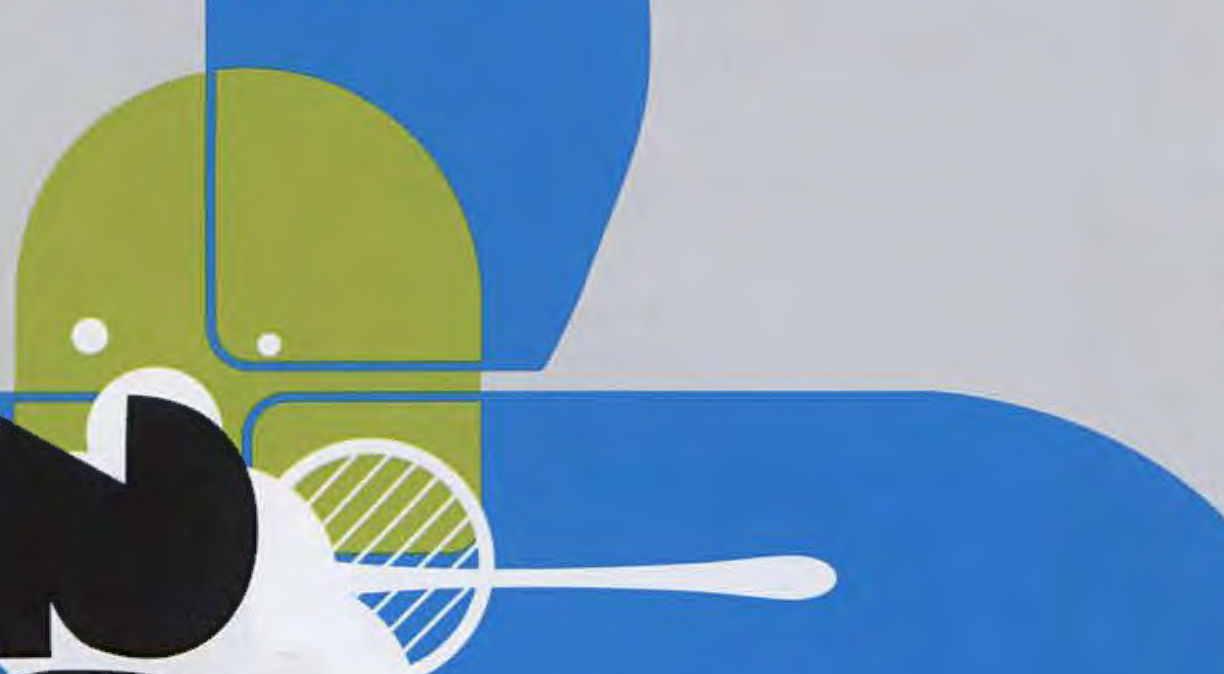



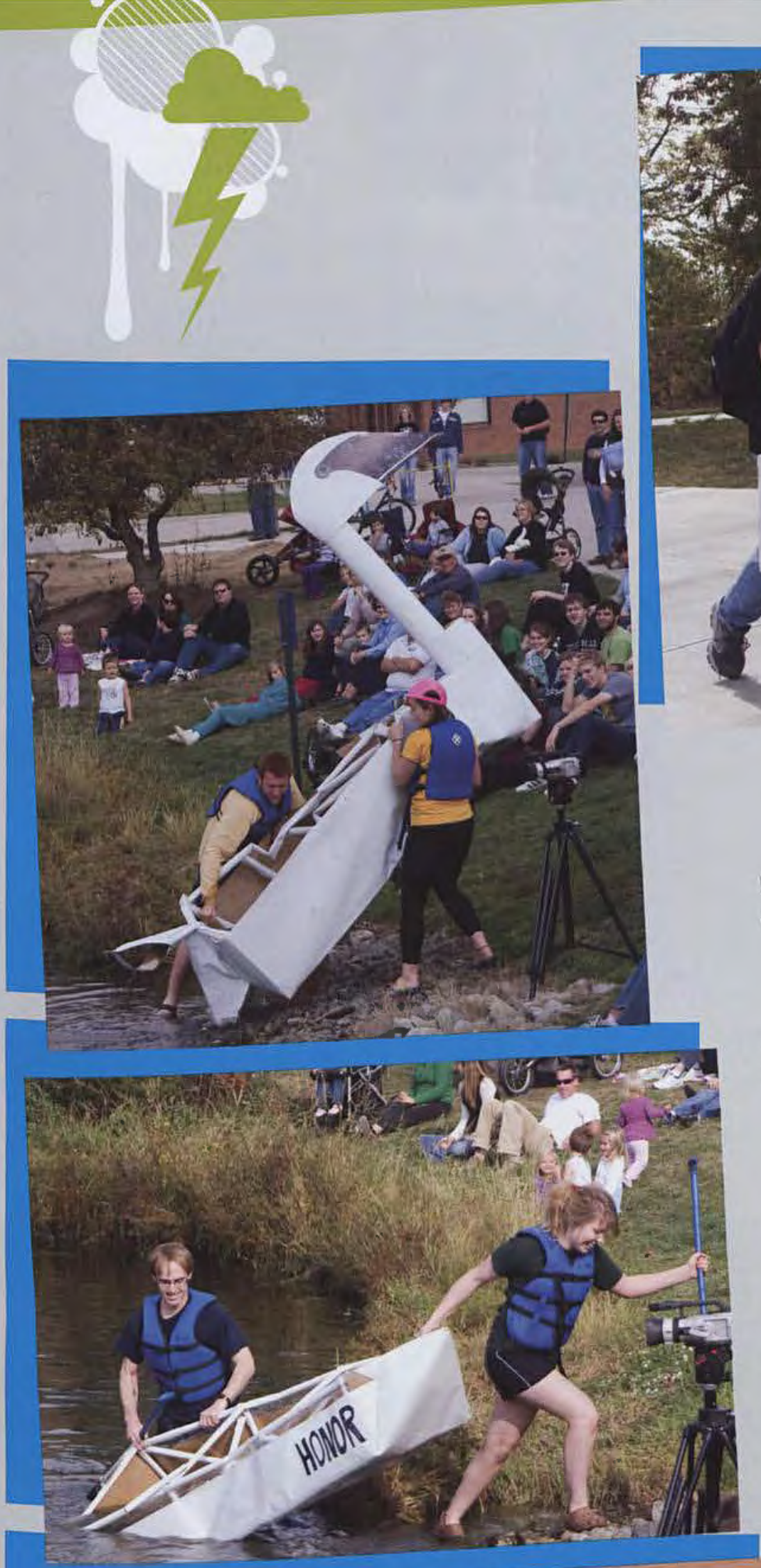

Freshman engineer Justin Engle carries his team's canoe to the lake, ready to watch it sink or swim.

Jacob Eldridge and Molly Fillion successfully carry what remains of their "CedarSwan."

Honors canoe all the way, solidifying that they're pretty much good at everything.

Bible depariment proves they too can float, as Andrew Gordon The Bible deparson paddle toward the finish line.

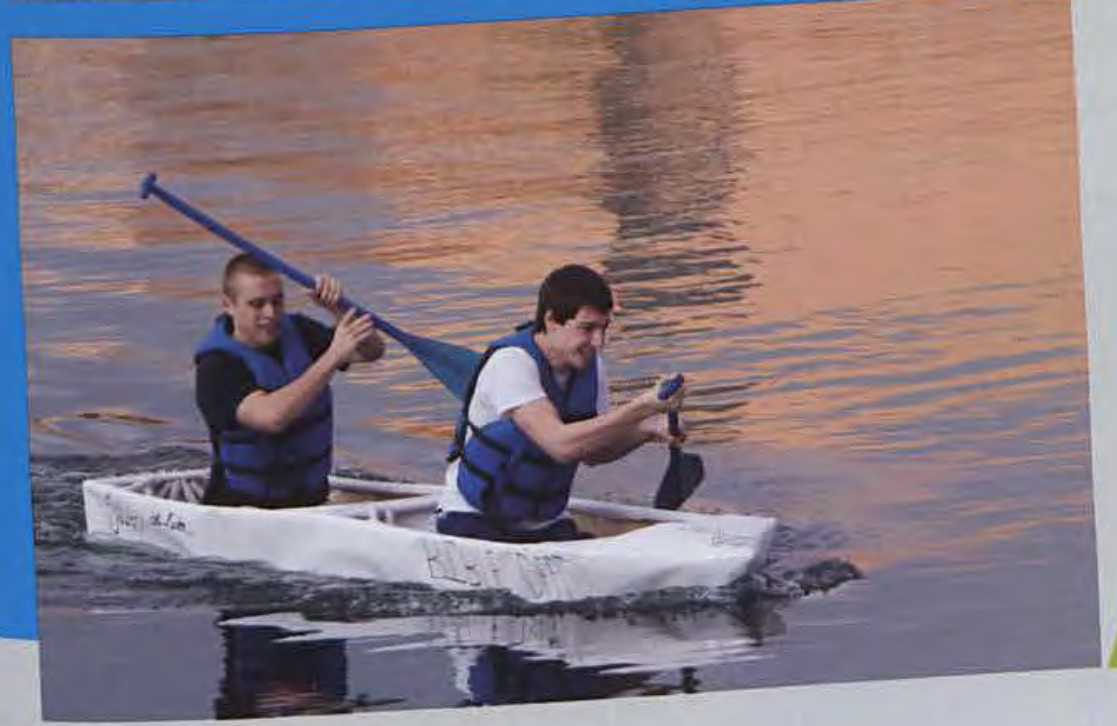



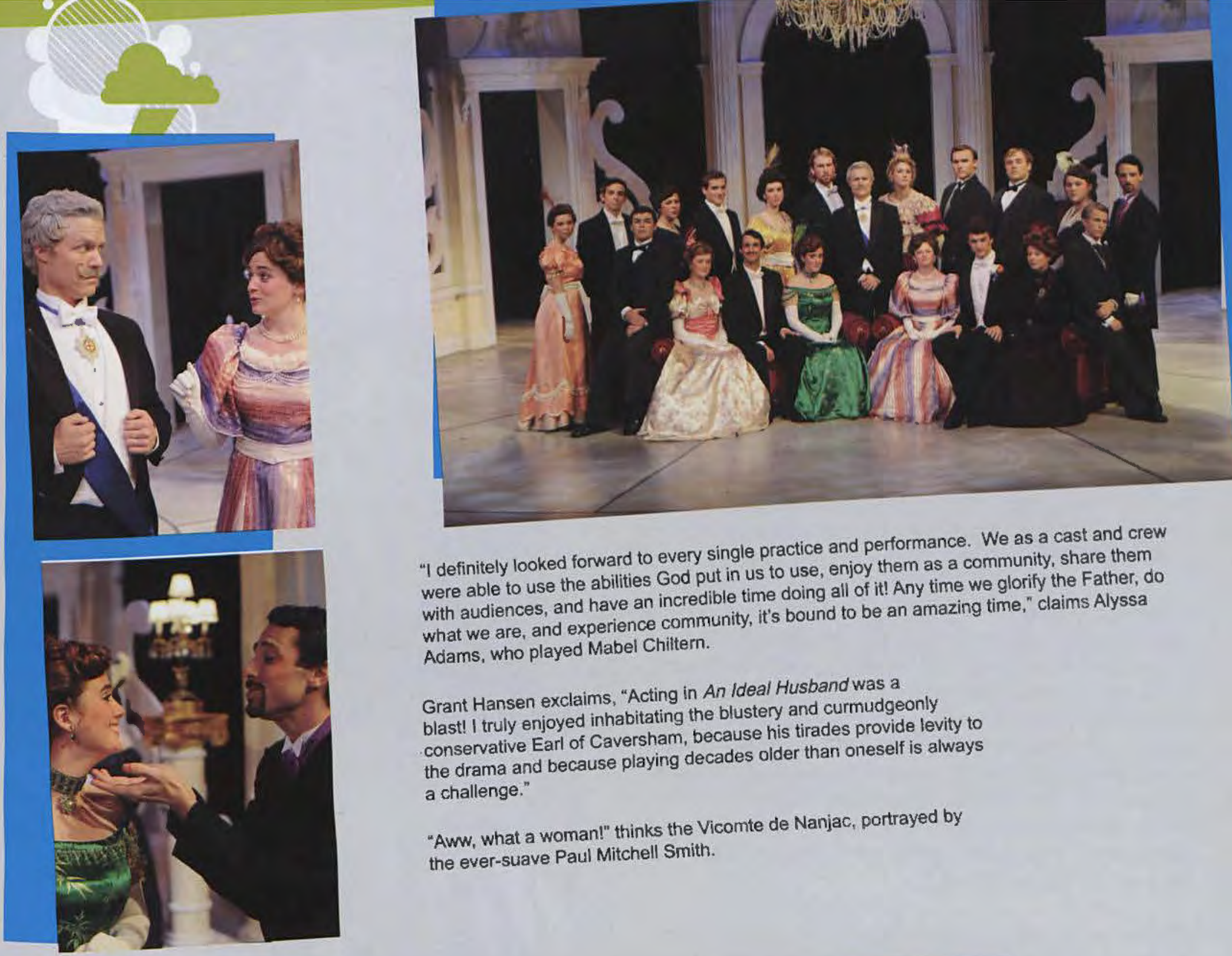

"I definitely looked forward to every single practice and perform as a community, share them were able to use the abilities God put in us to use, an it! Any time we glorify the Father, do with audiences, and have an incredible time doing all of it Any time we time," claims Alyssa

what we are, and experience comm
Adams, who played Mabel Chiltern.

Grant Hansen exclaims, "Acting in An Ideal Husband was a

ing blustery and curmudgeonly

blast! I truly enjoyed inhabitating the bluste his tirades provide levity to conservative Earl of Caversham, because his than oneself is always the drama and challenge.

"Aww, what a woman!" thinks the Vicomte de Nanjac, portrayed by the ever-suave Paul Mitchell Smith.

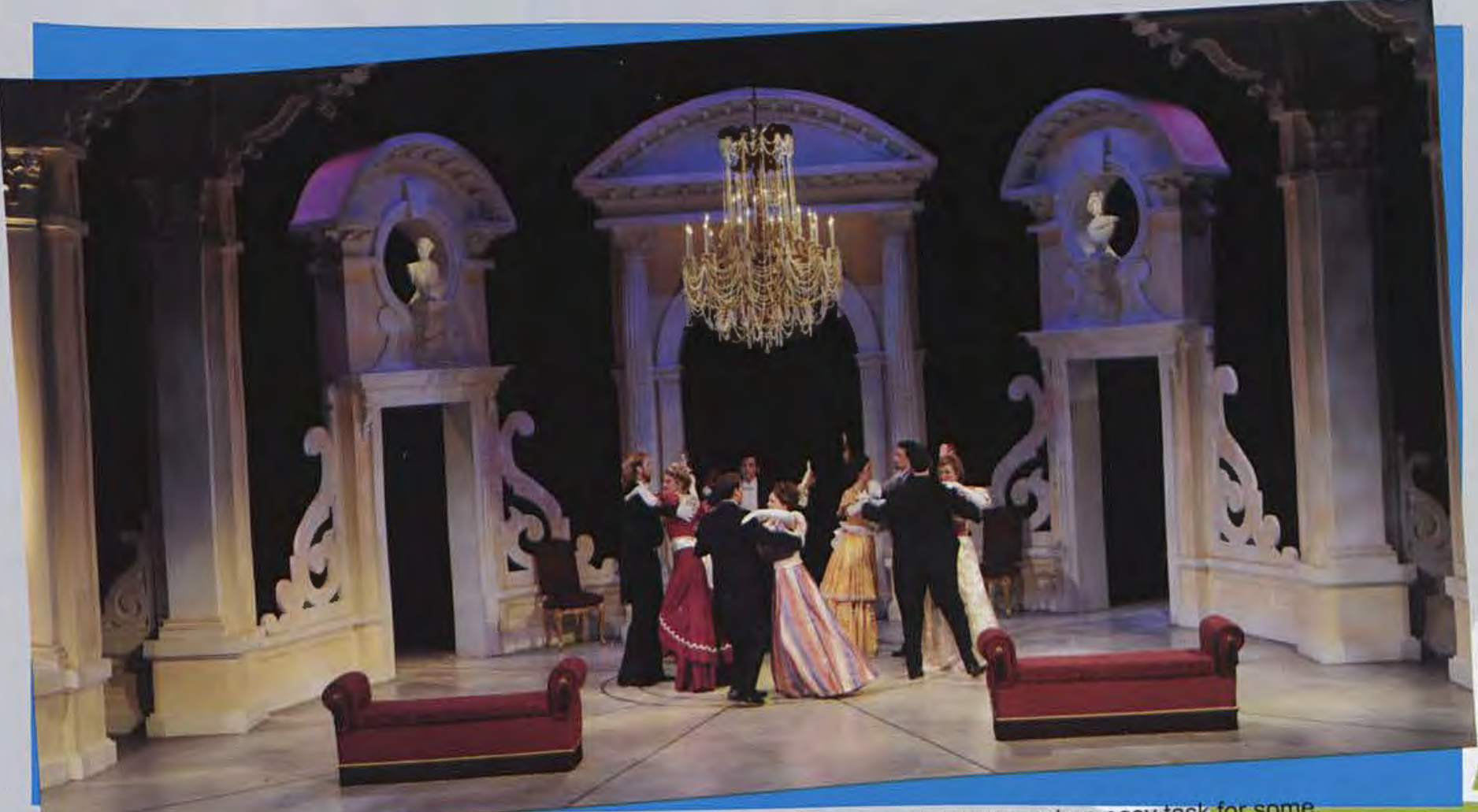

For the opening scene, many of the cast members had to learn how to ballroom dance,

"It took me forever for learn it, 'cause l'm such a klutz! Think 



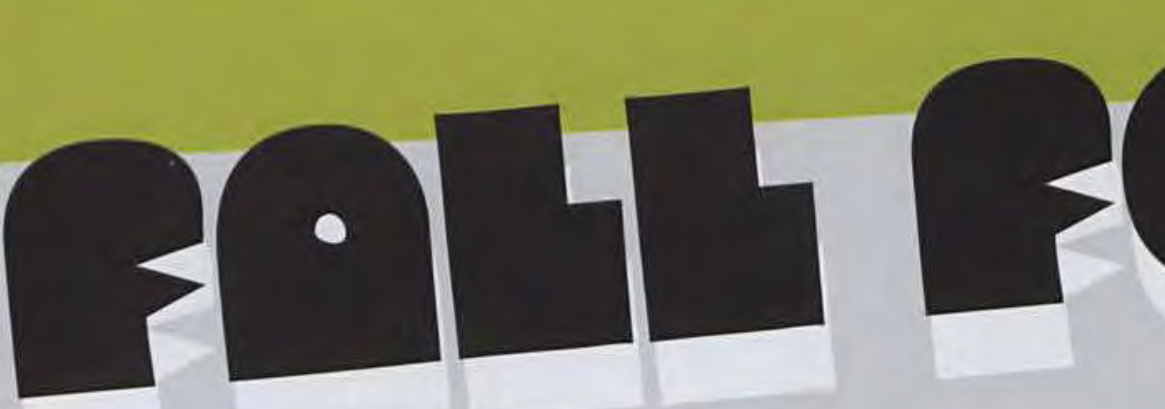

As leaves coated themselves in autumn colors, students swarmed back to campus to reap the responsibilities of their fall schedules. To keep summer in their spirits between classes and homework, SCAB and SGA provided students with festive activities celebrating the season.

SGA started the year off with excitement by introducing Mission Impossible: Cedarville, a campus-wide game attracting hundreds of students. Participants practiced stealth by creeping across campus without alerting hidden faculty and staff to earn points for their team.

SCAB added socialization and entertainment to the semester by hosting ALT weekends. "ALT weekends were planned with the goal of encouraging students to stay on campus and donate their money to a ministry chosen by SGA instead of spending their money off-campus," explained Rachel York. The monthly ALT weekends included open dorms, Wii games, and movie showings.

SGA continued the fun by presenting the annual Organizational Fair. For two and a half hours in the Field House on September 13, dozens of student organizations earned the interest of students with creative booths supplying games, food, and information. Attendees loved the many benefits of the Org Fair, such as inflatables and free stuff. As Abby Swaney put it, "The Org. Fair is a lot of fun! Everyone gets crazy and generous when it comes down to getting new members. It is great because there are fun things to do, lots of food, and opportunities to get involved in organizations that make a difference locally and around the world."

SGA encouraged a love of athletics with Moonlight Madness. The basketball extravaganza occurred on October 3 and used scrimmages and a dunk contest to ignite student support for the men's and women's teams. Morgan Callahan exclaimed, "I enjoyed the pre-game excitement that got us pumped for the season!" Moonlight Madness joined the fall festivities that made the semester memorable.

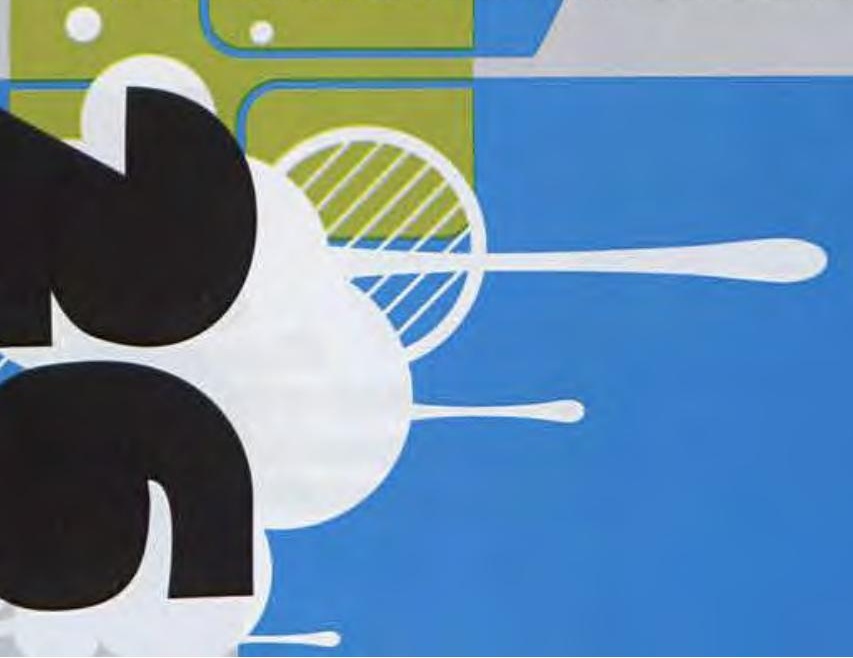

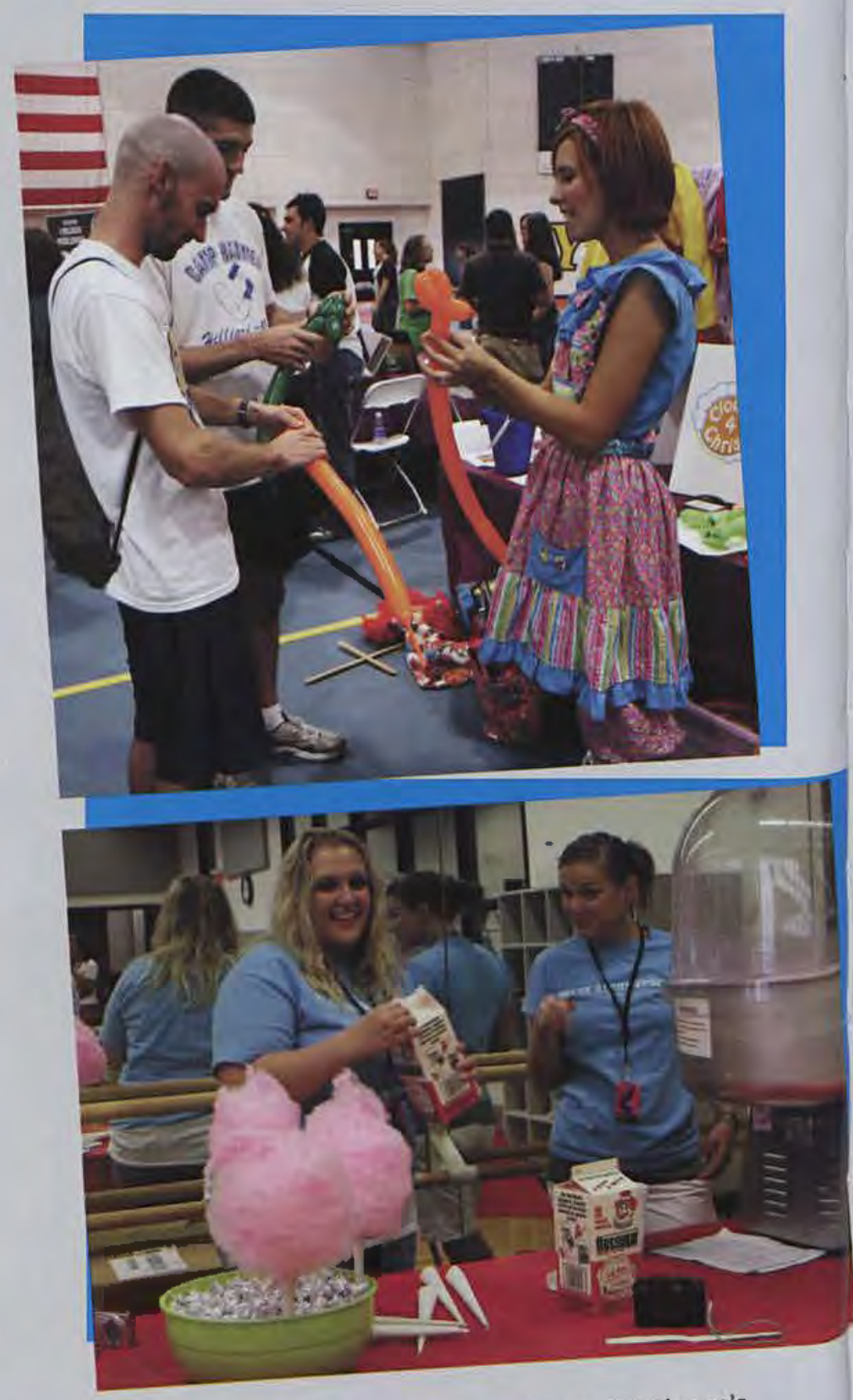

forist member Regina Hager does laps around men's Clowns for Christ member Seibert and Seth Campbell with her balloon track me
animals.

Cassandra Dobutovich exclaims, "Making cotton candy is something to do, and it is hilarious; it got everywhere! It's a perfect way to start the year off with friends." 

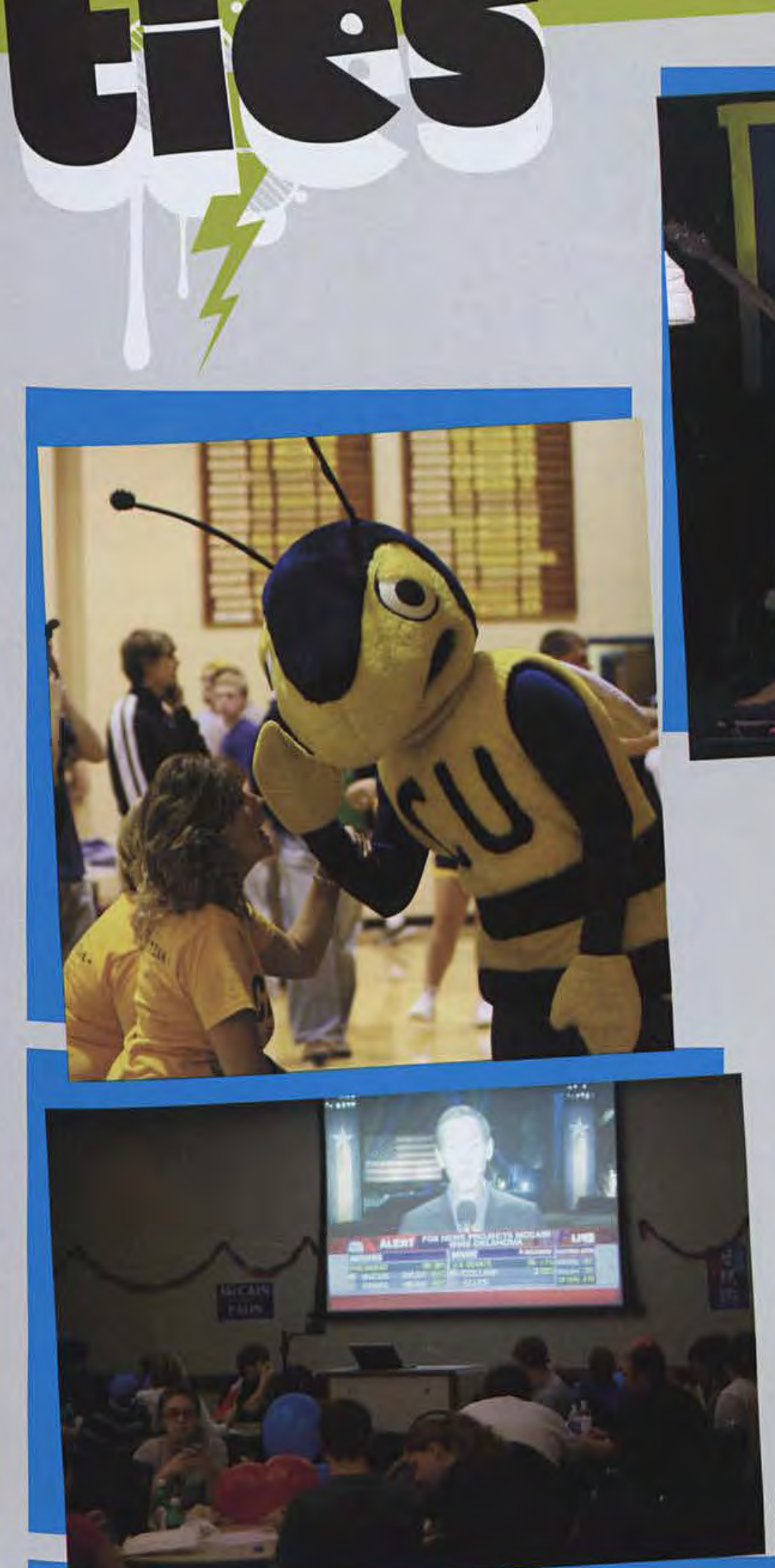

e freshmen are only willing At the New Student Talent Show, some their identities. Too bad we to show off their talent if they Caylor Dybwad, Matt West, Andrew recognize them as Jon Adler, Tawlor 17/19!

Comers, and Doug Brown of Lawor $17 / 19$

At Moonlight Madness, Dawn Scott tells the Bee, "I enjoyed watching both guys' and girls' basketball games, but I wish we have a bette turn out of fans this year." And the Bee wonders, "Should she tell that to the professors?"

the night of the presidential Students swarm the event roch state's selection is election, revealed.

Brave Cedarville students give blood and show no fear. Needles are nothing compared to papers. 


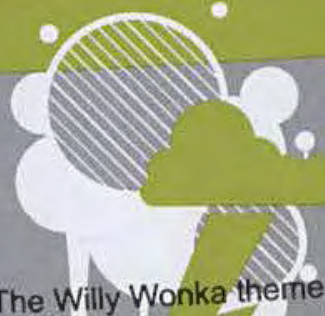

The Willy Wonka theme in Printy brought home the pnize then's dorms, thanks to a couple girls who seem to much self-tanning cream.
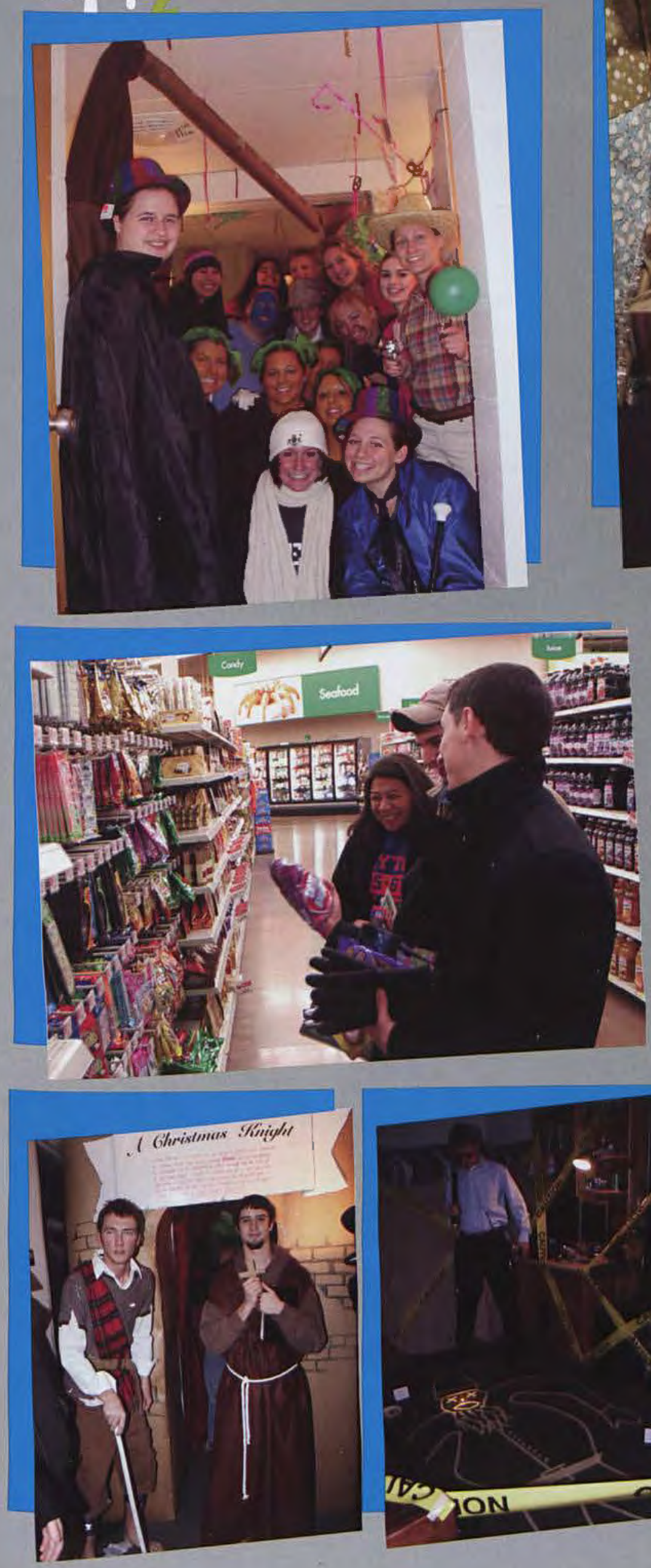

gurs left "home alone" in Lawlor. Lawlor + mischevious boys = ensuing chaos.

Cooper, Nate Linder, and Kena Mena take time to give back as they load up on goodies for their shoeboxes for needy kids. John explained, "It was a privilege to be able to provide children world with, possibly, the only gifts they've ever recei

Matt Fox and Austin Engel welcome their guests to "A Christmas Knight" in McChesney. 


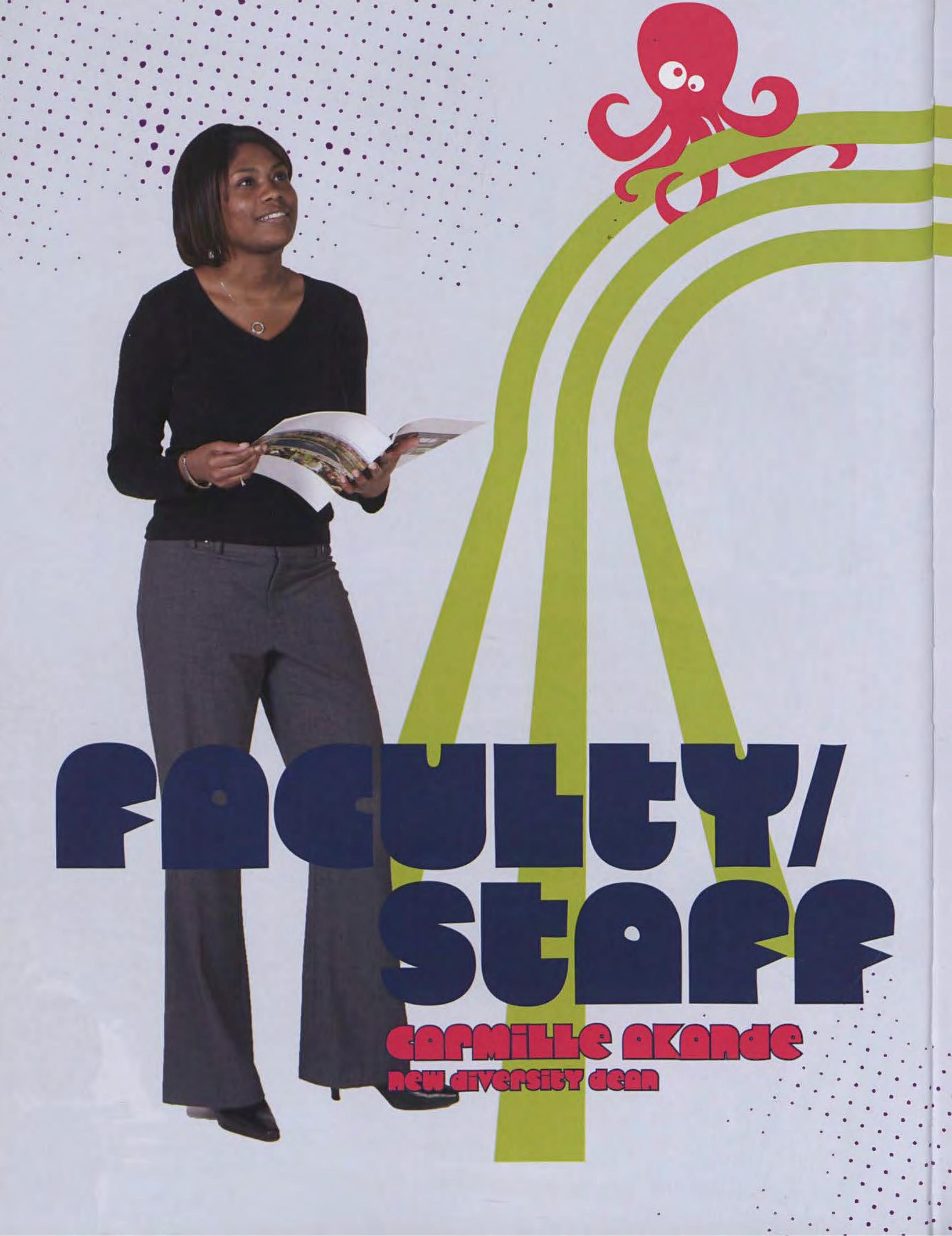


I saw the Lorraine Motel. It stood before me, stripped of the blood that stained the floor of the balcony so many years ago. Not even the brief, prickling chill of the cold breeze on my face could distract me as I gazed at the aqua-colored door-it was the one to the left, on the second story. Istood at leasta hundred feet away, but in my mind my hand felt the door's brittle exterior. I pulled my outstretched fingers from the frosty air, embarrassed, seeing before me the pictures I had gazed at during my childhood and wondering if this was simply a dream. It wasn't. reasoned, for if a fence did not stand between the motel and me, I could waik up those bitie, metai stairs that invite anyone and everyone to the second story. I could walk past those square windows on either side of each door, as big as each door, those windows that are shut to the outside world with white blinds - I could stand where Dr. King stood, vulnerable, unprotected, framed by the thin metal bars of the balcony walkway, just before he was shot and killed. I could stand in his place, and in a way I did-I do. He knew the risk he took so that I could have equal access to the workforce, to education, and to any opportunity available in America. He took the risk so that America could become a place where races could come together and love one another the way God intended.

My concentration broke as the Cedarville students filtered their way to the entrance of the Lorraine Motel-turned-Civil-Rights-Museum, but before I went in, my eye caught sight of a Scripture carved into a stone. The scripture was from Genesis, and it referred to Joseph. If you kill the dreamer, let's see what happens to his dream. As my eyes examined this simple truth, I found I knew the answer: the dream lives. God fulfills the dreams He has-and we can be the instrument, the tool that $\mathrm{He}$ fulfills them through. Just like Joseph. Just like Dr. King.

God's dreams are what propel me onward, and my journey with Cedarville's Annual Civil Right's Bus Tour is just one facet of my passions. When we see students of all colors and backgrounds, crammed together on a bus for five days, we begin to appreciate each other's differences and history; we see the glory of God at work in ways that He longs to work. This need for diversity transcends color, race, and ethnicity - it grows to encompass the entire body of Christ, and working towards unity is a dream from the very heart of God.

I have always been passionate about justice. My passion led me to the University of Dayton's Law School and eventually to Montgomery County Prosecutor's Office, where I worked as a Criminal Prosecutor. It also led me to Cedarville - a warm, friendly campus where people do - and should-feel welcome. I still act as an advocate as the Associate Dean for Multi-Cultural \& Special Programs, working with students who are from other cultures, have disabilities, live off campus, or come from an international background. I am here to assist and address any concerns students may have, meeting their needs and attempting to make their experience the best one possible.

Cedarville is working to create diversity by welcoming these students into our campus family, and my desire is to see Cedarville look more like the body of Christ by being representative of all ethnicities and celebrating the uniqueness of it.

Hopefully students will continue to cultivate an interest and appreciation for Dr. King's passion and dream.

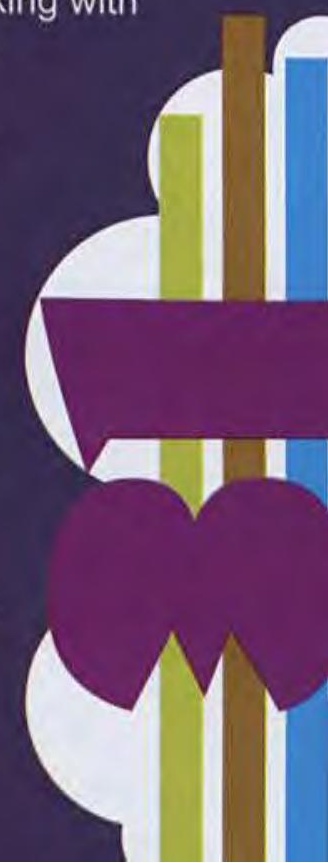


The 2008-2009 year at Cedarville has been a memorable one. Those of you who are seniors have appreciated the addition of the Center for Biblical and Theological Studies. You know how the Center has made a huge positive difference in your everyday life as a student. Those of you who are freshman have no idea what it was like without the new building. Needless to say, it is rapidly becoming the most recognizable building at Cedarville University.

-
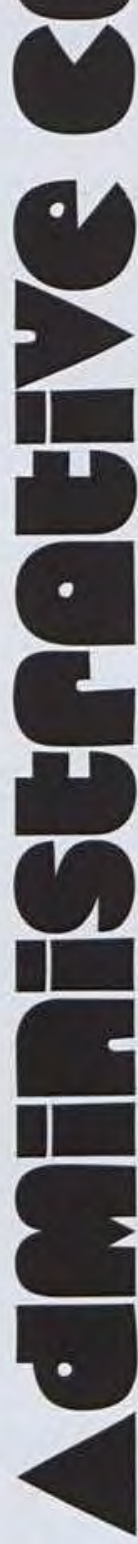

I am grateful that we at Cedarville never lose God's perspective of the importance of each individual. As I thumb through the yearbook, I look at each face and realize there is a story behind each photograph. Thanks so much for adding your life to the great history God is writing through Cedarville University. I hope that in the years to come you will look back on your time at Cedarville with fond memories of growth, challenges, friendships, and changes.

Our theme this year, "For the Long Journey: Equipped for Leadership and Service," has reflected the true heart of servant leadership that Christ demonstrated for us. I hope that as the years go by you will see God's hand in providing opportunities for you to lead and serve in a way that honors Him.

Don't forget, you are always a part of the Cedarville family!

God Bless Always,
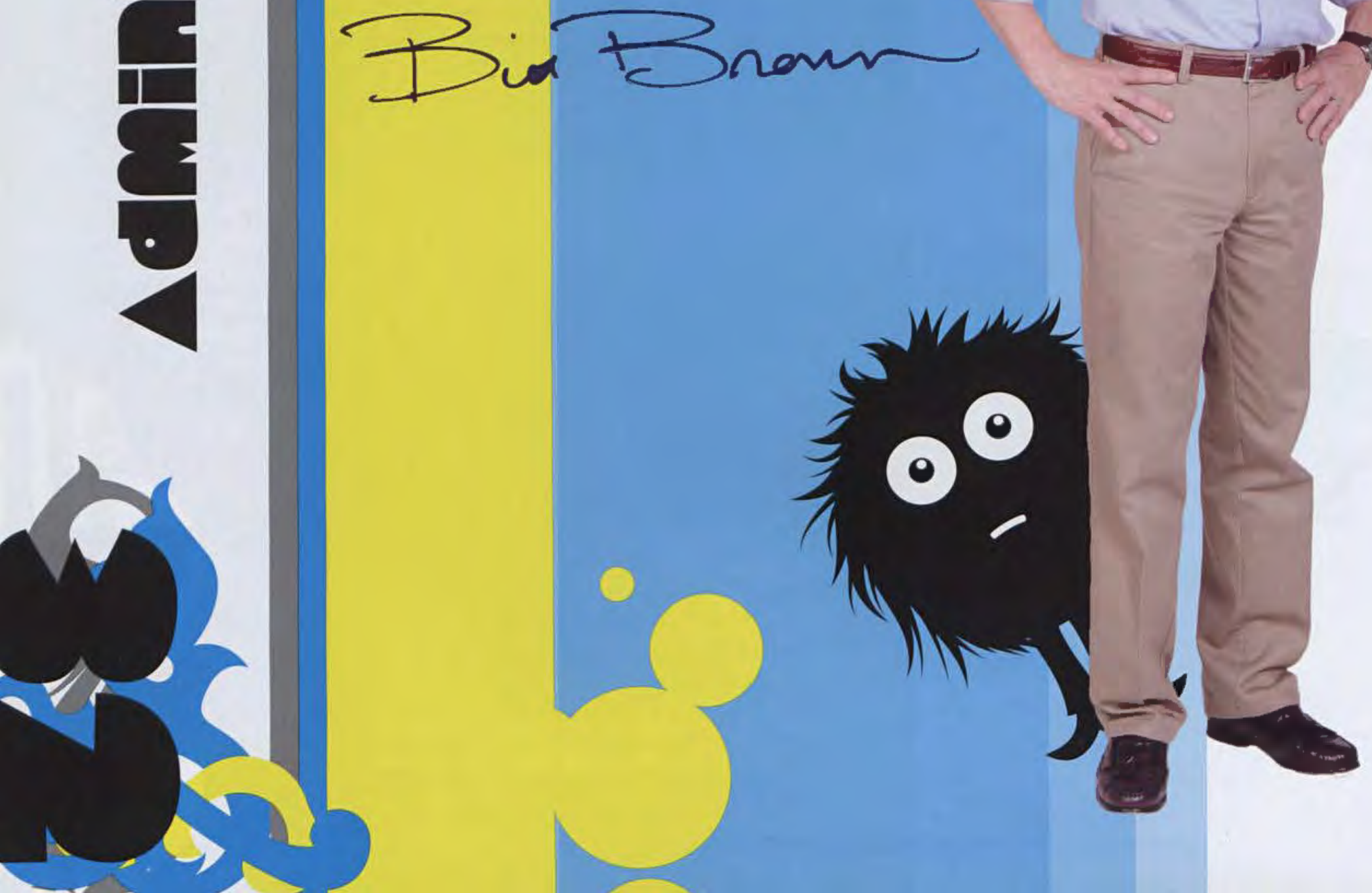


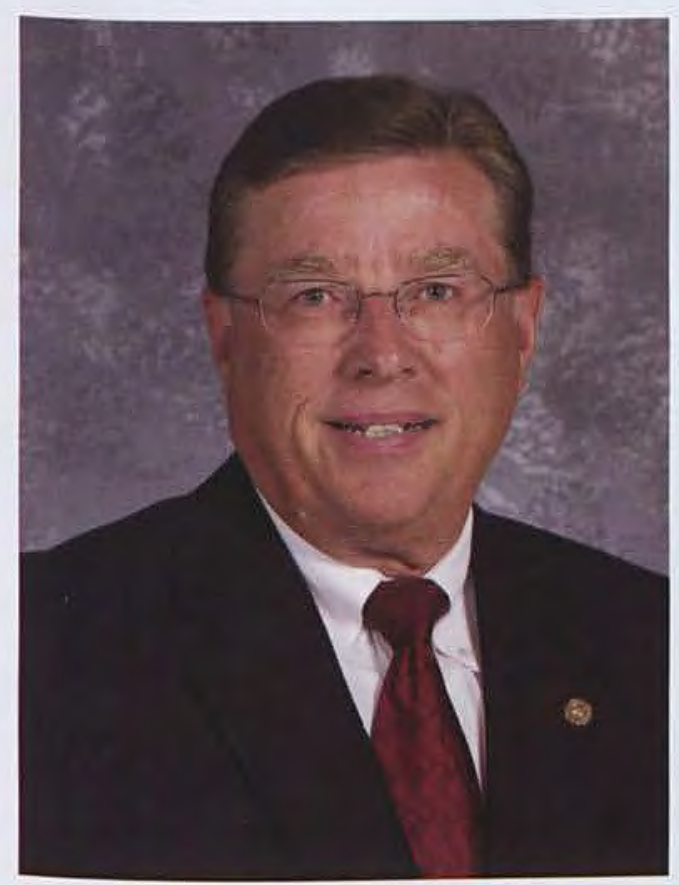

John Anglea

Vice President for Business

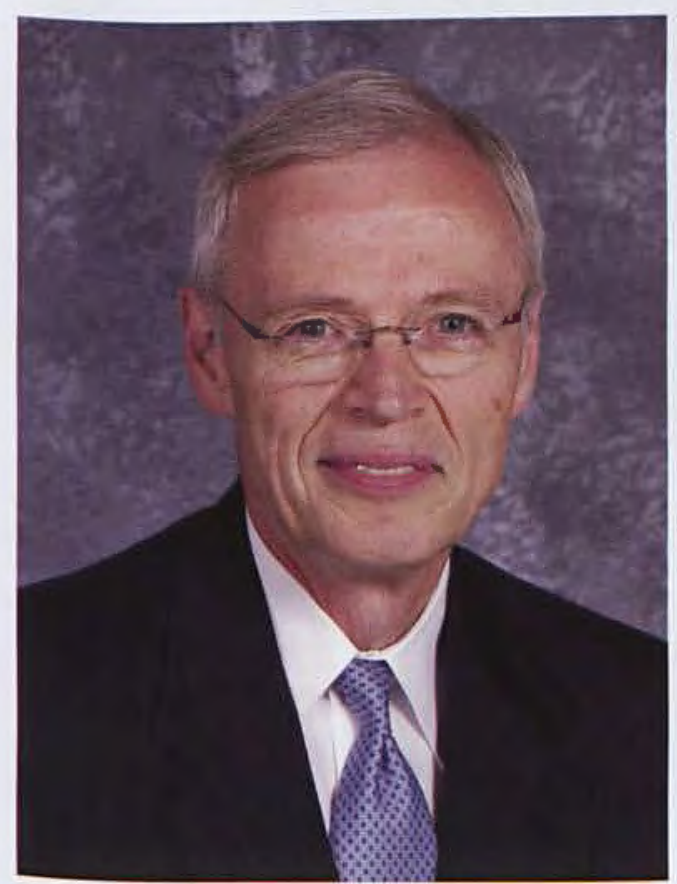

Robert Rohm

Vice President for Christian Ministries

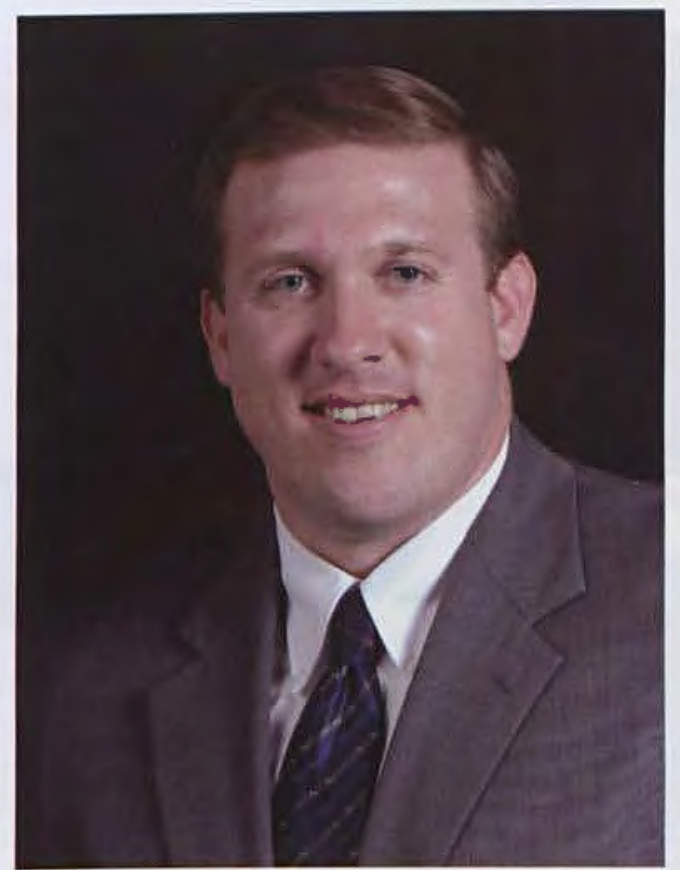

\section{Bill Bingham}

Vice President for Advancement

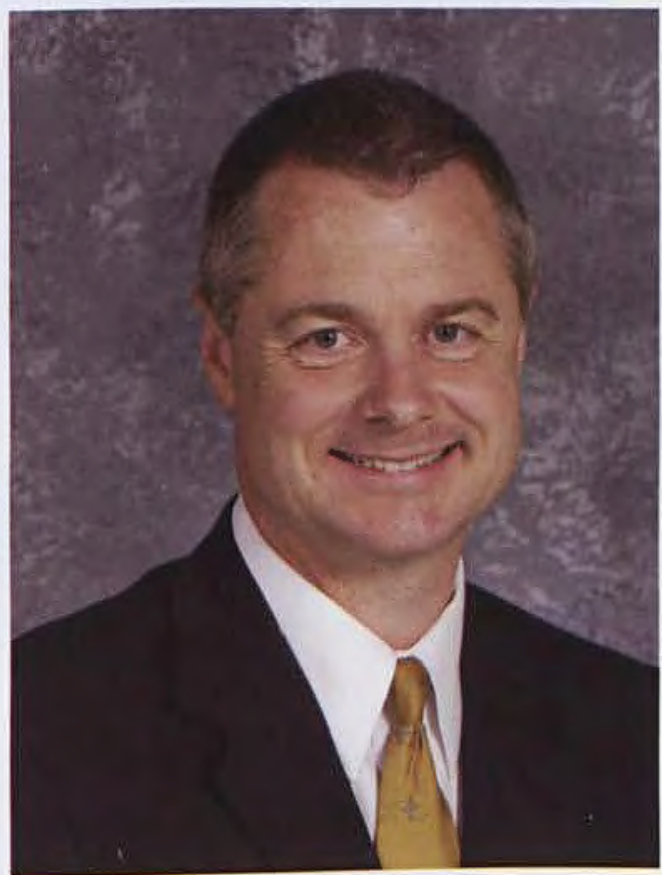

Carl Ruby

Vice President for Student Life

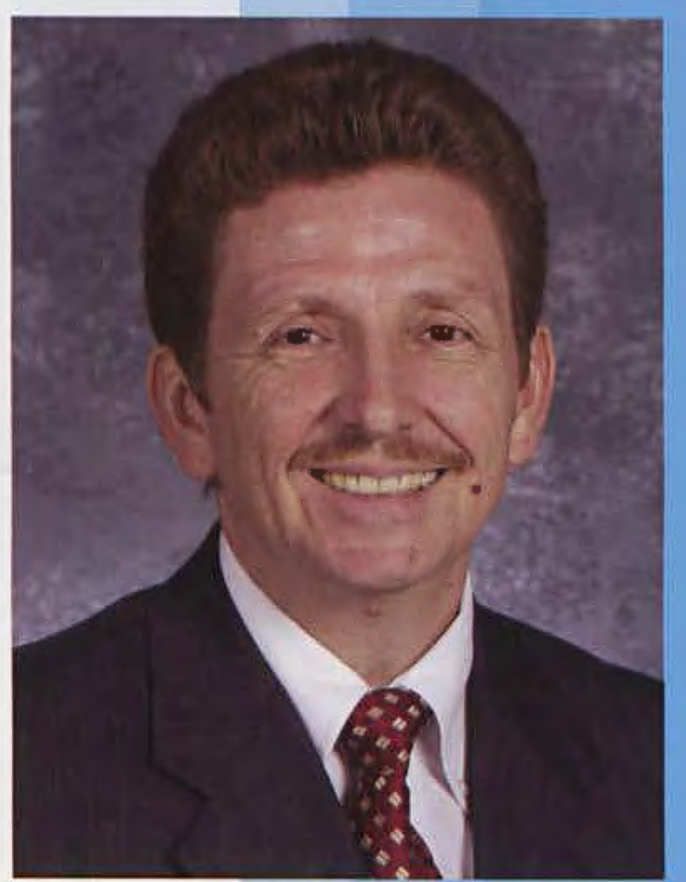

John Gredy

Provost, Vice President of Enrollment Management \& Marketing 


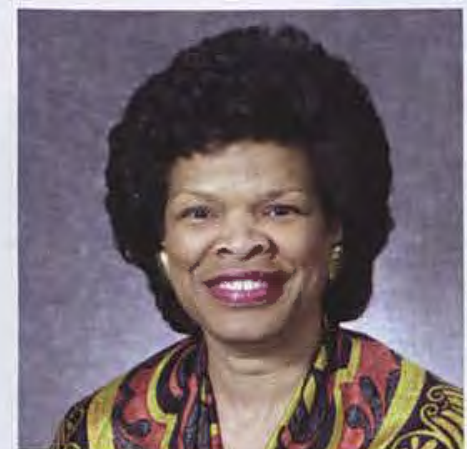

Dr. Deforia Lane

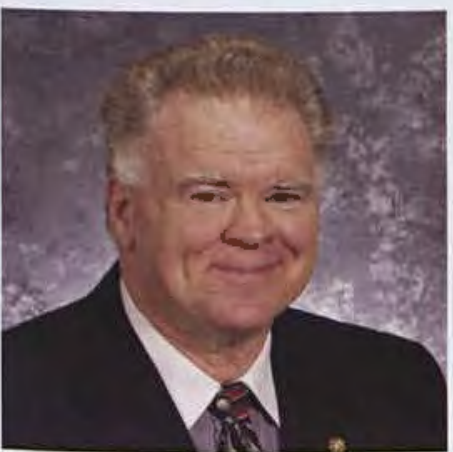

Dr. Paige Patterson

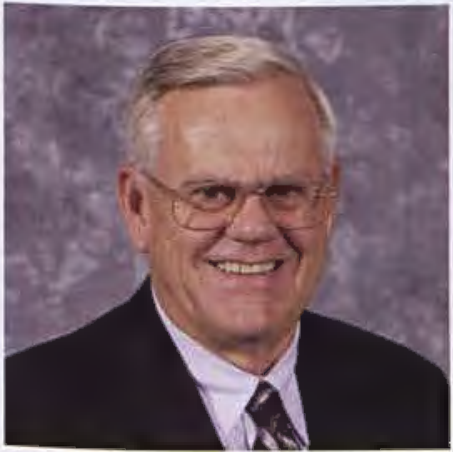

Mr. Al Stevens

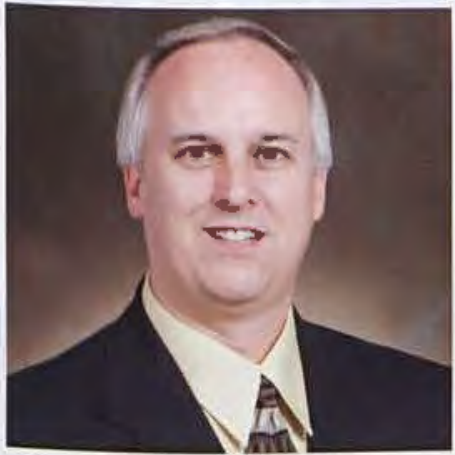

Rev. Jeff Willetts

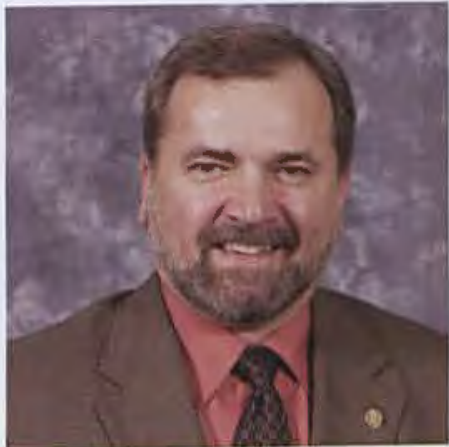

Dr. Michael Loftis

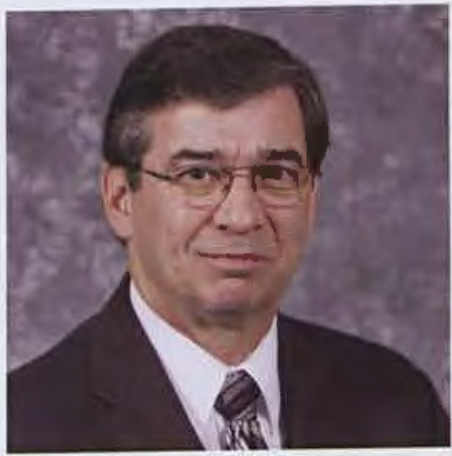

Dr. Bill Rudd

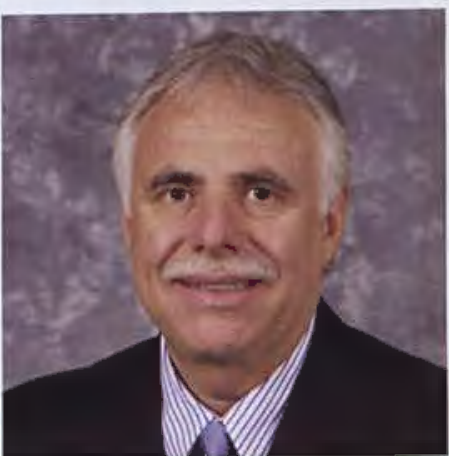

Hon. Robert Thomas

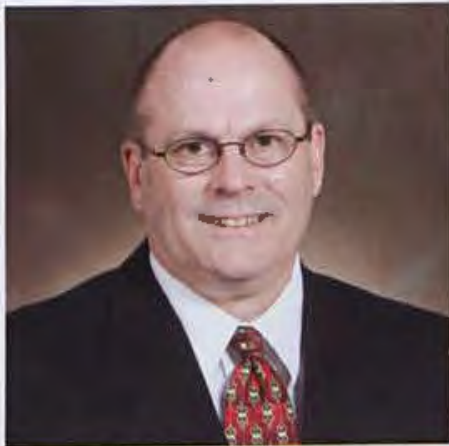

Mr. Bill Williams

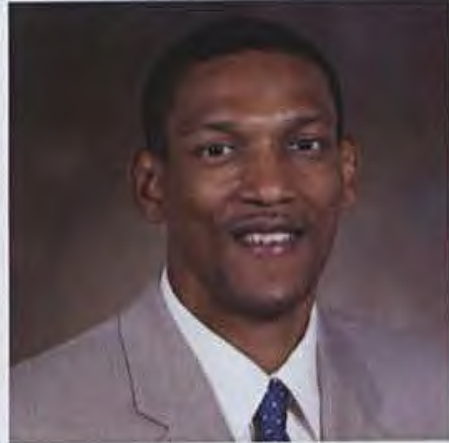

Dr. Dominic McKinley

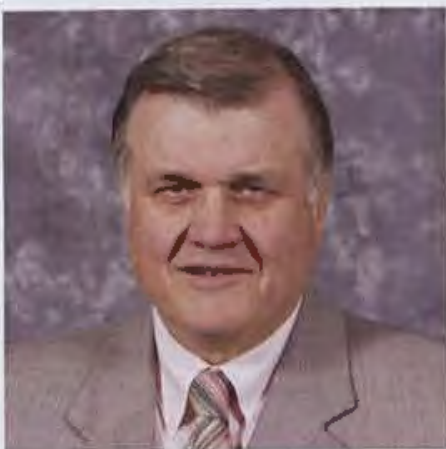

Mr. Lorne Scharnberg

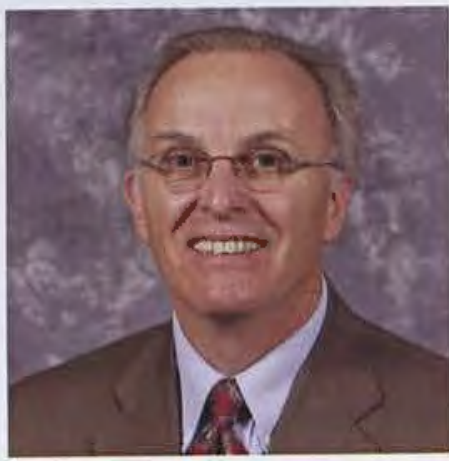

Dr. David Warren

\section{EAncertlï:}

Dr. Gene Apple

Mr. Fran Bresson

Mr. Gil Brueckner

Mr. Jim Carraher

Dr. Jack Cline

Mr. John Draxler

Rev. Joseph Godwin

Mr. Roy Guenin

Dr. Eddie Hawkins

Mr. Gene Miller

Rev. Wyn Olson

Rev. Lynn Rogers

Mr. Bill Smith

Dr. Robert Sumner

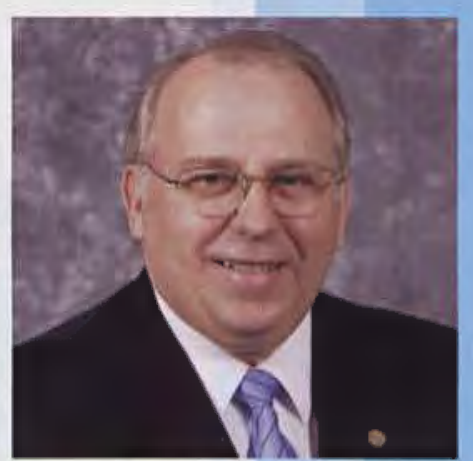

Rev. Randy Patten

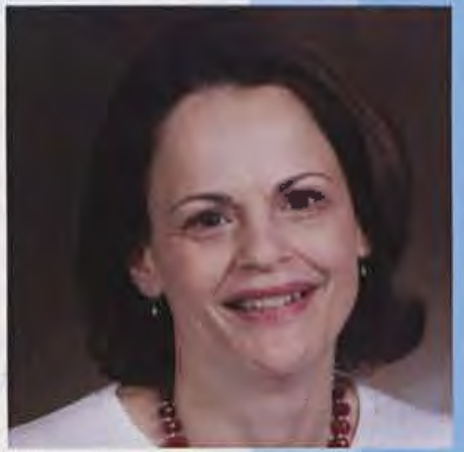

Ms. Debby Stephens

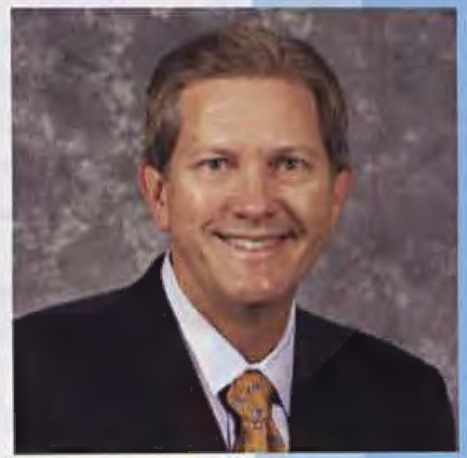

Dr. Hayes Wicker

\section{mot PHCtured:}

Dr. Randy Ross

Dr. Paul Vernier

Mr. Randy Wilcox 
Moving into the Center for Biblical and Theological Studies culminated many years of dreaming and planning. Several aspects of this facility highlight the vision of the school and Cedarville University.

"I am the Light of the World"-This theme is echoed throughout the structure. The open atrium and light wells bring natural light into every room. The central location on campus and Biblical Heritage display area highlight our continued commitment to the ancient text of Scripture coupled with a desire to bring it to bear on contemporary culture. Classroom design features, the commissioned sculpture and mosaic, abstract art, and the stained glass effect in the atrium all help to foster creativity and encourage active cultivation of the mind. Scripture verses etched in multiple languages on glass classroom walls emphasize the global nature of our task. This is a place to study the Word so that students are prepared to go out from Cedarville on a mission to bring the light of the Word to the contemporary world.

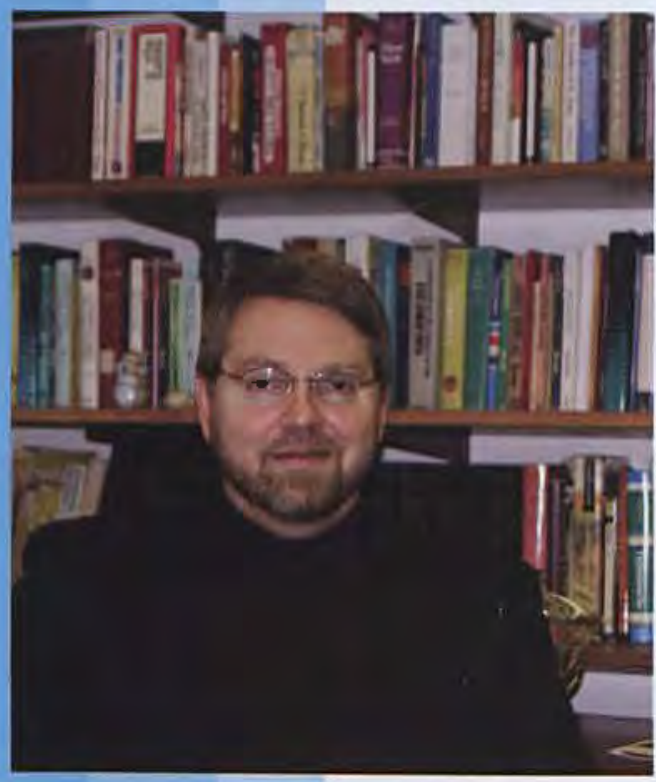

Dr. Thomas Hutchinson Dean

As the spiritual life is never experienced in theory or in a vacuum, we have attempted to create a space to give students real-world practice at the skills of communicating and ministering the truth of God's Word to others effectively. To learn the value of community, we have designed open, inviting spaces to study and class and collaboration built for engagement. We have already seen increased student interaction with each other and with faculty. We've been able to focus more on the value of community in the body of Christ and develop skills of teamwork.

We are grateful for those who have contributed to allow us to enhance our ministry and highlight our vision in such a tangible way on our campus.

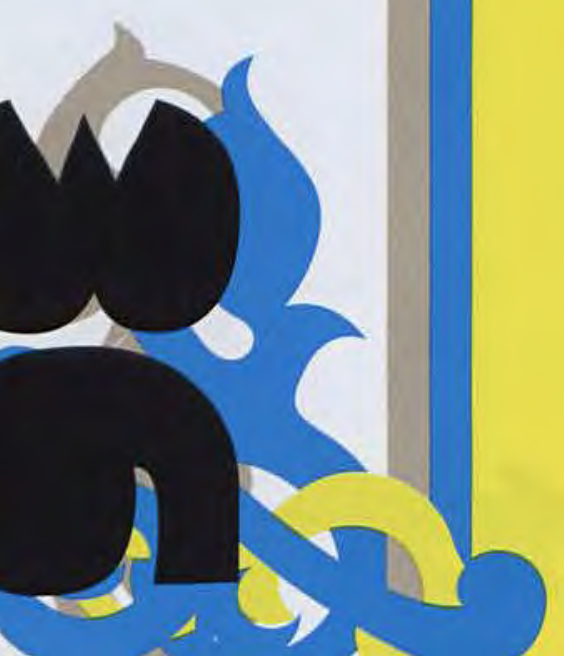

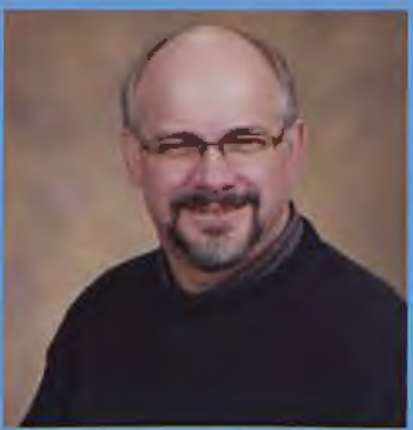

Rev. David Kisner Adjunct Professor

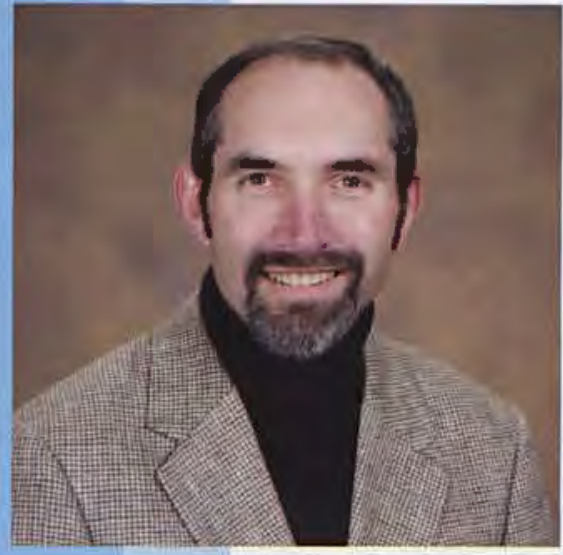

Dr. Chris Miller Interim Chair 


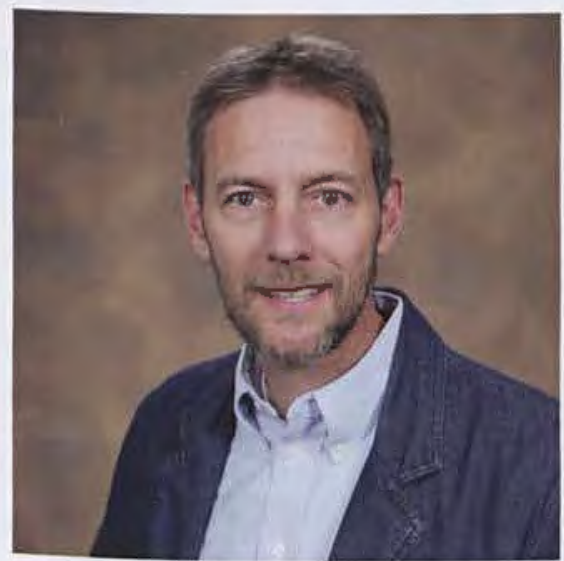

Dr. Donald Grigorenko

Interim Chair

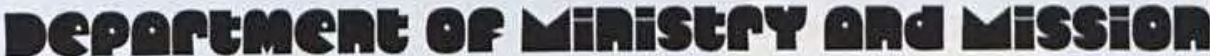

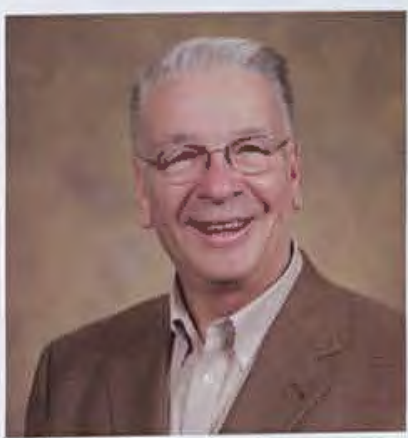

Dr. Richard Blumenstock Associate Professor

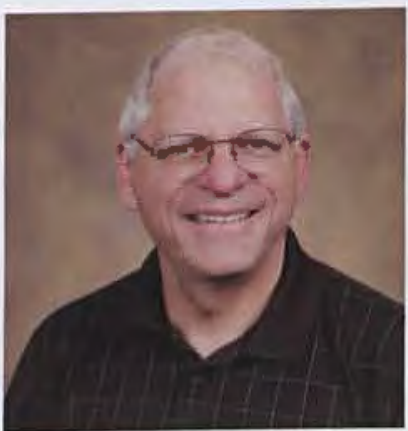

Michael Parrott

Assistant Professor

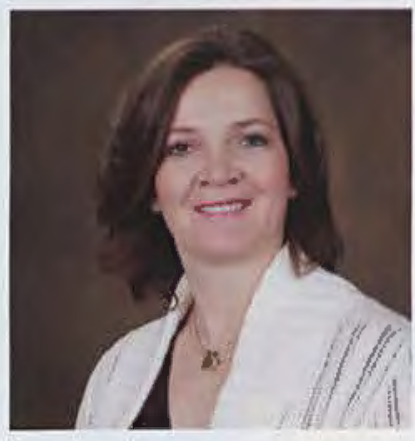

Dr. Joy Fagan

Assistant Professor

\section{hot pictured:}

Dr. Jeffrey Cook

Dr. -Scott Dixon

\section{Deperencer of wheoregr end PhHesepht}

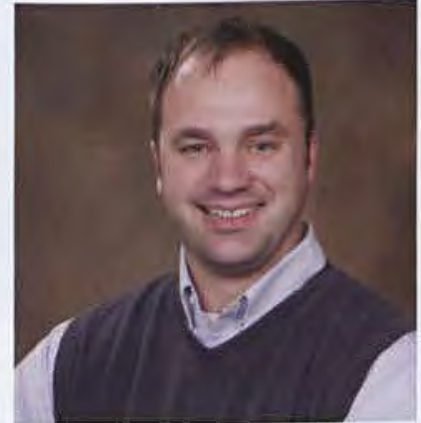

Shawn Graves Assistant Professor

not pictured:

Robert Gromacki Aaron James John White

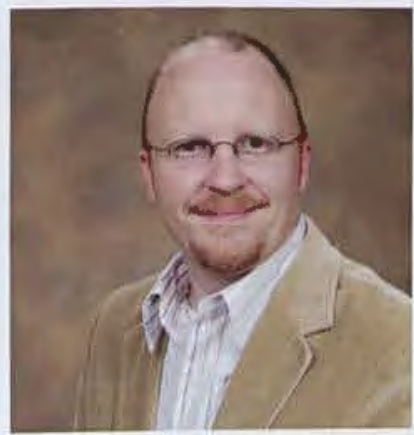

Dr. David Mills Professor

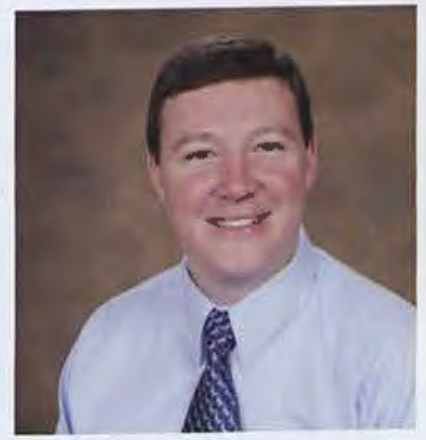

Ryan Peterson Assistant Professor

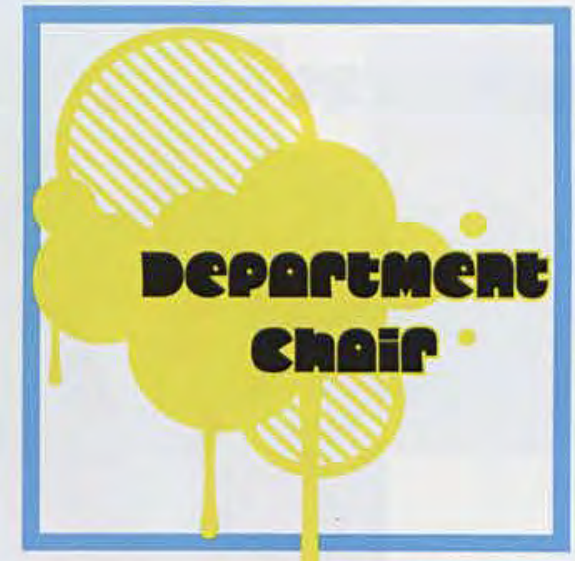

Dr. Gregory Couser Interim Chair

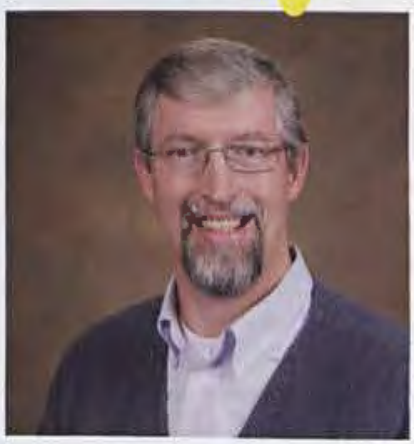

Dr. Carl Smith II Professor 
The School of Humanities saw students in two new major programs this year: Journalism and Worship. For the new Journalism degree, a modern Journalism Media Lab was created in the DMC next to the chapel. With the addition of the Worship degree, the department of Worship and Art became the department of Music, Art \& Worship.

The school has seen a fine record of student achievement during the 2008-09 academic year. Five of our Communication Arts students received awards at the National Religious Broadcasters Convention, a Graphic Design student won a national magazine design competition, a music student won a Piccolo artist competition, and our Society of Technical Communications chapter won their third Pacesetter Award. The Model UN team attended national competition at the United Nations for the very first time.

The School of Humanities presented numerous plays, concerts, recitals, art shows, and guest lecturers as

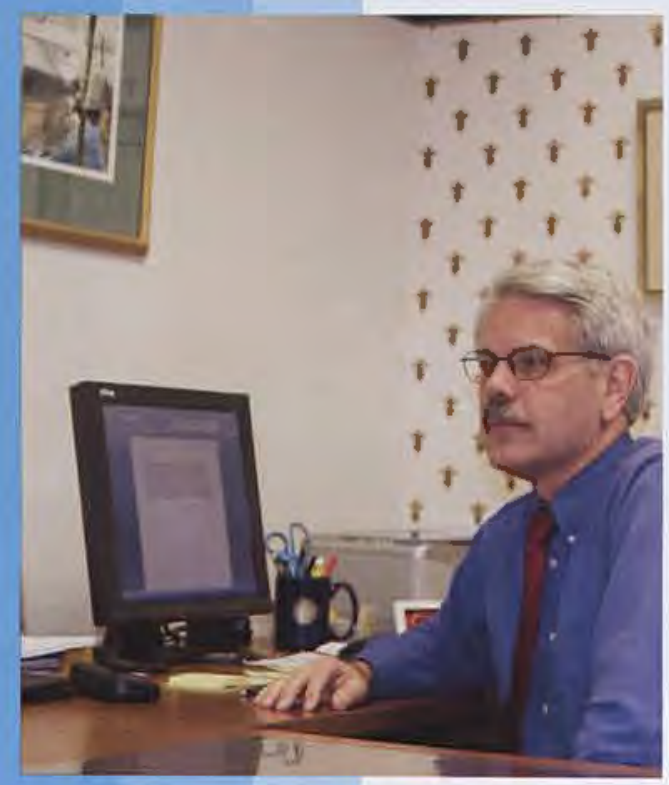

Dr. Steven Winteregg

Dean long celebration of the year " 1809 ," which was the birth year of such notables as Abraham Lincoln, Felix Mendelssohn, and Edgar Allen Poe. The school hosted a Society of Technical Communications Conference, an Ohio Writing Center Consortium, the National Association of Teachers Singing Student Auditions, and Marilynne Robinson, a Pulitzer-Prize winning novelist.
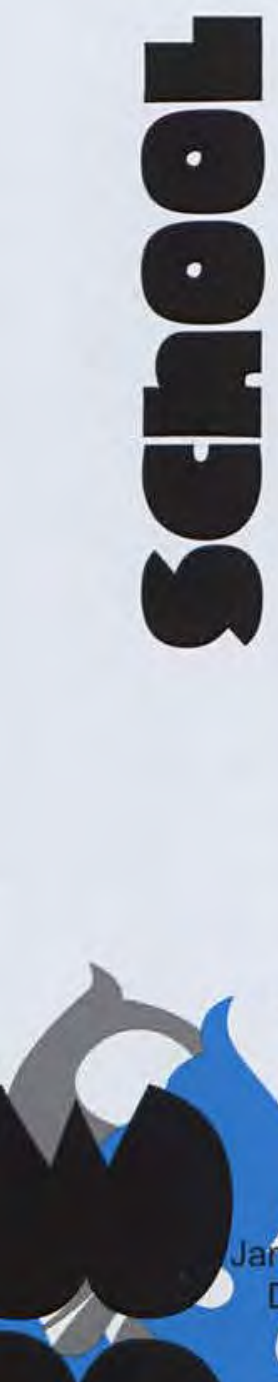

\section{net pictured:}

Dr. Wesley Baker

Robert Clements Derrick Green

Dr. Debrah Haffey James Kragel James Leightenheimer Dr.J. Michael Lopez Dr. Diane Merchant Matthew Moore Margaret Wheeler

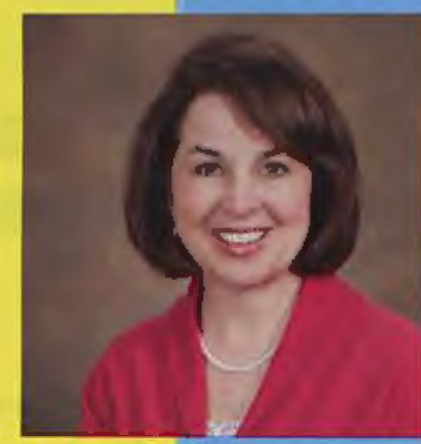

Rebecca Bake Assistant Professor

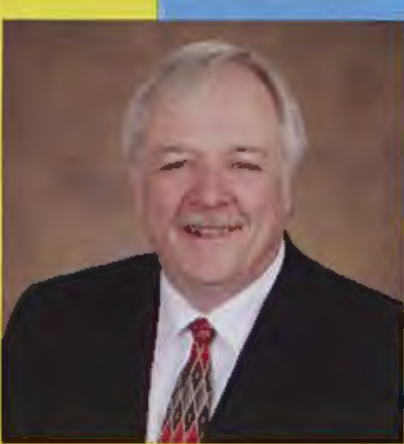

Kurt Moreland Associate Professor

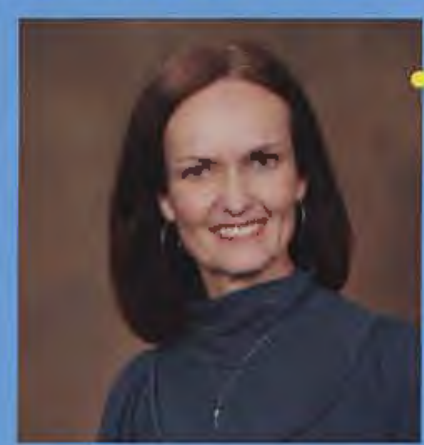

Mischelle McIntosh Assistant Professor

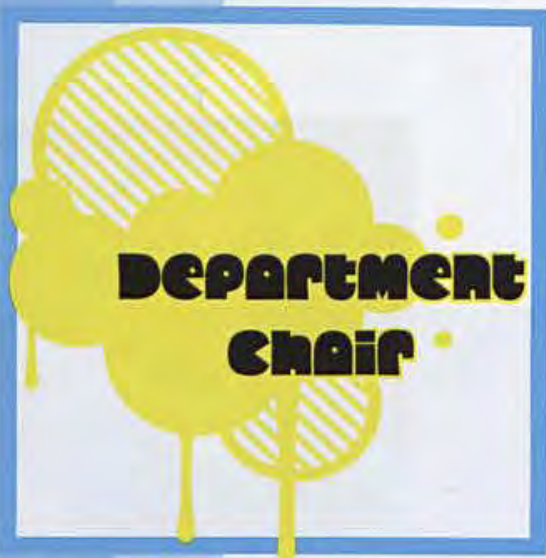

Dr. Charles Elliot Chair

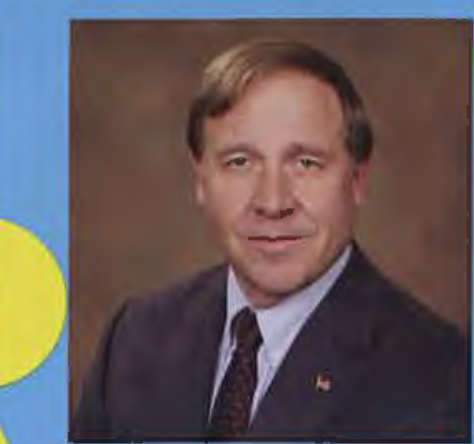

Dr. James Phipps Professor

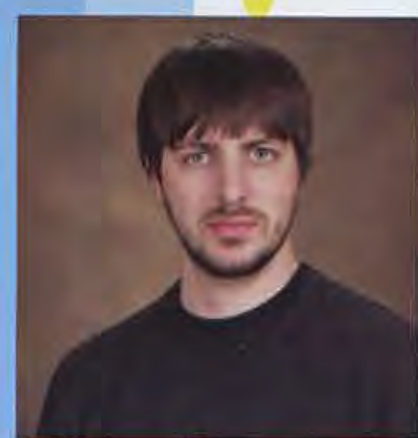

Timothy Phipps Asst. Technical Director 


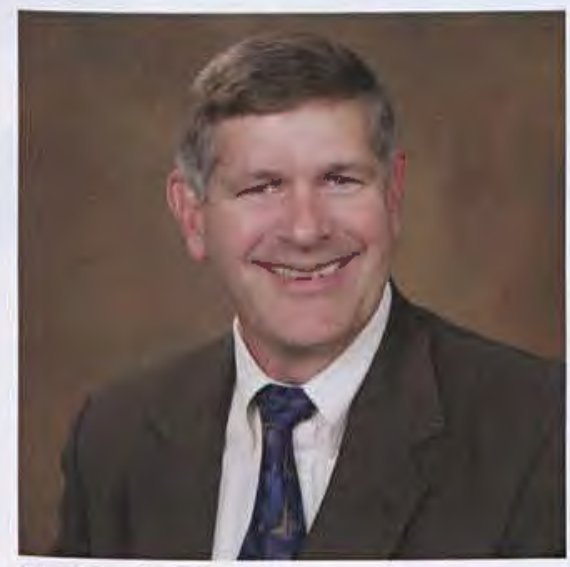

Dr. David Rich Interim Chair

\section{DCpartment ef metery end Governmget}

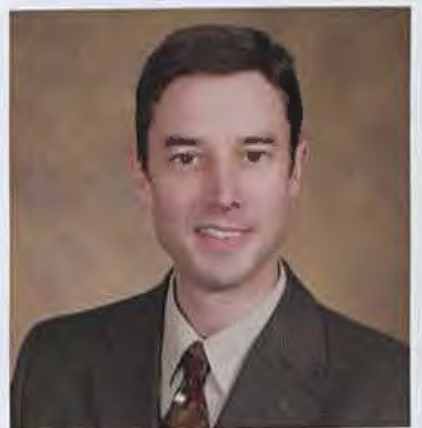

Dr. Thomas Mach Professor

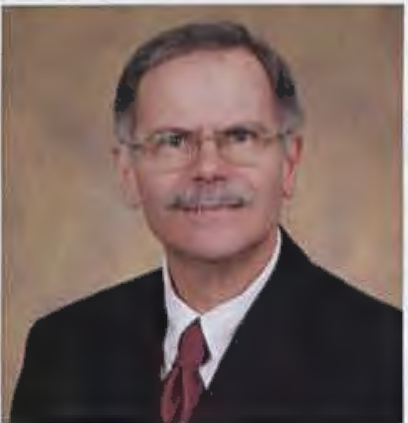

Dr. Kevin Sims

Professor
Ret pHetered:

Dr. Marc Clauson

Dr. Frank Jenista

Dr. Murray Murdoch

Dr. Mark Smith

\section{Depertment of menguege and thereture}

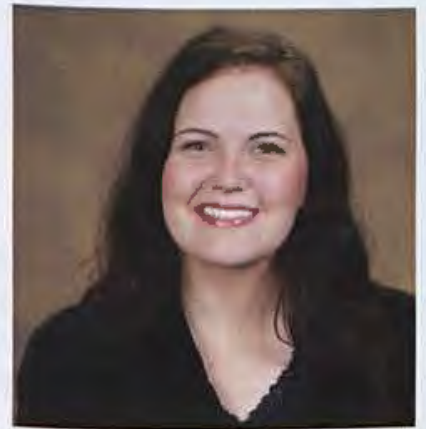

Dr. Helena Corder Assistant Professor

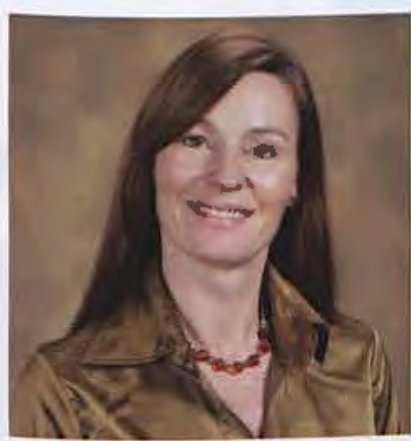

Dr. Melissa Faulkner Assistant Professor

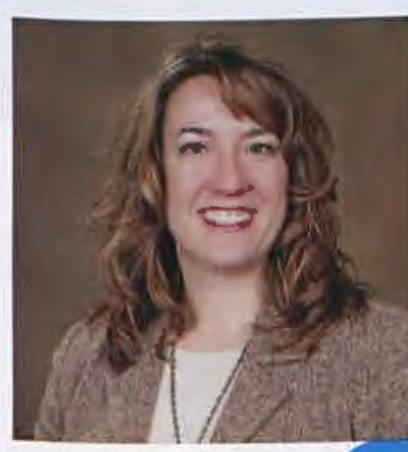

Cynthia Messer Assistant Professor

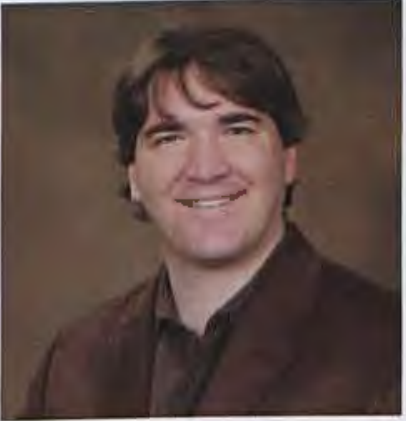

Dr. Donald Deardorff Professor

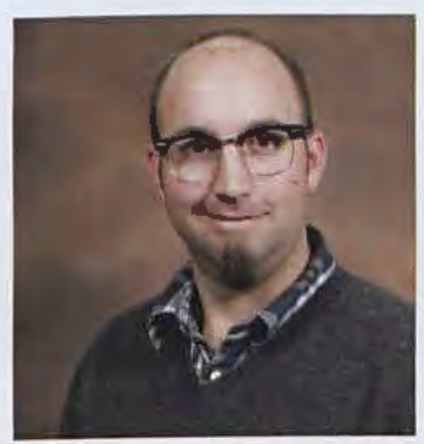

Ryan Futrell Assistant Professor

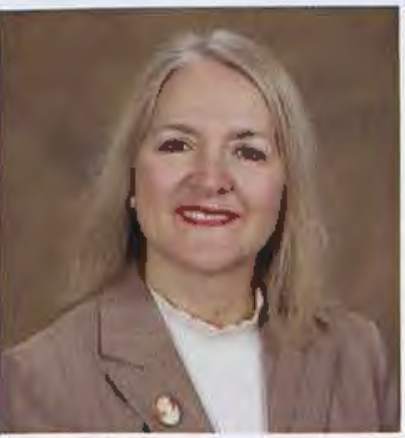

Dr. Annis Shaver Assistant Professor

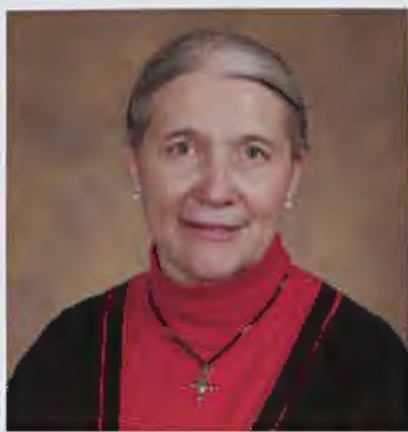

Louise Grandouiller Adjunct Professor

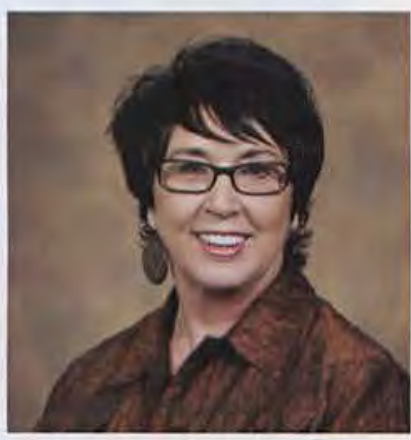

Sandra Harner Professor

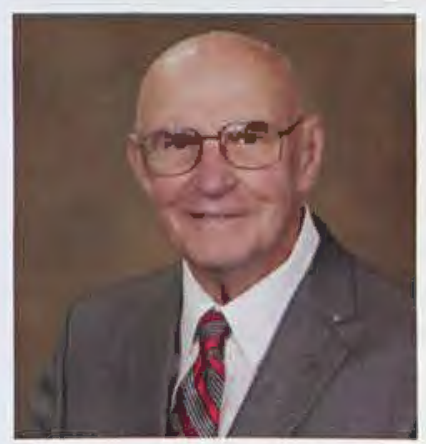

Edward Spencer Professor

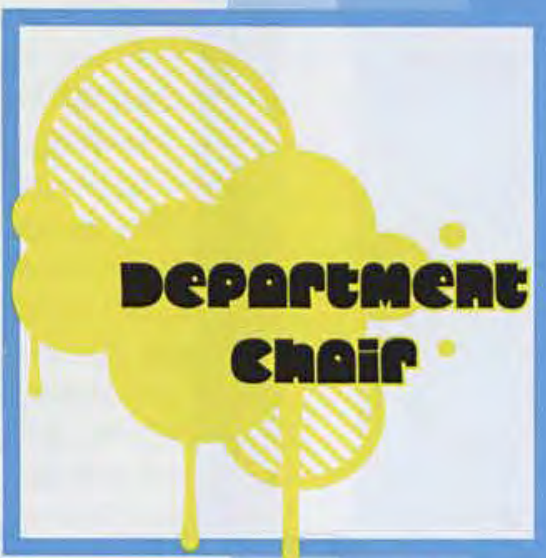

Dr. Kevin Heath

Chair

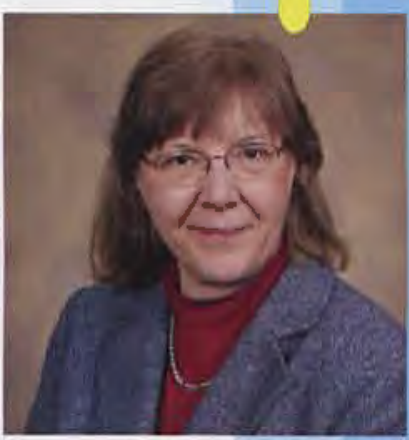

Dr. Barbara Loach

Professor

\section{net pictured:}

Gregory Belliveau

Dr. Scott Calhoun

Daniel Clark

Donald Humphreys

Julie Moore

Karen Power

Dr. Peggy Wilfong

Dr. Andrew Wiseman

Dr. Chimi Woo

Michelle Wood 


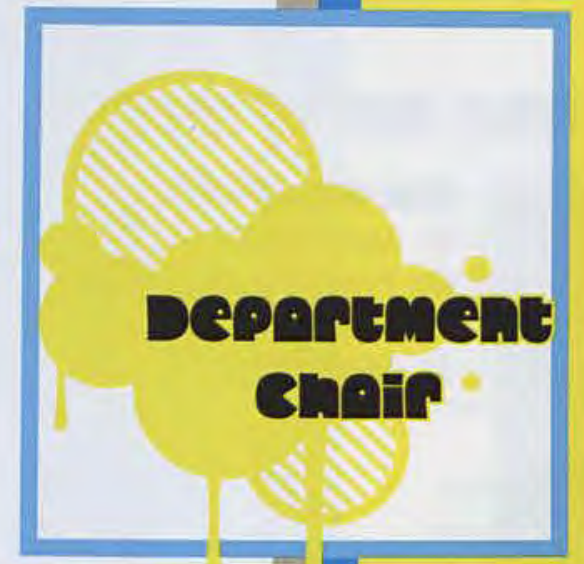

Beth Porter Chair

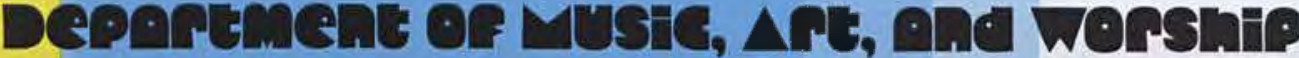

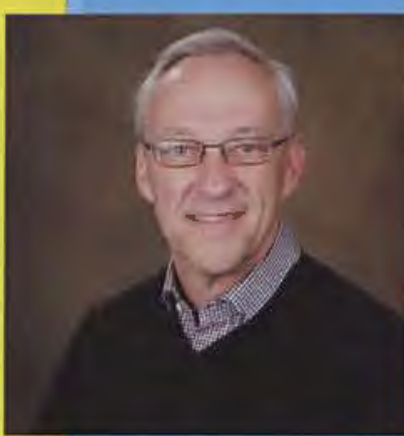

Terry Chamberlin

Associate Professor

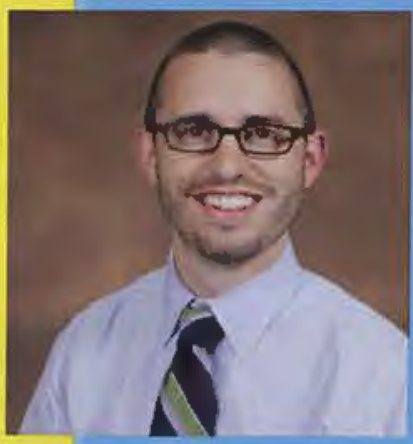

Aaron Gosser Instructor

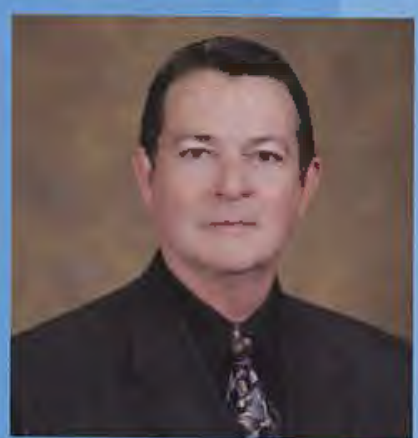

Dr. Charles Clevenger Professor

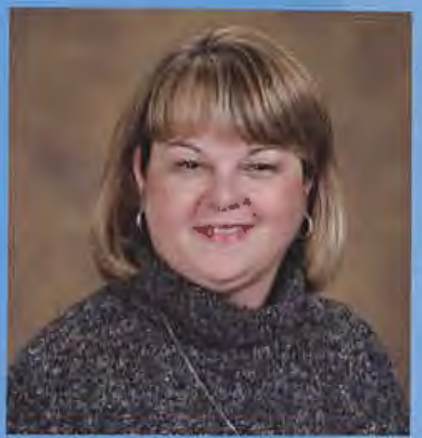

Pam Miller

Administrative Assistant

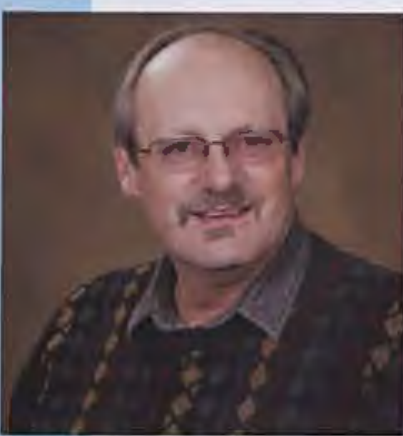

Michael DiCuirci

Professor

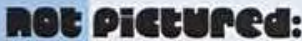

Connie Anderson

Dr. Lyle Anderson

Bruce Curlette

Laura Ferranti

Dr. Taylor Ferranti

Timothy Frame

Jun Kim

Dr. David Mattson

James Mellick

Dr. John Mortensen

Dr. Roger O'Neel

Charles Pagnard

Dr. Mark Spencer

Daniel Sternsher
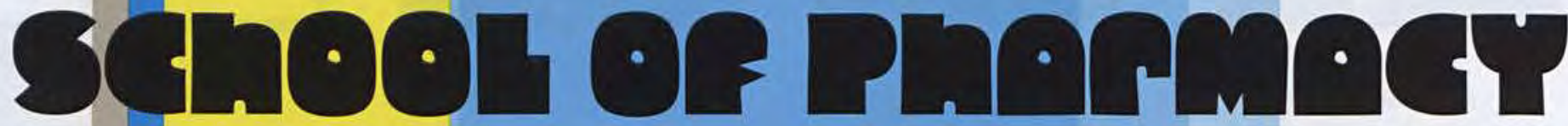

During the 2008-09 academic year, I was hired as

the Dean for the School of Pharmacy. Mr. David

Ormsbee was hired to coordinate and oversee the admissions for the School of Pharmacy. An Assistant Dean and Coordinator for Student Development were identified to begin for the 2009-10 academic year.

Recruitment continues for the identification of the

Department Chairs for Pharmaceutical Sciences and Pharmacy Practice. Interviews continue to identify the inaugural class, who will begin prepharmacy classes in Fall 2009. The School of Pharmacy Advisory Board continues to provide input in the development of the School of Pharmacy. The plan is to open the School of Pharmacy professional curriculum in the Fall of 2012.

As one of only a handful of Christian universities to offer a professional-level pharmacy program, Cedarville prepares students to bring their biblical, moral convictions to the complicated issues of pharmacy.

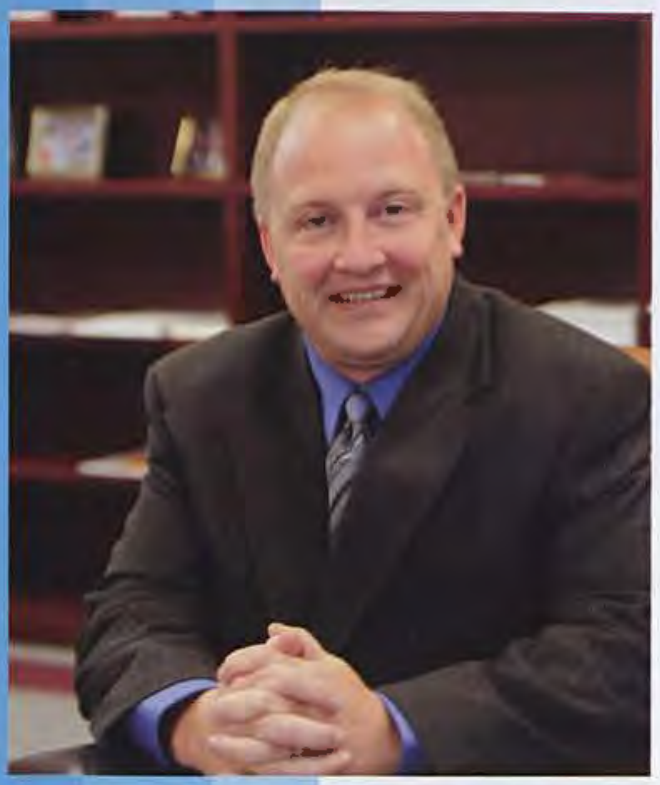

Dr. Marc Sweeney

With its legacy of quality Christ-centered academics, Cedarville will equip the next generation of compassionate, Christ-like pharmacists to influence the culture for Christ. 

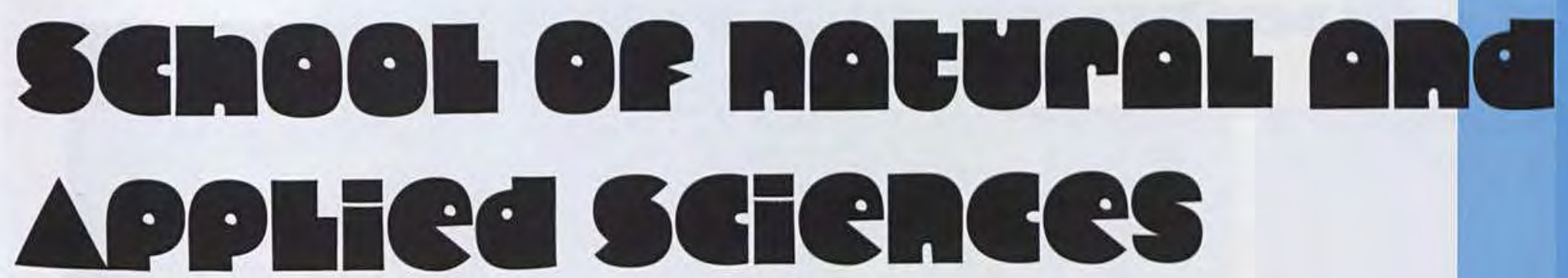

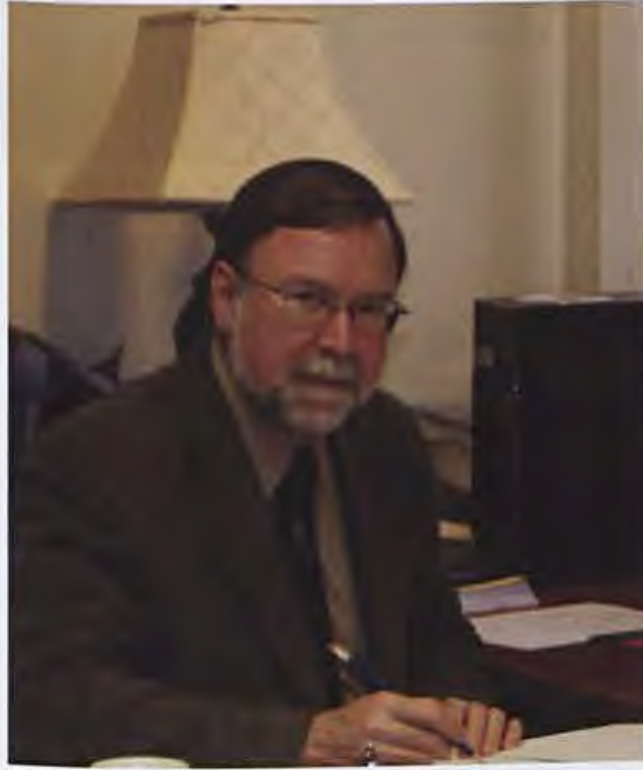

Dr. Stan Baczek

Dean
I love serving our Lord at Cedarville. Some say that being a university dean is one of the most sought-after positions of any profession. This may be due in part because nobody seems to be able to articulate exactly what a dean does. Therefore, there is tremendous opportunity to make the position yield to the person in that chair. At secular schools an obvious requirement of the position is that of fund raising, or more precisely acquiring federal and state grant monies to fuel the research engines that purchase release time for professors to accomplish knowledge discovery. This paradigm is not possible at Cedarville because we do not accept federal and state monies.

Therefore, I have chosen to focus my energies on serving the School's faculty so that they can see their dreams fulfilled within the context of the university mission. Although this has been a year of market uncertainties, we have moved forward on a number of fronts. The new geology major was approved. This unique major has been the dream of $\mathrm{Dr}$. Whitmore since the day he arrived on campus in 1991. An Actuarial Science minor was also approved. Two relatively new majors, Environmental Science and Forensic Science are flourishing. The Bioethics Center has been very active through the work of Dr. Sullivan and Dr. Salladay.

Restructuring of the BSN nursing major promises to make content delivery more efficient and position graduates for advanced studies. Engineering and Computer Science continues high enrollment and winning competitions. New faculty in both Computer Science and Electrical Engineering are filling needs with unique elective offerings. The Department of Business Administration has strengthened with a key Acting Chair and its Information Science major with a key faculty appointment. Did I say how much I love serving the faculty and students of Cedarville?

\section{Deportment of Bustaess $\Delta$ dmintstretton}

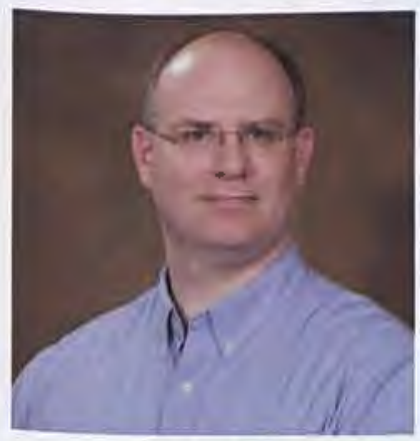

John Delano Assistant Professor

DeE PICt: ved:

Henry Anderson

Dr. Jon Austin

Charles Hartman

Dr. Andy Runyan

Dr. David Schmidt

Dr. Galen Smith

Dr. Sarah Smith

Susan Terkelsen

Dr. Ronald Walker

Dr. Bert Wheeler

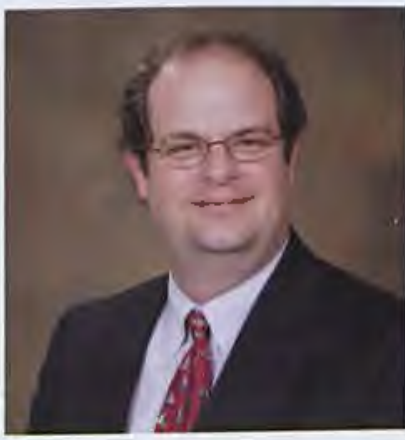

Dr. Jeffrey Fawcett Professor

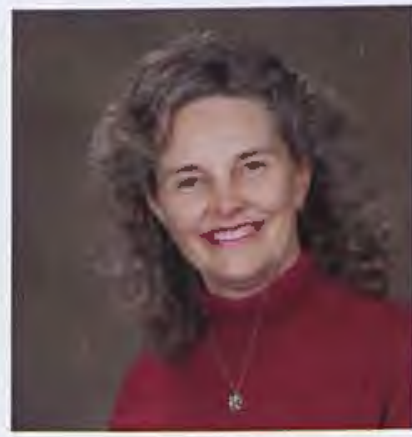

Virginia Huff Secretary

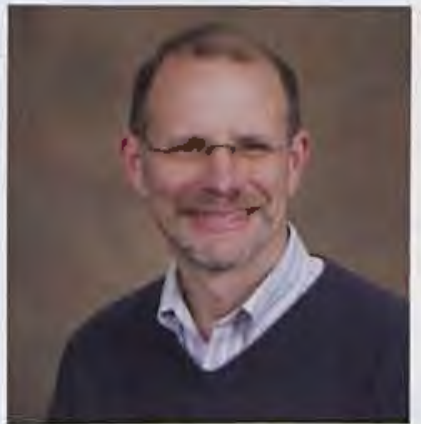

Jeffrey Guernsey Assistant Professor

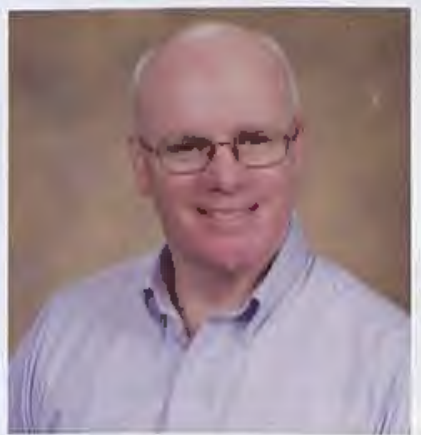

Dr. William Ragle Associate Professor

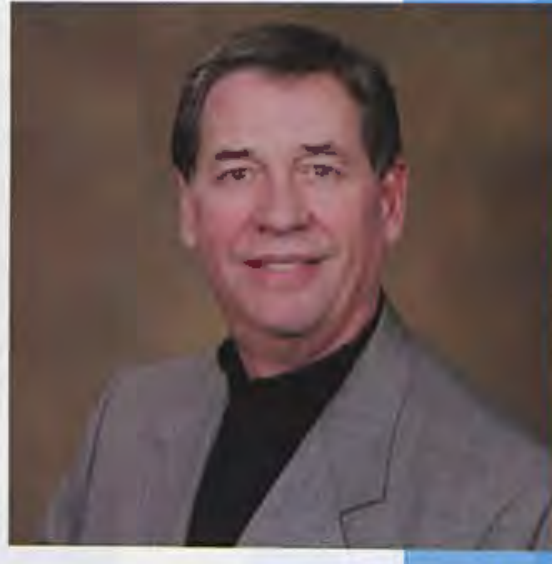

John LeBlanc Interim Chair

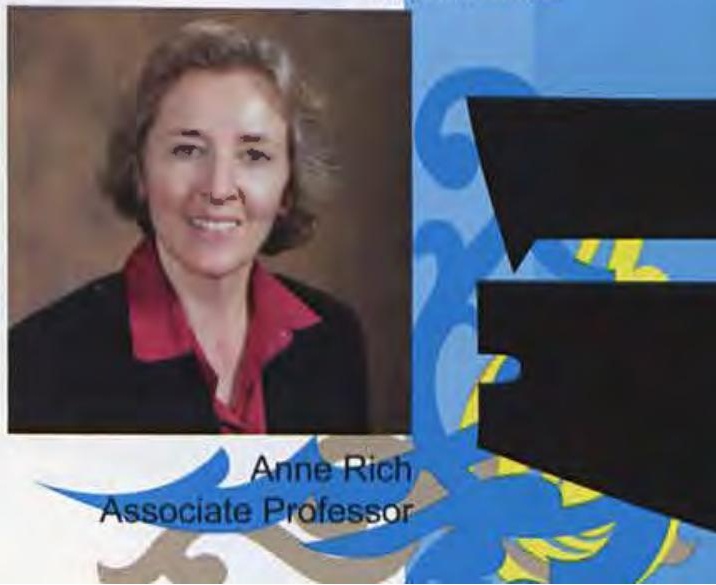


cemputer serence

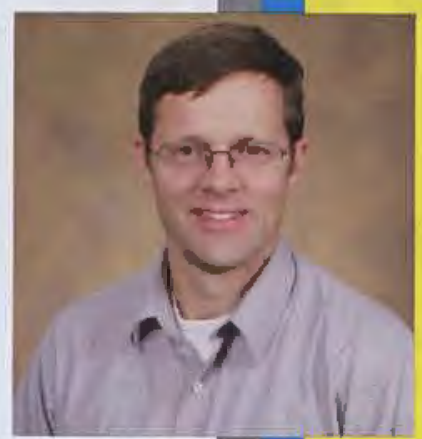

Dr. Gerald Brown Assistant Professor

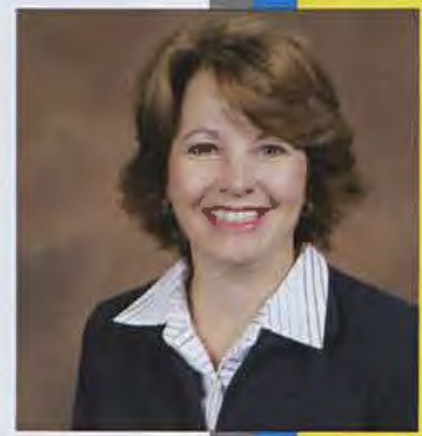

Cherie Hadra Administrative Assistant

not pretured:

Dr. Peter Burban

Dr. Timothy Dewhurst

Dr. Vicky Zhengwei Fang

Dr. Timothy Norman

Dr. Thomas Thompson

Dr. Timothy Tuinstra

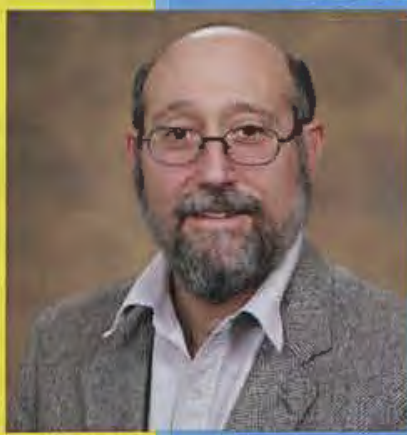

Dr. Robert Chasnov

Professor

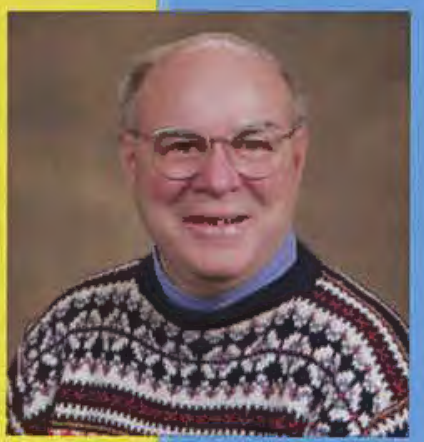

Dr. Harwood Hegna

Professor

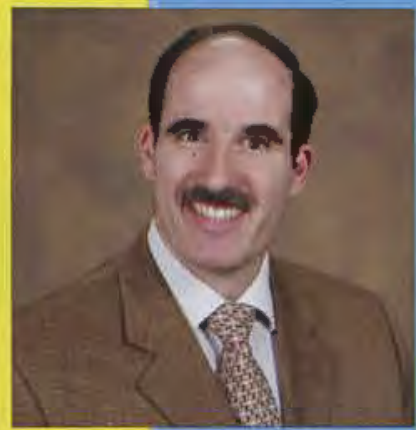

Dr. Keith Shomper Associate Professor

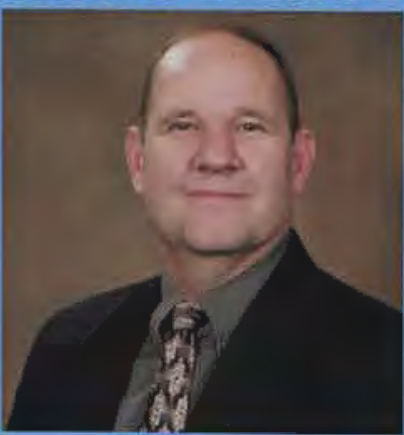

Dr. David Gallagher Professor

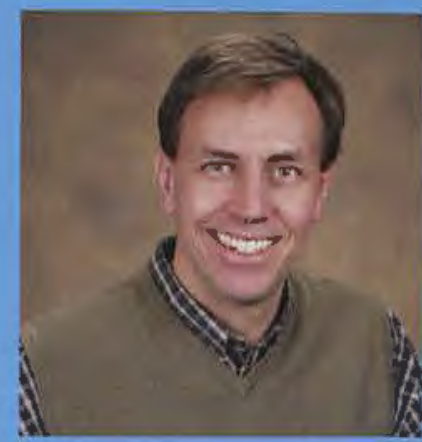

Jay Kinsinger

Assistant Professor

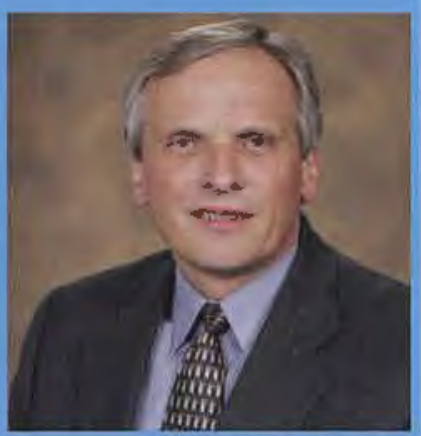

Dr. Jeffrey Shortt Professor

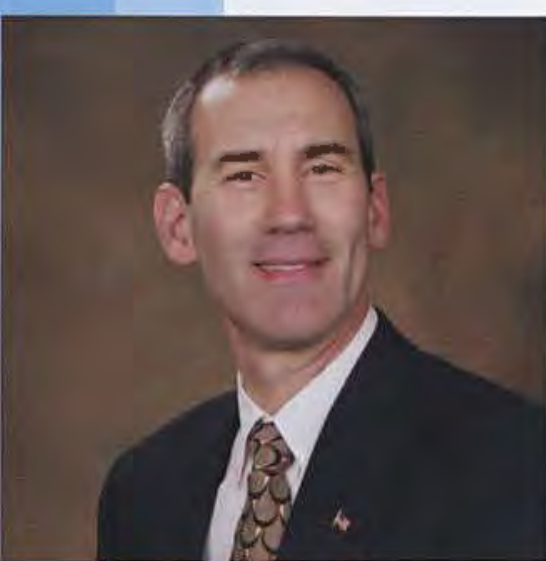

Dr. Samuel SanGregory Chair

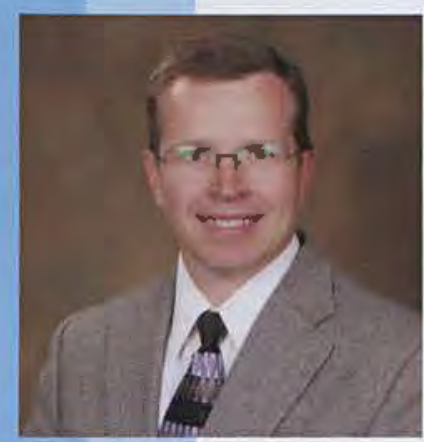

Dr. Clint Kohl Professor

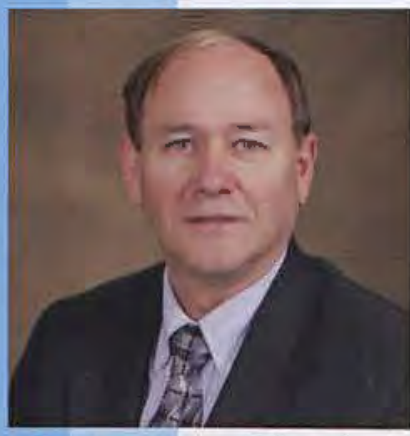

Dr. Lawrence Zavodney Professor

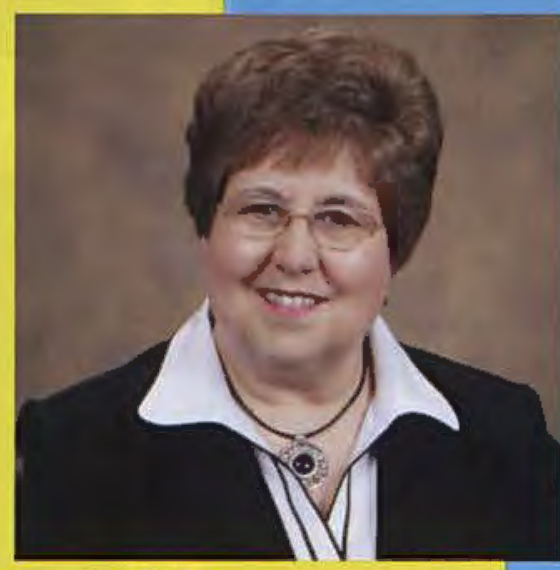

Dr. Janet Conway Chair

\section{Depersmcat or mupstar}

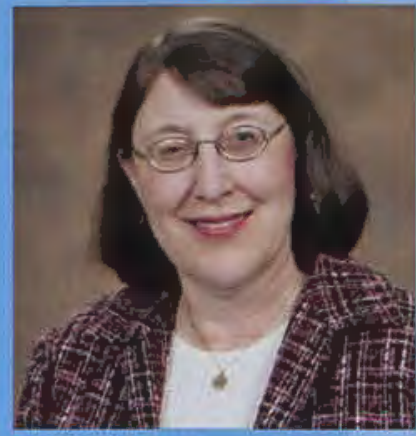

Dr. Lois Baker

Professor

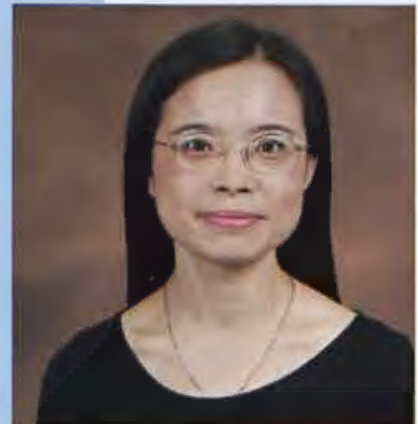

Dr. Chu-Yu Huang Associate Professor 


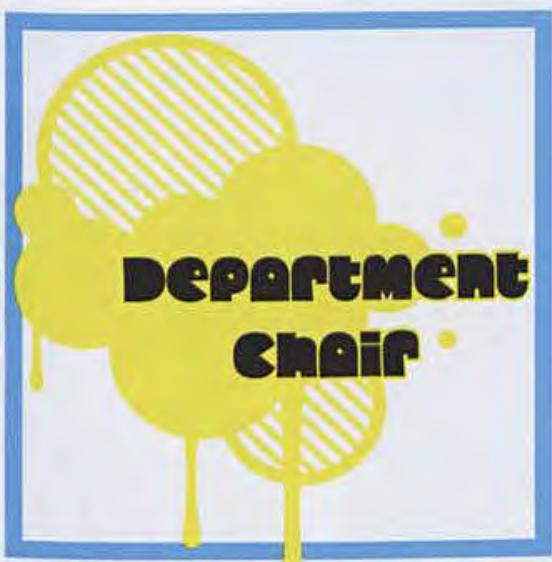

Dr. Dennis Flentge Chair

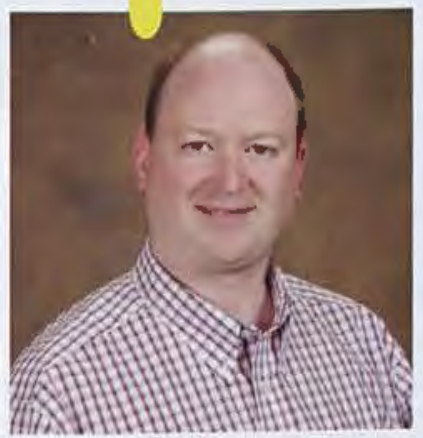

Dr. Darrin Frey

Associate Professor

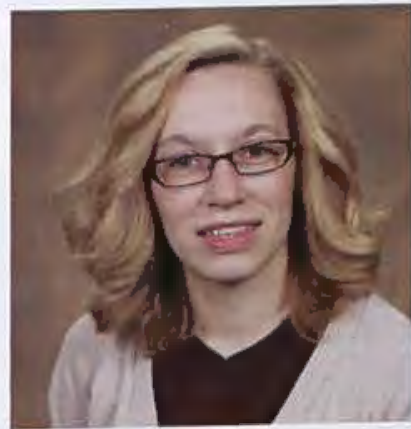

Christy Penrose

Administrative Assistant

Depertencht of seience and Methenetics

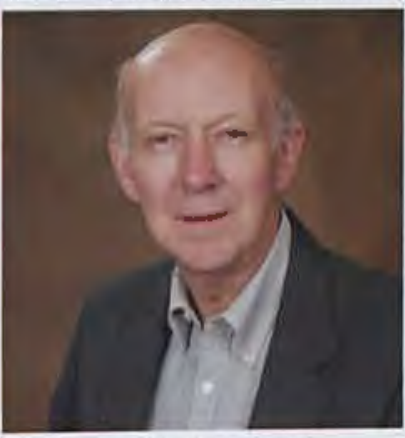

Dr. Donald Baumann

Professor

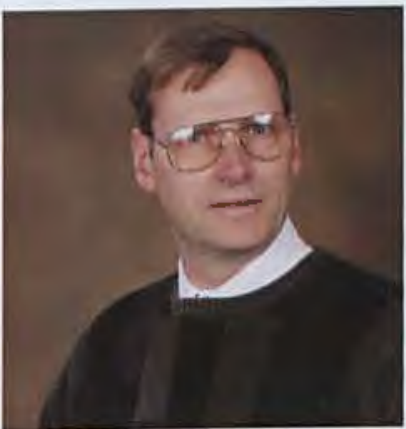

Dr. Steven Gollmer Associate Professor

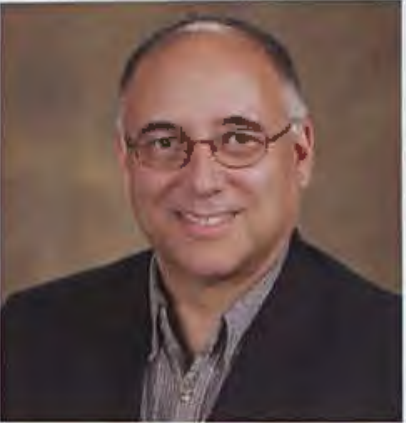

Dr. Kevin Roper

Associate Professor

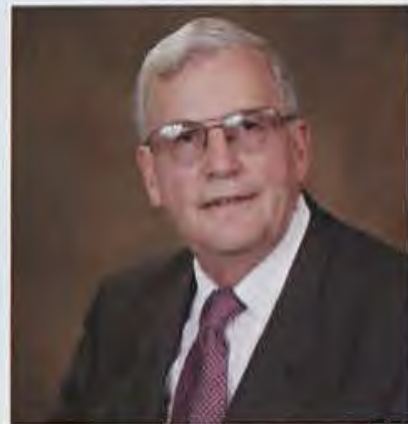

Dr. Edwin Braithwaite Professor

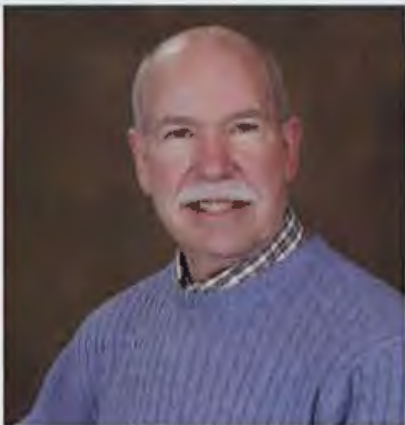

Dr. Larry Helmick Senior Professor

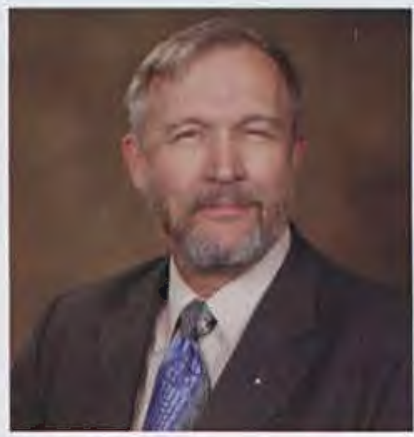

Robert Schumacher Assistant Professor
Ret PHeterred:

Dr. Xidong chen

Dr. Mark Gathany

Sarah Gilchrist

Nathan Hnatiuk Jennifer Hutchinson

William Jones

Dr. Heather Kuruvilla
Dr. Dali Luo Dr. Mark McClain Dr. Douglas Miller Dr. Terry Phipps Dr. Alicia Schaffner Dr. Dennis Sullivan Cynthia Wingert Dr. Otis Wright III

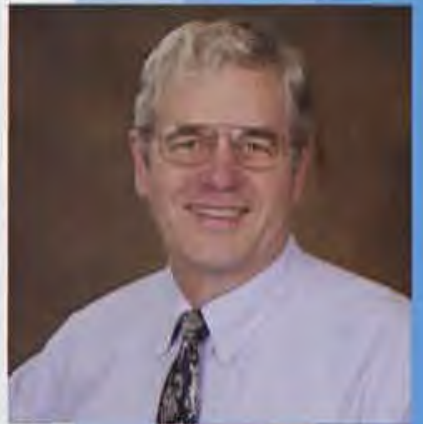

Dr. Leroy Eimers Professor

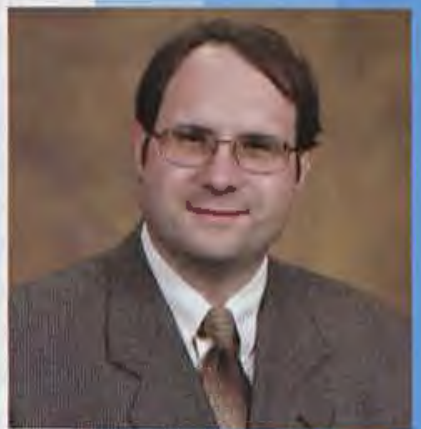

Dr. Aaron Hutchison Assistant Professor

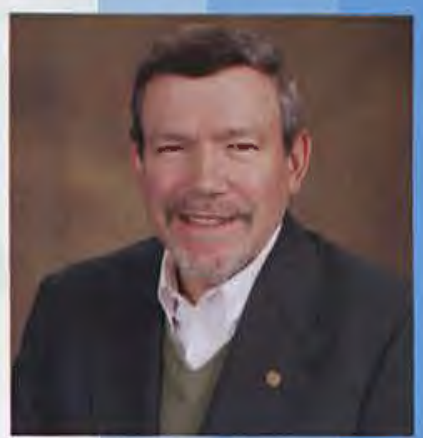

Dr. John Silvius

Senior Professor

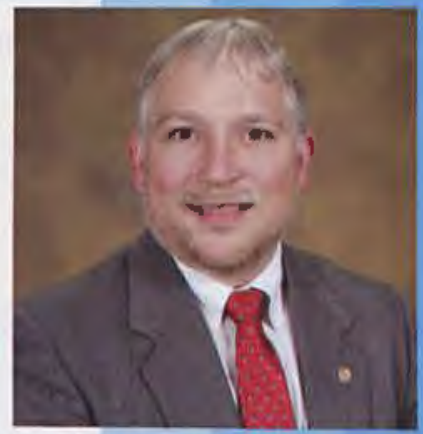

Dr. John Whitmore

Associate Professor
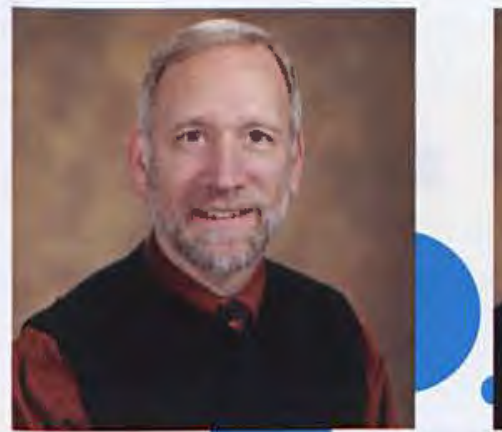

Mark Klimek Associate Professor

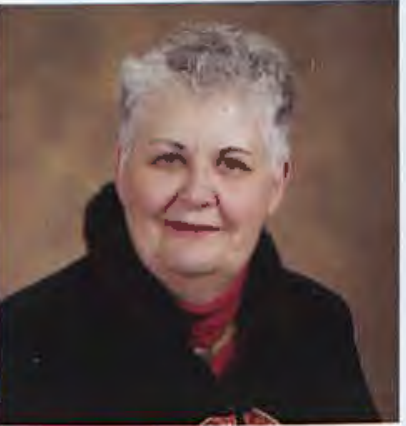

Dr. Judith Shrubsole Associate Professor
ROE PIC:

Carolyn Barnett

Karen Callan

Linda Cave

Dr. Sharon Christman

Connie Ford

J. Kimberly Higginbotham

Dr. Carrie Keib

Suzanne Lefever

Yvonne Lusk

Angelia Mickle
Janet Neal

Rachel Parrill

Dr. Susan Salladay

Marsha Swineheart

Amy Voris

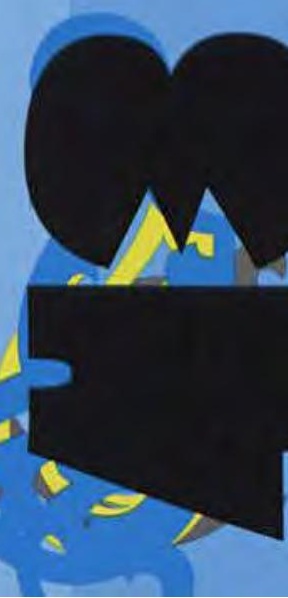



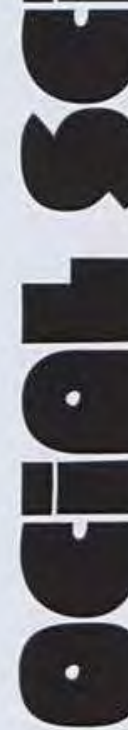

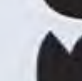
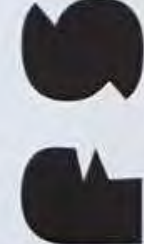

-
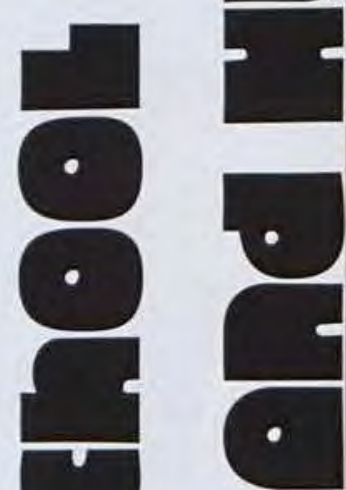

The 2008-09 academic year has been an exciting time for the School of Social Sciences and Human Performance as we have sought to serve our Lord by serving $H$ is students here at Cedarville. As the most unique School at Cedarville, with four academic departments and two co-curricular program departments in our make-up, we have been involved with almost every student in the University, seeking to help them become a well-prepared, more mature Christ-follower. To that end we provided more than 25 major and minor programs, several significant core curriculum courses like PACL, General Psychology, and Cultural Anthropology, and an exciting array of program offerings through our Campus Recreation Department. Our 14-team Yellow Jacket Athletic Department provided over 300 student-athletes intense competitive experiences where valuable lessons in living the Christian life were practiced and tested, and a significant number of fellow students, faculty, and staff cheered them on!

The Athletic Training faculty and staff provided both essential athletic training services for the athletic pro-

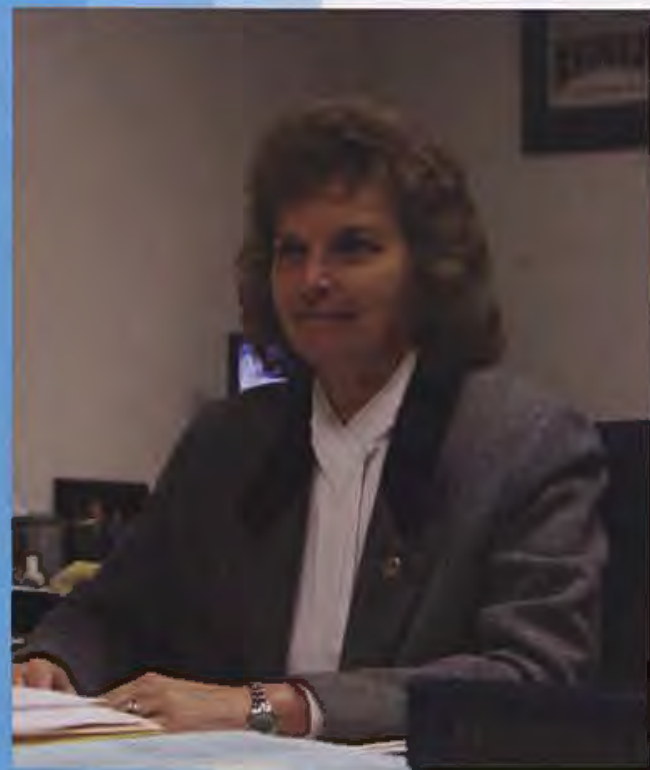

Dr. Pamela Johnson Dean gram, and also used it for key clinical education opportunities for athletic training students. Through various majors offered in our departments of Education, Exercise and Sport Science, Psychology, and Social Work, Criminal Justice and Sociology we have prepared cadre students ready to influence their world for Christ. We have prepared them to influence as licensed social workers, as certified teachers in more than 20 licensure areas, as certified athletic trainers, or through a fascinating variety of positions in criminal justice, psychology, sociology, exercise science, and sport management. Many go on to graduate school in these areas. We look forward to hearing what He does through them!

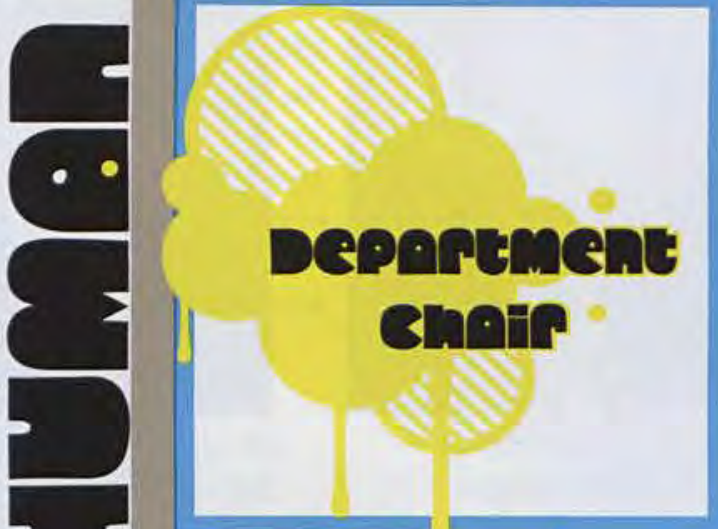

Dr. Stephen Gruber Chair

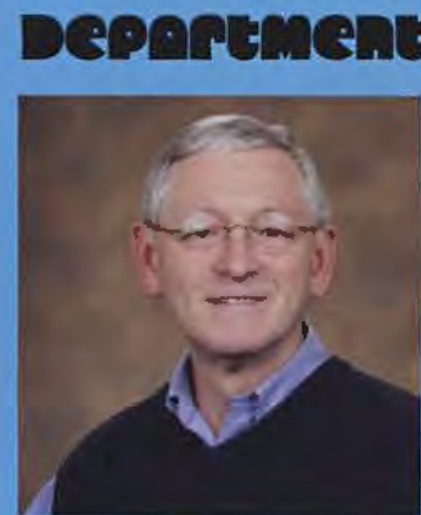

Dr. Merlin Ager Professor

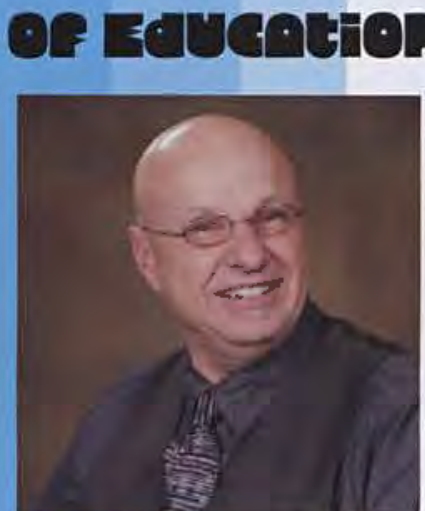

Dr. Timothy Heaton Professor not pictured:

Dr. Sue Baker

Dr. Eddie Baumann

Dr. Omer Bonenberger

Dr. Sally Castle

Terry Comers

Shelley Dugle
Dr. Dwayne Frank Margaret Grigorenko John Hess

Ruth Hess

Dr. Brenda MacKay Dr. Thomas Sweigard

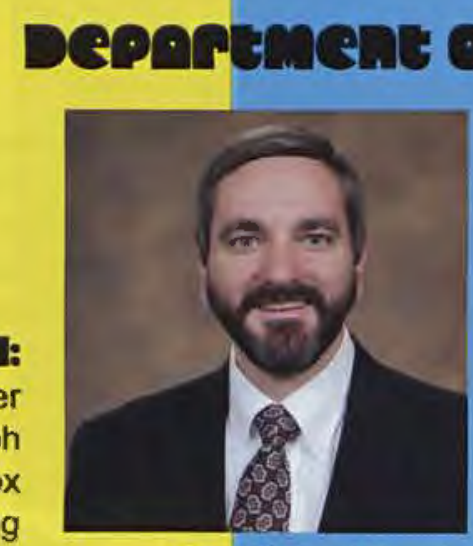

Dr. Michael Firmin Professor
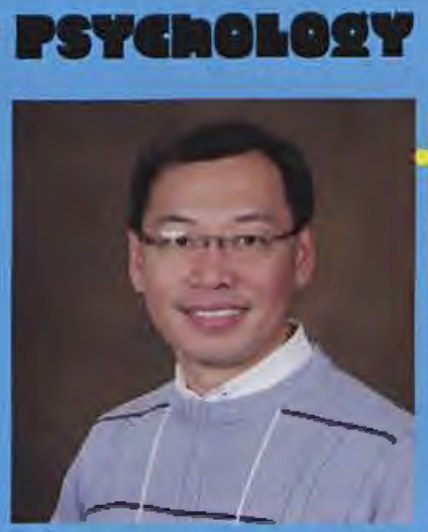

Dr. Luke Tse Associate Professor

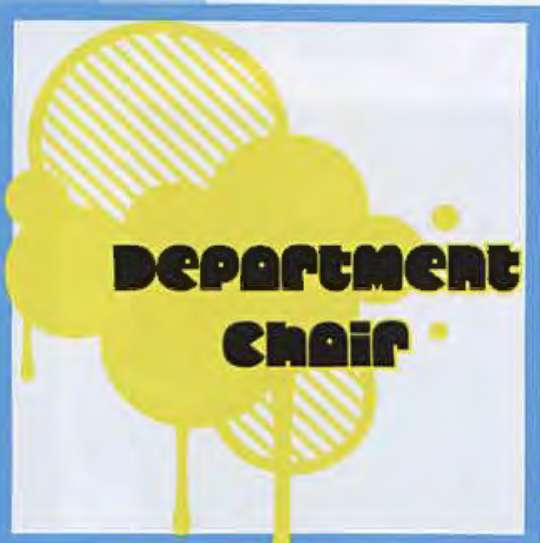

Dr. Milton Becknell Acting Chair 


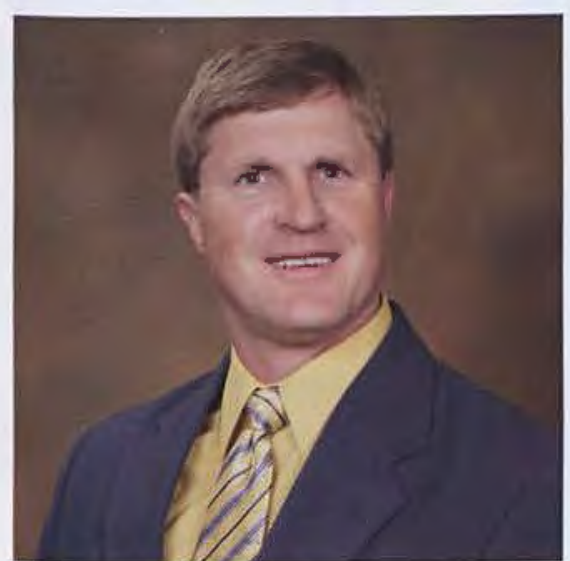

Dr. Evan Hellwing Interim Chair

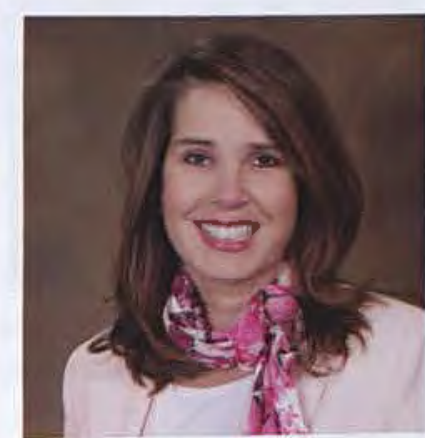

Dr. April Crommett Assistant Professor

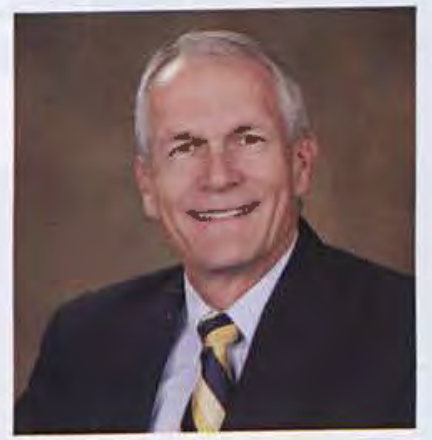

John McGillivray Associate Professor

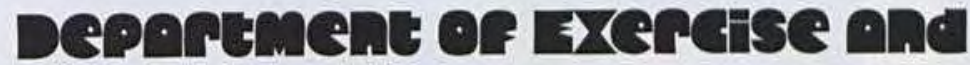 spert seichce}

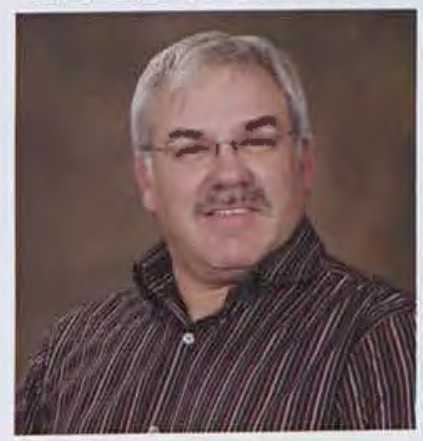

Benson Belleman

Assistant Professor

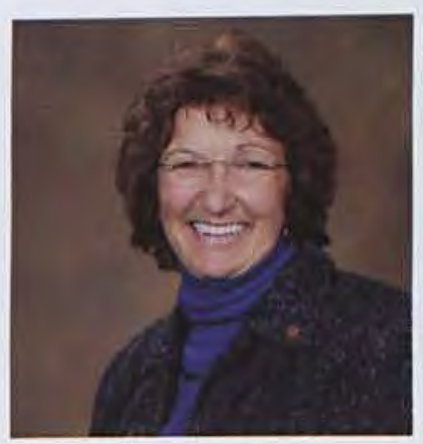

Kathleen Freese

Associate Professor

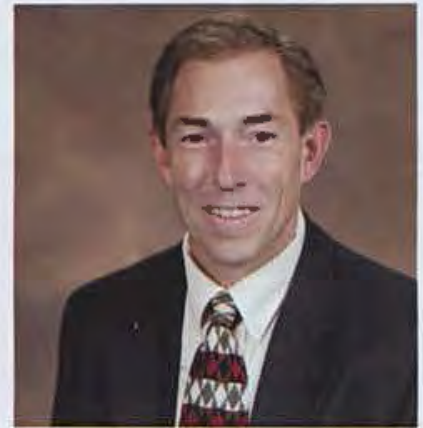

Paul Orchard

Assistant Professor

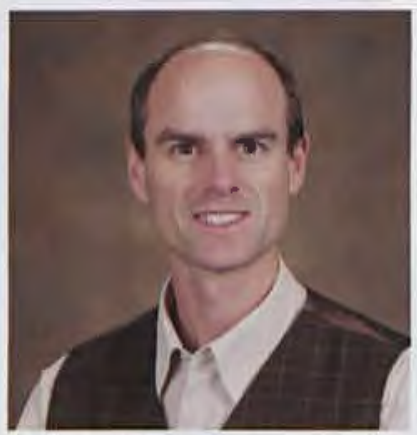

Jeffrey Bolender

Assistant Professor

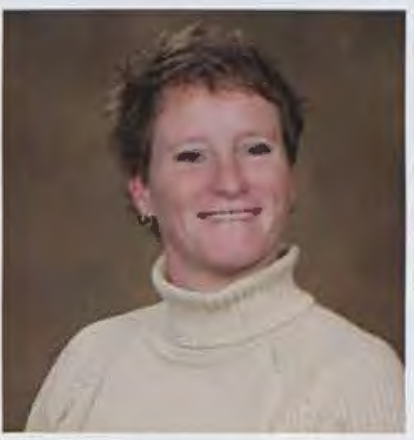

Lori Huckaby

Adjunct Professor

\section{not pratured:}

Robert Duchardt

Patrick Estepp

Dr. Alan Geist

Kirk Martin

Steven Patterson

Elizabeth Shearer

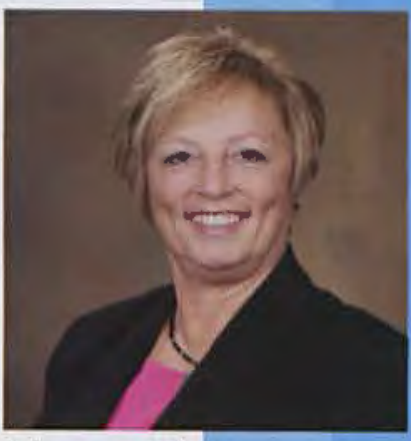

Theresa Clark

Associate Professor

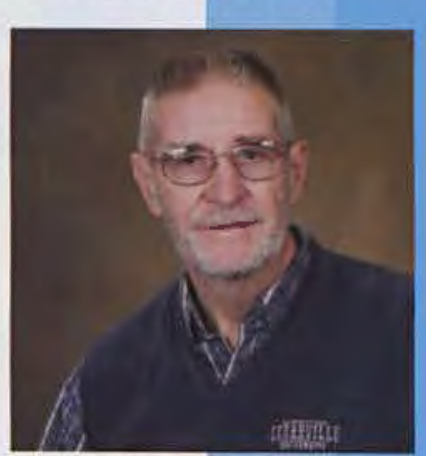

Elvin King

Associate Professor

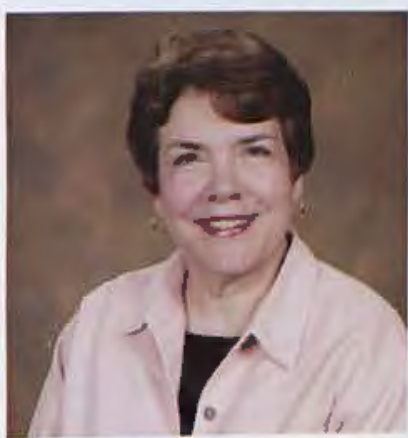

Sherrie Wood

Administrative Assistant Campus Recreation

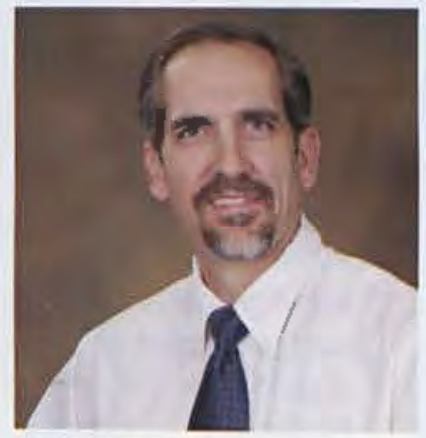

Mark Matthews

Director of Campus

Recreation and Facilities 


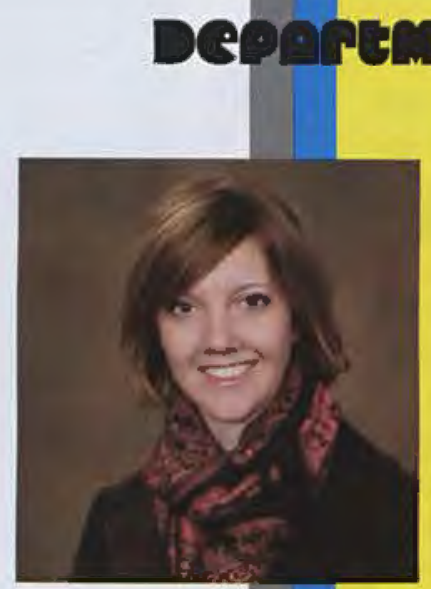

Julle Furj

Assistant Professor
Justice,

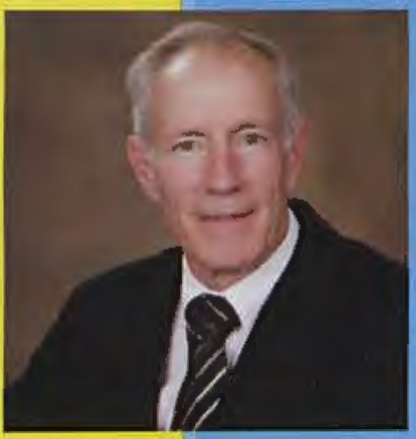

George Huff Associate Professor
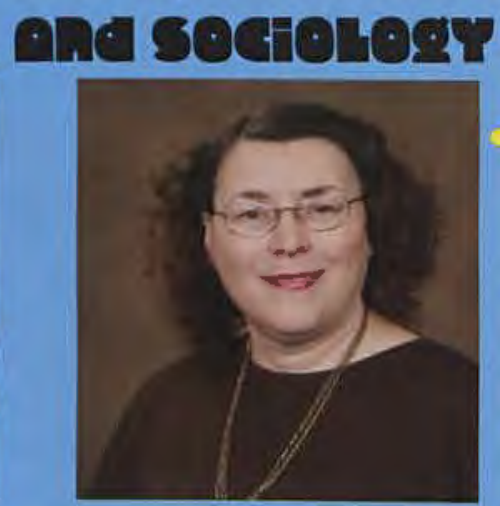

Esther Lanham

Administrative Assistant

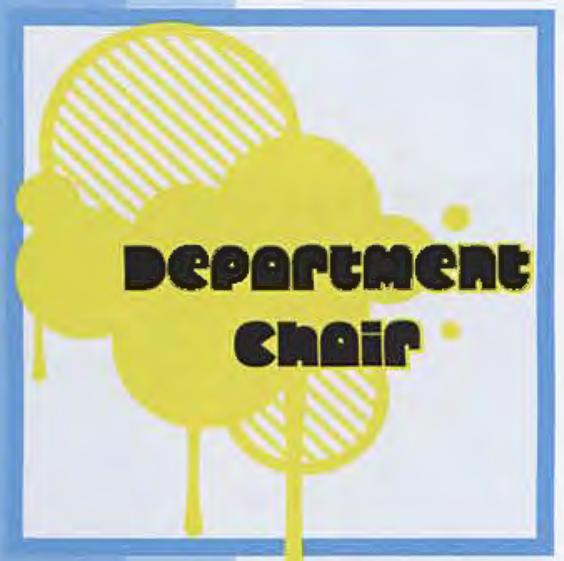

Dr. Nelson Henning

Chair

\section{not pratured:}

Christine Fulmer

Dr. Susan Warner

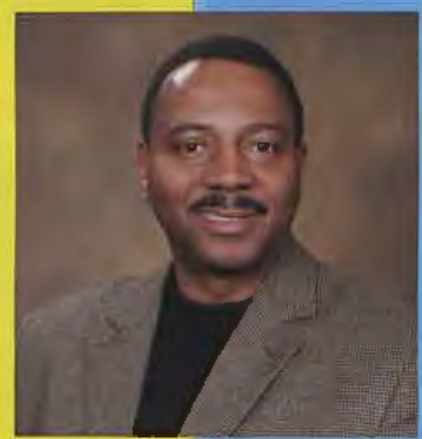

Patrick Oliver Assistant Professor

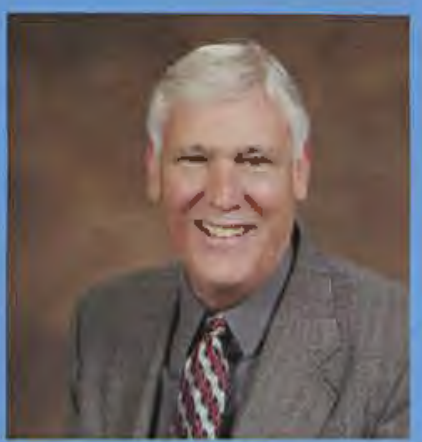

Dr. Robert Parr Professor

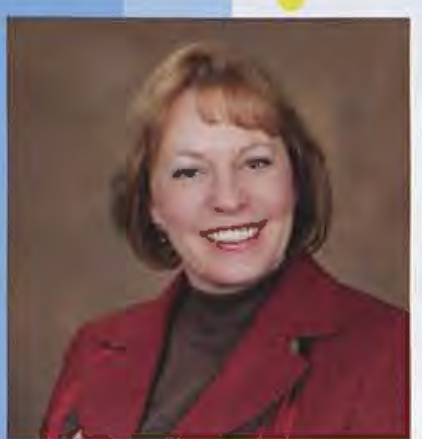

Debra Pitts Secretary
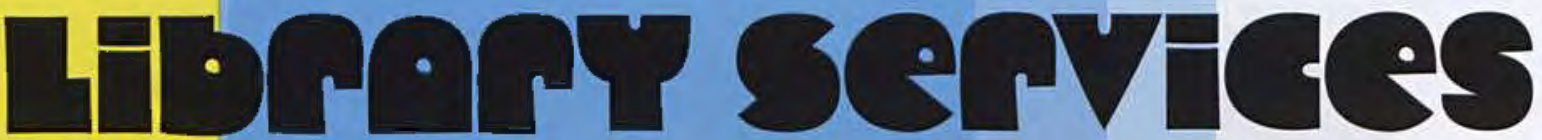

As students returned in the Fall, they found the Centennial Library in the midst of a major exterior grounds face-lift being completed in conjunction with the new Center for Biblical and Theological Studies. There were new walks and ramps, new steps, a new planting area and plaza, new exterior lighting, new glass in the vestibule, and new interior lighting in the vestibule.

All of this was intended to provide better access and visibility to the Library entrance.

As well, the Centennial Cartwheelers book cart drill team, composed of Library faculty and staff, participated in the Homecoming parade to the delight of all along the parade route. In the Spring, the results of the Noel-Levitz Student Satisfaction Survey indicated that the Centennial Library had the highest rating for library services of all the CCCU schools participating in the survey, affirming a high student satisfaction with Library resources and staff. During the year, Library faculty worked on the development of student research competencies and learning outcomes that will serve as a guide for the development of research assignments by faculty.

These competencies will document the research abilities that all students at the University should be able to master by the completion of their undergraduate career.

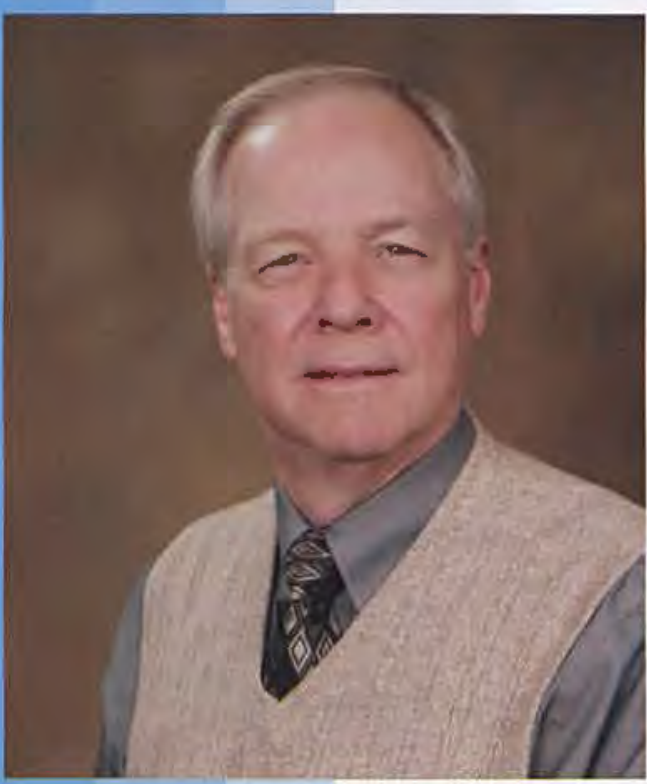

Lynn Brock Dean 


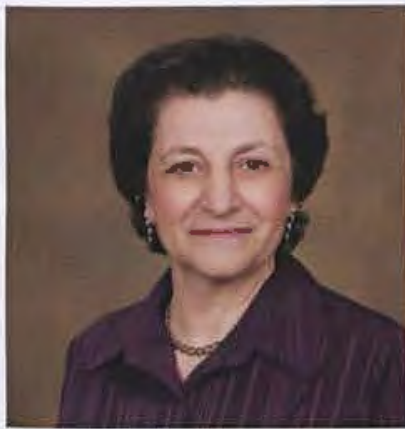

Fran Andrews

Office Manager

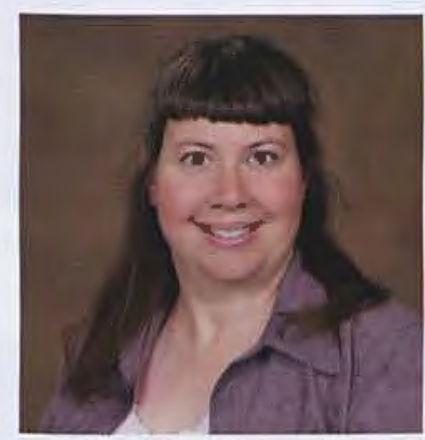

Julie Deardorff Director of Library Collection Services

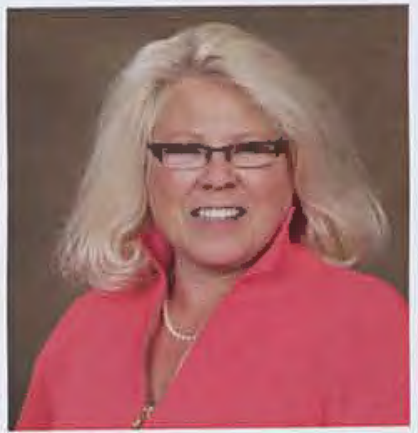

Janice Bosma

Associate Dean

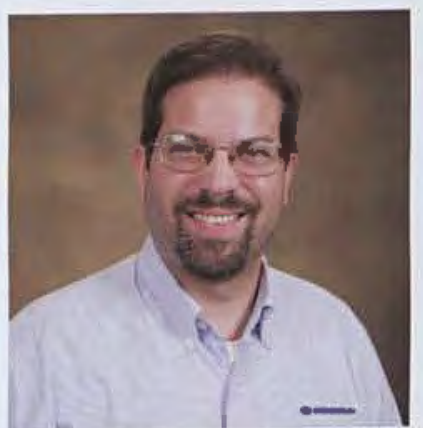

Scott Deetz

AV Services Manager

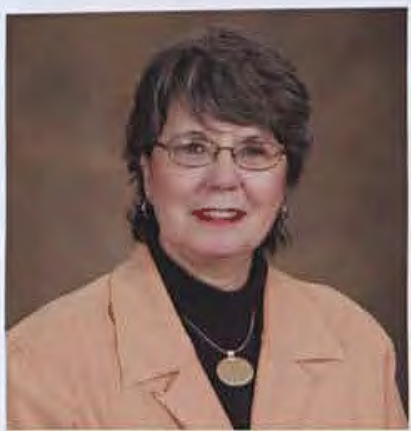

Lynne Funtik

Senior Reference Librarian

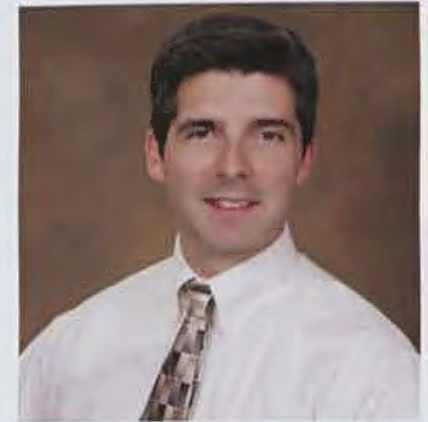

Brent Etzel

Instructional Services Librarian

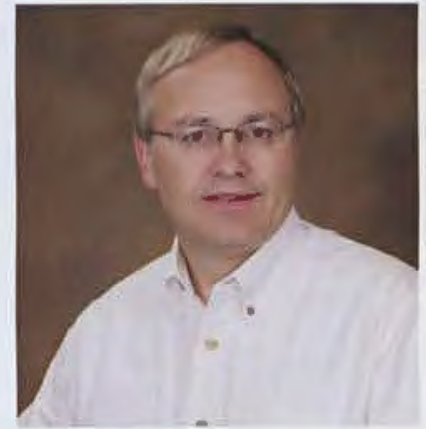

Jeffrey Gates

Instructional Services Librarian

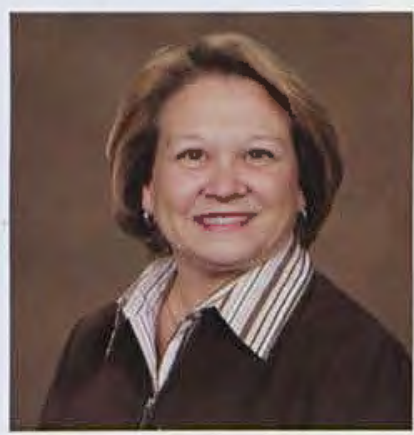

Andi Mounts

Assistant Circulation Manager

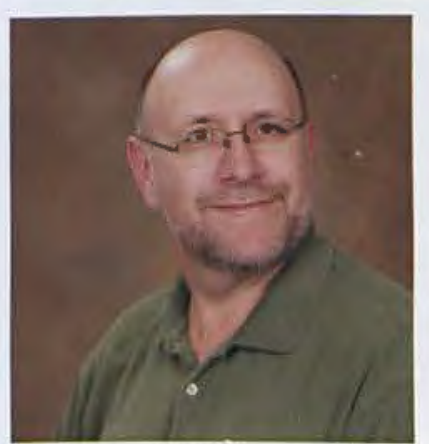

Richard Wiliams

Service Technician

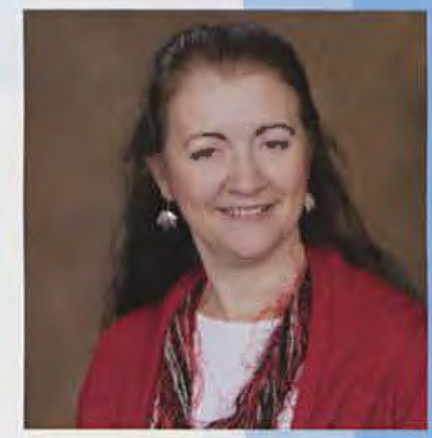

Tonya Fawcett

Director of Library Public Services

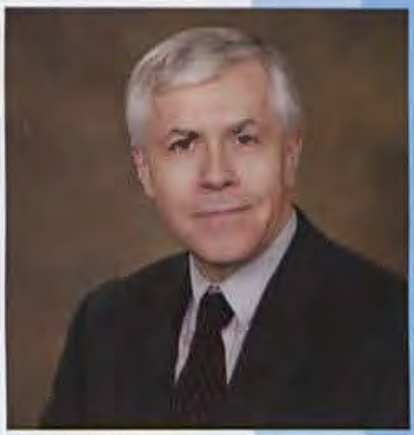

Greg Martin

Curriculum Materials

Center Librarian

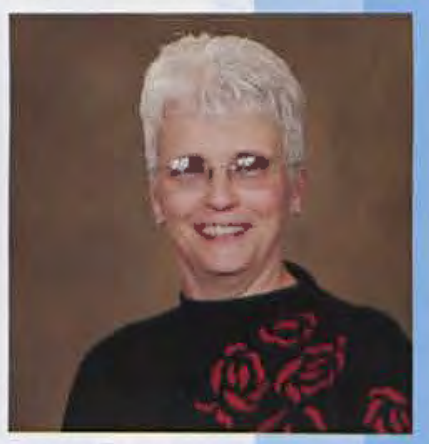

Patty Stutes

Desktop Publishing

Consultant
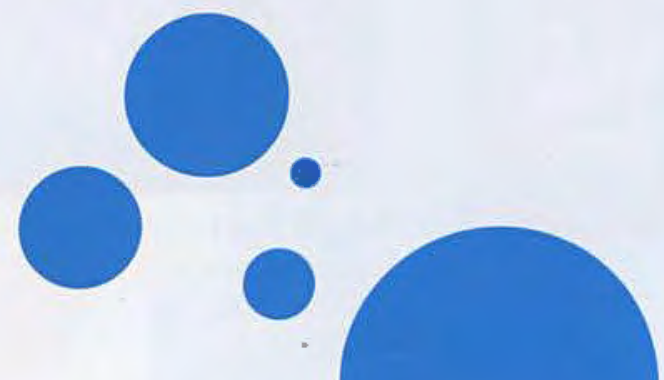

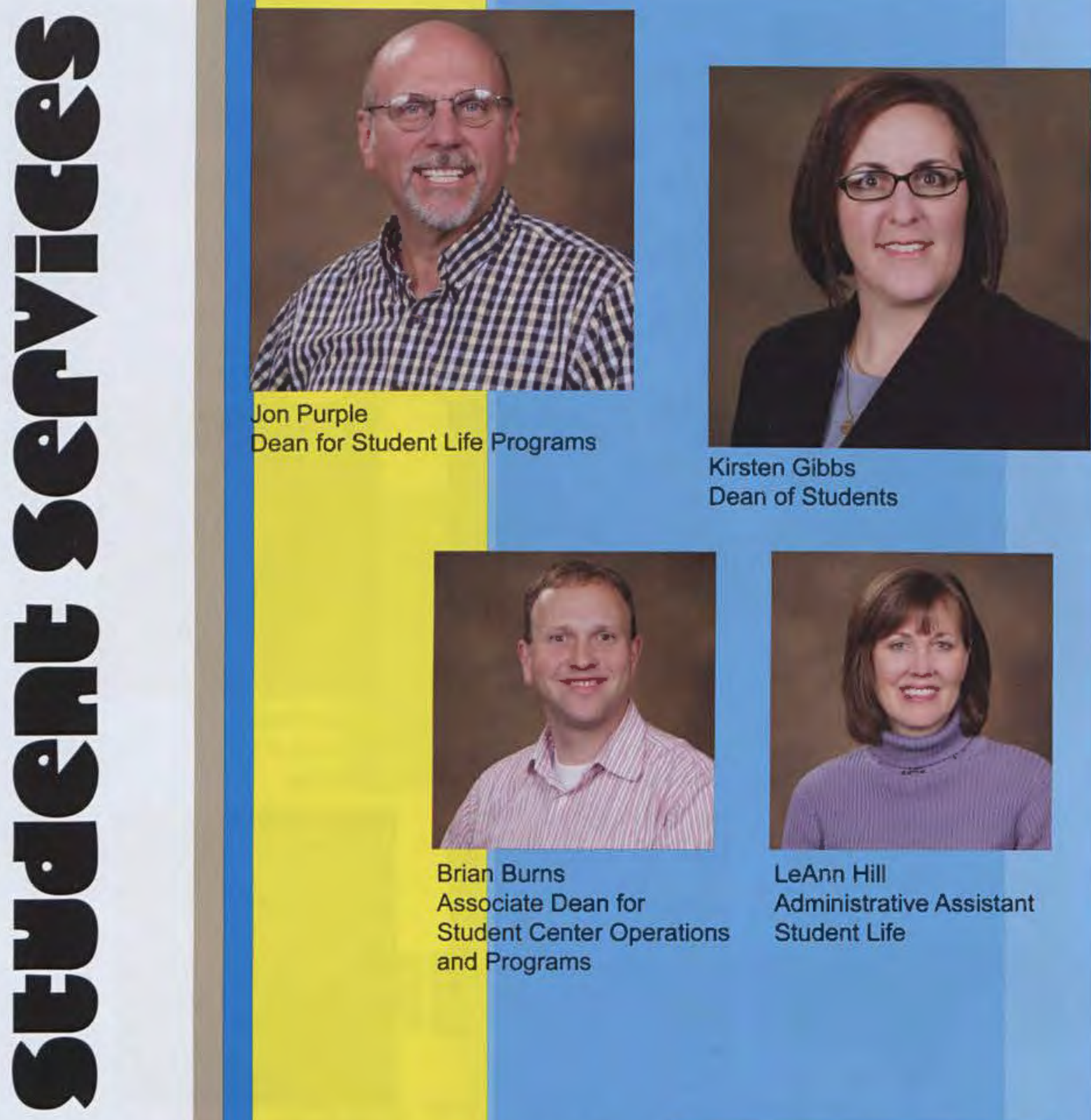

Dean for Student Life Programs

\section{Kirsten Gibbs \\ Dean of Students}

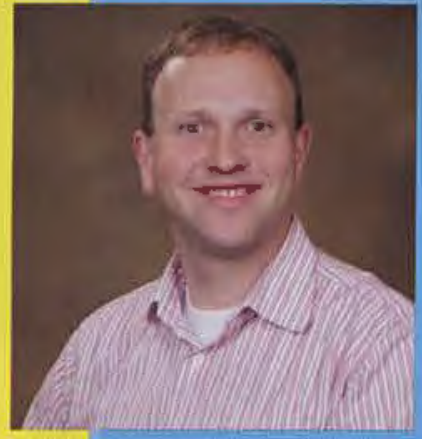

Brian Burns

Associate Dean for

Student Center Operations and Programs

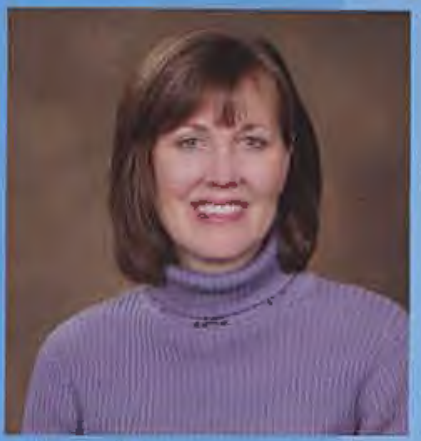

LeAnn Hill

Administrative Assistant

Student Life

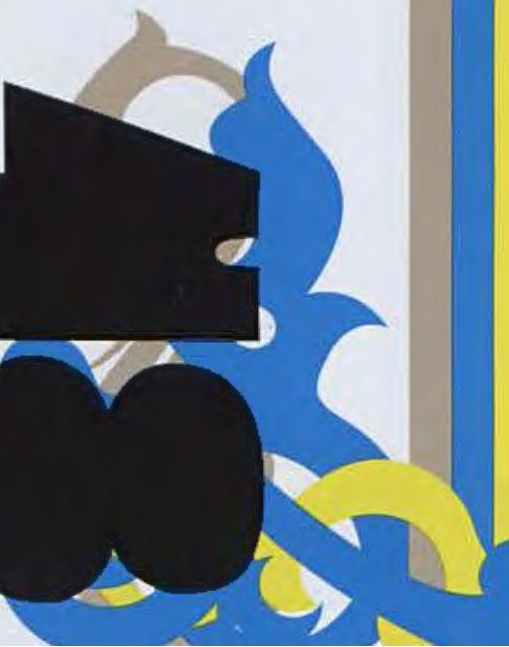

ceunseman scprices

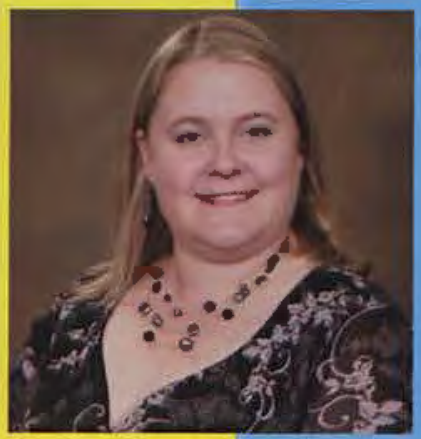

Jennifer Beck

Counselor

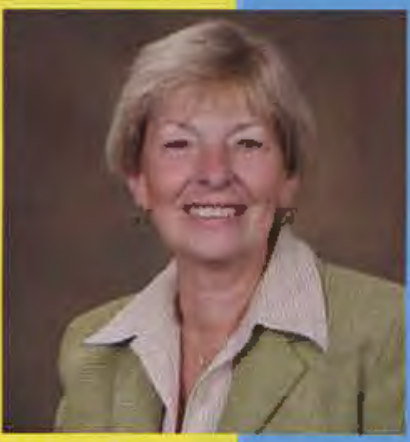

Helen Blumenstock

Secretary

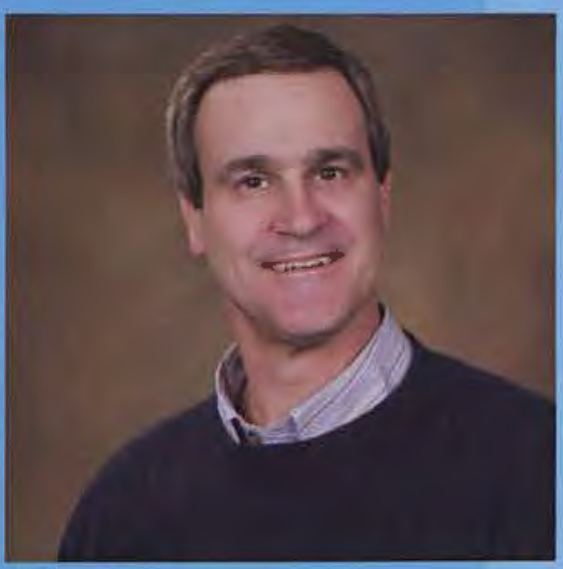

John Potter

Director

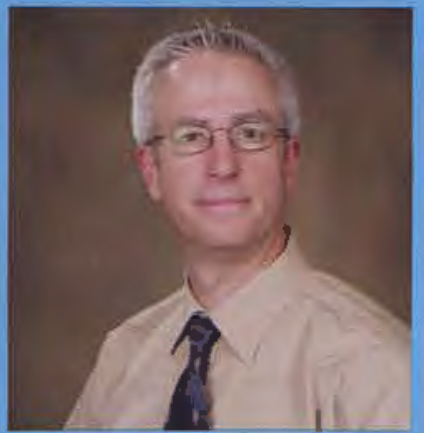

Jeffrey Fulmer

Counselor

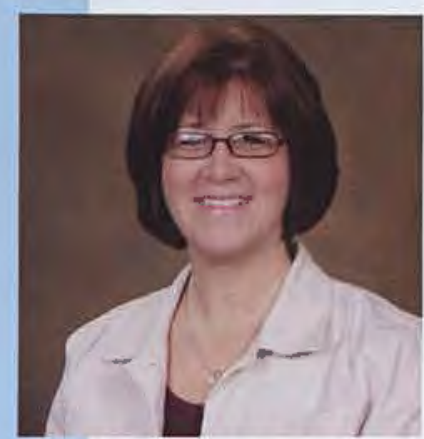

Elaine Keller

Administrative Assistant to Vice President of Student Life

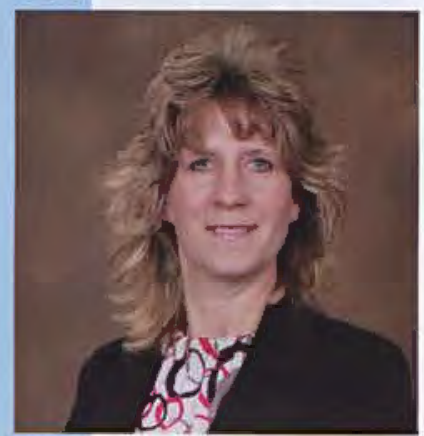

Dawn Scott

Information Center

Coordinator

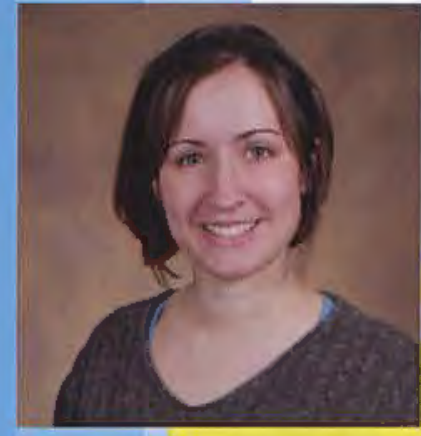

Laura Rost

Counselor 


\section{OMS}

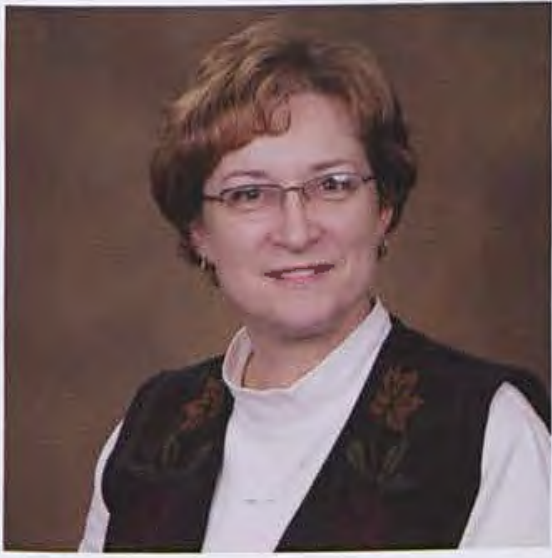

Debra McDonald

Director

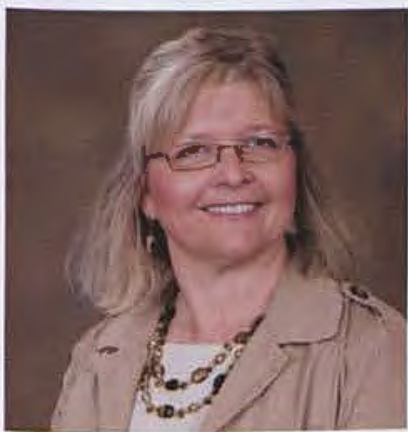

Kina Bostater

Medical Secretary

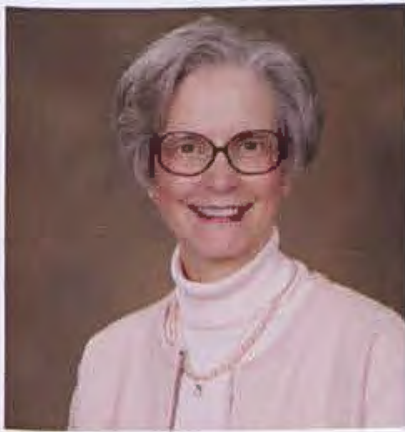

Murtha Kaercher

Student Insurance

Coordinator

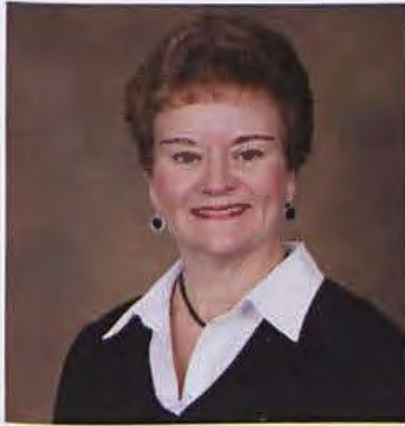

Beverly Robey

Nursing Supervisor

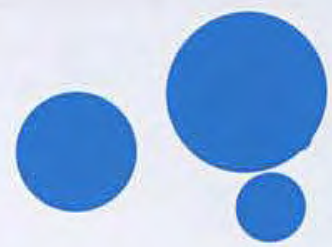

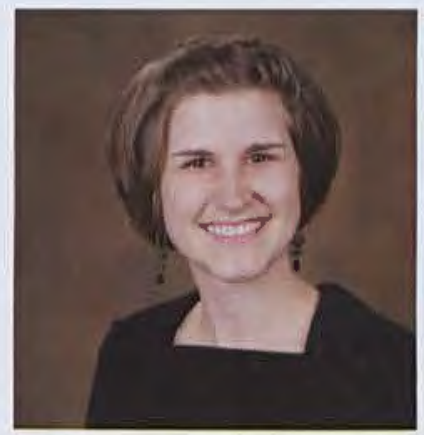

Jennifer Flynn Office Manager

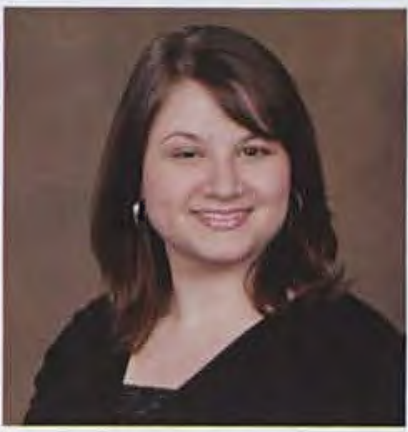

Laura Jolly Assistant Director

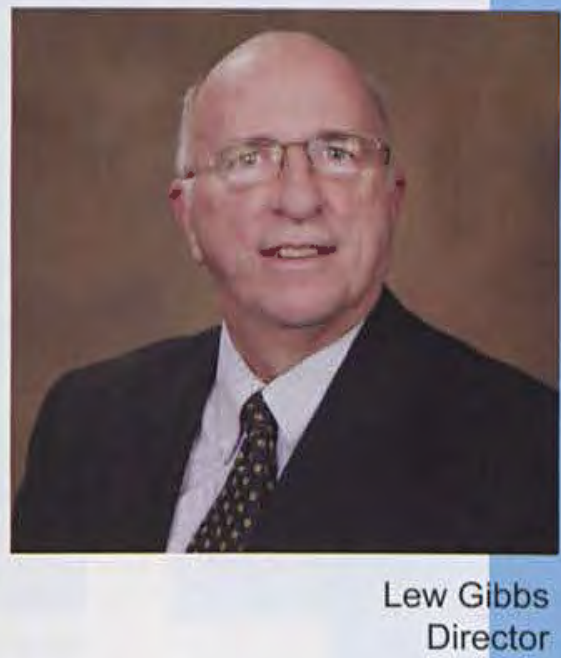

resident Dirceters

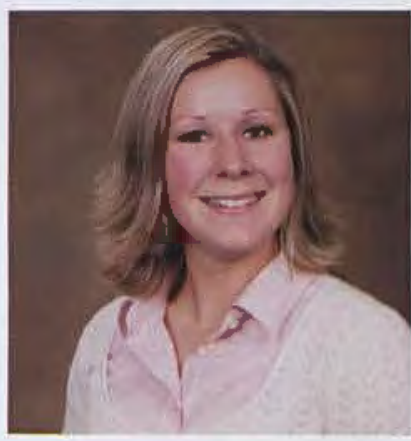

Lynsey Fabian
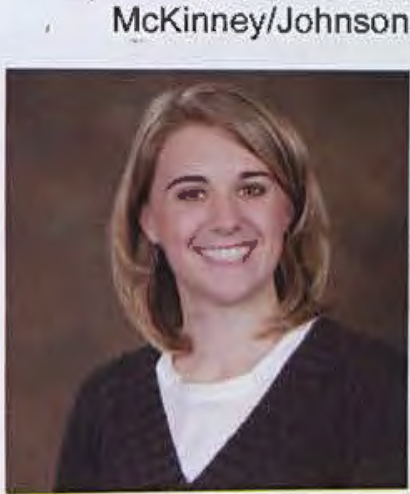

Sarah Niedermayer Maddox

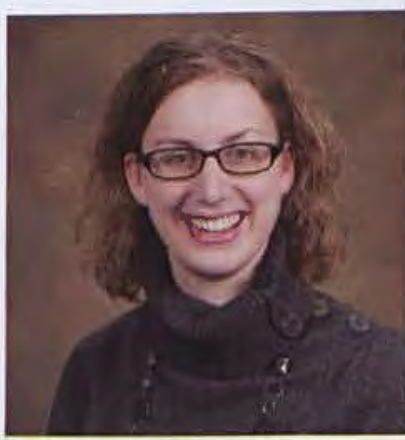

Becky Stowers

Faith

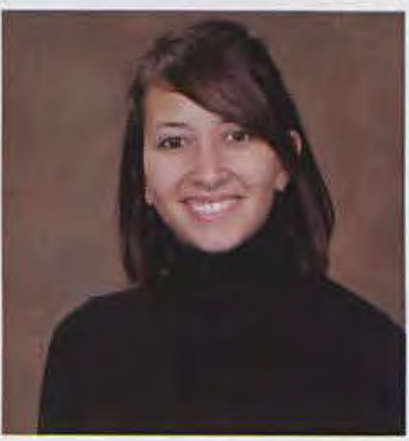

Kristie Febo

Willetts

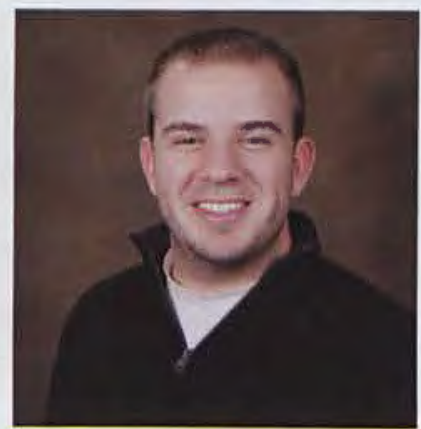

Joseph Poelzer McChesney/St. Clair

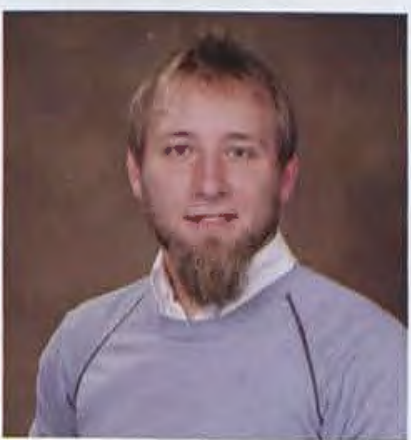

Matthew Thompson

Rickard

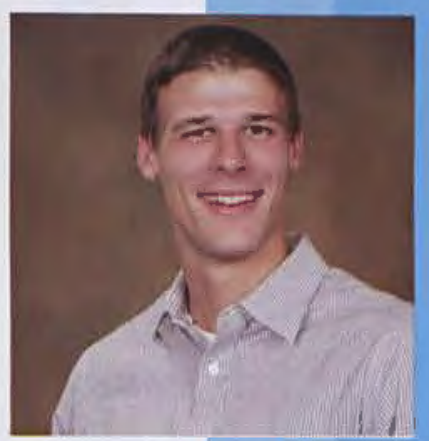

Anthony Mandela Lawlor

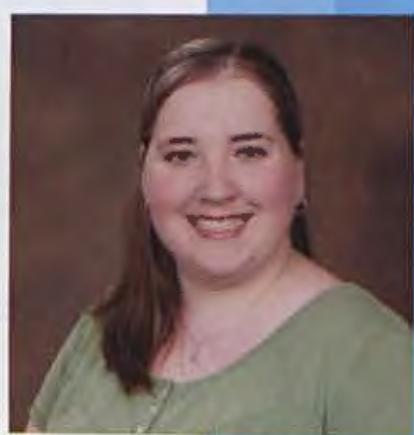

Ruth Poling Printy

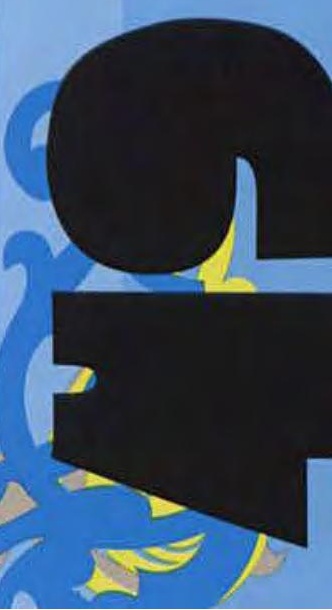



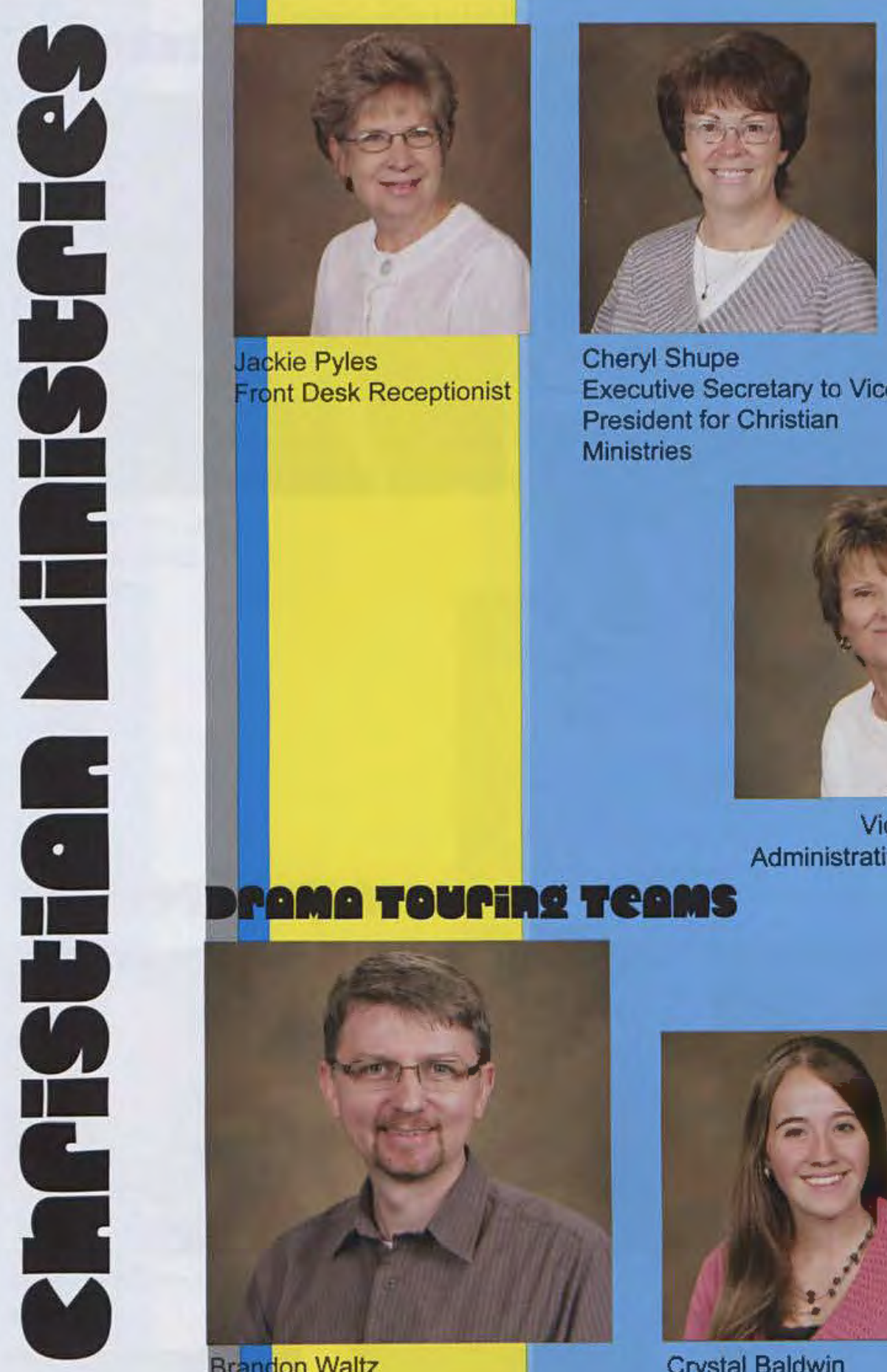

Jackie Pyles

Front Desk Receptionist

\section{Cheryl Shupe}

Executive Secretary to Vice

President for Christian

Ministries

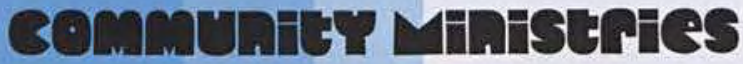

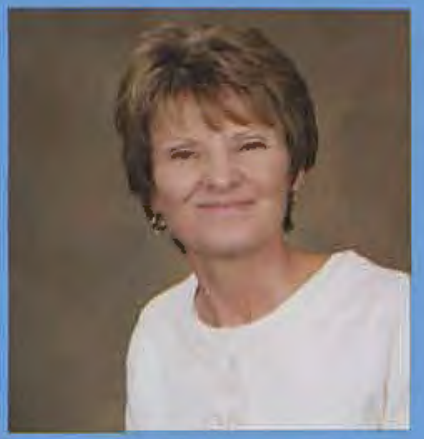

Vicky L. Hines Administrative Assistant

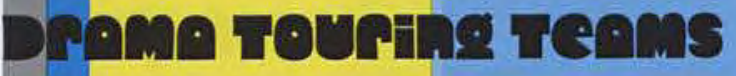

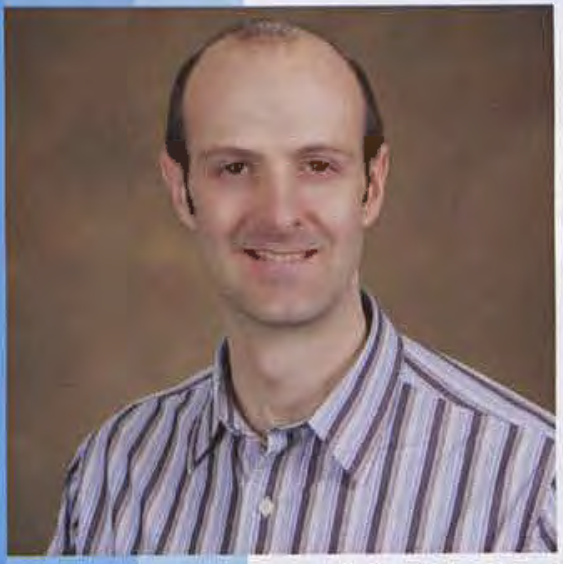

John Wambold

Director

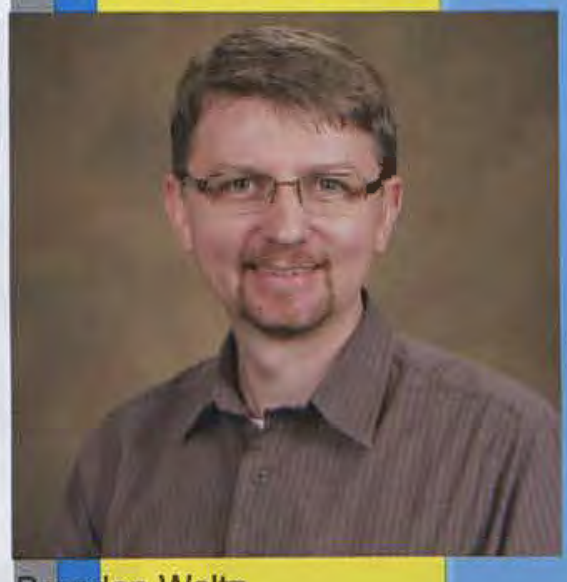

\section{Brandon Waltz Director}

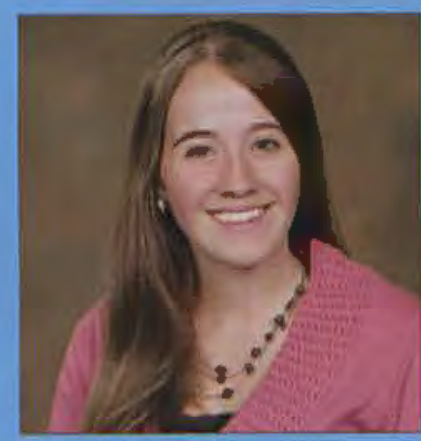

Crystal Baldwin

Administrative Assistant

\section{Diseipresnip Mitherices}
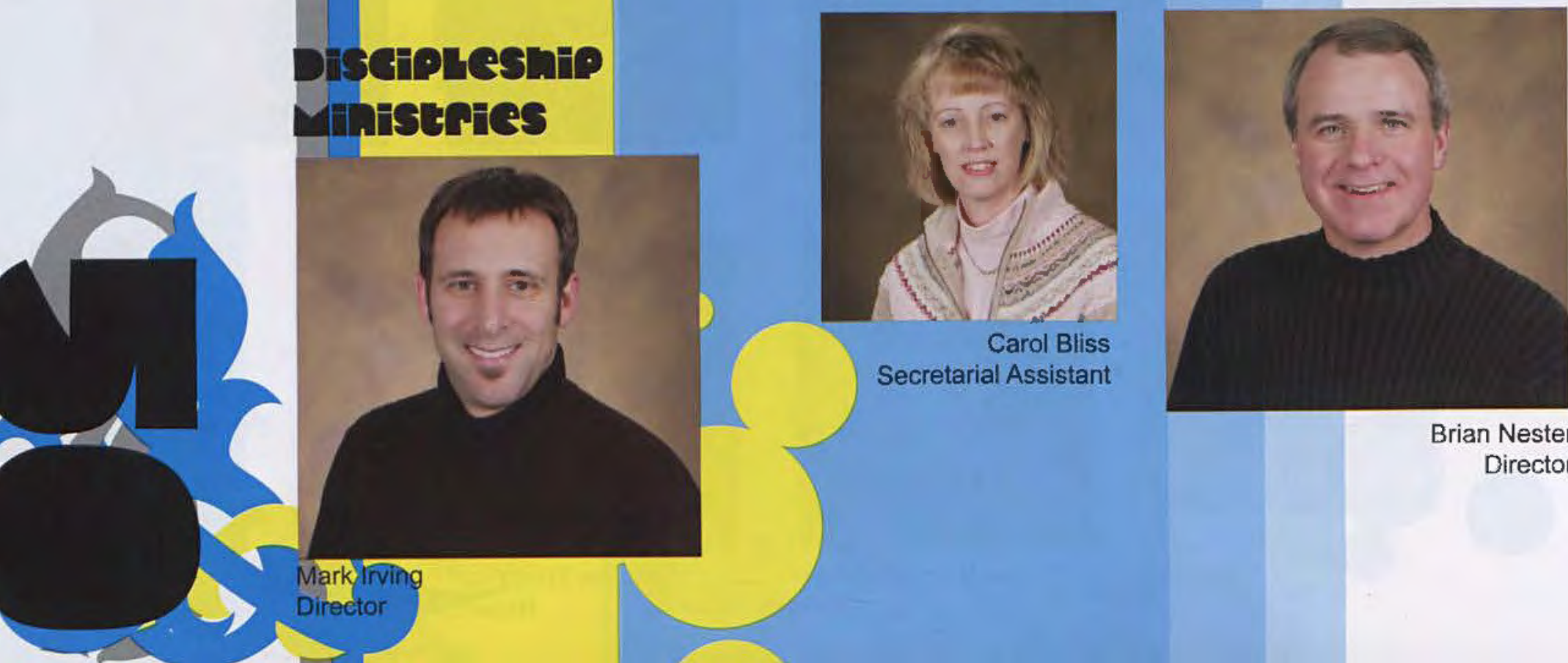

Brian Nester Director 

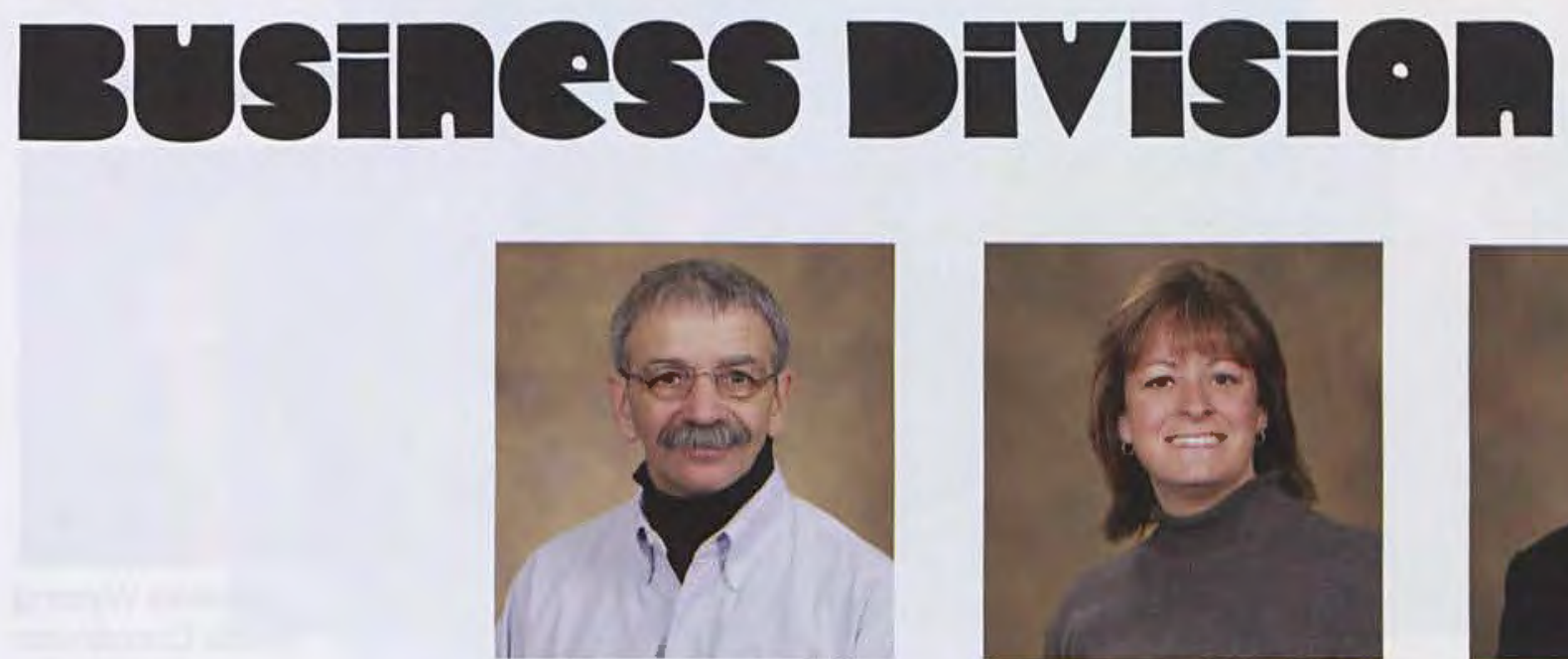

Bob Bielek

CAD Technician

\section{rest Office}

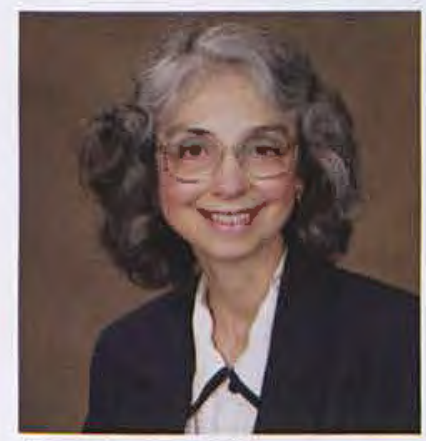

Bonnie McGillivray Supervisor

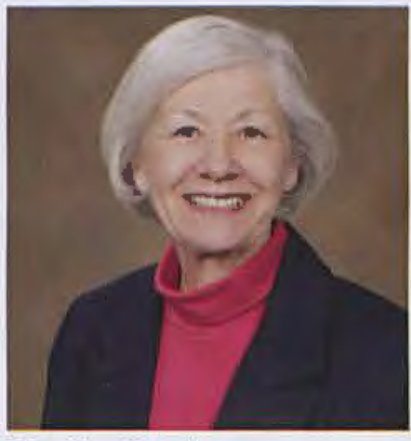

Phyllis Morris Postal Clerk

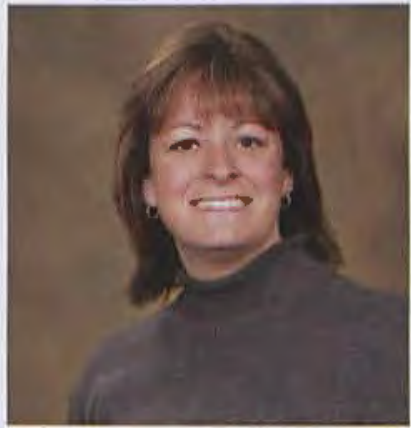

Cindy Weir

Payroll Supervisor

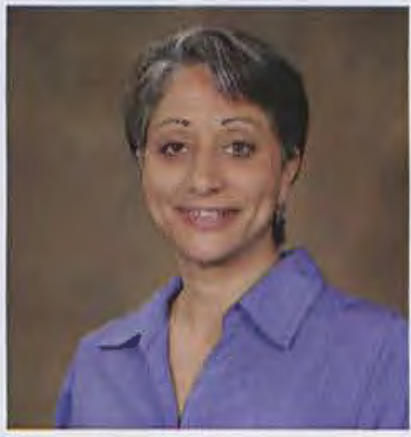

Diane Reid

Postal Clerk

\section{beorstere}

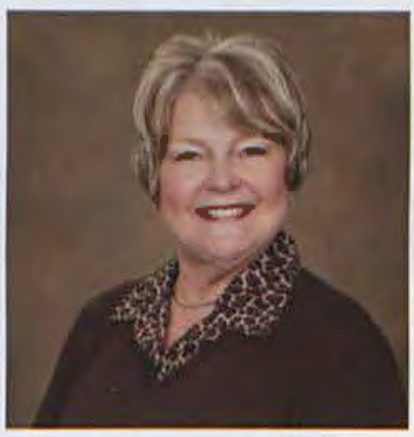

Jeanie LeBlanc Gift Buyer

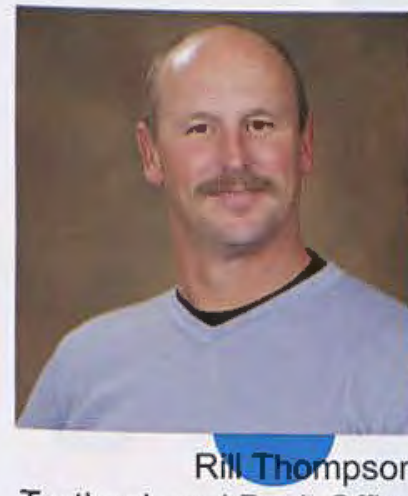

Textbook and Back Office Supervisor
Music and Sportswea Buyer

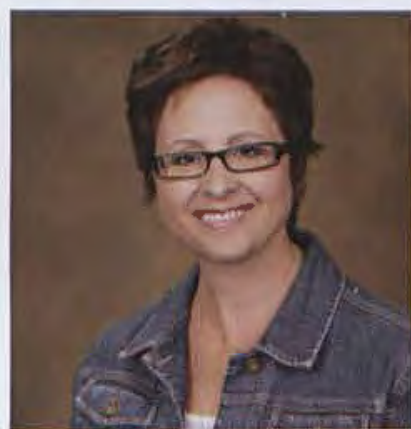

Ann Delange

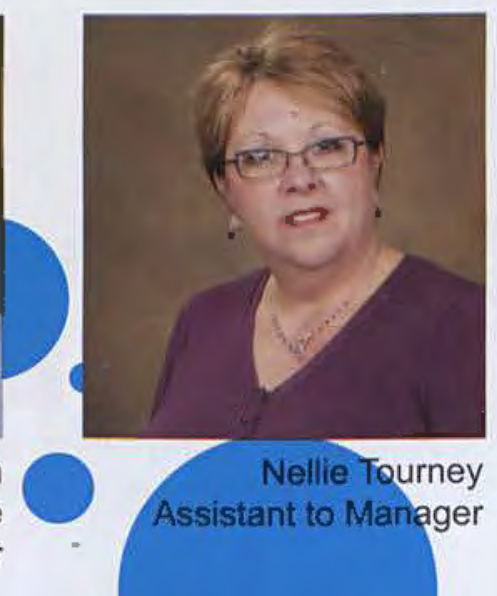

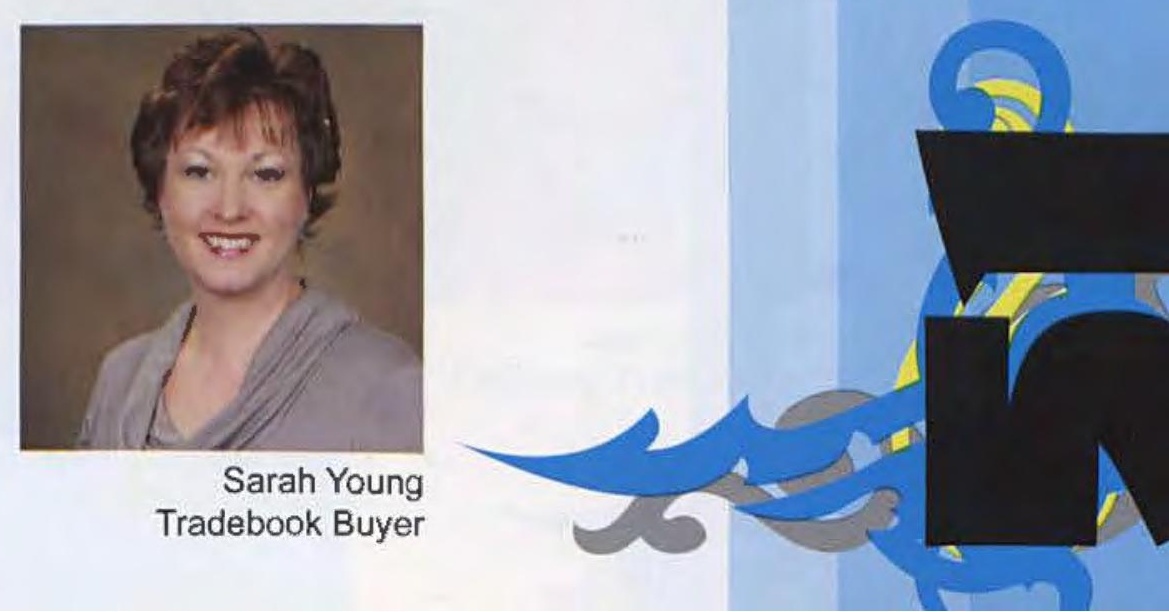




\section{Chupen end senoer rerotions}

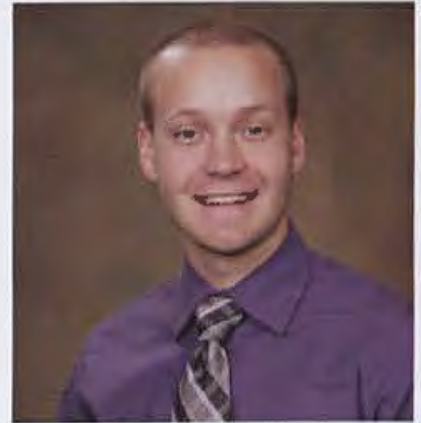

Scott Aker Assistant Director

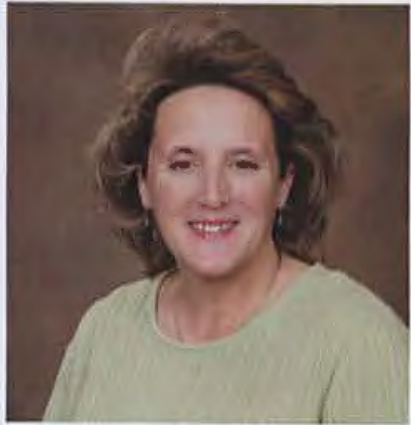

Joan Wilson Assistant Director

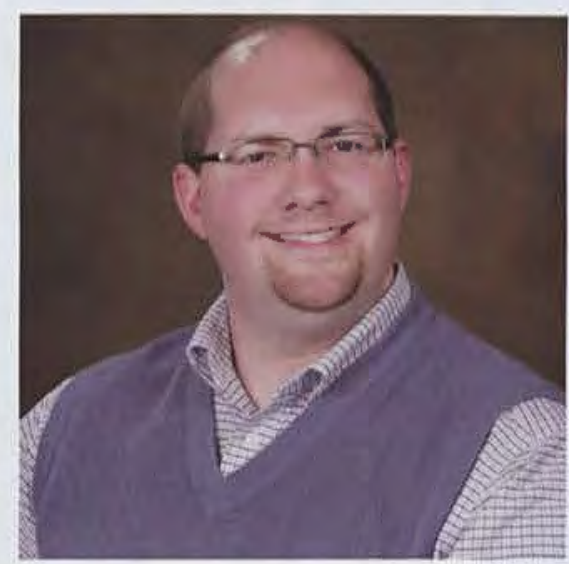

Joel Tomkinson Director

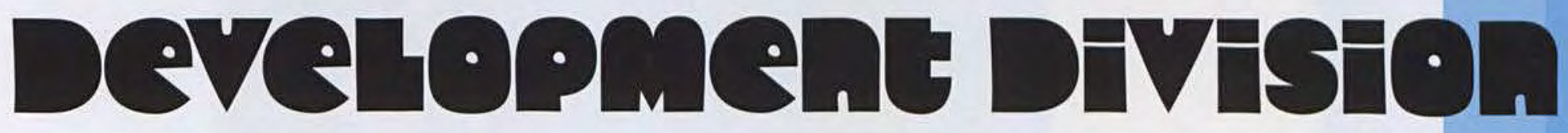

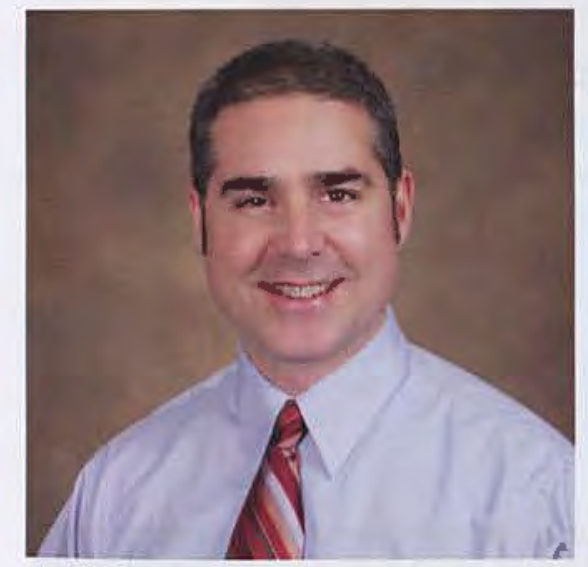

Jeff Beste

Director

\section{Arumat reretions}

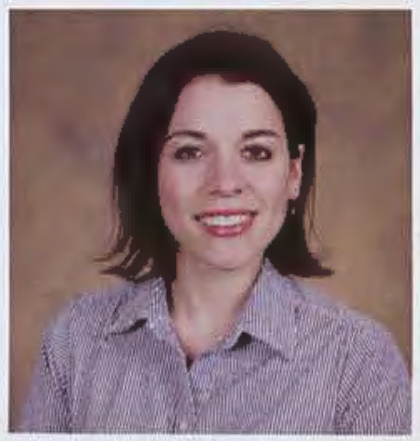

Michelle McCune

Assistant Director

\section{CDrredio}

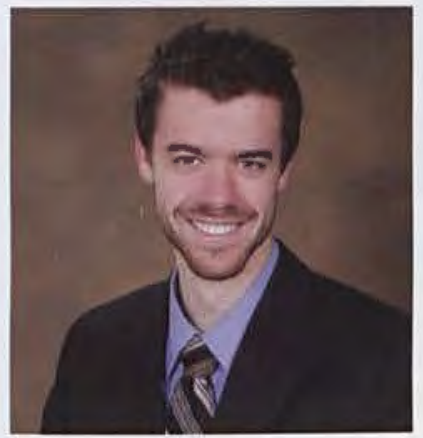

Jordan Link News Producer

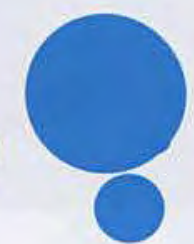

cedopvine rund

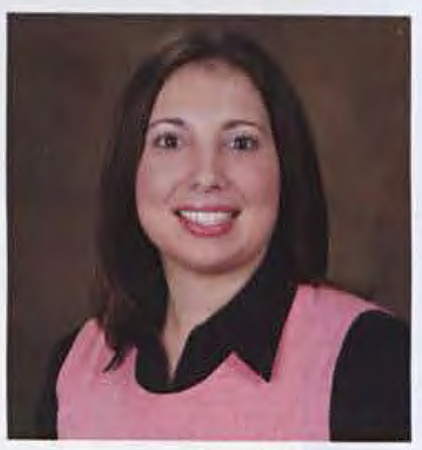

Jen Castellani Cedarville Fund Officer

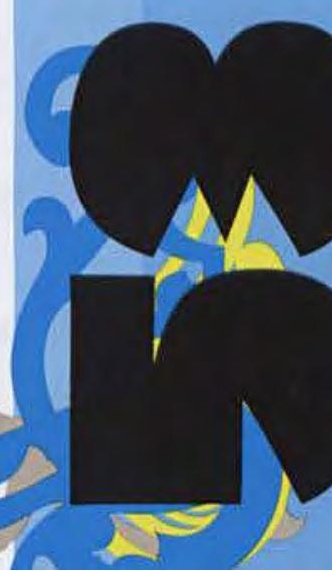


Cynthia K. Davis

Assistant to the Registrar
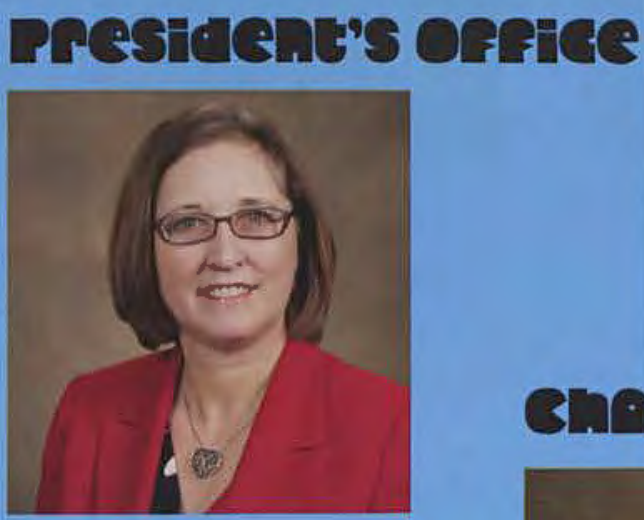

Carol George

Executive Assistant to the President

\section{Ghongrep's effice}

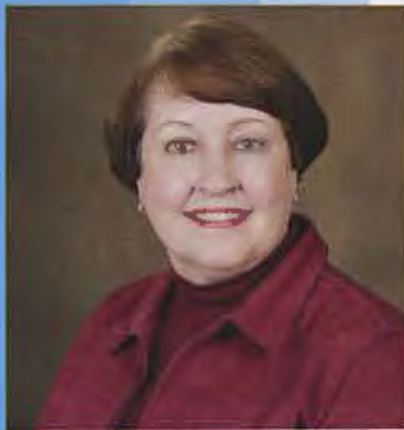

Lynn Rohm

Administrative Assistant

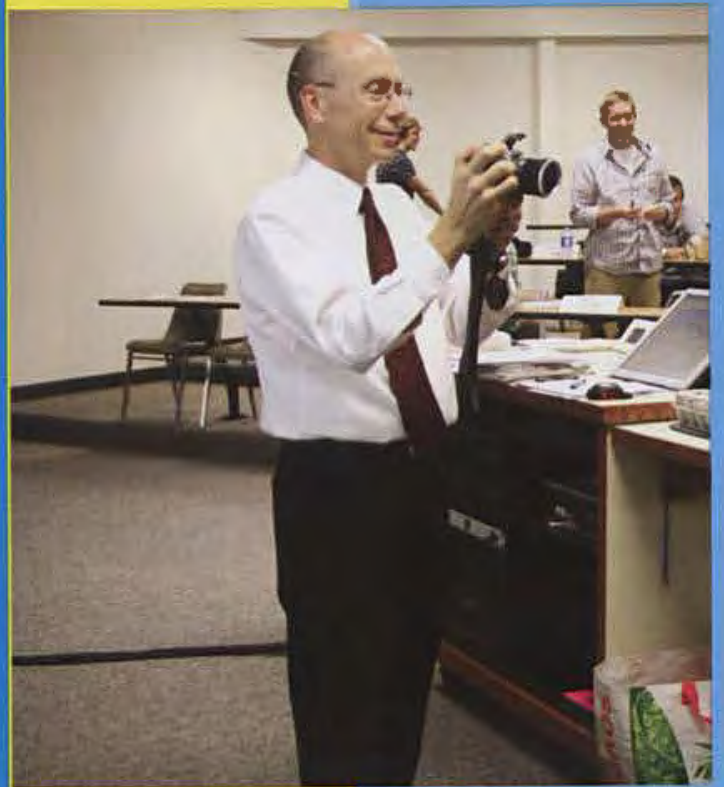

"Master Daniel Sterkenburg is the coolest adjunct professor in the whole DBA department!" says John Bertsche.

Peggy Grigorenko pours herself into relationships with students with a good time of fun and games.

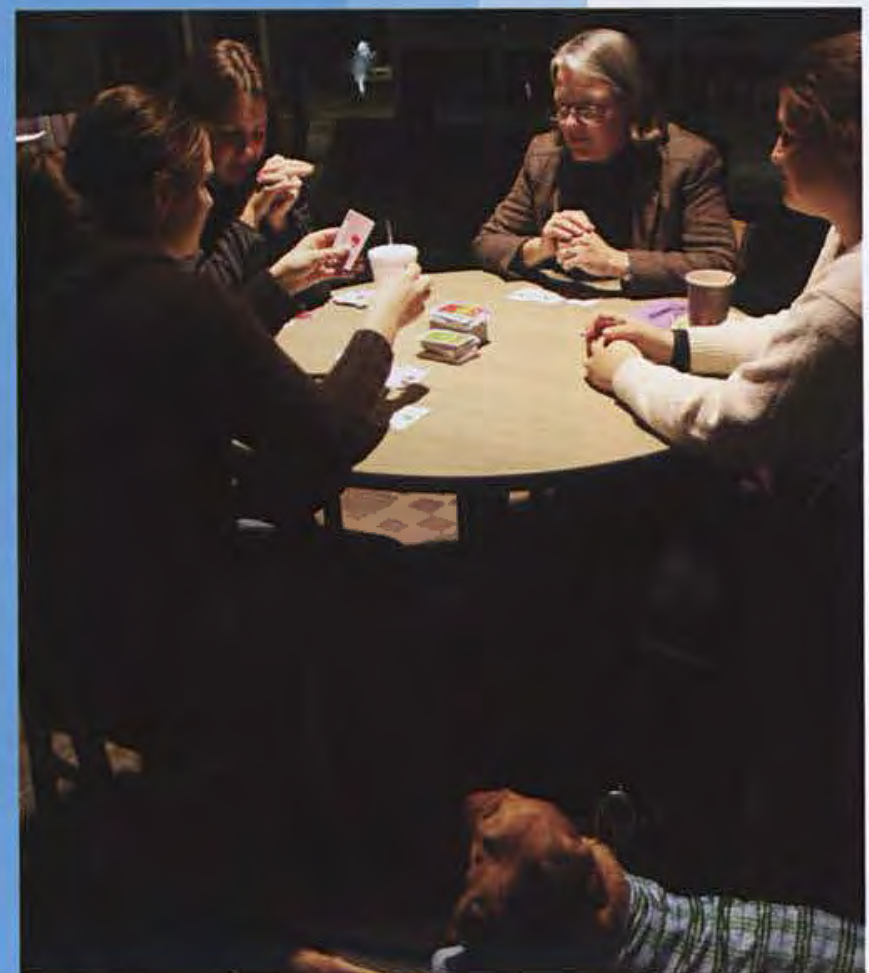




\section{A endenic Admentseroton}

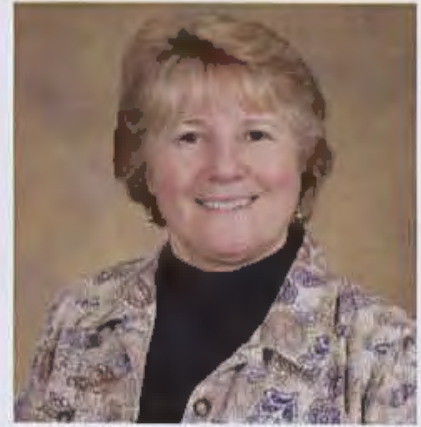

Joy Williams

Administrative Assistant Academic Systems

\section{computep sepvices}

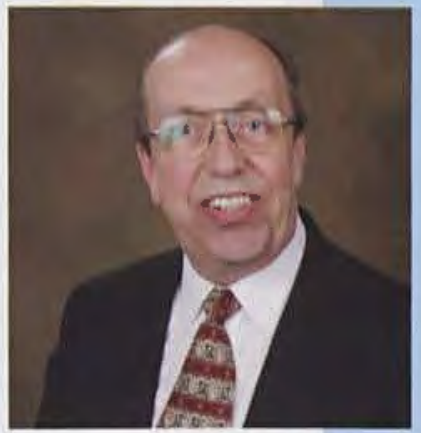

David Rotman Chief Information Officer

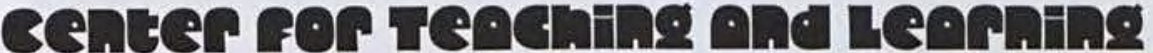

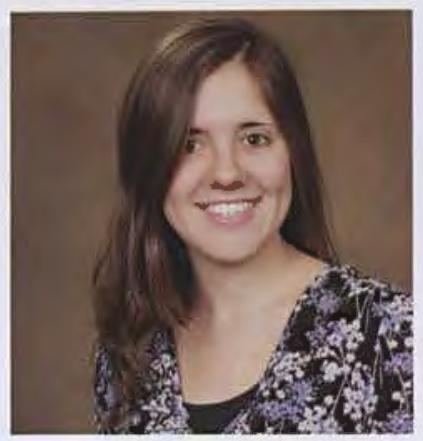

April Cooper

Educational Technology

Analyst

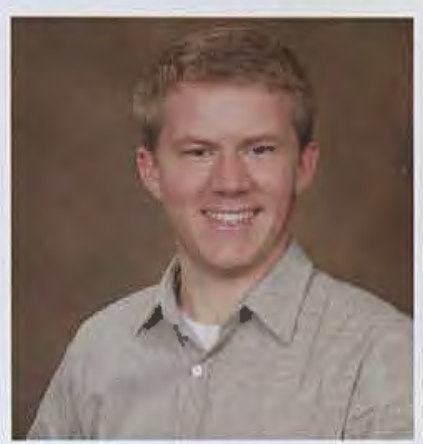

Phil Schanley

Educational Technology Analyst

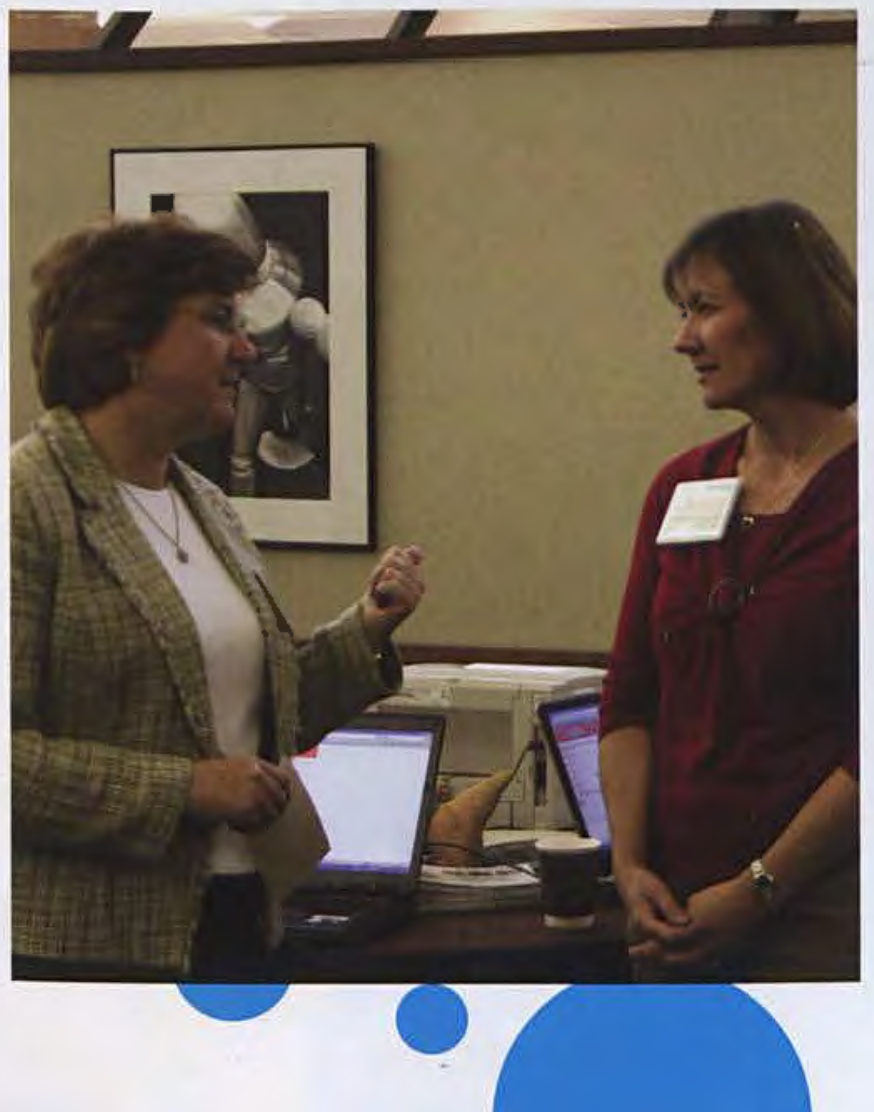

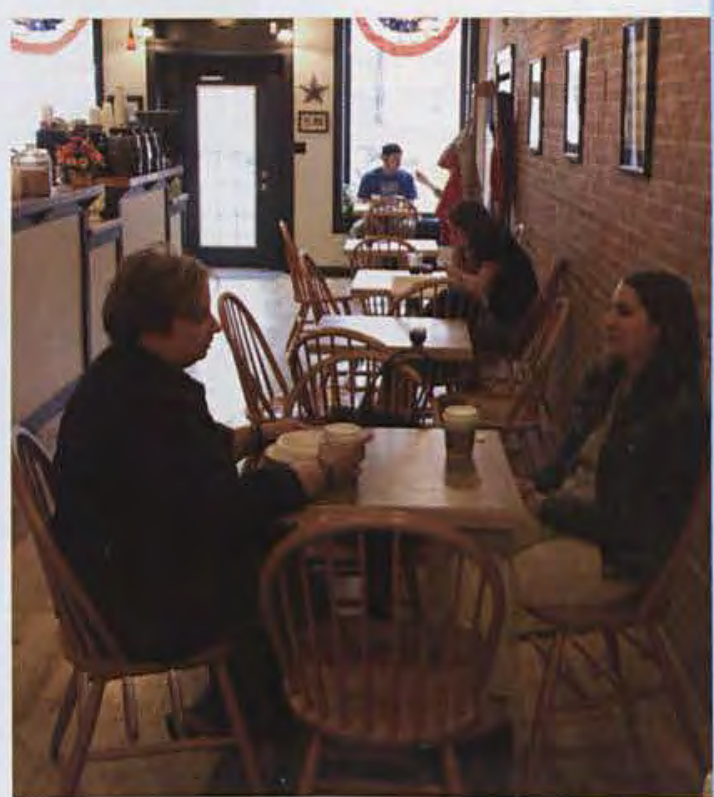

Julianne Sanburg meets with

Dr. Merchant at Stoneycreek Roasters for conversation and coffee.

Rebecca Kuhn and Kathy Miller of the Cove stand by to answer students' questions in the SSC.

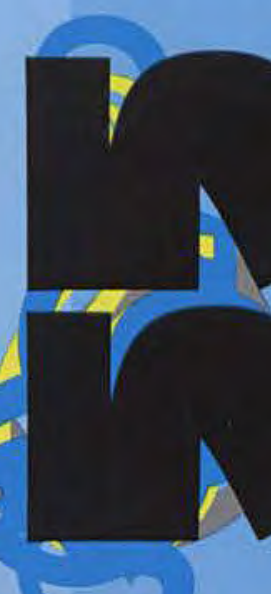


Mr. Bruce Grimes, adjunct professor of art, proves that he doesn't just teach things like ceramics, he has definite skills. He shows that here as he works on a project from raku firering night.

Erin San Gregory explains, "The group project in Worldview Integration really made me practice what I was learning in class. Some group members held very different opinions about our topic, so I got to apply what $\mathrm{Dr}$. Smith taught us about learning from people with different viewpoints."
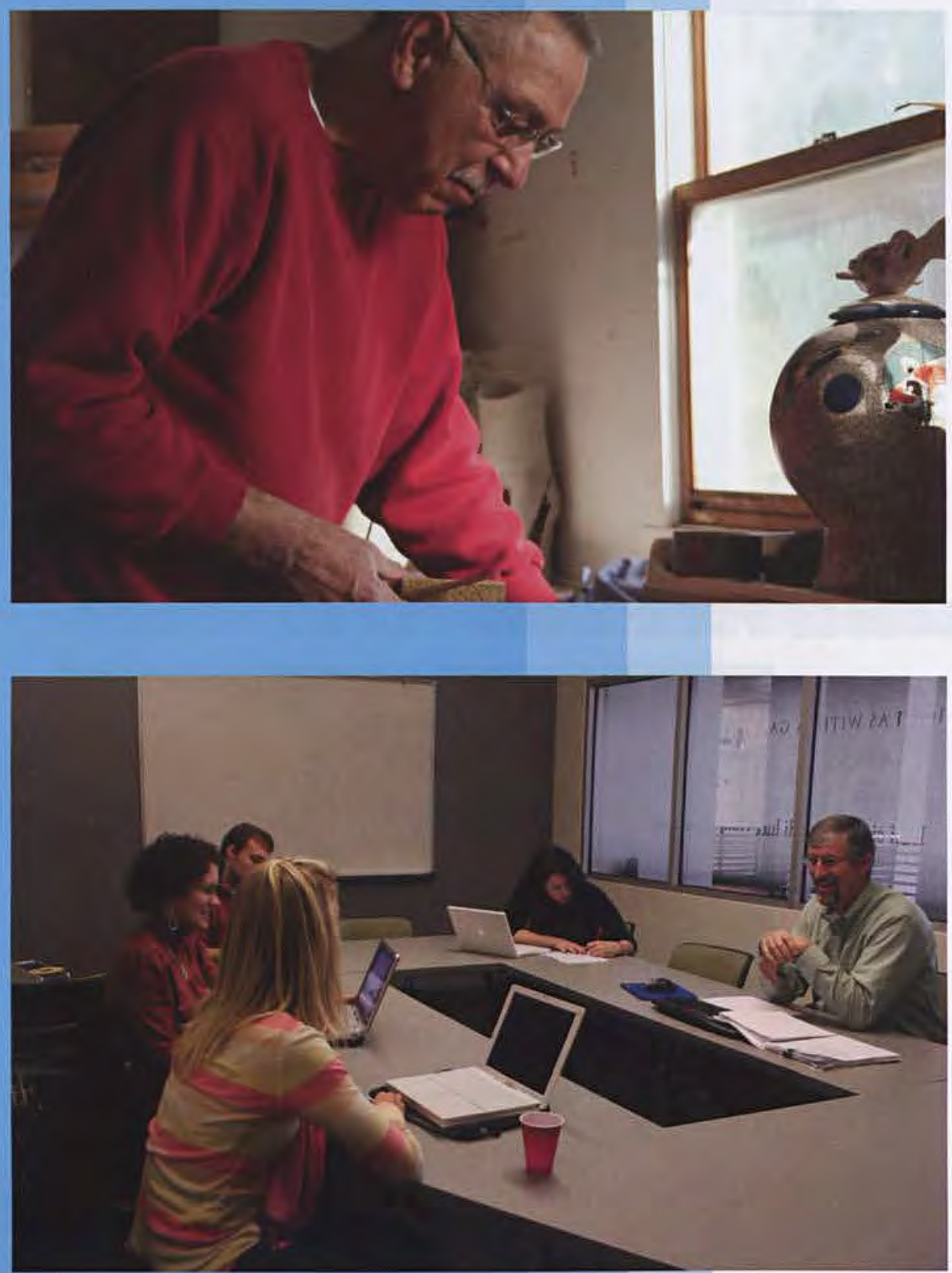

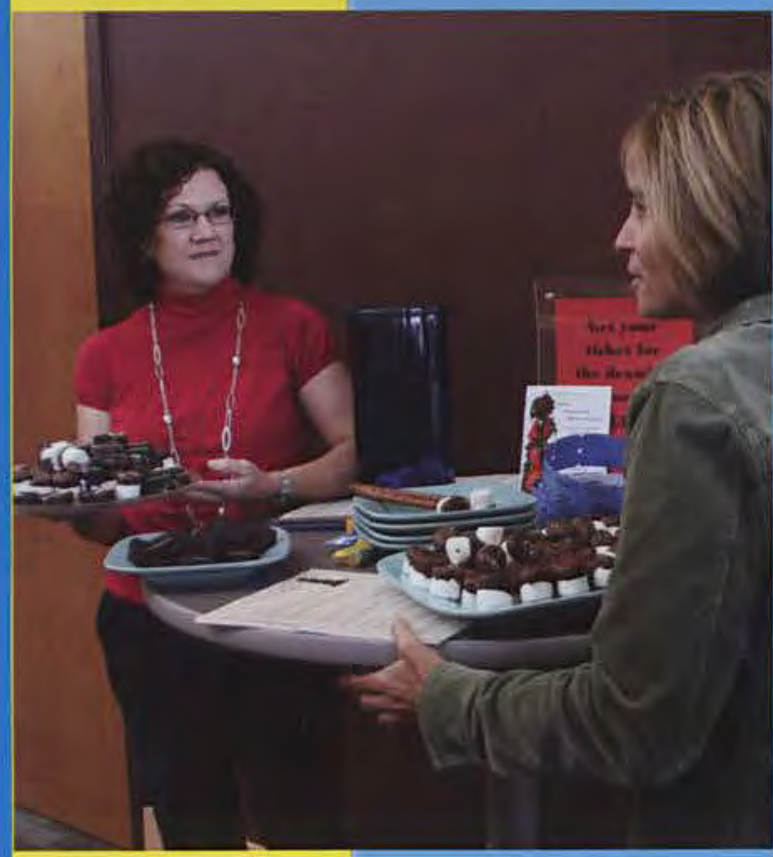

Staff members at The Cove serve up delicious treats for the Triple Chocolate Extravaganza.

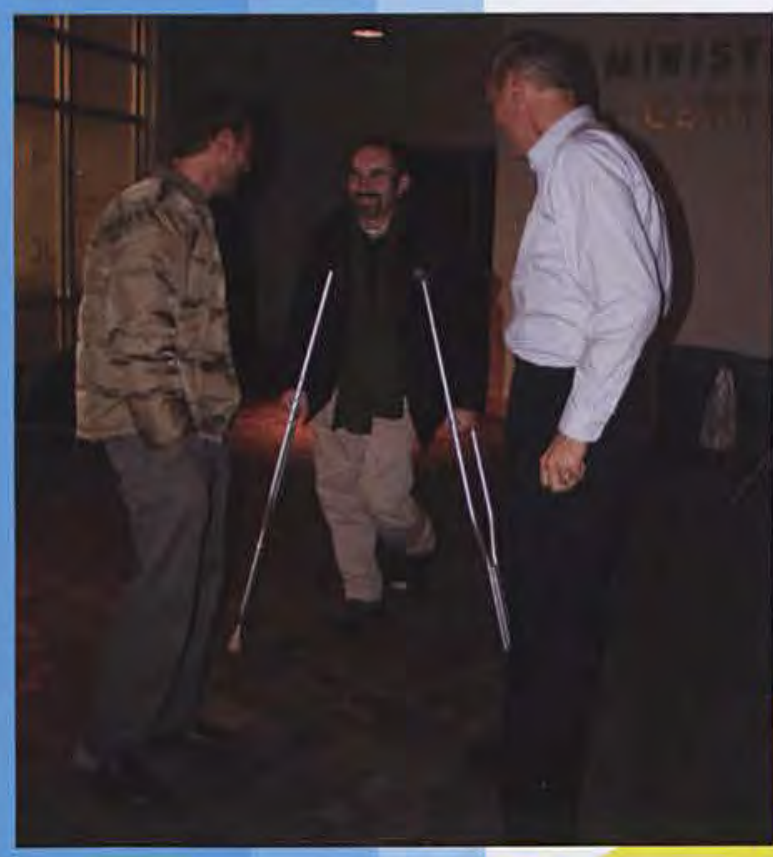

Jeff Reep and Dr. Grigorenko help make sure Dr. Miller doesn't get into any more trouble. 


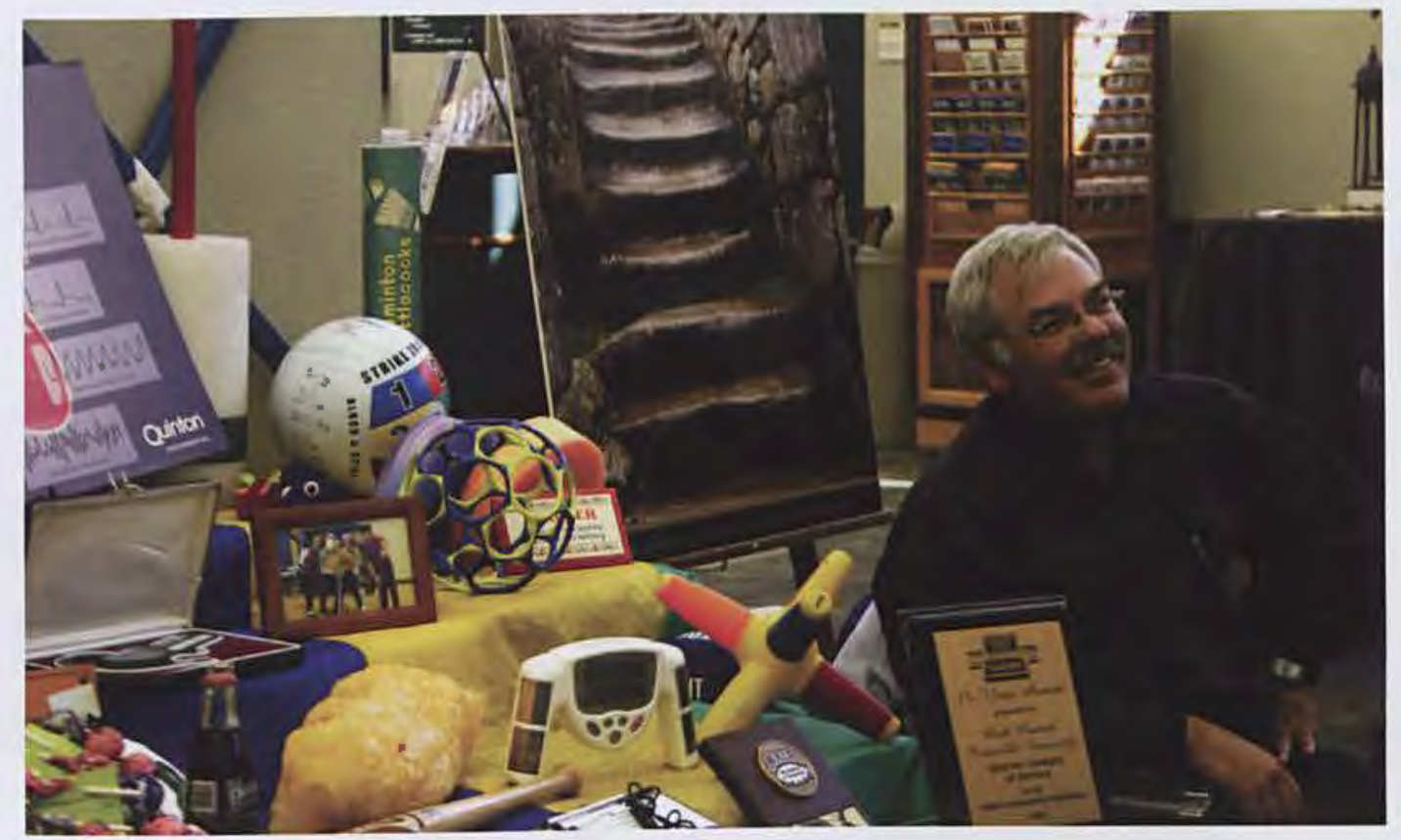

Ben Belleman helps at a Cove event by using his cheery smile and an array of props to appeal to inquisitive students.

Juthe furt is nenest, epen, end reek. she is Cncoupegine and inspiping as a professer but ovaris nerserf outside of chess. I rnow she cen be a suppert and advecetc in and out of chess fer me." - Mendr sueg.

"I tove DP. PMipes. we is se funary Cress with nim is ouwars a prot. wis rindness to me inside ond outside

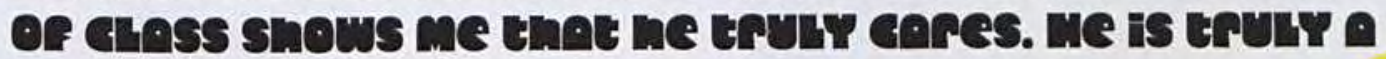
remertoble mon and an ineredible propesser"

- retherine watsen

"DR. Dugre nas been ene of the swectest investers inte my contege wife. mer instruction is precticet,

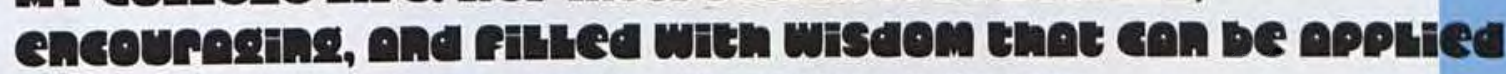

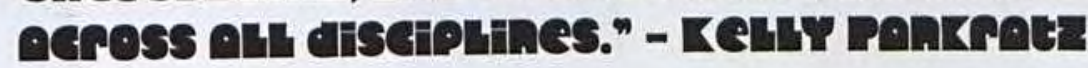

"DP. Fetes undepstends thet comere is a pressure end stress finied time in oup wives end understends the concept of erece. I rove, respect end edmire mim so mucn!n - Amber rpince 


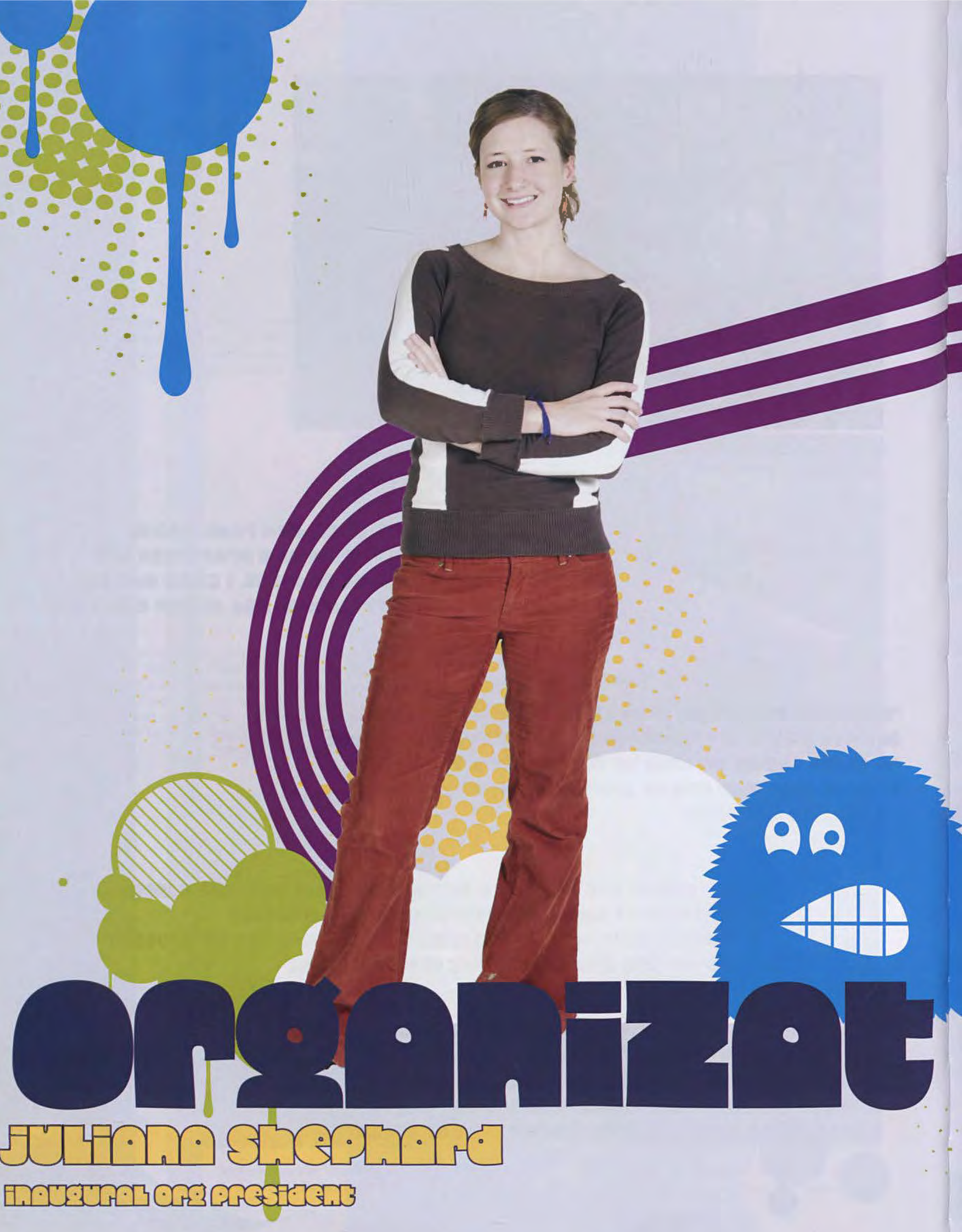


It was so hot outside. The fluid Egyptian sun rained down a steady stream of heat that left me sprinkled with sweat. If I focused on the affects of the sweltering atmosphere, I would thirst for relief.

But I was not focusing on the heat. Instead, I was drinking in every word my friend was speaking-words that stirred my heartstrings faster than a fan. She was telling me about the International Justice Mission, an agency that works to offer fair dealings to victims of violent oppression. The mission aids individuals around the world whose struggles with oppression come in the form of sexual exploitation, slavery, police brutality, and trafficking. These people cannot help but be affected by their sufferings, and every day, every moment, they ache for relief.

As I stood damp and breathless in burning afternoon light of Northern Africa, I felt one thing very strongly: the desire to be that relief.

I have always sustained a deep intolerance for injustice. When I was younger, I wanted to "kill the terrorists," I thought. But as I researched terrorism, I realized that many times people initiated terrorism because of injustices such as poverty and human trafficking. I was then driven to fight these injustices, and I committed myself to working with groups such as the International Justice Mission that would enable me to confront injustice long-term.

When I learned about the International Justice Mission, I saw that they were dedicated to being the relief that I longed to be, so I wanted to join them. I also wanted to help others get involved, and I needed common ground on which to find such people. Cedarville was that common ground.

I knew that as an institution, Cedarville shared the values of organizations such as the International Justice Mission and provided a welcoming system for establishing such organizations. Though Cedarville offers a great variety of organizations for student involvement, the point is not that the different orgs will compete with each other; rather, Cedarville intends for them all to create harmonious opportunities for ministry.

As 1 started the process of forming a branch of the International Justice Mission for Cedarville's campus, I found many others with similar desires for service that motivated them to seek the needy. I encountered students who wanted to plug in to an organization that provided the opportunity for them to develop their passions- passions for equality, for love, and mostly, for people.

Working with Cedarville's new International Justice Mission org enables me to see that the beauty of the body of Christ is the diversity of interests through which the Church reaches the vast range of needs in the world. Orgs here at Cedarville allow students to educate themselves about the issues of the world that concern them and to connect with other believers who share their concern. While I feel strongly for the issues of my org, I know that other orgs also champion great causes, so I would never pressure students to believe that is the most Christian thing to do. I will encourage student who end with victims of injustice to learn more with me, but as long as peokle are pan pating in groups that address the interests of their hearts,

I know that there can be unity in the Body that helps us combat the diverse problems of this world. I love the range of orgs on campus, and I pray that as Cedarville students continue to fuel their passions for goodwill, orgs like the International Justice Mission will persevere as sources of relief.

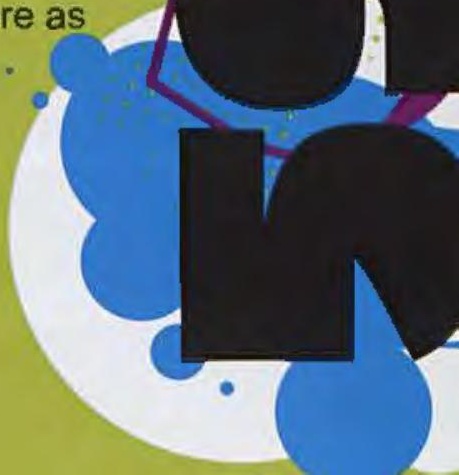



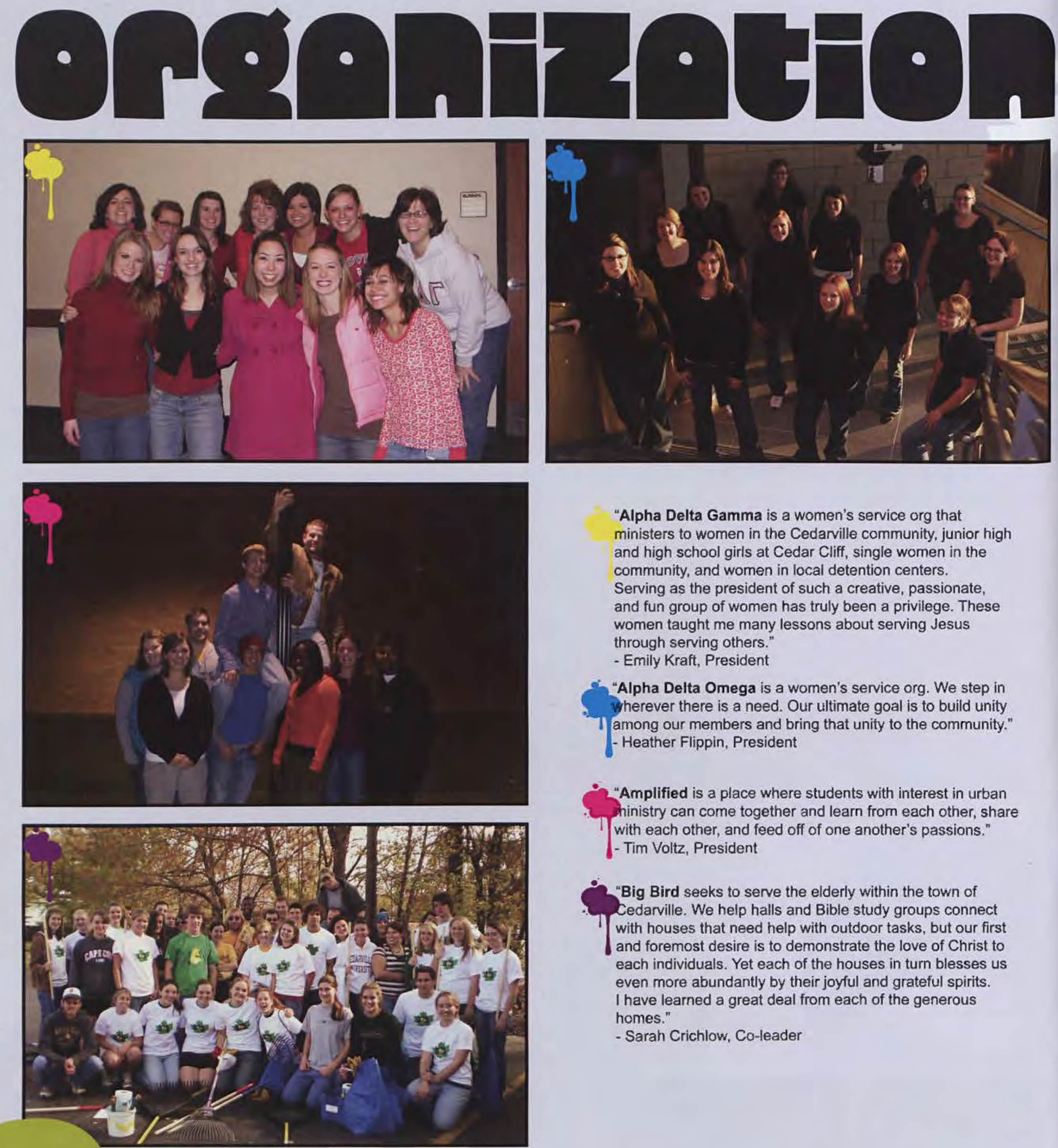

"Alpha Delta Gamma is a women's service org that ministers to women in the Cedarville community, junior high and high school girls at Cedar Cliff, single women in the community, and women in local detention centers.

Serving as the president of such a creative, passionate, and fun group of women has truly been a privilege. These women taught me many lessons about serving Jesus through serving others."

- Emily Kraft, President

S. "Alpha Delta Omega is a women's service org. We step in Wherever there is a need. Our ultimate goal is to build unity

I among our members and bring that unity to the community." - Heather Flippin, President

"Amplified is a place where students with interest in urban hinistry can come together and learn from each other, share with each other, and feed off of one another's passions." - Tim Voltz, President

"Big Bird seeks to serve the elderly within the town of Cedarville. We help halls and Bible study groups connect with houses that need help with outdoor tasks, but our first and foremost desire is to demonstrate the love of Christ to each individuals. Yet each of the houses in turn blesses us even more abundantly by their joyful and grateful spirits. I have learned a great deal from each of the generous homes."

- Sarah Crichlow, Co-leader 

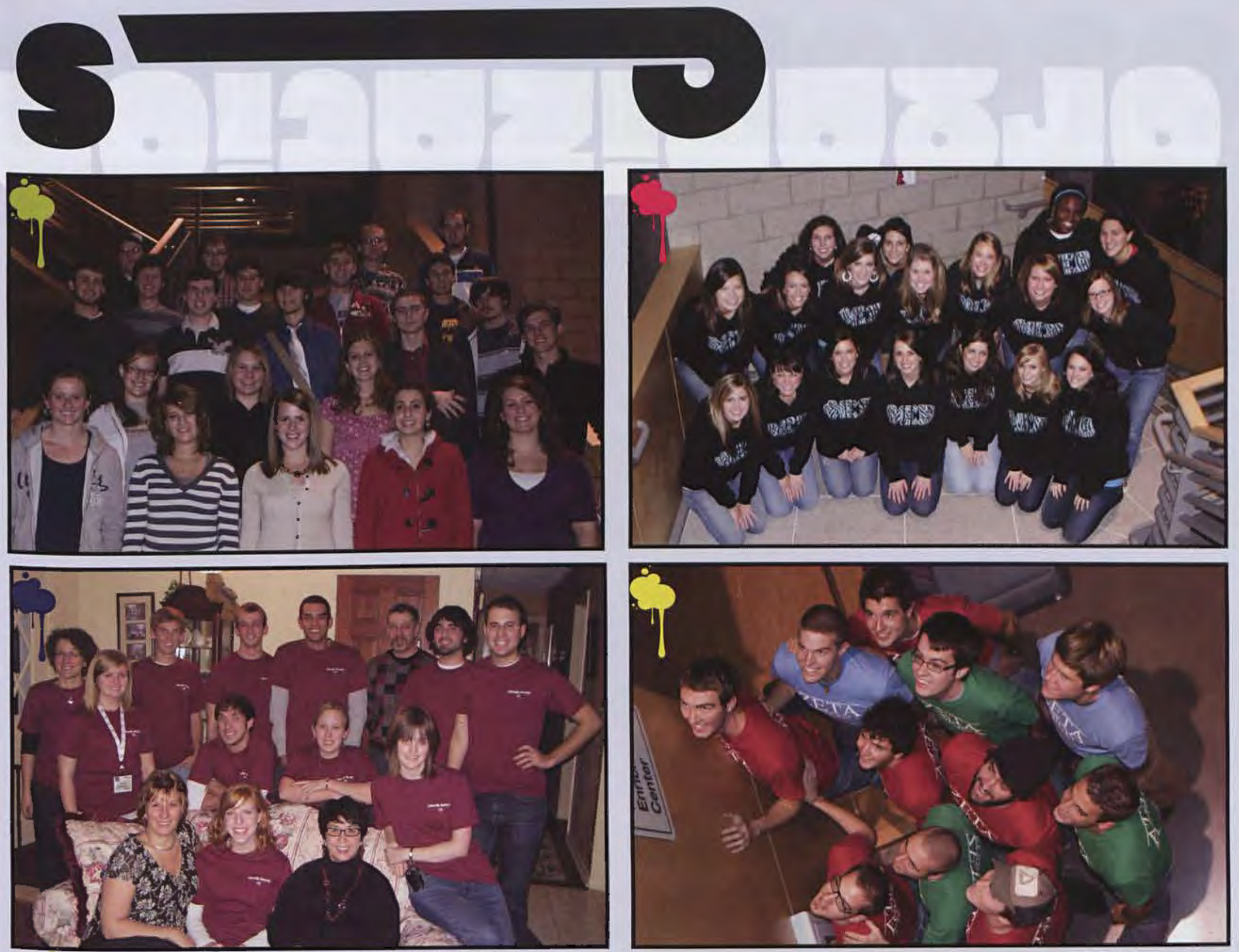

"The College Republicans strive to inform the students of

Cedarville and the surrounding community about current issues and the Republican point of view. We try to encourage students to consider these issues in light of a biblical worldview."

- Rachel Ross, President

in "The purpose of Pi Epsilon Beta is to reach out to women in the

community. We serve other women by reaching out to the school,

1) community, and world through service projects."

- Sandi Larsen, President

"STC was the best this year! We met with alumni from our program, took a trip to Nashville to tour companies, hosted a conference in the Fall, and even got to spend an evening with Mark and Cindy Clifford (in picture), the president and next year's president of STC International." - Adam Evans, President

"Zeta Pi Sigma is a group of guys that are about encouraging other guys on campus to be more like and follow Jesus Christ. We have a different type of friendship, because it allows for deeper accountability while still being able to have fun together."

- Matt Fox, President

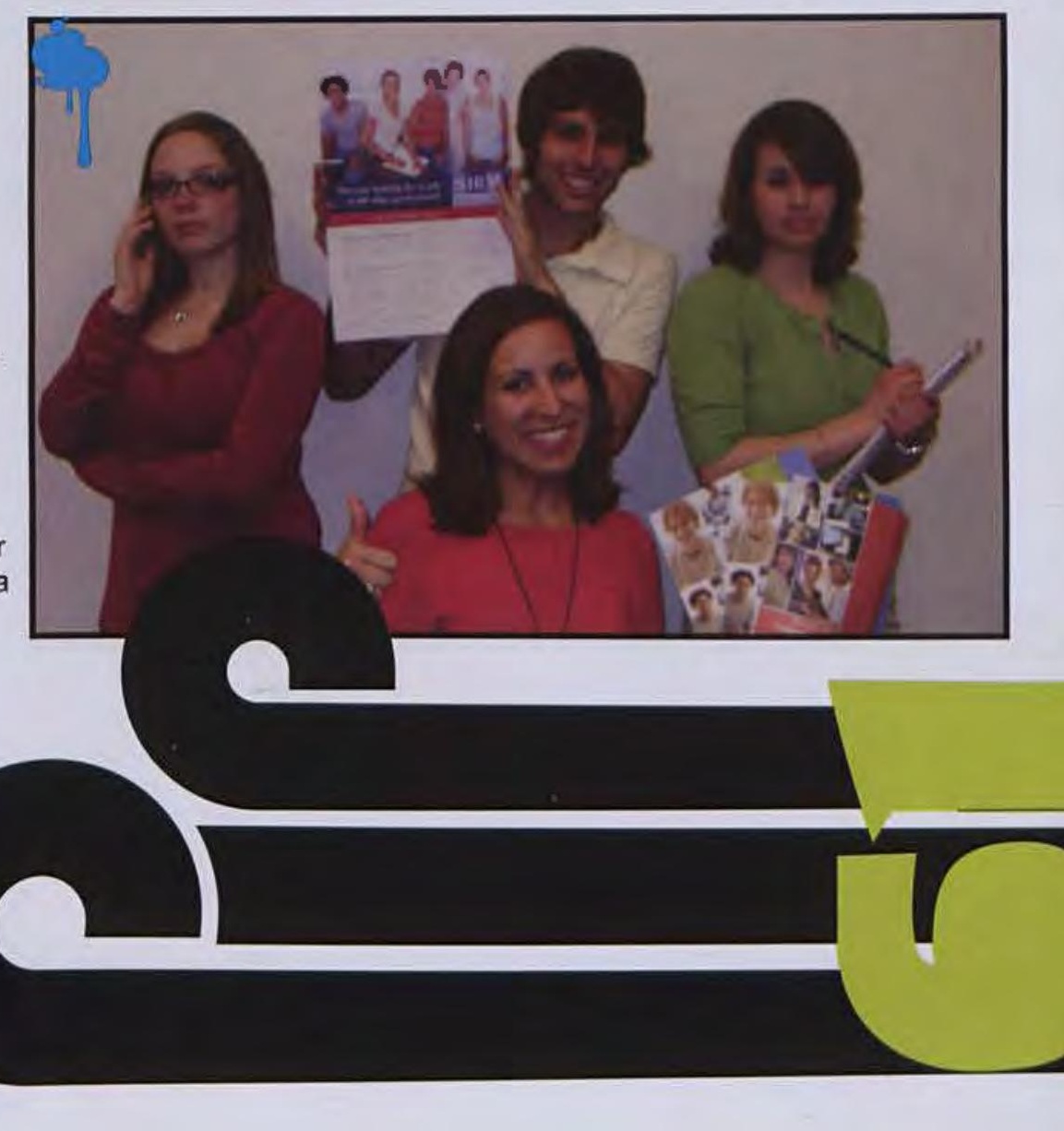



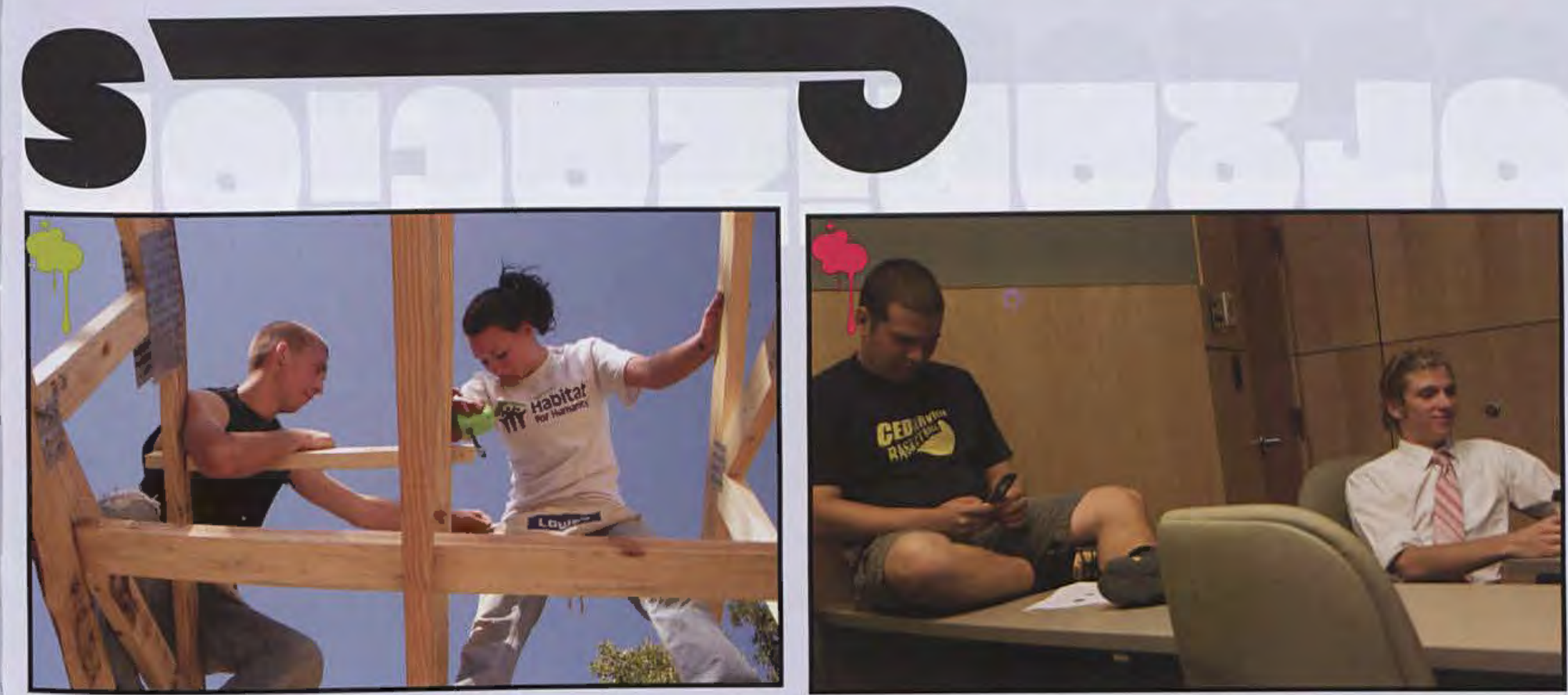

Sonathon Dias and Bethany Wailes, Habitat for Humanity volunteers, help build a house for a family in need.

- At the Alpha Sigma kickoff meeting, Caleb Speicher, Nathan Reed, and others discuss plans for the year. Nathan comments, "AS fosters discussion about issues that would not normally surface in a Cedarville classroom. For example, we tackled Universalism and the ethics of the War on Terror in 2008-2009. We think these conversations are vital to a liberal arts education, whether or not anyone buys into Universalism or opposes the War on Terror at the end of the day."

in Alpha Delta Omega helps out with the Women for Scholarship silent auction and fashion show as the event hostesses.

"The girls of ADO are so encouraging and fun to be around. I've been blessed so much by these girls and their willingness to serve those around us!" proclaims Heather Flippin.

At the Org fair, Psi Kappa Theta thought they'd hold some random competitions to incite students to learn more about the org. Shortly before this picture was taken, Kathryn Heckendorn and Emily Chiu discovered who can keep their hand or foot in a bucket of ice the longest. Kathryn was victorious!
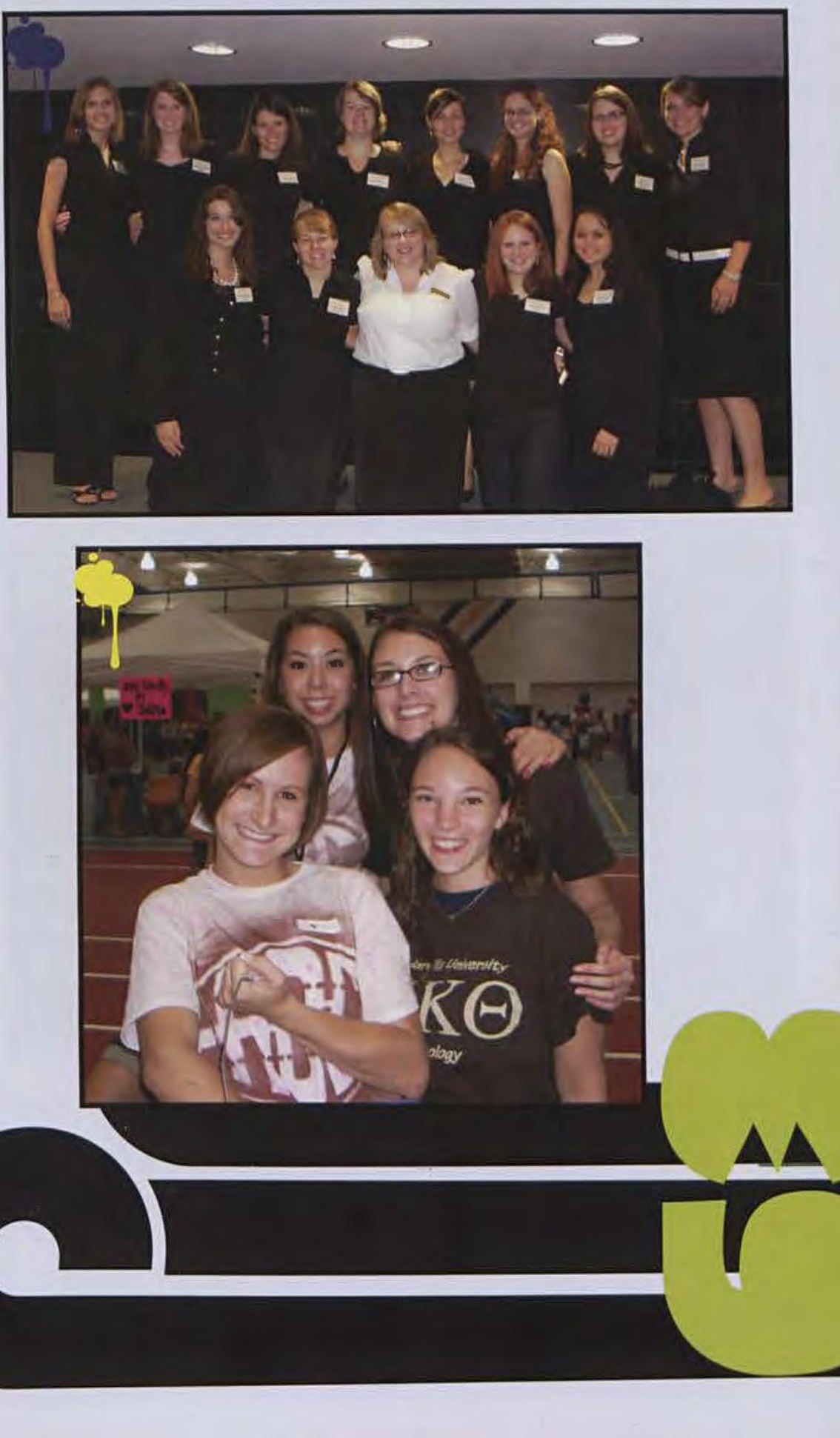
"Gamma Chi is a women's organization focused on being active in our local churches by serving those on campus and in our community. Our hearts are driven by the passion to be women of Christ, demonstrating this in our hands of service and our hearts of love."

- Rachel Latario, member

"Our mission in Sigma Phi Lambda is two fold: love God, love people. Brotherhood serves as a catalyst for 1 the former."

- Peter Ledger, President

\footnotetext{
"The College Democrats has challenged us to educate and . Critically engage leaders of today and tomorrow here at $\mathrm{CU}$. We believe that it is essential to your faith to know why you believe what you believe."

- Bethany Sierawski \& Sarah Jones, Secretary/Treasurer
}

"Tau Beta Pi is the national Engineering Honors Org; it strives to represent excellence in Engineering. At Cedarville, TBP supports and encourages underclass Engineers while trying to honor, through membership and various awards, upperclass Engineers."

- Jamin McCue, President
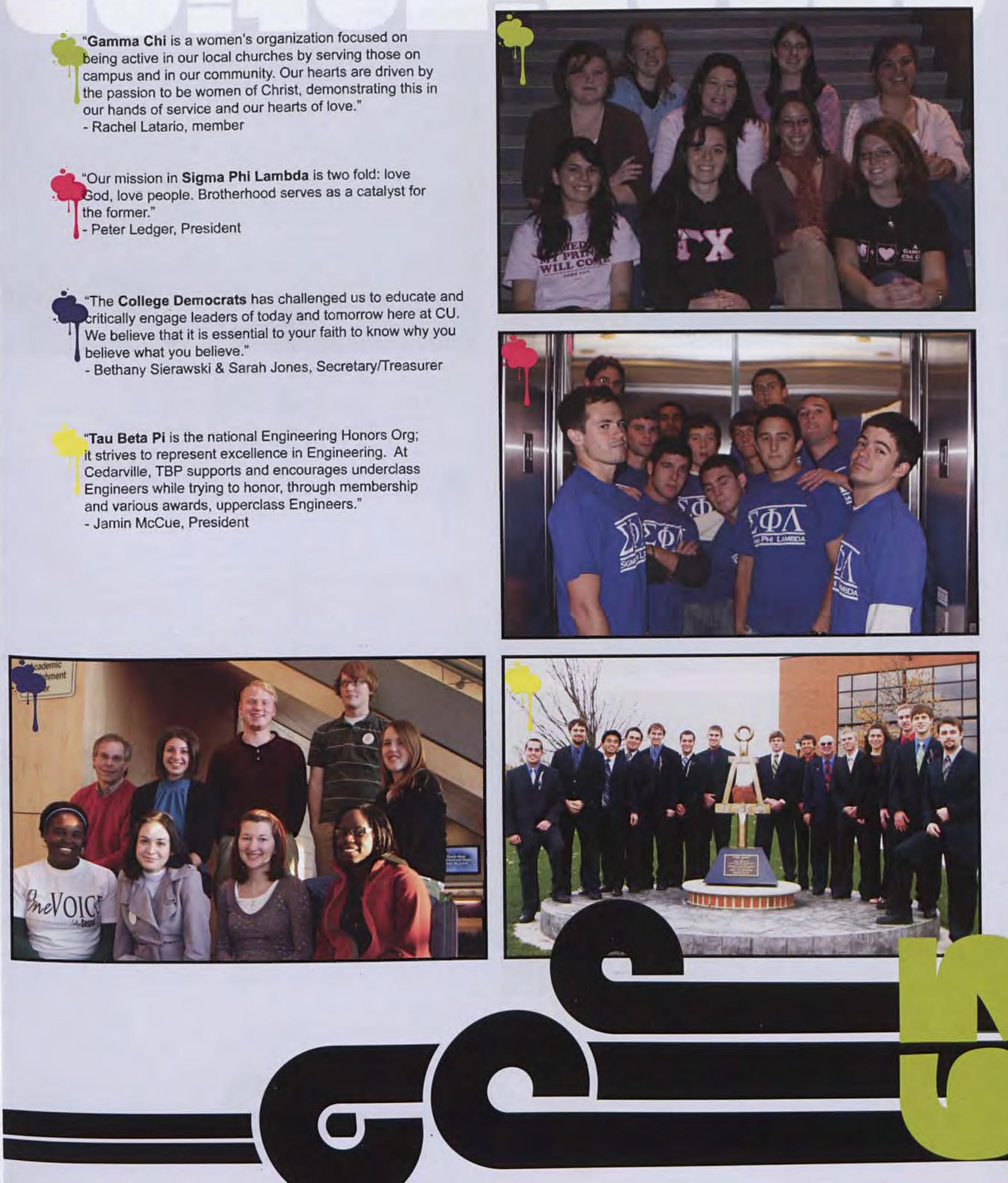

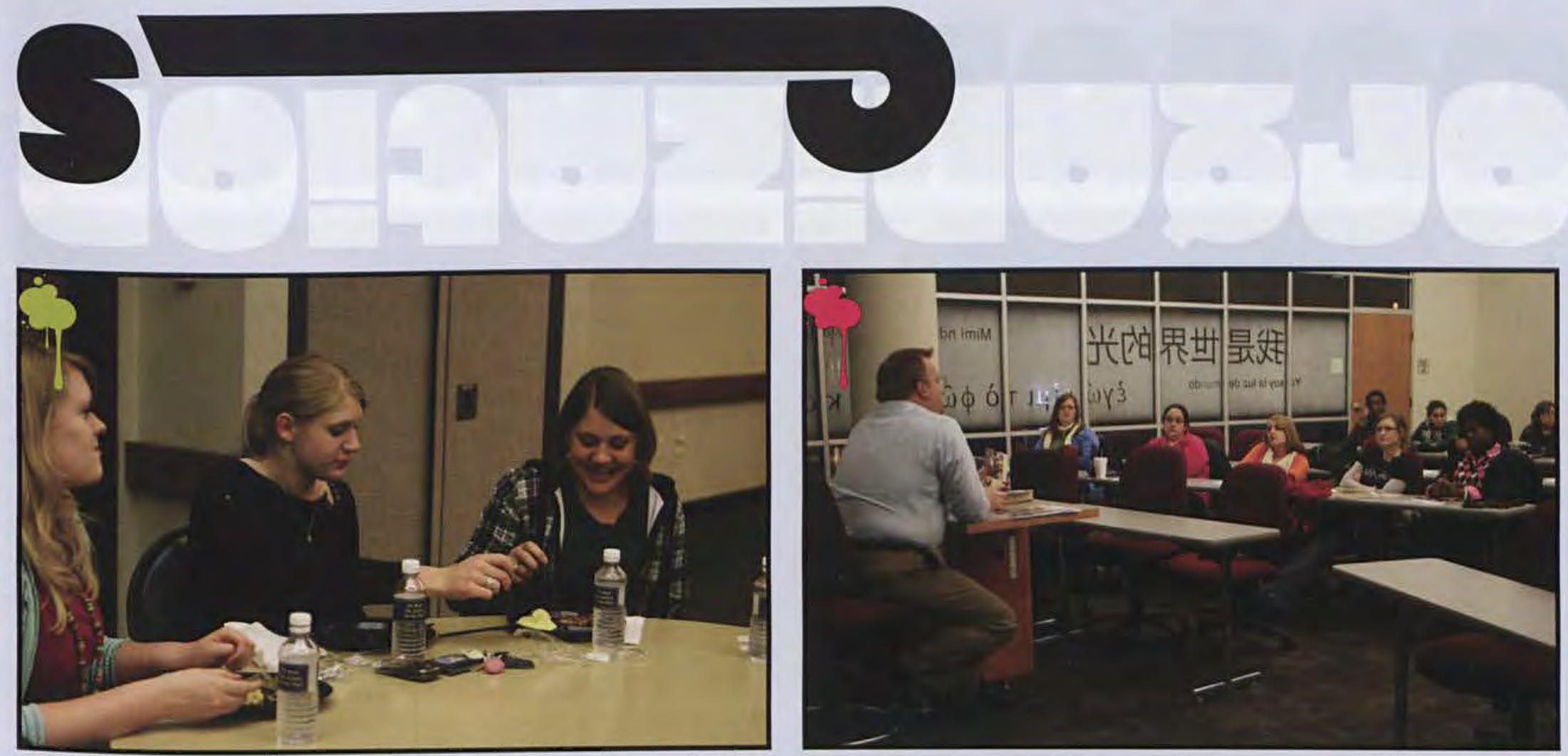

in Kate and Dresden Soules attend a Student Life cultural meeting for students with

Native American heritage. The event consisted of dinner and listening to Michael Jacobs, a Native American musician and recording artist, tell about his life. Dresden and Kate, as well as others, were invited because of their Native American heritage. Dresden comments, "it was one of those rare opportunities to meet one another."

Stacy Phillips, Sarah Blackridge, Ashley Groce, Juliana Shepherd, Briana Dupree, and Amber Prince attend an informational meeting for the ministry Garden of Hope.

Tim Miller shares about the months he spent homeless in D.C., Philly, and NYC with members of Amplified.

"Amplified is all about being a voice for the voiceless, specifically the urban poor. For those who have been marginalized, disenfranchised, forgotten or ignored, we seek to amplify their cries," comments Tim Voltz.

Bayley Nosal and Shaun Frazier engage in an SGA night of worship.
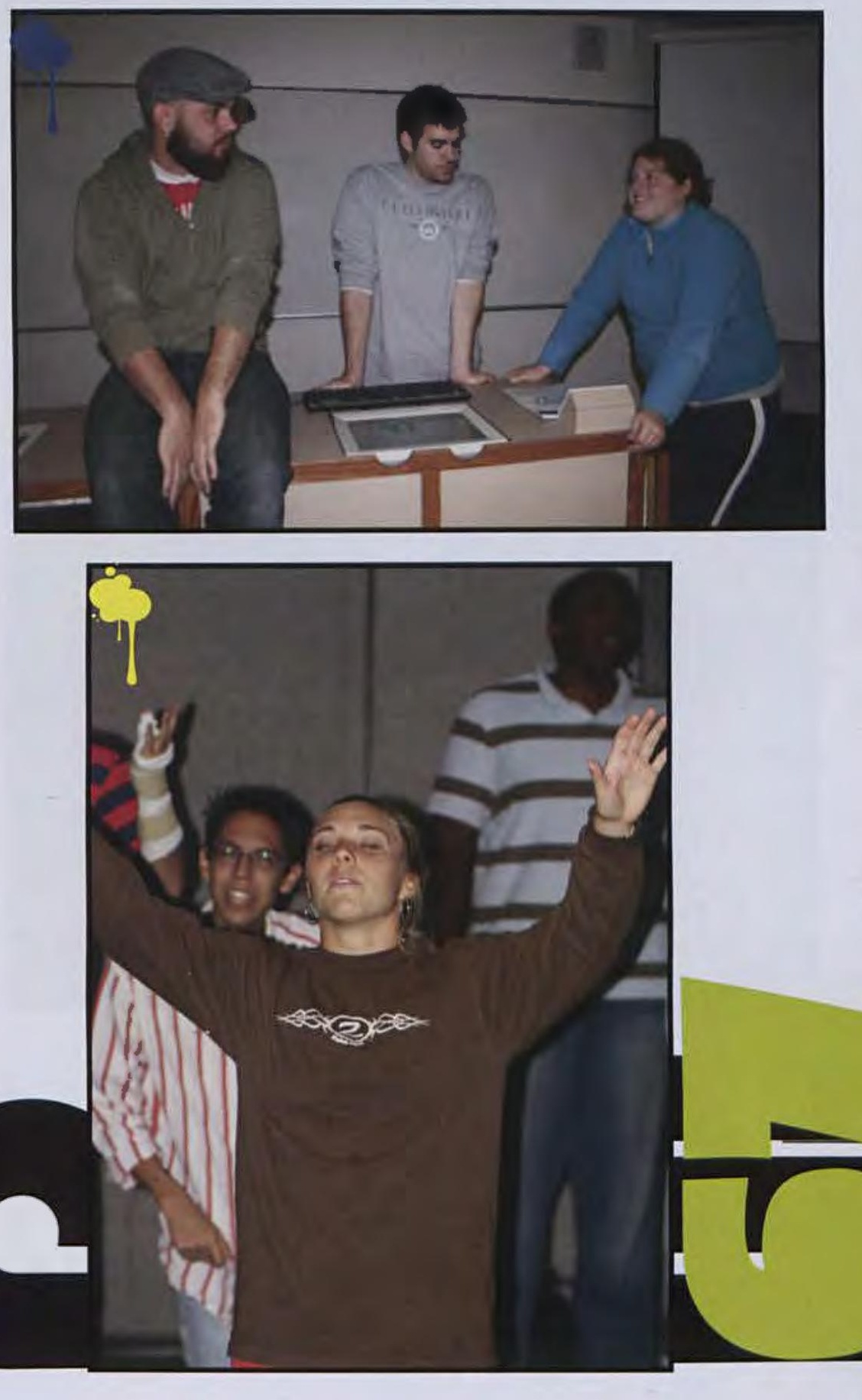

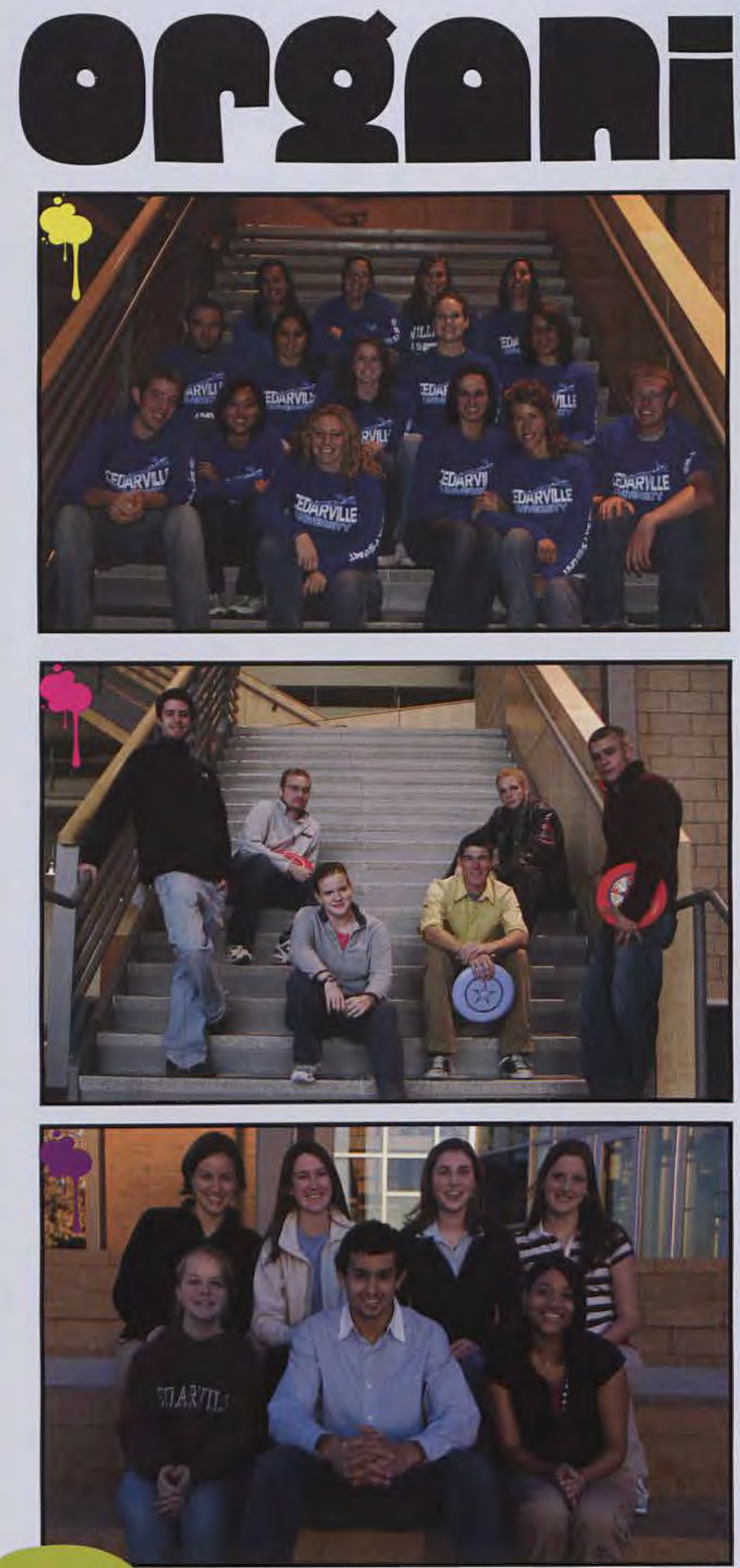
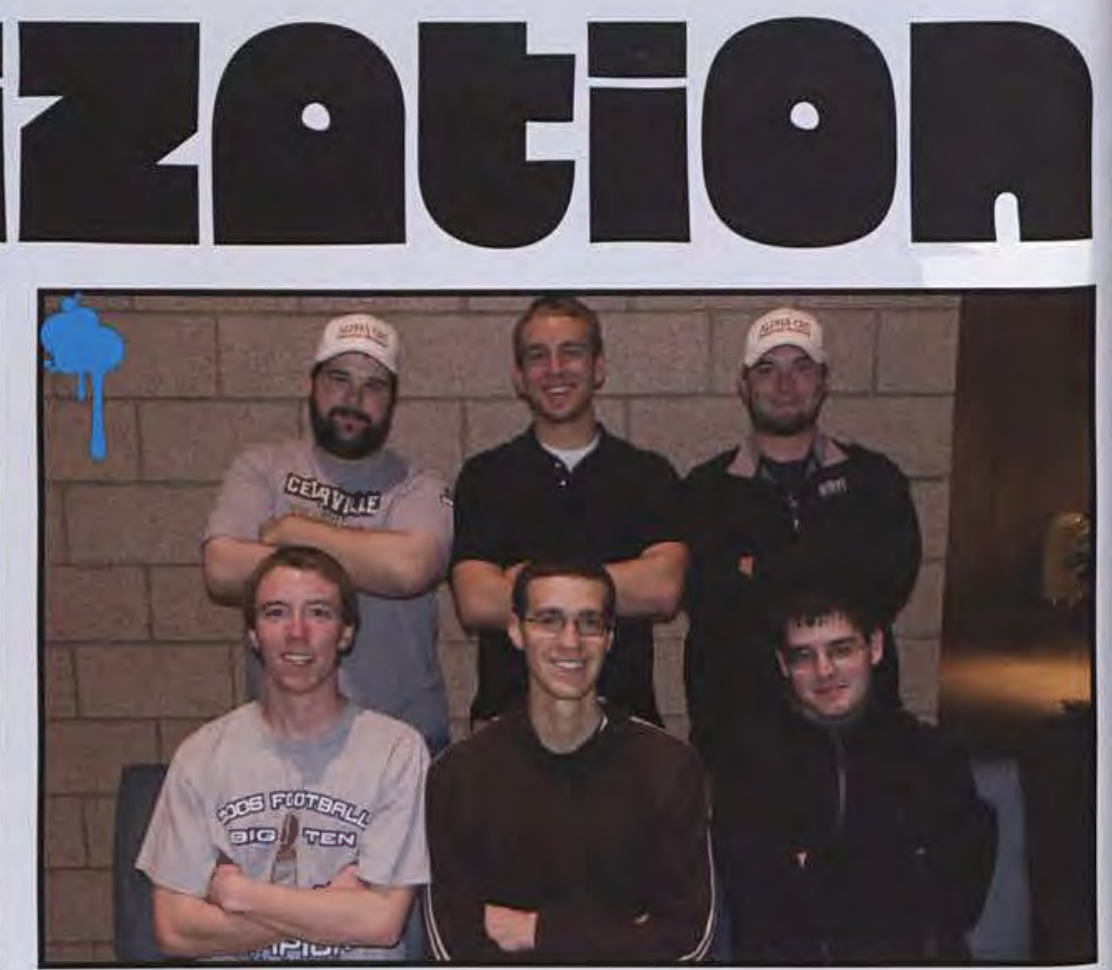

'The name 'Ten-24' is based off the verse in Hebrews, 'And let us consider how to stir up one another to love and good works,' and that's the goal of our org. Not only do we strive to encourage others through service projects, but we also plan some of the campus activities. - Michelle Zerminski \& Jeremiah Carsey, members

"Alpha Chi has a good mix of service and social aspects, and it's a great group of guys to be around. The clean-up service project after Ike was one of my favorite times with the org; service projects are always great." - Chris Bush, President

"Alpha Mu Beta is the Frisbee org on campus. We organize pick-up Frisbee games, set up tournaments, and have throwing clinics. Our goal is to unify the Frisbee community in good Christian fellowship. We would love to compete collegiately, but it hasn't happened yet." - Austin Engel, President

"Alpha Sigma Lambda is an organization that tries to raise deaf awareness on our campus, and engage the outside community to help minister to the deaf. We are using ASL as an opportunity to share the gospel with those who don't necessarily get to hear it." - Andrea Kiser, President 

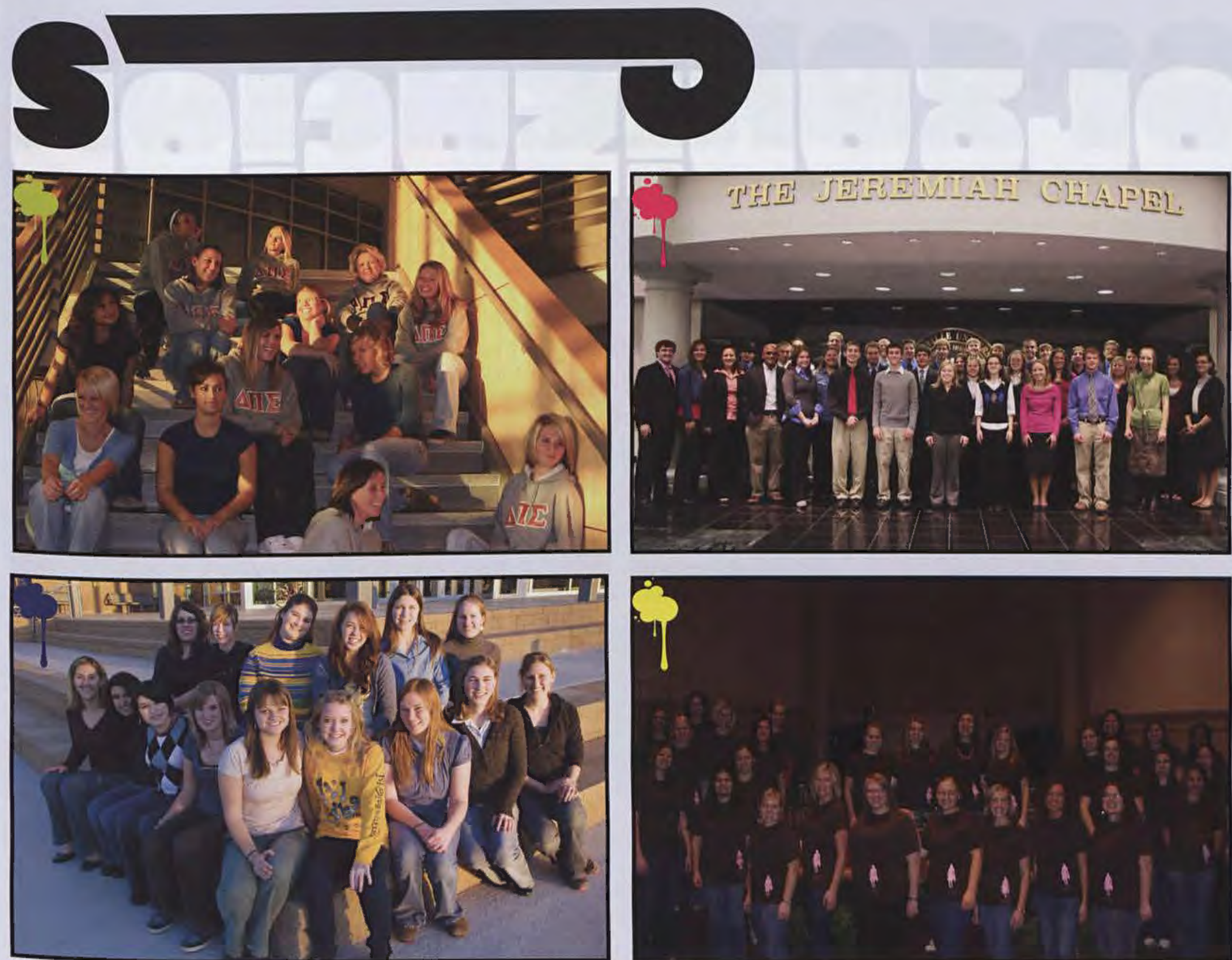

"Delta Pi Sigma is a women's service org that serves the faculty and staff here on campus. We have a very diverse group of girls. This year we're been focusing on sending encouraging cards to each faculty and staff member on campus." - Stacie Peden, President

"SIFE is an international organization, and along with other SIFE

chapters, our team seeks to educate others about the principles of

free enterprise. We feel so blessed to have been able to make a

difference on our campus, in our community, and even throughout the world through the teaching and practicing of free enterprise. - Amanda Graves, President

"The Society of Women Engineers exists to encourage fellowship among female engineers and computer scientists and to serve the community. It is encouraging to be able to share experiences with others who have been in your shoes." - Catherine Shanks, President

"Women in Business is a fantastic org for women who are thinking about going into business to join. We have three main areas of focus: learning, serving, and connecting." -Laura Quick, President
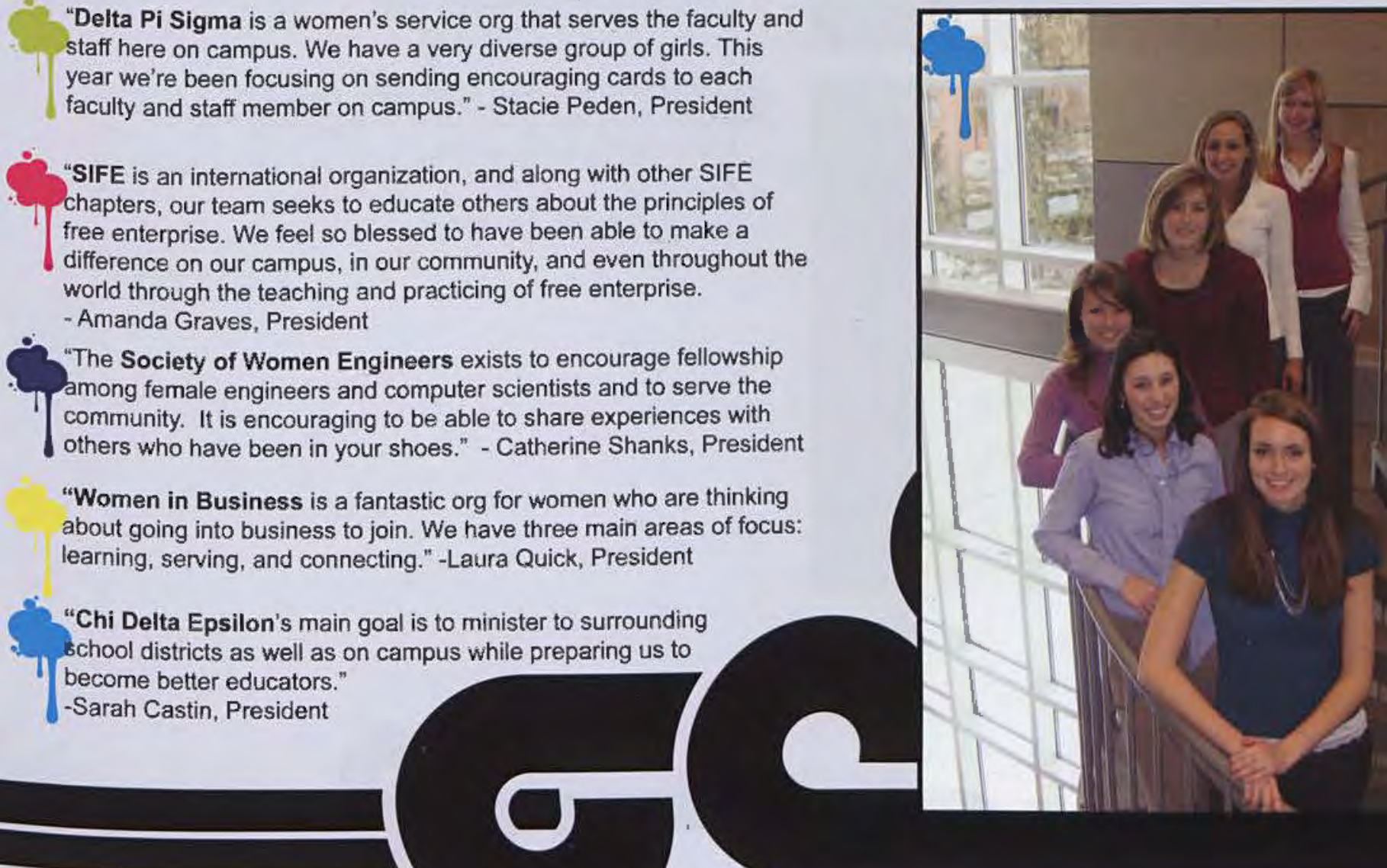

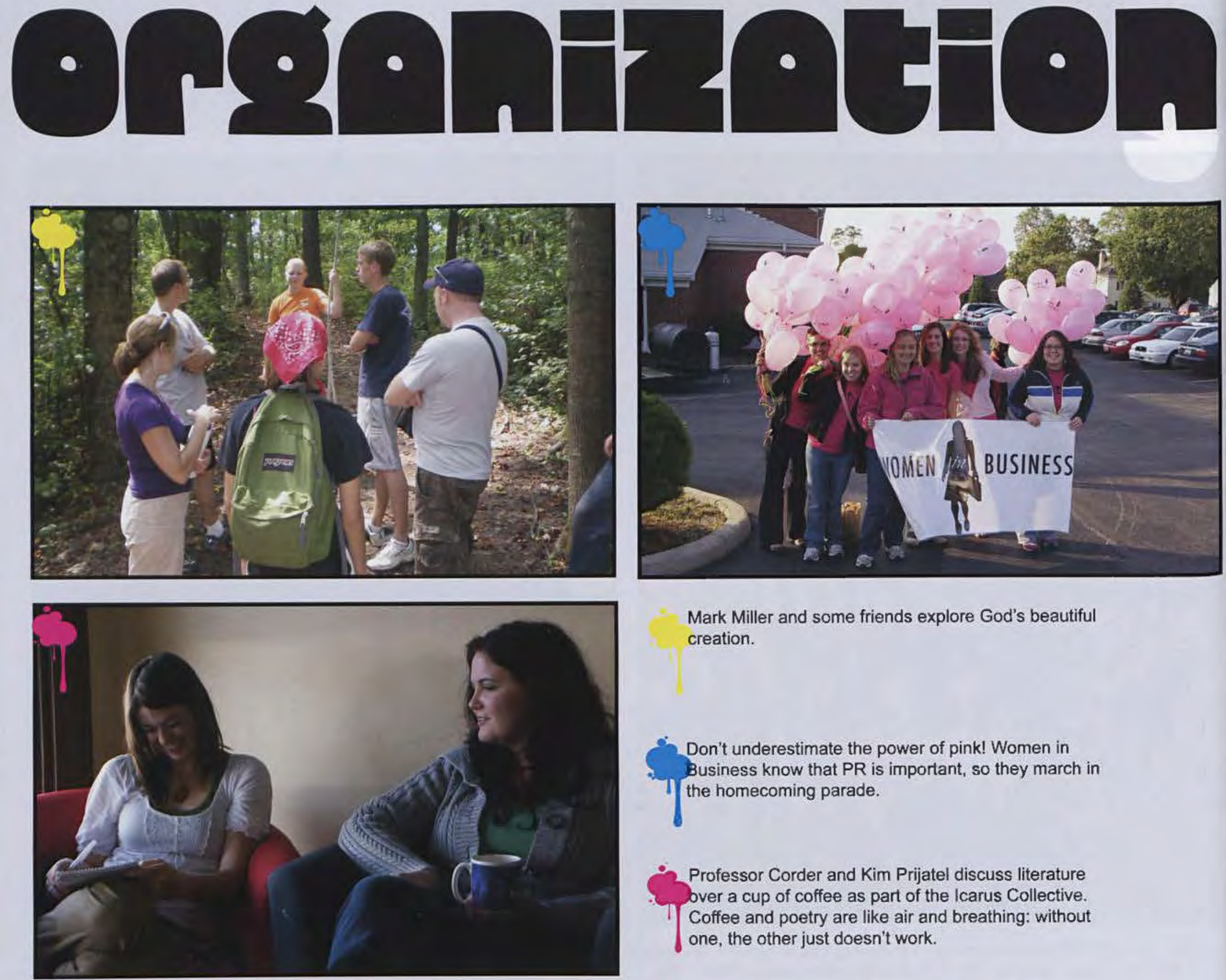

Mark Miller and some friends explore God's beautiful creation.

Don't underestimate the power of pink! Women in Business know that PR is important, so they march in I) the homecoming parade.

Professor Corder and Kim Prijatel discuss literature over a cup of coffee as part of the Icarus Collective. 7 Coffee and poetry are like air and breathing: without one, the other just doesn't work.
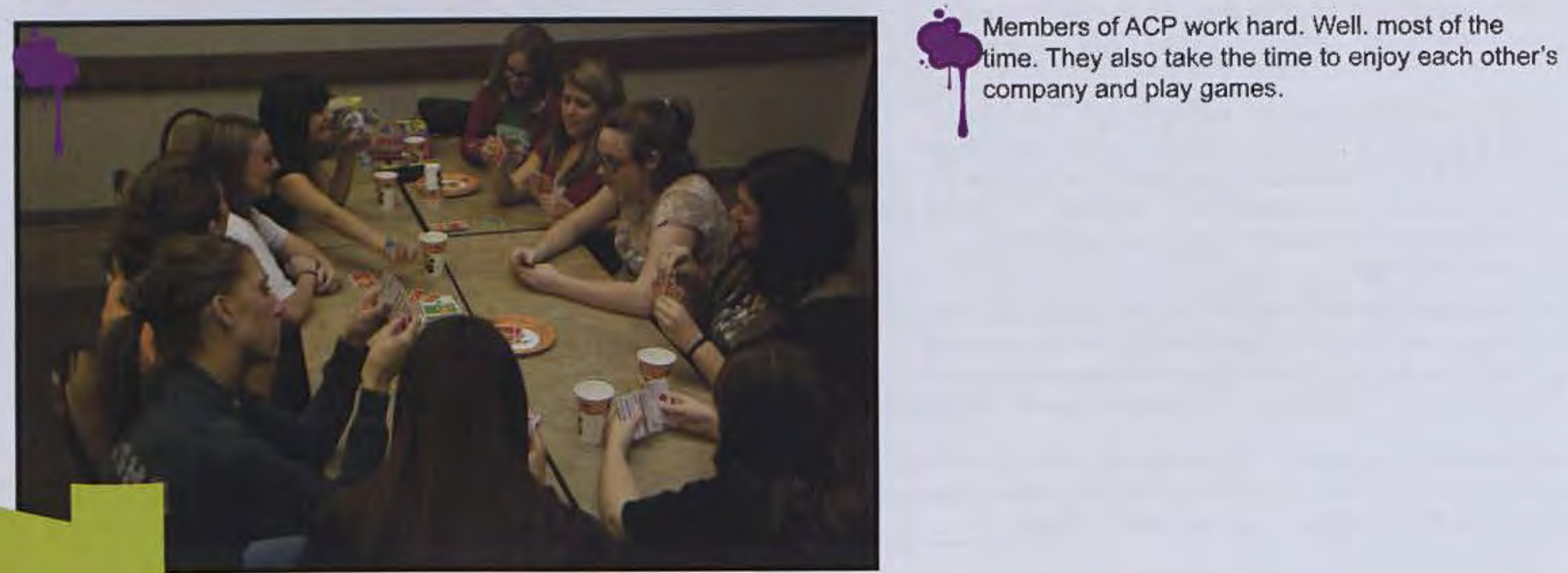

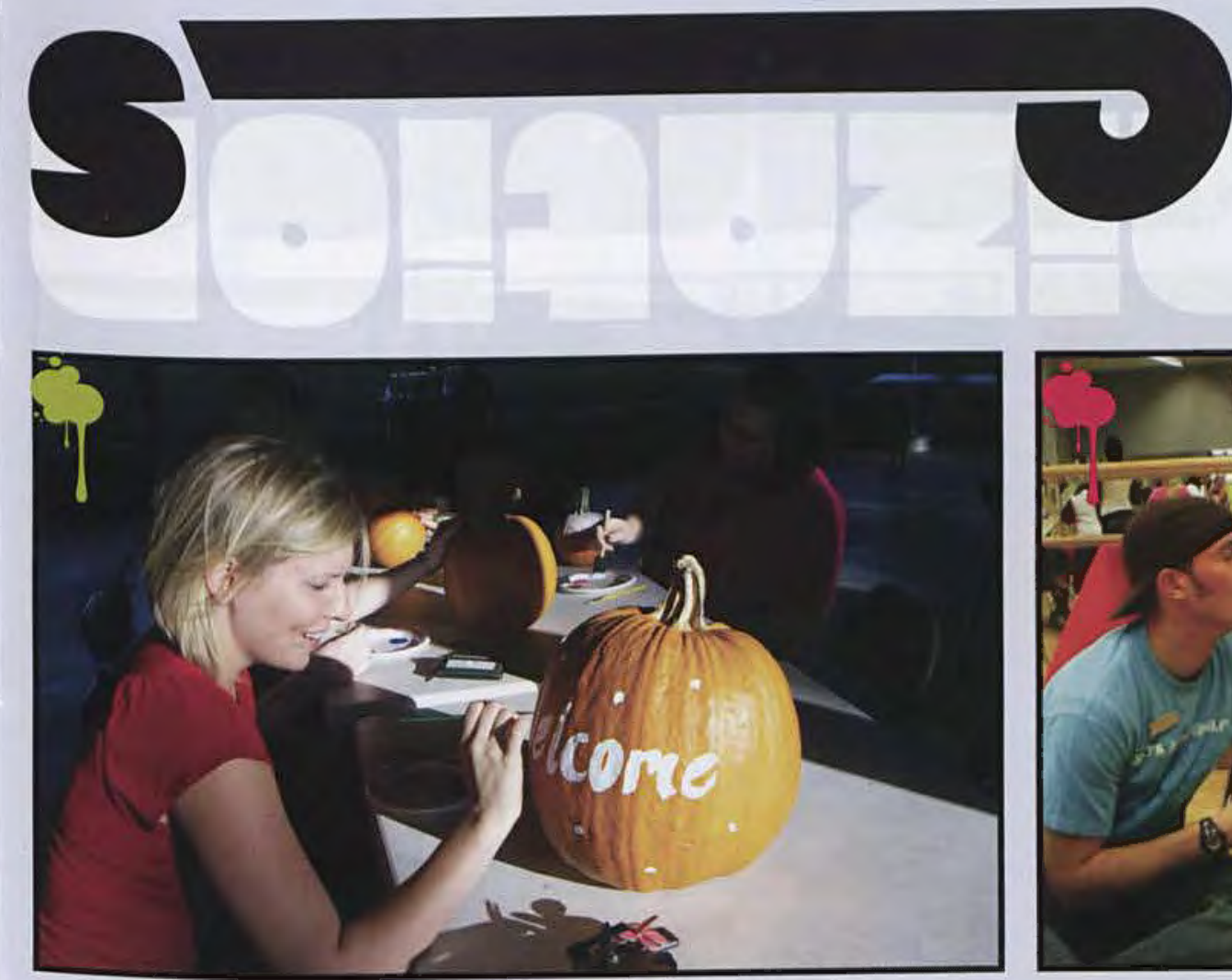

Members of Women in Business are busy painting pumpkins. Now if it was Men in Business, the pumpkins would probably be smashed in some inventive fashion.

DAE members offer free kisses at the Org fair... is that Cedarville appropriate?? You should have seen 7 the line of freshman for that table!

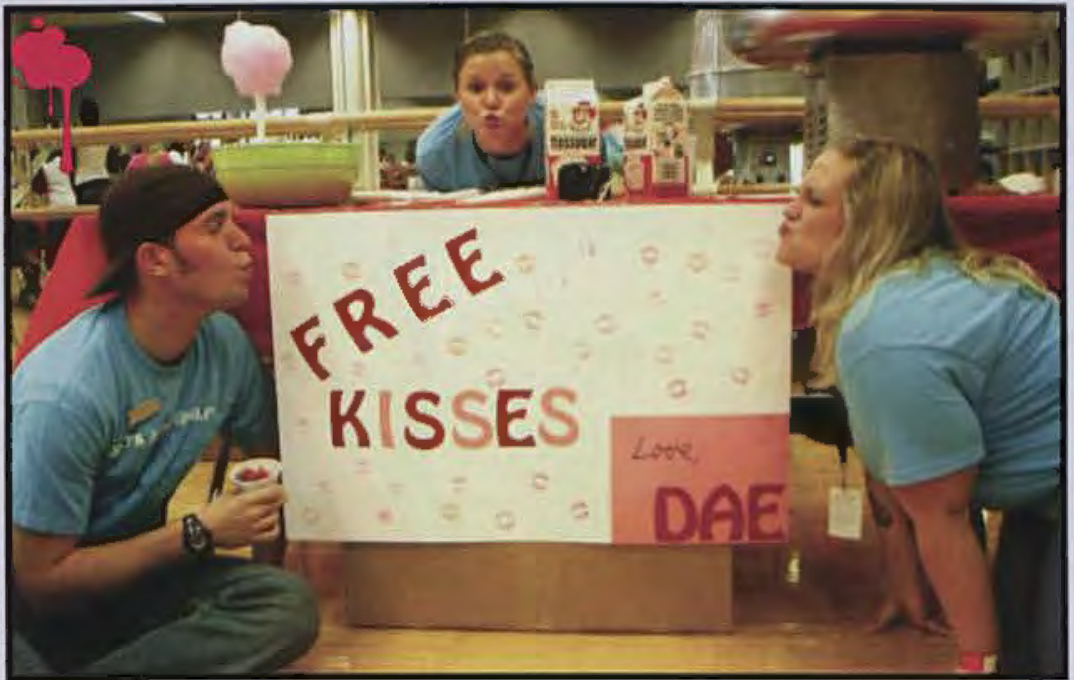
I

\footnotetext{
Ever wonder what to do with all that silverware? Women in Business take an evening to learn all the in's and out's of etiquette. Knife on the left, right?
}

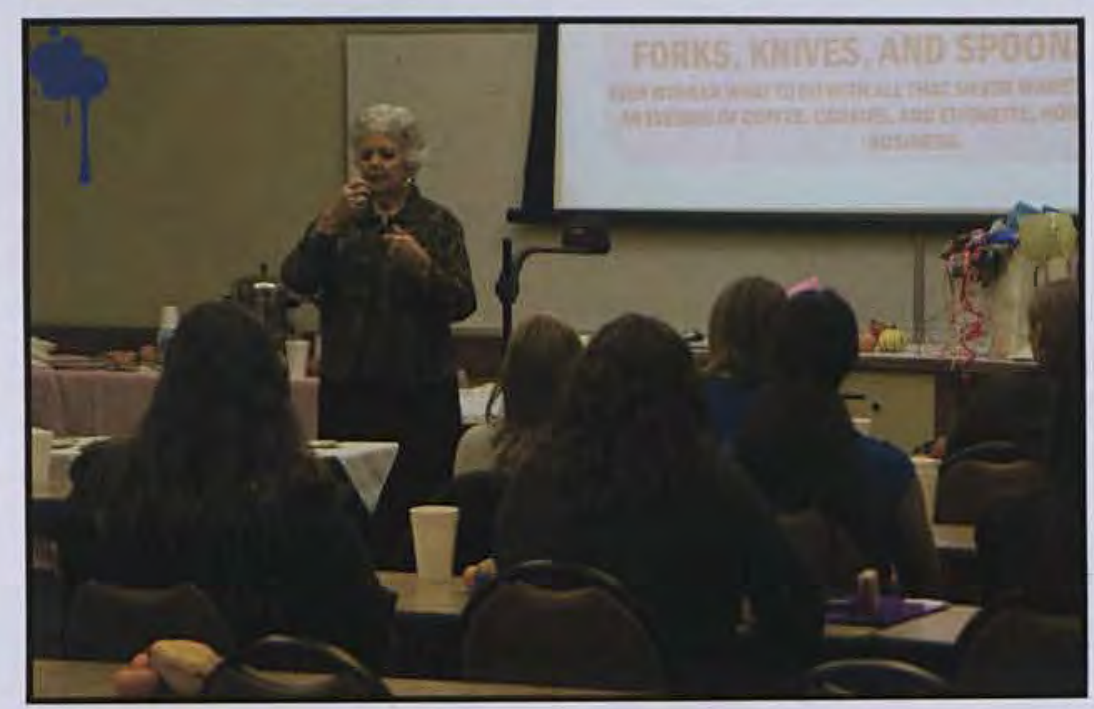

Orgs like Big Bird are involved in many service projects in the community. There's no better way to tell the community that we care than by sweeping leaves out of their garage! 

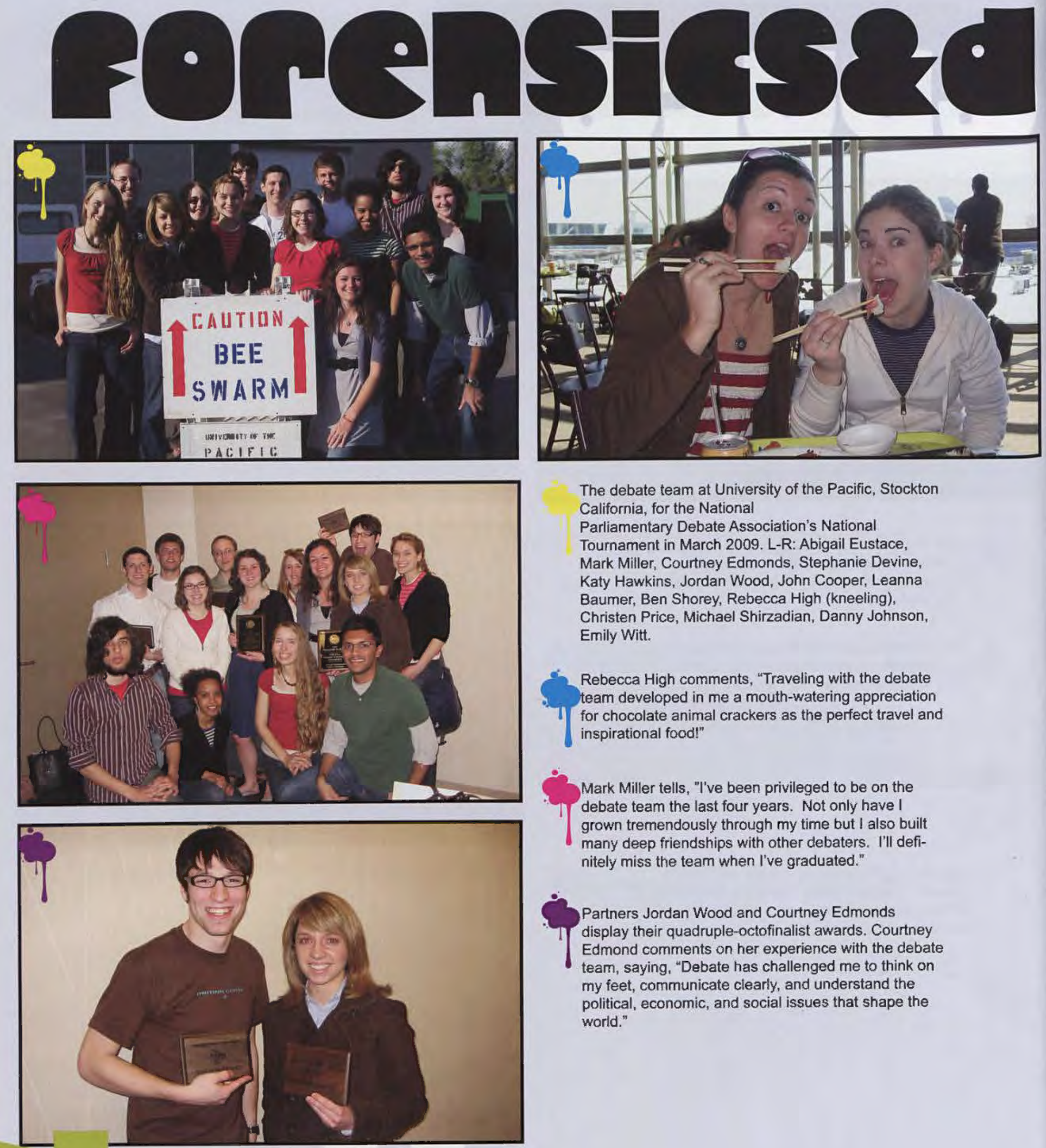

The debate team at University of the Pacific, Stockton California, for the National

Parliamentary Debate Association's National

Tournament in March 2009. L-R: Abigail Eustace, Mark Miller, Courtney Edmonds, Stephanie Devine, Katy Hawkins, Jordan Wood, John Cooper, Leanna Baumer, Ben Shorey, Rebecca High (kneeling), Christen Price, Michael Shirzadian, Danny Johnson, Emily Witt.

Th Rebecca High comments, "Traveling with the debate team developed in me a mouth-watering appreciation

If for chocolate animal crackers as the perfect travel and inspirational food!"

Mark Miller tells, "I've been privileged to be on the . debate team the last four years. Not only have I grown tremendously through my time but I also built many deep friendships with other debaters. I'll definitely miss the team when I've graduated."

Partners Jordan Wood and Courtney Edmonds display their quadruple-octofinalist awards. Courtney Edmond comments on her experience with the debate team, saying, "Debate has challenged me to think on my feet, communicate clearly, and understand the political, economic, and social issues that shape the world." 


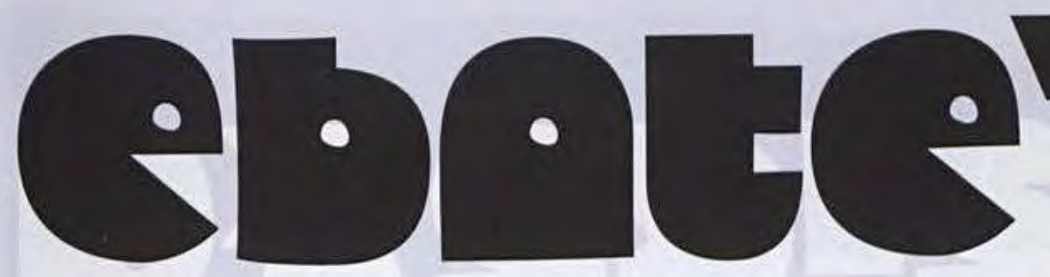

Debate partners John Cooper and Mark Miller proudly hold their award for being triple octafinalists - meaning they were in the top 64 of about 600 teams participating in the tournament. John Cooper remarks, "It was a great experience, in my final year of debate, to compete and win at the highest levels and to develop lifelong friendships with amazing people, both on the team and from other schools. It's been a great ride."

They may be able to agree in the debate room, but

they can't quite seem to agree on where that swarm is...

My only regret is that we never

employed Michael's brilliant strategy known as the 'punch me in the face' response," explains Katy

Hawkins (left). "Its simplicity is not the least of its many advantages: our two opponents would be allowed to punch Michael in the face, provided they conceded the round to us first."
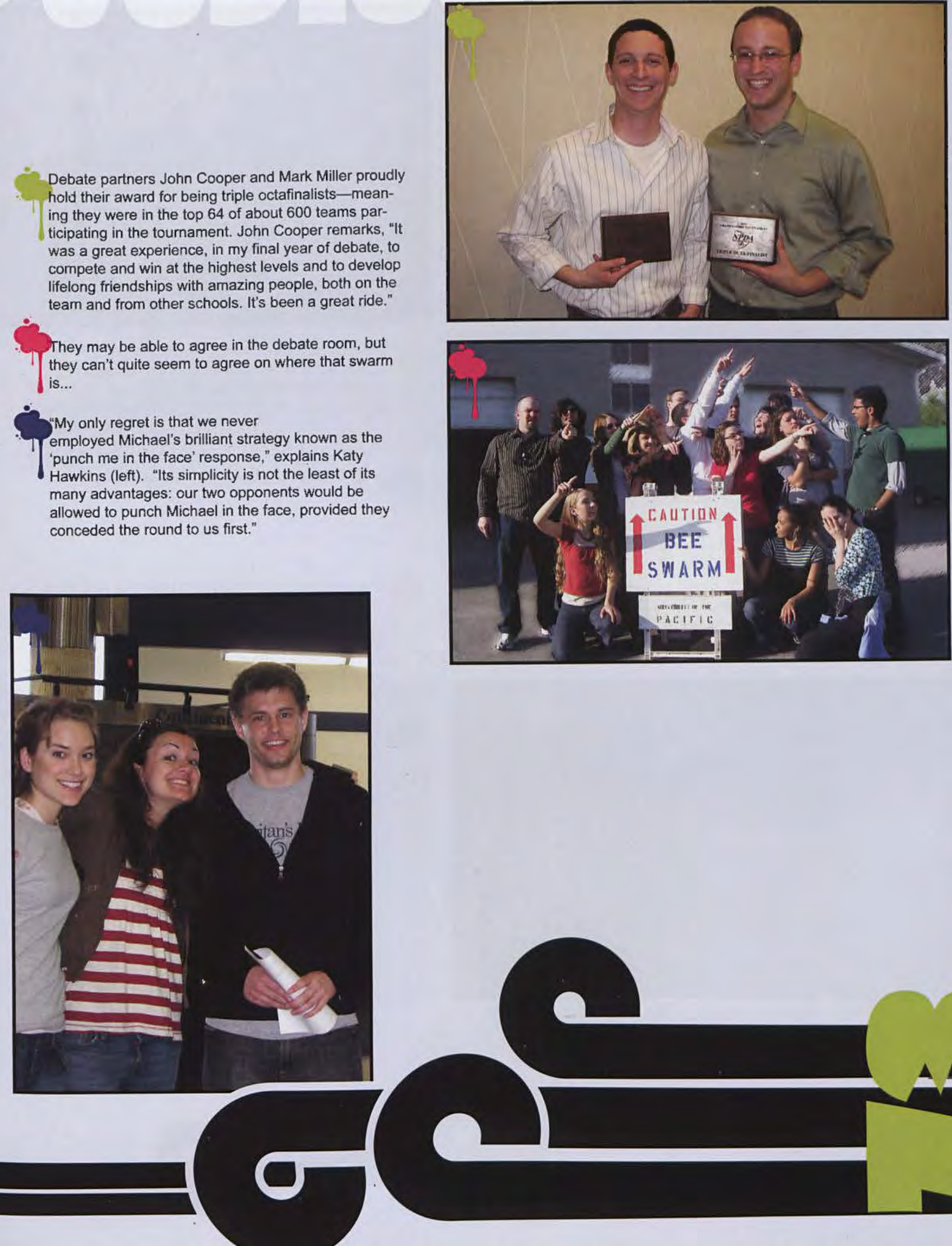

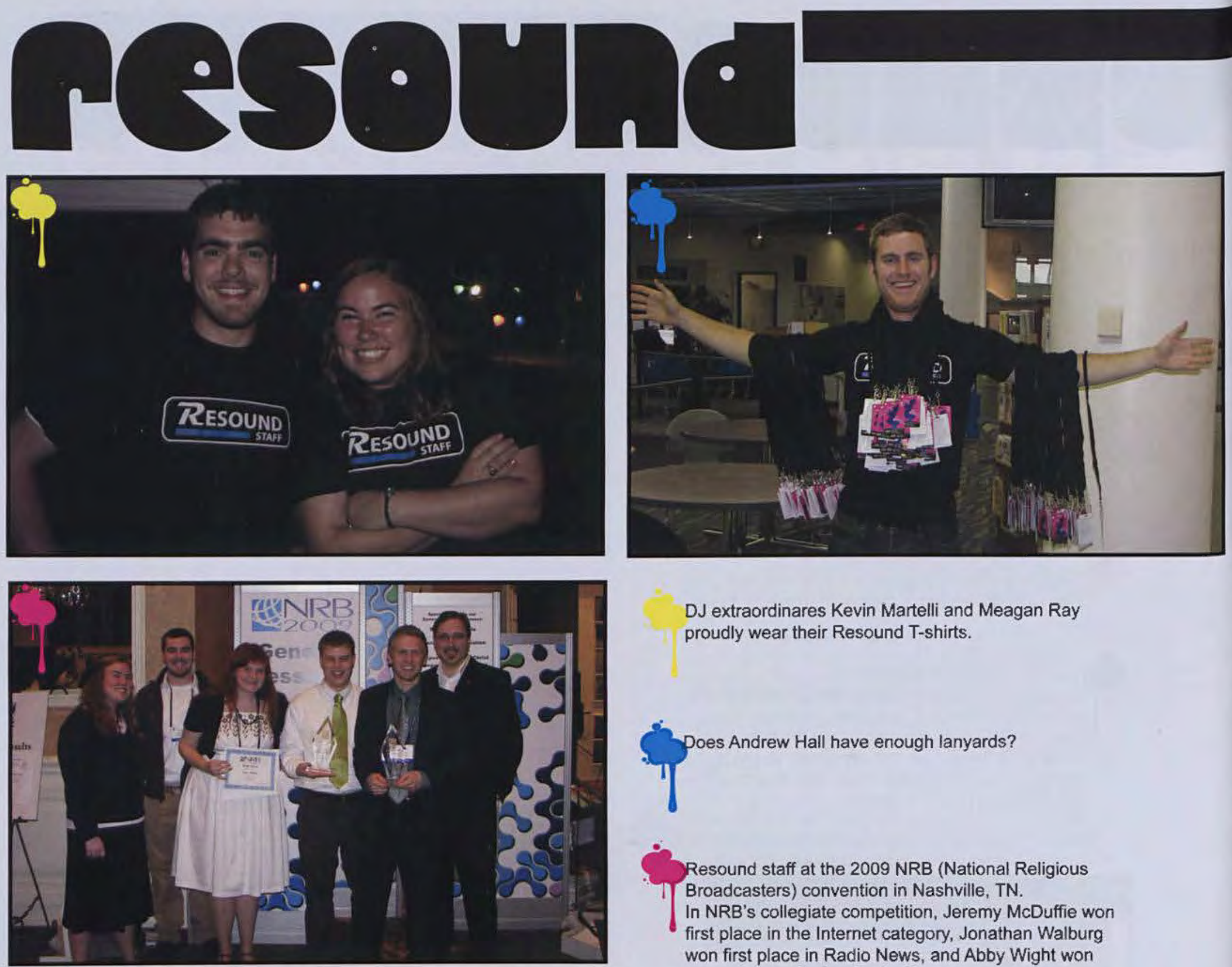

DJ extraordinares Kevin Martelli and Meagan Ray proudly wear their Resound T-shirts.

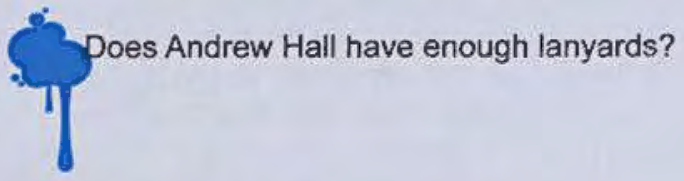

Resound staff at the 2009 NRB (National Religious
Broadcasters) convention in Nashville, TN.

In NRB's collegiate competition, Jeremy McDuffie won first place in the Internet category, Jonathan Walburg won first place in Radio News, and Abby Wight won second place for her Radio Demo.
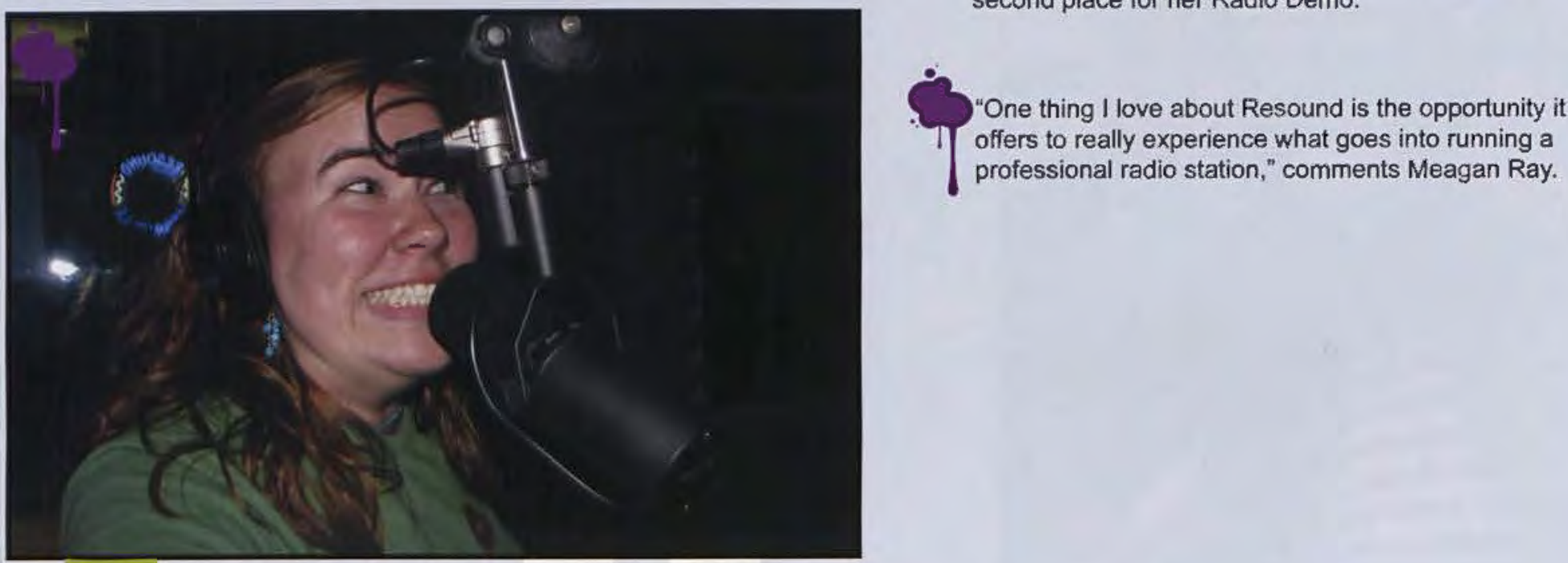
The Resound team tries to stay warms in the winter months. Music may warm your soul, but a heavy coat helps warm the body, too!

1) and Meagan Ray watch as the Presidential elections unfold, ready to report the results to the student body

Seen here working hard in the studio with Jarrod Steinmetz, Andrew Hall mentions, "Working for

Resound throughout my time at Cedarville was irreplaceable. It was especially enjoyable for me, being a Bible major, because I got to meet several Electronic Media majors and our faculty adviser, Mr. Lightenheimer. They are a hard-working, fun-loving, excellence-oriented group of people. I will continue to

They're like one big, happy family! At least Jordan Knoedler, Meagan Ray, Dave Stephens, and Abby Wight think so.
Resound isn't just about great music. Jesse Jones benefit from those relationships for the rest of my life."
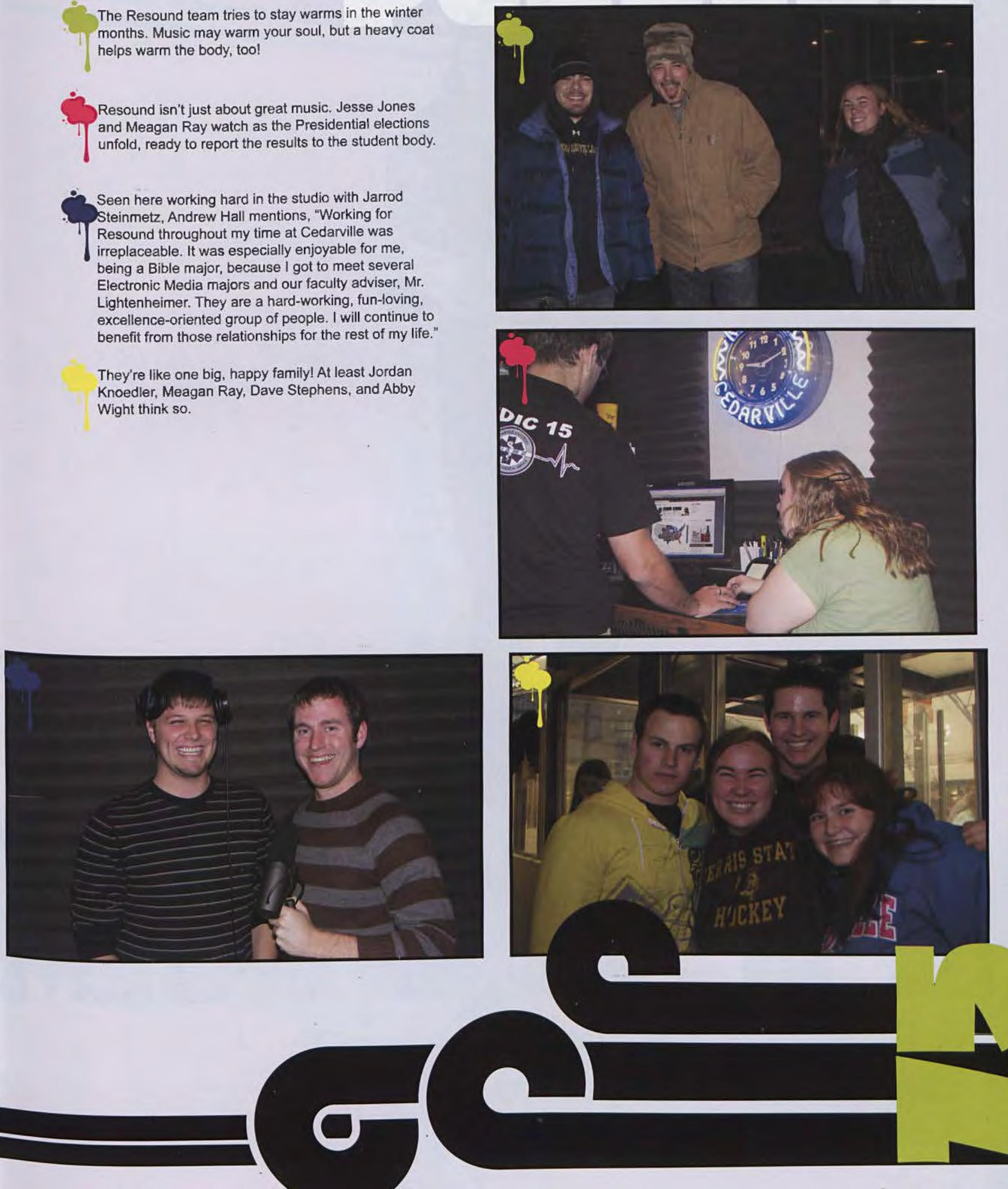


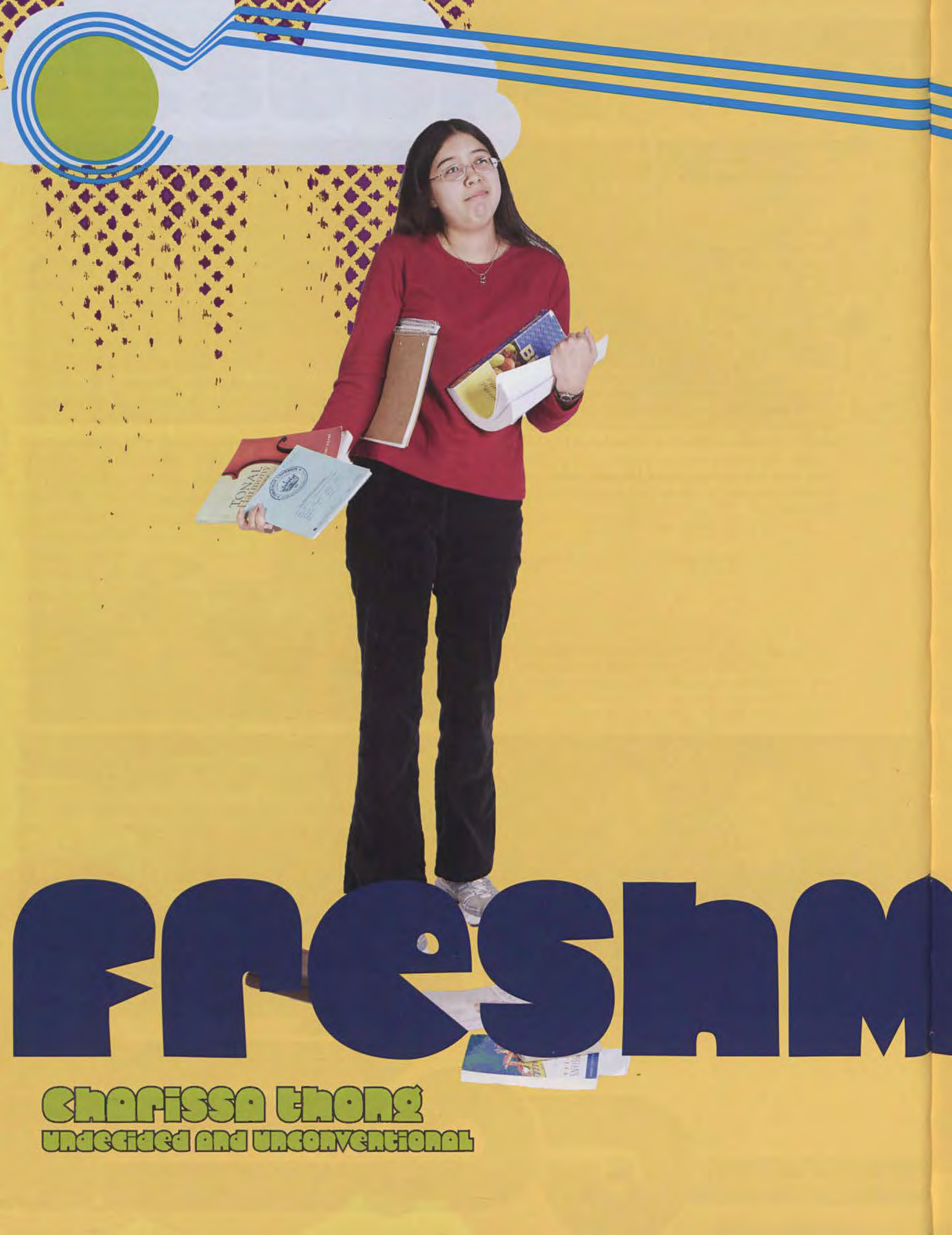


I remember the first day of school. It was probably one of the scariest "first days" I've ever had. I woke up wondering if the flurry of Getting Started Weekend was actually over; my brain was already experiencing processing overload. I thought back to Pastor Rohm's "Giants in the Land" sermon and began to feel like I, a freshman, was one of those grasshoppers. I left Maddox in plenty of time to make it to my first class. My first class! Though I was scared, the sound of that made me more than a little excited. I am finally getting to do what I came here to do! Which building is it in? I wondered. Oh yes, that's right, the Apple Technology Center. In plenty of time for class, I quietly walked down the enormous hallway and entered the spacious room. I sat very quietly and neatly organized my books, pen, and paper - ready.

As the students began to file in, it wasn't too long before I noticed that all of my fellow students were boys. Isn't this Intro to Literature? Shouldn't this be co-ed? The longer I waited, the more I felt out of place. Finally, right as the teacher was about to speak, I double checked my yellow jacket planner and realized, Oh no! I'm in the wrong room! My heart began to race as I threw my books together and darted out. I knew that every eye must have been staring right at me! Though stuck in the middle of a cornfield, there were definitely giants in the land.

Thankfully, my entire freshman experience wasn't this scary. Looking back on my first semester, as I now round finals week, I already have several things which l've grown to love and appreciate about Cedarville. The two words I could best use to describe Cedarville are challenging and caring.

Cedarville offers me a very challenging education. I was homeschooled for the majority of my education because my family has lived overseas for most of my life. Now, I love actually being in the classroom! I have learned so many new and interesting things in my humanities class. Before, I didn't even realize how much I loved the arts; thanks to some amazing professors, my mind has been challenged and expanded. Probably the most difficult part of the academic transition from high school to college has been learning how to take class notes. Sometimes it is difficult to know what is most important to write down; I think l'm starting to get the hang of it now, however.

The minute I stepped onto Cedarville's campus, I knew it was different from the other colleges I visited. Now, looking back, I realize that many freshmen have had similar experiences. Cedarville was the most friendly and caring college that I have visited. I knew I wanted to be a part of a program where the students love the school and each other. Cedarville was a perfect fit. The unit style dorms have been one of my favorite parts of the school. Not knowing any one, the unit-style has really helped me to fit in and make friends quickly. Often times, my units will get together to watch a movie, go off

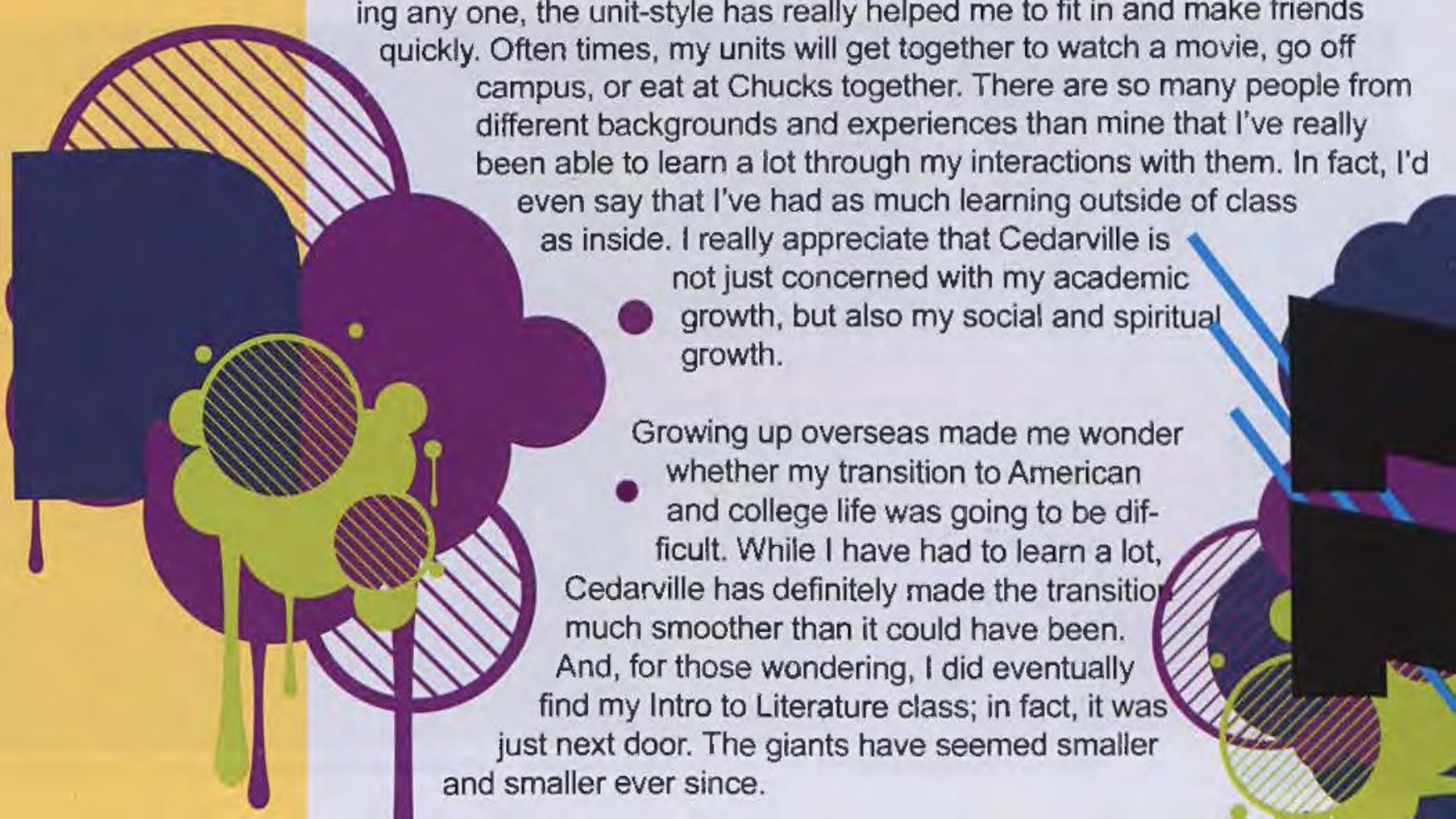




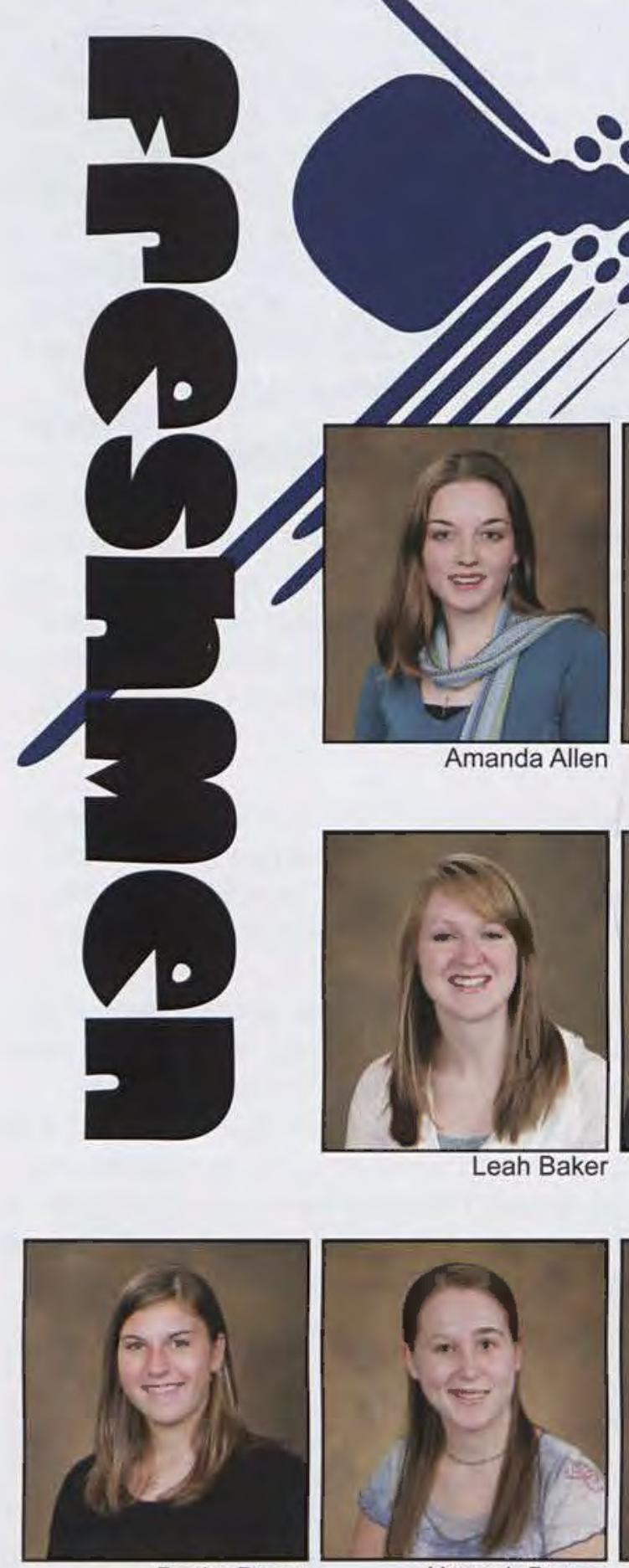

Becky Baron

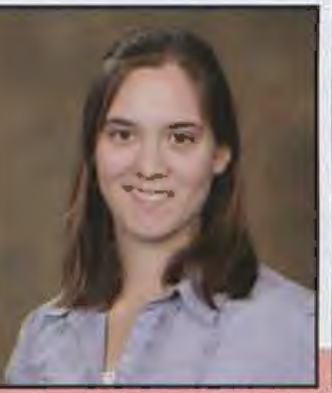

Rachel Beachy
Hannah Barron

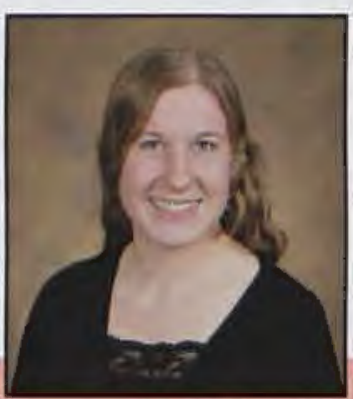

Molly Bearden
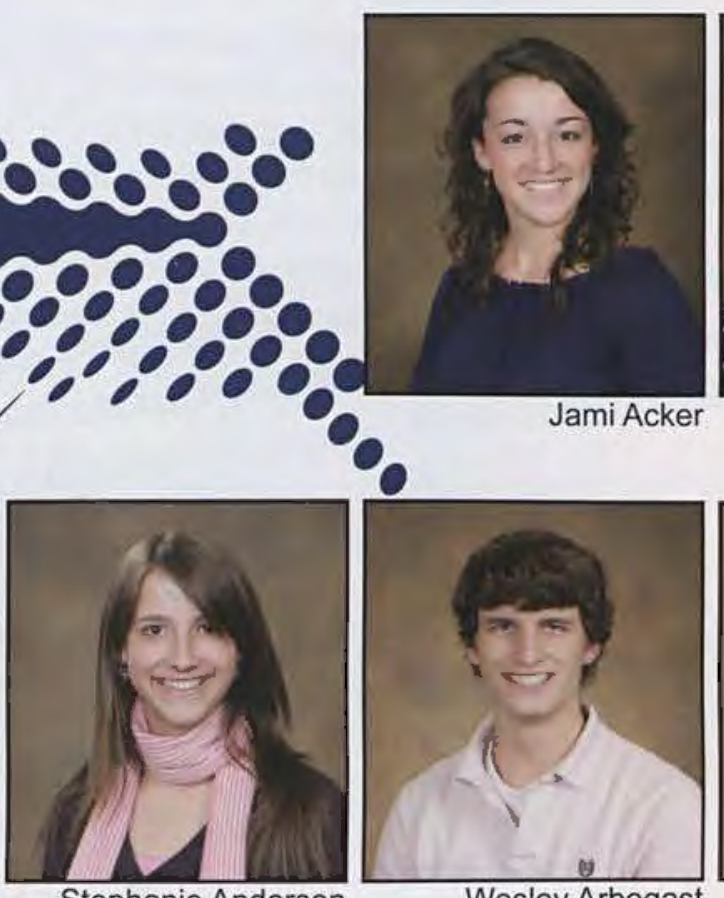

Stephanie Anderson

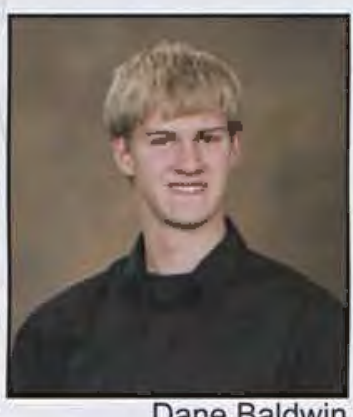

Dane Baldwin

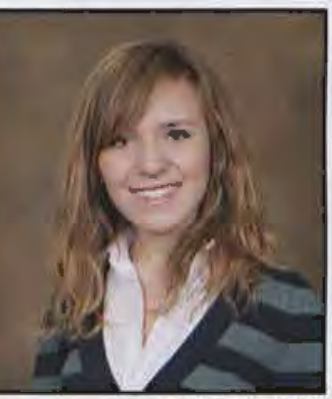

Erin Bartley

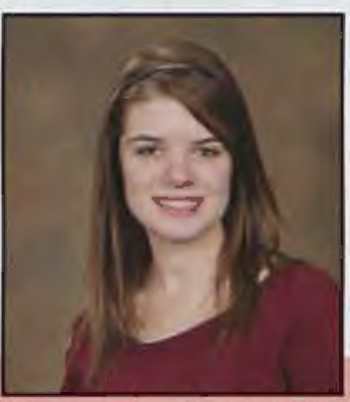

Lauren Bell

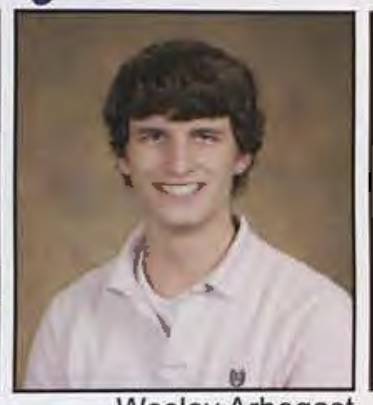

Wesley Arbogast

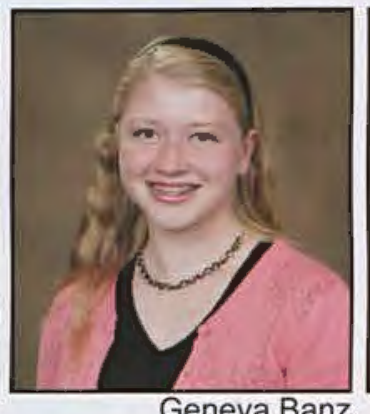

Geneva Banz

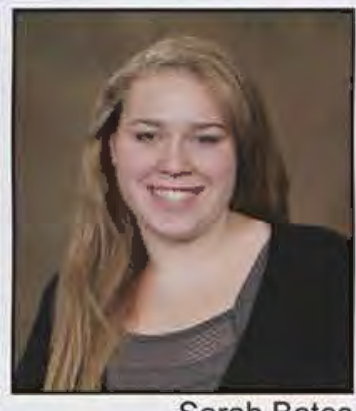

Sarah Bates

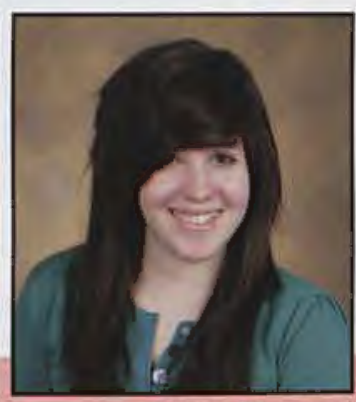

Katie Bernard

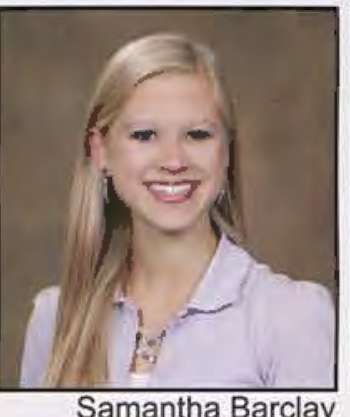

Samantha Barclay

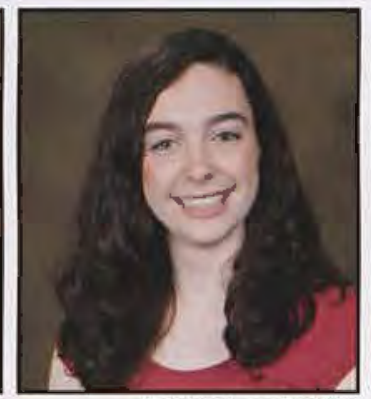

Christine Battis

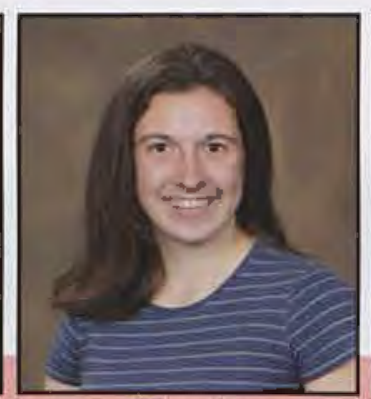

Mary Bernecker

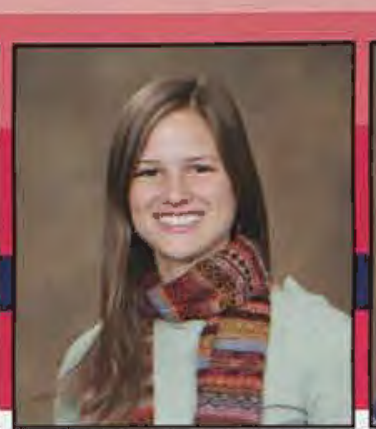

Michelle Blackstone

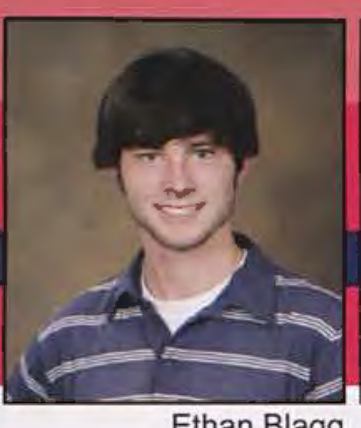

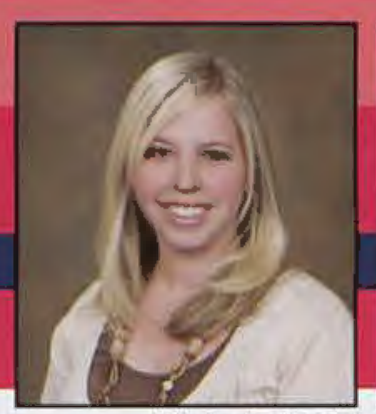

Stefanie Betori

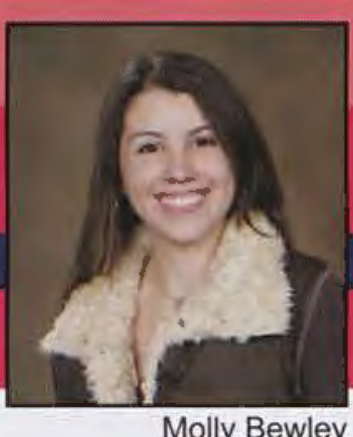

Molly Bewley

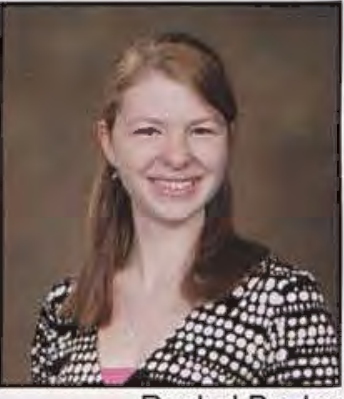

Rachel Baylor

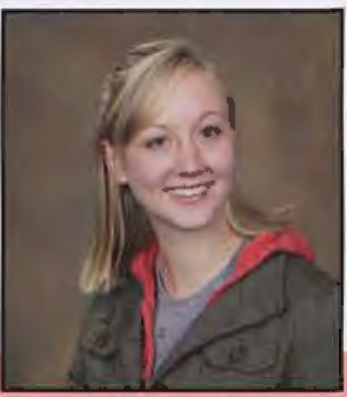

Kathleen Bertagnoli

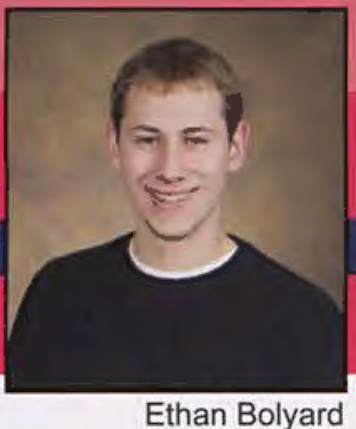




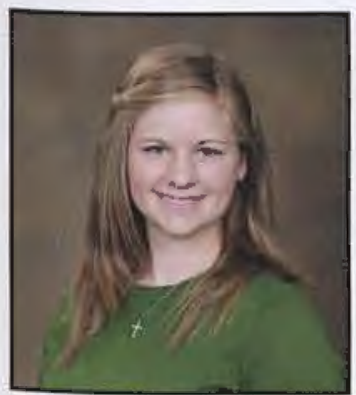

Sarah Bowlsby

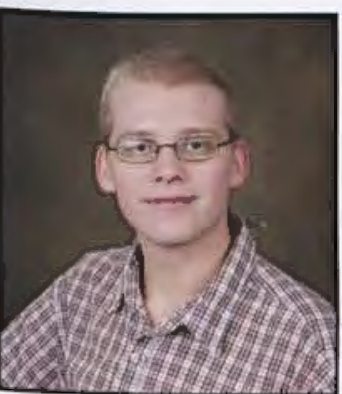

Dennis Brodzinsk

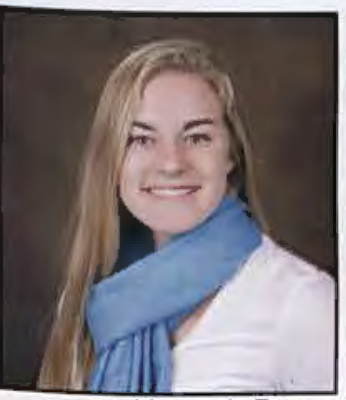

Hannah Bryan

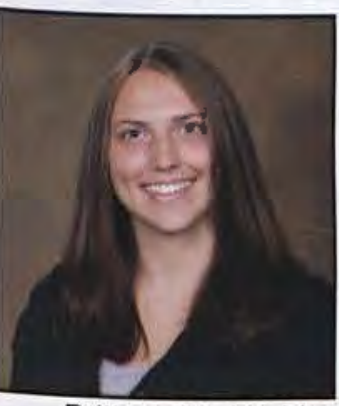

Brittany Burkholder

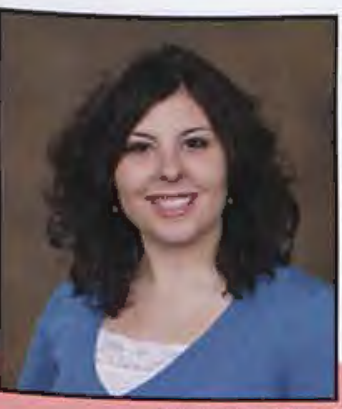

Lindsay Carr

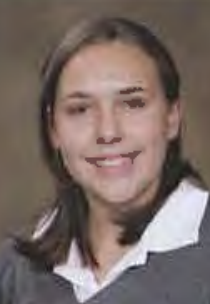

Heather Boyd

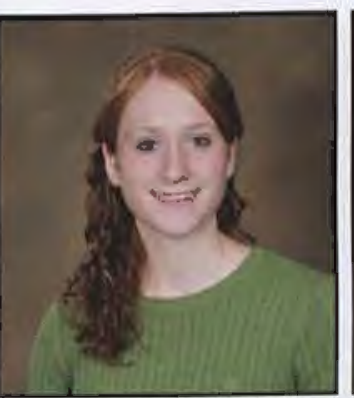

Andrea Brown

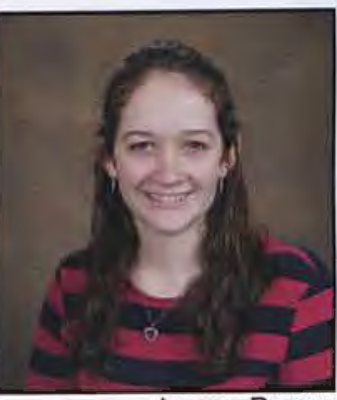

Laura Bryan

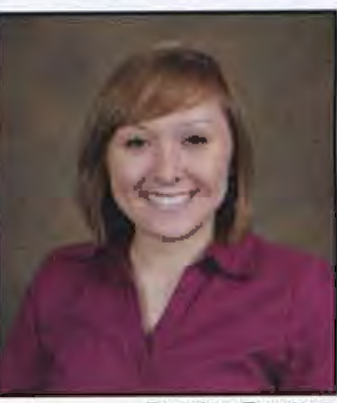

Becky Burton

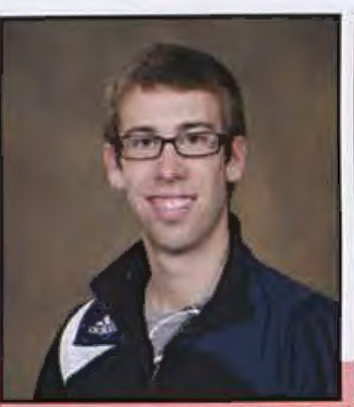

Robbie Carter

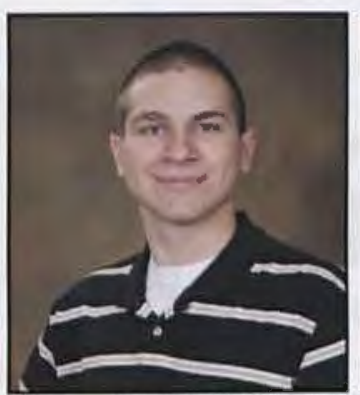

William Brannon,

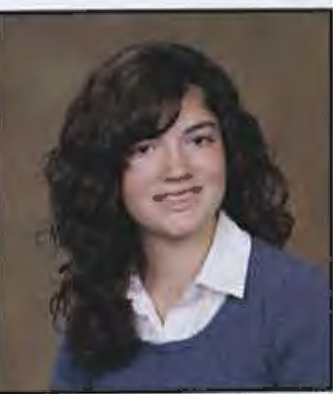

Breanna Brown

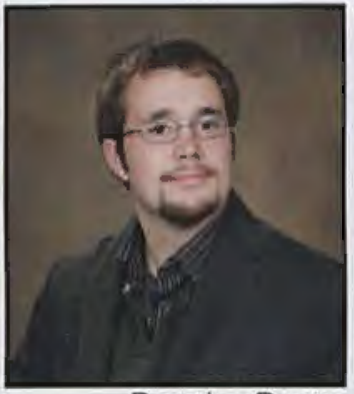

Douglas Brown
Alexandria Bryce
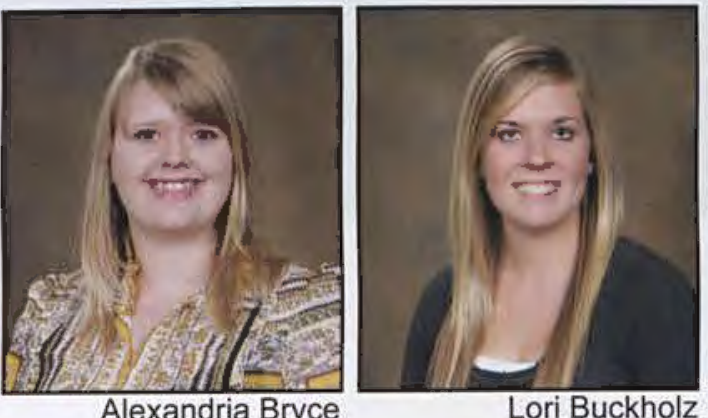

Lori Buckholz

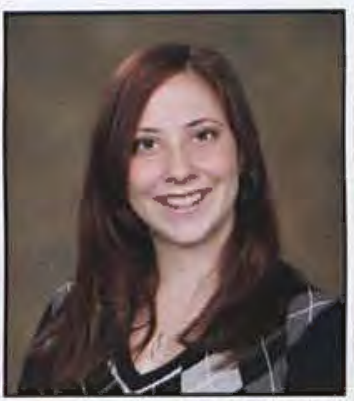

Olivia Byers

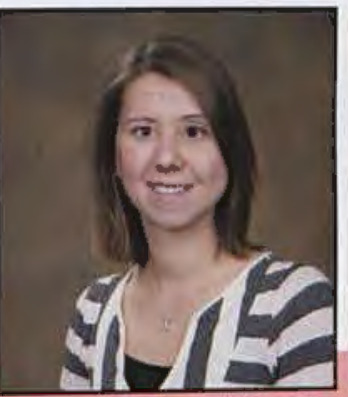

Margaret Casillo
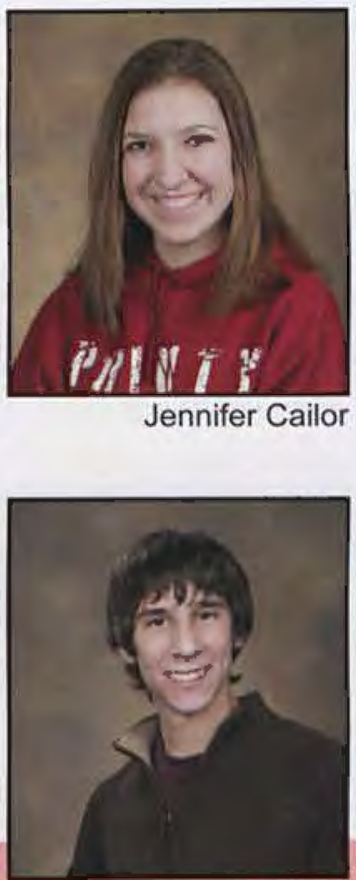

Joseph Cathey

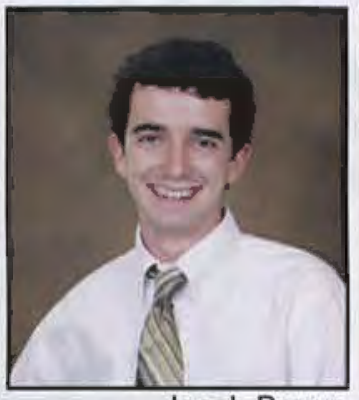

Jacob Brown
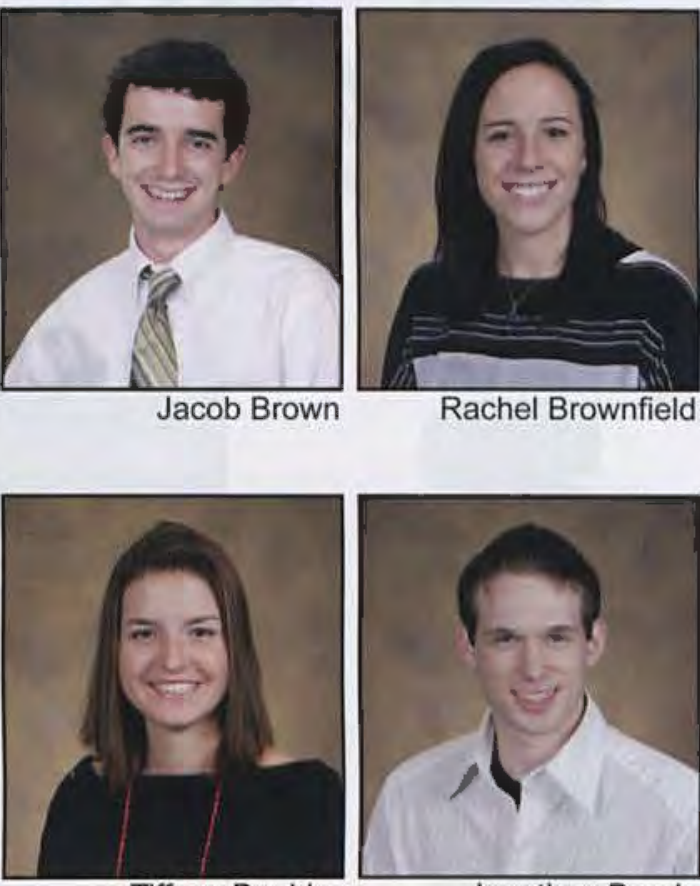

Tiffany Buehler

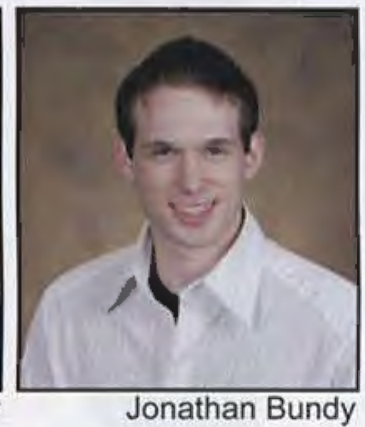

Jonathan Bundy

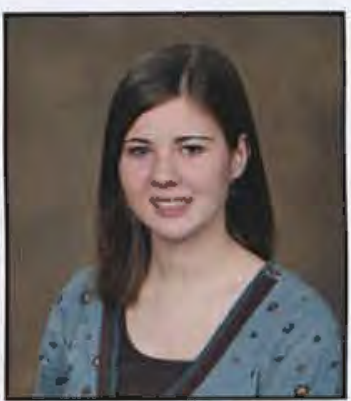

Hannah Campbell

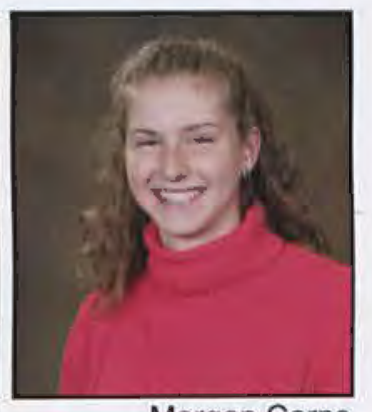

Morgan Carns

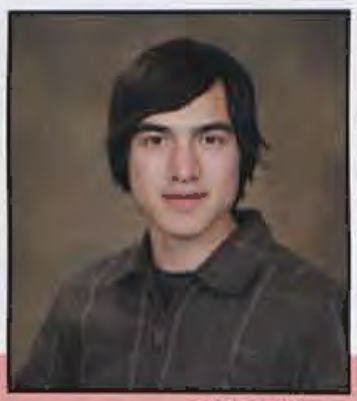

Tyler Chan

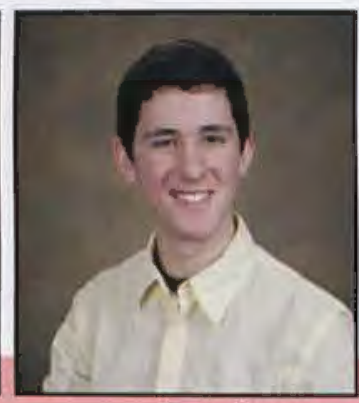

Ben Chasnov
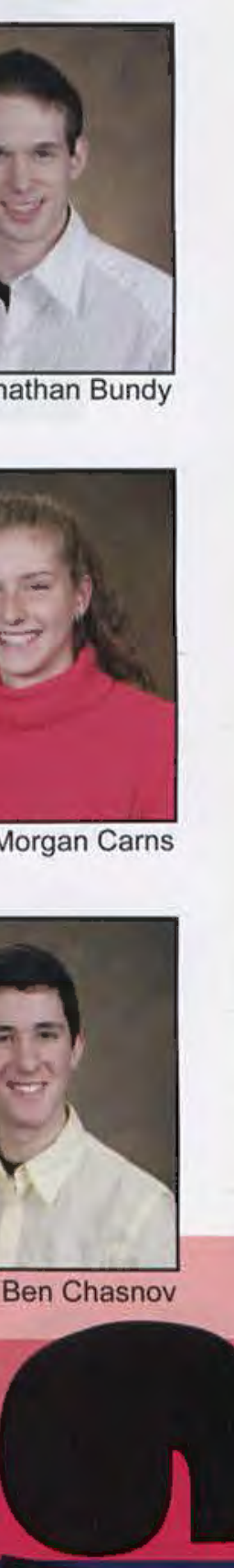

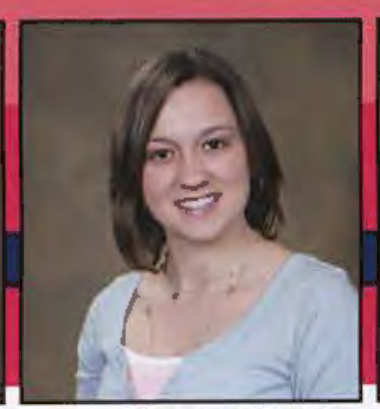

Jessica Chester

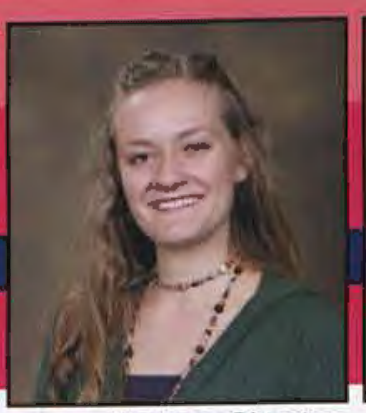

Melissa Cheston
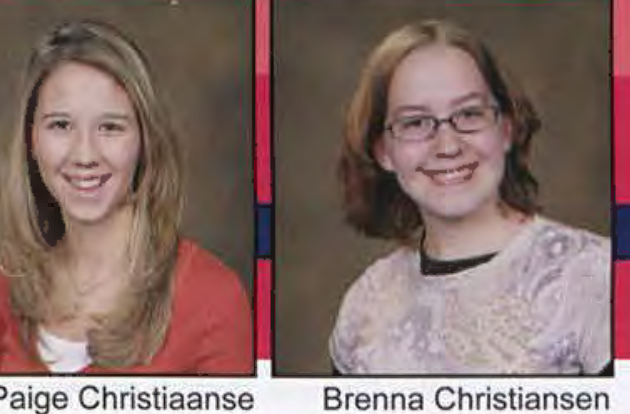

Brenna Christiansen 


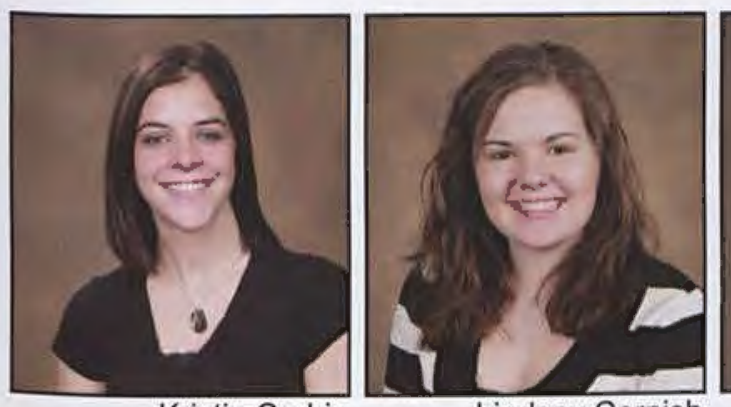

Kristin Corbin

Lindsay Cornish

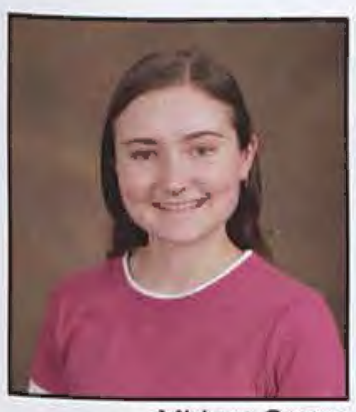

Miriam Crane

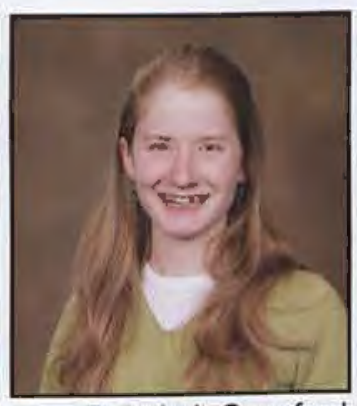

Rebekah Crawford

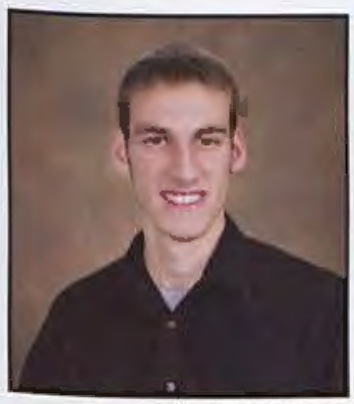

Mark D'Anna

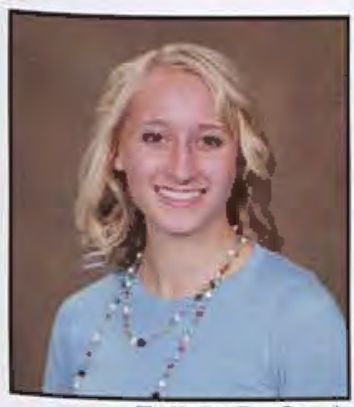

Felicia DeCook

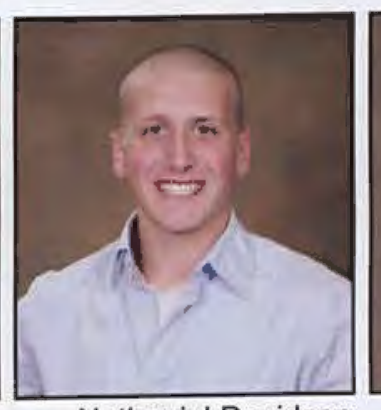

Nathaniel Davidson

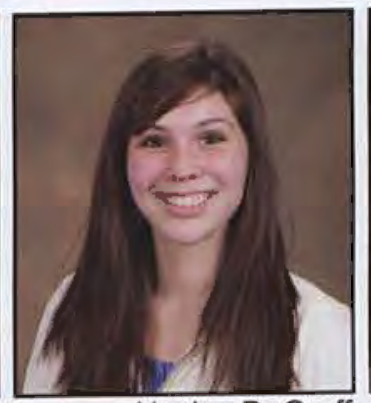

Hayley DeGraff

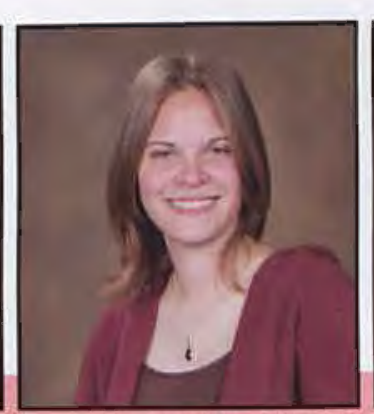

David Dion

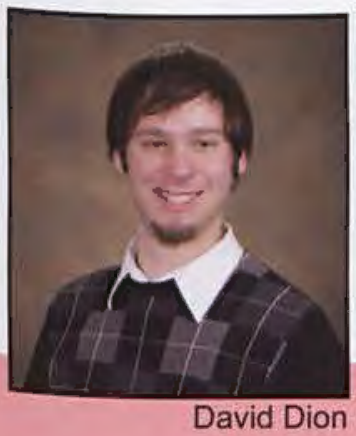

Jennifer Dixson

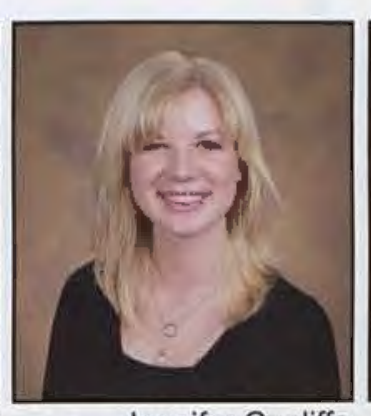

Jennifer Cunliffe

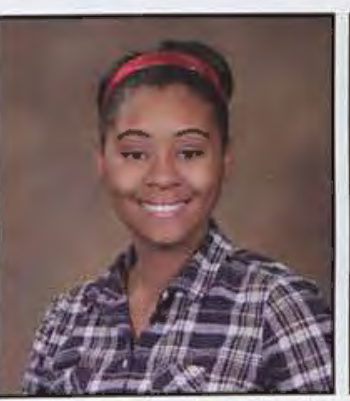

Andrea Davis
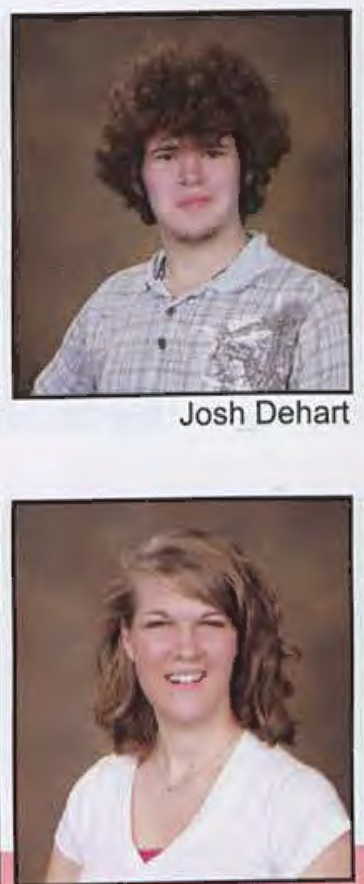

Rebekah Dodson

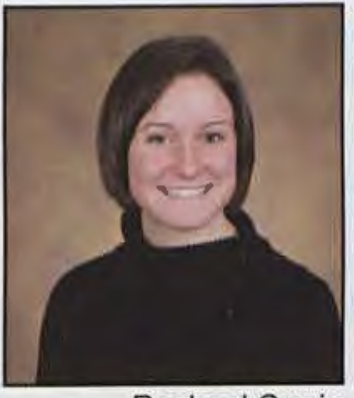

Rachael Currie

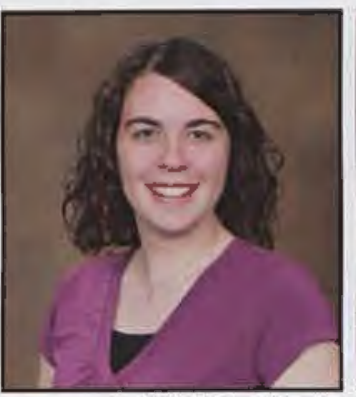

Virginia Davis
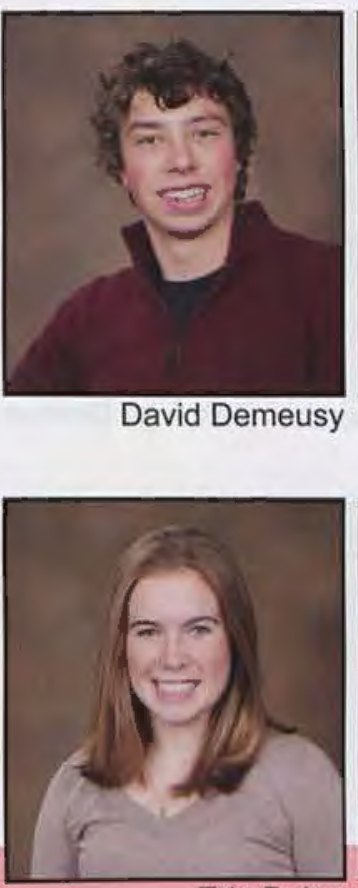

Erin Dolan

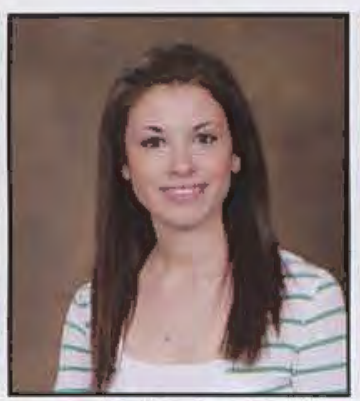

Katherine Dayton

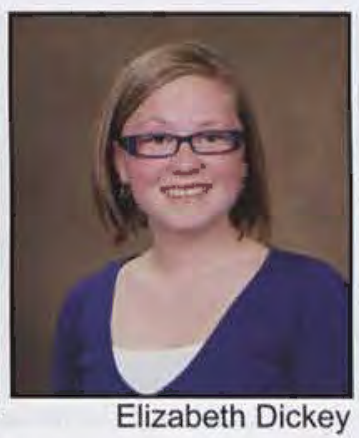

Elizabeth Dickey

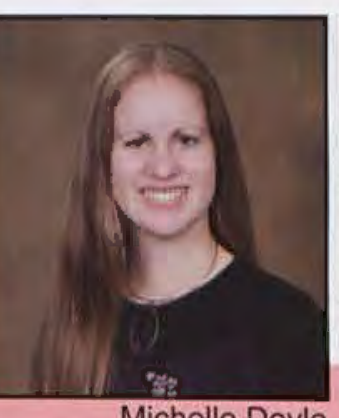

Michelle Doyle

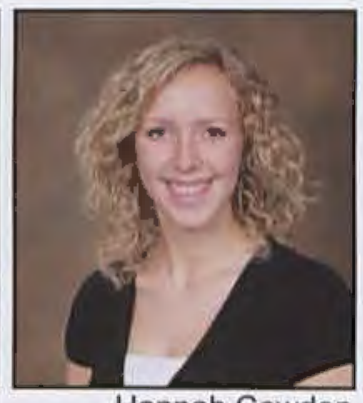

Hannah Cowden

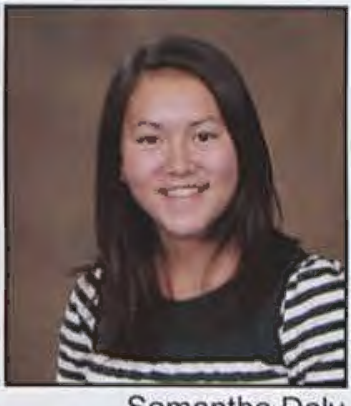

Samantha Daly

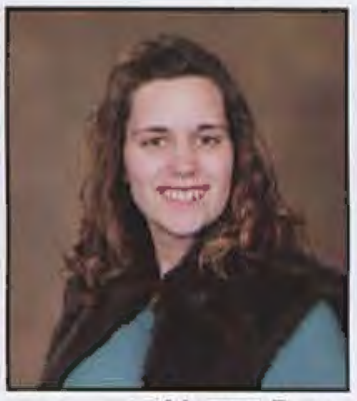

Megan Dean
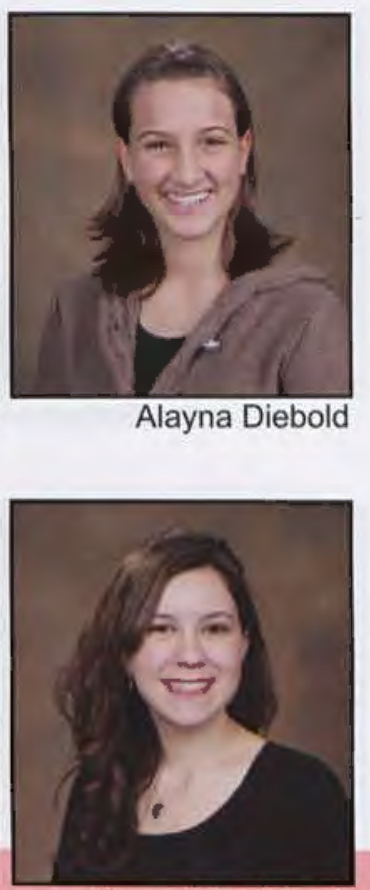

Hannah Dreisbach

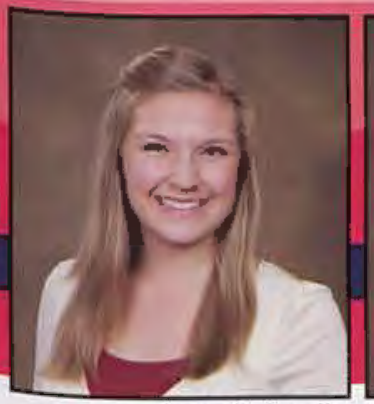

Jean Drimalla

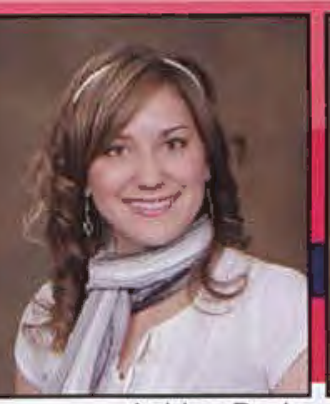

Ashley Duda

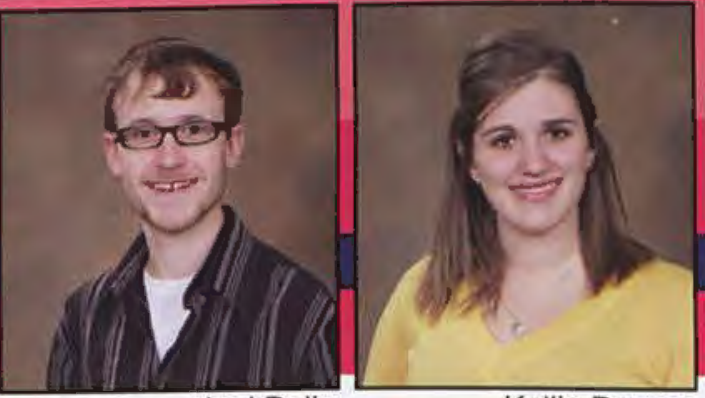

Joel Dulin
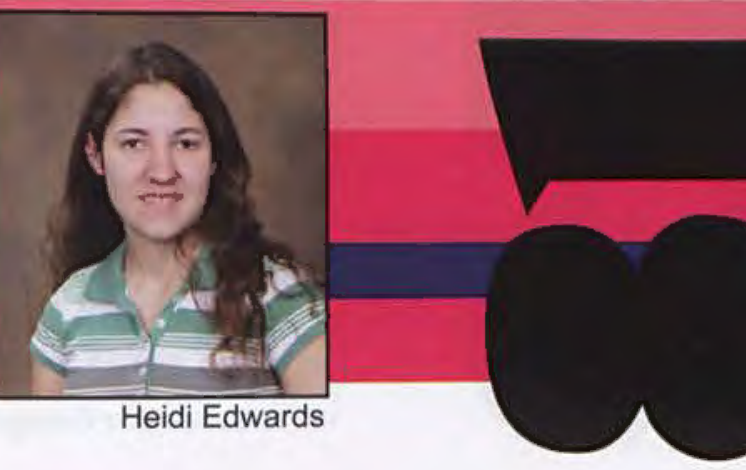

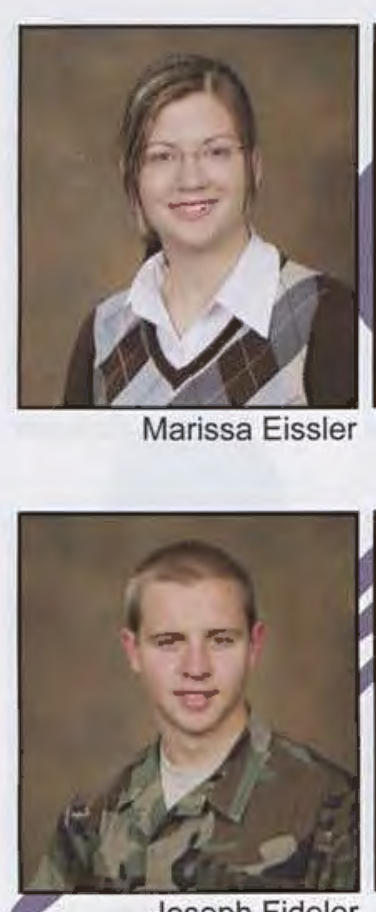

Joseph Fideler

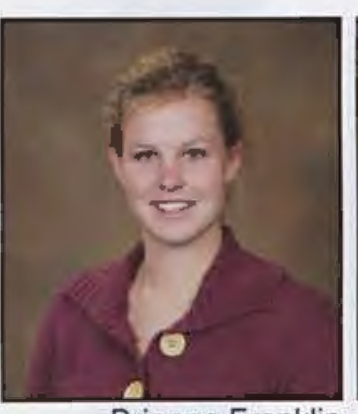

Brianna Franklin

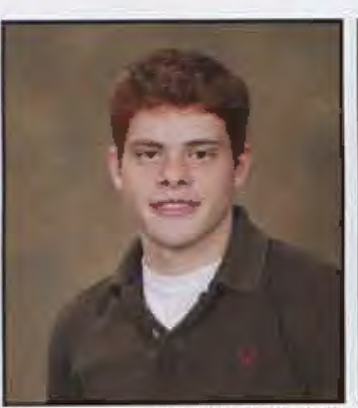

Joe Gallagher

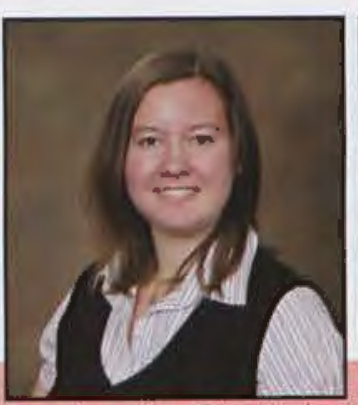

Jennifer Giambalvo

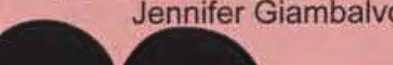

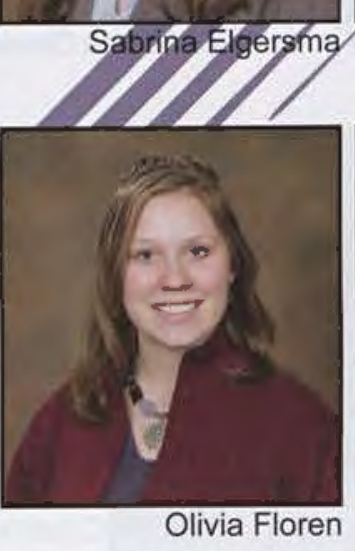

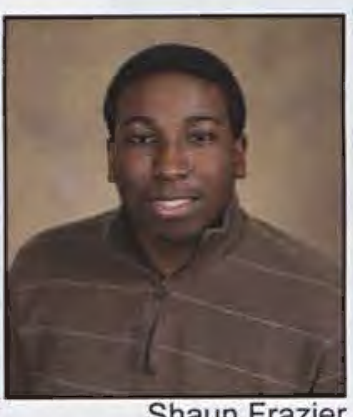

Shaun Frazier

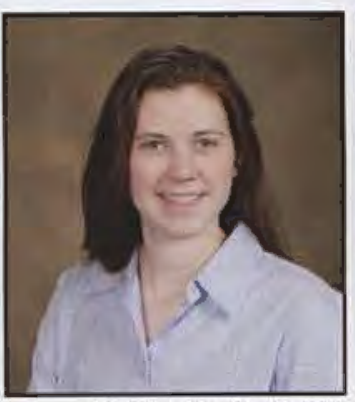

LeeAnne Gammie

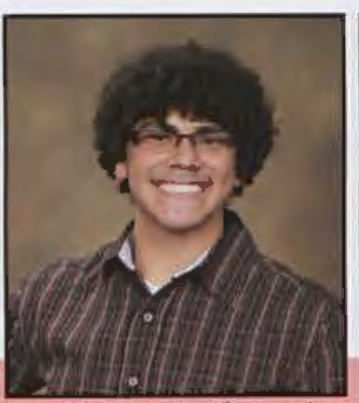

Alexander Gonzalez

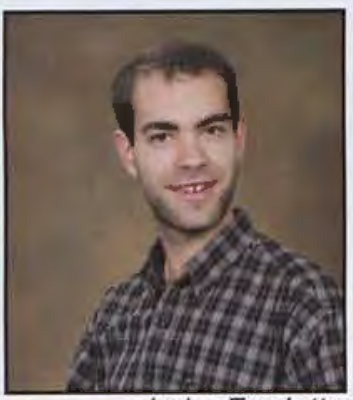

Luke Fredette

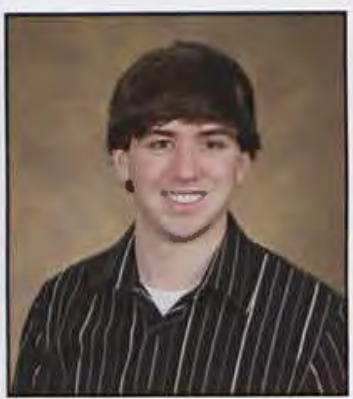

Jennings Gardner

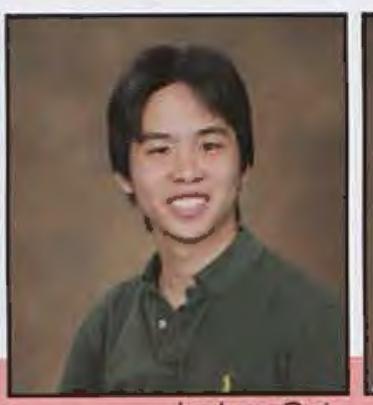

Joshua Goto

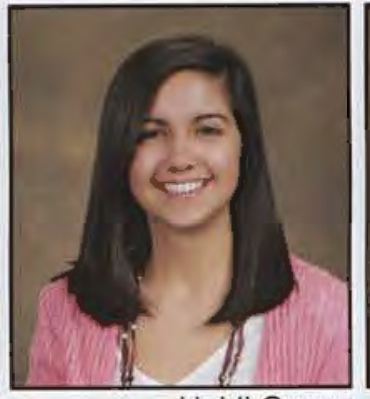

Heidi Gavza

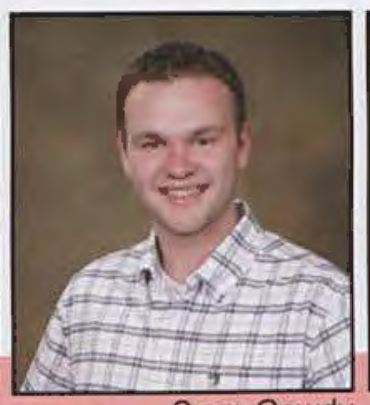

Sean Gowdy
Justin Feczer
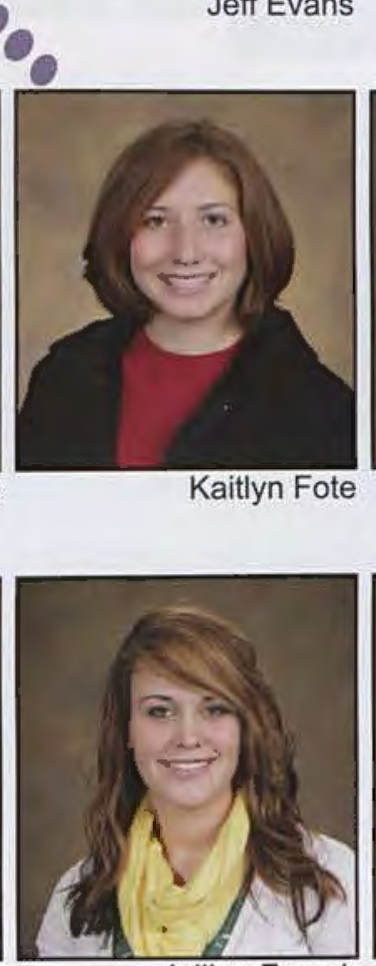

Jullian French

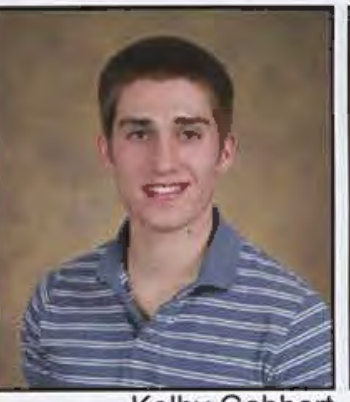

Kolby Gabbert

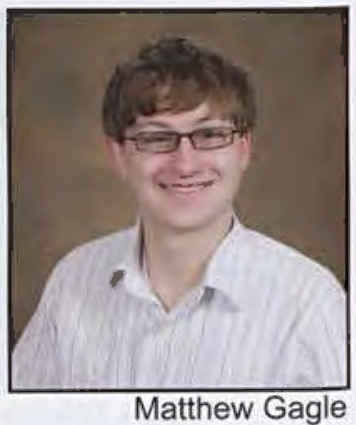

Matthew Gagle

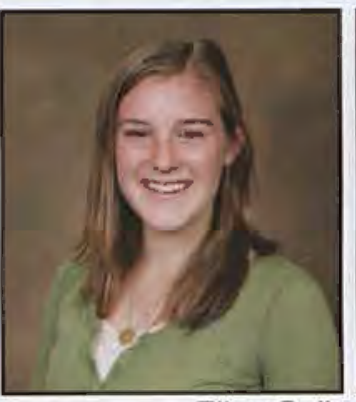

Ellen Geib

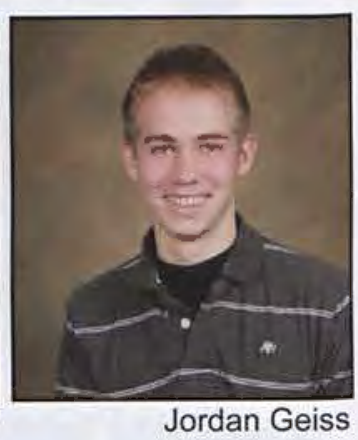

Jordan Geiss

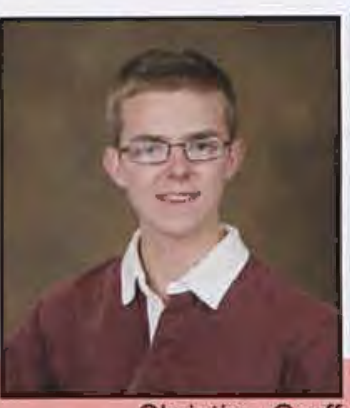

Christian Graff

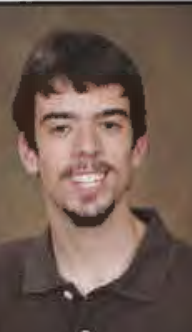

Kyle Fox

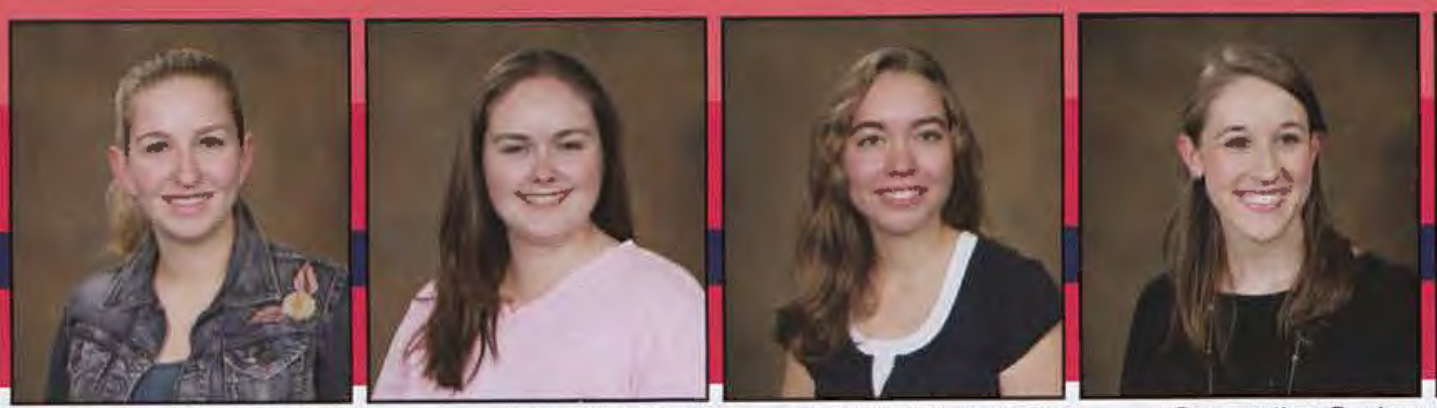




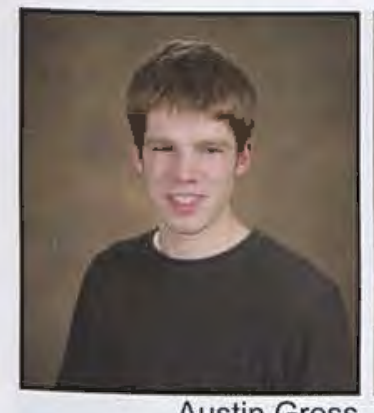

Austin Gress

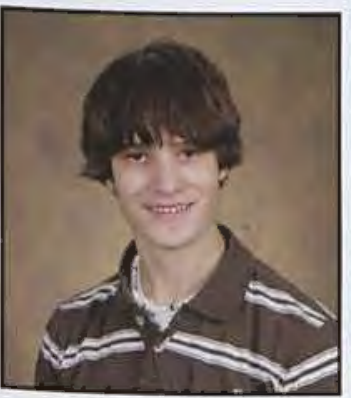

Brent Hagy

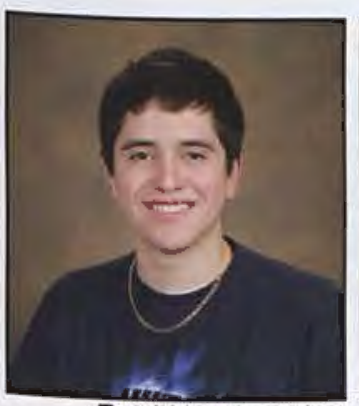

Paul Hembekides

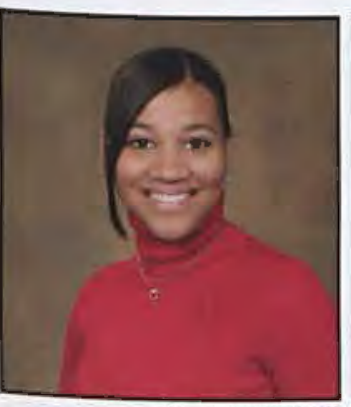

Candace Hill

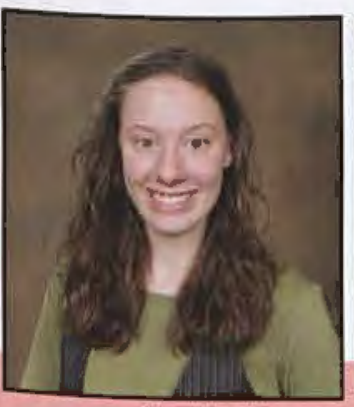

Noelle Hooper

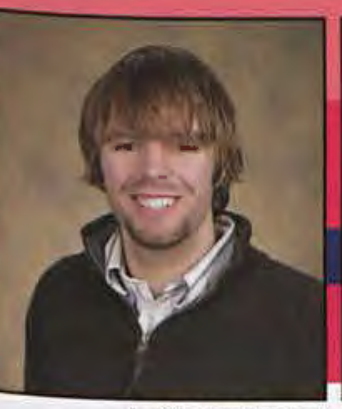

Leif Jacobsen

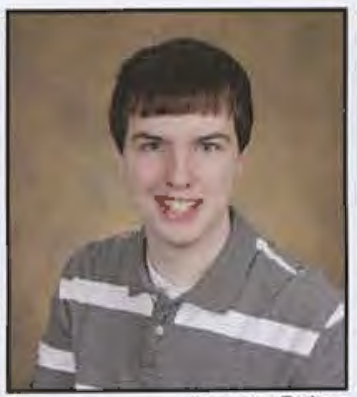

Jason Grier

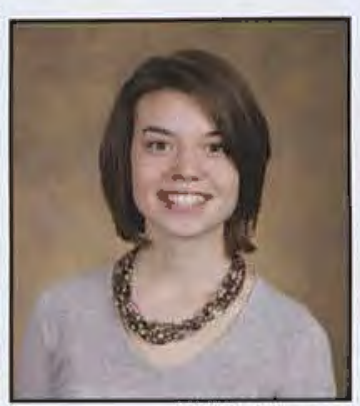

Faith Hamm

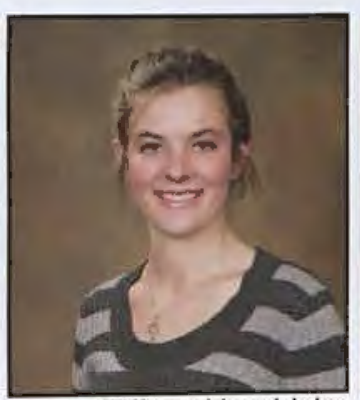

Tiffany Hendricks

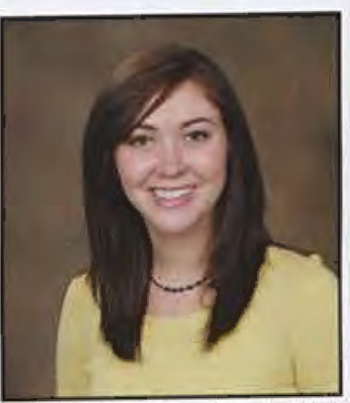

Stephanie Hill

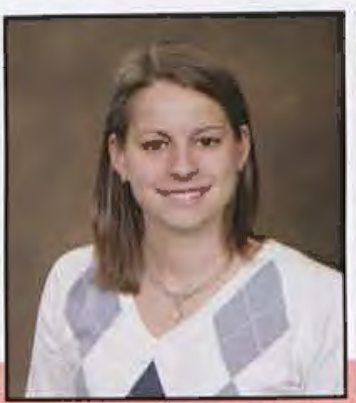

Rachel Horst

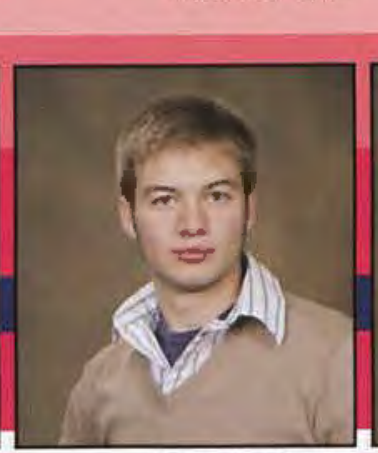

Joseph James

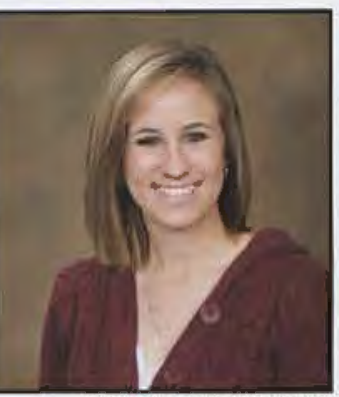

Stephanie Griffith

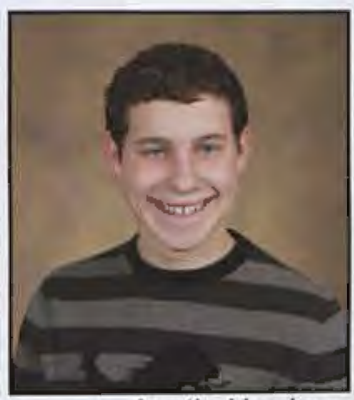

Austin Harrison

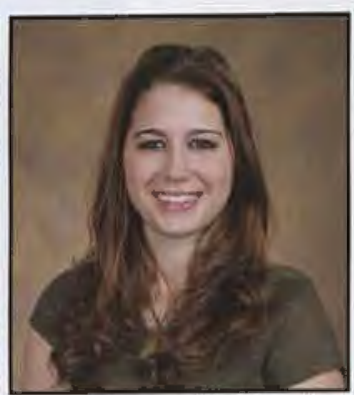

Laura Henkenius

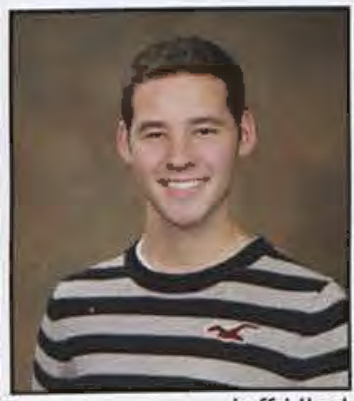

Jeff Hlad

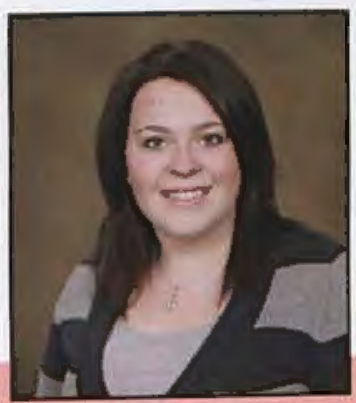

Lisa Humberson

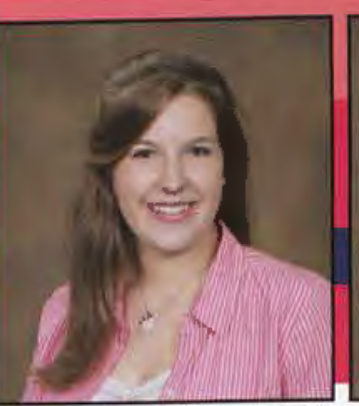

Ashley Janczewski

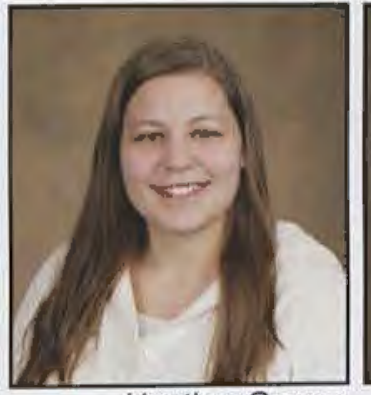

Heather Grosso

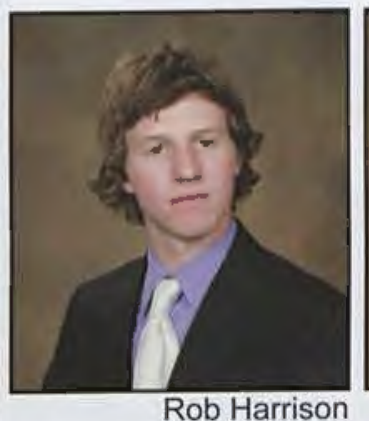

Rob Harrison

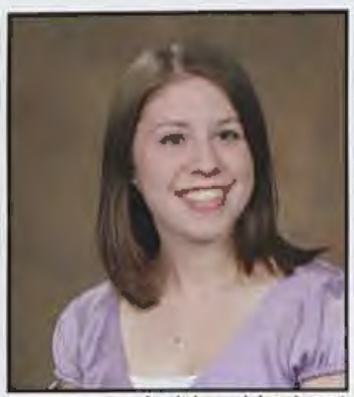

Ashley Herbert
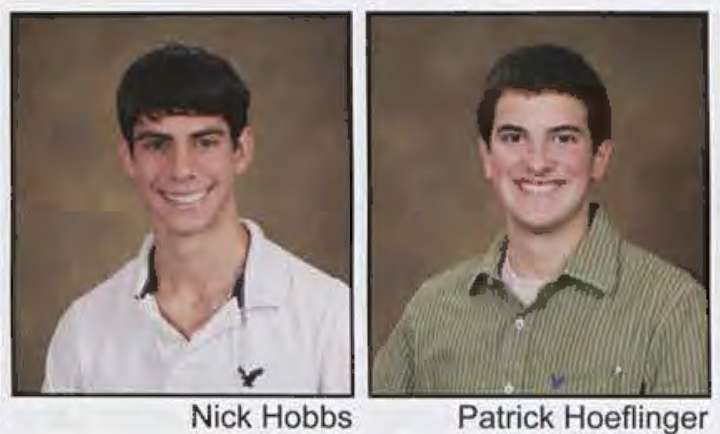

Patrick Hoeflinger

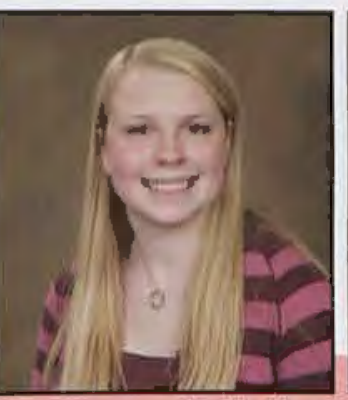

Katie Hunt
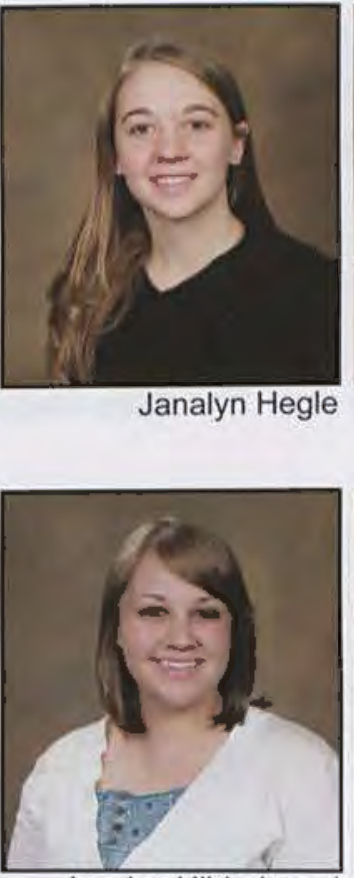

Jessica Hilderbrand

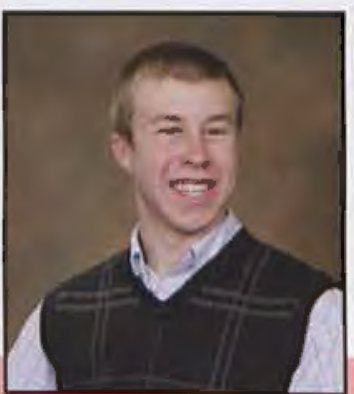

Craig Hunter
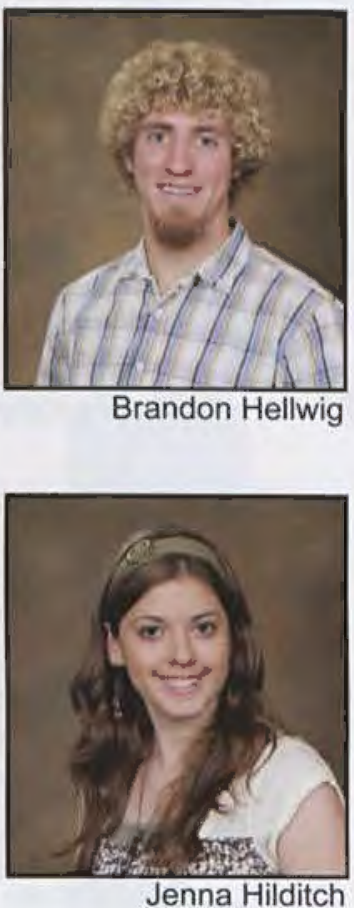

Jenna Hilditch

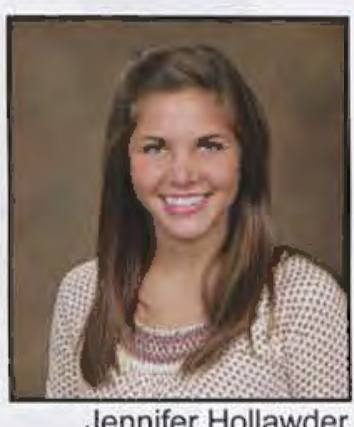

Jennifer Hollawder

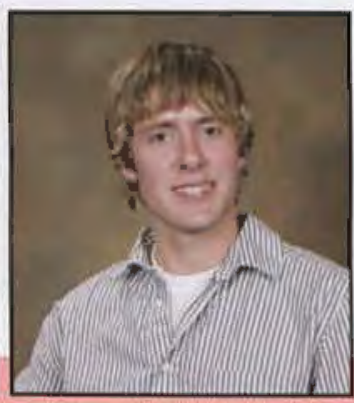

Caleb Ingram

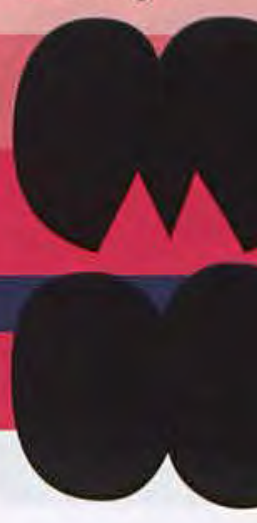




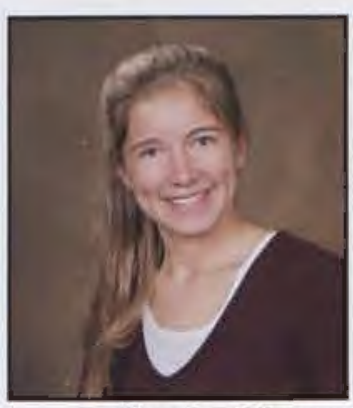

Kendra Johnson

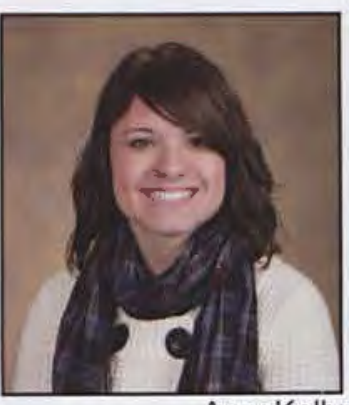

Amy Kelly

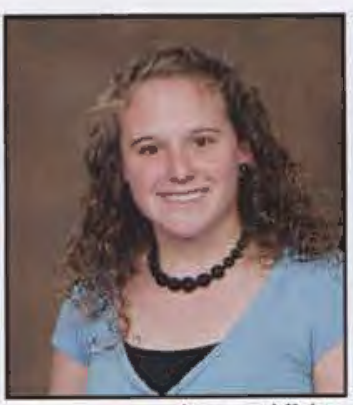

Laura Kirby

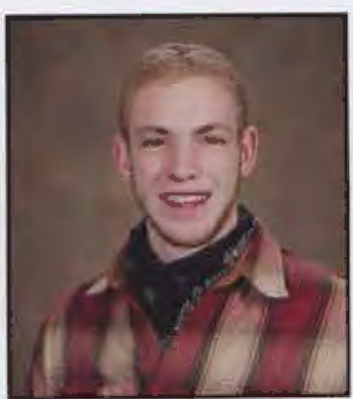

Jesse Kuiken

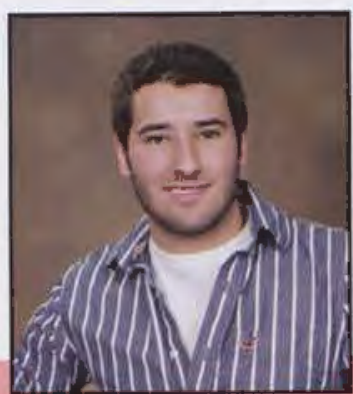

Evan Lavoie

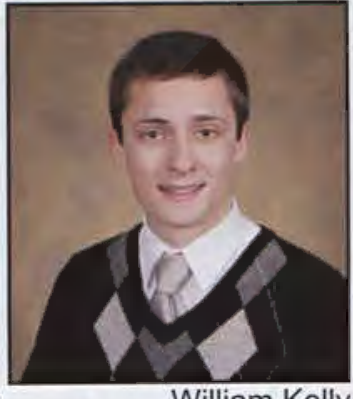

William Kelly

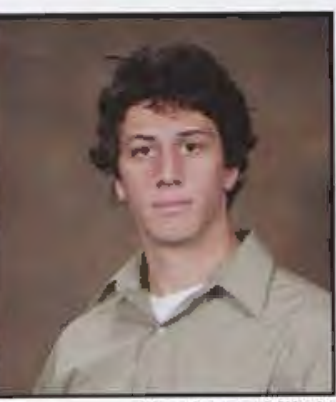

Zachary Klink

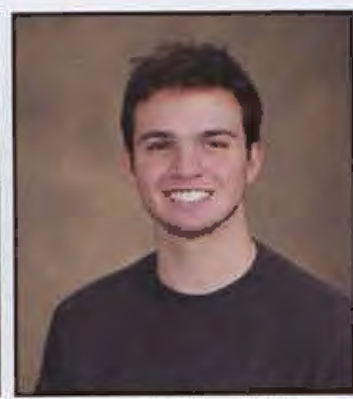

Michael Lacey

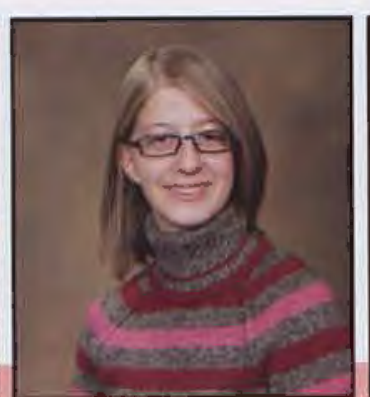

Sarah Lawton
(1)

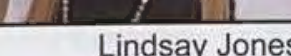

Lindsay Jones

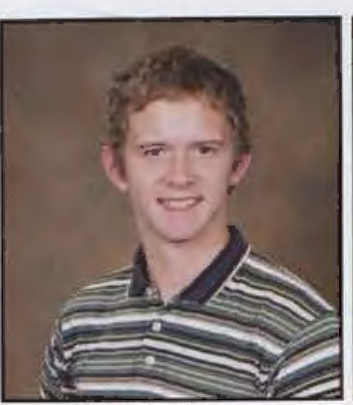

David Kemmenoe

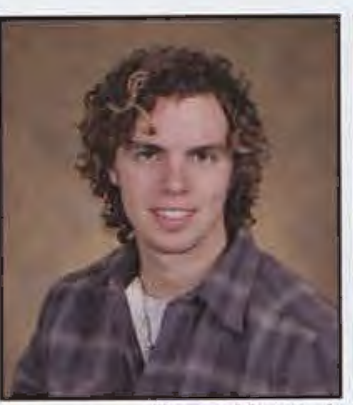

Ryan Knepel

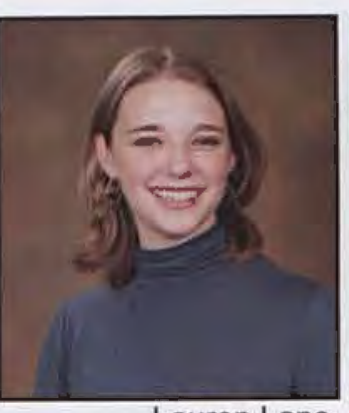

Lauren Lane

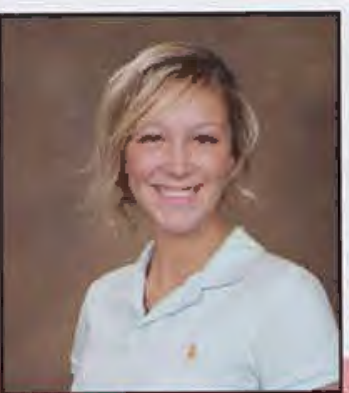

Rachel Lee

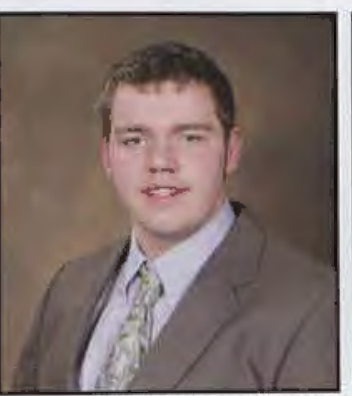

Nicholas Kerner

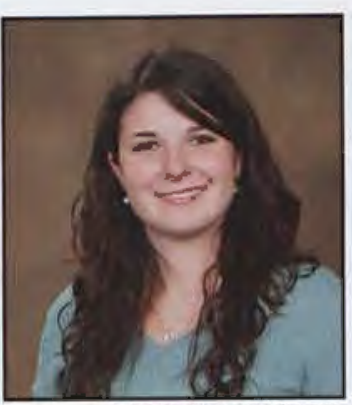

Rebeccah Knott

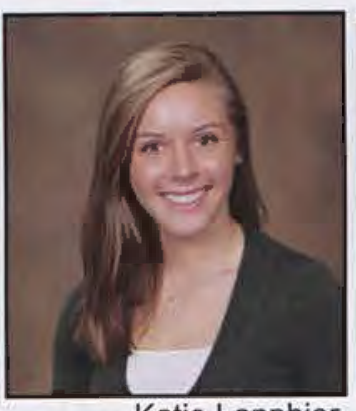

Katie Lanphier

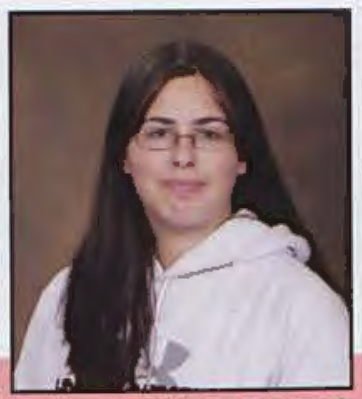

Caryne Leonard

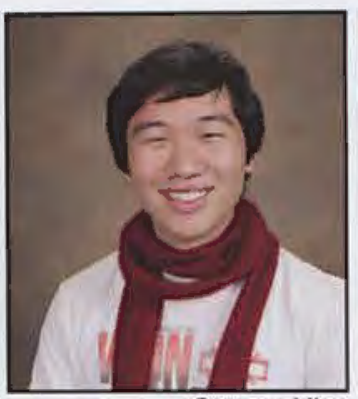

Seung Kim

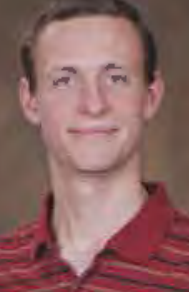

Matthew Koranek

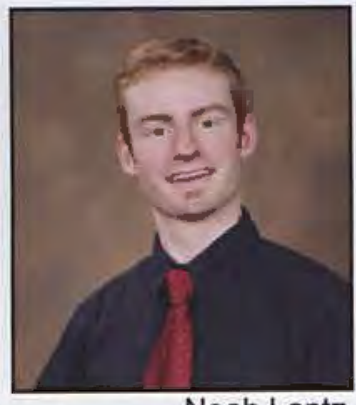

Noah Lantz

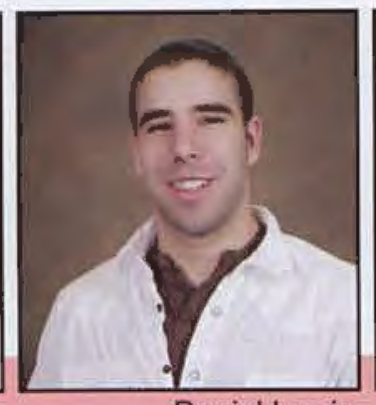

Daniel Lewis

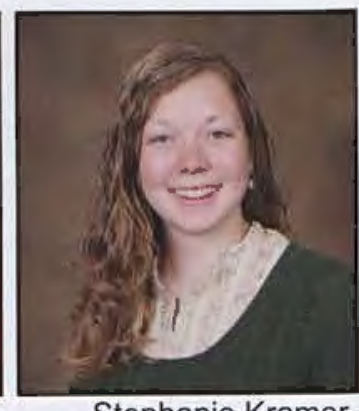

Stephanie Kramer

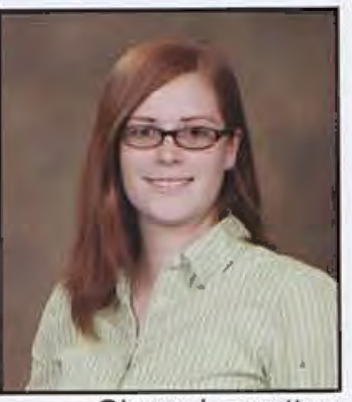

Ginger Lassetter

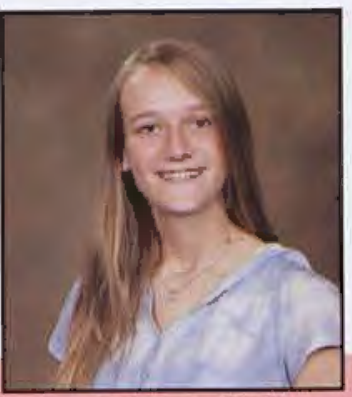

Melody Lightner
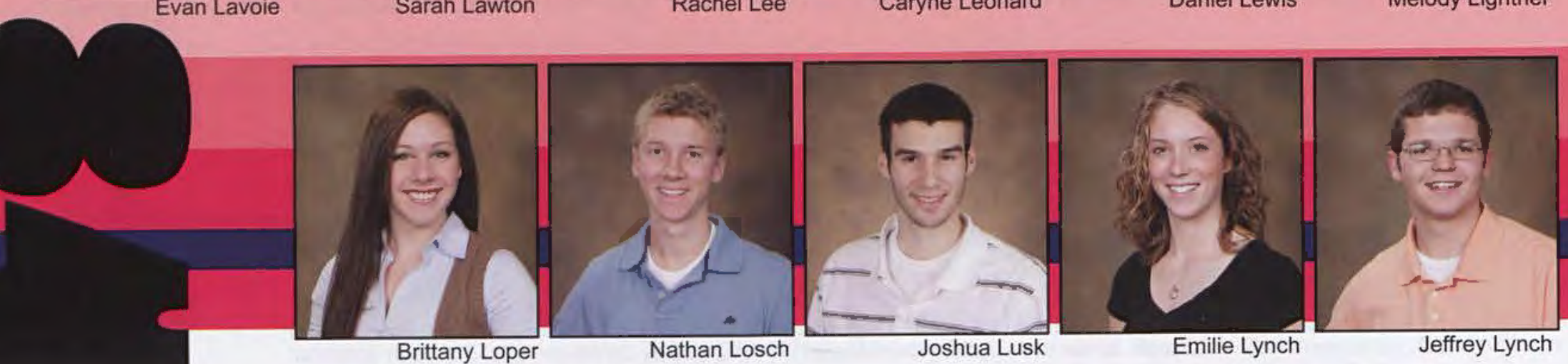

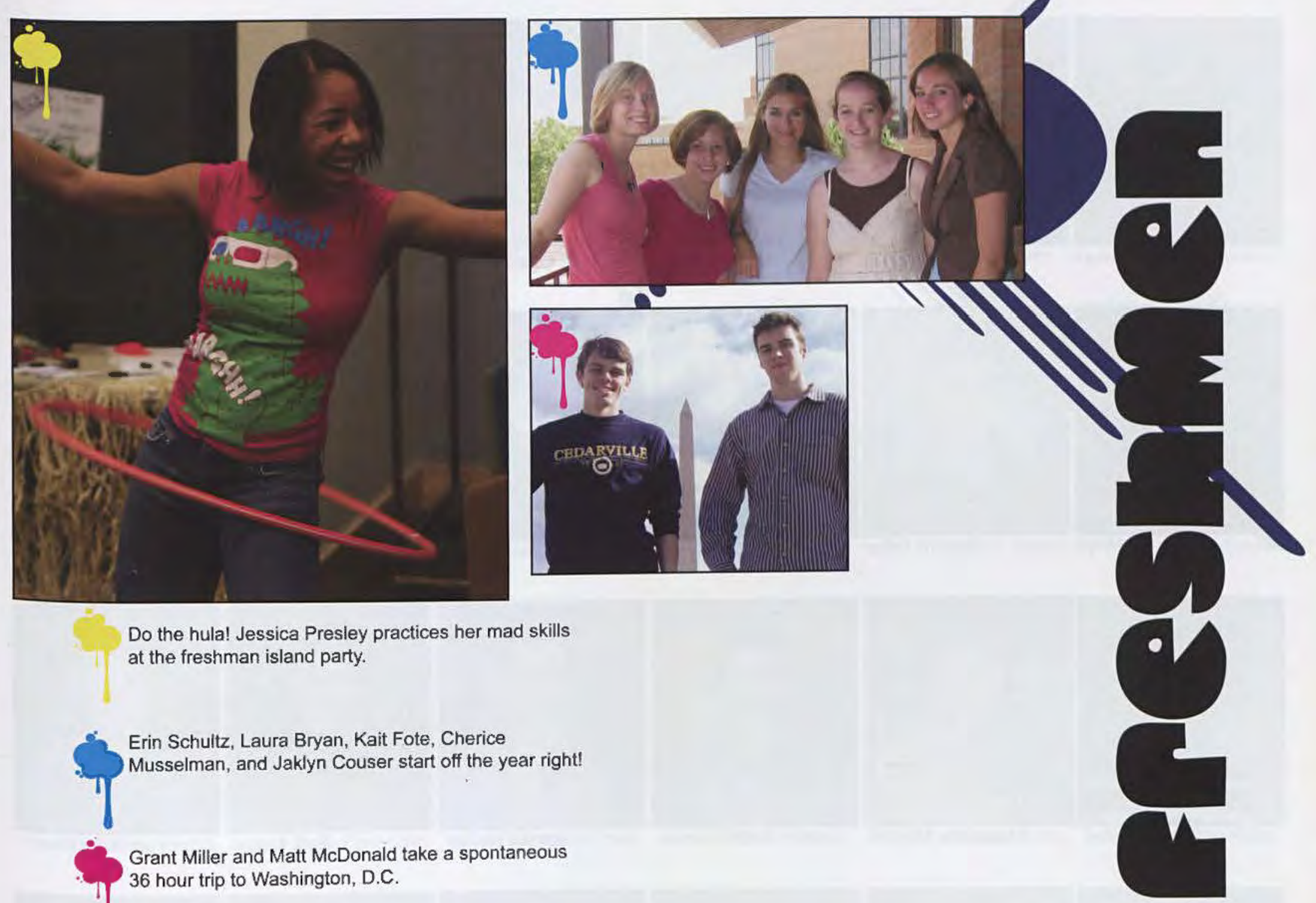

Do the hula! Jessica Presley practices her mad skills at the freshman island party.

6. Erin Schultz, Laura Bryan, Kait Fote, Cherice

Musselman, and Jaklyn Couser start off the year right! 1

Grant Miller and Matt McDonald take a spontaneous

36 hour trip to Washington, D.C.

"This first year has been so amazing and God has taught me so much, I can't wait to see what He has in store for me and my class in the next three

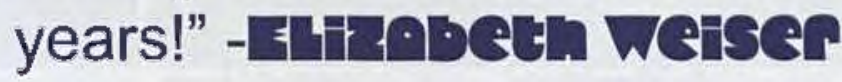

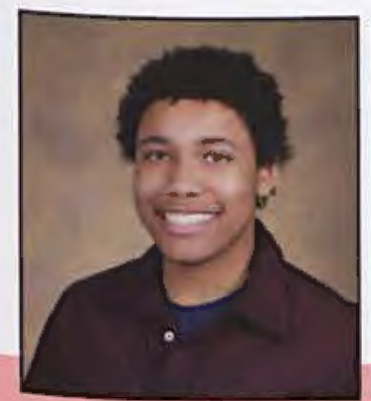

Kyle Mack

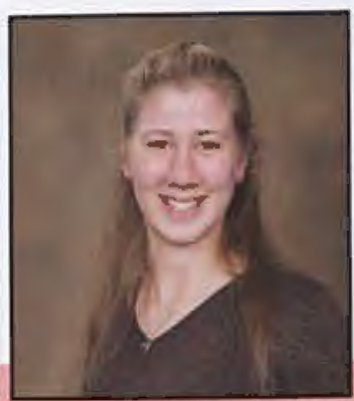

Allison Mackenzie

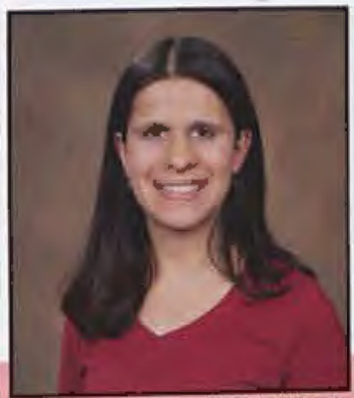

Sarah Maithel

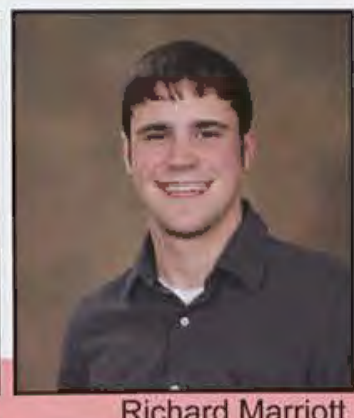

Richard Marriott

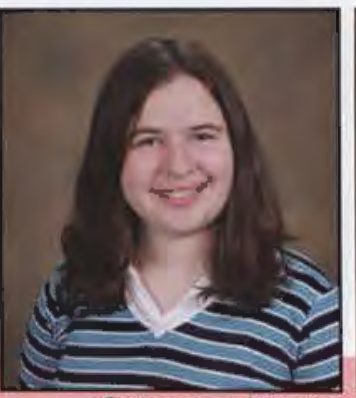

Catherine Martin

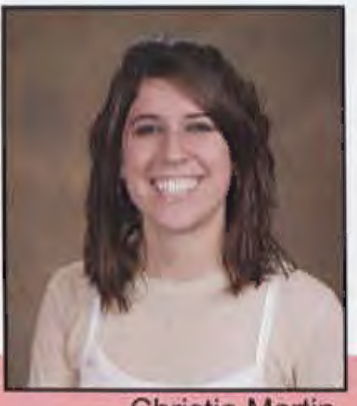

Christie Martin

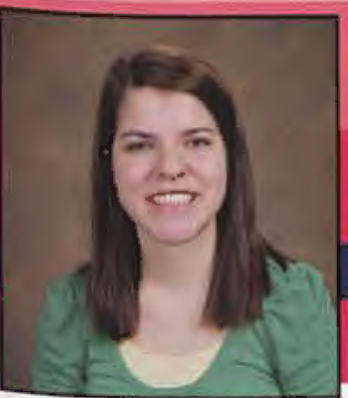

Alyssa McClure

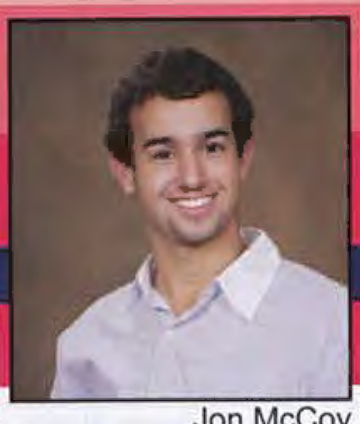

Jon $\mathrm{McCoy}$

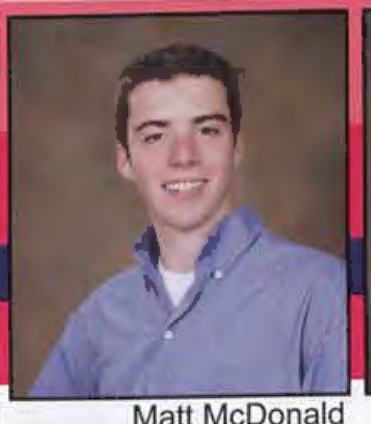

Matt McDonald
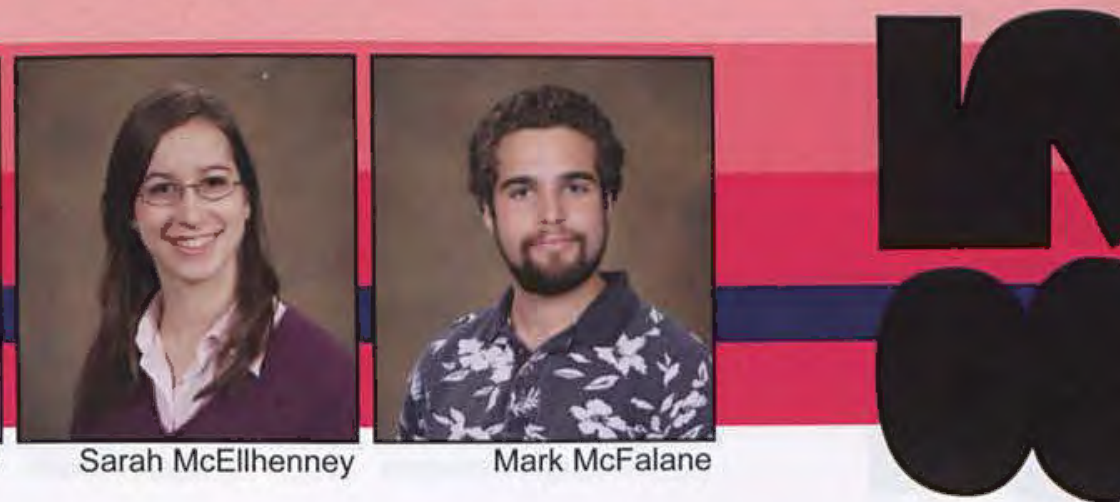


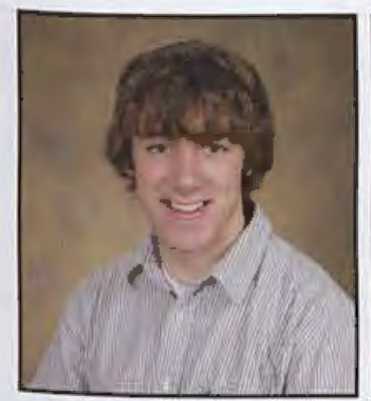

Jordan Oakes

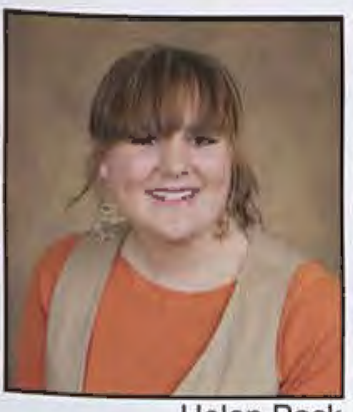

Helen Pack

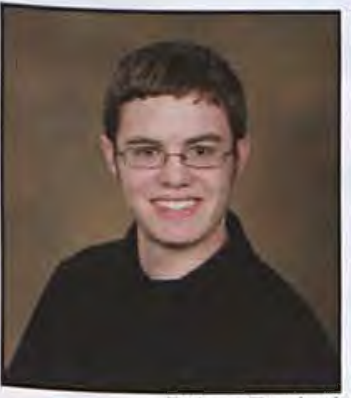

Brian Perhai

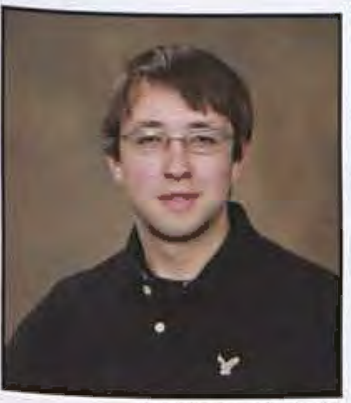

Thomas Poore

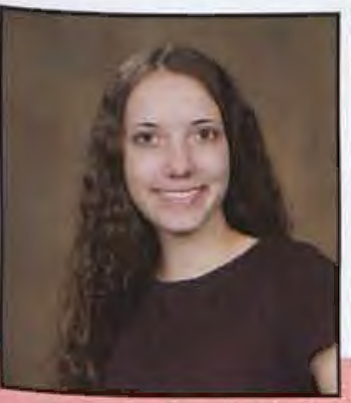

Sarah Rader

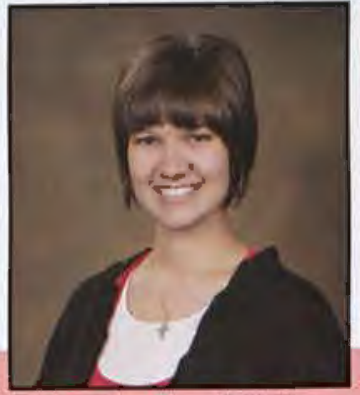

Jessica Rathburn

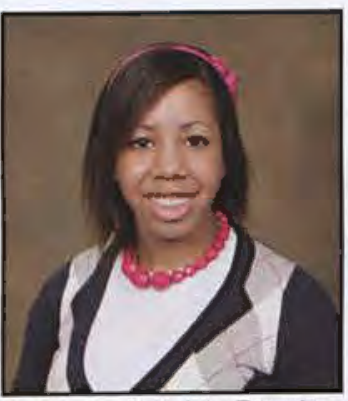

Jessica Presley

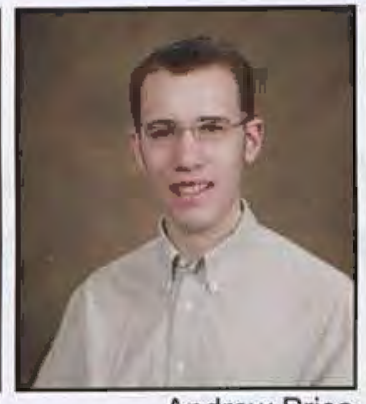

Andrew Price

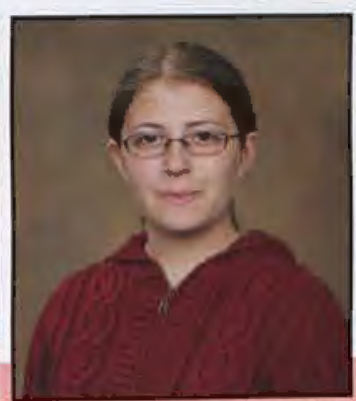

Brittany Rea

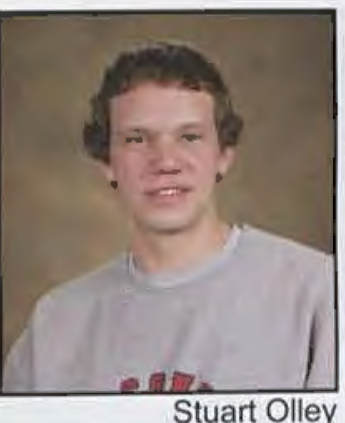

Stuart Olley

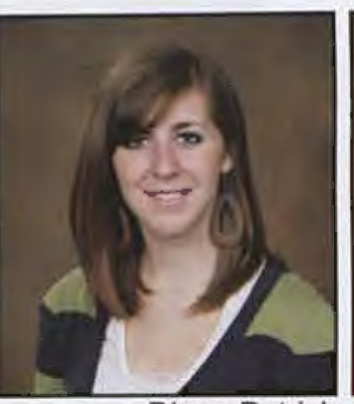

Diana Patrick

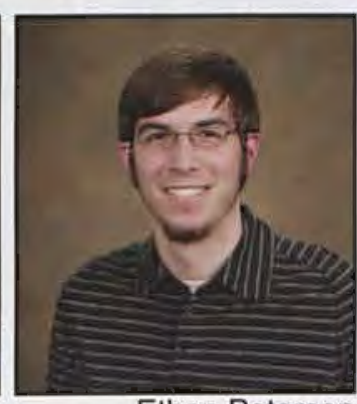

Ethan Peterson
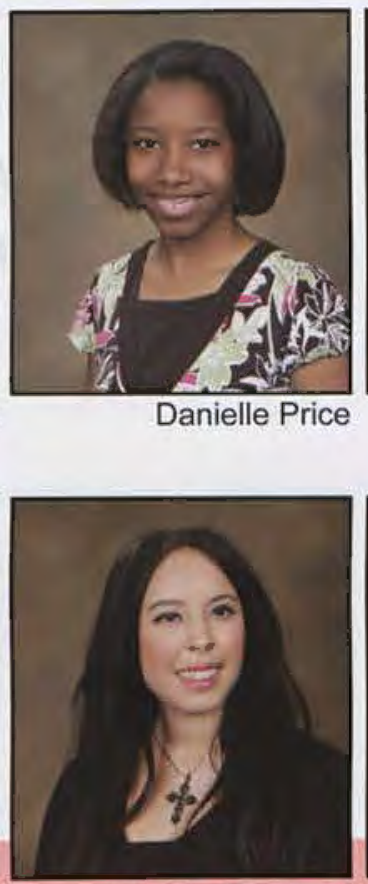

Claudia Reategui

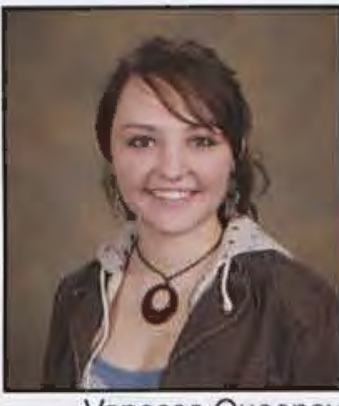

Vanessa Queeney

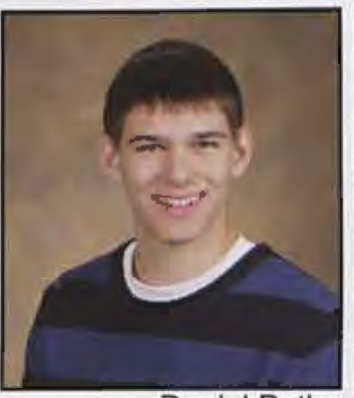

Daniel Petke

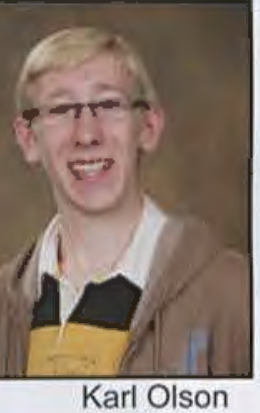

Karl Olson

Corey Ommundsen

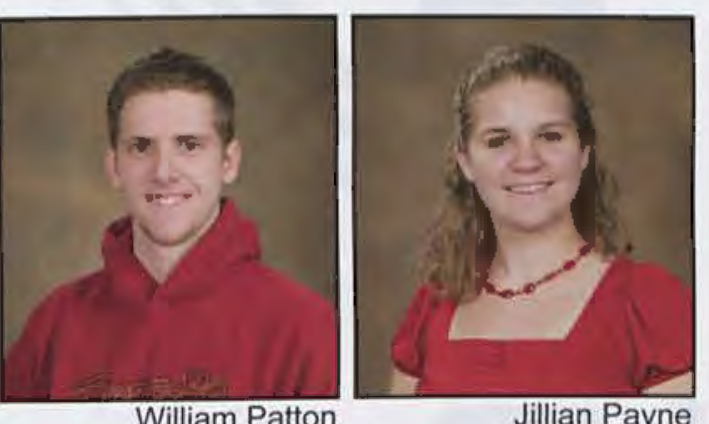

Jillian Payne

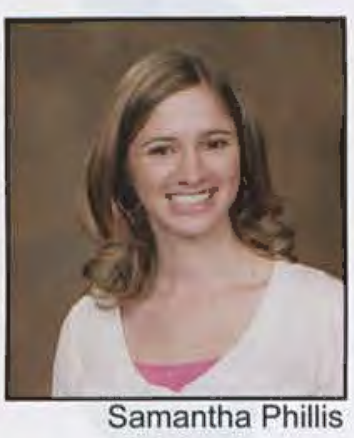

Samantha Phillis

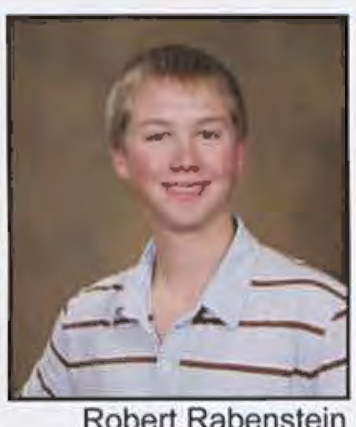

Robert Rabenstein

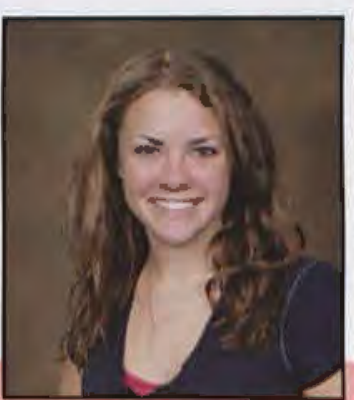

Susan Rienzo

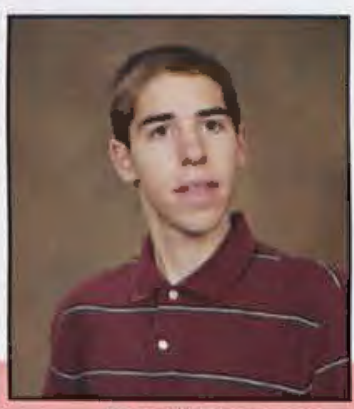

David Riggleman

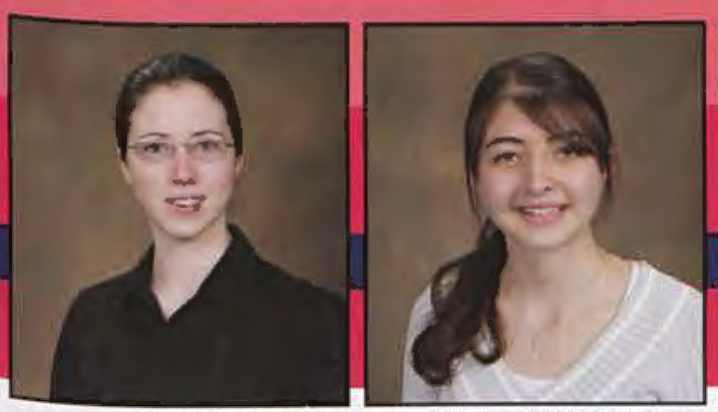

Jennifer Risser

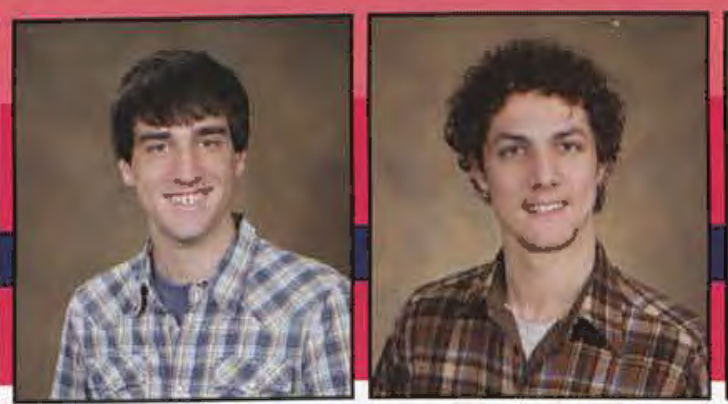

Tim Ronco

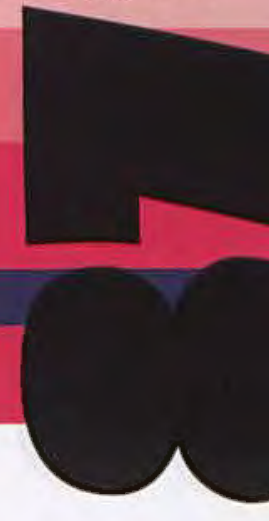




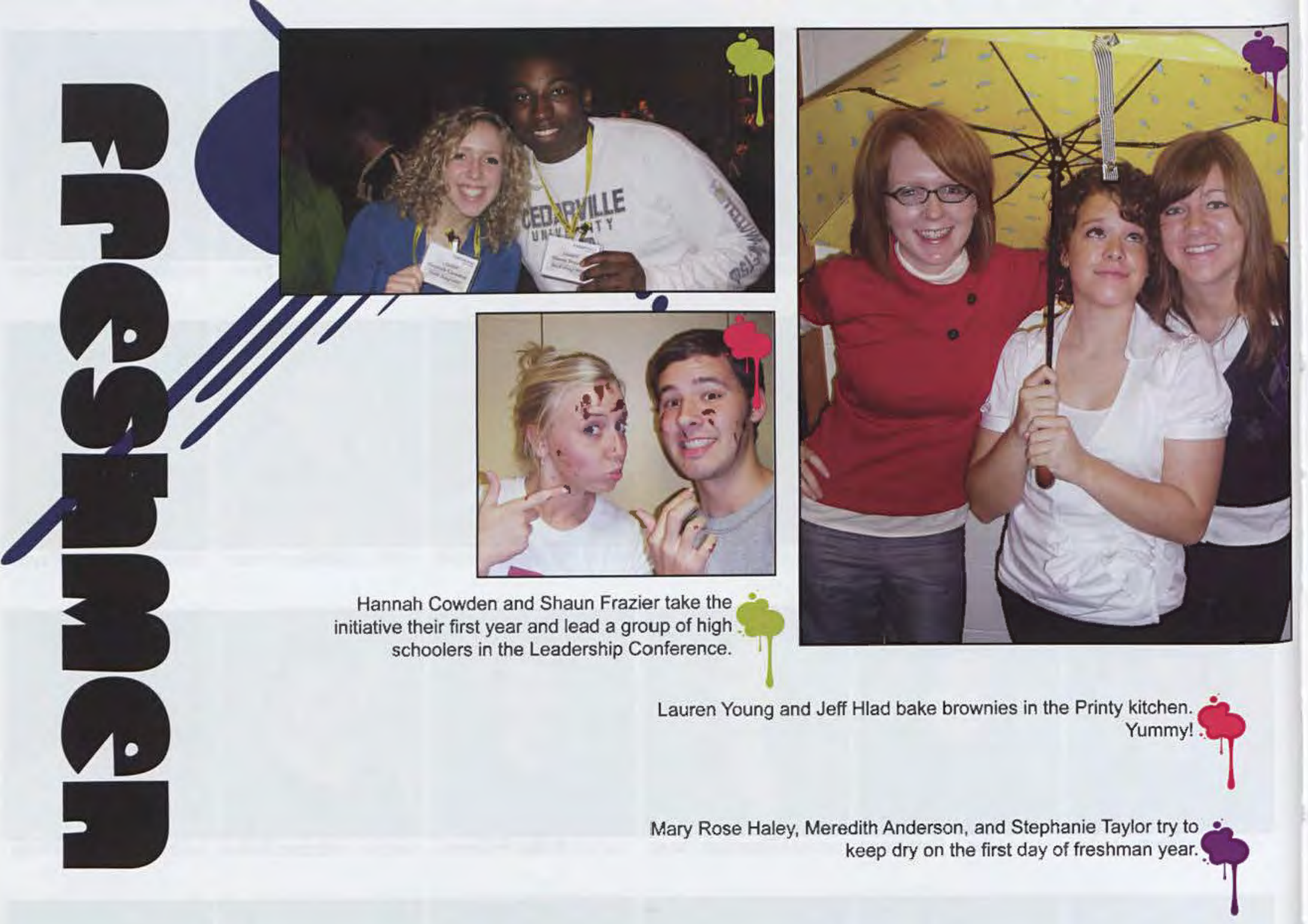

"Freshman year is like braces: awkward, painful,
and life changing." -

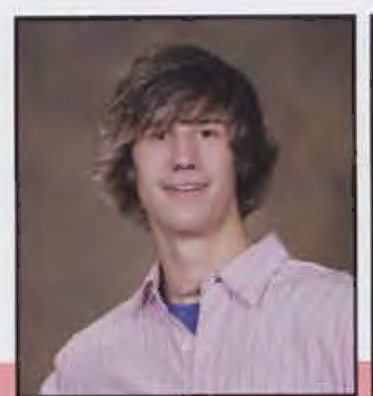

Niles Rosner

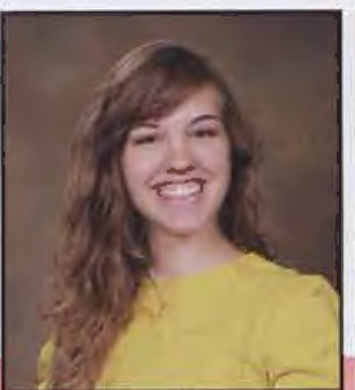

Sarah Rothhaar

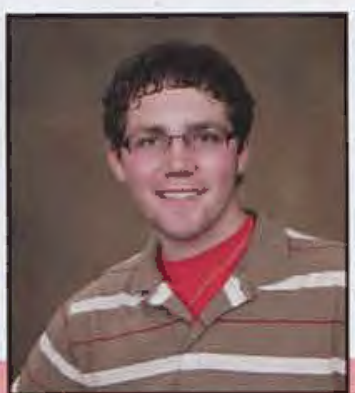

Garrett Rucinski

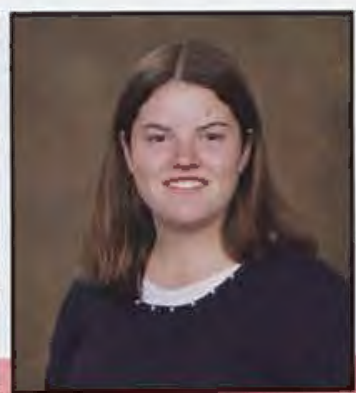

Bridget Russell

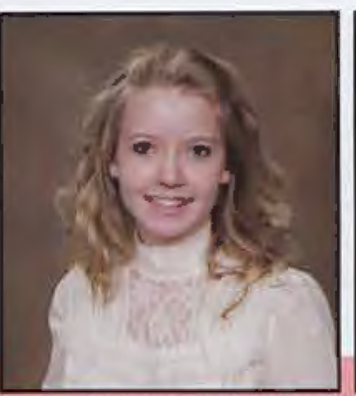

Stephanie Russell

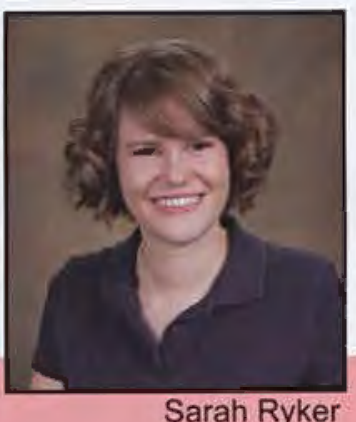

Sarah Ryker

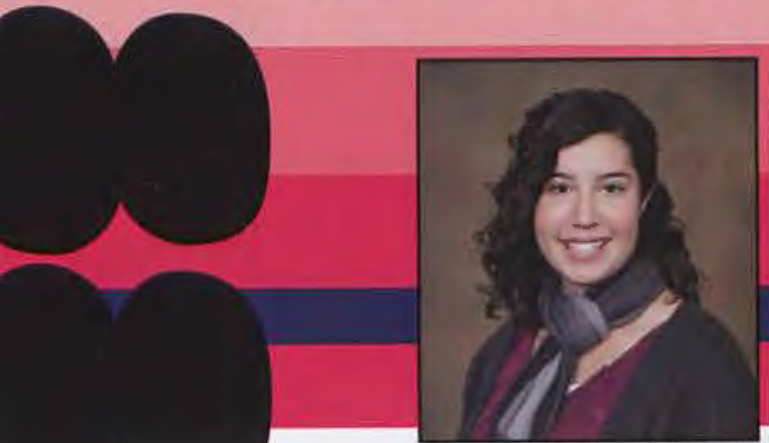

Sarah Sahulcik

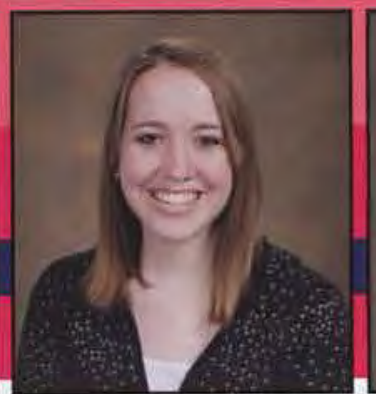

Stephanie Salzler

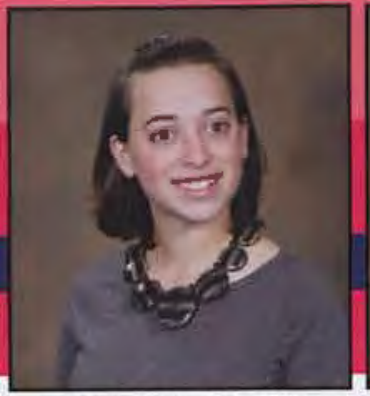

Gabrielle Sanfilippo

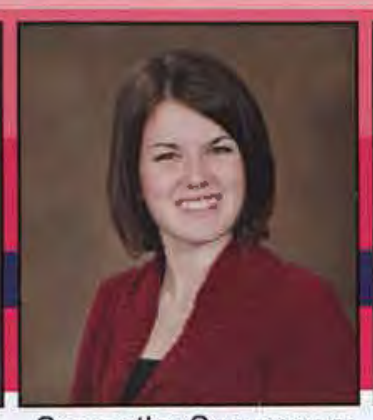

Samantha Sangregory

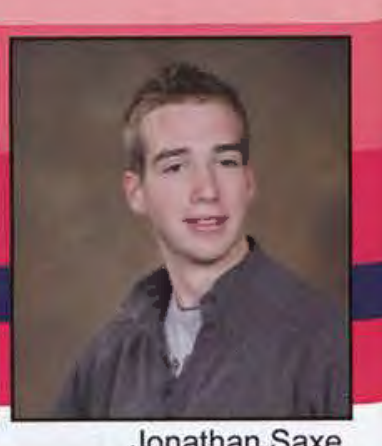

Jonathan Saxe 


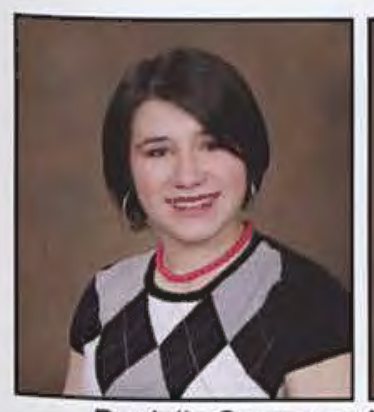

Danielle Scarpone Matthew Scheerschmidt

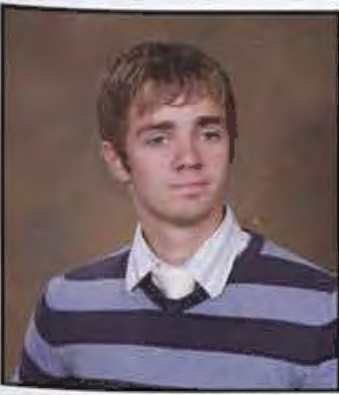

Seth Scott

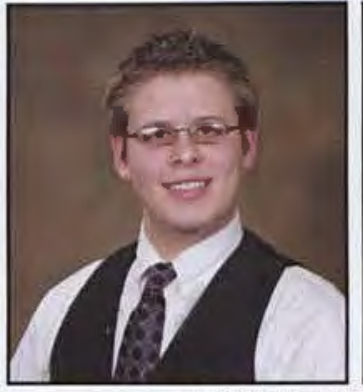

Tyler Scott

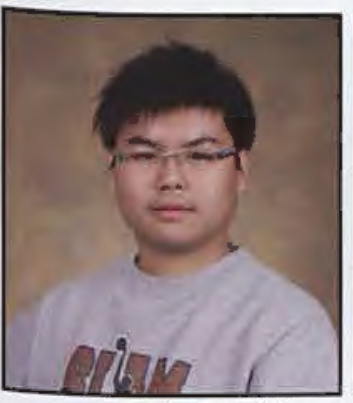

Heman Shum

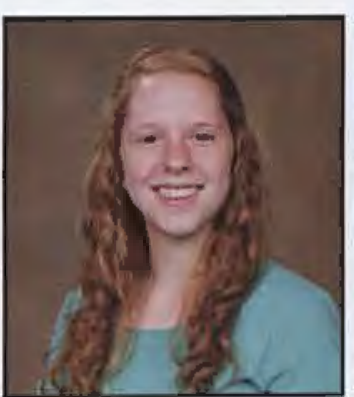

Laura Simpson

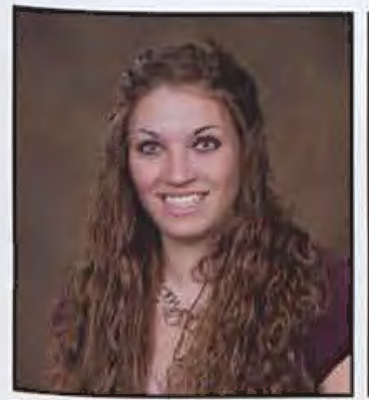

Kendra Smith

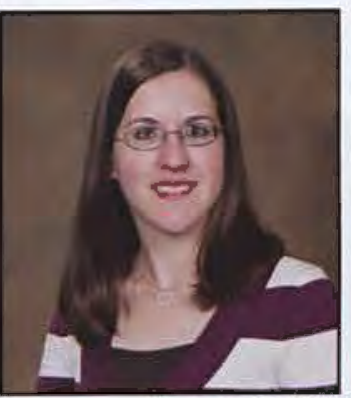

Megan Smith

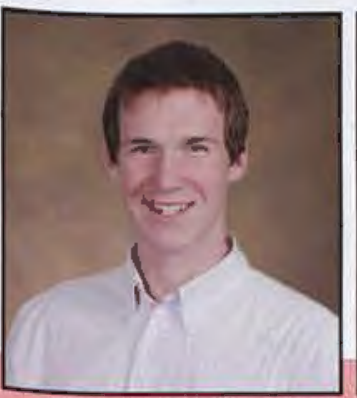

Christopher Spurlock

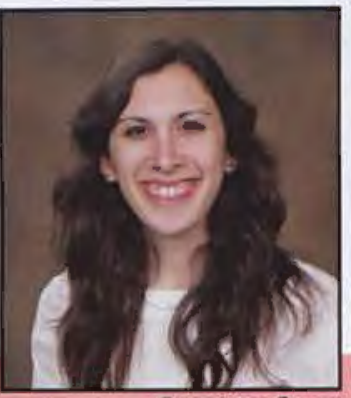

Cammy Sray

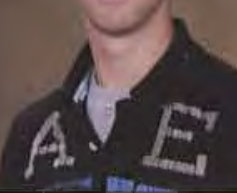

Paul Schindler
당

군

Katherine Schmidt

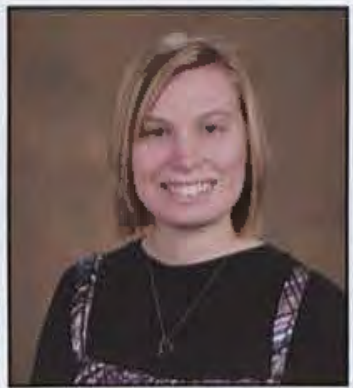

Erin Schultz

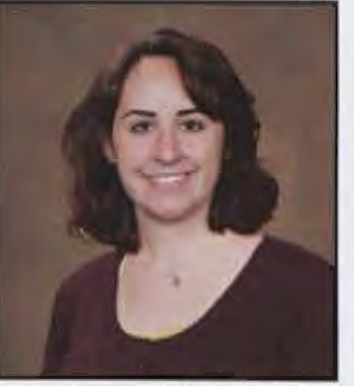

Megan Scott

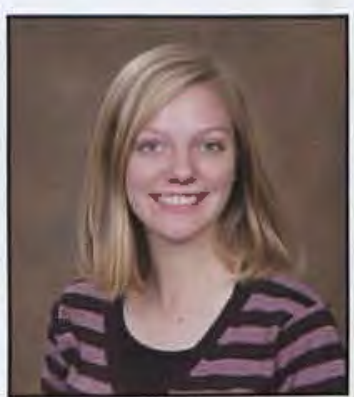

Bethany Servi

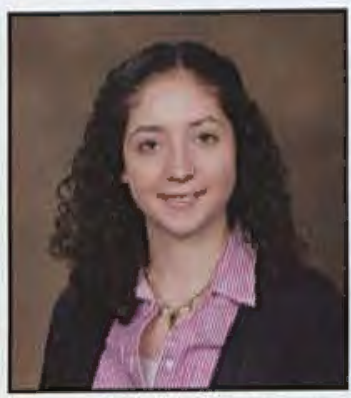

Emily Severance

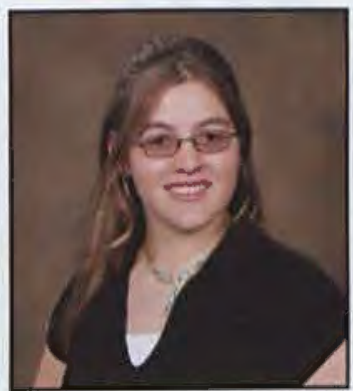

Susanne Seybold

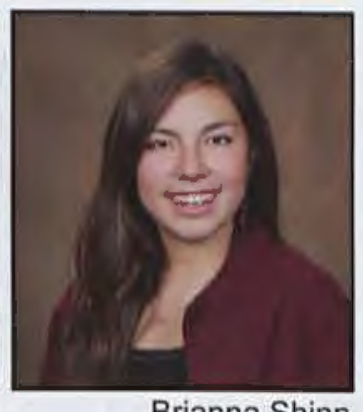

Brianna Shinn

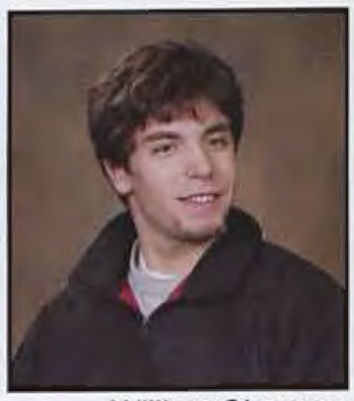

William Simpson

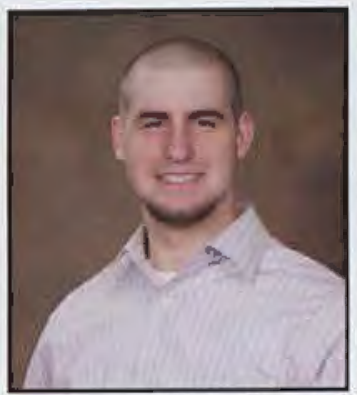

Ryan Sjoquist

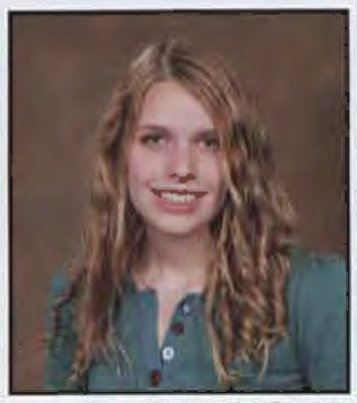

Amanda Smith

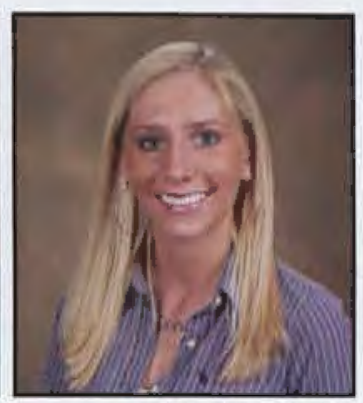

Jessica Smith

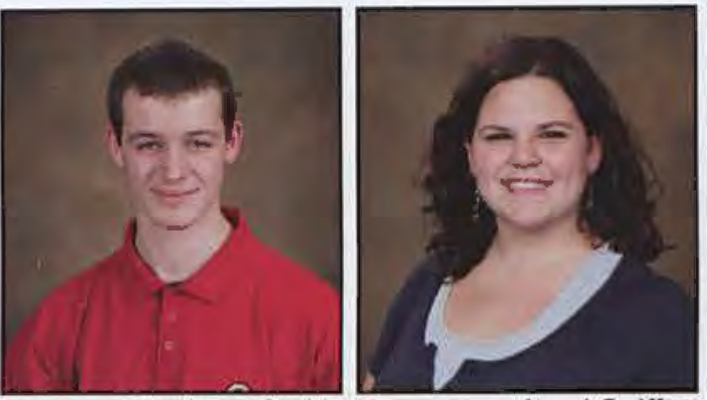

Zachary Smith

Apryl Sniffen

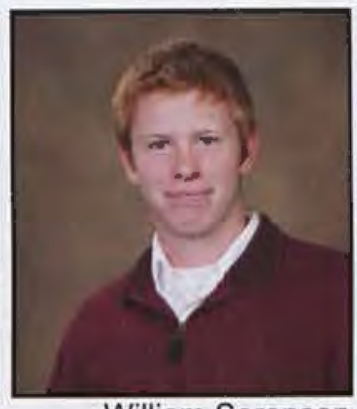

William Sorensen

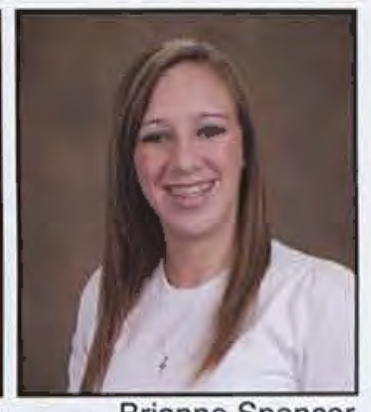

Brianne Spencer

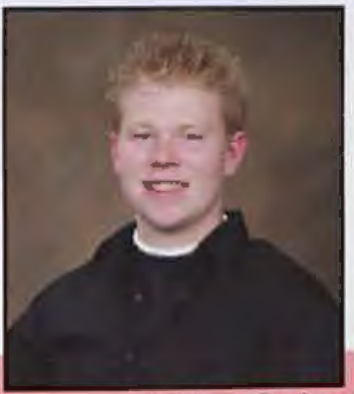

Joshua Staley

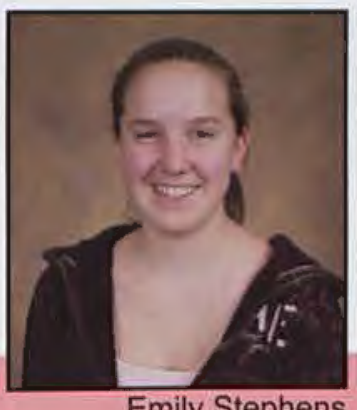

Emily Stephens

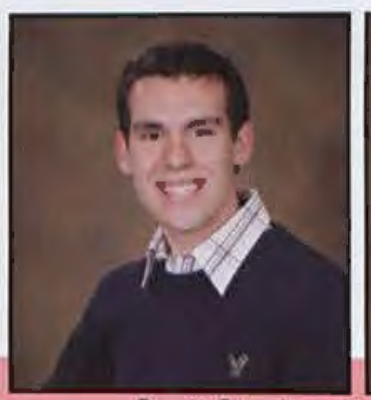

Scott Stephens

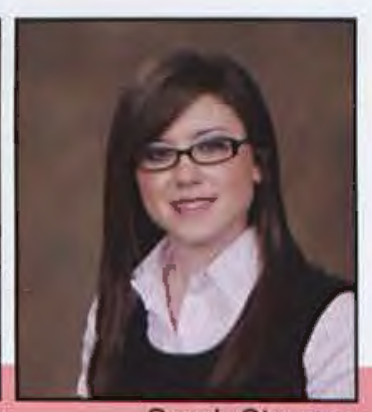

Sarah Stevens

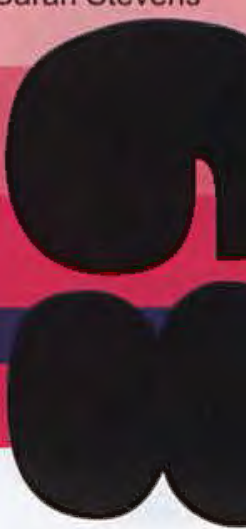



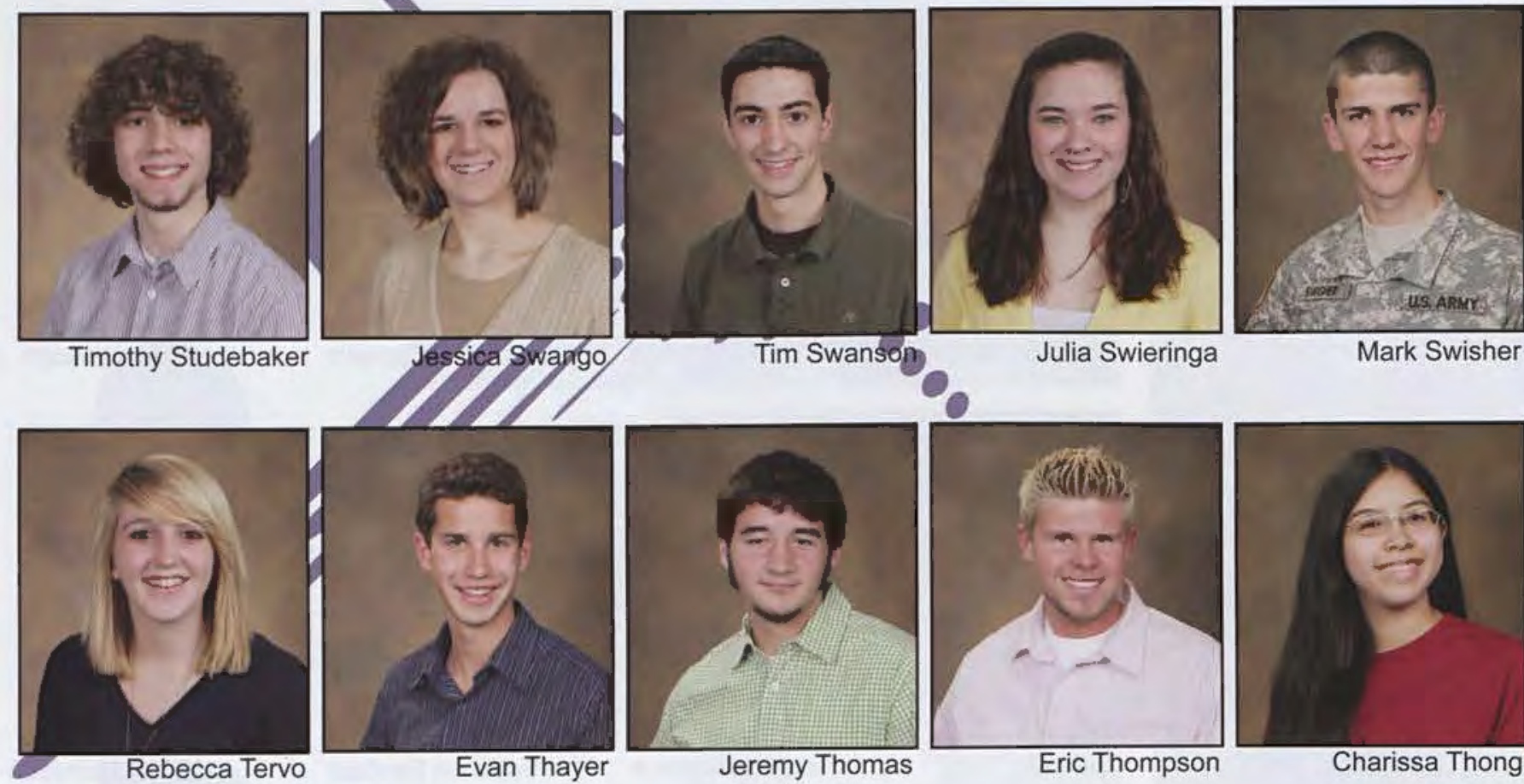

Mark Swisher
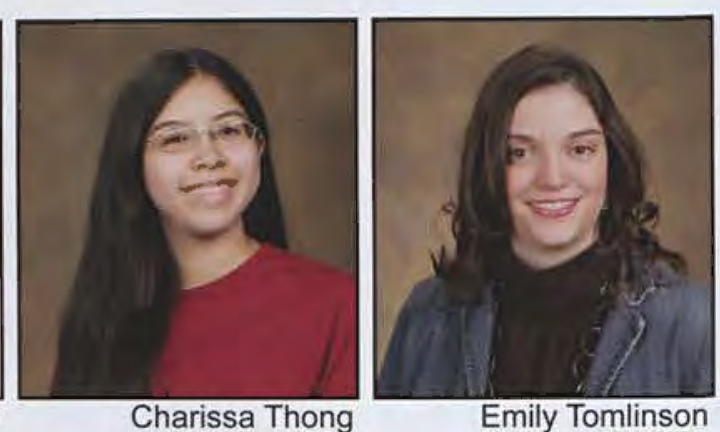

Emily Tomlinson
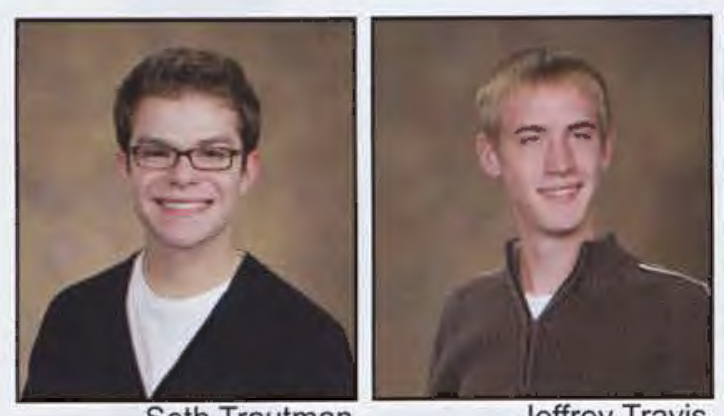

Jeffrey Travis
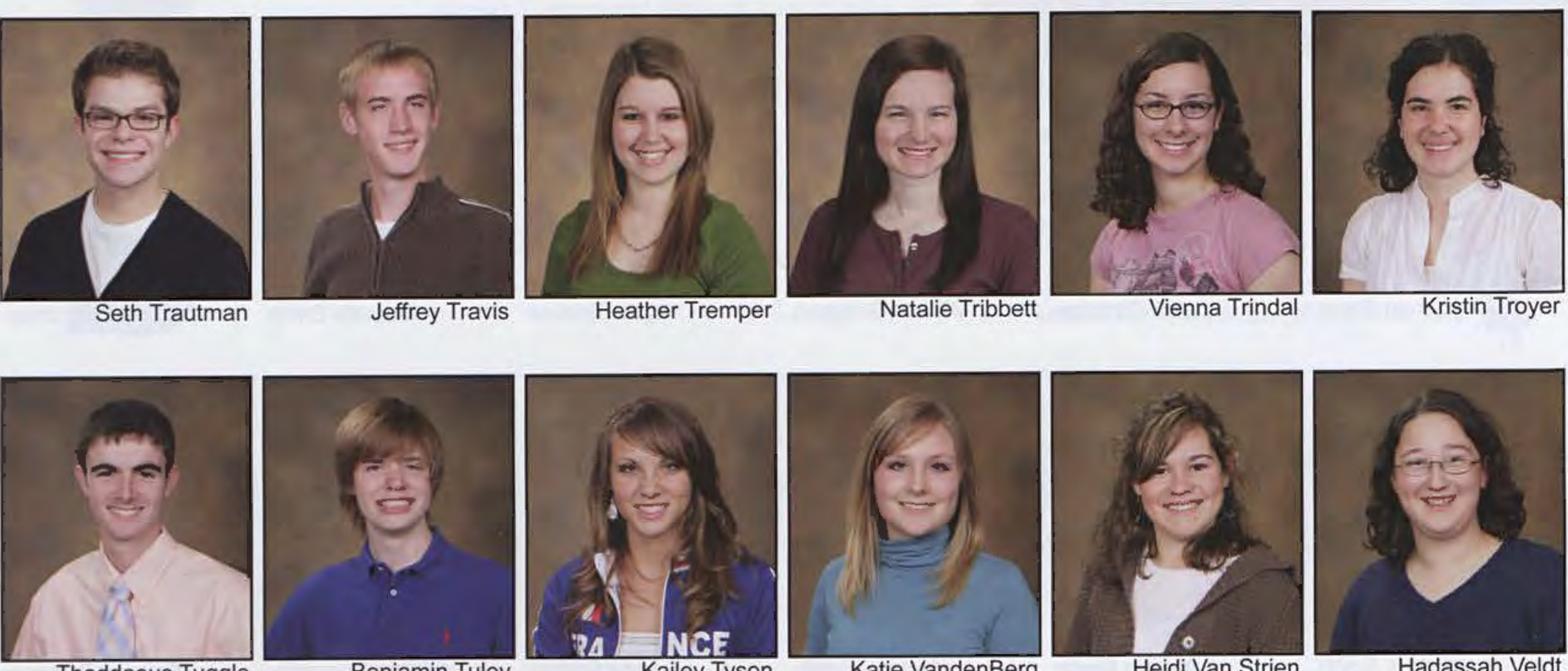

Thaddaeus Tuggle

Benjamin Tuley
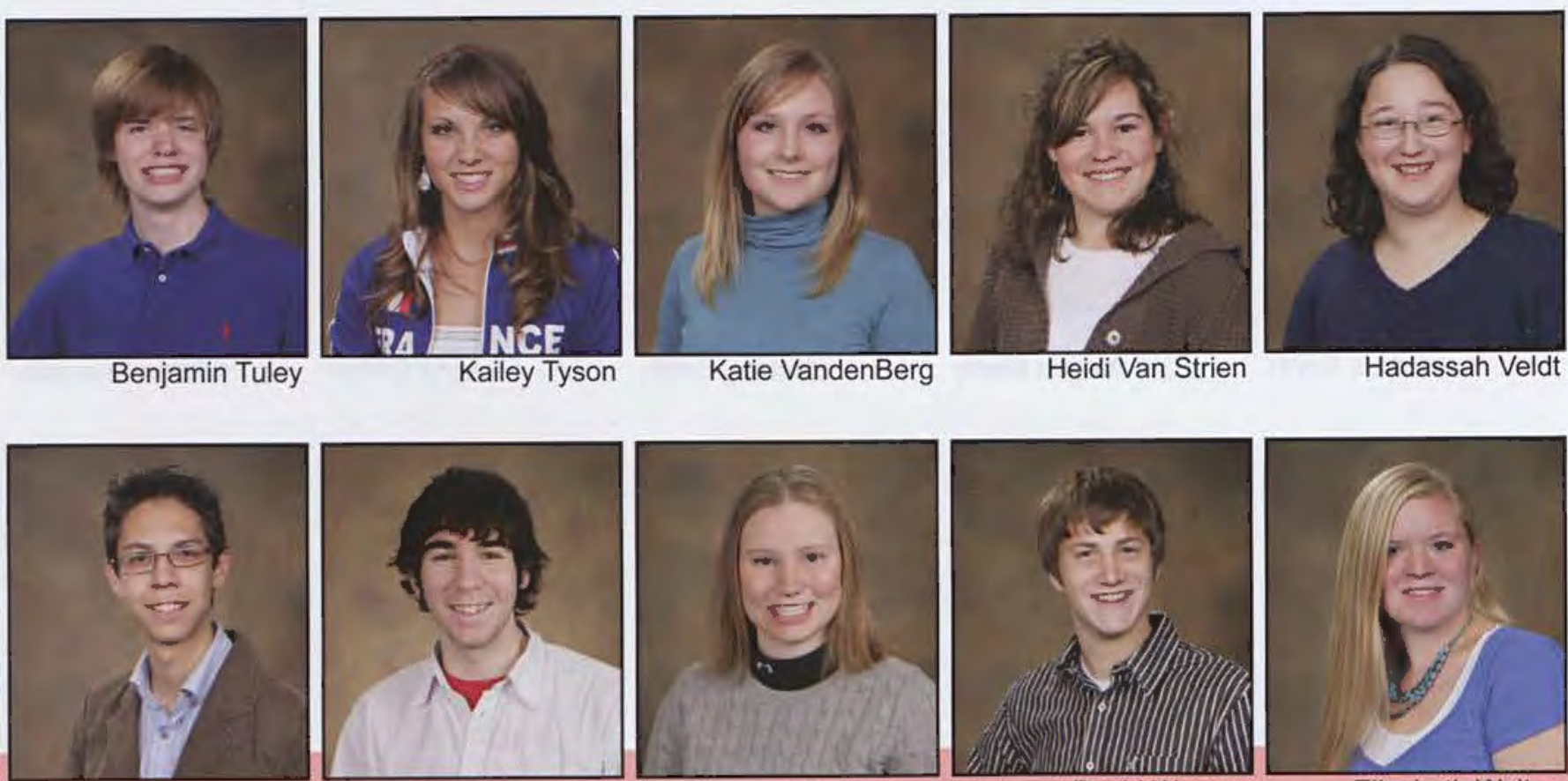

Abraham Vivas

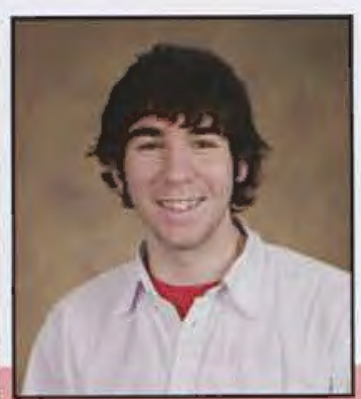

Christopher Voltz

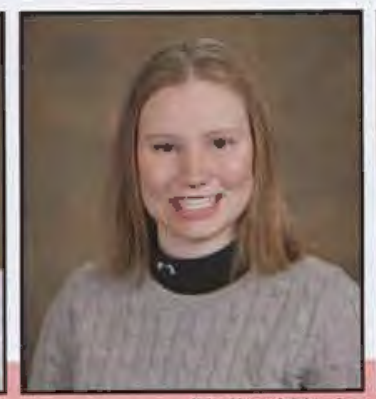

Kelby Wade

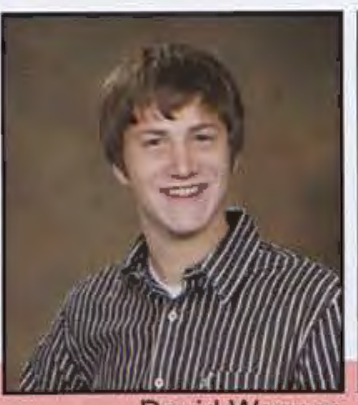

David Wagner

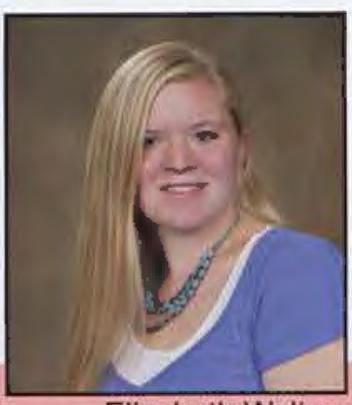

Elizabeth Walton
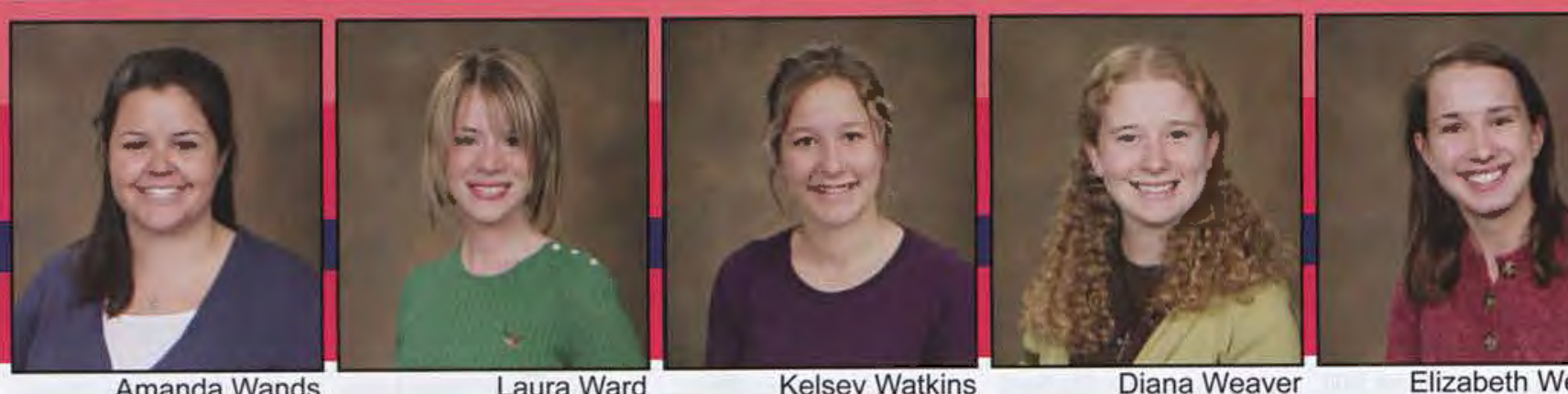


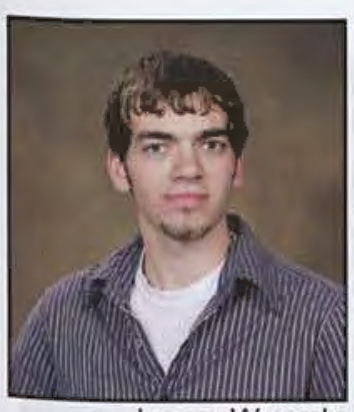

Lucas Wemple

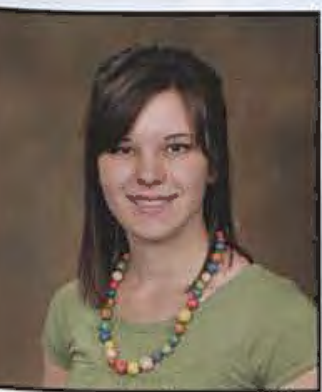

Krista Wiles

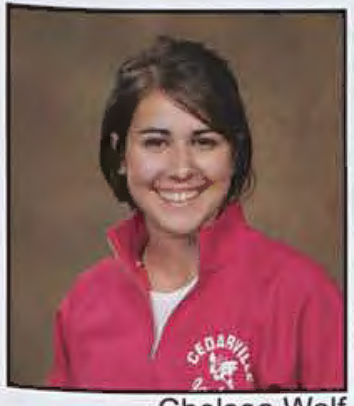

Chelsea Wolf

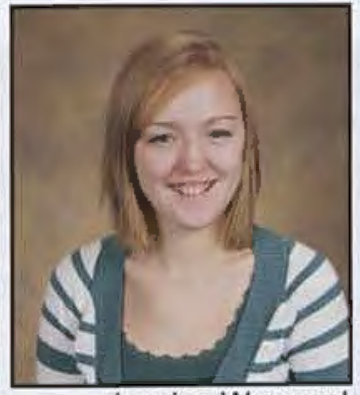

Jessica Wengerd

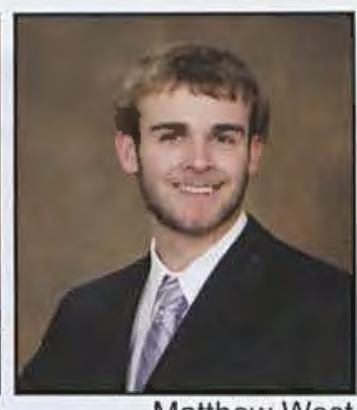

Matthew West

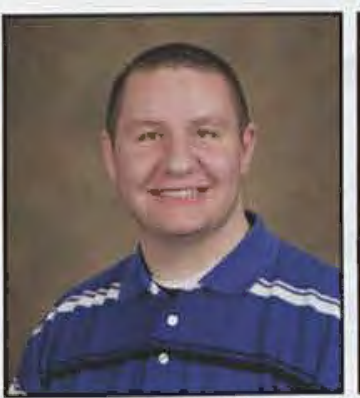

Chris Whitaker

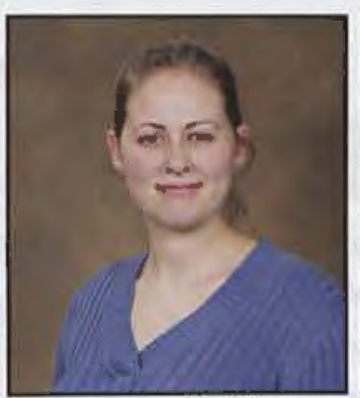

Devin Wicker

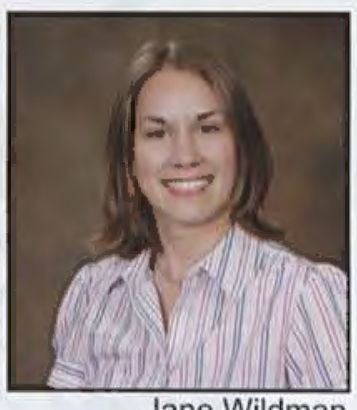

Jane Wildman

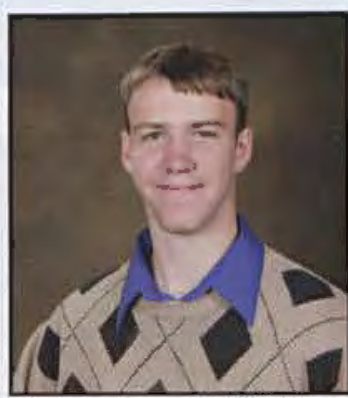

Jeremiah Wilkins

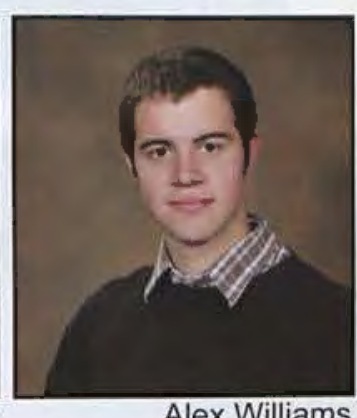

Alex Williams
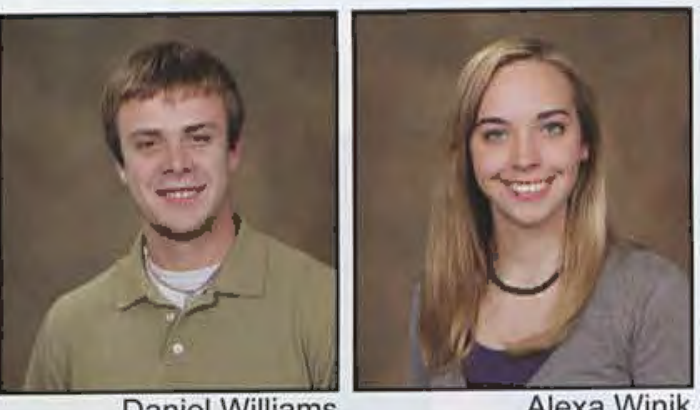

Alexa Winik

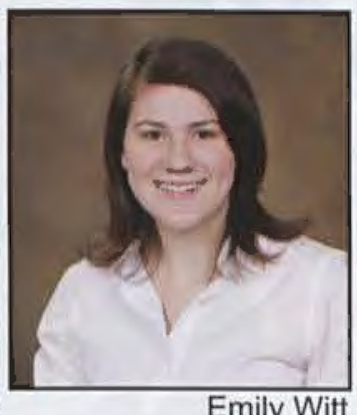

Emily Witt
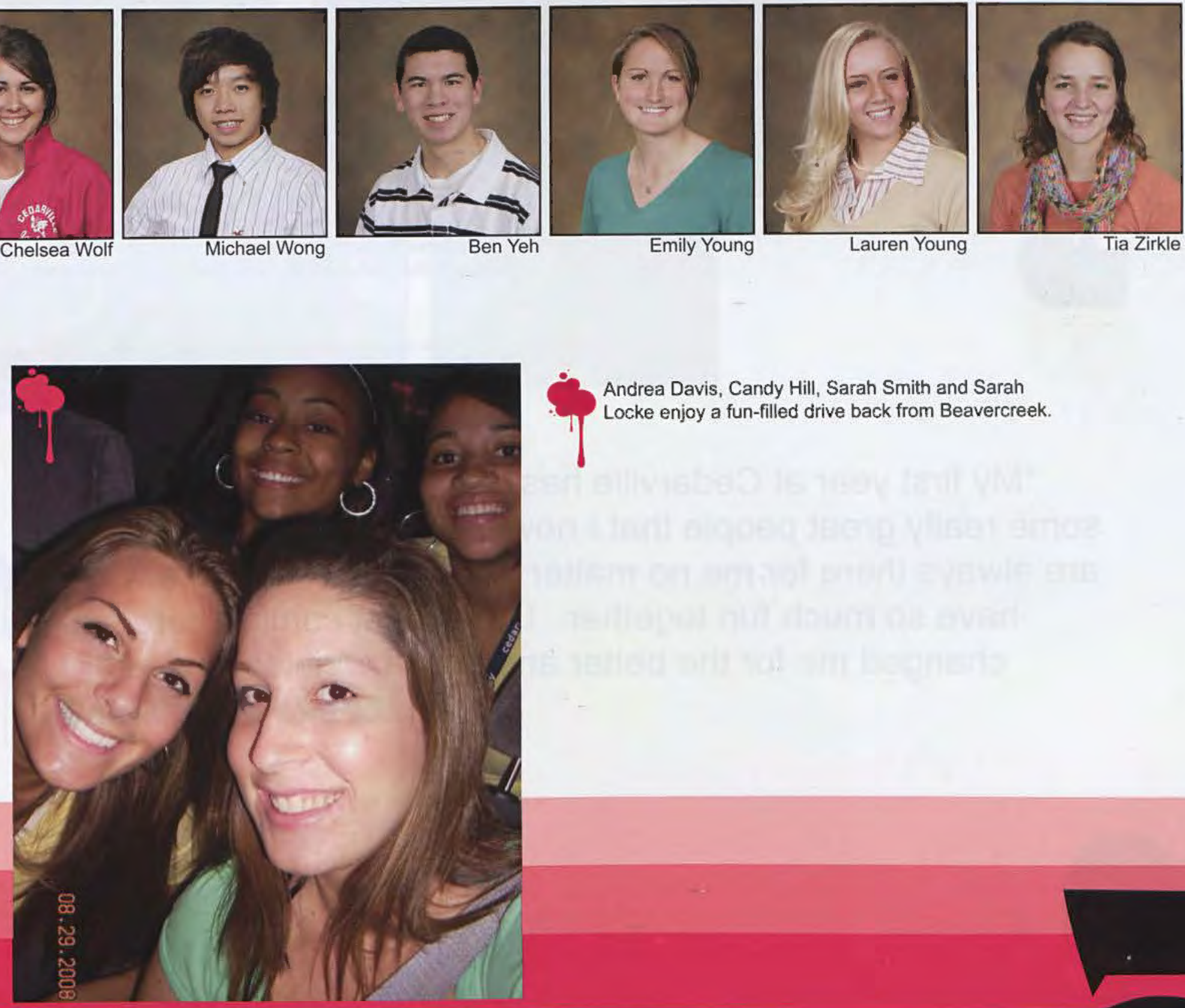

Andrea Davis, Candy Hill, Sarah Smith and Sarah
Locke enjoy a fun-filled drive back from Beavercreek

1 Locke enjoy a fun-filled drive back from Beavercreek. 

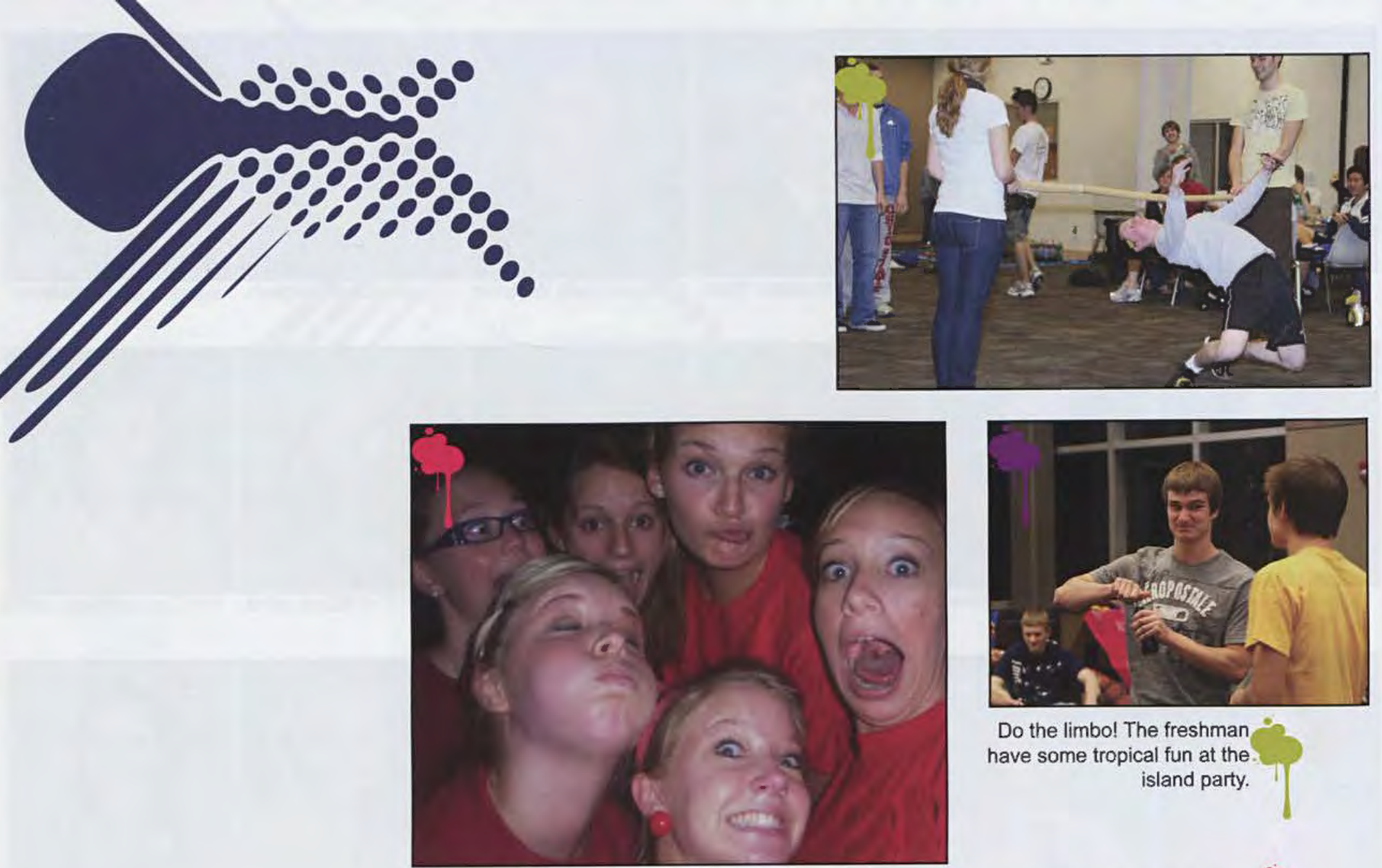

Do the limbo! The freshman
have some tropical fun at the.
island party.

This Printy unit is excited for Printy Wars! Stefanie Betori, Stephanie Russell, Brianne Spencer, Elizabeth Dickey, Rachel Horst, and Jean Drimalla. Stefanie Betori thinks it was "fun times!!" Pop? Soda? Who cares? Just get that bottle open and
chug it down!

"My first year at Cedarville has been amazing. I have met some really great people that I now call my true friends. They are always there for me no matter what I need and we always have so much fun together. I know that coming here has changed me for the better and I can't wait for next year." - Moperpet cestrio 
"I love being surrounded by people who love God and want to spend every minute serving God! God has answered prayer in so many ways and have given me an amazing group of friends that have gotten me through so much this year."-rendre smith
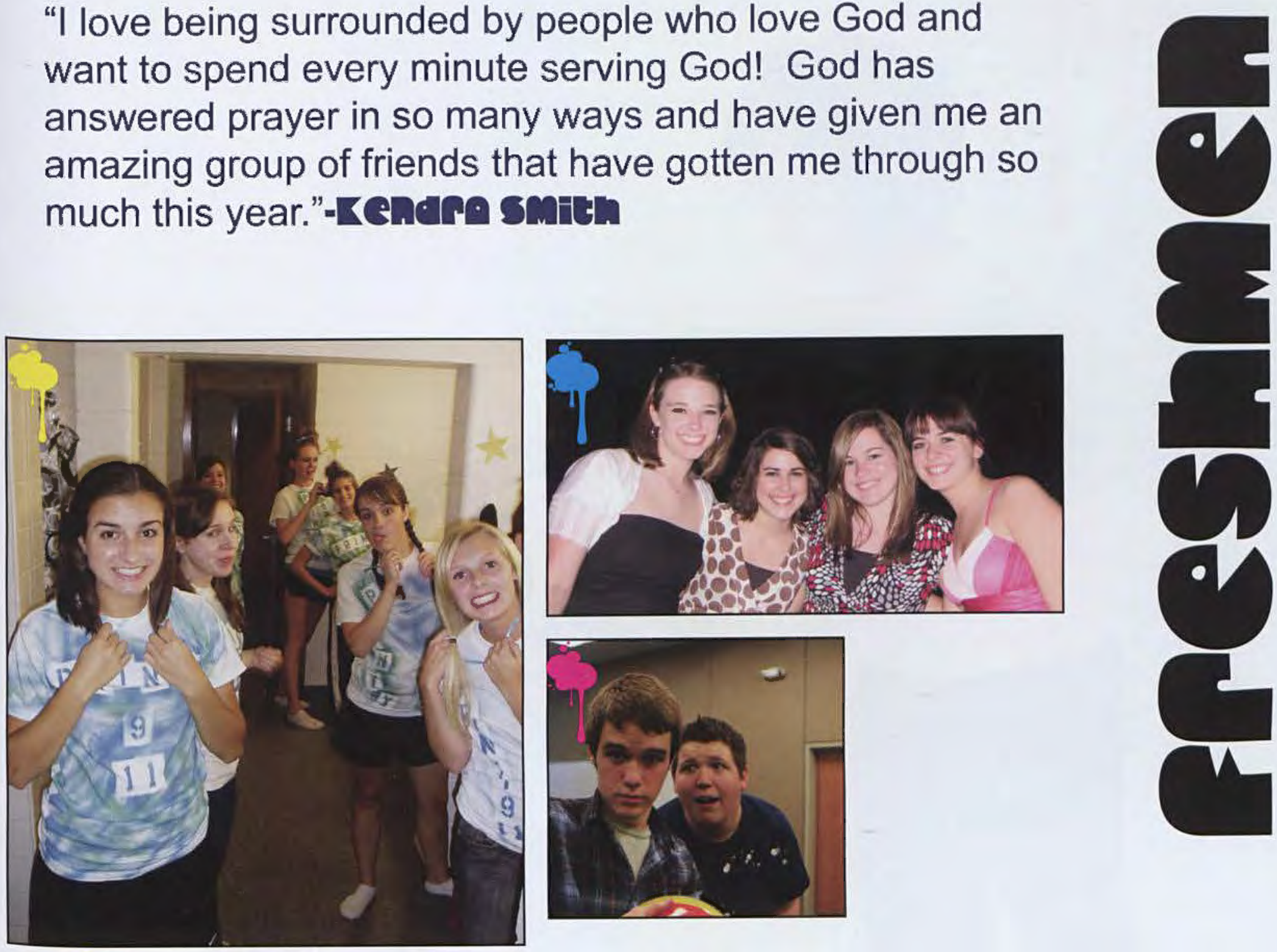

Printy units 9 and 11 prepare to dominate in Printy wars.

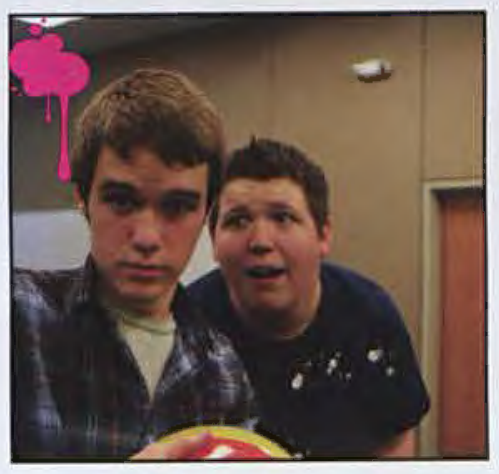

Lauren Lane, Chelsea Wolf, Heidi Van Strien, and
Rachel Parrott are dressed up Elliv.

Adam Brandt's attempt to look cool for the camera is ruined by his friend. Though some might argue that it enhances the shot. 

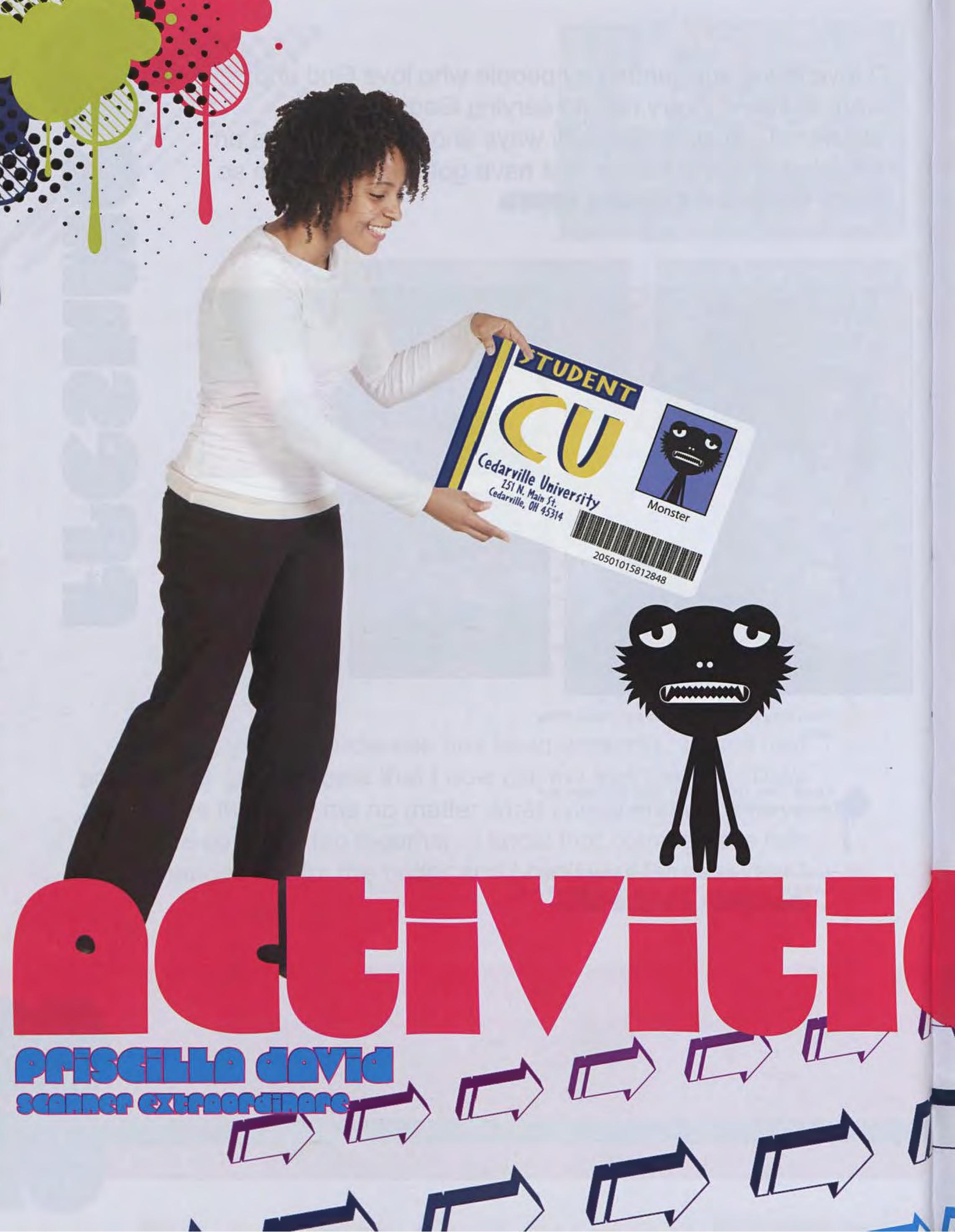
As hard as they tried and as many "open dorms" as they may have crashed, the reality that Cedarville men were never able to comprehend fully the fascinating world that existed inside a girl's dorm. Only the ladies who spent their memorable college years in these estrogen-saturated hallways can adequately articulate what the experience of living in the dorms was really like. From Maddox to Murphy, these girls could testify that each dormitory had a culture as unique as the ladies that called them home. While each dorm had its own individual personality and atmosphere, some sounds could be heard echoing from most rooms regardless of location: late night roommate talks, uncontrollable giggling, and "interpretive movement" partiesto name a few. Looking back on an especially crazy moment, Juliann Lamb laughed, "There's nothing like seeing fifteen girls run through an entire dorm during a power outage!"

Aside from the random, girly excitement that was constantly alive and well in girls' dorms, there was a definite undercurrent of love and support. That attitude of feminine camaraderie emerged in spontaneous prayer times for a sister in need, surprise engagement parties involving silly string attacks, and studies of God's Word together. Elyse Looyengoed enjoyed this wide variety of bonding moments, and said, "I love living with so many sisters-in-Christ! Hall pizza parties, prayer times, and having someone there to listen are times I will always cherish." After living in Printy for one year, Jess Burkhardt shared that, for her, life in the dorm was "amazing... Living in a unit has been one of the best experiences I have ever had."

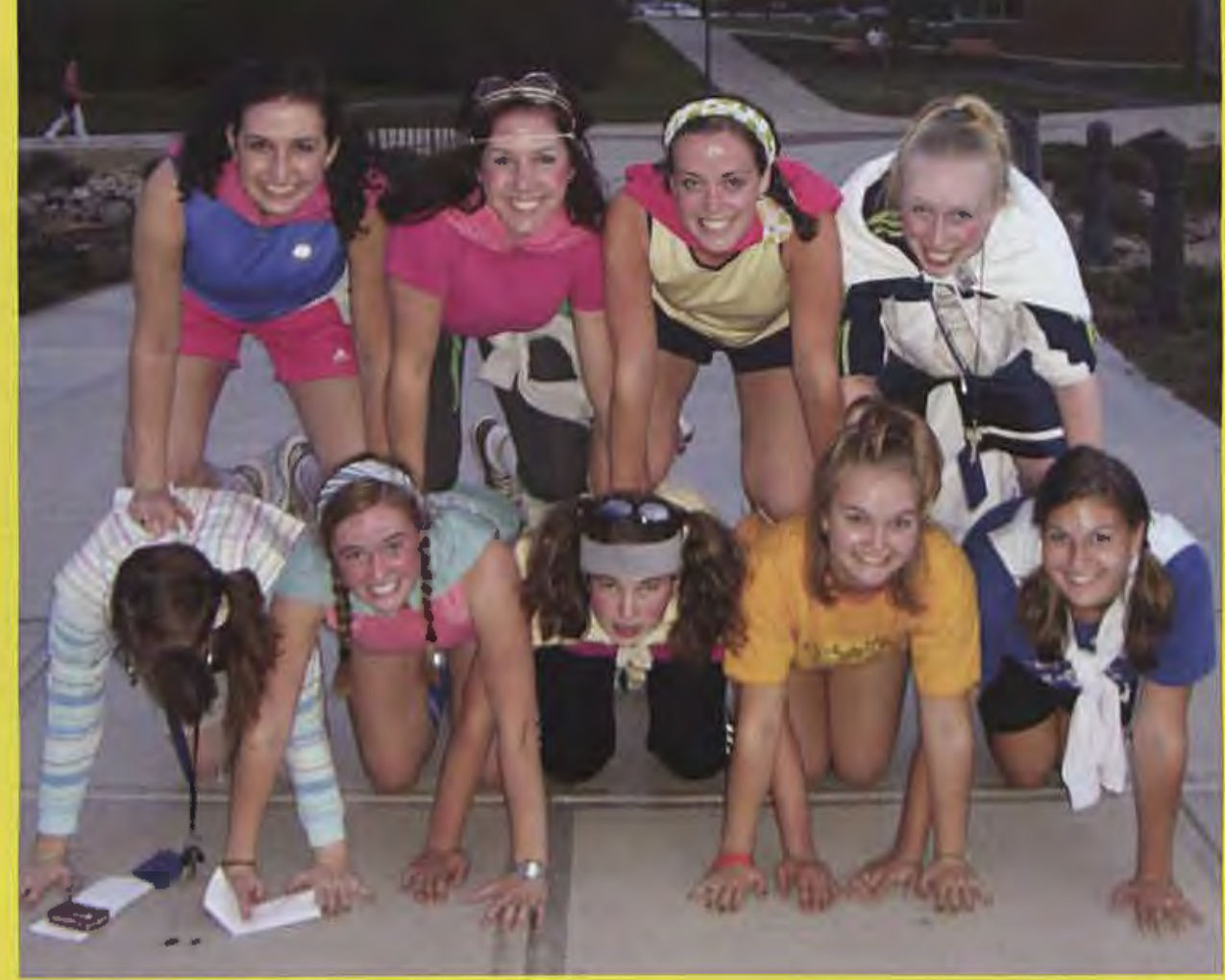

Jessica Wynalda knows from experience,

"There's no better way to build unit-unity than to do a crazy-fun dorm picture-scavenger hunt dressed as Supernerds!"

Pizza... Check. Laptop with movie... Check. Friends... Check.

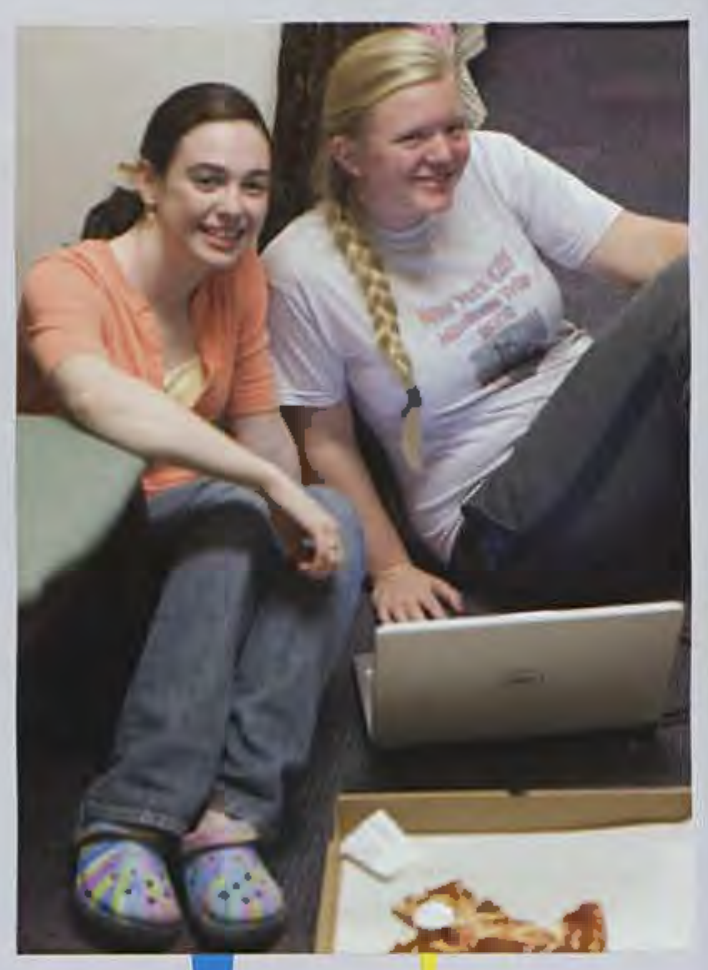

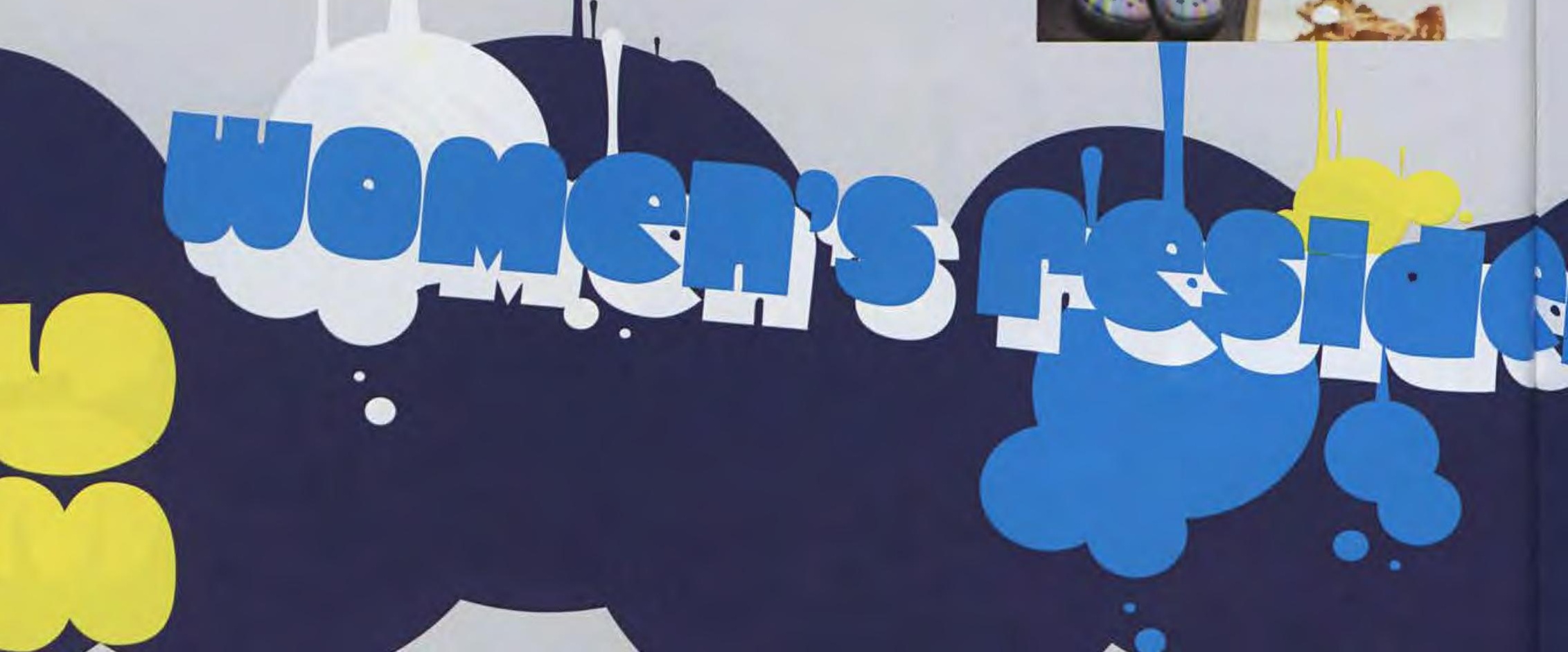


Lynsey Fabian and her McKinney and
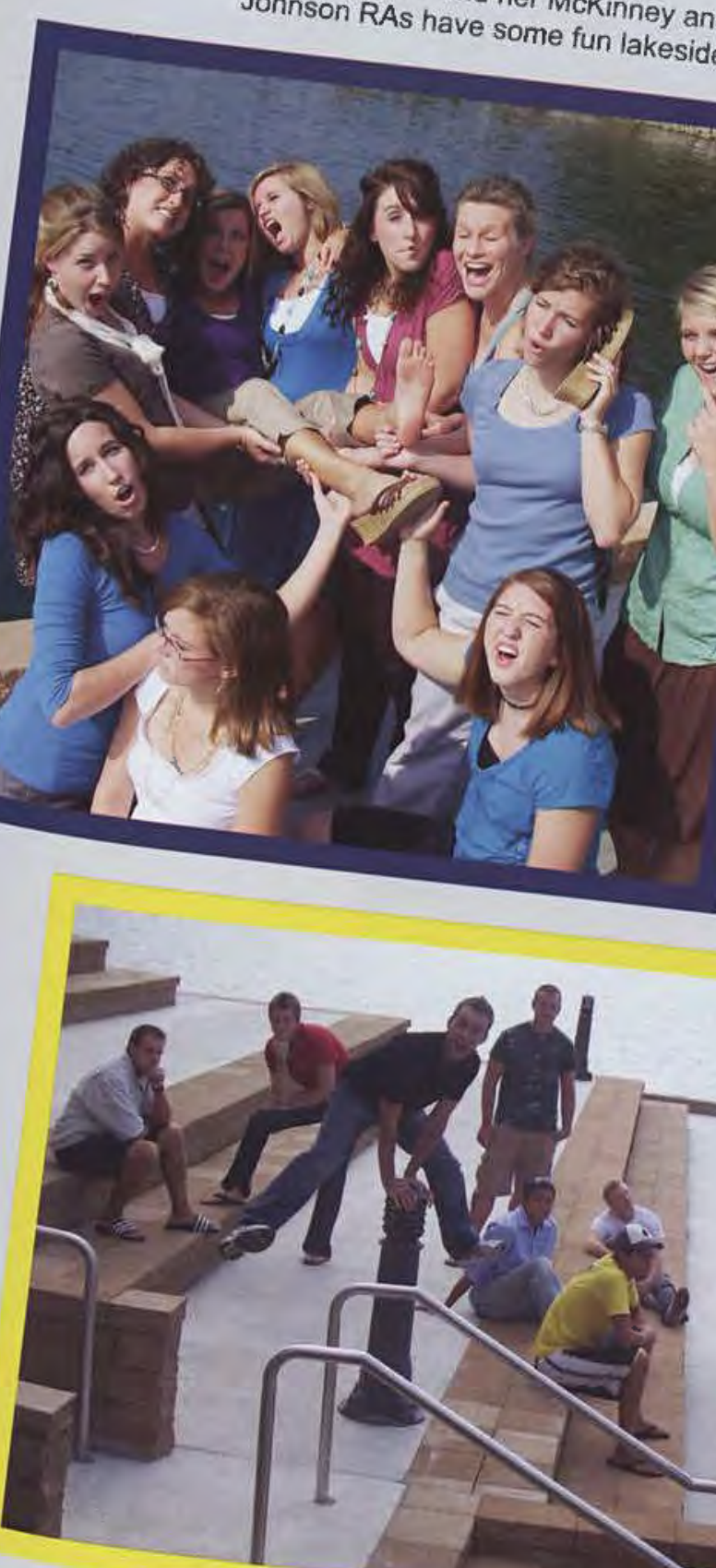

Joe Poelzer stops to loot with his St. Clair and look suave and serious Meanwhile, Matt Fox looks Steven LoCicero is trying acrobatic and

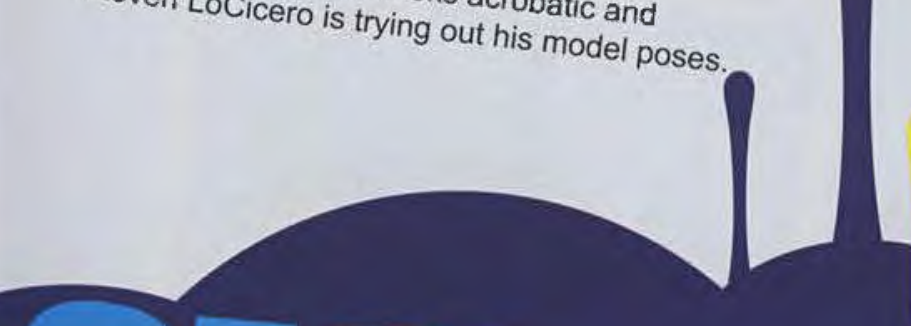

"Being an RA has been

the 16 men in my been a life-changing

alongside. GO LAWLOR!" exclaims Daniel Hess.

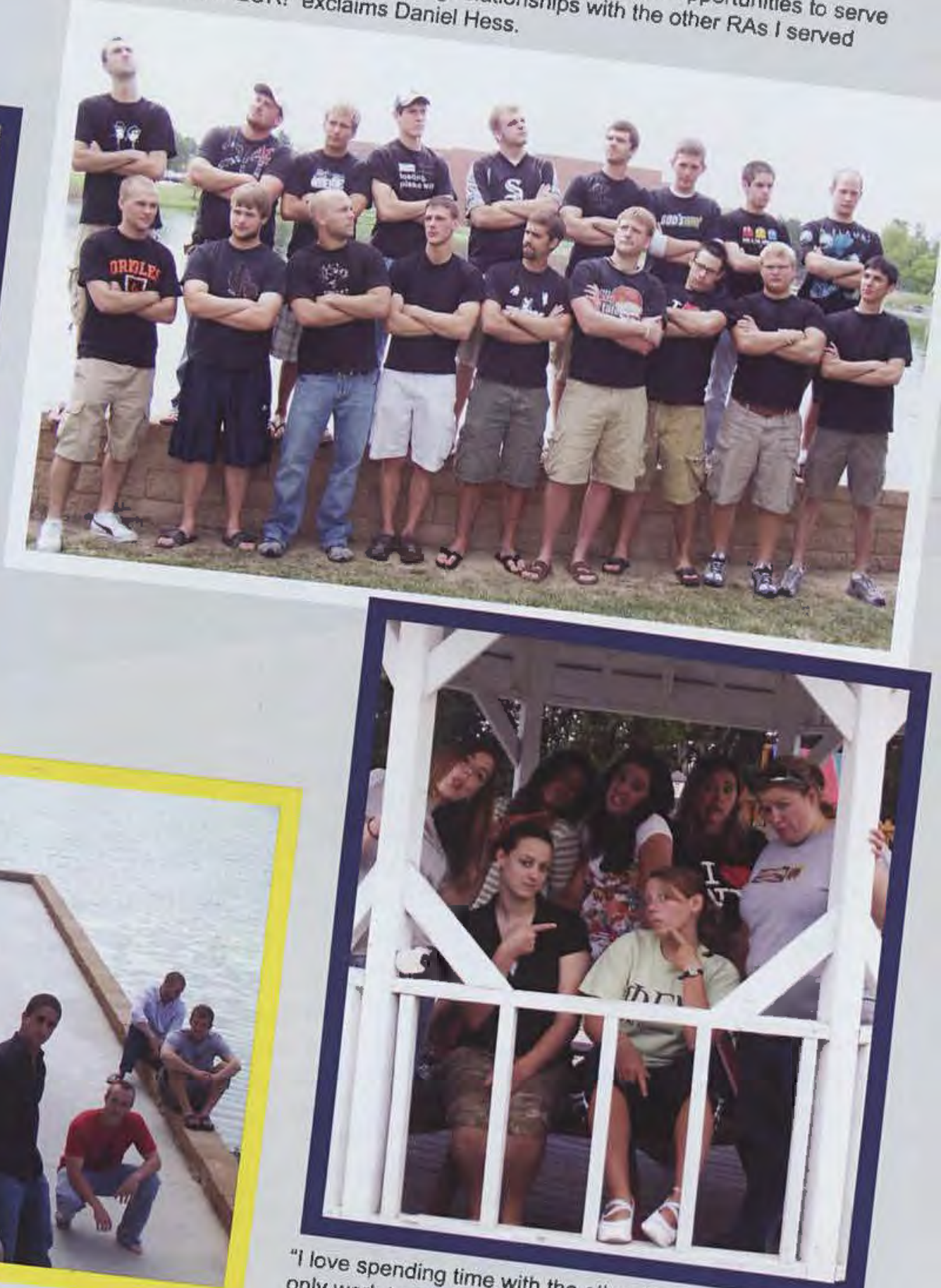

"I love spending time with the other RA's in Printy basis," says Rachel Gilin.

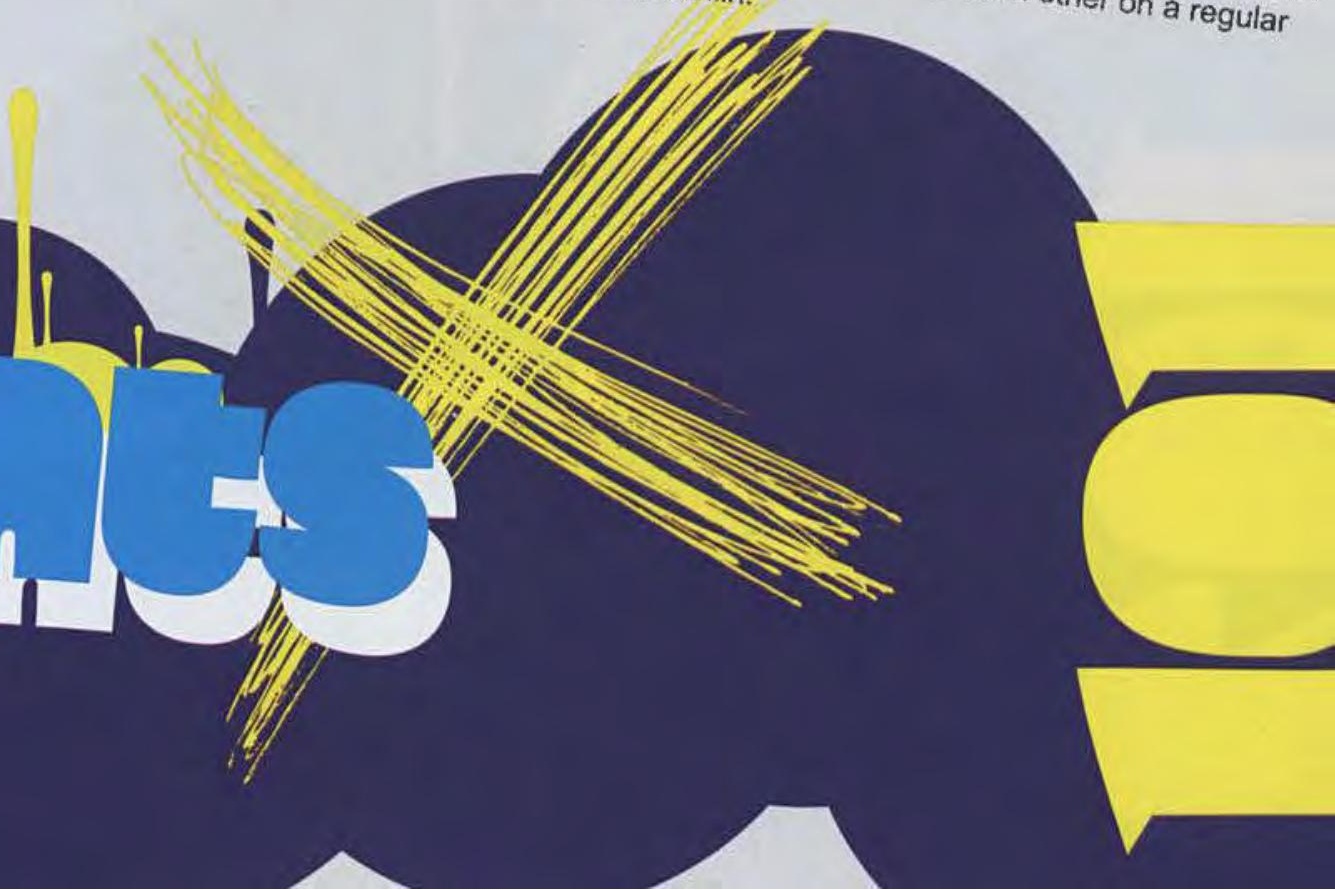




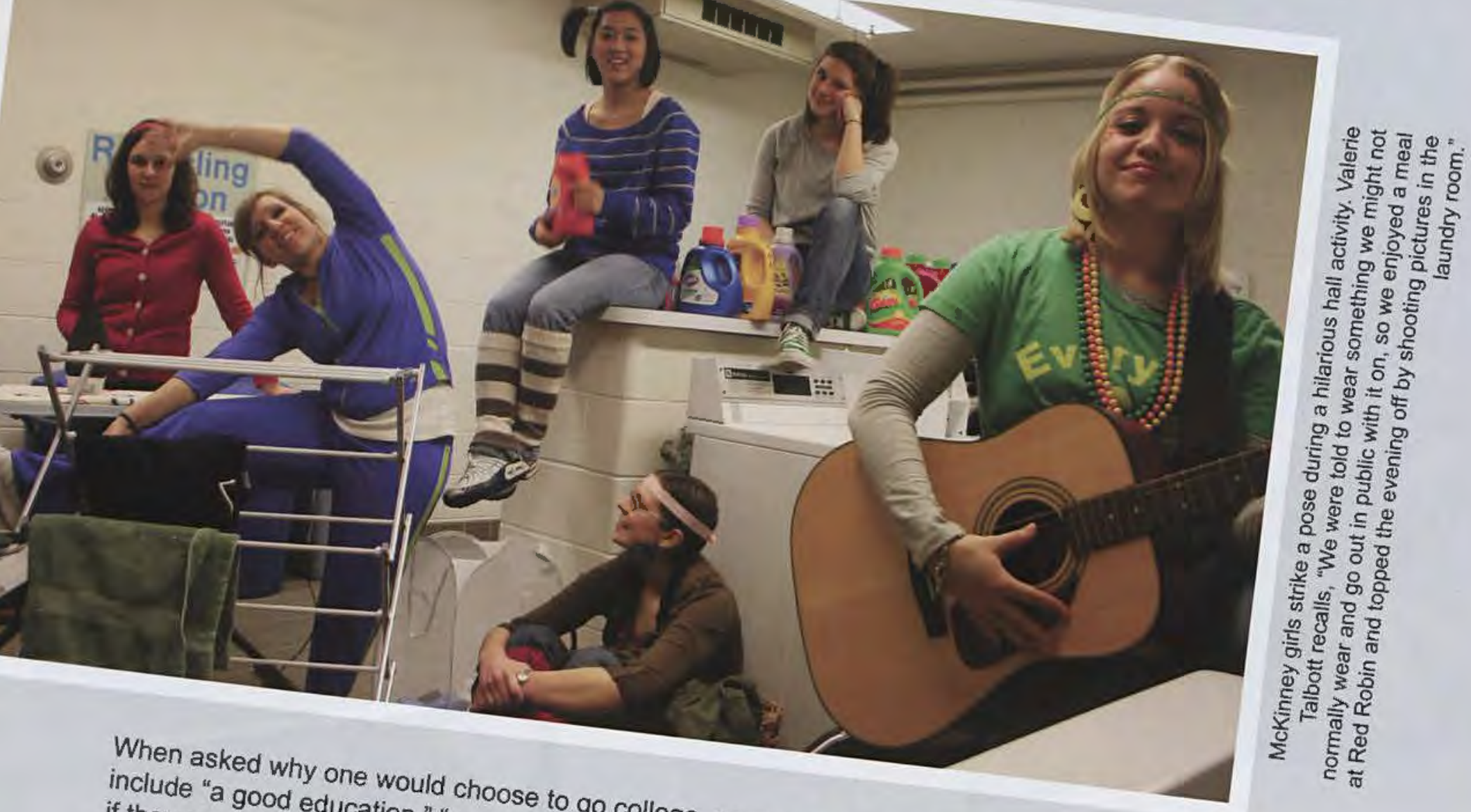

if the "a good education," choose to go college, conscien

people they will meet honest, students will are in this uncertain econts will often give sensible answers that of community in meet. Living in the dorms admit that the main reason thy," or "valuable life t-shirts that each action. The dorms at Cedan campus was definitely on they look forward to univerience." But vided a comfortable stent acquires each year. to this fact, saying. "Dironment for people to gread they were places to sleep and to store therience this sense to love the girls in my unife has been one of grow closer, not only to of excitement and encoura millions of $\mathrm{CU}$ the women. Ryan Martz so much - we have bo very best parts of each other, but to God. Magement that procamaraderie between agreed, "Living in the donded so well!" This my experience at Cedarville. was a chance that Cedarvilian brothers." The chans has provided an optant relationship building I never expected quence, "This is a time of mille students shouldn't take to share a hall op unitunity to grow spiritur wasn't only for ing of becoming closer of my life that I will nev't take for granted. Rall or unit with many other likelly and to build how much you driver than mere acquaintever forget." After living in the York didn't and said that as a cople ine crazy, you still love. "The unit becomes like your, many dorm mates had the be- 


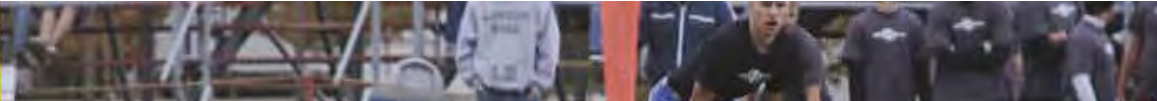

competitors doctable RAs, Anna Sloan and her rer whatever it takes to win Printy Wars.

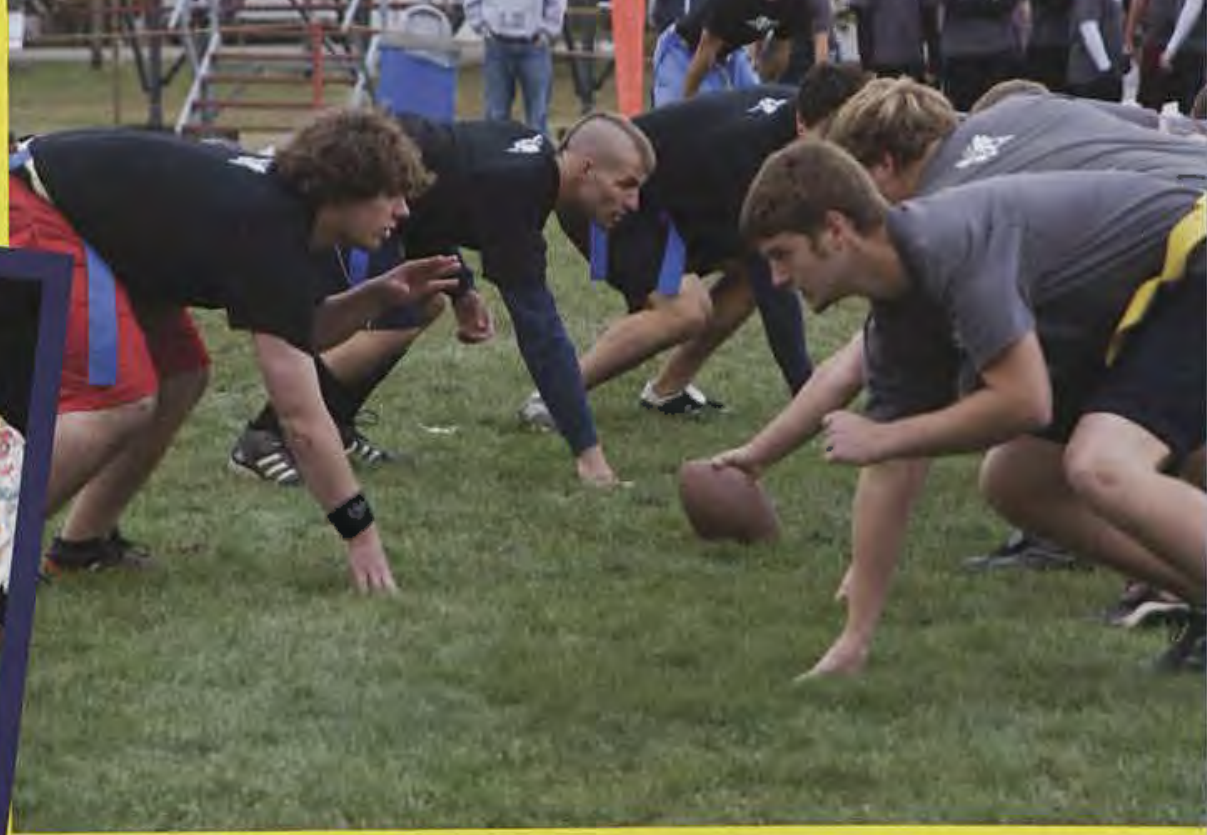

4) $19 \operatorname{nith}^{2}$

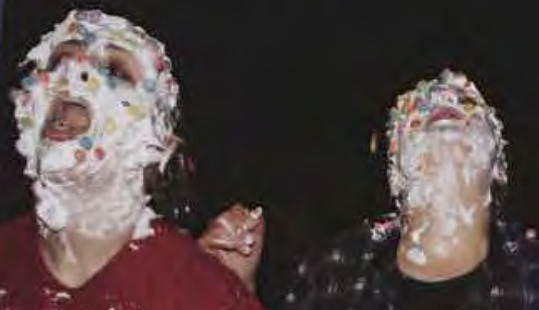

Ar.

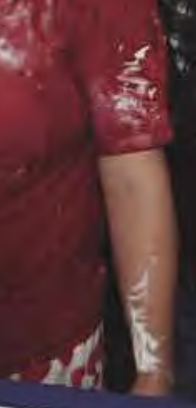
Many gather in the Lawlor lounge to stay
up to date with Michael's antics on The
Office.

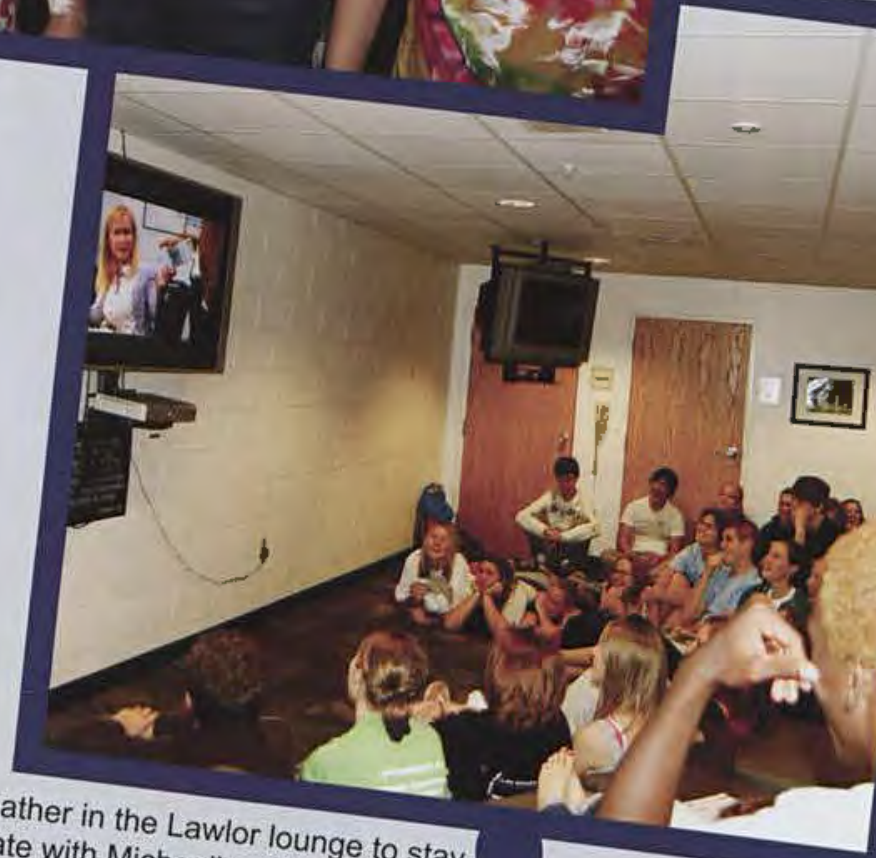

(n)

Cedarville men struggle to gain a competitive edge during the annual Lawlor/Hill football game.

"Chugging a 2 Liter of Sierra Mist, scarfing down whe

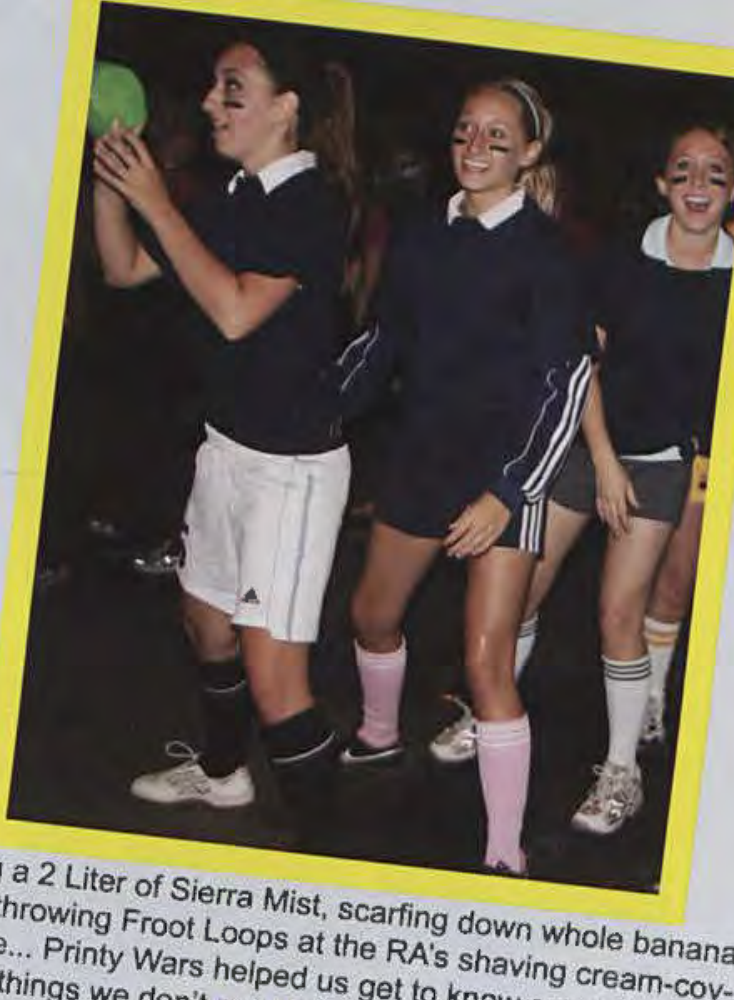

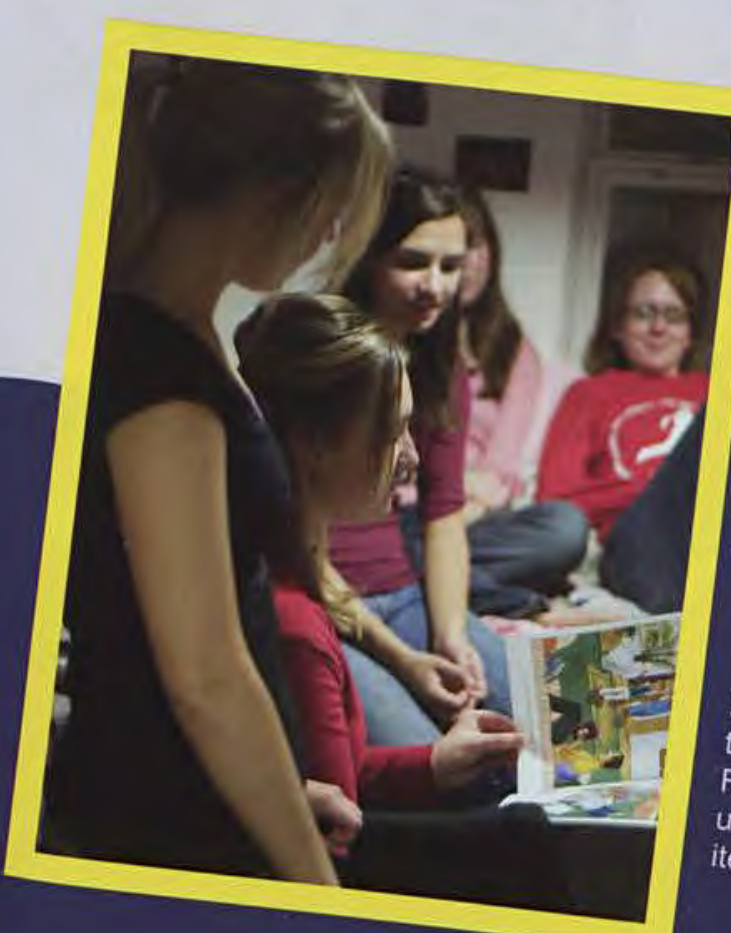
ered face while doing things wry Wars helped us RA's shaving cream-cov-

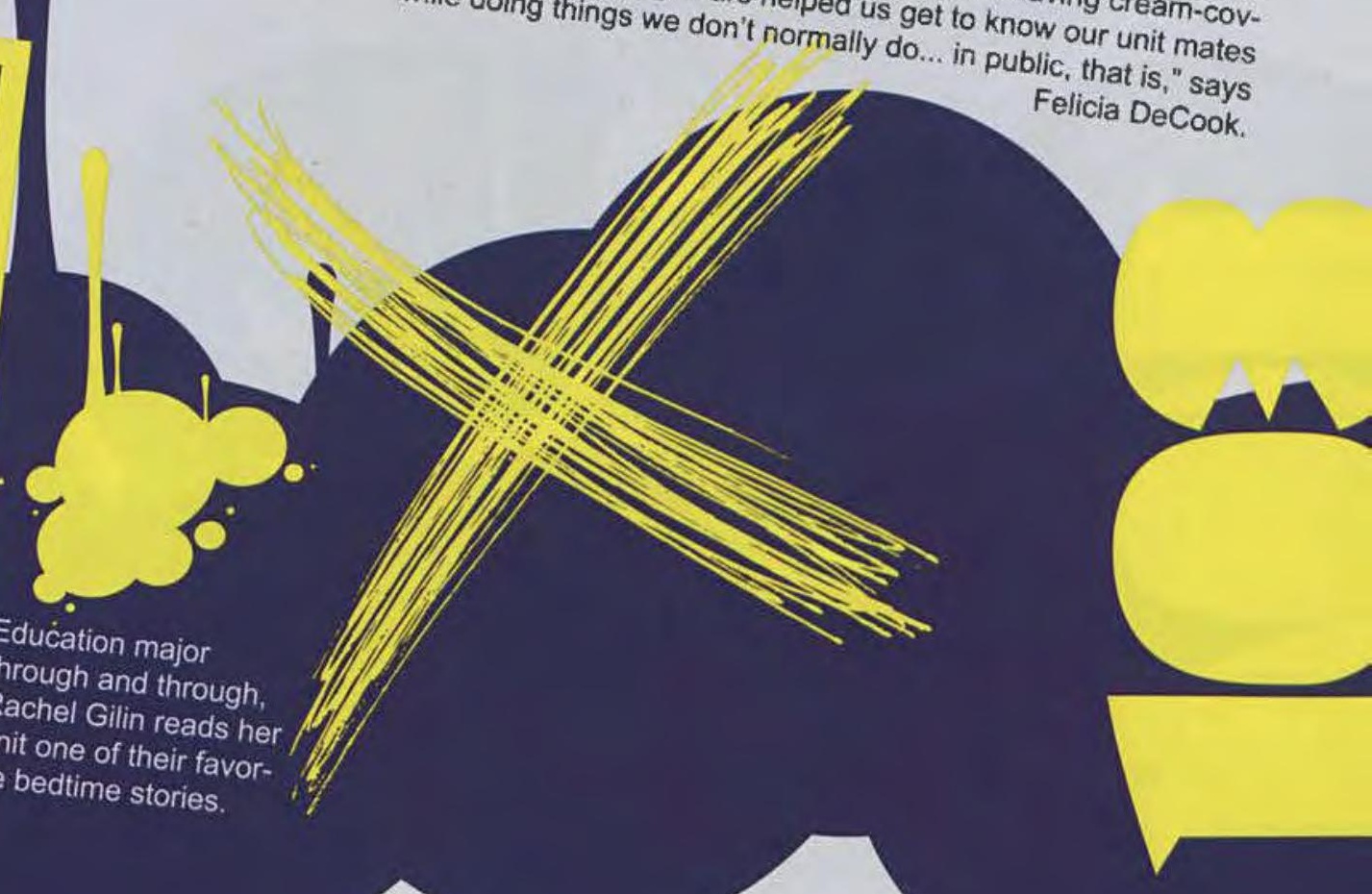




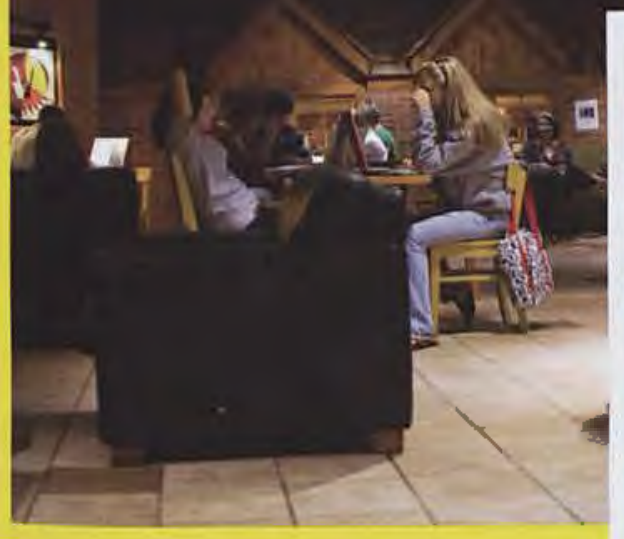

A studying, gabbing, coffee-drinking crowd always buzzes outside the Hive while the SSC is open.

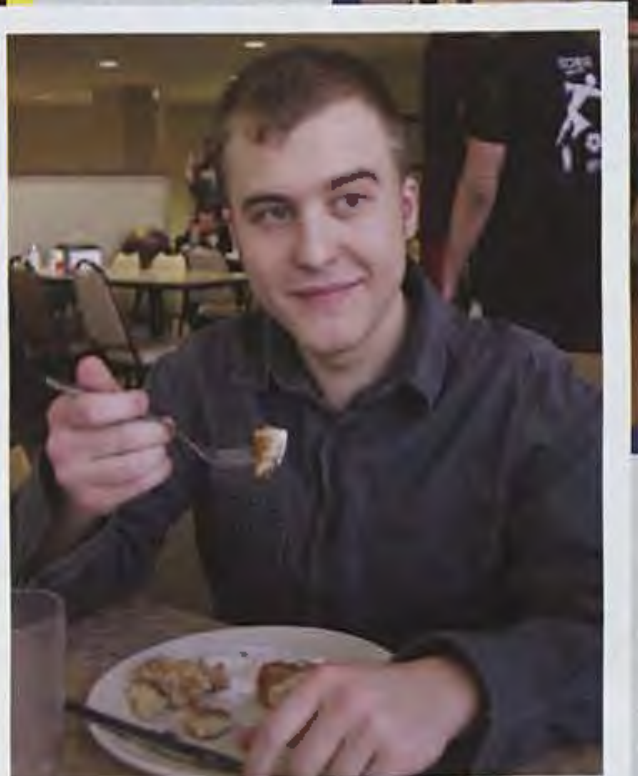

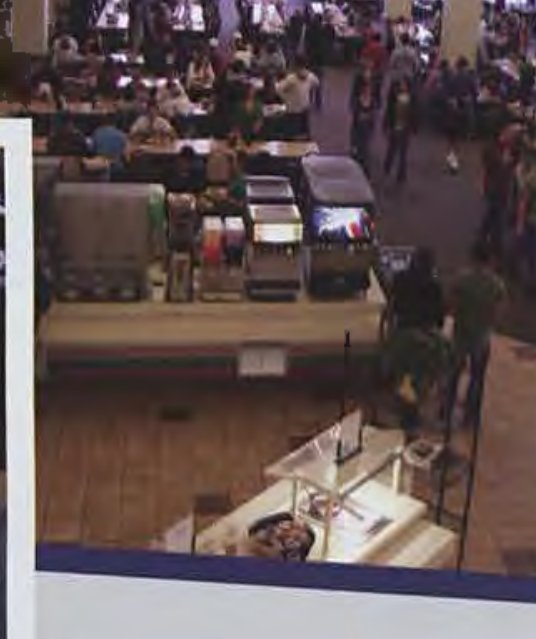

"Satisfaction at Chuck's simply requires some patience, creativity, and a healthy appreciation for the spice rack," admiots Steve Paimer.

Though the newly acquired demonstration line continued to win the trust of students, Chucks had become, oddly enough, a place of confusion. Where did the trays go? Why were the napkins brown? And why were all of the napkin containers now avenues of some sort of blind dating service? We may never truly understand the answers to these questions.

Junior Jared Simpson commented on the blind-date messages on the napkin containers, one in particular revealing a "captivating" nursing major looking for a "wild at heart" man. "I would totally respond to one of these!" he said with a smile. "My little brother has, and he's had seniors answer. Too bad he's taken now."

Sophomore Jack Campbell still scratched his head in confusion when he remembered a certain dinner experience. "We had a brother-sister dinner in upchucks, and as we sat there, someone at our table yelled out, 'Nathan Deakin, stand up!' So, eating his cup of ice cream and not understanding at all what was going on, he stood-fully trusting that his friend had his best interest in mind. But as he stood, the entire table started clapping. Looking around, we saw that ALL of Up-Chucks was clapping for Nate. And in a matter of seconds, the entirety of Chucks was clapping for my dear friend, Nate. The reason for the clapping no one kporys to this day."

Despite confusion and conspiracy theories, Chucks' dedication to quality food and environmental rejuvenation keeps students coming back for seconds. And thirds.

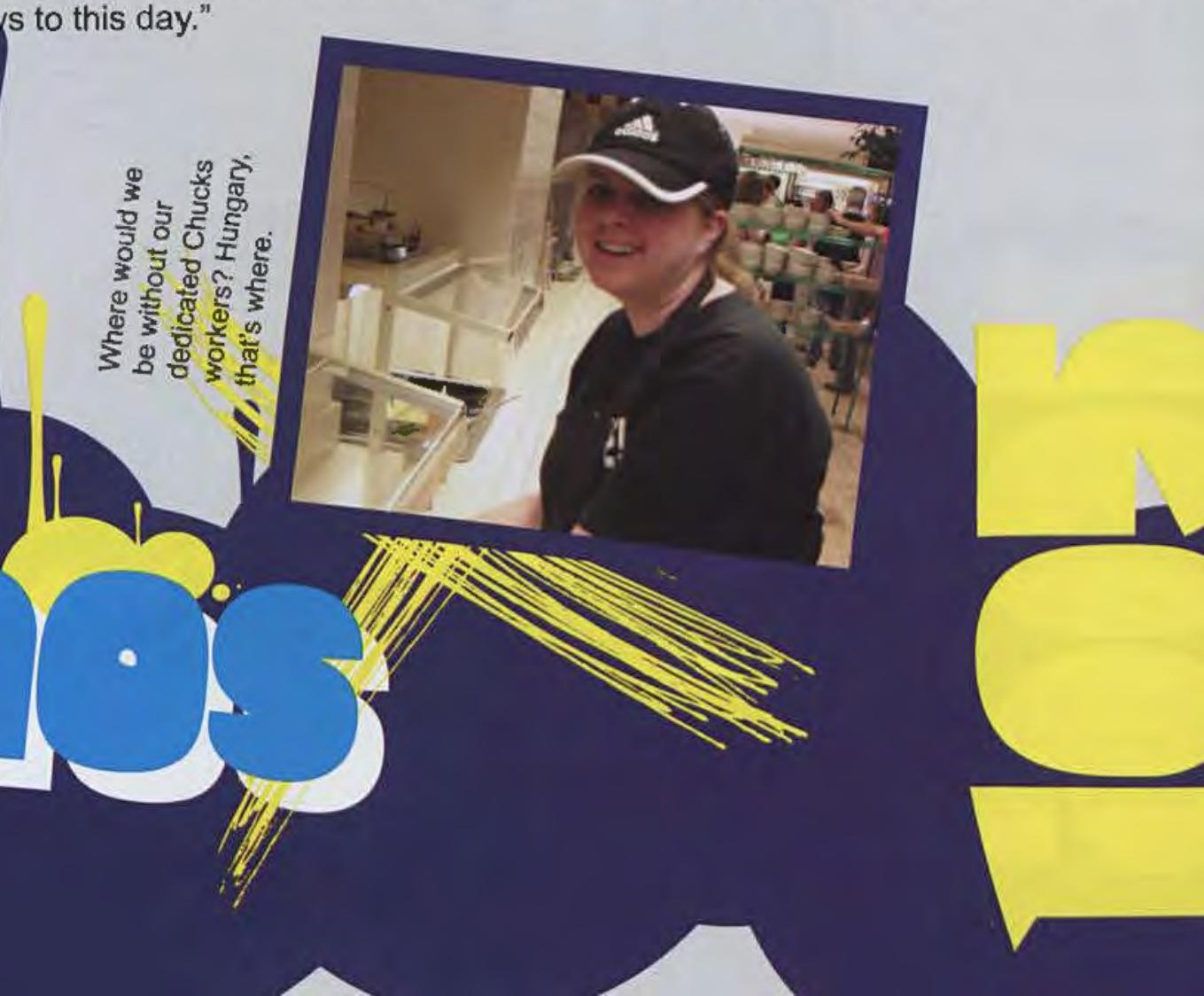


8:00 ENS, 9:00 CBTS, 10:00 Chapel, 11:00 Tyler, 12:00 Chuck's. Every MWF. Callie Mcinturf admitted, "I may sound like a geek here, but the best thing about going to class is learning and being challenged in new ways. I love it!" She was not the only "geek"sounding student.

The two best things about classes according to Tonisia Jackson were that "We get both sides in our education. We have the Christians' aspect, but also the world's view on things. So we know why we view and believe things the way that we do. And we start every class with prayer, LOVE that!" Most classes make lasting impressions and change the way students think at the 'Ville.

All these classes piled up four years of many academic, Biblical, and of course, worldview lessons. Anna Hejl has learned that "Cedarville is not a place where we should get academically or spiritually fat, but an opportunity for us to become academically and spiritually fit for the endeavors we will face in the future." So from 9:00 to 2009 , students did mental workouts and kept coming back for more.

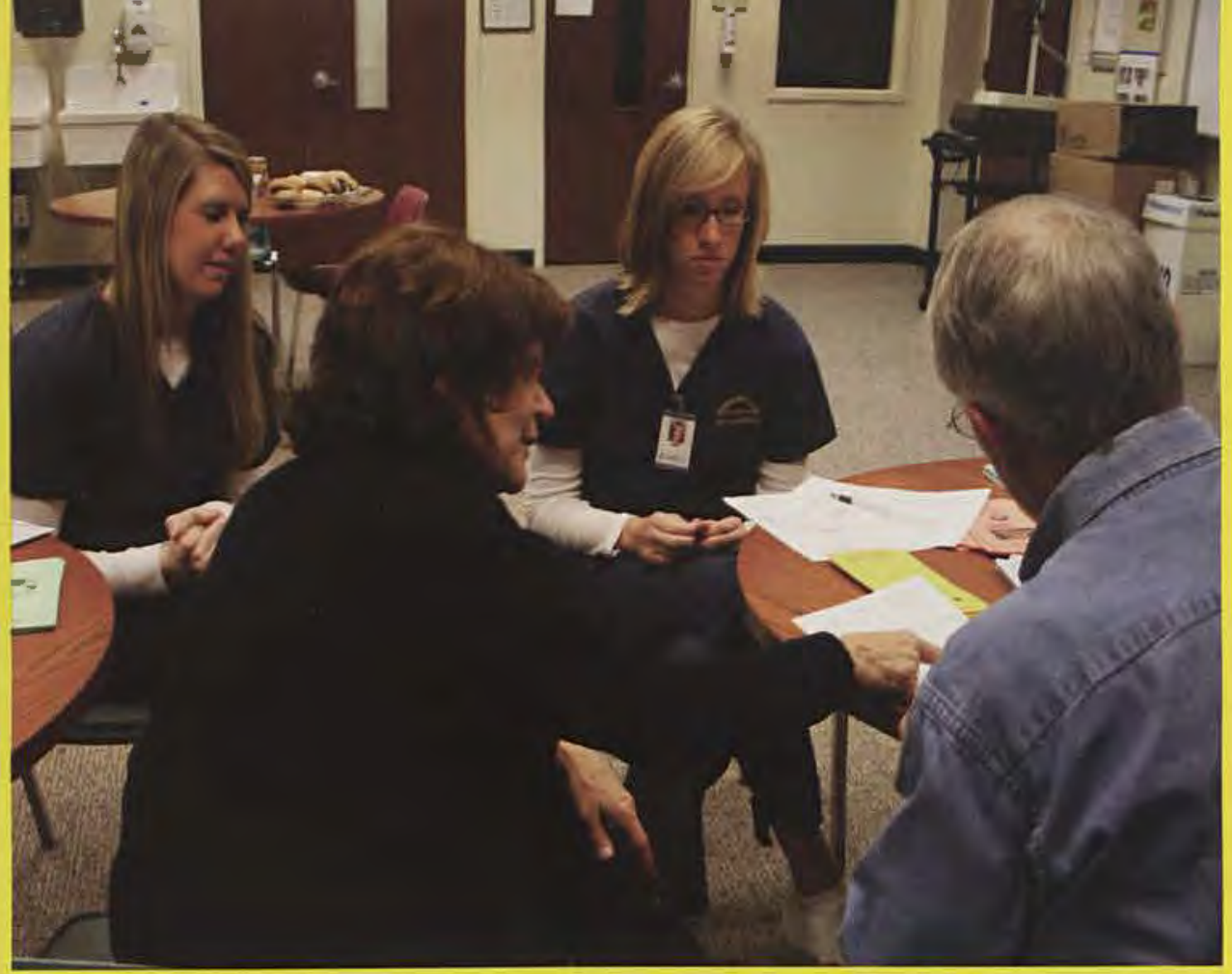

Dr. Carl Smith dares his student to apply concepts to real life.

Well, you have to practice somehow! These nursing students try to get the hang of poking in a needle. Who wants to be their live practice buddy?

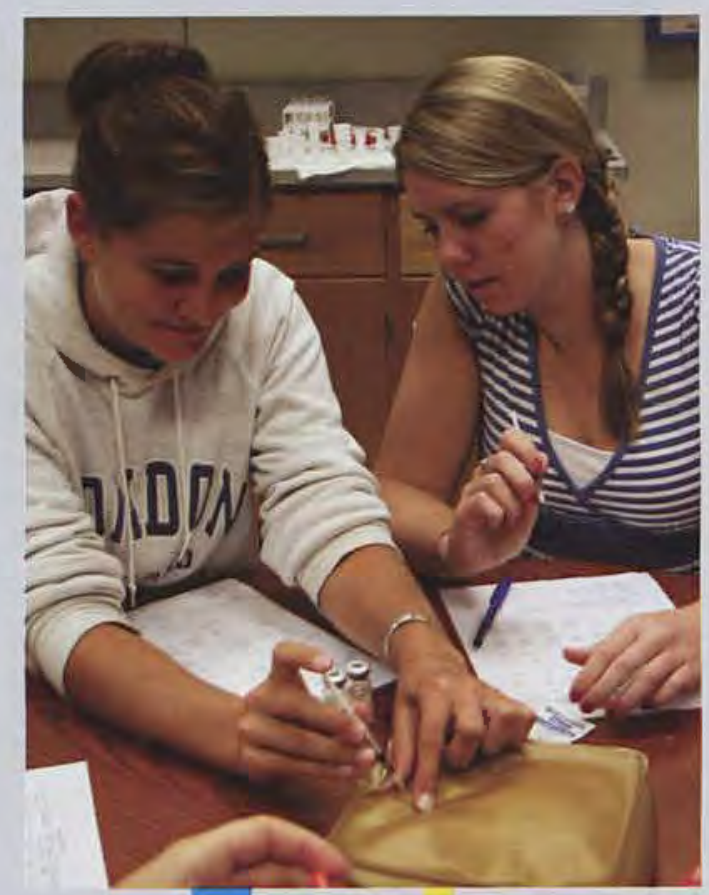


Brent Jackson thinks that "By senior year, all the students and professors have
gotten to know each other so well. It's much more like getting to have an hour-

Sit and listen to a lecture? Or do some
hands-on experiments?

these students prefer.

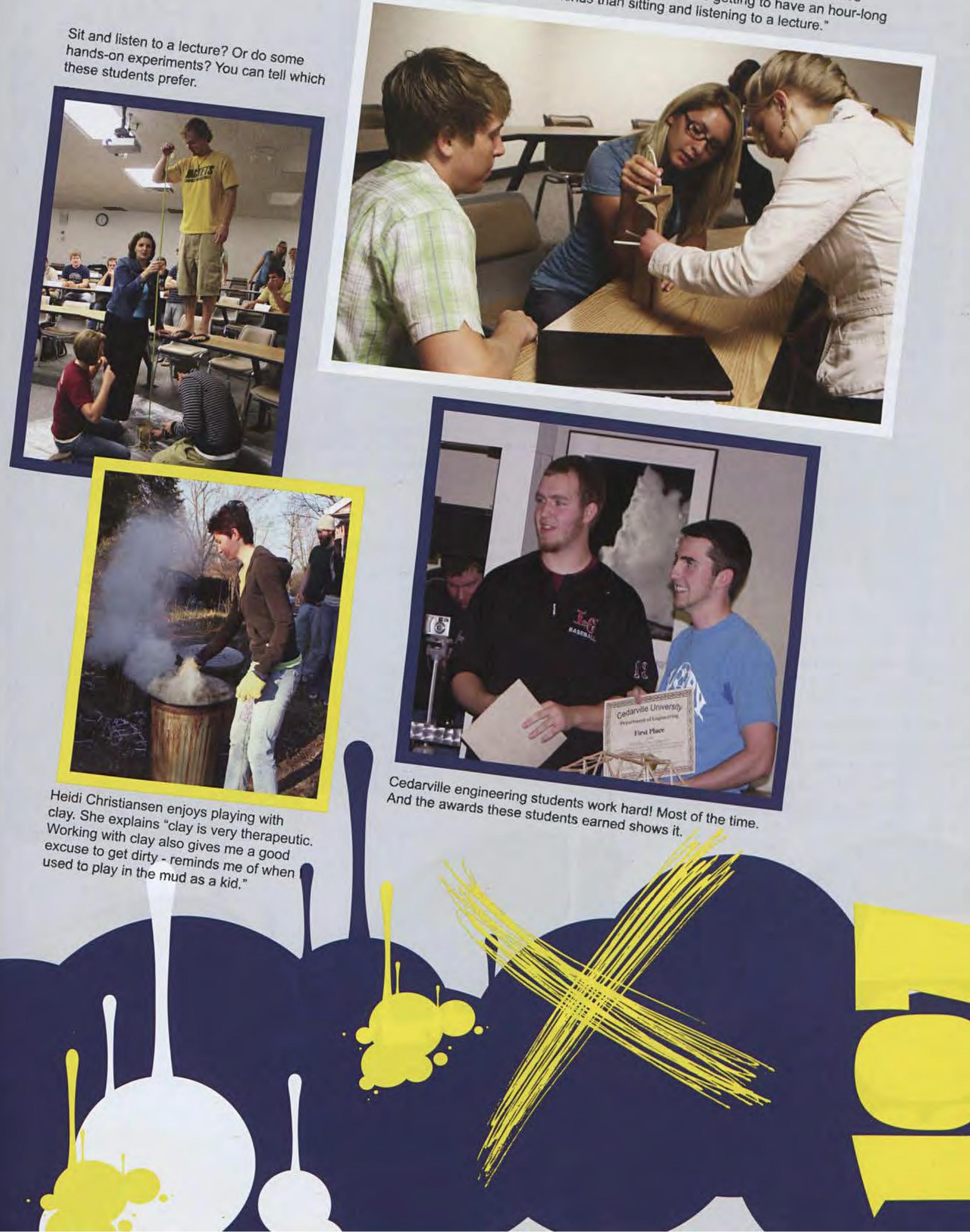

ren sitting and listening to a lecture hour-long 
Intramural soccer teams enjoy the versatility

outdoor field options.

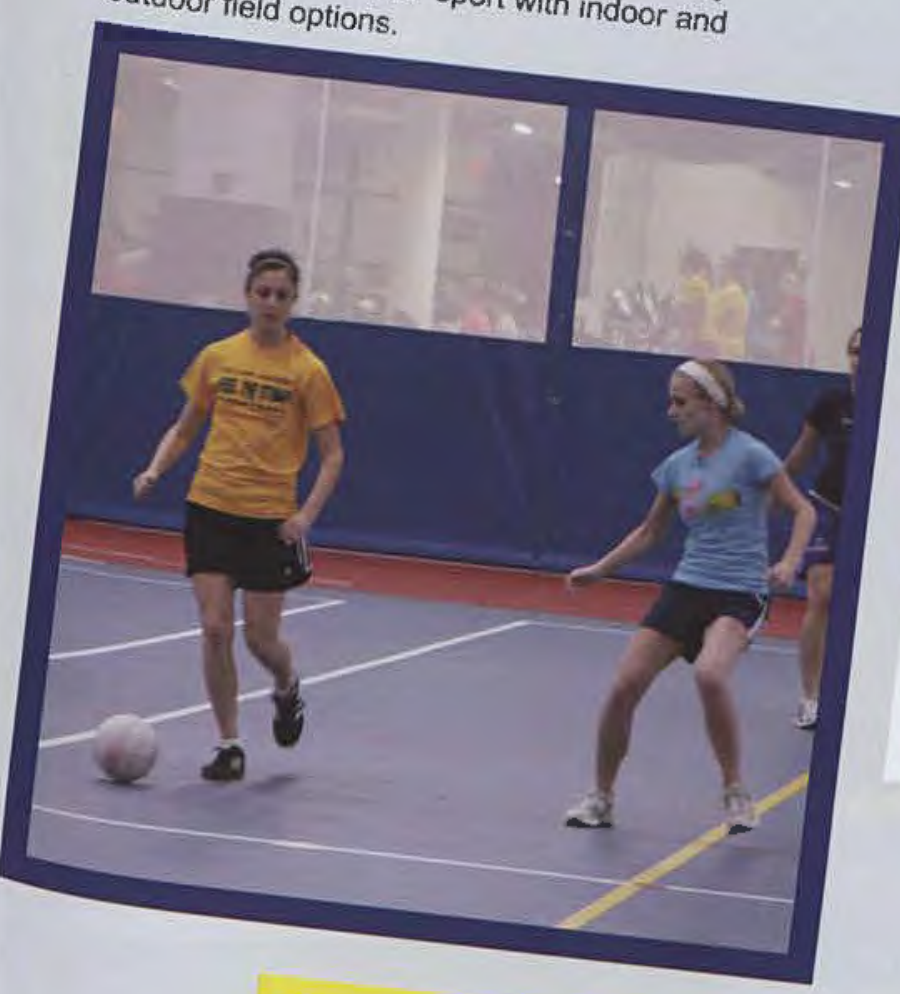

each other after an ultim, Joshua Tucker,

timate frisbee game. Nister. ofy
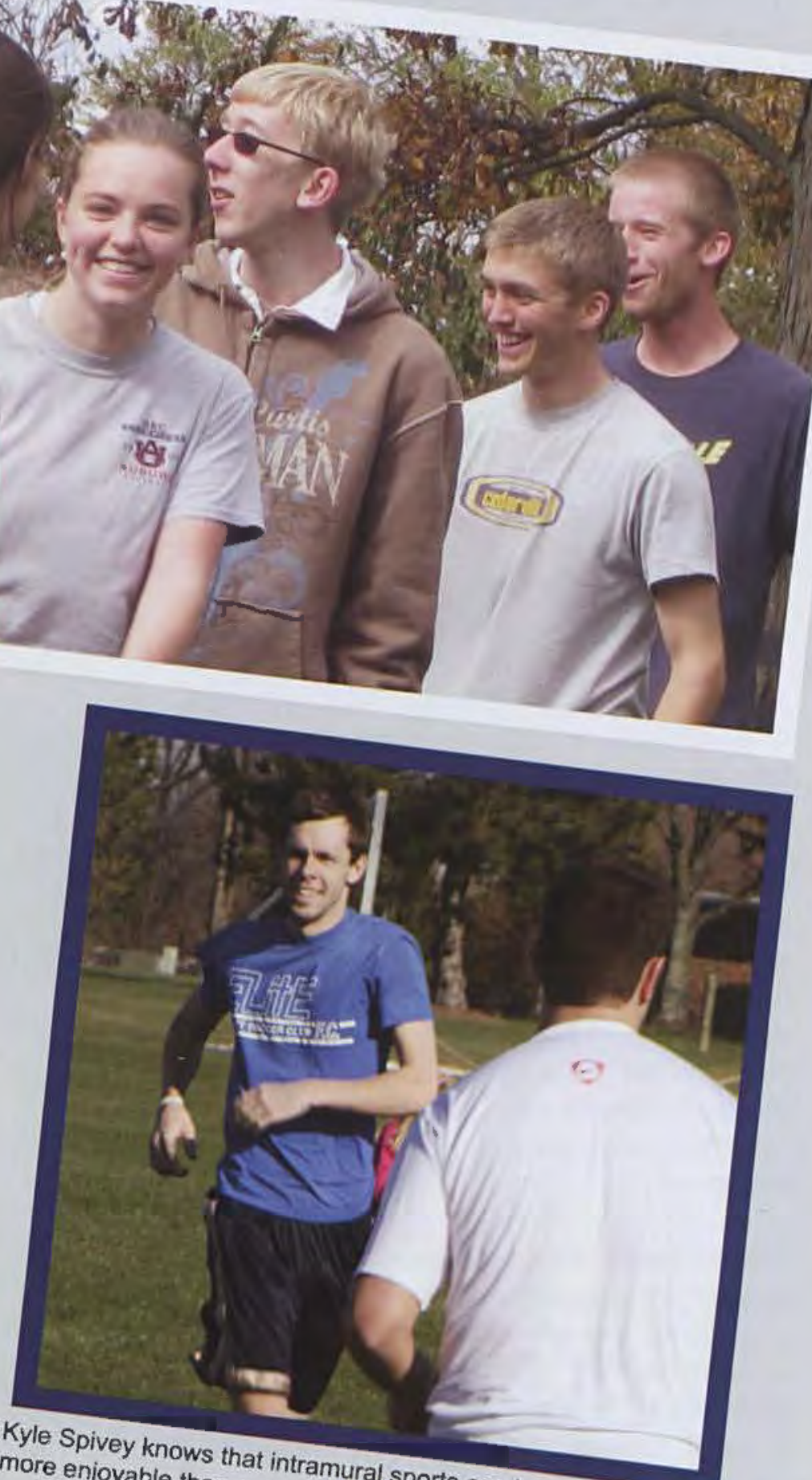

Rebekah Grafton, Kimberly Flynn, Lydia Briggs,

Tiffani Gaston celebrate

Championship.

Musser,

rmin, and

Softball

more enjoyable than a regular trip to the can be much

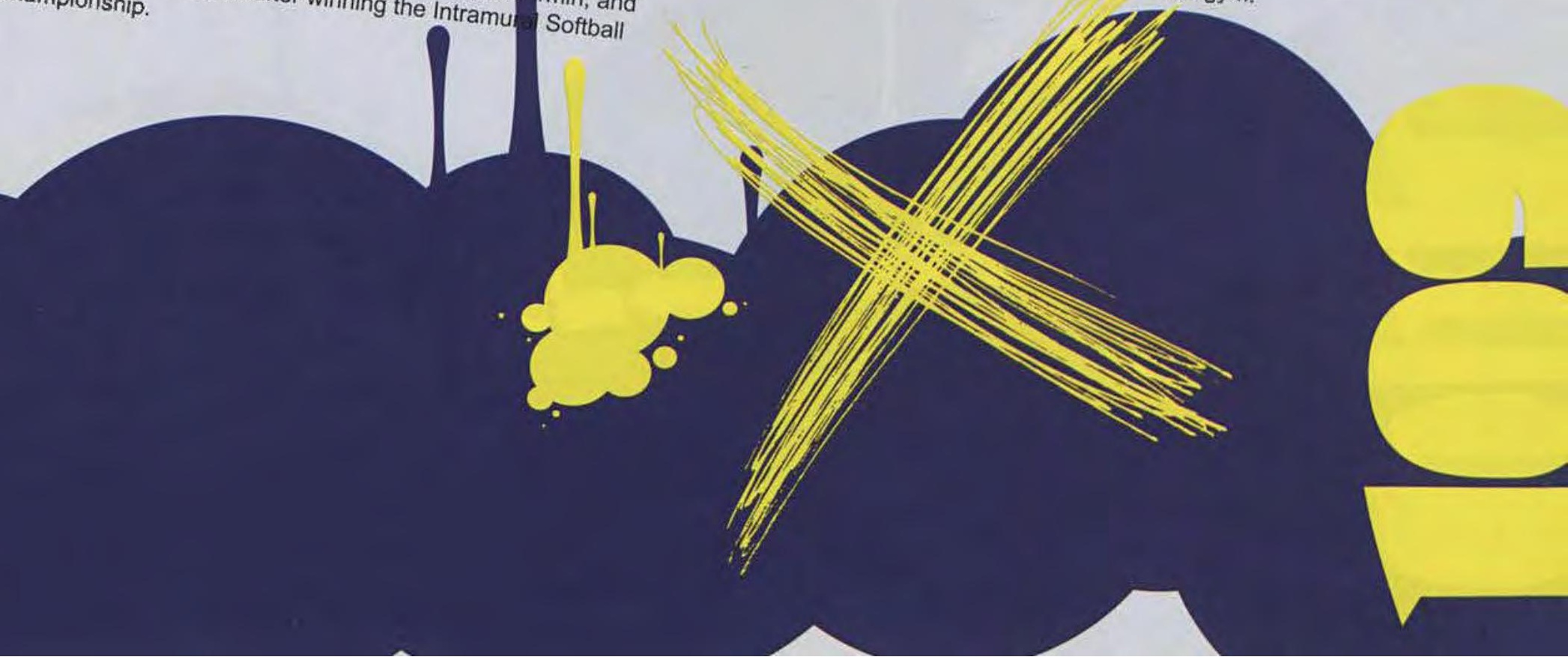


Whether it's on the stage or simply on display, both the theatre and art departments enjoy showcasing the talents of their seniors. Tom Worrall, a senior theatre major, performed The Boys Next Door by Tom Griffin. Worral explained, "It is a comedy about four mentally handicapped men and their caretaker and their lives. It shows how the worlds of the mentally handicapped and normal people differ but more how they are similar in the aspect that we all share moments of joy, anguish, love, and disappointment."

Mike Domeny chose to do an oral interpretation of the novel The Ordinary White Boy by Brocke Clark. "This project provided a fantastic capstone to my work with oral interpretation through three years of forensic competition. I worked with my friend, coach, and mentor Matt Moore. He was instrumental in helping me bring to life all seven characters in this story."

On the fine art side of things, every year the graduating seniors create a show that acts as their capstone project. "It's the best artwork that we've done during the four years at Cedarville, both inside and outside of class," explains art major Kate Bell. "It's not really something that we work on specifically just our senior year, like other research projects, so there's generally not a specific 'project'-it's more a showing of the work we've done. We plan and set the show up ourselves, and we decided to also have a sort of opening night reception with ours, as well-with food, drinks, and music."

Characteristic of both of these departments is a love for the faculty. "They exhibit a genuine concern for the growth of their students, and in doing so, they develop relationships with $s$ that extend beyond the classroom-they are friends," reveals Domeny. "Cheesy? Maybe. But true."

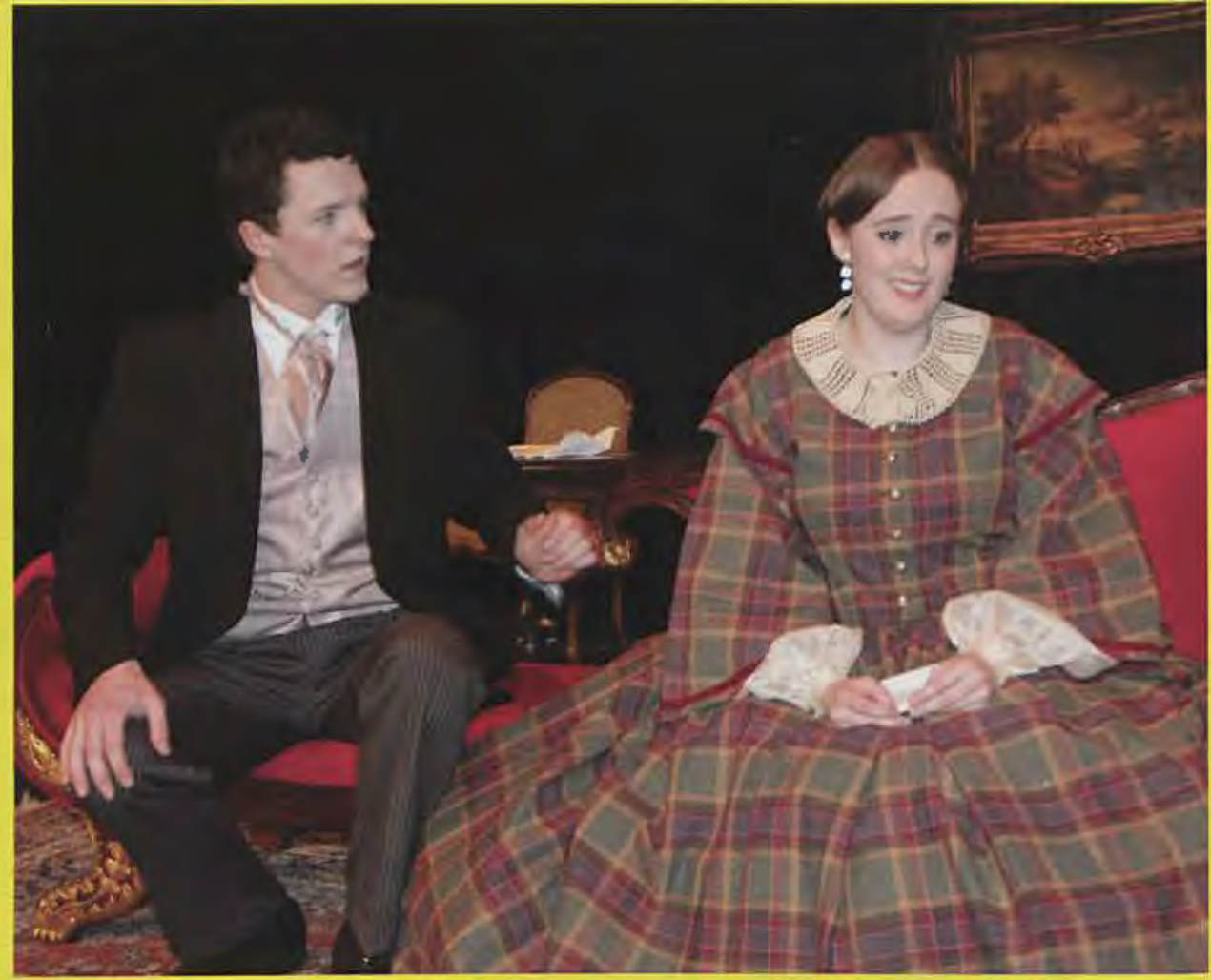

Alex Clements and Jordan Hickling perform The Heiress. "Morris is in the middle of wooing dear Catherine," explains Hickling. "Her behavior at the moment is quite awkward and quite oblivious to everything, but Morris is quick and is smooth talking his way into a fortune."

Mike Domeny performs The Ordinary White Boy. "It was a blast to really dig into this text and bring these characters and this story to life with nothing but a stool to sit on and a bucket of words. But the best part was having all my best friends and family there to watch and enjoy this capstone project."
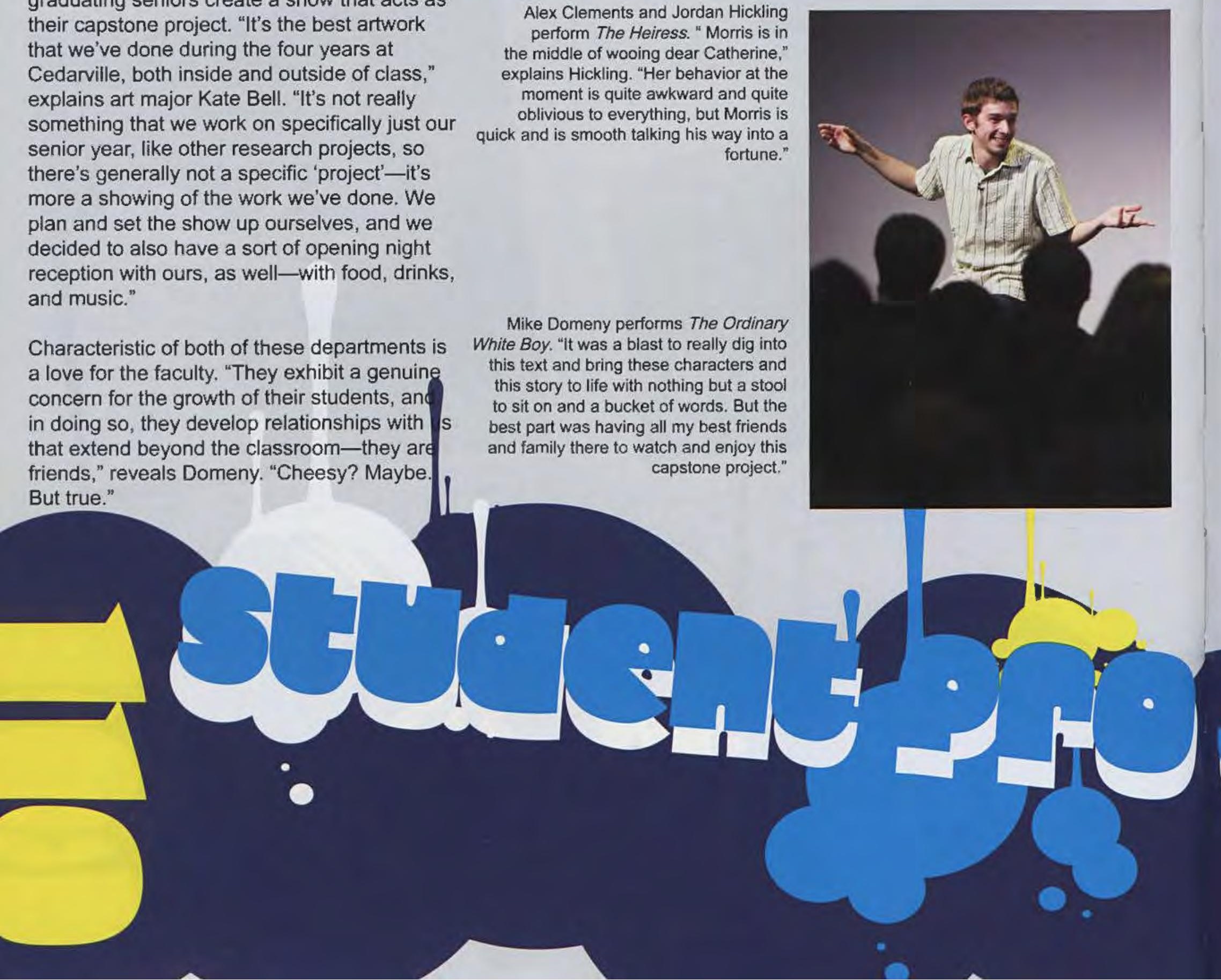
David Swick and Gabriel Pyle play two senior project.
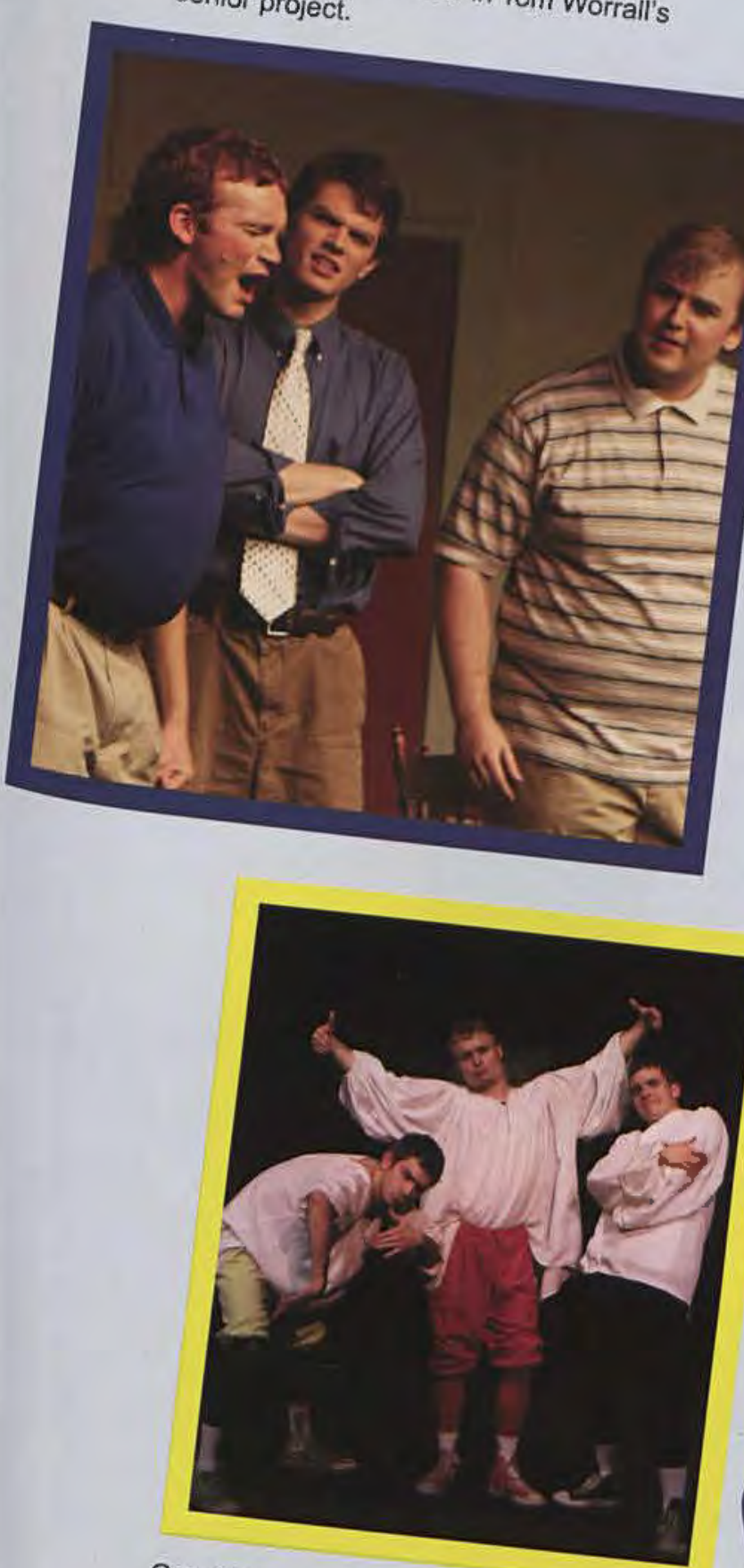

Wrant Hansen, along with Mike Domeny and Shakespeare's Othello? Hilarity. That's what," con

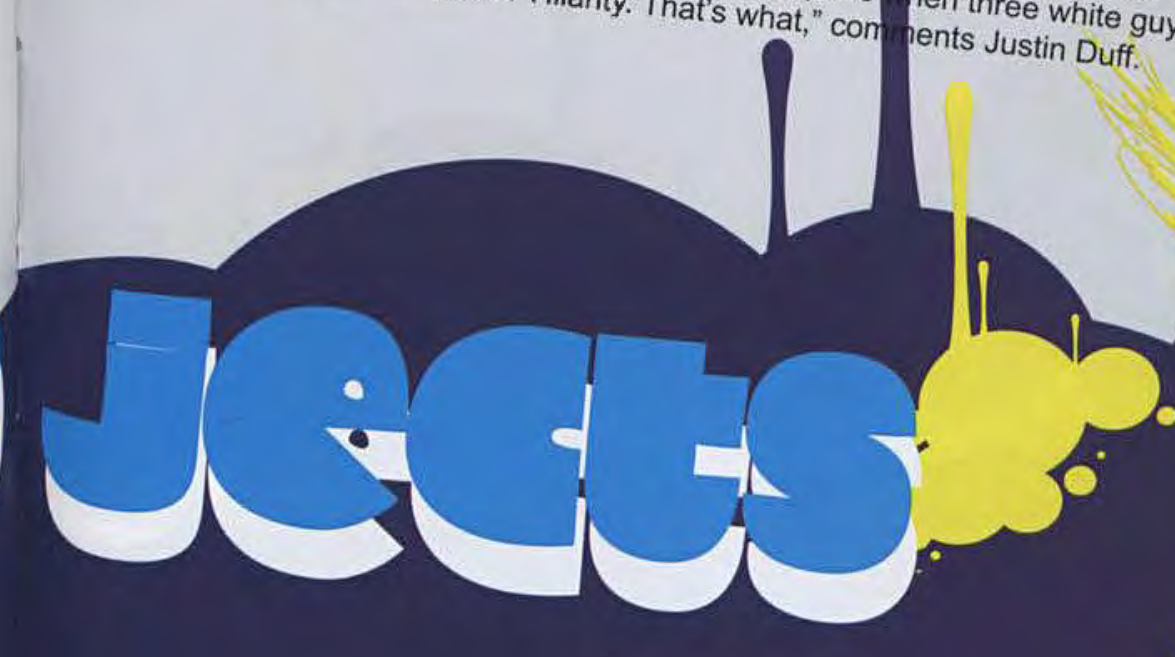

A student appreciates Sarah Gneiser's artwork "This Is How It Works," "I did the

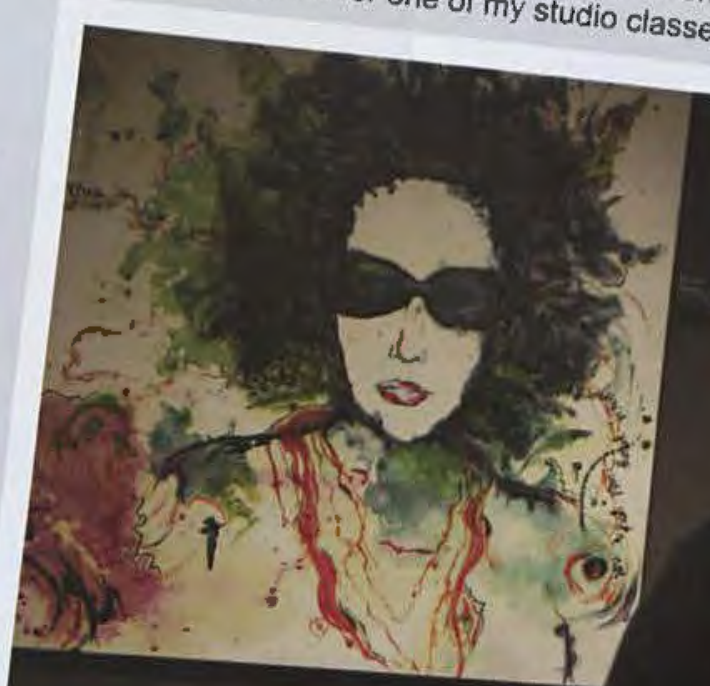

0

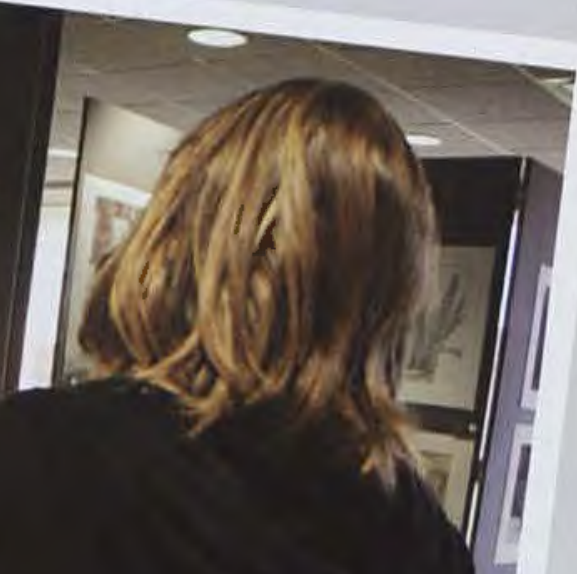

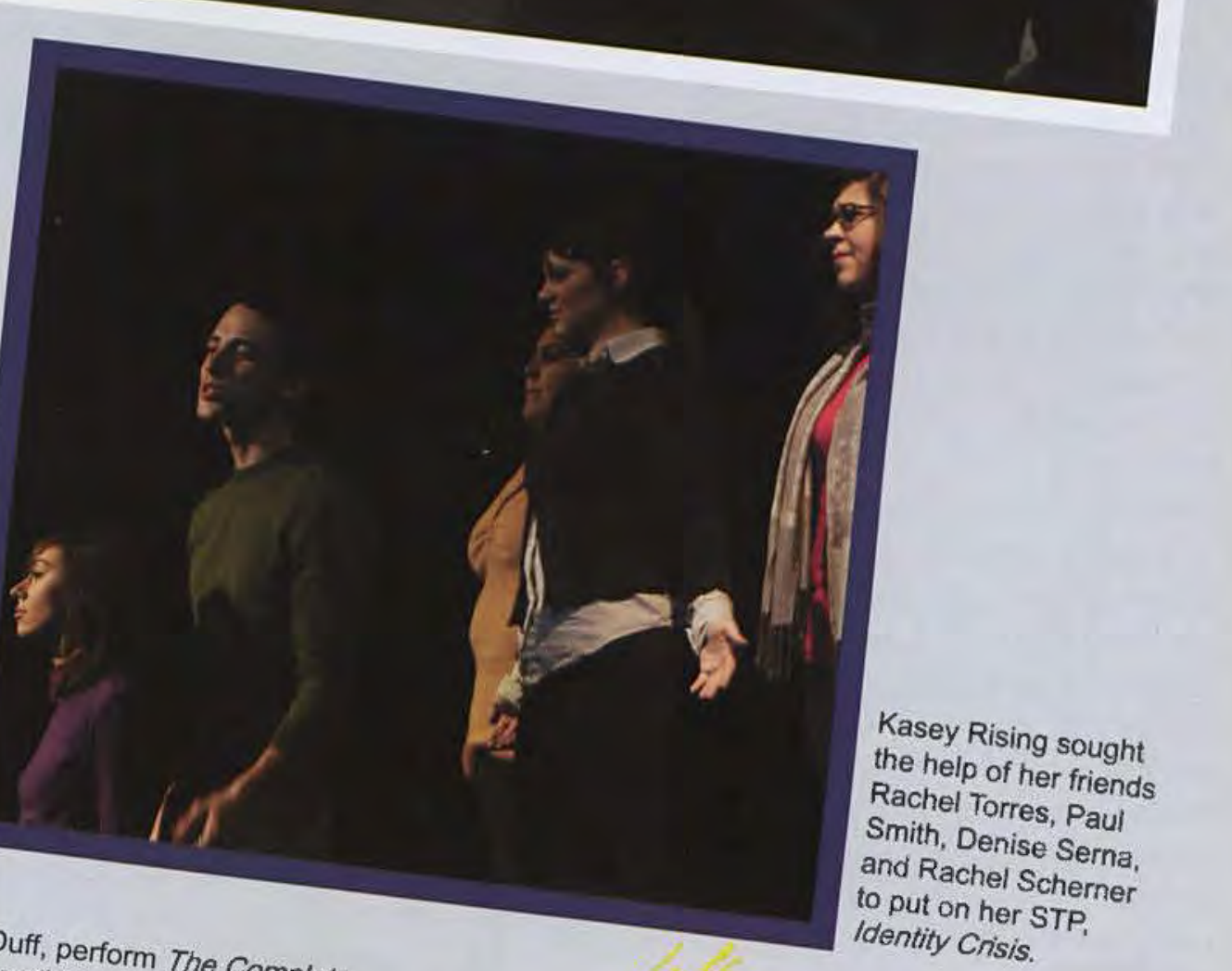

Duff, perform The Complete

ents Justin guys try to rap 
White board aficionados simplify complex
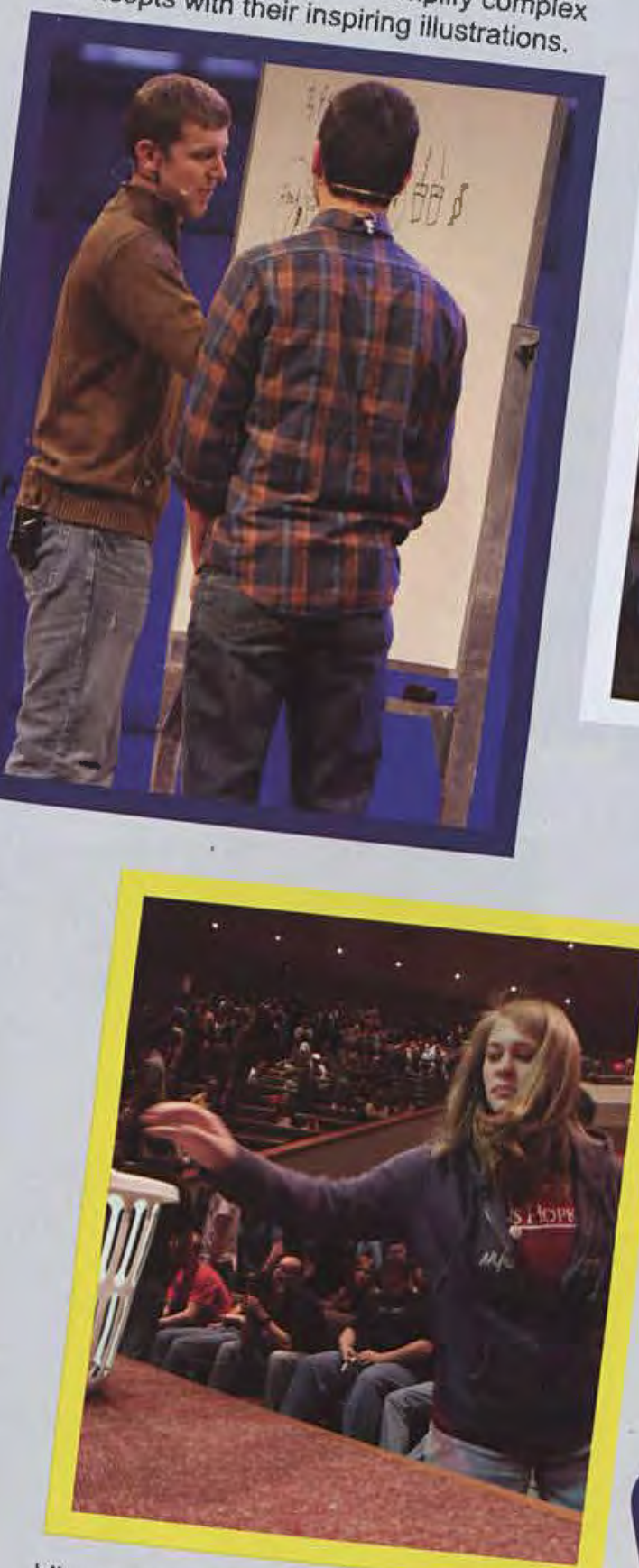

Like many other generous Cedarville

Students, Anna Sloan gives to the
Preemptive Love Coalition offering
Alex Carison chats with friends

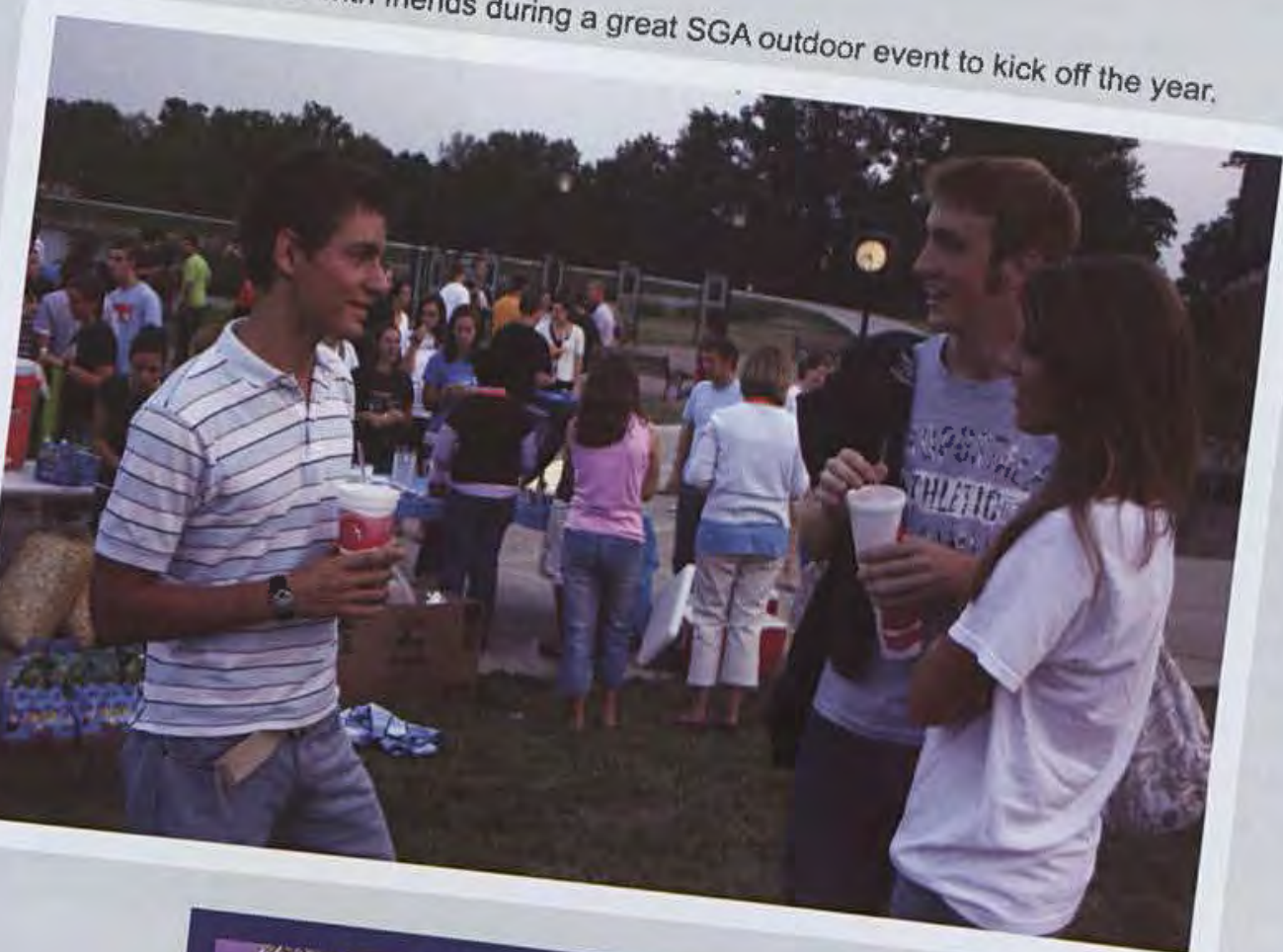

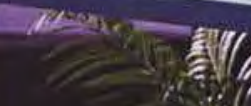

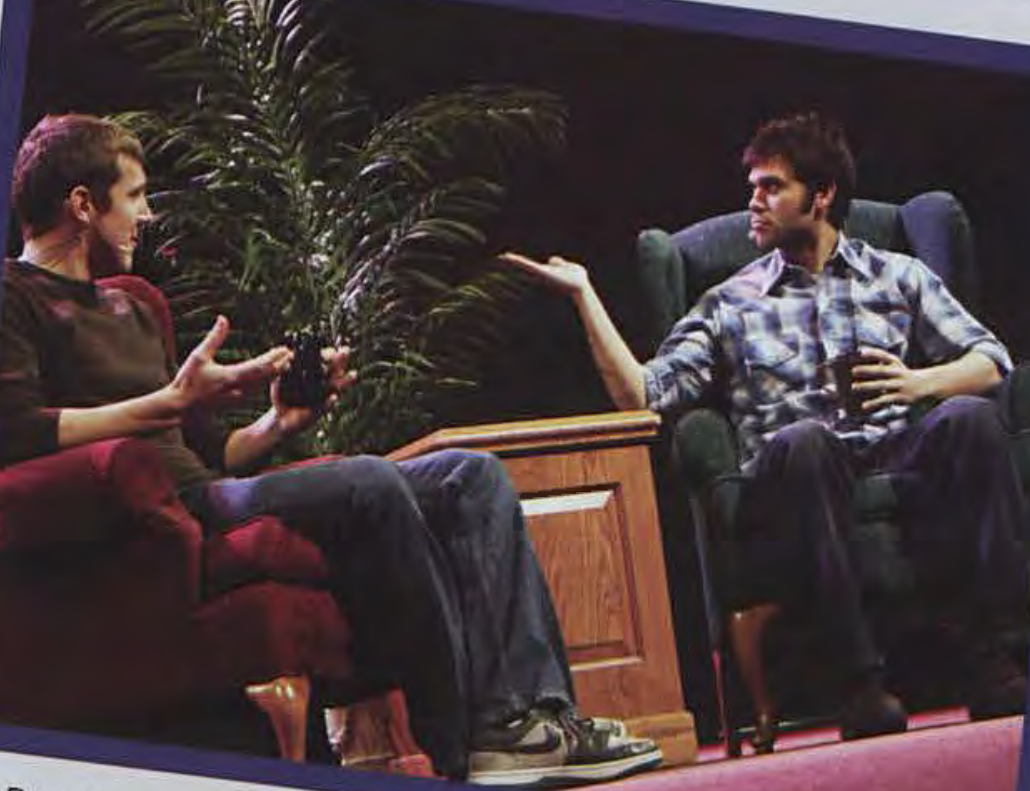

Matt Dearden and Jon McGill take some time to discuss the
deeper meaning of life.

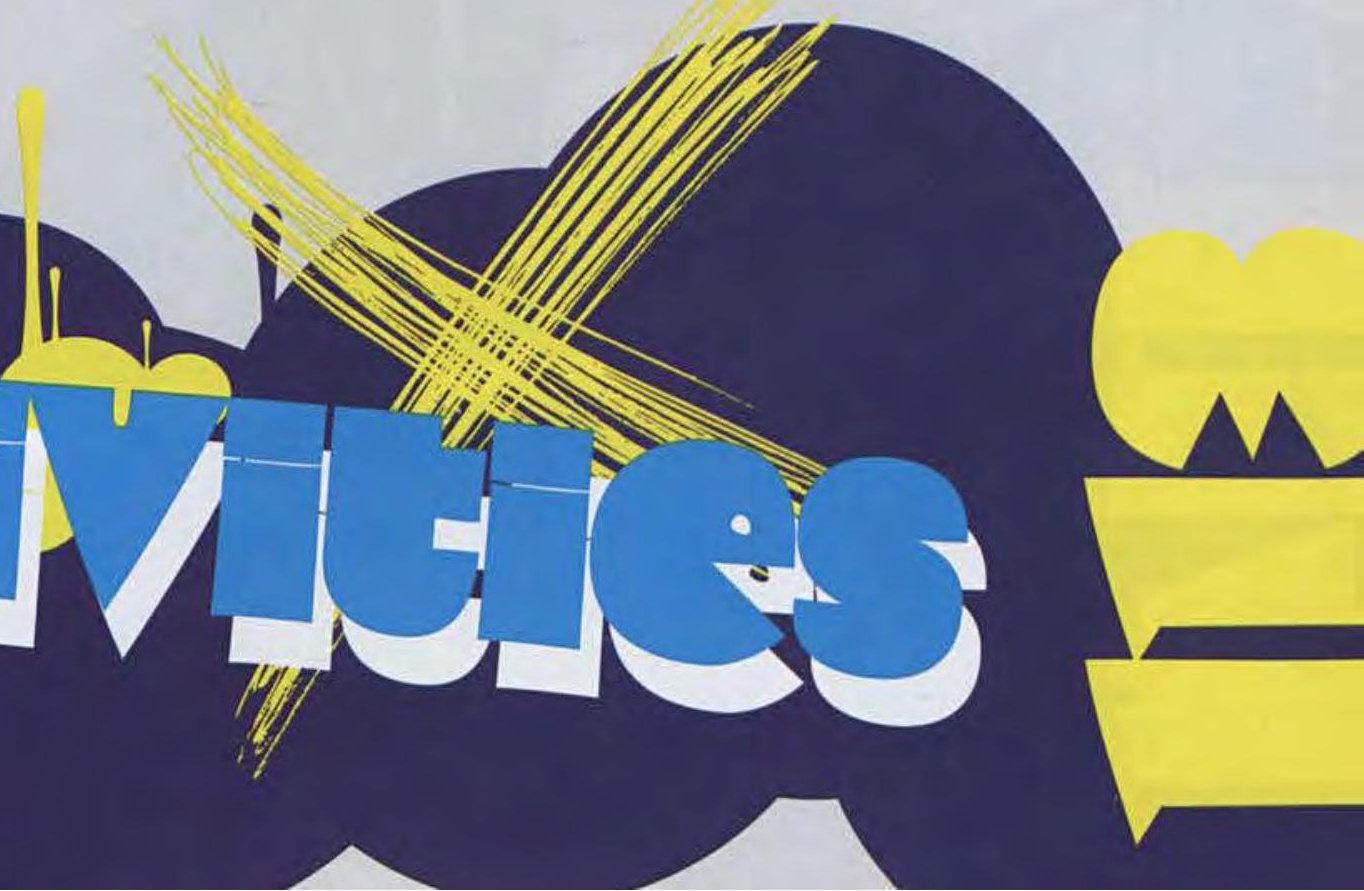


Former president of Moody Bible Institute. sage insight.
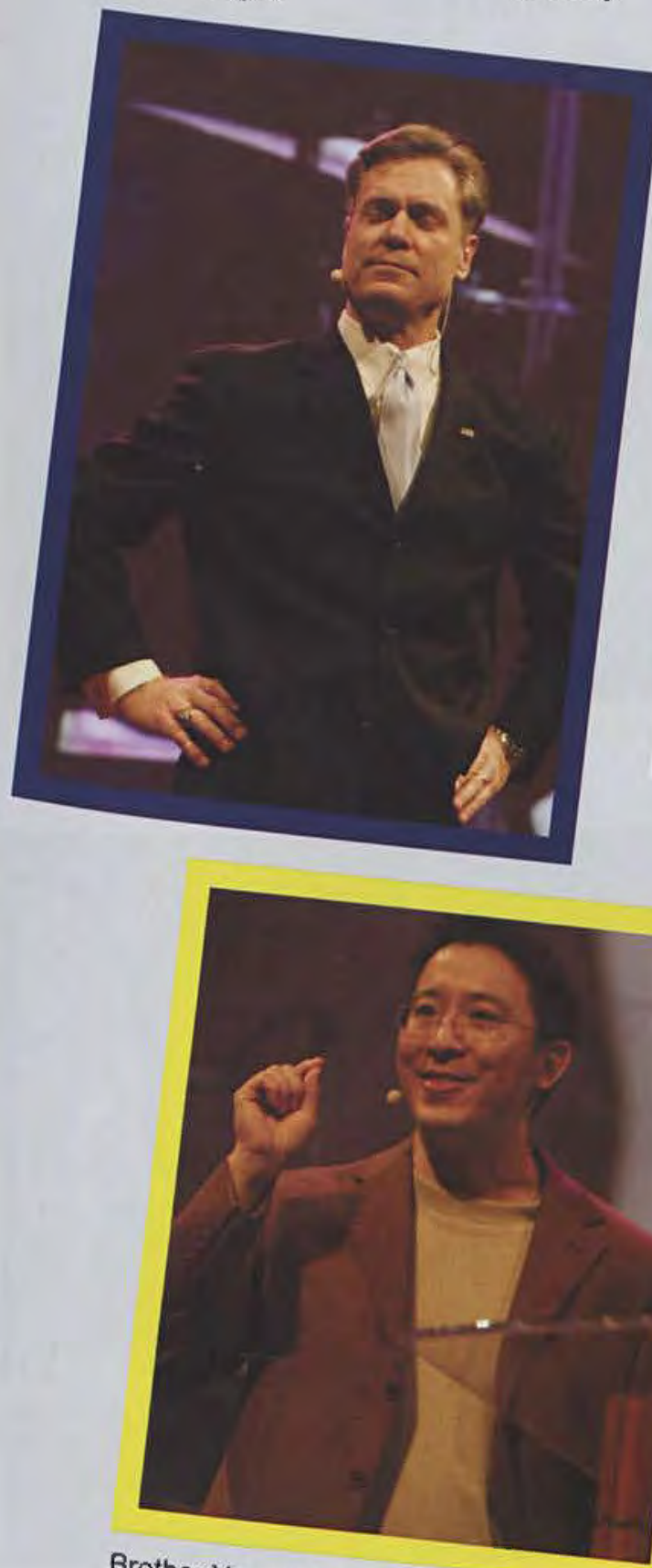

took his time in amazing story of his lo to share the students of Cedarville.
Katie

believers. the Chinese

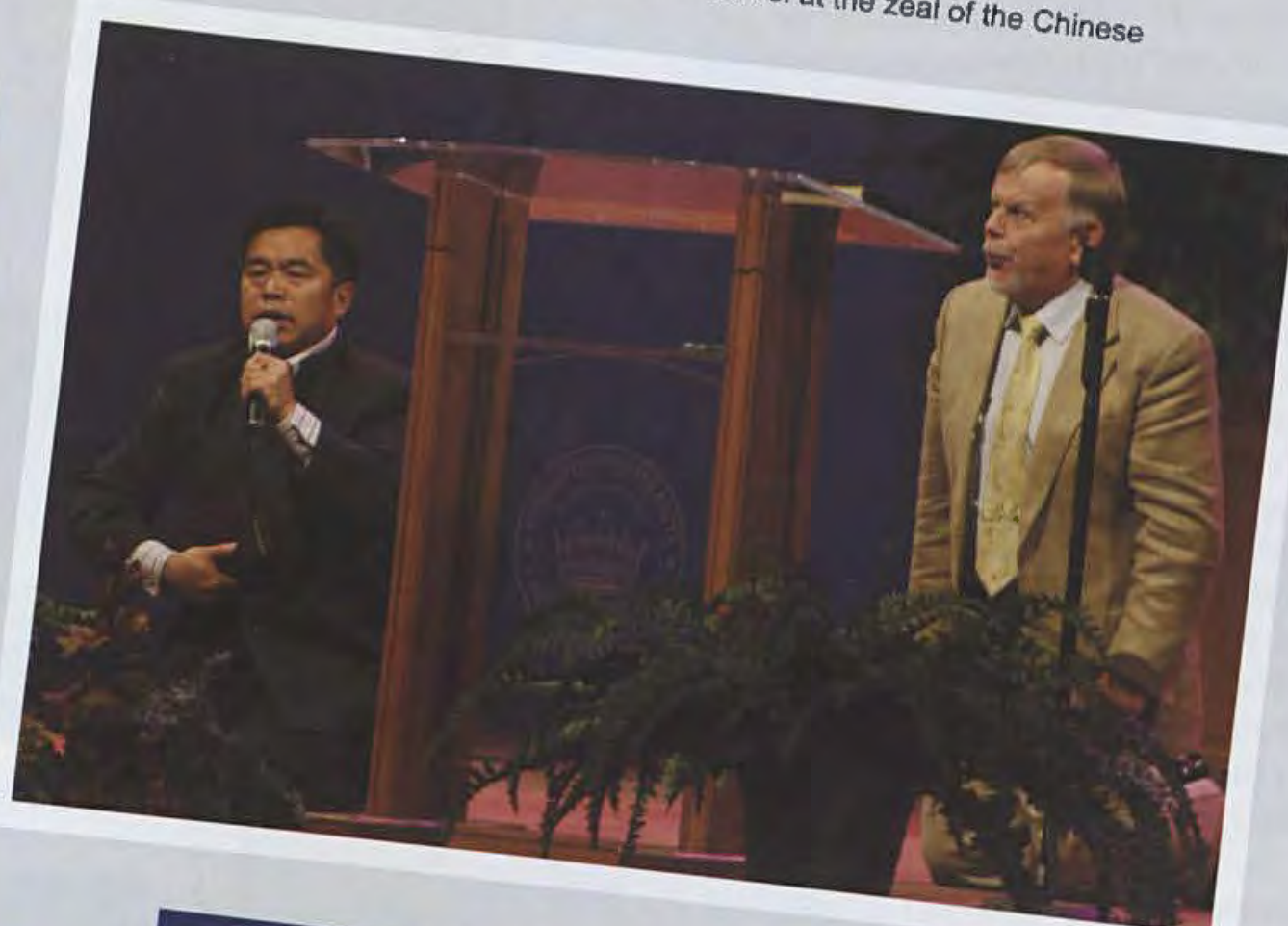

Once again, Dr. Brown mentions Nehemiah's blog. Unfortunately, students struggle to Google it online.

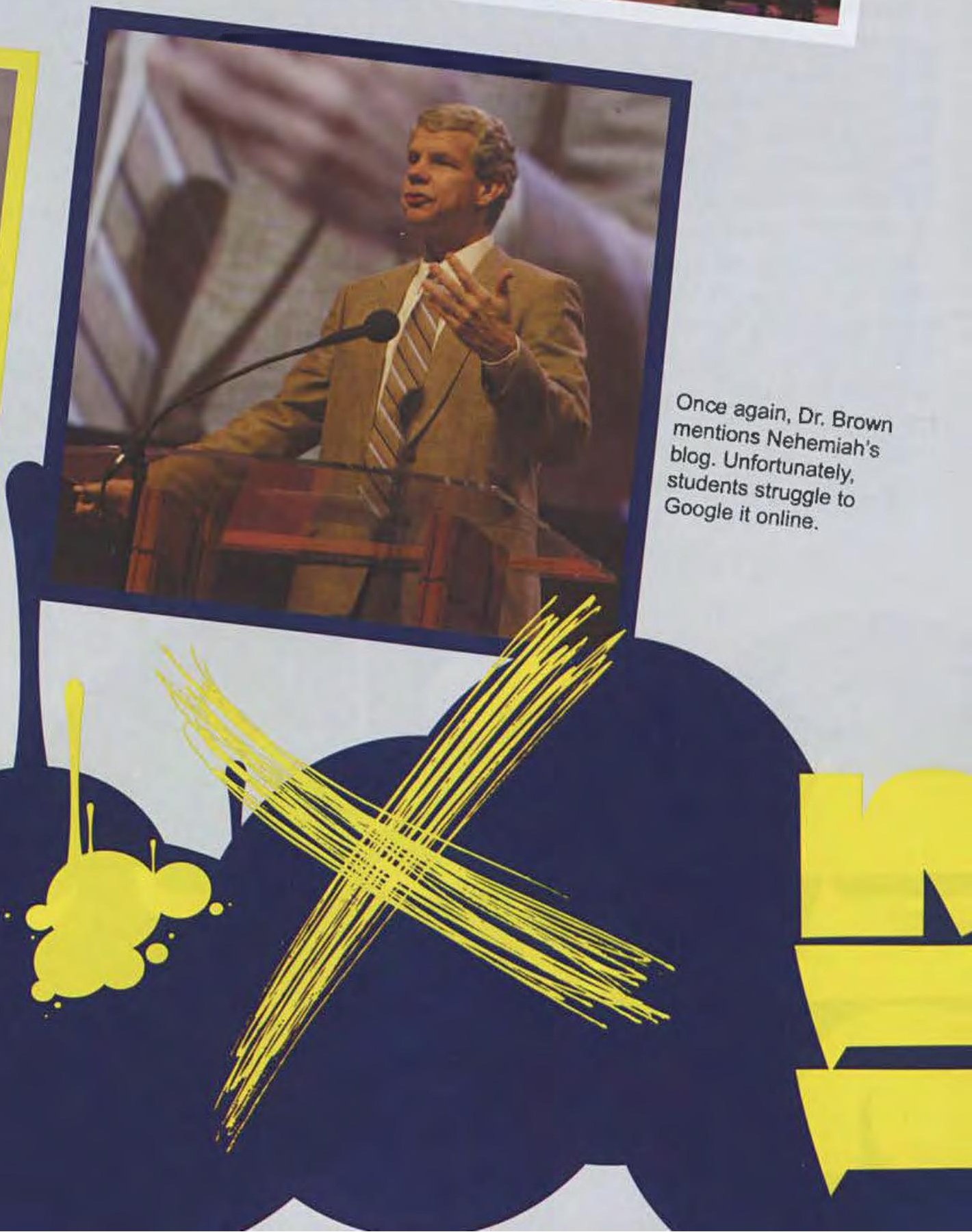


Not surprisingly, Cedarville University worked hard this year to bring in some incredible musical talent to the campus for both students and the surrounding community to enjoy. In October, David Crowder Band brought it all to the stage as they led the students in worship using predictable instruments such as guitars and drums as well as some more innovative and unexpected music makers like the classic keytar and a revamped Guitar Hero controller. As anticipated, David Crowder and his team put on a fantastic show. Band members not only ministered from the platform, but also took some time to sit down with one of Resound Radio's staff members. "David Crowder was a really fun guy to sit down with. He disclosed to me that he has not shaved his beard since the Y2K. After that, I attended the concert which was all around an amazing concert. I mean honestly, how many artists do you know that can play the Mario theme song on a guitar hero guitar?" shared Jesse Jones.

Propelling spring semester into motion was Leeland's concert promoting their newest CD release, "Opposite Way." Jesse Jones was able to spend time with this talented group of guys as well. He commented on the experience, saying, "These guys were an absolute blast to hang out with before the show. They are all really down to earth and kind of crazy guys, even crazy enough that at one point in their career they practiced in a funeral home. The show was even better than hanging out with the guys though.

Unlike a lot of artists, Leeland sounds exactly the same in concert as they do on CD, which made it one amazing concert."

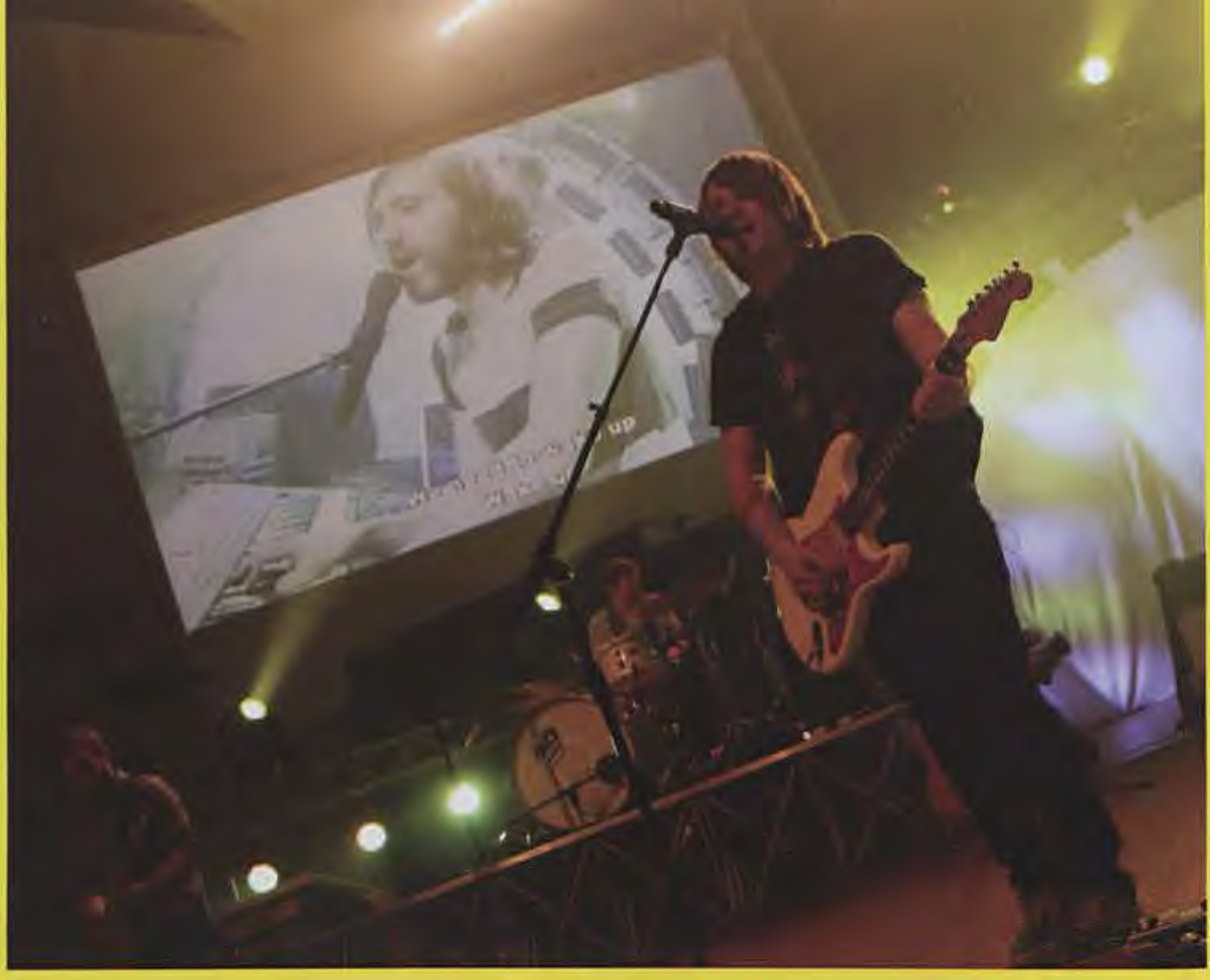

"Leeland has the most amazing voice ever," declares Sarah Ryker.

"The David Crowder Band concert wasn't just a concert-it was a night of worship. You could tell it wasn't about them. It was awesome to see such a talented, well-known, fun-loving band want to give all the praise and glory to Jesus," Amanda Allen shares.

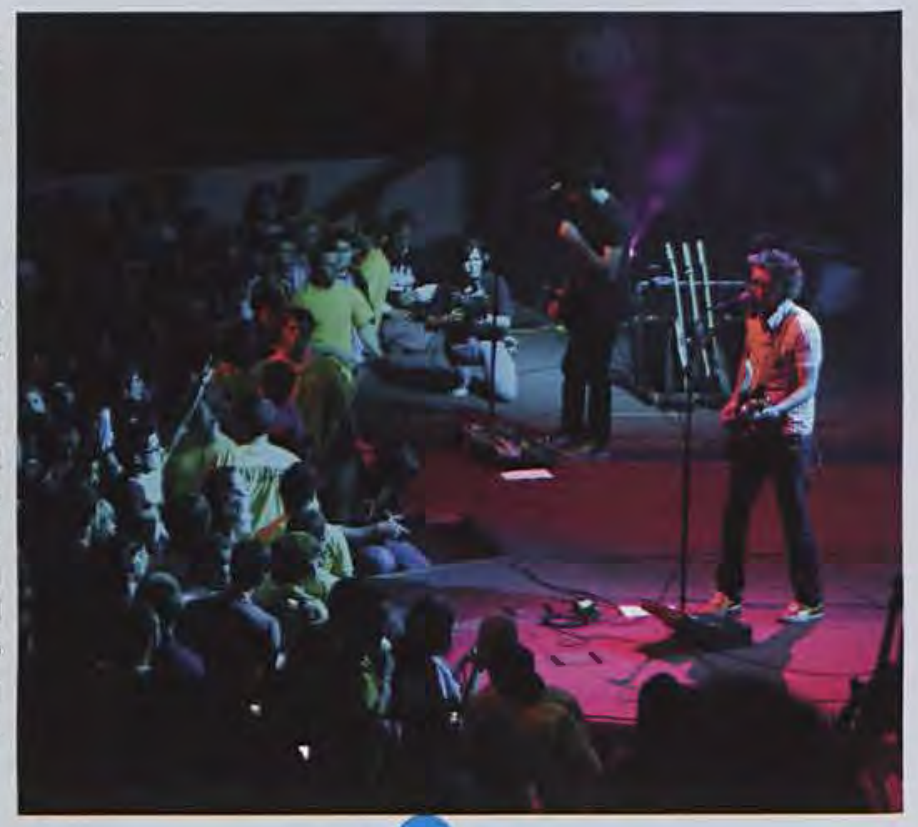

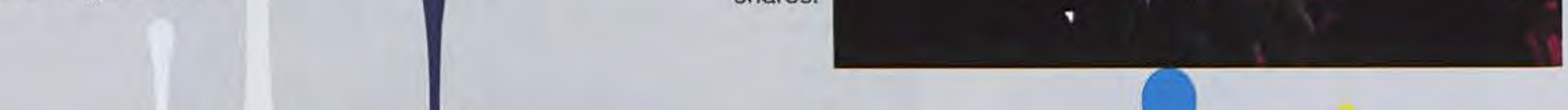

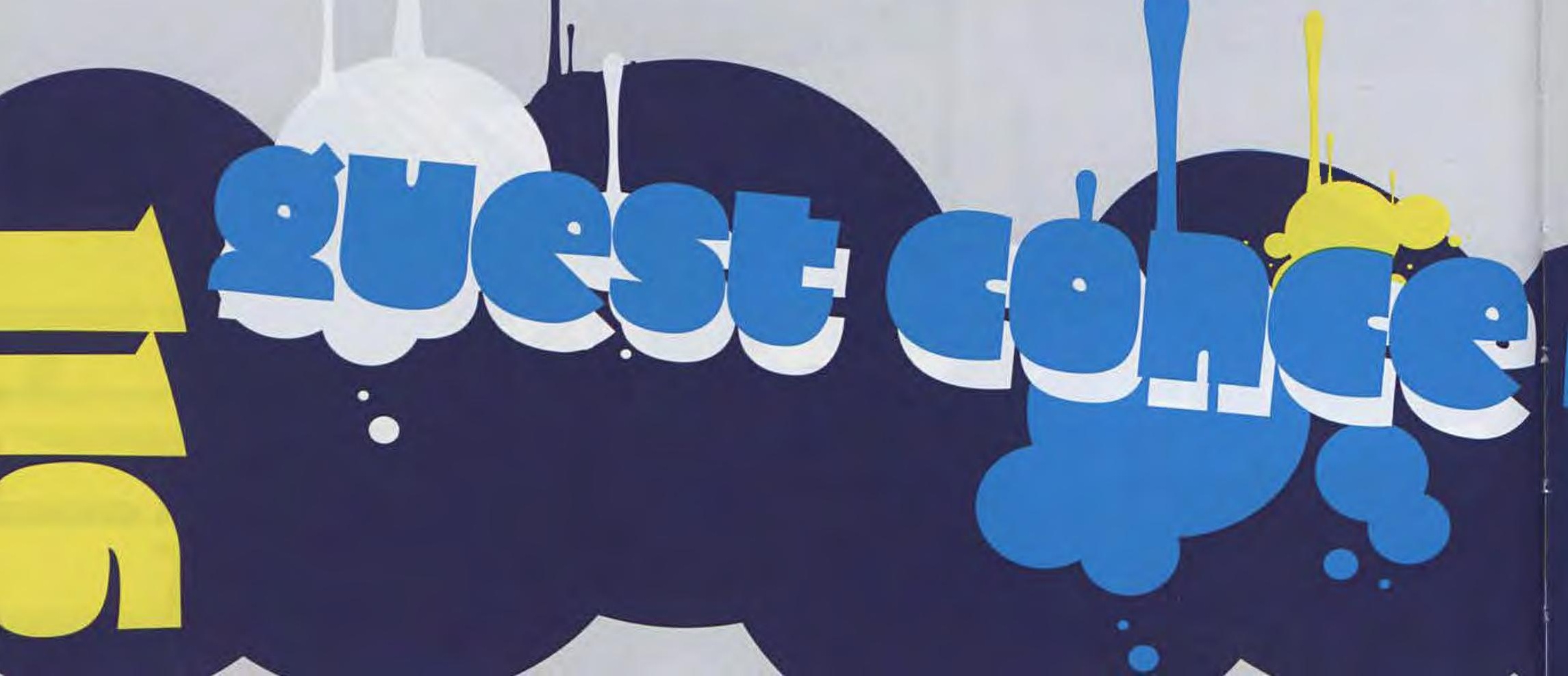


"I got to touch David Crowder's beard!" gushes lucky student Bri Mottashed.
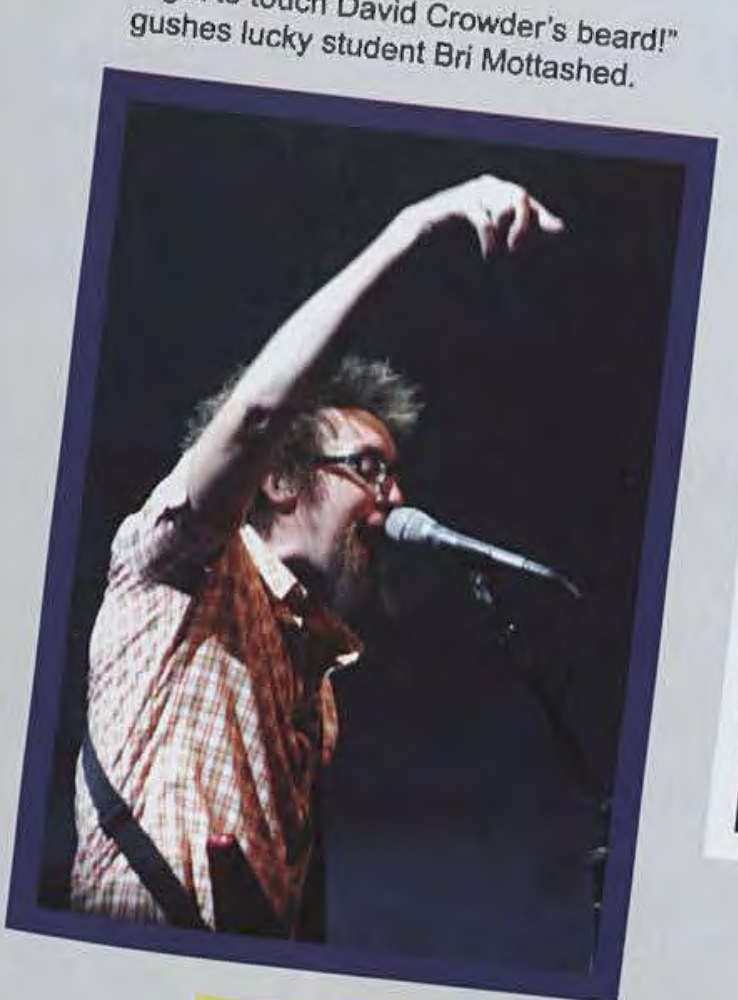

couldn't help but wonder if it was an act of God to make us focus on went out, I
the praise, rather than the musicians themselves."
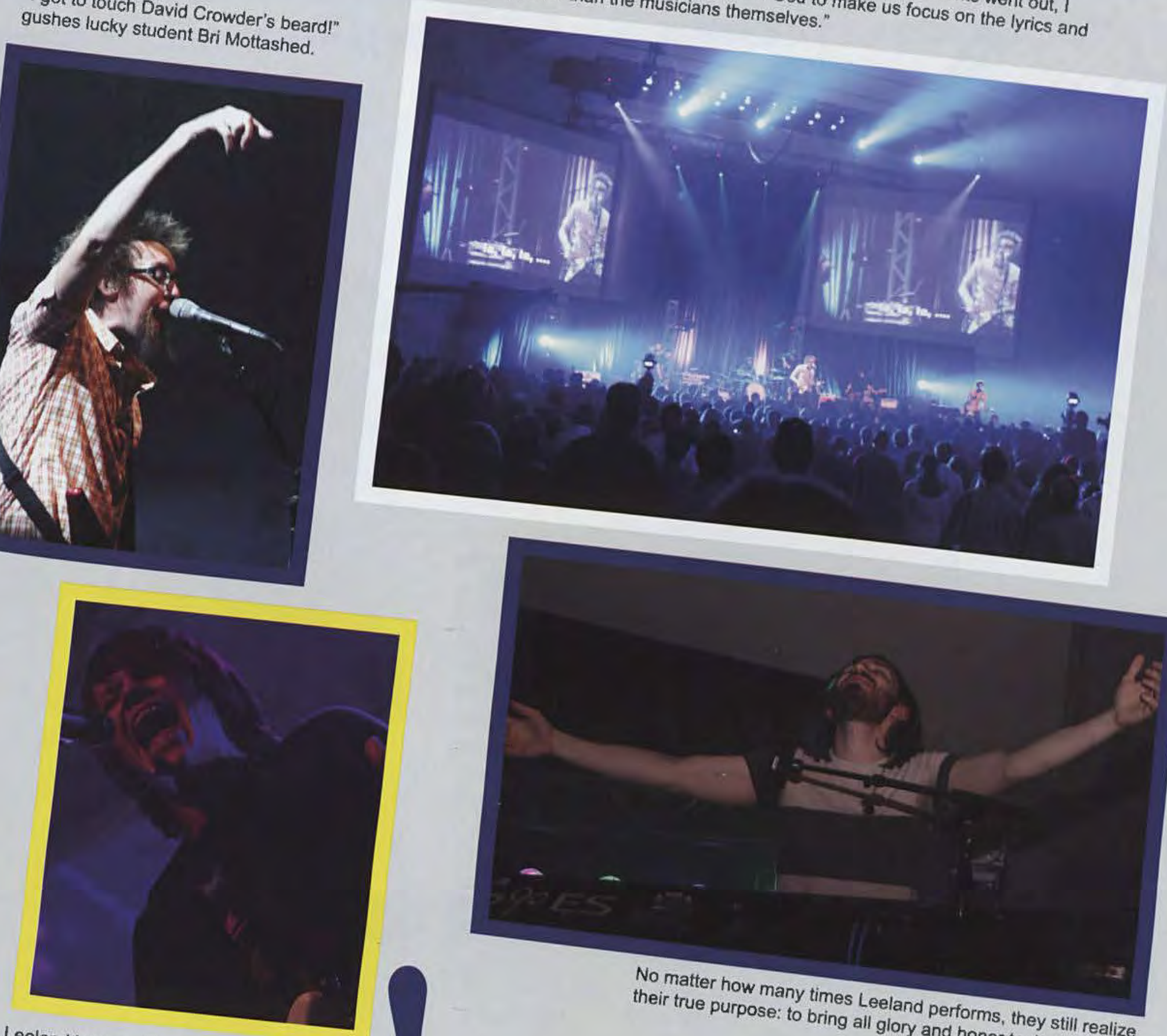

Leeland belts out the "Sound of Melodies."

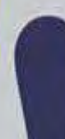

No matter how many

their true purpose: to times Leeland performs, they still realize re
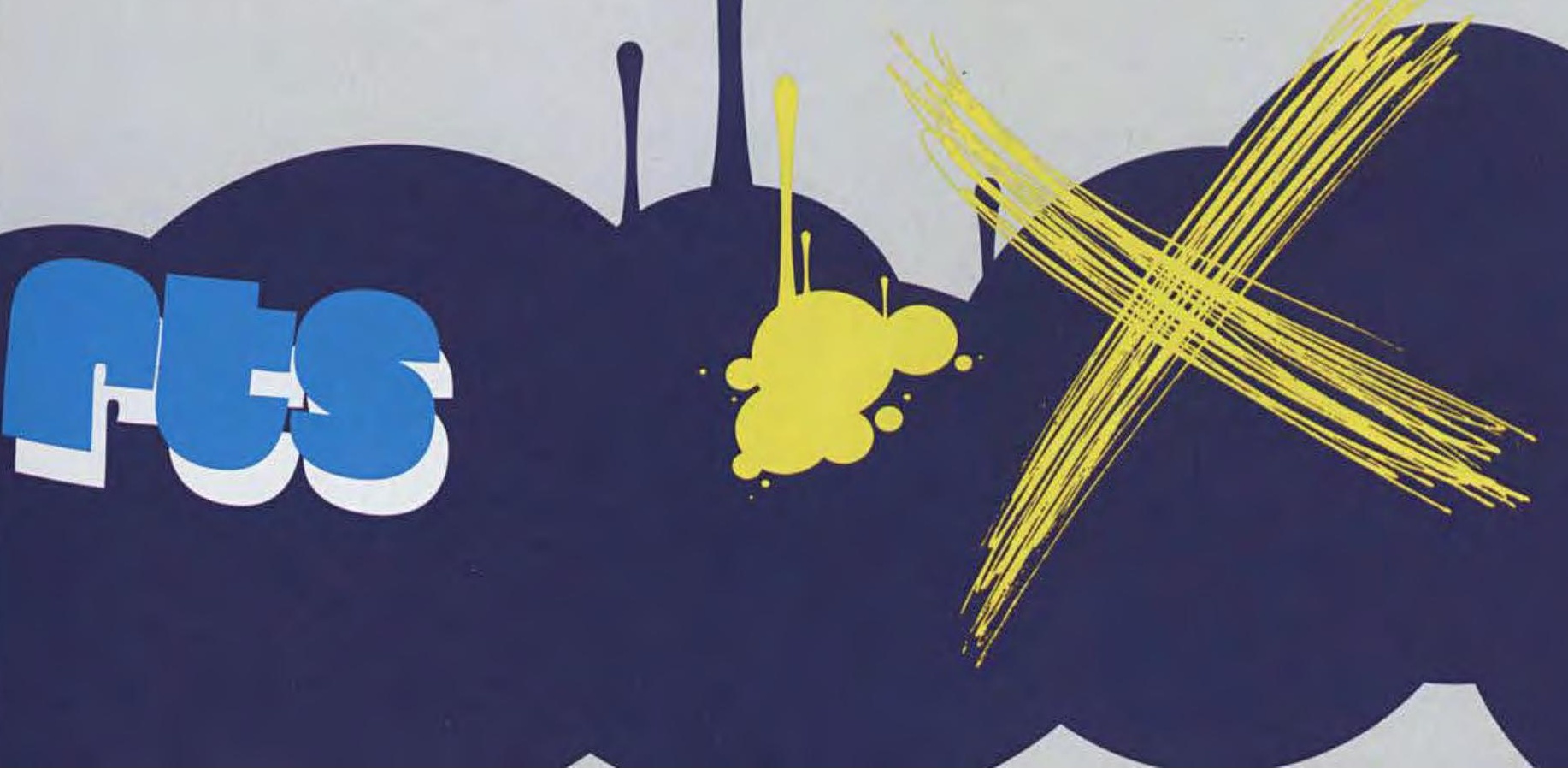


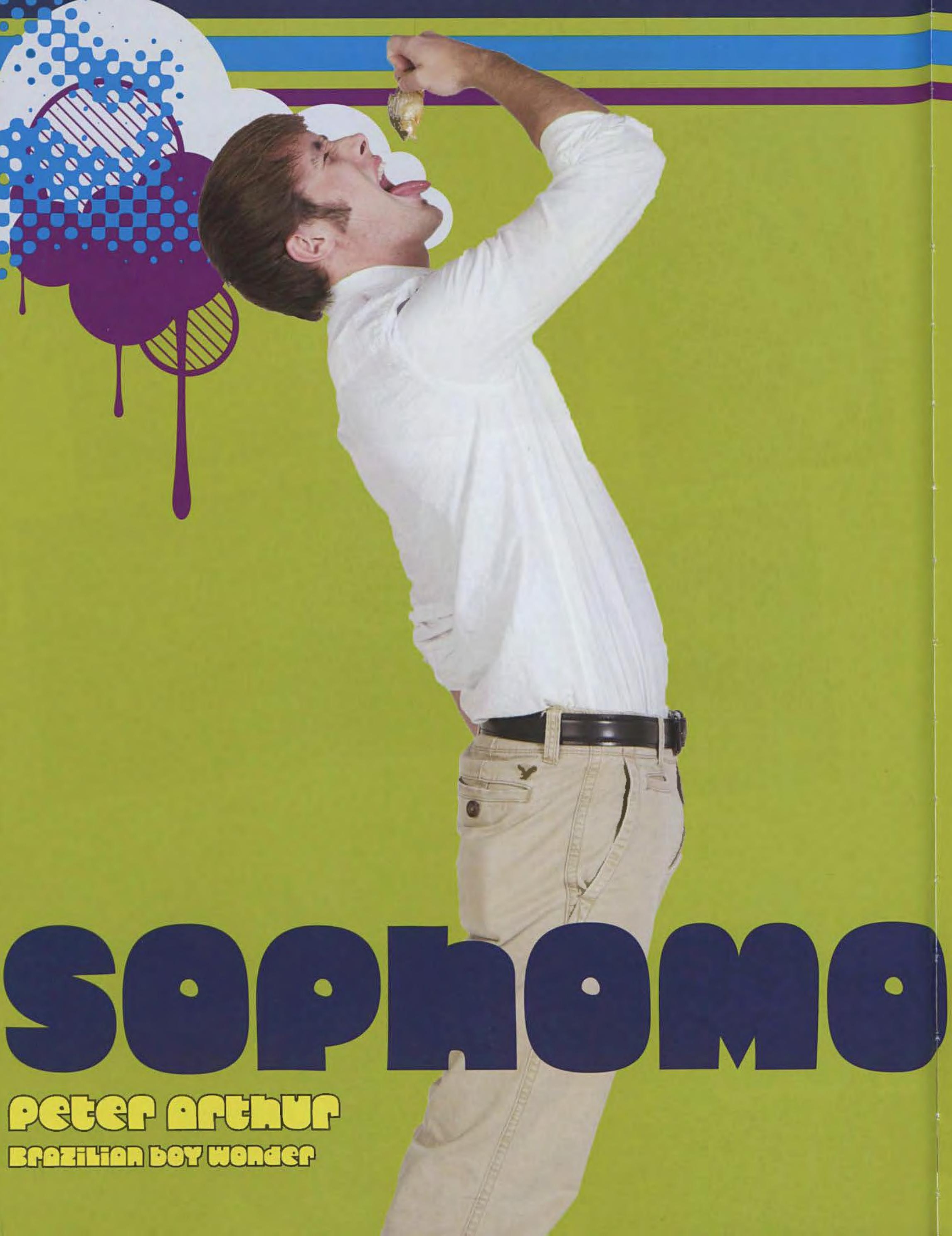


When I close my eyes in those moments between consciousness and sleep, I can see São Paulo on the backs of my eyelids. I can hear the noise of the Brazilian city, smell the familiar scent of home. When I open my eyes again, though, I only see the ceiling of my dorm room. I am still in Cedarville.

My dad was an engineer. After working for a few years in the field, he realized that the products he was developing would last at most forty years and then become futile, merely progress for progress' sake. He wanted to develop something that was lasting, that was meaningful and never futile. With a heart for the lost and the goal of church planting, my parents took my brother and moved to São Paulo, Brazil, where I was born there a year later. São Paulo is among the largest cities in the world but as its population grows, so does the allure of corruption and immorality. Don't get me wrong - it is a great place to live, and it is my home. From the nearby sandy beaches and rolling terrain to the remnants of the Atlantic Rain Forest north of the city, São Paulo bursts with natural character. It houses the most important financial district in Brazil and abundant opportunities for successful careers, with São Paulo the tallest sky scrapers in Brazil and a bustling Downtown. The culture is unique - it has a distinct blend of Portuguese, Italian, and other European influences. In this culture the arts flourish and soccer thrives. These worldly achievements, though, are coupled with immorality. I think my dad got it right when he decided to be used to make a lasting impact. So here I am, a sophomore at Cedarville, being trained to tell the lost citizens of São Paulo about the One who finds, forgives, and restores.

I came to Cedarville to pursue a degree in engineering, the same field in which my dad is trained. I quickly realized, though, that I should be headed in a different direction, one that encompasses my life-long passion for writing, as well as people. Now majoring in writing, I am preparing for a life on the mission field, for a profession in which I can tell people that there is hope. I, like my dad, realized that I should develop something that lasts.

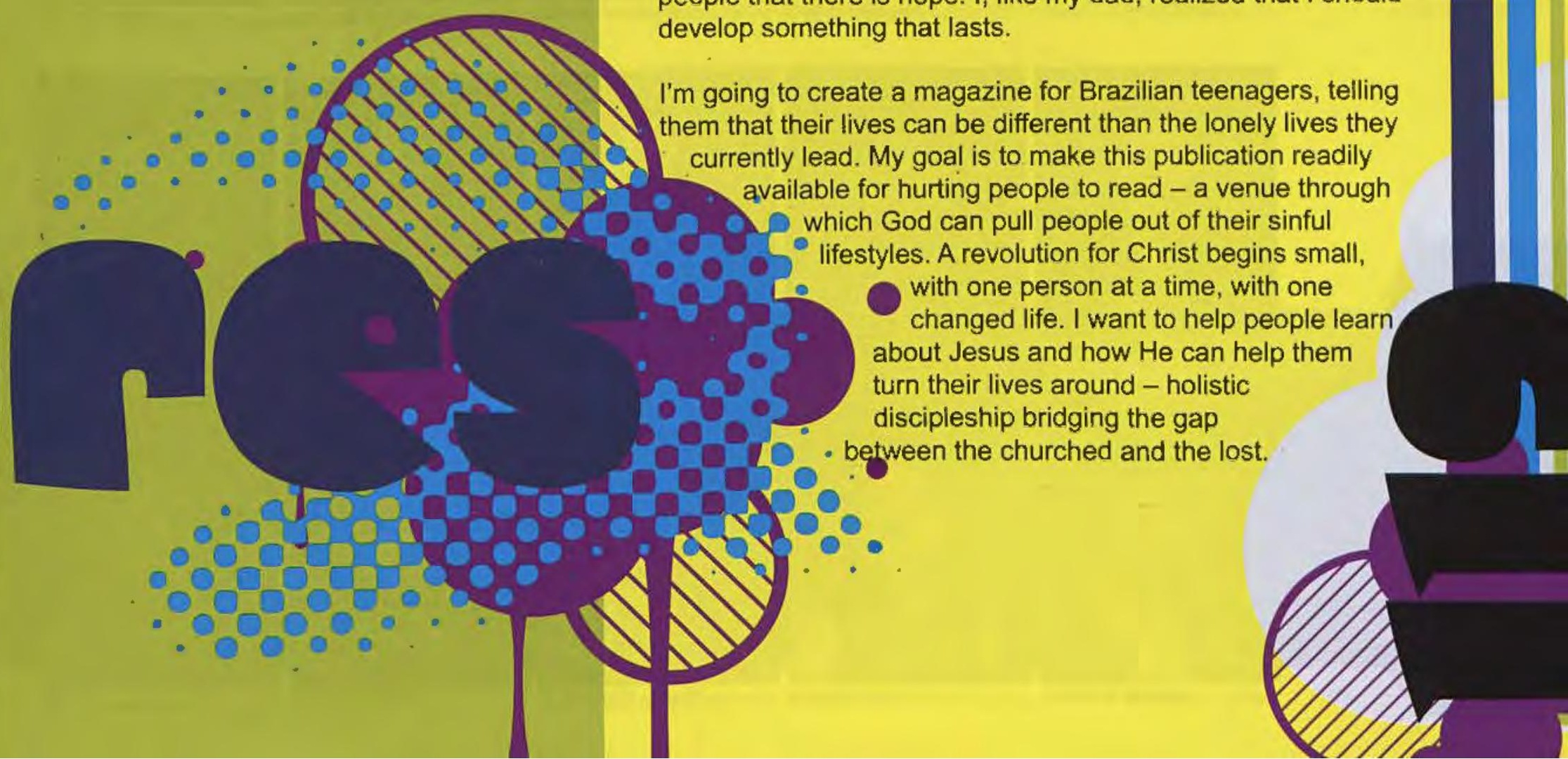




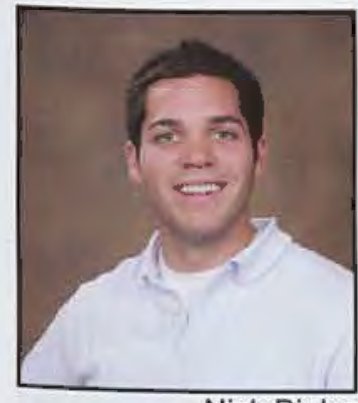

Nick Bigler

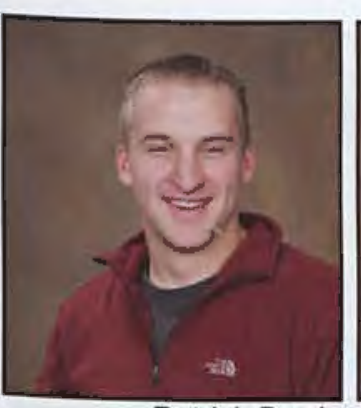

Patrick Brady

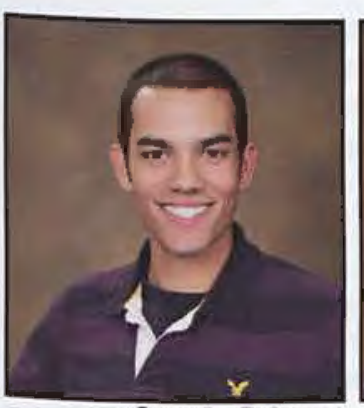

Corwin Briscoe

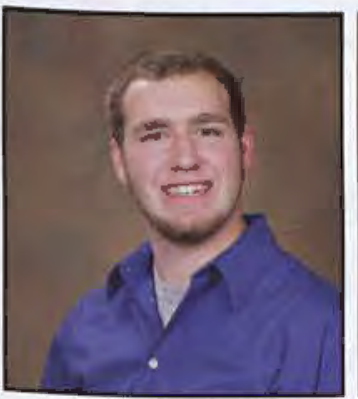

Nathaniel Brown

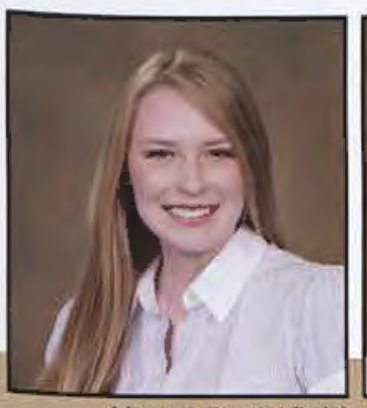

Karen Butterfield

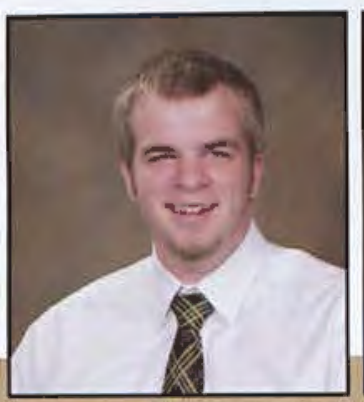

Jack Campbe

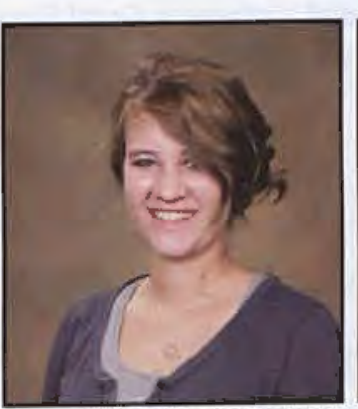

Micaela Buesgens

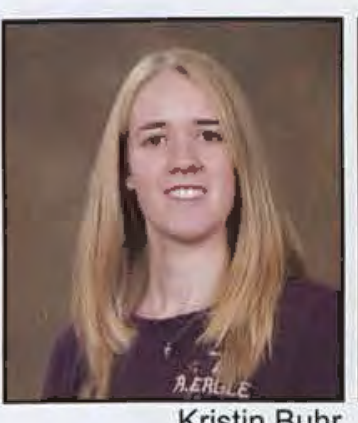

Kristin Buhr

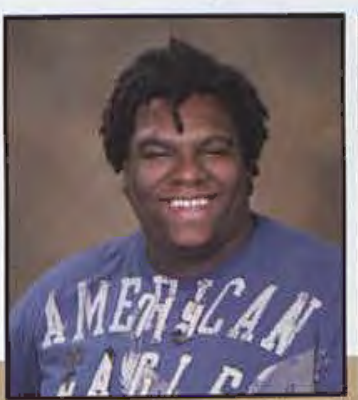

Jovaughn Carmichael

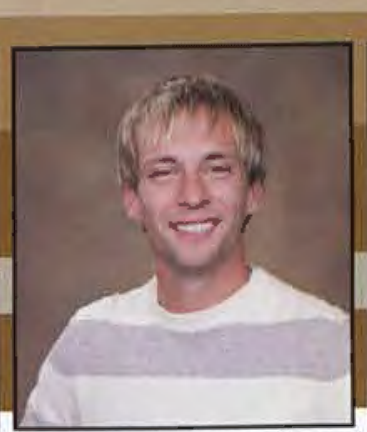

Joshua Chasteen

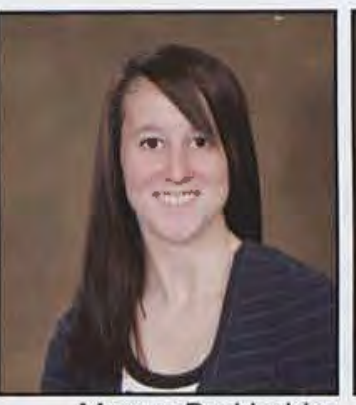

Megan Burkholder

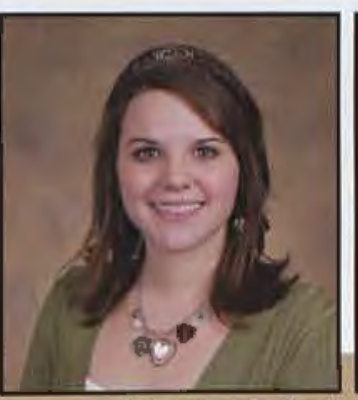

Stephanie Carr

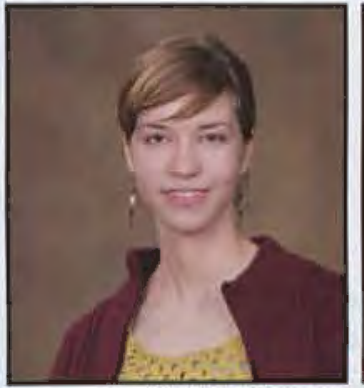

Hanna Bowes

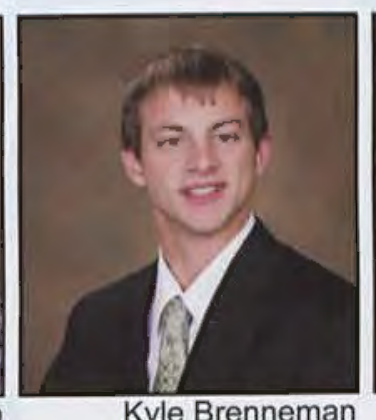

Kyle Brenneman

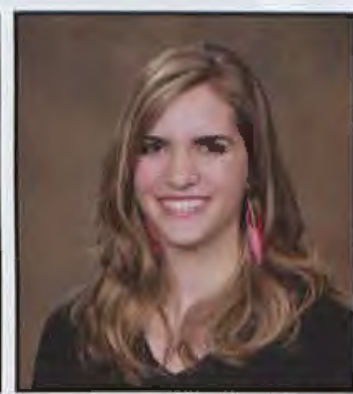

Lydia Briggs
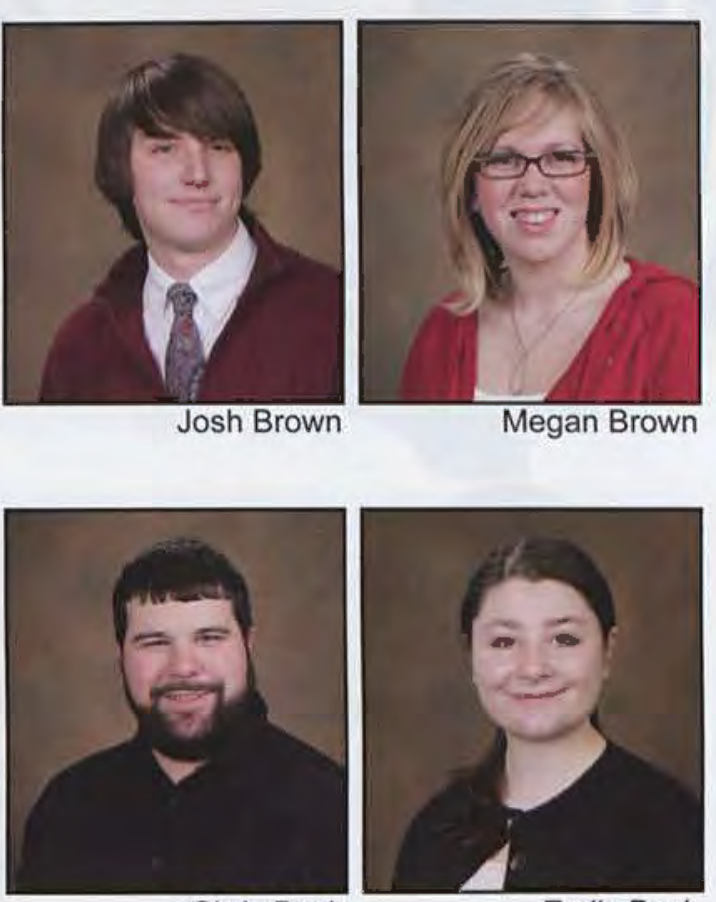

Chris Bush
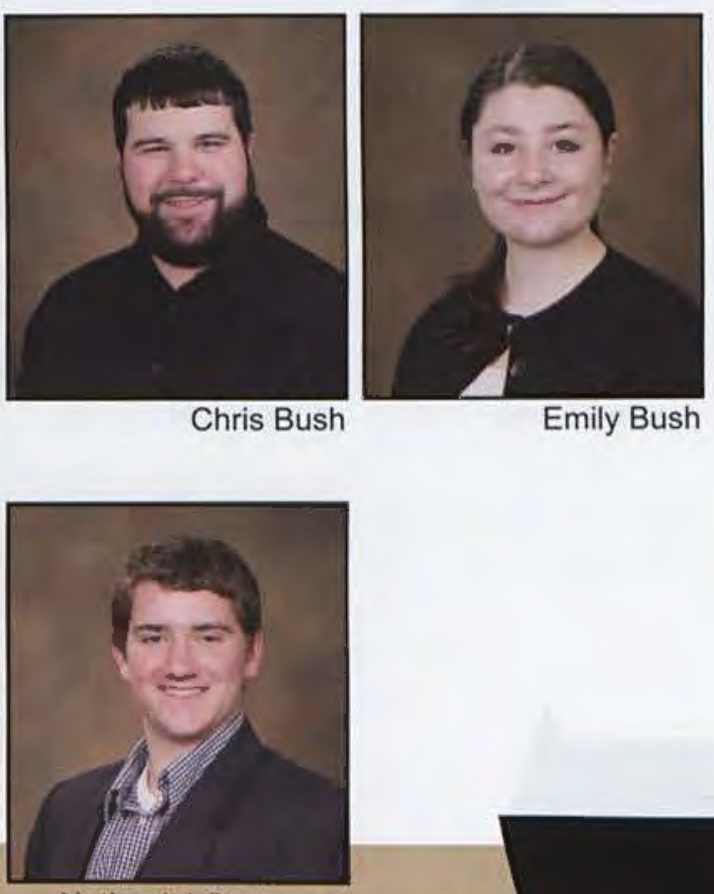

Nathaniel Casement

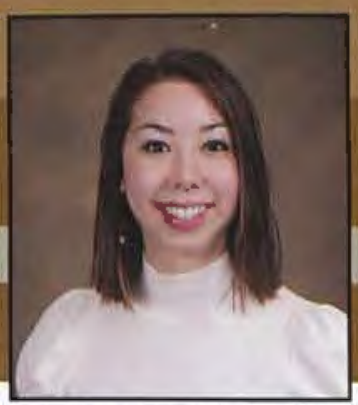

Emily Chiu

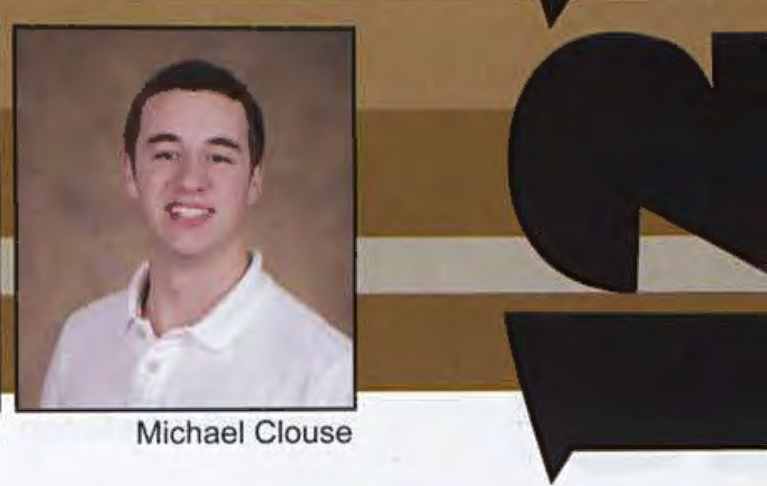





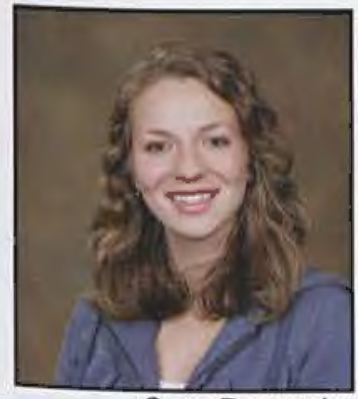

Sara Daransky

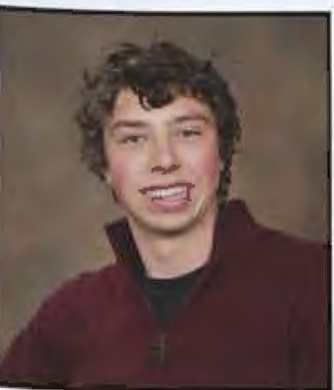

David Demeusy

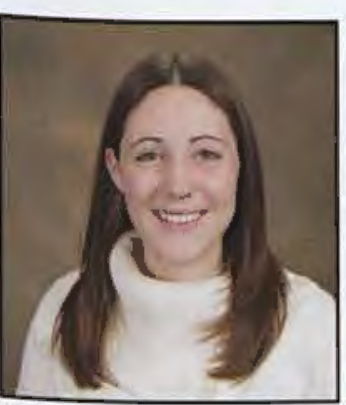

Kristina Dorbritz

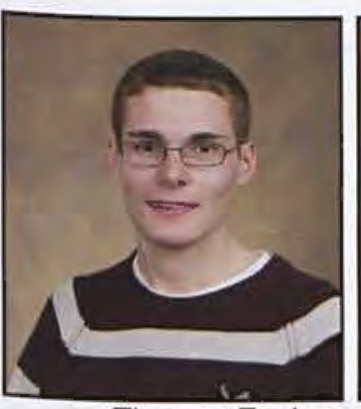

Thomas Eachus

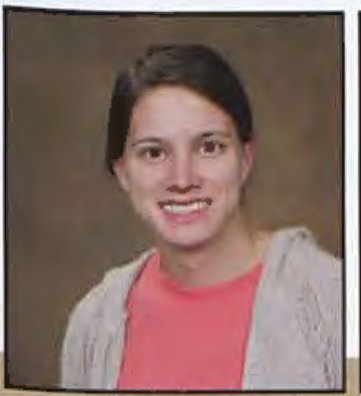

Johanna Ensslen

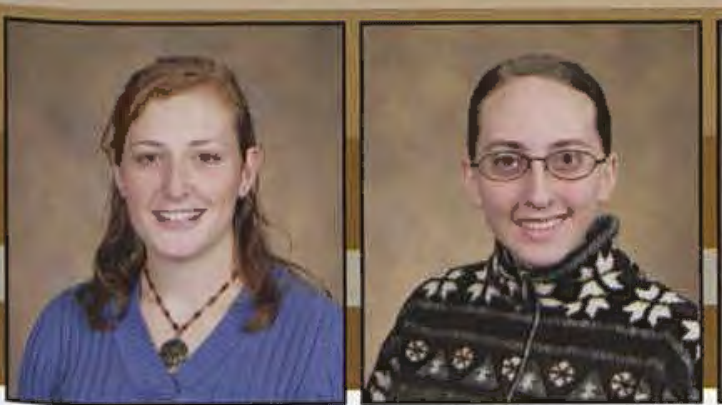

Julianne Everswick

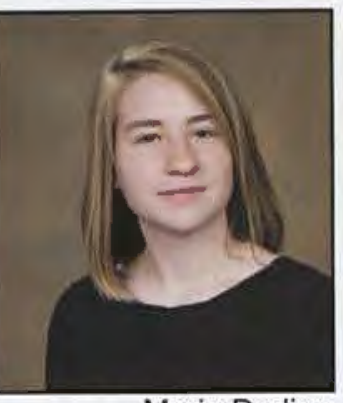

Marie Darling

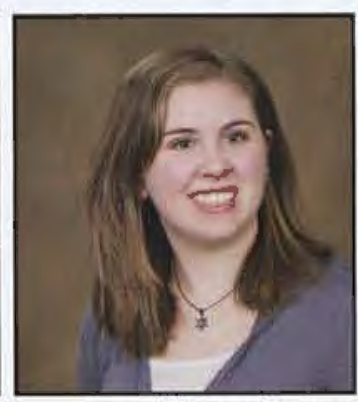

Alyssa Denen

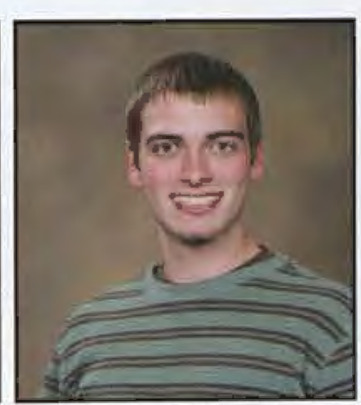

Adam Downs

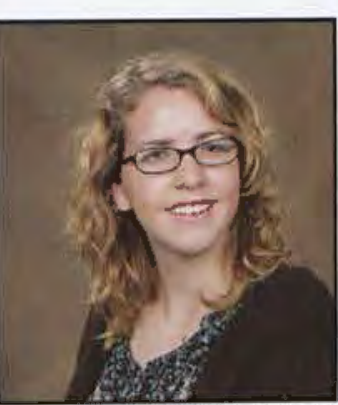

Danielle Early

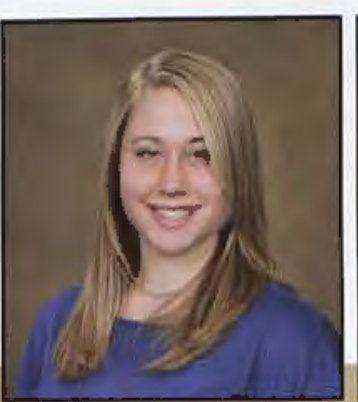

Kara Enterline

Jason Erdmann

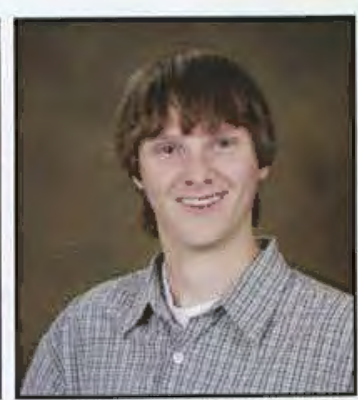

Stephen Devereaux

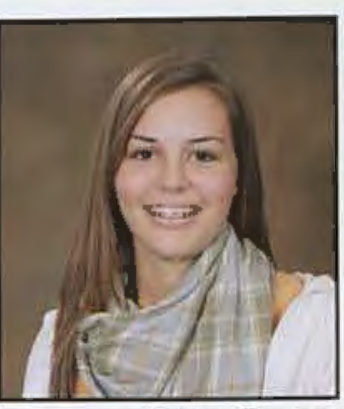

Laura Dreyer

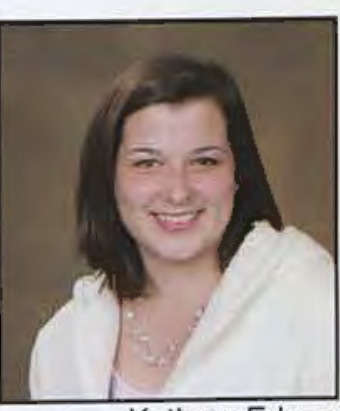

Kathryn Edens

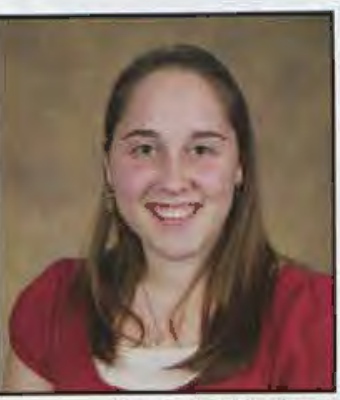

Kendra Dewese

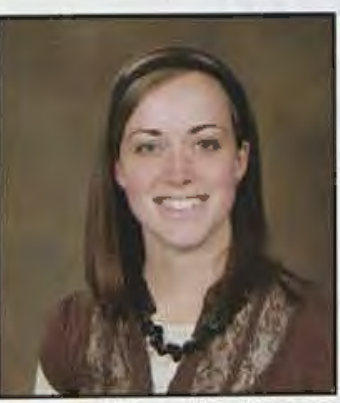

Meredith Dreyer

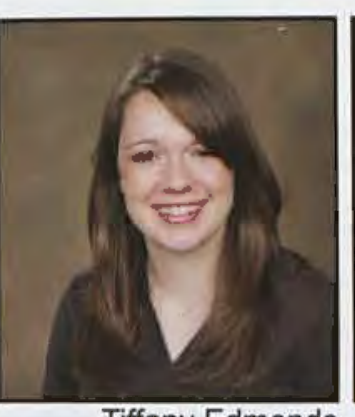

Tiffany Edmonds

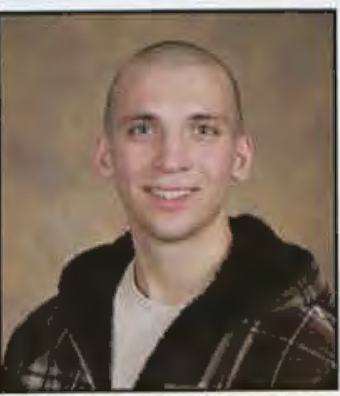

Jonathon Dias

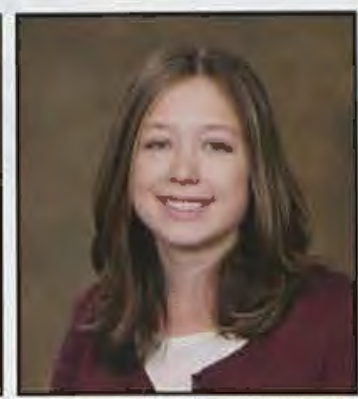

Allison Dunalp

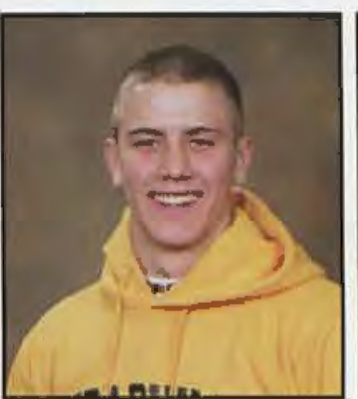

Malachi Ege

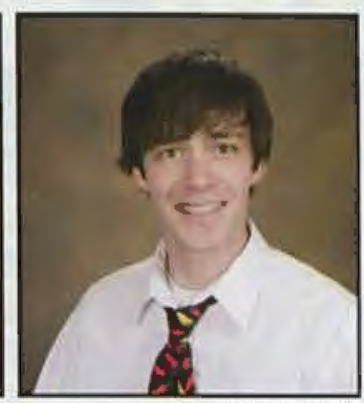

John Dunn

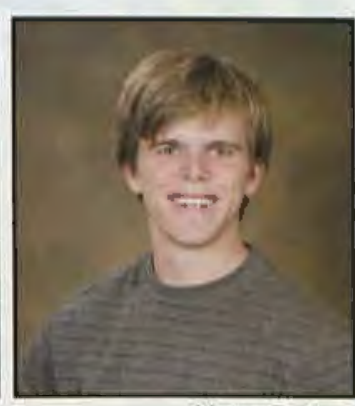

Nate Dolan

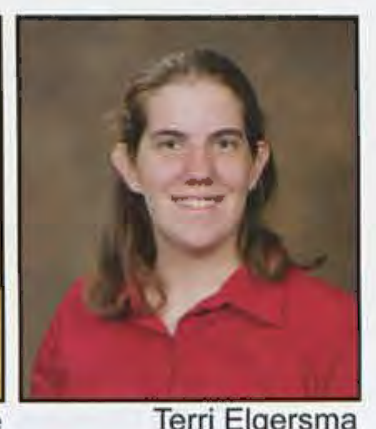

Terri Elgersma

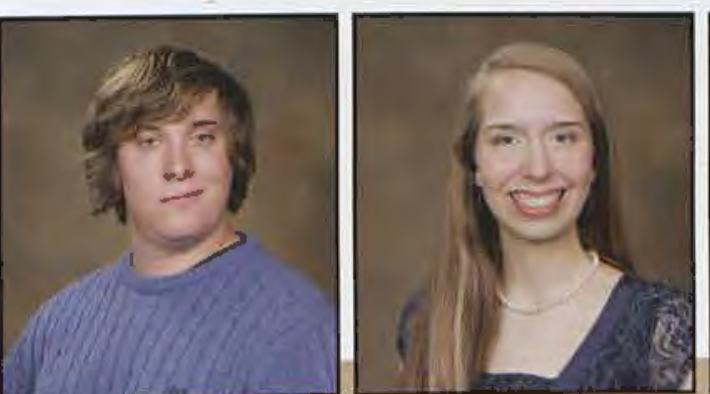

Abigail Eustace

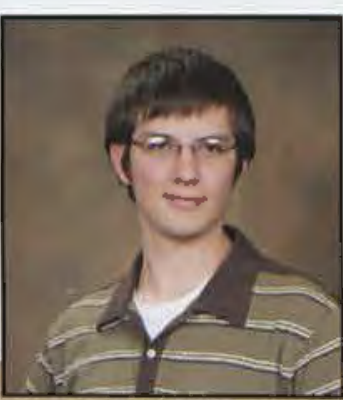

Matthew Evans 



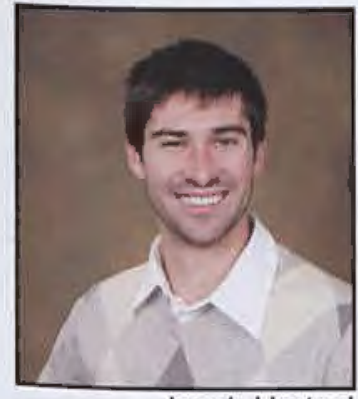

Jacob Hartsel

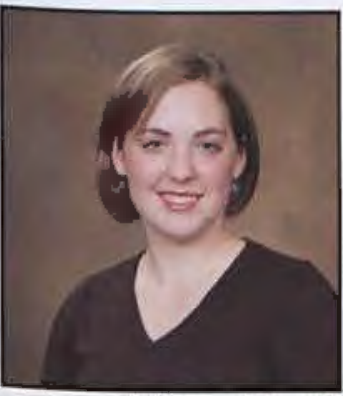

Julianne Heady

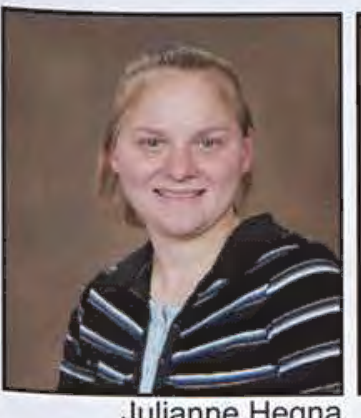

Julianne Hegna

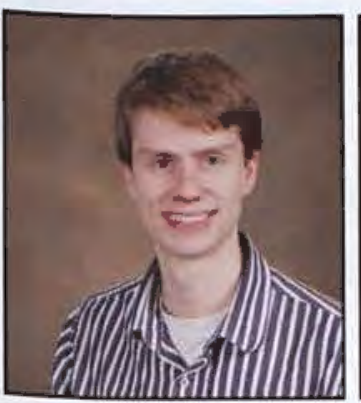

Gregory Hicks

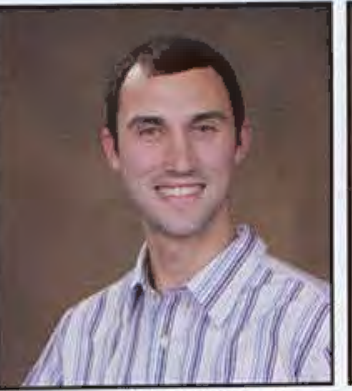

Mark Hiteshew

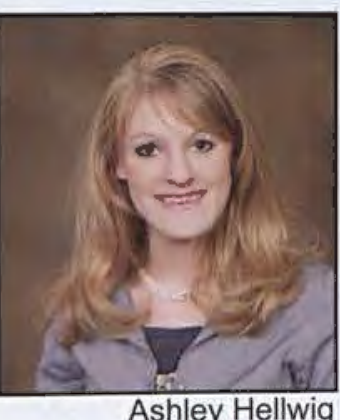

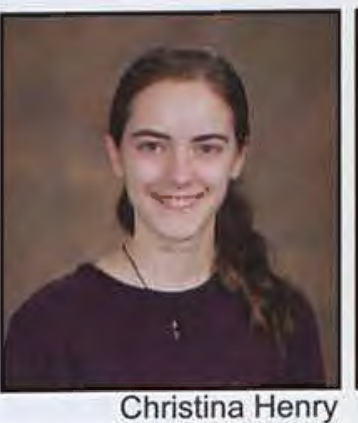
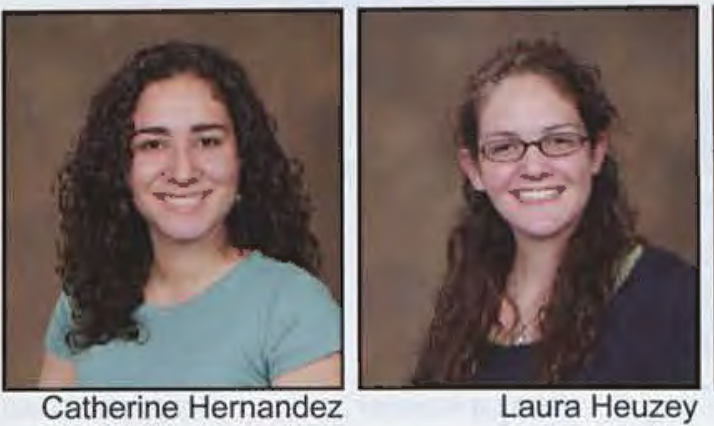

Laura Heuzey

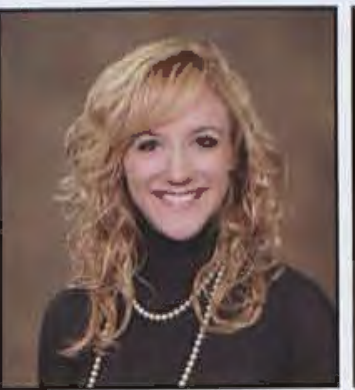

Chloe Heckman

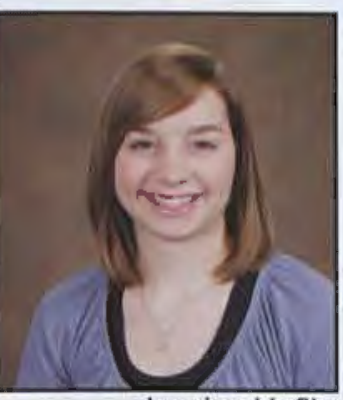

Jessica Heflin
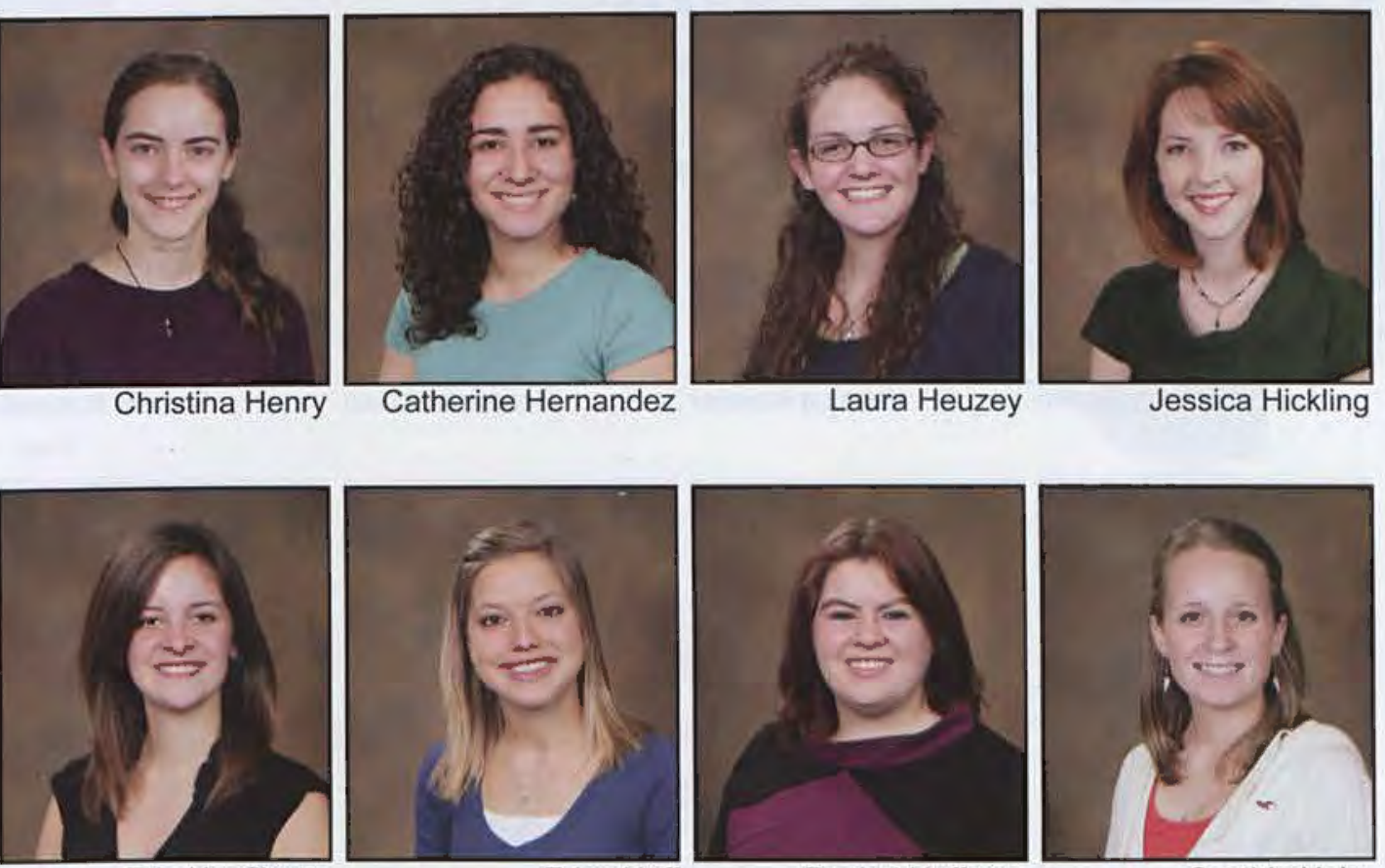

Erin Hoeflinger

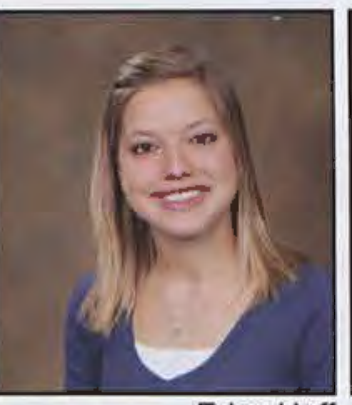

Erica Hoff

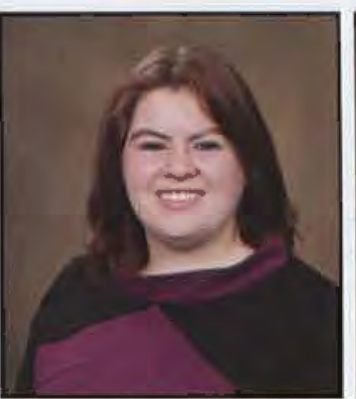

Rachel Hoffman

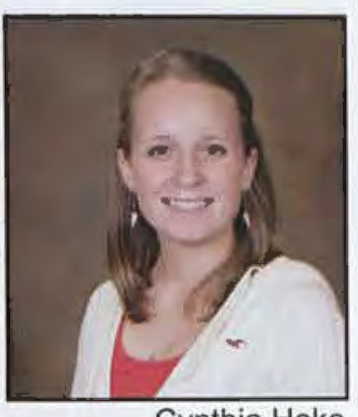

Cynthia Hoke

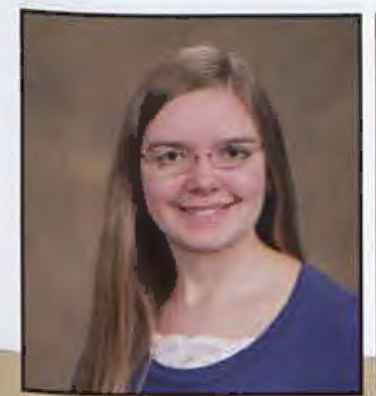

Evonne Hoogland

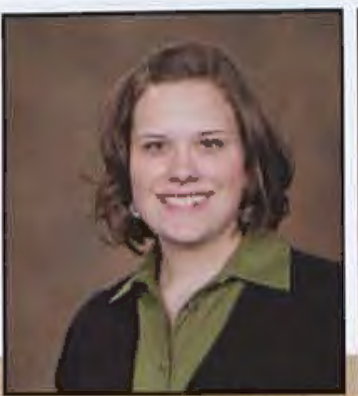

Jenny Howel

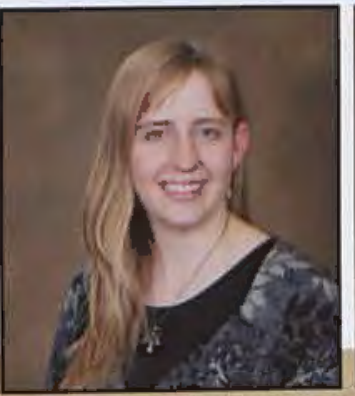

Eliane Hoyt

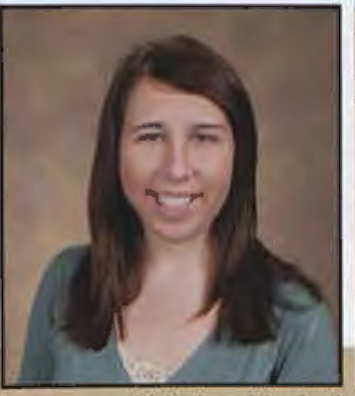

Kristina Hrabal

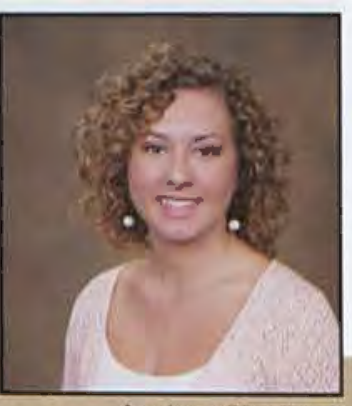

Audrey Huebert

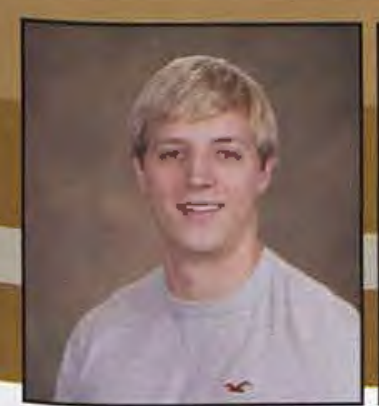

Samuel Huebner

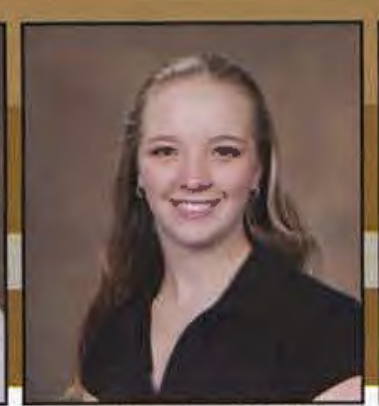

Heather Huffaker
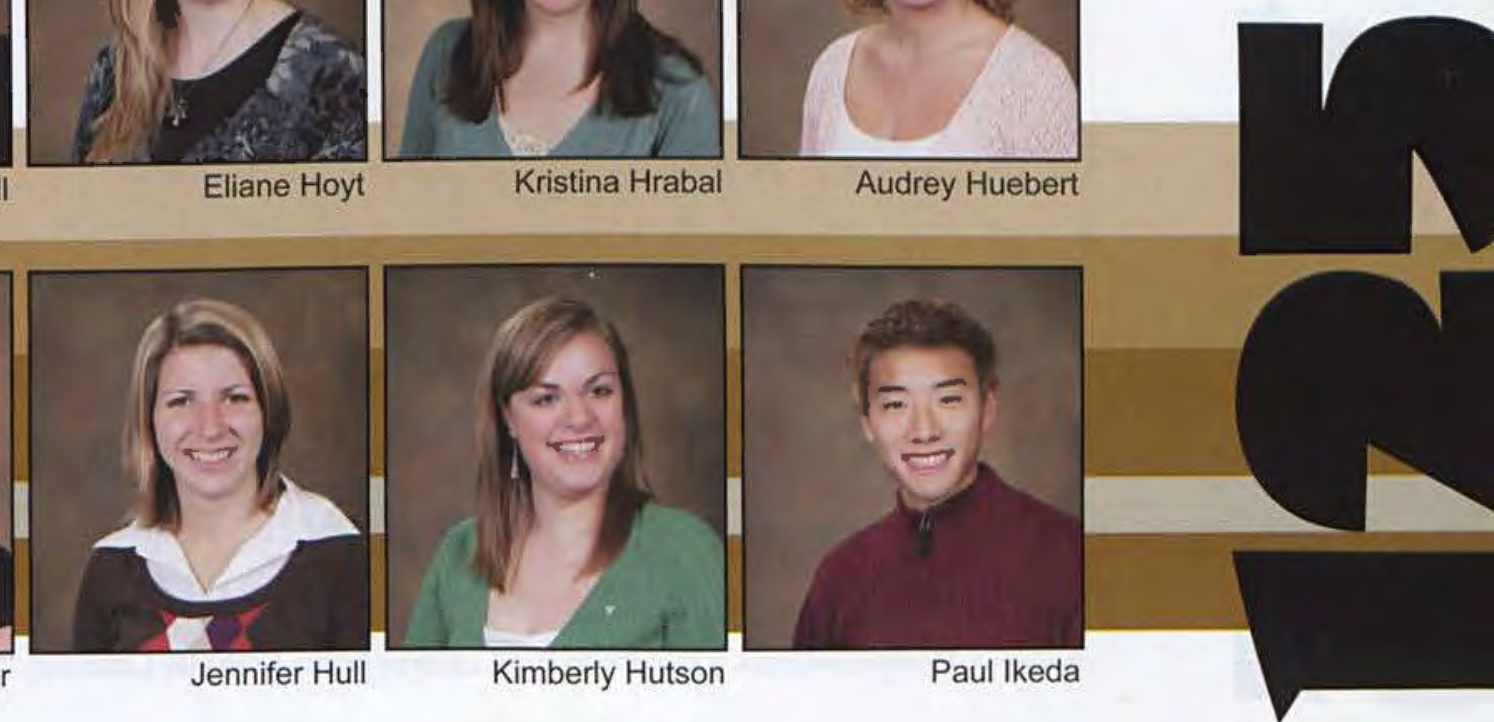


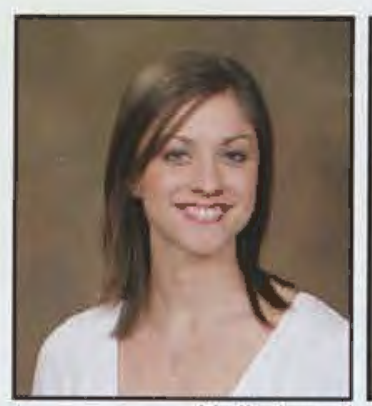

Holly Israel

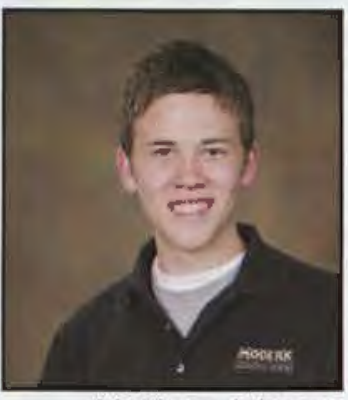

Matthew Johnson

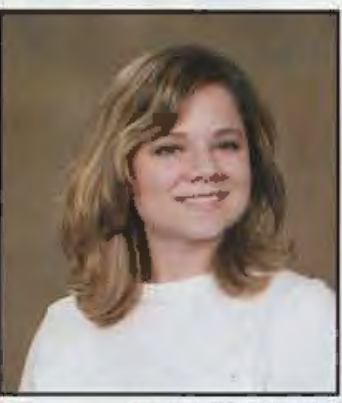

Lindsey Kepiro

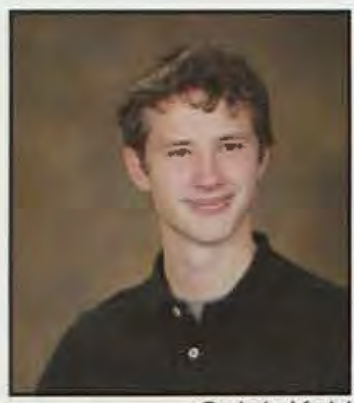

Caleb Kahl

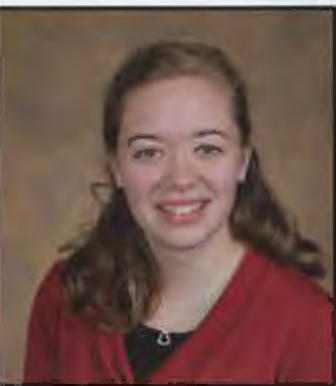

Hannah Jones

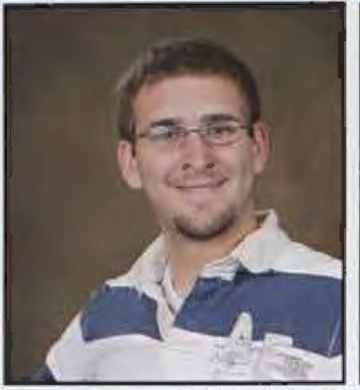

Jesse Jones

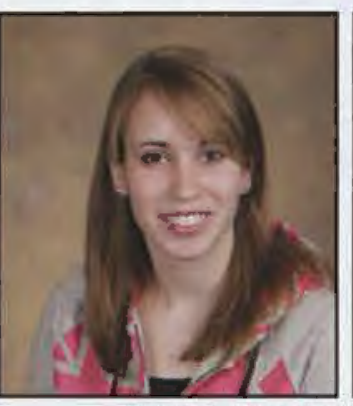

Jessica Kessler

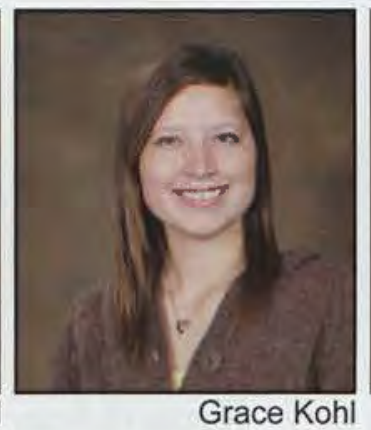

Grace Kohl

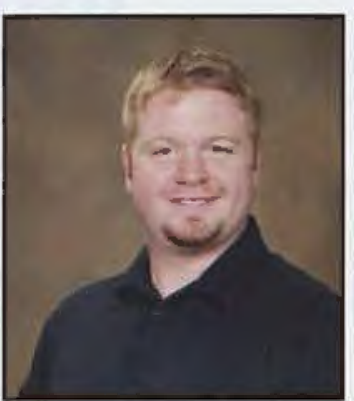

Benjamin Kilian

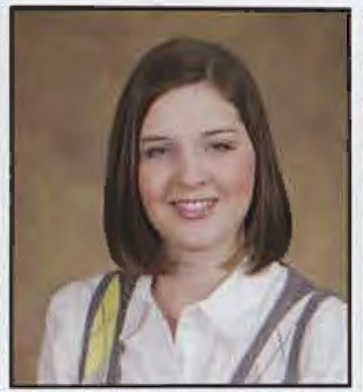

Hannah Juhant

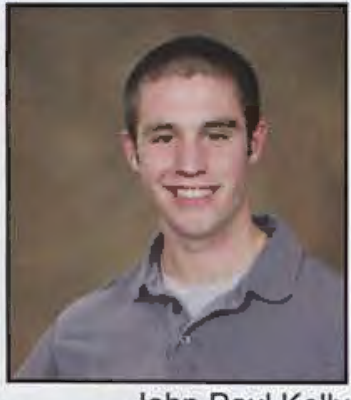

John Paul Kelly

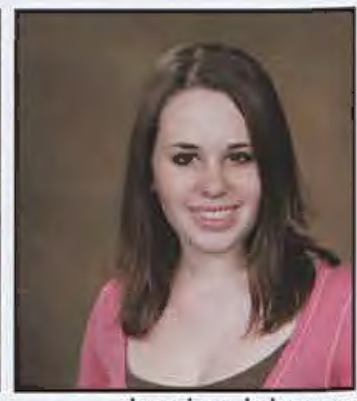

Jessica Johnson

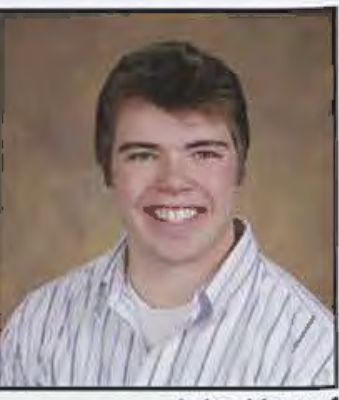

Jake Kempf

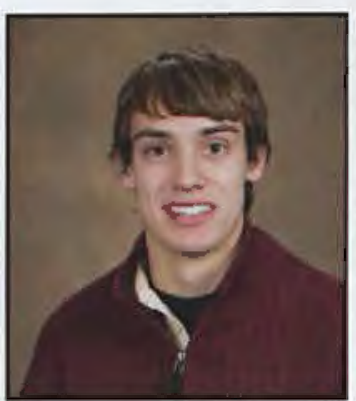

Andrew Knosnik

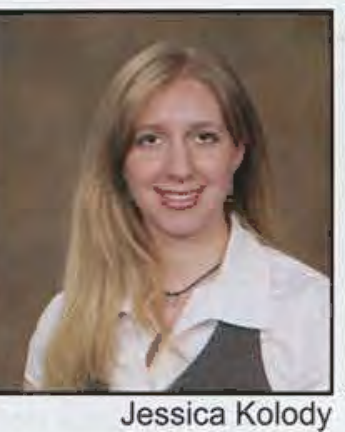

Jessica Kolody

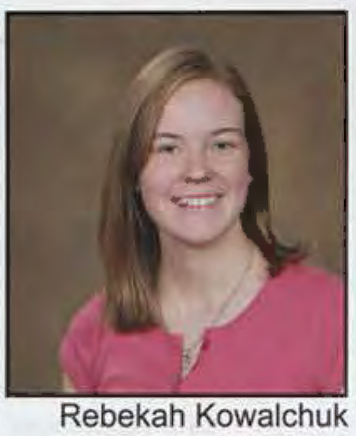

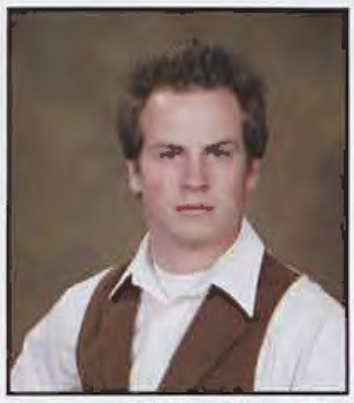

Jordan Knoedler

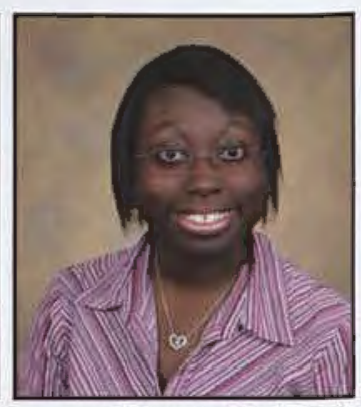

Sharon Korbinah

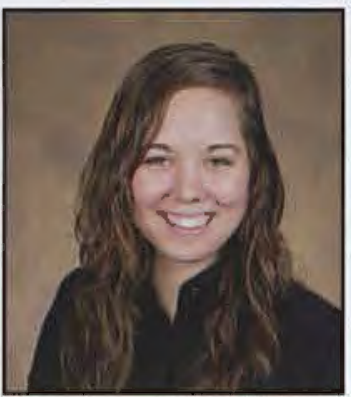

Laura Kramer

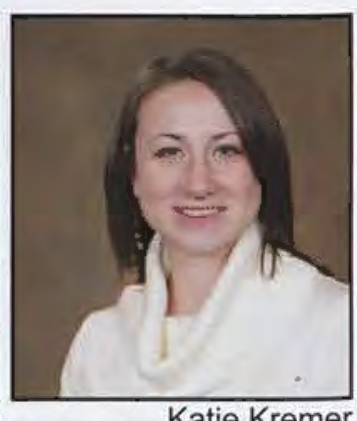

Katie Kremer

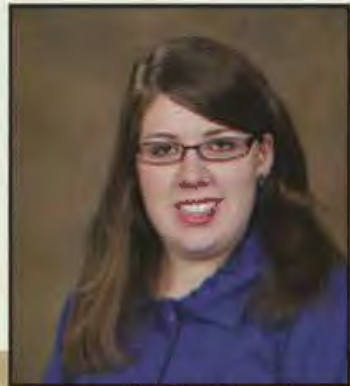

Krista Kroninger
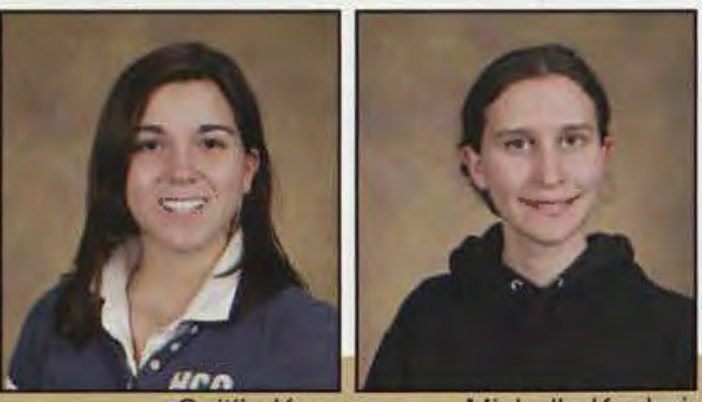

Michelle Kushnir

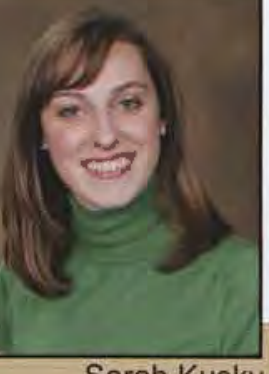

Sarah Kusky

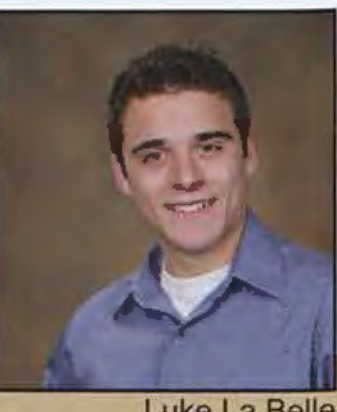

Luke La Belle
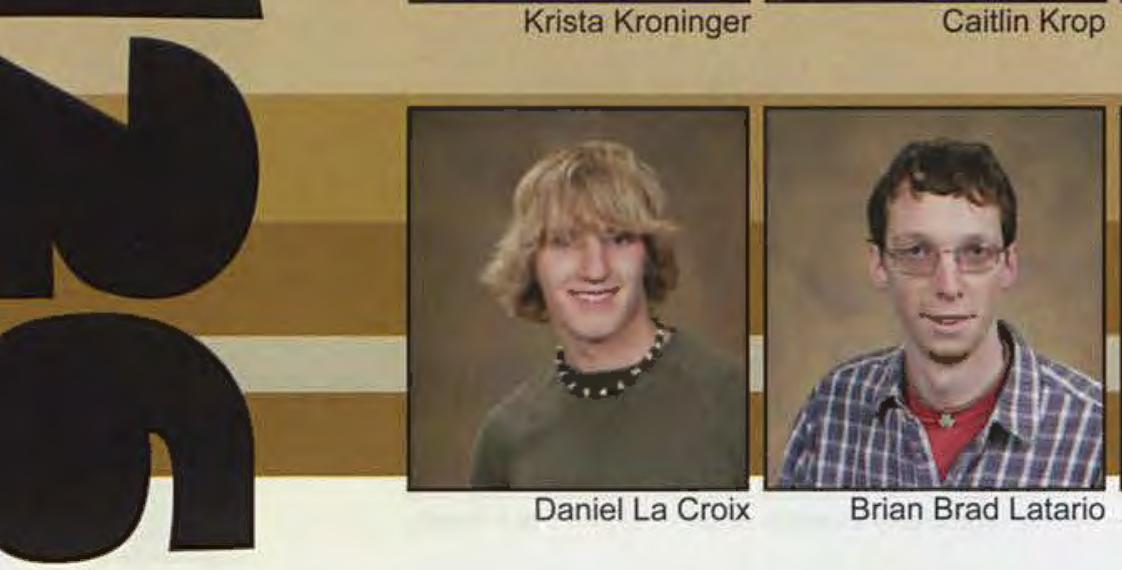

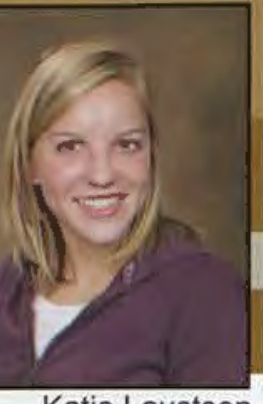

Katie Laustsen

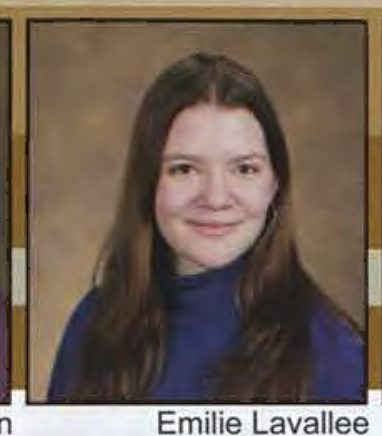

Emilie Lavallee

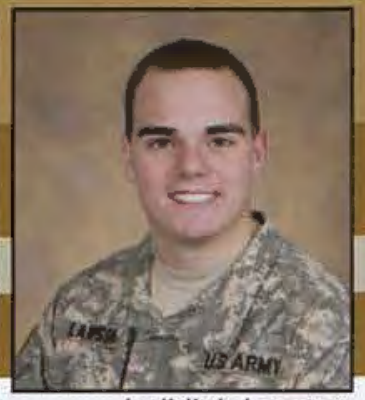

Jedidiah Lawson 

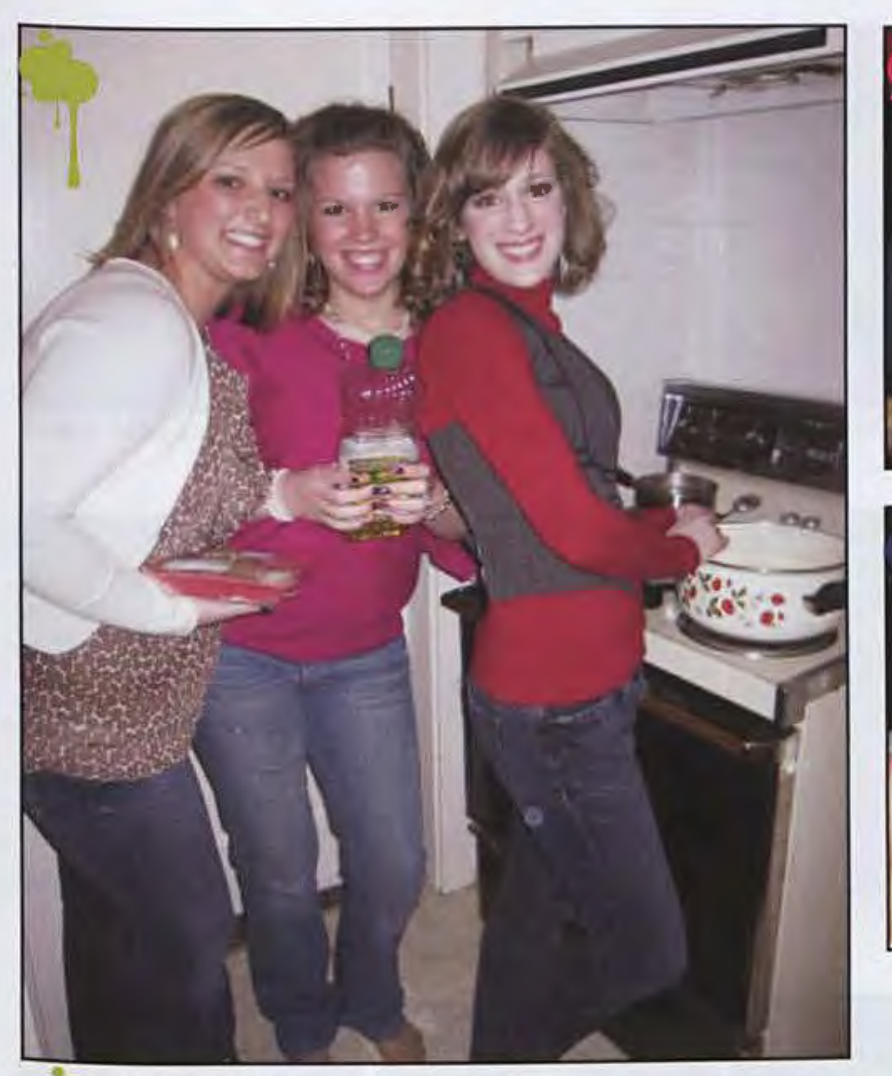

Rachael Wasem, Mary Lewis, and Ali Blatherwick spend some time in the kitchen cooking Valentines dinner,

in Tim Lukasiewicz and Nathan Flack share a tasty sundae.

3

Ah, how cute.

Bethany Wailes and Abby Eisenlohr tell, "We were

.1 tough skater chicks...had tats, longboards, and

serious attitudes!"
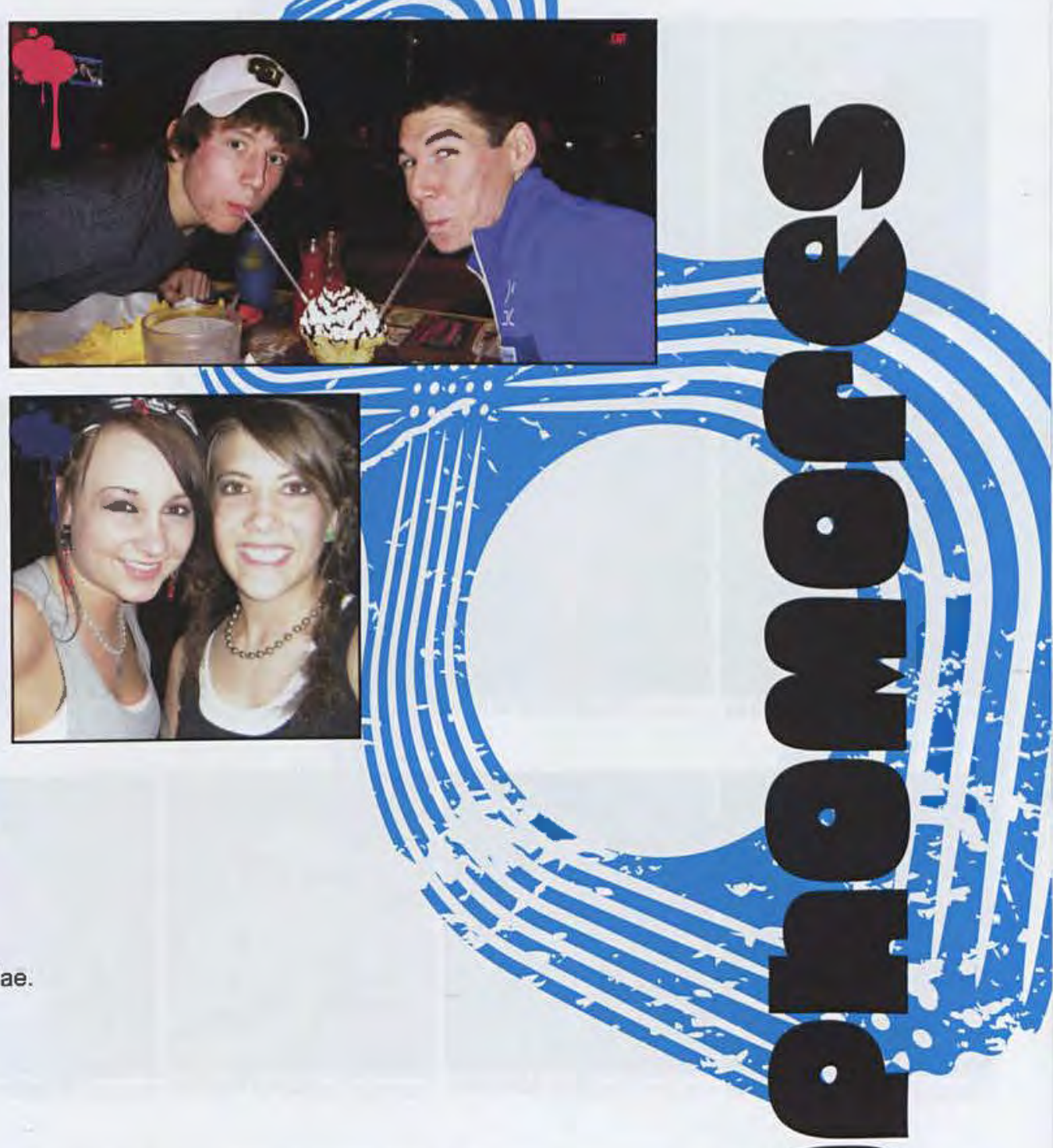

"Take freshman year with all of its awkwardness, add amazing friends, constant laughter, and double the workload, and, voila,

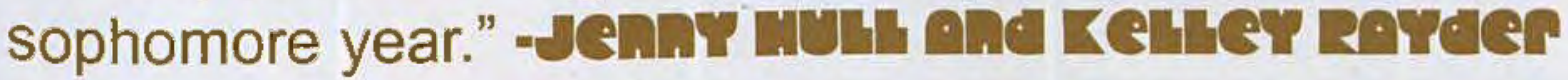

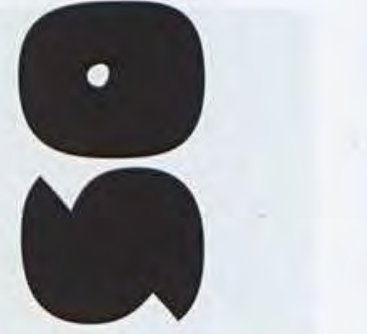

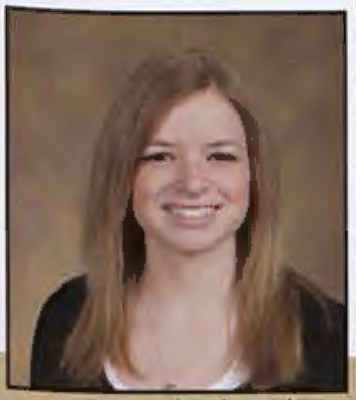

Larissa Lear

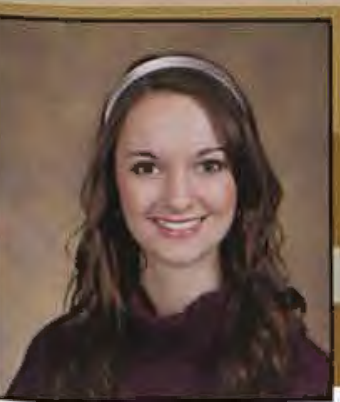

Heidi Lockridge

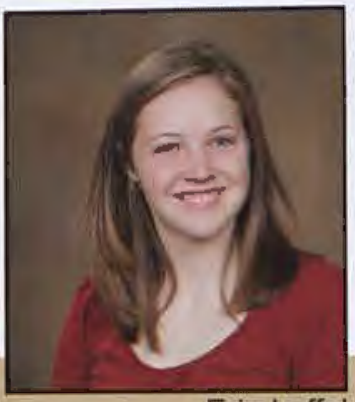

Erin Leffel

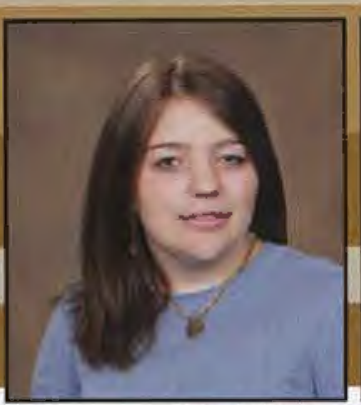

Sarah Lowry

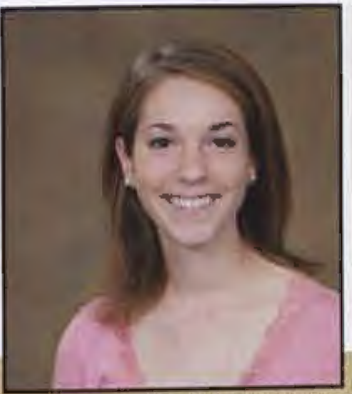

Gail Lehmann

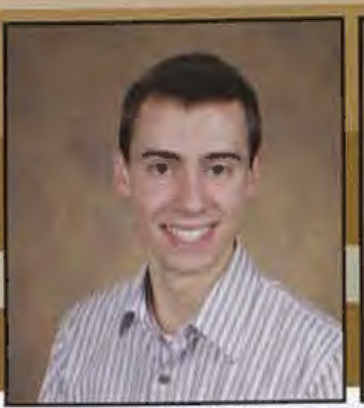

Daniel Lyman
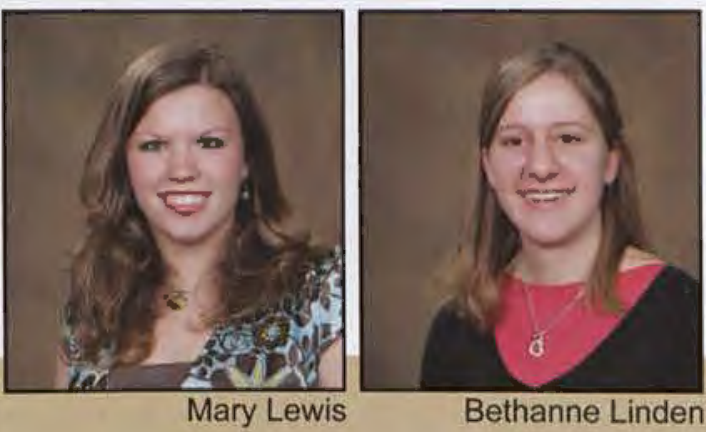

Bethanne Linden

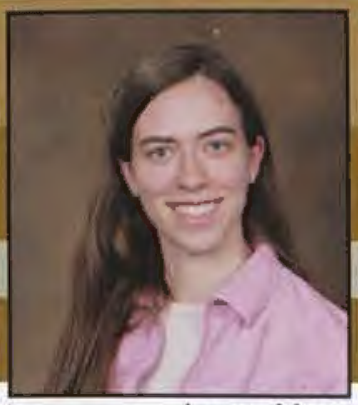

Laura Mace

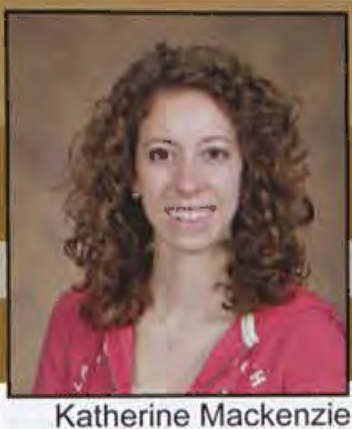

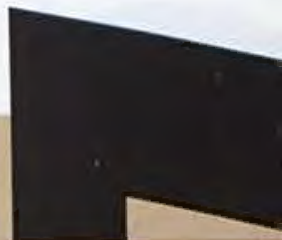
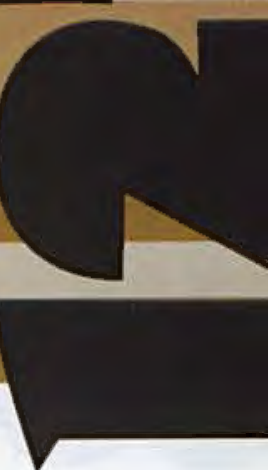


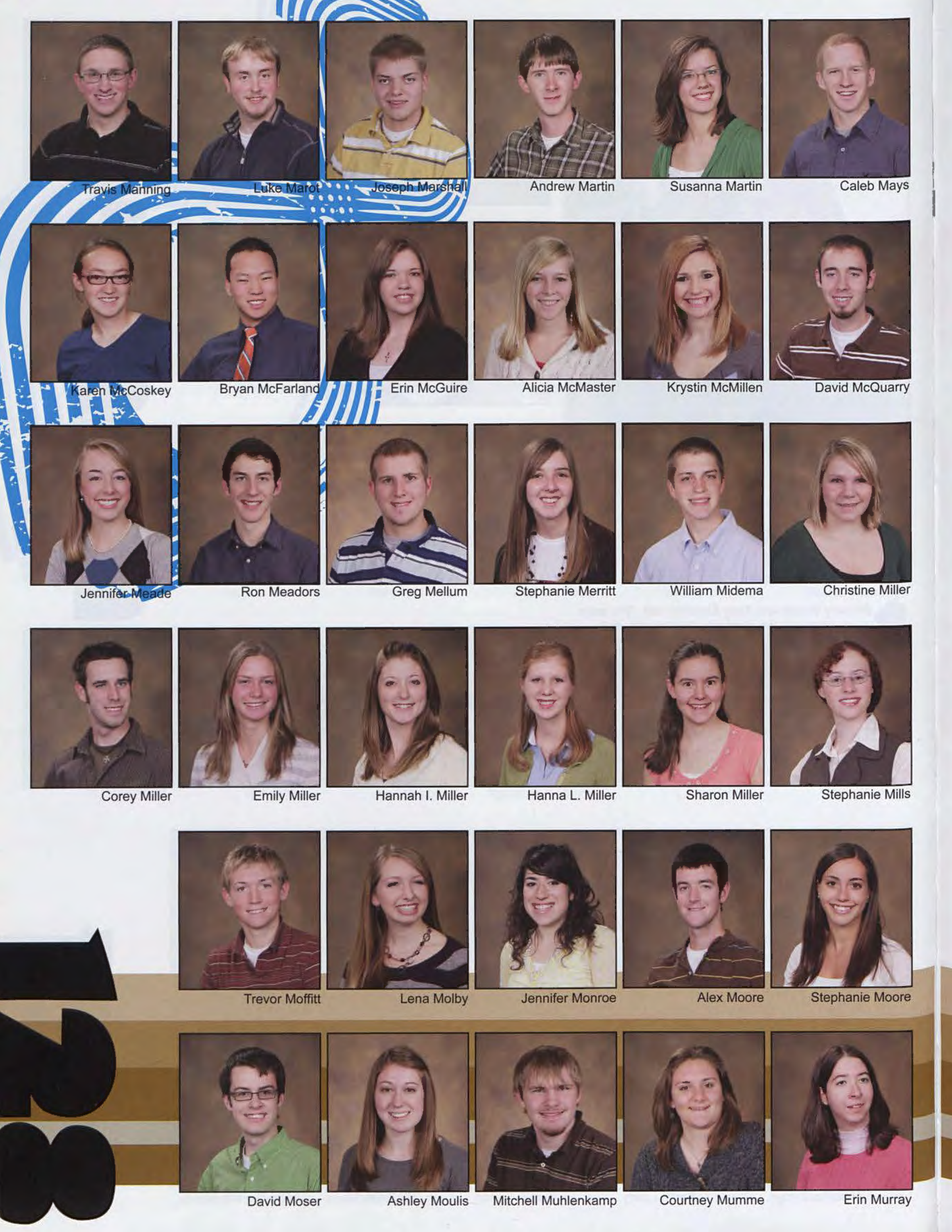




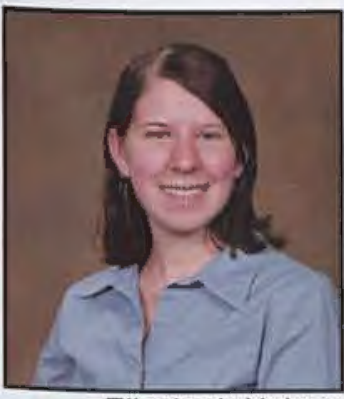

Elizabeth Nelson

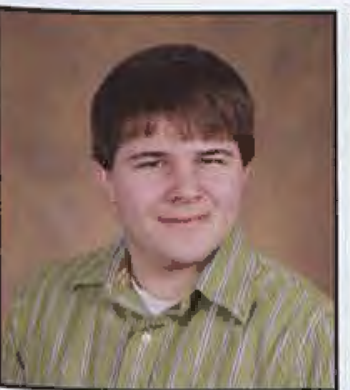

Thomas O'Connor

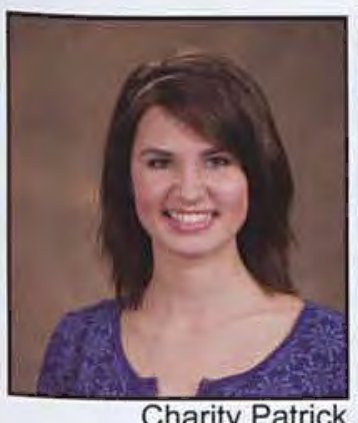

Charity Patrick

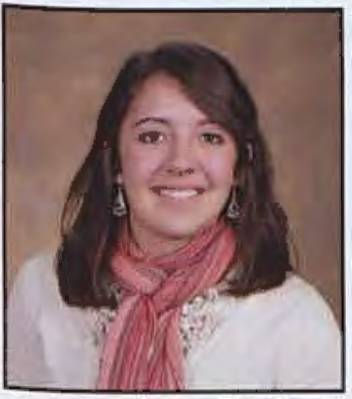

Anna Plasterer

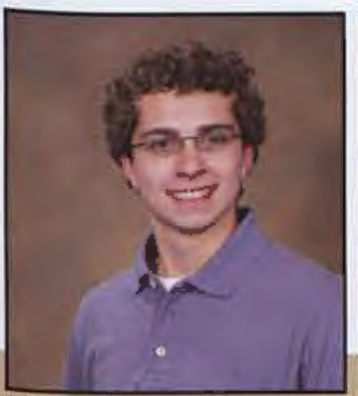

Stephen Pupillo

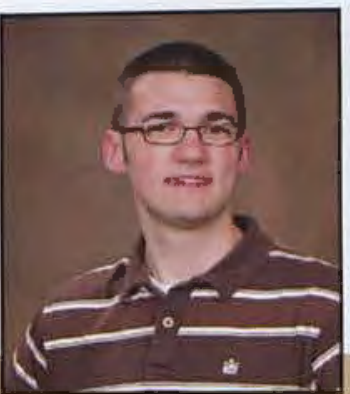

Adam Putinski

Adam Putinski
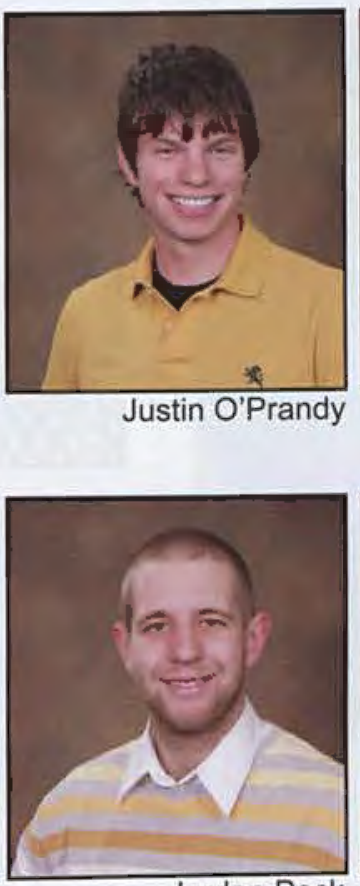

Jordan Peck

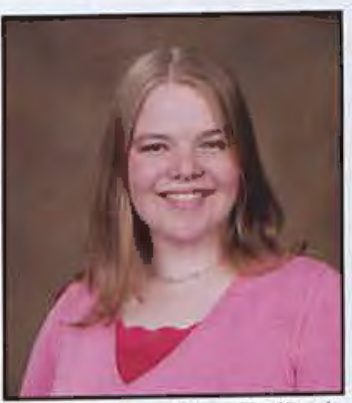

Lisa Pollock

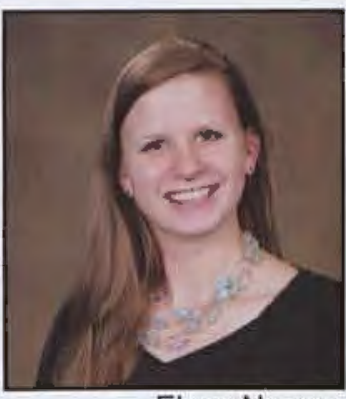

Elyse Nemec

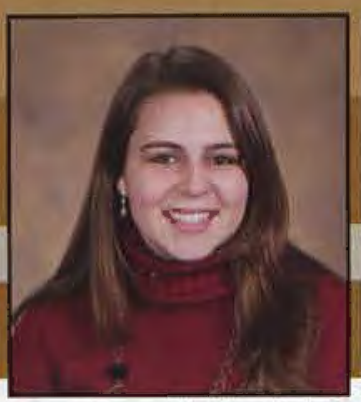

Taylor Ralph

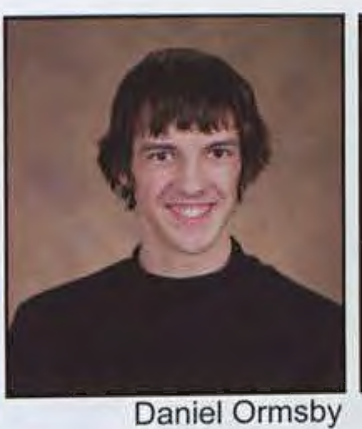

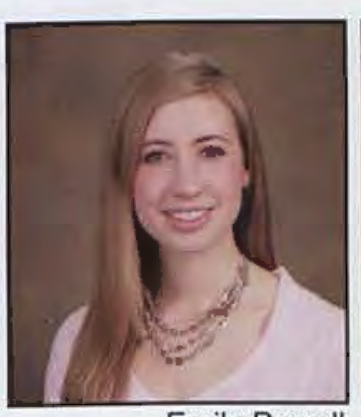

Emily Powell

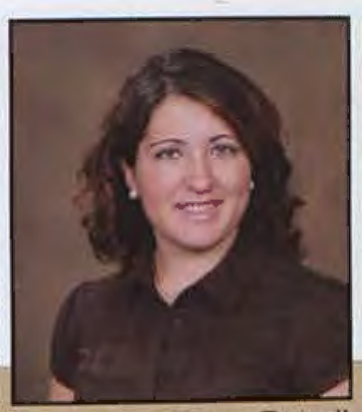

Tracy Putrel

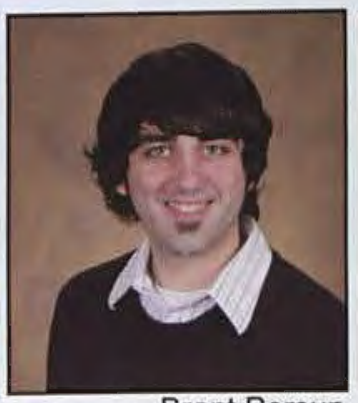

Brent Persun

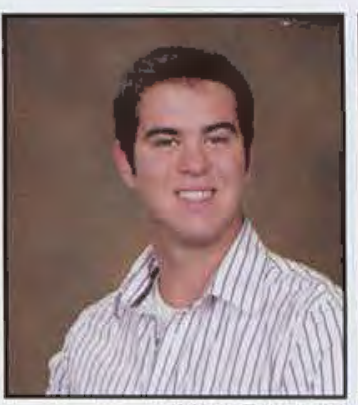

Alex Pressly

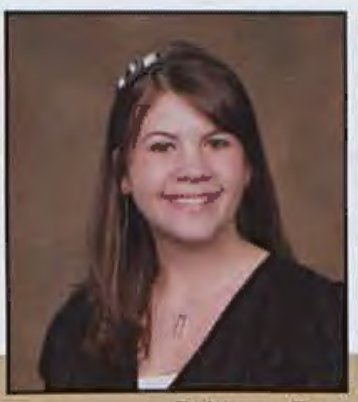

Brittany Putt

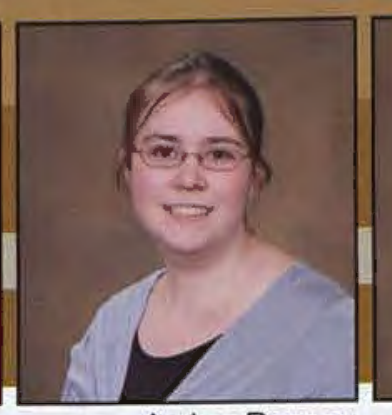

Amber Ranney
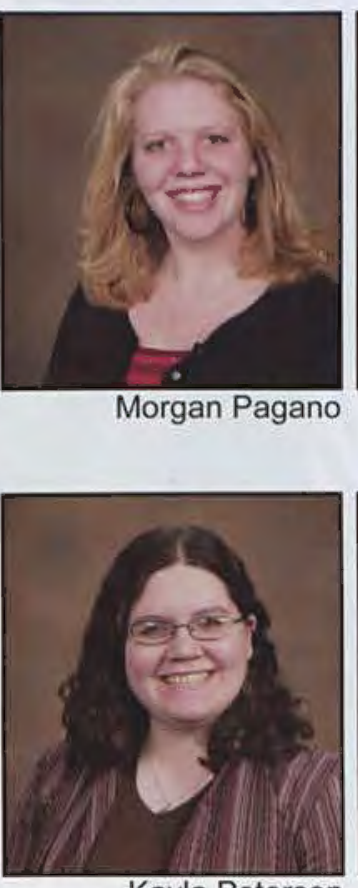

Kayla Peterson

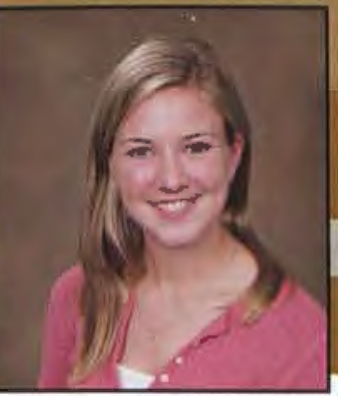

Jessica Rarick

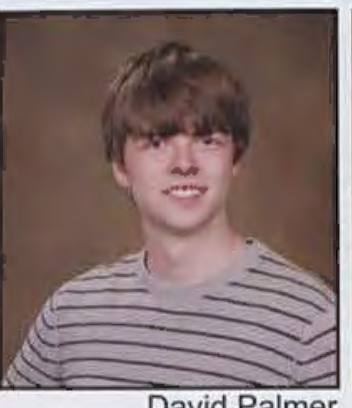

David Palmer

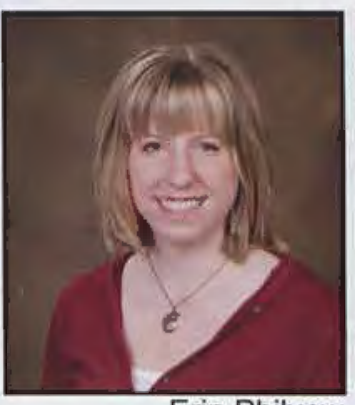

Erin Philyaw
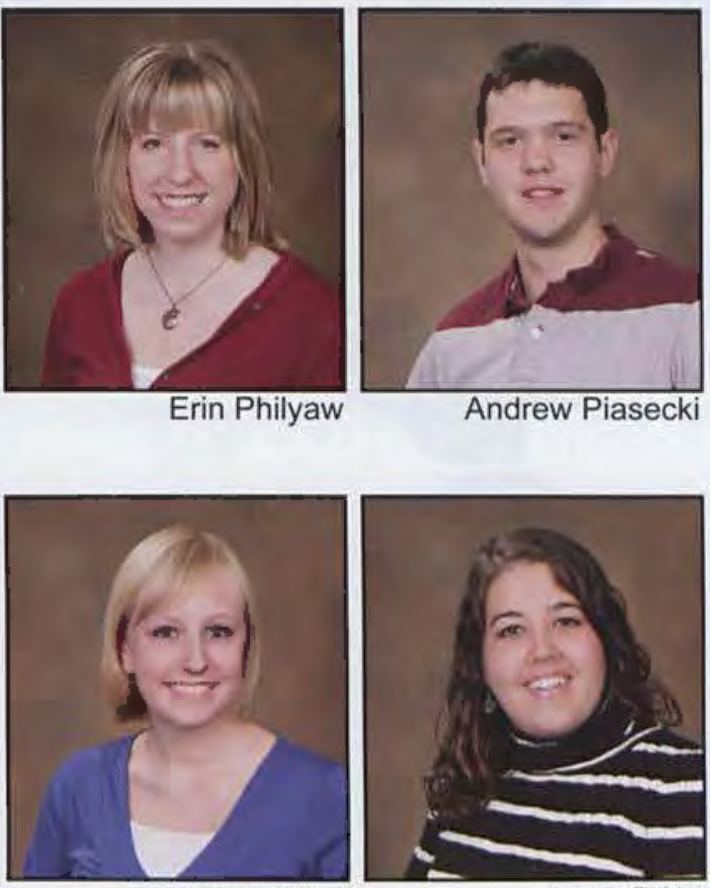

Krista Price

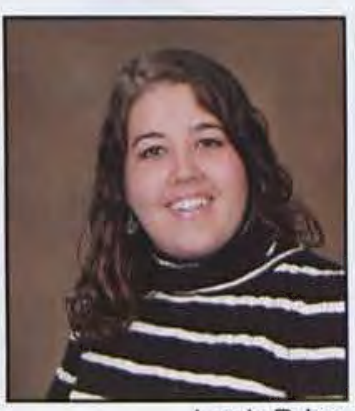

Leah Price

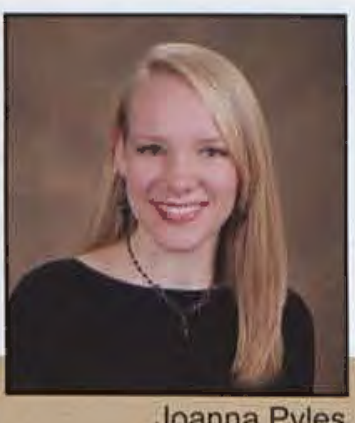

Joanna Pyles
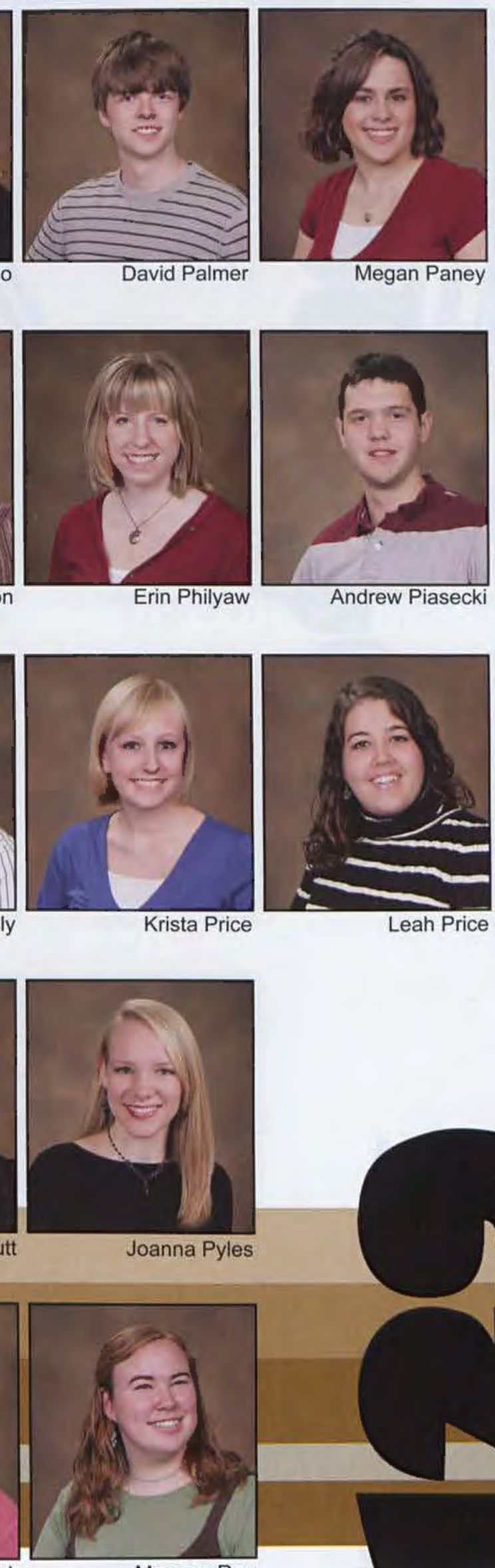

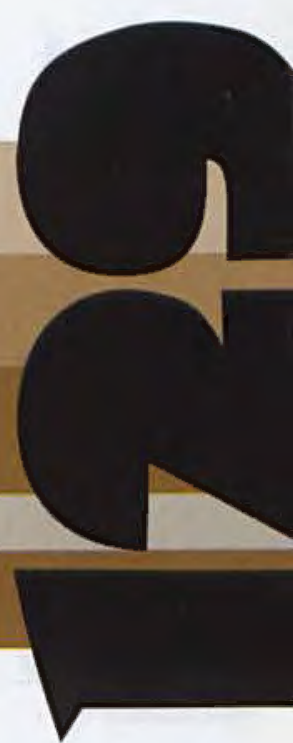





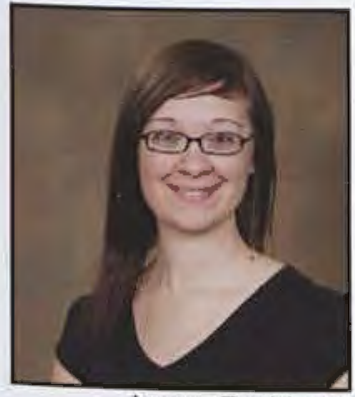

Jenna Rothhaar

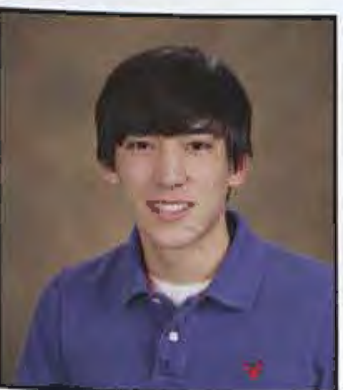

Josh Schindehette
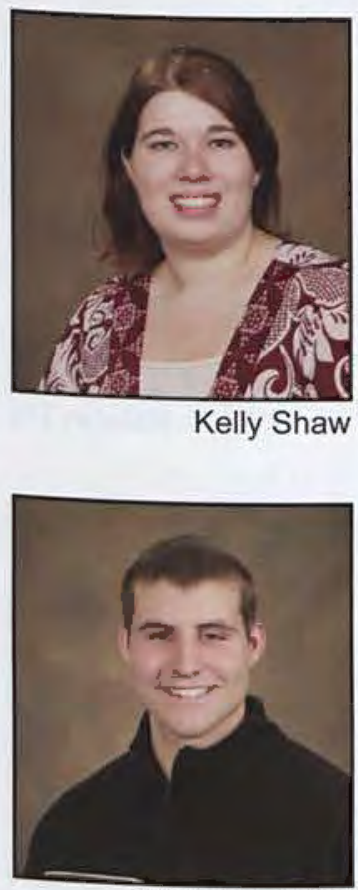

Nathan Sioquist

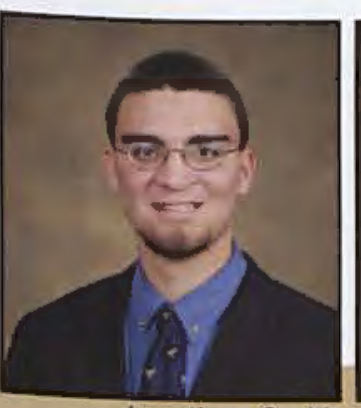

Jonathan Smith

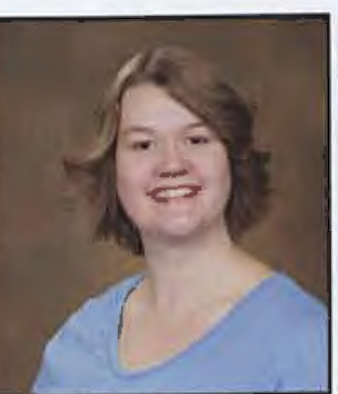

Stacie Schmidt

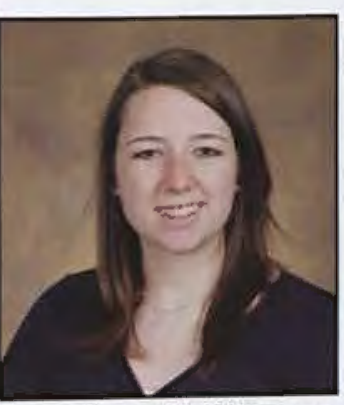

Amanda Sherman

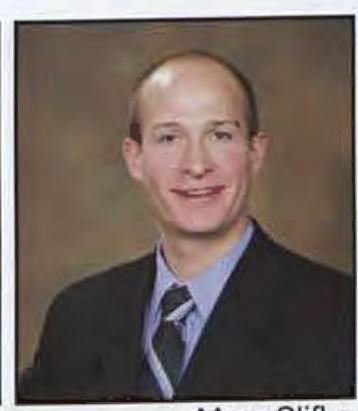

Mary Slifka

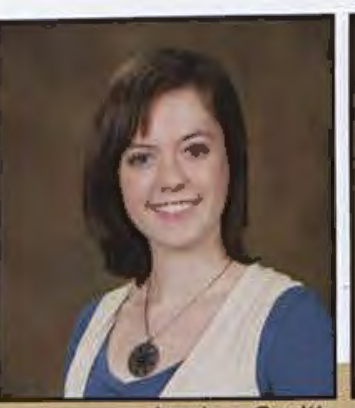

Jordan Smith

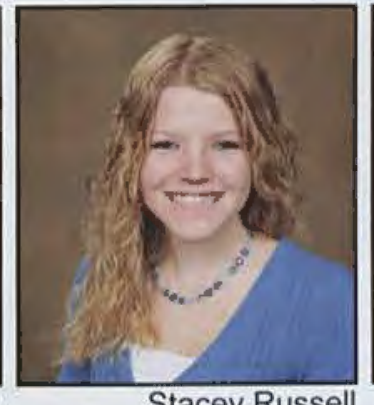

Stacey Russell

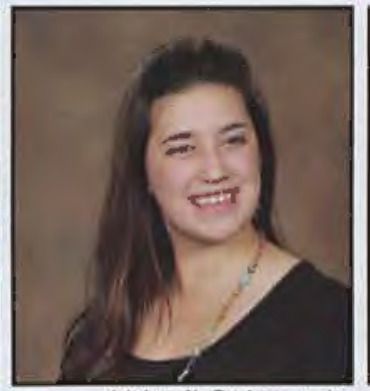

Abigail Salaverria

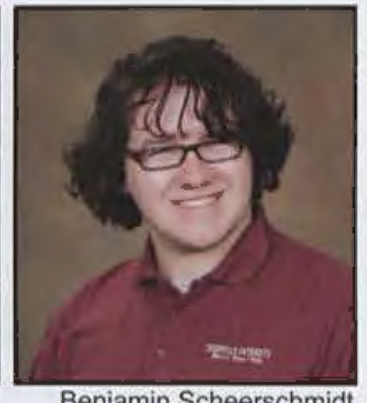

Benjamin Scheerschmidt
Christina Self
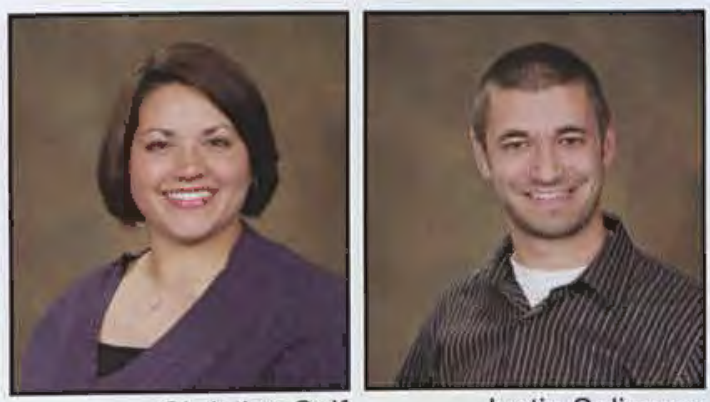

Justin Seligman
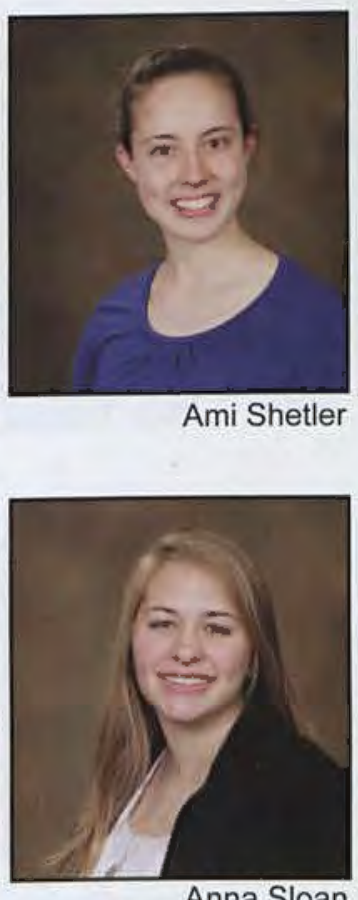

Anna Sloan

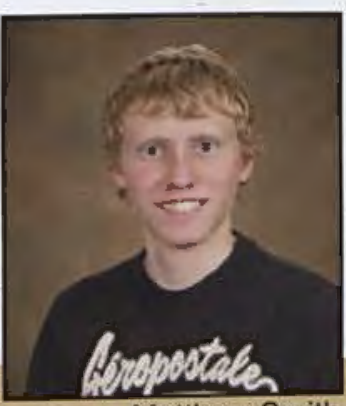

Matthew Smith

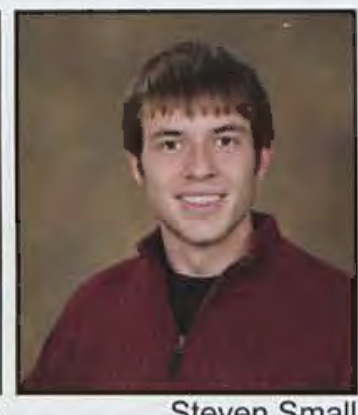

Steven Small

Rebecca Smith

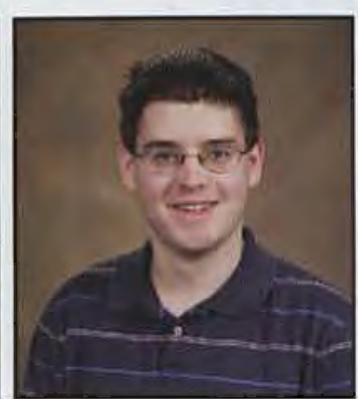

Peter Shimeall

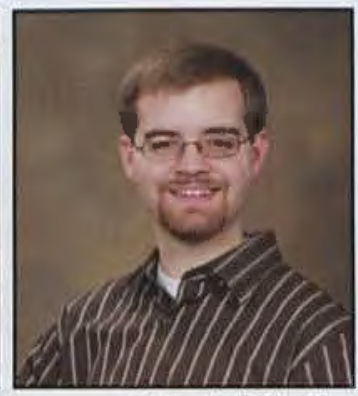

Daniel Shaffer

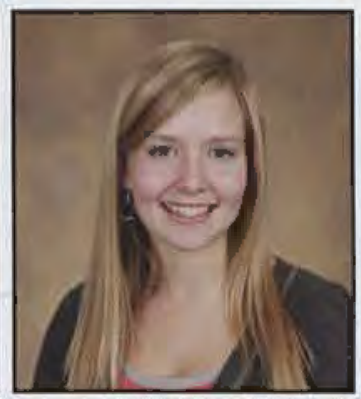

Danielle Sigman

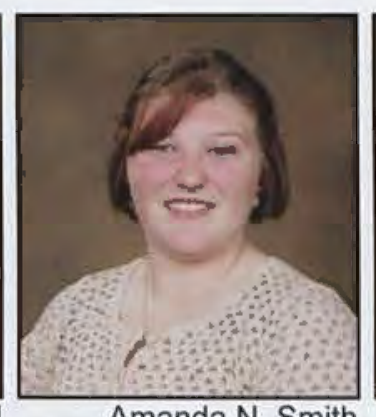

Amanda N. Smith

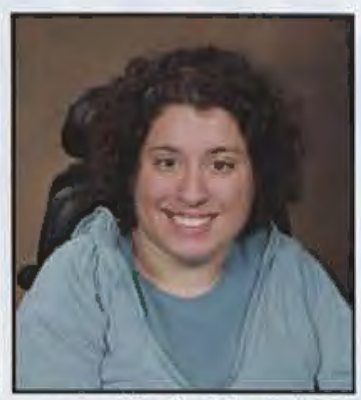

Emily Shanahan

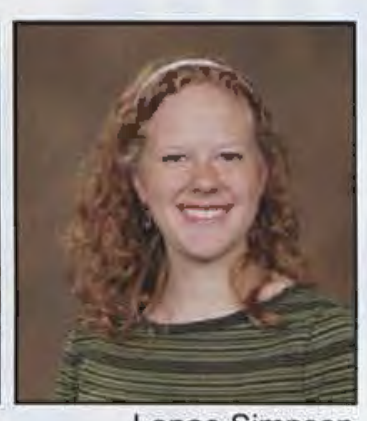

Lenae Simpson
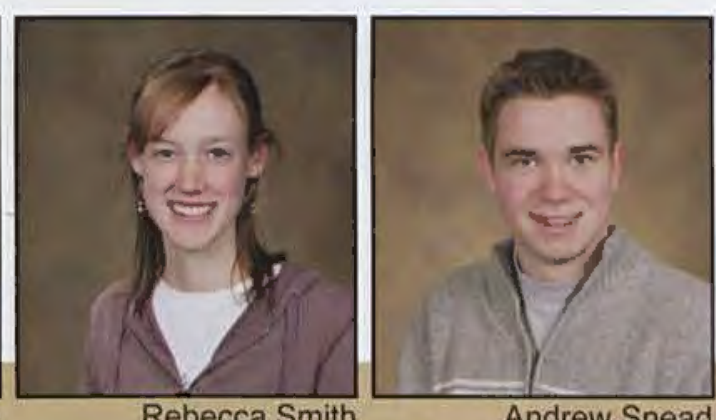

Andrew Snead

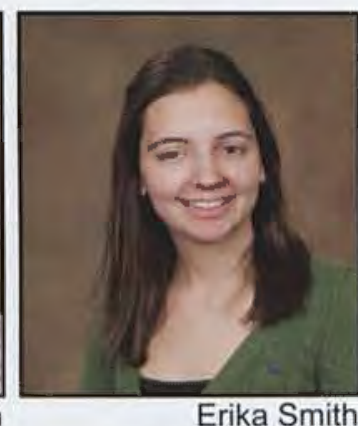

Erika Smith

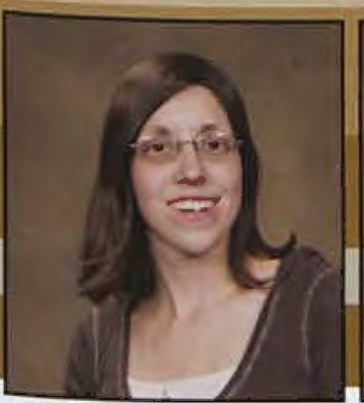

Amanda Snyder
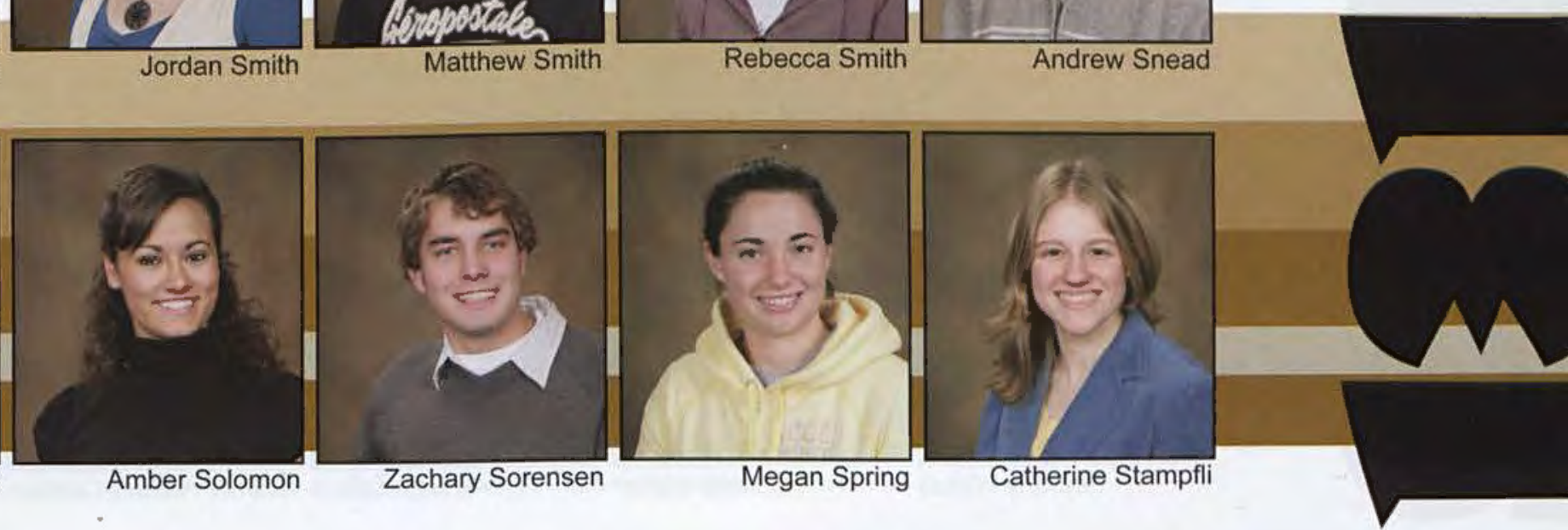


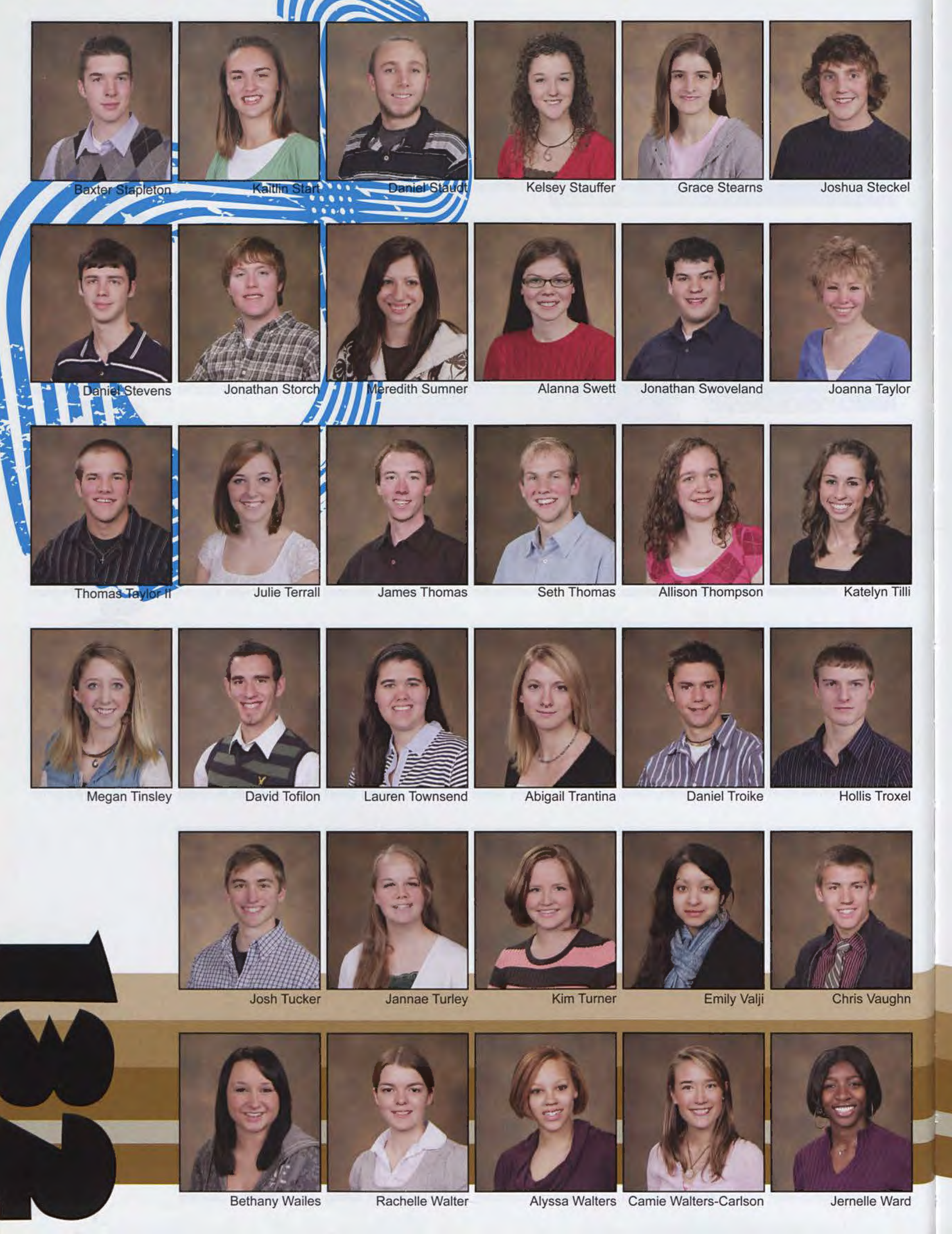



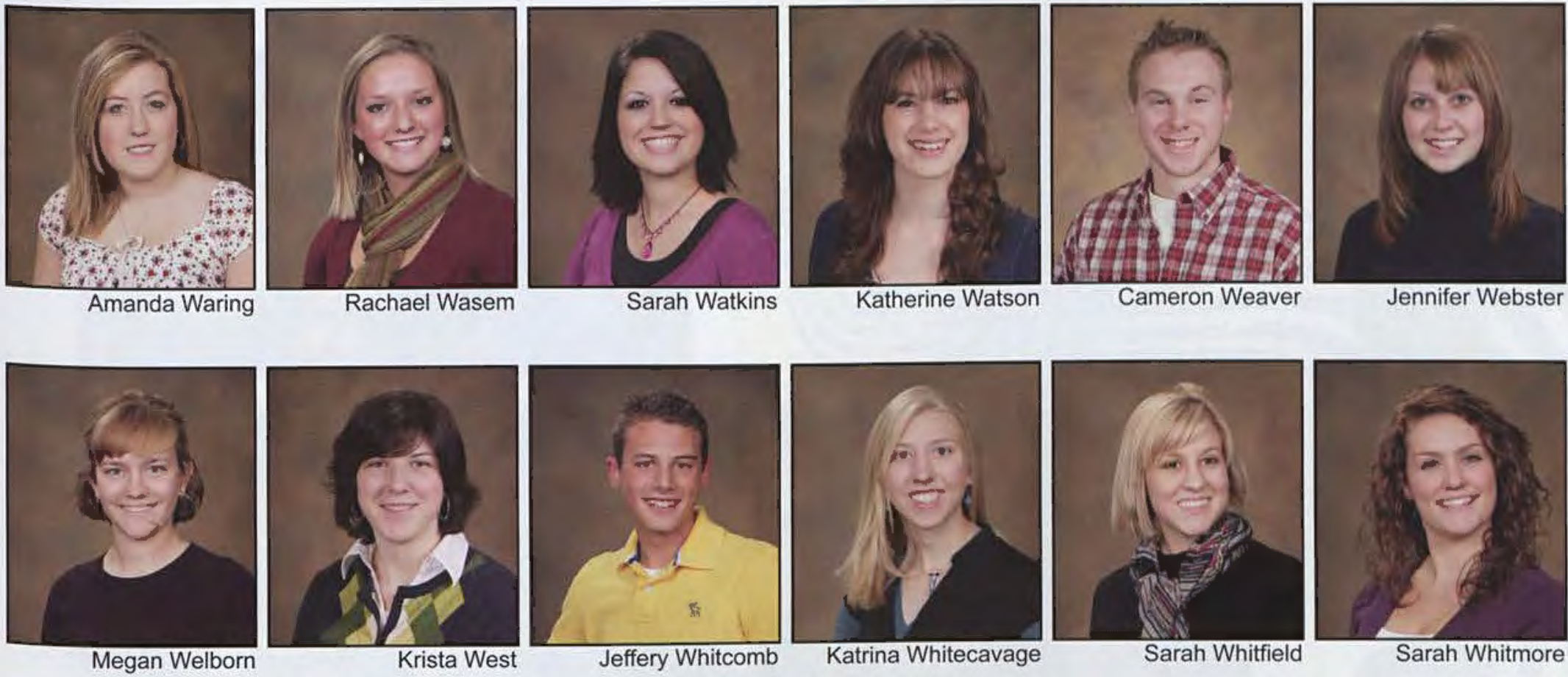

Megan Welborn

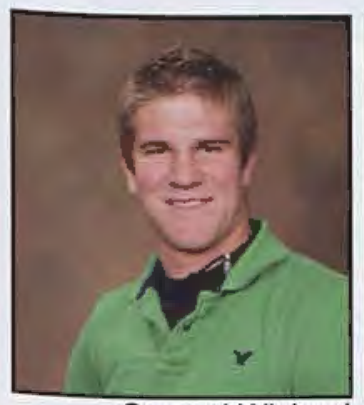

Samuel Wichael
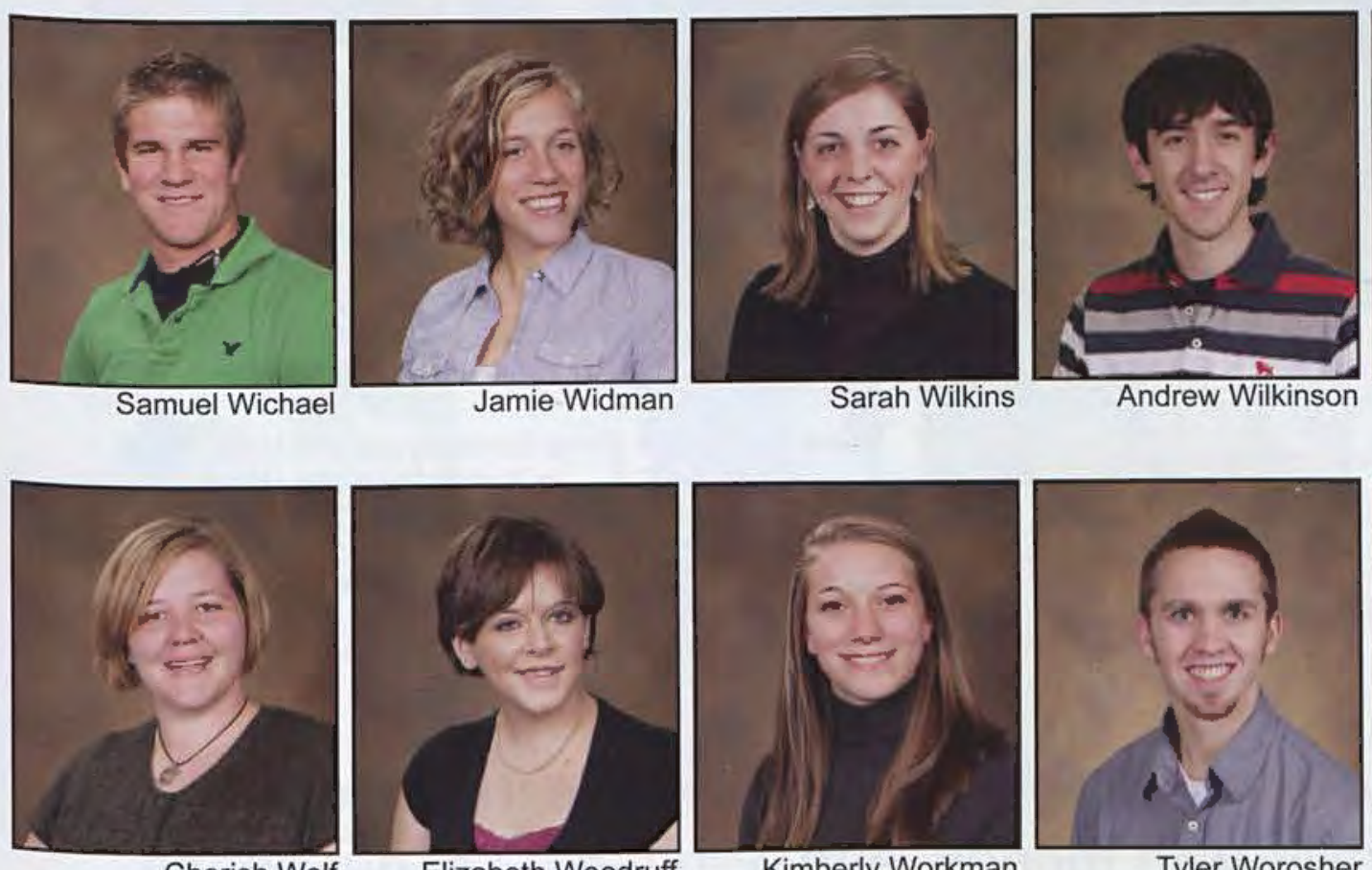

Cherish Wolf

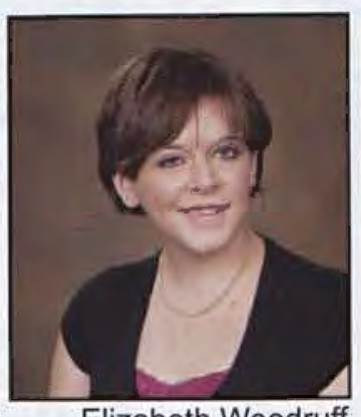

Elizabeth Woodruff

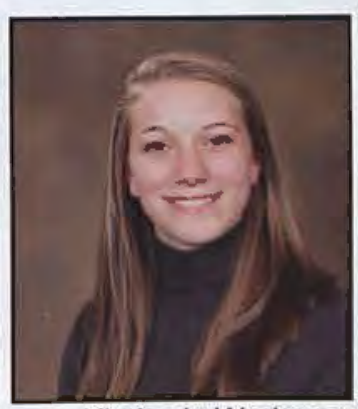

Kimberly Workman

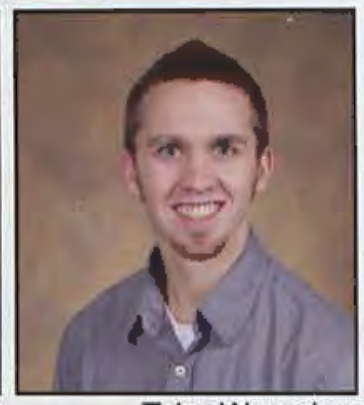

Tyler Worosher

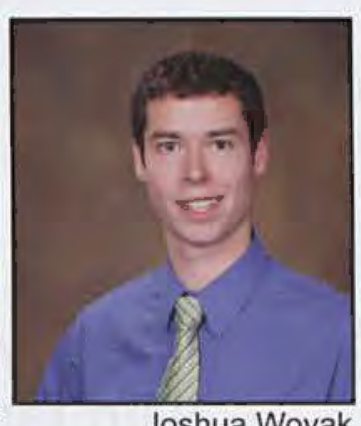

Joshua Woyak
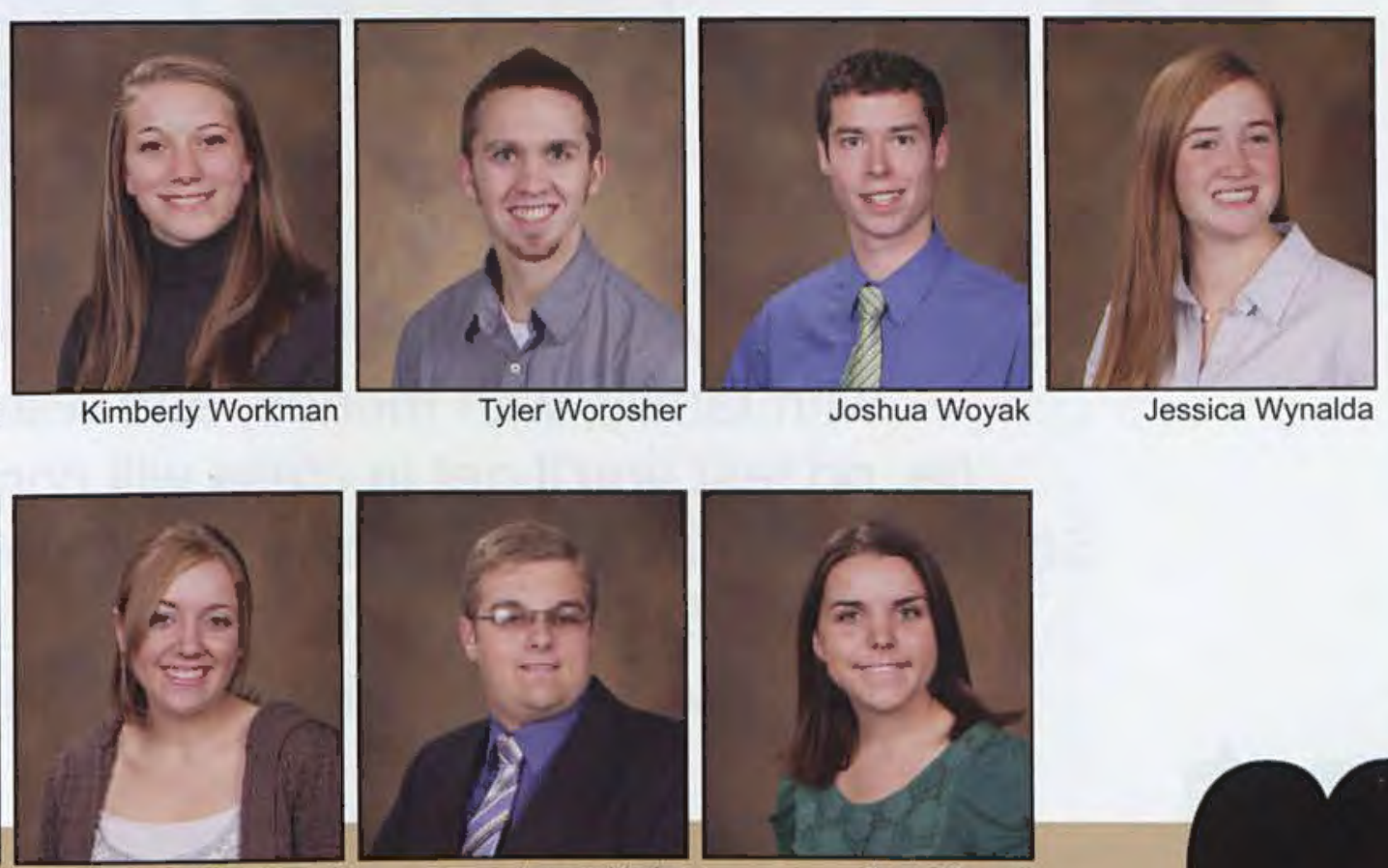

Janelle Yoder

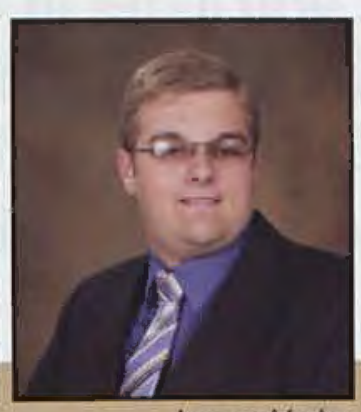

Lance Yoder

Caitlin Yoder
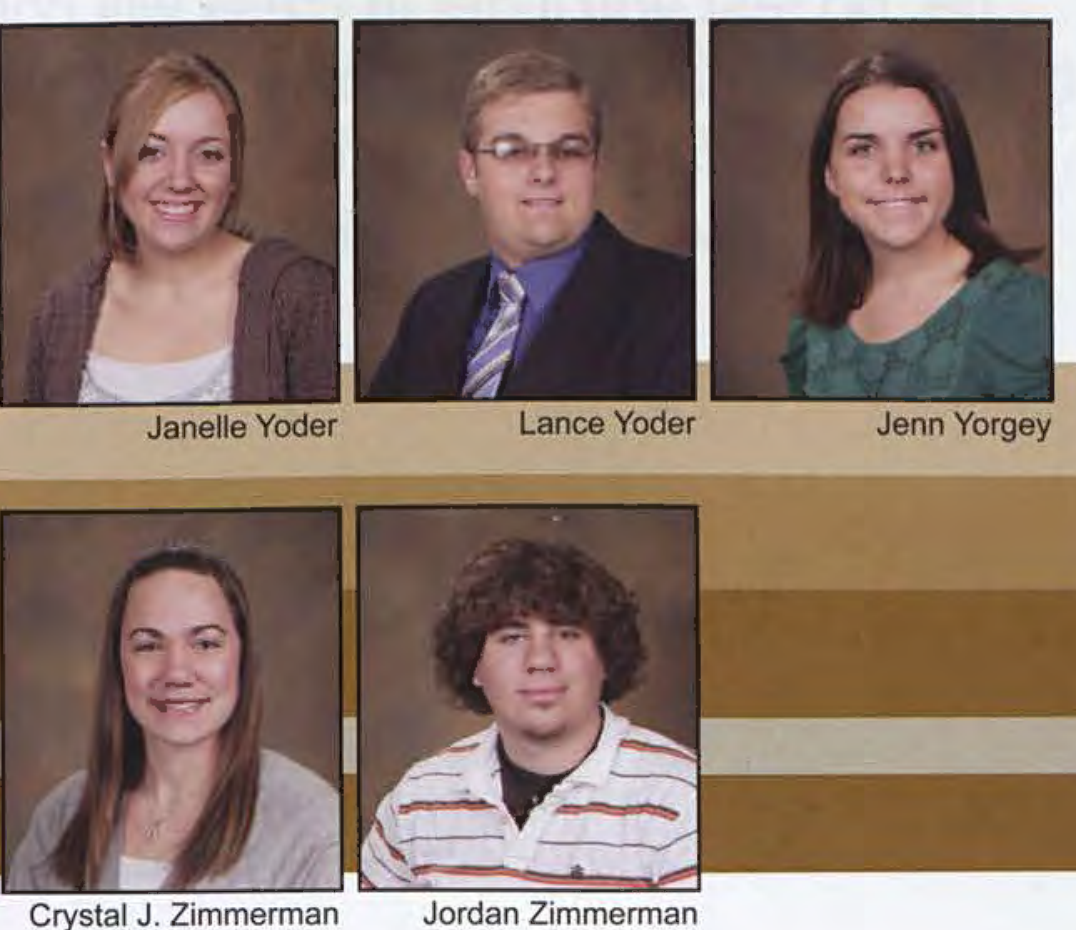

Jenn Yorgey

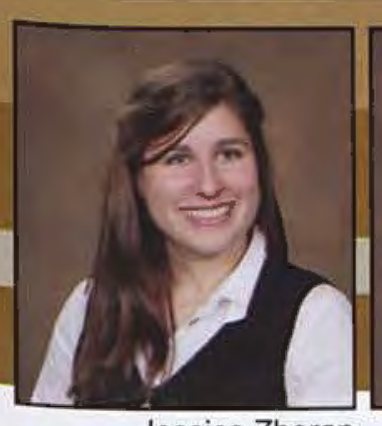

Jessica Zboran

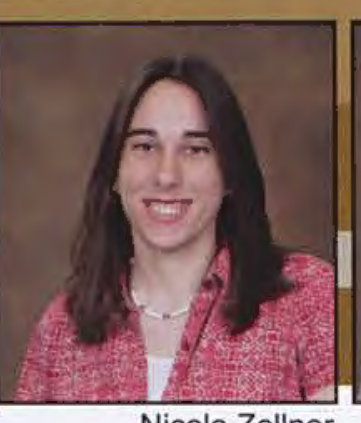

Nicole Zellner
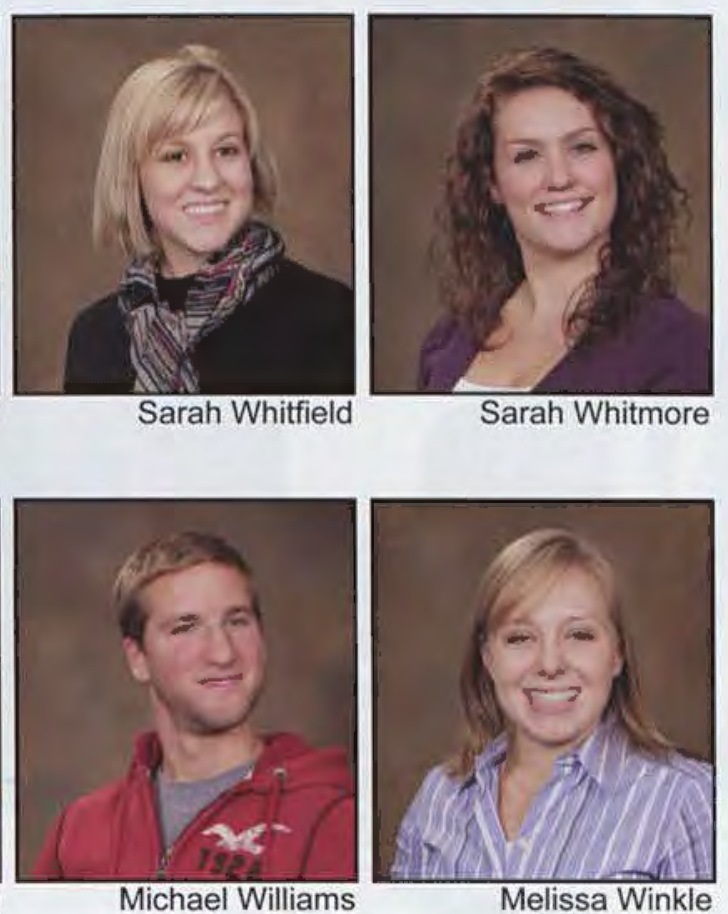

Sarah Whitmore

Melissa Winkle 

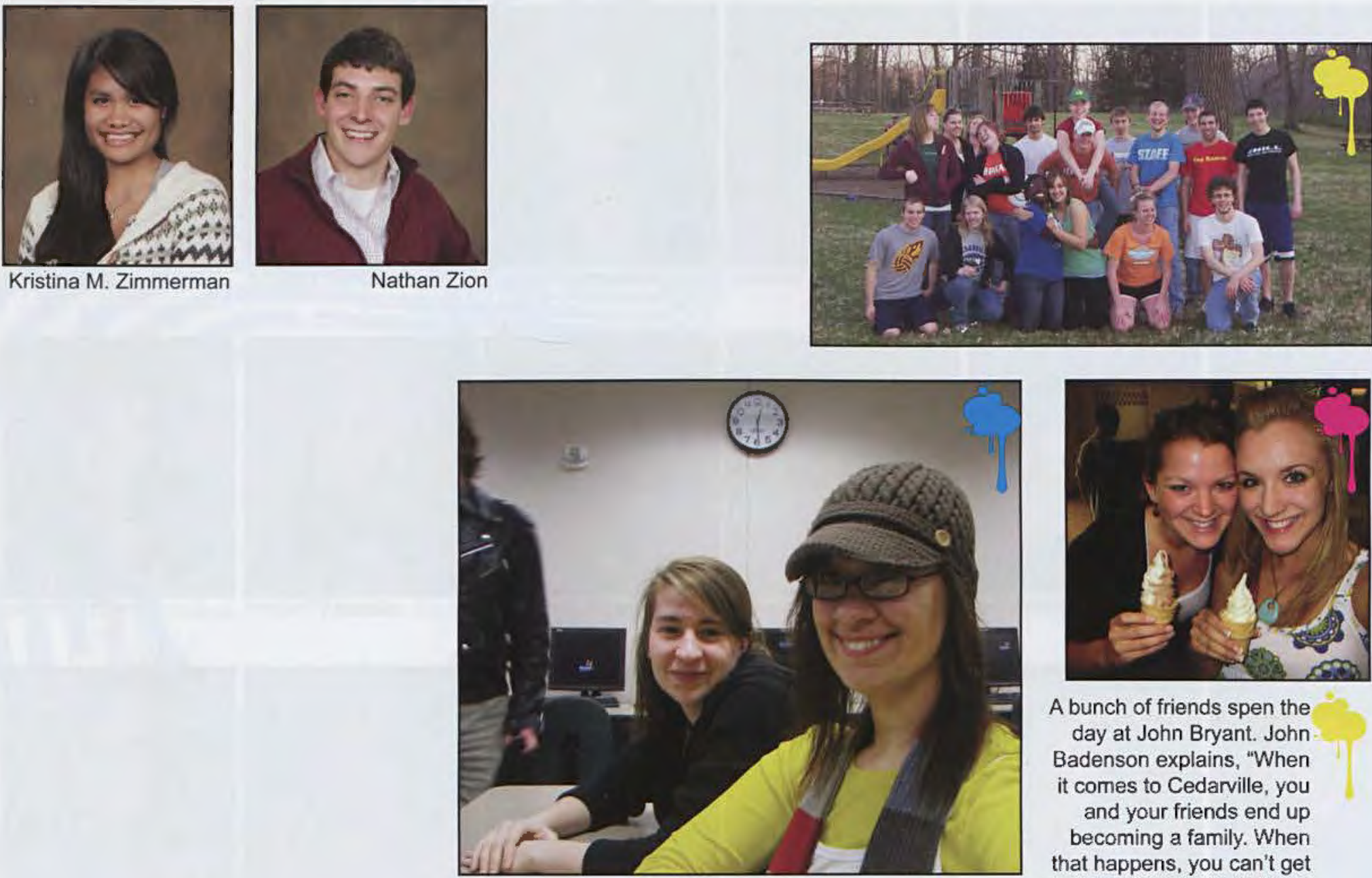

A bunch of friends spen the day at John Bryant. John Badenson explains, "When it comes to Cedarville, you and your friends end up becoming a family. When that happens, you can't get enough of each other. A true friend is forever family."

Marie Darling and Jenna Rothhaar just love being in Survey Research class!

Lauren O'Neal and Chloe Heckman enjoy some of Chuck's famous soft-serve. (Bet you didn't know it's made of soy!)

"Sophomore year gives you the chance to test relationships with family, with friends, and with God. I'm not gonna lie, no test you'll get in class will compare to those tests. Sophomore year will stretch you, but looking back, I wouldn't trade it for anything. I'm excited to continue the ride at Cedarville as a Junior next year!" -Tecie Bremencer 


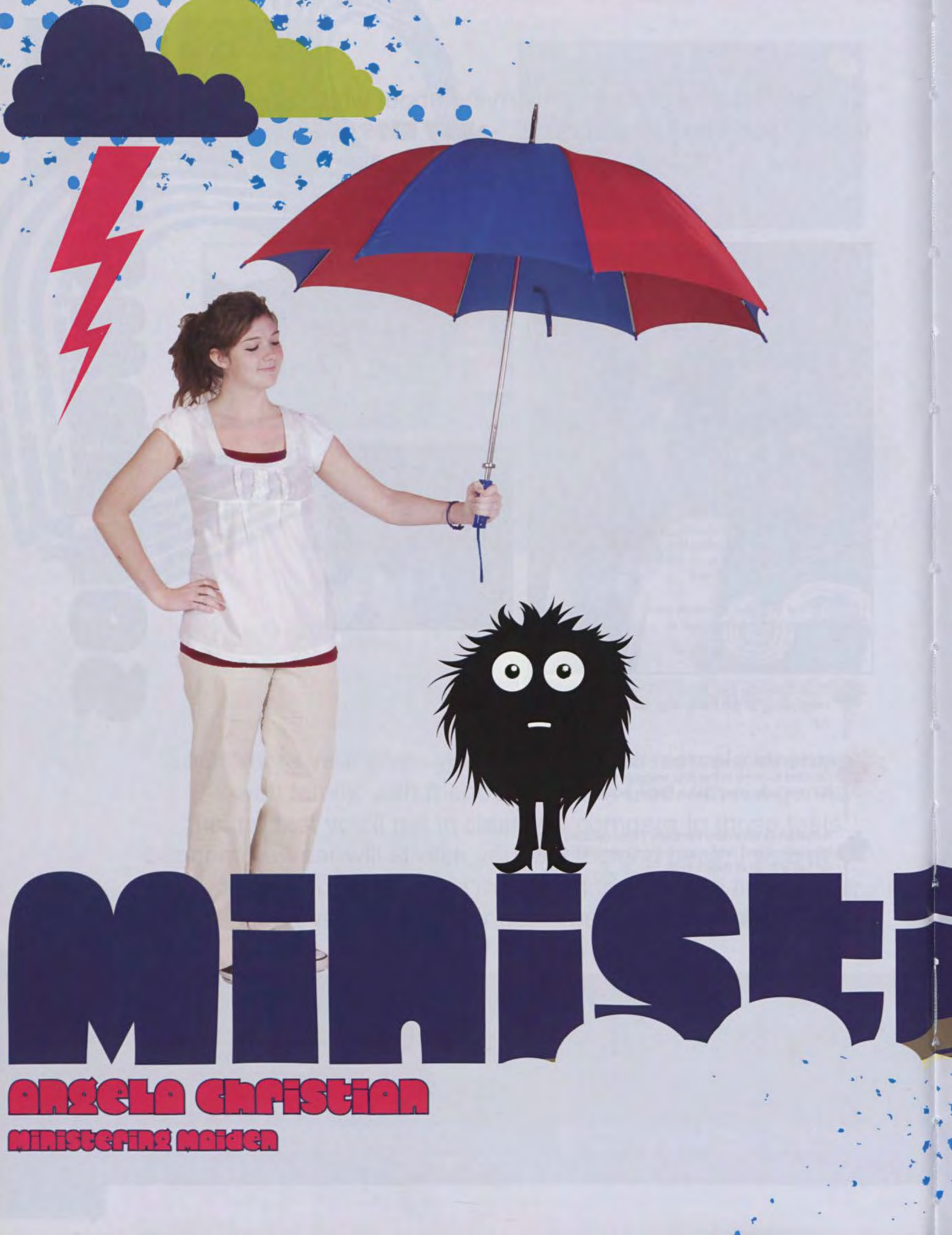


She wore the heavy head covering and the modest dress of a typical Jordanian

Muslim woman. The dark drapery veiled the shape of her body, but a hint of makeup brightened her face. She approached my team excitedly, her curious eyes questioning us as we talked about Christ.

Suddenly she spoke, gesturing frantically. "I have had a dream about this man, who you call the Christ," she said. "In my dream, I am huddled in a dark place, beaten furiously by attackers. A sharp knife cuts my skin, and I fall to the ground in pain. The light begins to fade, and I feel the life draining from my helpless body. Suddenly, a bright figure rises from the ground in front of me, dressed in brilliant white with a rod in his hand. His beckons me gently, lifts his voice, and looks me kindly in the eyes, saying, 'I am the Christ, and I want to give you new life.' The dark colors around me suddenly blossom into a deep green, and I feel an overwhelming sense of peace and joy. This is my dream. Ever since, I have been trying to discover who this man is, but no one can give me answers."

In a quiet corner of a room, hushing our voices to protect this woman's safety, my team and I explained to this wide-eyed Muslim woman that Jesus Christ wants to fill her with abundant life. It's the little conversations like these, one-on-one and vulnerable, in which the Spirit moves most mysteriously.

I used to walk the streets of Springfield on Friday nights with the Jeremiah Project, carrying copies of the gospel of John and extra food from the Hive. The faces I loved to see were the Springfield Christians who partnered with us - Christians who didn't have the opportunity to attend Bible classes and chapel like I did but who were filled with zeal for the Lord. One woman in particular, Lynn, sticks out to me. Lynn found Christ later in life and began passionately sharing her faith with the down-and-out in Springfield. Christians often want to leave their hometowns and find a glamorous new place to serve, but Lynn felt content to minister on her local streets. She consistently helped to organize the Jeremiah Project, showing such patience for our irregular attendance and short-lived passion for the city. Lynn couldn't walk campus sidewalks with us and sit down at Chuck's each day surrounded by believers. She needed the body - the body of the man in the brilliant white robe who appeared to the Muslim woman in Jordan - and for a time, that body walked the streets of Springfield on Friday nights with leftover food from the Hive.

During the summer, I see the Jordanian woman's wide eyes and Lynn's need for encouragement in the children of inner-city Detroit. Inner city kids at the day camp I work for crowd around me, their eyes begging for my affection - affection they do not receive from the frustrated parents who drop them off each day in exchange for peace and quiet.

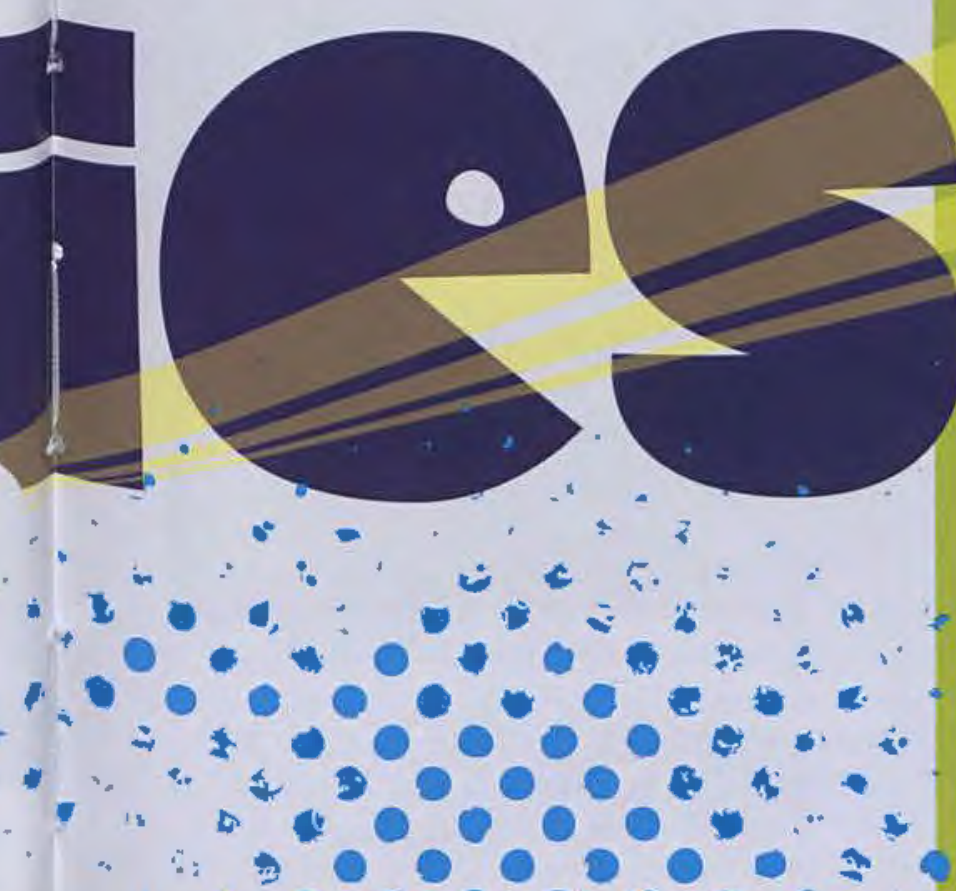

When I used to attend Urban Light in Springfield, I saw these Detroit kids' faces in the poverty-stricken men and women who came for the free meal we offered. I tried to engage them in conversation. Often I talked to Bill, a regular at Urban Light. He would hobble toward me on his crutches and talk to me about his family, his veteran status, and his financial needs. Bill would talk for hours if I was willing to listen.

When I think about ministry, I push aside thoughts of programs and projects. Instead, I think about Bill. I think about Lynn in Springfield. "I think about my Muslim sister in Jordan. These people are not my projects, and they are not my ministry. They are people hungry for Christ - people that I love. b.

I believe that the word "ministry" puts a gap between me and the people I want to serve. I yeam to erase that gap. And I yearn to fill it with Jesus.

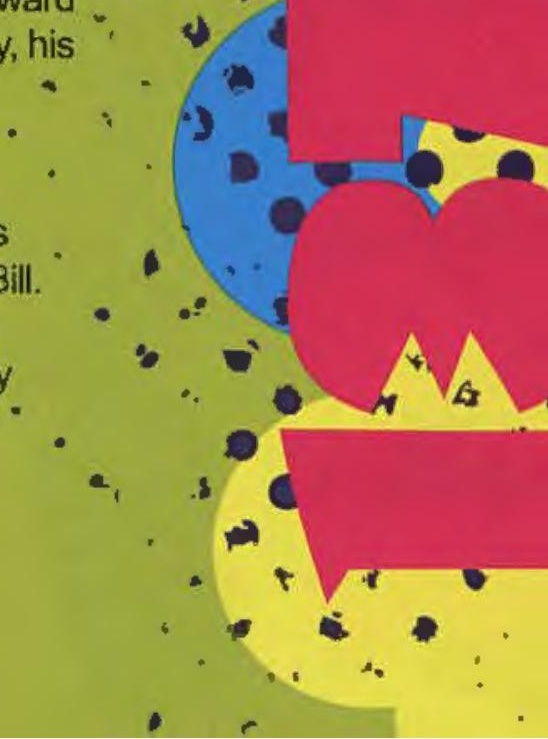




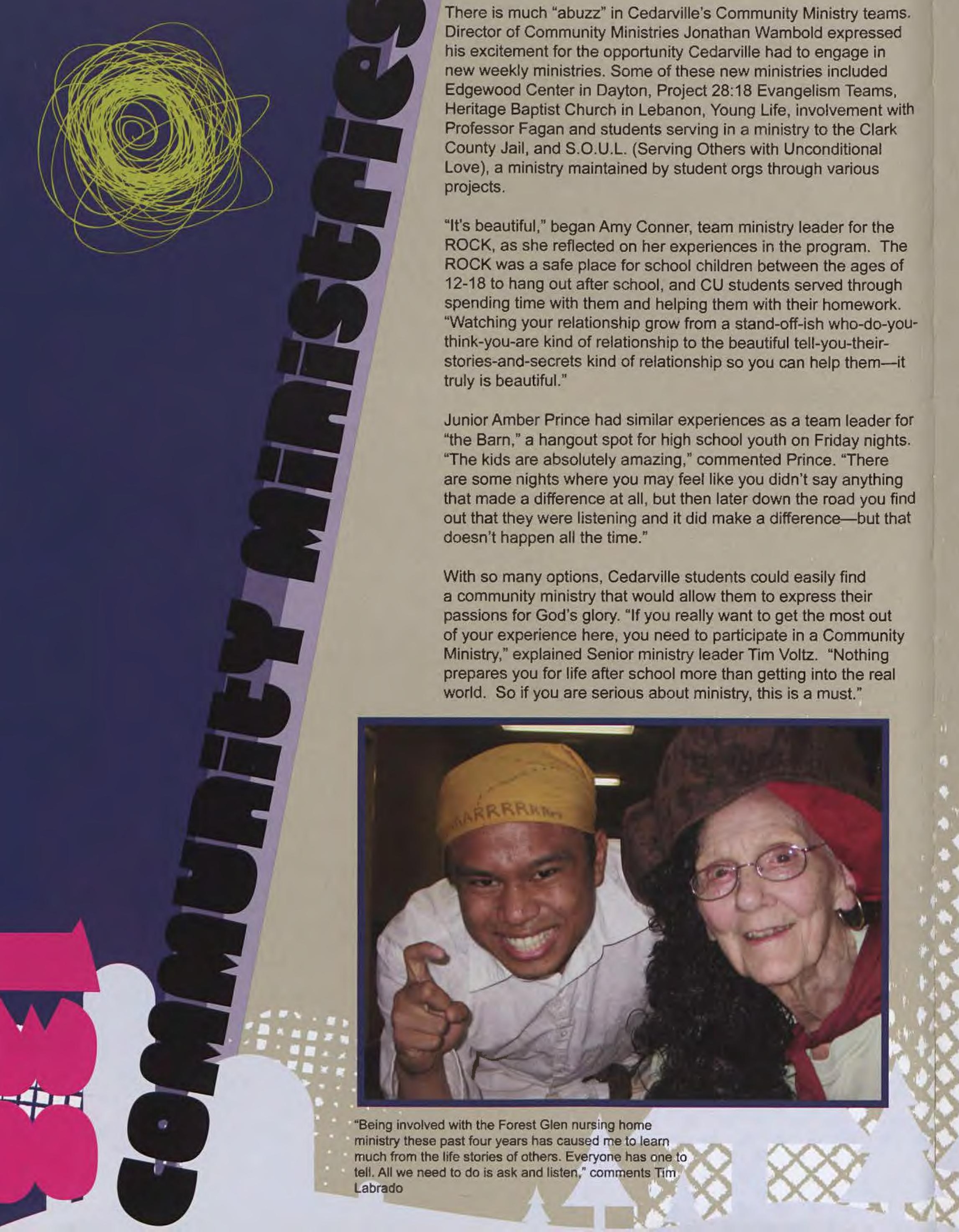



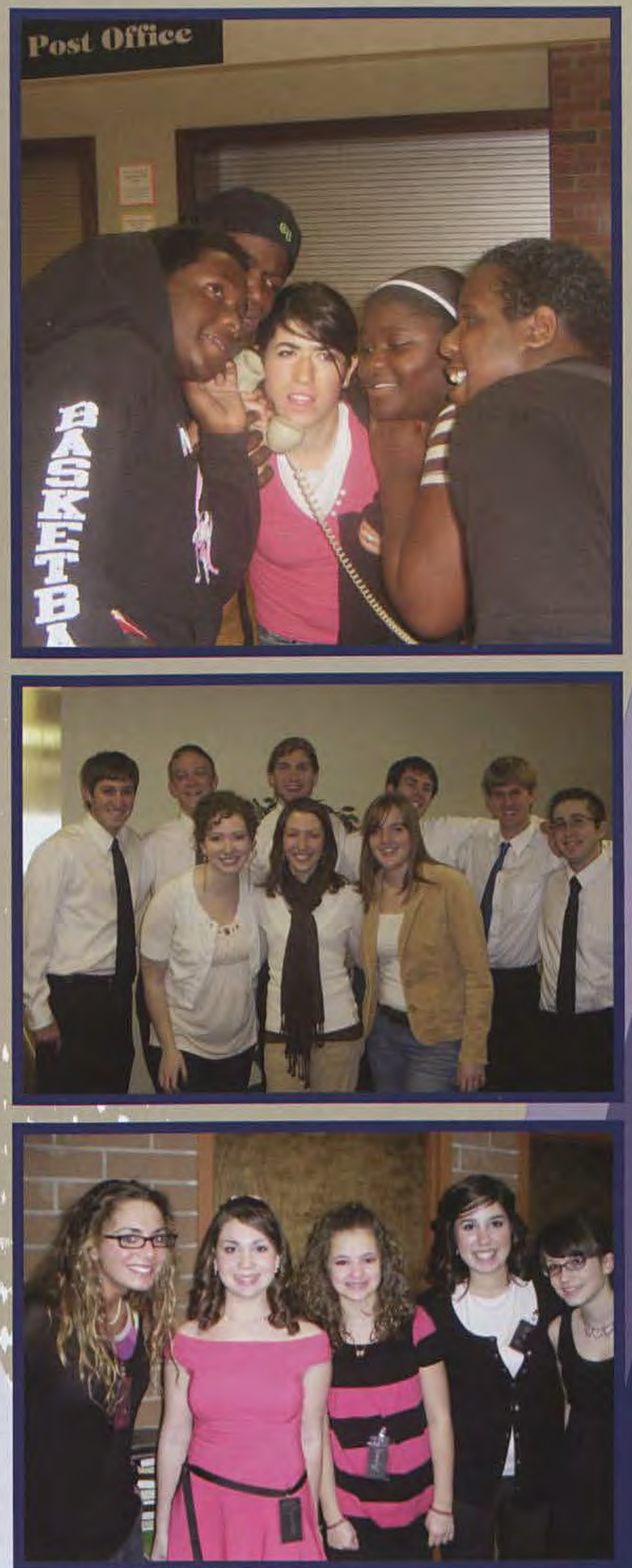

Cedarville students like Jenny Monroe host youth irom All Nations Bible Fellowship for a weekend and participate in a picture scavenger hunt.

Collin Barrett says, "Our goal for the weekend was to provide the youth with positive Christian rolemodels for a weekend and to give them exposure to the university setting. It was an amazing weekend of developing relationships and growing closer to our Savior (while having a blast)."

Nathan Reed says, "The most rewarding aspect of my ministry experience has been the way that the kids I teach repeatedly deconstruct my college experience. They remind me that crayons and skits are just as indispensable to my life as research papers and final exams."

Veronica Wilkins comments, "STARS has definitely always been a highlight of the week. Middle school can be such a difficult time, and I think that's why I feel for this group. While we all enjoyed the

Valentine's Day banquet, just spending time with the girls, building relationships and trying to impact their lives positively in some small way, is by far the best part." 


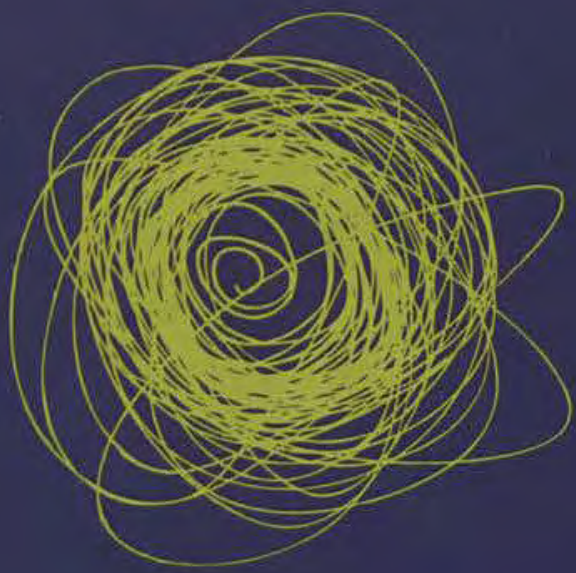

Katie Schmidt tells, "I got involved with Adopt-A-Grandparent because I work at a nursing home in Illinois and was surprised at how much I missed seeing the residents everyday once I got to Cedarville. My favorite Adopt-AGrandparent experience was when Renee, my partner, and I threw a little party for our adopted grandma's 70th birthday. She never really had birthday parties growing up, and we were more than happy to celebrate with her."
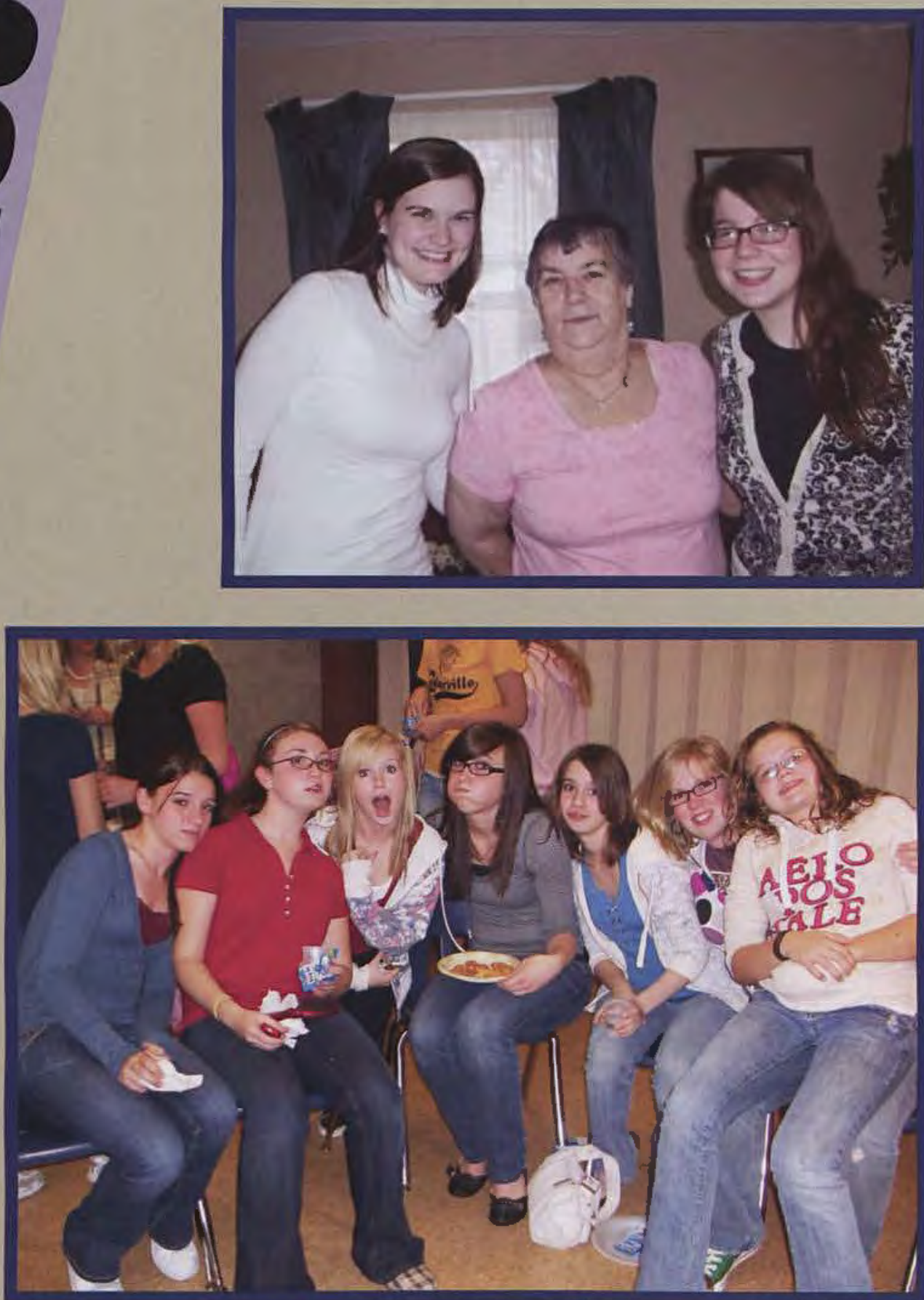

"STARS is an awesome program for junior high girls. I am blessed to be a part of this team and these girls' lives to try and influence them in a positive way. I wish I had had a mentoring group like STARS when I was in school! What a great ministry!" exclaims Megan Brown.
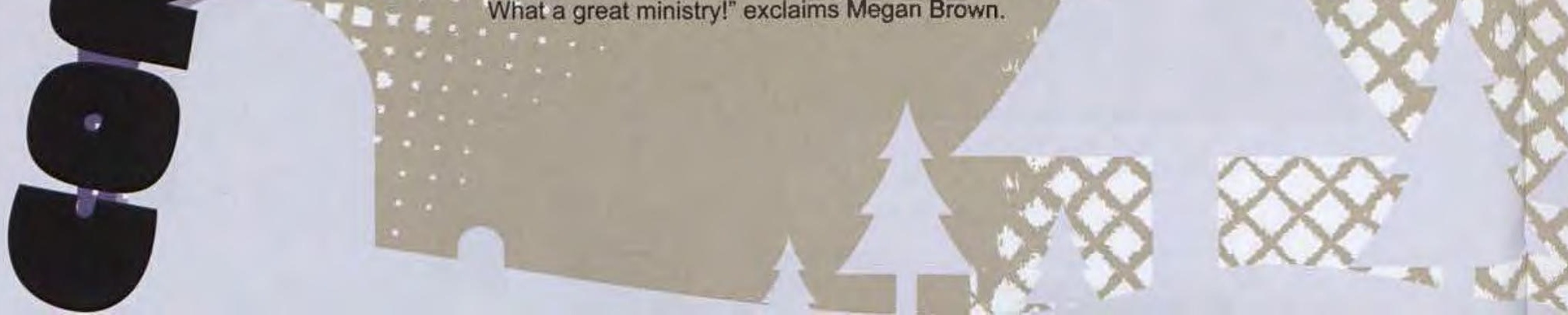
Kaitlin Brewer says, "There's always the argument over whether ministry benefits the person doing it or the person receiving it. Visiting the people at the nursing home does, I believe, greatly enhance the lives of both. I love visiting my 'family' at the nursing home, and their smiles reveal their joy at seeing me."
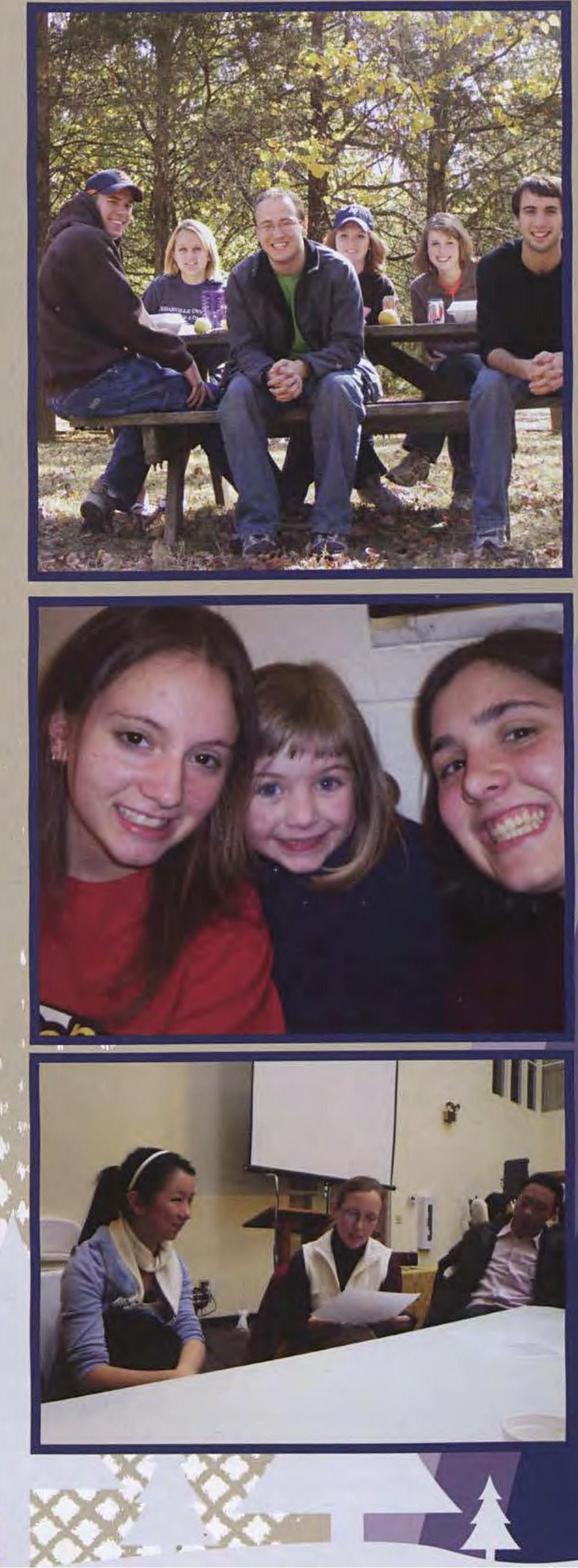

Katie Marburger leads a Chinese ESL

conversational group at Agape Chinese Evangelical Church in Fairborn. says. "As a leader I have been challenged both spiritually and in my leadership skills. It has been great o see God at work in the students that I worked with. Truly this ministry has been one of the highlights of my year at Cedarville."

"Awana is such a great way to serve God and be involved in the community when you are a college student. Valerie and I both work with the kindergartners, and it is such a blessing to see them learning verses and growing in spiritual things," comments Sara White

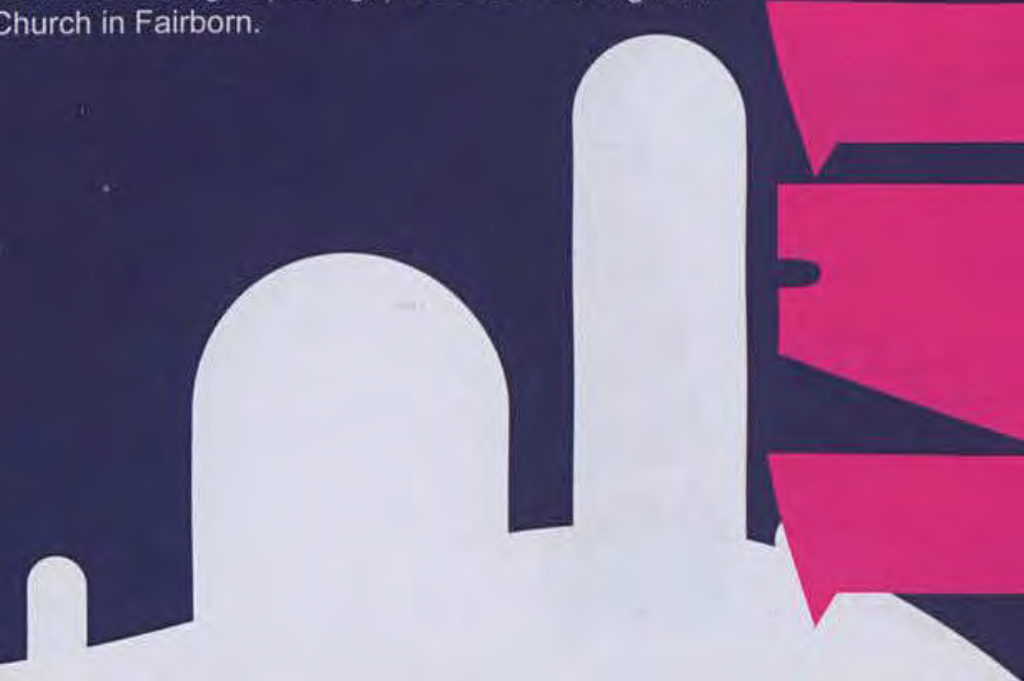




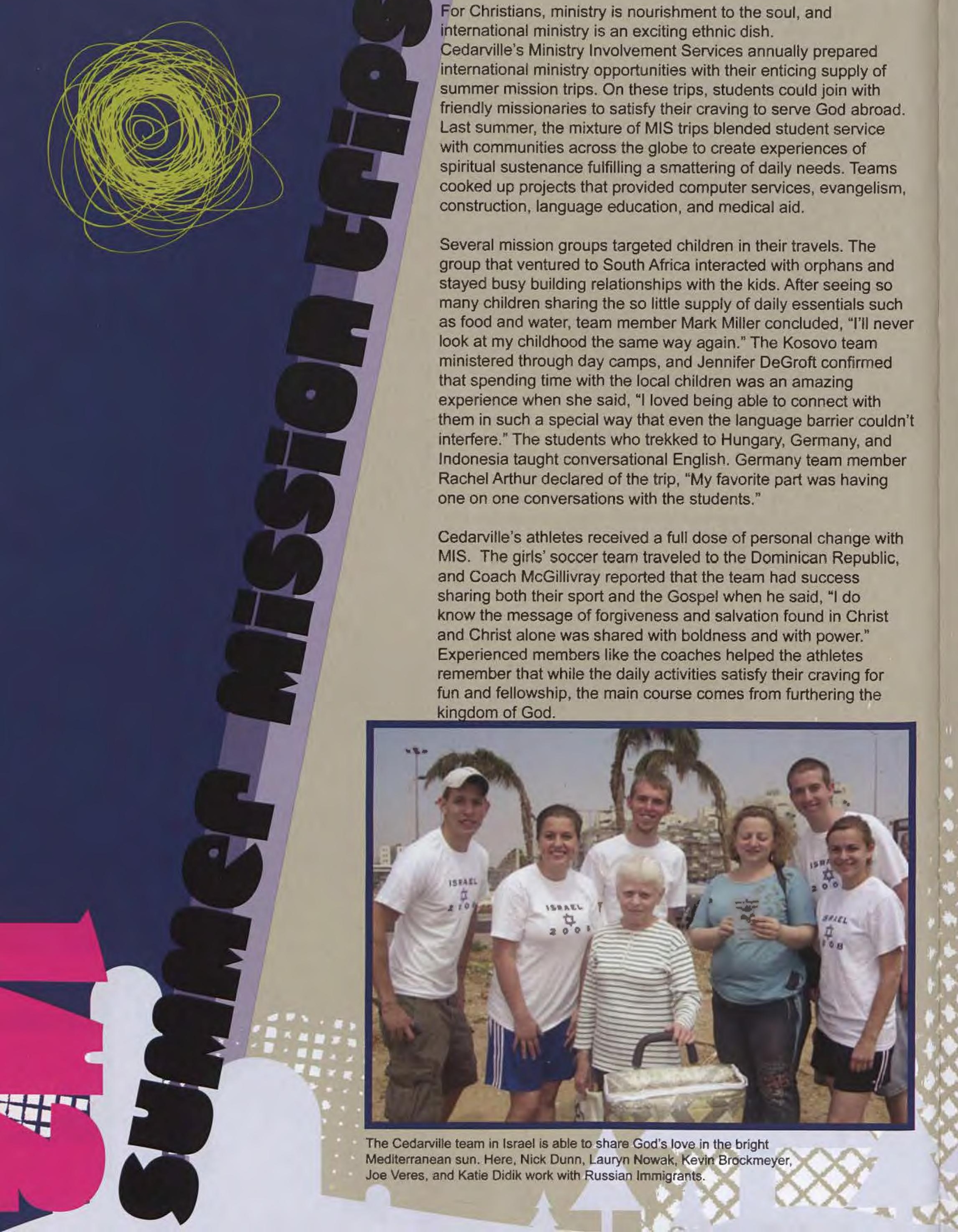


After spending time with the children of South Africa, Sarah Porter

reports, "There is nothing like serving the Lord. Be it overseas or right next door, answer His call!"
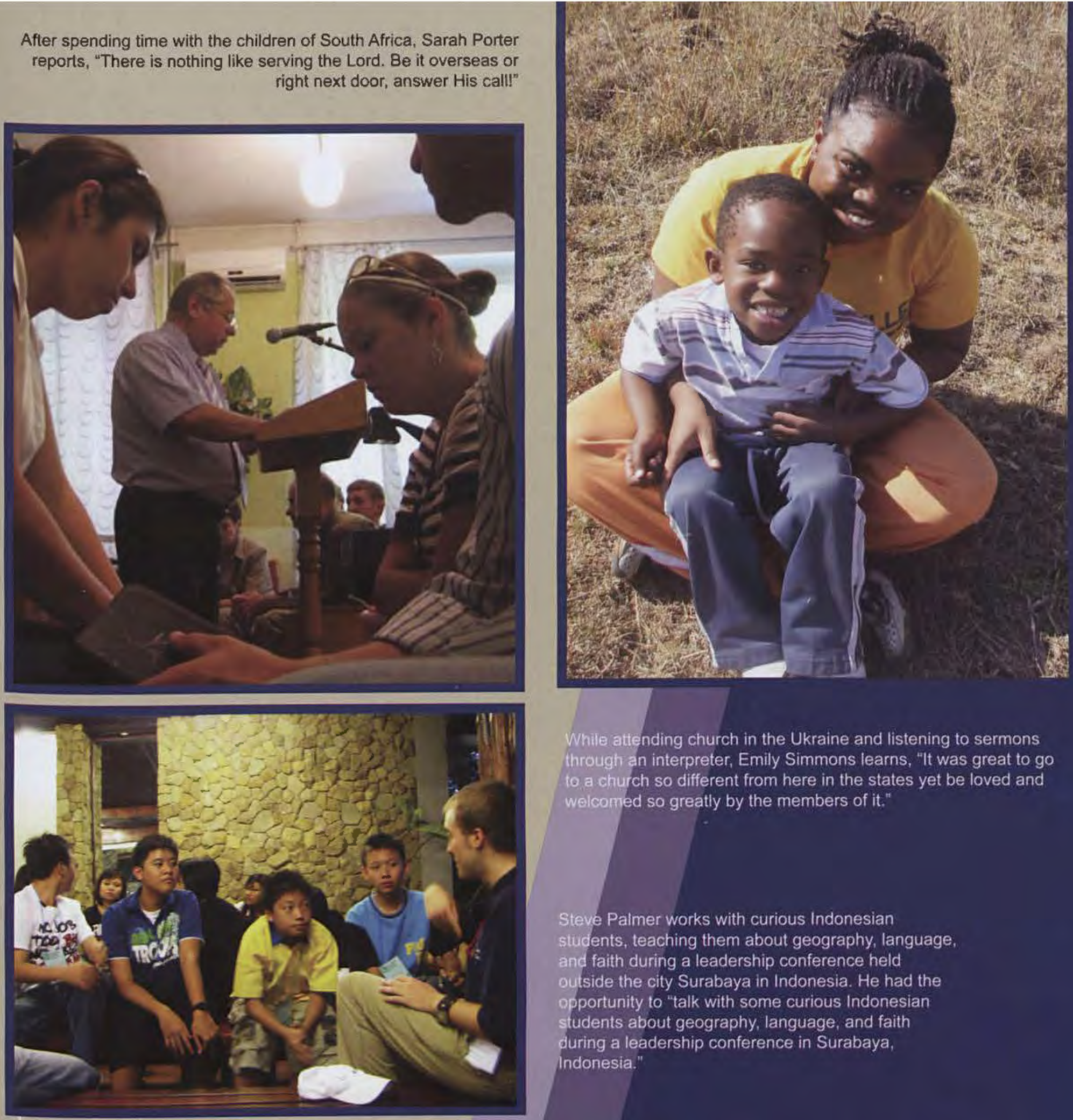

While atiending church in the Ukraine and listening to sermons through an interpreter, Emily Simmons learns, "It was great to go to a church so different from here in the states yet be loved and welcomed so greatly by the members of it."

Steve Paimer works with curious Indonesian students, teaching them about geography, language, and faith during a leadership conference held outside the city Surabaya in Indonesia. He had the opportunity to "talk with some curious Indonesian students about geography, language, and faith during a leadership conference in Surabaya, indonesia."

While jumping for joy with the rest of the group, Jessica Robb states, "In South Africa, we were truly humbled by the joy of the children at Bethesda who came from destitute situations. Their joy was contagious!"

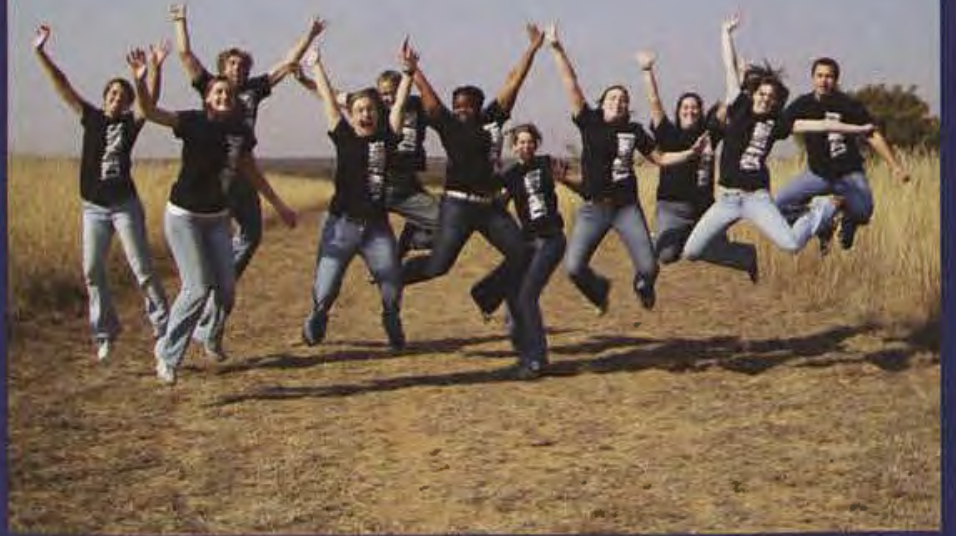




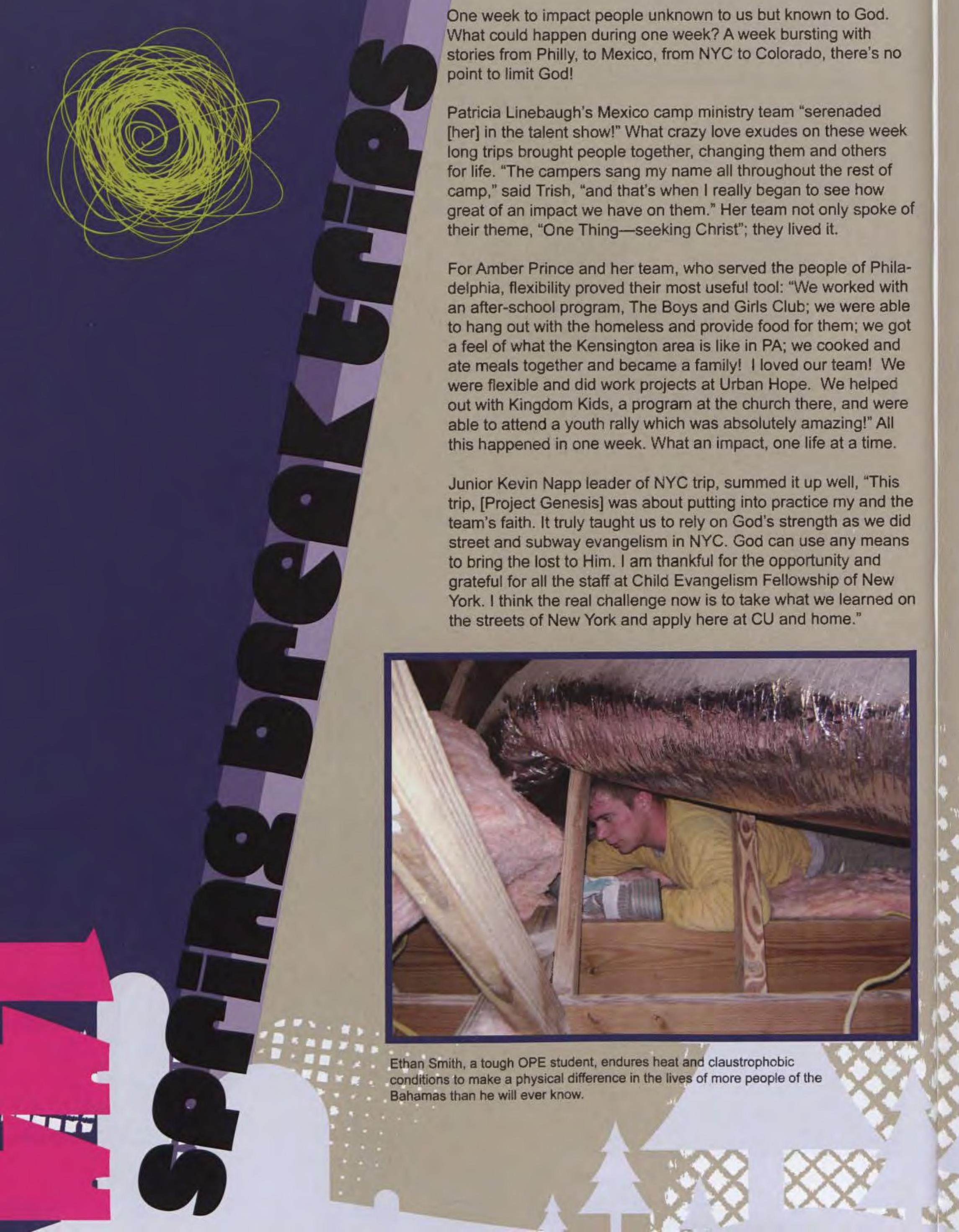


Lauren Ciarrone, Amy Oulette, Stacy Phillips, and Erin Leffel help out at Open Door Ministries in Denver, CO, and will never forget the blessings they receive from giving.
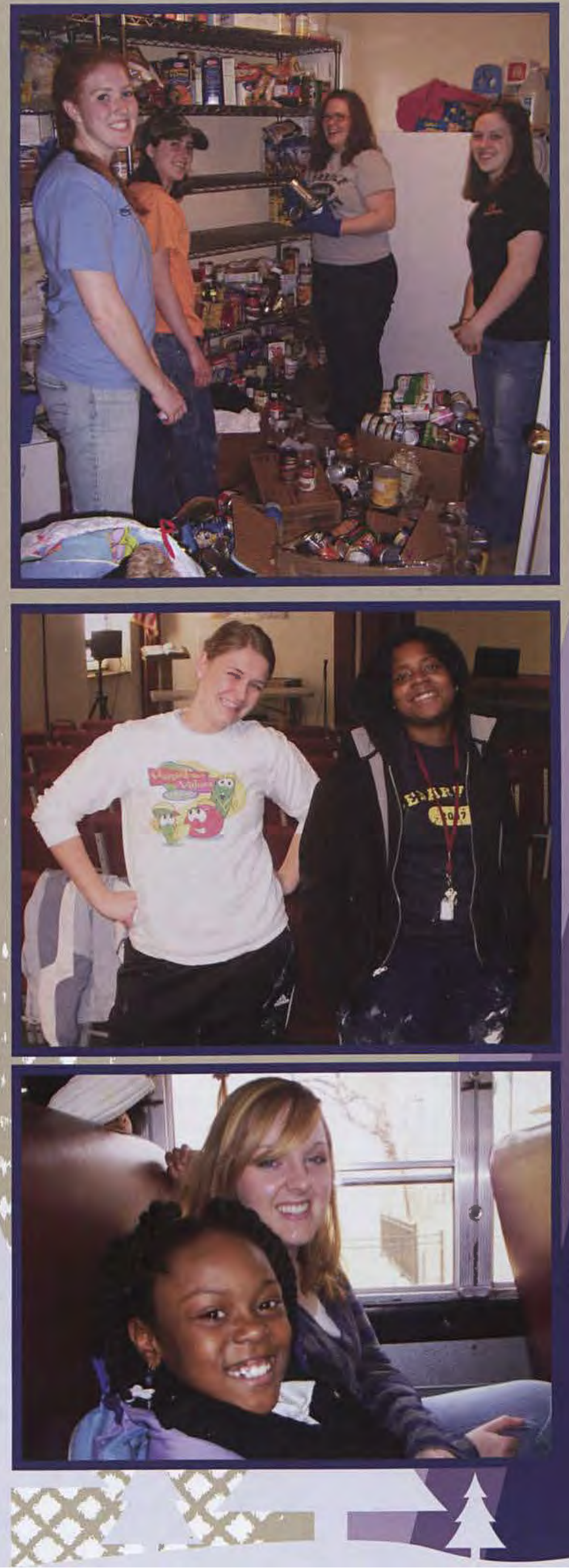

After a long week together, students bond so tightly they know how to avoid the "posed family portrait" photos.

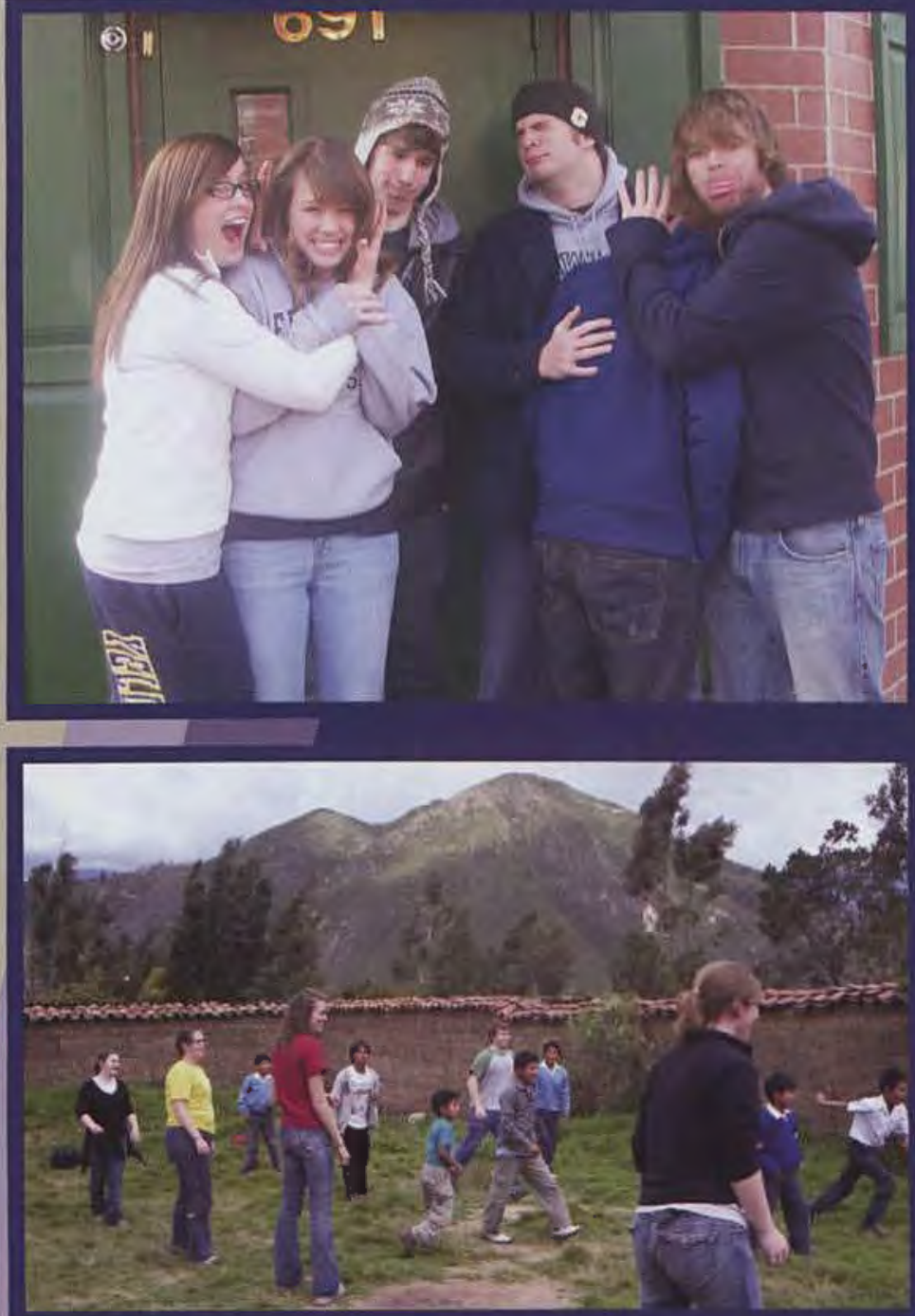

Students in Peru, like Emily Bush, try to play soccer

with some of the local kids. Who do you think won?

Stephanie Grant and Briana Dupree take a well-deserved break from painting the church, Urban Hope, in Kensington, PA.

Jessica Losch tells, "Our team of four girls got to go to Chicago for the week and work with By The Hand Club for Kids in Altgeld-Murray. We spent the week with kids from first to seventh grade, picking them up at school, helping them with homework and just loving on them. We had a great time both with the kids at the program, as well as during those times we were able to go downtown and explores? Our eyes and hearts were definitely opened to the ministry opportunities in the inner city thro gighourt fitls week.

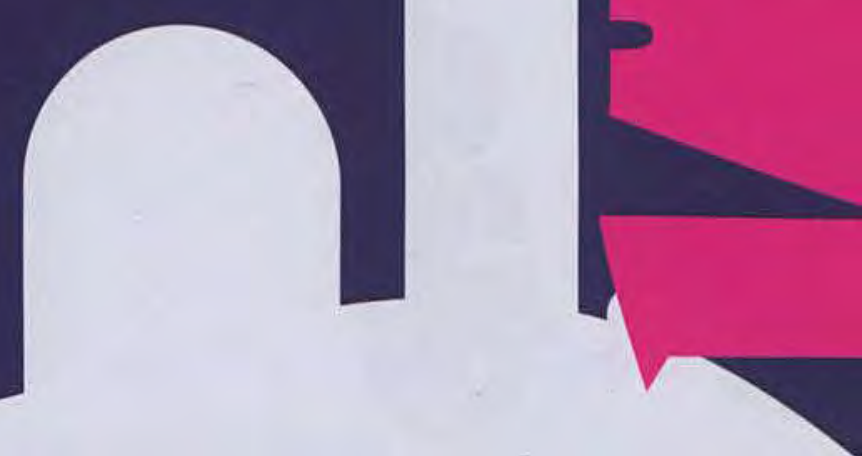




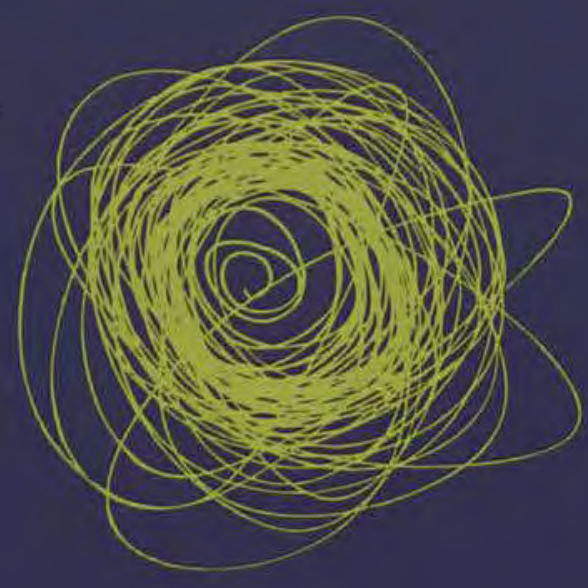

Satisfy My Thirsty Soul. The Enemy Within. Matthew. Crazy Love. Ephesians. These books are just a sampling of the many rich studies Discipleship Ministries offered to Cedarville students this school year.

Small groups of students gathered all across campus each week under the leadership of Discipleship Small Group Leaders, studying God's Word and encouraging each other as microcosms of the Body of Christ. DSGLs also met in small groups led by Discipleship Council members. The Discipleship Council studied the Gospels this year under Mark Irving's leadership and spent a week in Dearborn, Michigan over Christmas break, ministering to its Muslim population.

Of course, not every Bible study meeting this year was pious and reflective. Kristen Ensminger says, "For one of our community building times we played Spoons, with forks. I never knew how competitive my group of girls could be, and I'm pretty sure at least two people were bleeding by the end. What a way to build community!" Every other week, discipleship groups took part in community building activities, which varied from trips to Stoney Creek Roasters, to vicious games of Spoons.

These times of fellowship shaped Godly friendships that sharpened students spiritually. "It was awesome to come to Bible study, exhausted from studying, yet leave completely refreshed and rejuvenated at the splendor of God and the fathomless grace He's given to me," said Susanna Martin. Kelly Pankratz joined a discipleship group as a freshman that met this school year for the third year in a row. She said, "It has been one of the biggest blessings and most memorable group of friends of my time at Cedarville. Nowhere else do I find the freedom to share my shortcomings, laugh until I cry, dig into the Word with godly women, and be challenged in new ways to follow Christ."

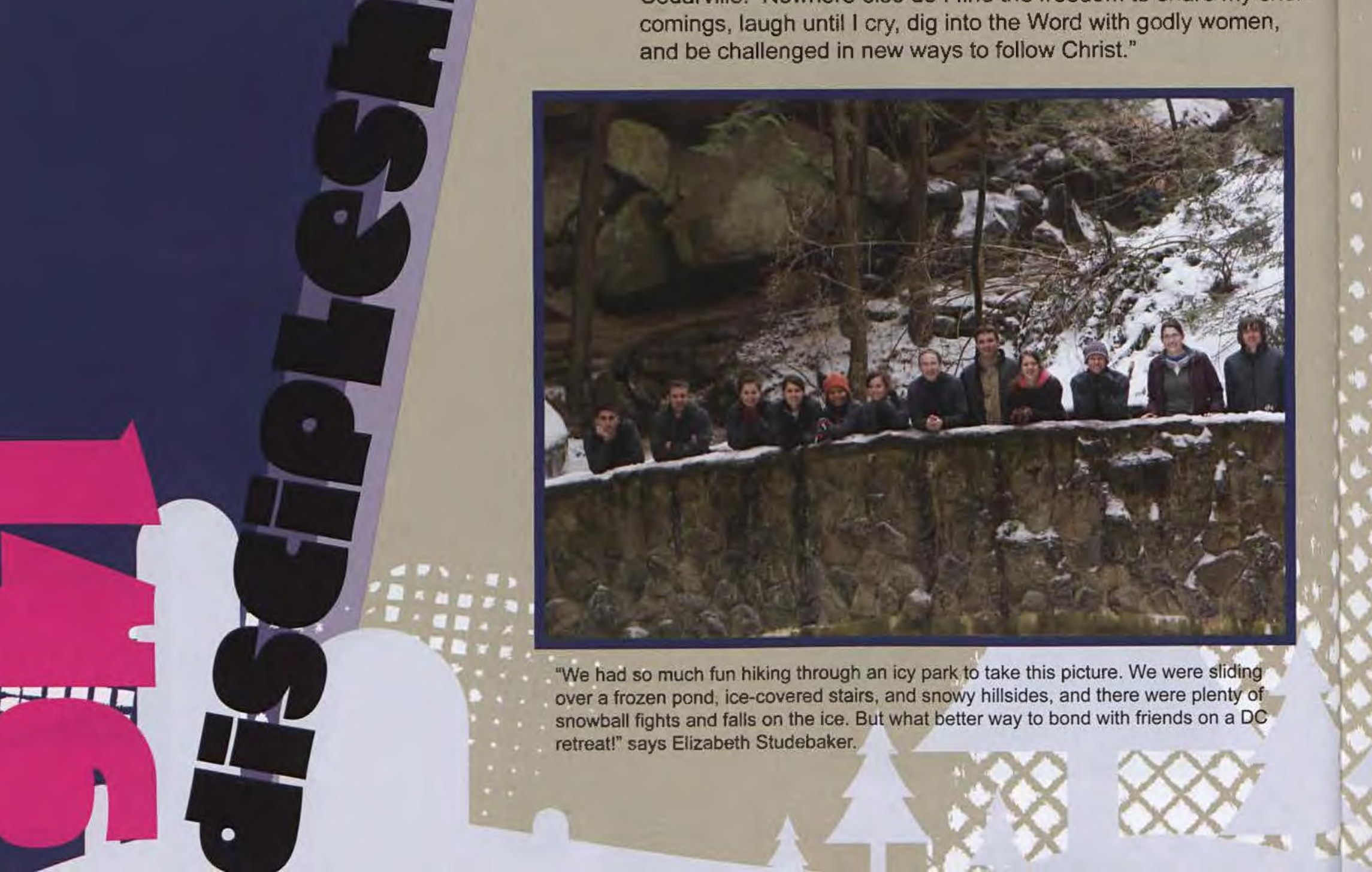


Sarah Thompson says, "Bible study has been an amazing encouragement! No matter how my week had gone, I knew that l'd have Bible study on Wednesday night and that l'd leave feeling relieved of my stress no matter what!"
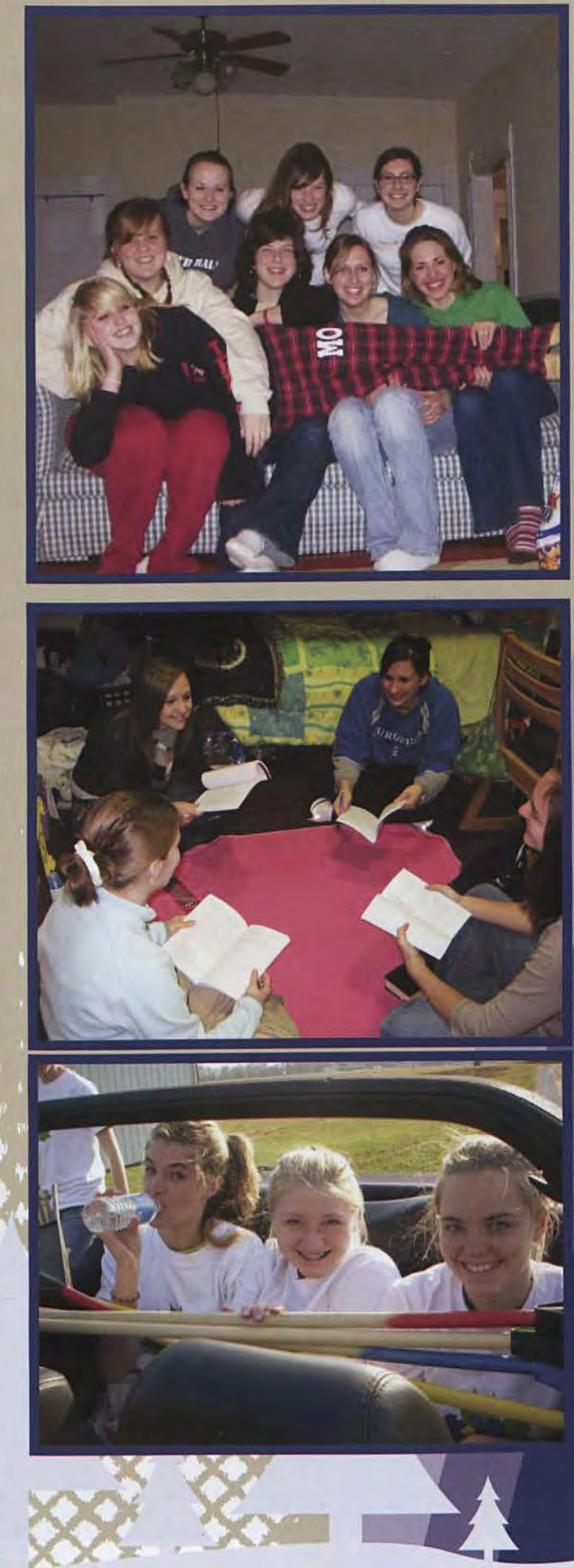
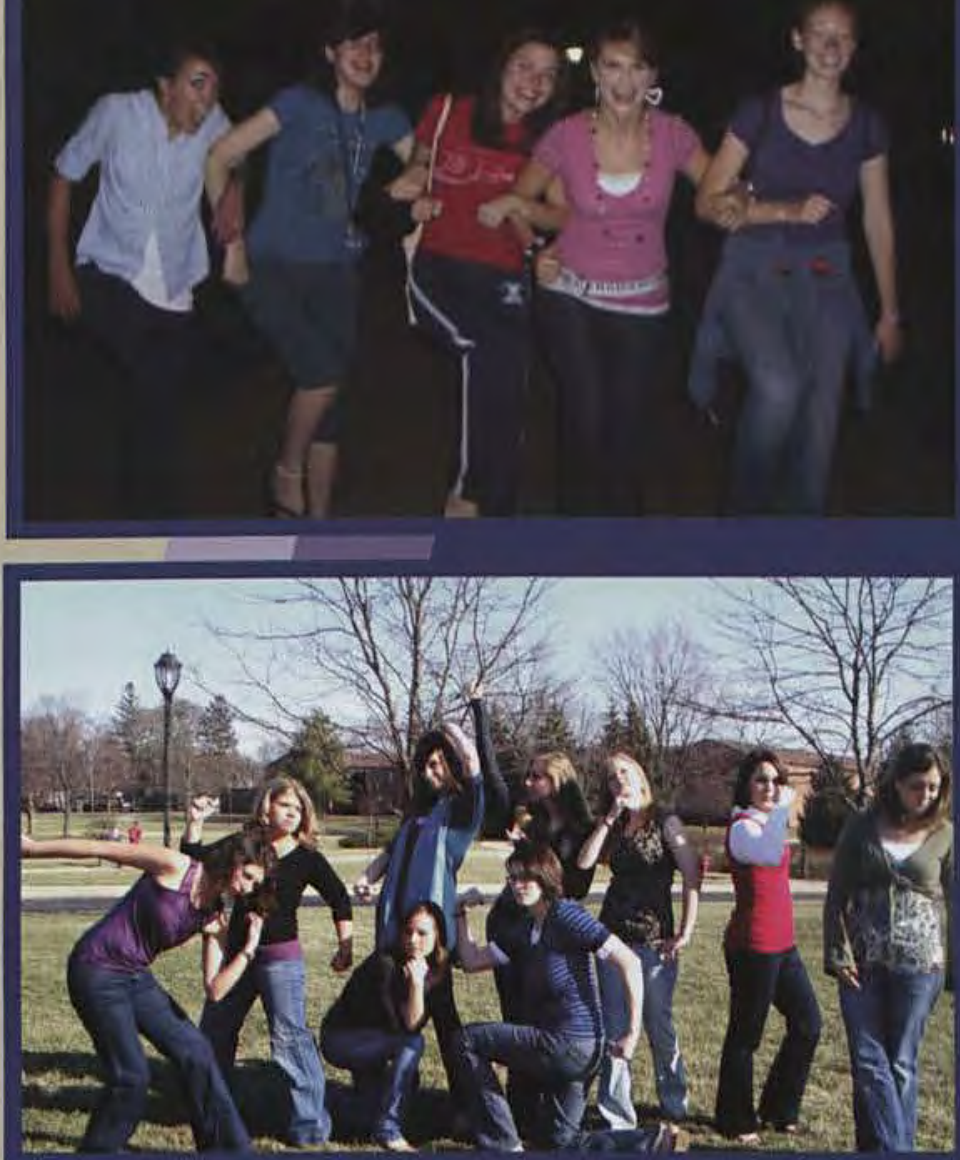

"The first night we all met, Becca set up a photo scavenger hunt for our Bible study to get to know each other. We had a blast taking pictures of us skipping around campus or proposing to random strangers!" says Rebekah Cvetich.

"Doing those Greek word studies has rubbed off on us." says Amanda Roller, as she and her group pose as Greek goddesses. Or try anyway.

A DSGL group gathers in a dorm room to study God's Word.

Tiffany Hendricks, Geneva Banz, and Tiffani Gaston pause for a quick photo before heading out to rake leaves with the Big Bird project. 


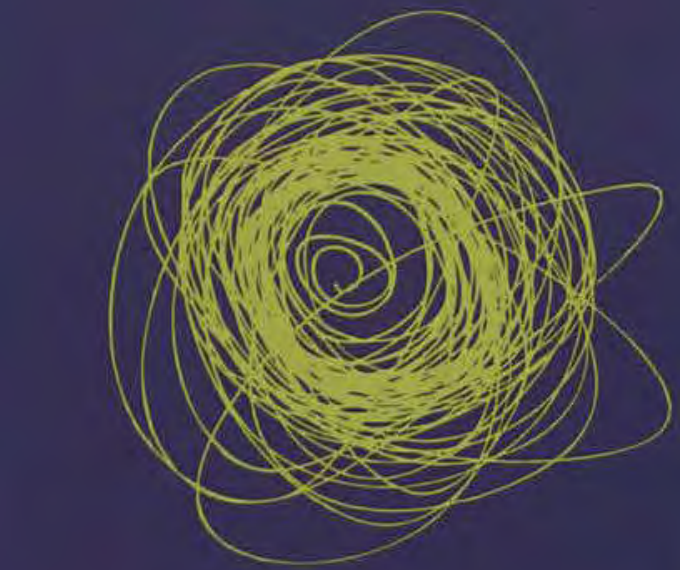




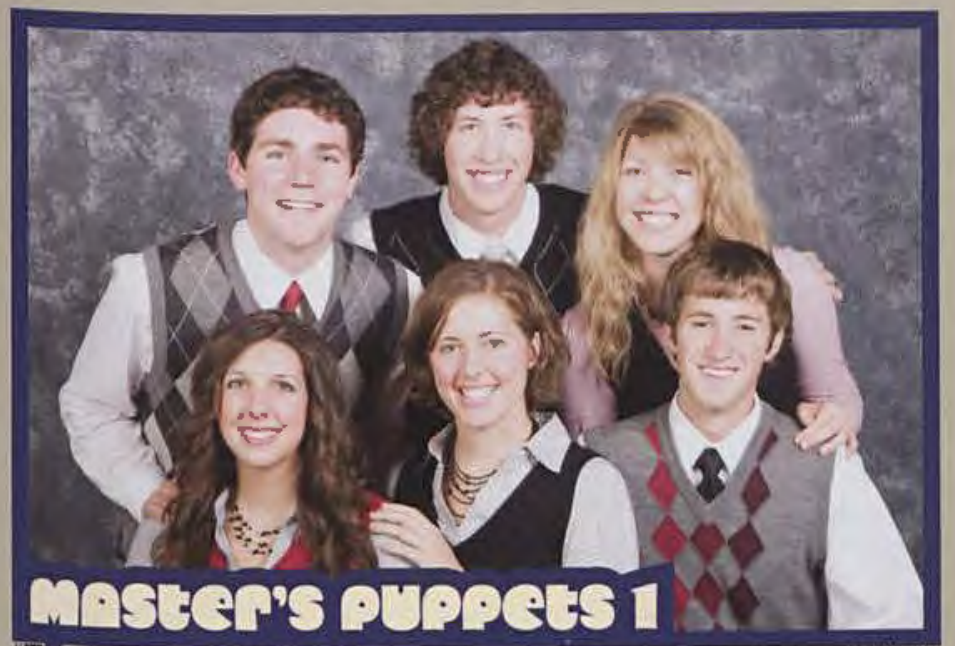

"The puppet team has been such an awesome experience. I think most of all; it has improved my communication skills with people of all ages. Though this is a children's ministry, so much of it is working with adults and teenagers too. I am so thankful for the opportunity to serve." -Chase Baldwin

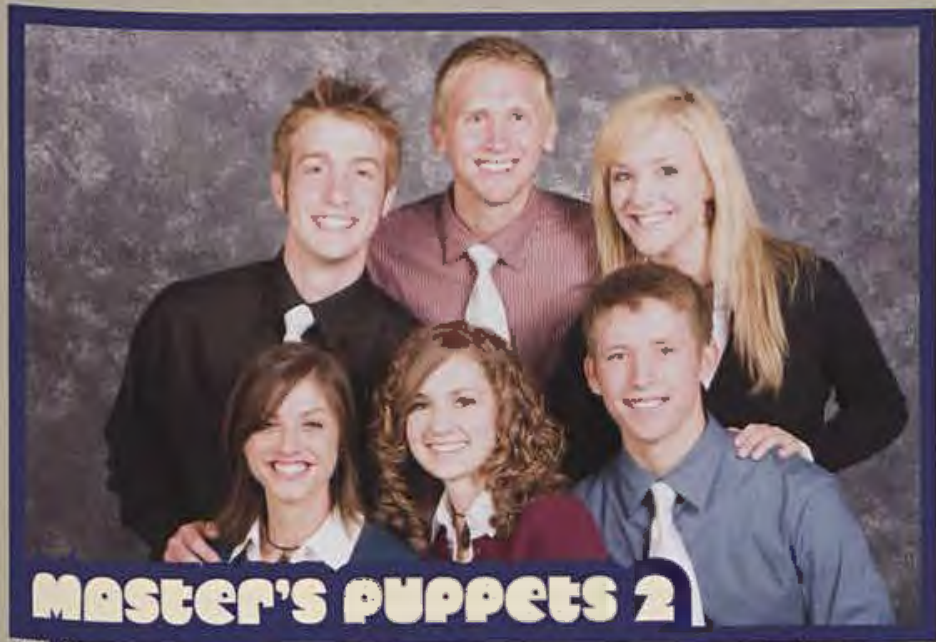

"It's been awesome to see God use this ministry as a bridge to many life-changing relationships. I've had the unique opportunity to serve alongside other believers - both in churches across the country, as well as on my traveling team. It's definitely a part of my Cedarville experience that I'll never forget."-Jonathan Walburg

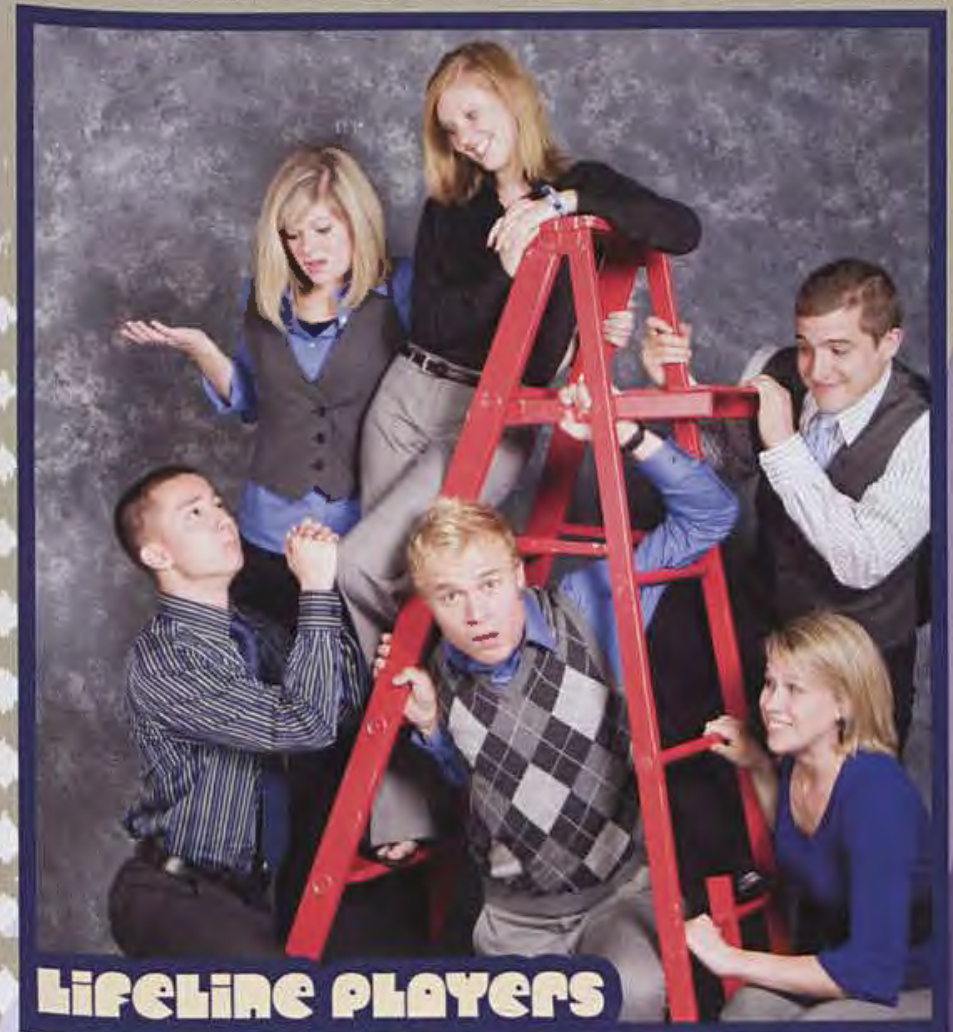

"Drama ministry has been an exciting opportunity to encourage the body of Christ. I'm amazed at the hospitality and generosity each church shows a team of college students they've never met befor -Erin Blackburn.

\section{OP G-member}

Unted in OUR Geren

the neve ef cinpst

impece for mis rins

- Shroe mectron

\begin{abstract}
The Lifeline players lake advantage of their annual retreat in Brownsburg, Indlana, to just have some fun! "Your teammates become vour family. You travel together, perform together, grow together, and minister logether, says JoyAnna McBride.
\end{abstract}

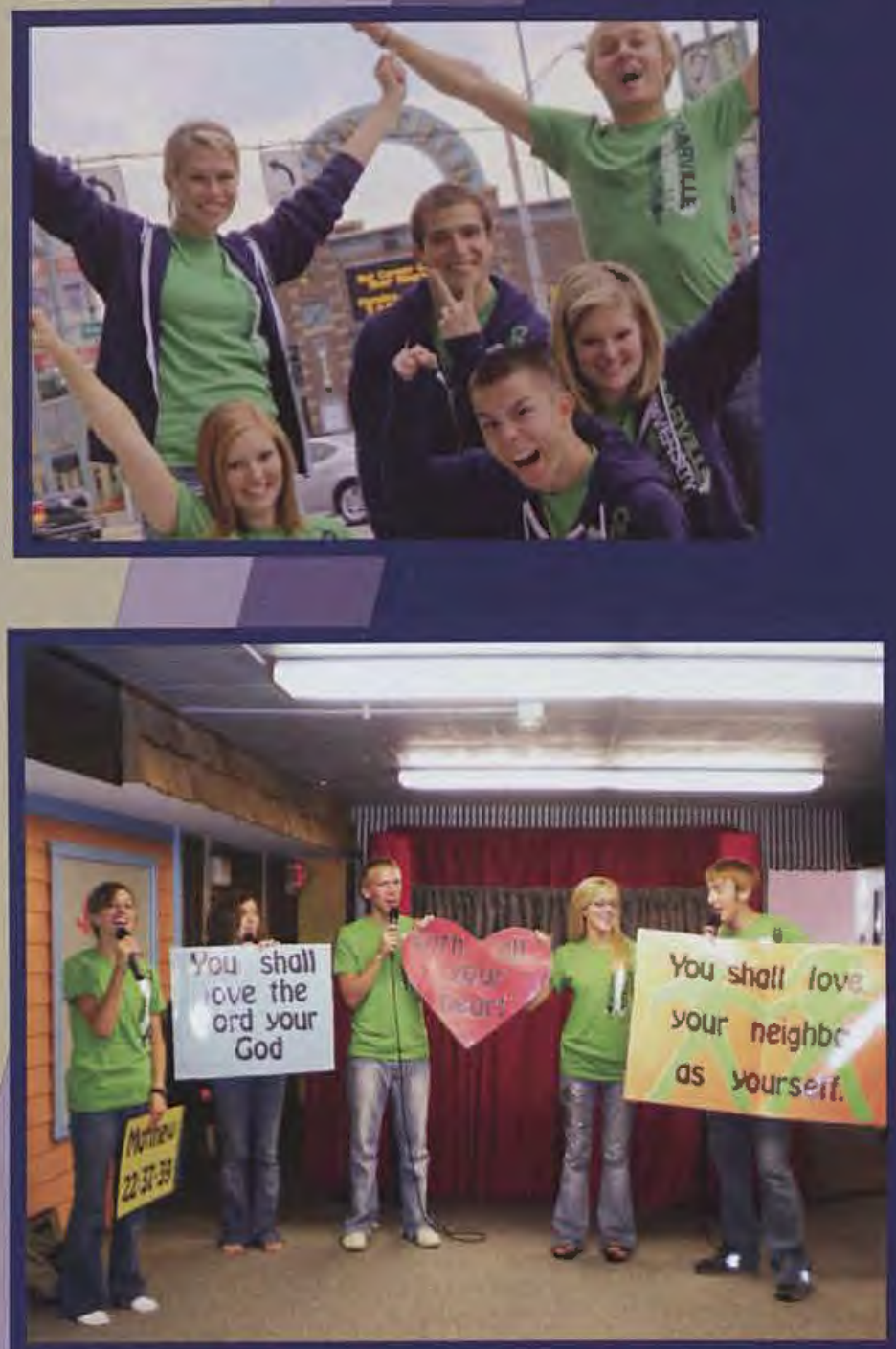

The Master's Puppets team shows they can handle any props in this children's program.

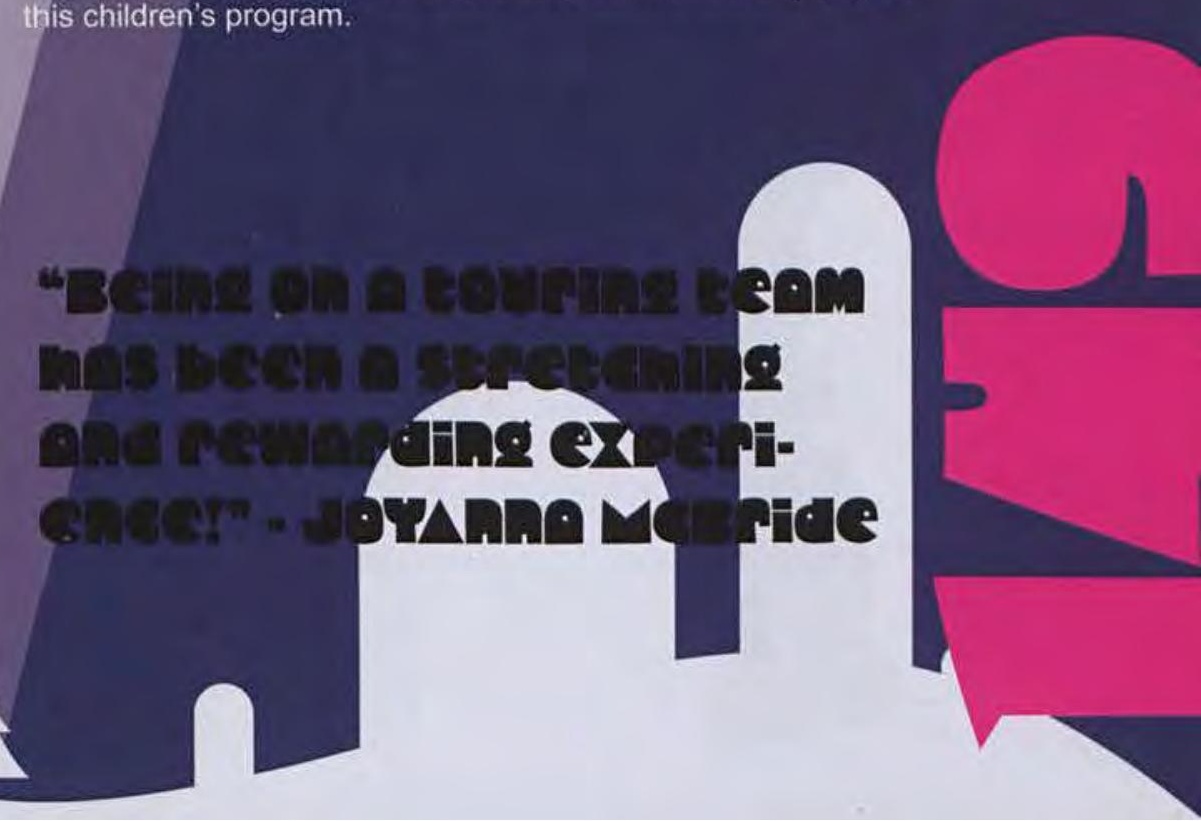




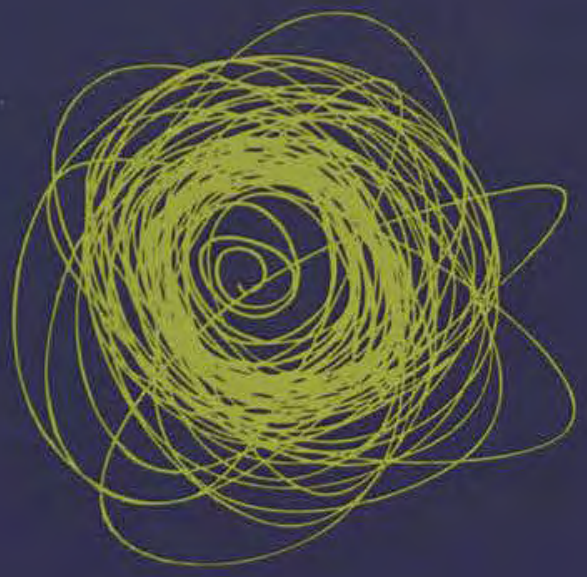

2. 사 갈

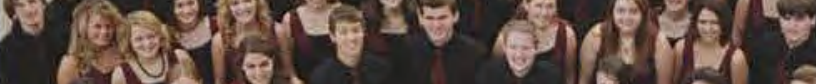

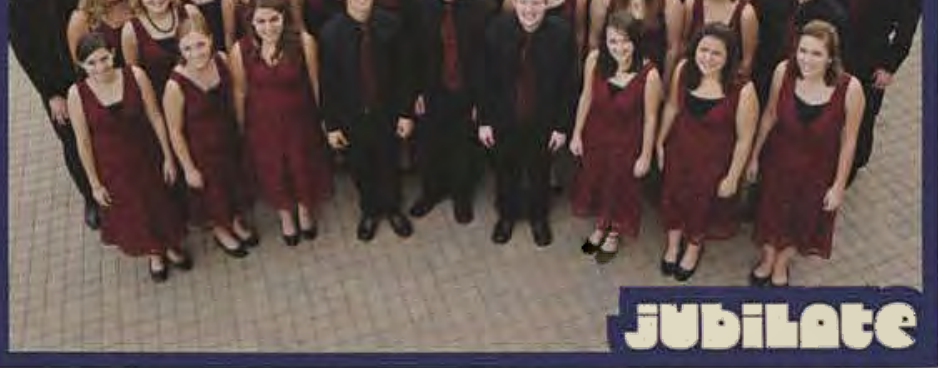
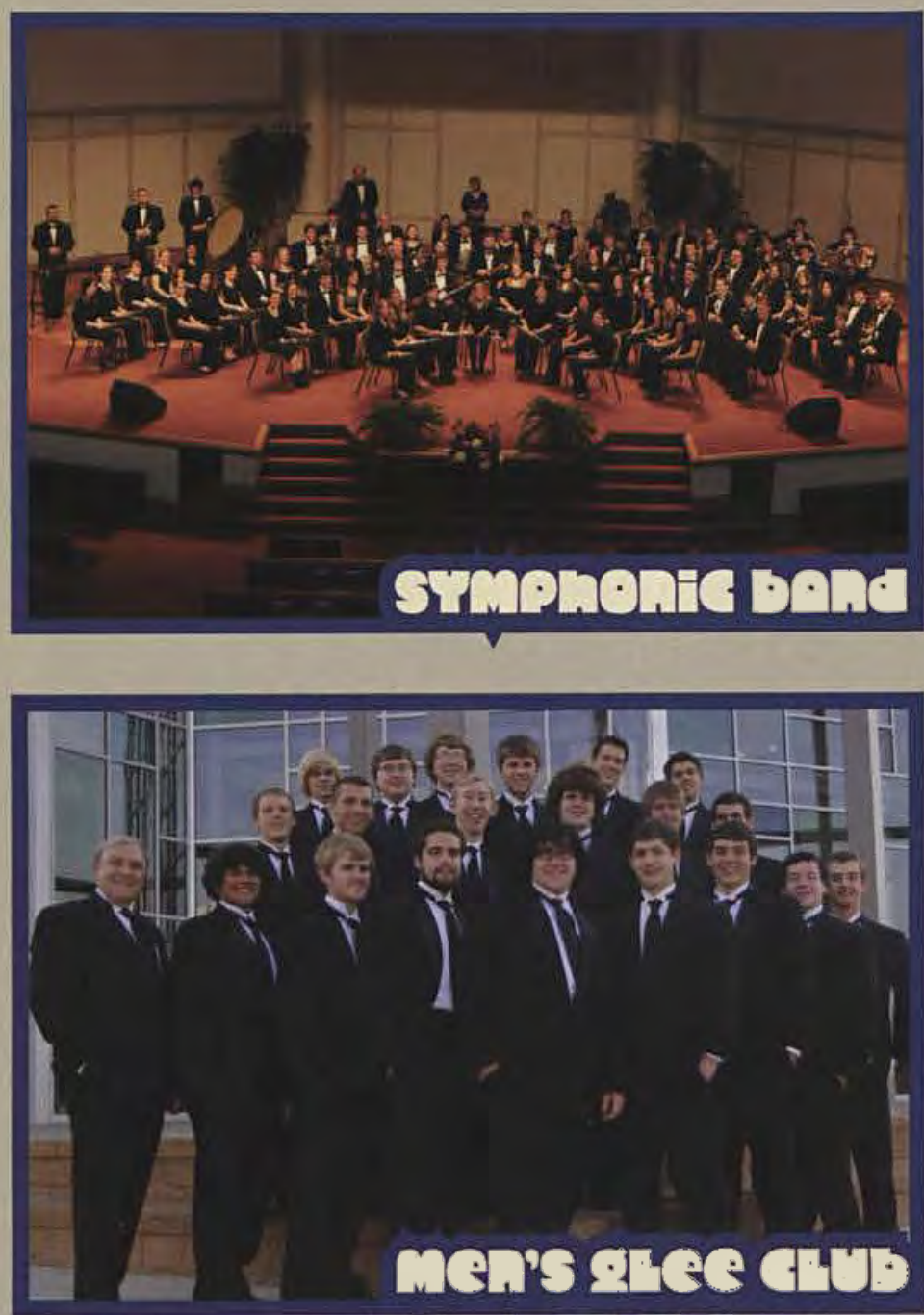
John Carey adds a little pizazz to the symphonic band with the xylophone.
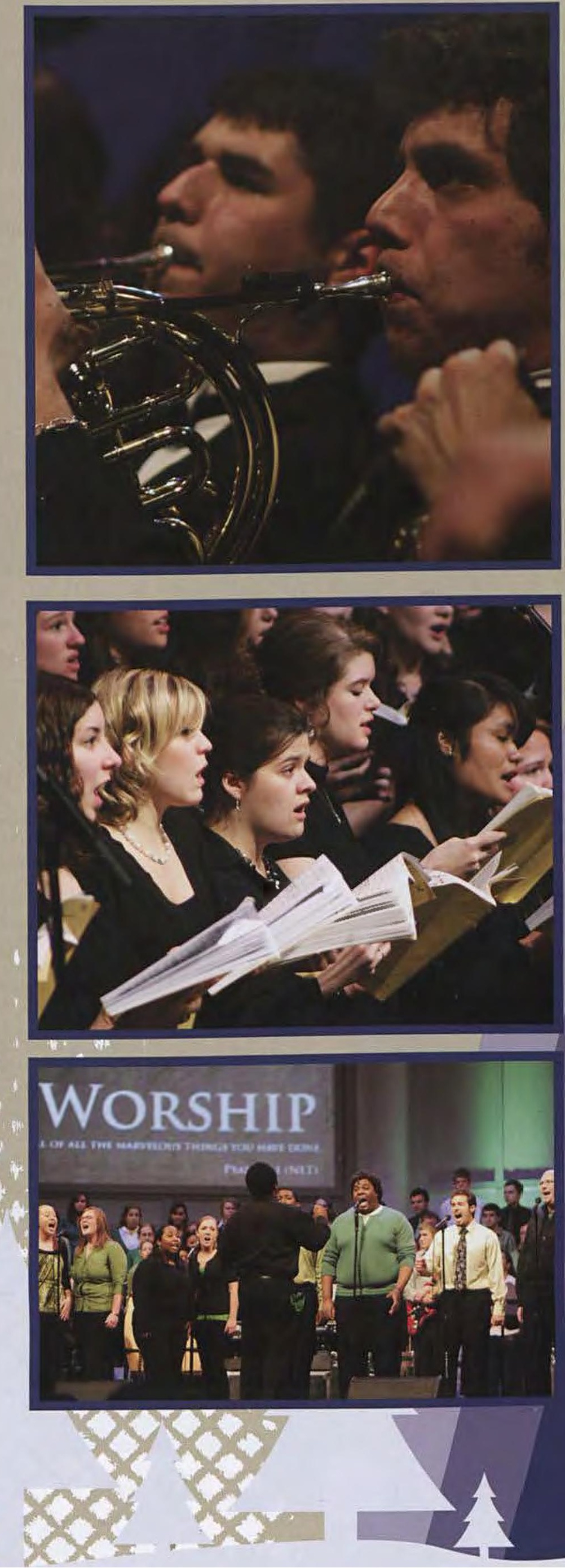

d
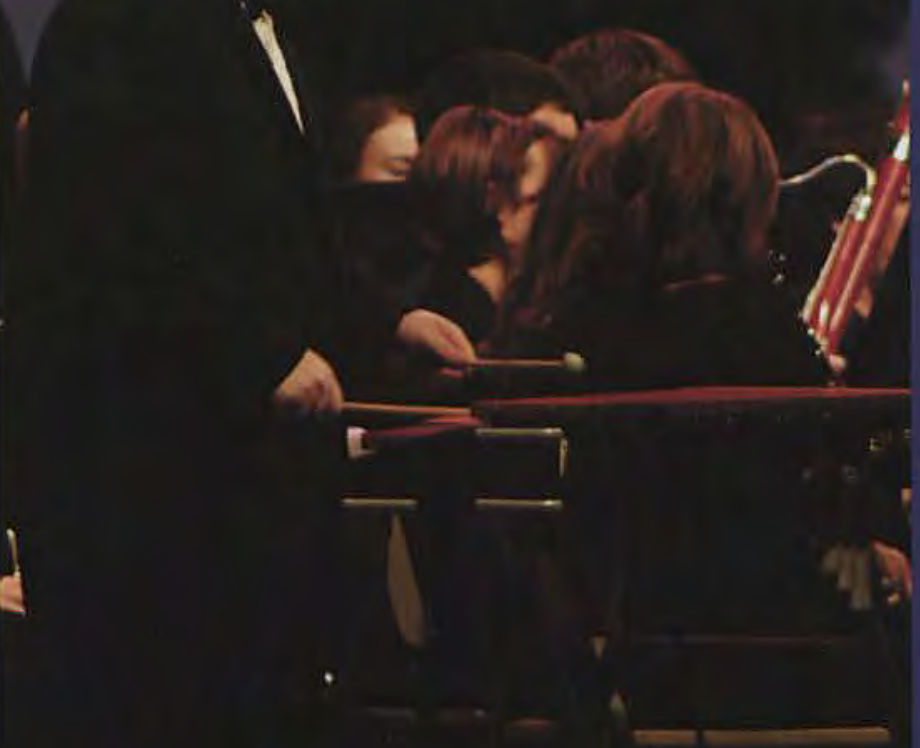

We are a family that comes together to praise an Almighty God and to be a light for Him through the gift of music," reflects Lena Molby.

"One Voice is an awesome time to get away for the melee of life and just praise God in a relaxed and chill atmosphere," comments JC Carmichael. 

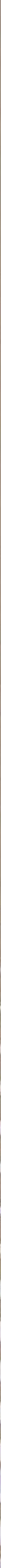
Matt Fawcett concentrates on playing his piece perfectly.
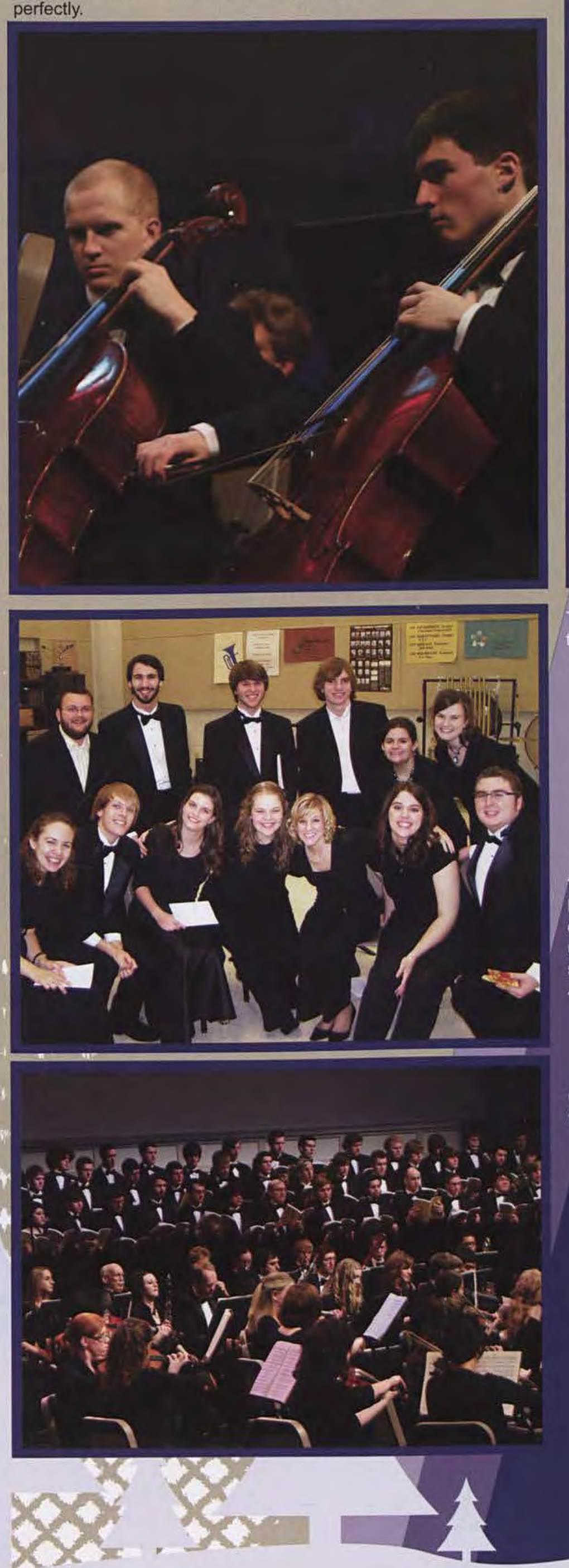

Two Cedarville men serenade the audience with their cellos.

After the Masterworks performance of Mendelssohn's Elijah, which included all choral ensembles and the University orchestra, a group of us who were caught-up on the "post-concert cloud" stayed and fellowshipped for hours afterwards," says Julie Pickens.

Cedarville's many talented musicians often come together with impressive collaborations. 

to hear more about the students' personal lives. As a teacher, I look forward to hearing about what all my little second graders are experiencing and learning. One cherub student, a little girl with a thick hill-billy accent, excitedly raises her hand to share exactly what she did over the weekend.

"Well, my cousin is pregnant and she's real big, and she went to the bathroom this weekend, and she looked down, and the feet were hanging out! She had to go to the hospital and they pulled a baby out! It was great!"

Such is an everyday experience during my teaching field esperience. Where I taught, students are not required to wear shoes or dress a certain way because the teachers do not want to interfere with various learning styles. I was interested to see what kind of cultural divide would exist between Cedarville and this community in the elementary classroom, and I am discovering that it is even more drastic than I originally anticipated. We use a wind chime to get the students' attention so that I feel like Mother Nature. Discipline and classroom management are considered to be hindrances to learning, so the classroom often seems more like a zoo of second graders than an academic endeavor. Yet, I learn a lot from the students and teachers: namely, compassion.

They are all very unique students, delicate and yearning for praise. They are searching for who they are and attempting to define themselves. This experience is teaching me a lot because it is so different from anything I knew or anything Cedarville could have prepared me for. It is very humbling to be criticized. I am in this weird middle ground where I am a student and learning and yet also desire some sense of competence as a teacher. Dissolving my pride and being able to take that constructive criticism is a good lesson for me to learn.

It's nice to know I have a lot of field experiences in which to refine my skills. Junior year is such a fun year because I am finally getting into it, but am not yet held fully accountable, so the pressure isn't as intense, allowing me to learn, stretch, and grow. I don't teach the whole day, only two lessons, so this allows me time to observe and learn. Some of my responsibilities include phonics activities, read-a-louds, writing workshops, transitioning times, and guided reading with different focuses.

Most of all, this junior year field experience has made me excited about student teaching. It's so good to be in the classroom, especially when I know this will be my little home for however many years I'll teach. By this point, I know through prior experiences that I want to be a teacher, so field experience is more a confirmationthan an indicator that this is where I want to be.

I eagerly anticipate my upcoming student teaching internship, yet I doubt that anything could exceed my diverse experiences thus far. 


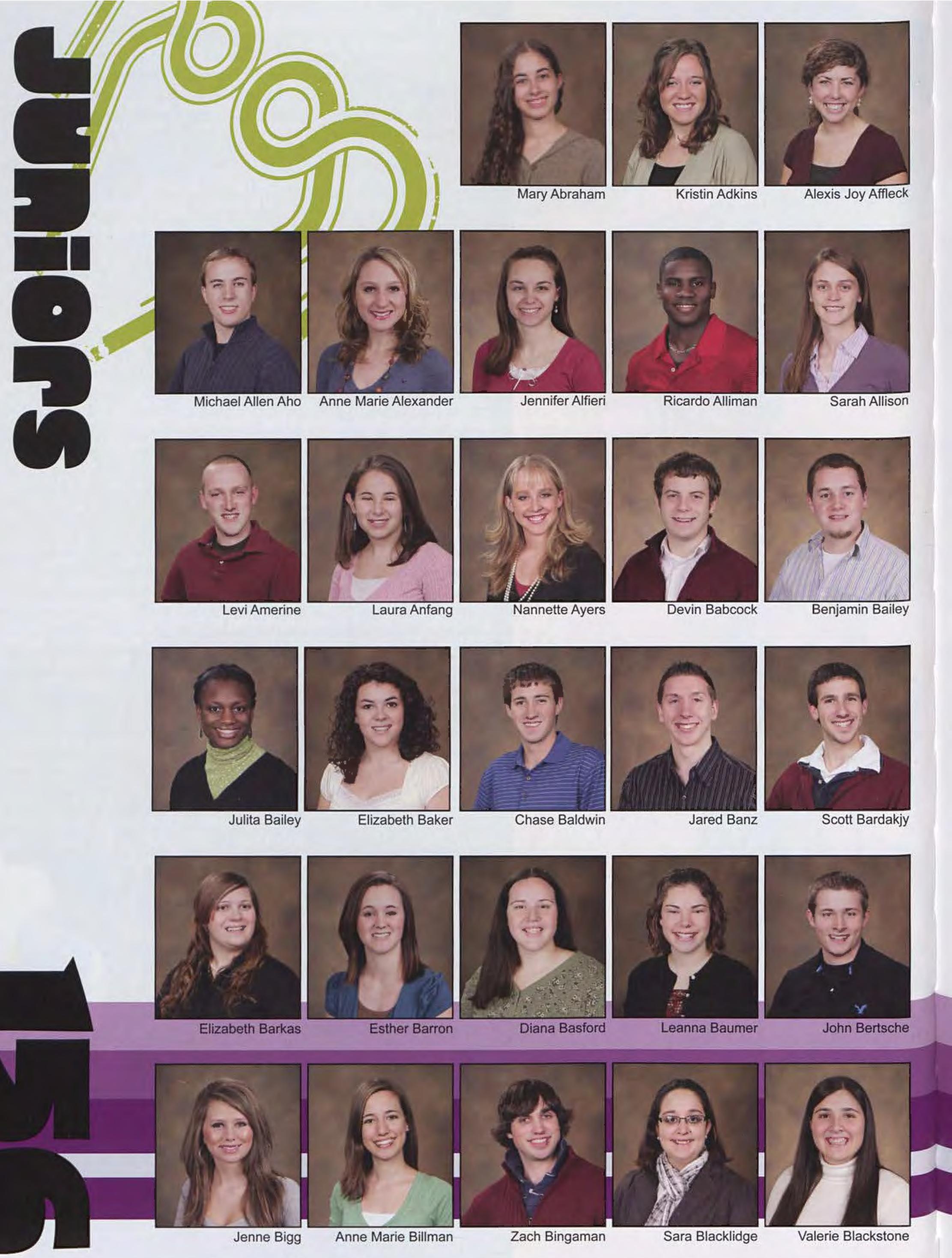




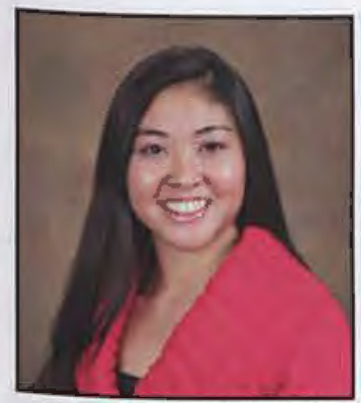

Katie Bliss

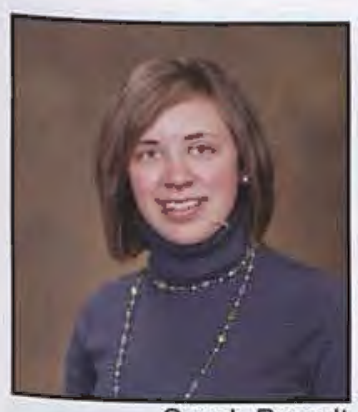

Sarah Brandt

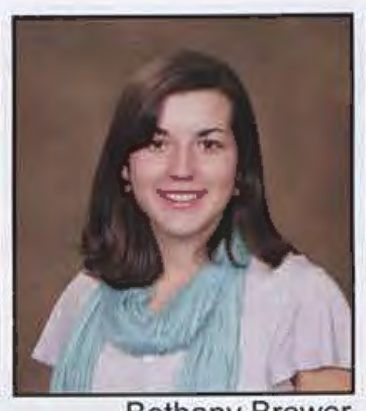

Bethany Brewer

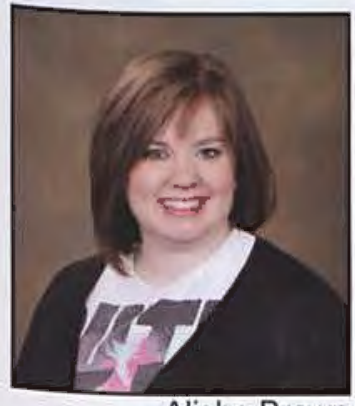

Alisha Brown

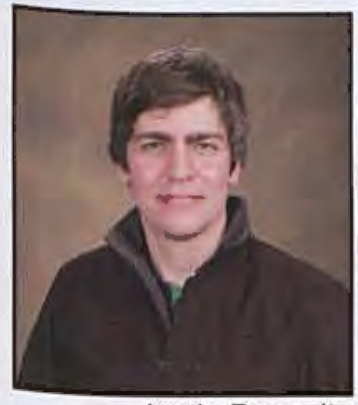

Justin Busenitz

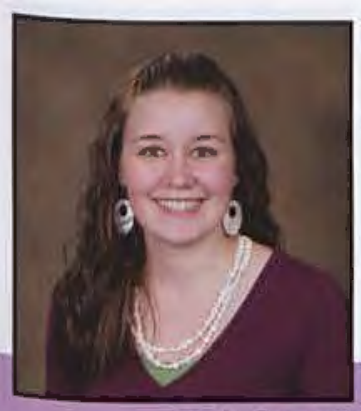

Leah Casper

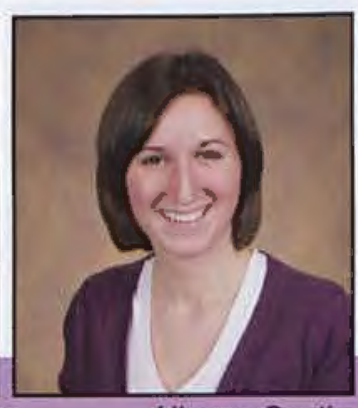

Allyson Castle

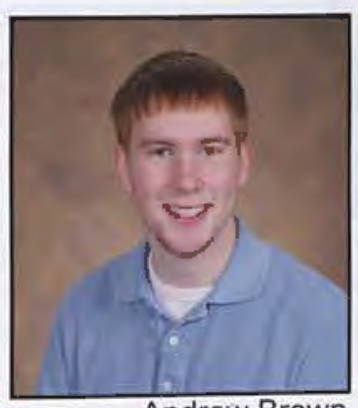

Andrew Brown

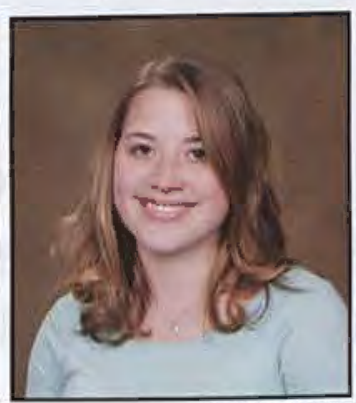

Kayla Butz

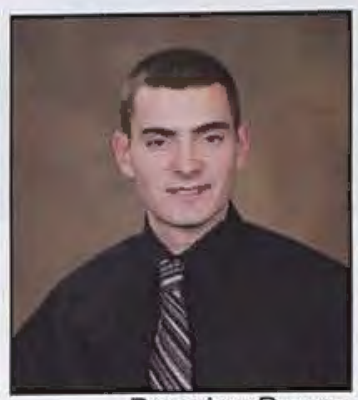

Brandon Brown

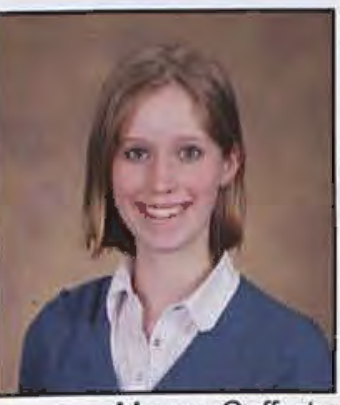

Megan Cafferty
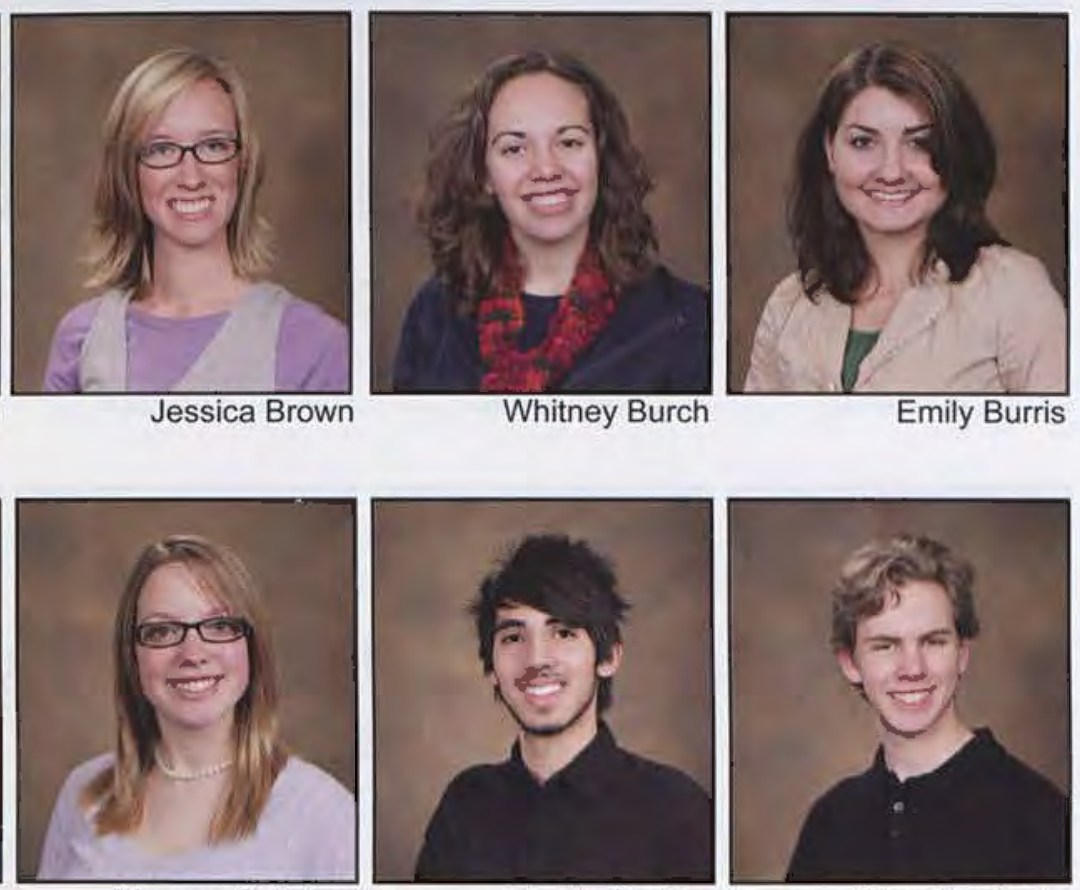

Morgan Callahan

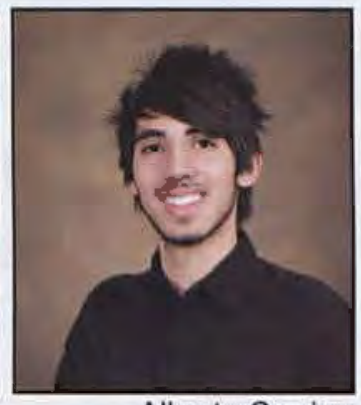

Alberto Carrion

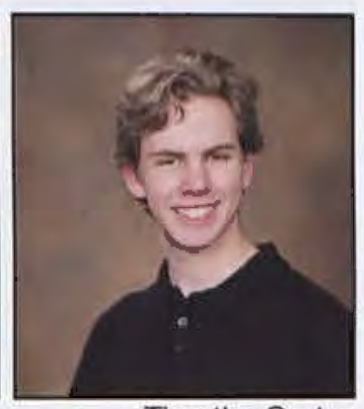

Timothy Carter

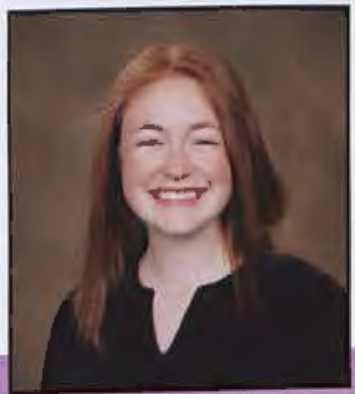

Hannah Cathers

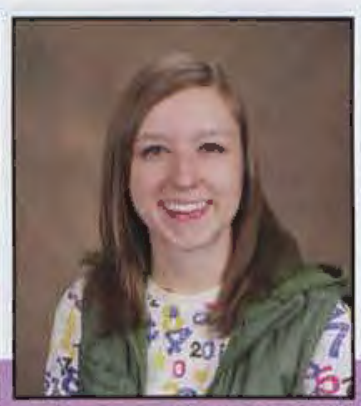

Catie Caudle

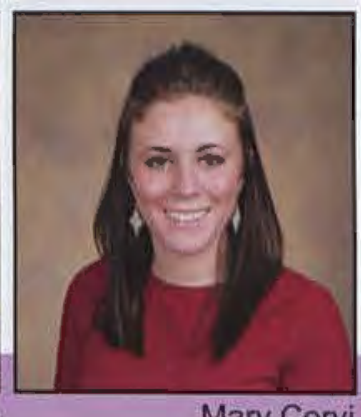

Mary Cervi

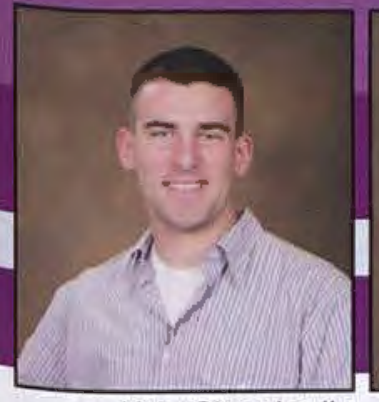

Kyle Chamberlin

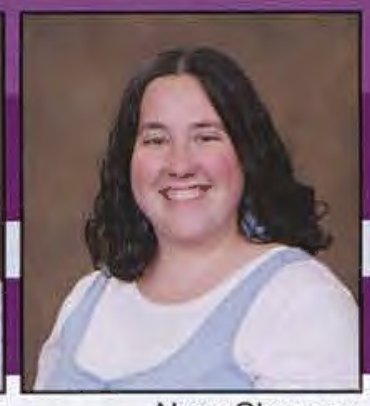

Nora Chasnov

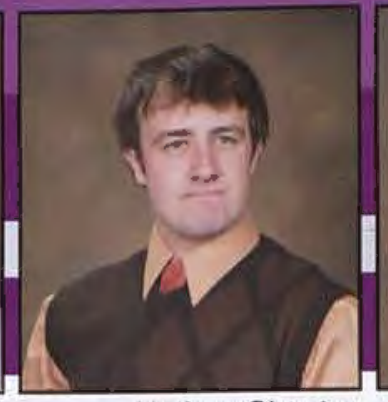

Nathan Chester

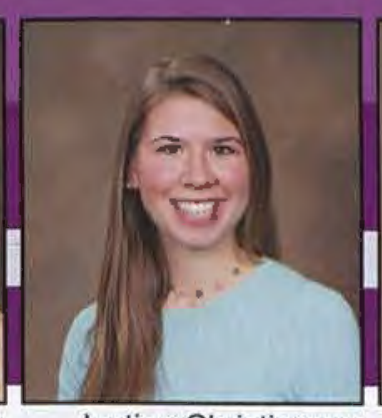

Justine Christiaanse
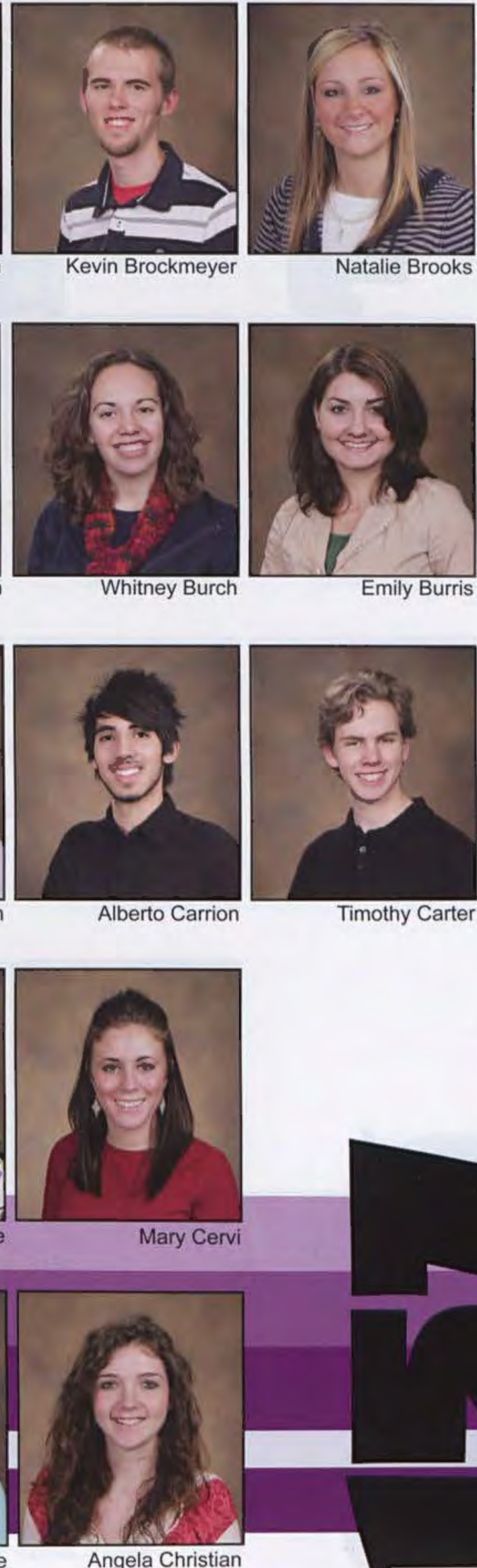

Natalie Brooks

Emily Burris 


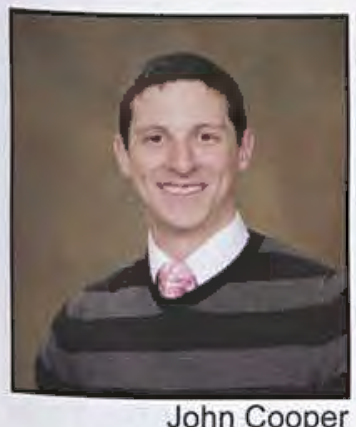

John Cooper

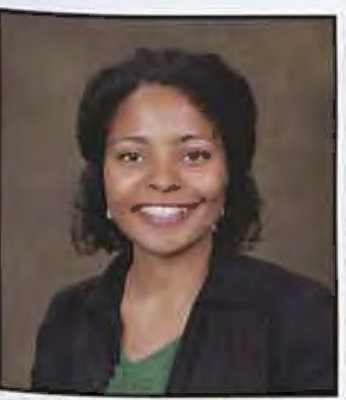

Priscilla David

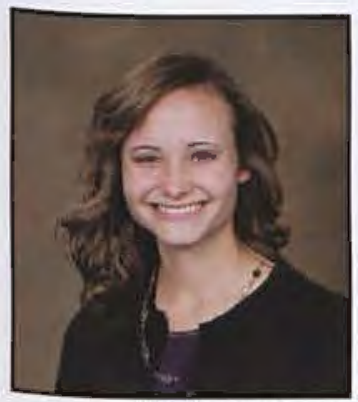

Tricia DeKorne

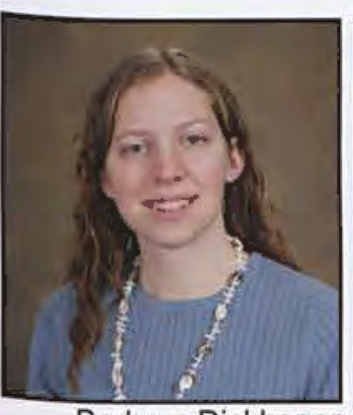

Barbara Dickhoner

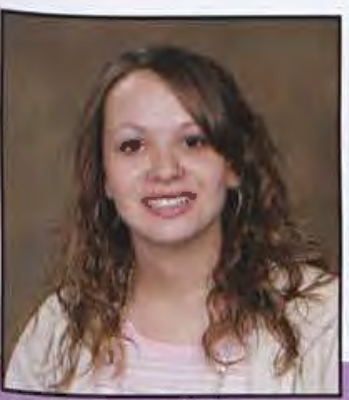

Dorianne Eaves

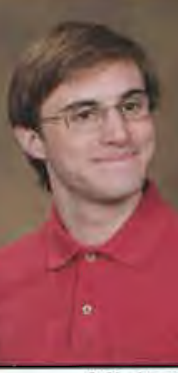

Michael Cox

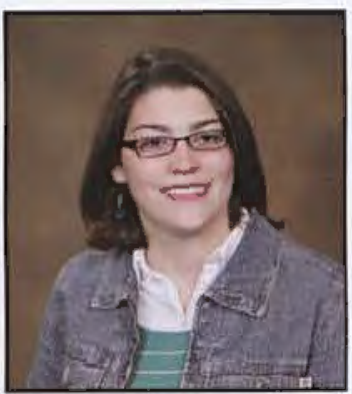

Courtney Davis

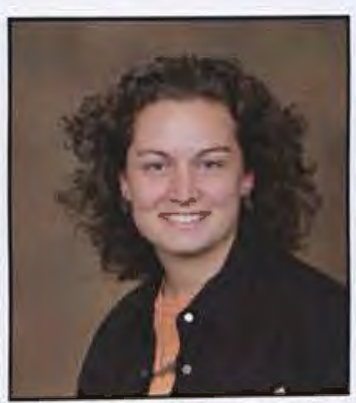

Kristen DeLong

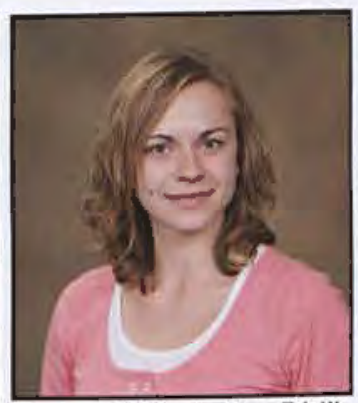

Yekaterina Didik

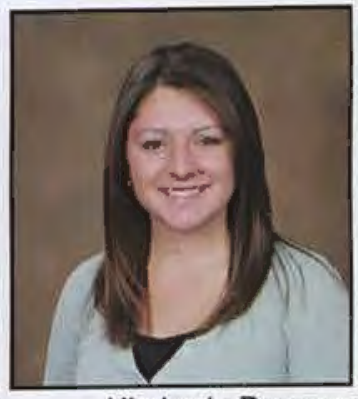

Kimberly Deseno

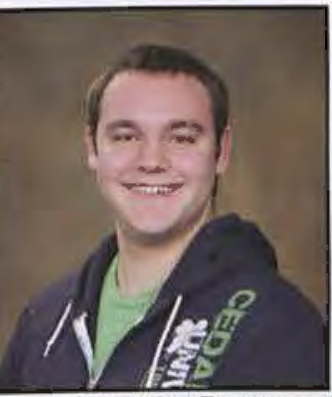

Timothy Doenges
Rachel Cox

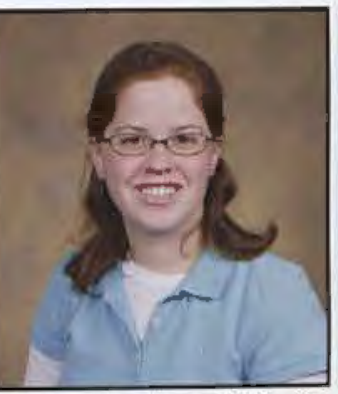

Jewell Davis

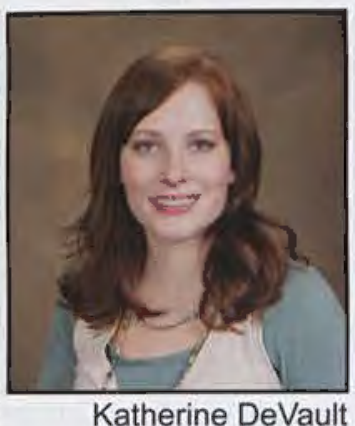

Katherine DeVault

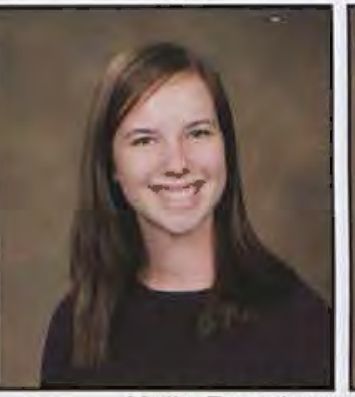

Kelly Donohue

Lisbeth Cummings

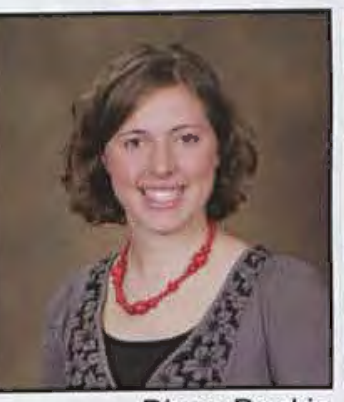

Diana Deakin

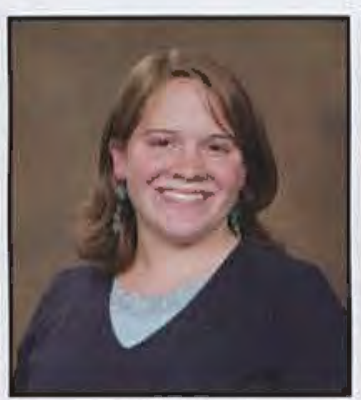

Carolyn Deemer

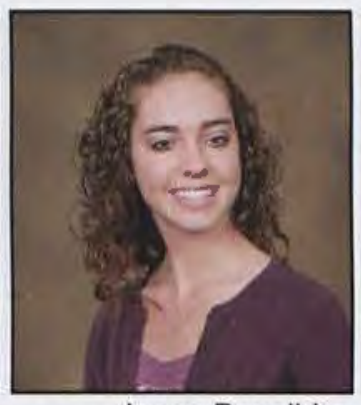

Jenna Develbiss

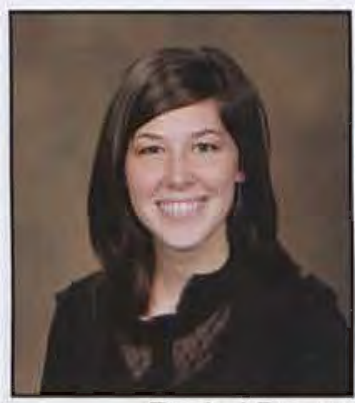

Rachel Duarte

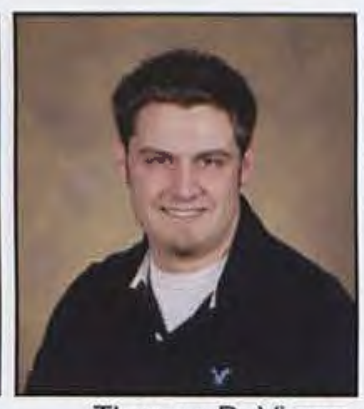

Thomas DeVinney
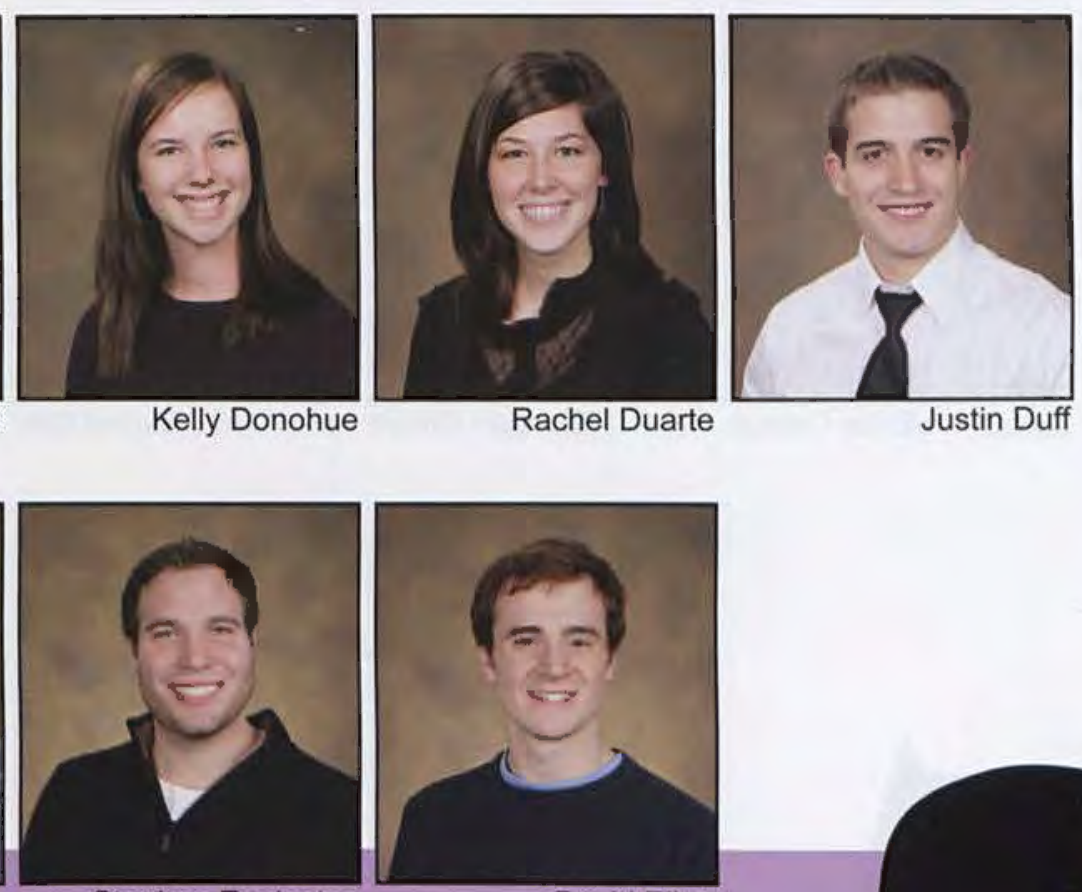

Stephen Eggleston

Meredith Edwards

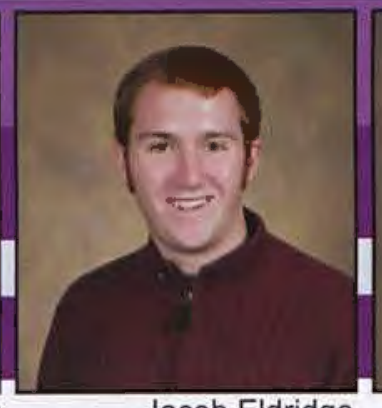

Jacob Eldridge
Sarah Egenreider
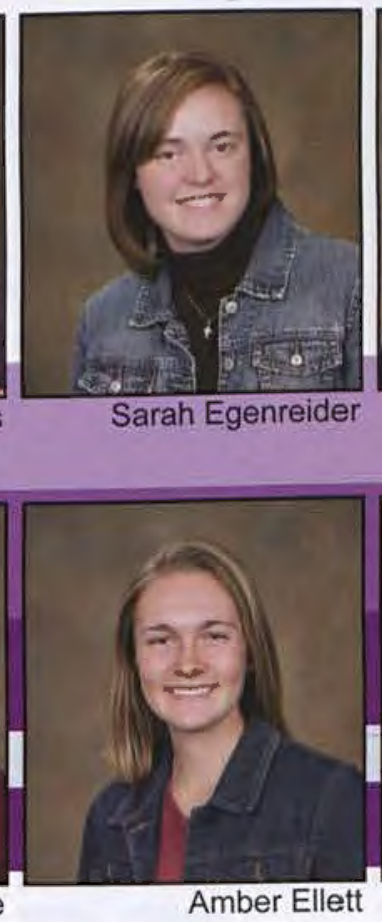

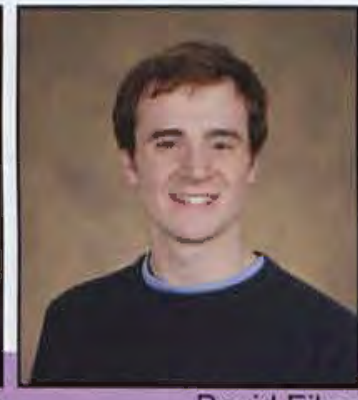

David Eiker
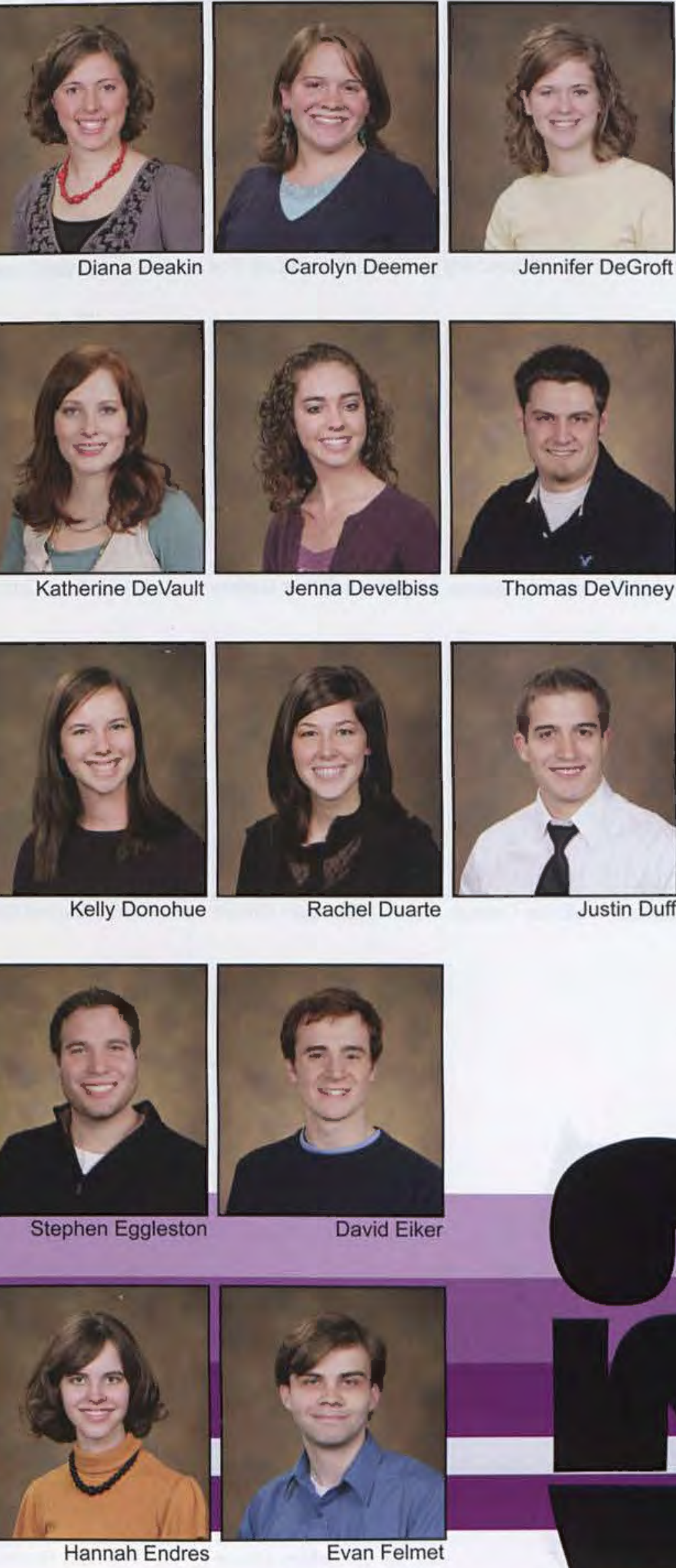

Jennifer DeGroft

Justin Duff

Hannah Endres 

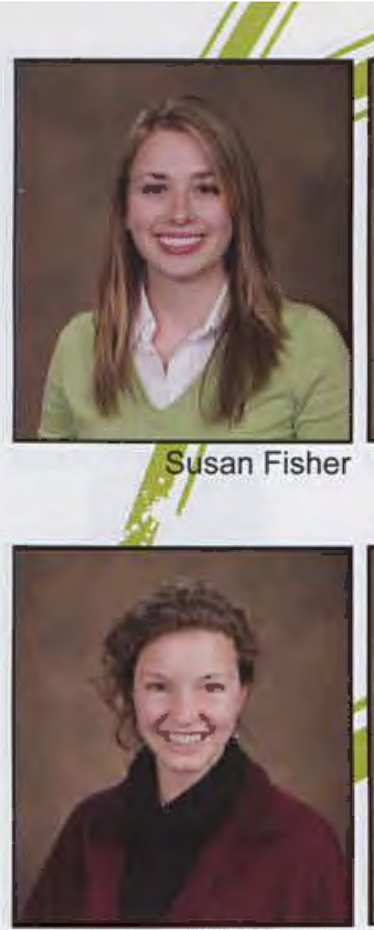

Jill Forsberg

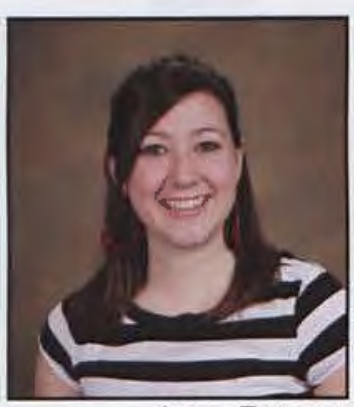

Anna Futoran

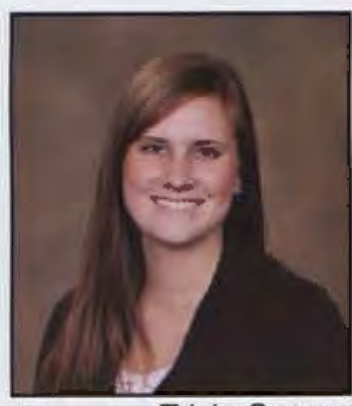

Tricia George

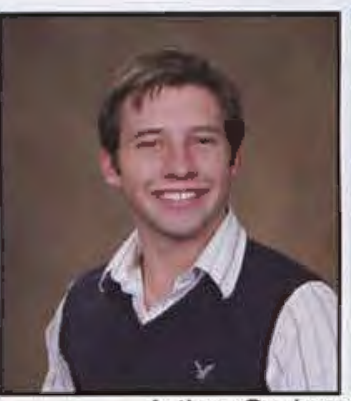

Arthur Garlow

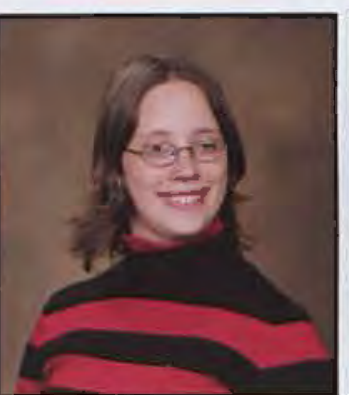

Rebekah Gifford

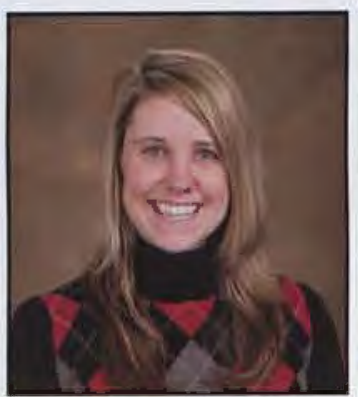

Amy Garner
Dan Floren
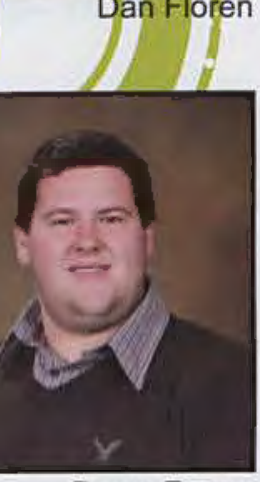

Bryan Frame

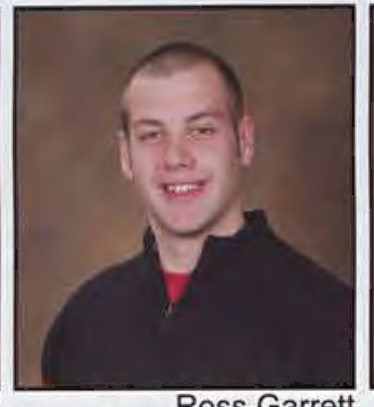

Ross Garret

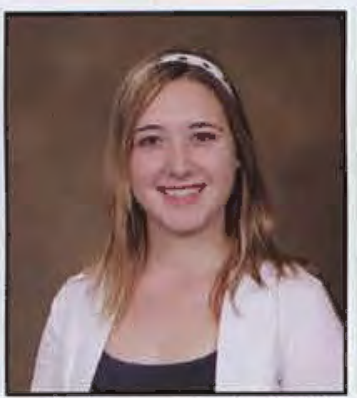

Rachel Gilin

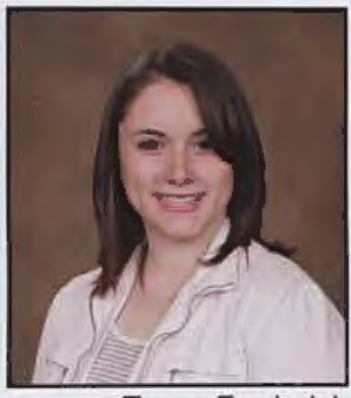

Tanya Frederick

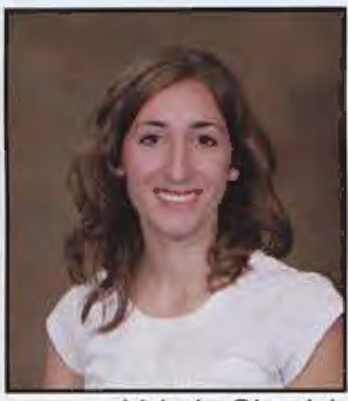

Valerie Gingrich

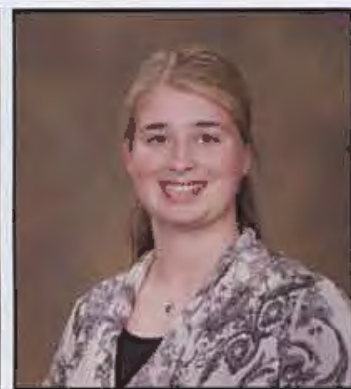

Martha Flow

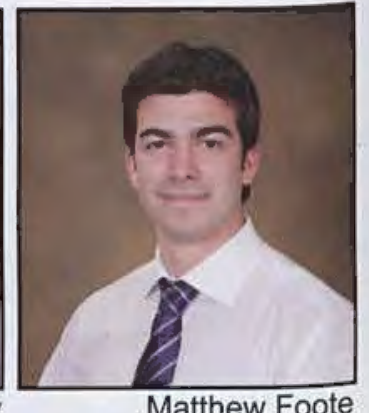

Matthew Foote

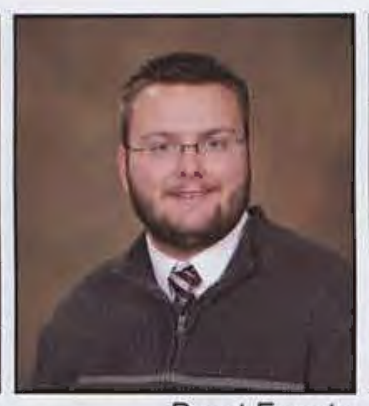

Brent Fugate

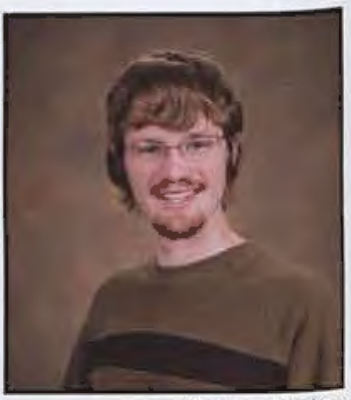

Dalen Fultz
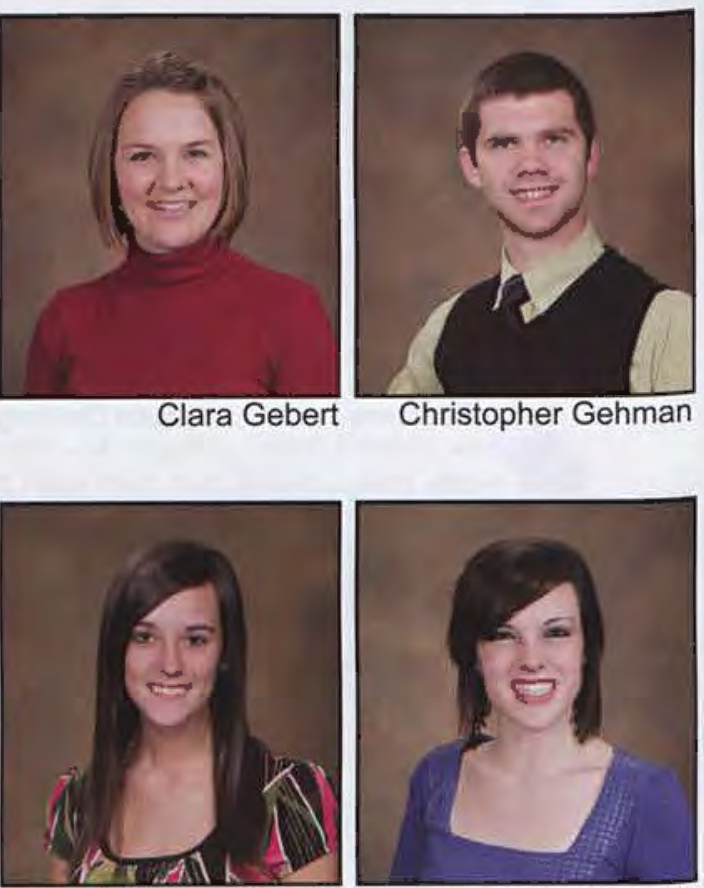

Bethany Godwin

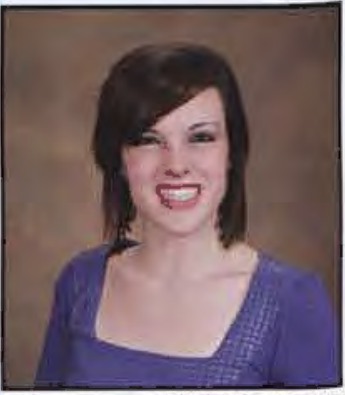

Erin Gollihue

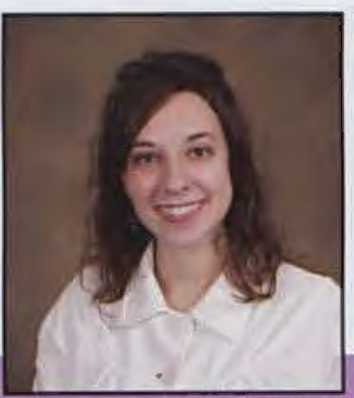

Erika Goodrow

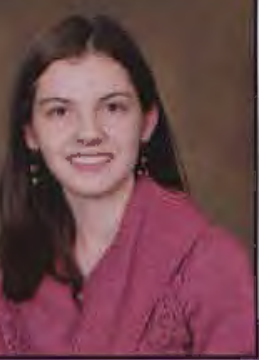

Amanda Graves

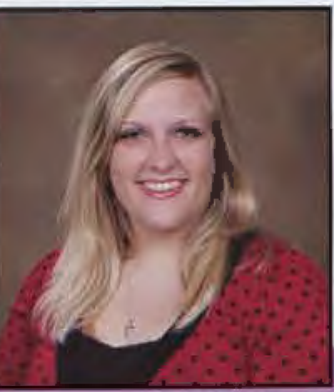

Lisa Gray

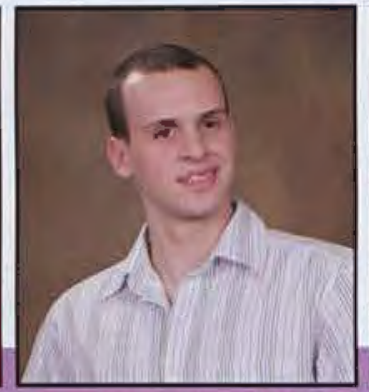

Stuart Green

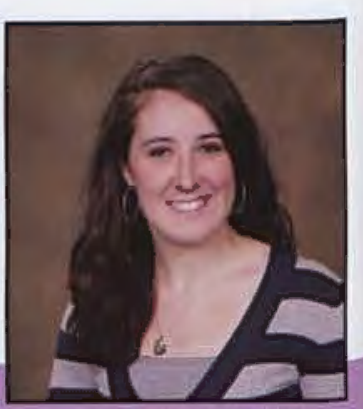

Rebecca Gregg

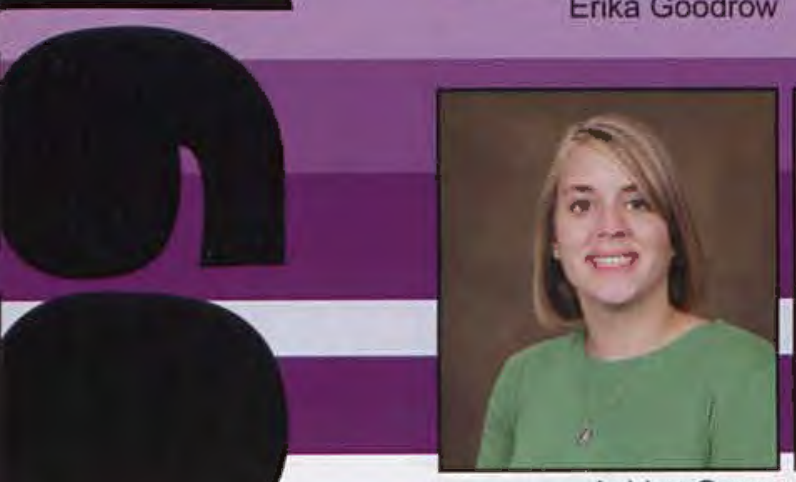

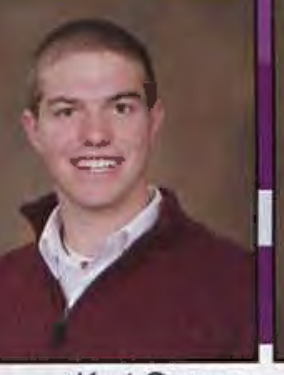

Kurt Groman

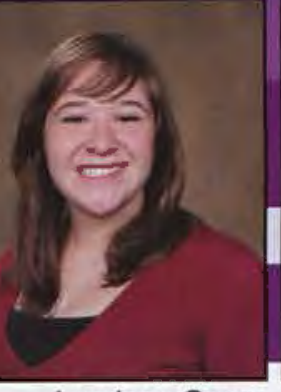

Lyndsey Gvora

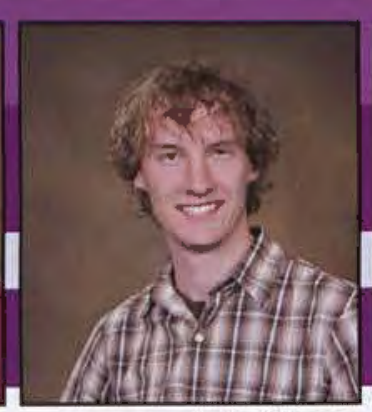

John Hagen

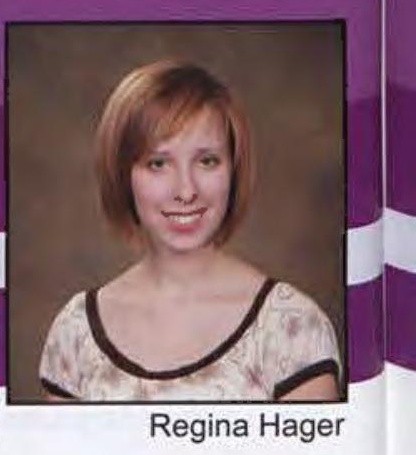




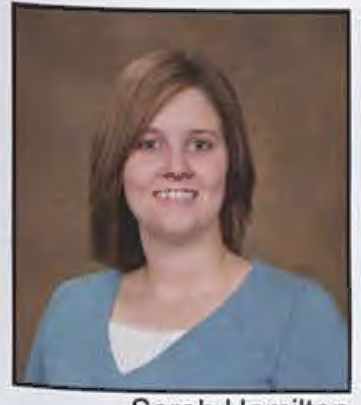

Sarah Hamilton

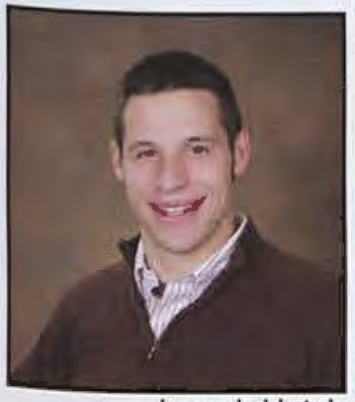

Joseph Hatch

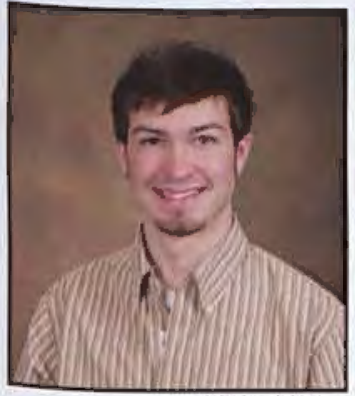

Kyle Hess

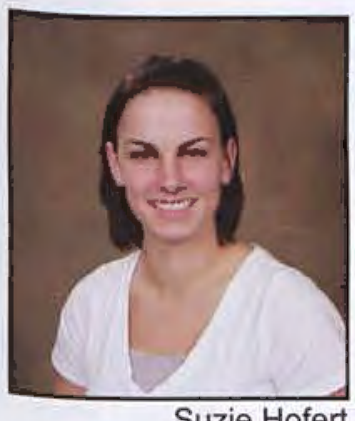

Suzie Hofert

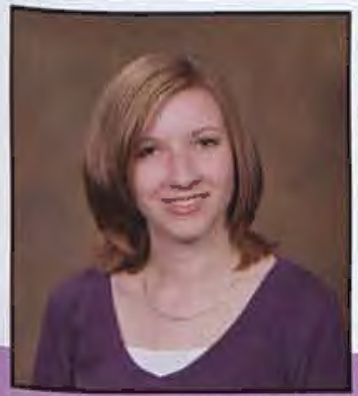

Caitlin Hull

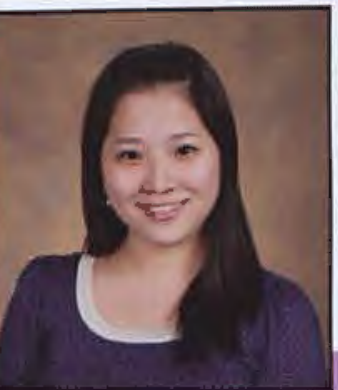

Jungah Hyun
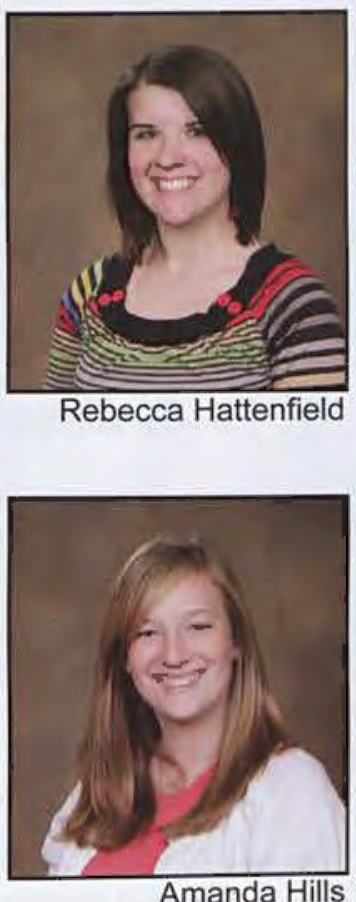

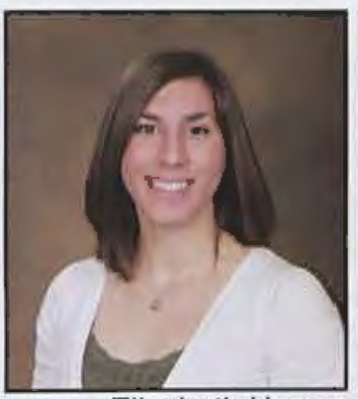

Elizabeth Hanna
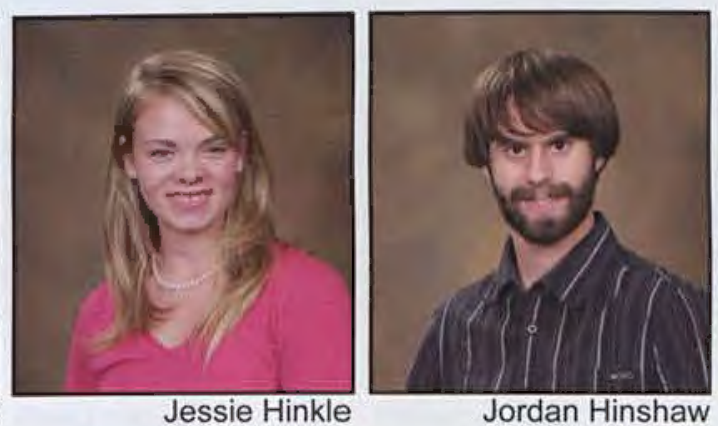

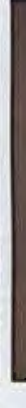

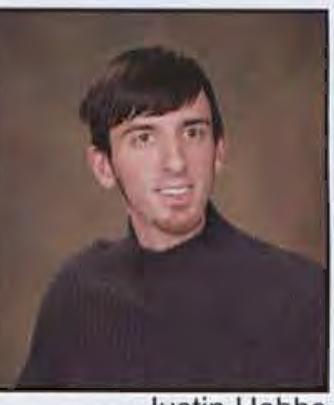

Justin Hobbs
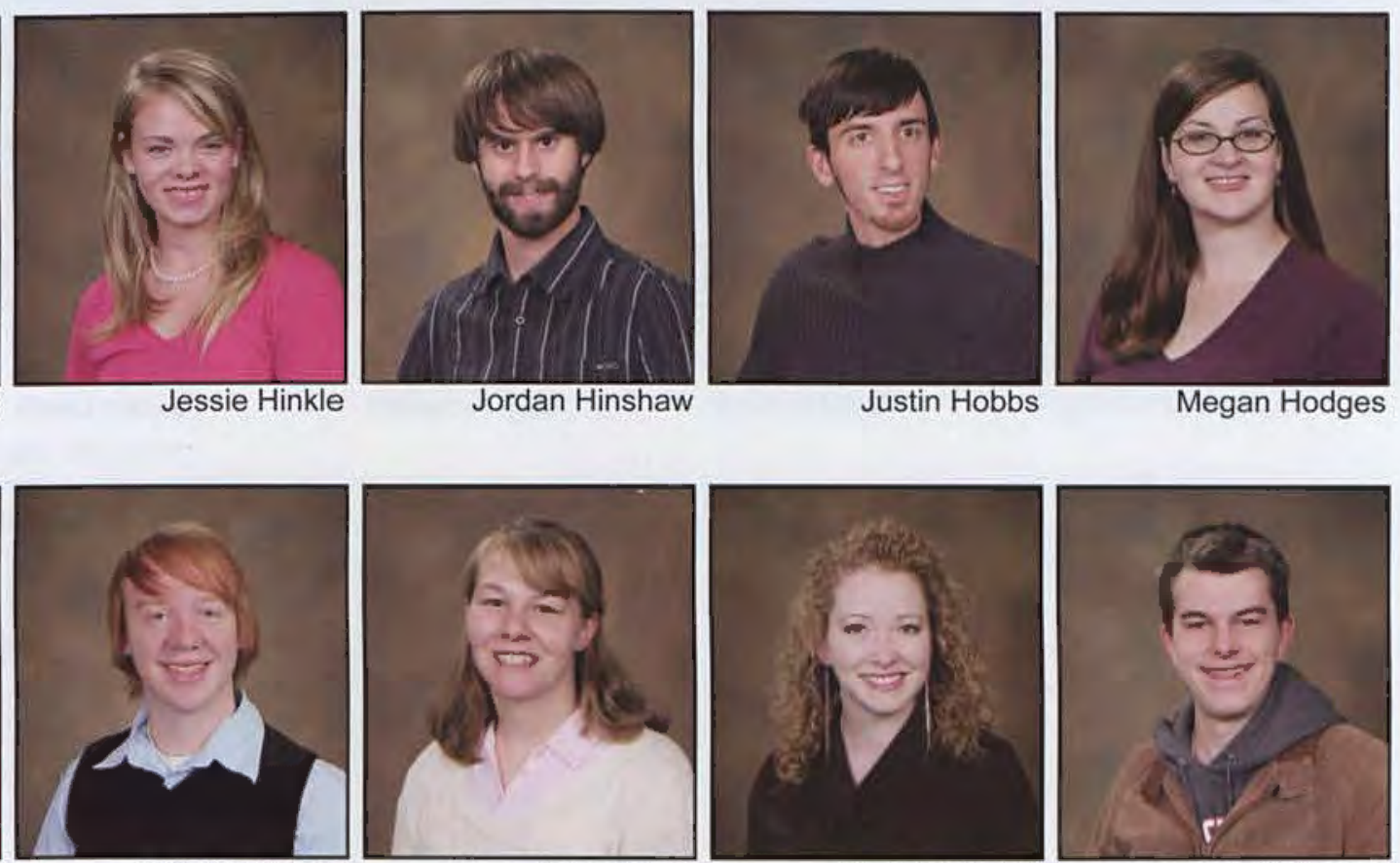

Grant Holloway

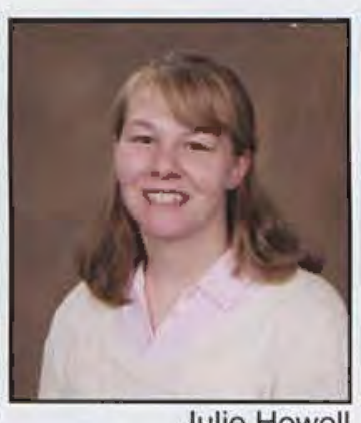

Julie Howell

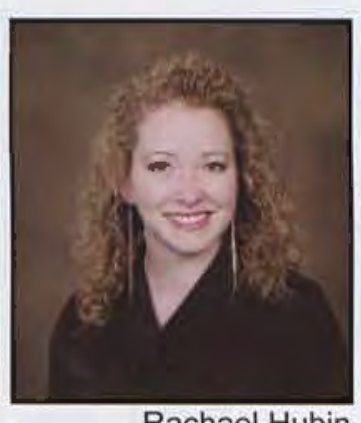

Rachael Hubin

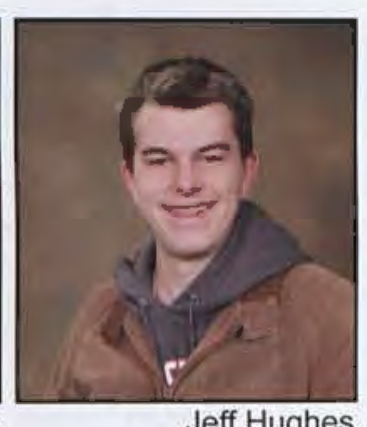

Jeff Hughes

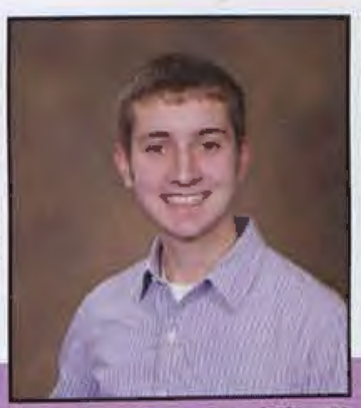

Brandon Inkrott

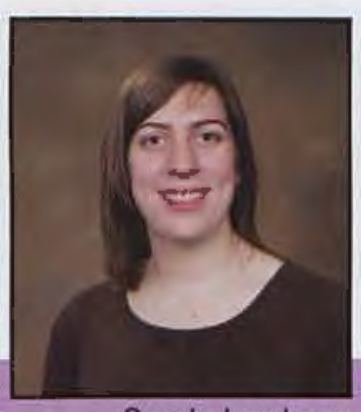

Sarah Jacobson

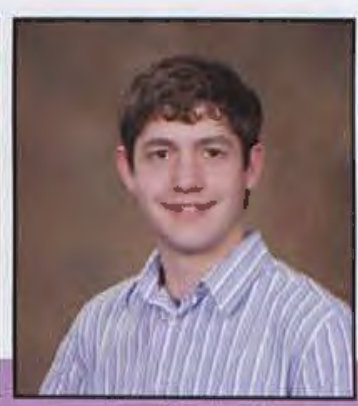

Kevin Jenson
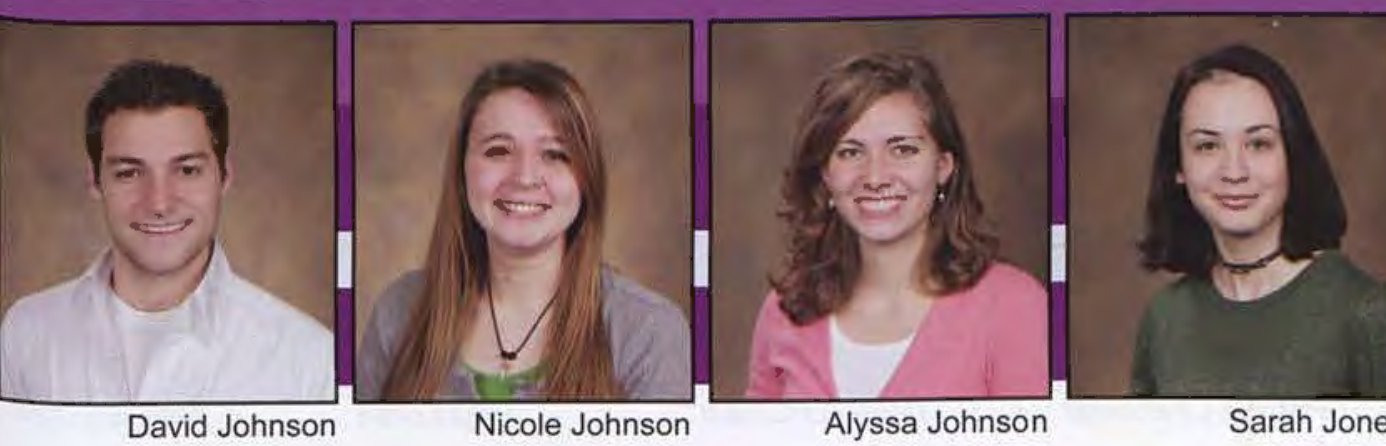

Sarah Jones

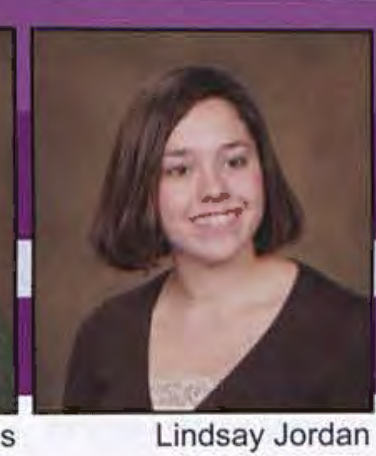




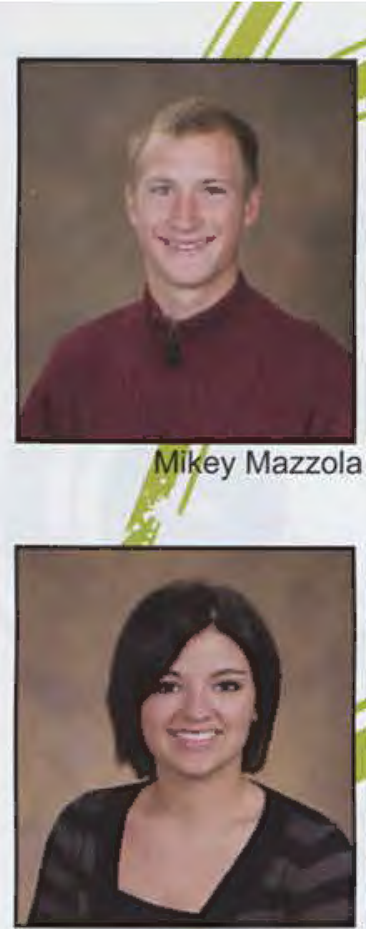

Callie McInturf

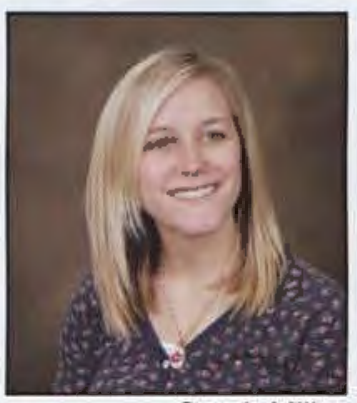

Sarah Miller

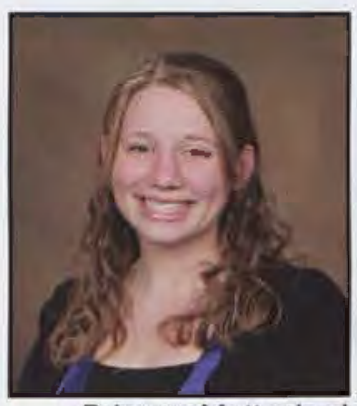

Brianna Mottashed

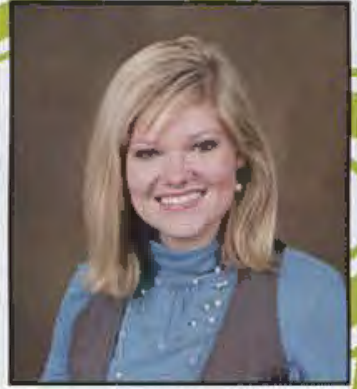

Joyanna McBride

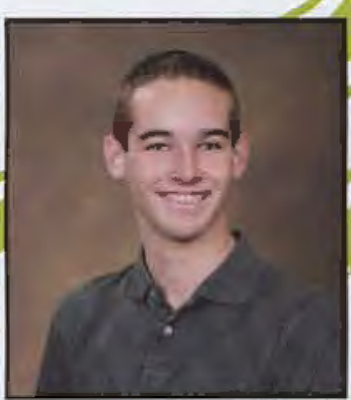

Daniel Merkh

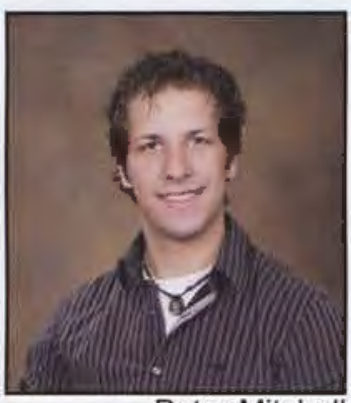

Peter Mitchell

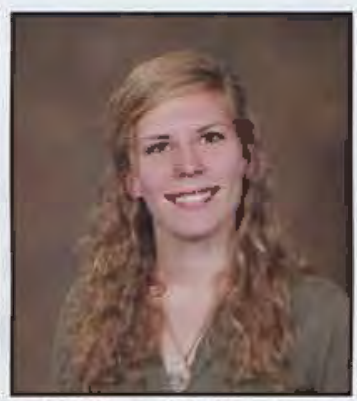

Whitney Muhlenkamp

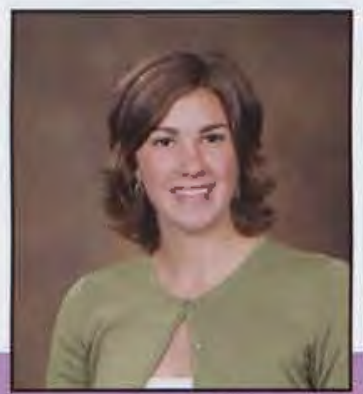

Cristin Murschel

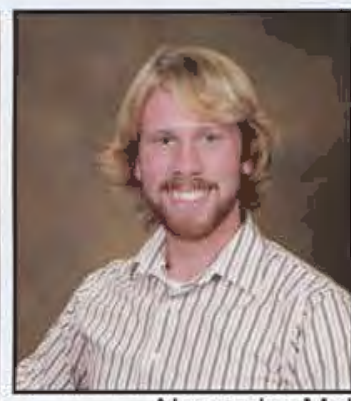

Alexander $\mathrm{Mo}$

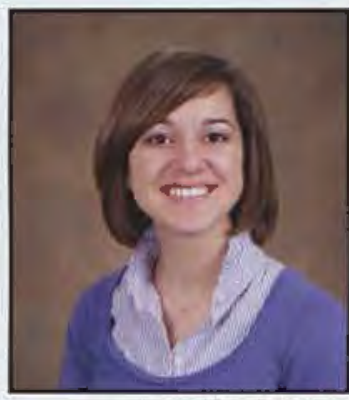

Jennifer Mukes

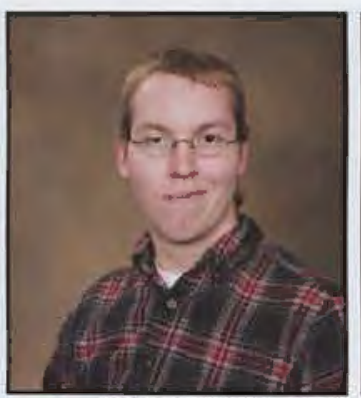

Lucas McConnell

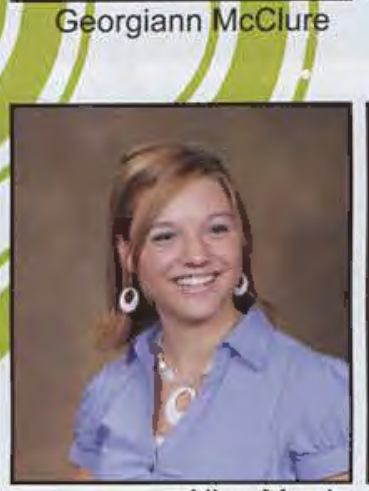

Alise Merrin

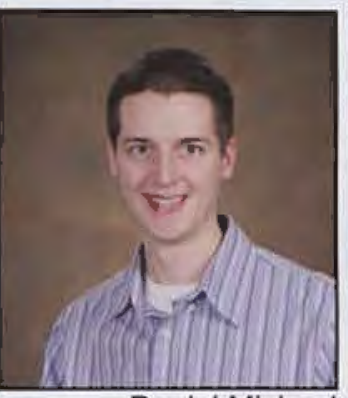

Daniel Michael

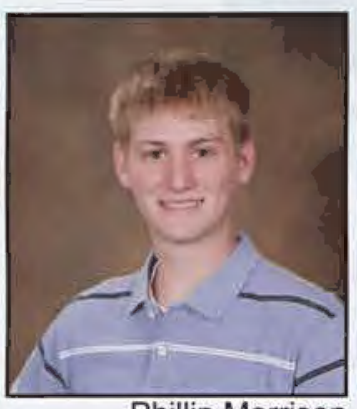

Phillip Morrison

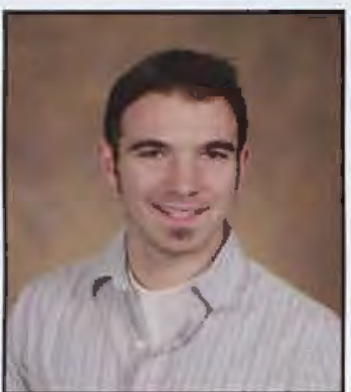

Gerhard Mullican

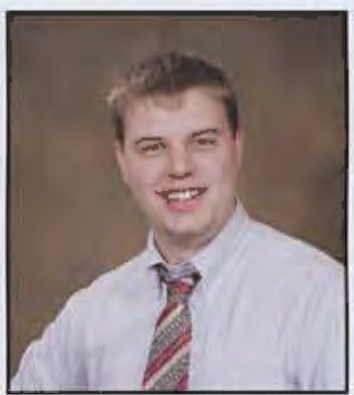

Jeremy McDuffie

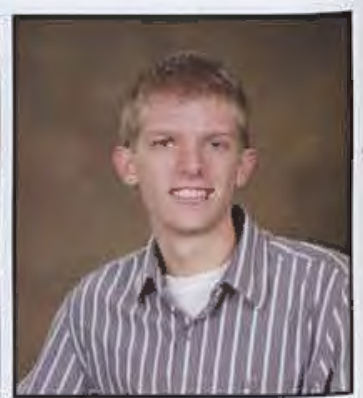

Miles McGee
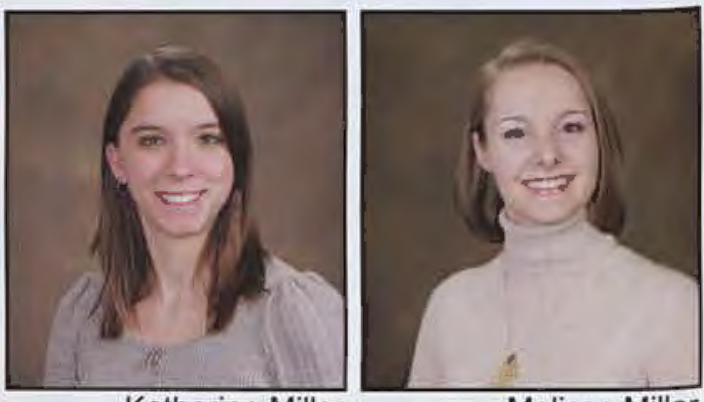

Katherine Miller

Melissa Miller
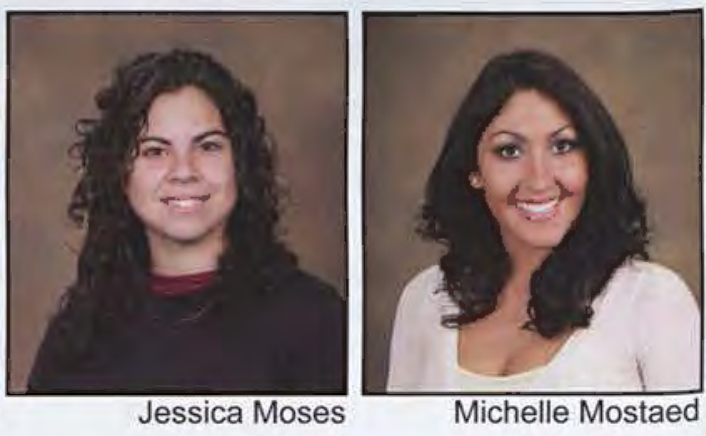

Jessica Moses
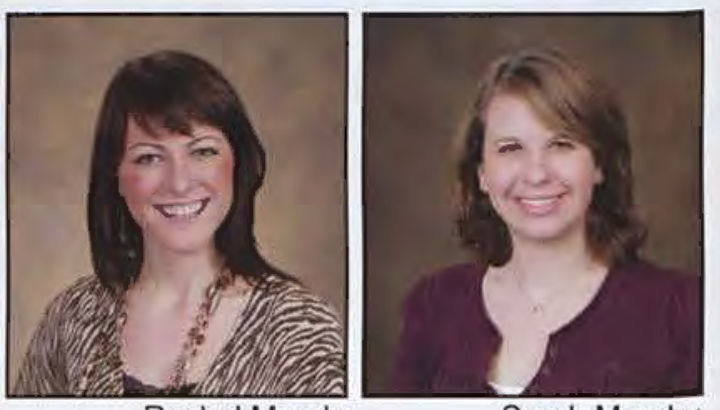

Rachel Mundy

Sarah Murphy

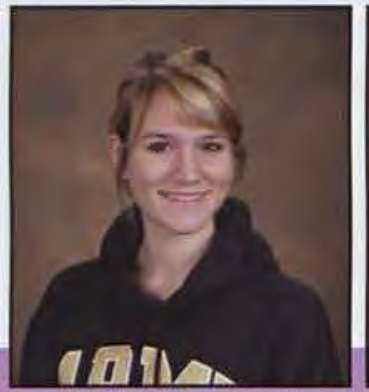

Brittany Musseiman

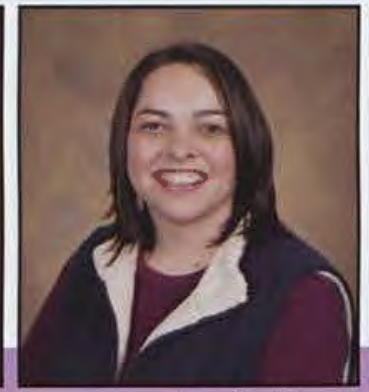

Janelle Myers

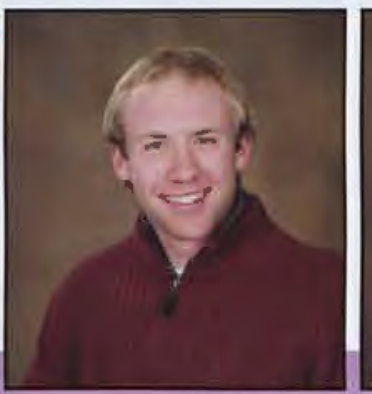

Kevin Napp

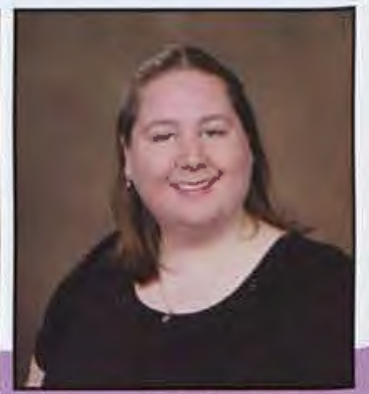

Jennifer Nelson
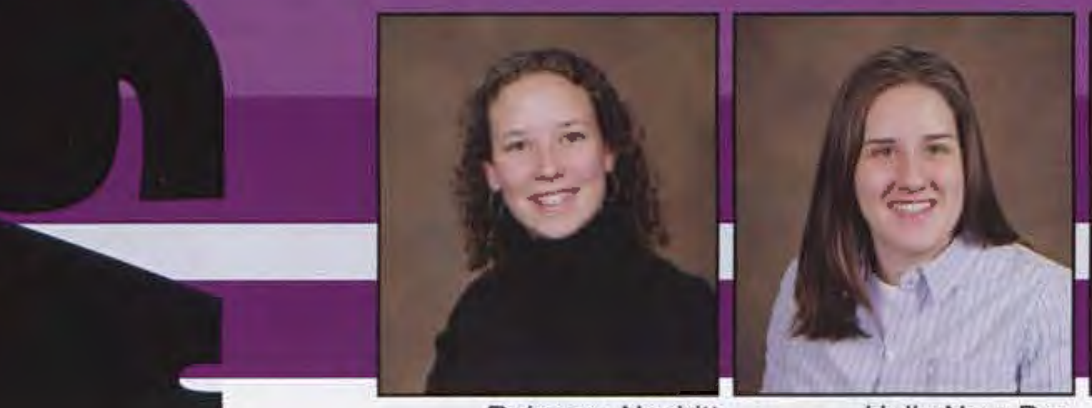

Holly New-Day
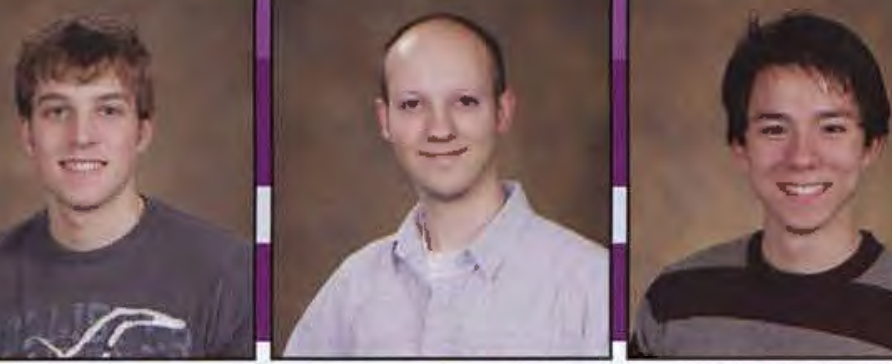

Chris Newman

Justin Newman

Daniel Nichols 


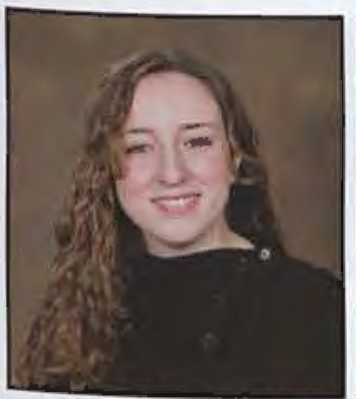

Hannah Niday

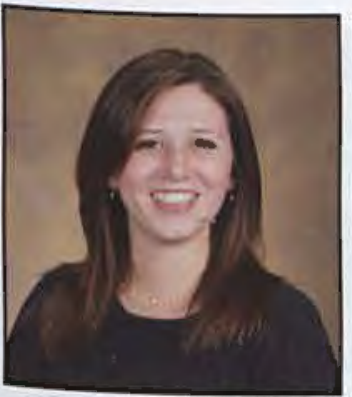

Erin Olsen
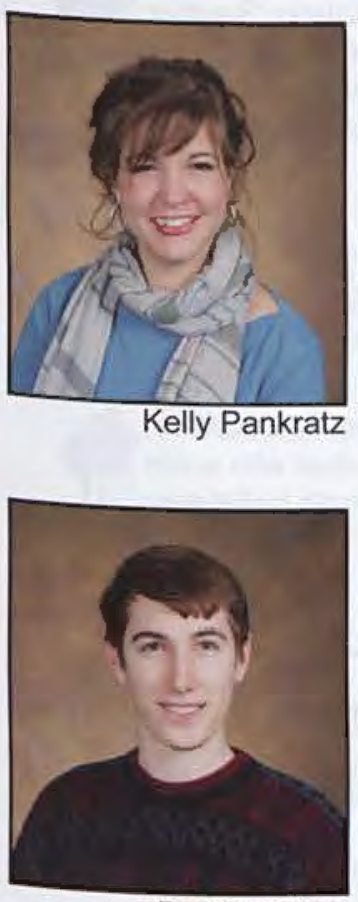

Daniel Peters

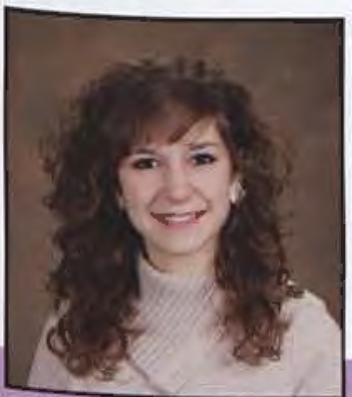

Danielle Pruzaniec

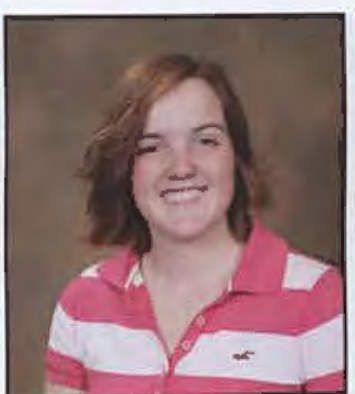

Jerilynn Nine

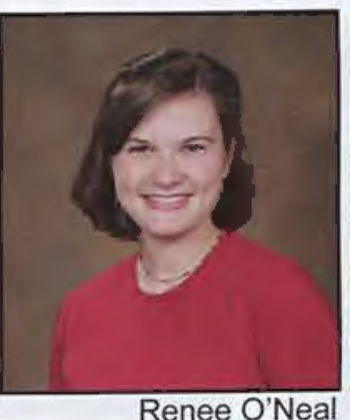

Renee O'Neal

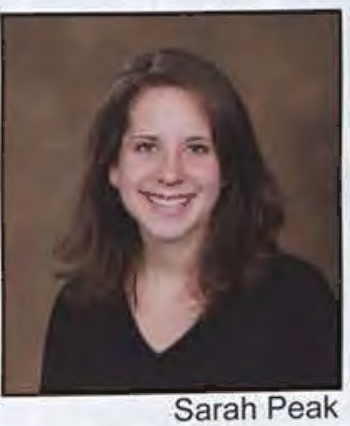

Sarah Peak

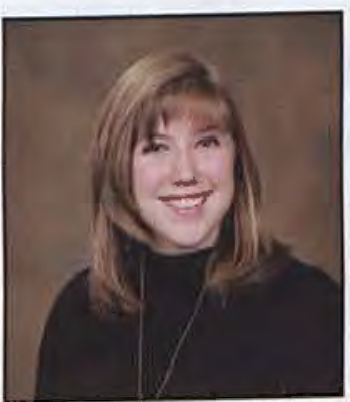

Jillian Pierce

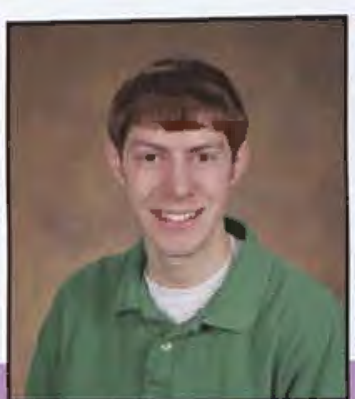

Aaron Ramsey

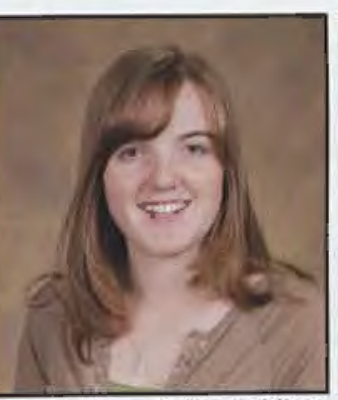

Juliana Nine

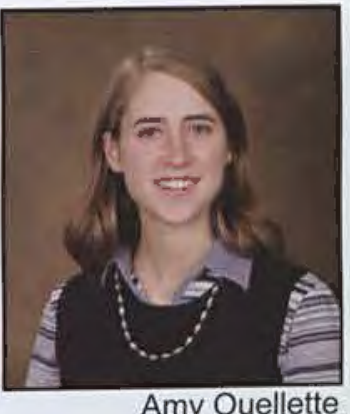

Amy Ouellette

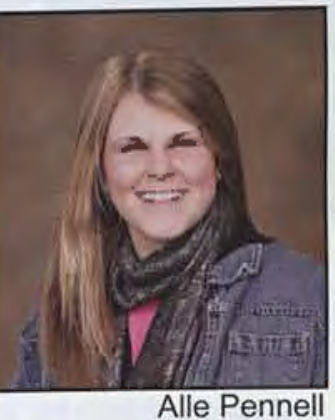

Alle Pennell

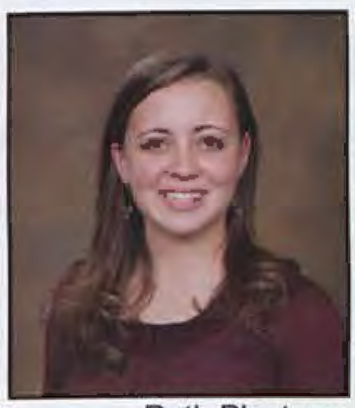

Ruth Plasterer

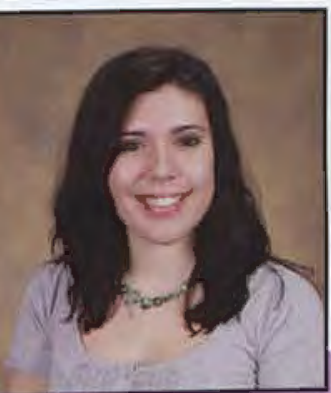

Kristen Recupero

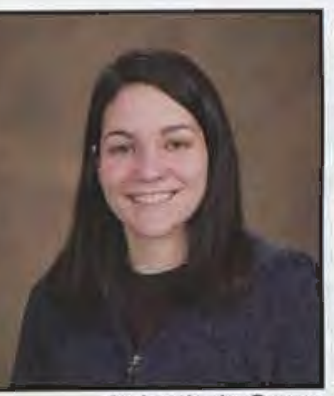

Asheritah Oana

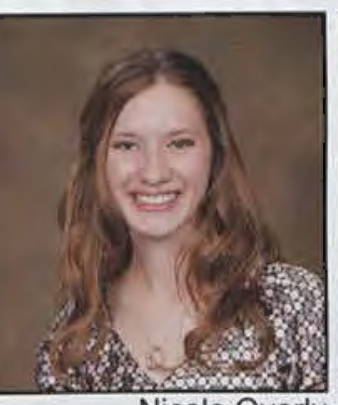

Nicole Overly

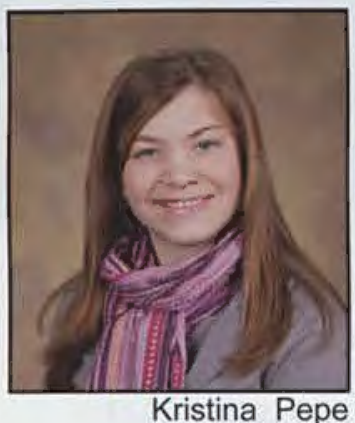

Kristina Pepe

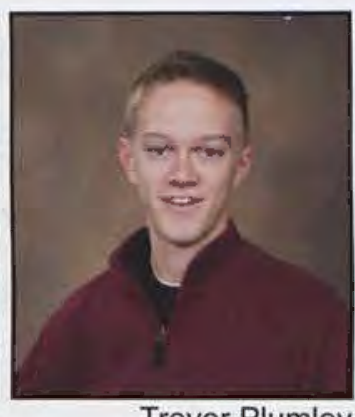

Trevor Plumley

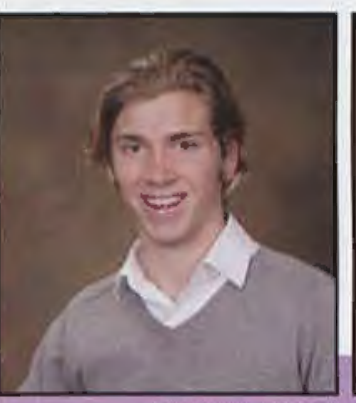

Nathan Reed

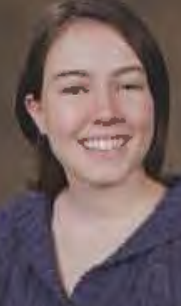

Hallee Palmer

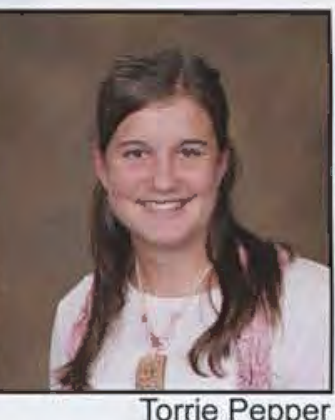

Torrie Pepper

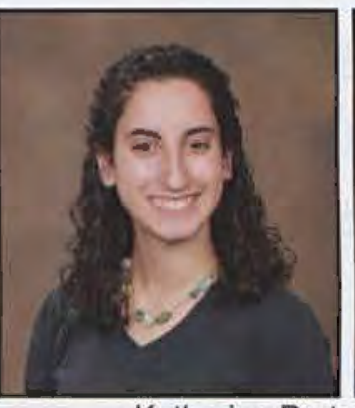

Katherine Port

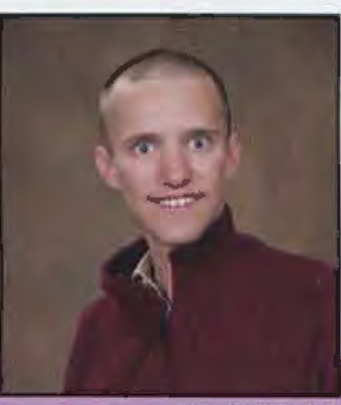

David Reke

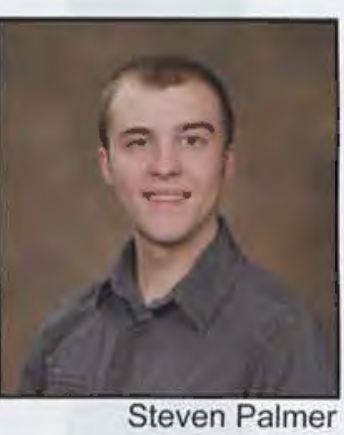

Steven Palmer
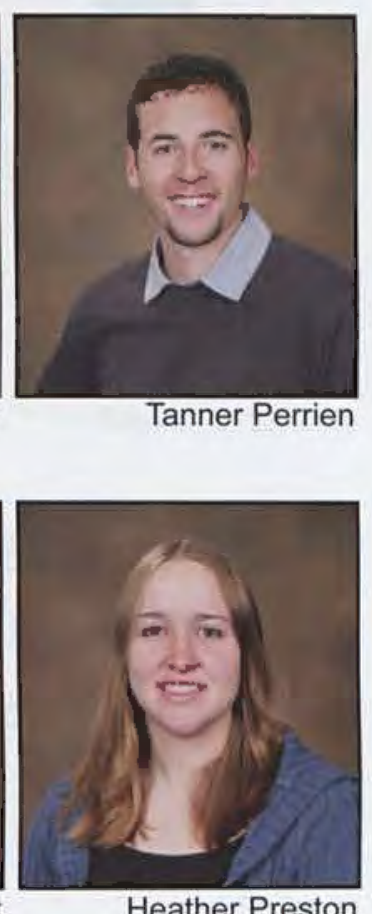

Heather Preston

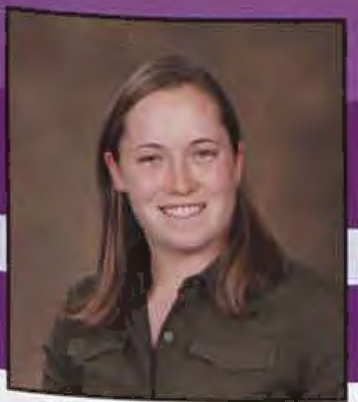

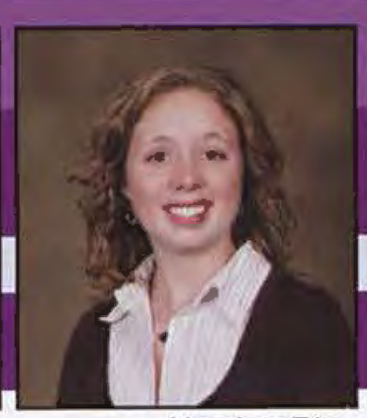

Heather Rice

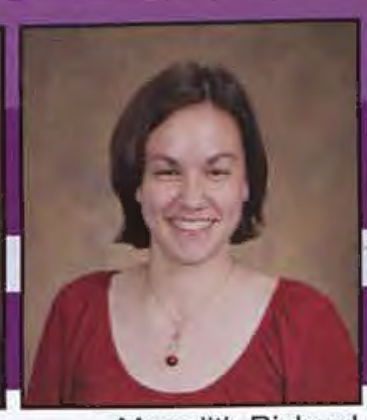

Meredith Richard

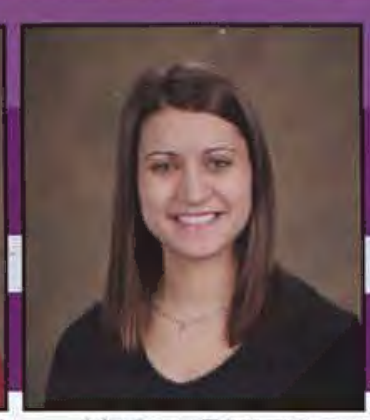

Lindsey Richardson
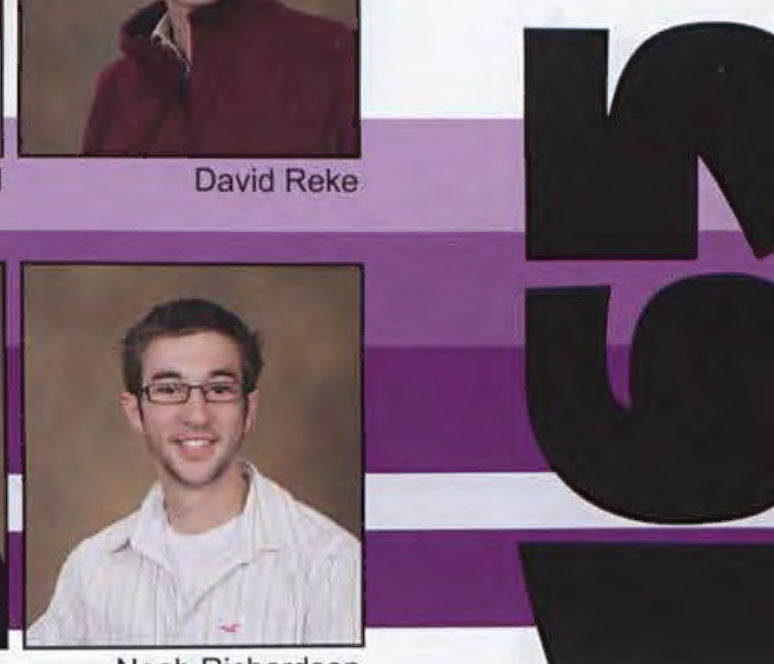

Noah Richardson 


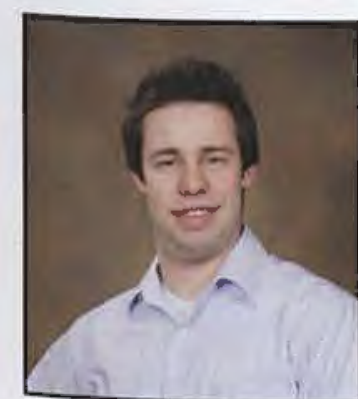

Justin Salzman

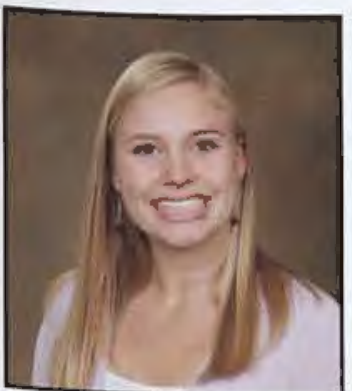

Sarah Schaszberger

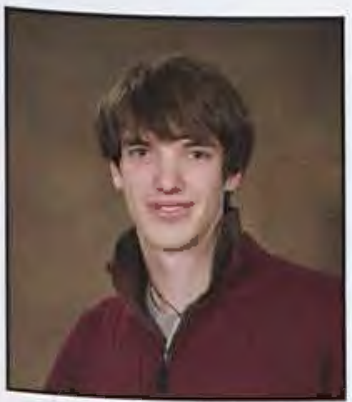

Peter Scull

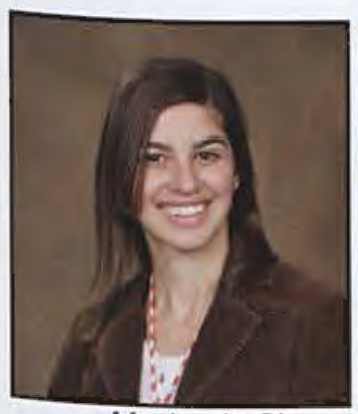

Mychaela Sherry

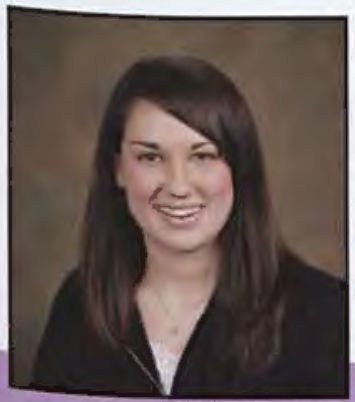

Julia Skelton

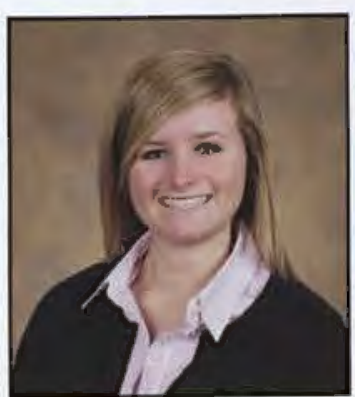

Genelle Schedlauer

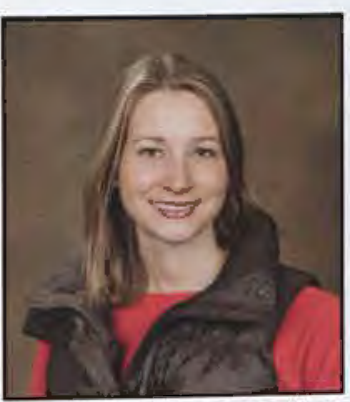

Samantha Seifert

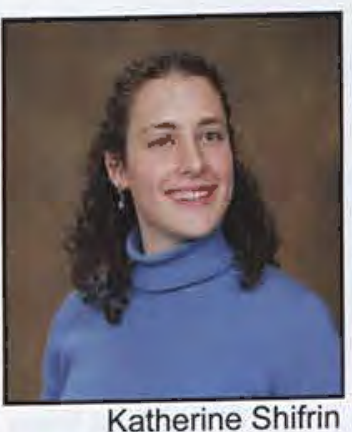

Katherine Shifrin

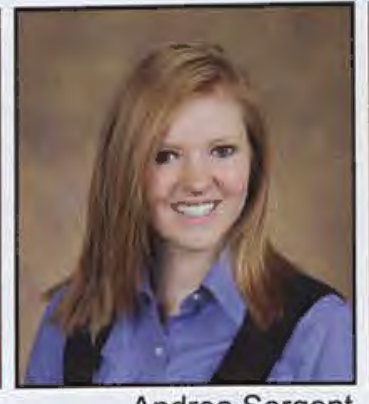

Andrea Sergent

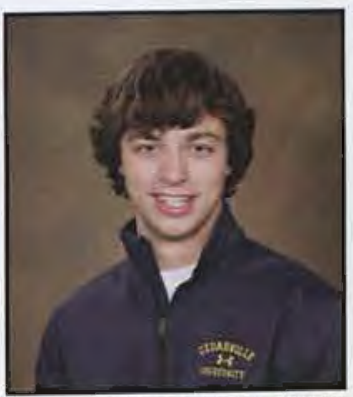

Grant Schlabach

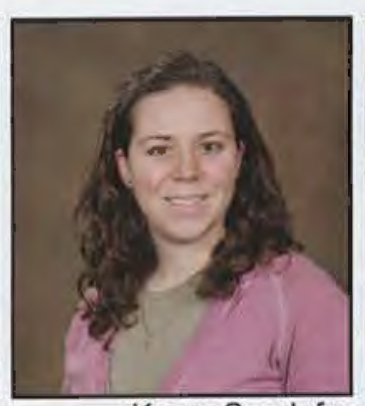

Karyn Sandefur

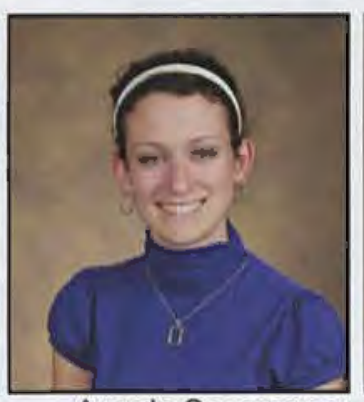

Angela Sangregory

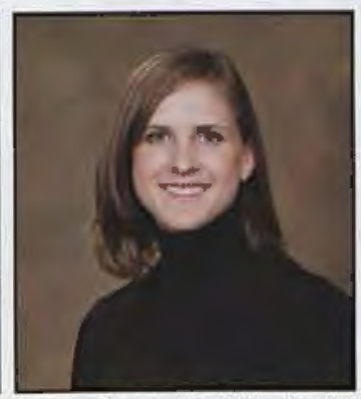

Ann Marie Schlindwein

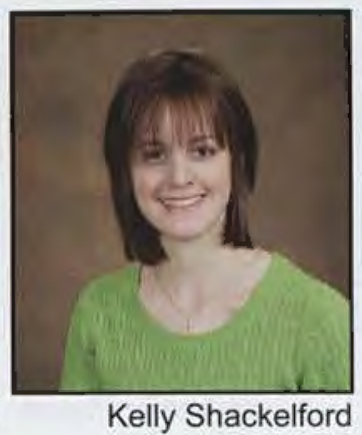

Kelly Shackelford

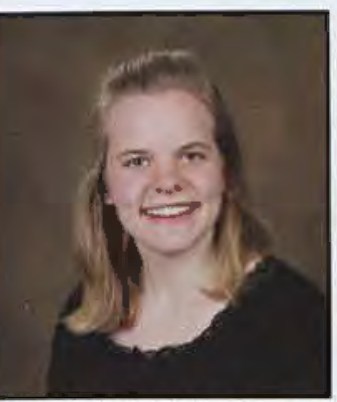

Rebekah Shinabarger
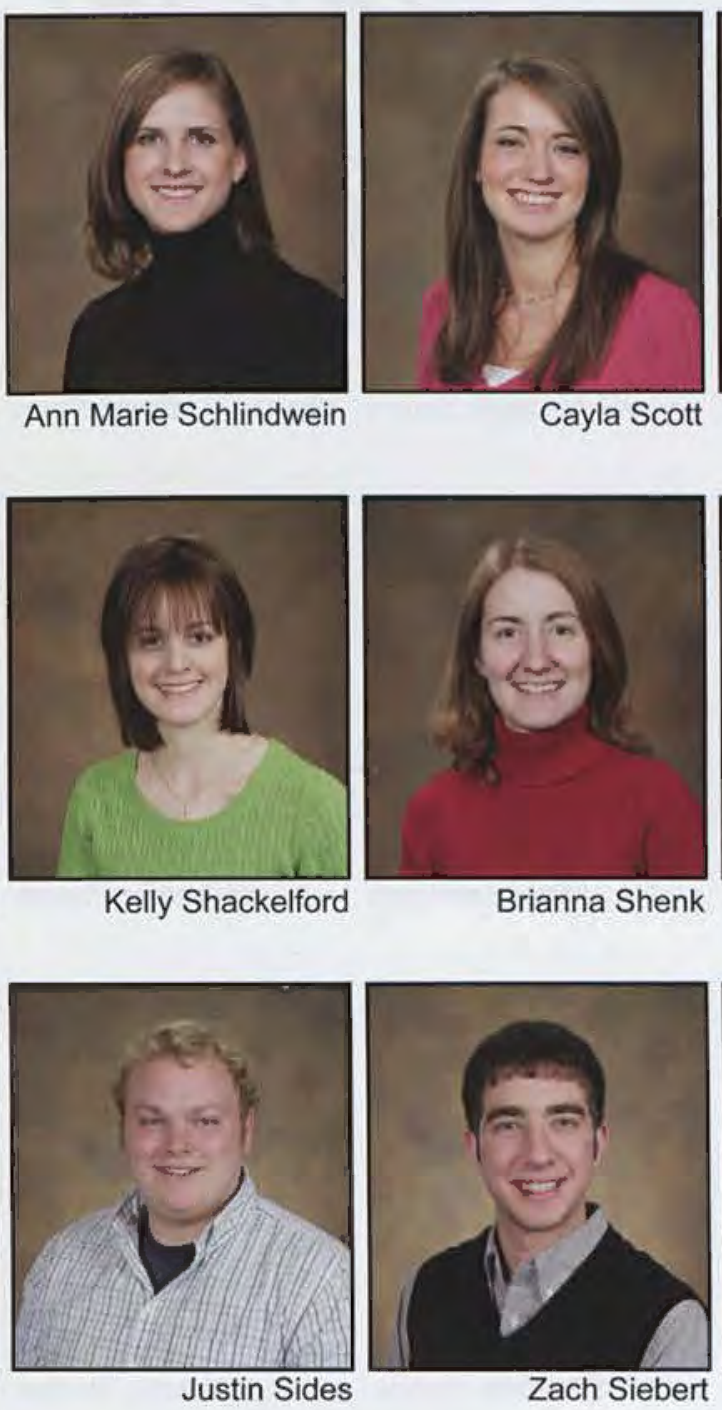

Cayla Scott

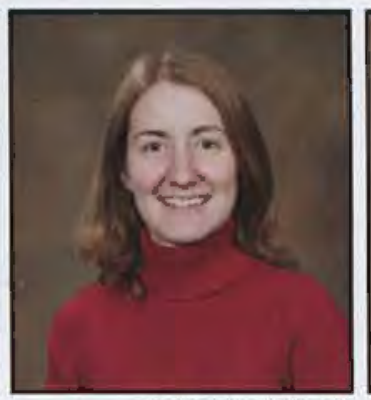

Brianna Shenk

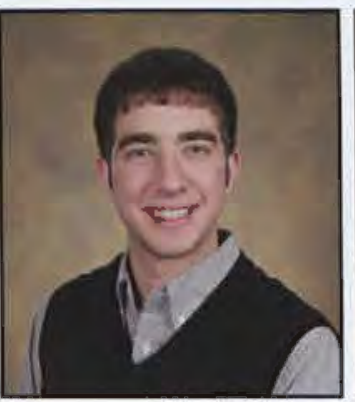

Zach Siebert

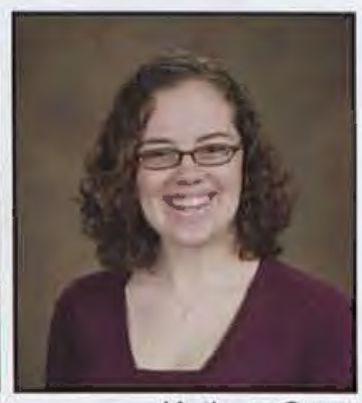

Kathryn Scott

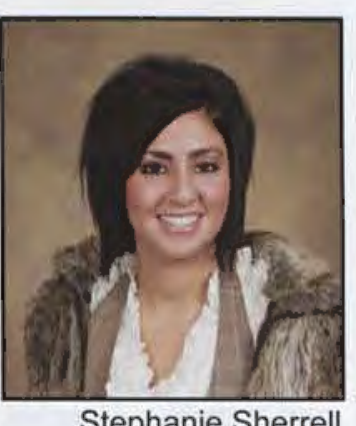

Stephanie Sherrell
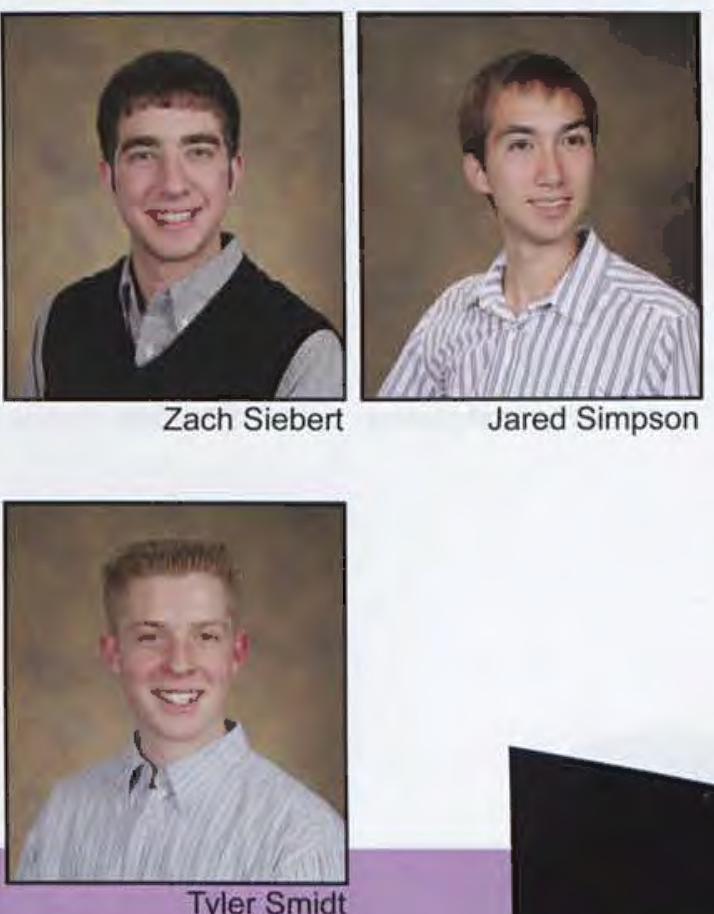

Jessica Skinner
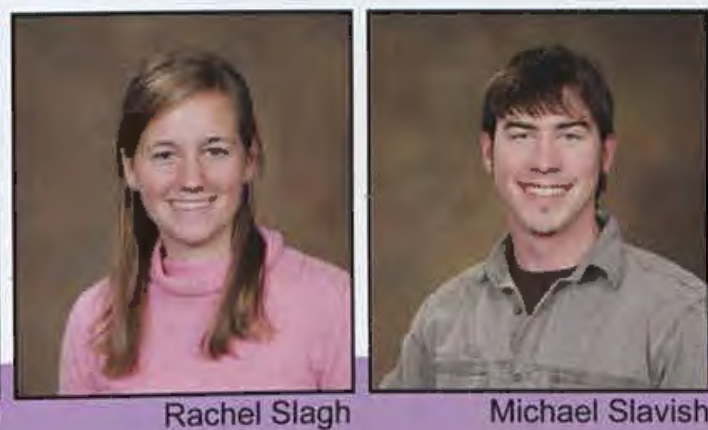

Tyler Smid

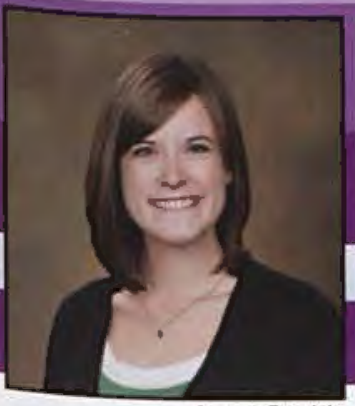

Amy Smith

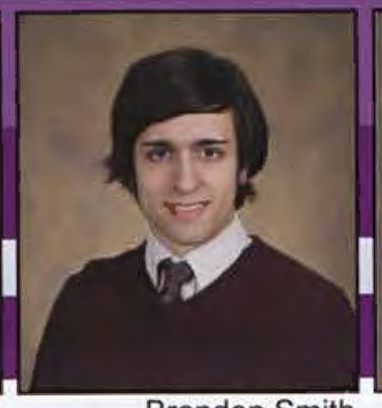

Brandon Smith

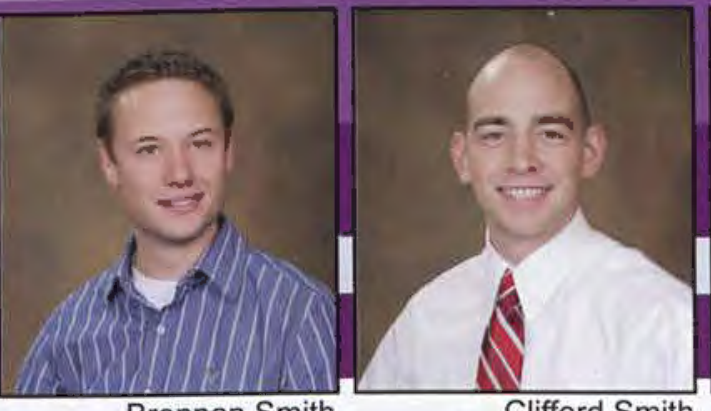

Brennan Smith

Clifford Smith

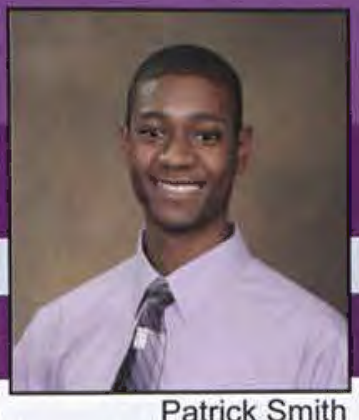

Patrick Smith 


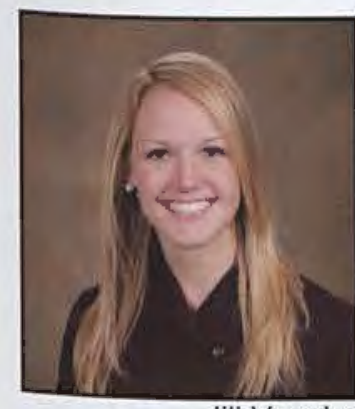

Jill Vaughn

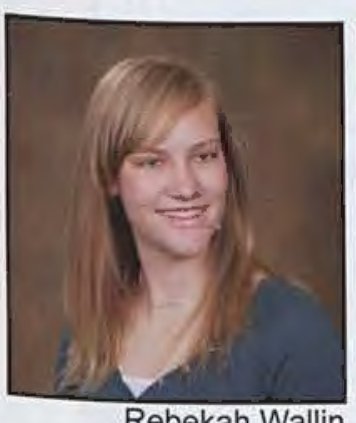

Rebekah Wallin

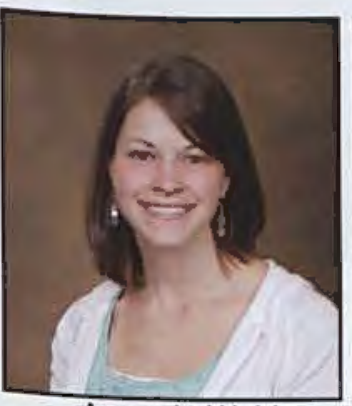

Amanda Weidman

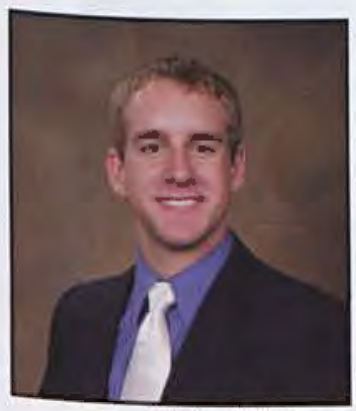

Chris Williams

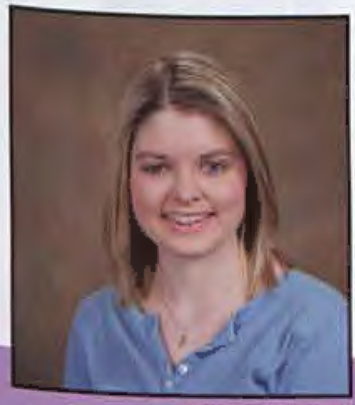

Anna Wood

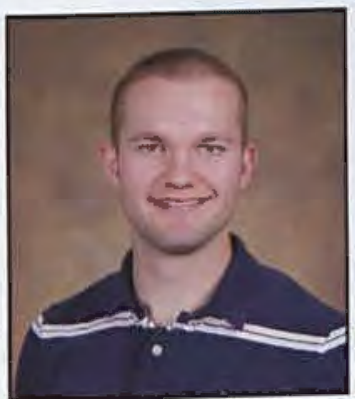

Mark Williams
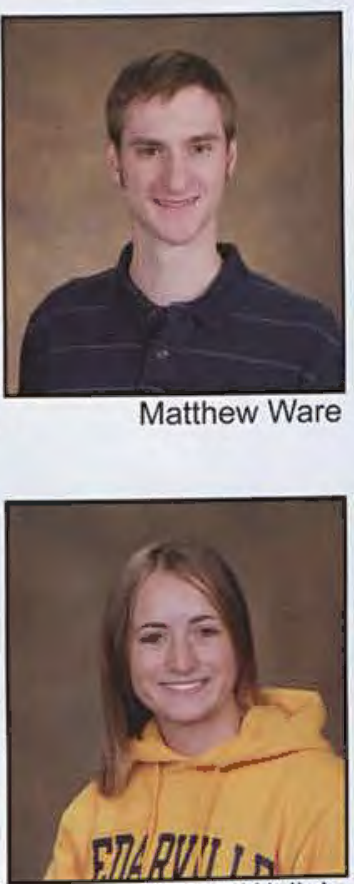

Janna Weilein

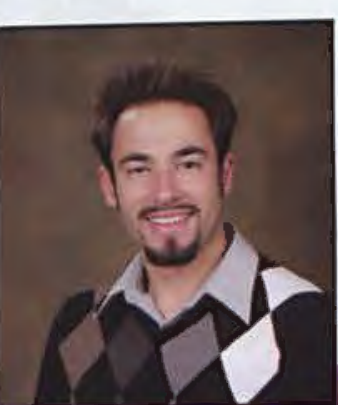

Darren Wurz

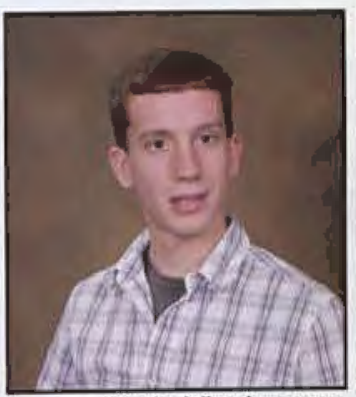

Chris Vinciguerra

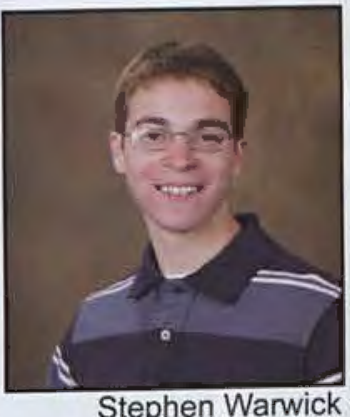

Stephen Warwick

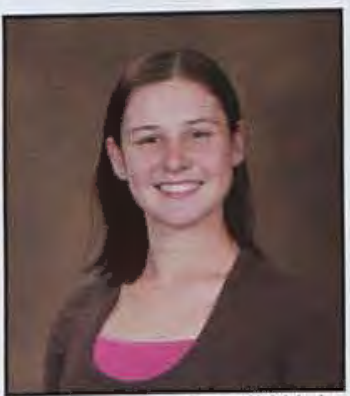

Cyndi Wein

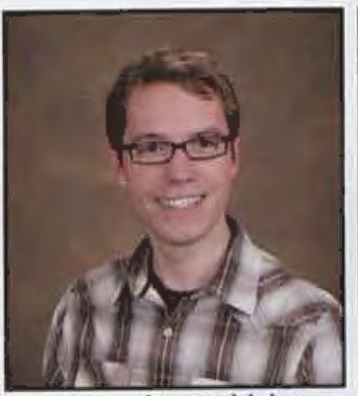

Aaron Volenec

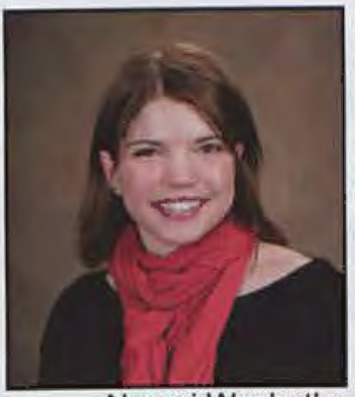

Naomi Washatka

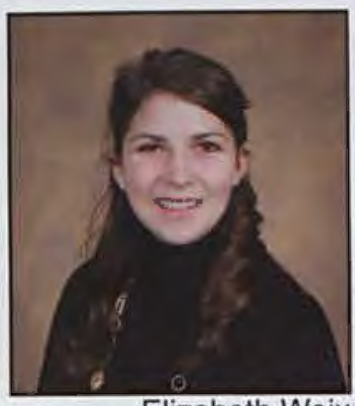

Elizabeth Weix

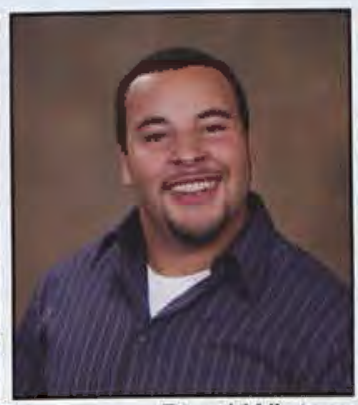

Daryl Winters

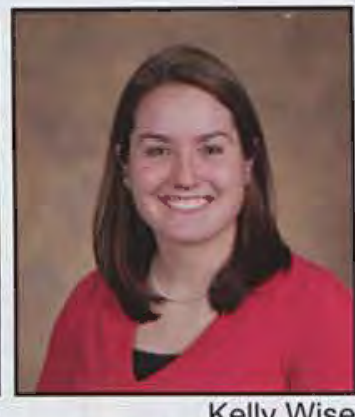

Kelly Wise

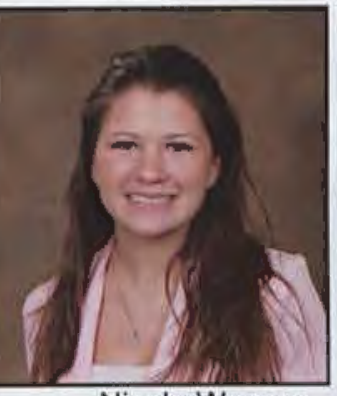

Nicole Wagner

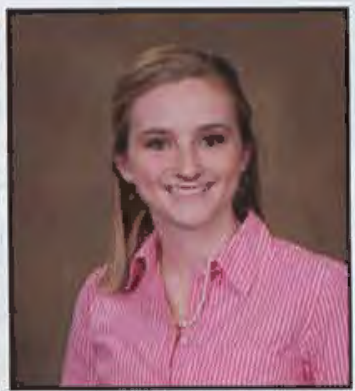

Dakotah Waugh

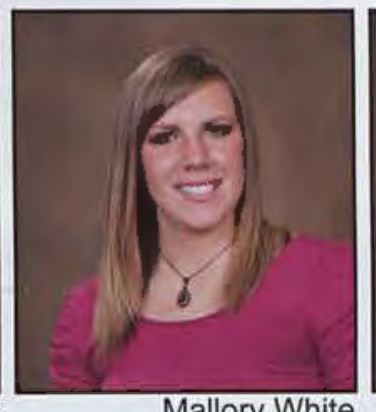

Mallory White
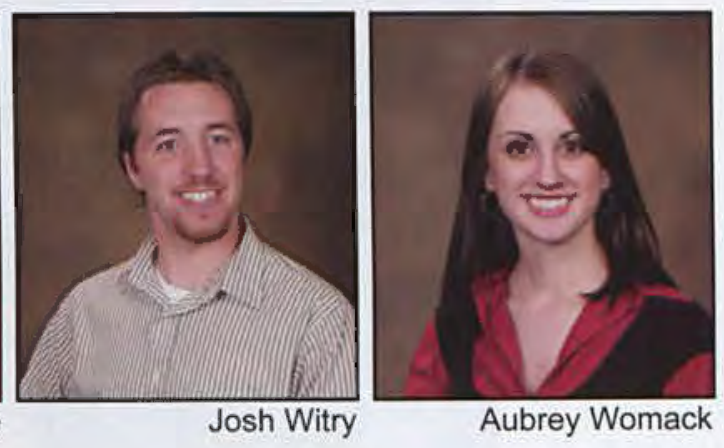

Jonathan Walburg

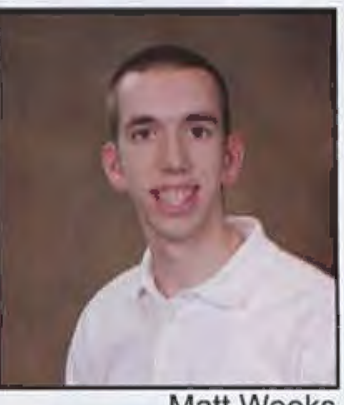

Matt Weeks

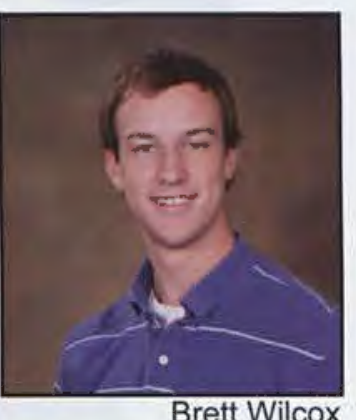

Brett Wilcox
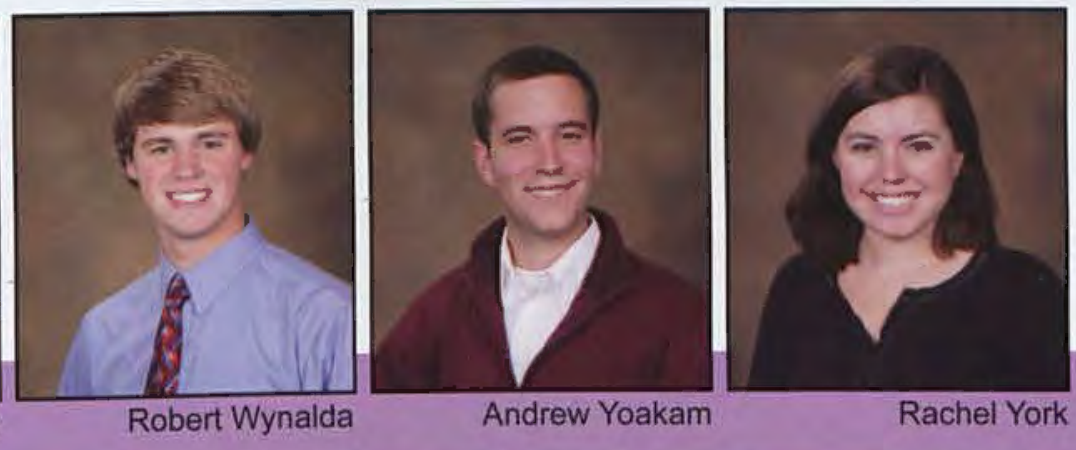

Rachel York

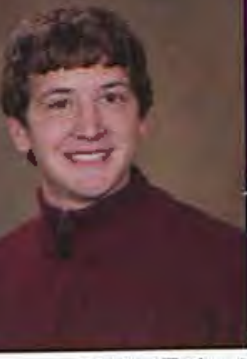



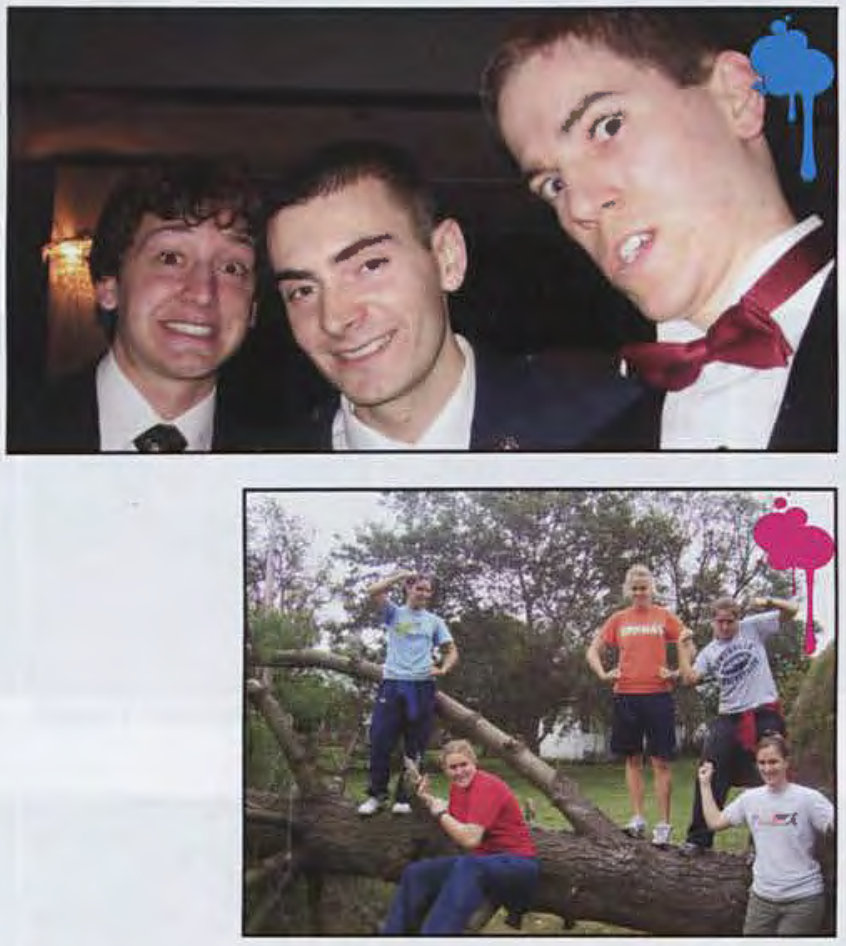

Brandon Salsbury, Brandon Brown, and Ryan Zeiset try to spice things up at the Air Force ROTC Dining Out formal event.

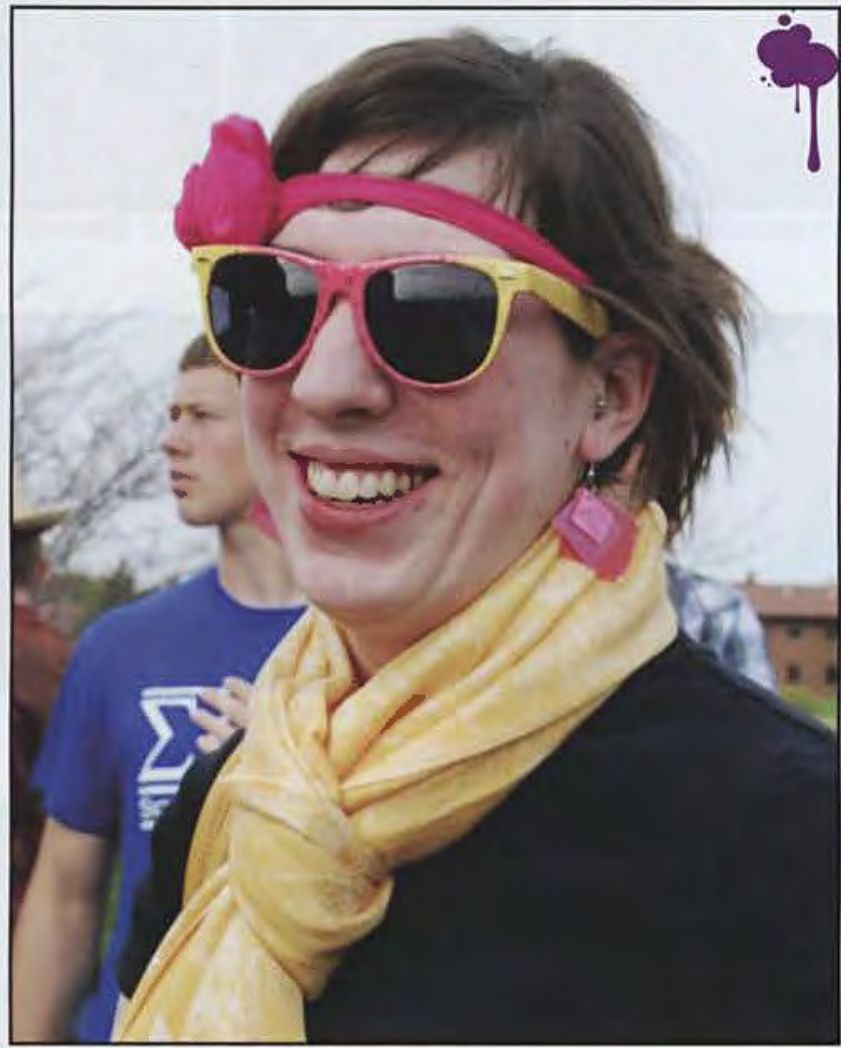

Sarah Jacobson looks all dressed up for a night on the town

Carissa Parmerlee, Megan Van Schepen, Rachel Arthur, Kelly Wise, and Juliana Heerschap do a little grunt work after the "black out hurricane" hit the community.

"My junior year was much like a rigorous, deep-tissue back massage-agony in the present, payoff in the long run?" -decr wereen 
"Junior year was the best! Campus felt like home all year, and my classes were more exciting and challenging than ever. I think I appreciated everything more this year: the professors, the students, the academic buildings, the gym. I just became better friends with Cedarville."

-Amefida roberts
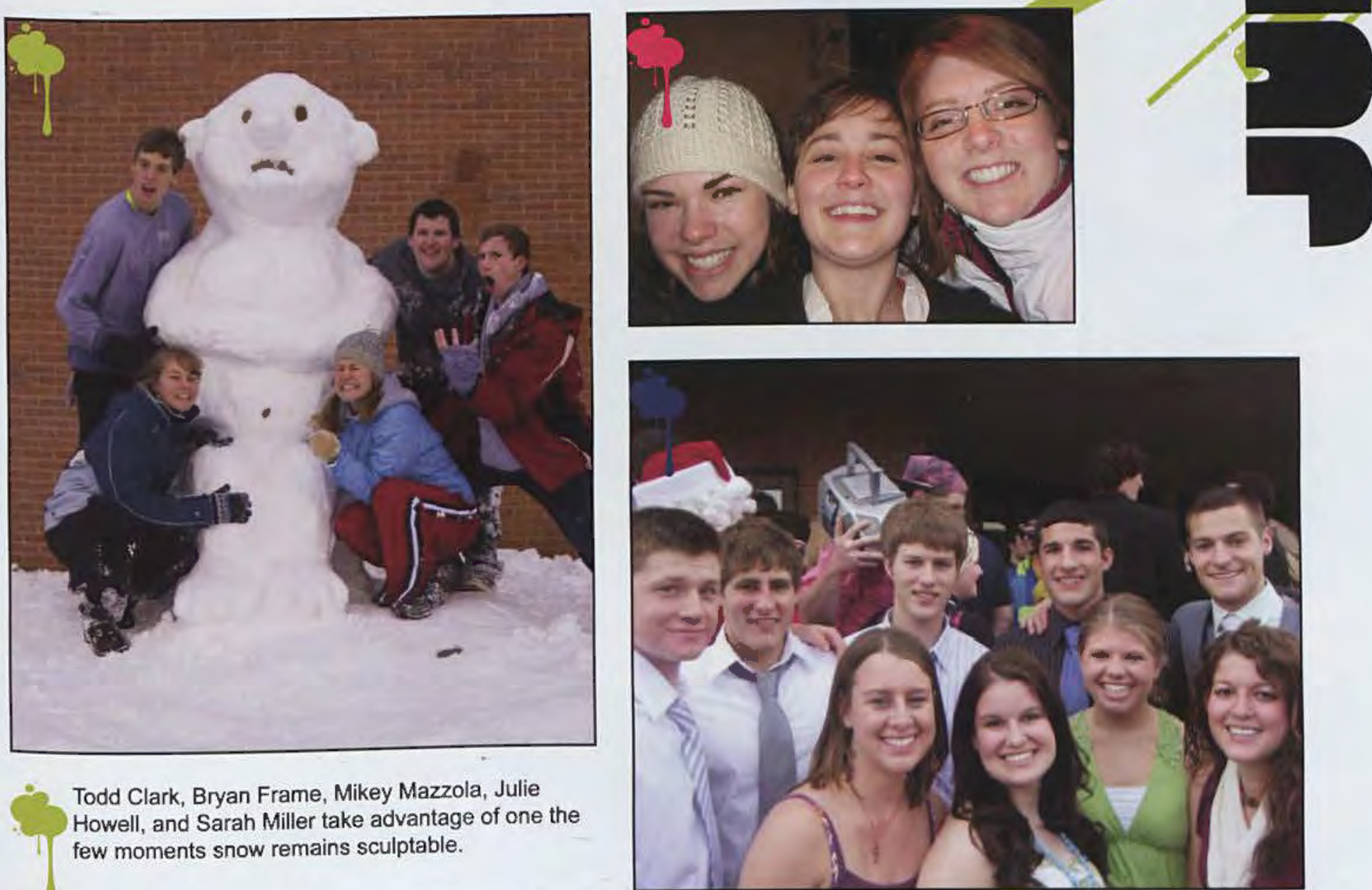

- Leanna Baumer, Molly Fillion, and Christine Keeports Howell, and Sarah Miller take advantage of one the few moments snow remains sculptable.

take an evening out at the Greene.
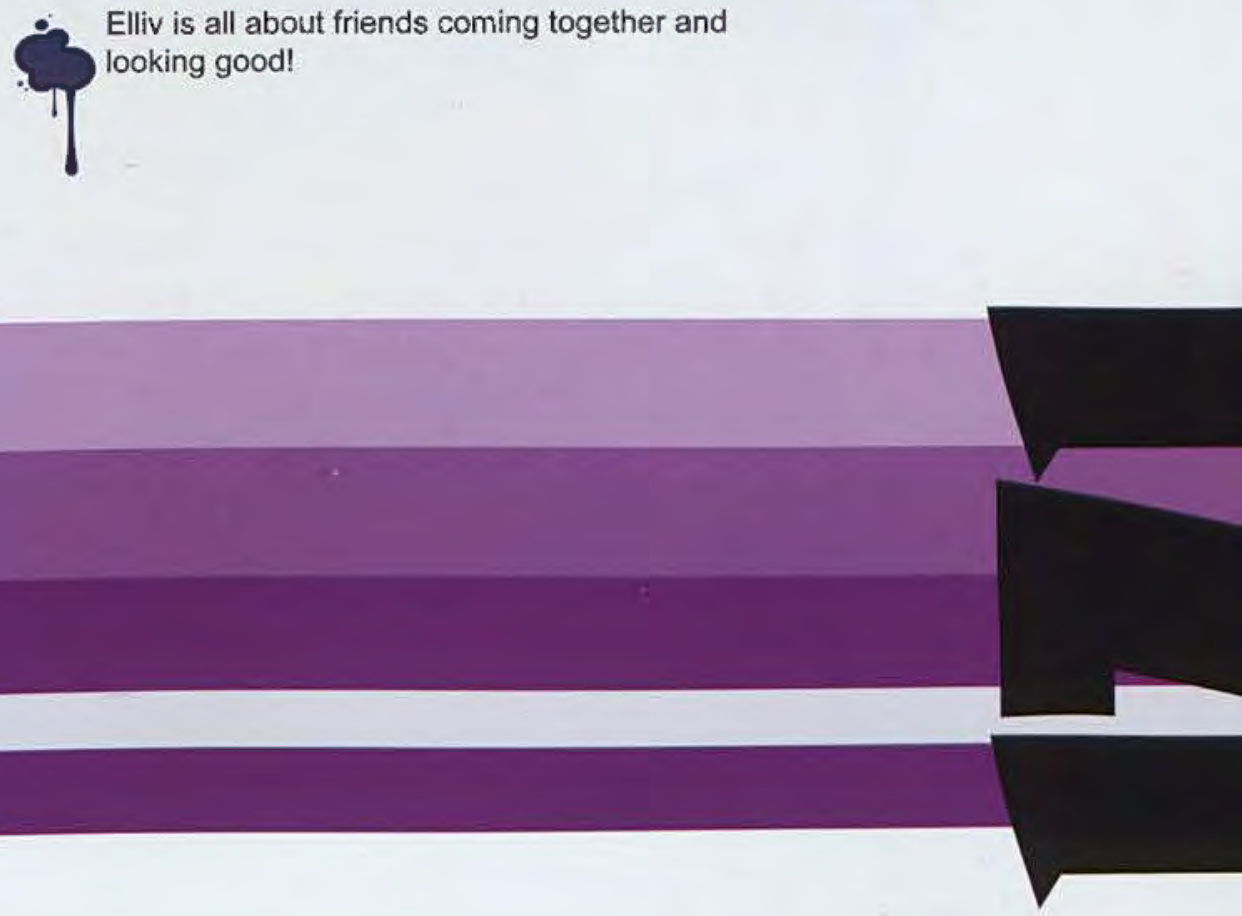


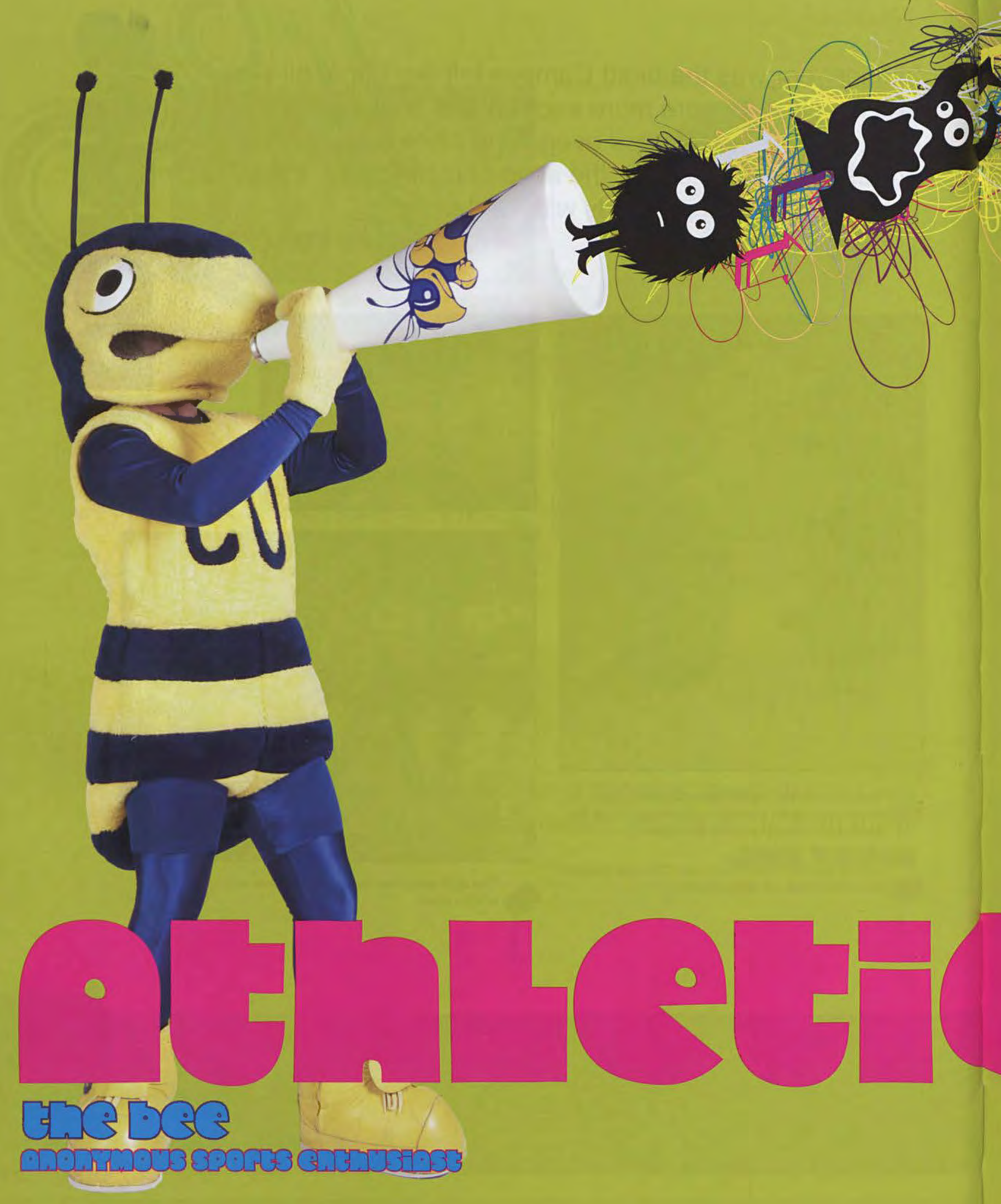


The moon was high and heavy in the black Ohio sky, but its light stopped short of Callan's brick walls. Muted florescent orbs hung hazy in the dimly lit darkness filling the athletic center box. Showered in the bright beam spotlight, I landed in center court. I would start the night off right. But from the shadows emerged two audacious figures. A saber-brandishing cavalier and a cougar of daunting size charged toward me with bloodthirsty eyes. Posers! How dare they taunt me and mess with my crowd! I would not stand for it. So I rushed at them and with one iron blow, they were flat on their backs. I stood on my fallen foes and raised my arms to the surrounding crowd. I knew the court was mine again. No one can take on a Yellow Jacket.

And so the season begins. The electric atmosphere reverberates with the drone of the massive assembly, swarming blue and yellow. I thrive on the thunder-clapping fans stuffing the stands with spirit. Their enthusiasm sends me spinning with dreams of thrilling competition and sweet victory. They make my job as mascot so easy.

Each spectator is important. Each voice echoing in the cheering chorus sustains the community that draws us and the goal that unites us. Like hundreds of worker bees humming in unison, they lift up the chant:

"JACKETS!" With every mind focused on the purpose of the game, the reason to play, I expend my energy with confidence. I step onto the court, the field, the track, and know that these moments will be worthwhile. I exercise my skills and demonstrate my talent with my team behind me, the team that extends past the bench and even past the bleachers to every one who supports this university. Wrapped in these colors, I wear them with pride and gratitude, because I represent something bigger than myself. I am the representative of a mighty force, a combined will stronger than my own. I count it a privilege to defend the name of Cedarville Athletics.

Tiny children stare up into my black eyes in awe and fascination. Their grins glisten beneath my own permanent smirk, and they urge their parents to document this moment in megapixels. I appreciate their eagerness to remember. I hope they do remember, and that their memory sustains their support. As I love how much Cedarville thrills their hearts now, I would love these same hearts to return to this gym and take it upon themselves to stand for Cedarville with athletic ability. I hope that one day these little fans will grow into big players for the university we love.

As the parents snap pictures, I am grateful that I can see them from the other side of the camera. Once they drew the crowds, made the plays, flew the colors from their backs. They still come to the court, but with children rather than tennis shoes in tow. Or they come with grandparents, who first sweat on this wood and first put names on these walls. Though their knees might now give way, there was a time when they could stand through the whole game, or play the whole game.

We all say we are here for the game. The players admit that they just want to play. But as I fly through the stands and they fly about the court, I think we are here for more than a demonstration of skill in competition. The players do not do everything for the love of the game; they do everything for love. The game might ask them to yell, to fight, to cheat. Love asks them to show respect, responsibility, integrity, servant leadership, and sportsmanship. 

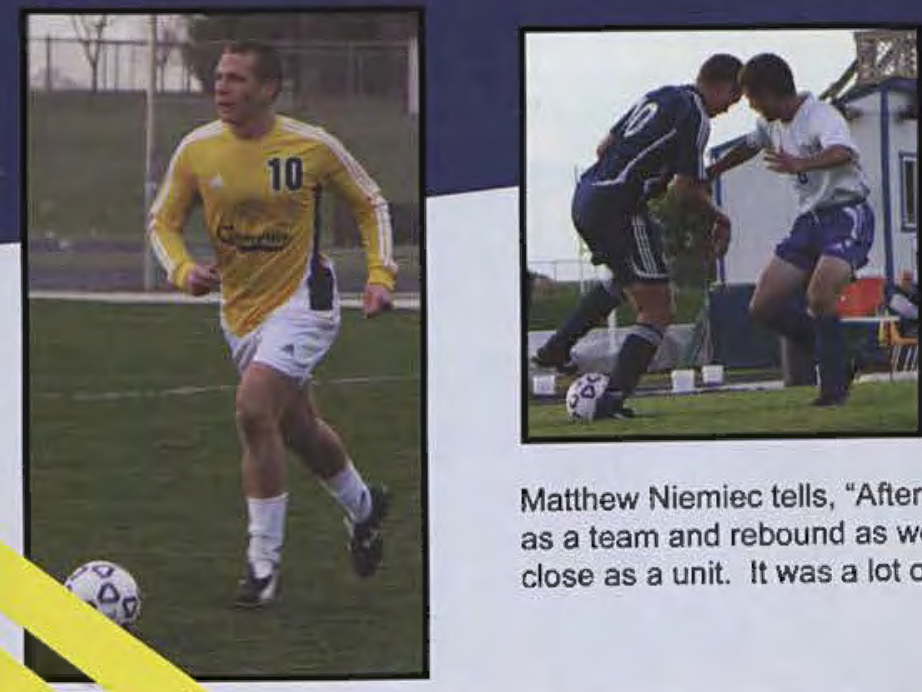

The CU soccer team respectfully walks with their opponents at the start of a game.

Matthew Niemiec tells, "After a poor start to the year, it was good to see us unite as a team and rebound as we did. We battled through a lot of stuff, and grew close as a unit. It was a lot of fun, and I can't wait for next year."

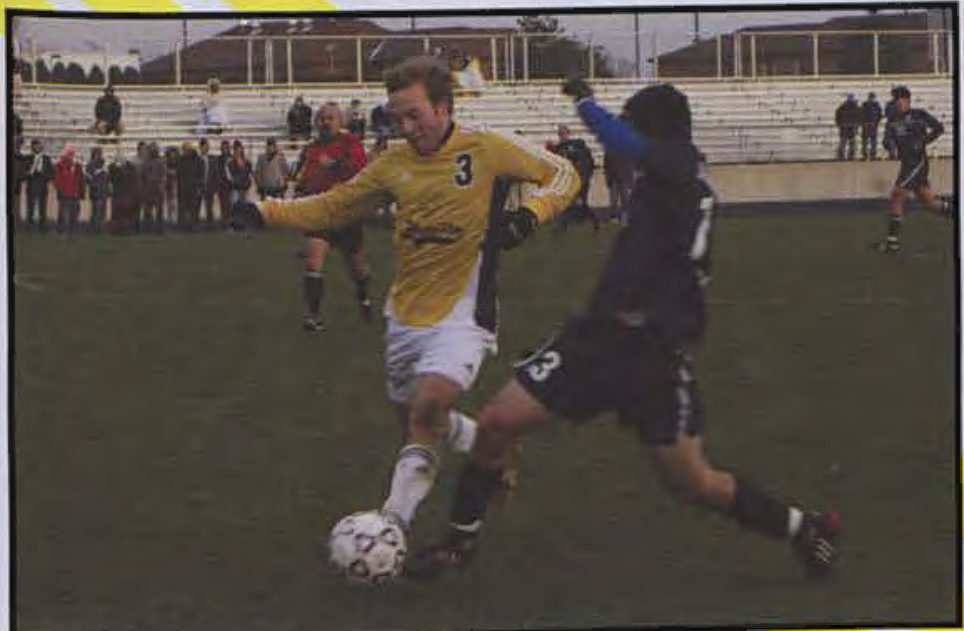

Ryan Thurman defends the ball from an attacker.

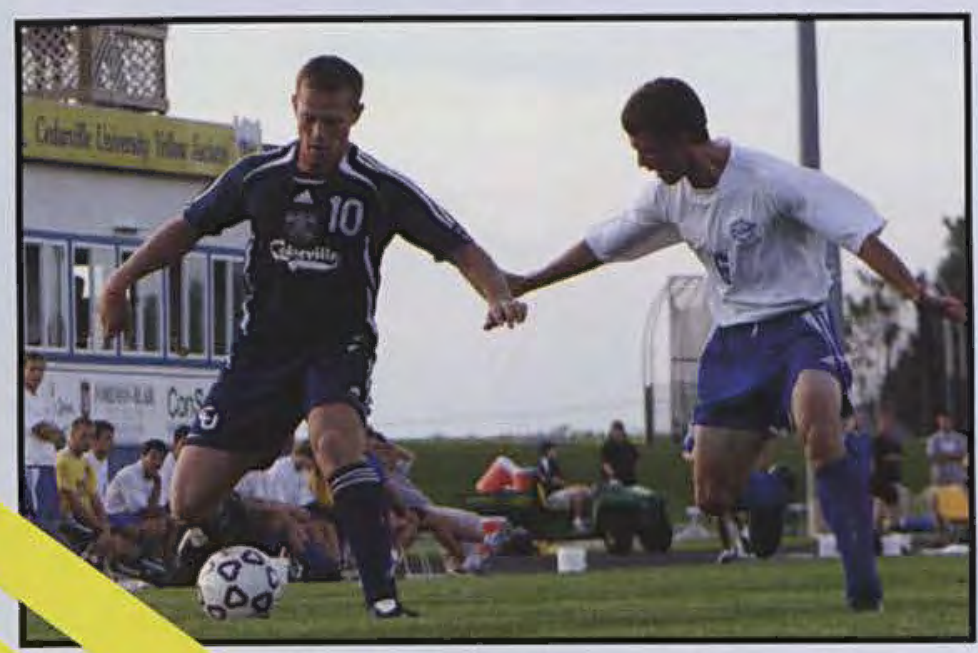

Matt Niemiec works to out-dribble a defender.

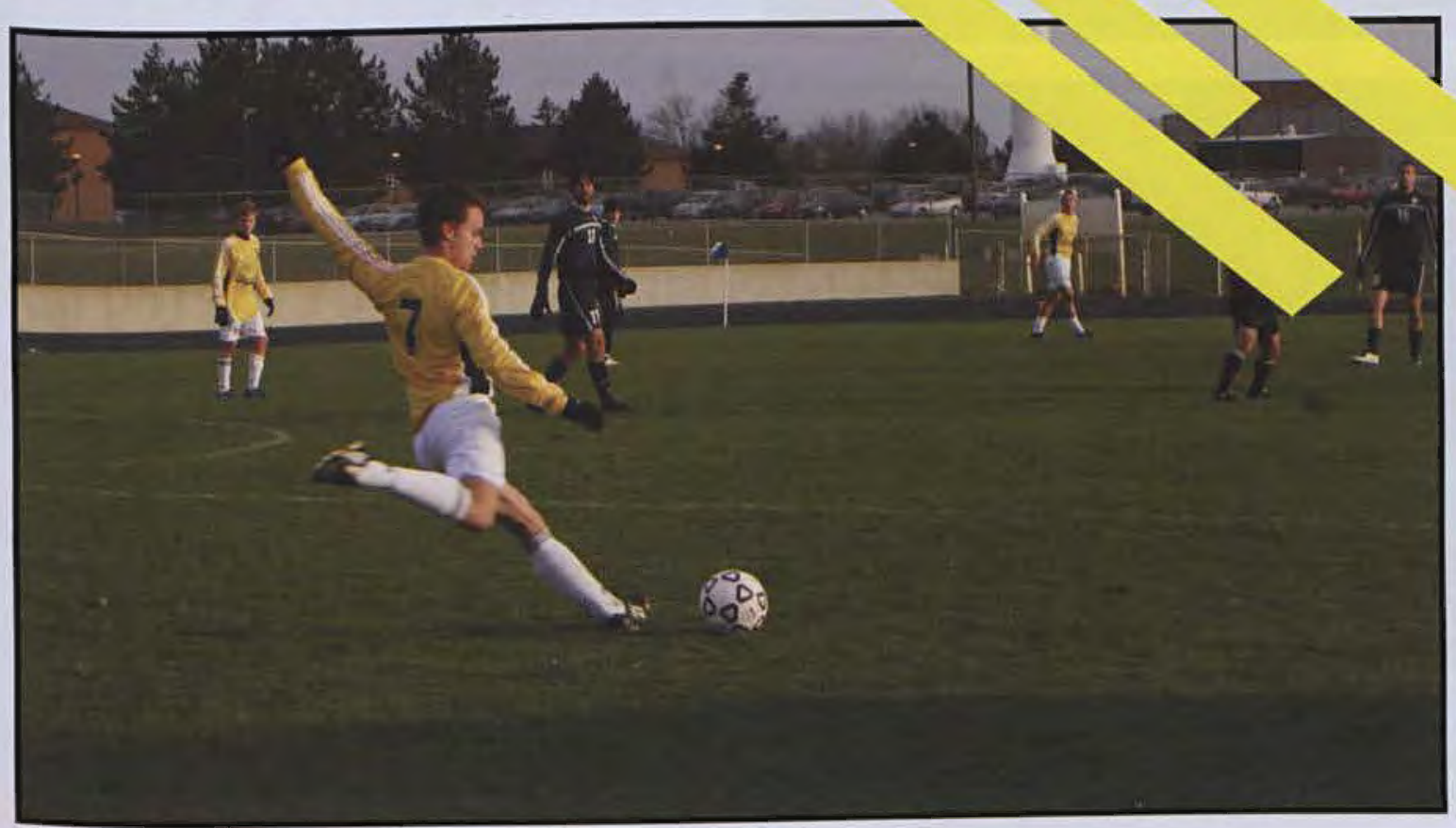

Tyler Waltz rears back for one massive shot
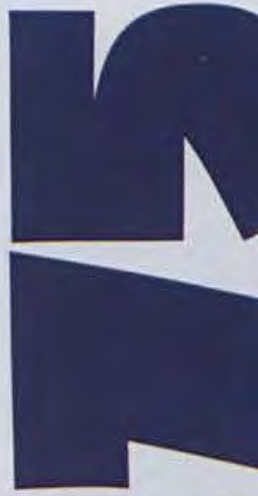
Left to right. Front row: Katie Koch, Erin Hayes, Kelly Wise, Hannah Wailes, Bethany Rigg, Lisa Burgman. Second row: Lacie Condon, Dresden Erin Landers, Jaimie Matson, Erin Lan Brownfield. Watkins, Sarah Browniter Megan Spring, Becky Burton row: Jamie Widman. Third row: Jam, Jill Carroll. Bethany Wailes, Jil Carnes. Amber Laing, Brianne Rachel Karen McCoskey, Rachel.

Brownfield, Alison Schach Fourth row: Assistant Coach Krista Mattern, Assistant Coach Julie Stauffer, Head Coacher, John McGillivray, Torns, Courtney Kelsey Watkins, Raubuck, Brown, Lindsay Revin Roper, Assistant Coach Keviner Bob Associate Athletic Trainer Buchardt.

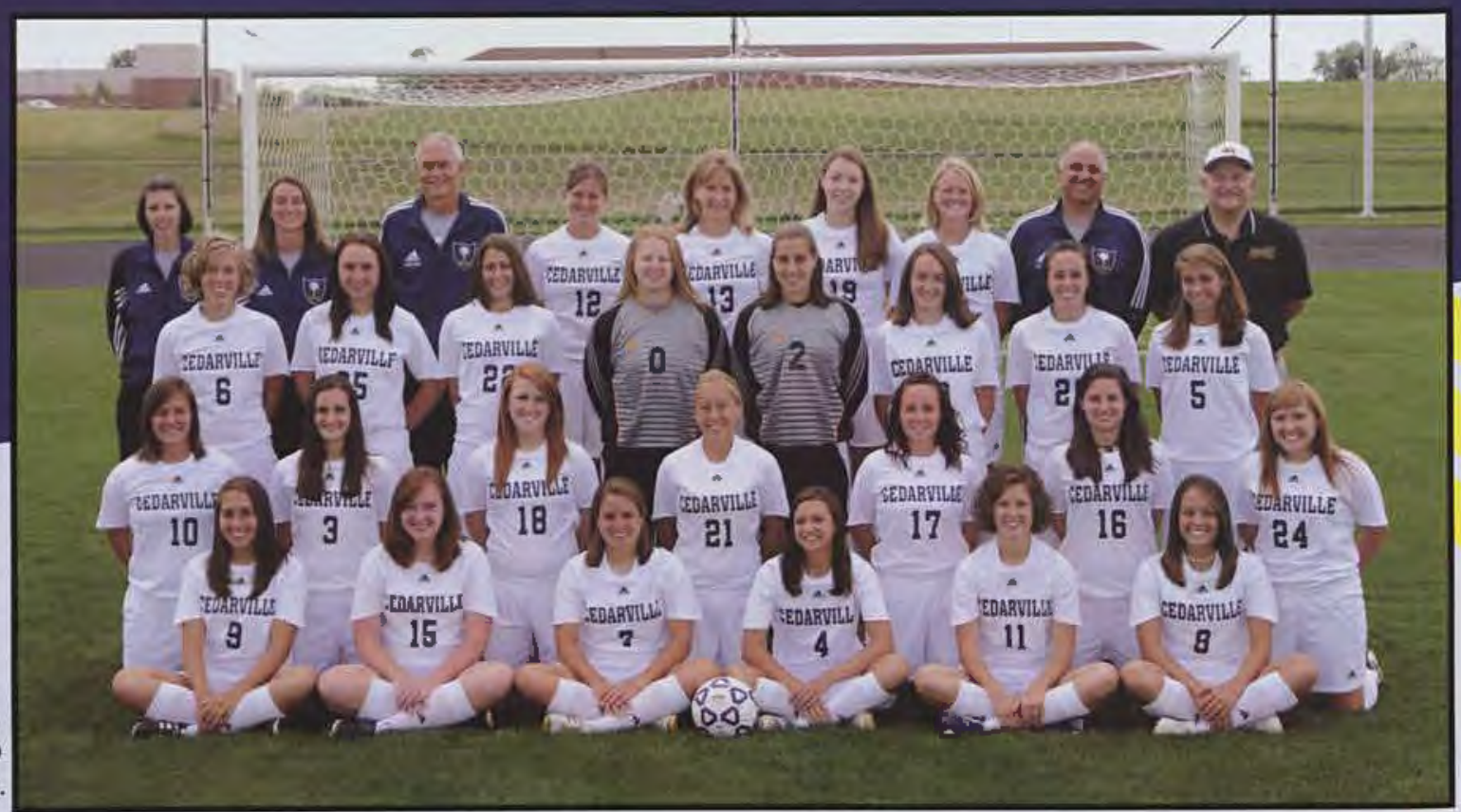

Huntington

W 1-0

Grace

W 5-0

Taylor

Carlow

Indiana Wesleyan

Malone

Mount Vernon Nazarene

Ursuline

Walsh

Shawnee State

Campbellsville

Ohio Dominican

Notre Dame (Ohia)

Daemen

Rio Grande

Point Park

Roberts Wesleyan

Houghton

Mount Vernan Nazarene*

Grace**

Spring Arbor**

W 3-2

W 4-1

L $1-3$

W 8-2

W 2-0

W 3-0

L. 1-2

W 3-0

L $0-1$

T $0-0$ (2ot)

L $0-2$

T 1-1 (2ot)

W 2-0

W 5-1

L 1-2 (2ot)

L $0-4$

L $0-5$

W 2-0

L $1-4$

*American Mideast Conference Tournament

*NCCAA Midwest Regional and injuries, the team achieved several team goals," tells Coach John A. McGillivray.
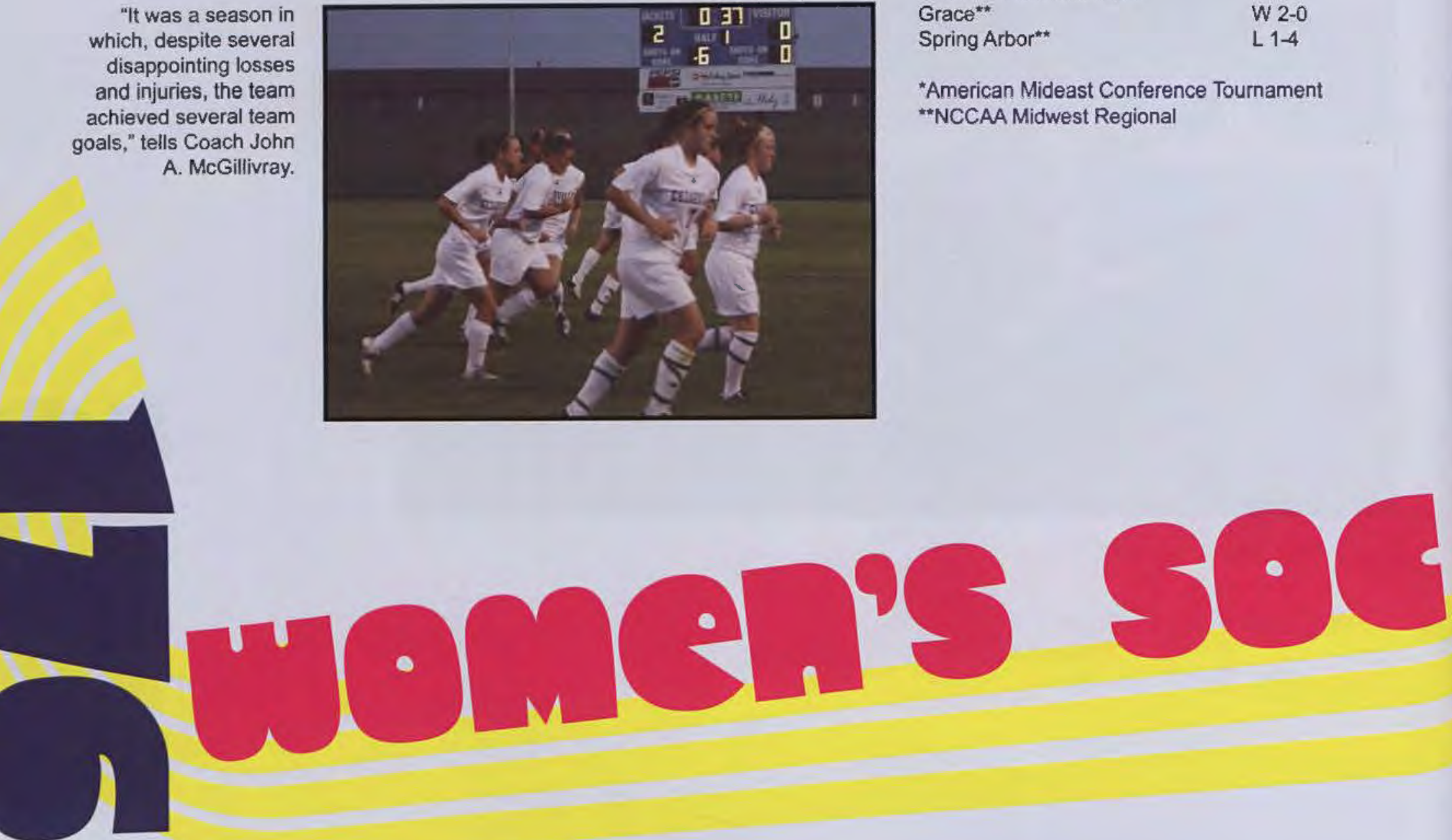


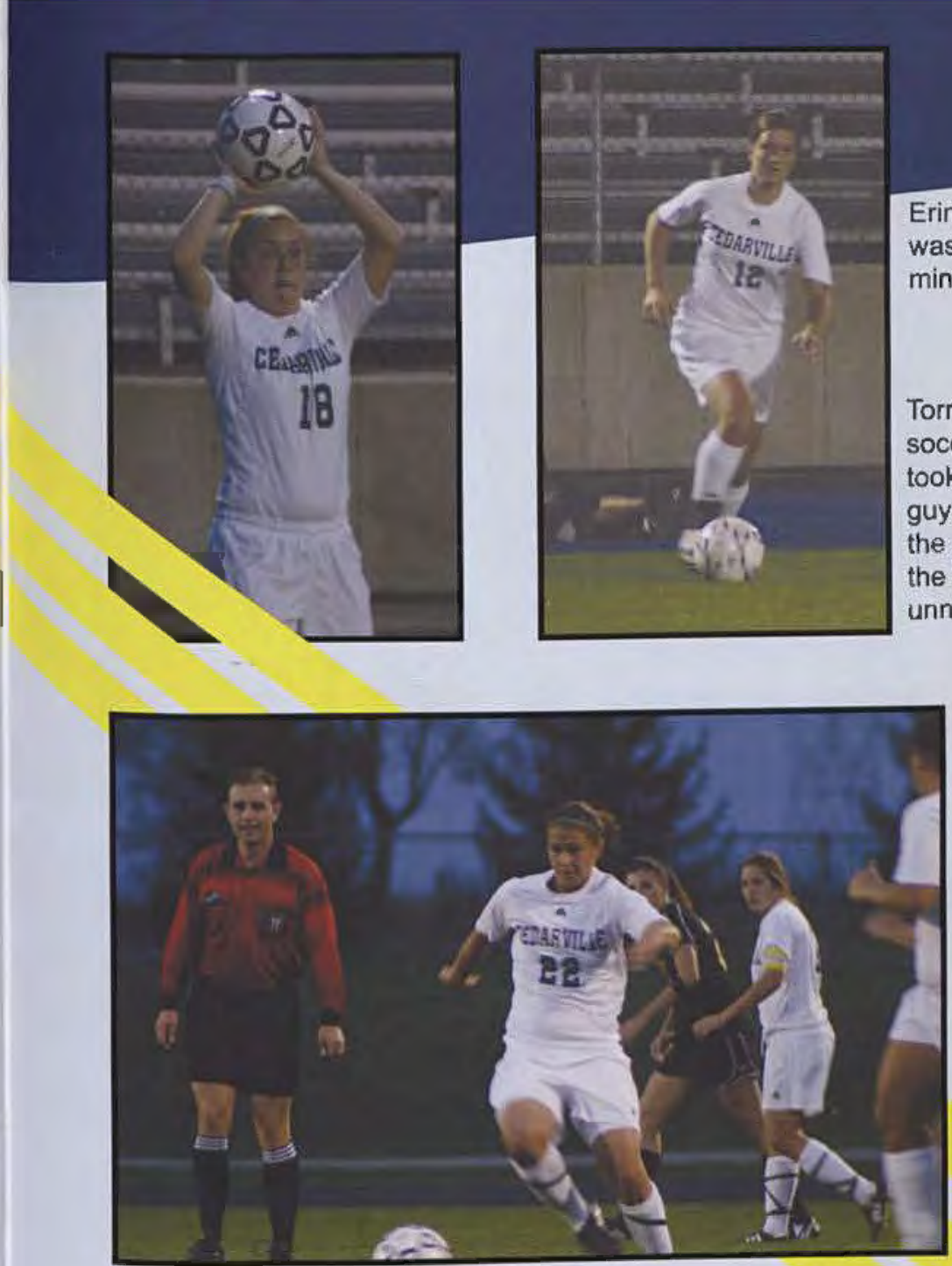

Jill Carroll makes her way through the opposing players.
Erin Landers comments, "After last year with numerous injuries, it was awesome to see how God used our hard work, faith, and determination to achieve good results and goals."

Torrie Pepper reveals, "Well, the bus ride to New York with the men's soccer team was definitely the most interesting trip our team ever took this season. It was kinda cool watching our team support the guys when they played after they sat and cheered for us, however, the yelling of anarchy and pounding the windows on the bus half the time we were watching Beauty and the Beast was a little unnecessary."

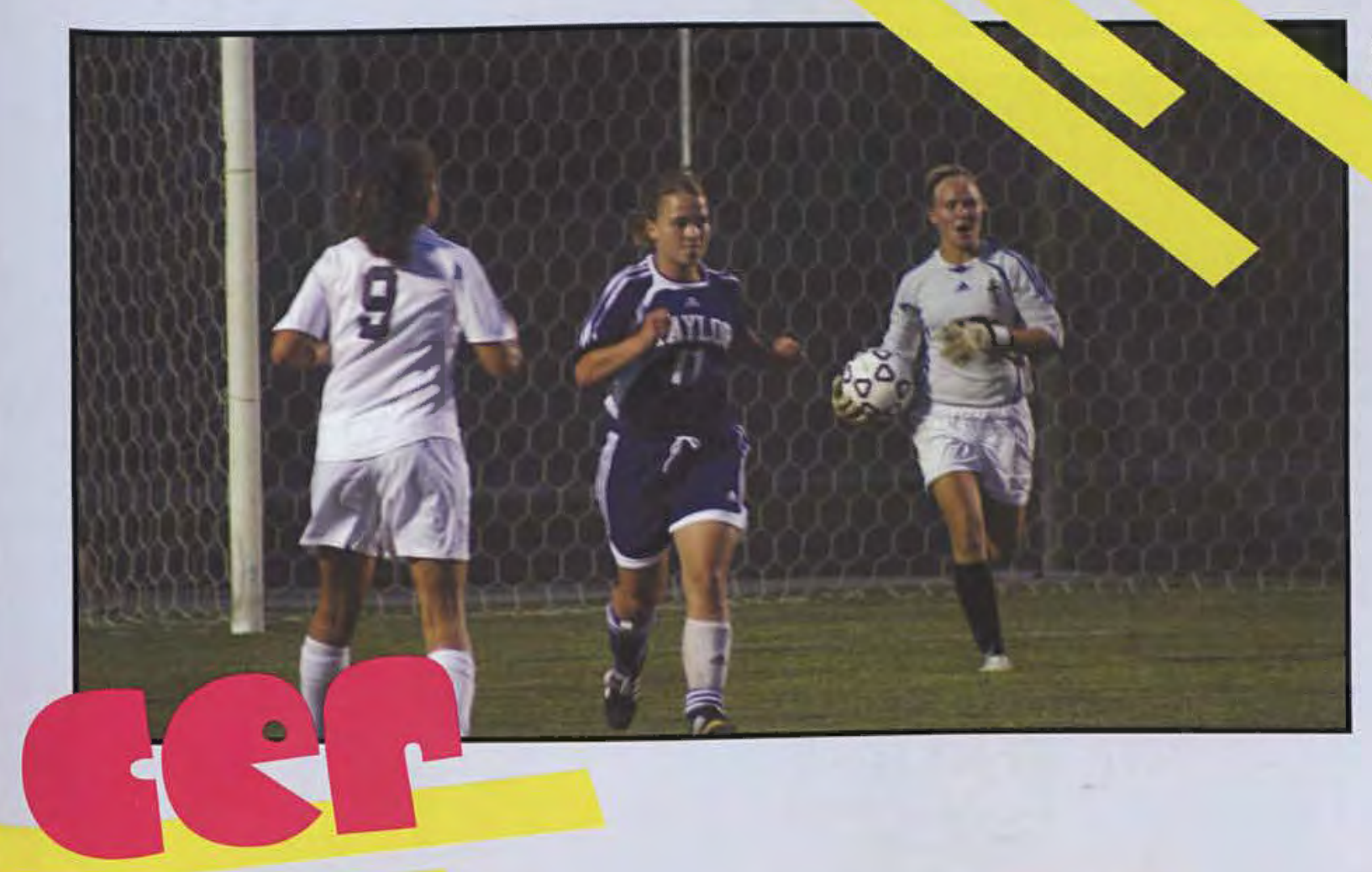

Rachel Brown waits for the keeper to send the ball back onto the field.

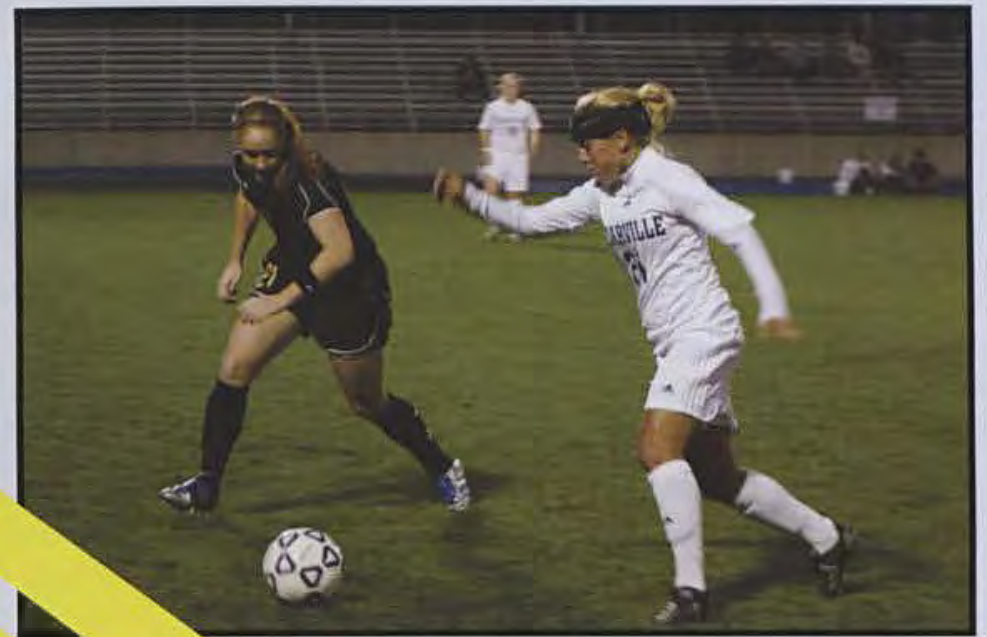

A Lady Jacket works to get the ball through her defenders. 

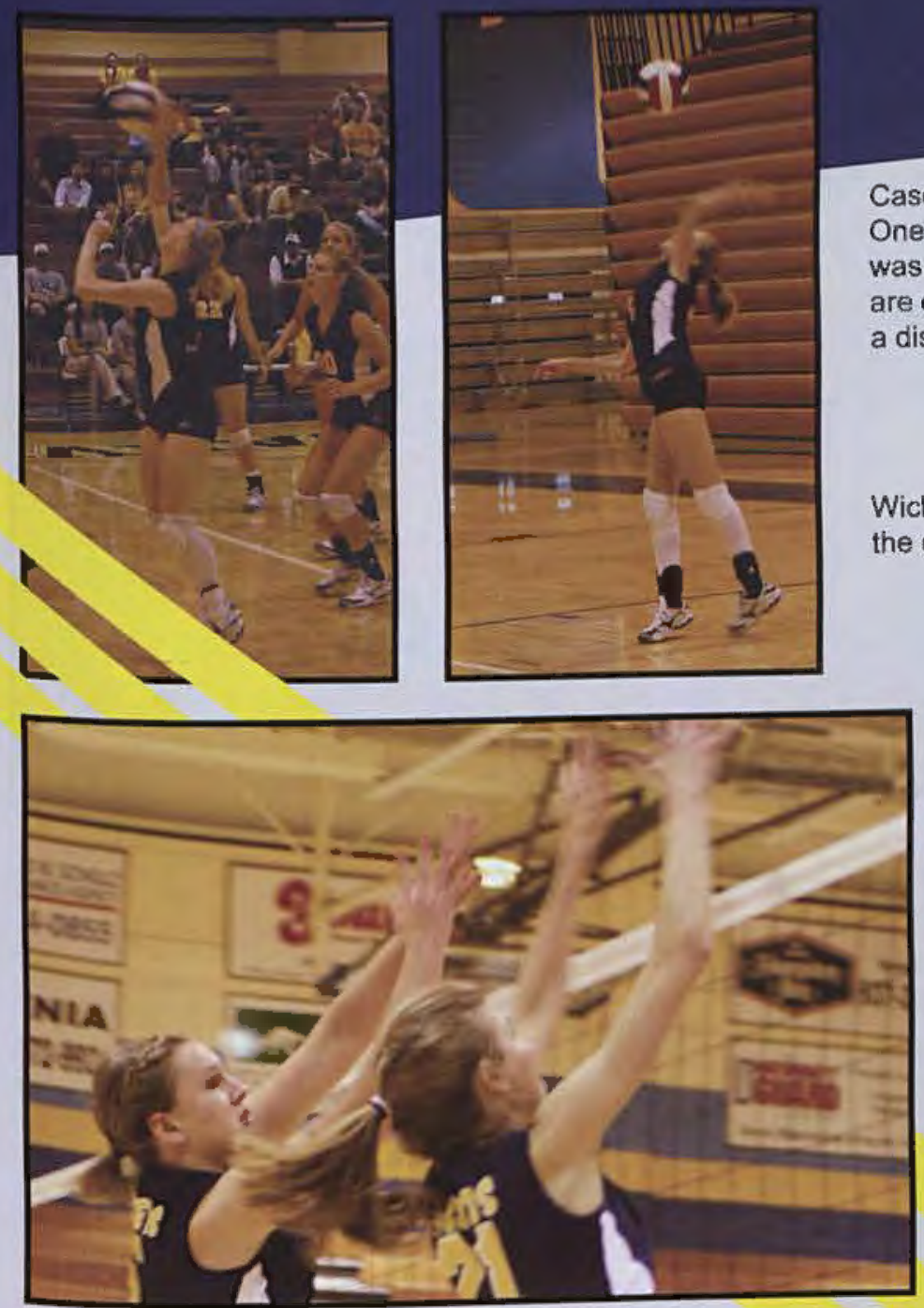

Powerful net players like Justine Christiaanse help Cedarville dominate on defense.
Casey Hinzman says, "It is so amazing playing for an audience of One. Losing 3 players last season and getting a new coach again was frustrating. But when God's hands are at work, the possibilities are endless and winning at the NCCAA championship was definitely a display of this."

Wicked serves were strong weapons that Cedarville presented on the defensive front of each match.

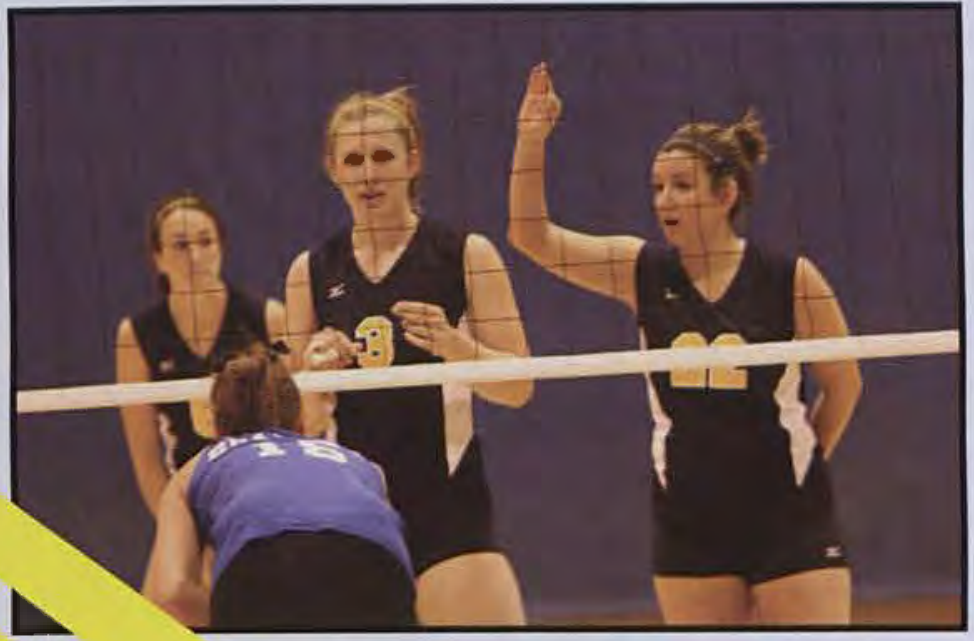

Kylee Husak shares her thoughts about the 2009 season: "There's something amazing about a year where you completely surprise everyone with how you play. This year was such an opportunity. Not only did God allow us to play hard and play together above and beyond what we thought possible, but he provided us with an opportunity to witness at the National Tournament through an honor call."

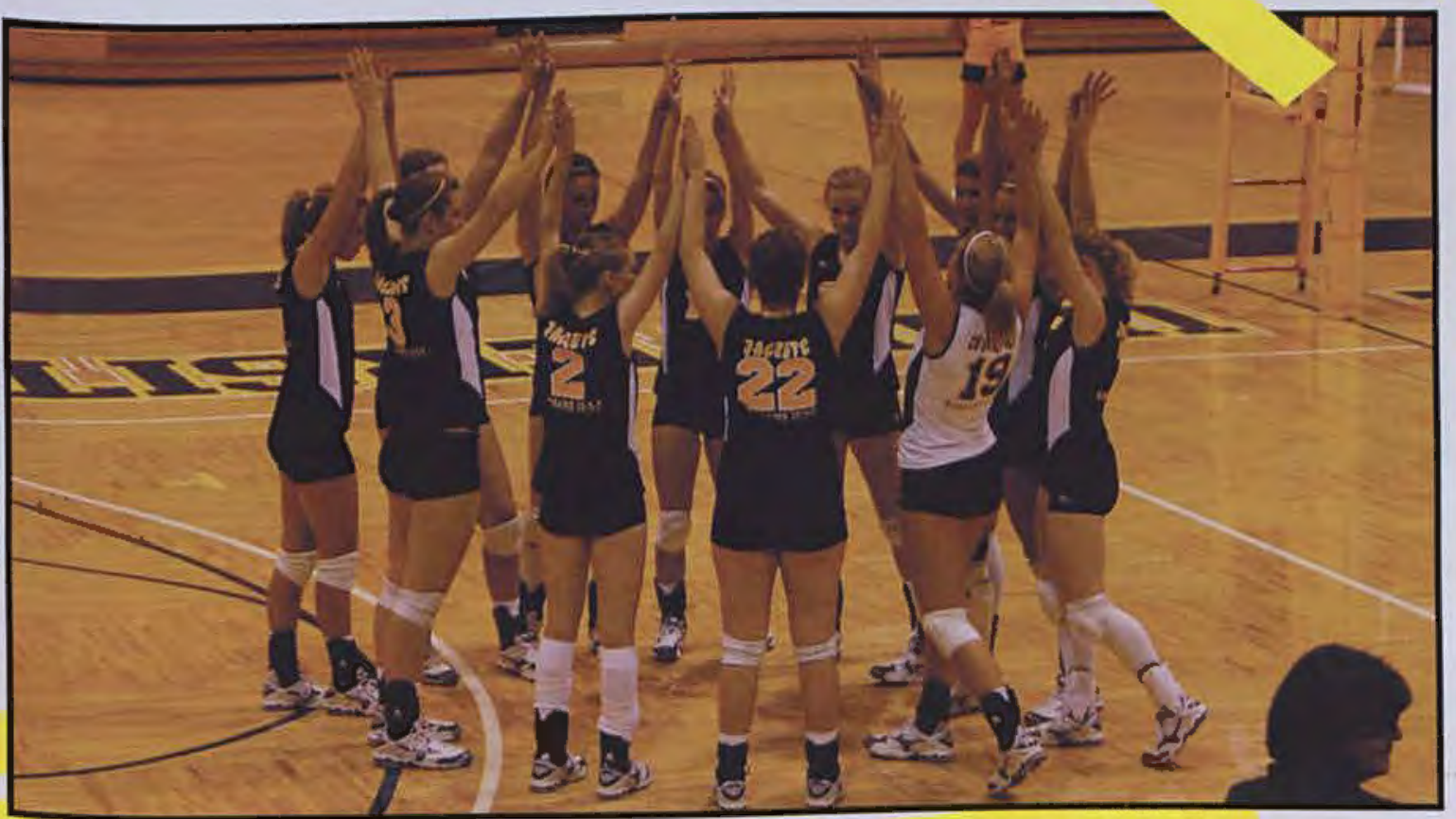

Liz Aker really loves the common purpose she shares with her teammates: "This season was intense with high points and low points, not unlike other college sport seasons. However, the most memorable thing I will take away from this season is how our entire team truly developed and grew as volleyball players and followers of Christ. What a great way to end my CU career!"

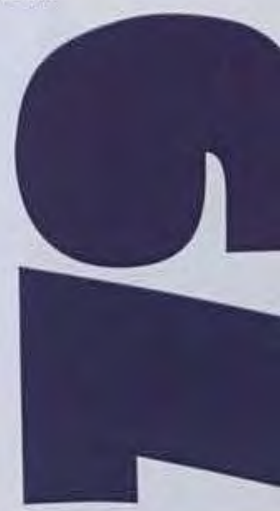


Left to right. Front row: Josh Wiseman, Chris Vaughn, Ethan Blagg, Jud Brooker, T.J. Badertscher, Jordan Davies, Seth Campbell. Middle row: Head Coach Orchard, Team Chaplain Paul Orchard, Assistant Coach Bud May, Ass Zachary Klink

Steve Powers, Zachary Kathey, Luke Clore, Joff Evans, Brian Cheney, Jow: Evan Kevin Kuhn. Back Michael Thayer, Hollis Troxel, Micha, Alex Lacey, Matt Silveira, Alex

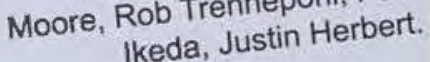

"Cedarville Cross

Country has been a rewarding experience

because it has

encouraged and

promoted running our best for the glory of God," explains freshman Evan Thayer.

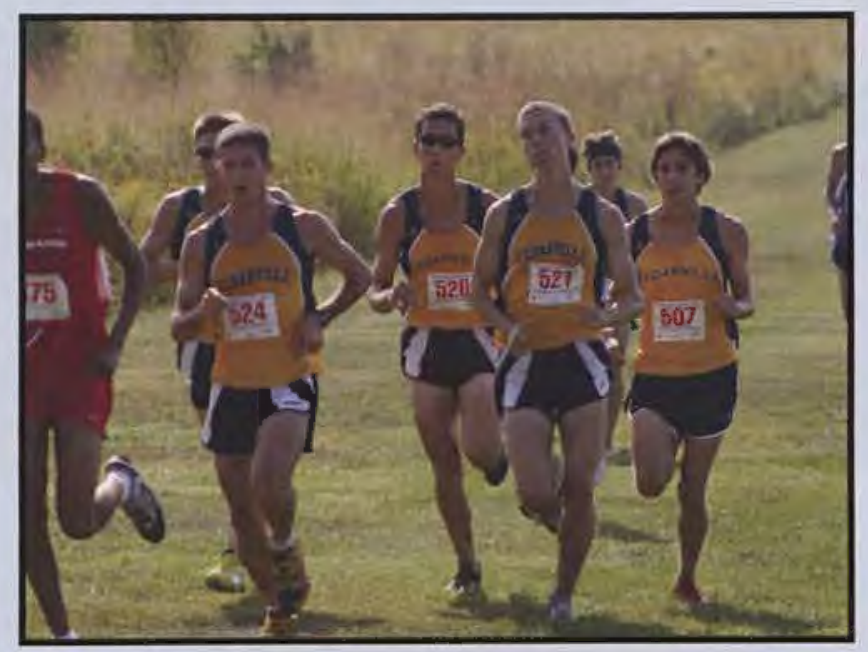

Joseph Cathy remembers teammate Justin Herbert saying, "Guys just remember why we're out here, to run for God, just like we yell every race. So, let's get out there and do what we came to do, run for Him."

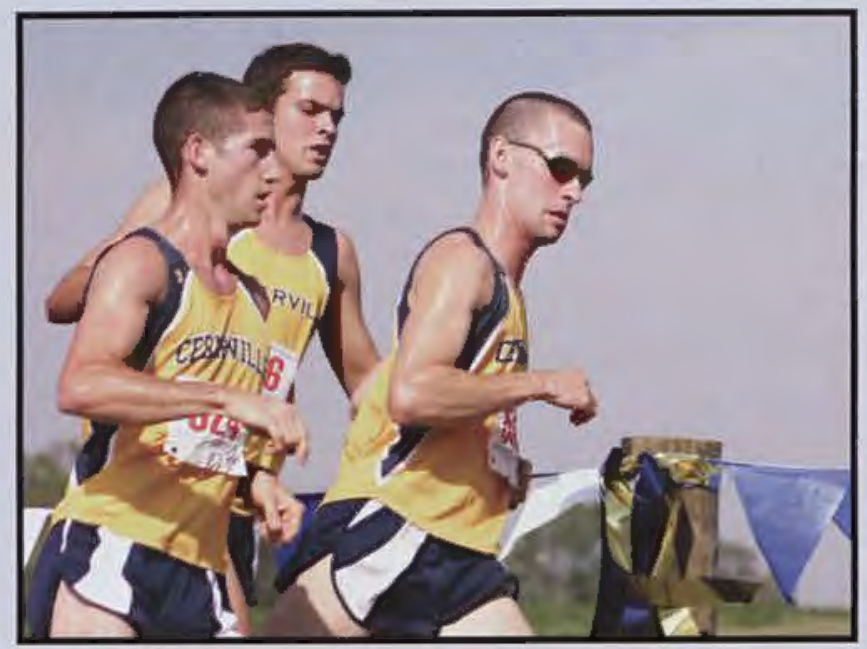

Wittenberg Invitational

18th Annual

Friendship Invitational

Second Annual Cedarville Open

All-Ohio Intercollegiate

Aquinas Invitational

American Mideast Conference

NCCAA Championship

NAIA Championship
1 st of 4

1 st of 20

3rd of 7

24th of 42

6 th of 22

2nd of 13

3rd of 18

19 th of 32
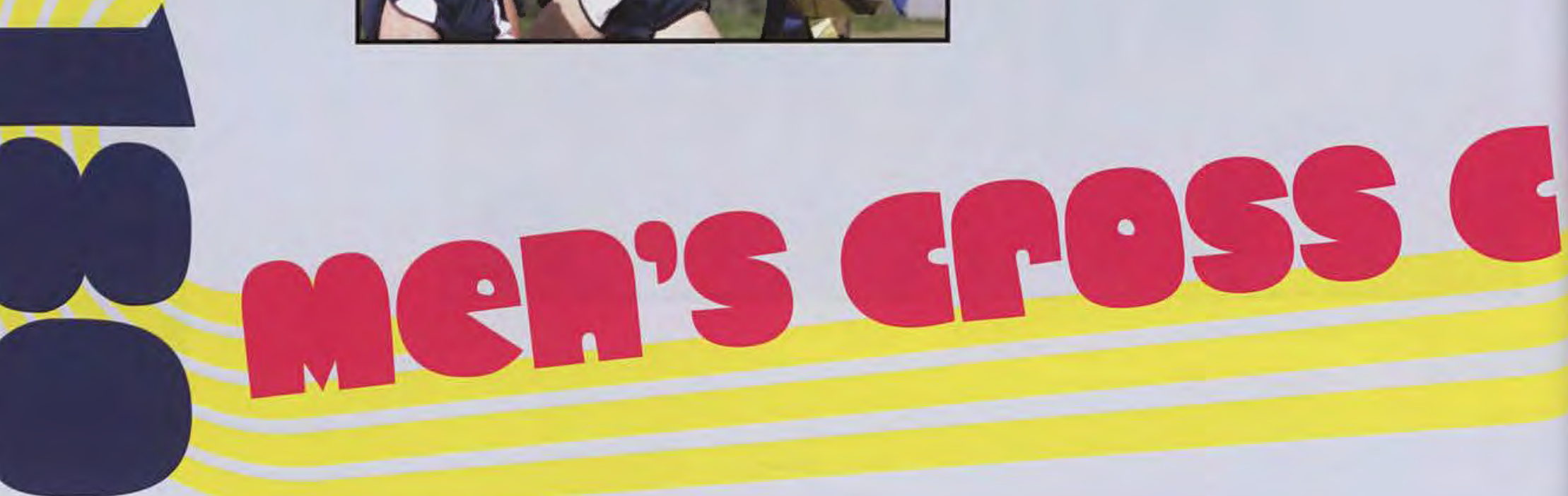


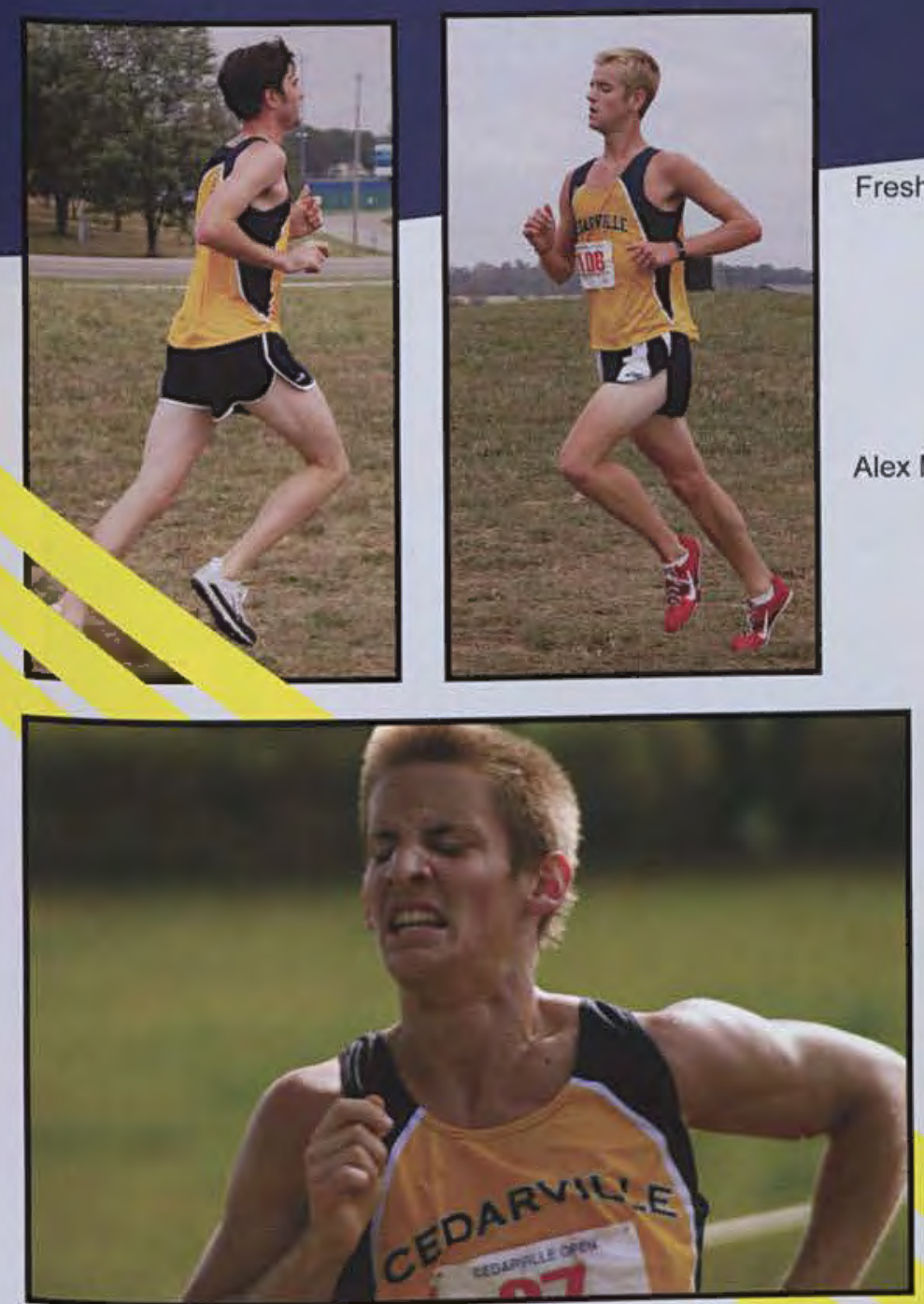

Freshman Brian Cheney works hard for each breath as he runs the race.

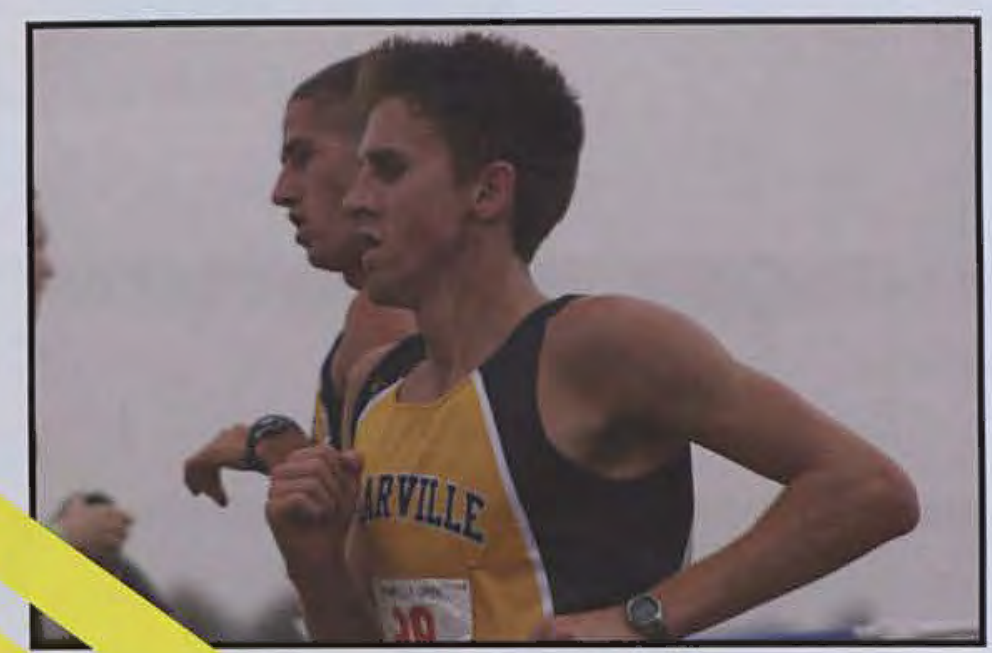

Sophomores Jordan Davies runs with teammate Josh Wisemans.

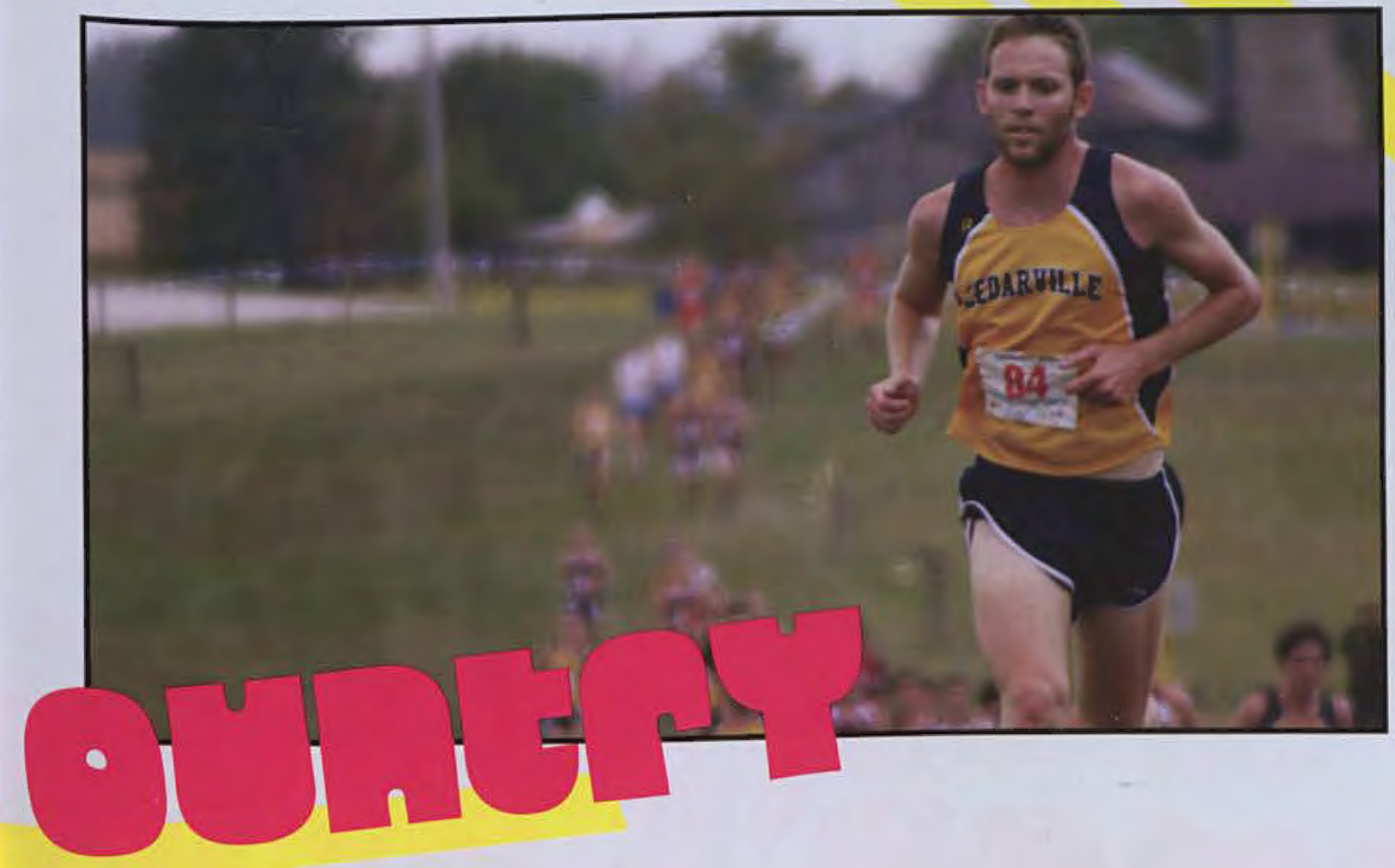

Senior Jud "Chubbs" Brooker reflects on the past year, "We are extremely close group of guys, and love just having fun and hanging out. I am definitely going to miss this group of guys and all the fun we've had developing inside jokes and making ourselves look like idiots."

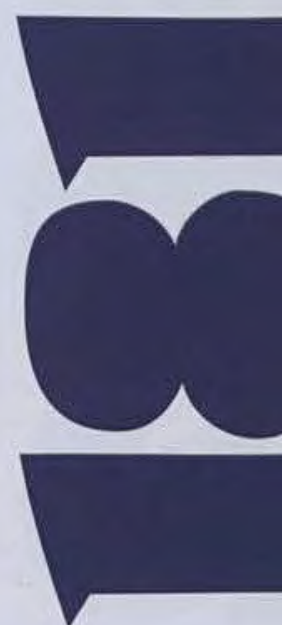


The Lady Jackets show off the many awards they won during the season.

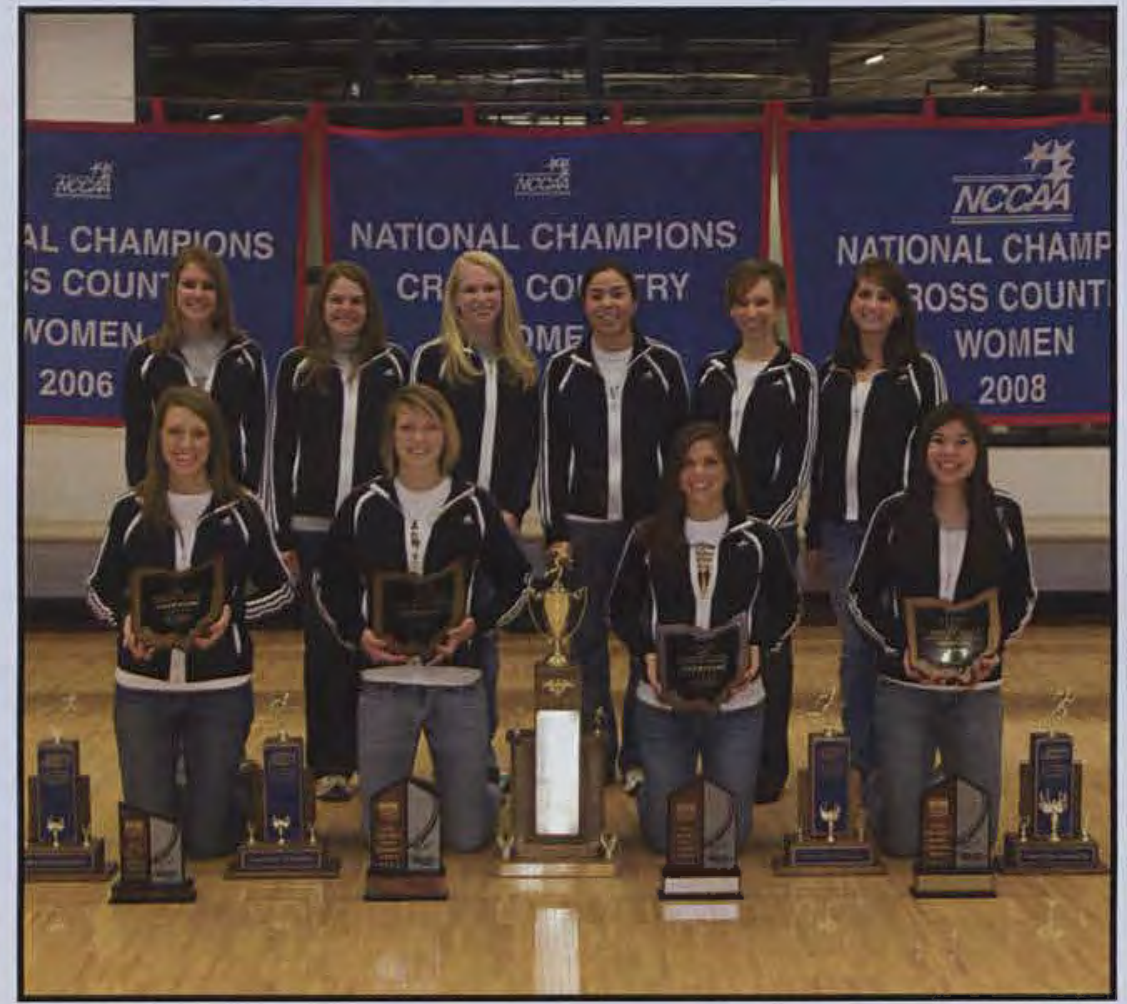

Shawnee State Invitational

1st of 9

Wisconsin-Parkside Midwest Open 1st of 17

Second Annual Cedarville Open

All-Ohio Intercollegiate

Aquinas Invitational

American Mideast Conference

NCCAA Championship

NAIA Championship 1st of 6

1 st of 41

1st of 20

1 st of 14

1 st of 19

2nd of 32

\section{1}

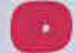

. . .

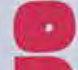

5

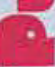

nd of 32 

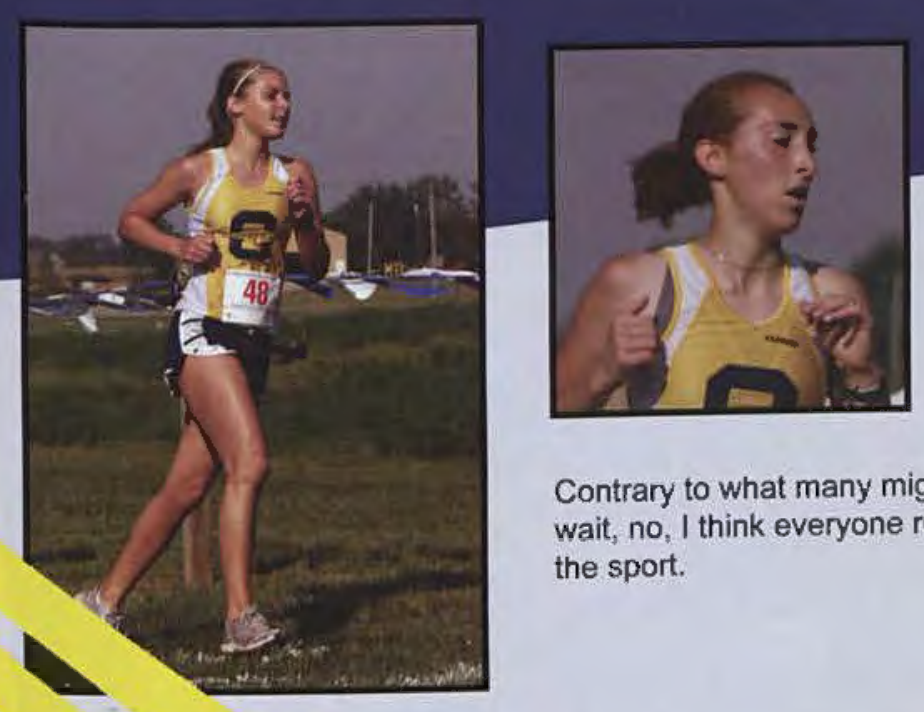

Despite the hard work, she just won't stop. Keep your eyes toward that finish line!

Contrary to what many might think, cross country is hard work. Oh wait, no, I think everyone realizes the dedication the ladies put into the sport.

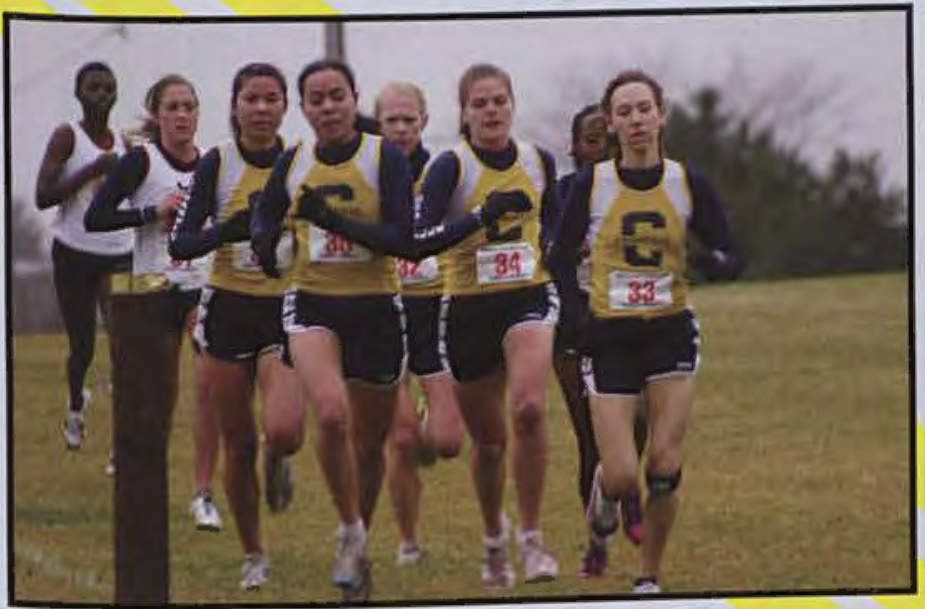

The team works to stay ahead of the oppenents.

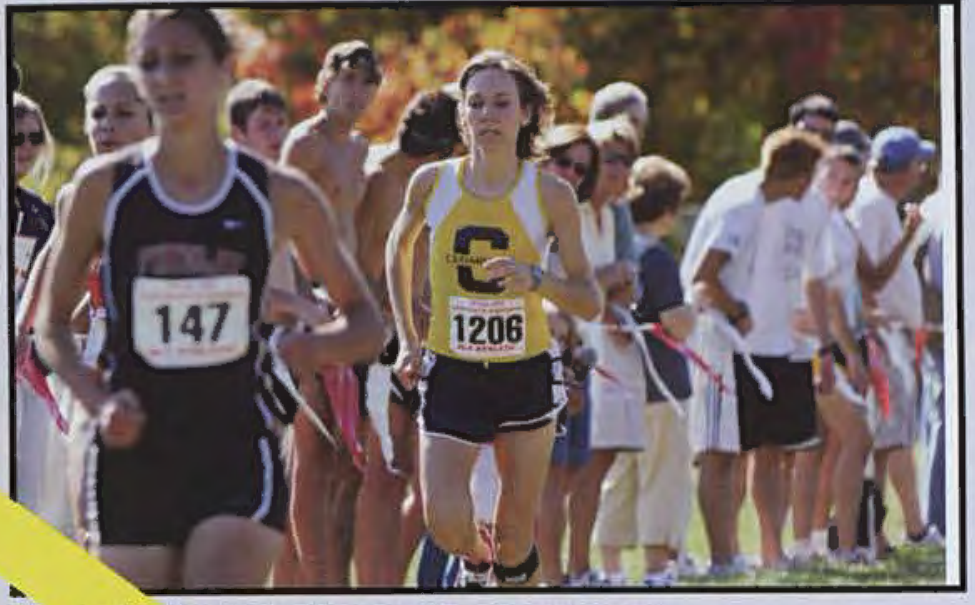

Nicole Santos works to pass the other runners.

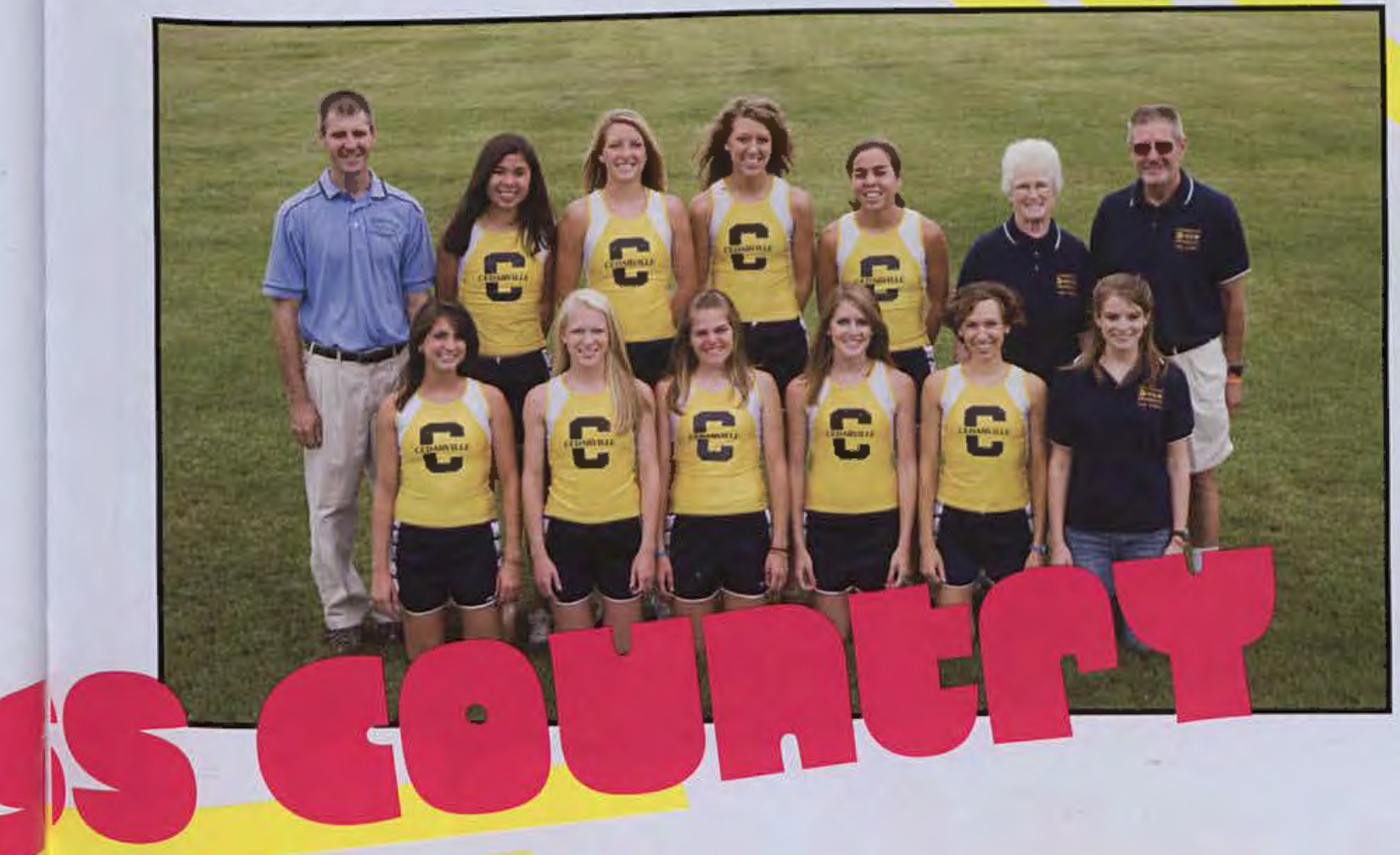

Left to right. Front row: Emily DeRusha, Carolyn Cann, Jennifer Hollander, Rachel Wong Back row: Bethany Davies, Brittany Simpson, Elisabeth Pyles, Lydia Wong, Nicole Santos, Stacey Keller. 
(7):

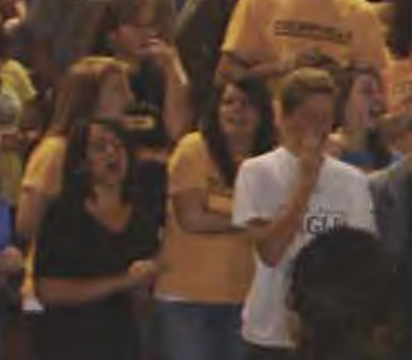

Sabrina Elgersma cracks a pun, "The drum secto
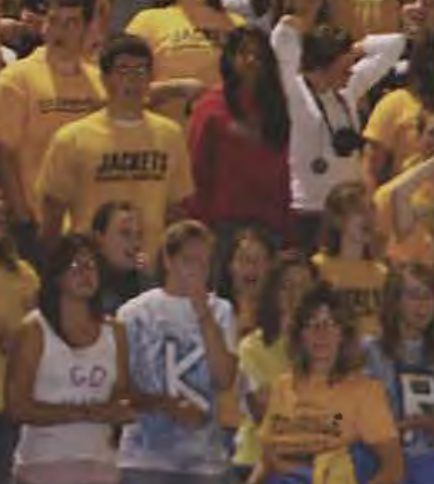

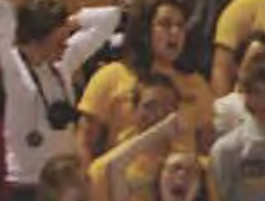

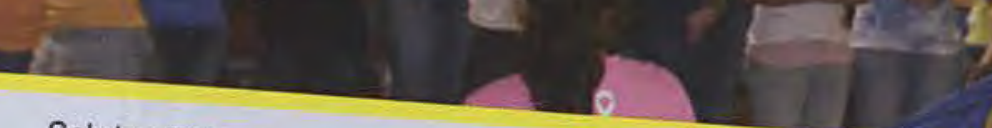

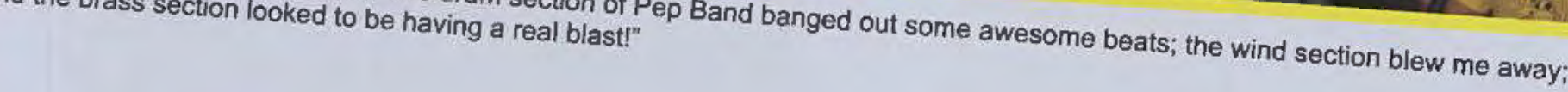

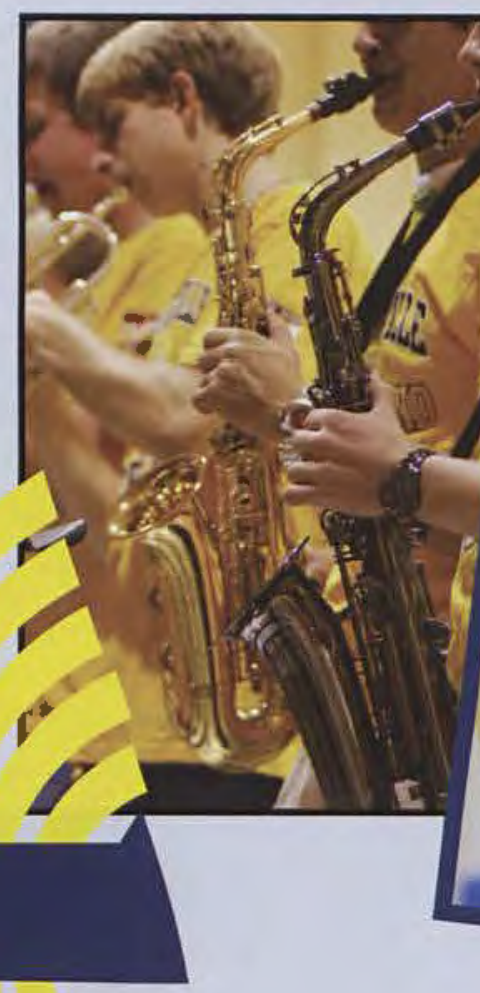

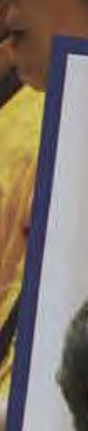
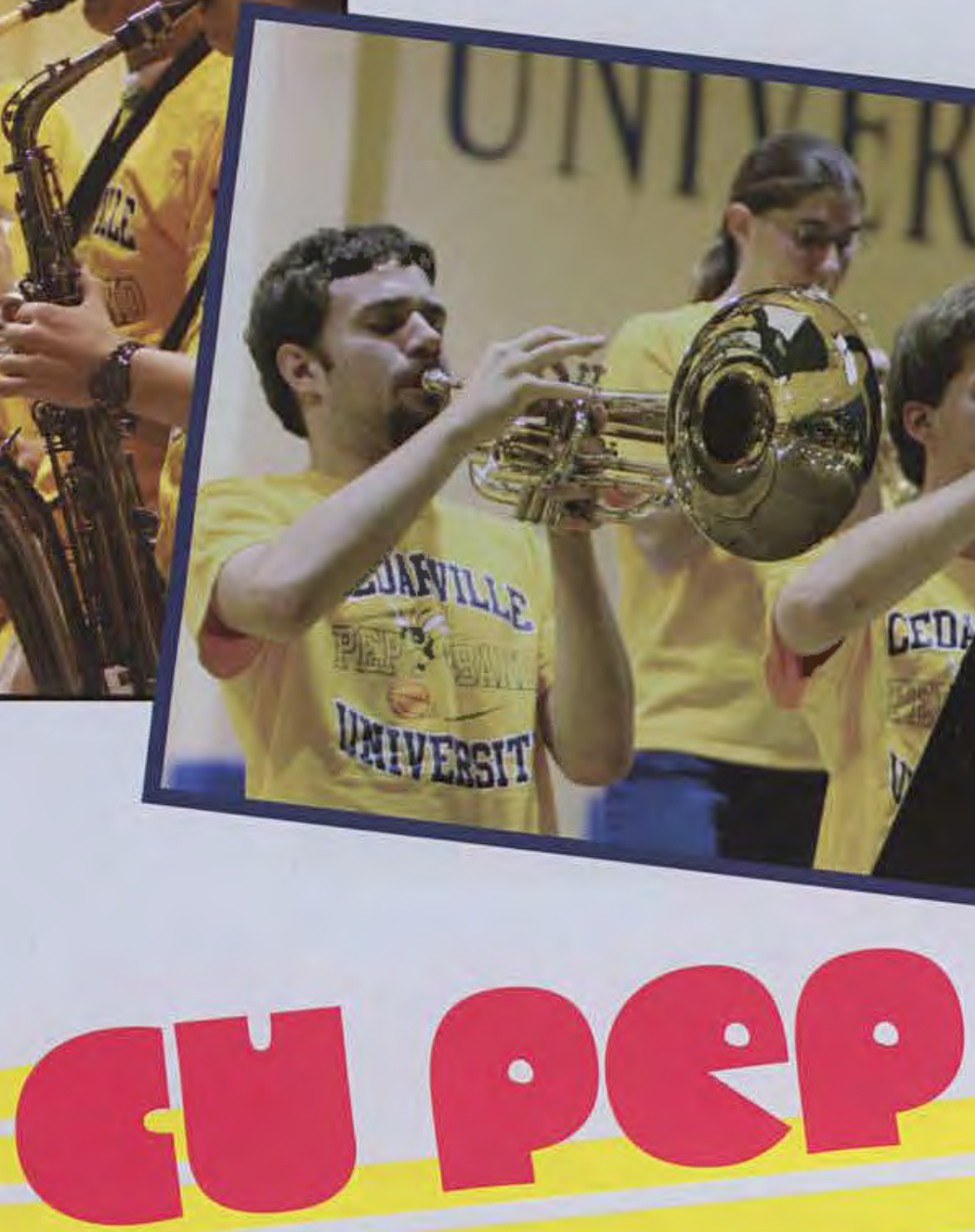

The Cedarville Jacket is great at two things: Breaking out fabulous dance moves and conquering other mascots. 

Left to right Front row: Daniel Kohavi, Haddon Anderson. Chris Beals, Grant Walker.

Ryan Reep, Derrick Hannon. Joe Osborn. Middle row: Head Athletic Trainer Chris Cross, Assistant Coach Loren Kuhn, Assistant Coach Andy Allgrim, Head Coach Pat Estepp, Assistant Coach Jason Beschta, Student Assistant Eric Chisholm. Student Assistant Ryan Mosher. Back row: Adam Riehle, Ricardo Alliman, Adam Clouse, Daniel Rose. Brandon Sok, Christopher Walker, Matt Harner
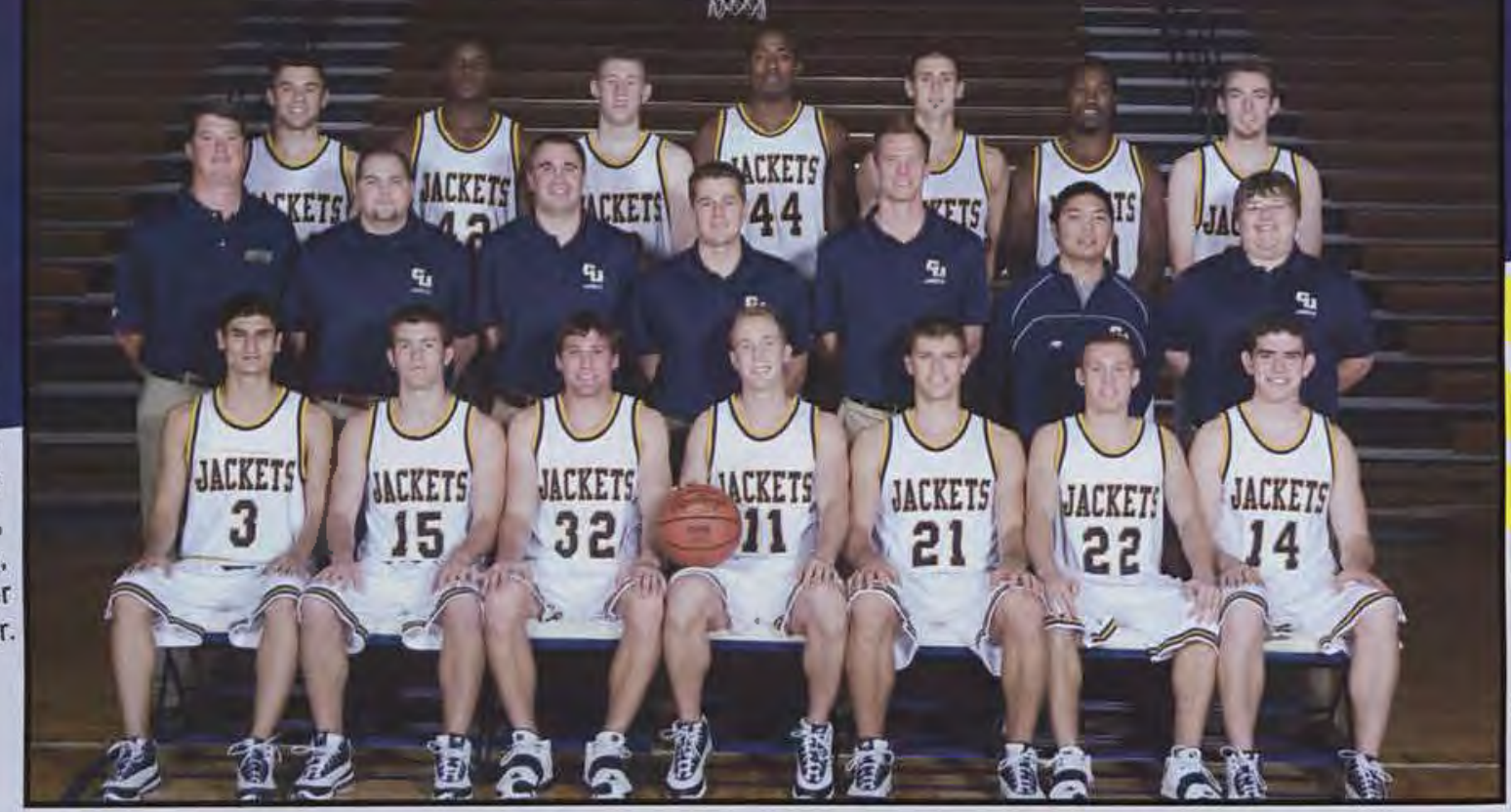

The Jackets have an impressive record on the road, winning 12 out of 13 games. The road warriors are fierce components even without the encouragement of their faithful fans.

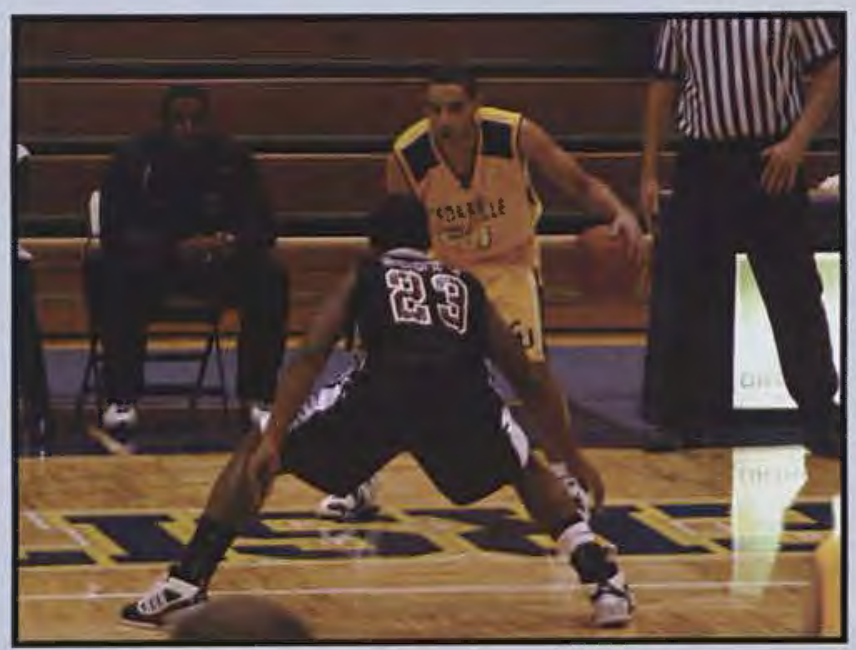

This player beats his defender baseline to the basket, contributing to their $27-4$ record. Despite being in one of the most competitive conferences in the NAIA, the Yellow Jackets are still able to challenge their opponents, providing exciting games for their fans.

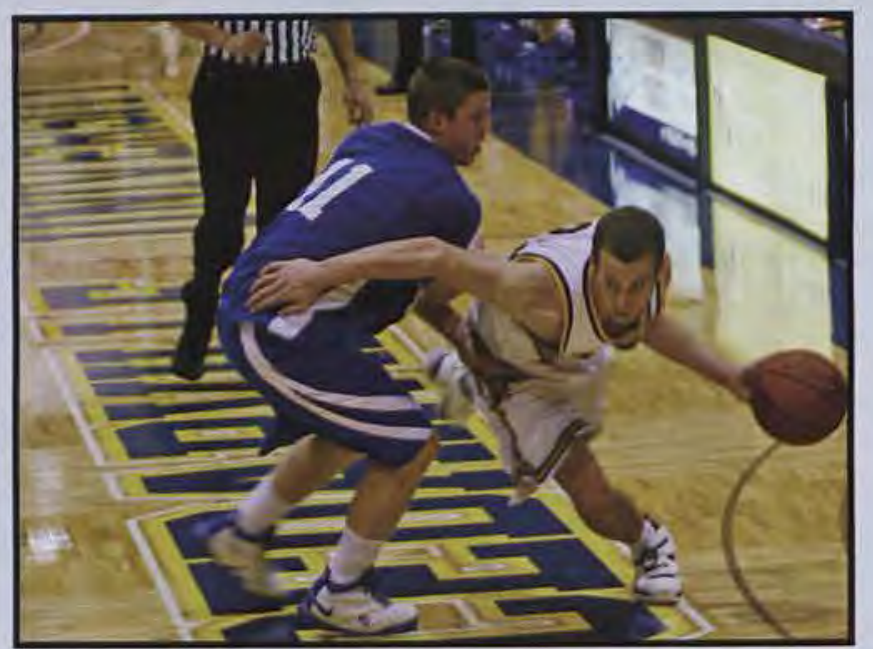

Miami-Middletown

Ohio Christian

Ozarks

Madonna

Covenant

Taylor

Shawnee State

Lake Superior State

Rio Grande

Wilberforce

Wittenberg

Trevecca Nazarene

Tennessee Temple

Bryan

Windsor (Ont.)

Algoma (Ont.)

Rio Grande

Ohio Dominican

Walsh

Mount Vernon Nazarene

Malone

Northwestern Ohio

Daemen

Mount Vernon Nazarene

Shawnee State

Wilberforce

Notre Dame (Ohio)

Point Park

Houghton

Roberts Wesleyan

Sioux Falls*

*NAIA Division II National Tournament
W 99-76

W $78-48$

W $67-50$

W $81-65$

W 74-47

W 68-59

W 74-62

W $74-69$

L 65-70

W 79-58

W $80-53$

W $75-63$

W $102-46$

W 51-43

W 77-54

W 100-51

W 66-64

W 87-65

W 70-66

W $70-68$ (ot)

W 69-63

W 71-58

W 81-67

L 71-76

L 50-61

W 70-63

W 64-53

W 76-58

W 69-53

W 71-66

L 63-66

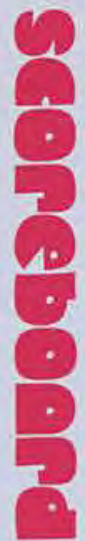

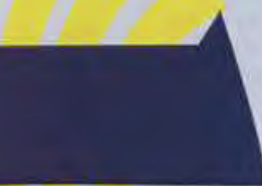
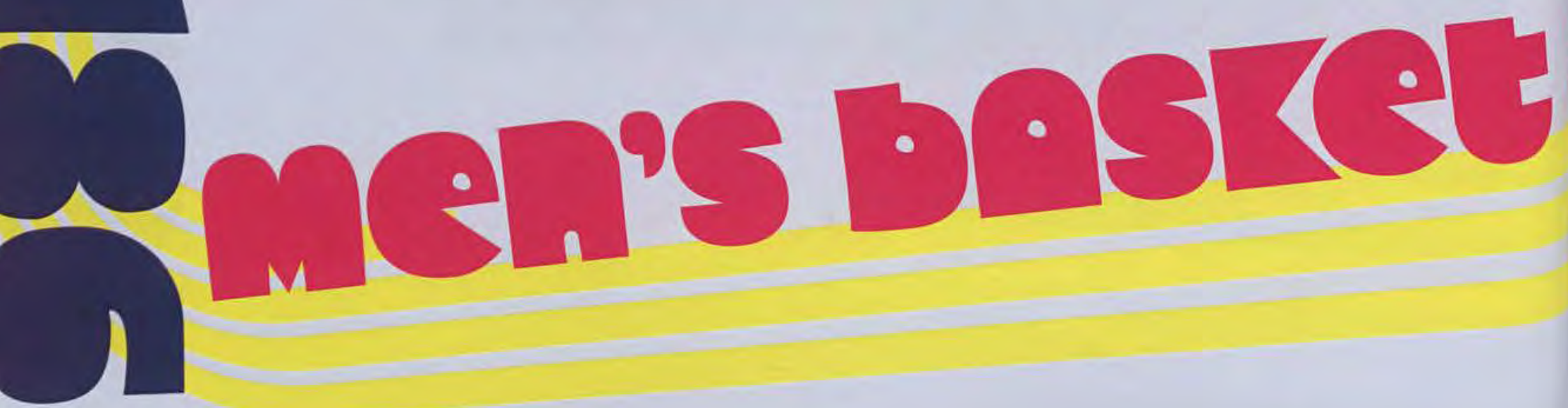

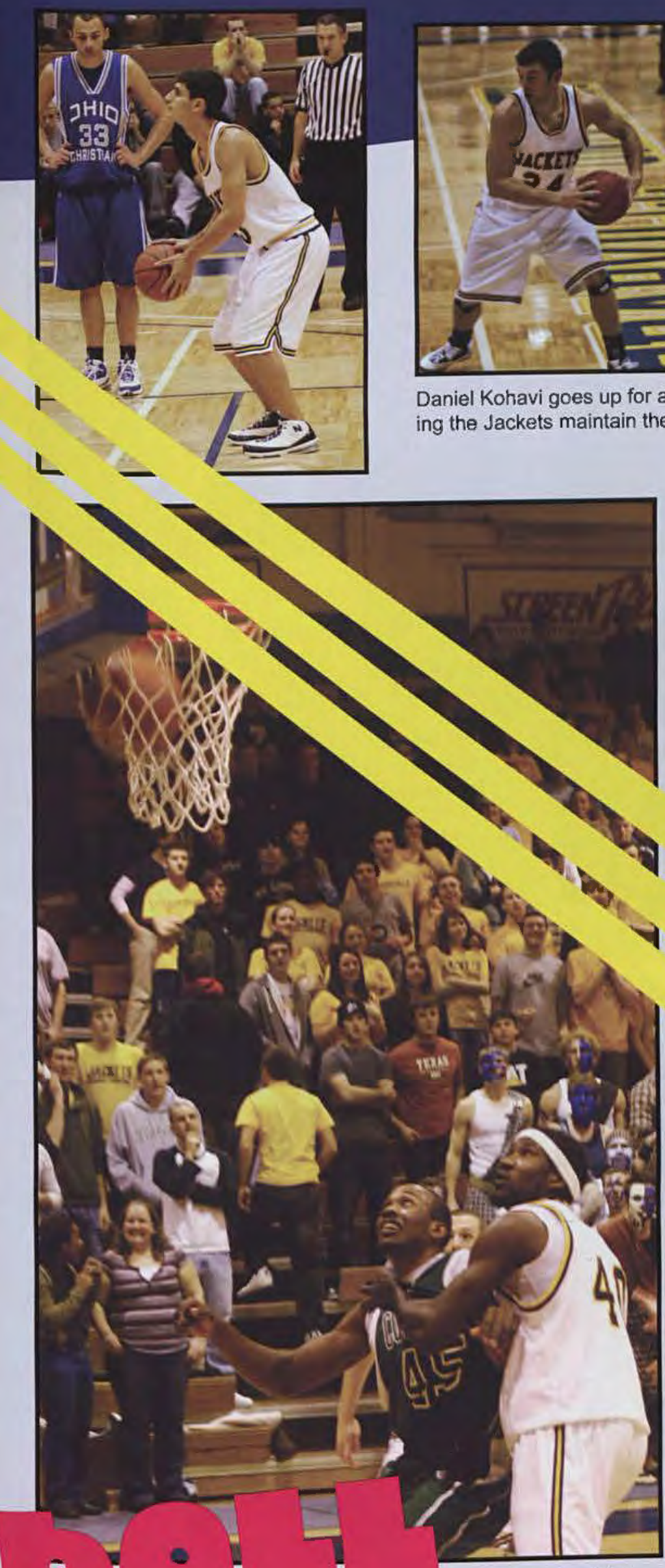

Christopher Walker competes for the rebound.

Daniel Kohavi goes up for a free throw at this home game, help-

ing the Jackets maintain their $11-2$ home record.
"Our team was very successful on the court this year, but I believe our greatest success was the deep relationships we built with each other. I have never been part of a team who loved each other more than our team did this year," says Adam Riehle.

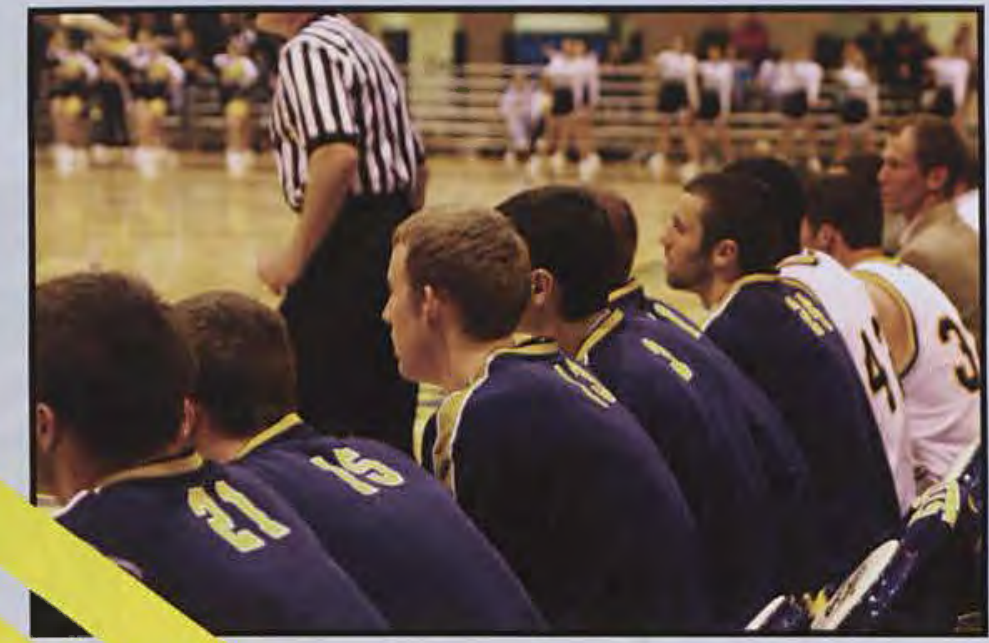

"There are two things that keep us going throughout the season: the motivation to honor God with our effort, and the banana pudding during our pre-game meals at Golden Corral," says Brandon Sok.

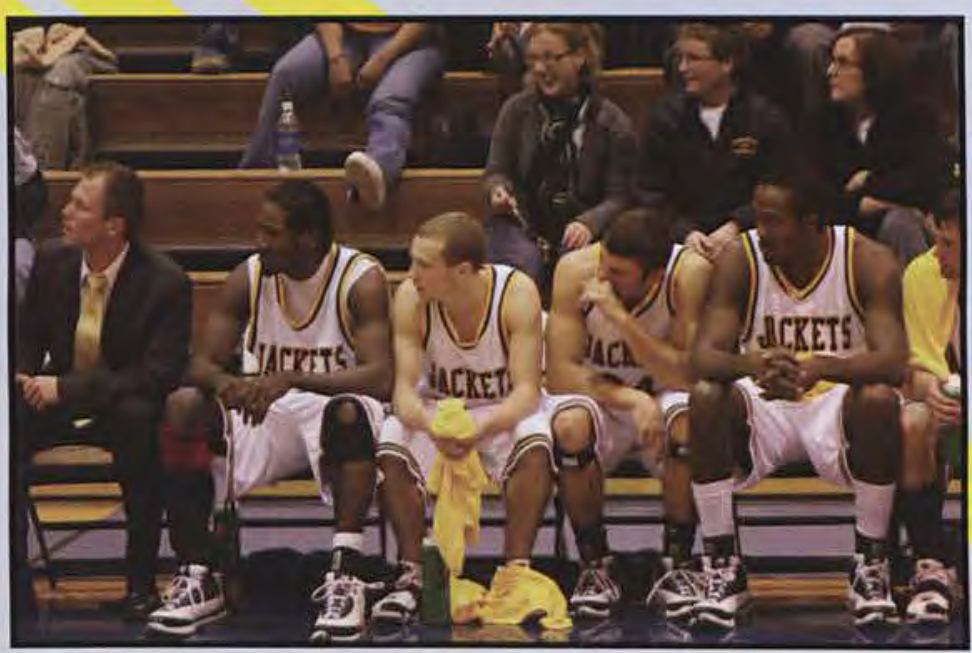

Adam Clouse remembers, "Everything was going great on our road trip to Mt. Vernon-we had just enjoyed a buffet dinner, and the girls were winning their game. Then Coach asked where the jerseys were..." 
Cayton, Julie Utz. Middle

Smith, Caitlyn Mauch, Lydia Miller, Student Assistant Stephanie Latham. Back Assistant Coach Dan Schetter, Head Coach Kirk Huckaby, Assistant Coach
Lacie Condon, Rache

Hurley, Bayley Nosal, Alisha

Lee, Alison Lemon, Kara

ow: Student Athletic Trainer

Julie Brandt, Stefanie Rod-

gers, Aubrey Siemon, Jenna row: Head Athletic Trainer Chris Cross, Assistant Coach Heather Grooms, Martin, Assistant Coach Lori Lynsey Fabian.

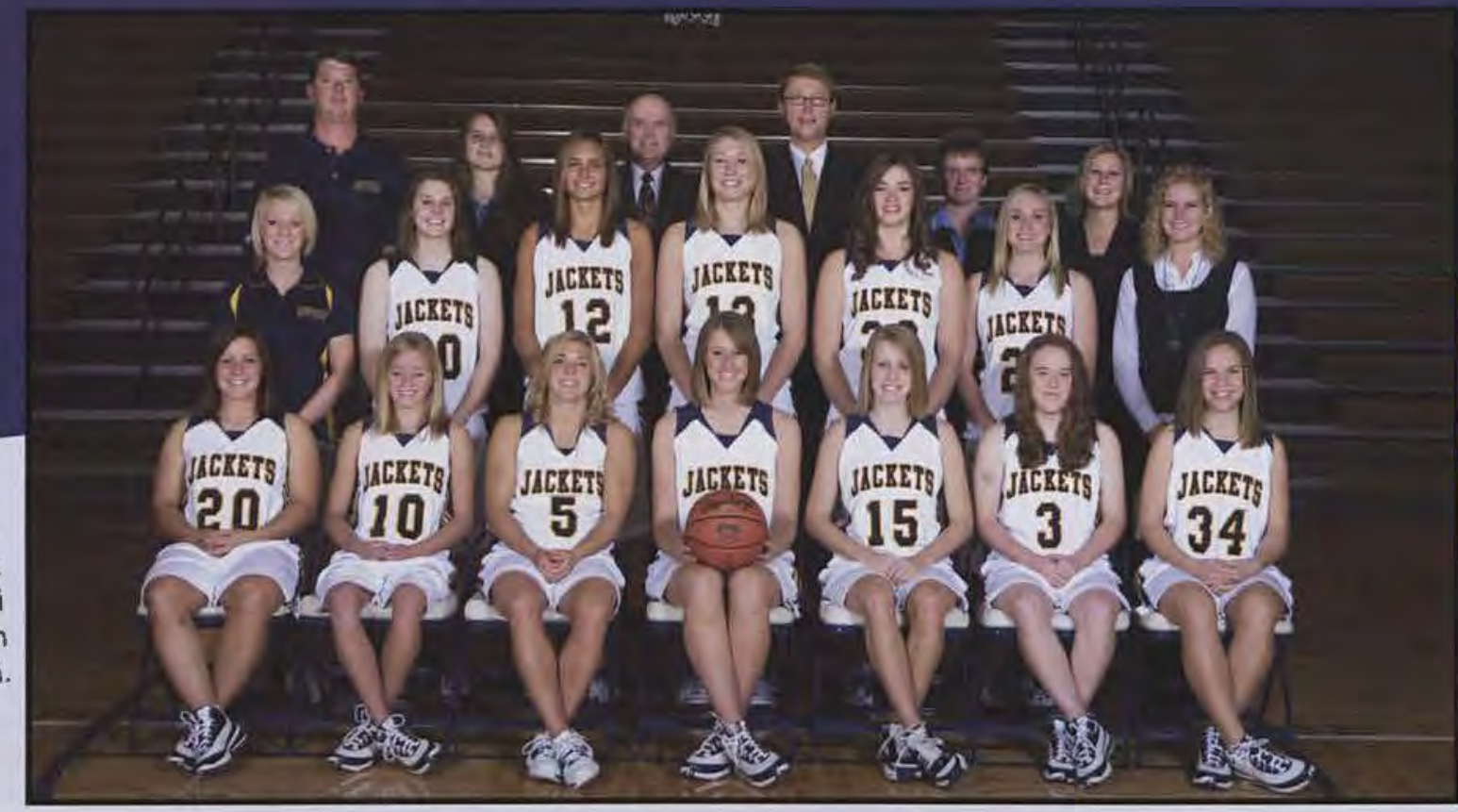

The Lady Jackets play

against some fierce

competition in their

division but end their

season winning nearly two-thirds of their games.

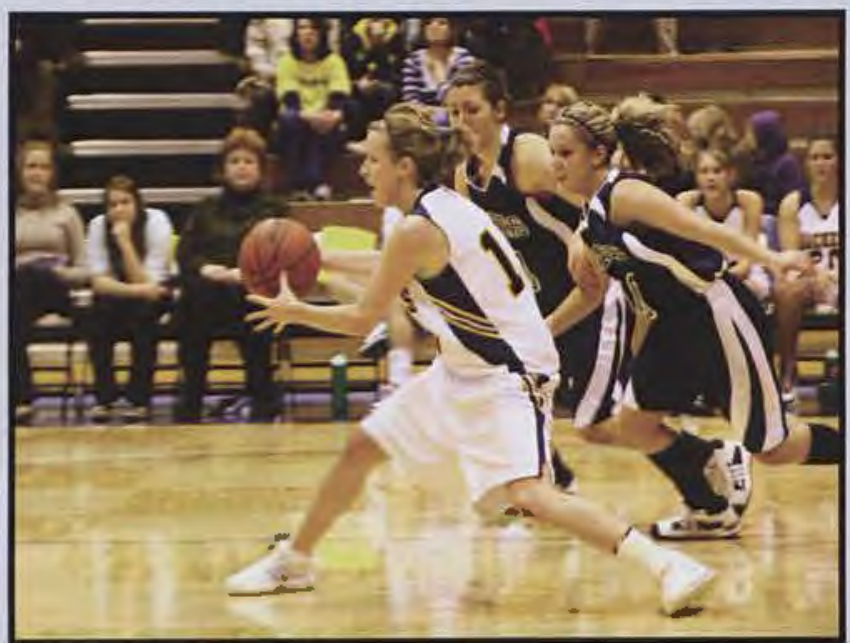

Guard Rachel Hurley,

known for her agility and ball handling skills, dribbles the ball down the court to set up the offense while forward Aubrey Siemon sets a screen on the opposing team's defense.

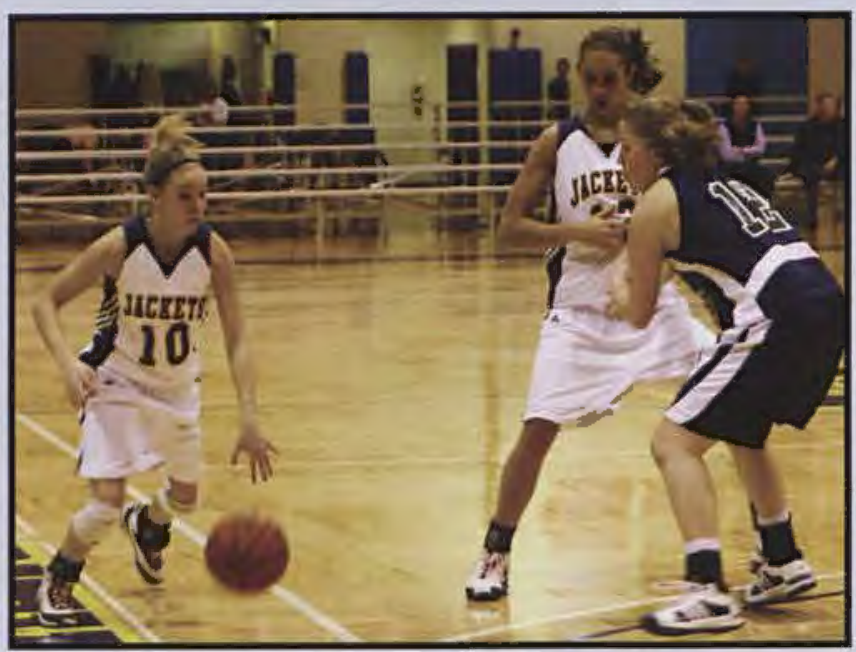

Point Park

Malone

Taylor

Shawnee State

Central State

Urbana

Wilberforce

Urbana

Madonna

Saint Francis (III.)

Brewton-Parker

Saint Francis (ind.)

Rio Grande

Ohio Dominican

Walsh

Mount Vernon Nazarene

Malone

Northwestern Ohio

Daemen

Mount Vernon Nazarene

Shawnee State

Wilberforce

Notre Dame (Ohio)

Carlow

Point Park

Ursuline

Houghton

Roberts Wesleyan

Shawnee State

Bethel (Ind.)*

W 85-71

W $75-40$

W 63-56

L 57-68

L 64-82

W $90-54$

W 76-64

W 84-54

W $77-70$

L 57-67

W 70-47

W 73-61

L 57-62

L 70-74

W $77-62$

W 84-68

W 81-63

W 86-71

L 66-73

L 84-88

W $91-56$

W 90-63

W $94-39$

W 68-60

W 78-56

W 62-55

W $80-54$

L 47-59

L 63-67

*NAIA Division II National Tournament
W 75-55
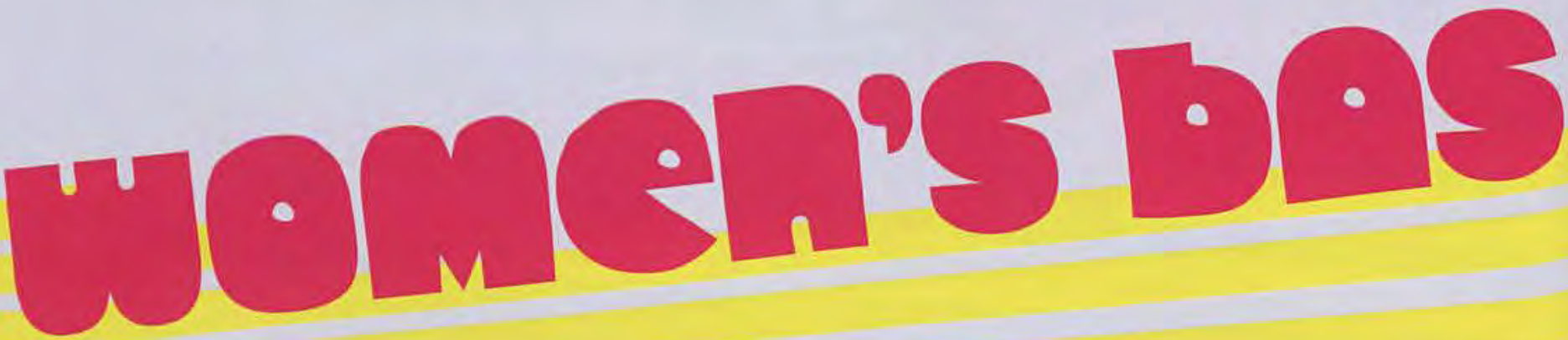

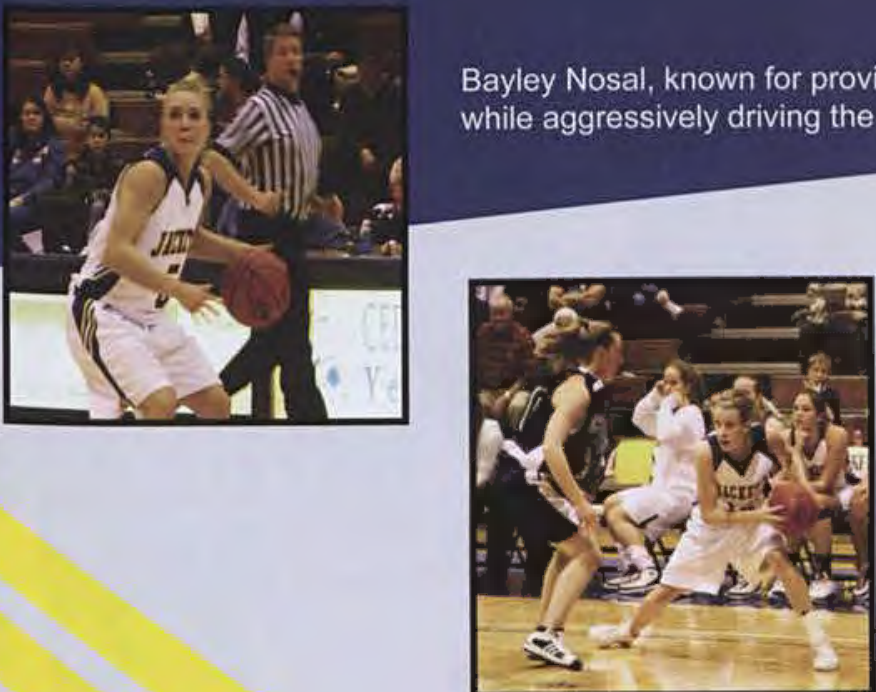

"This year we were challenged to be doers of the Word and not just hearers. Our team took this challenge and executed," says Aubrey Siemon.

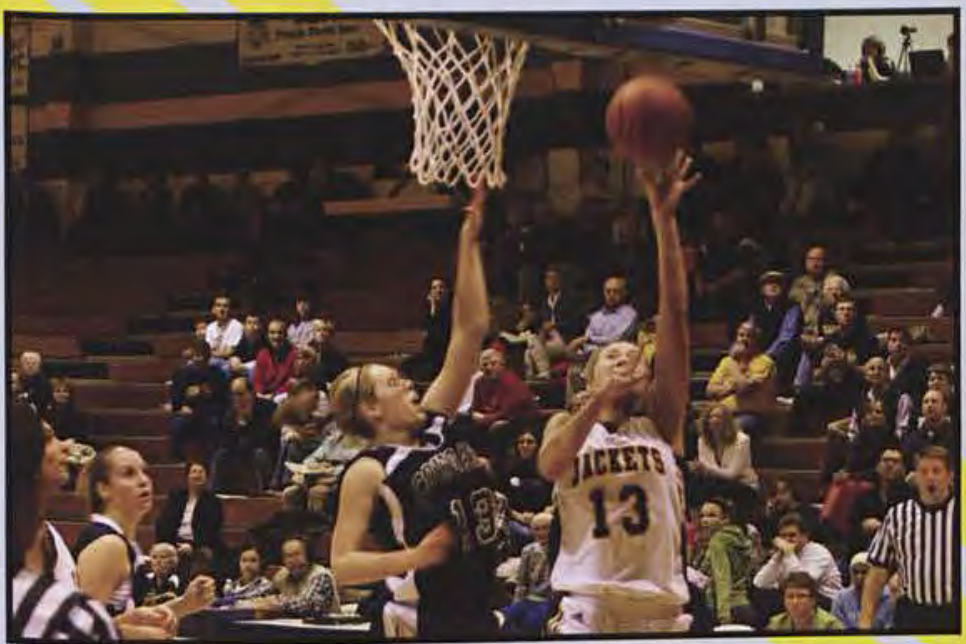

Ali Lemon says, "I had a blast this year. The whole team grew closer with each other and worked exceptionally hard all season. I was excited to share the experience with the girls of making it back to NAIA Nationals."

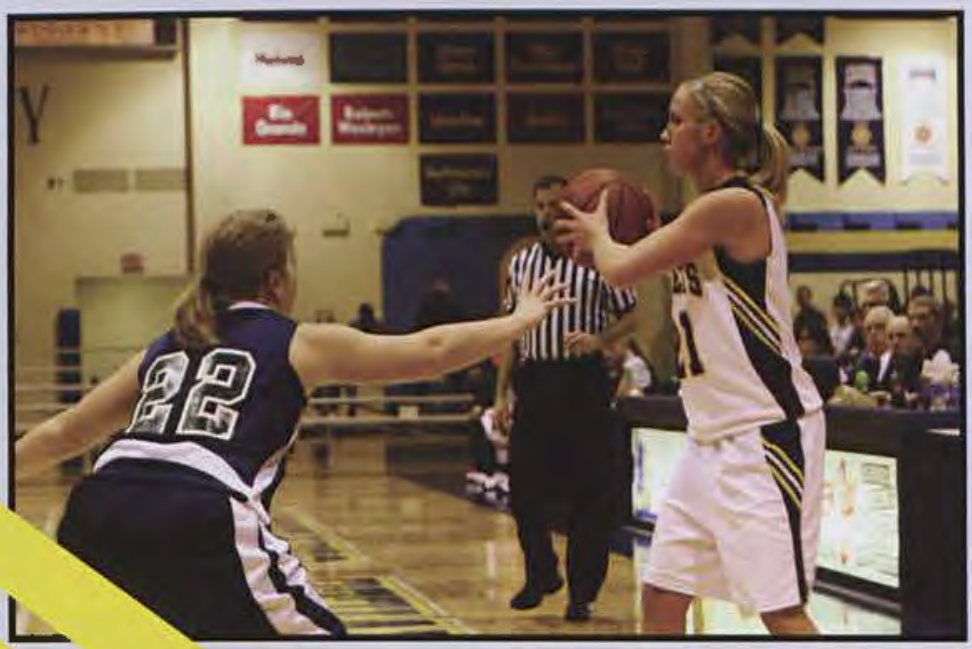

Lydia Miller attentively watches her teammates for an open pass at this home game. While at home, the Lady Jackets are encouraged by many faithful Cedarville fans who always come decked in blue and gold.

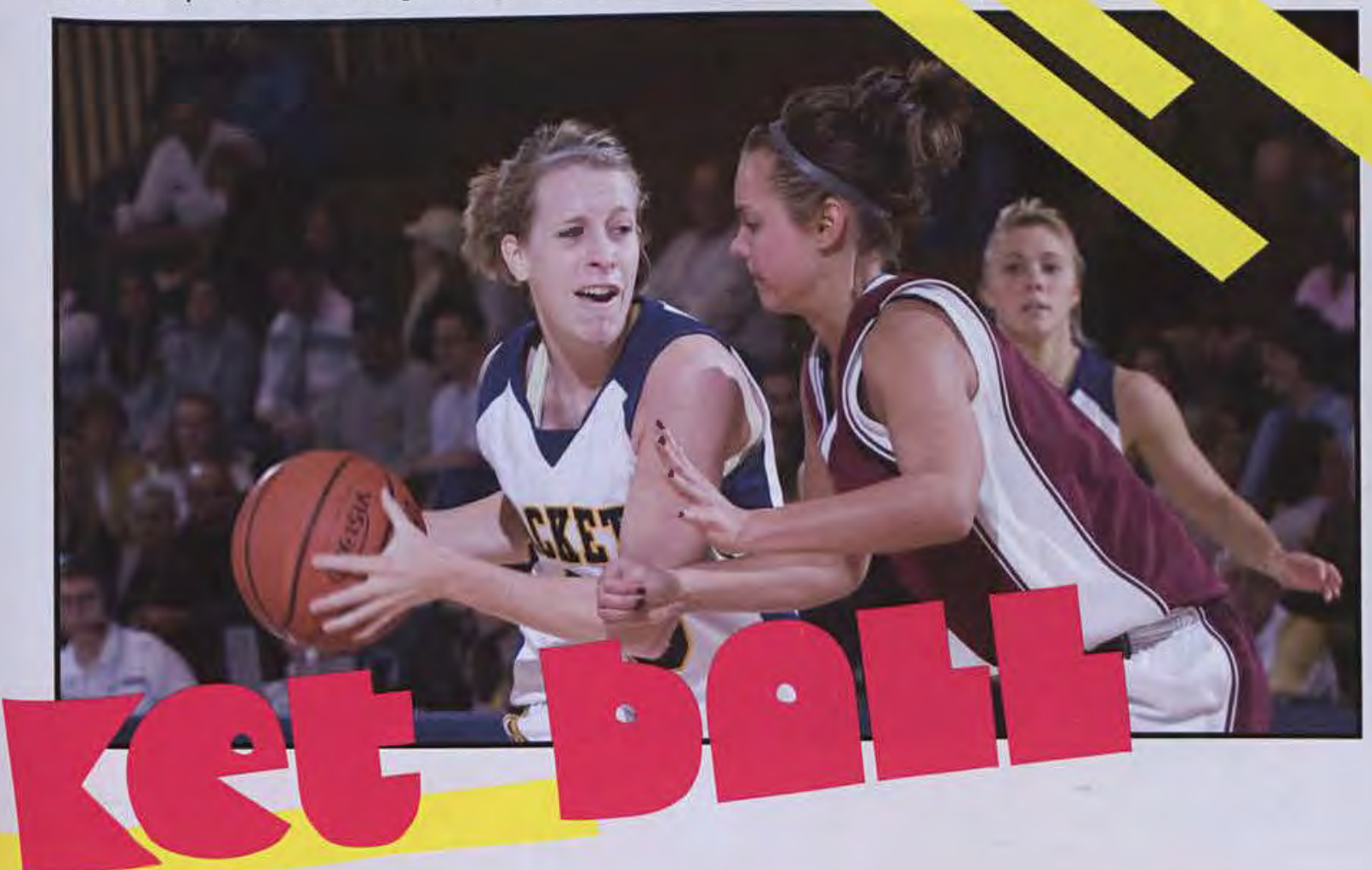

Ali Lemon looks for a pass while being tightly guarded by Walsh defender, Leah Seaman. The Lady Jackets play their games wholeheartedly, with passio for the sport and for representing Christ through their words and deeds. 
Lauren Young and David Duerrwaechter show their team spirit with a difficult lift.
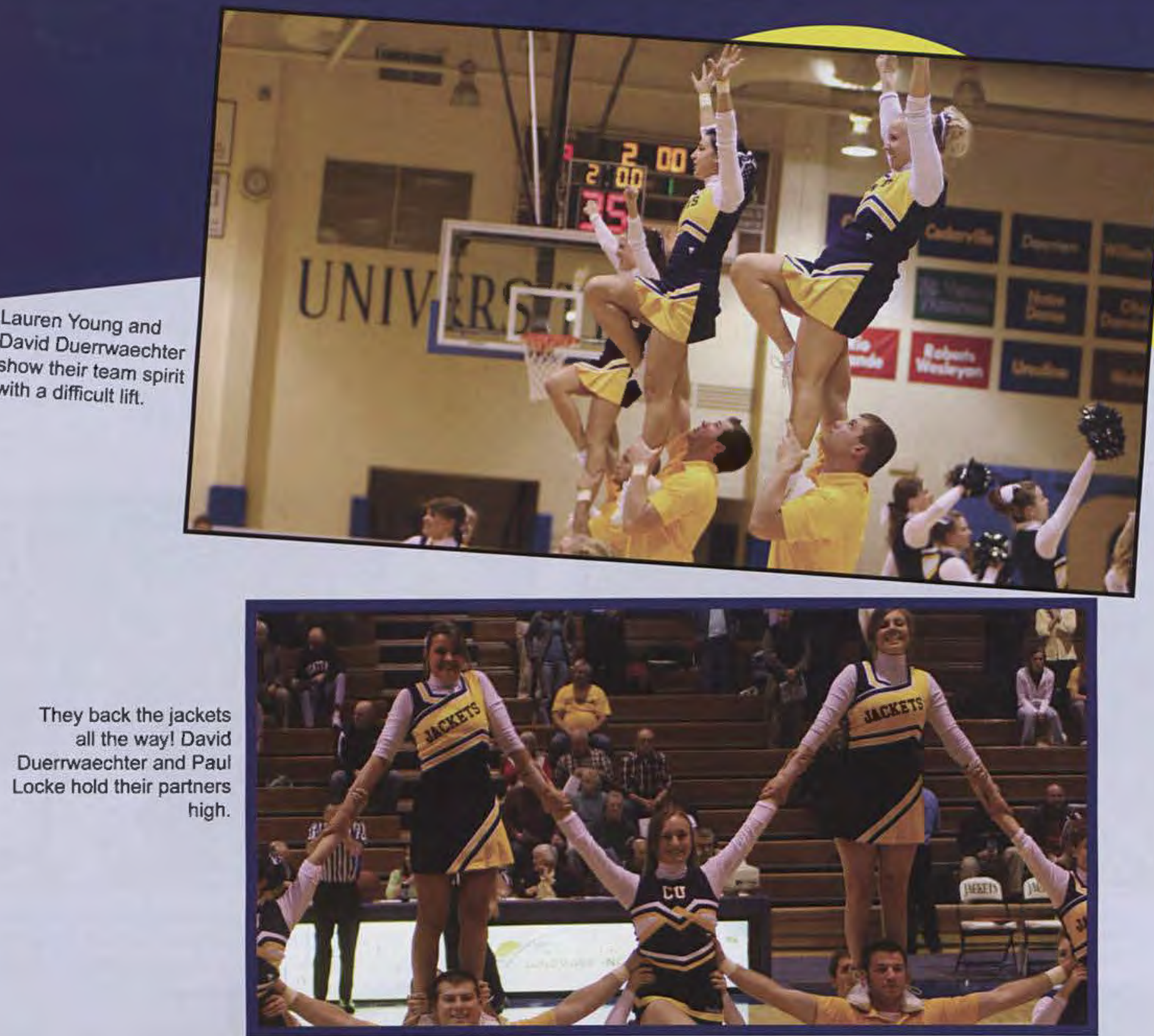

\section{Rob Anfang} admits that cheerleading, "was definitely a test of my manhood."
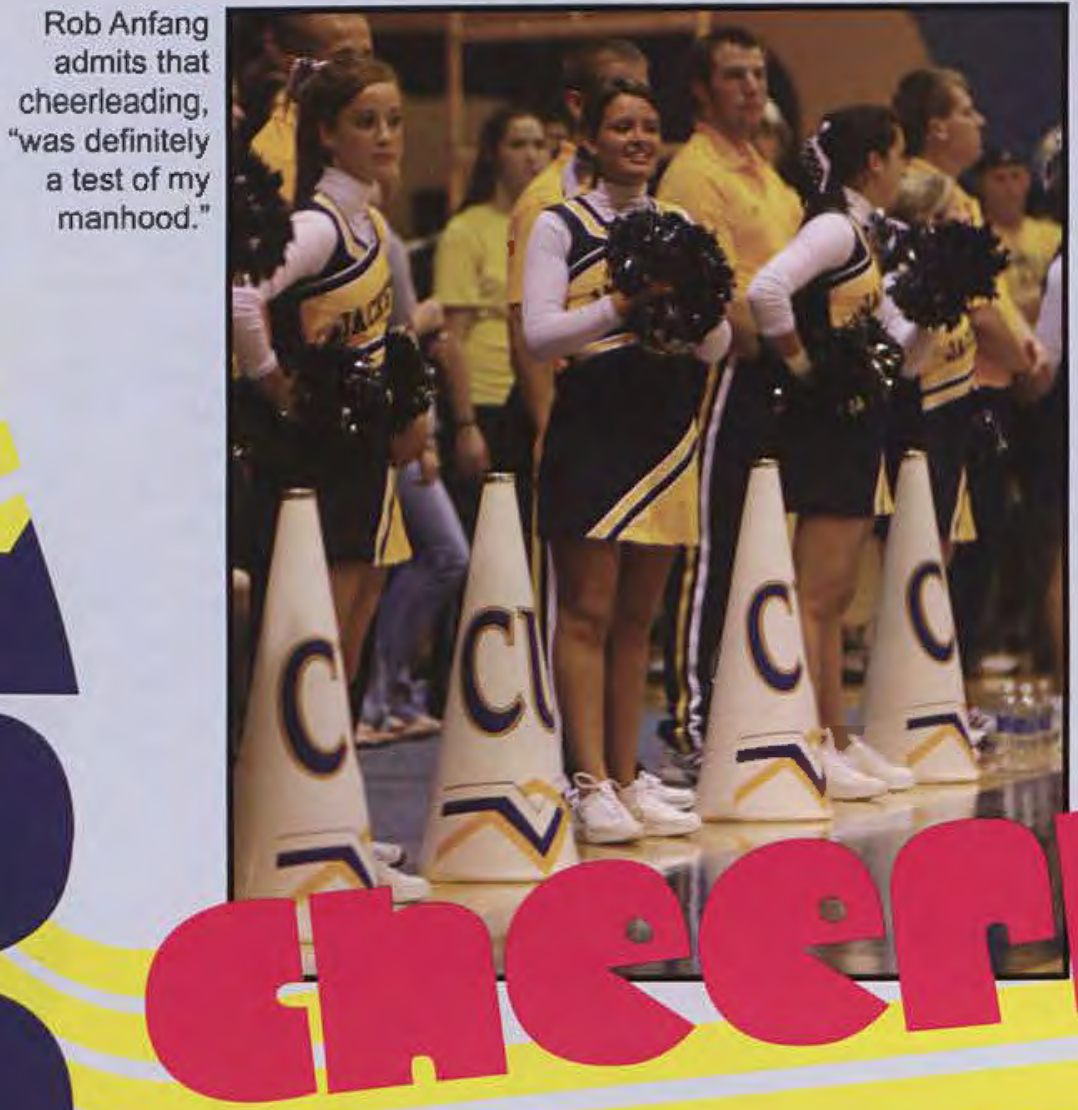

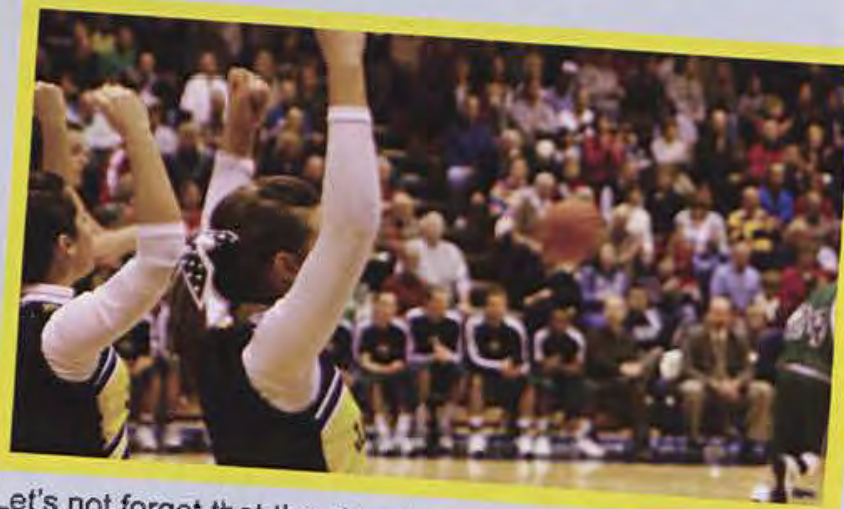

Let's not forget that the cheerieaders are

really there for the fans!

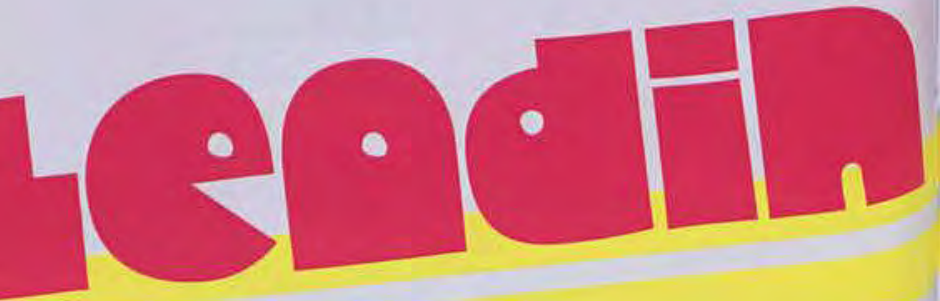




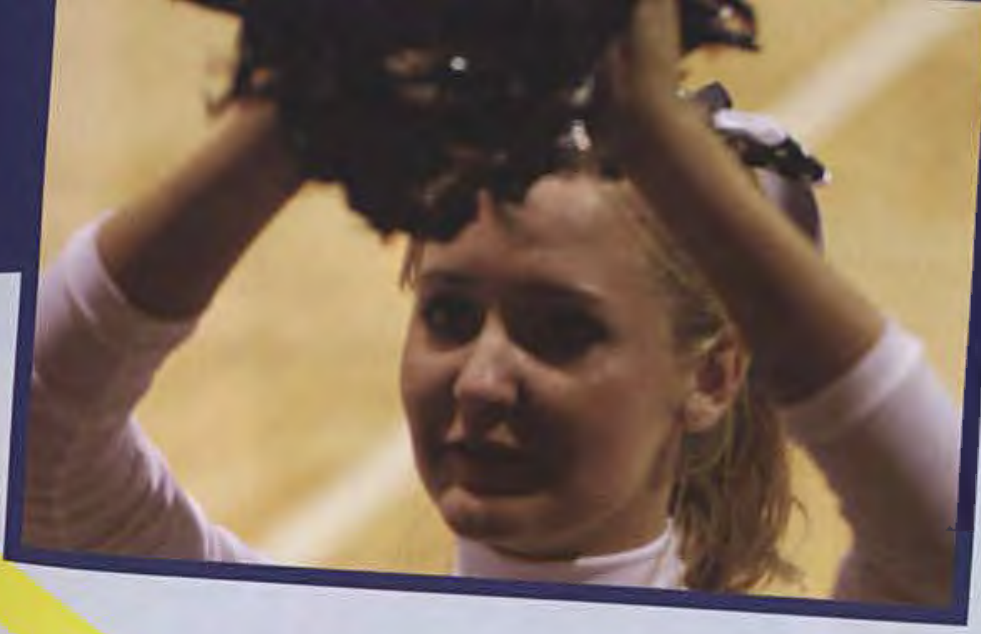

Abigail Morris cheers her

heart out.

\section{"Cnecricedine mos begn swe ter thes reer, the enmes, the prectices, ond a chence to suppert our senoon." -Jessien prester}
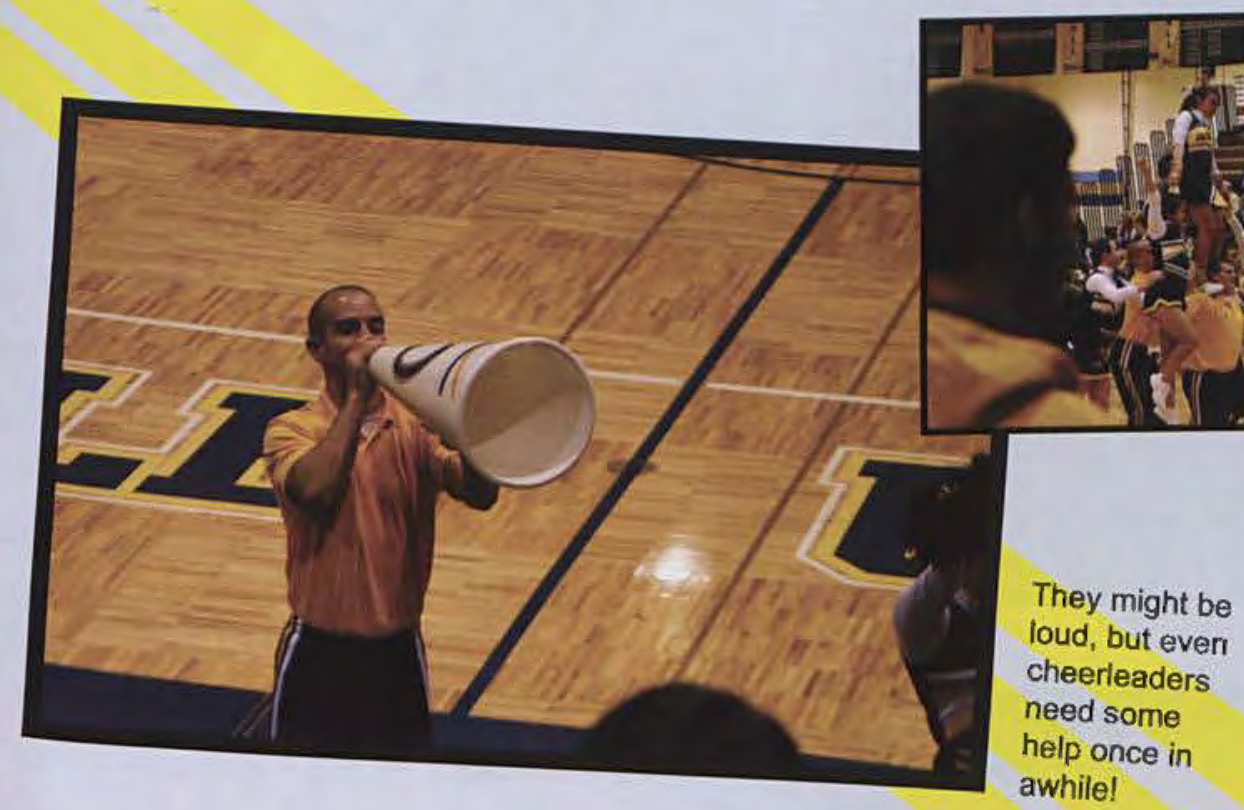

"Everyone on

the team worked

together to

accomplish many

of our goals. I

was proud to be

on a cheerleading

squad made up

of good friends,"

comments

Jessica England

They might be

loud, but even

cheerleaders

help once in

awhile!

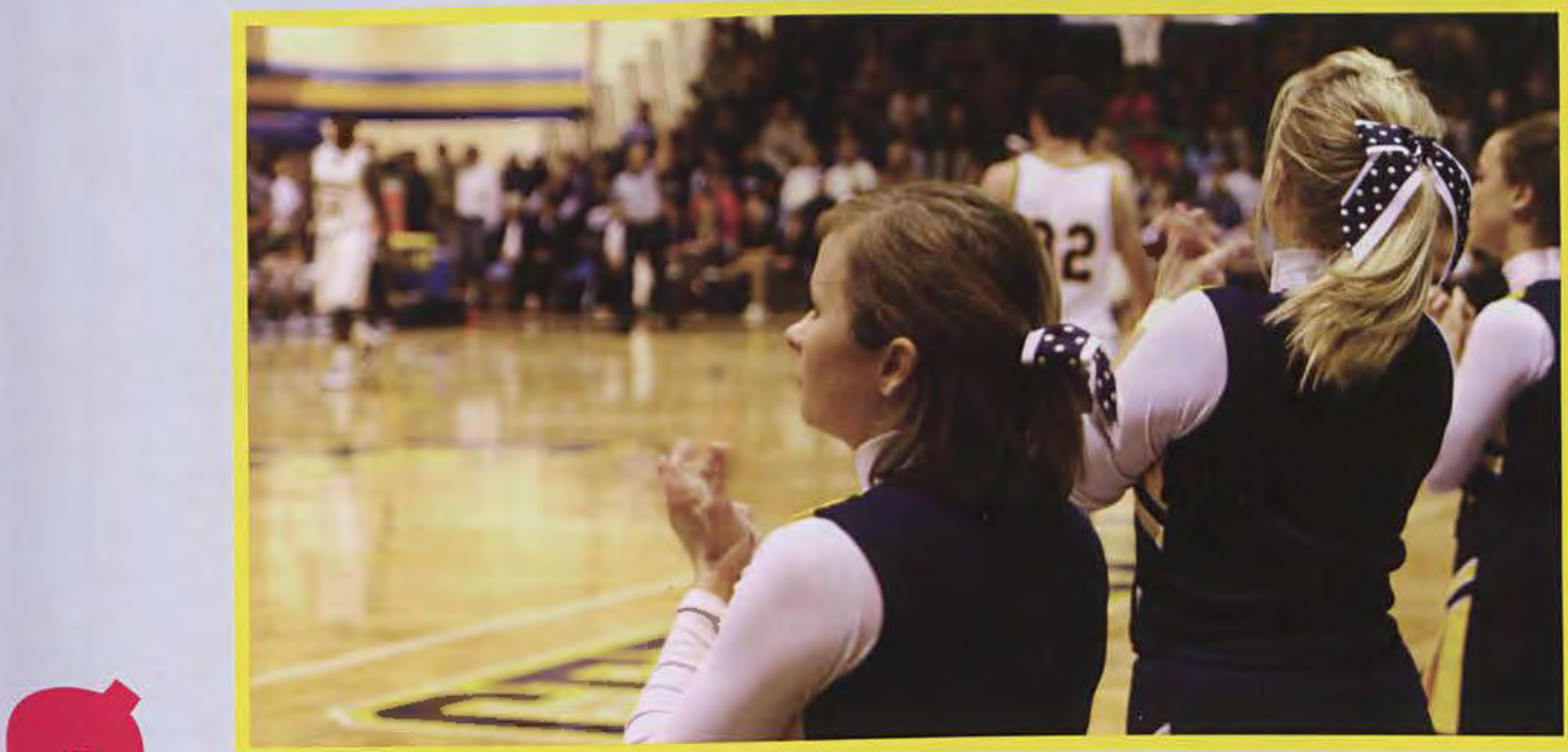

The girls watch the game anxiously, sometimes even forgetting to clap!

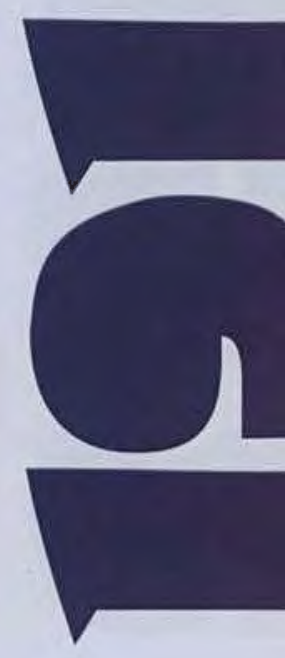


Trevor Bowman explains, "The past few months have been an eager time for all of us. We have put in a lot of hard work and practice to prepare us for the next couple of months and we are really excited to see what God has in store for us."

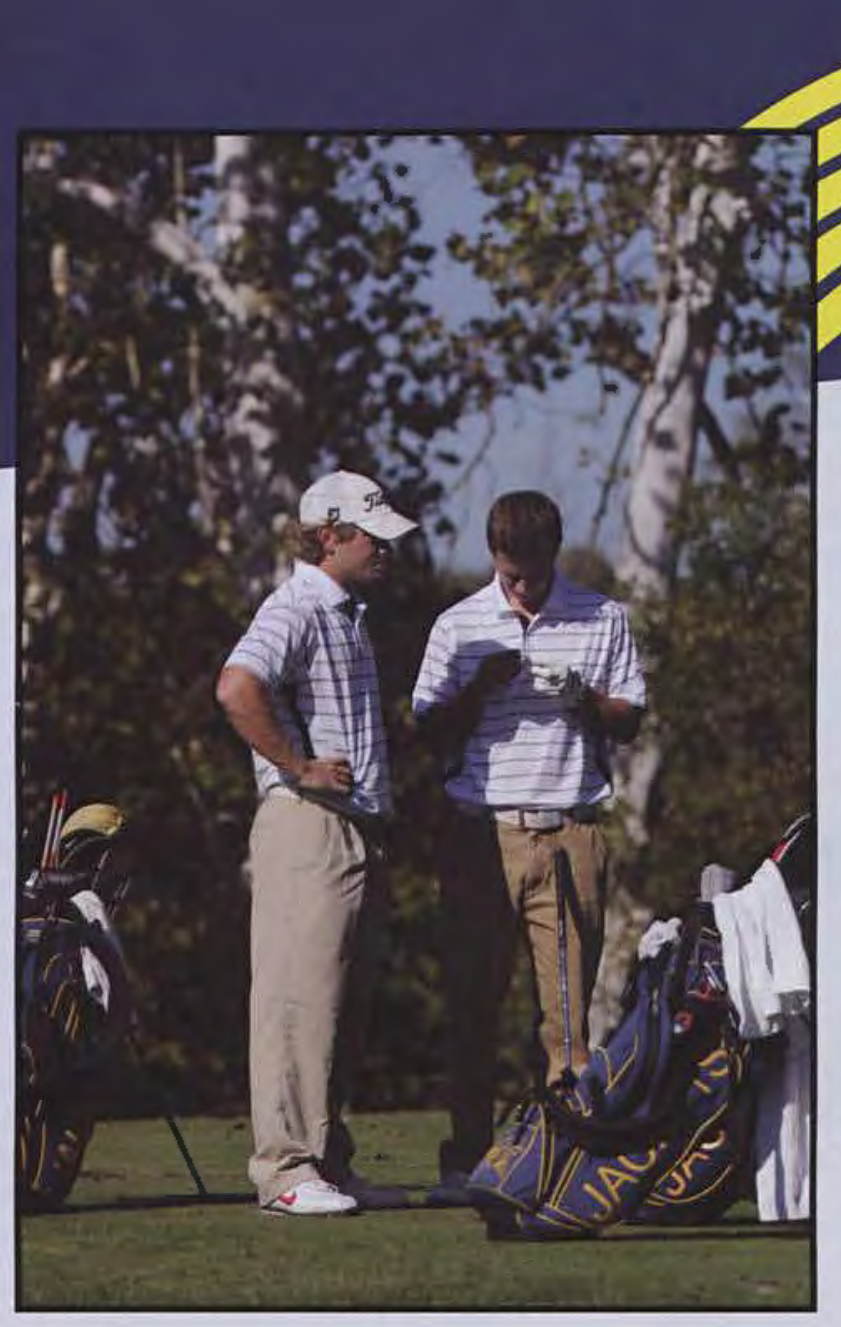

Brendan Ojala goes in for the swing. He shot a season best at 75 .

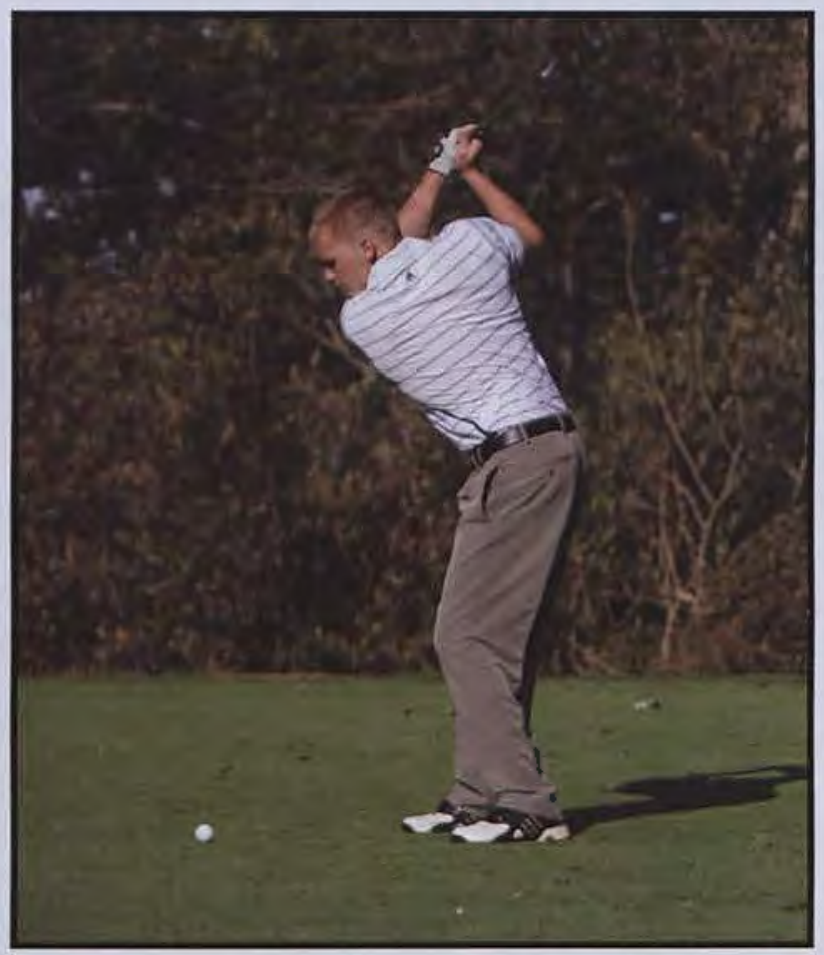

Kisor Classic

Lindenwood Invitational

Bethel (Ind.) Classic

Wittenberg Invitational

NCCAA Championship

Shawnee State Invitational

Southeastern Kentucky Invitational

NAIA Classic

28th Annual

Cedarville Invitational

AMC Championship
T2nd of 9

6 th of 23

1st of 14

2nd of 15

T5th of 18

1st of 6

6 th of 19

T4th of 15

3rd of 13

2nd of 10

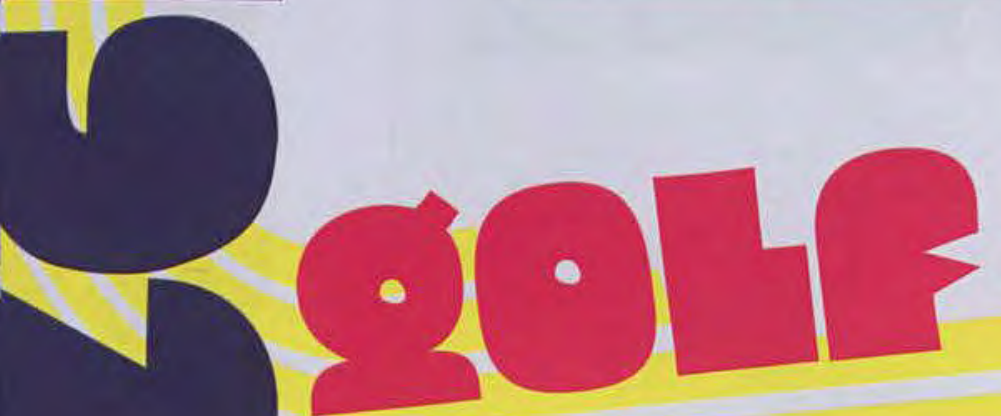




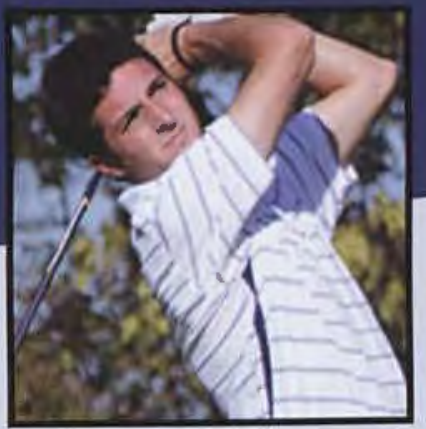

Brett Bigler knows how to follow through. He followed through with a season best of 79 .

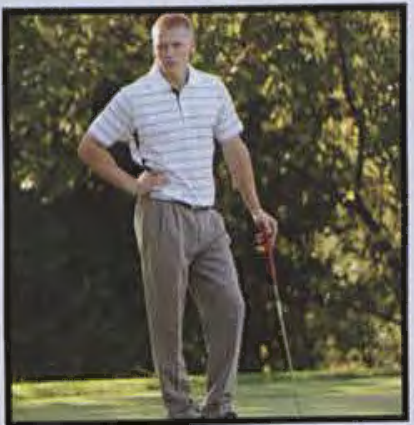

Brendan Ojala strikes a pose. As a senior, he managed an average stroke of 80.81 for the season.

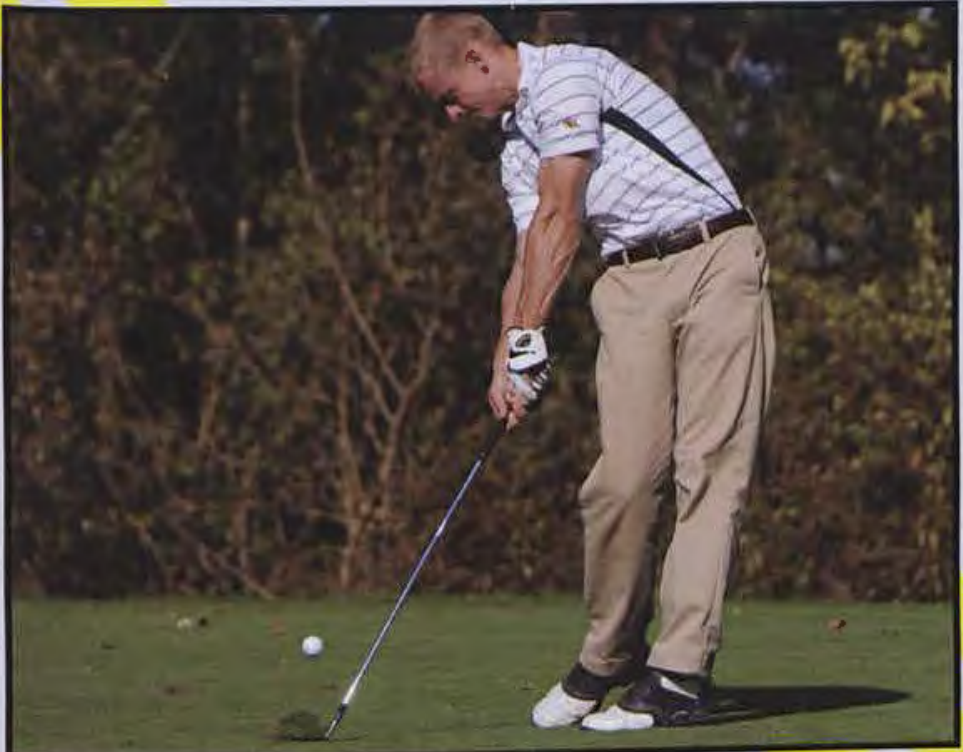

"We have such a close group of guys that are all thankful to use this opportunity to play golf for God's glory together." says Dan Atkeson.

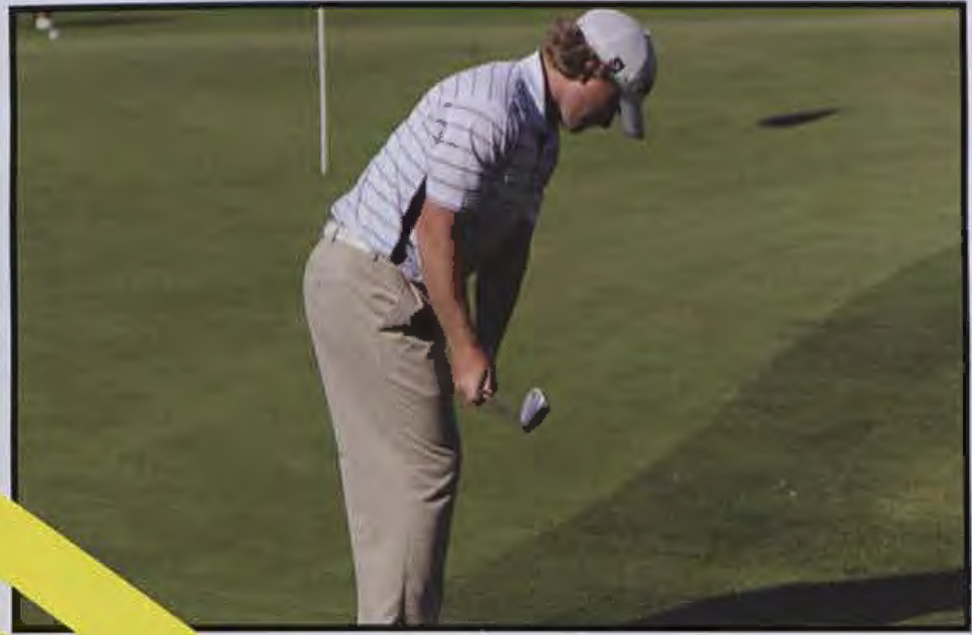

"Everybody on this team is capable of contributing and the competition amongst ourselves as a team is continually driving us to improve," says Trevor Bowman.

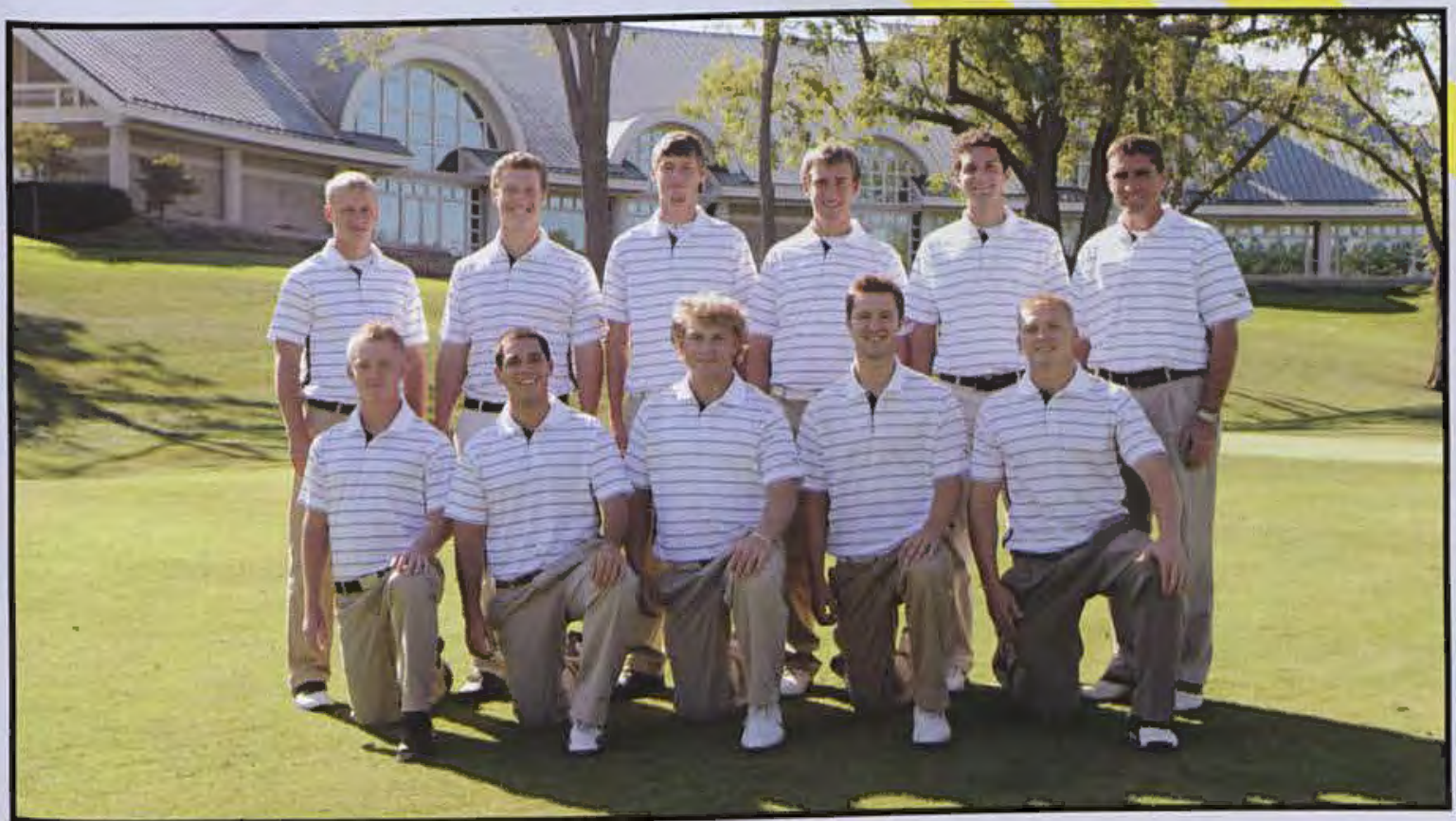

Left to right. Front row: Matt Krogstad, Nick Bigler, Trevor Bowman, Trent Roach, Brendan Ojala. Back row: Dan Atkeson, Brigham Michaud, Chris Bennington, Matthew Yeiter, Brett Bigler, head coach Ryan Bowen.

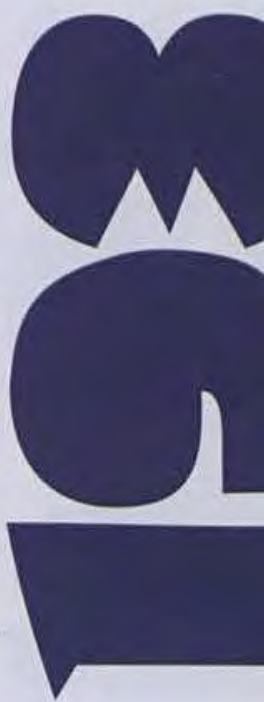




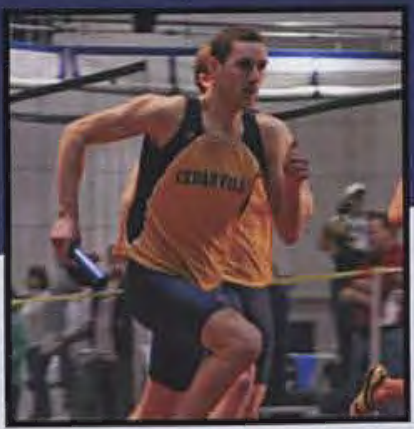

Kevin Kuhn hustles to pass off the baton to his teammate.

While being a team player in the relay, Kevin made a

season best of $26: 38$ at the $8 \mathrm{~K}$

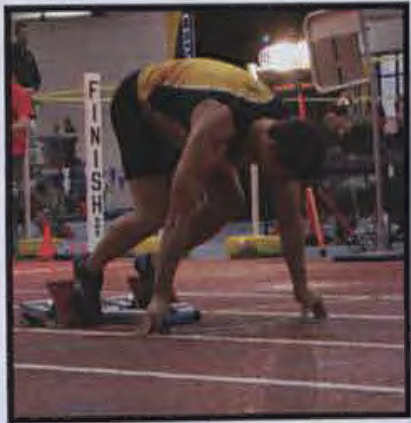

Brandon Tress takes his mark before the race. Brandon championed the American Mideast

Conference 110 meter hurdle champion with a career-best time of 14.90 seconds.

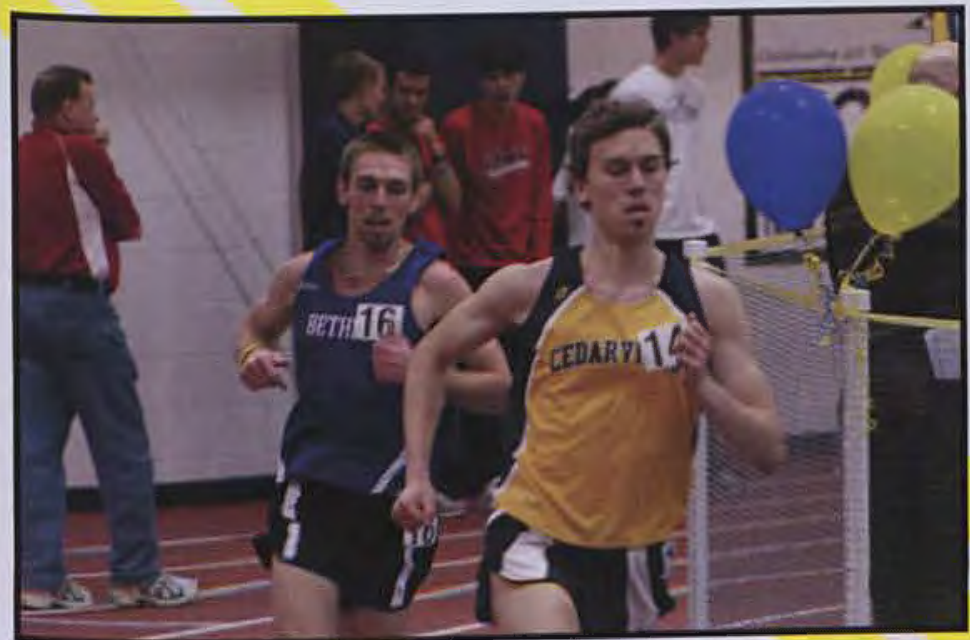

Rob Trennepohl stays focused during his race.

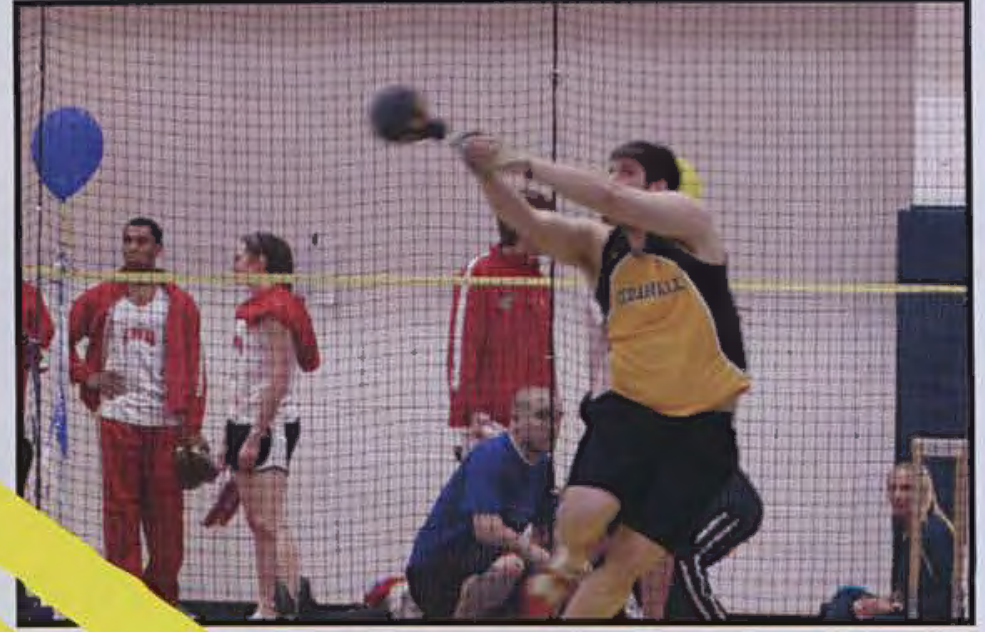

Christian Maat heaves with all of his might.

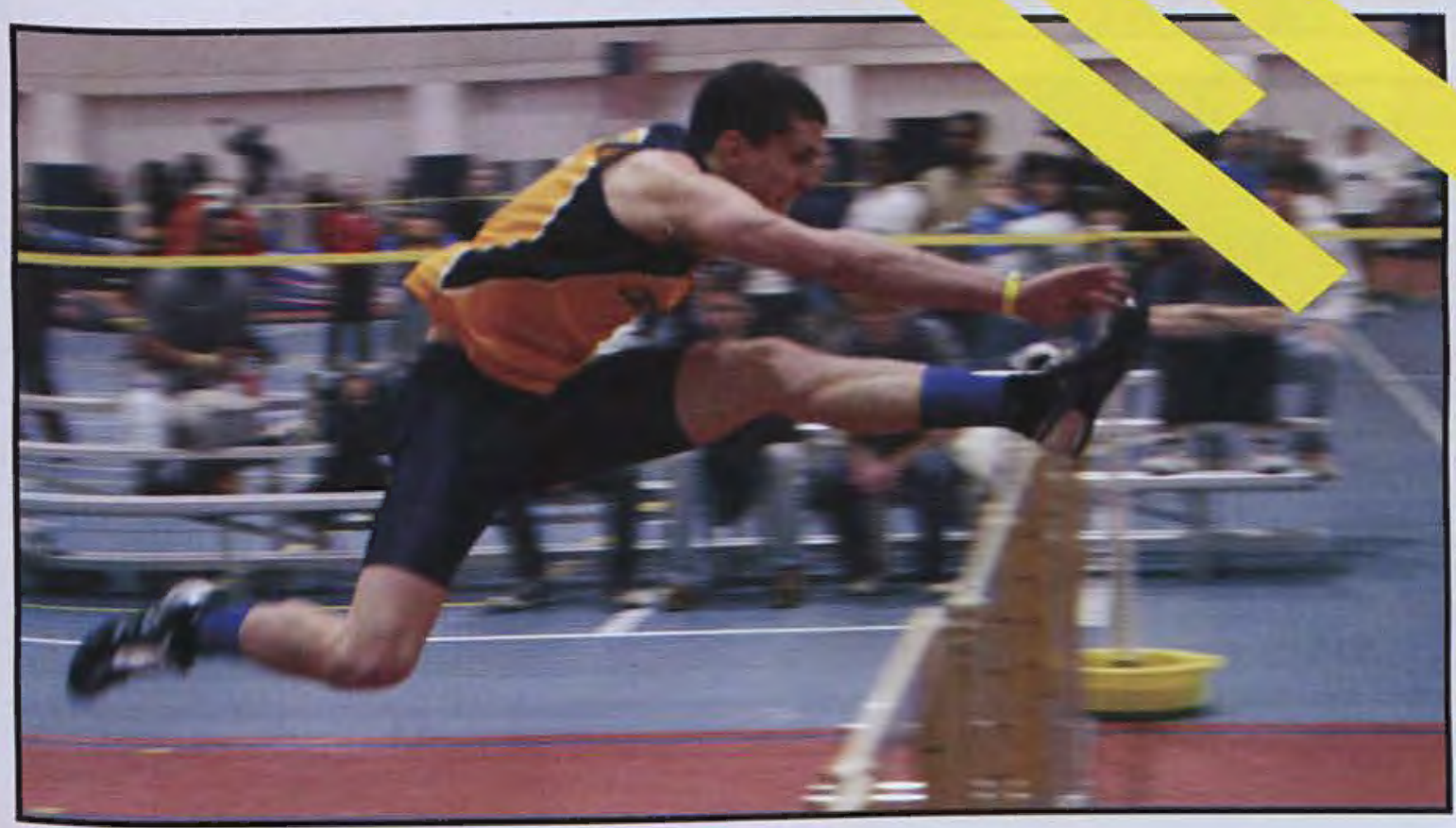

Brandon Tress expertly leaps over the hurdles.

"Hitting hurdles isn't fun, unless you break one!" laughs Jason Scott.

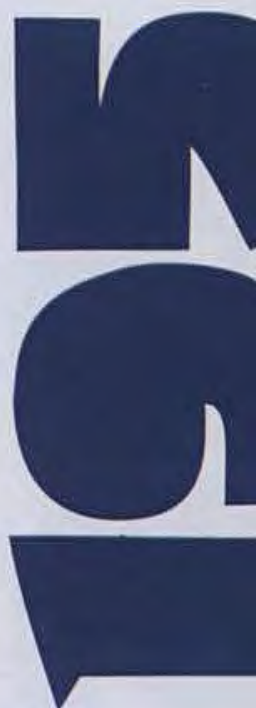


Left to right. Front row: Brittany Tara Leaman, Kristin Simpson, Tara Leam Pyles, Nicole Corbin, Elisabeth Pyles, Jennifer Santos, Sarah Steven Keller. Middle Hollander, Stacey Keller. Mhawn row: Assistant Coach Shach Phil Graves, Assistant Coach Paul Scott, Assistant Koach Ashley Orchard, Laura Kiror, Bethany Herbert, Katie Lanphuett, Meagan Davies, Chelsea Gruet, Mead Carns, Kelsey Watkins, Assistant Coach Jeff Bolender, Aack row: Coach Jason Scott. Back Smith, Sarah Grener, Jmily DeRusha, Marla Rice, Emanda Smith. Sarah Hannay, Amanda Cann Alexa Winik, Rachel Wong,

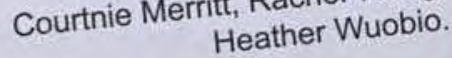

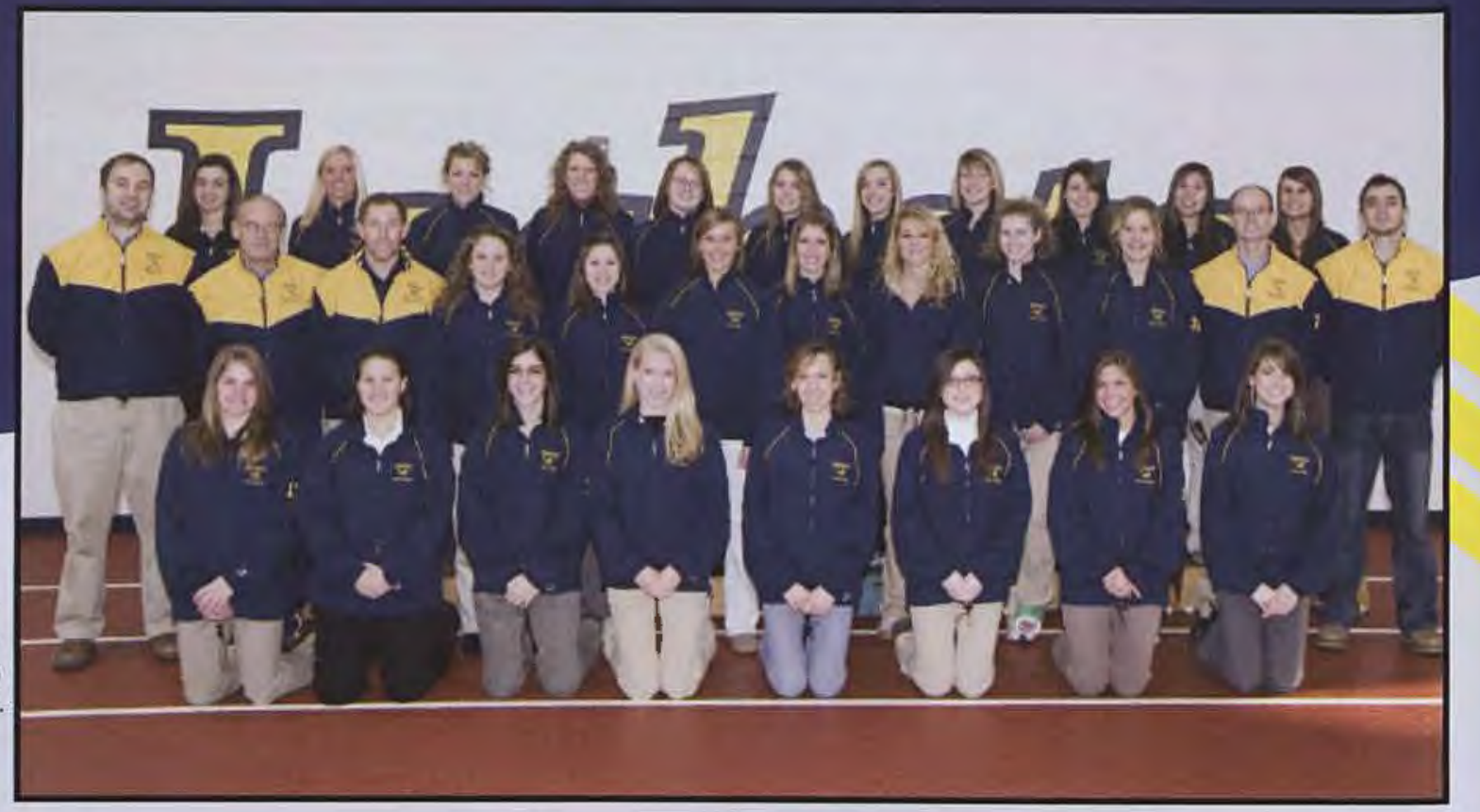

Heather Wuobio sets up a high jump. Heather produced 13 individual

indoor points and six during the outdoor season as a high jumper.

Bethany Davies runs a relay. She placed 9 th at both the Shawnee State Invitational (19:54) and the CU Open (19:49).
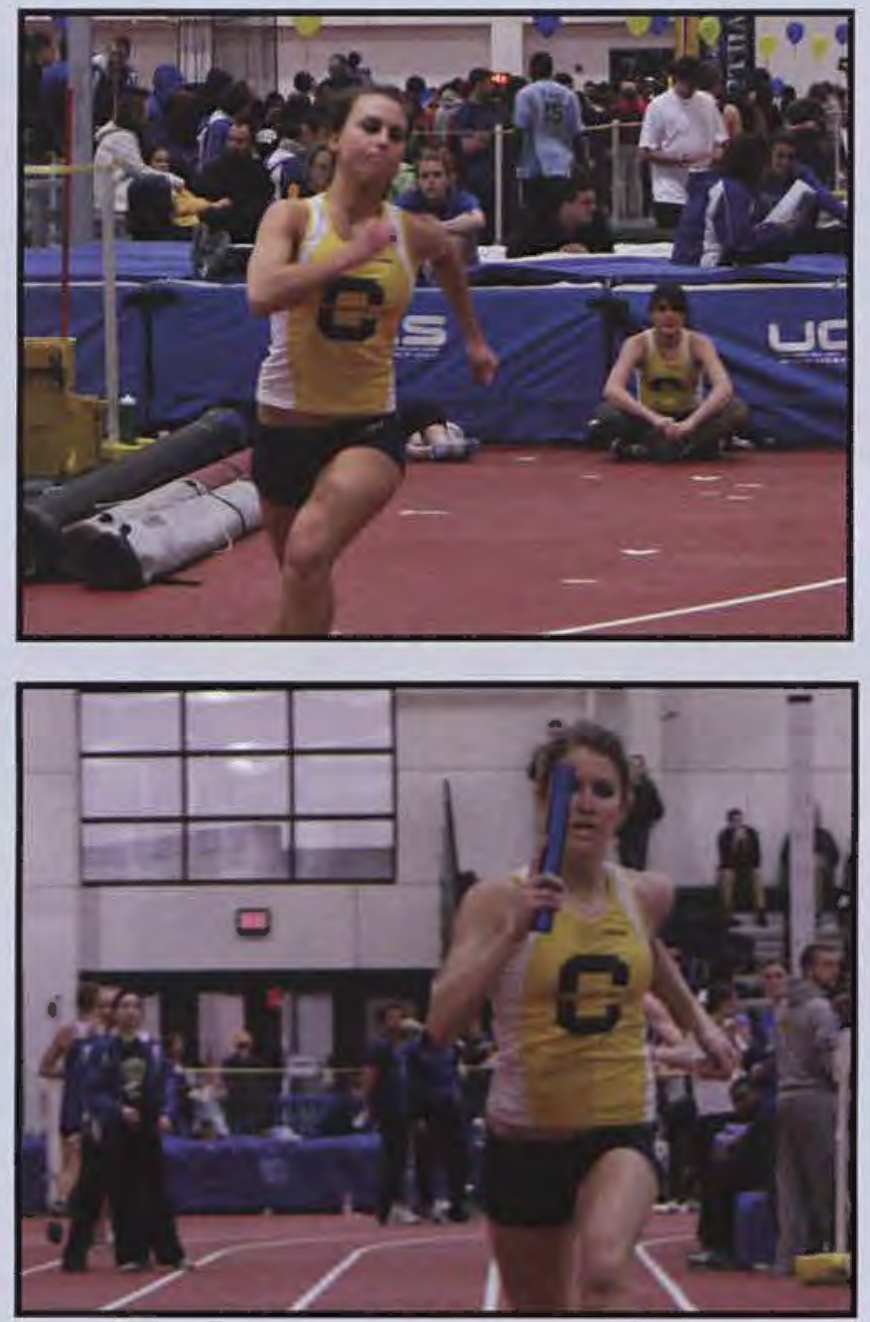

Cedarville Invitational

Indiana Wesleyan Classic

Indiana Relays

DePauw Invitatonal

Findlay Open

NCCAA Indoor Championship

NAIA Indoor Championship

Emory Invitational

Cedarville Open

Cincinnati Southern Ohio Cup

Tennessee Relays

Miami Invitational

American Mideast Conference

NCCAA Outdoor Championship

Tiffin Qualifier

Cedarville Challenge

NAIA Outdoor Championship 1st of 11

Non-Scoring

Non-Scoring

7 th of 11

Non-Scoring

1st of 12

7 th of 89

2nd of 19

1 st of 6

3rd of 8

Non-Scoring

Non-Scoring

2nd of 8

3rd of 15

Non-Scoring

Non-Scoring

13th of 106
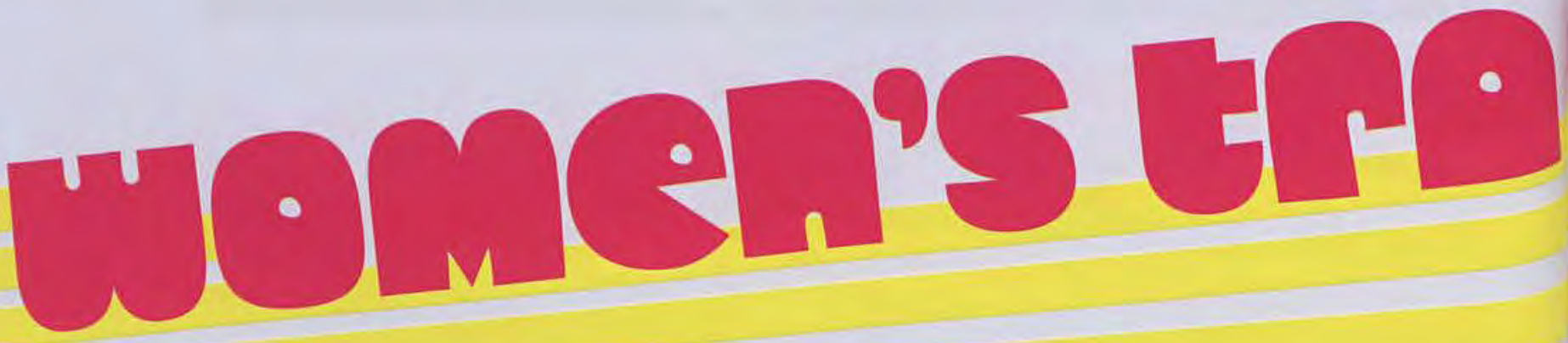

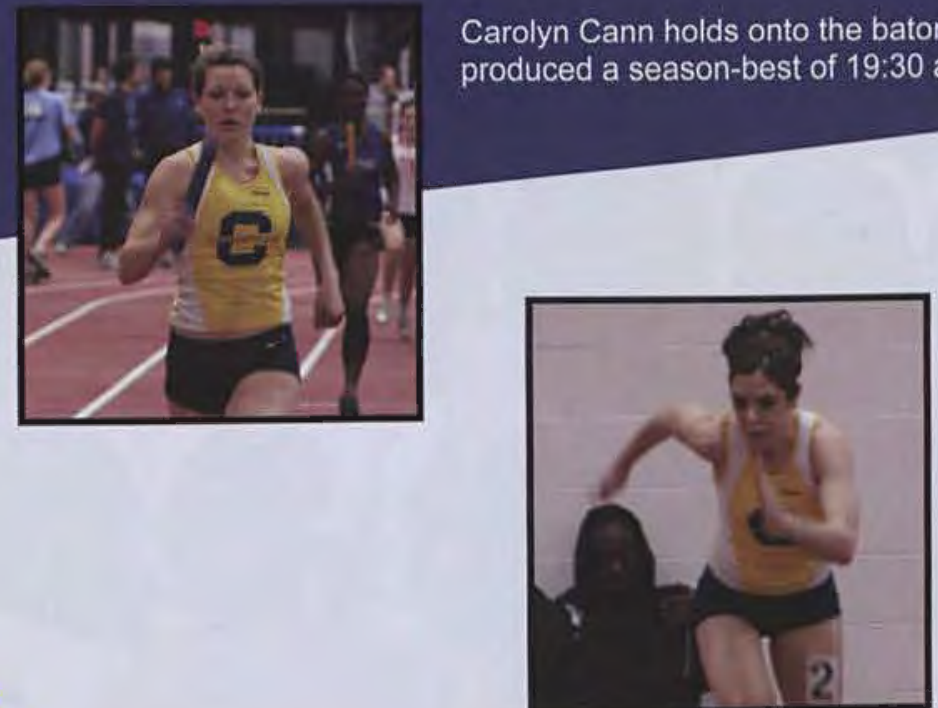

Kristin Corbin sprints toward the finish line. As a freshman, Kristin brought her considerable high school experience to the team.

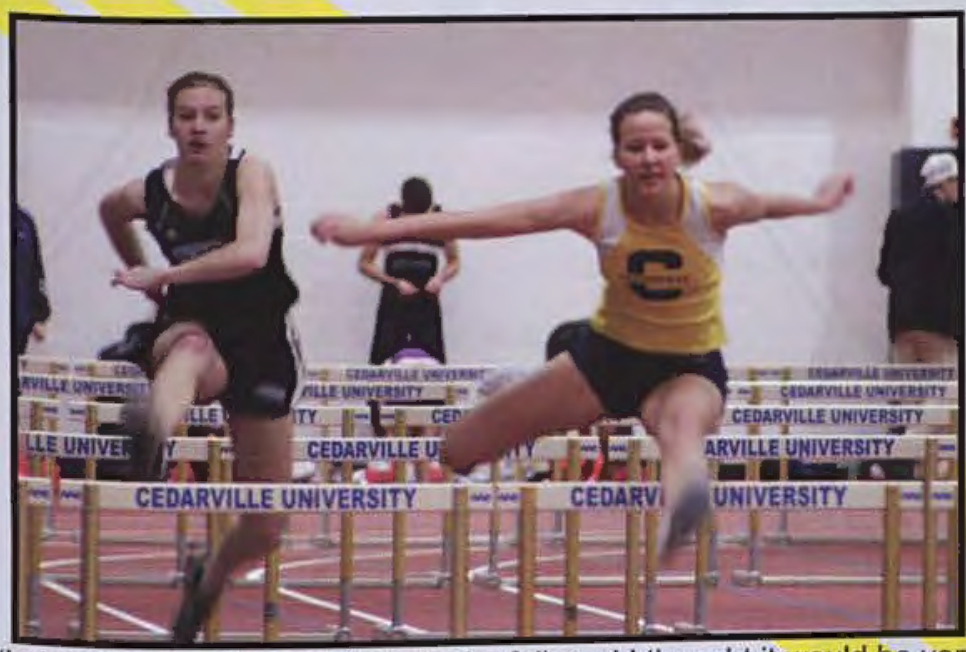

"I played soccer for Cedarville in the fall, and I thought it would be very hard to make friends joining the track team halfway through the season. But it wasn't. They welcomed me with open arms, and I am so glad I joined," says Kelsey Watkins.

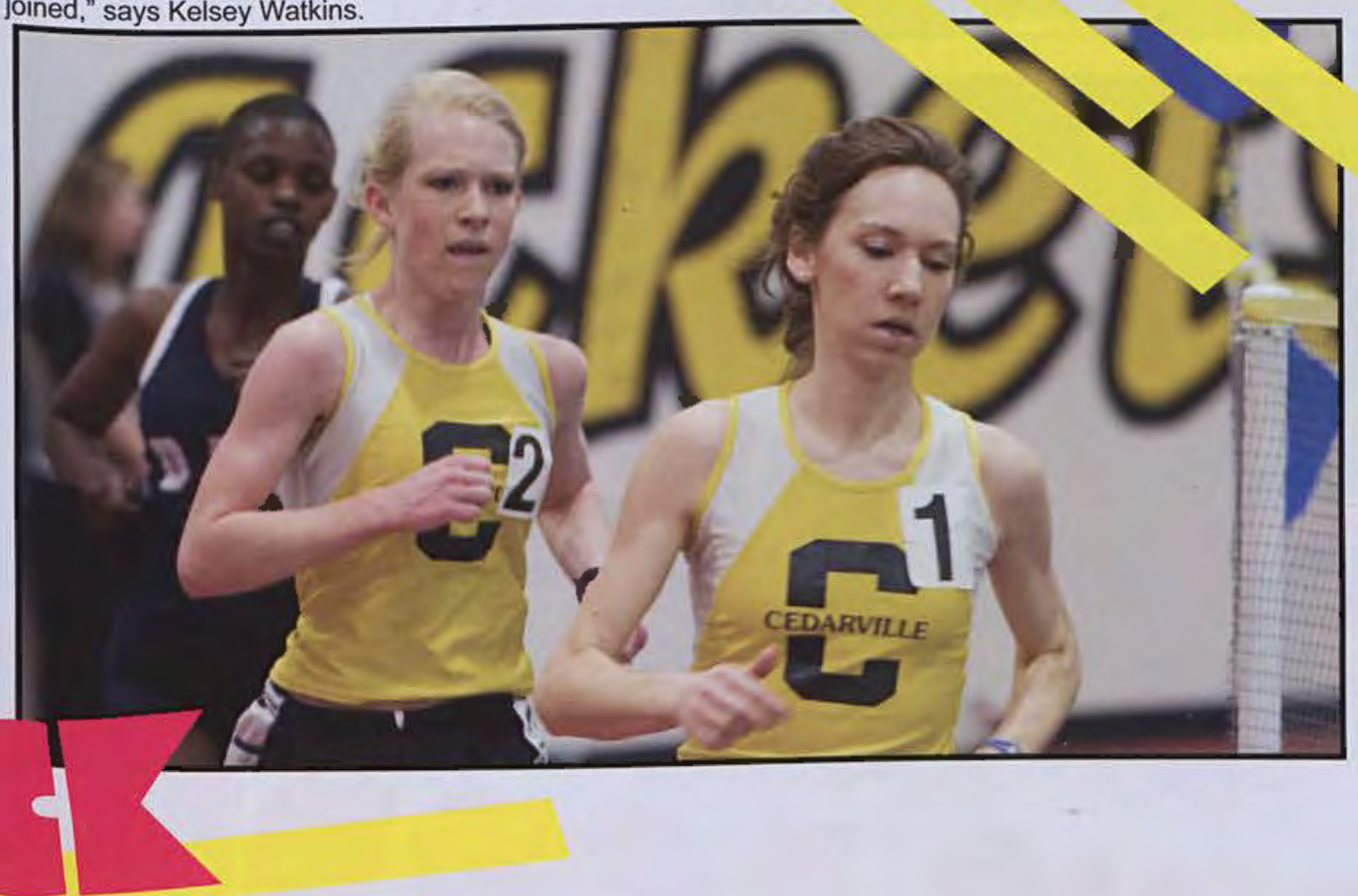

Elizabeth Pyles and Nicole Santos stick together on the track. 

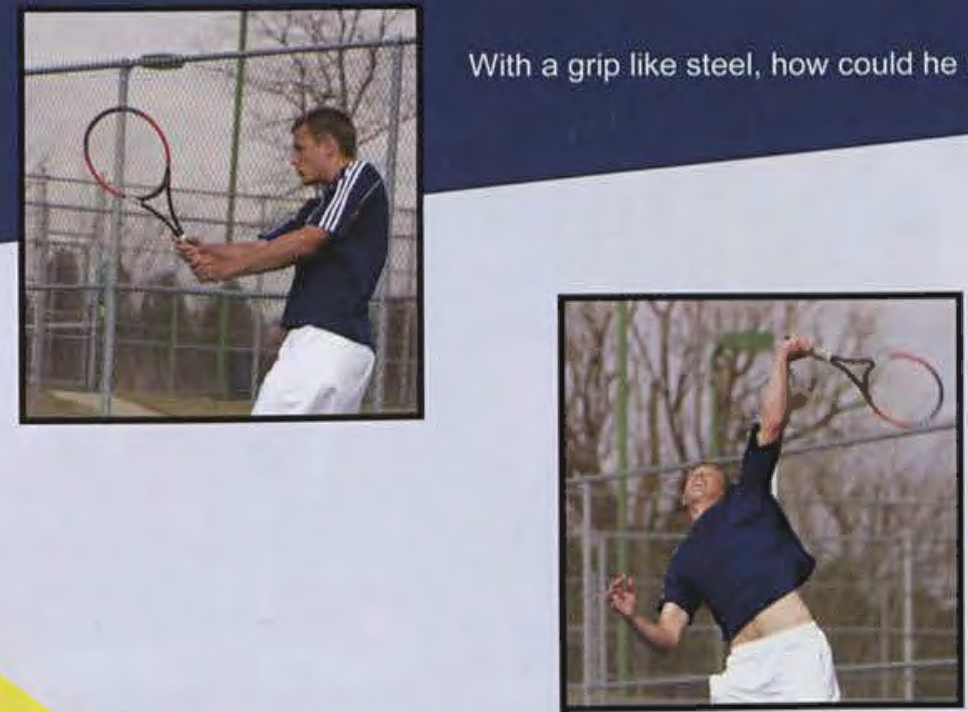

His reach is like Mr. Fantastic's!

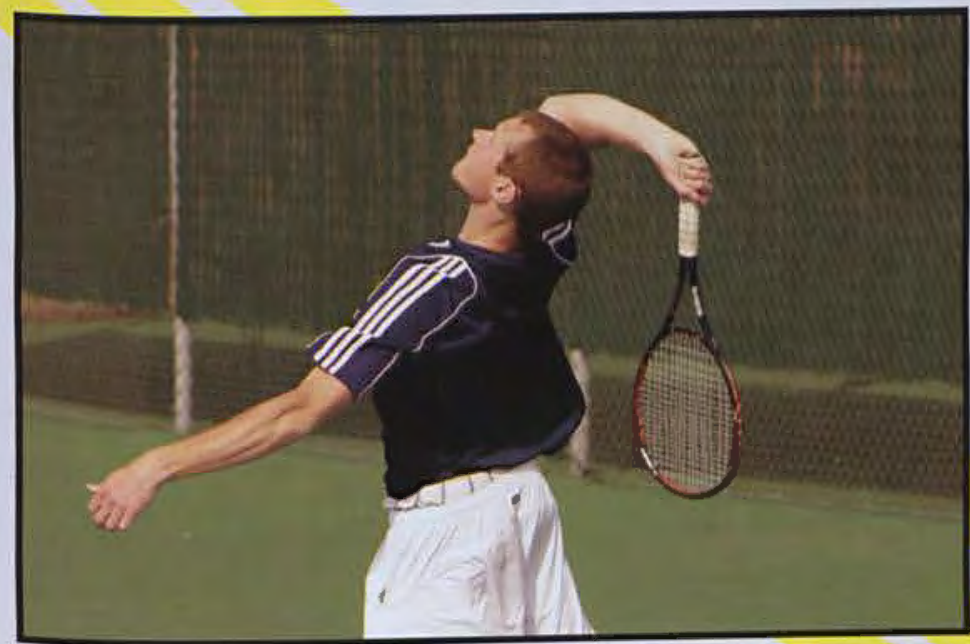

Impressive! His arm is like coiled spring, ready to attack.

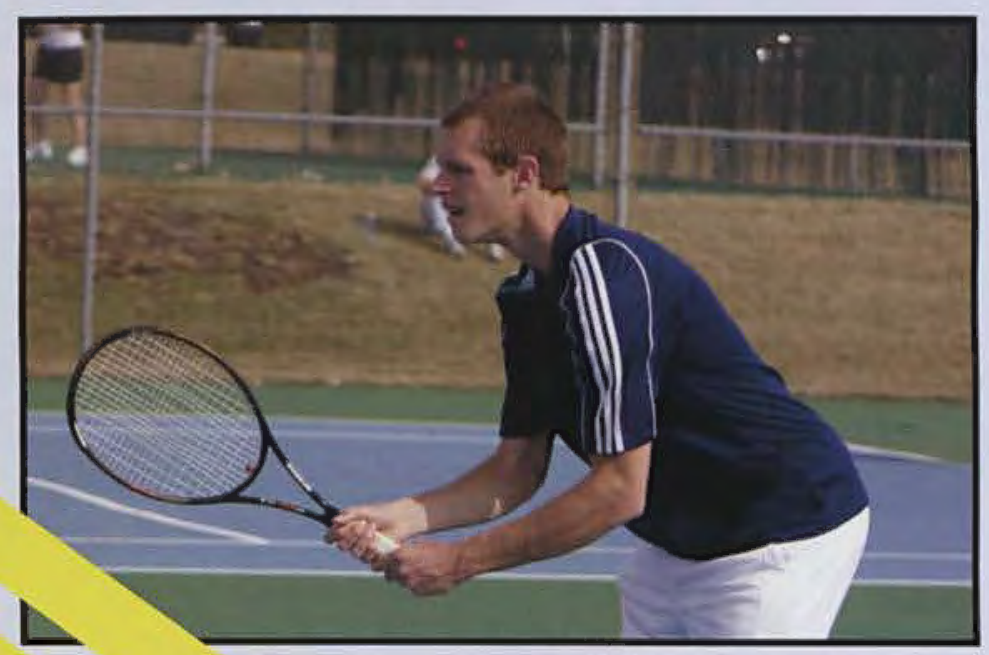

Yellow Jackets are always ready to sting.

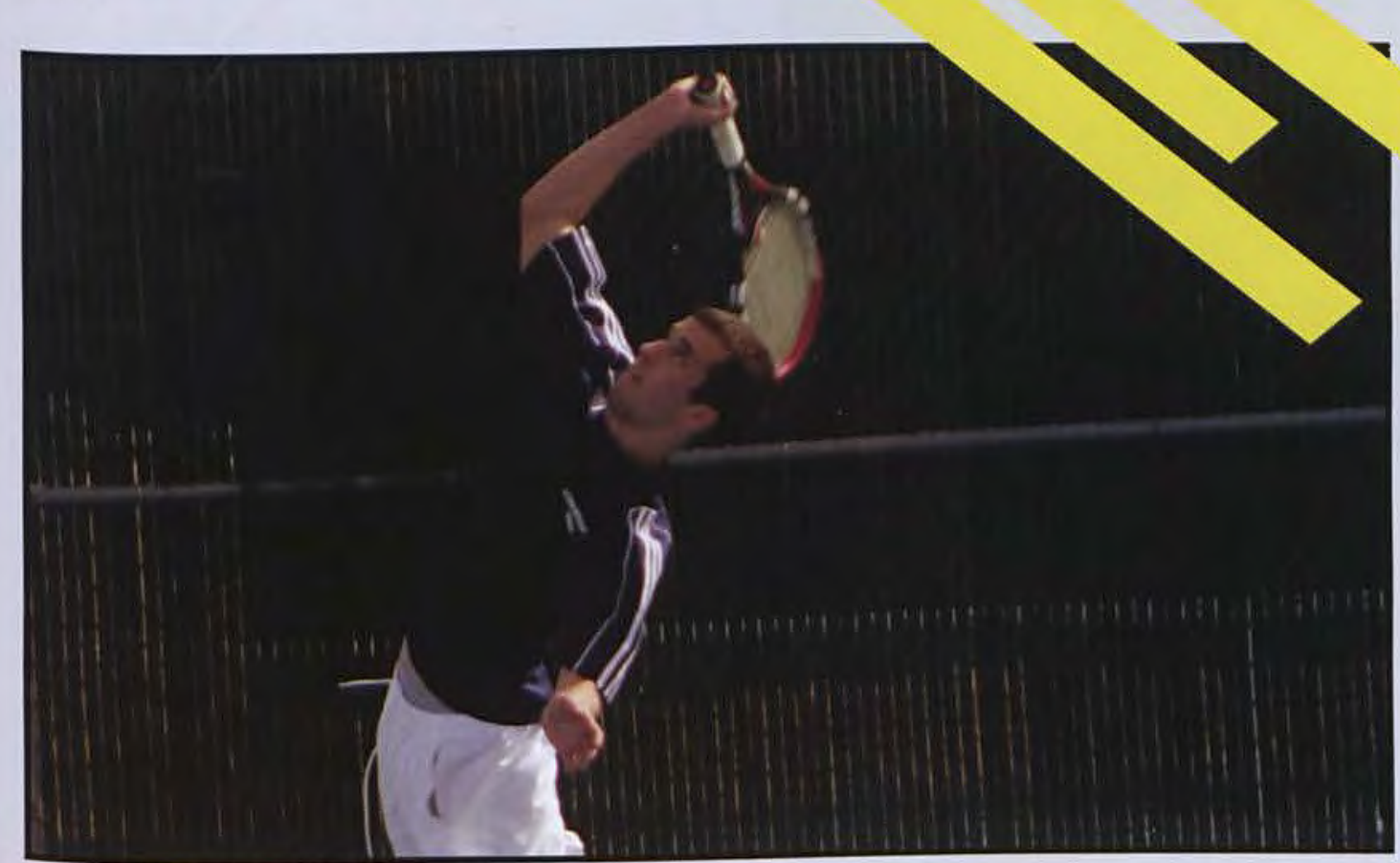

Zach Rost swings for the ball. With that swing, the opponent won't even see the ball coming. 
Left to right. Front row: Georgiann McClure, Joy Kellogg, Cindy Hoke, Carissa Parmerlee, Kristina Hrabal. Back row: Team Chaplain Becky Kuhn, Lauren Williams, Anna Strayhorn, Sarah Andersen, Rachel

Arthur, Associate Head Coach Dr. Pam Johnson. Head Coach Dr. Dee Morris. .

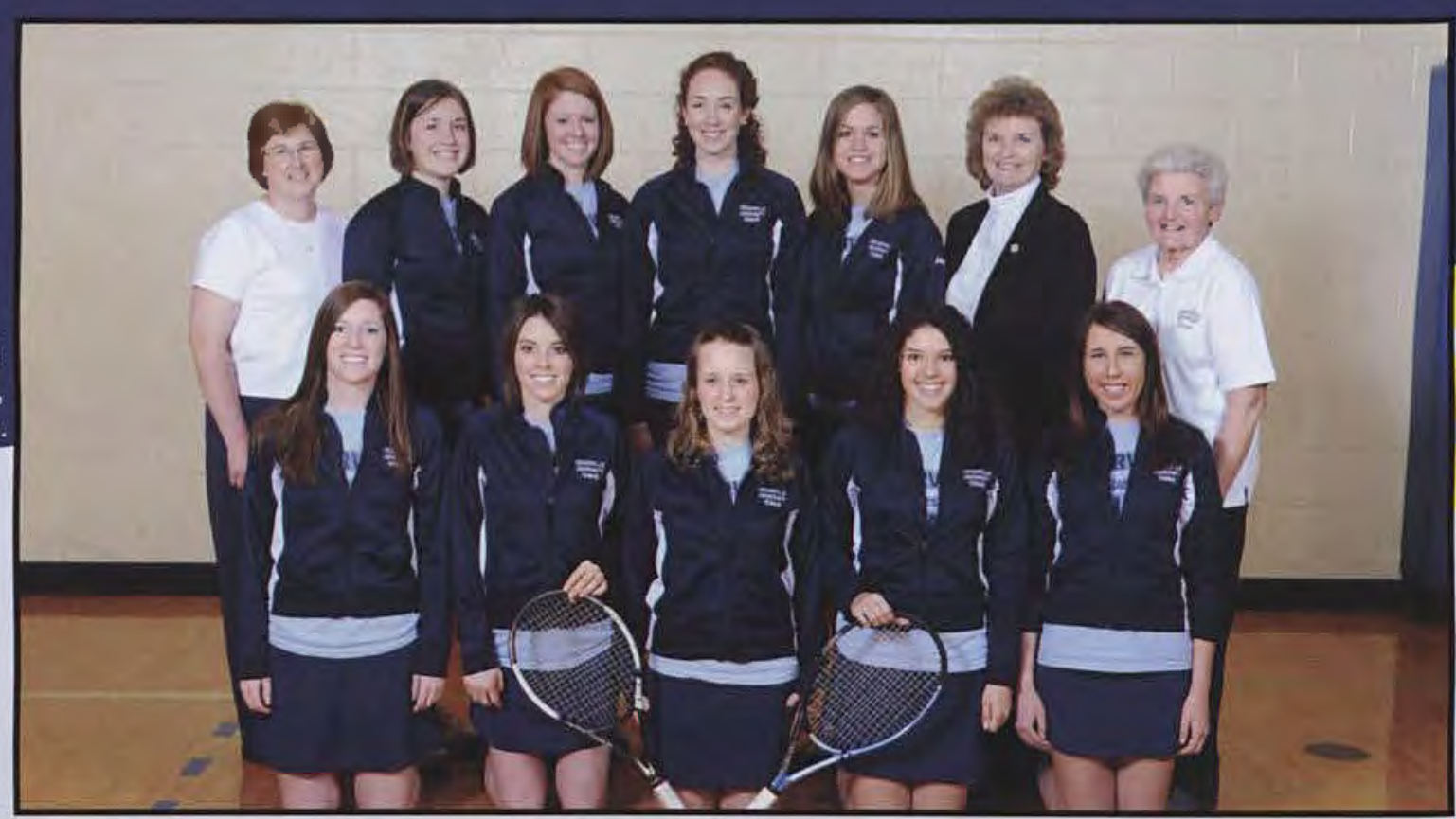

Carissa Parmerlee goes in for the shot. About the year, she says, "I have loved my team this year! They have been such an encouragement to me! We'll miss you Limbs and PNTC1!."

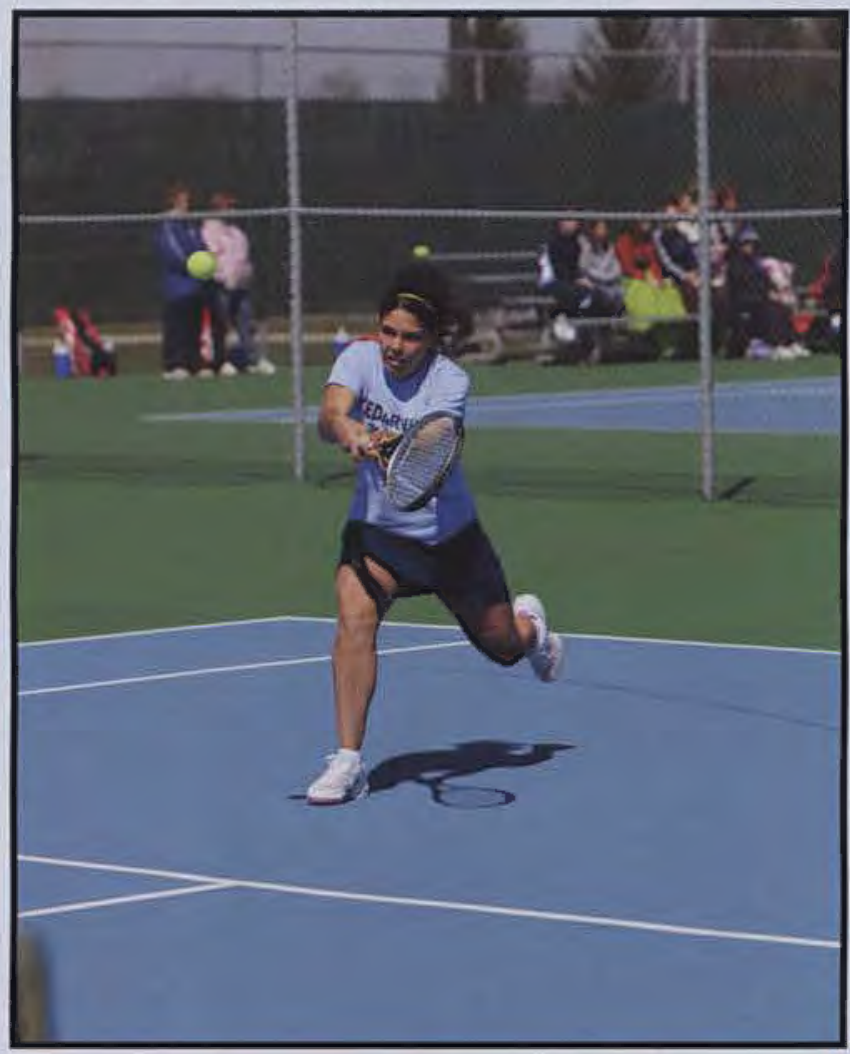

Huntington

Southeastern

Wilmington

Cumberiands (Ky.)

Marietta

Carlow

Grove City

Shawnee State

Malone

Roberts Wesleyan

Walsh

Marian

Ohio Dominican

Northwestern

Indiana Southeast

North Greenville
L 75-77

L 4-5

W 6-3

L 1-8

W 7-2

W 9-0

L 2-7

W 9-0

W 6-3

W 7-2

L $1-8$

L 4-5

L O-9

L 4-5

L 2-7

L 3-5

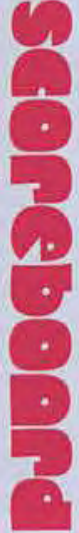

1. 

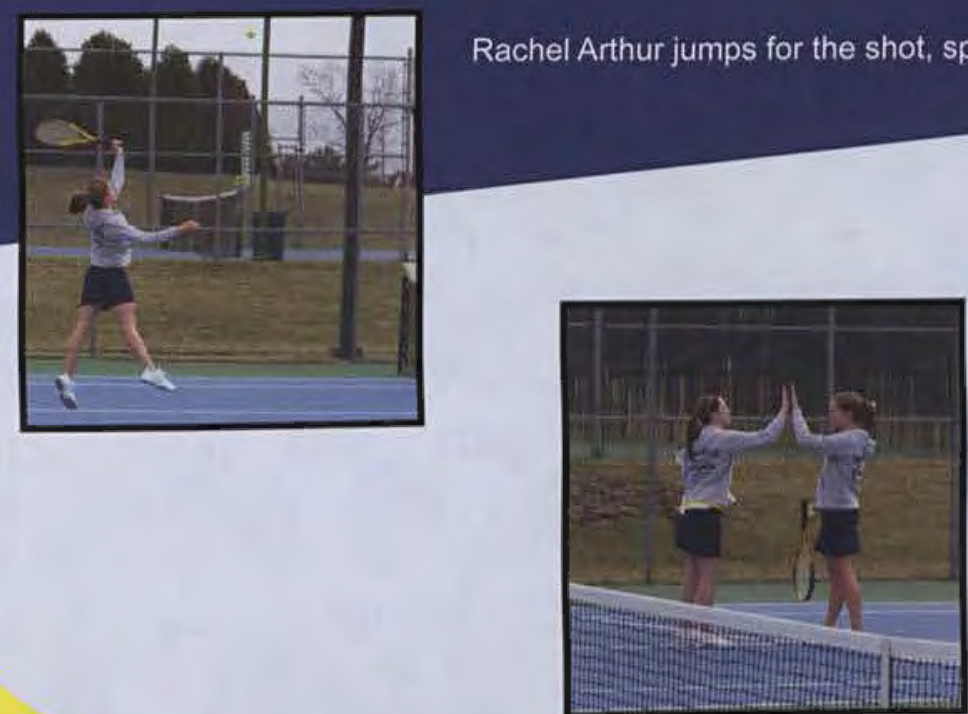

Georgiann McClure and Rachel Arthur exchange a high-five during a match.

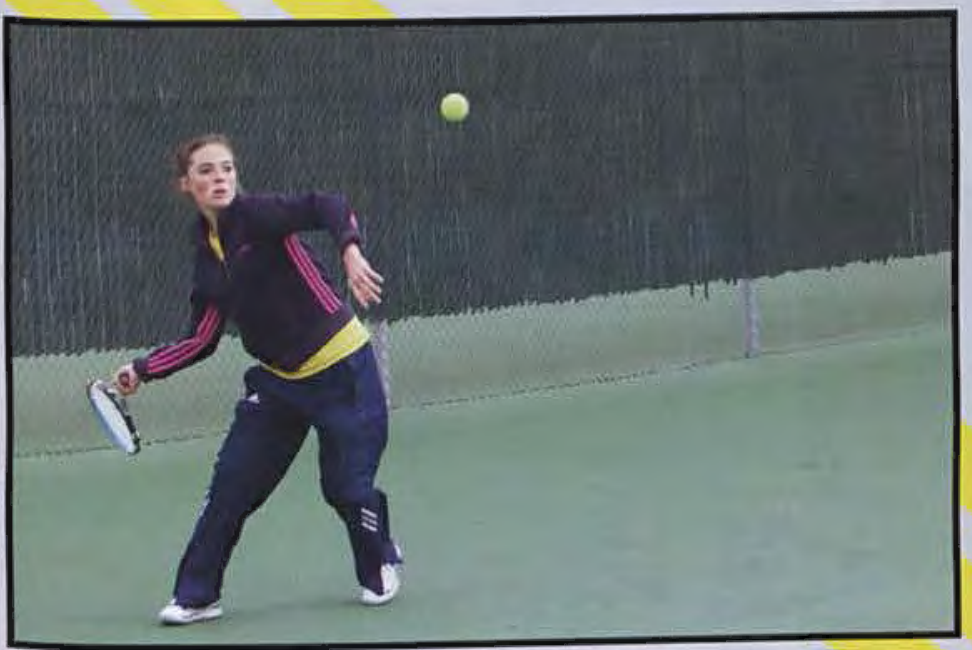

Joy Kellogg takes a swat for the ball, ready to send it flying.

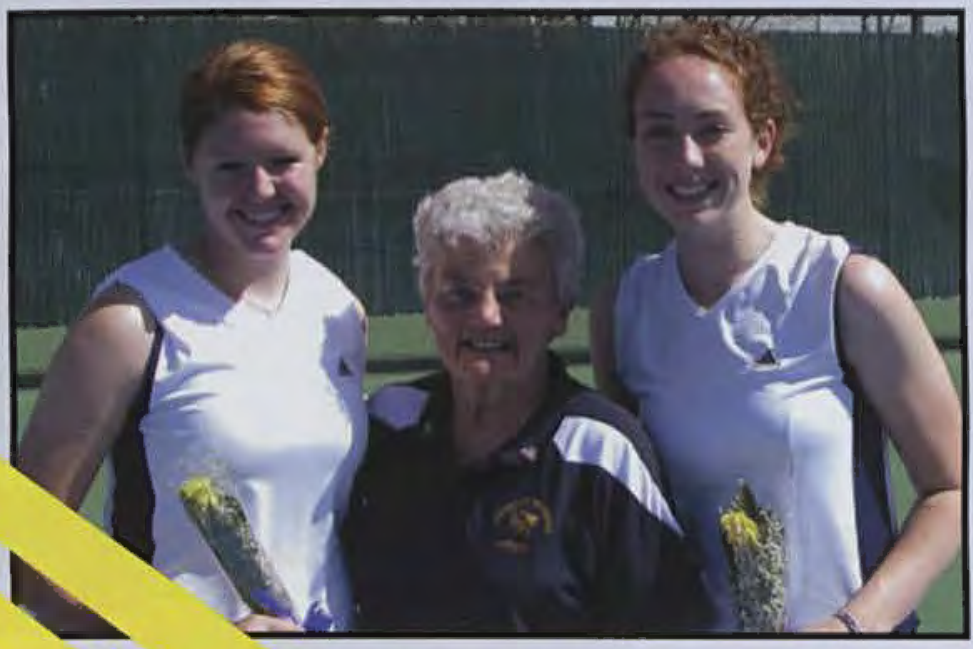

Anna Strayhorn and Sarah Andersen smile with head coach Dr. Dee Morris.

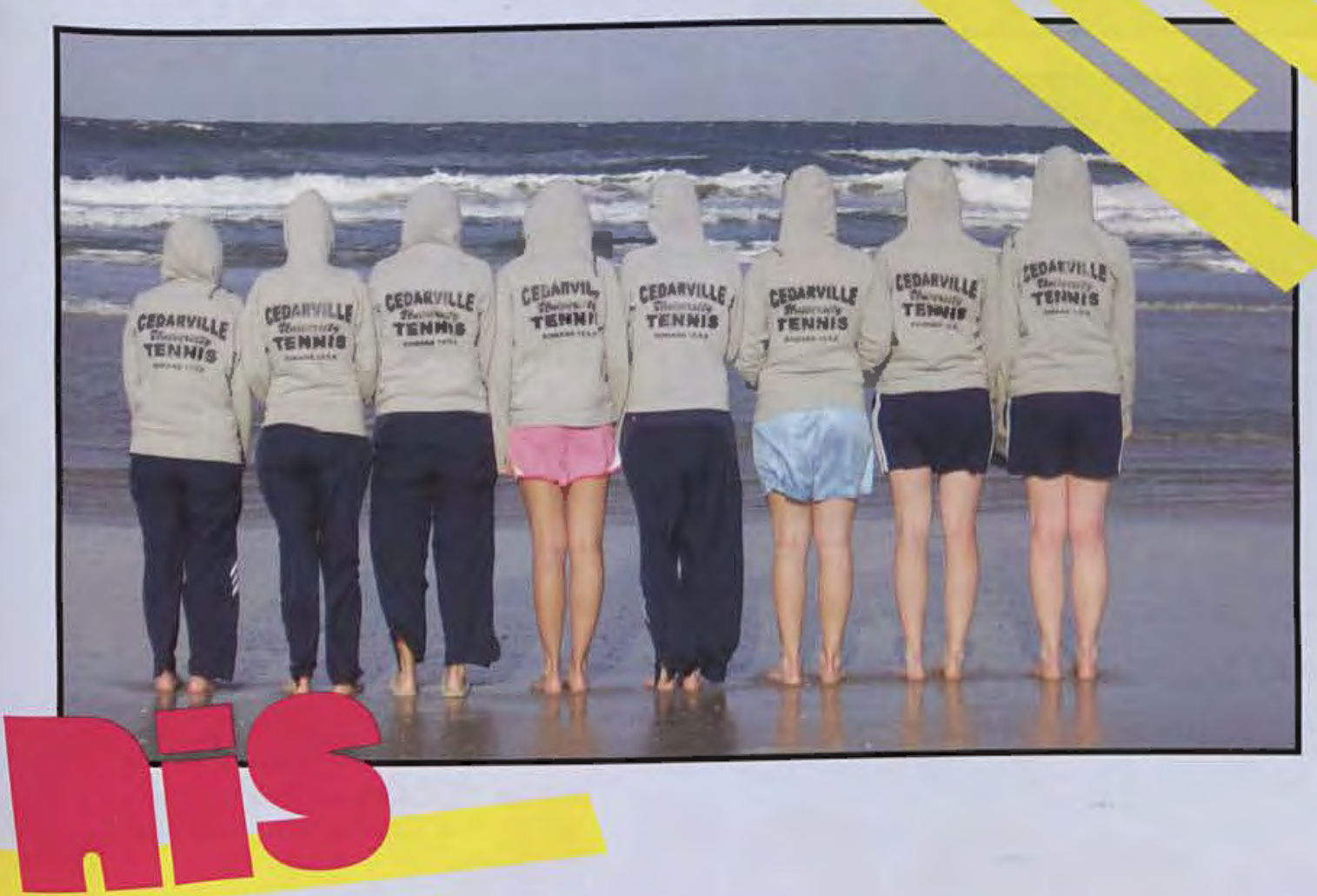

The team shows their Cedarville tennis pride. "What I will remember most about my tennis experience this year is the fact that we went from being teammates to friends. The amount of encouragement that others provided to me both on and off the court was such a blessing!" comments Anna Strayhorn.

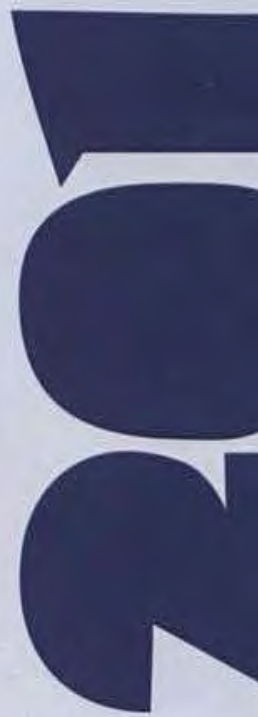



Taylor, Paul Hembekides. Second row: Brandon Young. Student Athletic Trainer Jon Bright, Alex Beelen, Dan Petke, Josh Sisson, Nathan Petke, Aaron Cocks, Clay Wallace, Aaron Coach Kiel Bryan, Assistant Cow: Assistant Boynton. Back row. Ahompson, Coach Rill Thompson, Willett, Colby Stolzfus, Josh
Left to right. Front row: T.J. Tyler Rost, Kyler Ludlow. Juan Martinez, Dave Valle. Clint Price, Brady Workman. Jordan Shumaker, Micah Wilson, Luke Freshwater. avid Convertini. Third row: Andrew York, Brian Brown, Nathan Davenport, Mas, Josh Sandlin, Head Coach Manes.

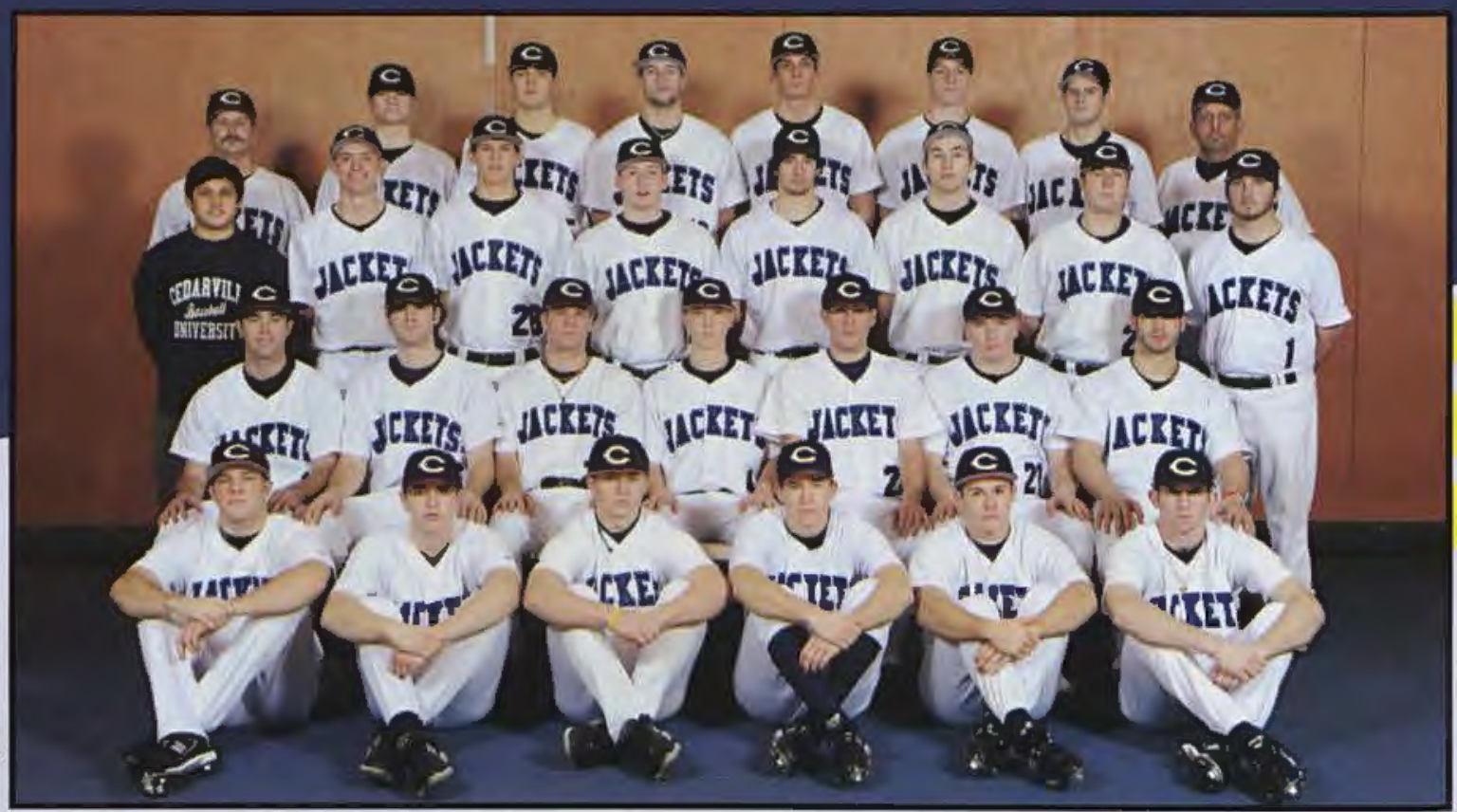

Emmanuel

Covenant

Indiana Wesleyan

Southeastern

Clearwater Christian

Evangel

Concordia (Mich.)

Bethel (Ind.)

Indiana Wesleyan

Saint Xavier

Dakota Wesleyan

Saint Xavier

Bethel (Ind.)

Grace

Point Park (DH)

Point Park (DH)

Ohio Northern (DH)

Notre Dame (Ohio) (DH)

Notre Dame (Ohio) (DH)

Juan Martinez gets ready for the pitch.

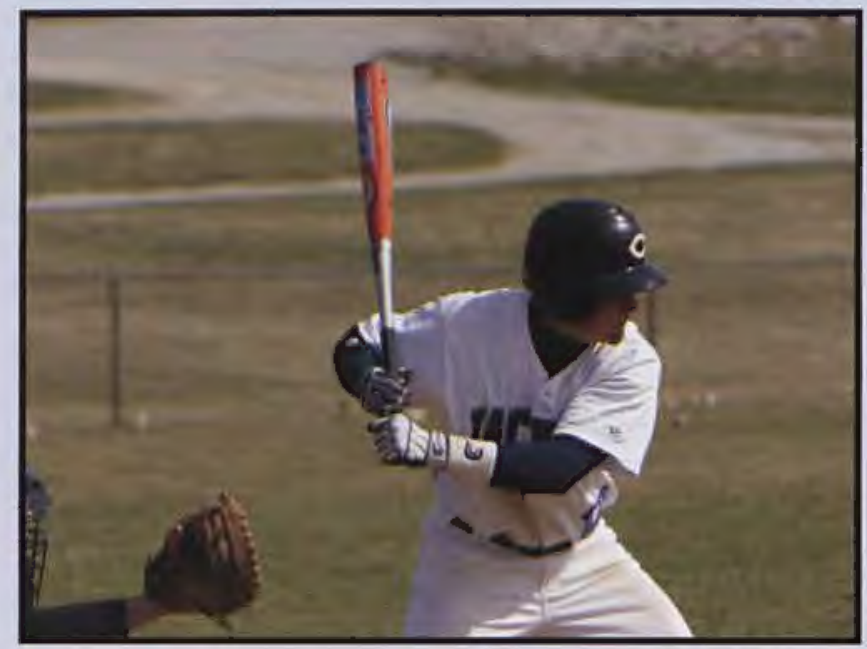

Earlham

Malone (DH)

Malone $(\mathrm{DH})$

Ohio Christian (DH)

Ohio Dominican (DH)

Cincinnati-Clermont

Ohio Christian (DH)

Walsh (DH)

Walsh $(\mathrm{DH})$

Rio Grande (DH)

Rio Grande (DH)

Wilmington

Mount Vernon Nazarene (DH)

Mount Vernon Nazarene (DH)

Shawnee State (DH)

Shawnee State (DH)

Grace (DH)*

Mount Vernon Nazarene**

Oklahoma Wesleyan**

Geneva**

Malone $^{* \star}$

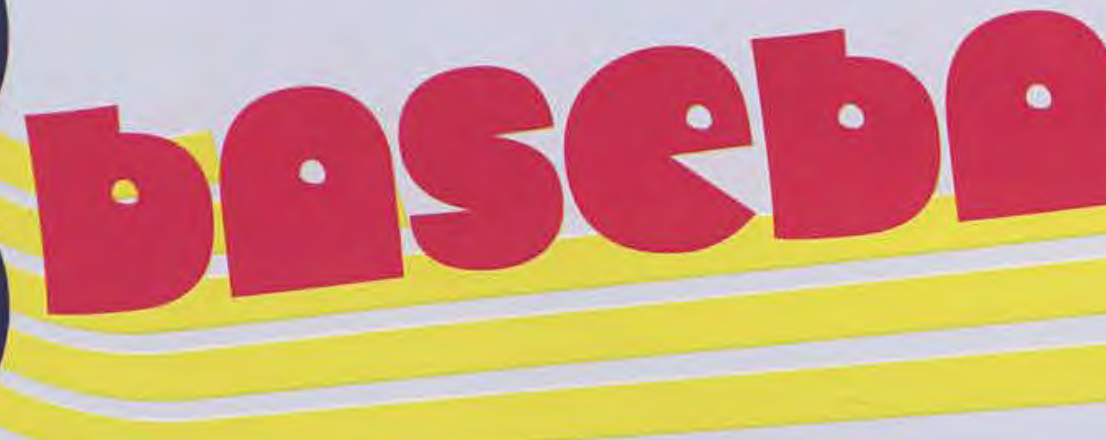

L 1-4

W 5-4 (12)

W 13-0

L 6-17

W 14-3, L 6-7 (9)

L $0-9$

W 9-3

W 3-1

W 13-3

W 8-7

L 4-9

L $0-5$

W $12-5$

W $12-3$

W 8-0, L 4-7

W 10-0, L 3-7

W 5-3 (8), L 5-6

L 2-7, W 9-4

L 0-3, W 6-3

W 6-3

L 2-5, L 2-4

L. 1-6 (10), L 0-5

W 9-1, W $10-0$

W 4-1, L 5-12

W 3-2

W 13-3, W 10-0

W 11-5, L 2-6

W 8-3, L 3-5

L 5-7, L 3-14

W 6-5, L 8-13

W 11-4

L 9-14, L 3-7

W 5-4, L 2-5

L 1-11, L

W 2-1, W 11-1

L $0-5$

W 8-5

W 5-2

L 3-6

*NCCAA Midwest Regional **NCCAA National Tournament
W 1-0, W 6-0 


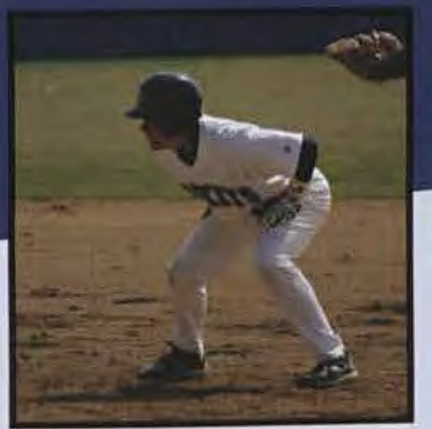

Juan Martinez crouches ready, eyes on the ball. In describing their season, Juan says "Speed limit." We assume that's an inside joke.

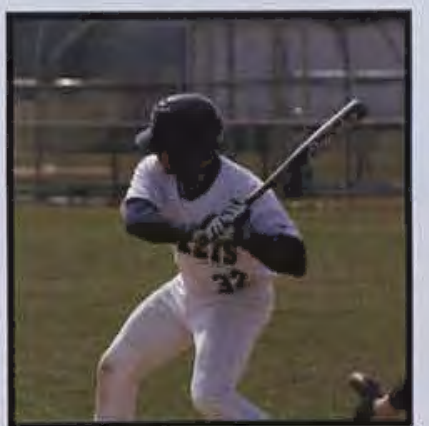

David Convertini describes one of the games, "After the 'Brawl' with Rio Grande, Coach Manes gathered us together and said with a stern face, "That was entirely way too much fun,' and the team replied with a Braveheart scream."

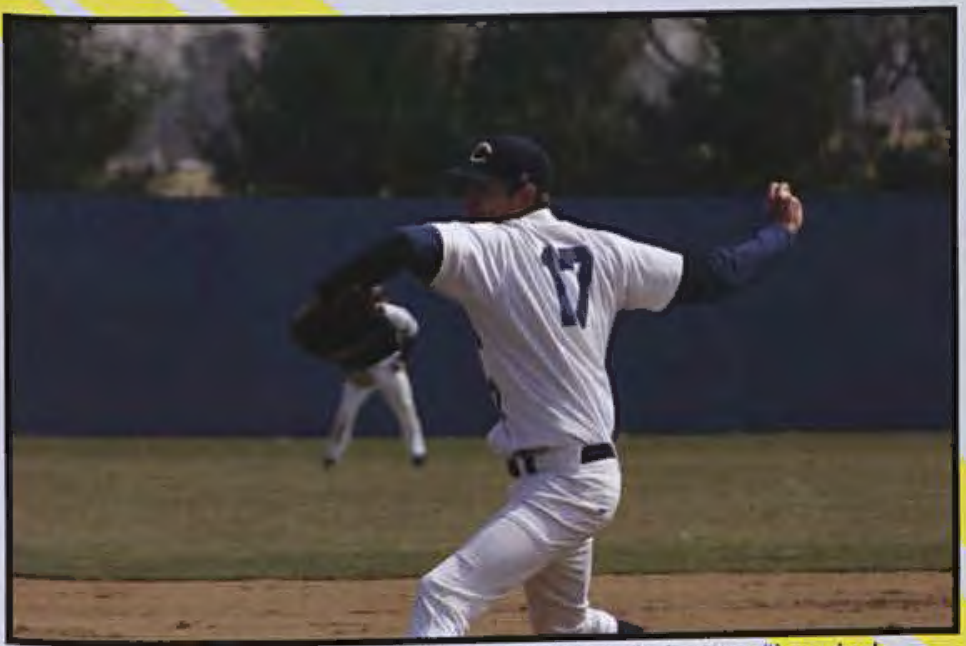

Matthew Willett pitches to the opposing team's batter. "I ended up getting great baseball experience, awesome friendships, and memories that will last a lifetime," says Matthew Willett.

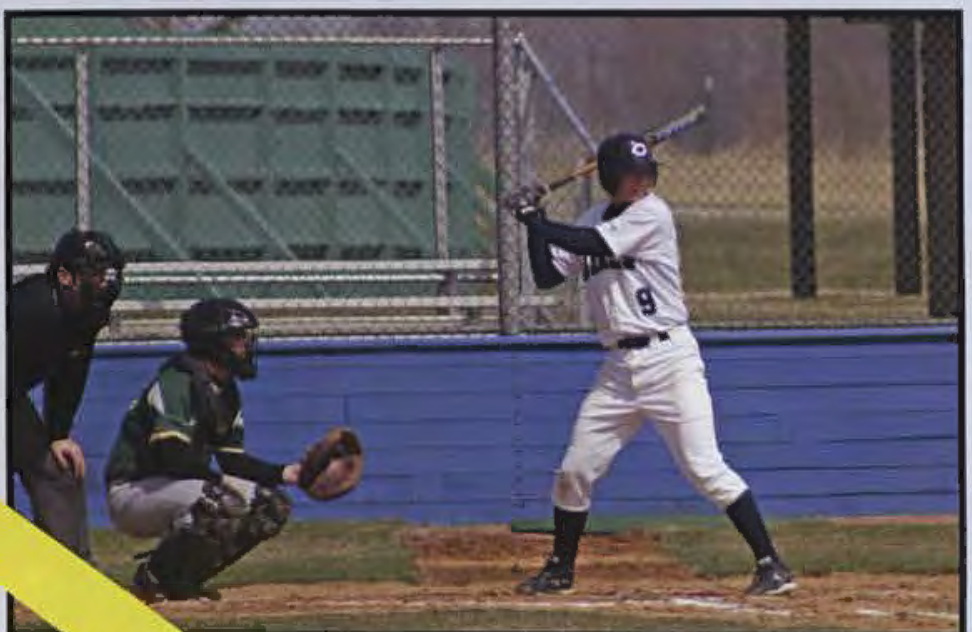

Alex Beelen says, "This was a year of firsts; it has been a process. At times we've struggled, and at times we've succeeded, but through it all we have had a great time playin' ball."

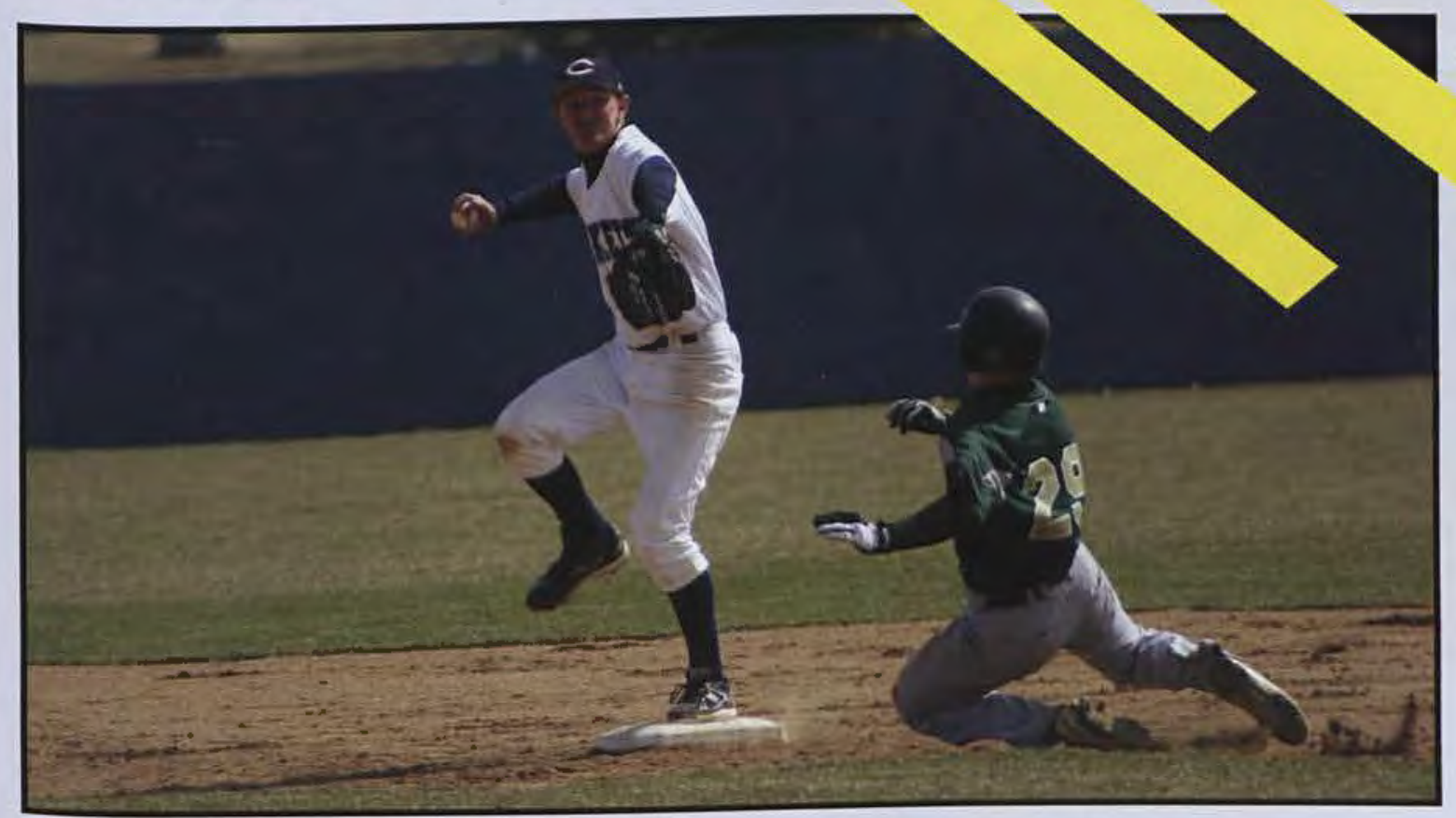

Alex Beelen fields the ball cleanly and attempts to turn to get the double play. 
Left to right. Front row: Christina Zorn, Grace Komar, Sarah Hoffman. Charissa Rowe, Sara

Koepke, Sarah Harnica Middle row: Jillian French, Lexi Mitchell. Emily Young, Jenna Fox Mallory White, Leah

Baker, Stephanie Griffith Cortni Combs. Back row: Assistant Coach dy Herron, Assistant Coach Dave Stewart, ead Coach Wes Rowe, Assistant Coach Joy Asan, Assistant Coach Dreese, Student Manager Liz Vallis.

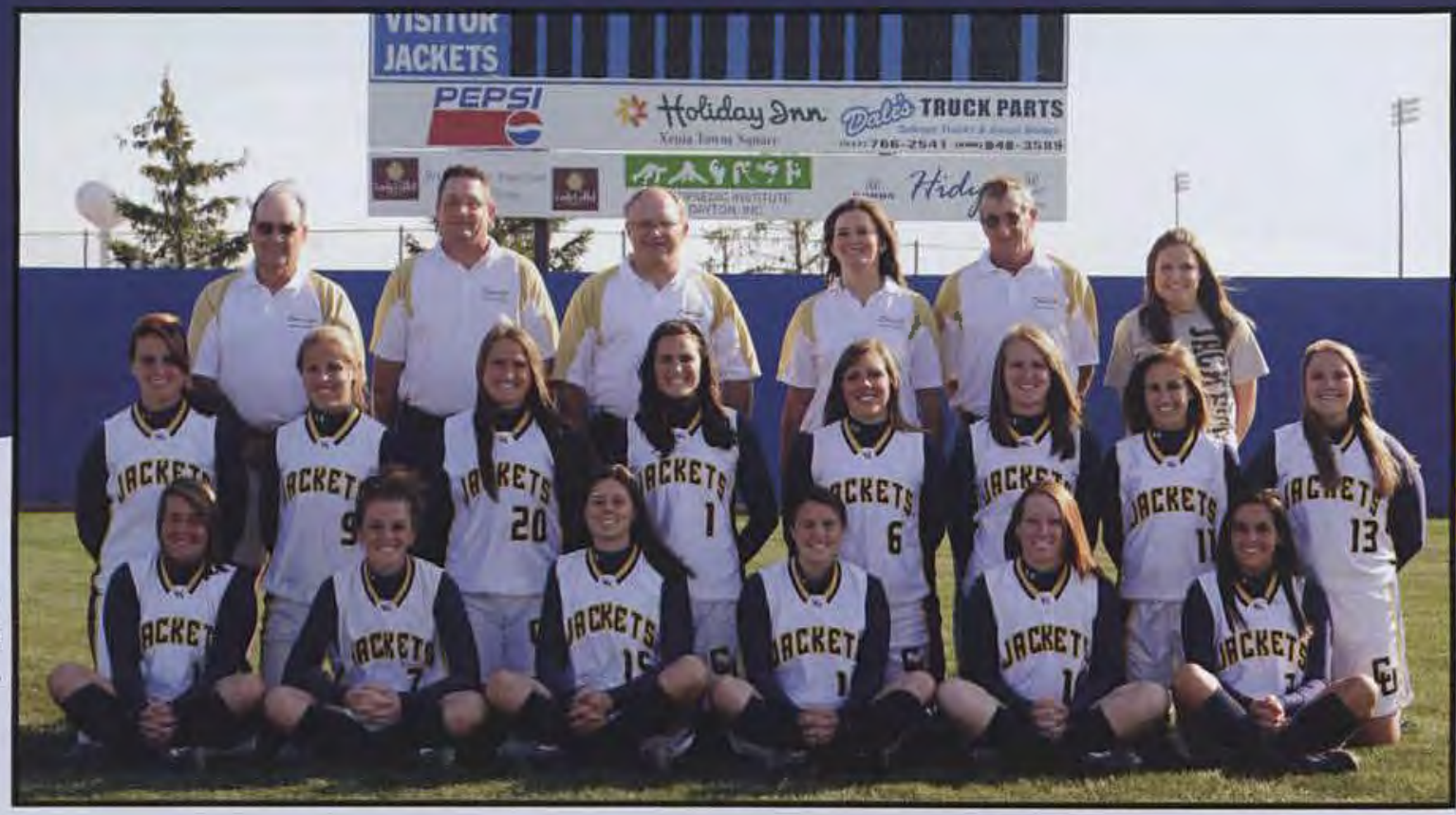

Saint Mary-of-the-Woods King's

Marywood

Ursuline

Saint Anselm

Dakota Wesleyan

Lees-McRae

Taylor

Indiana Wesleyan

Spalding

Marian

Adrian

Siena Heights

Rochester (Mich.)

Rio Grande

Notre Dame (Ohio)

Shawnee State

Walsh

Mount Vernon Nazarene

Ursuline

Carlow

Point Park

Ohio Dominican

Malone

Spring Arbor*

Indiana Wesleyan*

W 7-1

L 3-13

W 6-0

L 7-10, W 5-4

L 0-9, L 1-5

L 2-10

L 0-5, W 6-4

L 0-4, L 0-8

L 0-8, W 4-2

L O-4, L O-3

L 0-7, L 0-4

W 9-1, W 9-7

W 14-6, L 4-8

W 5-4, L 2-5

L $2-4$, L $0-9$

L $2-9$, L 1-3

L 3-9, L 5-13

L 0-5, L 0-5

L 4-6, W 2-1

L 2-5, W 4-2 (8)

L 2-5, W 7-0

L 0-11, L 2-6

W 6-5 (10), W 4-1

L 2-6

L $0-8$

"NCCAA Midwest Regional
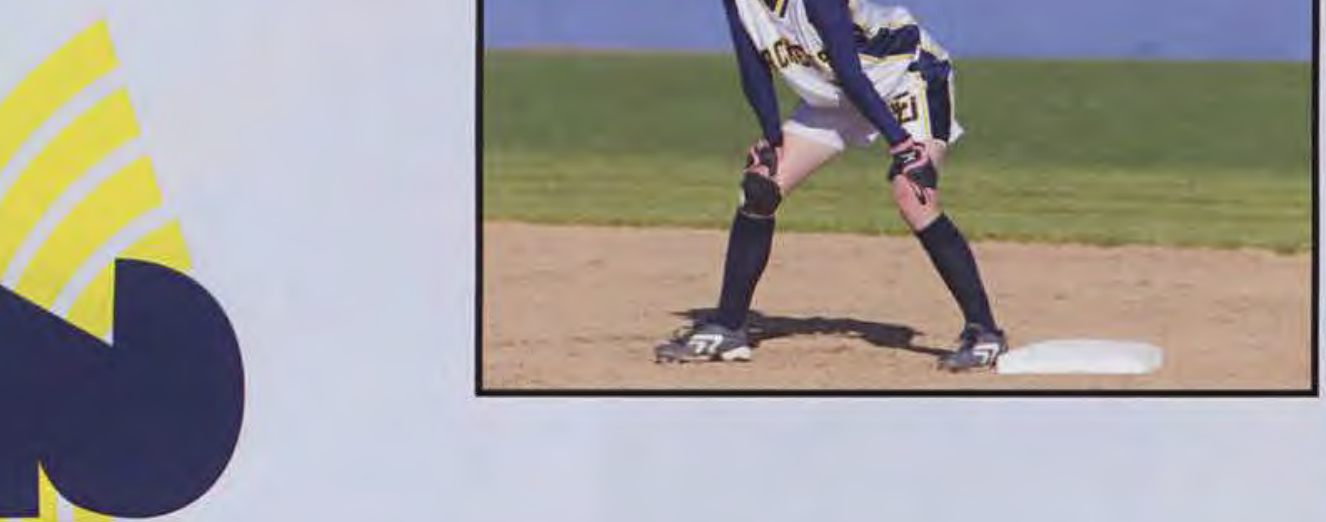

\section{portunity to steal.}
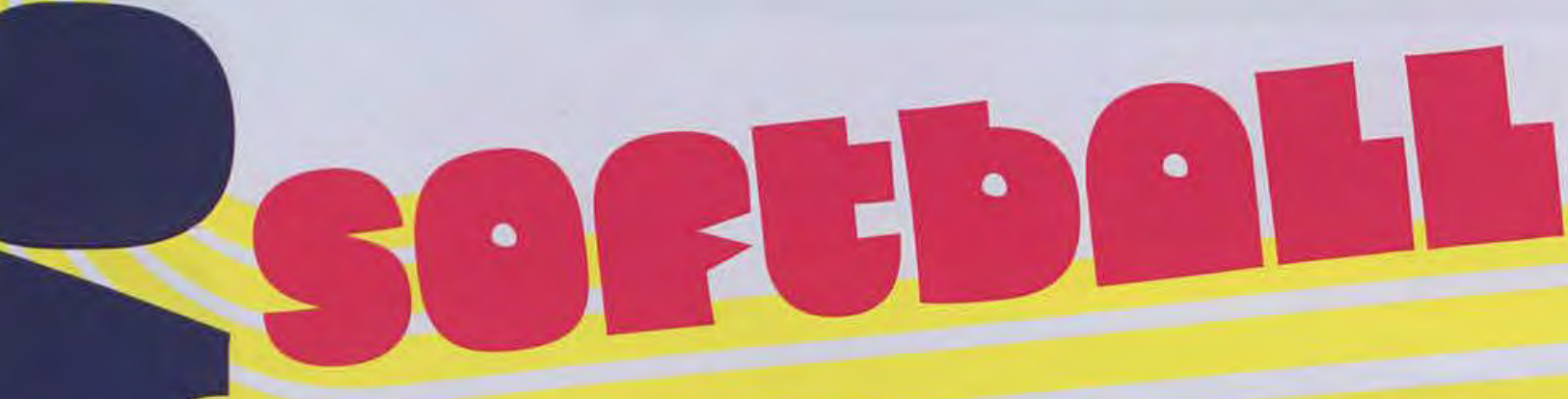


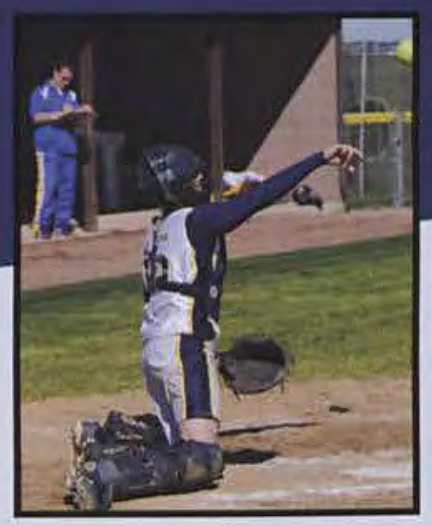

Sarah Hoffman is always ready behind the plate for whatever action

comes her way.

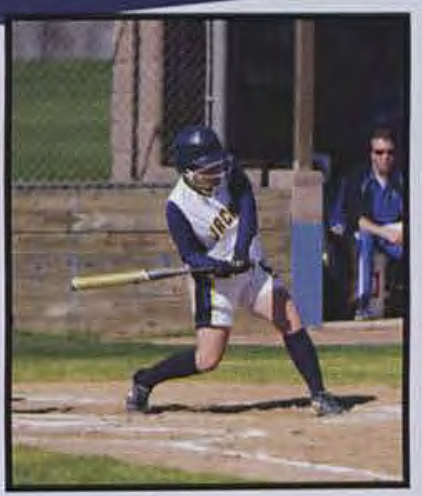

Swing, batter batter! That look's like a homerun swing to me.

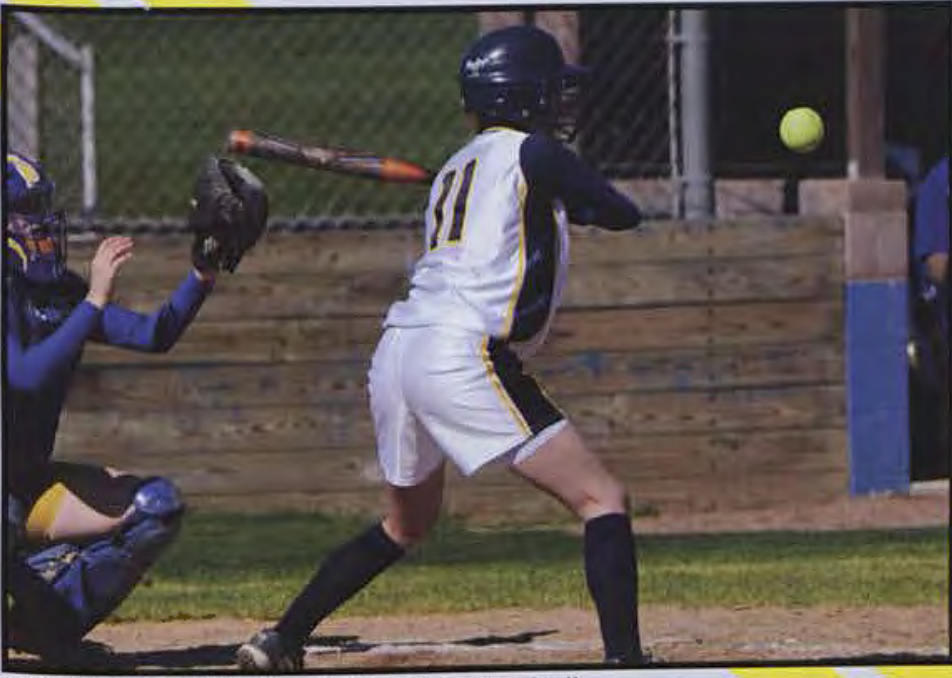

Stephanie Griffith has her eye on the ball and prepares to give a mighty swing.

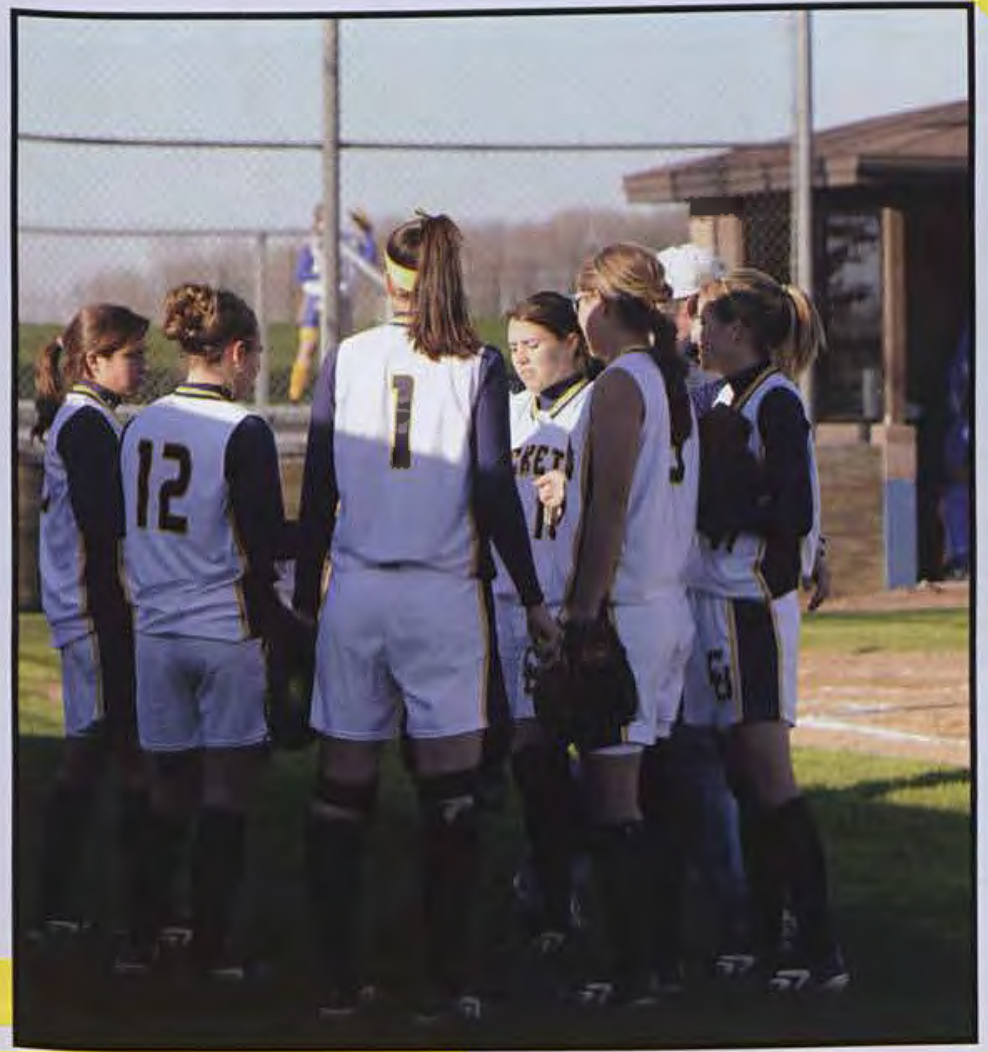

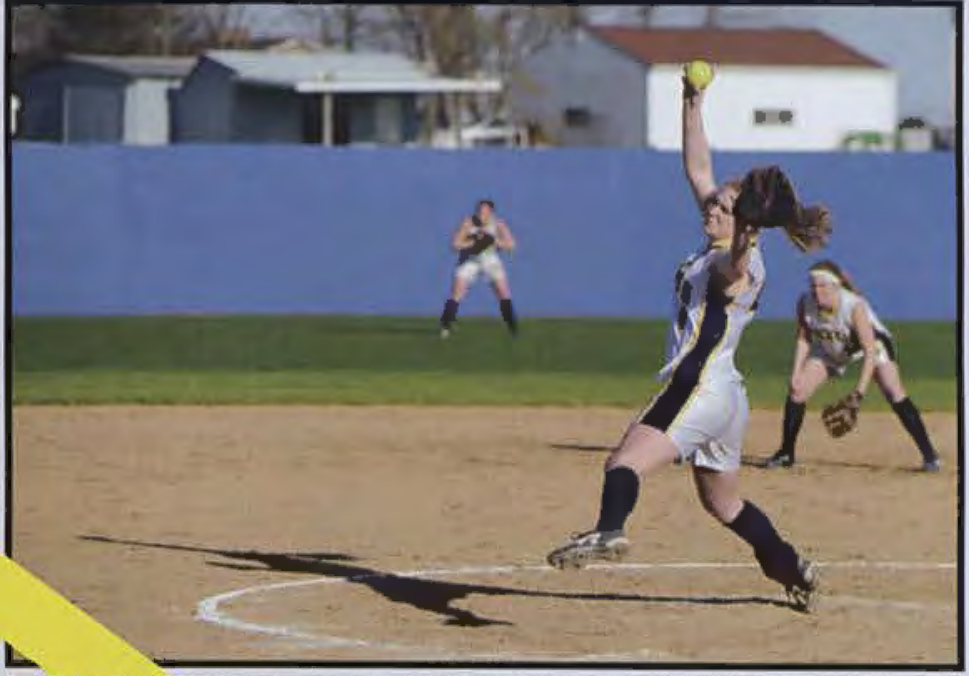

All batters better be on their game when Leah Baker is on the mound.

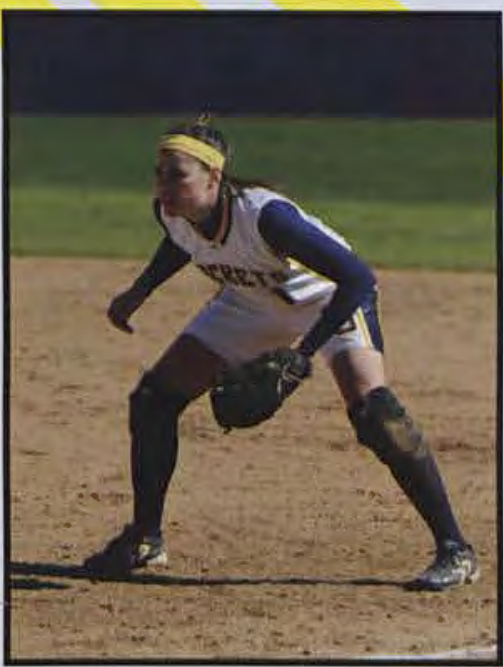

Jenna Fox is determined not to let any ball get by her.

The girls circle up to get ready for the game.

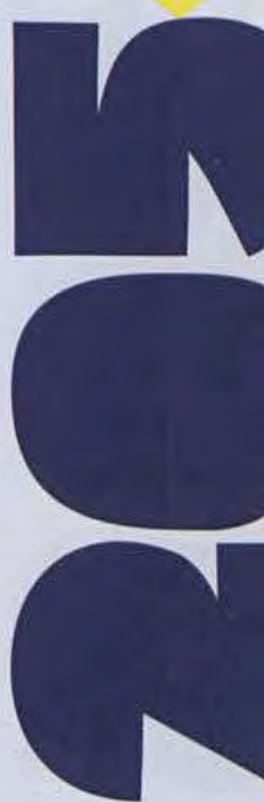


Adam Clouse thinks that "Because JV is more about player development and typically less competitive than the varsity, it allows the to focus more on fellowship and investin each other's lives. Besides beating the \#1 ranked JUCO team in the nation, the highlight for me was being able to spend quality time establishing relationships with the guys.

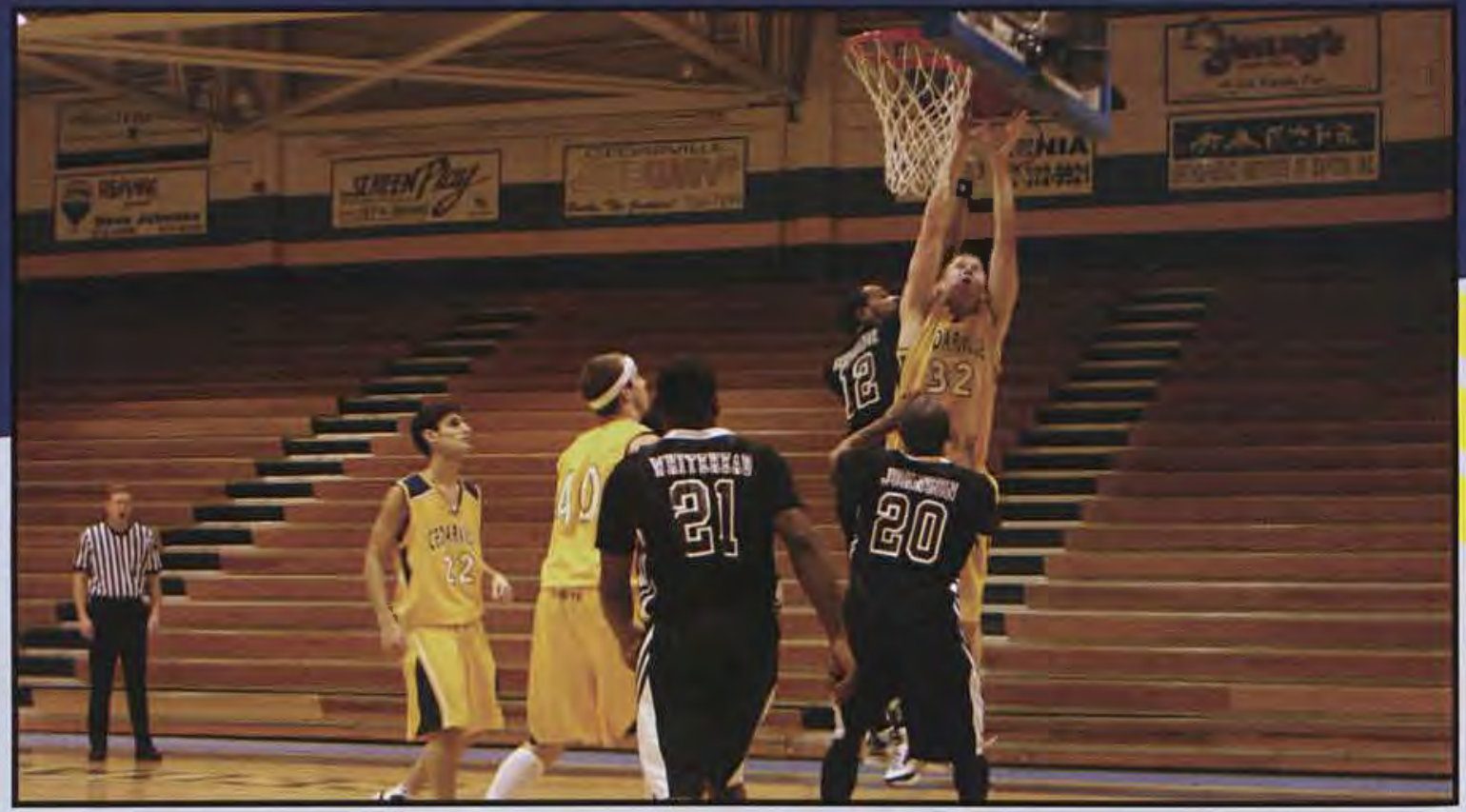

Women's JV basketball can sometimes be heated, but there's always time for a smile.

"Playing JV soccer with the women's soccer team was a wonderful, stretching experience. It was a great way

to play a sport we all loved, develop great friendships, and worship our Lord together," comments Kaitlyn Fote.
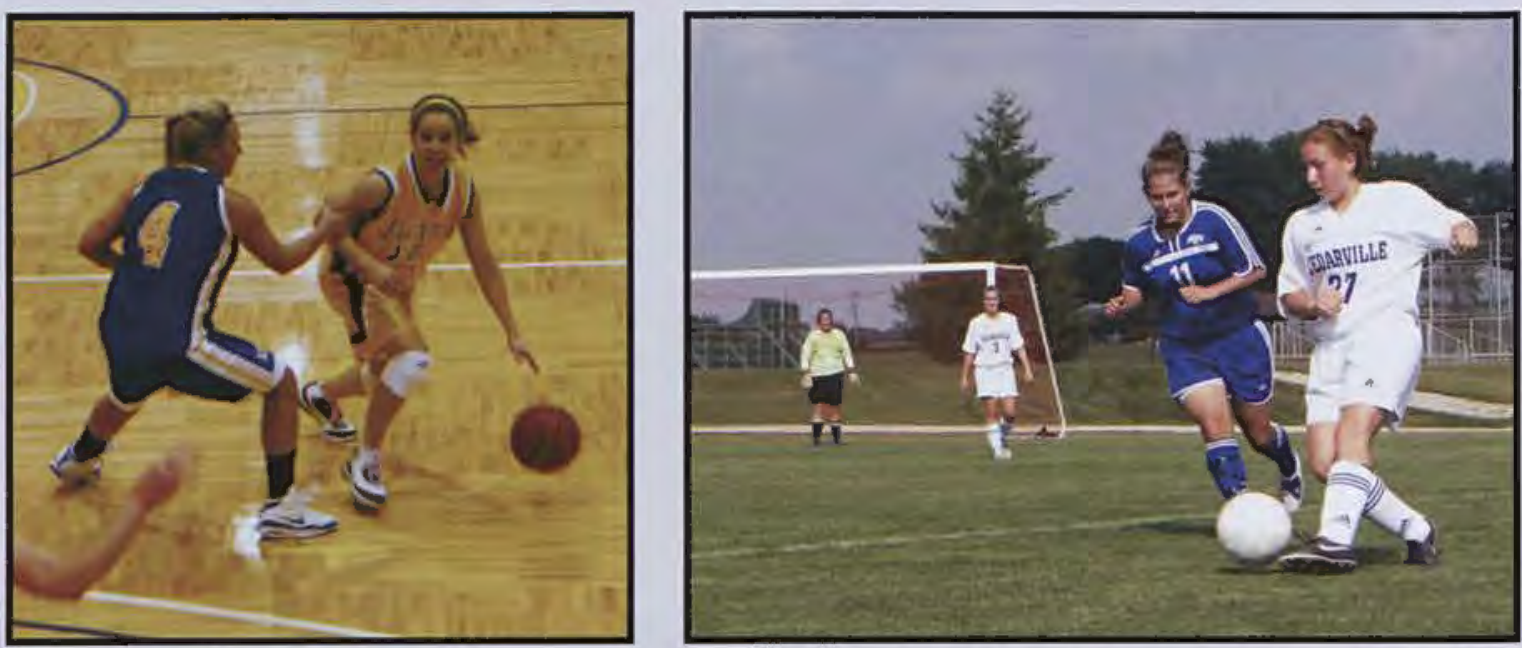

Women's JV Soccer Left to Right, back row: Holly VanderZouwen, Lindsay Raybuck,

Courtney Brown, Plasterer Anna, Coach Krista Mattern Front row: Katelyn Reuther, Becky

Burton, Kaitlyn Fote, Karen McCoskey, Natalie Tribbett, Mary Bernecker
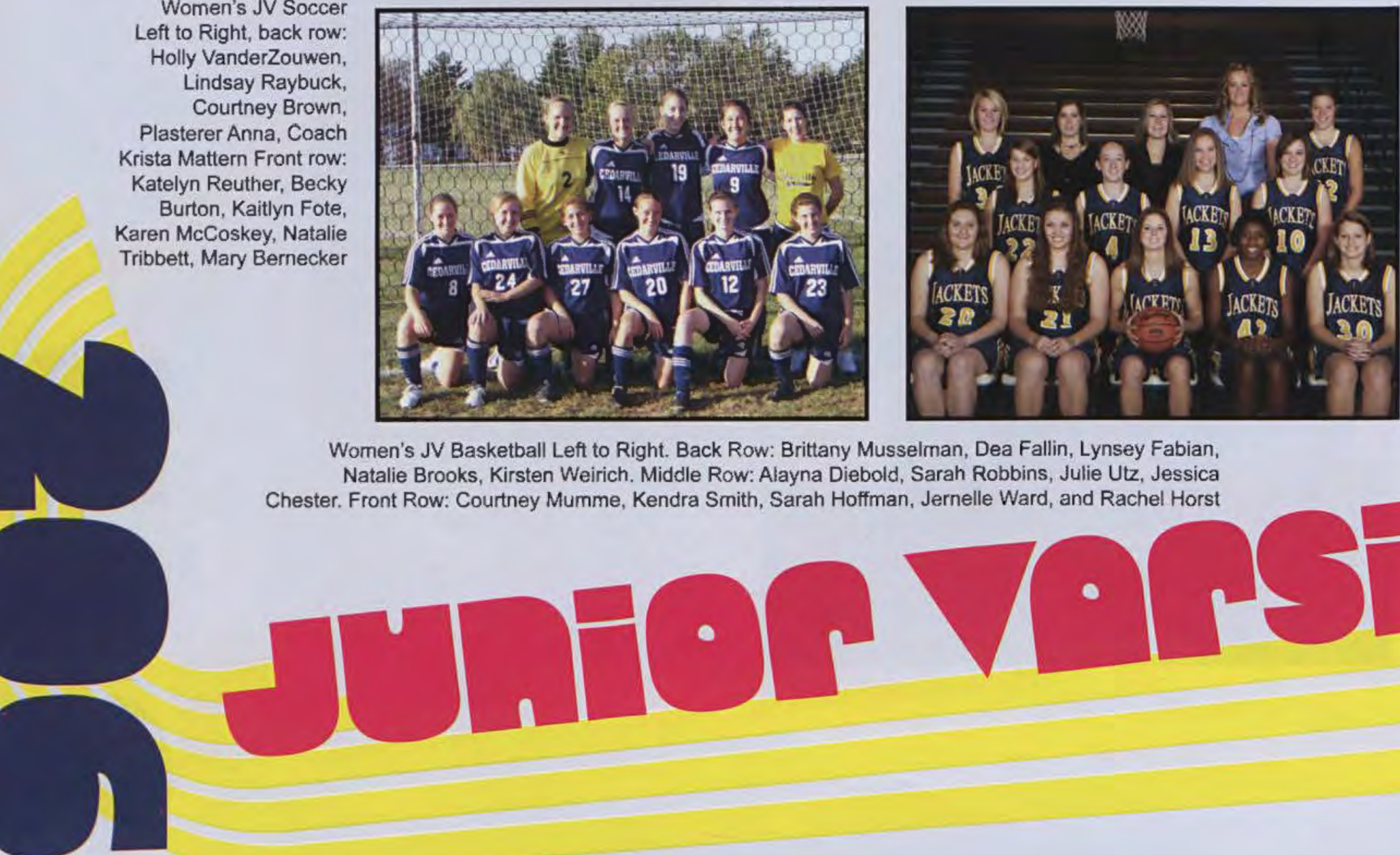

Women's JV Basketball Left to Right. Back Row: Brittany Musselman, Dea Fallin, Lynsey Fabian, Natalie Brooks, Kirsten Weirich. Middle Row: Alayna Diebold, Sarah Robbins, Julie Utz, Jessica Chester. Front Row: Courtney Mumme, Kendra Smith, Sarah Hoffman, Jernelle Ward, and Rachel Horst
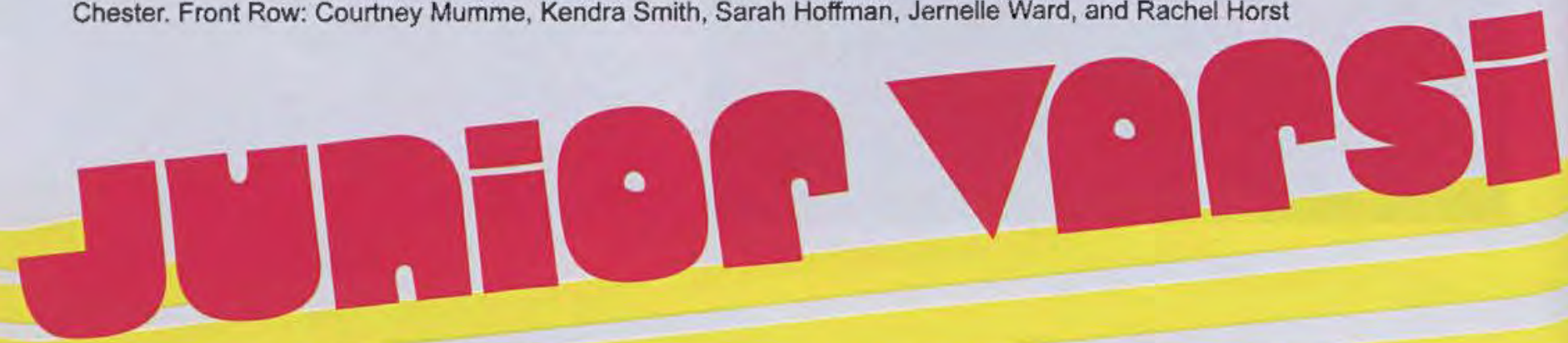


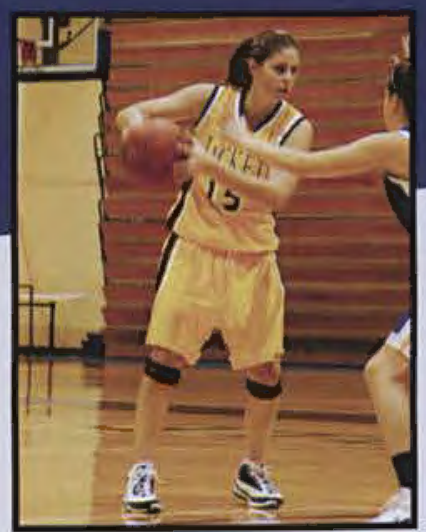

"I played four years of JV basketball and loved it! Needing to balance school with playing softball, there really was no reason for me to play JV basketball besides my love for the game, my coach, and teammates. Each fall I looked forward to the season starting and enjoyed every minute of it!" comments Sarah Hoffman.

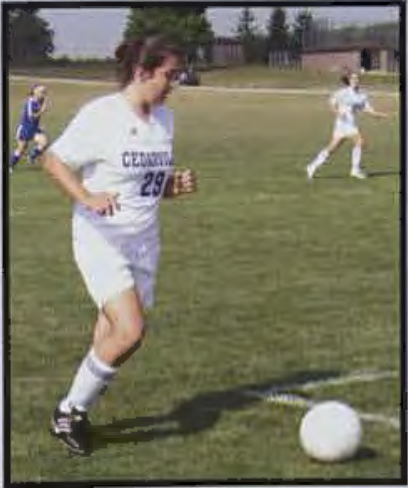

"Playing for the J.V. soccer team was a great experience and time of growth. Weekly Bible studies and constant encouragement from tearnmates and coaches allowed for spiritual growth alongside our physical growth" says Anna Plasterer.
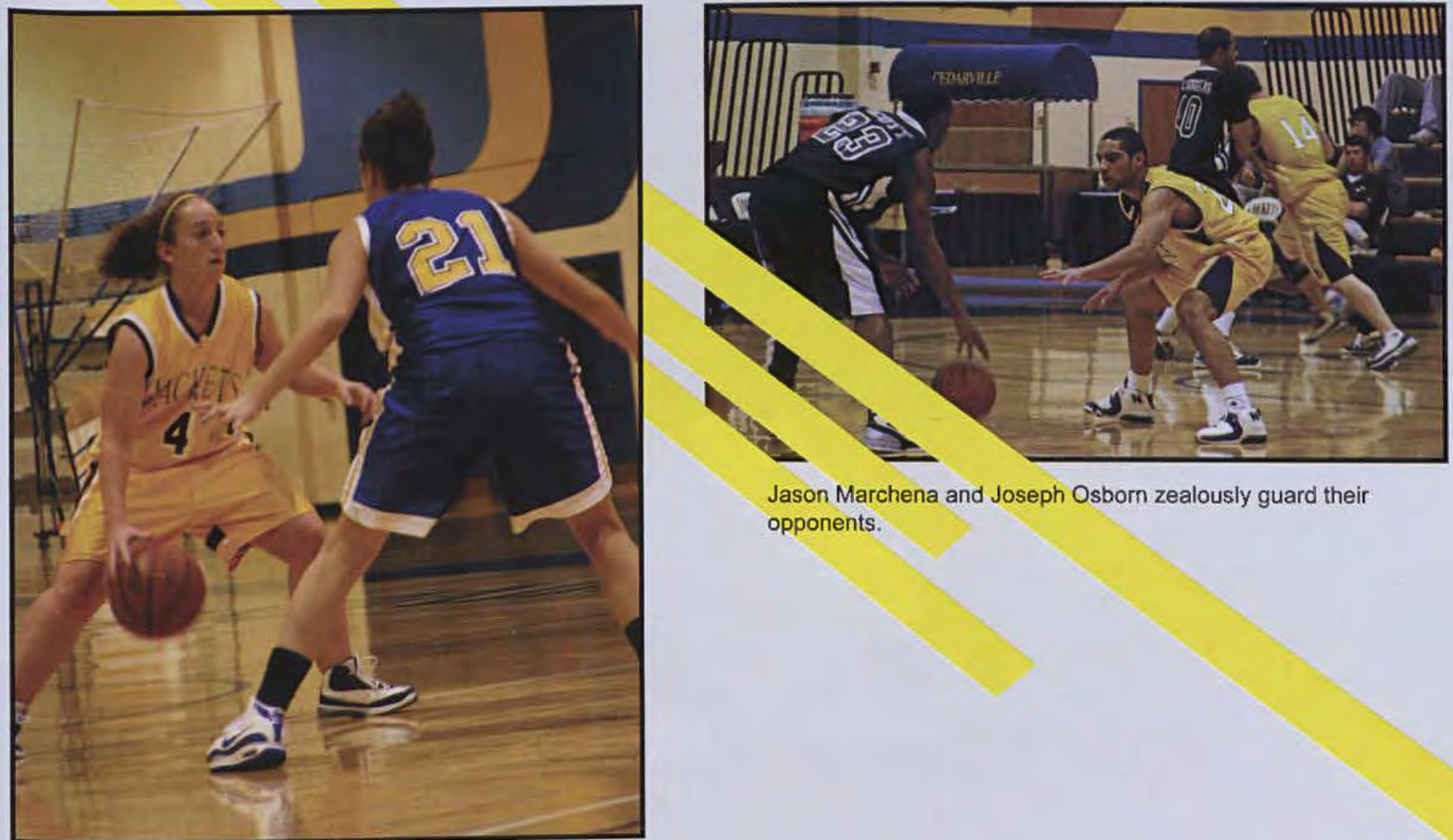

Jason Marchena and Joseph Osborn zealously guard their opponents.

Sarah Robbins blazes a trail to the basket.

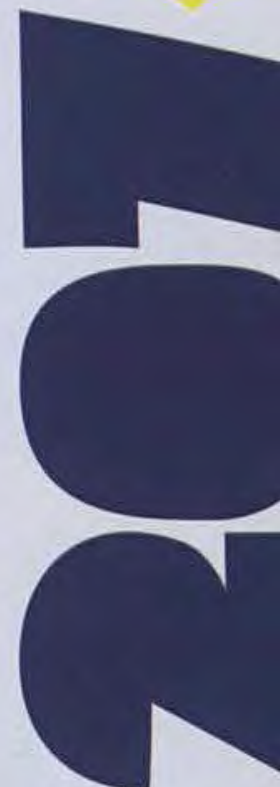



I think back to my first week as an intern at the American embassy in Costa Rica. One of the ambassadors hosted a party for an ambassadorial inauguration. His mansion sat outside the bustling city of San Jose. The scene seemed to jump out of a movie: sleek black cars glided up to the doors, leaving men and women dressed in dashing tuxedos and startling gowns at the door. I noticed several bulky security guards wandering the grounds, protecting the elite of Costa Rica. Waiters slid through the crowd, carrying trays of champagne and hors d'oeuvres. As I walked through the double-door entrance, a scene of pure elegance greeted me. The foyer led into a ballroom with an arching ceiling. Deep red curtains reached from the top of the tall windows to brush gently on the floor. I tried not to look too much like a kid in a candy shop as my coworkers - Stacy, Kim, Joel, and Eric - and I moved about the room. "Oh, look, that's Kathrine Rodregez. She's a model, big deal around here."

I glanced where Eric was looking and spotted a tall women with dark hair piled on her head. A brilliant green dress hugged her frame. A thick line of shimmering jewels adorned her neck. As I took in the model, Stacy spun me around.

"Here Adam, I want you to meet Bob. He's works for NASA."

As I shook hands with the astronaut, I could almost see the far reaches of space shimmering in his dark eyes. He asked me how I liked Costa Rica, and we made small talk about politics.

I met more Costa Rican celebrities and politicians - some major players - all through the night. At one point, Stacy brought me to an elderly man in a double-breasted suit and a large purple sash. Even as I reached for his outstretched hand, I heard Stacy say "President." Was I meeting the President of Costa Rica? His face crinkled into a smile as he greeted me. Trying to stay calm, I ushered a response. He also asked me how I liked my stay in San Jose before turning to more dignitaries. I drifted away with Stacy, a little heady from the encounter.

All of my time in Costa Rica wasn't spent at parties though as I spent most of my time as a technical writing intern at the consular section of the embassy. The consulate is the face of the embassy, dealing with everything from passports and visas to deported Americans. I loved my time in San Jose though, able to help American citizens with their various issues and troubles.

I never would have gotten to Costa Rica without my time at Cedarville; here I truly found my voice in writing as I studied technical and professional communications. Coming in as a freshman, I never dreamed of going to Costa Rica, but a lot has changed since my first day on campus. Cedarville has helped me to develop not only academically, but spiritually and emotionally as well. I've been able to get involved with tons of different things: from being a writing tutor to working in the PR department to acting as president of the technical communication organization! As I look past graduation, I don't know what exactly to expect. Marriage and a job, I know that much. But beyond that, I'm not too worried; I know I'm prepared.

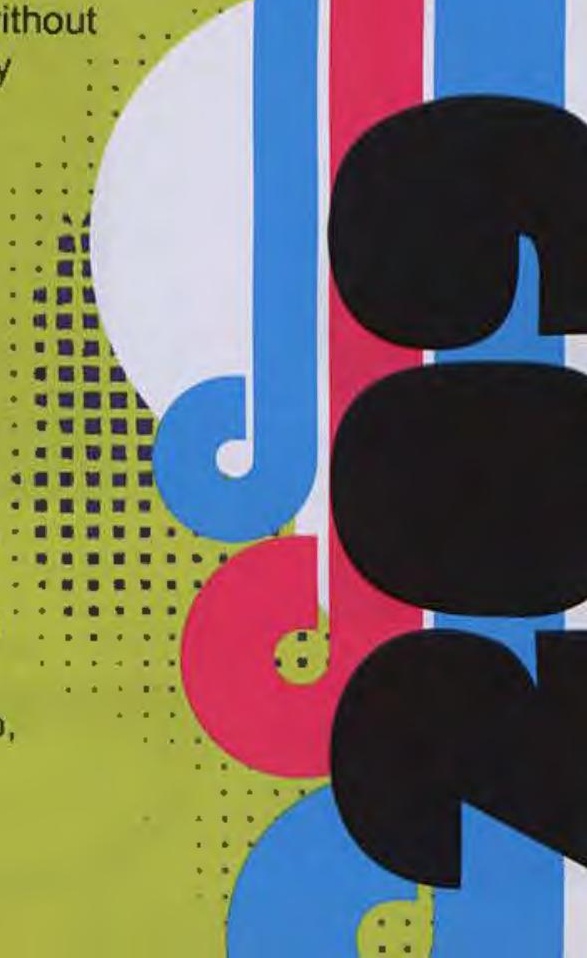



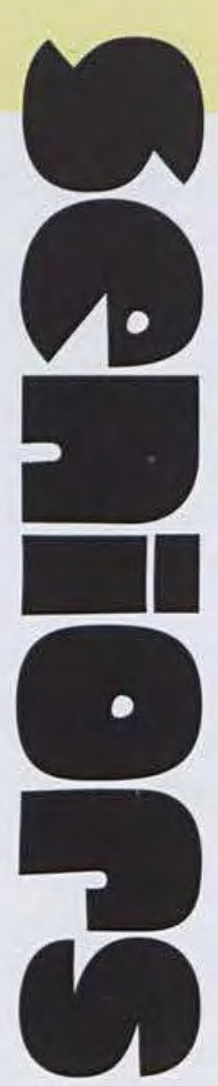

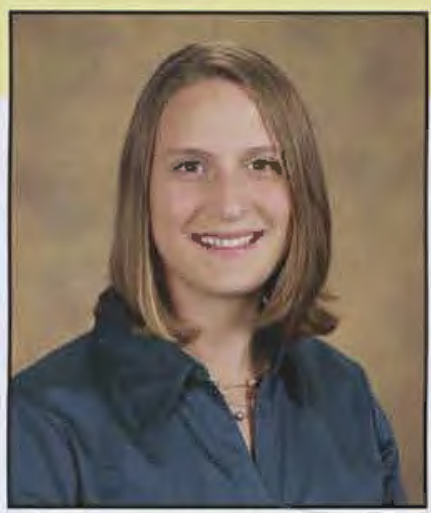

Rebekah Abbey Nursing

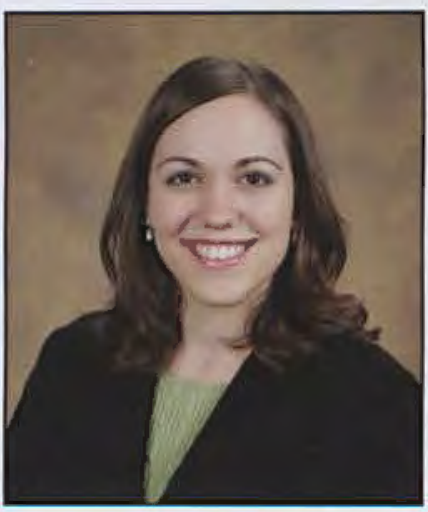

Rebekah Adams

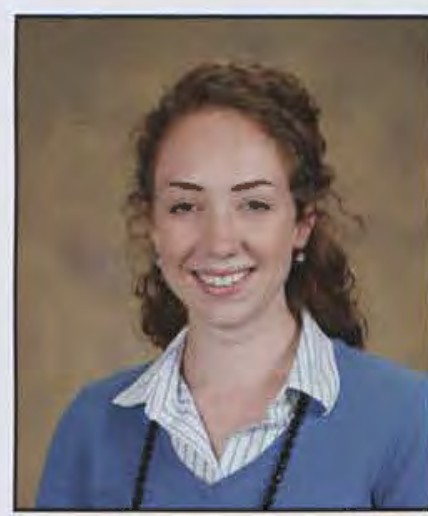

Sarah Andersen Social Work

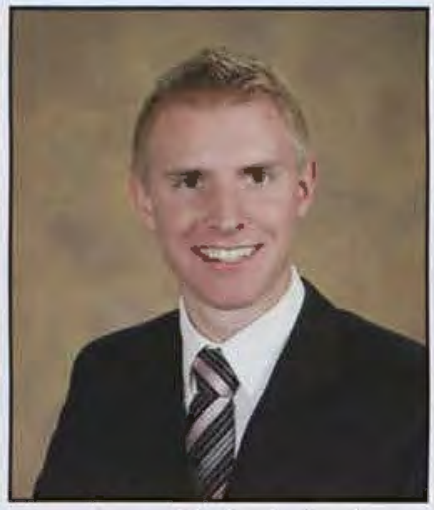

Benjamin Anderson Psychology Finance

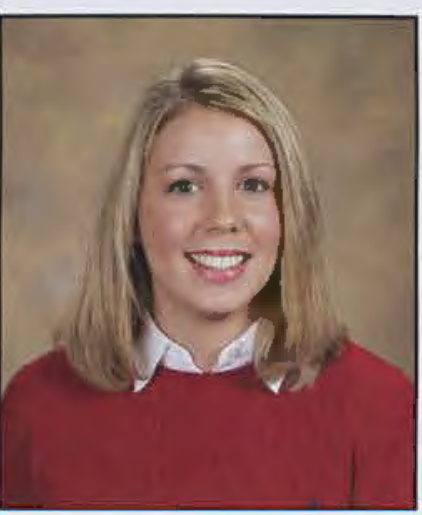

Olivia Aker Early Childhood Education

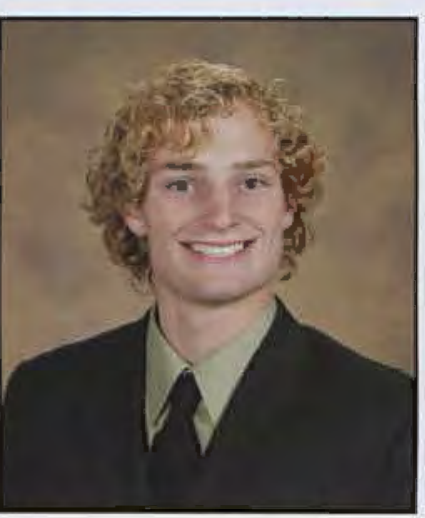

Eric Andrews Finance

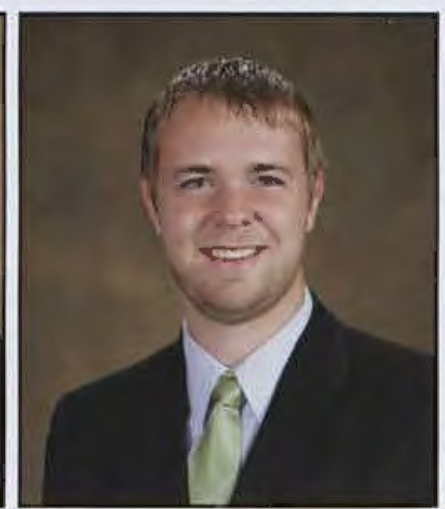
Andrew Arthur English

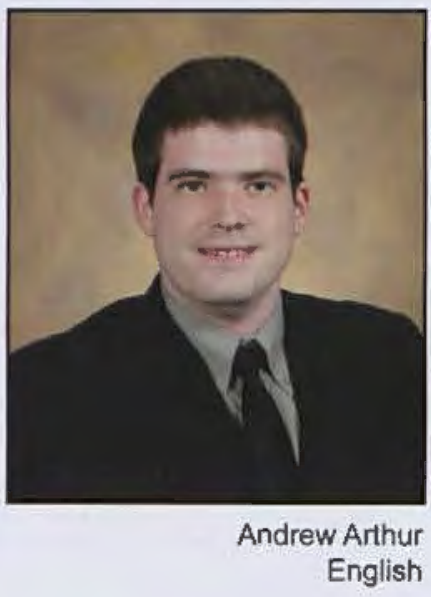

Jeremy Auyer

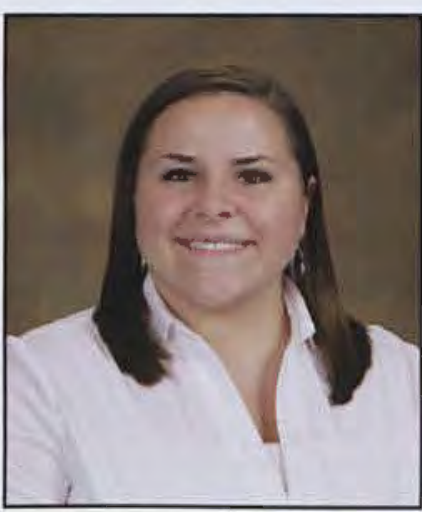

Allison Babbitt Sport Management

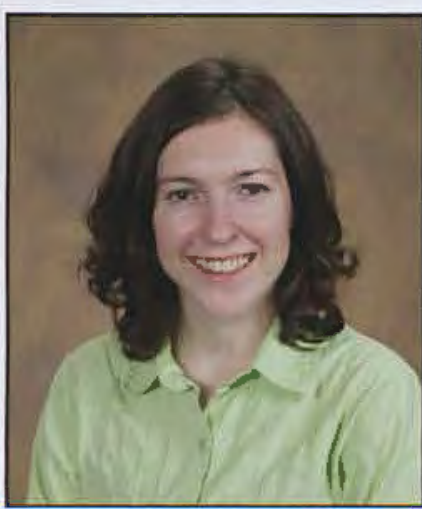

Brietta Allen Social Work

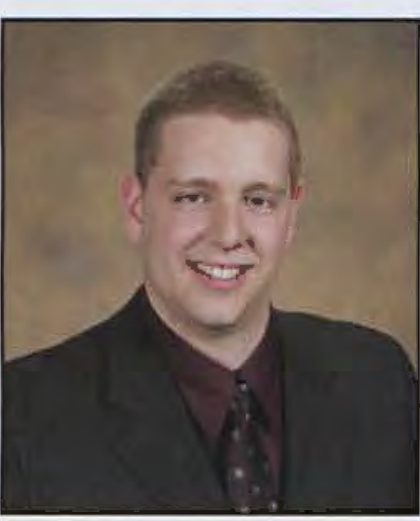

Drake Angle Computer Science

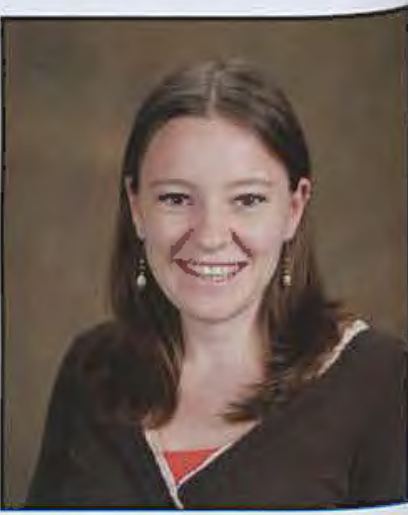

Holly Amarante Mathematics

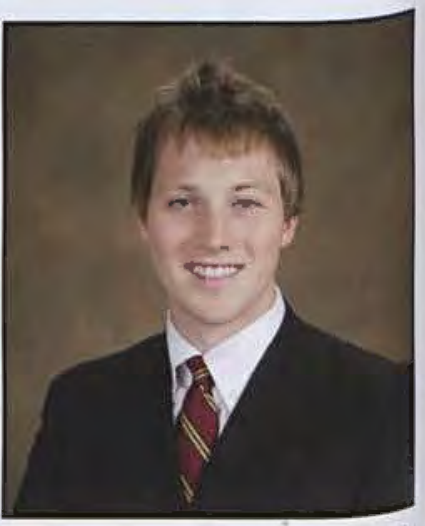

Nicholas Arch Finance

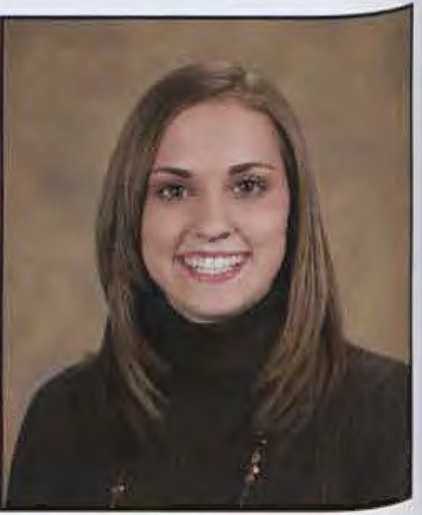

Brittany Baranowsk Nursing 


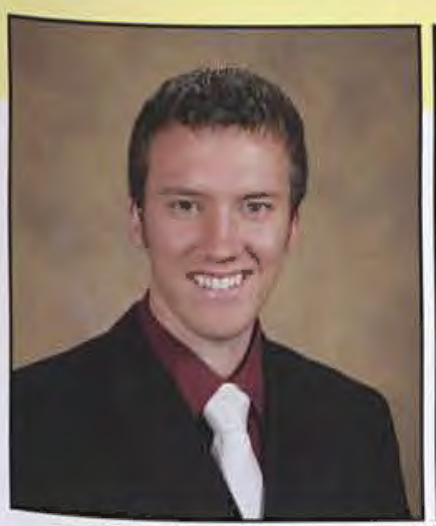

Jacob Baranski Mechanical Engineering

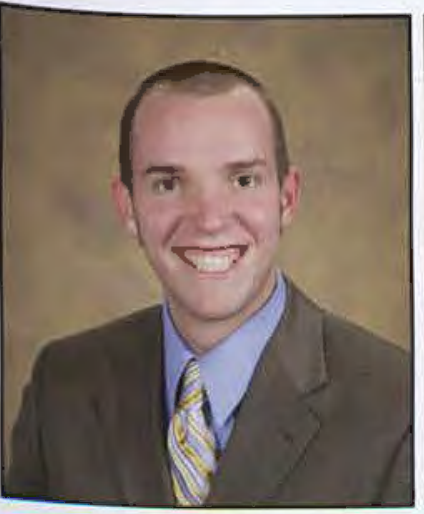

Mark Becknell Nursing

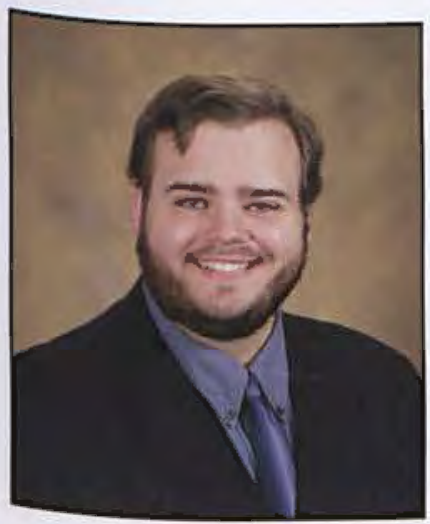

Kevin Bennett Applied Psychology

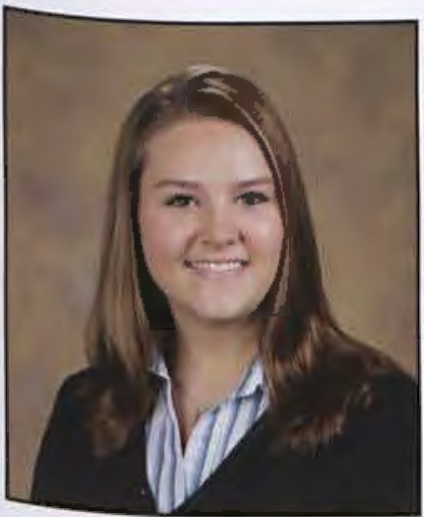

Jennifer Bidwell

Early Childhood/Multi-Age Special Education

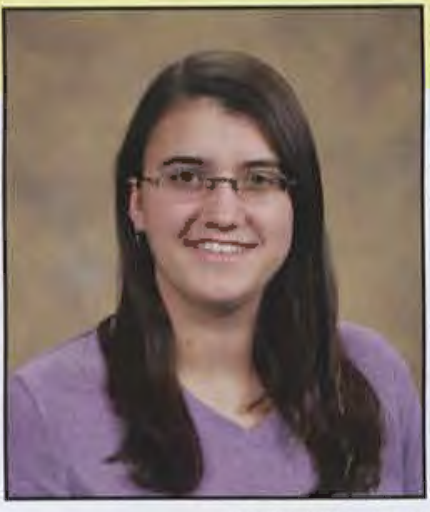

Rachel Barnard

Chemistry

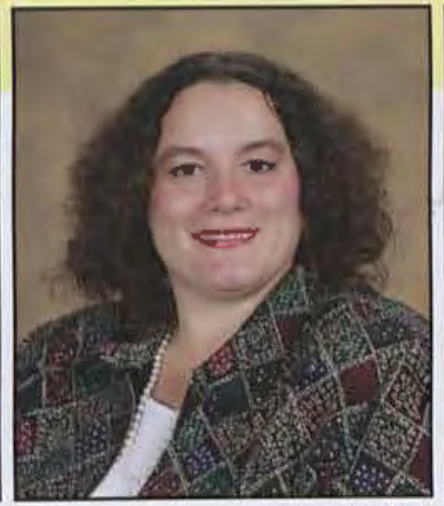

Betty Bartlett

Nursing

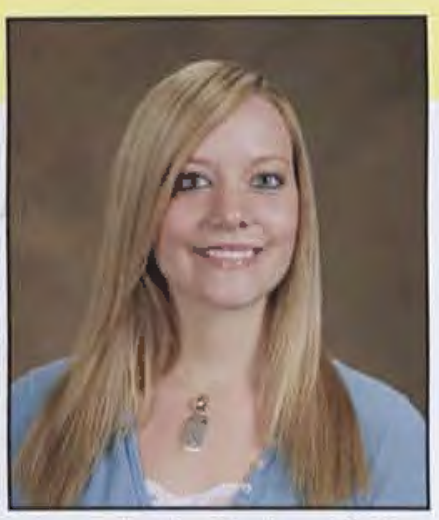

Jessica Bashore-Conley Multi-Age Spanish Education Spanish

Amanda Beesley Social Work

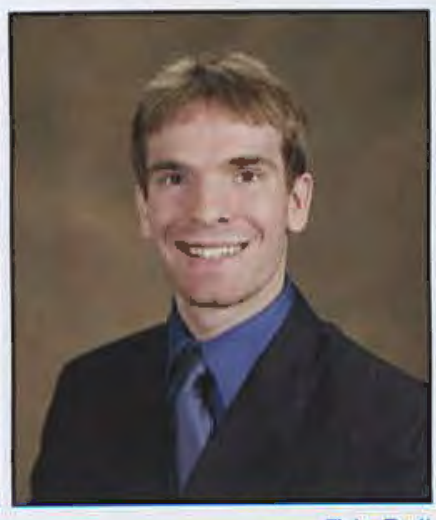

Eric Bell Mathematics

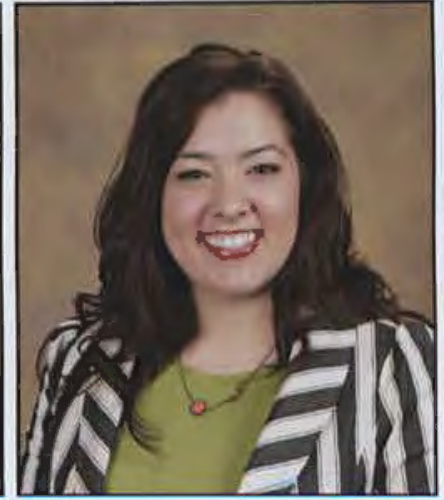

Katie Bell

Studia Art

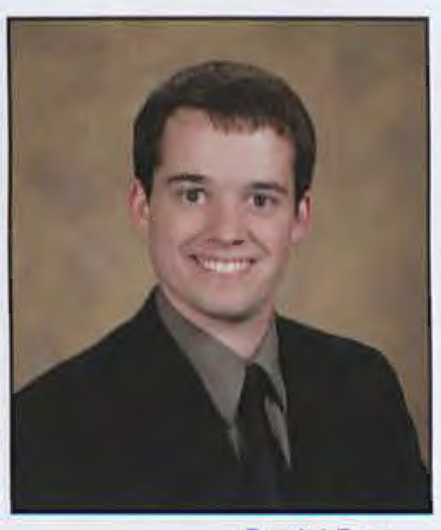

Daniel Benner Comprehensive Bible

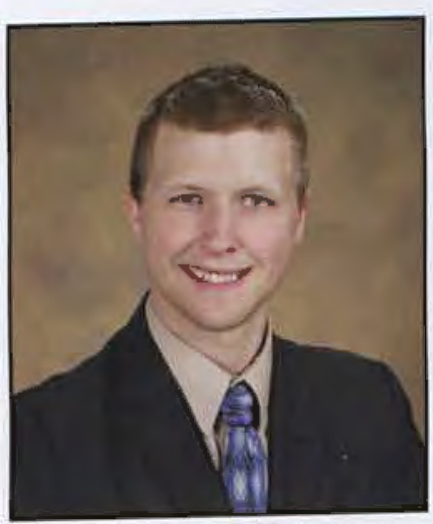

David Benson Computer Science

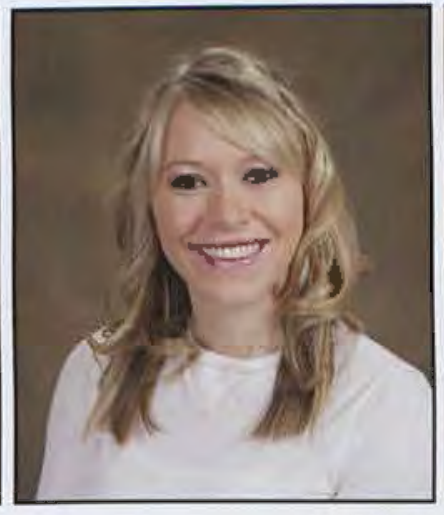

Erin Benz Nursing

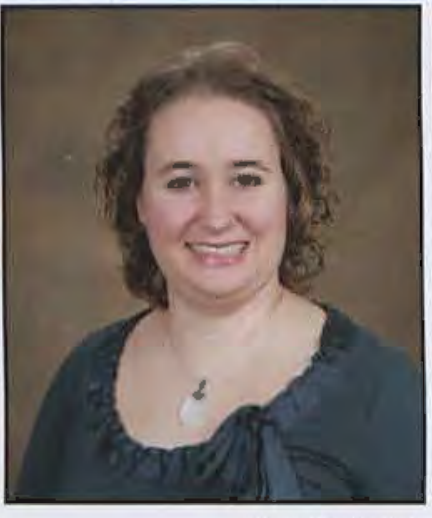

Eva Bertsche Nursing

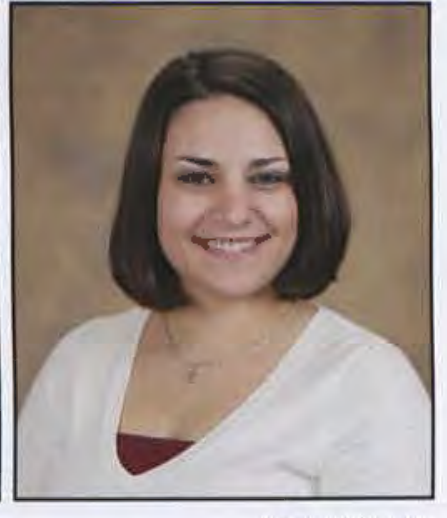

Laura Besaw Media Communications

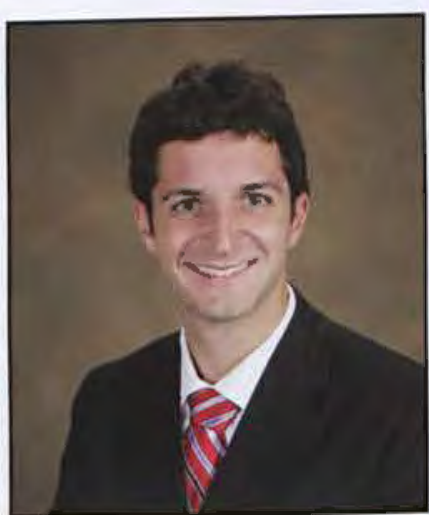

Brett Bigler Accounting/Finance

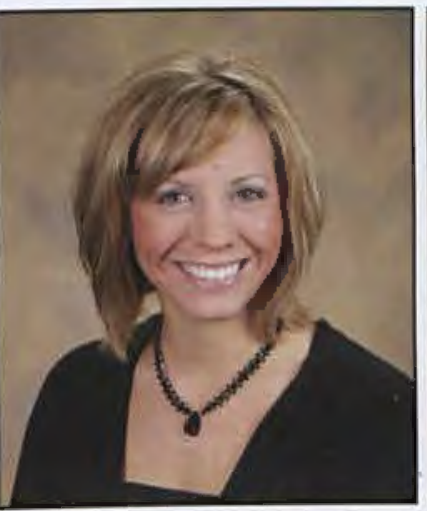

Kathryn Billhartz Nursing

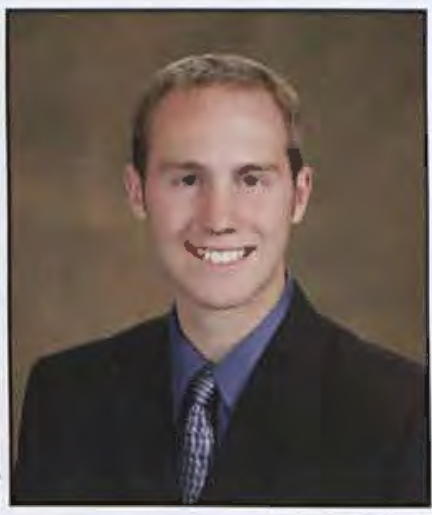

John Black Theatre 


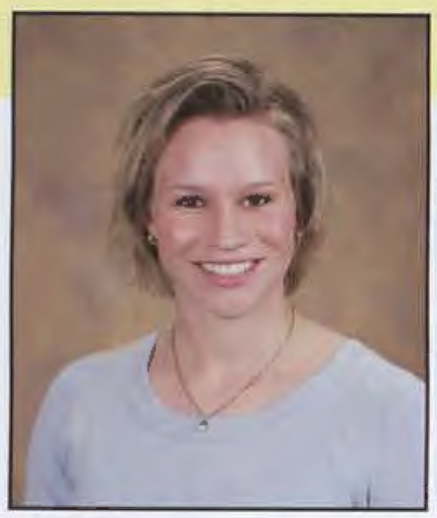

Kelly Blackburn Business Management

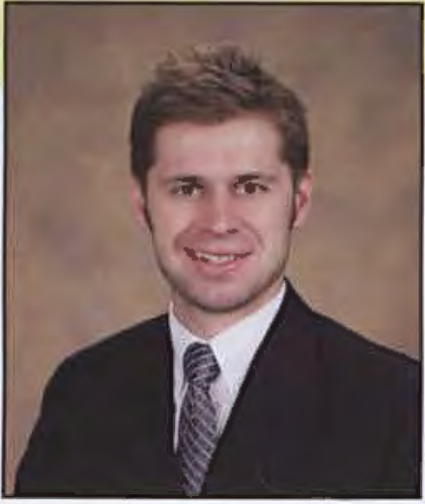

John Blake

Electrical Engineering

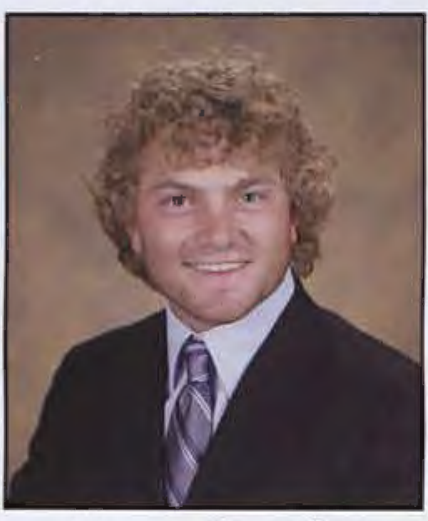

Trevor Bowman

Karissa Bollenbacher International Studies

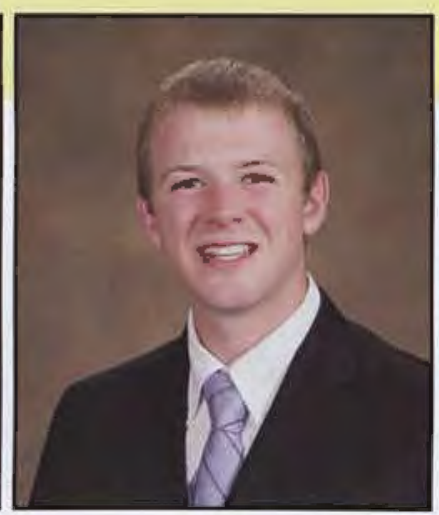

Hugh Blocker III Athletic Training

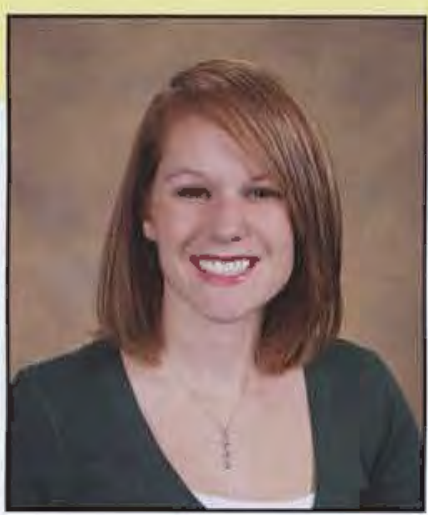

Heidi Bochman Social Work

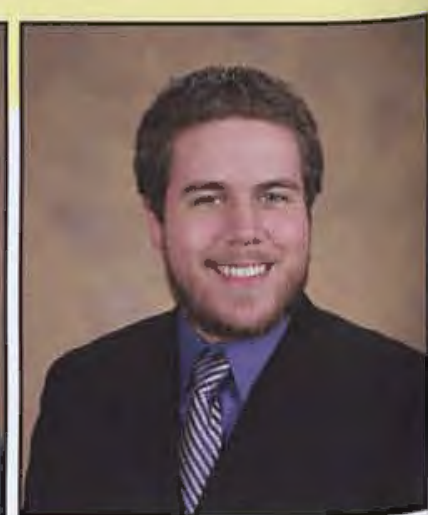

Joseph Bolander International Studies

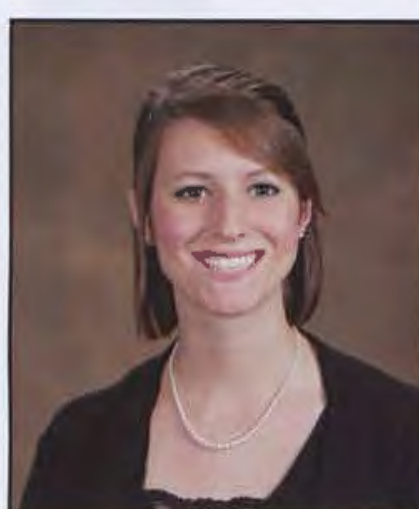

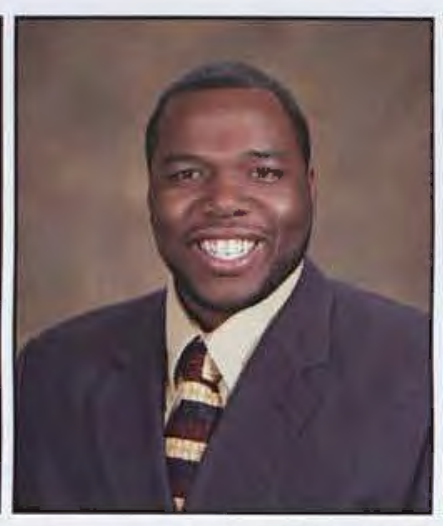

Roderick Bradley II Early Childhood Education

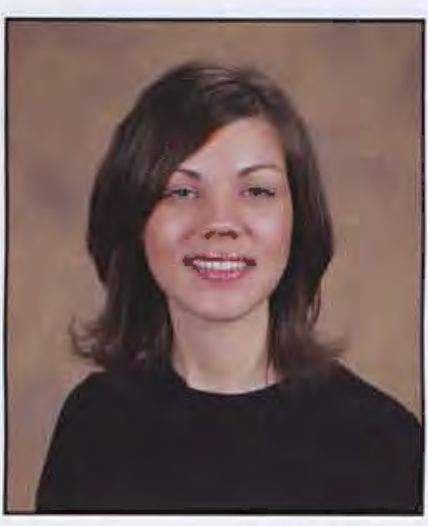

Bonnie Brady Applied Psychology

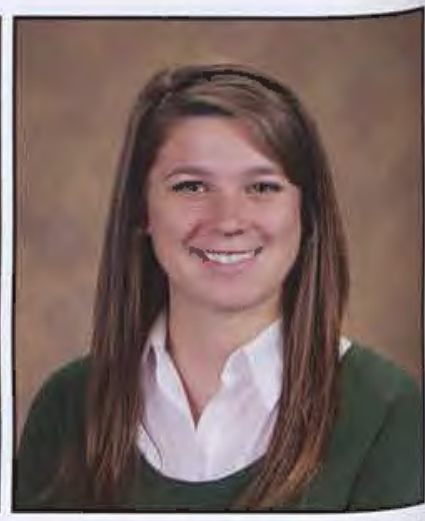

Amy Brand Special Education

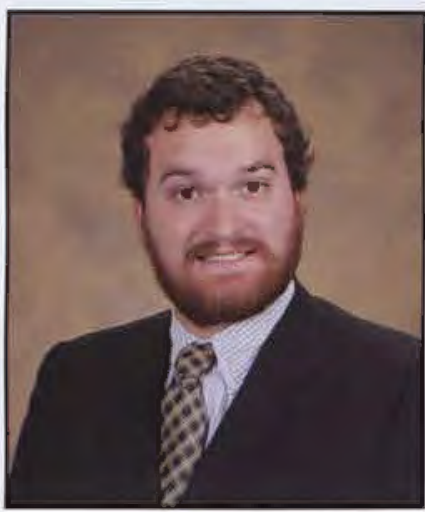

Mike Brandes Electronic Media

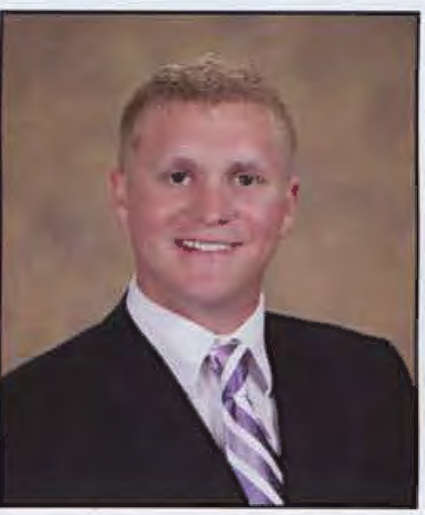

Peter Brock Athletic Training

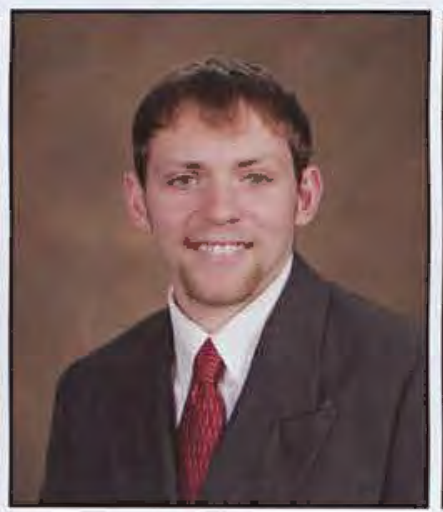

Judson Brooker Multi-Age Physical Education

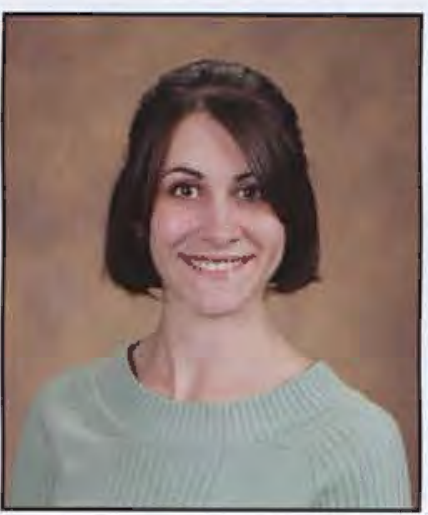

Anna Brooks

Nursing

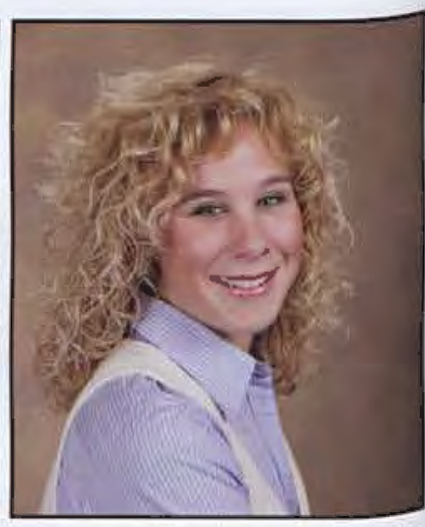

Janelle Brooks Integrated Social Studies Education

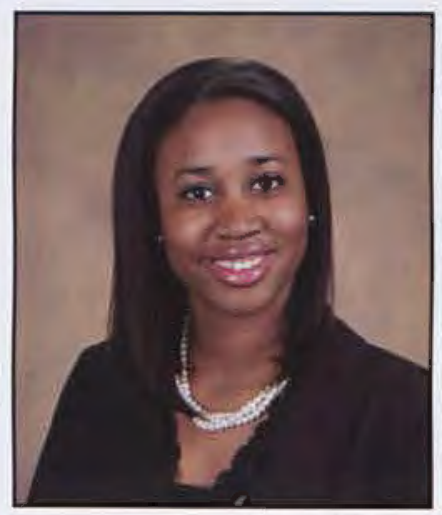

Alexis Brown Electronic Media

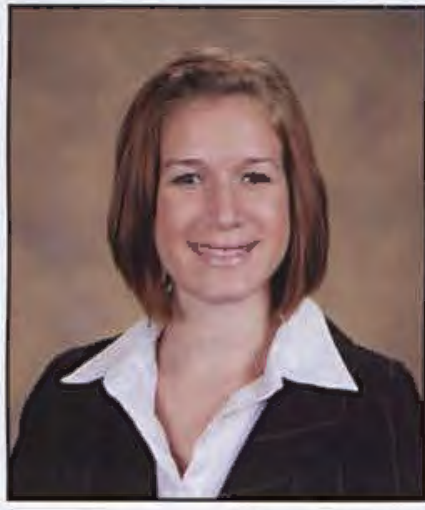

Gracia Brow

Church Music Ministries

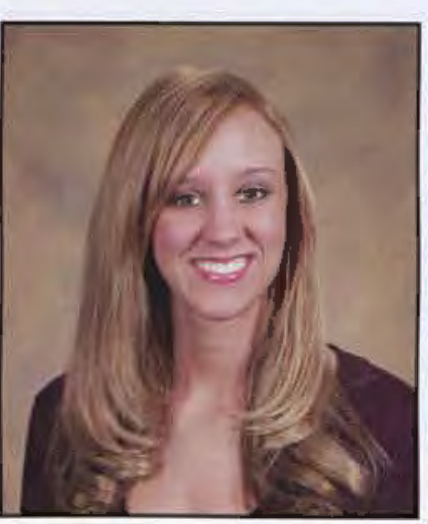

Lauren Brown Social Work

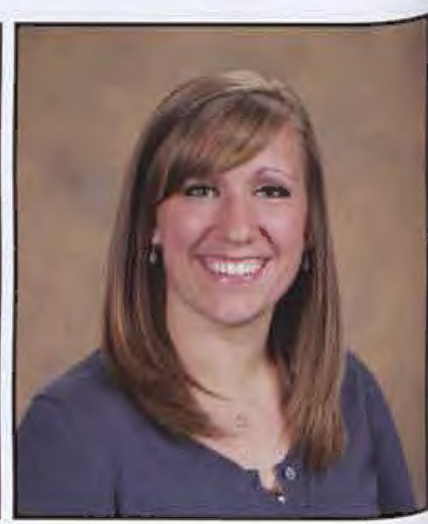

Reba Brown Nursing

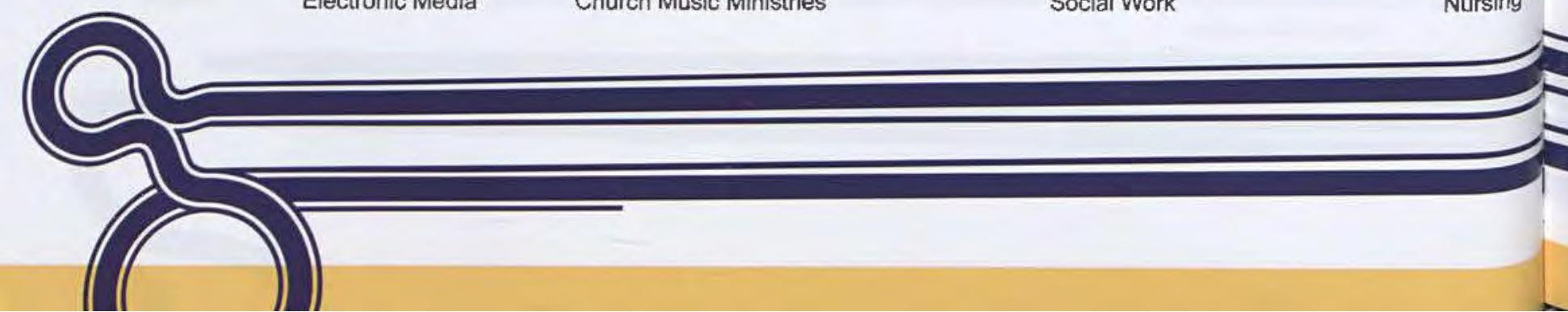




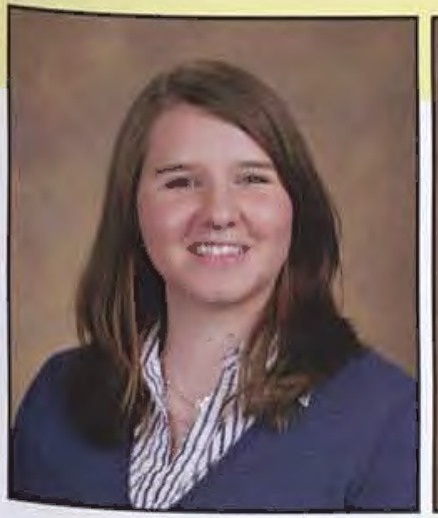

Kimberly Broesder Criminal Justice

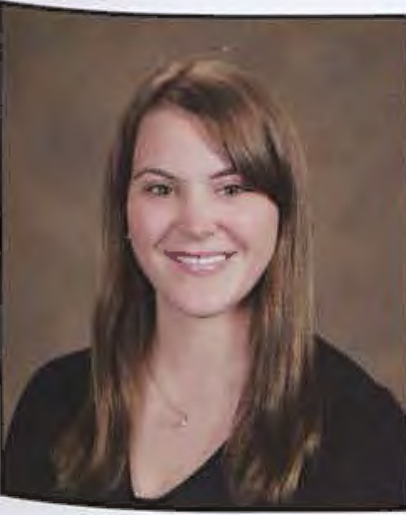

Mary Anne Caldwell

History

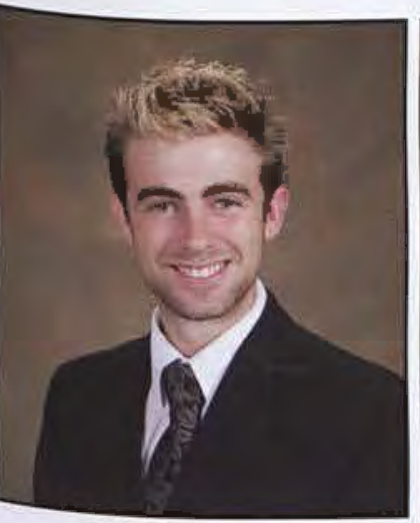

Jace Carter

Mechanical Engineering International Studies/Missiology

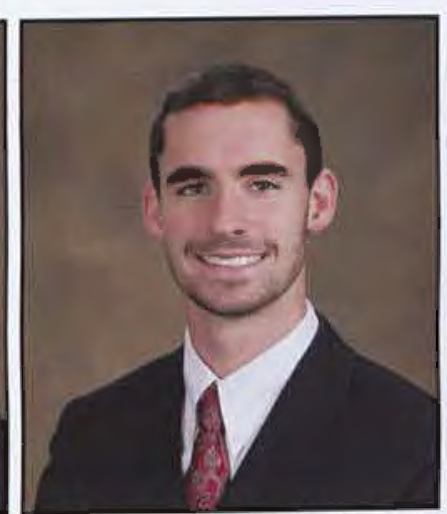
Accounting/Finance

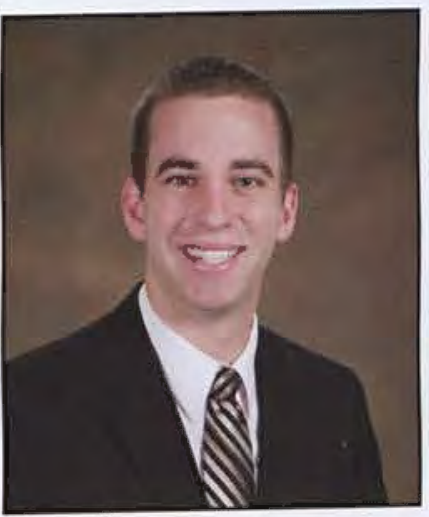

Thomas Cary
Seth Campbell

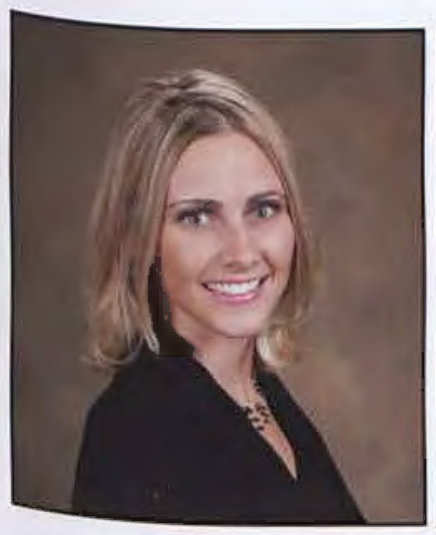

Sarah Cheatham Nursing

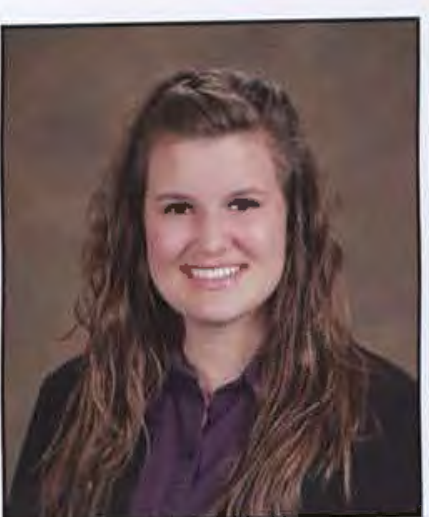

Melanie Cherland

Psychology Comprehensive Communication

So Mang Cho

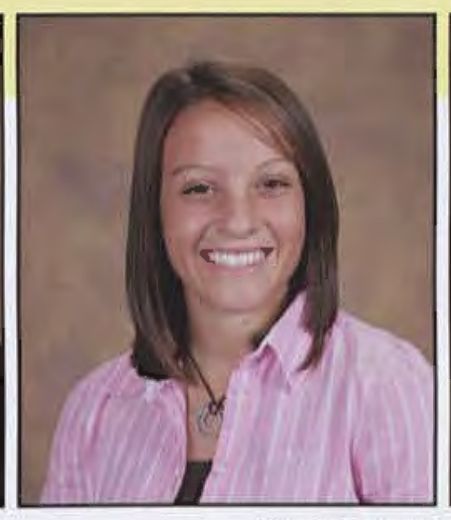

Lisa Burgman

Technical and Professional Communication

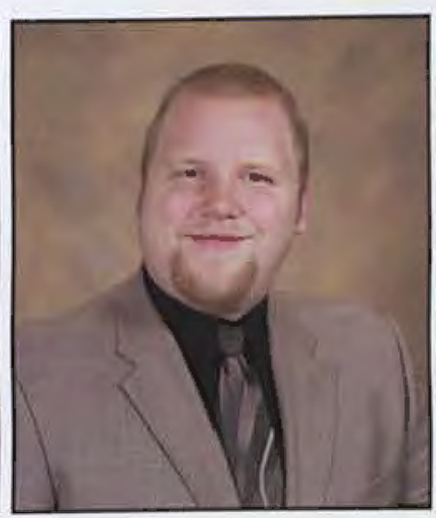

John Carey

Music Performance

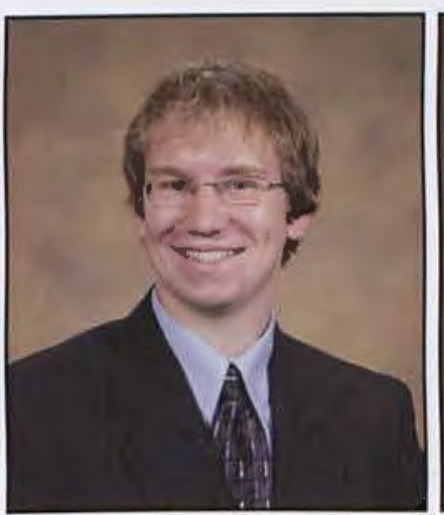

lan Capser Vocal Performance
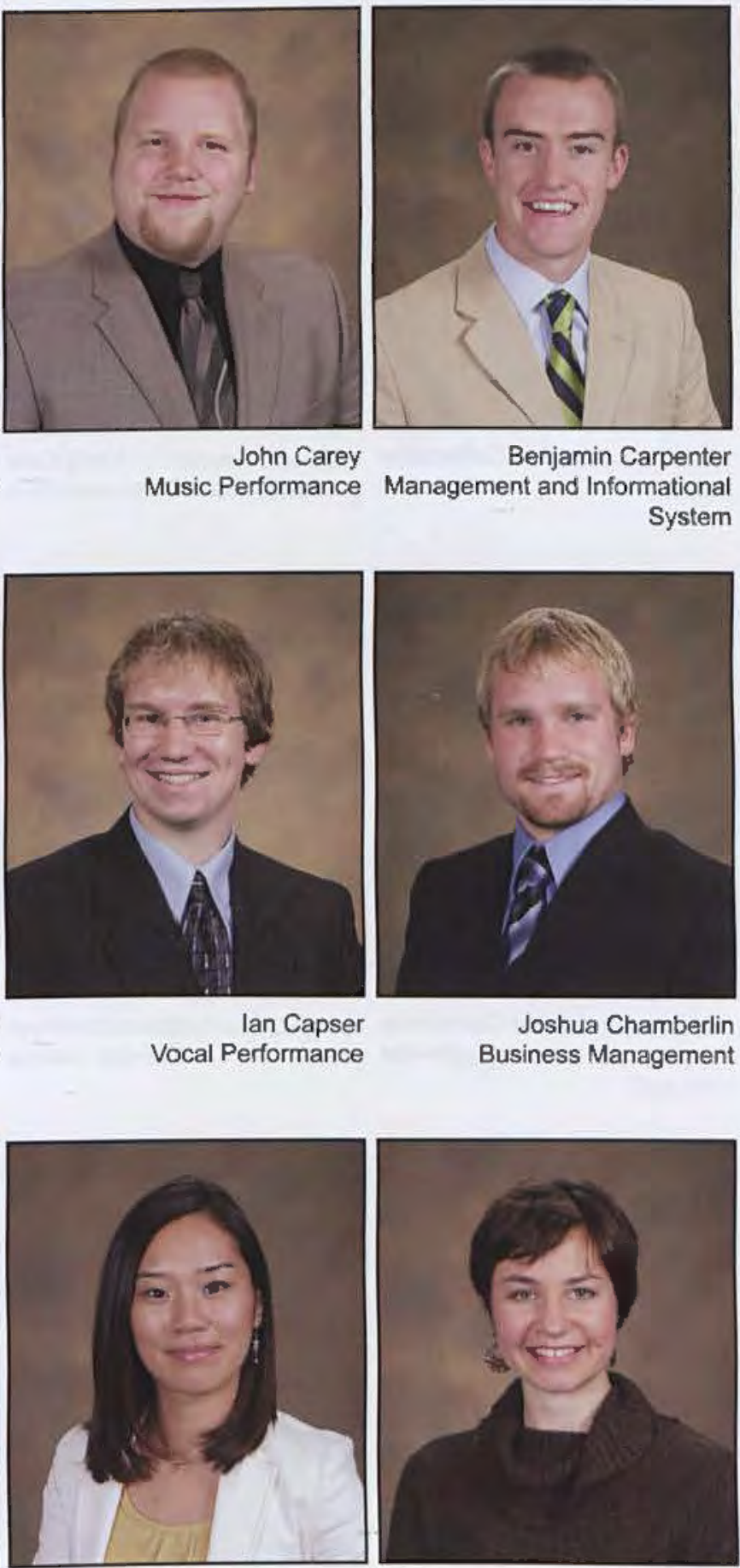

Benjamin Carpenter Management and Informational System

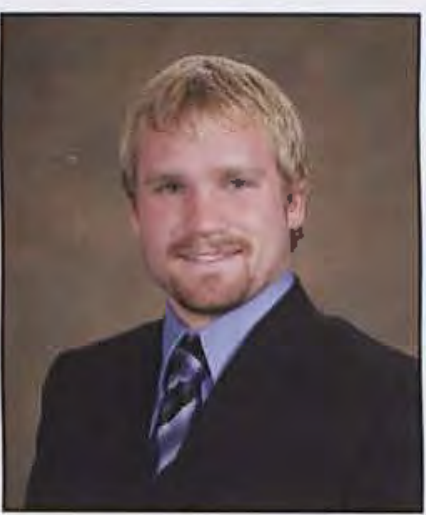

Joshua Chamberlin Business Management

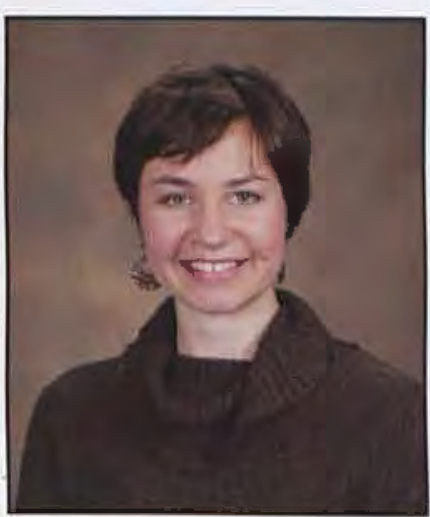

Heidi Christiansen Studio Art

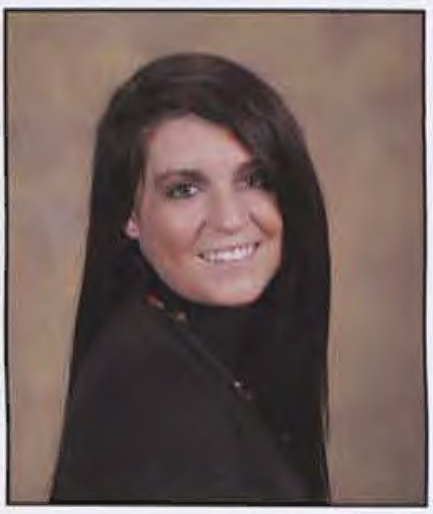

Ashley Carter Nursing

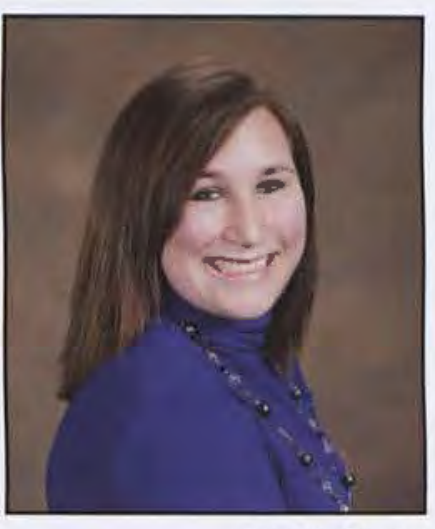

Cherith Chase Spanish

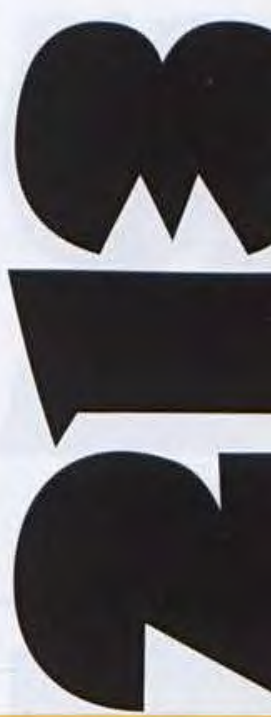




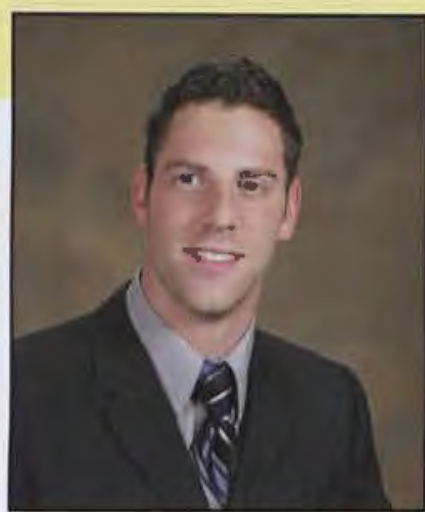

Nathan Ciolek Electrical Engineering

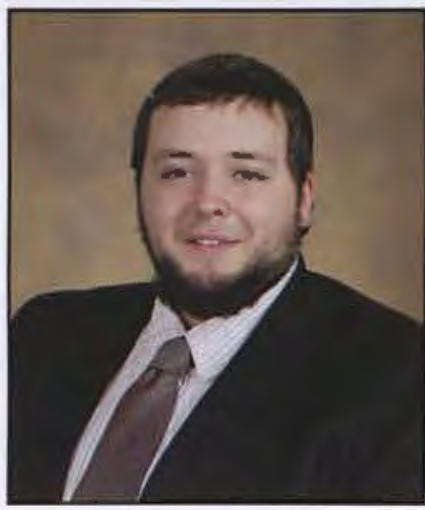

Timothy Cochran Jr. Youth Ministries Christian Education

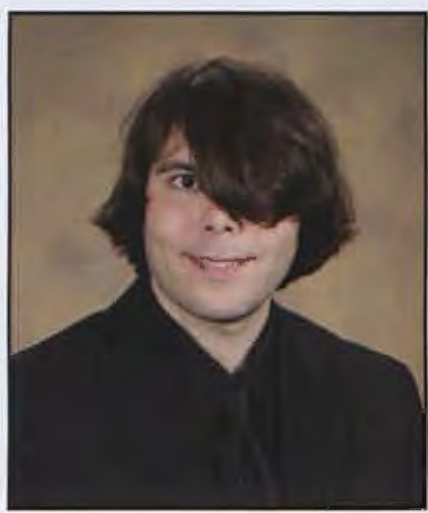

James Colton Graphic Design

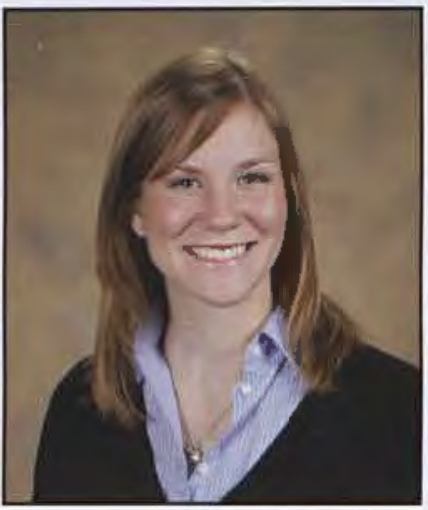

Kari Coffindaffer Marketing

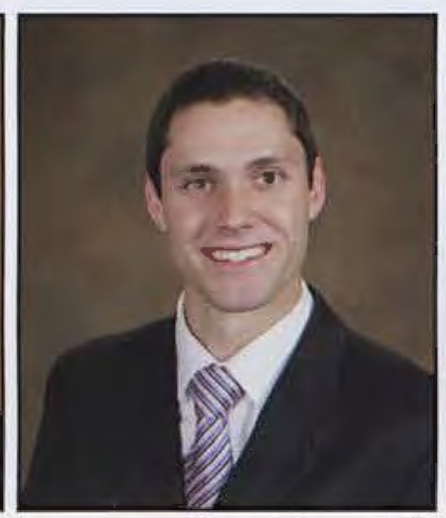

Everett Compitello

Pre-law

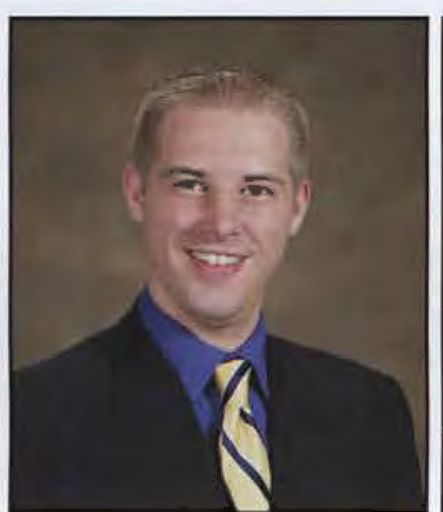

Shaun Cookson Criminal Justice

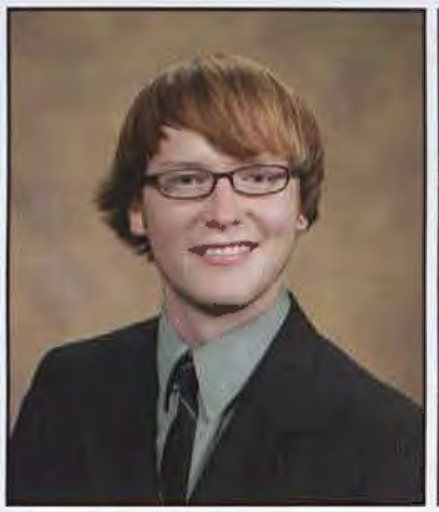

Kraig Cole Intercultural Communication

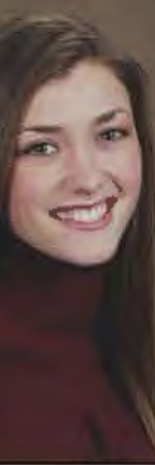

Kaydrie Clymer Music

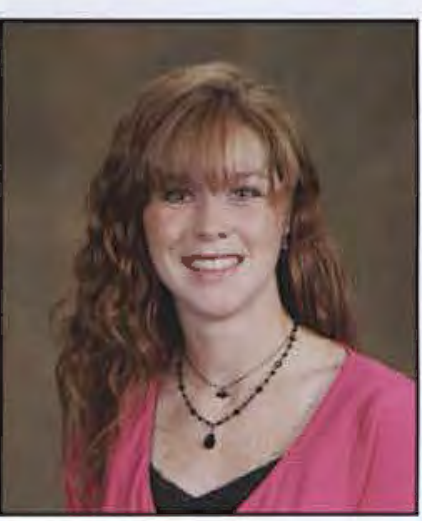

Sarah Coleman Early Childhood Education

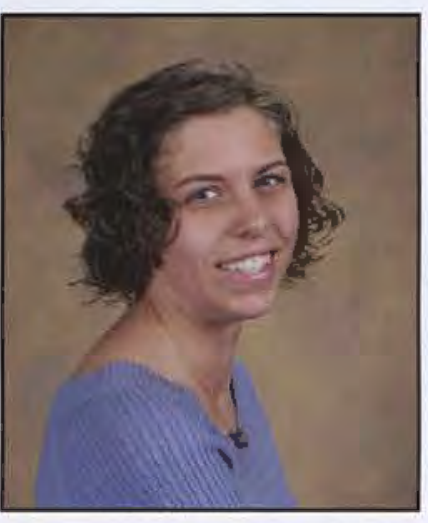

Laura Cornell Early Childhood Education

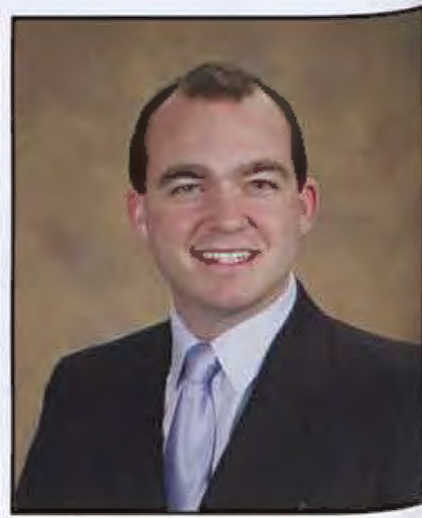

Andrew Costerisan Biology

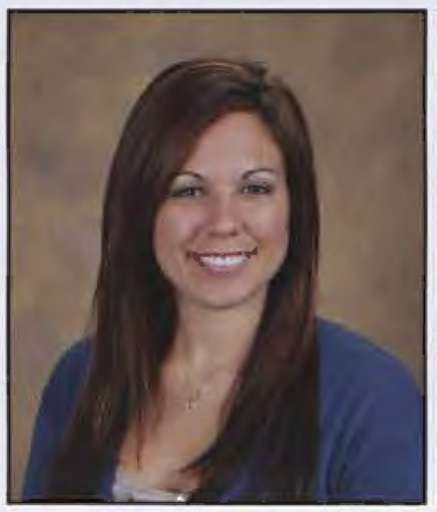

Bethany Cottings Biology

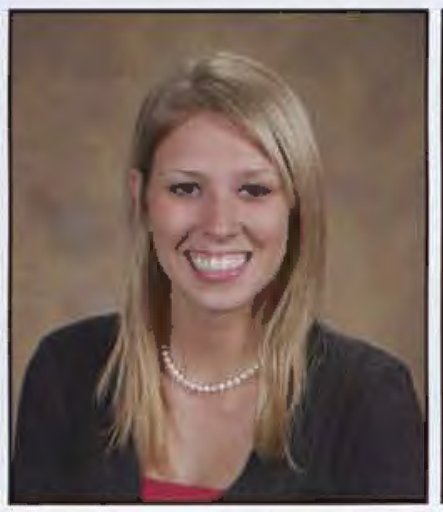

Laura Cover Sociology

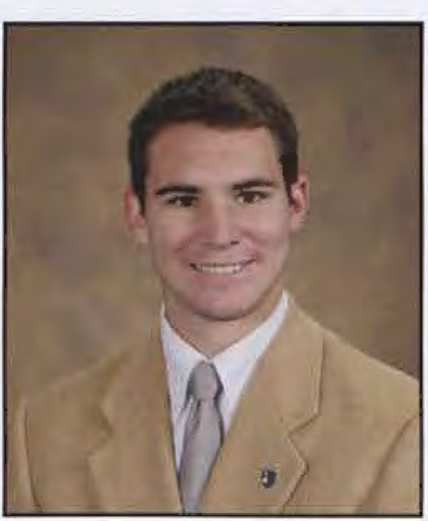

Timothy Cox International Studies-Business

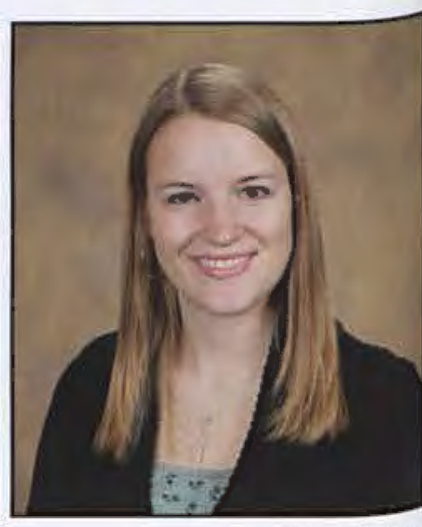

Sara Craig Keyboard Pedagogy
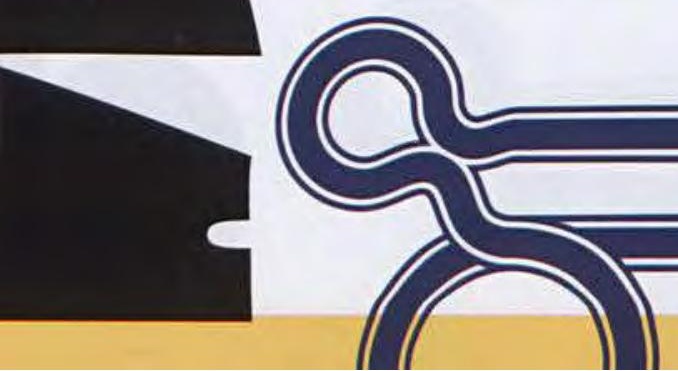


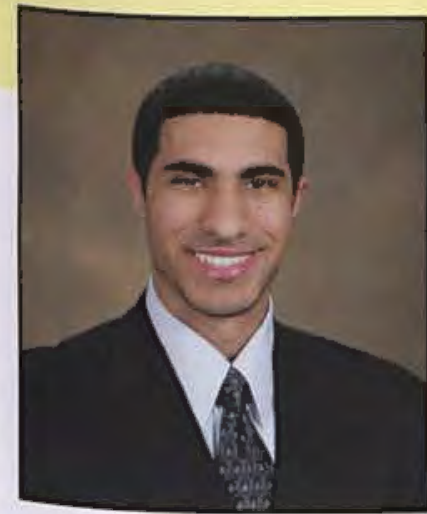

Scott Crawford

Mechanical Engineering

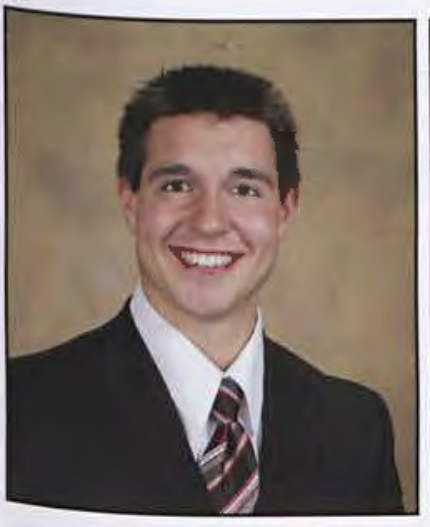

Benjamin Curtis Pre-Seminary

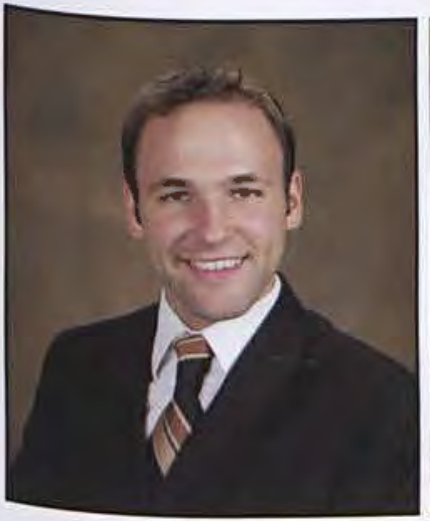

Andrew Davis History/Political Science

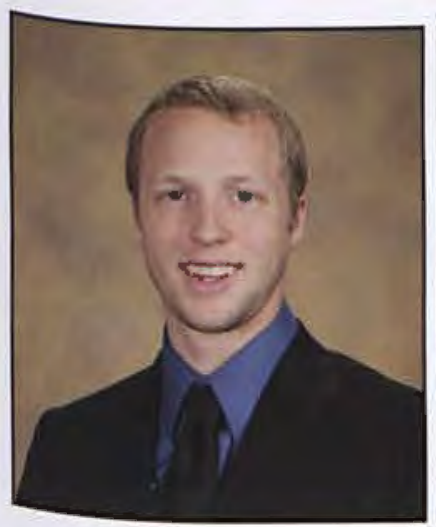

Samuel DeLong History

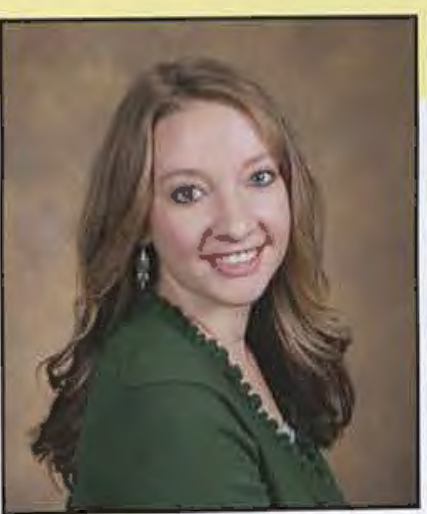

Sarah Crichlow Biology

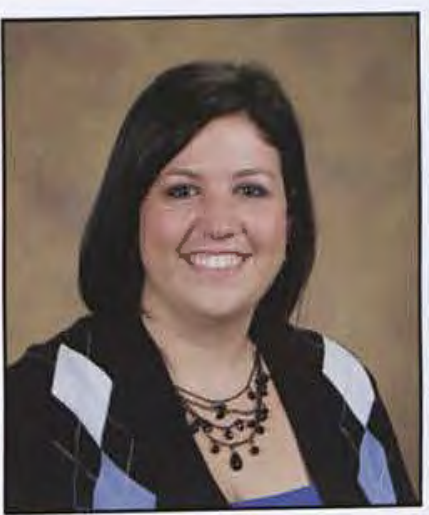

Amanda Cyrus

Social Work

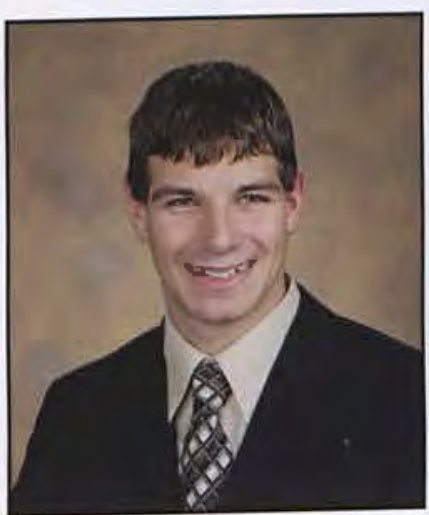

Dan Davison American Studies

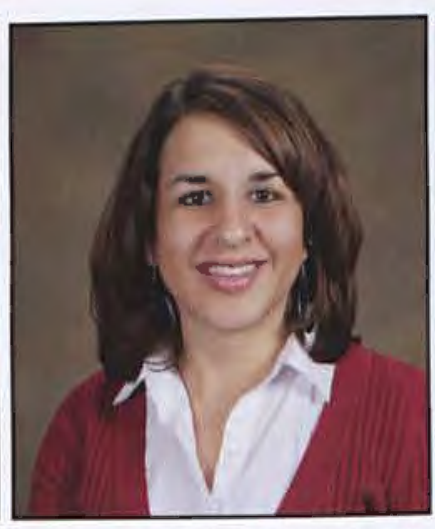

Elizabeth Dennis Nursing

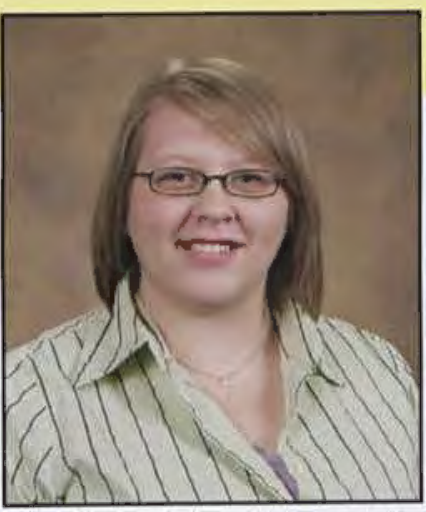

Lisa Crowder

Organizational Communication

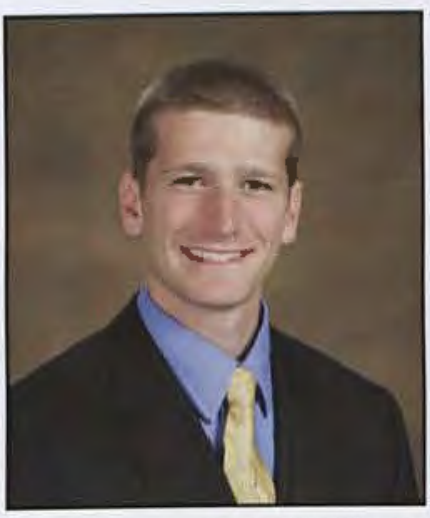

Robert Davidson Accounting

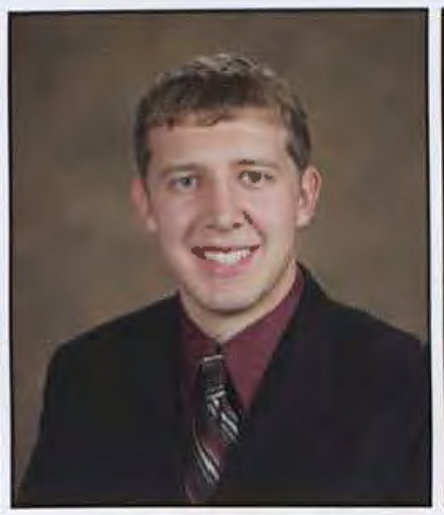

Matthew Dearden History

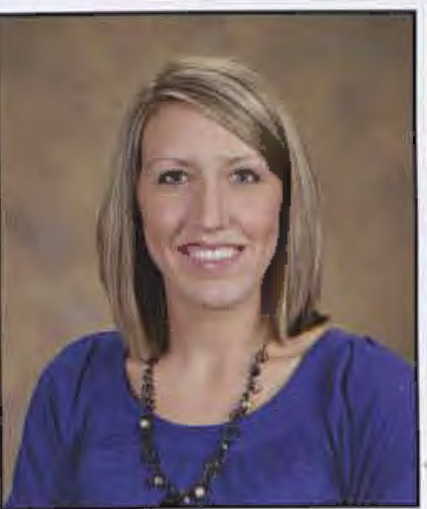

Shannon DeRusha Nursing

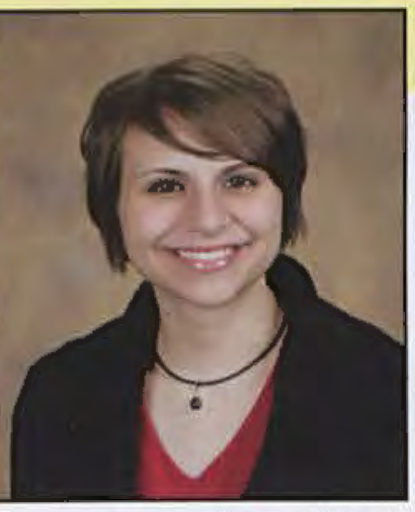

Renee Cuenin English

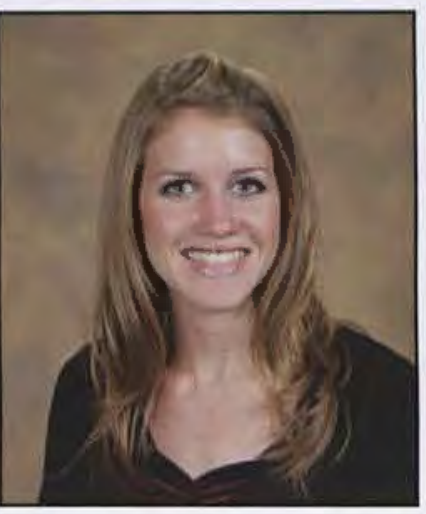

Bethany Davies Nursing

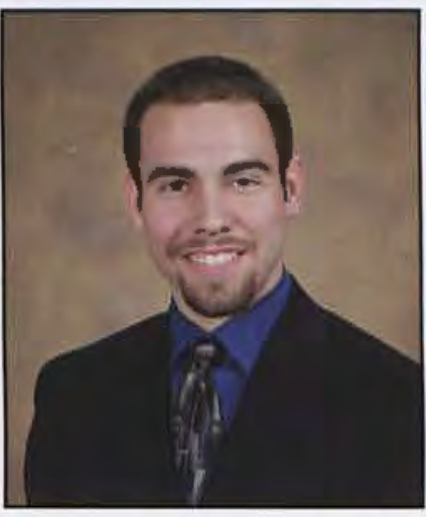

Jonathan Decker Integrated Social Sicience Education

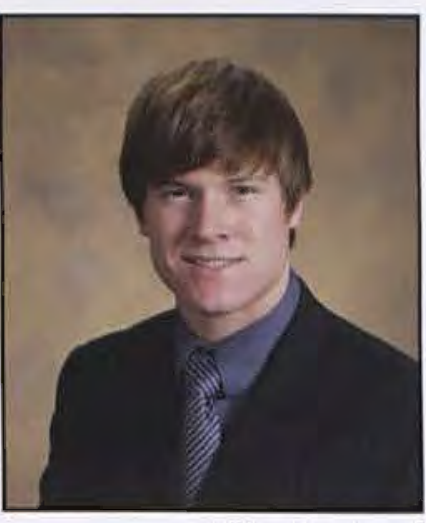

Tobias Dewhurst Mechanical Engineering

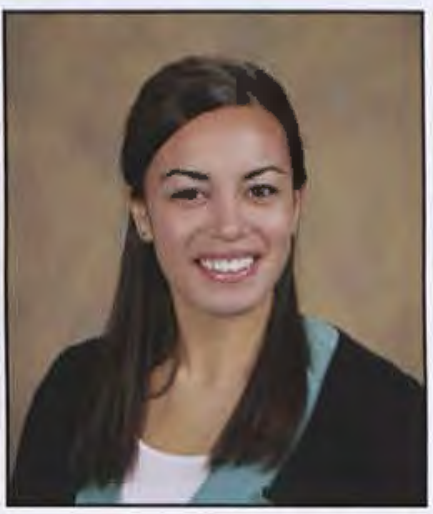

Cynthia Davies Psychology

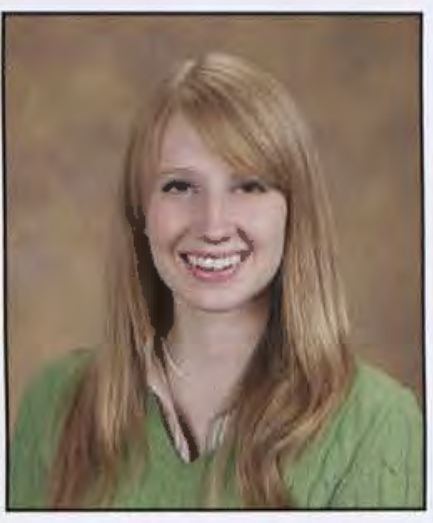

Leslie DeLong Middle Childhood Education 


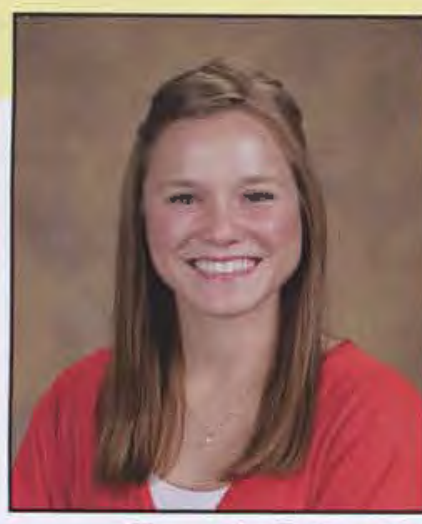

Cassandra Dobutovich Social Work

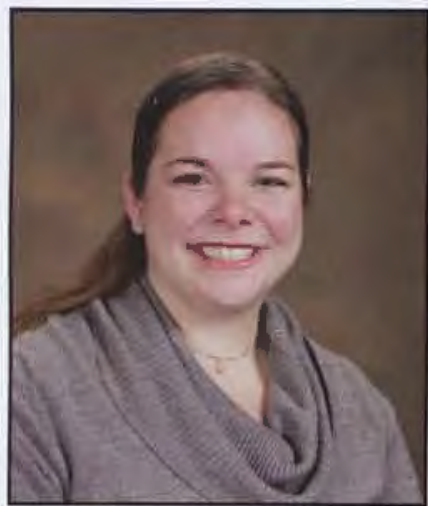

Emily Driscoll

Theatre

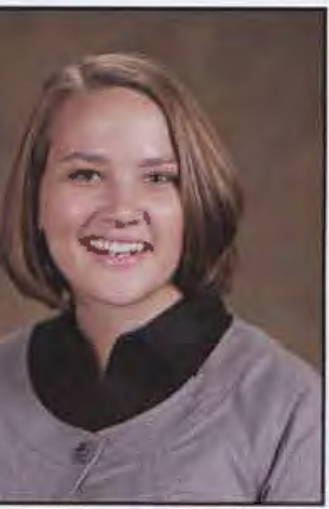

Bethany Duncan Social Work

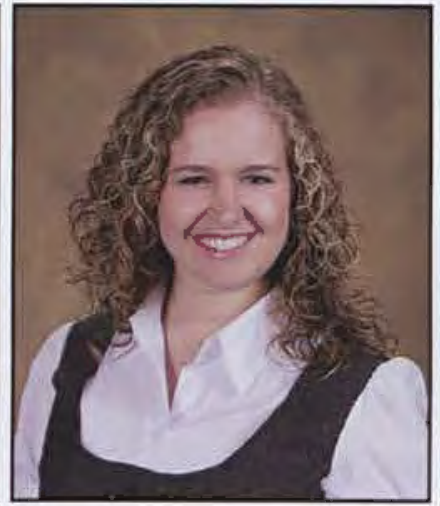

Kaitlin Dunnevan Integrated Language Arts Education

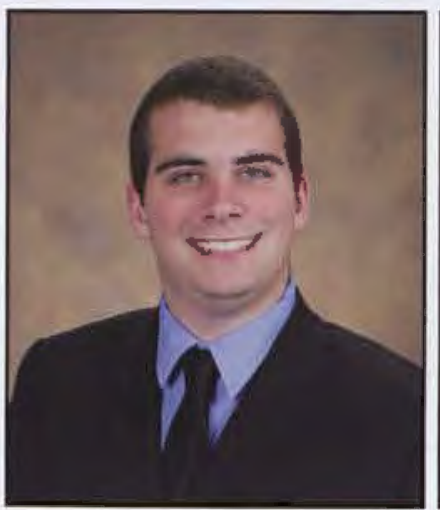

Matthew Eberle

Accounting

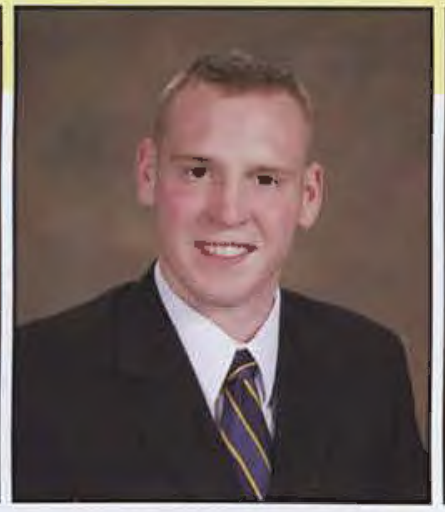

Benjamin Donaldson Criminal Justice

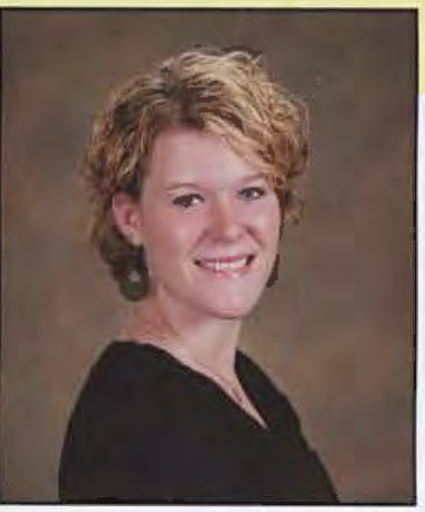

Ericka Donaldson Biology/Pre-Med

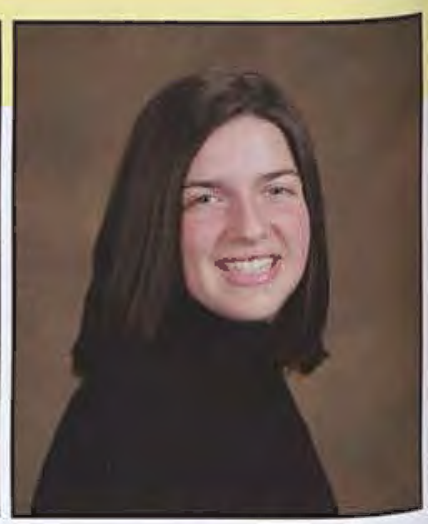

Abby Douglas Middle Childhood Education

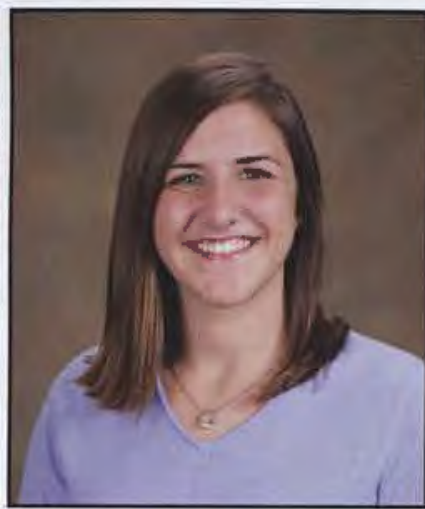

Rebekah Drum Biology/ Pre-Physical Therapy

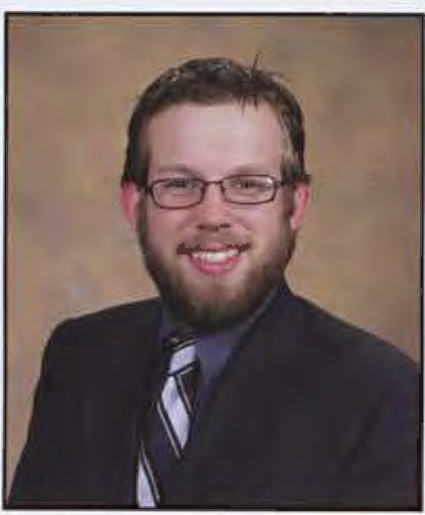

John Dunning Youth Ministryl Christian Education

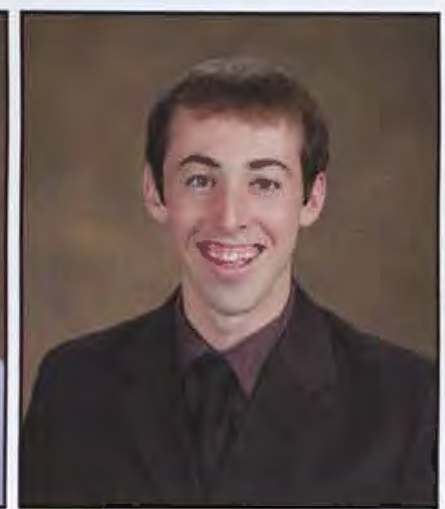

David Duarte Marketing

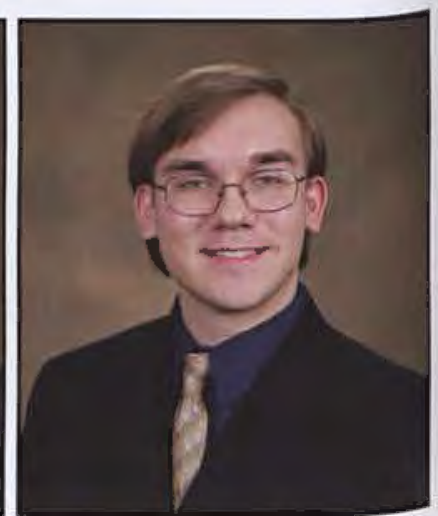

Ezra Dulis Electronic Media

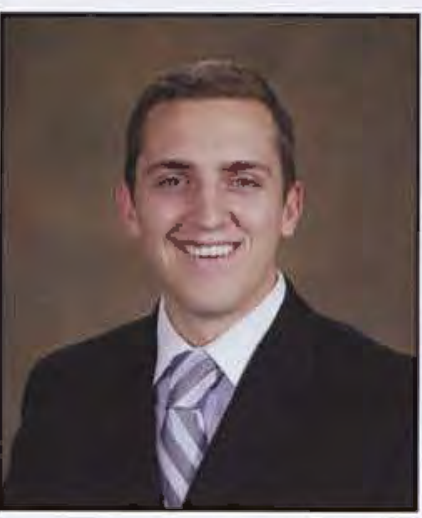

Alexander Durbin Finance

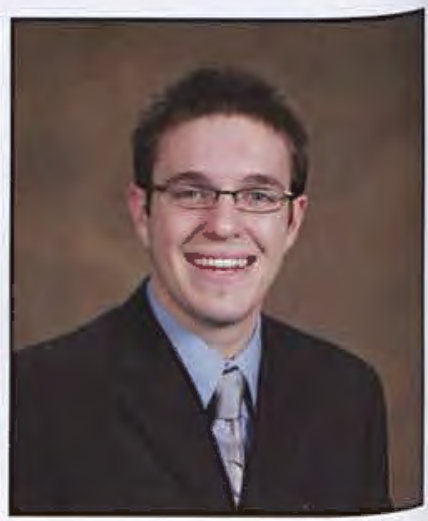

Christopher Earls Comprehensive Communication

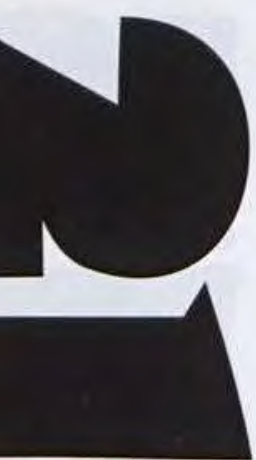

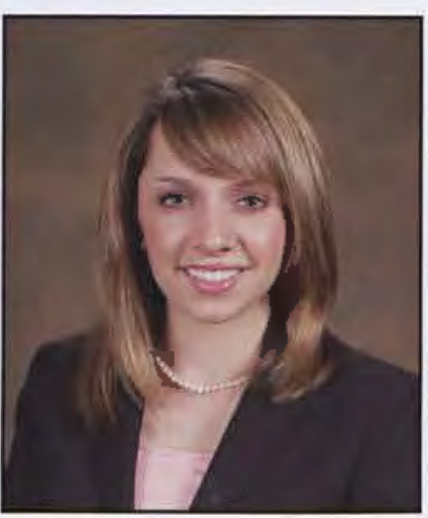

Courtney Edmonds History

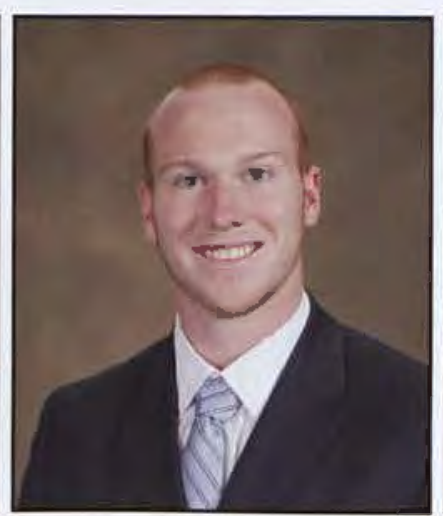

Kaleb Eldridge Technical and Professional Communication

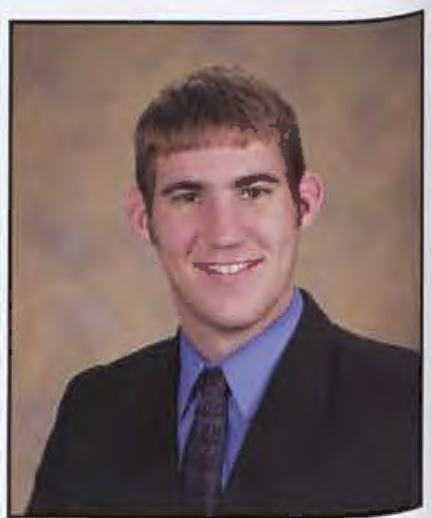

James Elgersma Mechanical Engineering

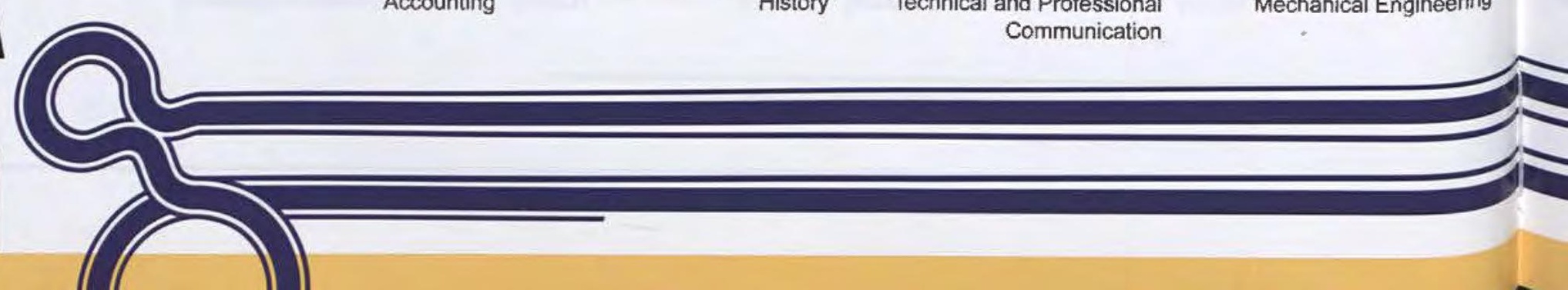




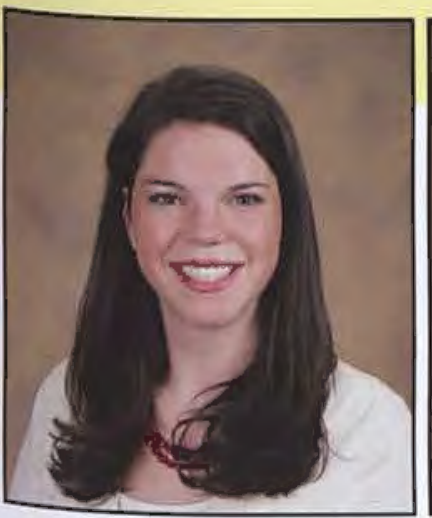

Ashley Elliot

Early Childhood Education

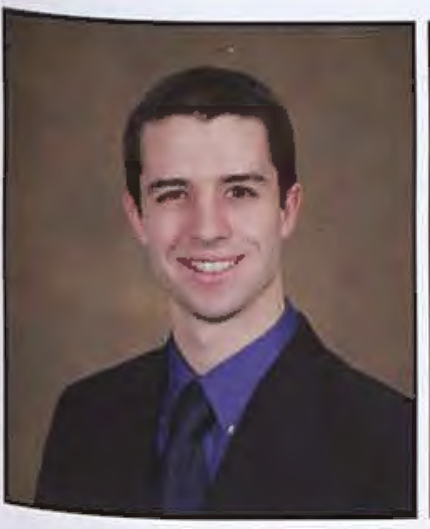

Austin Enge Youth Ministry/ Christian Education

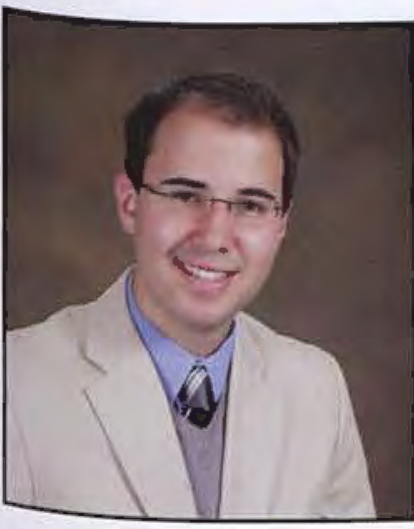

Adam Evans

Technical and Professional

Communication/Spanish

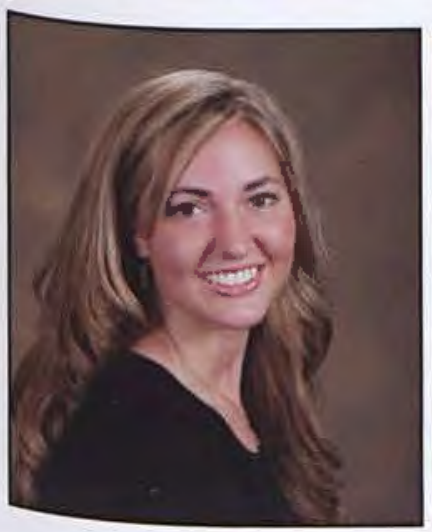

Kristen Ferguson Biology

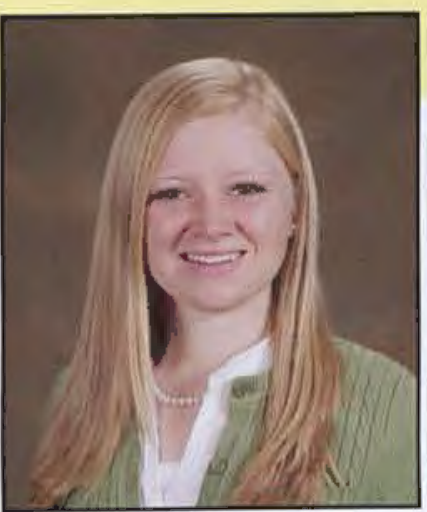

Camaren Elliott History

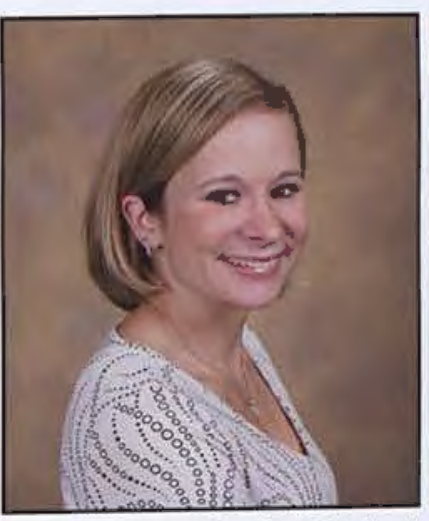

Jessica England Business Administration/ Accounting

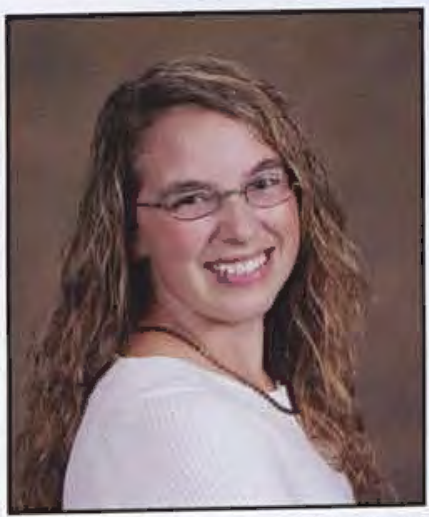

Lacy Ewers Special Education and Early Childhood Education

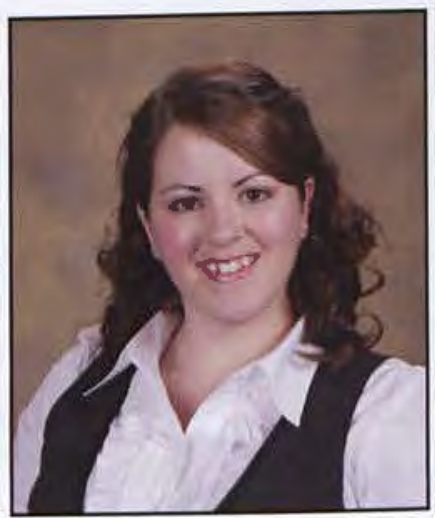

Talitha Ferrell Nursing

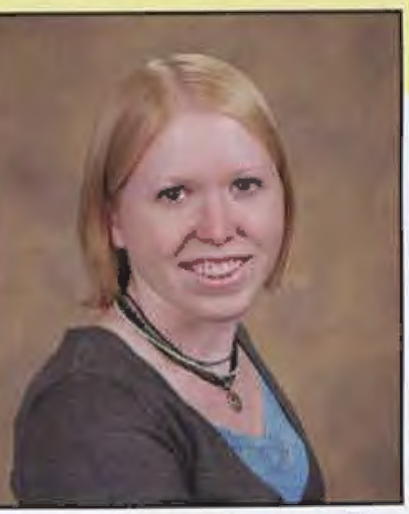

Kimberly Elliott English

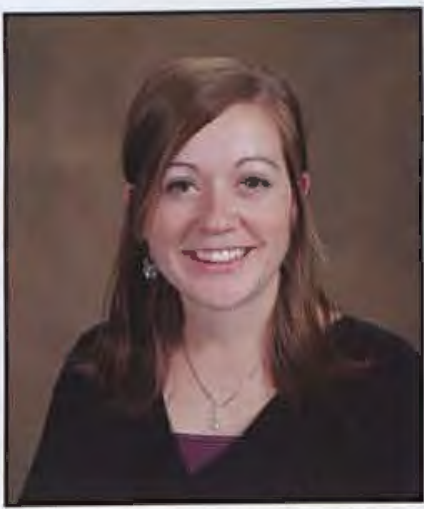

Kristen Ensminger Early Childhood Education

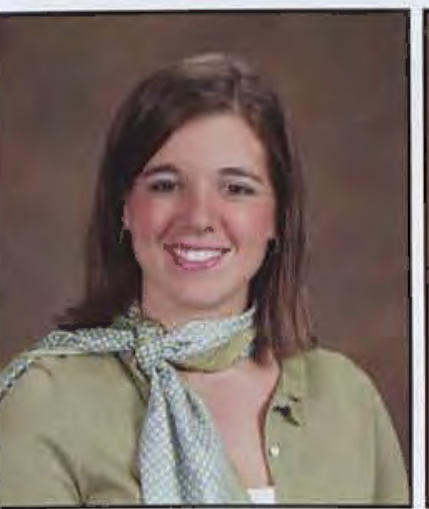

Dea Fallin Nursing

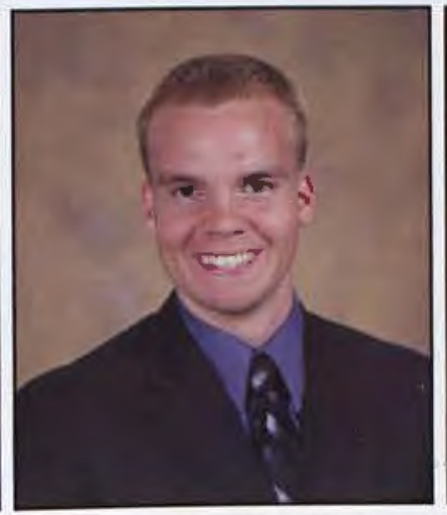

Jonathan Fleetwood

Early Childhood Education

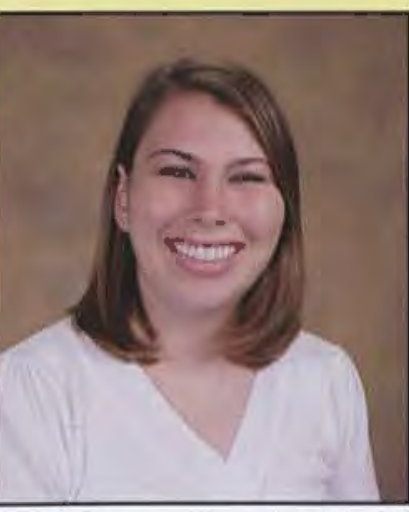

Jennalee Elmore Biology/Pre-Med

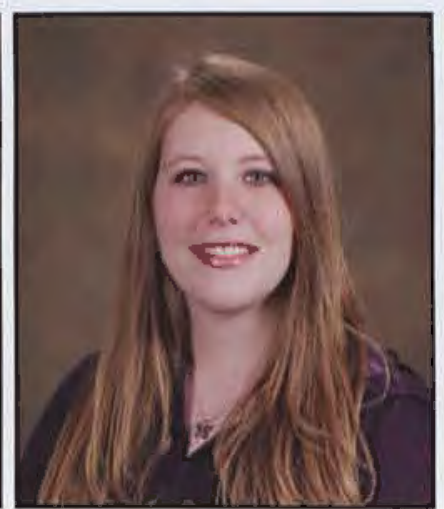

Jenna Erlandson Nursing

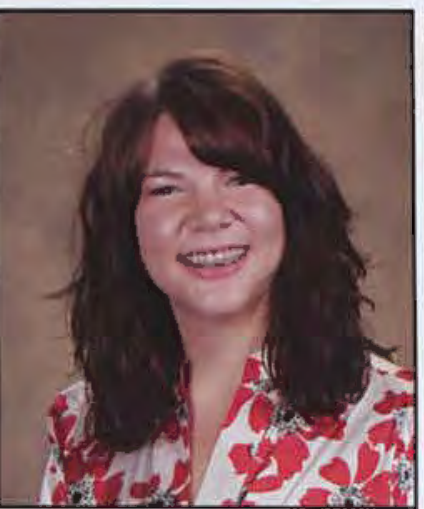

Megan Fansier

Nursing

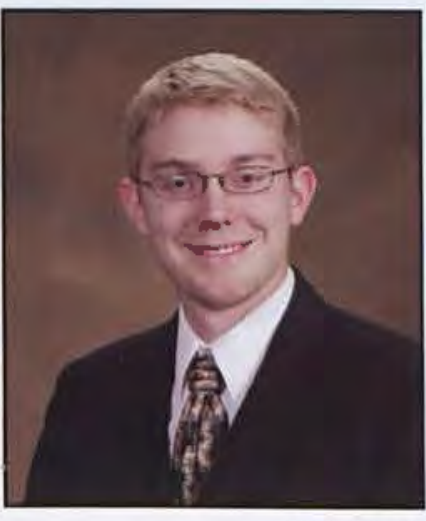

Matt Focht Graphic Design

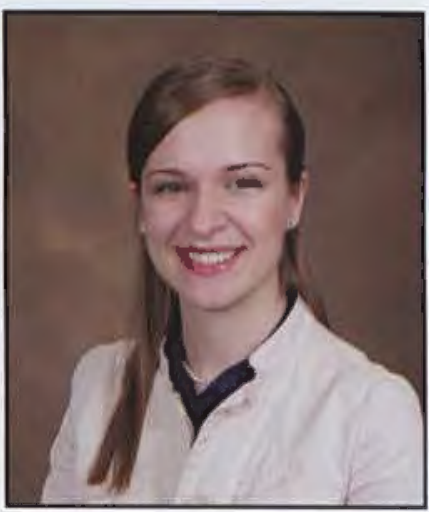

Sarah Erlandson Keyboard Pedagogy
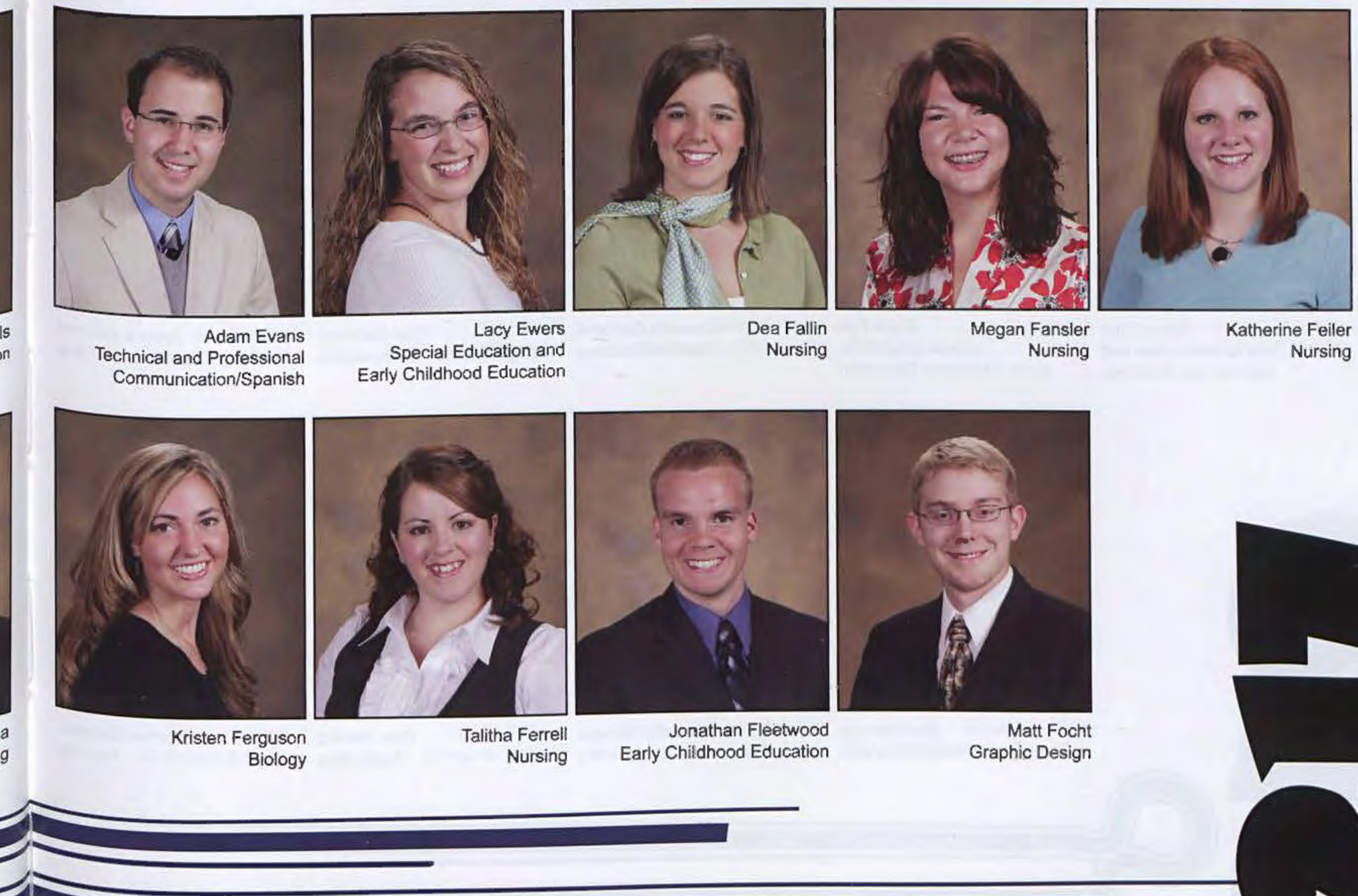


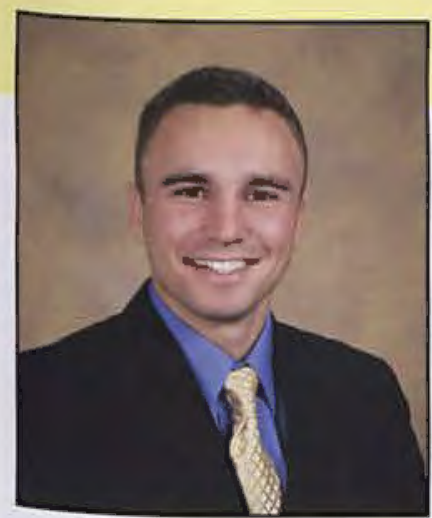

Benjamin Giglio Criminal Justice

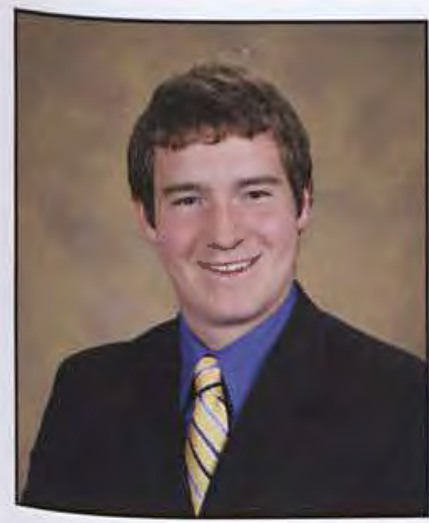

Daniel Godinez Management/Finance

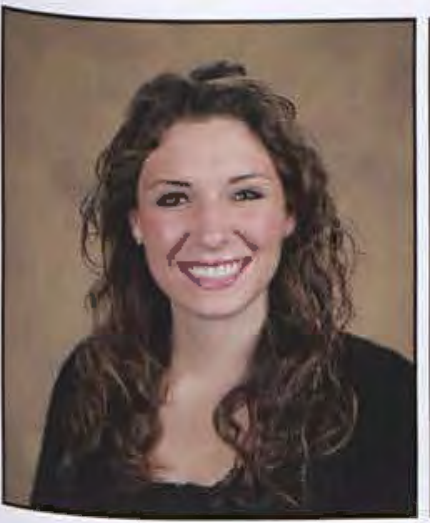

Jennifer Grant Nursing

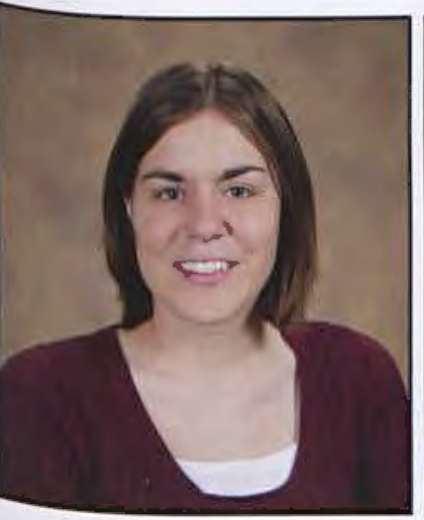

Amy Greenfield Chemistry Education

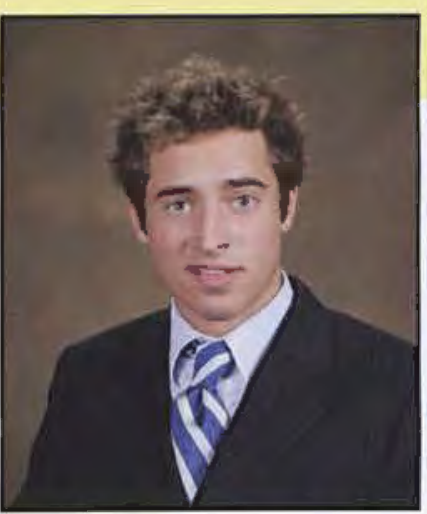

Joshua Gigliotti History/Political Science

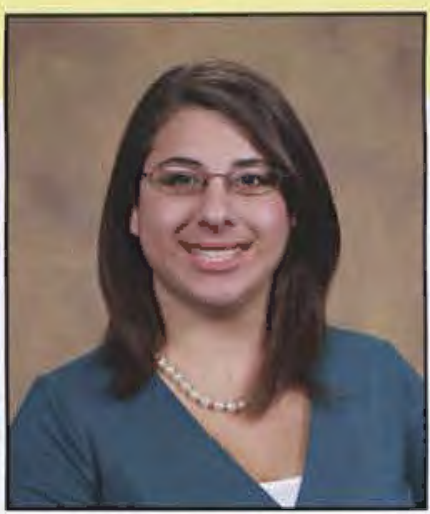

Serenity Gingrich Pre-Law/Public Administration

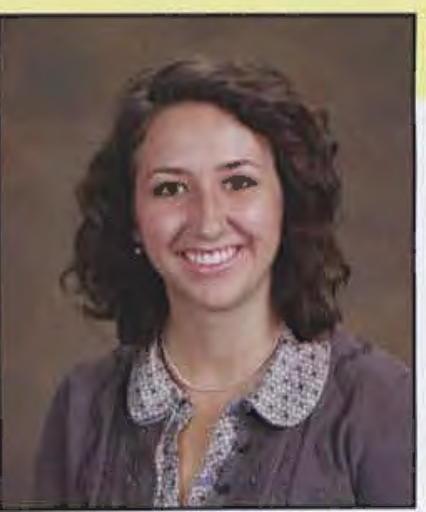

Ashley Gleichauf Social Work

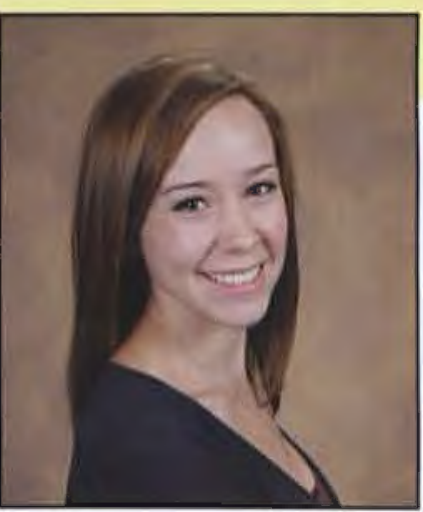

Sarah Gneiser Studio Art

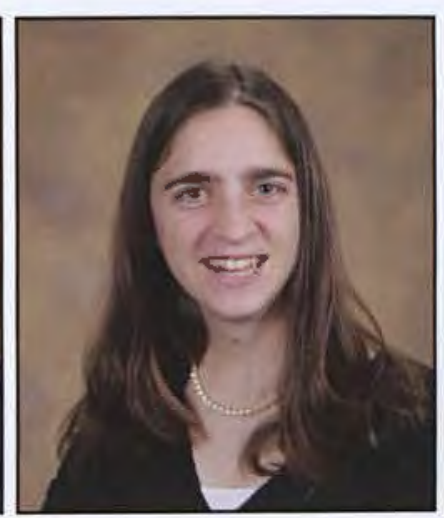

Terri Goodman Social Work

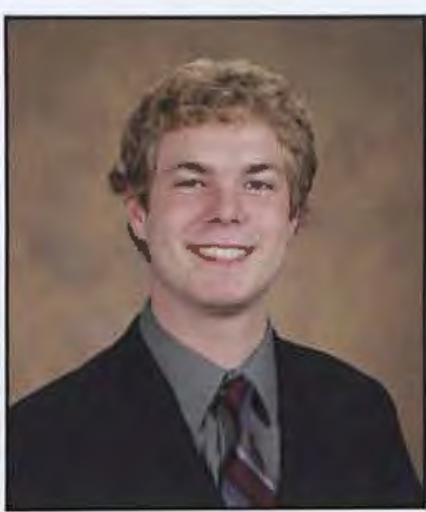

Jonathan Gorrell Criminal Justice

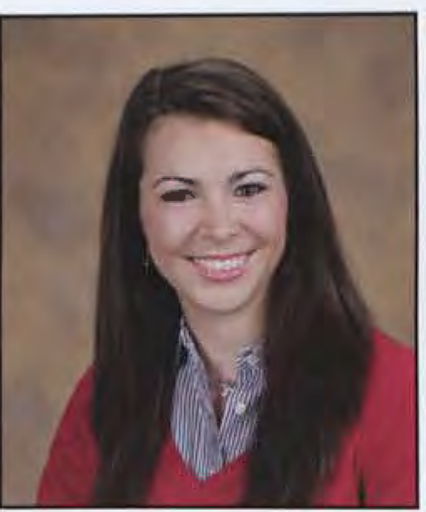

Emilie Gouch Accounting

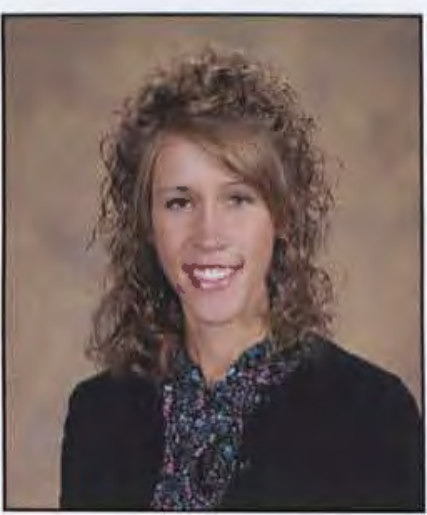

Michelle Graeff Biology/Pre-Med

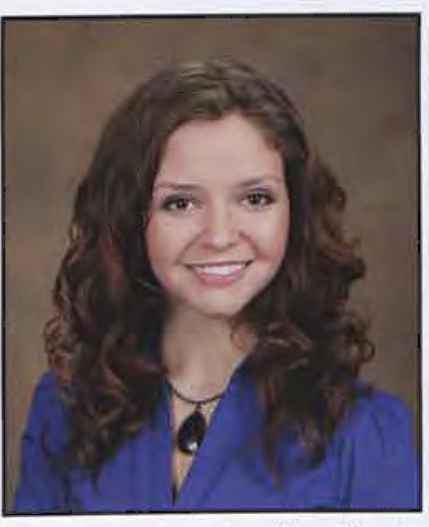

Amy Gredy Business Management

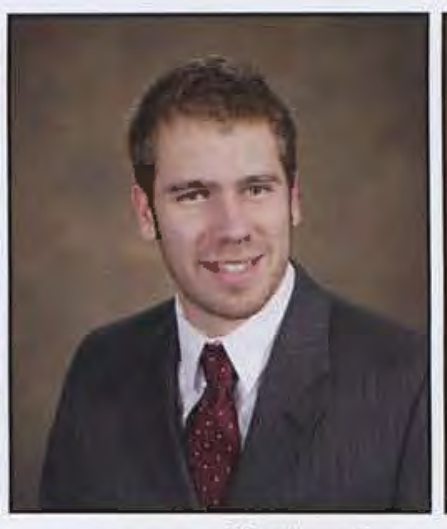

Matthew Green Mechanical Engineering

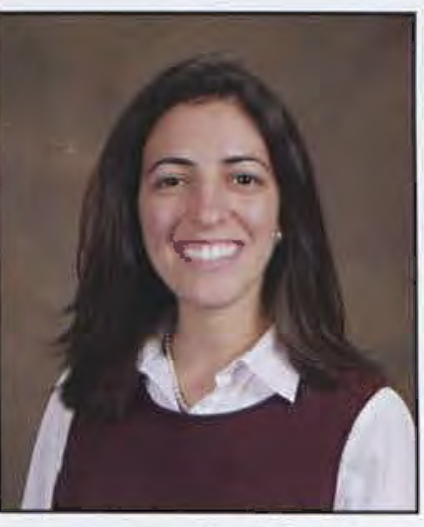

Leah Greenberg Nursing

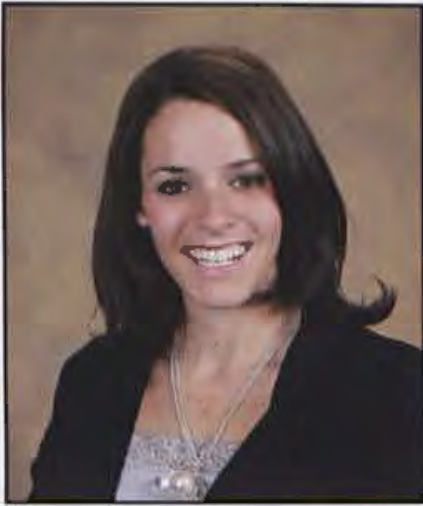

Sarah Greenberg Nursing

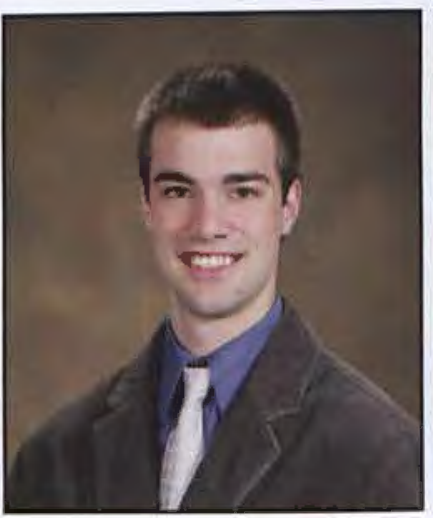

Gabriel Greve Computer Science

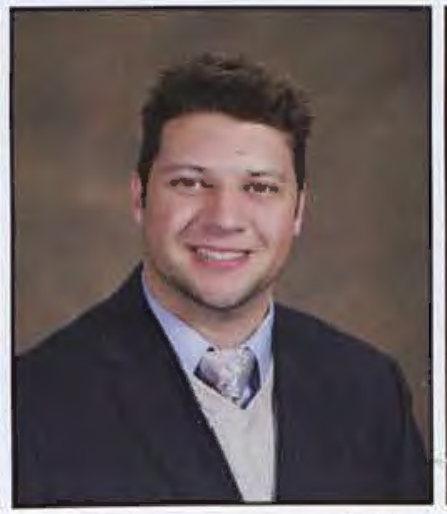

Joshua Griffith Music

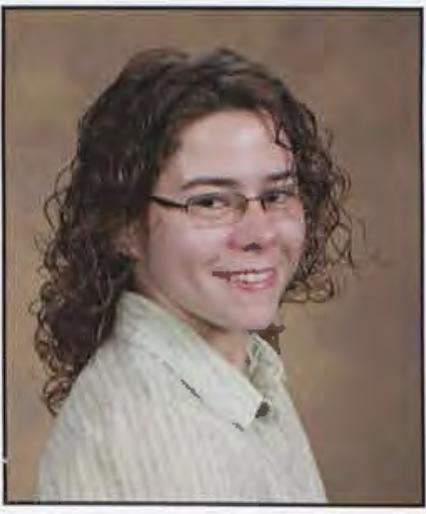

Kelsey Griswold Early Childhood Education

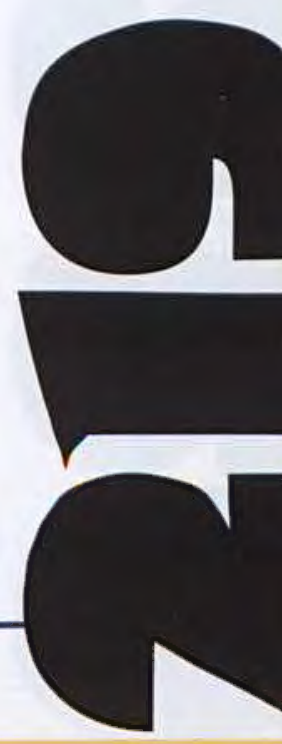




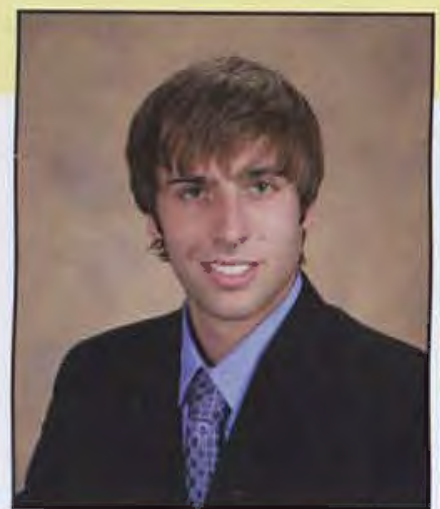

Adam Groff

Organizational Communication

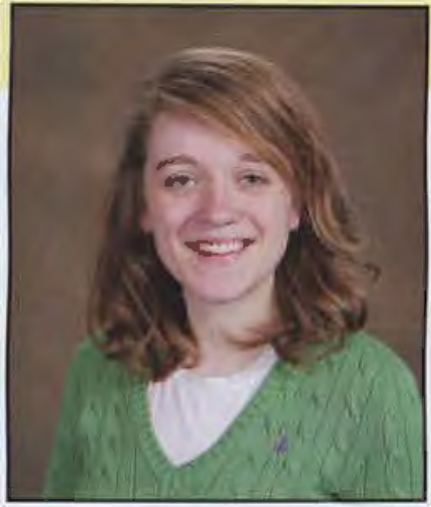

Kathleen Guenther

English

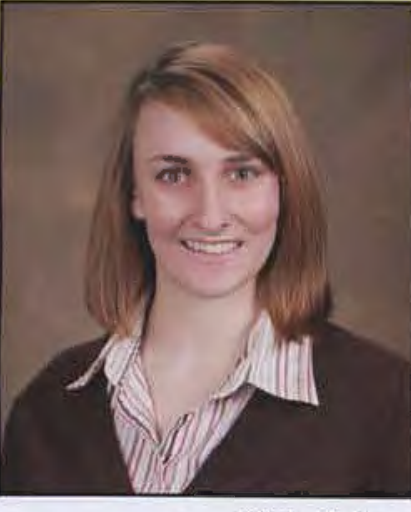

Krista Haine

Technical and Professional Communication

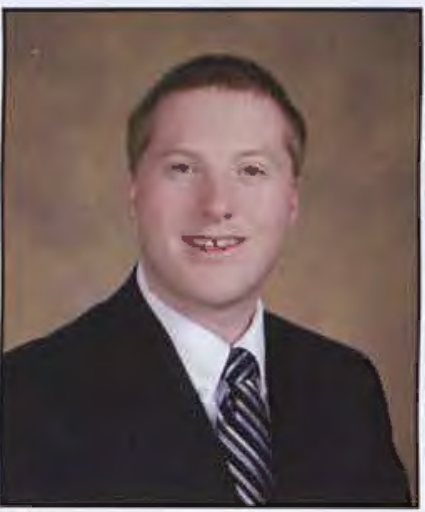

John Hamilton Electrical Engineering

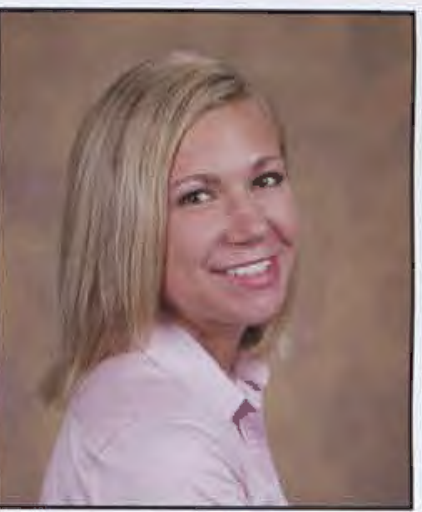

Kristin Hamminga Nursing

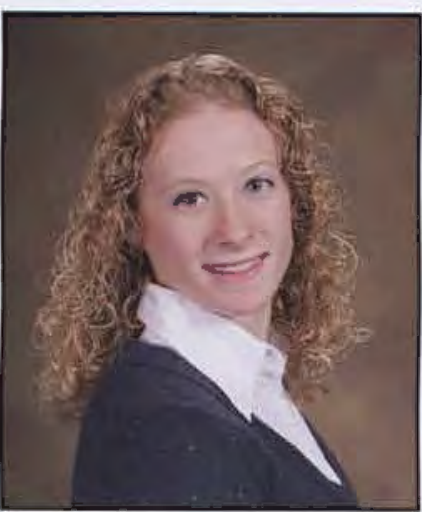

Maija Hampton Social Work

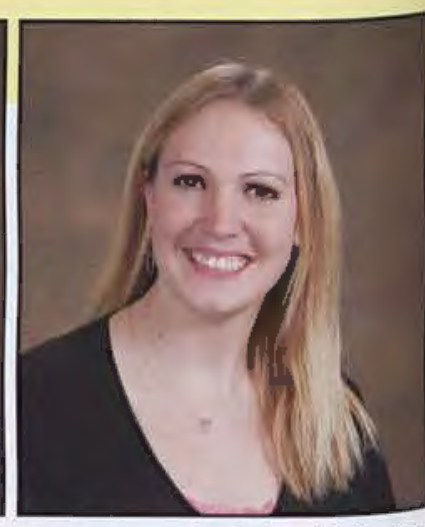

Laura Halladay Andrew Hall
Youth Ministry Sports and Exercise Studies

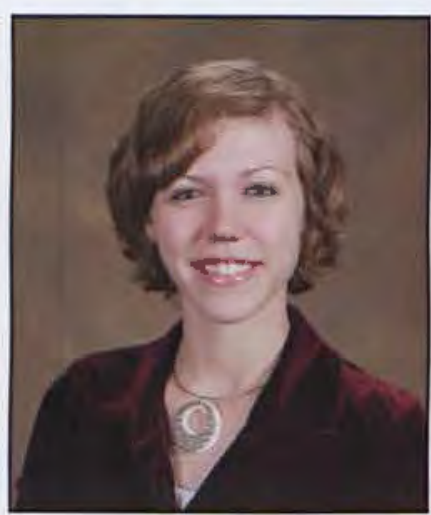

Charity Hancock Integrated Language Arts Education

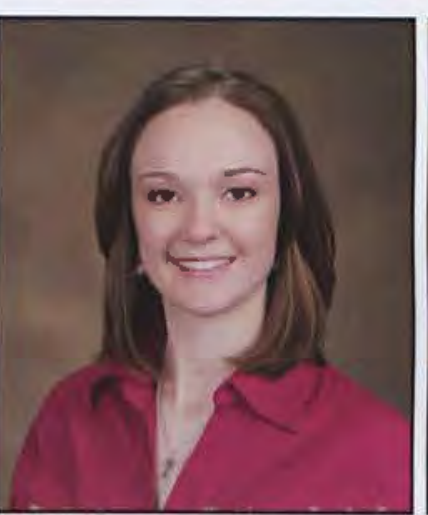

Amy Hanna Psychology

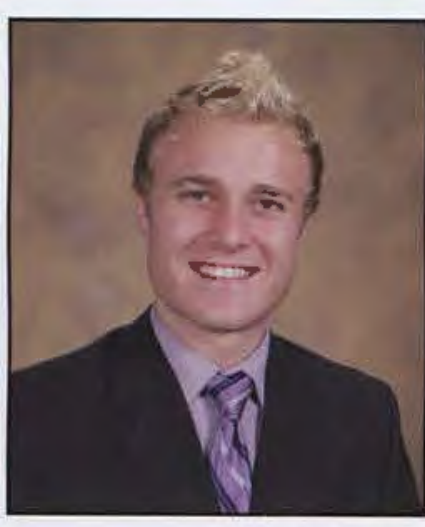

Grant Hansen Theatre/Communications

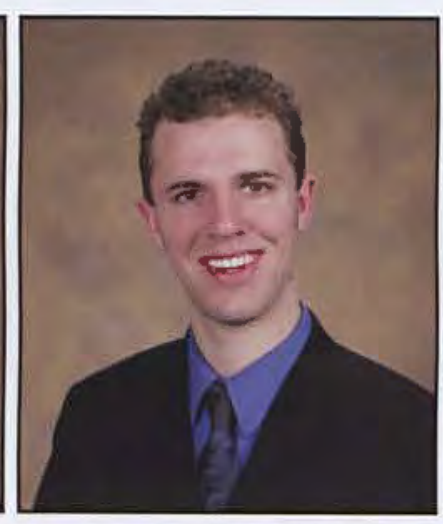

Timothy Harenda Music Composition

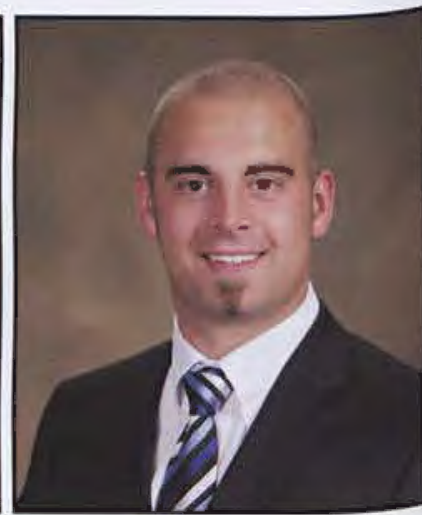

Caleb Hanby Athletic Training

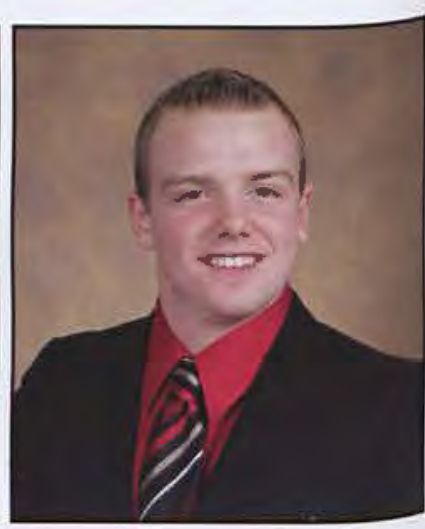

Kyle Haring Pre-Seminary Bible

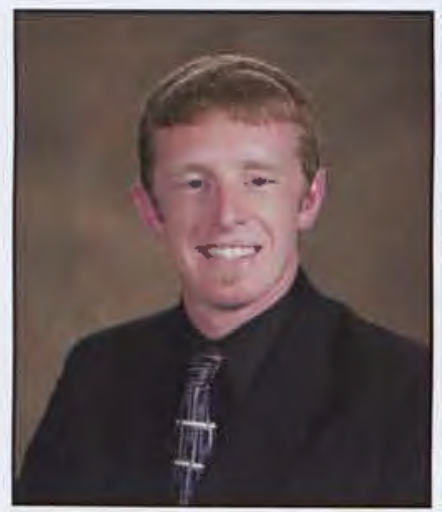

Chris Harmon Middle Childhood Education

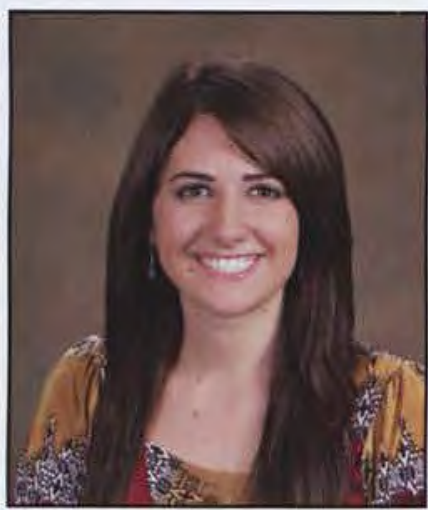

Sonya Harris Early Childhood Education

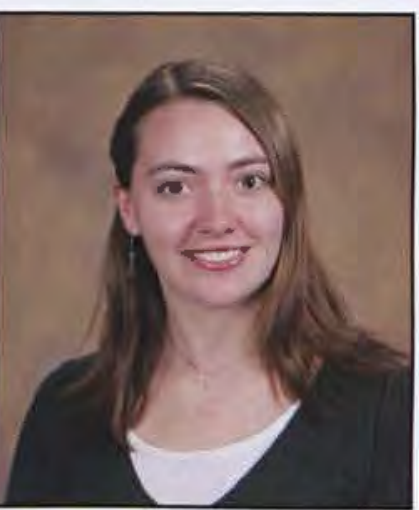

Autumn Harrison Chemistry

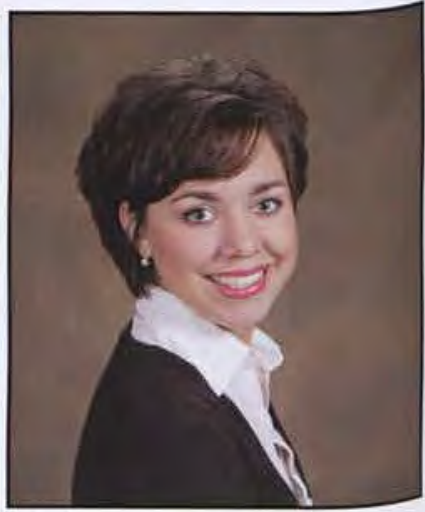

Alison Hatch Nursing

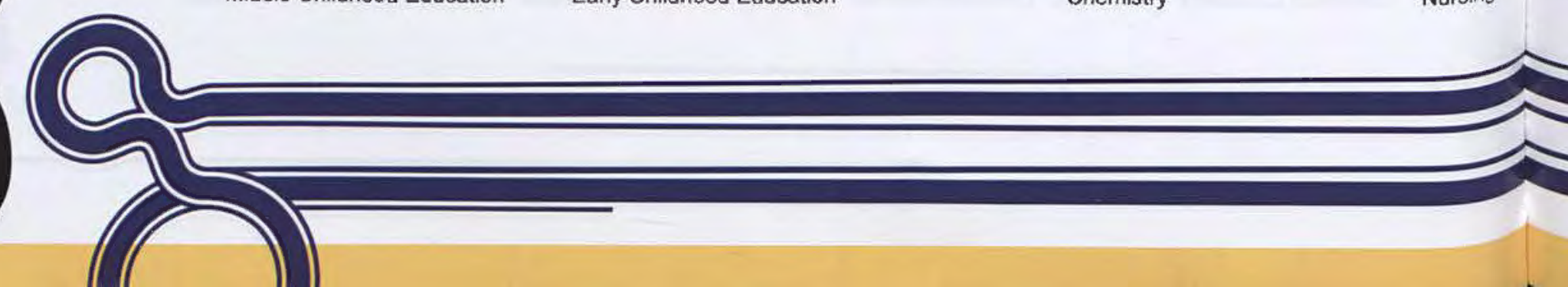




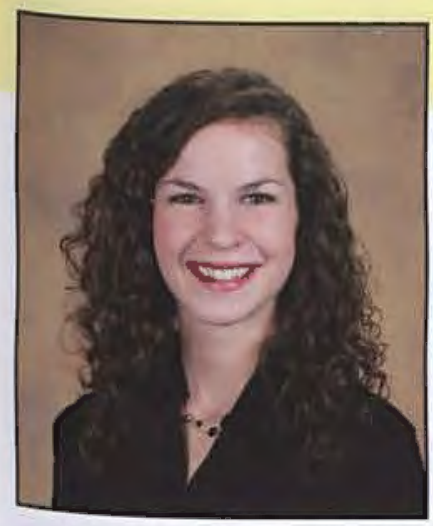

Ann-Marie Haug

Middle Childhood Education

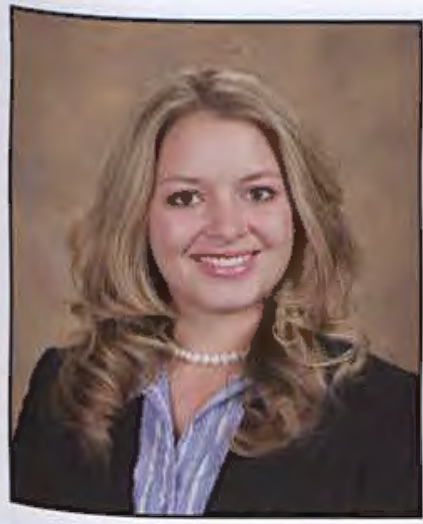

Elizabeth Hays

Nursing

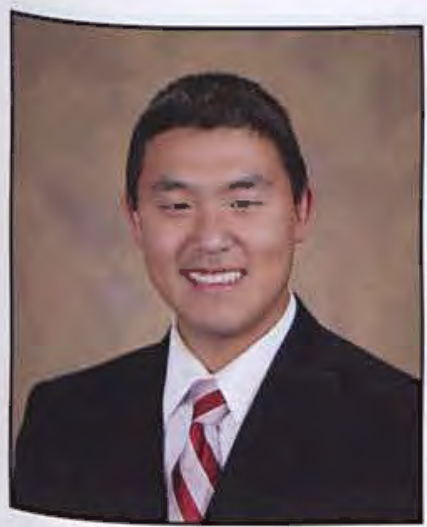

Nicholas Hendricks Finance

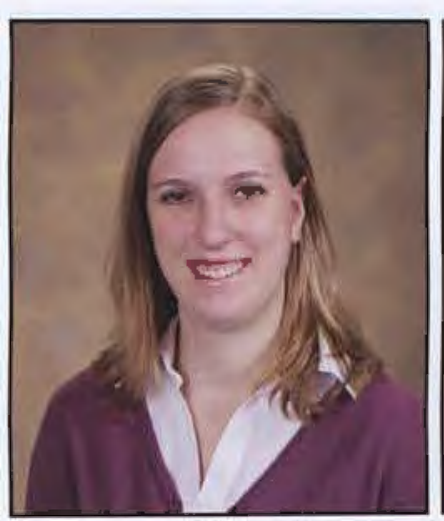

Juliana Heerschap Comprehensive Communications

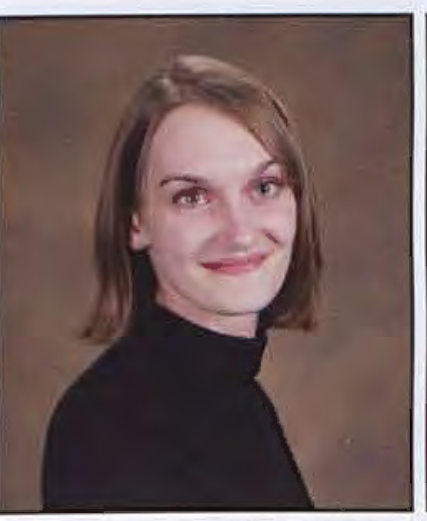

Rachel Herbolt Nursing

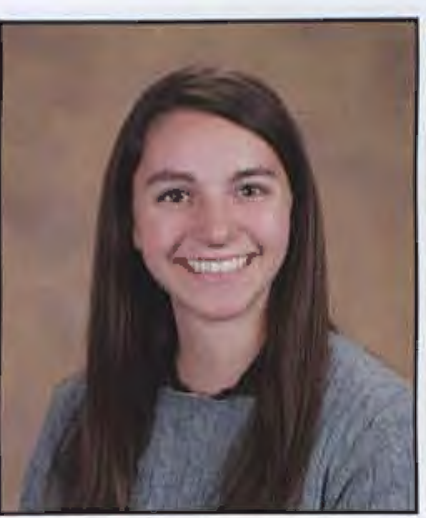

Beth Hegarty Integrated Mathematics Education

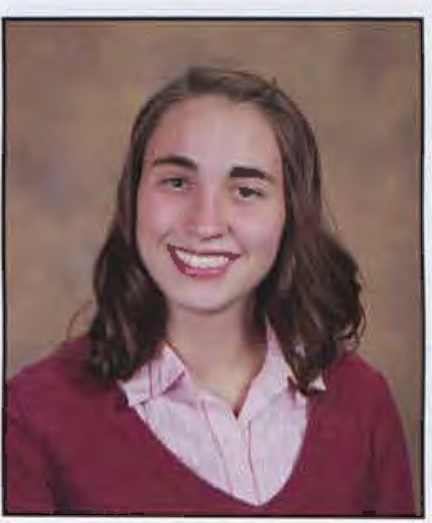

Katie Higgins Early Childhood Education

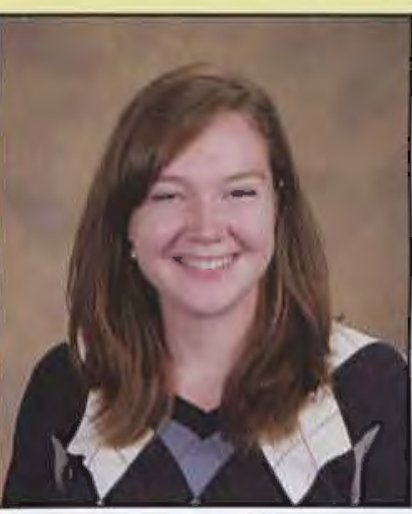

Erin Hayes English

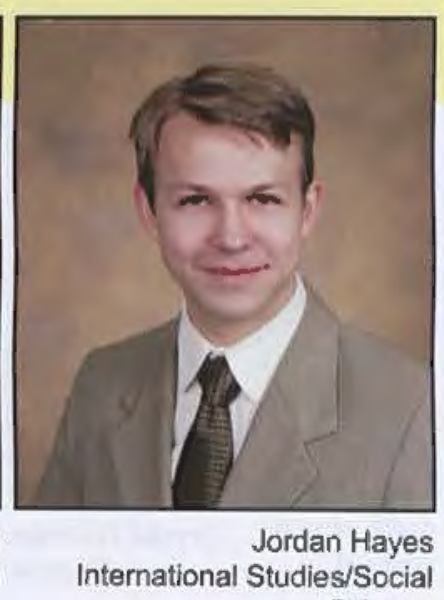

Science

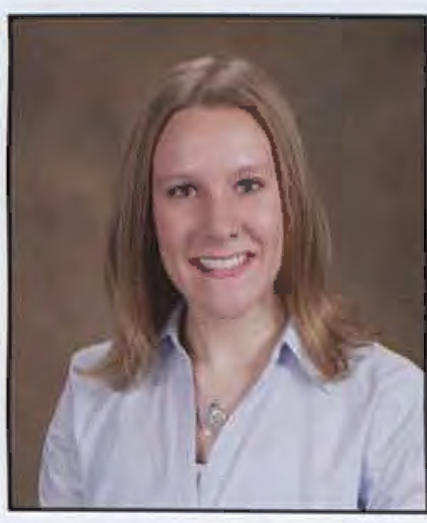

Anna Hej

Environmental Biology

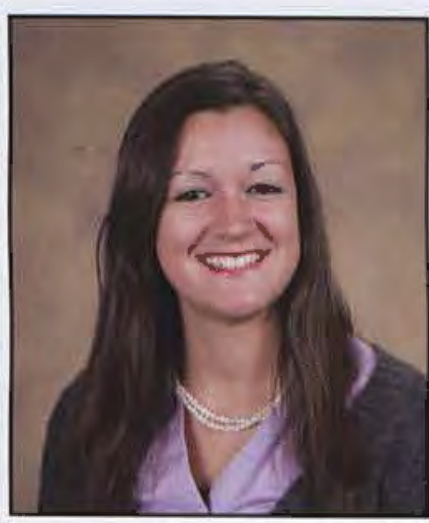

Rebecca High International Studies

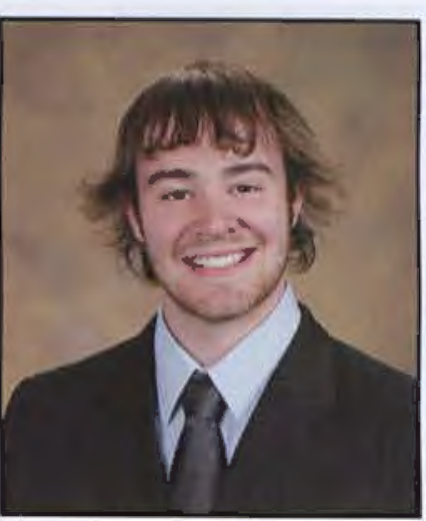

Joseph Hemphill Electronic Media Audio Production

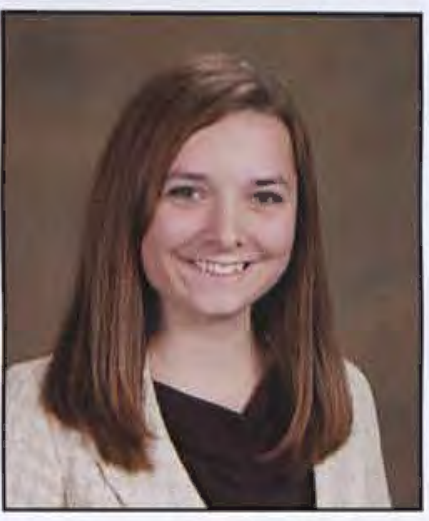

Michelle Hildebrand Christian Education

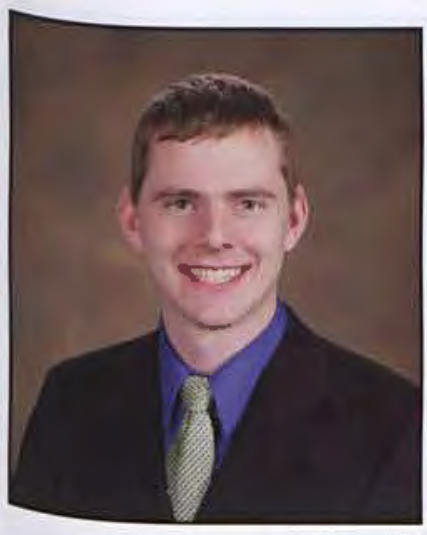

Ben Hill

Mechanical Engineering

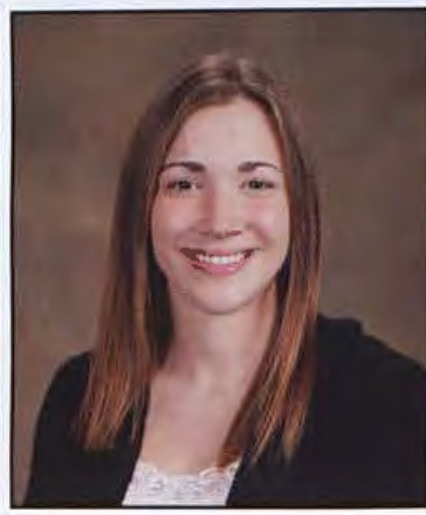

Hannah Hindle Social Work

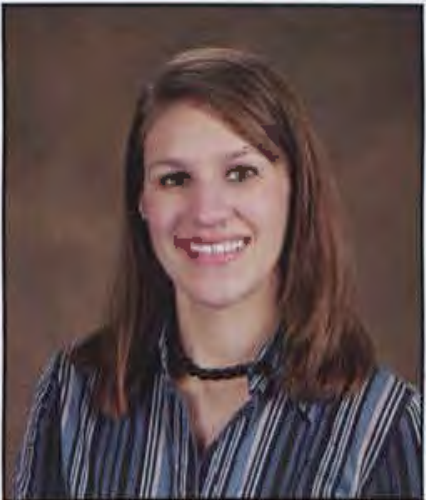

Katlyn Hiteshew

Organizational Communication

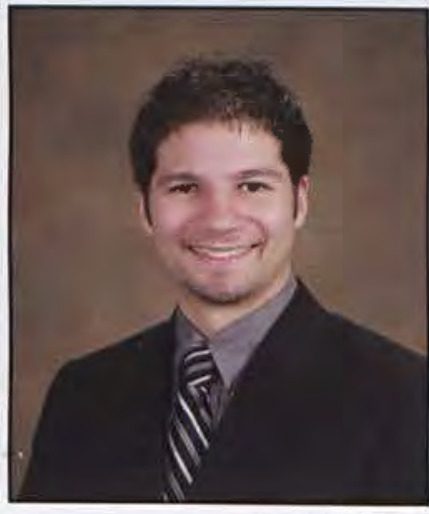

Seth Hobbes Criminal Justice

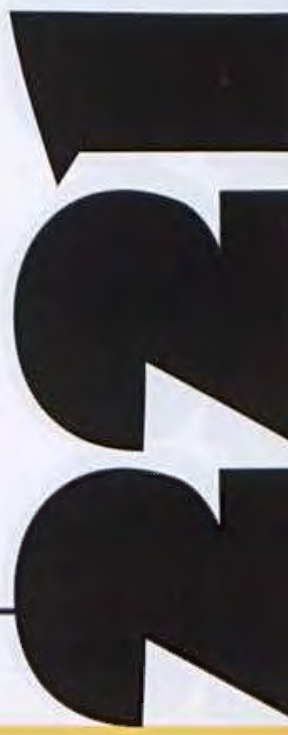




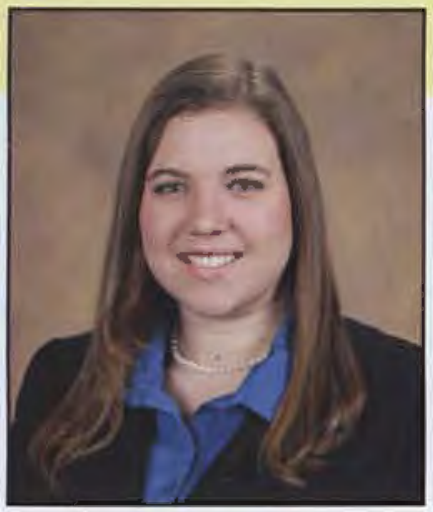

Crystal Hoffman Finance

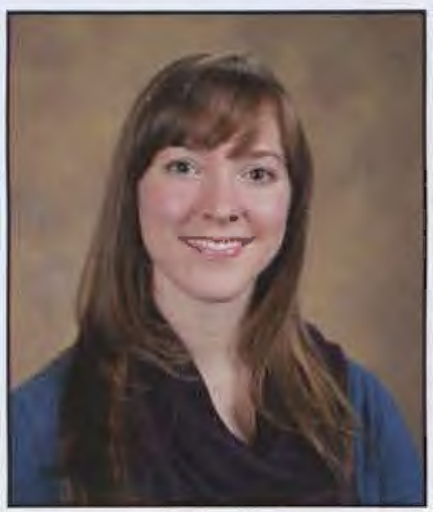

Kristen Horton Nursing

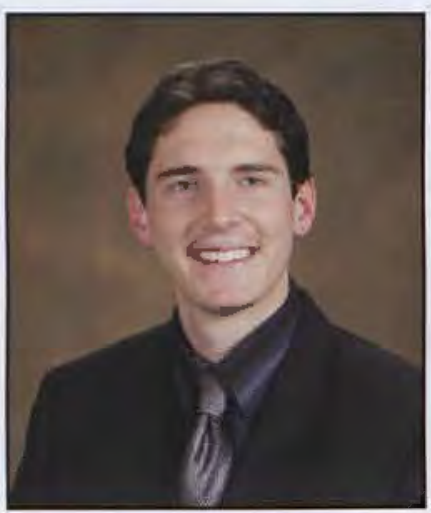

Timothy Hoyt Mechanical Engineering

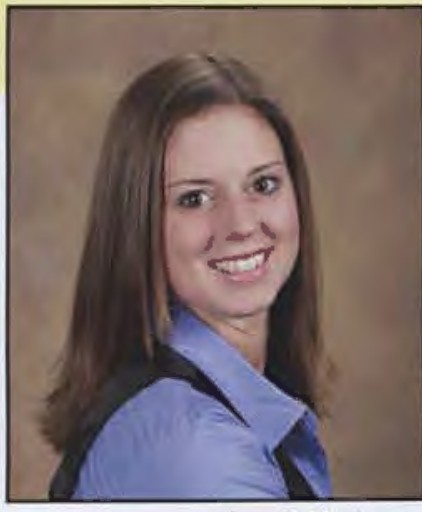

Sarah Hoffman Psychology

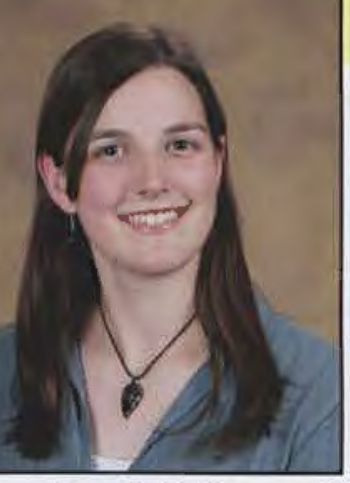

Hannah Hollingsworth Biology

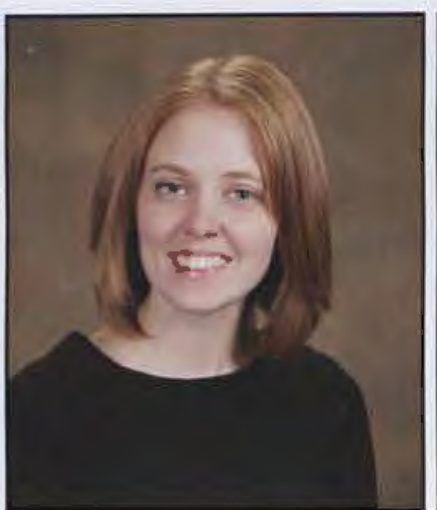

Sarah Hosan Early Childhood Education

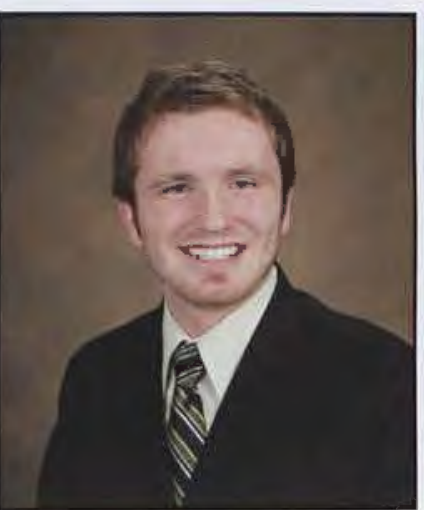

Maxwell Hounshell Youth Ministries/Christian Education

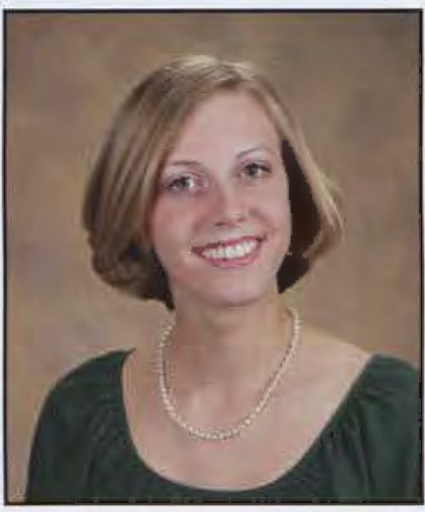

Jessica Huber Nursing

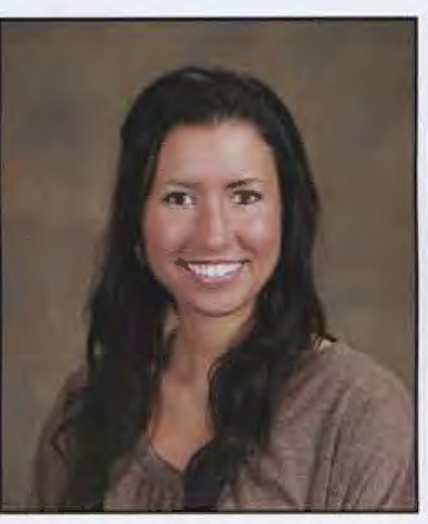

Kari Hufhand Special Education/ Early Childhood Education

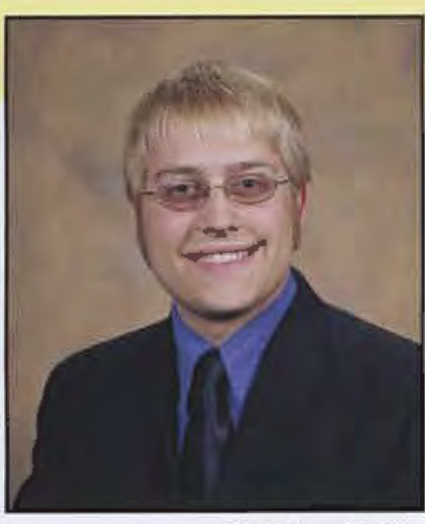

Matthew Hollis Mechanical Engineering

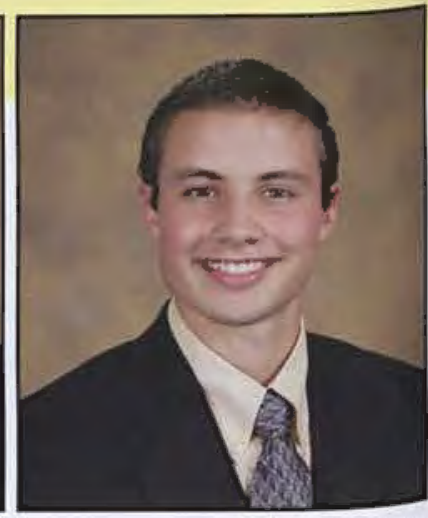

Samuel Hoppe Chemistry

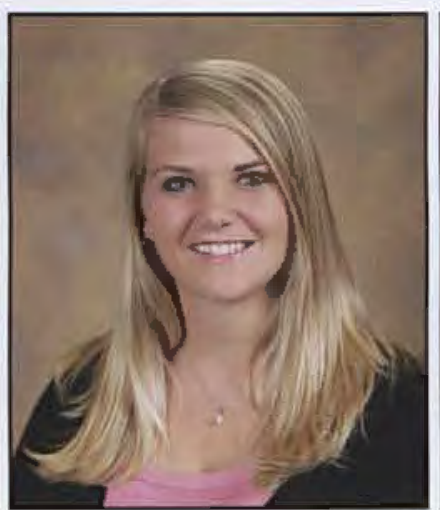

Angela Howard Early Childhood Education

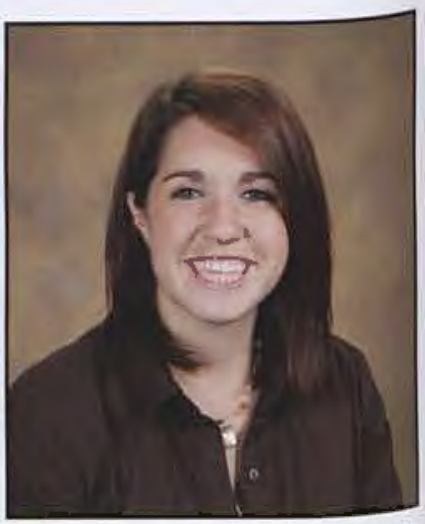

Chelsea Howard Nursing

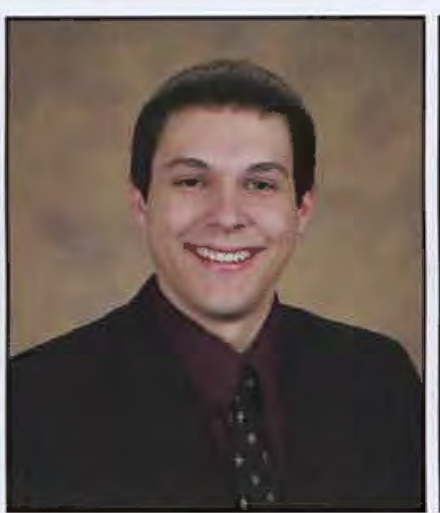

William Humphrey Mechanical Engineering

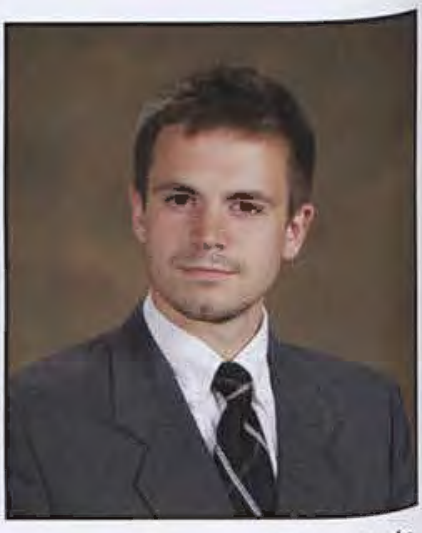

Ryan Hyde Integrated Social Science Education

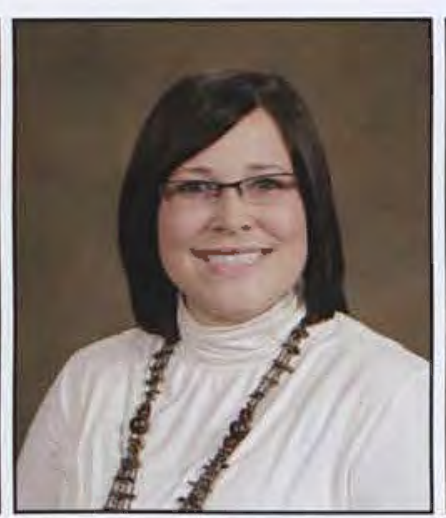

Clarissa Jackson

Aaron Ikehara Electrical Engineering

Brent Jackson

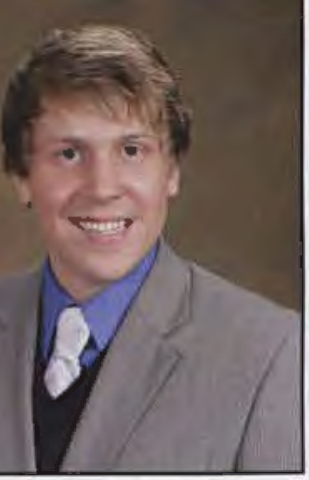

Management/Marketing

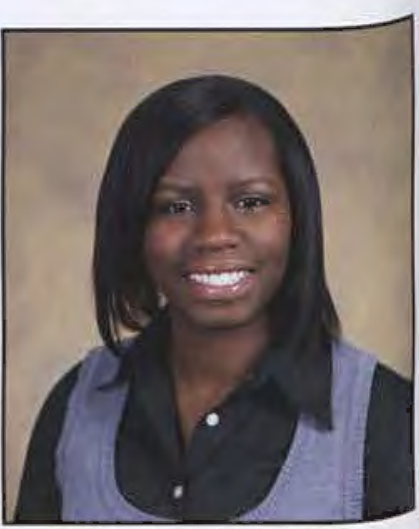

Tonisia Jackson Psychology
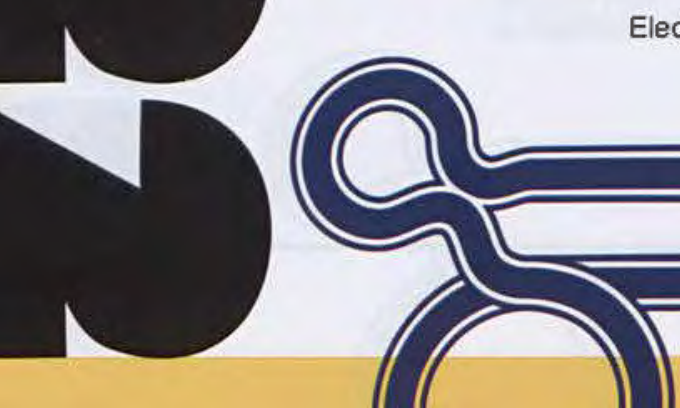


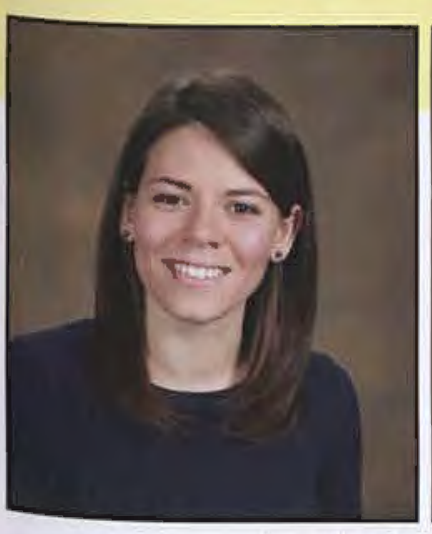

Amy Jacobs Biology

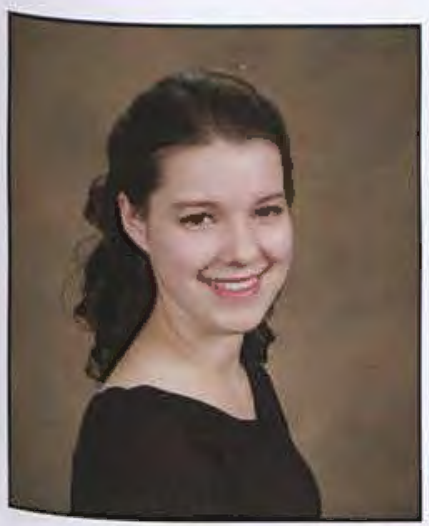

Gwendolyn Jobson Music Education

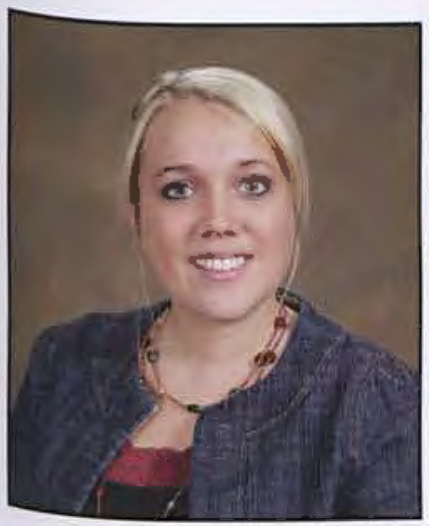

Amber Jones

Early Childhood Education

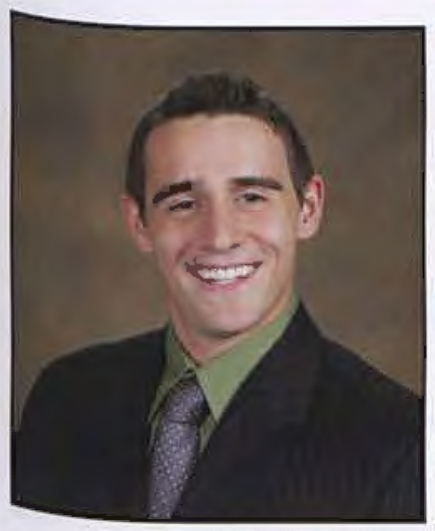

L. Anton Kilburn Biology/Edcuation

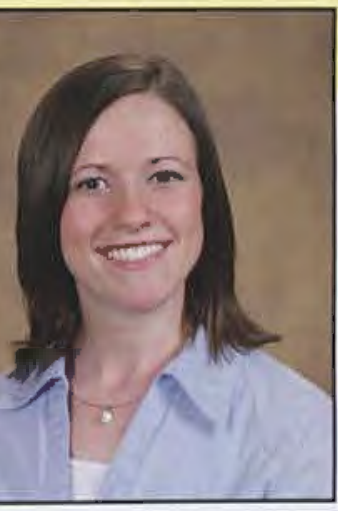

Natalie Jasper Nursing

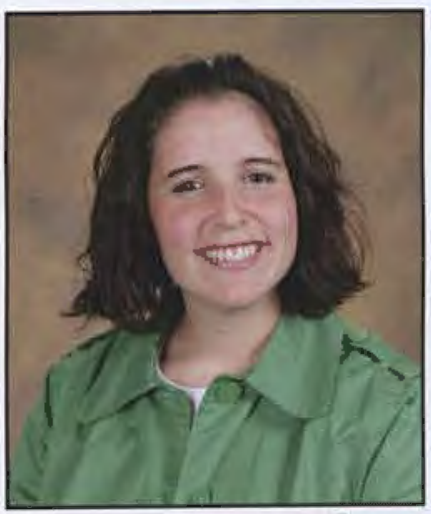

Jori Johnson Special Education/ Early Childhood Education

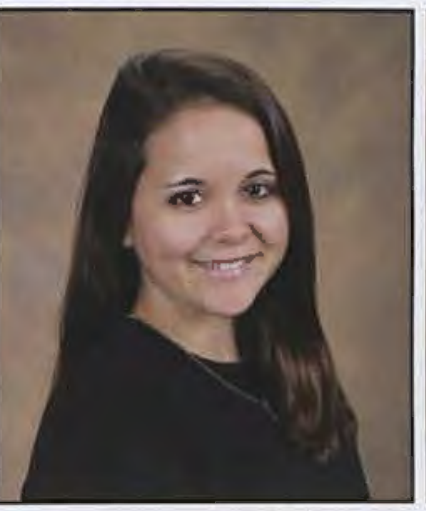

Julie Johnson

Middle Childhood Education

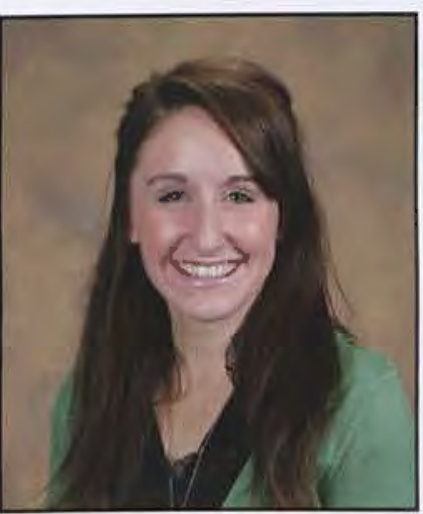

Grace Jones Communication Arts

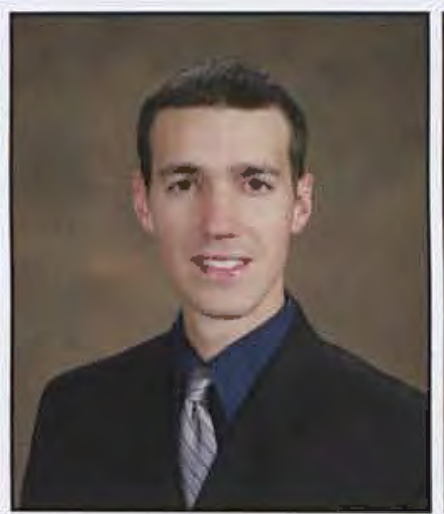

Jonathan Juhl

Physics

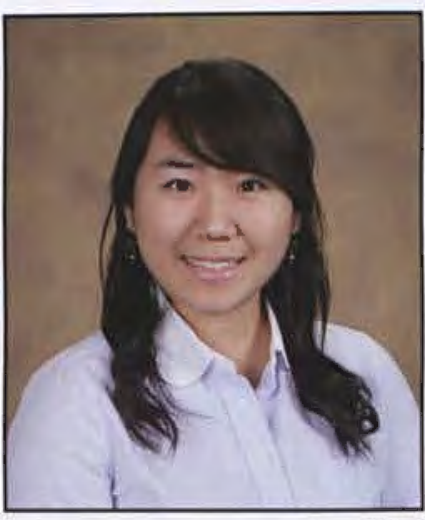

Jinsil Kim

International Studies/ Social Science

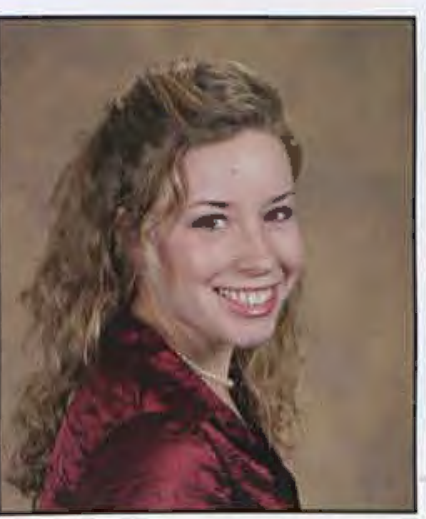

Laura Kindt

Integrated Language Arts Education

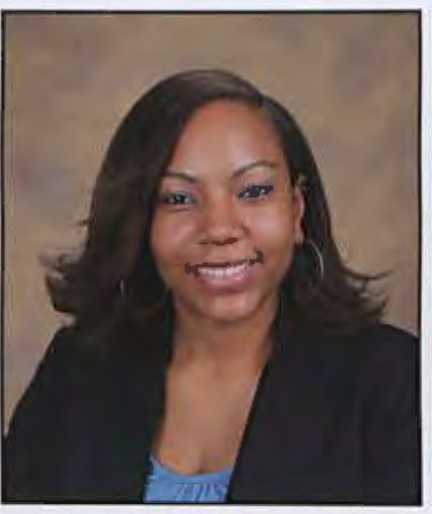

Amanda Jolly Communication Arts

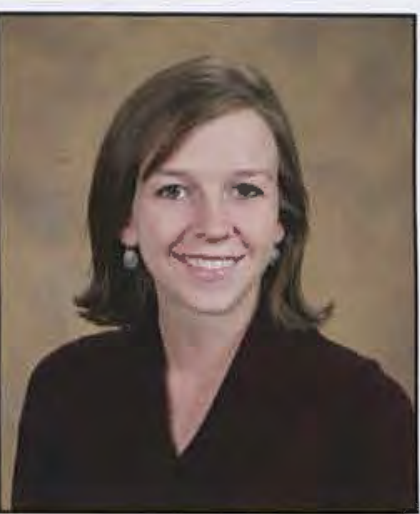

Amanda Kapp Music Education

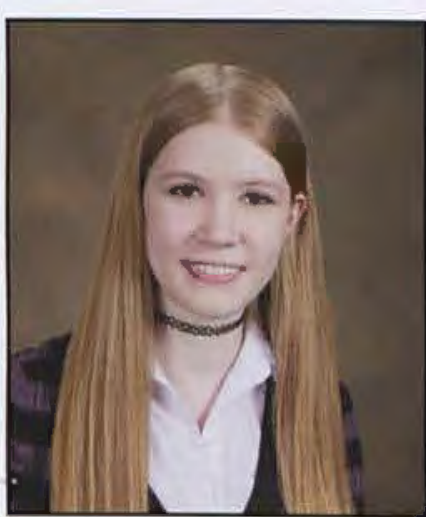

Shannen King Graphic Design

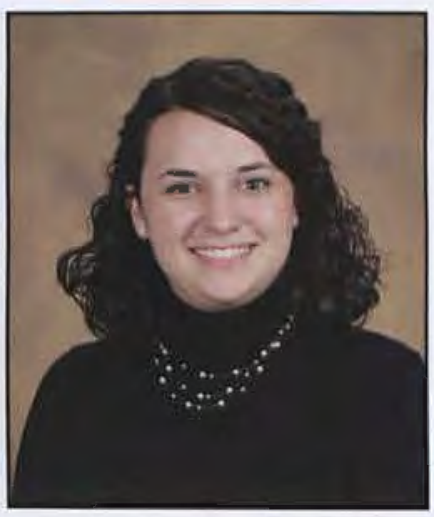

Allison Jones Nursing

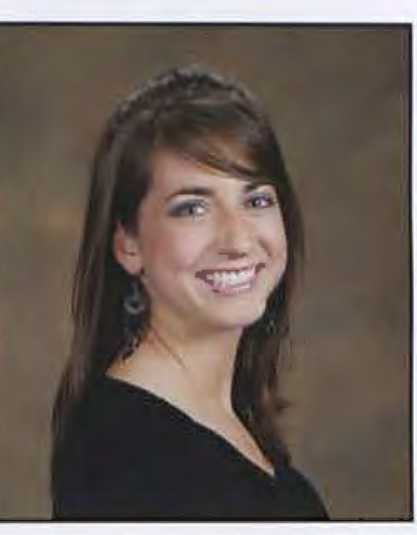

Stacey Keller Vocal Performance

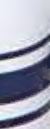




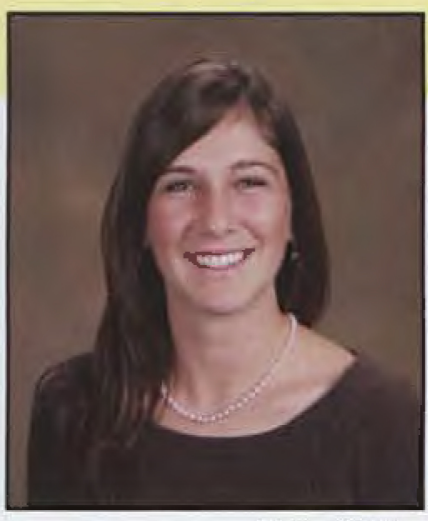

Abigail Kirby Criminal Justice

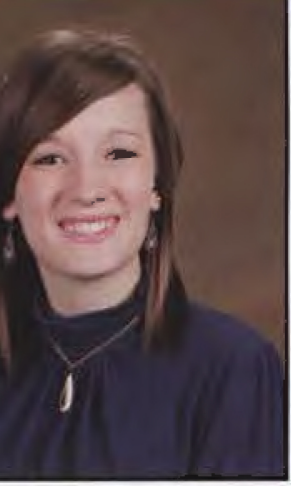

Kathryn Klimek Studio Art

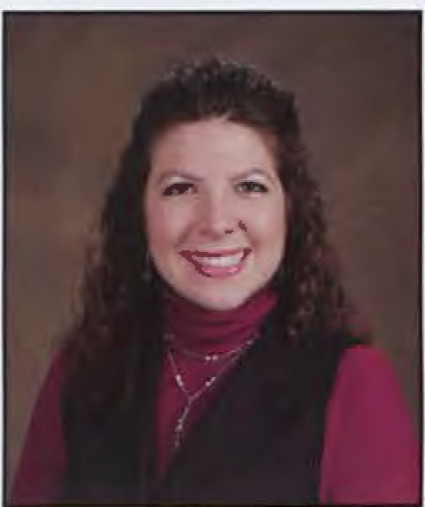

Christine Knott Mathematics

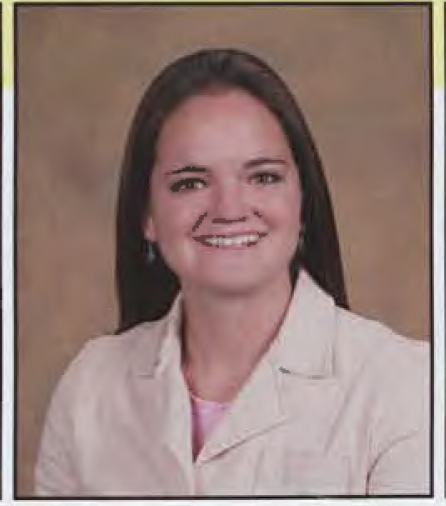

Marie Kirby Athletic Training

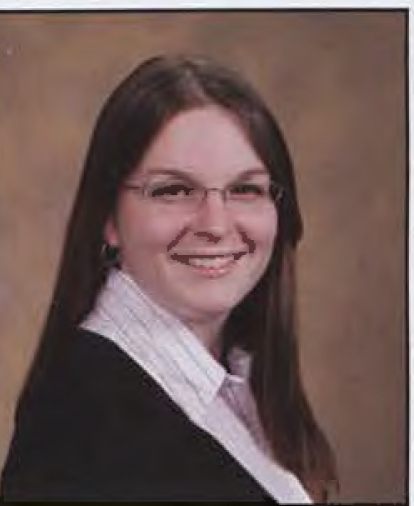

Courtney Kline Finance/Accounting

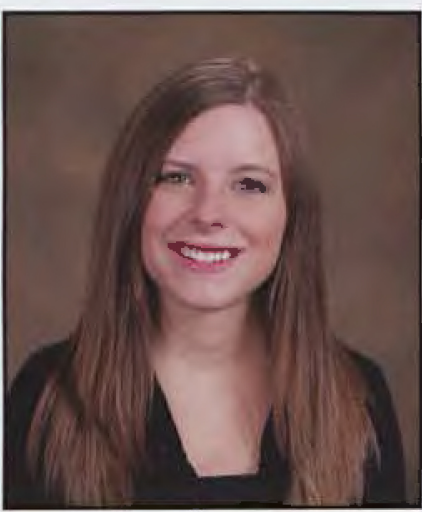

Rebecca

Finance/Accounting

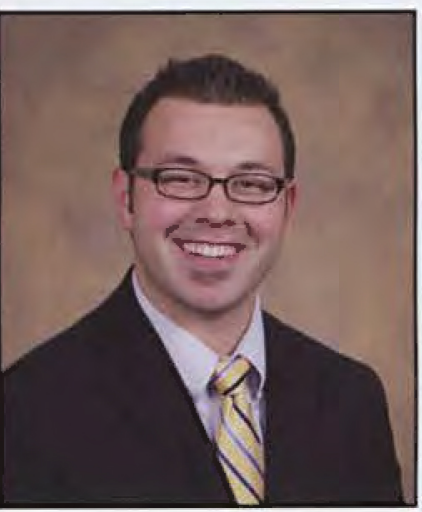

Daniel Kloosterman Integrated Langage Arts Education

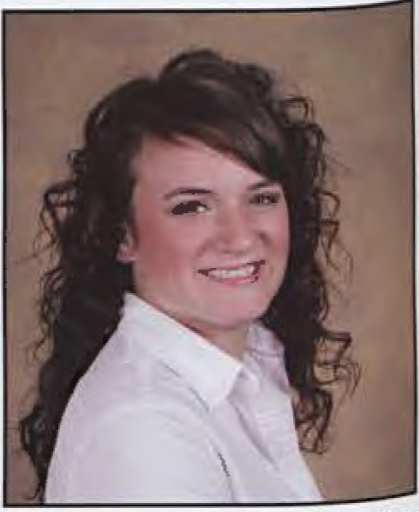

Brittany Knoedier Psychology

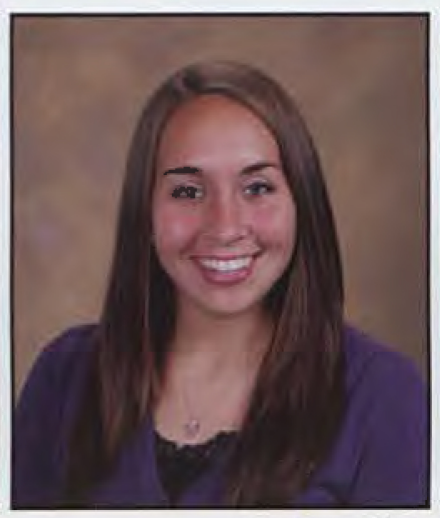

Kathryn Koch Multi-Age Physical Education

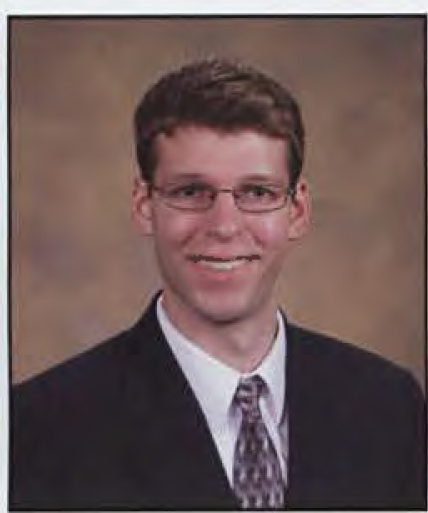

Timothy Kolody Mechanical Engineering

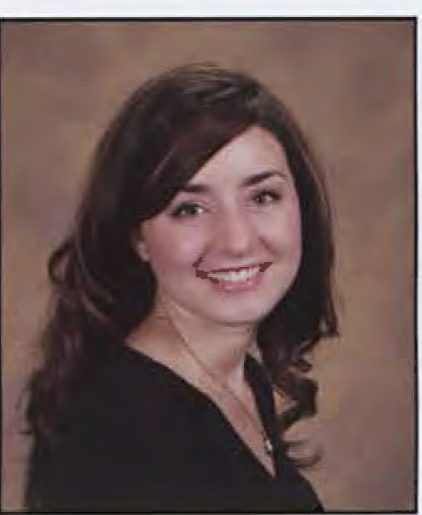

Valerie Kowalczyk Nursing

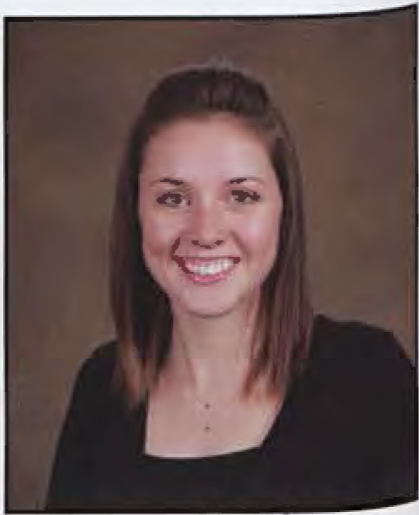

Krista Kowatch Communications

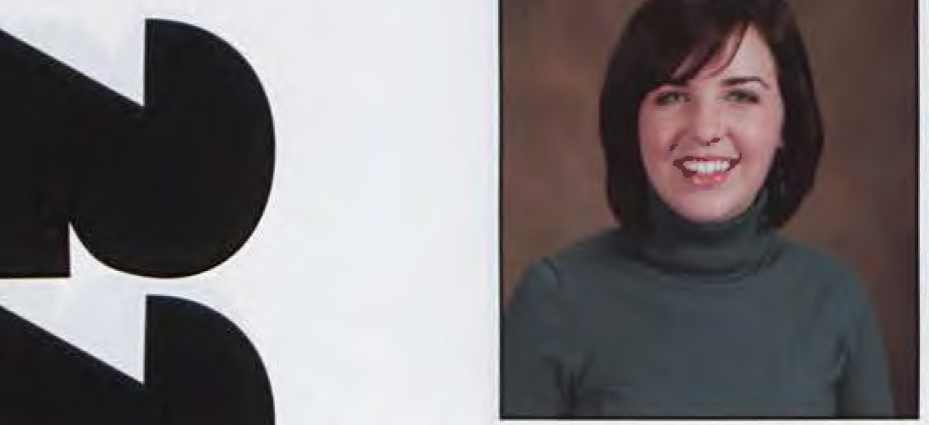

Emily Kraft Organizational Communication

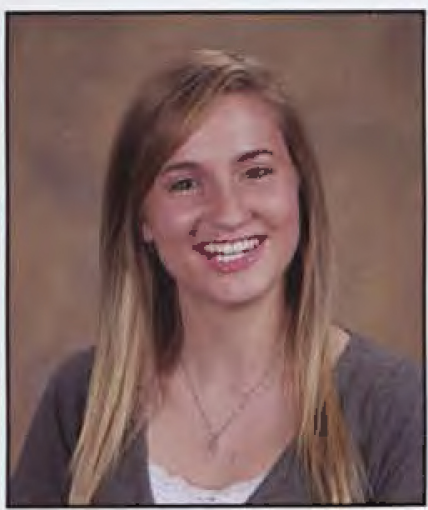

Michelle Kroll Early Childhood Education

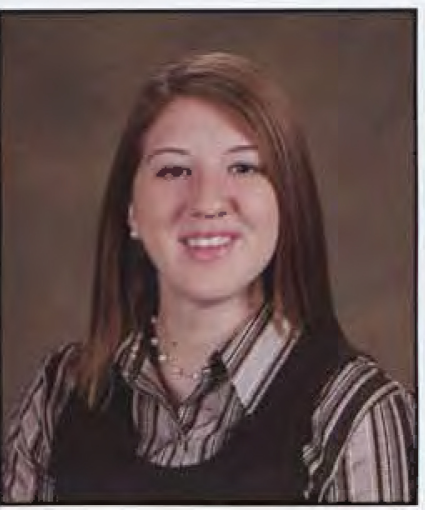

Michelle Kropf Youth Ministry! Christian Education
Benjamin Kleis

Philosophy/History
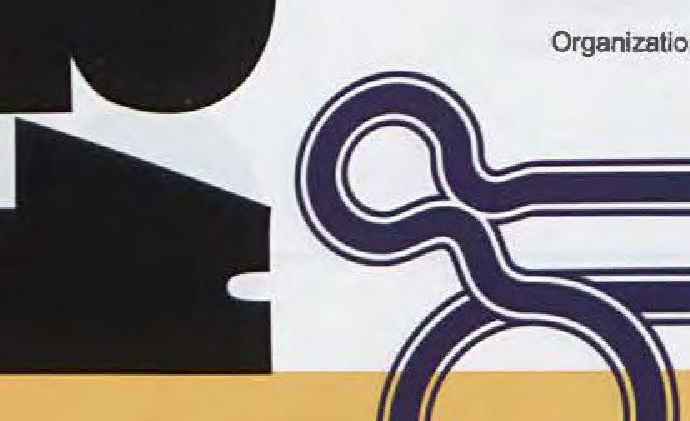


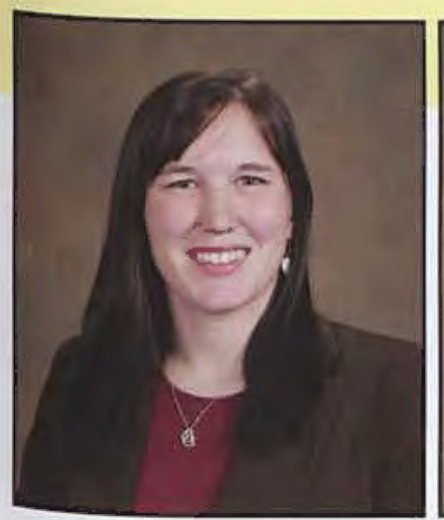

Lauren Kurtz Electronic Media

Timothy Labrado

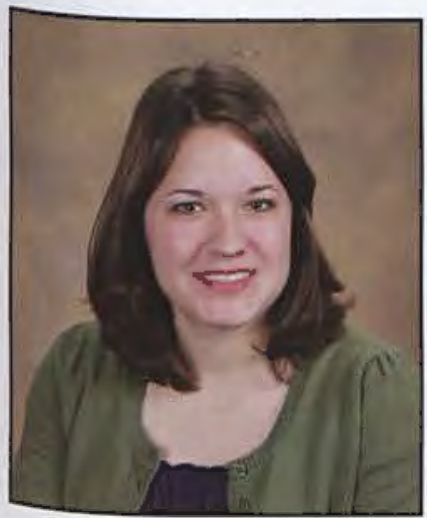

Rhoda Lambright Nursing

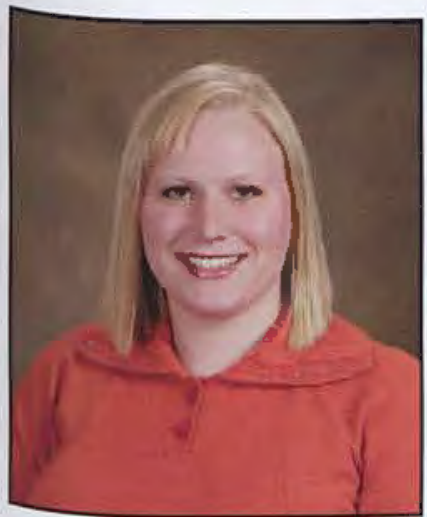

Weslea Law

Technical and Professional Communication

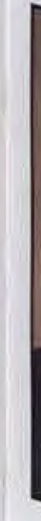

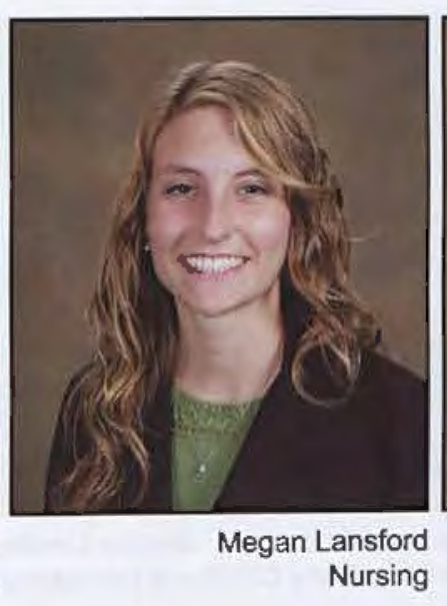

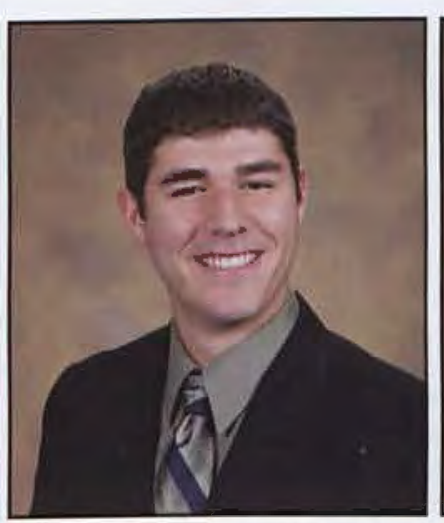

Joel Lawrence

History and Political Science International Studies

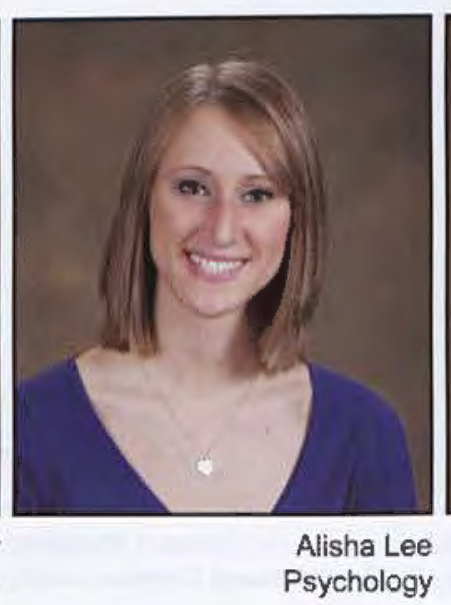

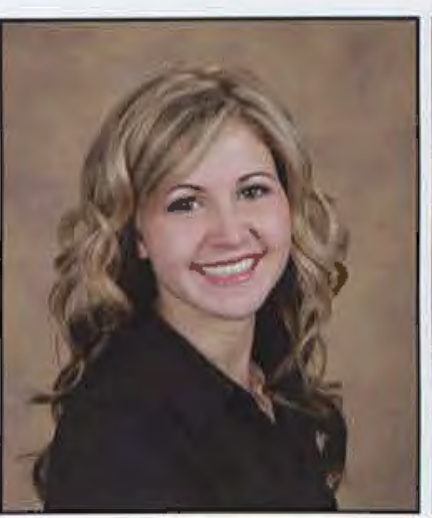

Sandra Larsen Marketing

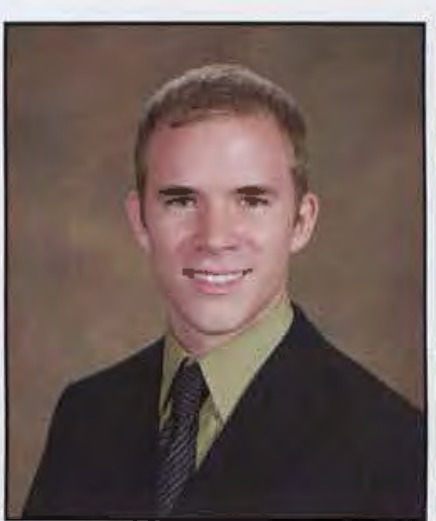

Jesse Lear Marketing

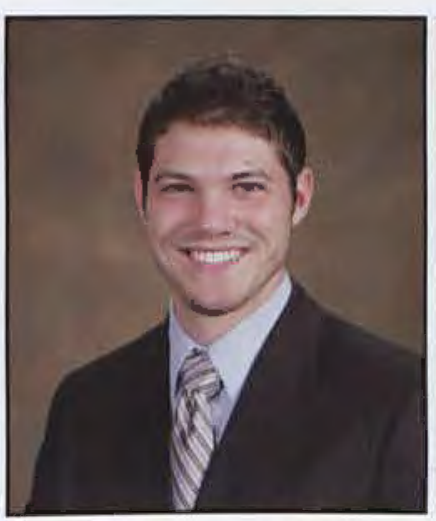

Jordan Leffel Alisha Lee Psychology
ManagemenV Business Management
Peter Ledger Graphic Design

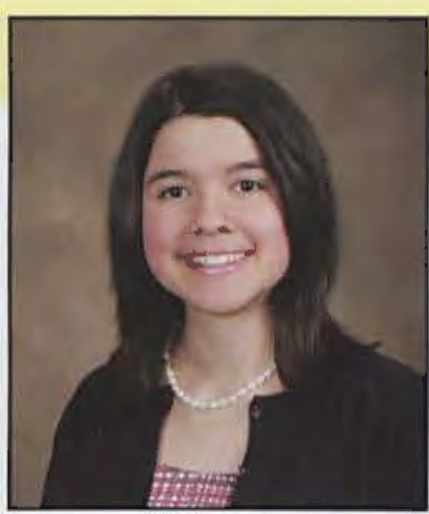

Natalie Lagar Early Childhood Education

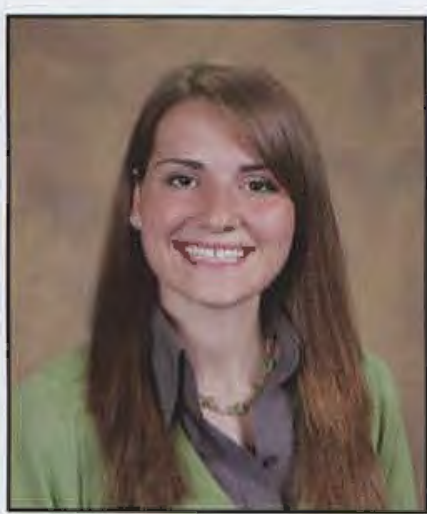

Alyssa Laswell Biology

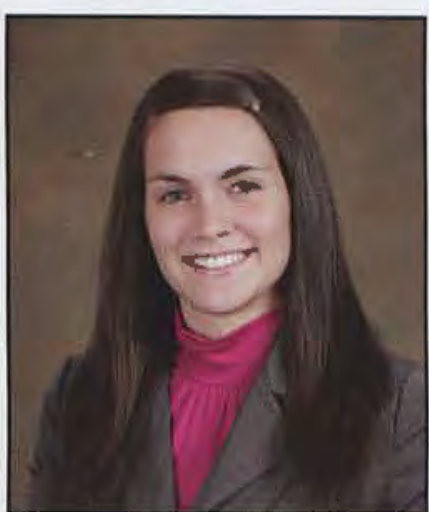

Rebecca Leary Accounting

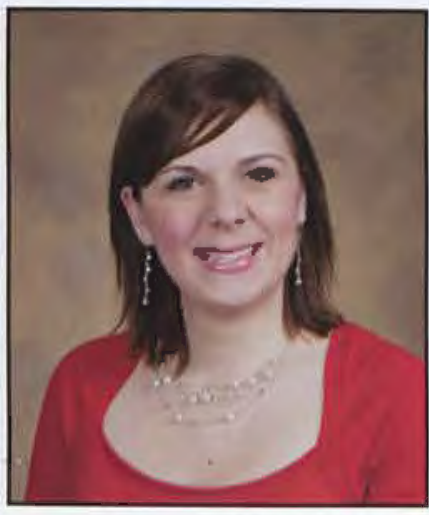

Hannah Leitch Social Work

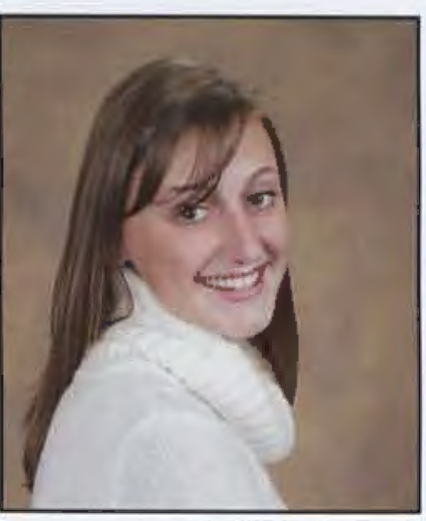

Ashleigh Laswell Early Childhood Education

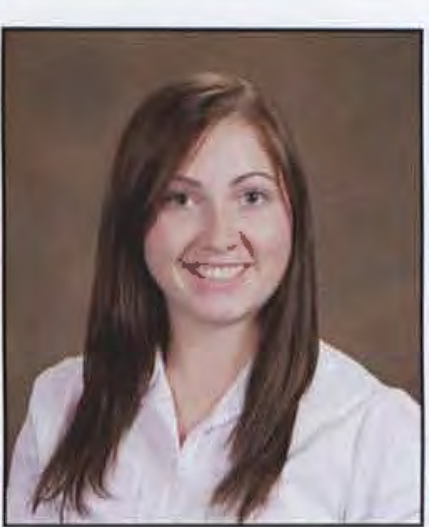

Alicia Lease Nursing Amber Laing
Multi-Age Physical Education 


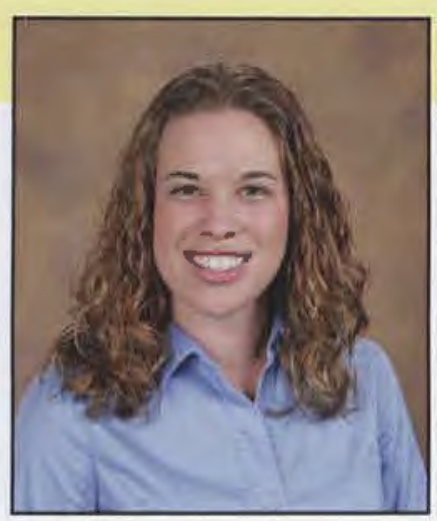

Christina Leonard Organizational Communication

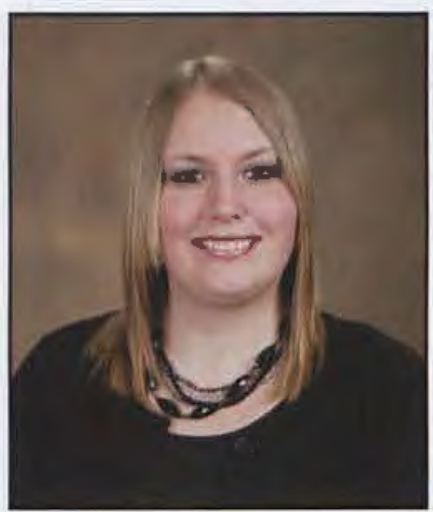

Jana Linville Graphic Design

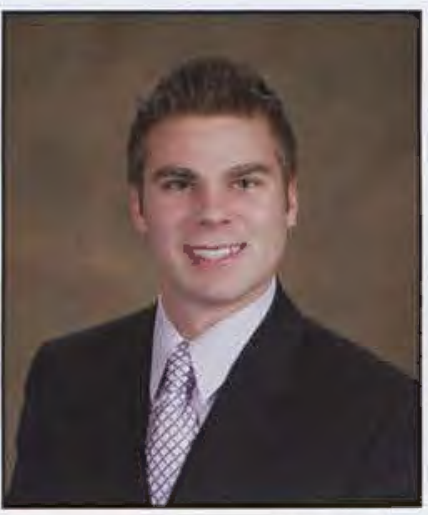

Ryan Lustig Business Marketing

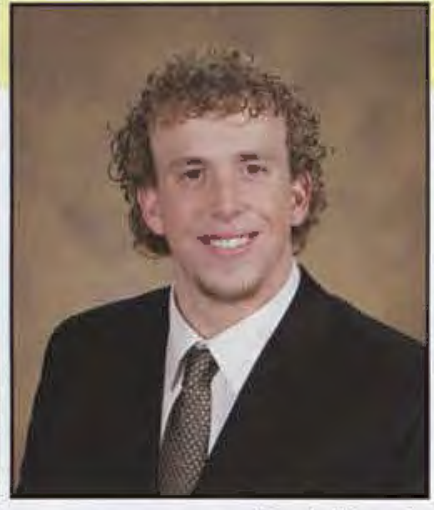

Daniel Lewis Music Performance

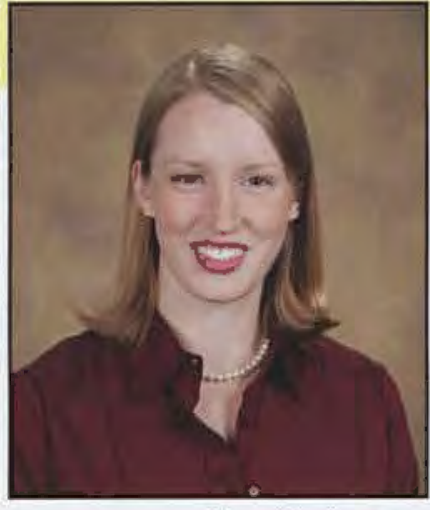

Kara Lindemann Nursing
Kyle Linden Computer Science

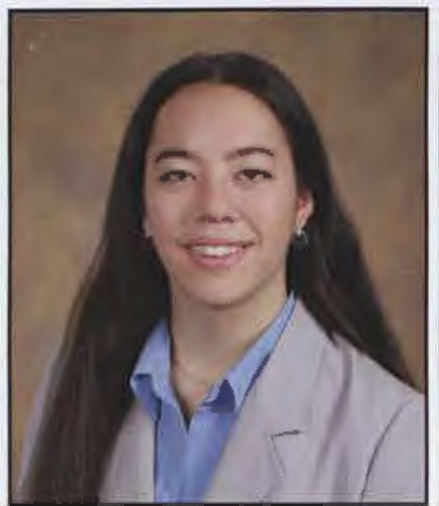

Annette Liu Nursing

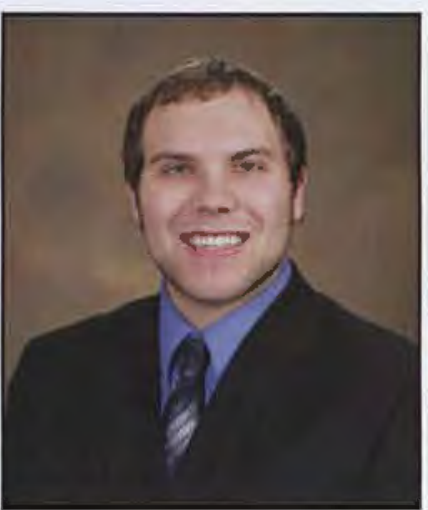

Dustin Long

Youth Ministry/Christian Education

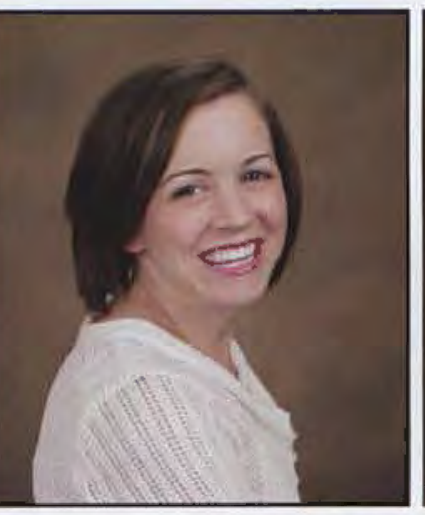

Rachel Luther Theatre

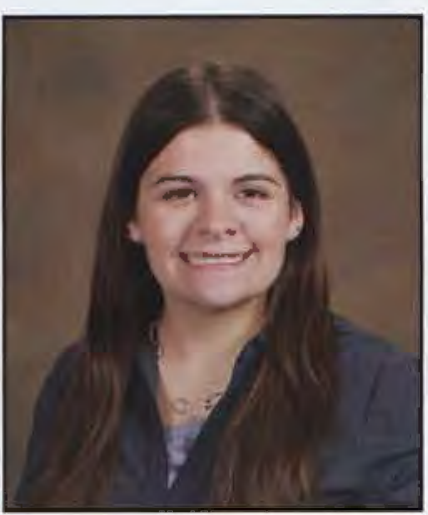

Kathryn Lutz Music

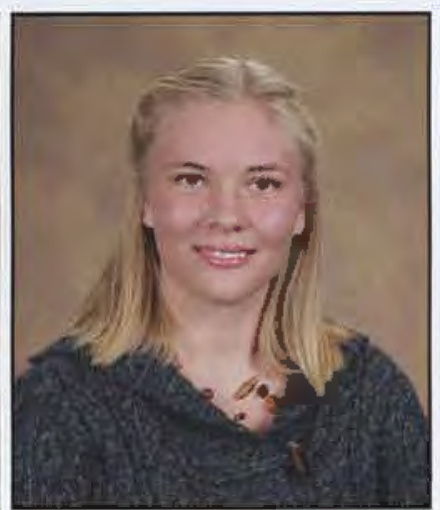

Jessica Losch

Early Childhood Education

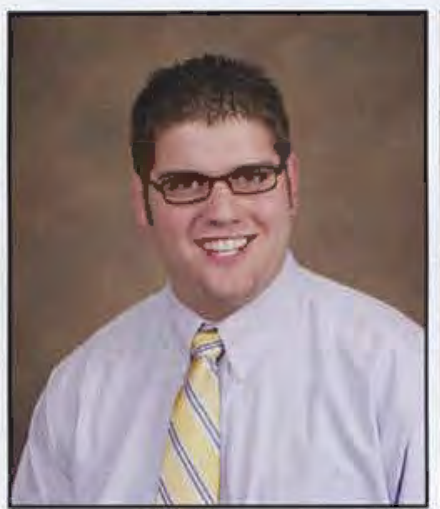

Christian Maat Accounting

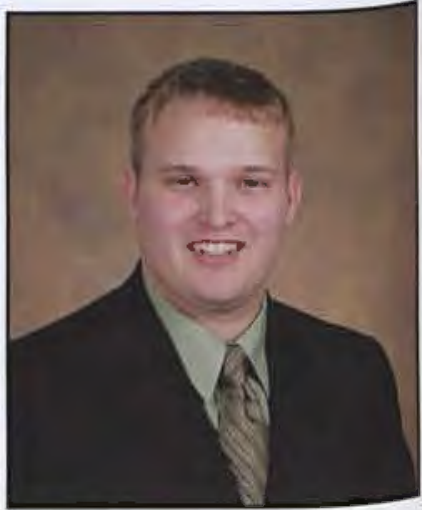

Kyle Lowry Psychology

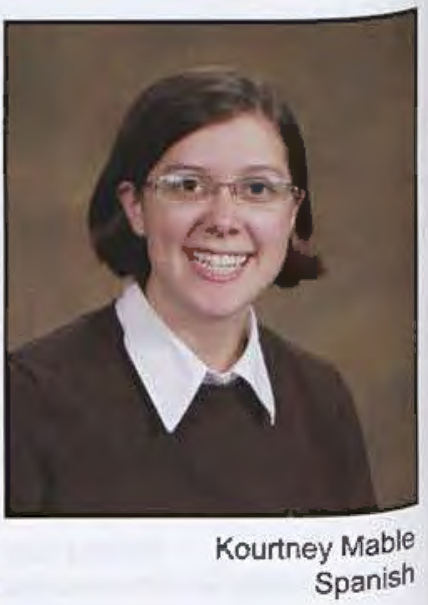

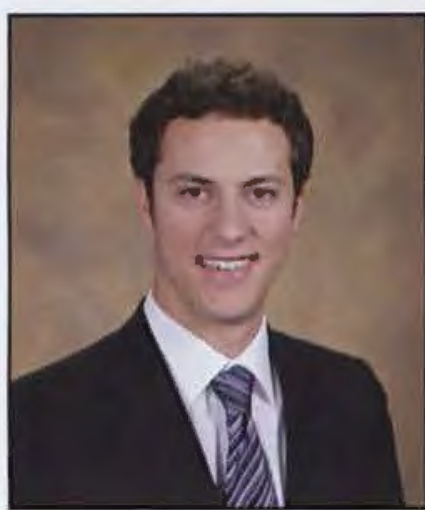

Matthew MacKenzie History/Psychology

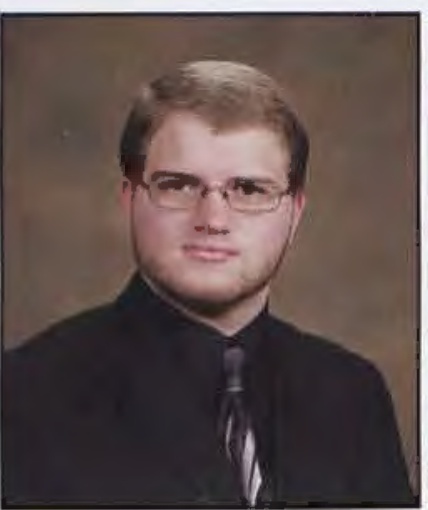

Marcus Manchester History

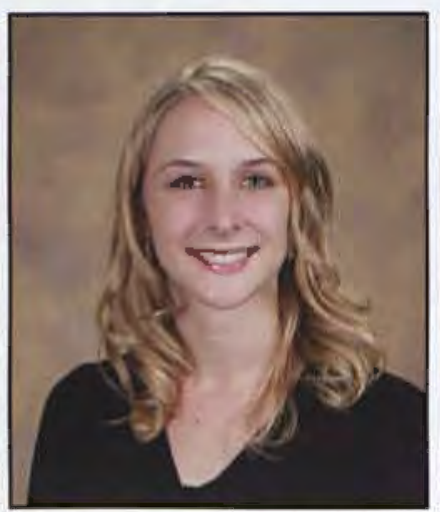

Kristen Mansker Organizational Communication
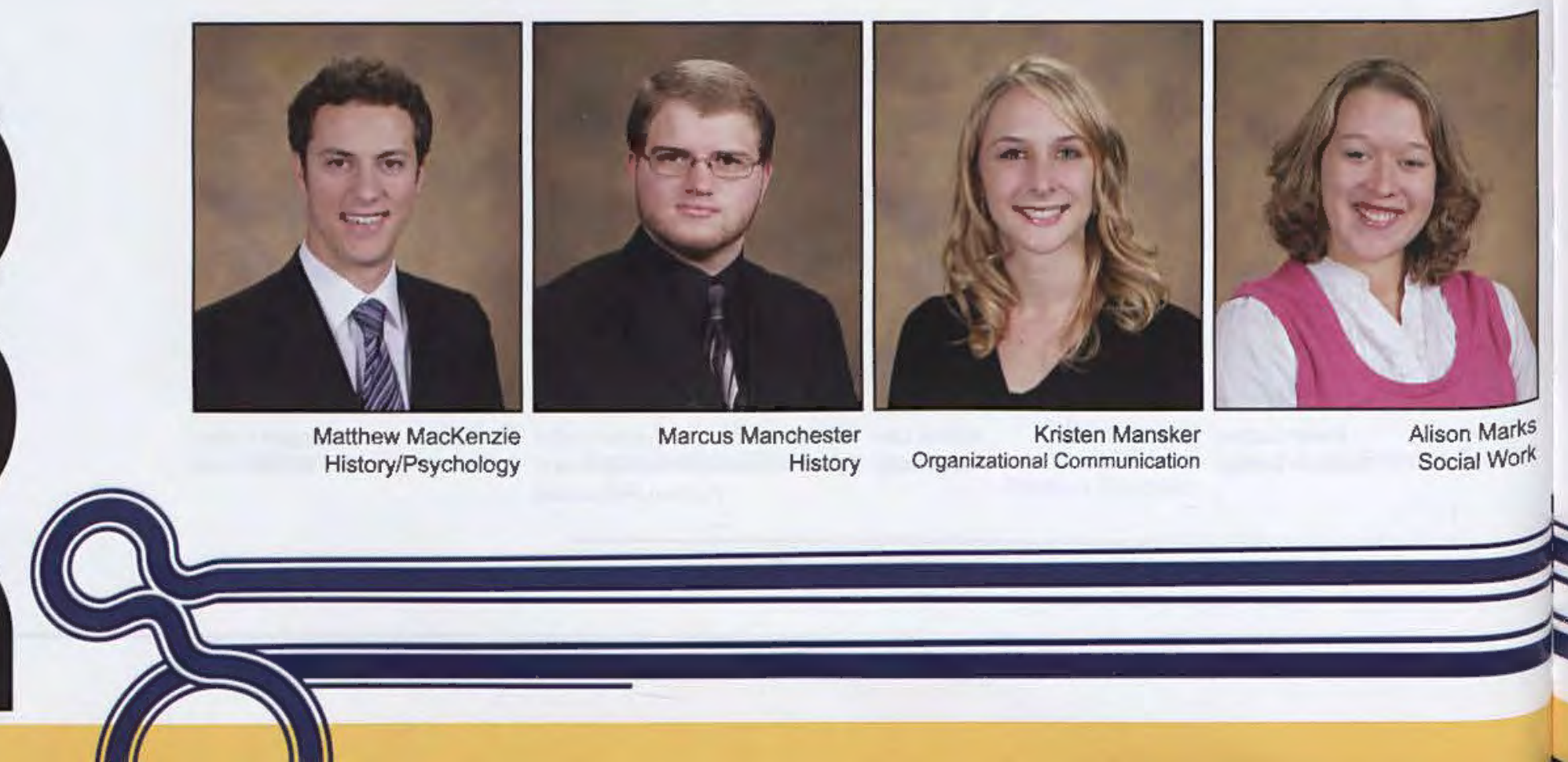


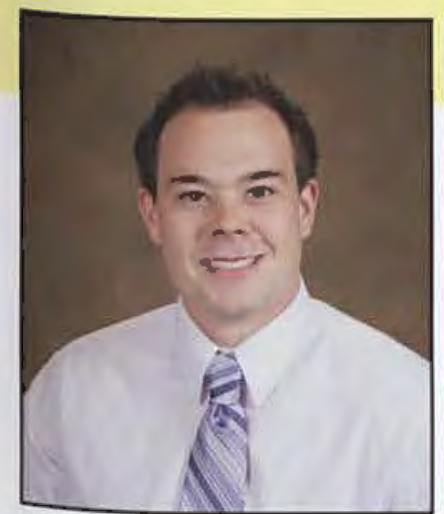

Justin Marshburn Exercise Science

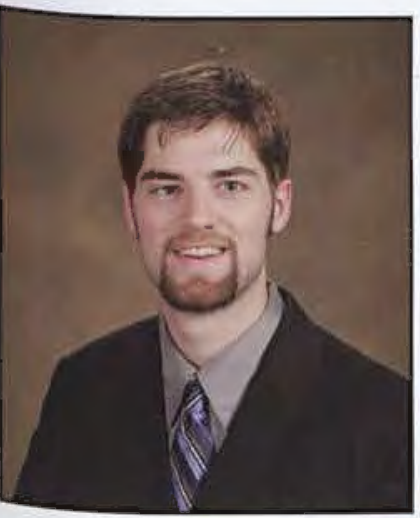

Nicholas Matthews Electronic Media

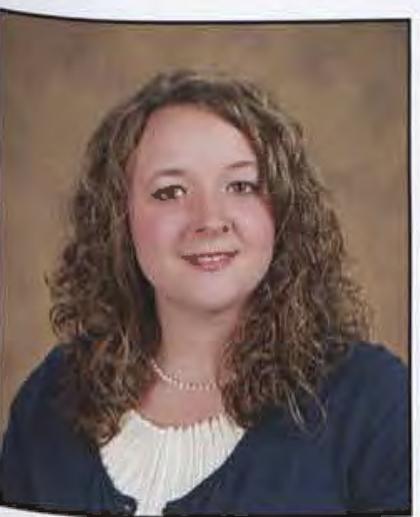

Casey McCown Nursing

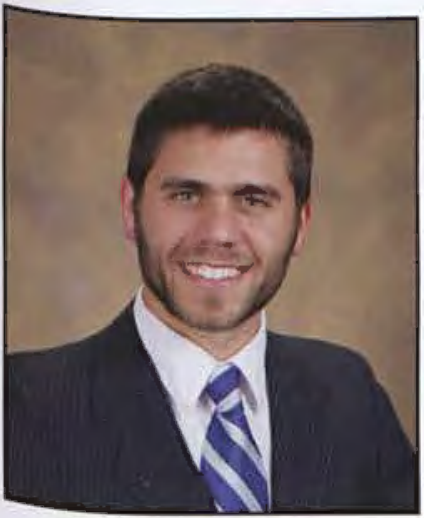

Jonathan McGill Pre-Seminary Bible

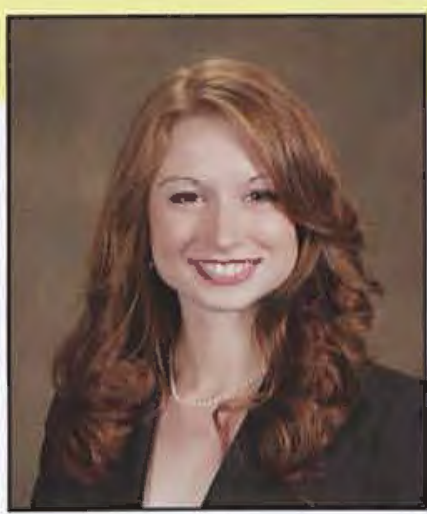

Amanda Martin Cross-Cultural Nursing

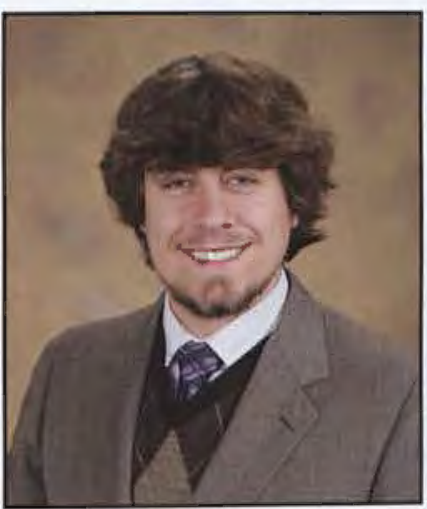

Isaac Mayeux English

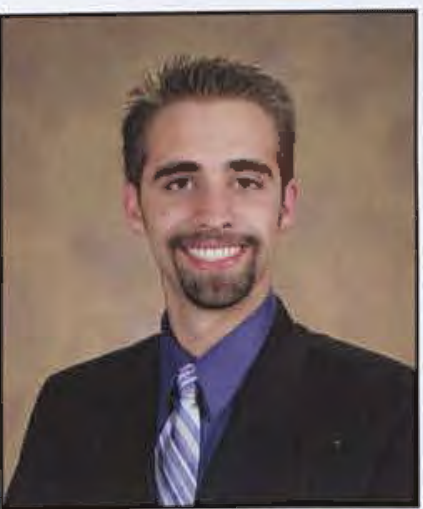

Michael McCoy Computer Science

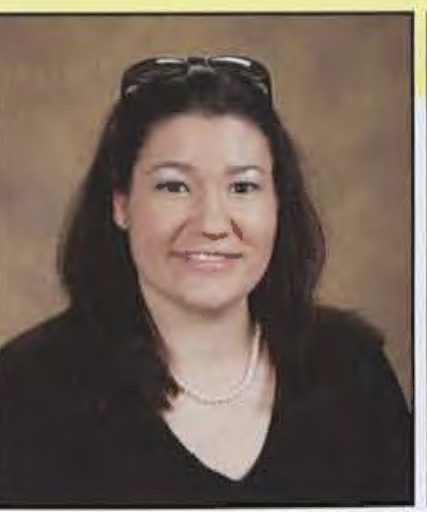

Rachel Martinez English

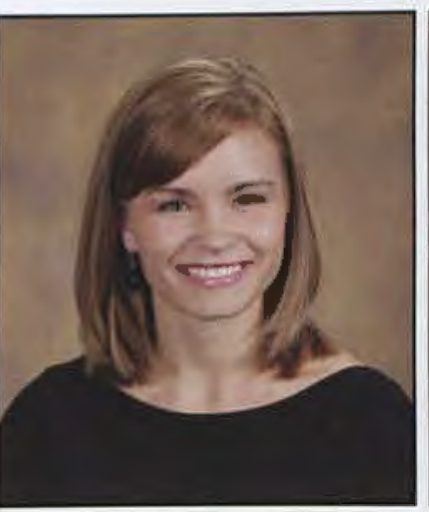

Carrianna McCay Nursing

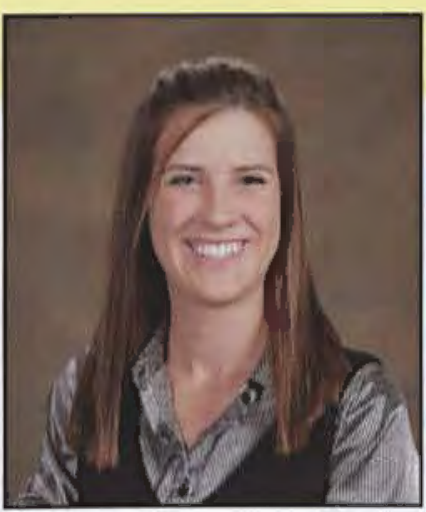

Bethany Mast Early Childhood Education/ Multi-Age Special Education

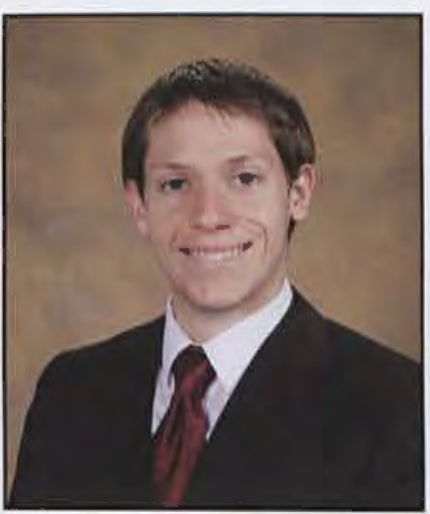

Nathan McCloskey English

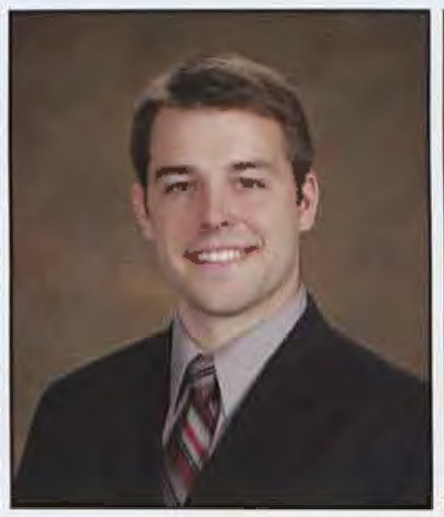

Jamin McCue Electrical Engineering

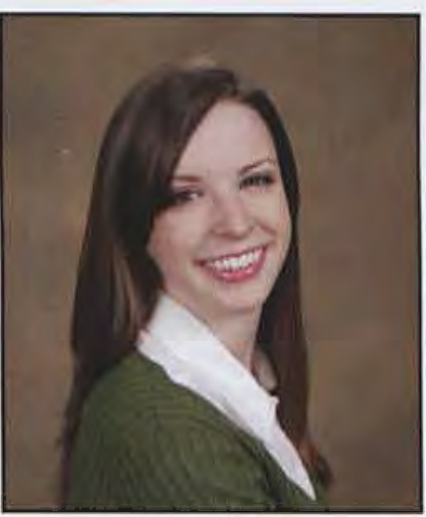

Jennifer McDonald Early Childhood Education

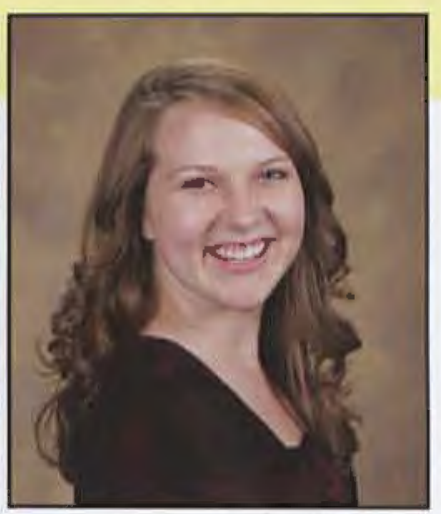

Sarah Matney Organizational Communication

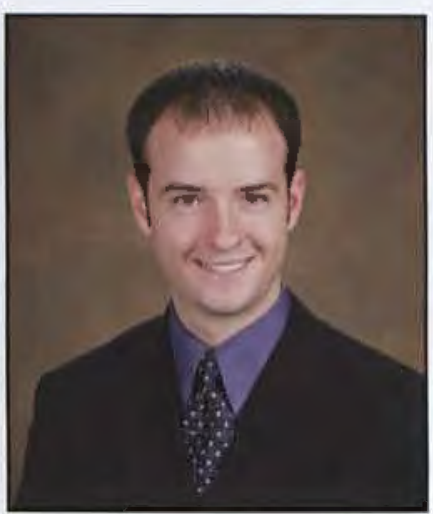

John McClure Mechanical Engineering

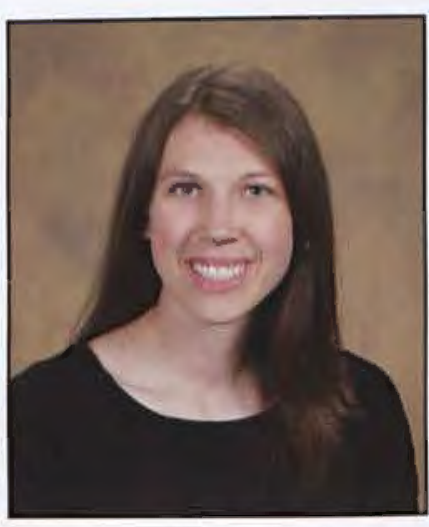

Kristine McGunnigal Marketing

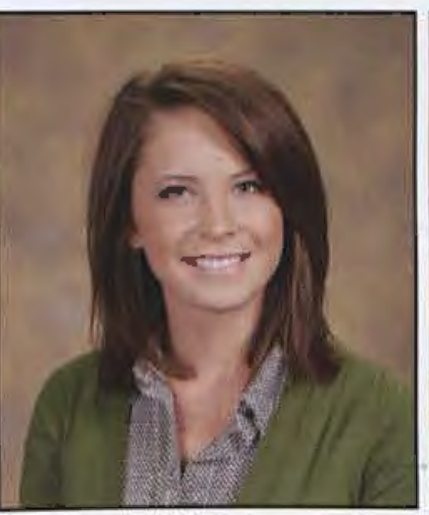

Courtney McHugh Graphic Design

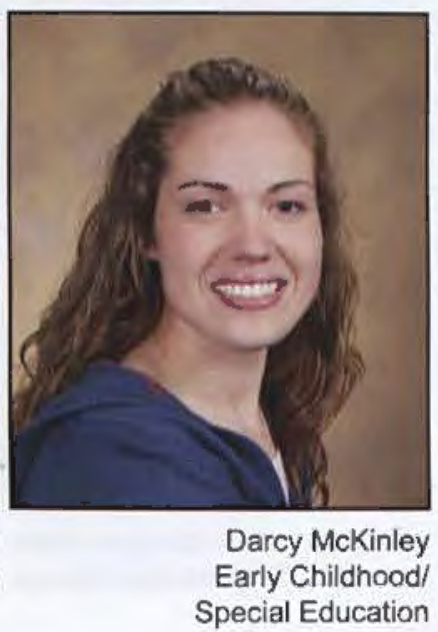

Heather McGee History

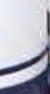

Special Education

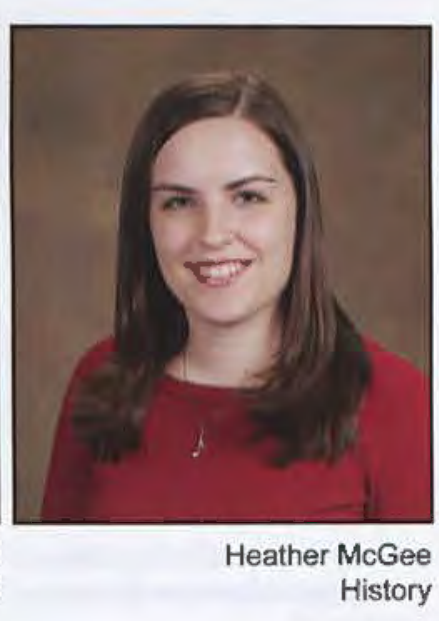




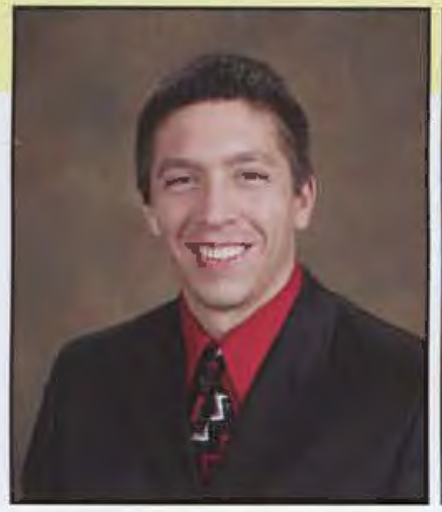

Nathan McLeese Mechanical Engineering

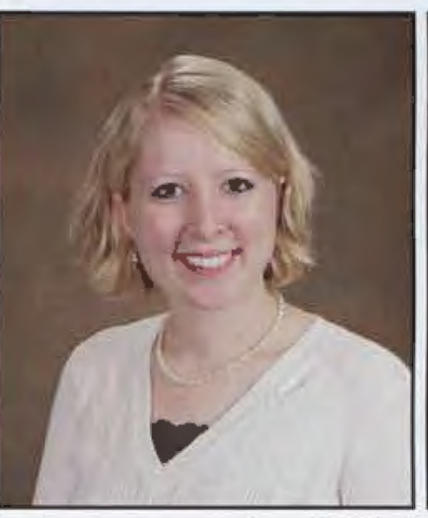

Lynn Mellum Keyboard Pedagogy

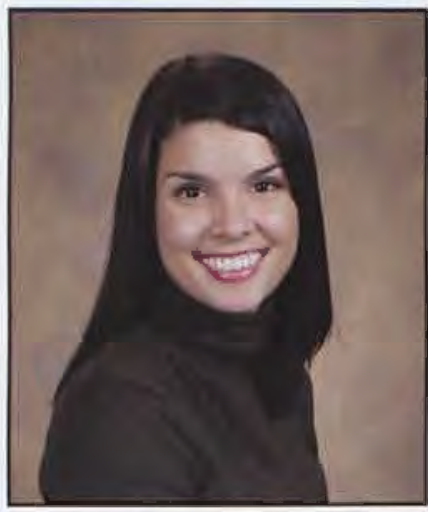

Sterling Meyers Comprehensive Communications

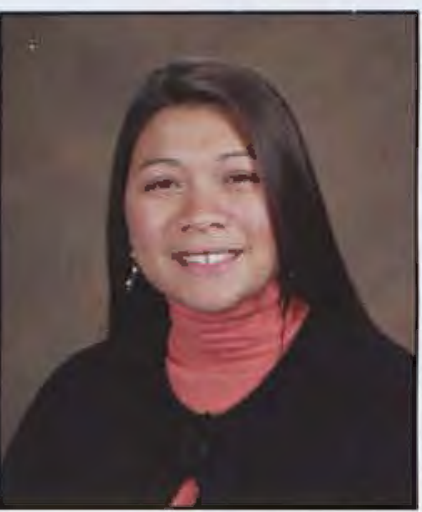

Kena Mena

Psychology

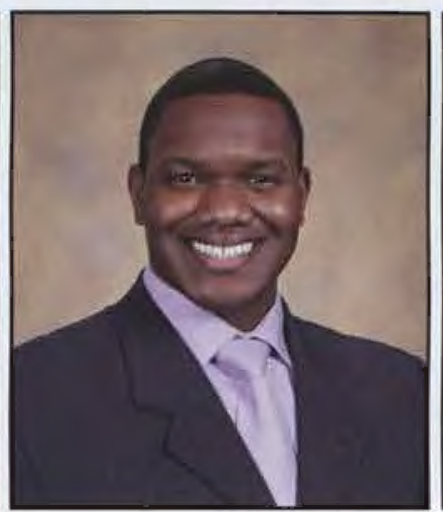

Andrew Mensah

Psychology

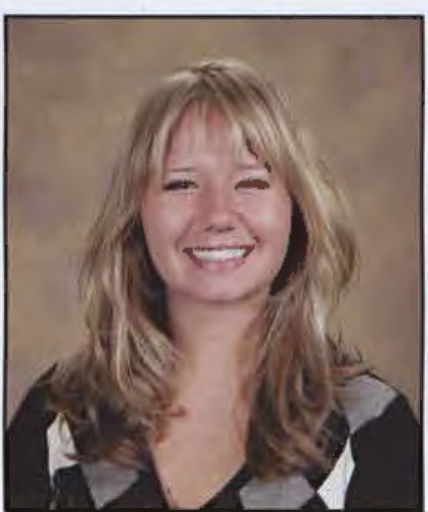

Heather Merrell

Psychology

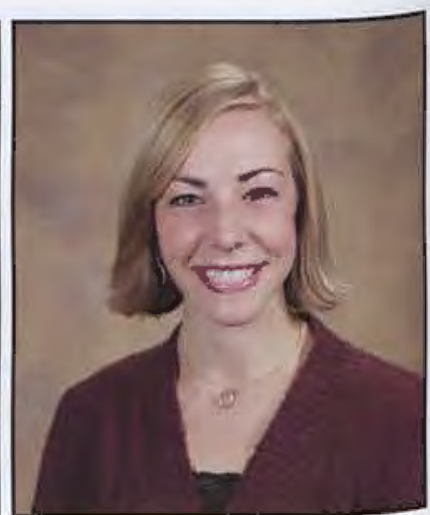

Megan Mertus Business Management

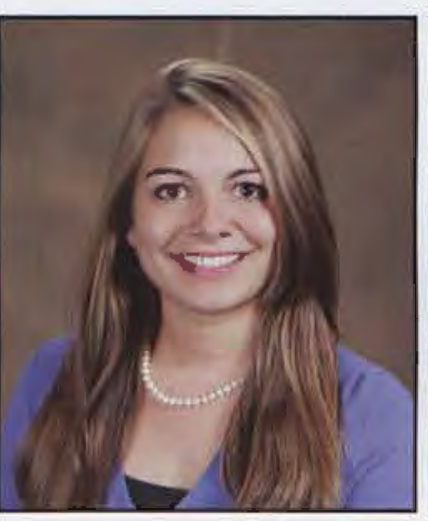

Hannah Mihm Nursing

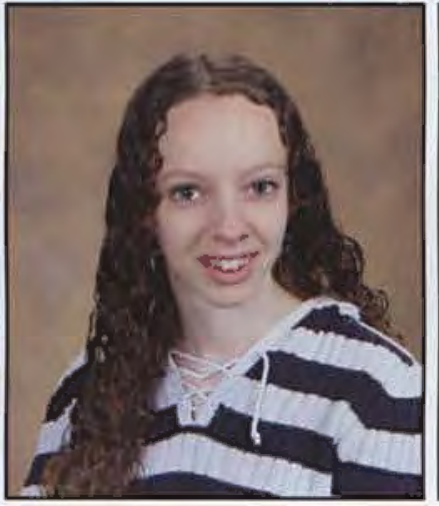

Becky Millar Nursing

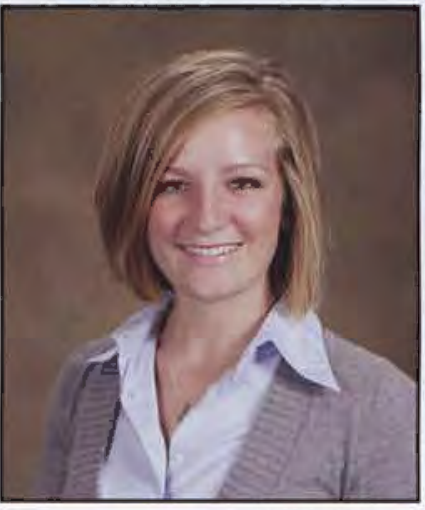

Emily Millay Graphic Design

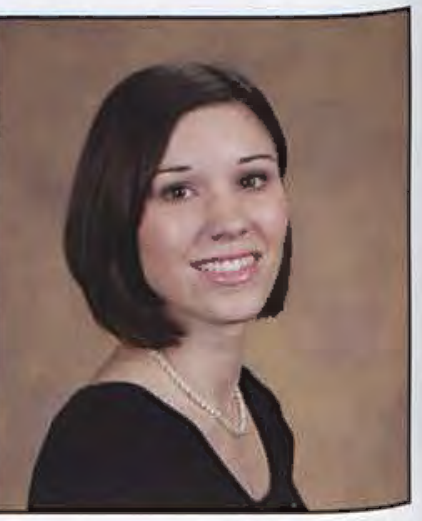

Bethany Miller Nursing

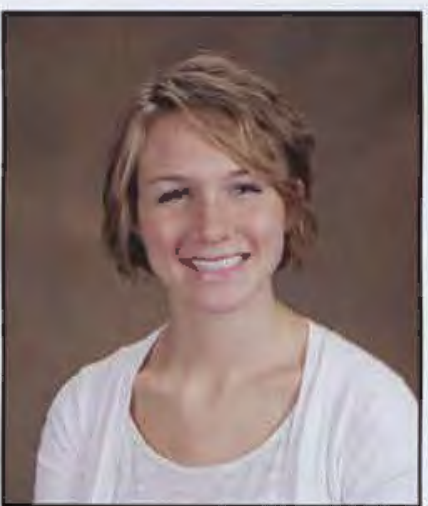

Christina Miller American Studies

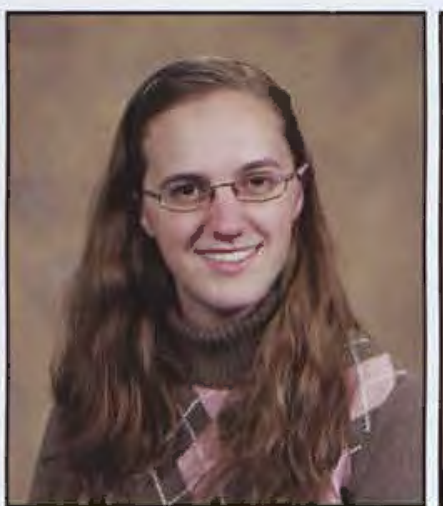

Kelly Miller

American Studies

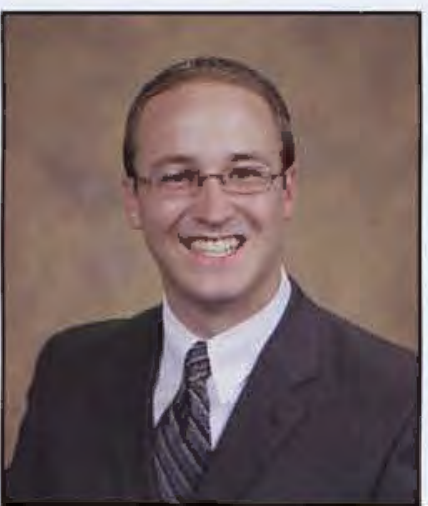

Mark Miller

Preseminary Bible/

Philosophy

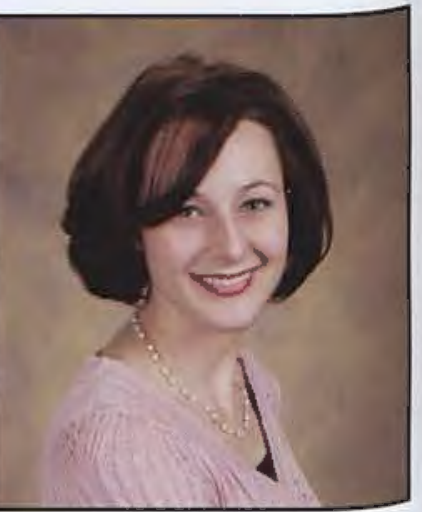

Rachel Milliman Early Childhood Education
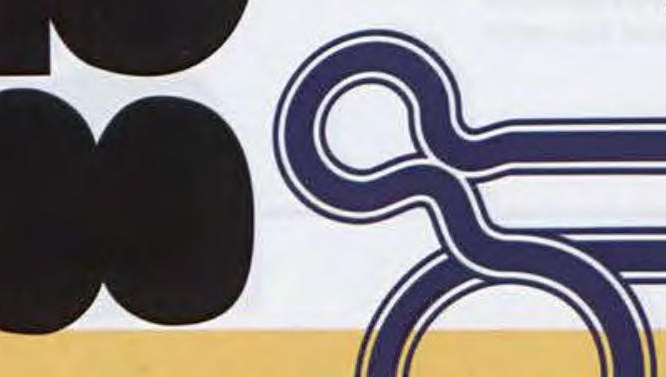


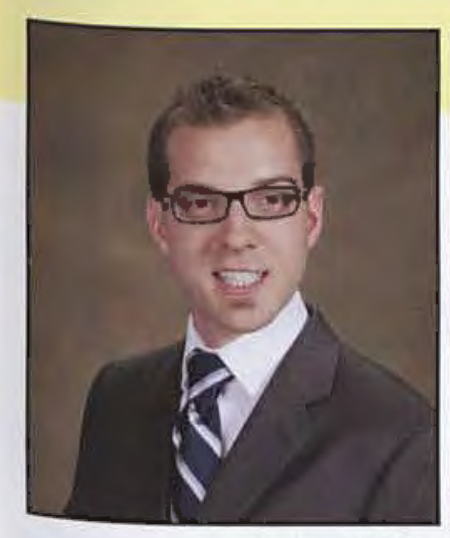

Joshua Mohler Exercise Science

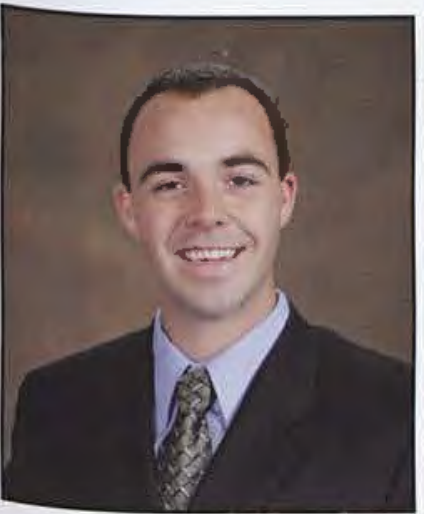

Stan Moran Music Education

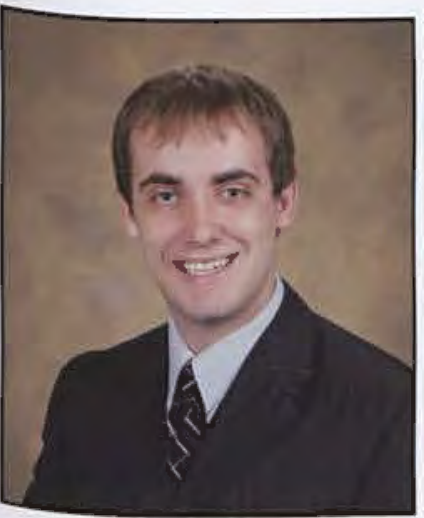

Joshua Nearhoof Biology

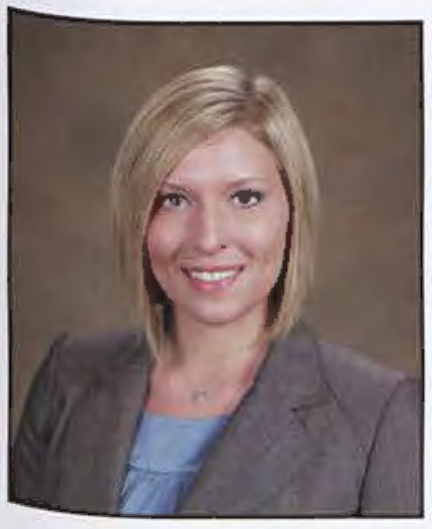

Sara Ney

Political Communication

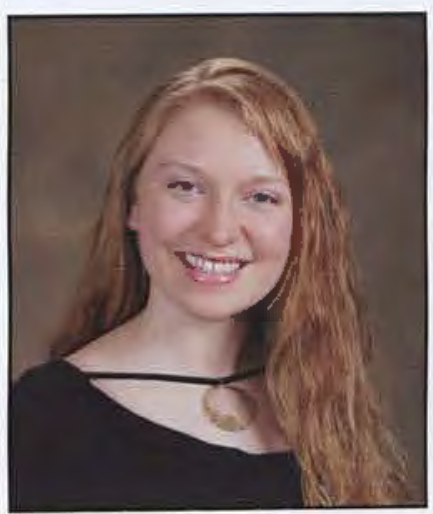

Alicia Moss Nursing

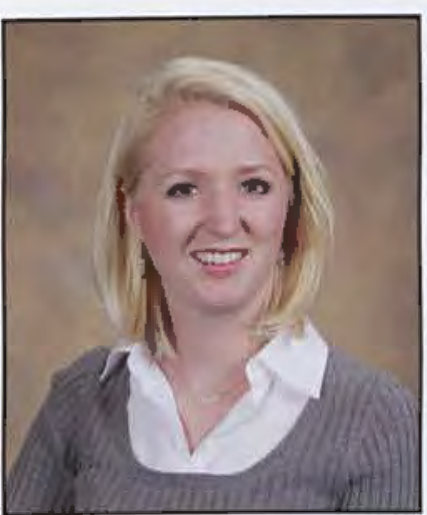

Annalise Nelson Nursing

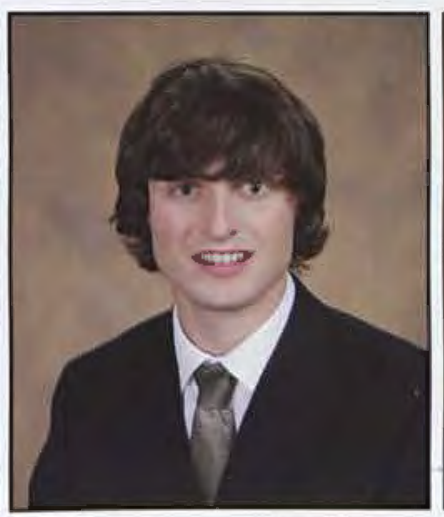
Emily Noble Sport Management

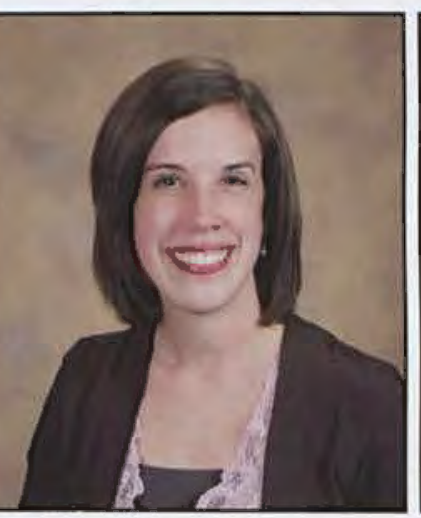

Megan Nelson Nursing

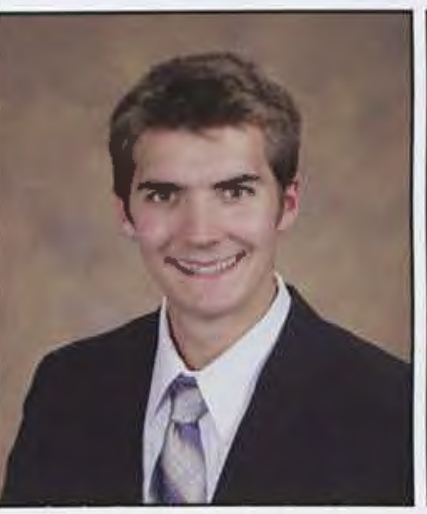

Jason Moss Accounting and Marketing

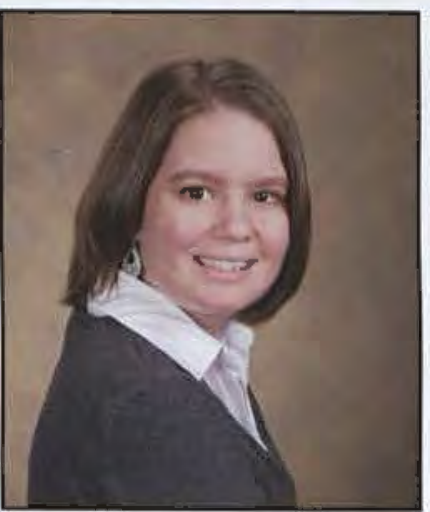

Micaela Neuhart Early Childhood Education

Laura Mullikin Social Work

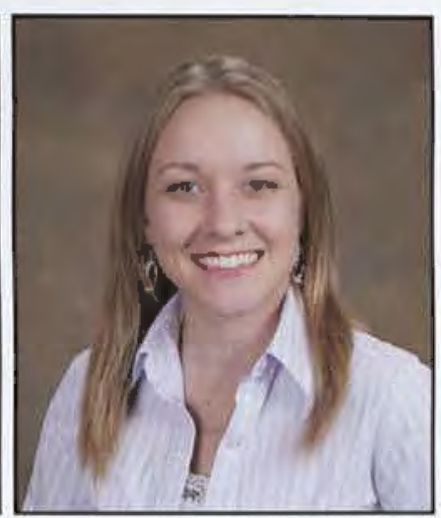

Danielle New-Day Business Management/ Accounting

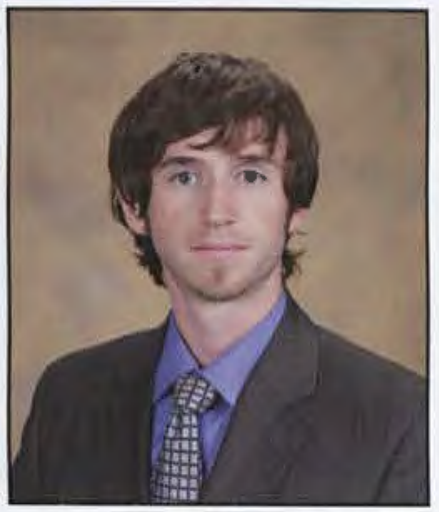

Bradley Muschott Electronic Media
Garrett Noble

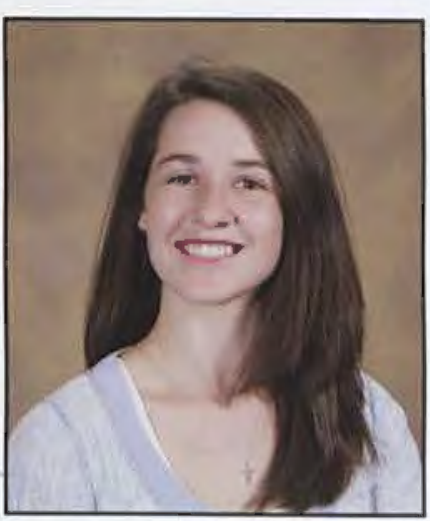

Nicole Normandeau Mechanical Engineering

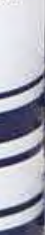




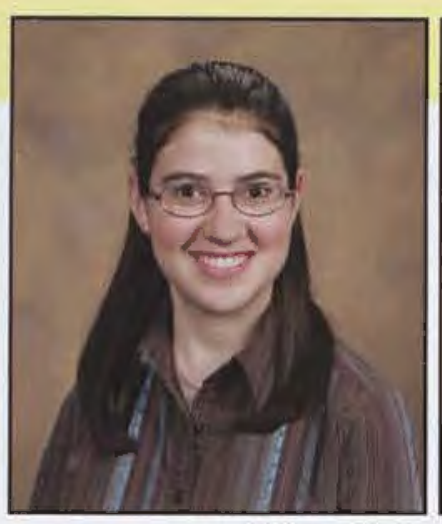

Brittany North Environmental Science

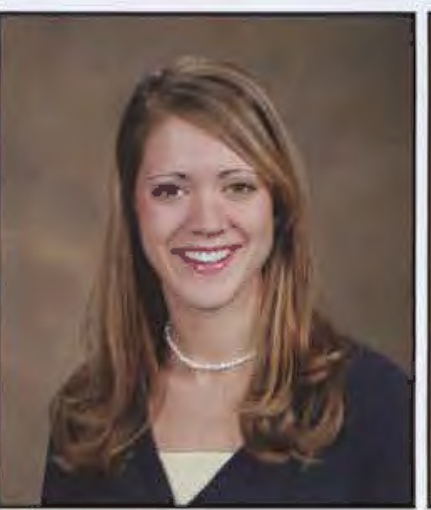

Christine O'Neal Exercise Science

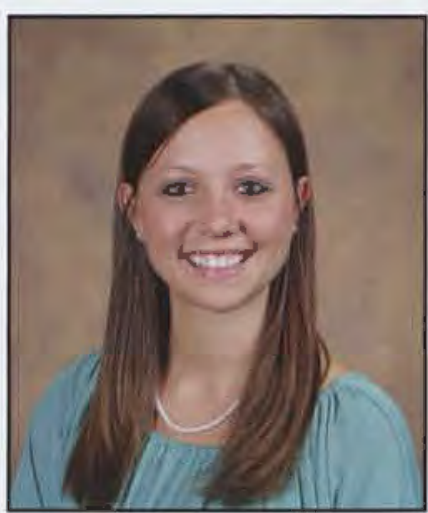

Cassandra Peletis Psychology

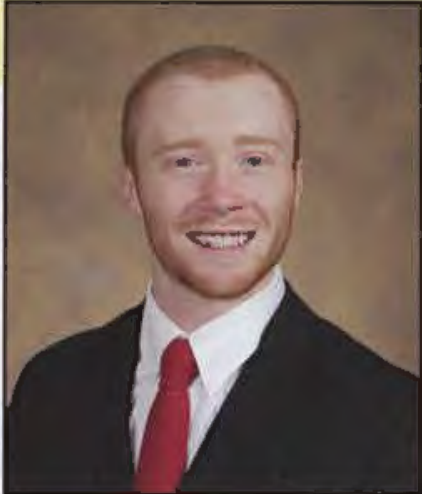

Kelly Nowels Media Communication

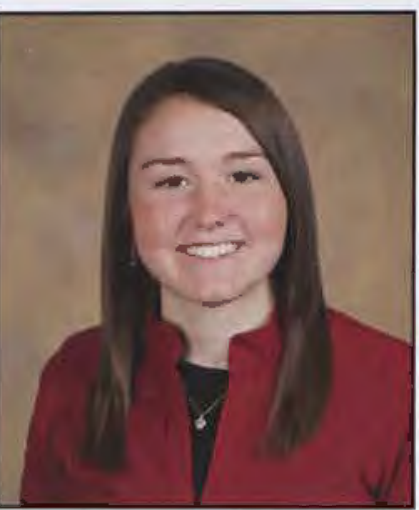

Melody Ortiz Early Childhood Education

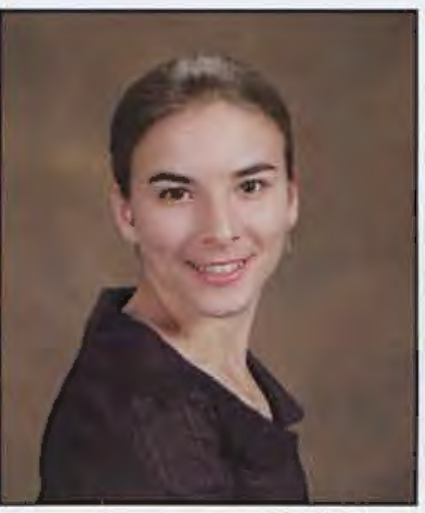

Karie Pepe

Biology

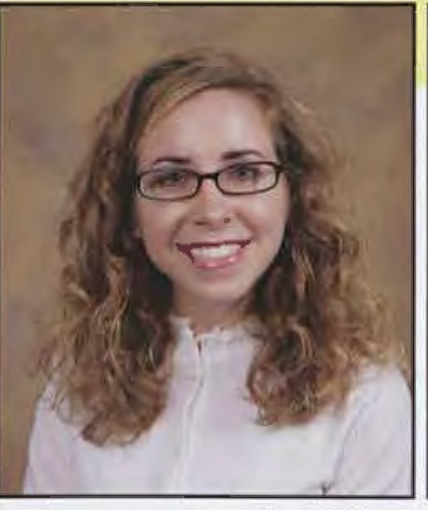

Lucy Olney Nursing

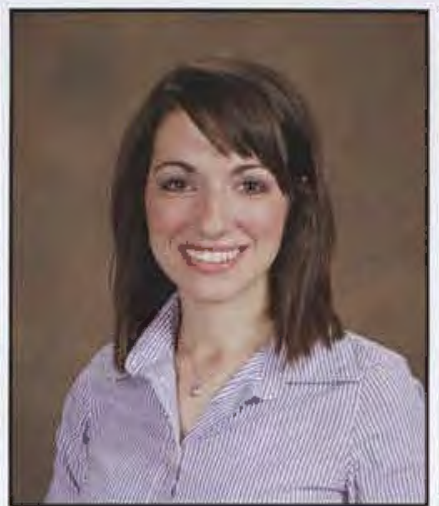

Krista Paoloni Exercise Science

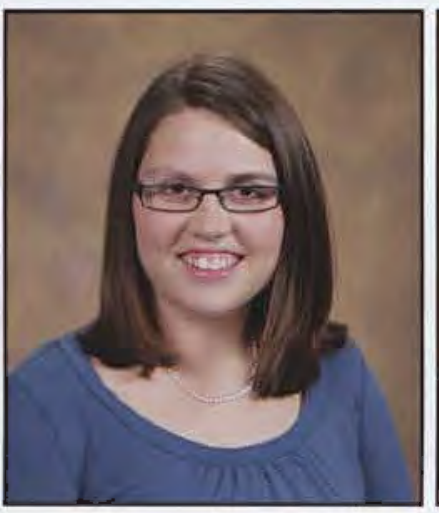

Kelly Perrel Social Work

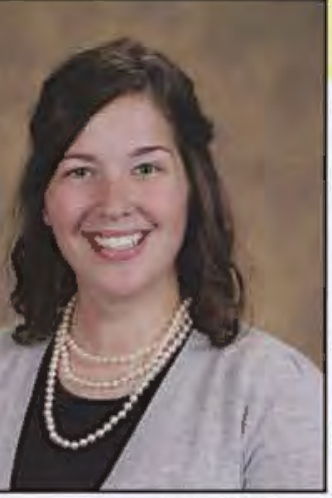

Rebekah Olsen integrated Langauge Arts Education

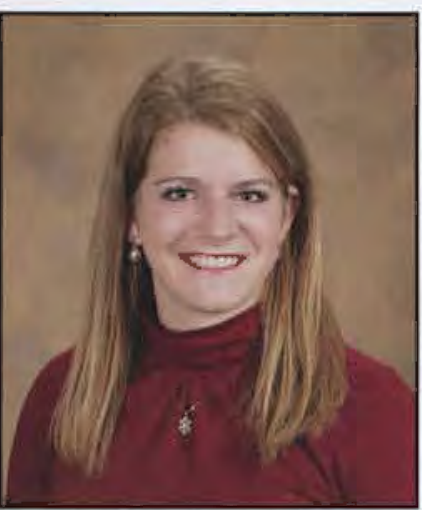

Laura Parker

Nursing

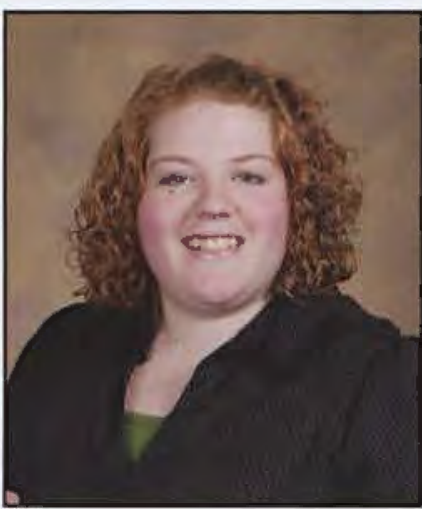

Rebecca Perry Psychology

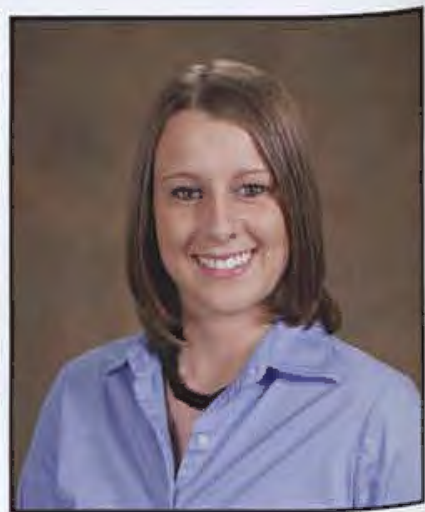

Stacie Peden Sociology Public Administration

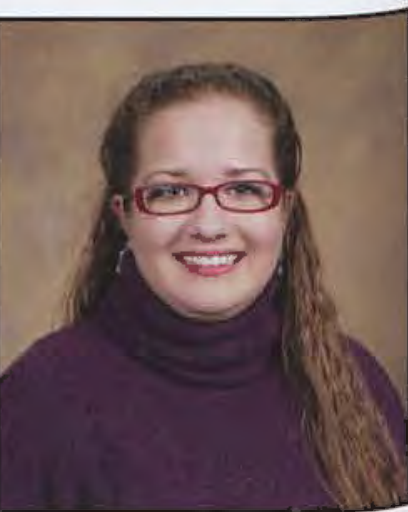

Trisha Peters Integrated Langauge Arts Education

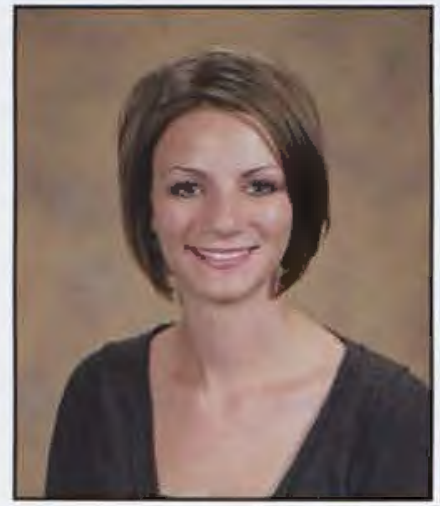

Andrea Phillips Nursing

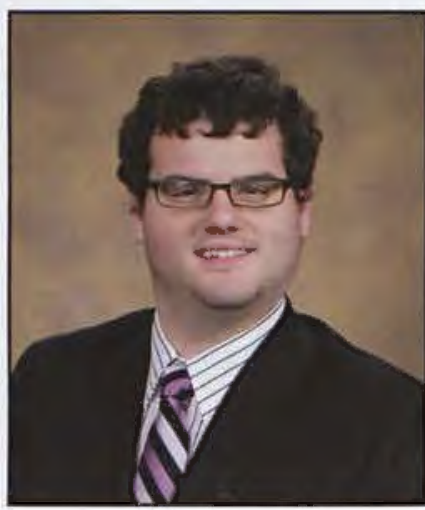

Drake Philyaw Accounting

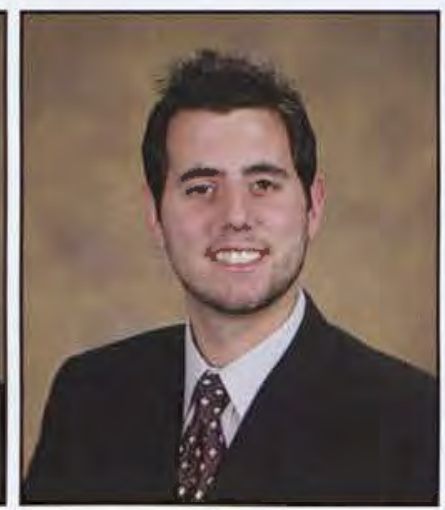

Kyle Plante Criminal Justice

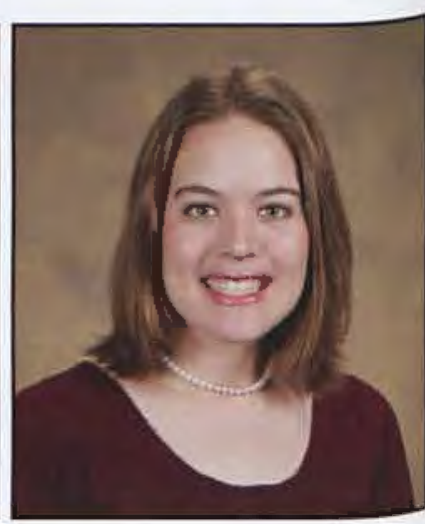

Laura Polston Middle Childhood Education

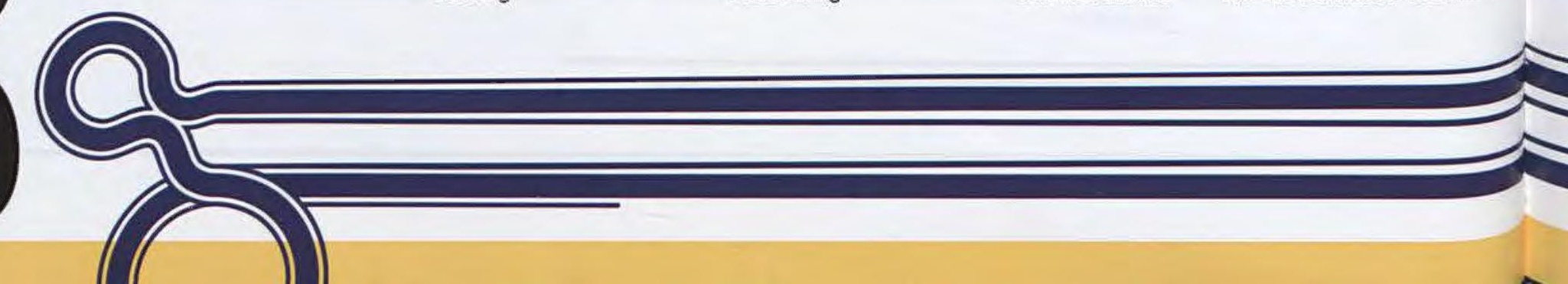




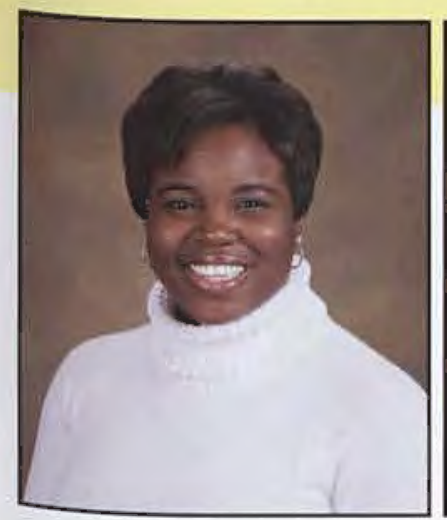

Charmaine Porter Organizational Communications

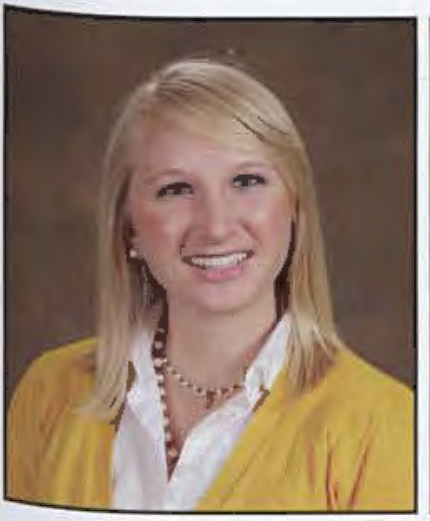

Elisabeth Price

Christian Education/Youth Ministry

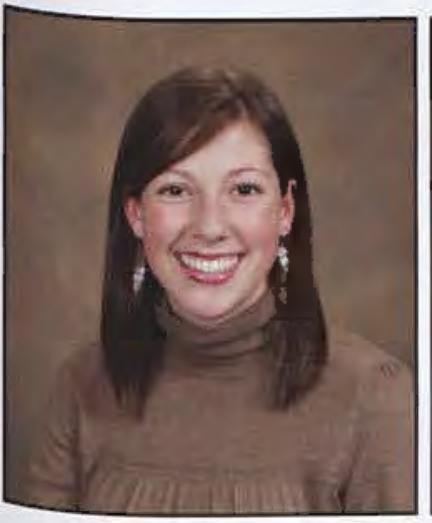

Jessica Quaid

Sociology

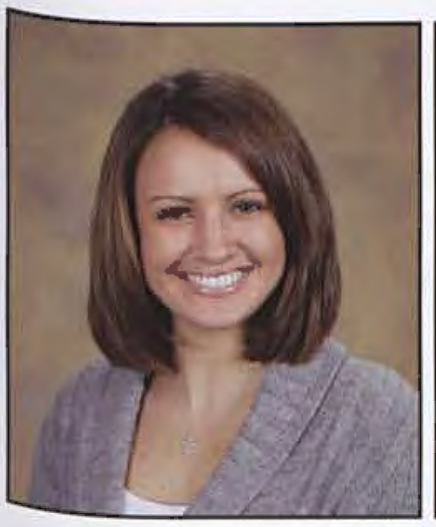

Rachel Reed

Nursing

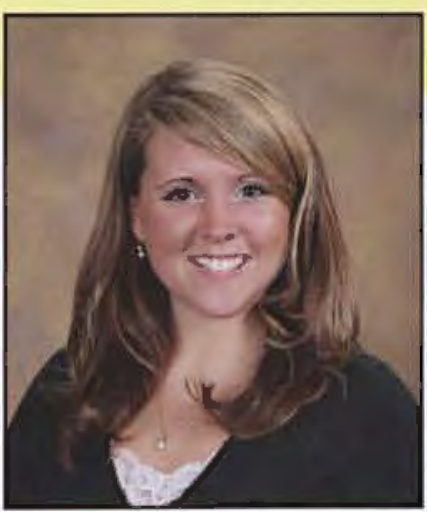

Jennifer Powell

Nursing

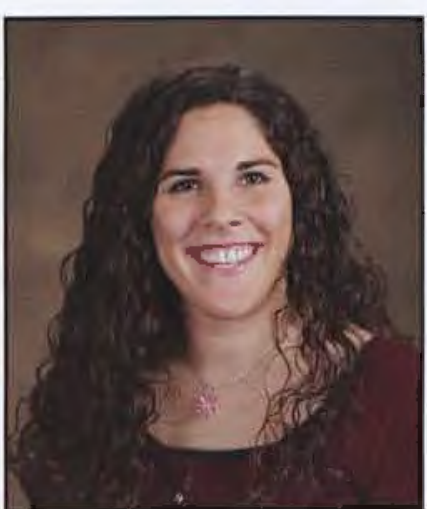

Lisa Beth Prins

Electronic Media

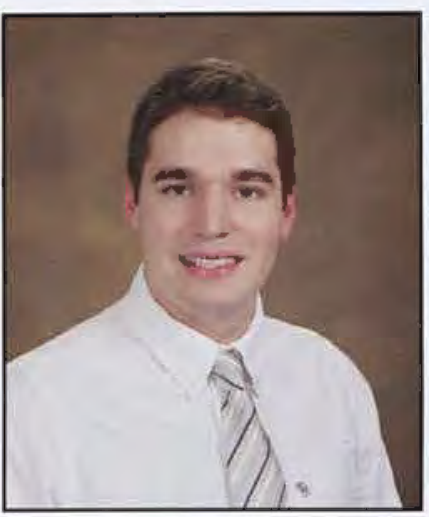

Jonn Rafoss

Business Management

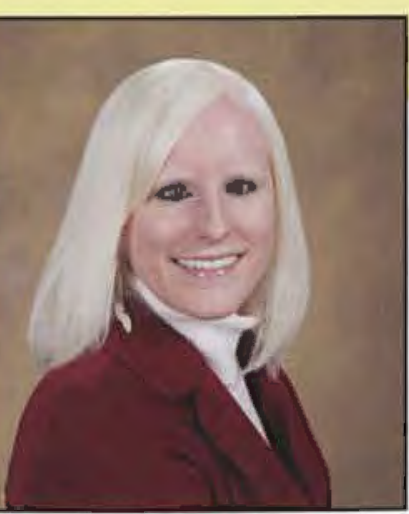

Amy Price

Communication Arts/ Spanish

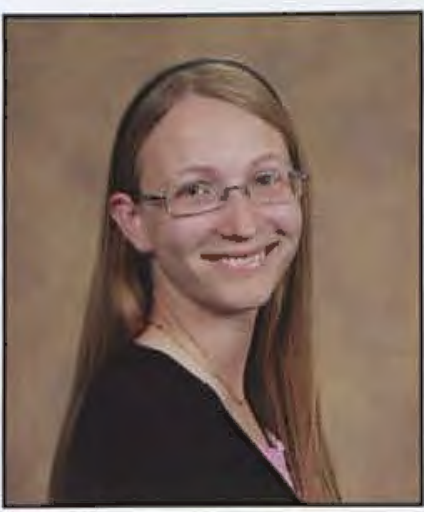

Pamela Proctor Froensic Science

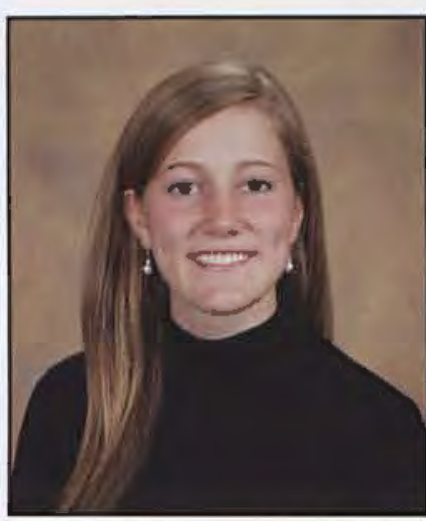

Amy Rambiku Early Childhood Education

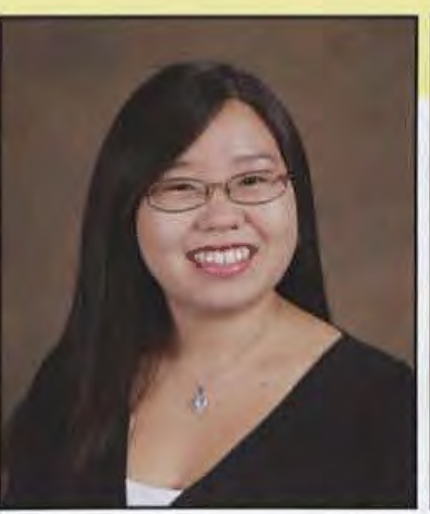

April Price English

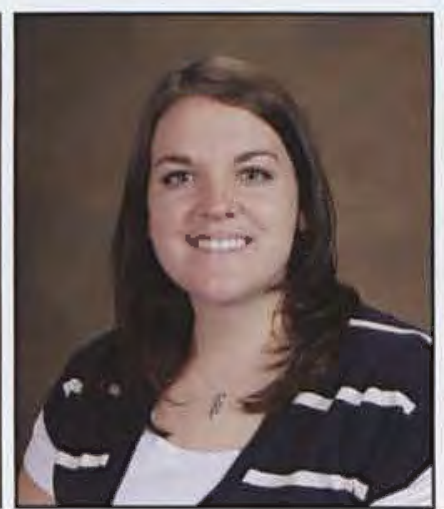

Anna Pyles

Middle Childhood Education

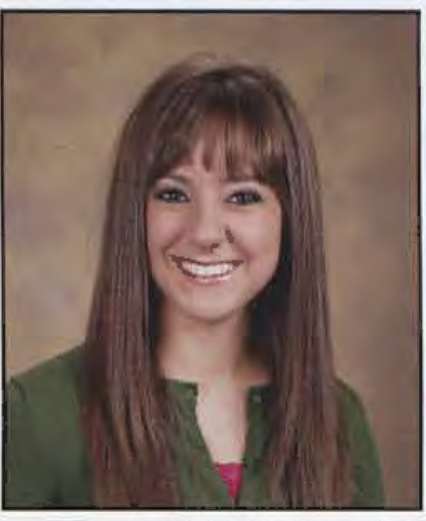

Brittaney Randolph Middle Childhood Education

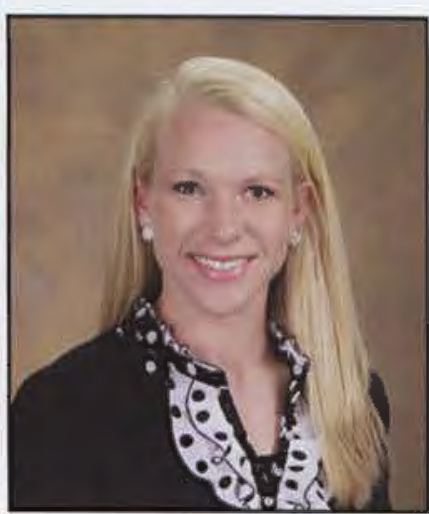

Elisabeth Pyles

Early Childhood Education

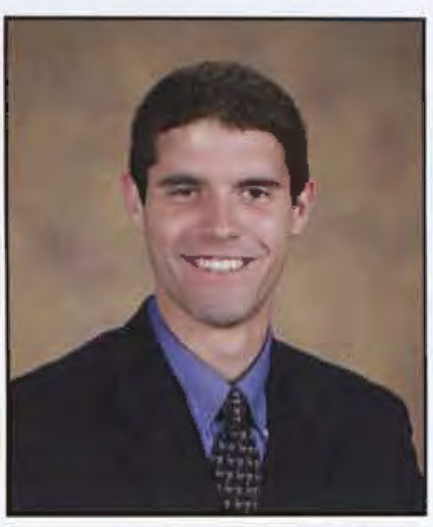

Mark Ratliff Integrated Math Education

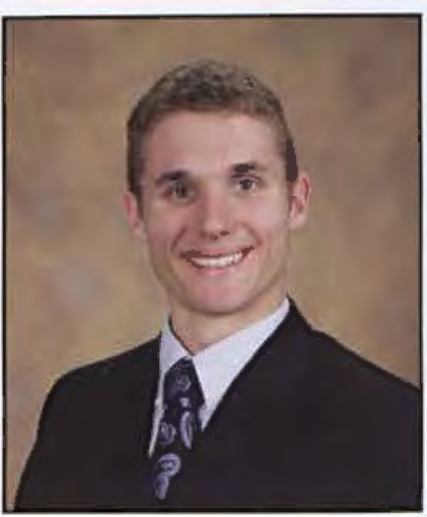

Corey Reeder International Studies/Missiology

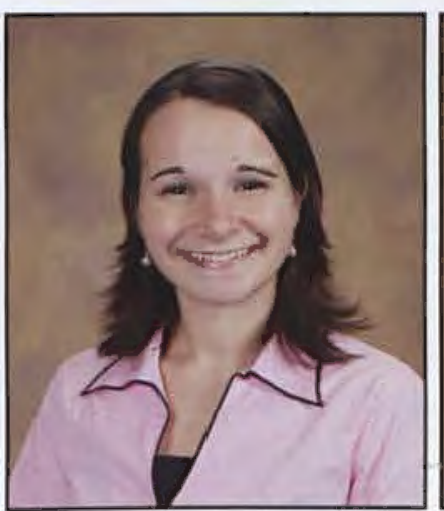

Jillian Reeder International Studies/Missiology

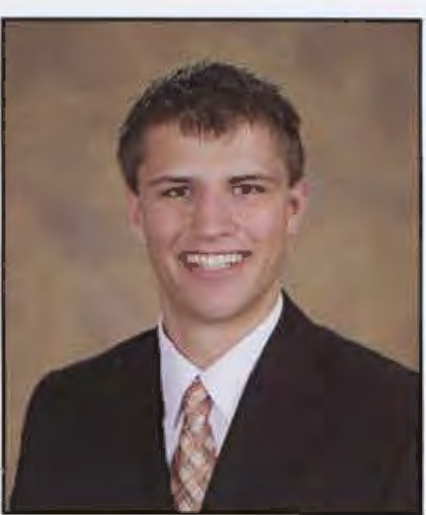

Ryan Reep Mechanical Engineering

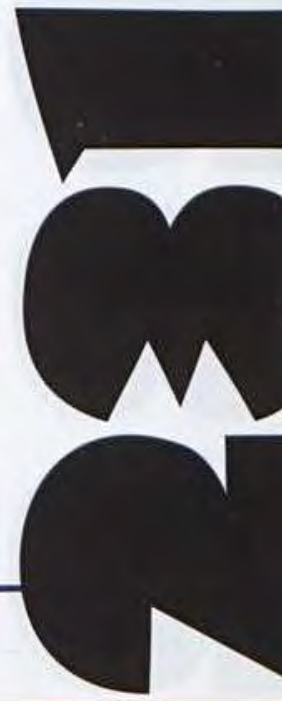




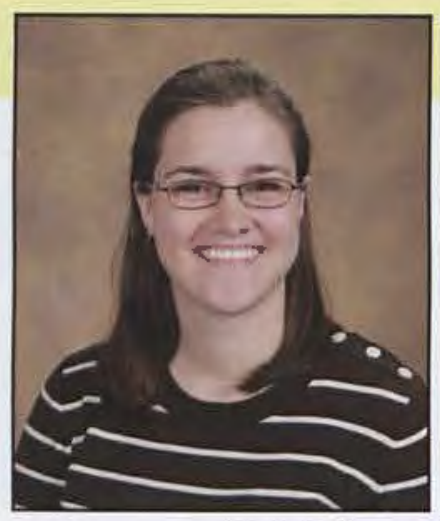

Krista Reilmann Middle Childhood Education

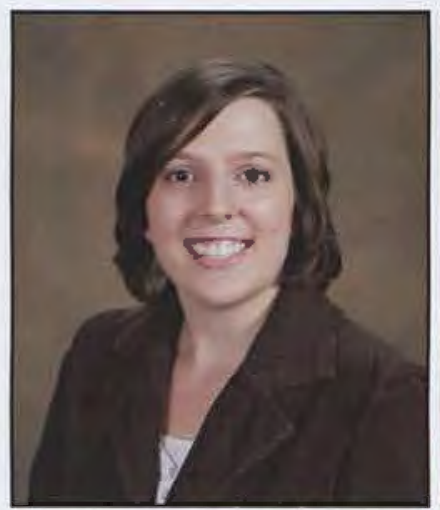

Danielle Robertson Nursing

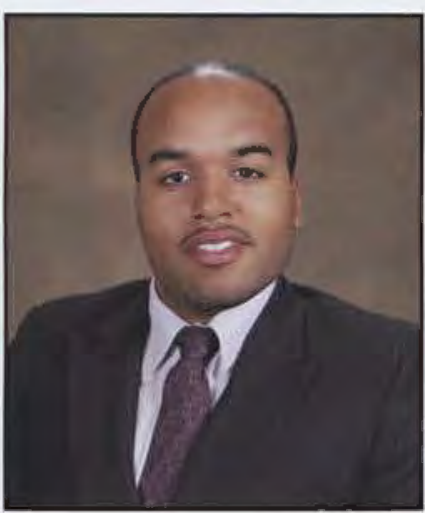

Eber Rosario Spanish

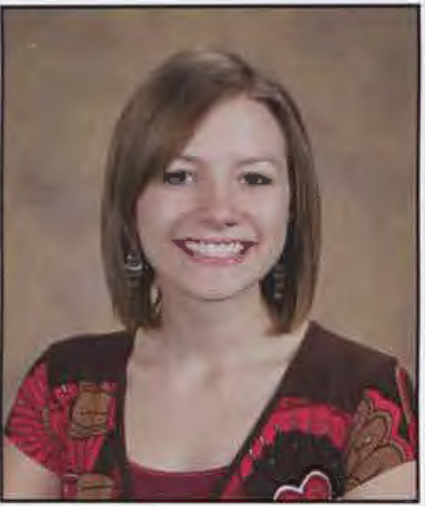

Amanda Roebuck Preseminary Bible

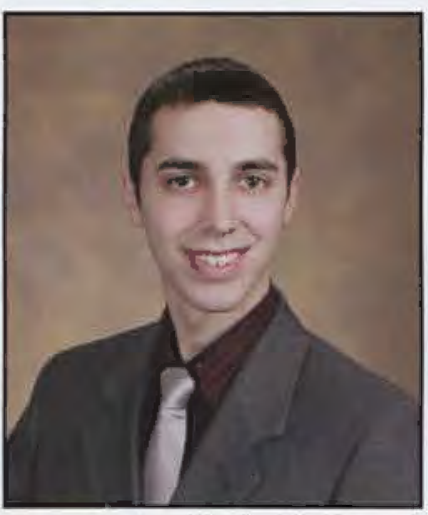

Benjamin Roscup Technical and Professional Communication

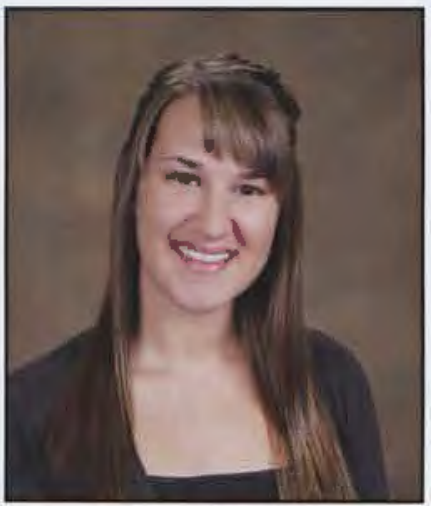

Emily Sammons Music Education

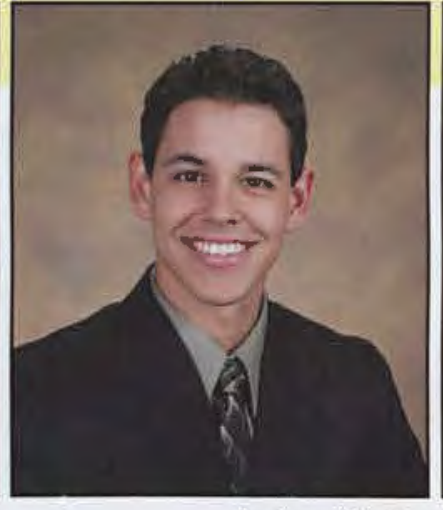

Andrew Renke Youth Ministry

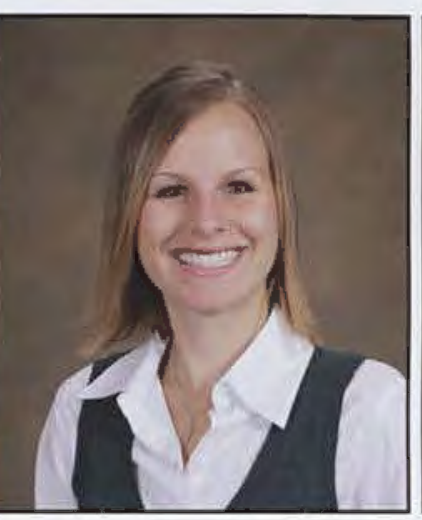

Amanda Roller Comprehensive Bible

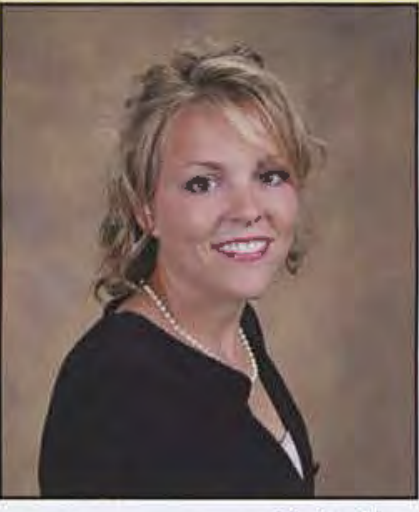

Marla Rice Biology

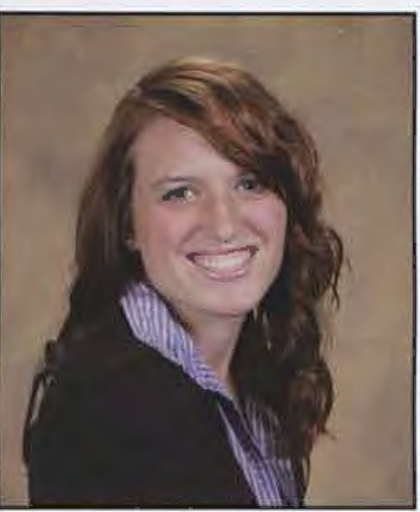

Michelle Romage Psychology

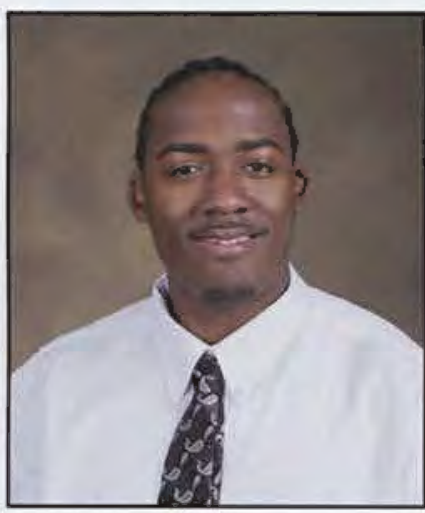

Daniel Rose Business Management

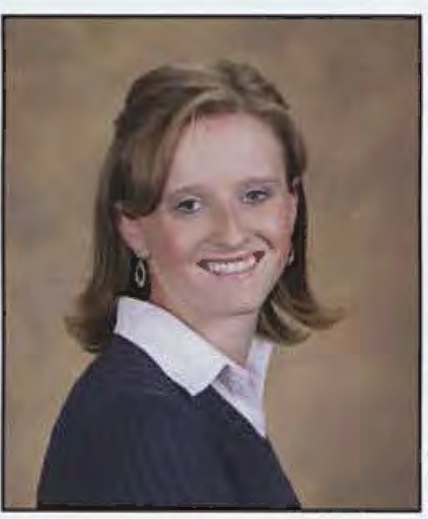

Rachel Ross Political Science

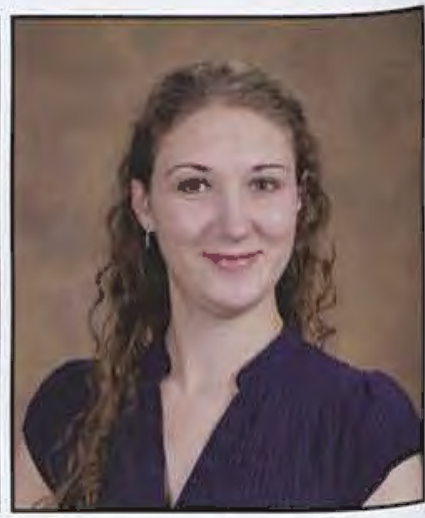

Kristina Ronan International Studies Theatre Design

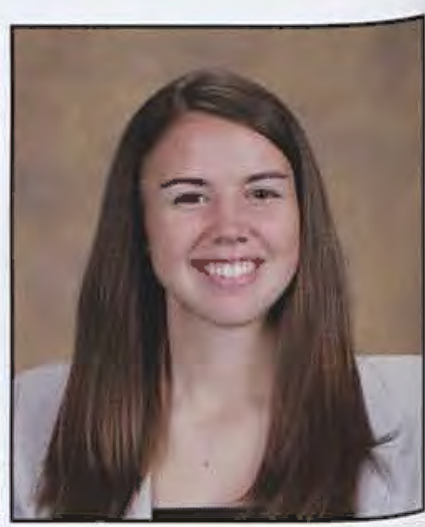

Charissa Rowe History-Political Science Pre-Law

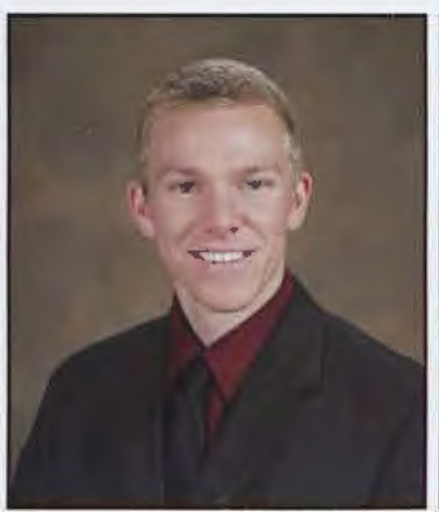

Ryan Samuelsen Mechanical Engineering

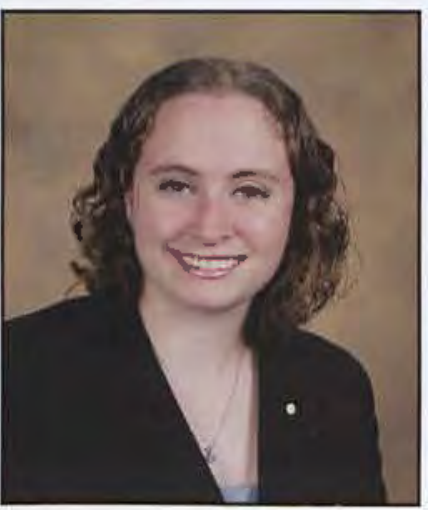

Angela Sanders Political Science

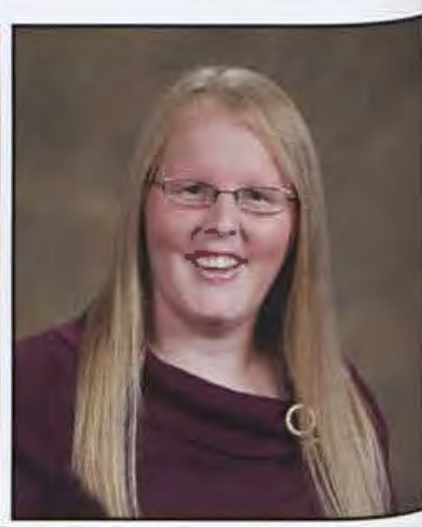

Emily Sandlund Nursing
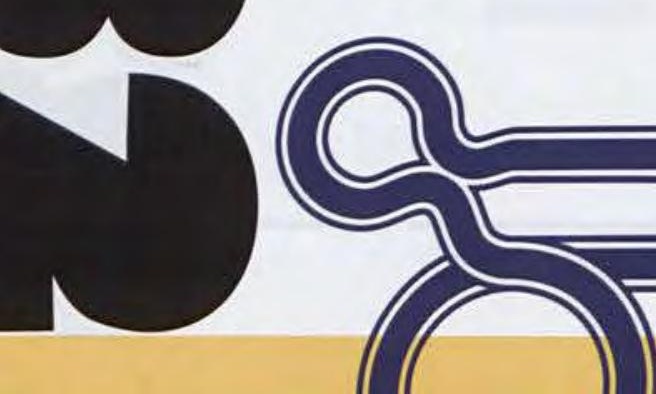


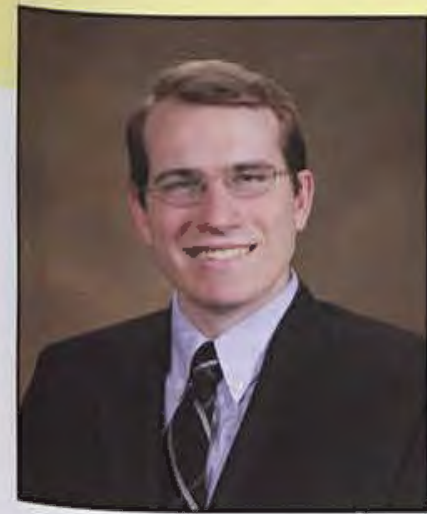

Timothy Sanford Pastoral Studies

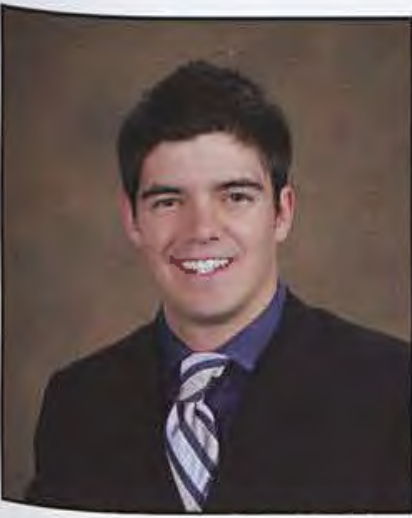

Bradley Schellin Integrated Social Studies Education

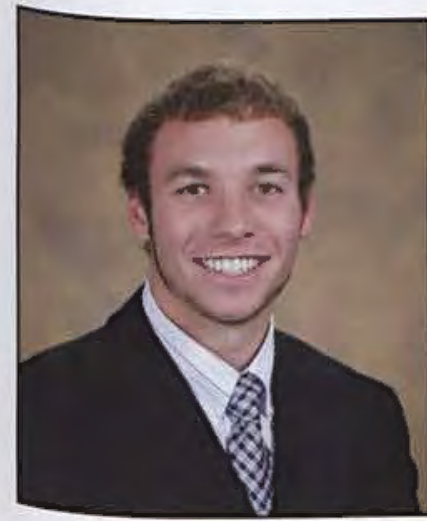

Tyler Scott

Finance

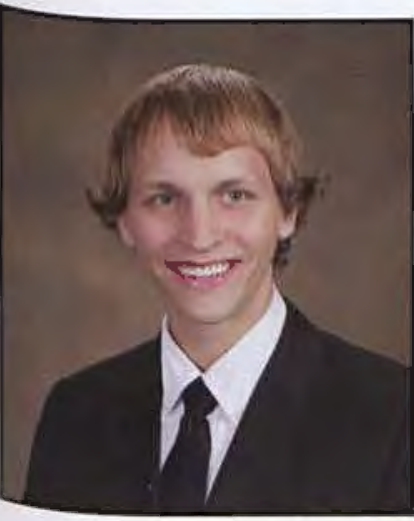

Nathan Sheppard Electronic Media

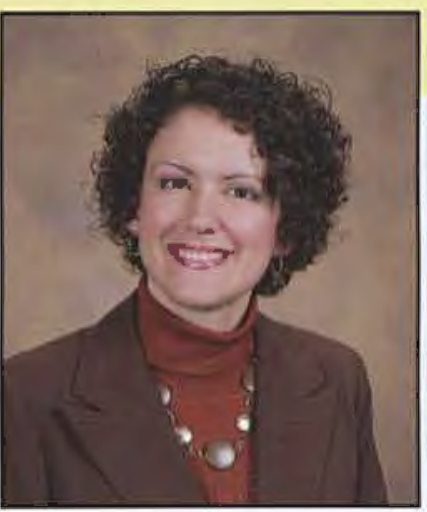

Erin SanGregory

Technical and Professional

Communication

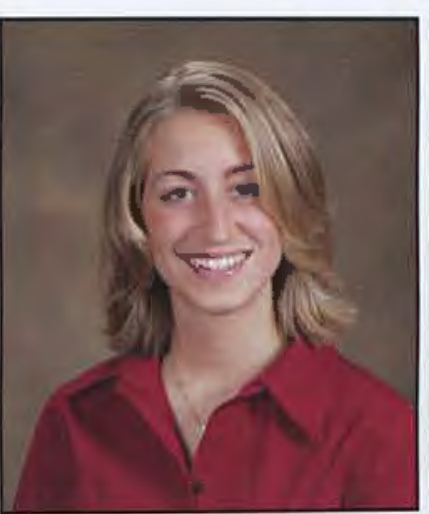

Jessica Schlener

Nursing

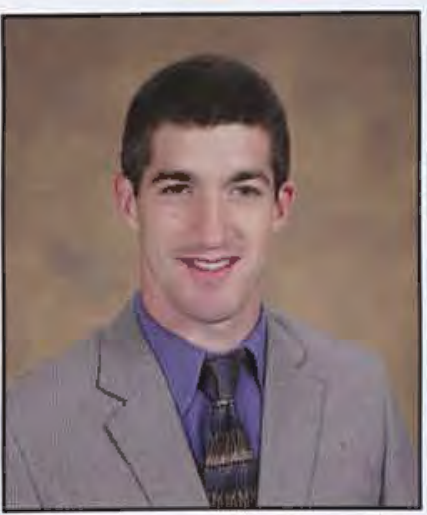

Jordan Seibert Criminal Justice

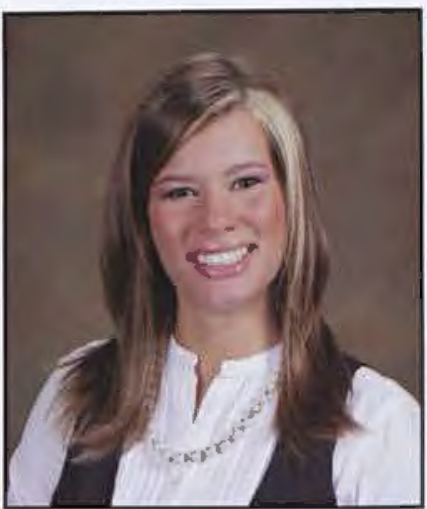

Rachel Sherwood Social Work

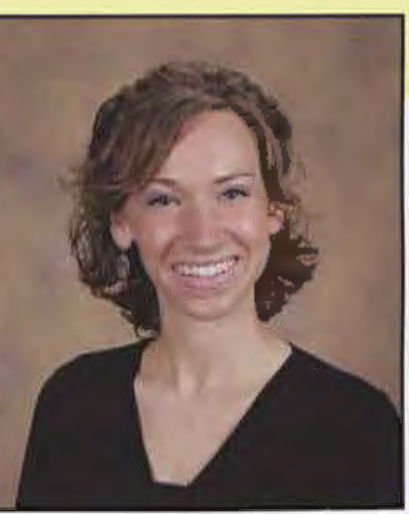

Nicole Santos

Nursing

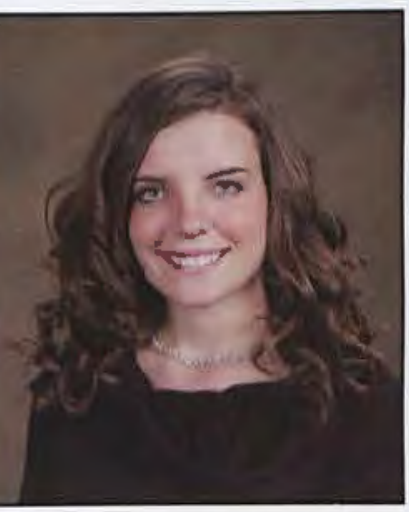

Tiffany Schlueter Nursing

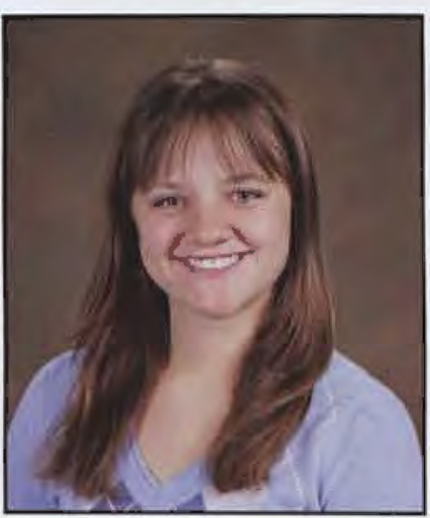

Catherine Shanks

Mechanical Engineering

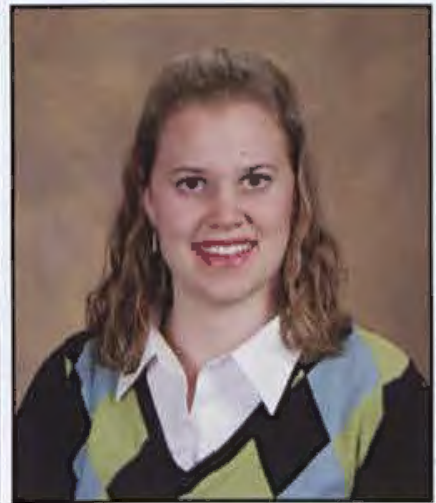

Rachel Shinabarger

Nursing

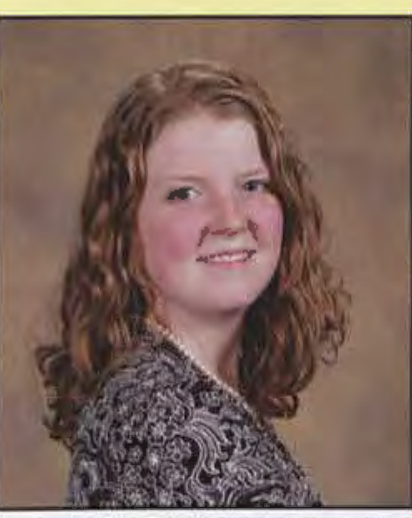

Kaitlin Sattler Special Education

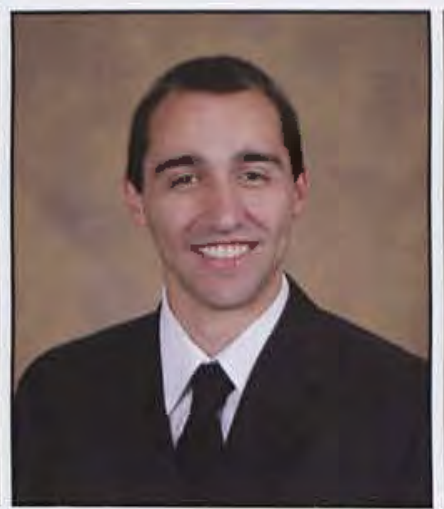

David Schoen Biology

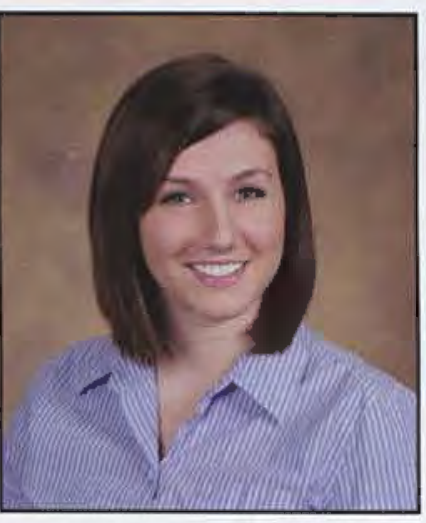

Sarah Sheers

History/Politcal Science

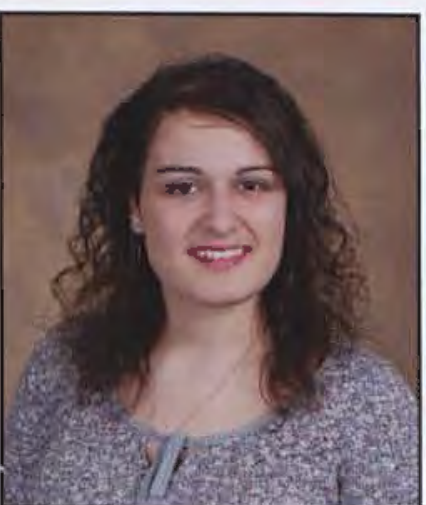

Rebekah Shomper Early Childhood Education

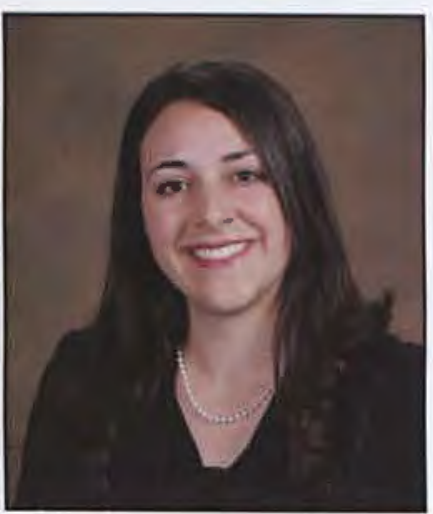

Jessica Scott Biology

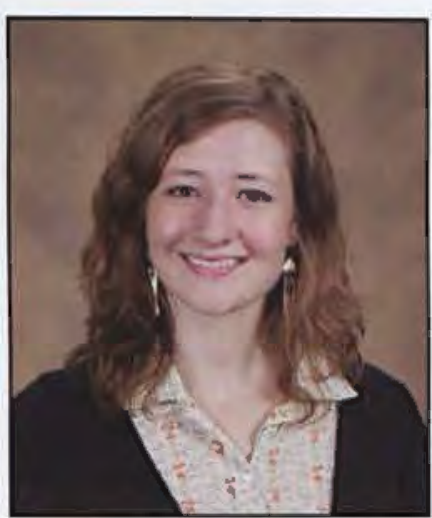

Juliana Shepherd International Studies/ Social Science

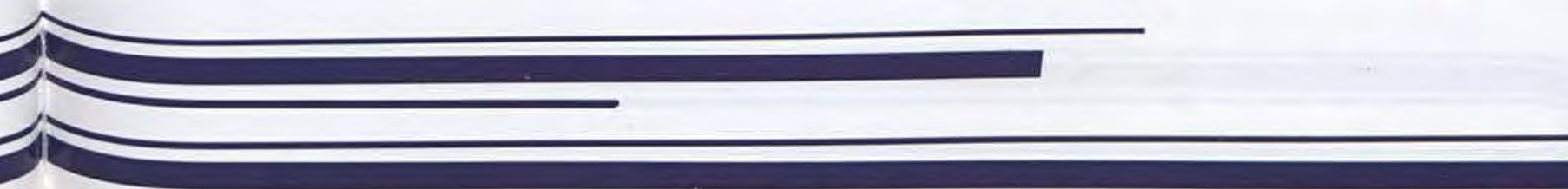




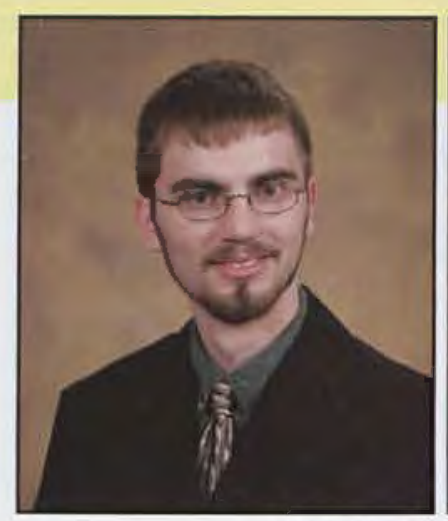

Mikal Shorts Mechanical Engineering

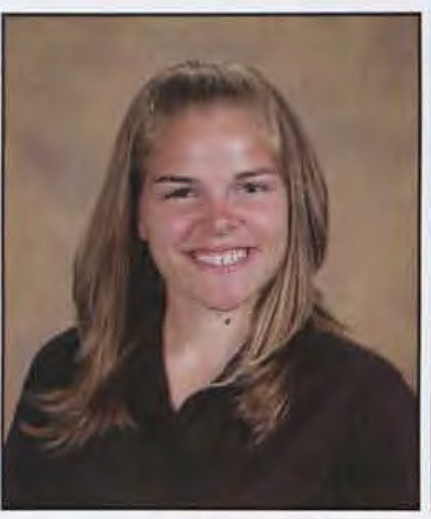

Brittany Simpson Early Childhood Education

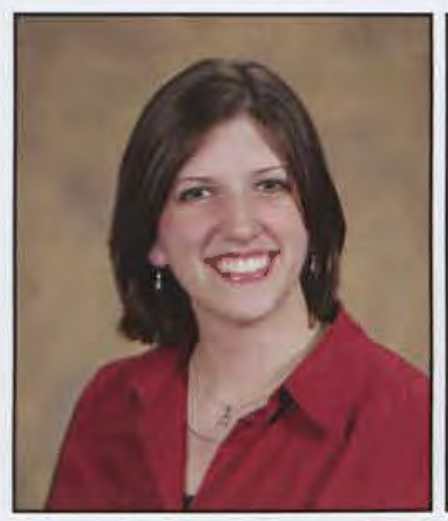

Elisabeth Small Psychology

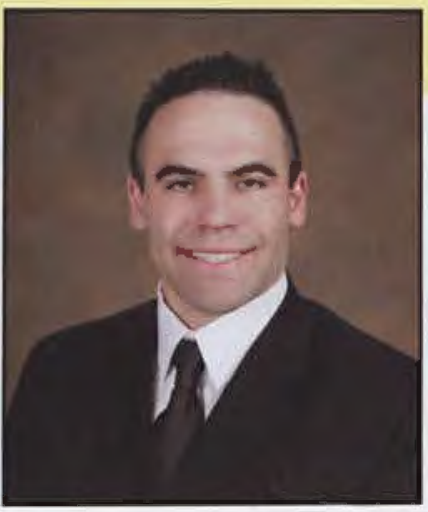

Andrew Shriber Mechanical Engineering

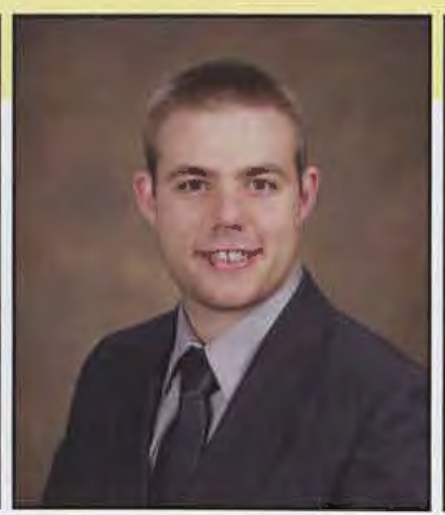

Jordan Siefkes Sport Management

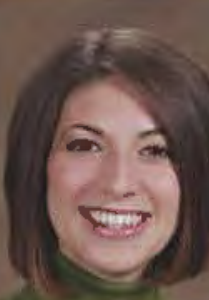

Bethany Sierawski

Nursing

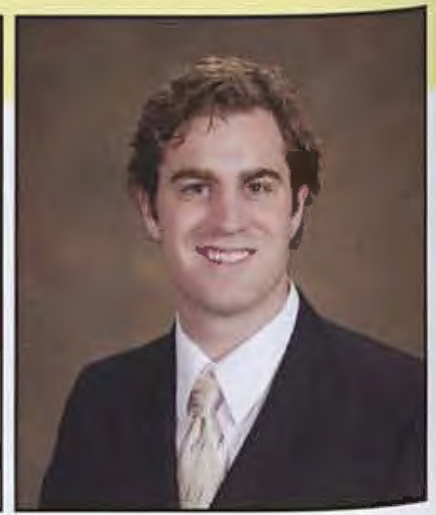

Trenton Simon

Mechanical Engineering

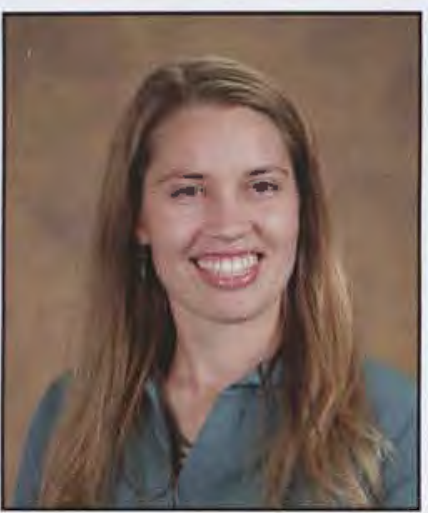

Grace Sims Athletic Training

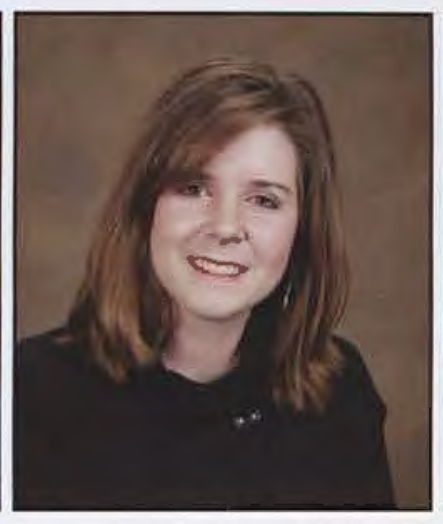

Kelly Sims Early Childhood Education

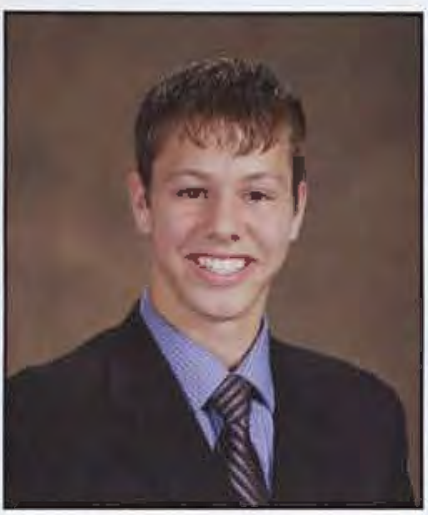

Benjamin Sincock Church Music Ministries

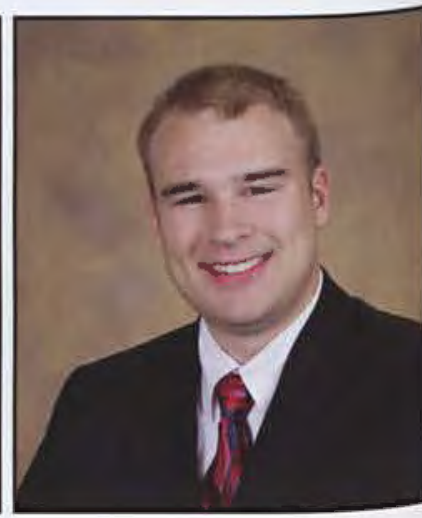

Timothy Sloan Physics

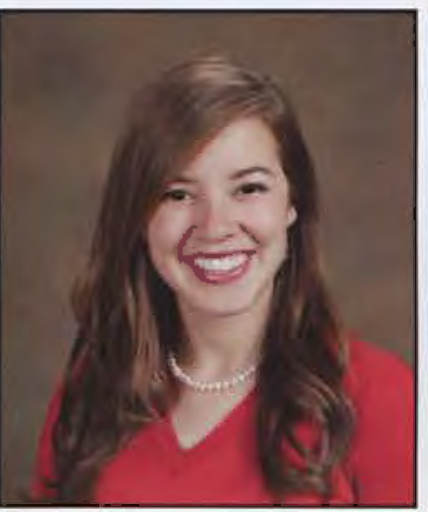

Stephanie Small Biology

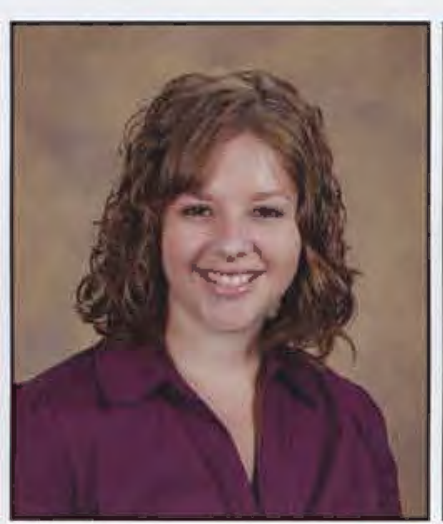

Amanda Smith Nursing

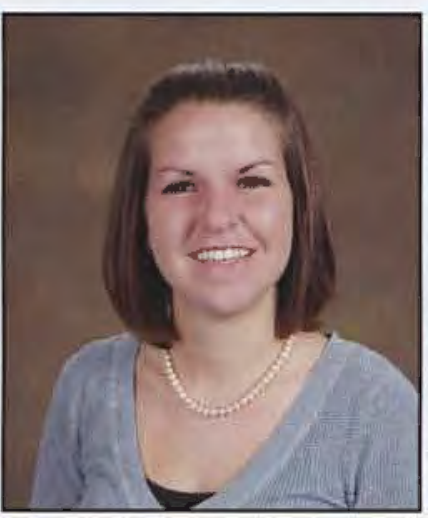

Amie Smith

Global Economics and International Busniess

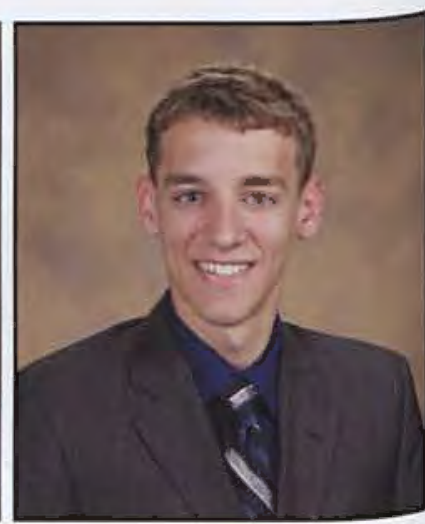

Eric Smith Computer Science

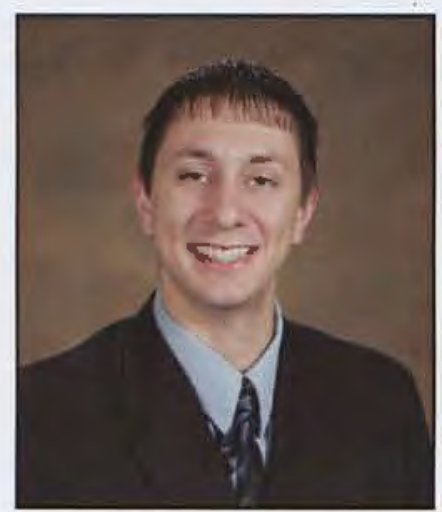

Jacob Smith Biology

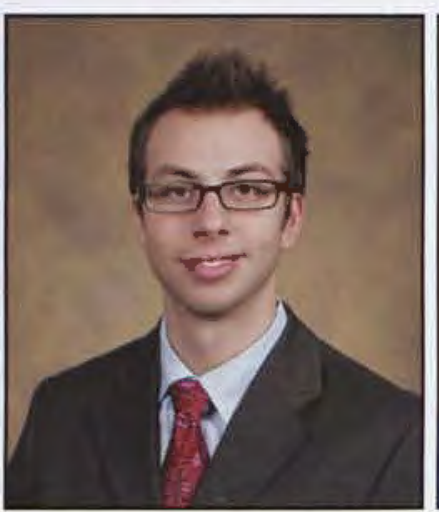

Kyle Smith Graphic Design

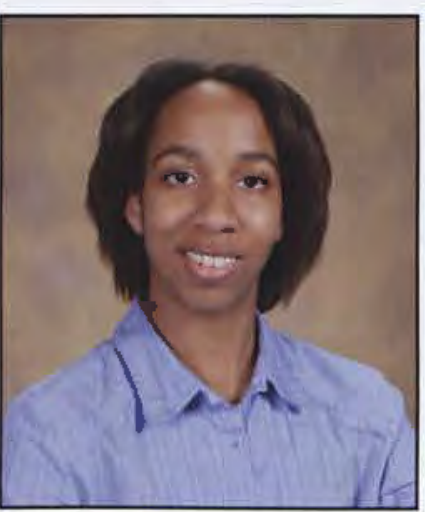

Lydia Smith Electronic Media

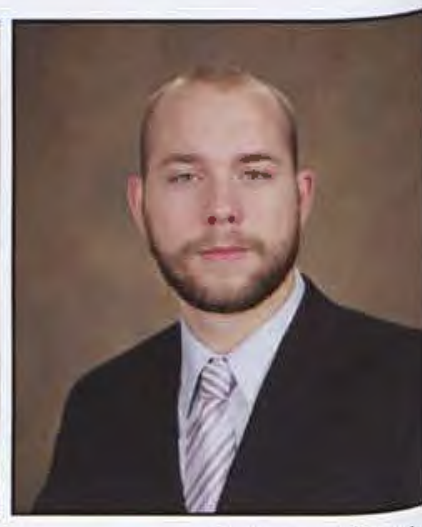

Nathanael Smith Multi-Age Special Education 


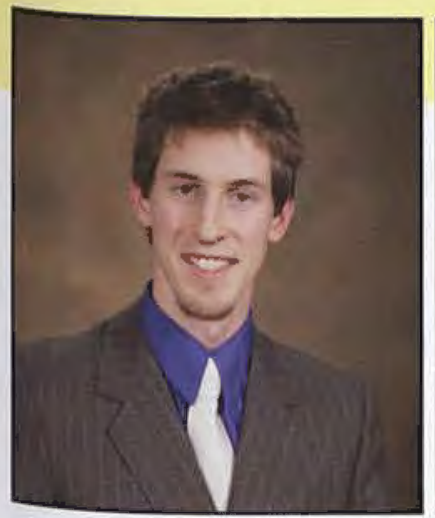

Peter Smith

History

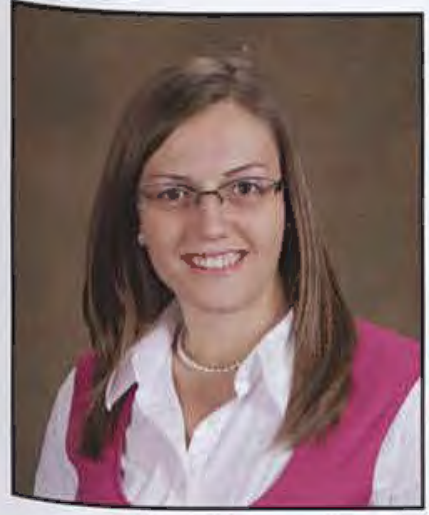

Stephanie Stahl Finance

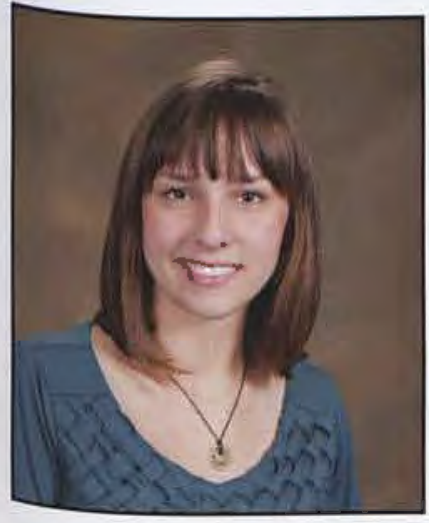

Charis Steffe Social Work

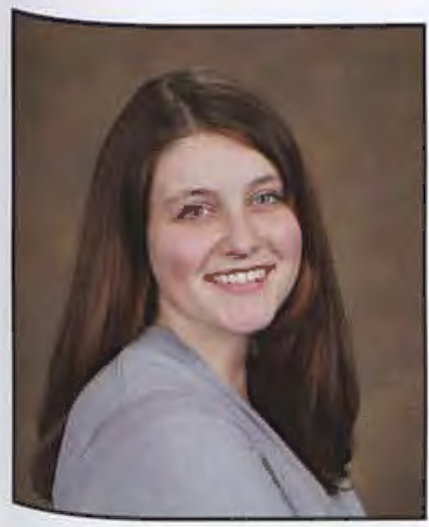

Ashleigh Stewart Integrated Language Arts/

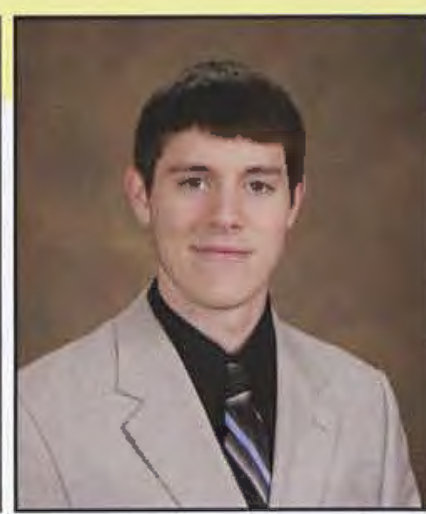

Ryan Smith Exercise Science

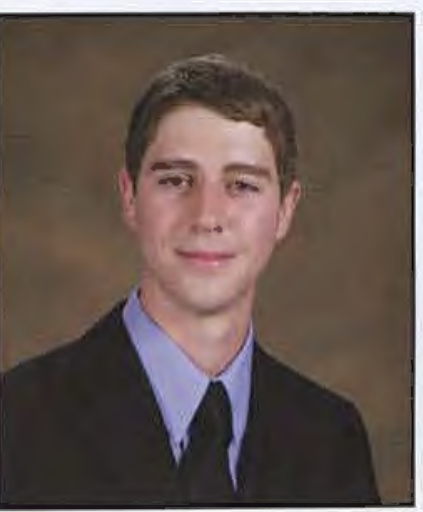

Aaron Stauffer Electronic Media

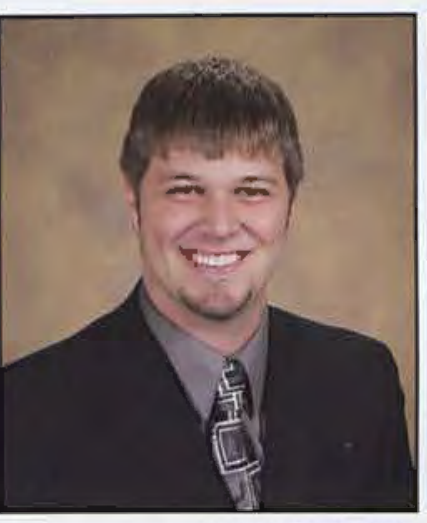

Jarrod Steinmetz Integrated Math Education

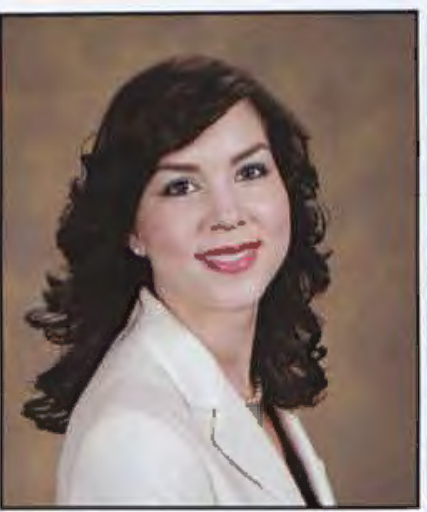

Erica Stikeleather Electronic Media

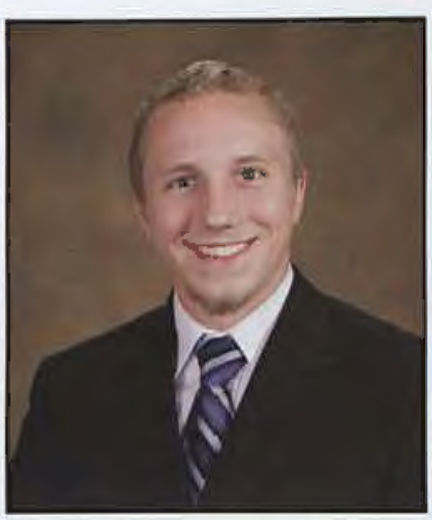

Jeremy Steckel Media Communications

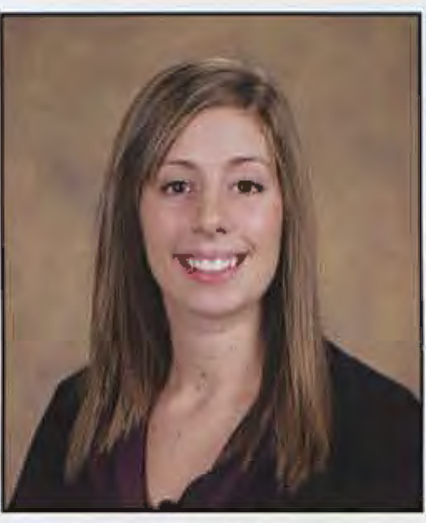

Jessica Steinmetz

Early Childhood Education

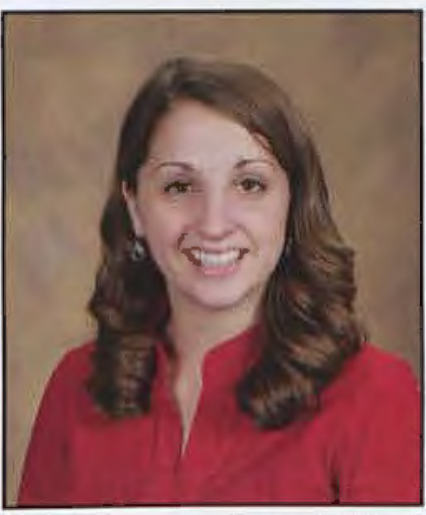

Amanda Stock

Early Childhood Education

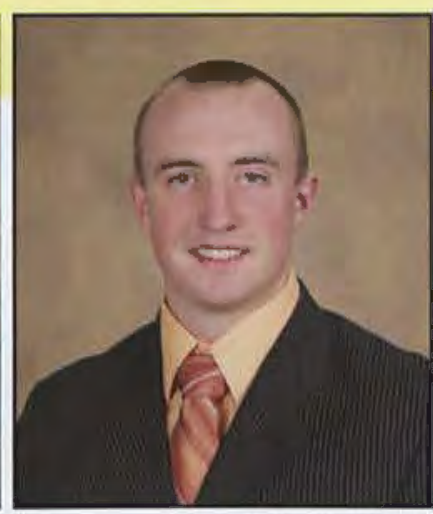

Timothy Smith Criminal Justice

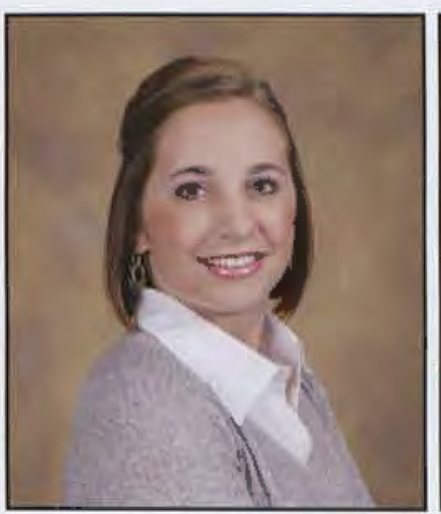

Meghan Steele Social Work

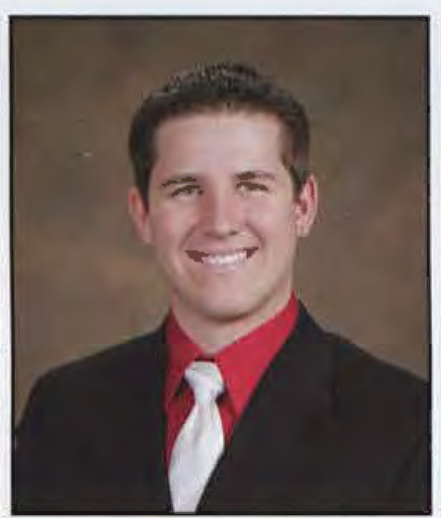

David Stephens Electronic Media

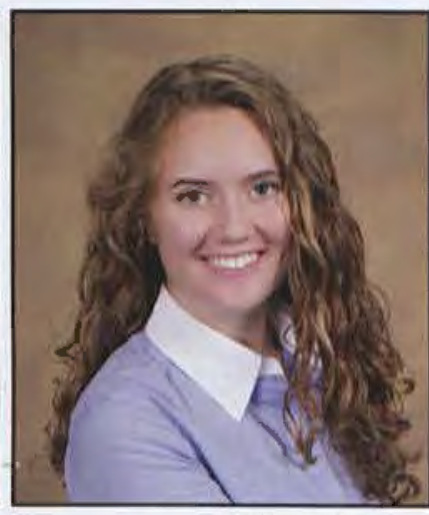

Macy Straton Mathematics

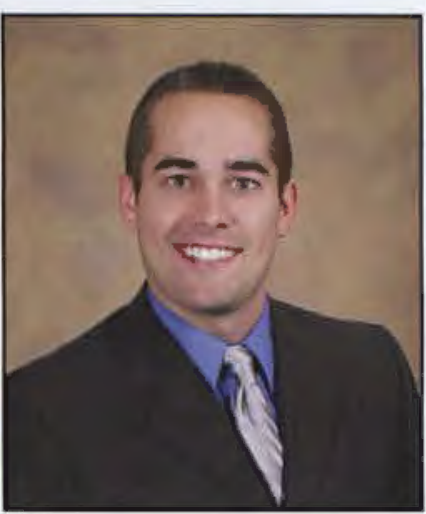

Michael Steele Mechanical Engineering

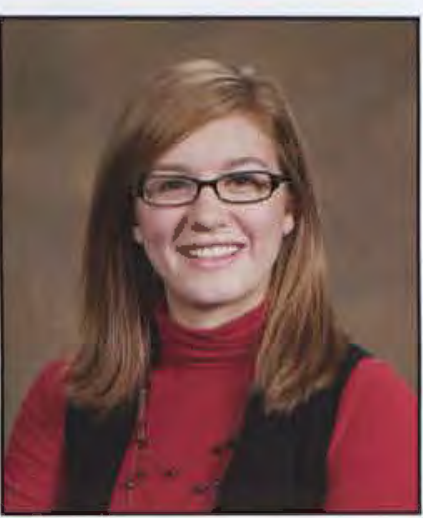

Jennifer Stern Early Childhood Education 


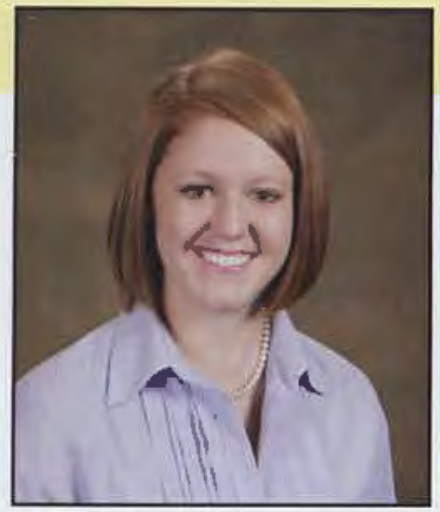

Anna Strayhorn Graphic Design

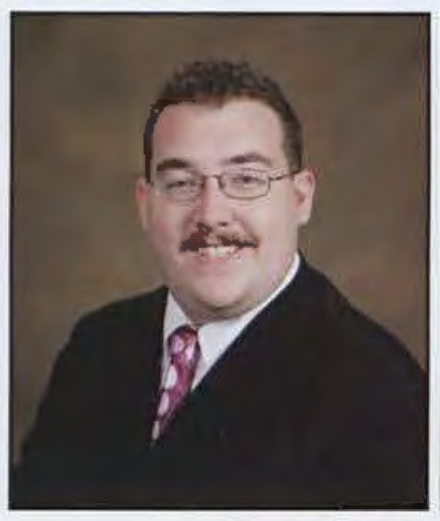

Steven Tackett Preseminary Bible

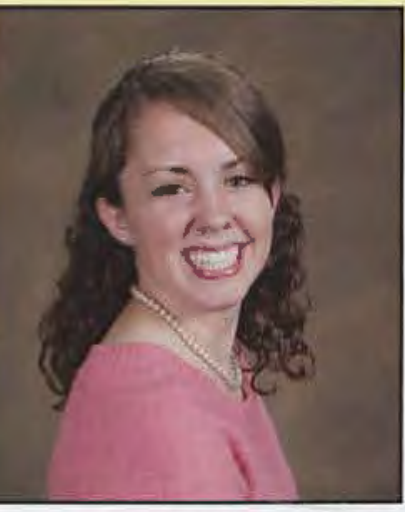

Elizabeth Studebaker Applied Psychology

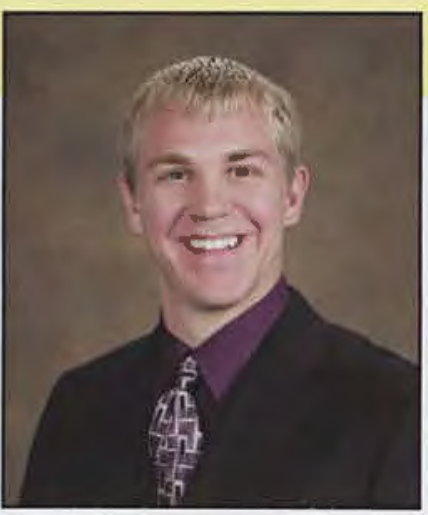

Caleb Suendsen Electronic Media

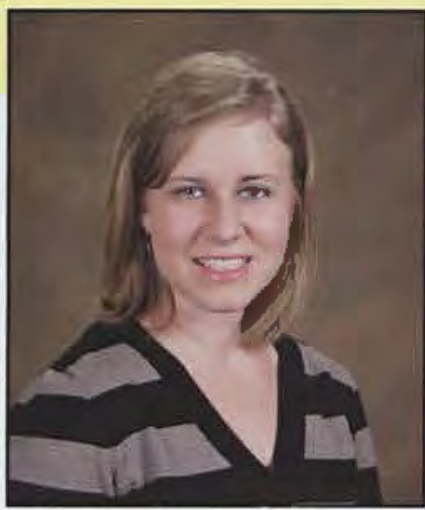

Jessica Swayze Comprehensive Communication

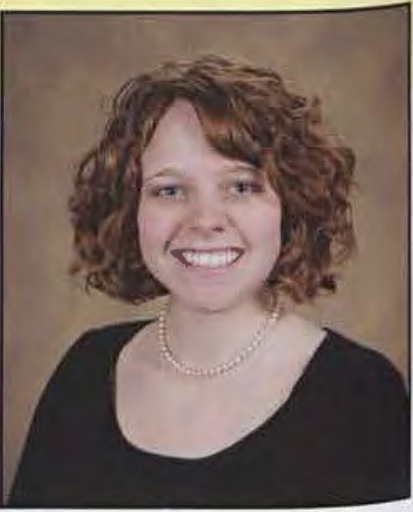

Brittany Sylvester Christian Education

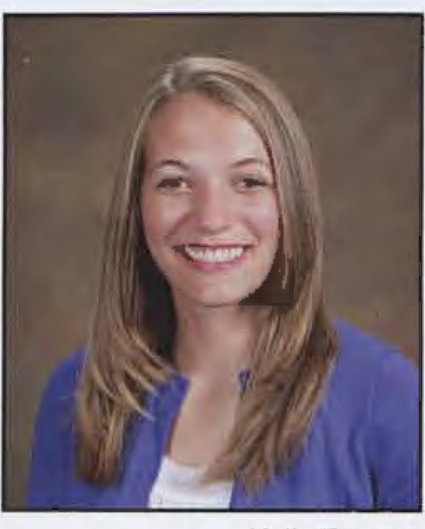

Kelly Teague History and Politcal Science

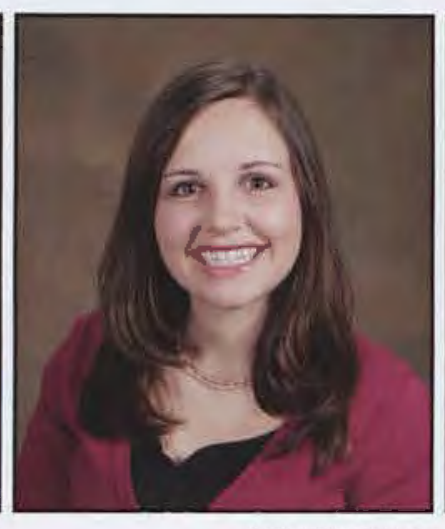

Katee Temple Biology

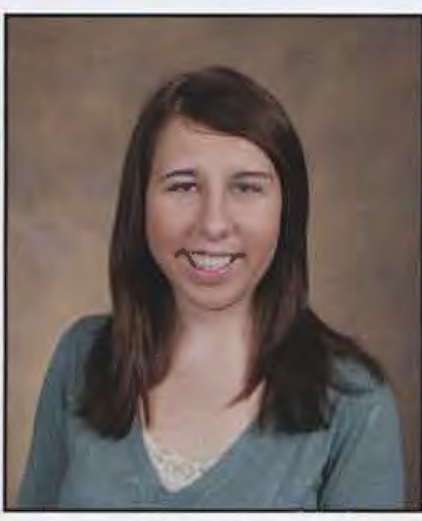

Kelly Theiss Marketing

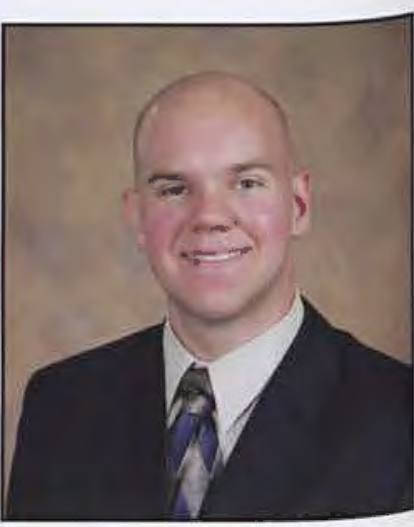

lan Thomas Mechanical Engineering

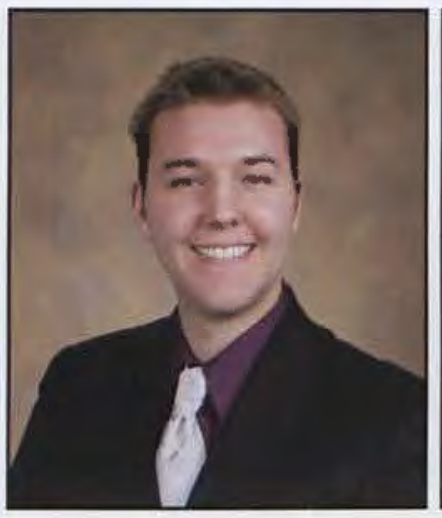

Steve Tormey Mechanical Engineering

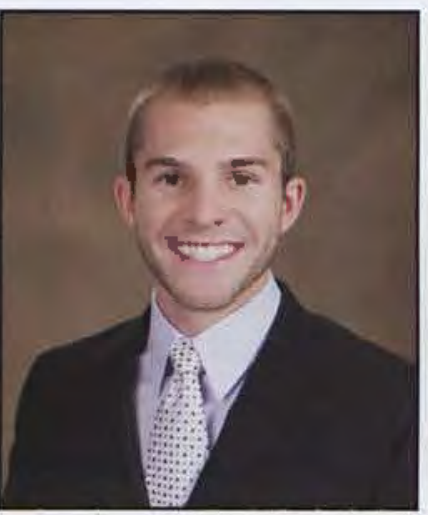

Christopher Travis Finance

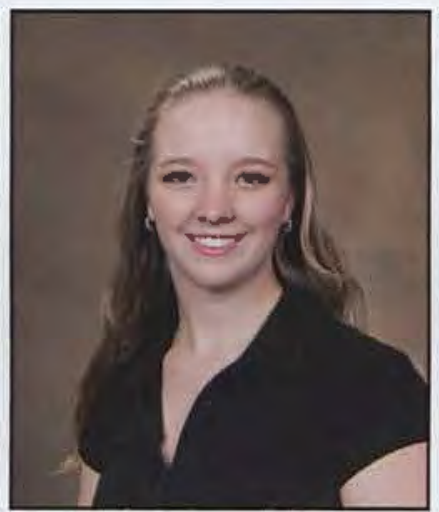

Justine Trego

Biology

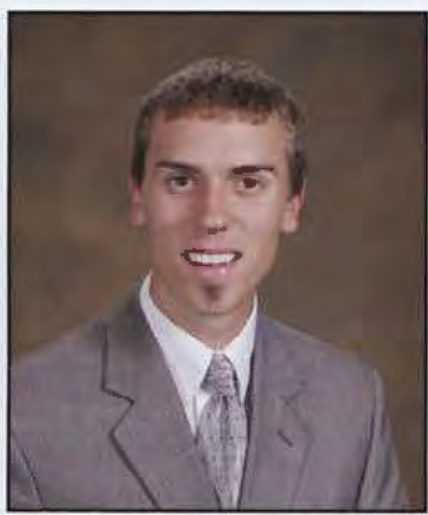

Robert Trennepohl

Finance and Accounting

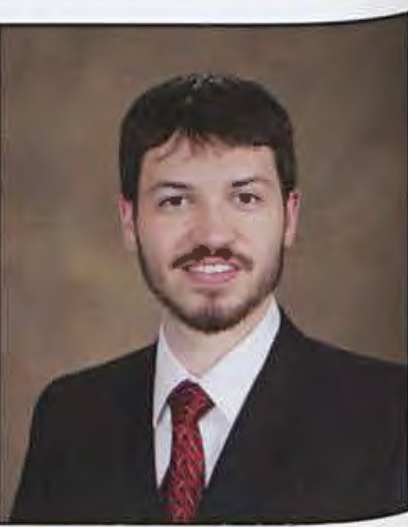

Brent Trowbridge Integrated Life Science

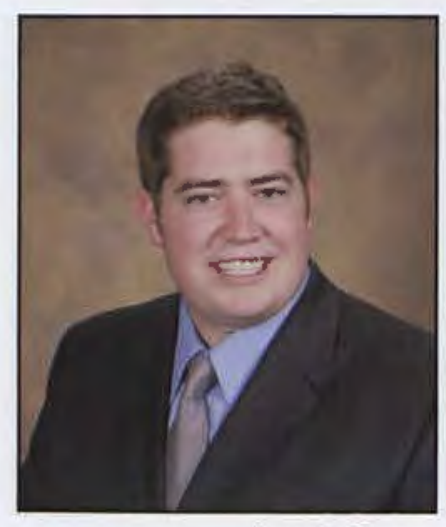

Joseph Turner International Studies/Social

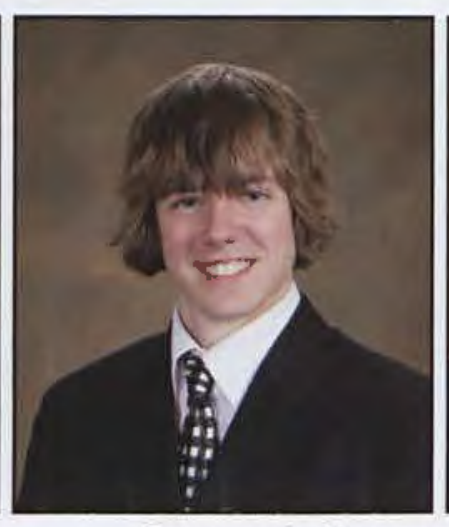

Peter van der Hoeven Computer Science

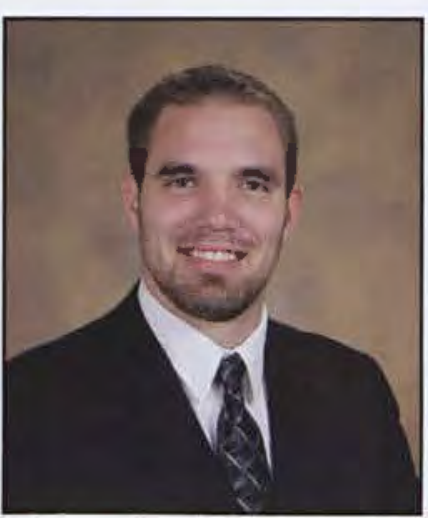

John van der Wa Finance

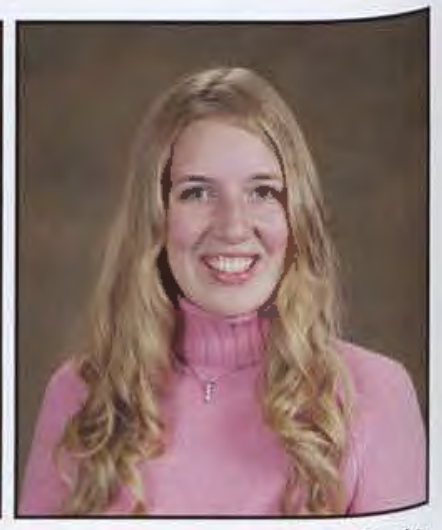

Emily VanDette Music Education 


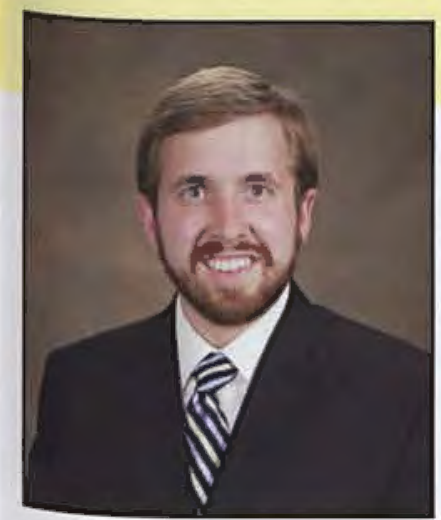

Ethan VanDette

Multi-Age Special Education

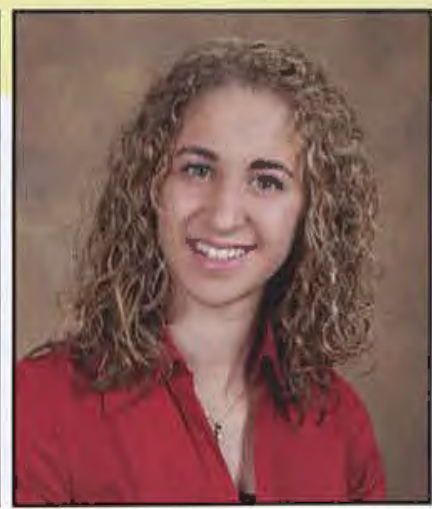

Emily Van Matre

Nursing

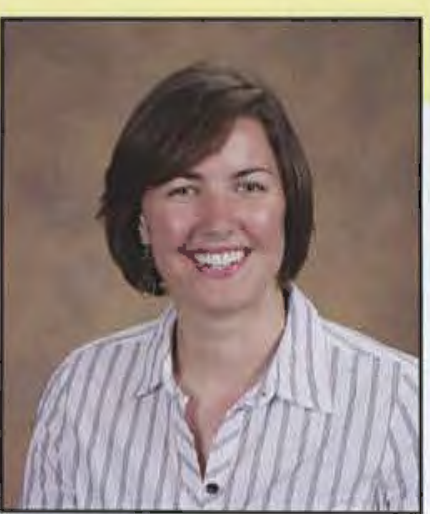

Amy Vanover

Nursing

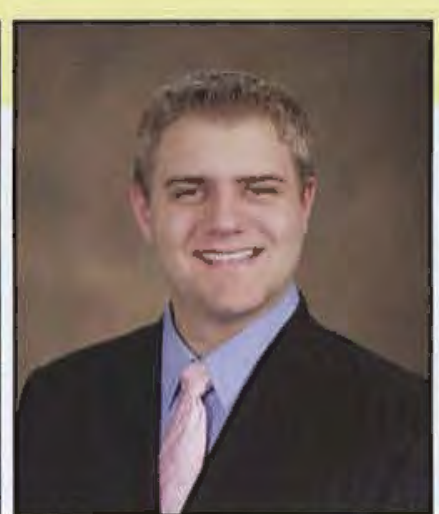

David Van Schepen Mechanical Engineering

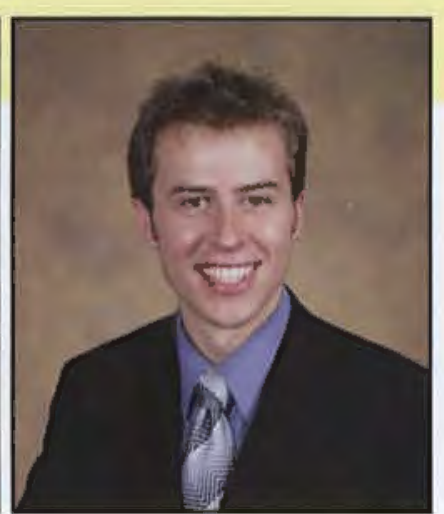

Alexander Vaughan Chemistry

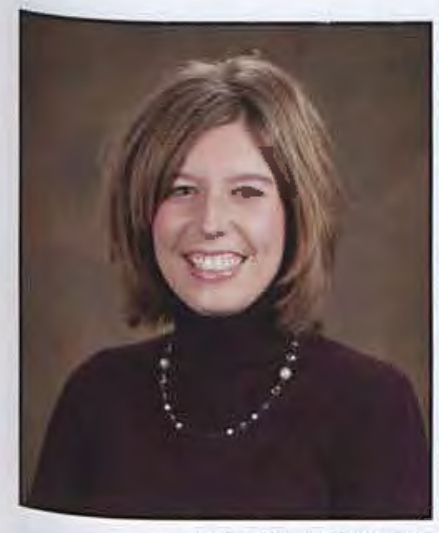

Sherri Velahuizen Christian Education

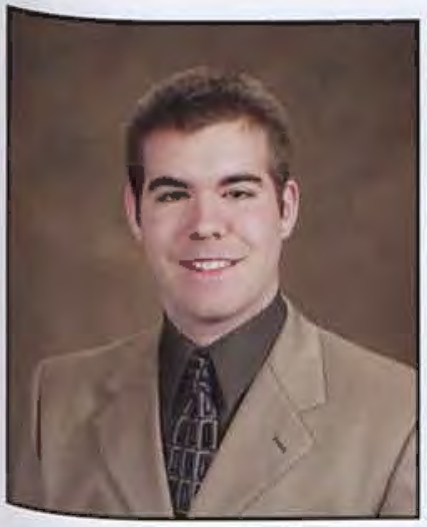

Timothy Voltz Comprehensive Bible

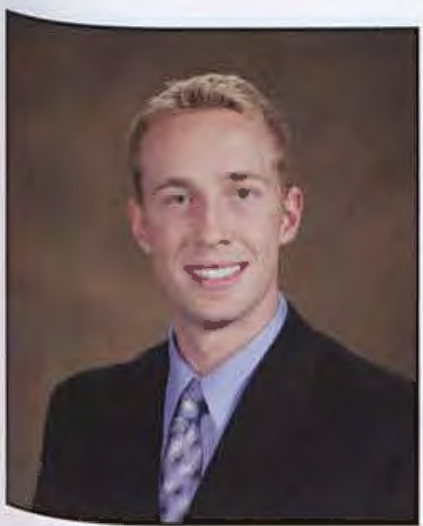

Grant Walker Biology

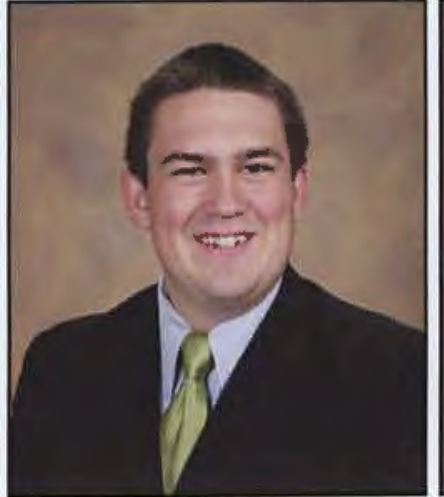

Mordecai Veld Mechanical Engineering

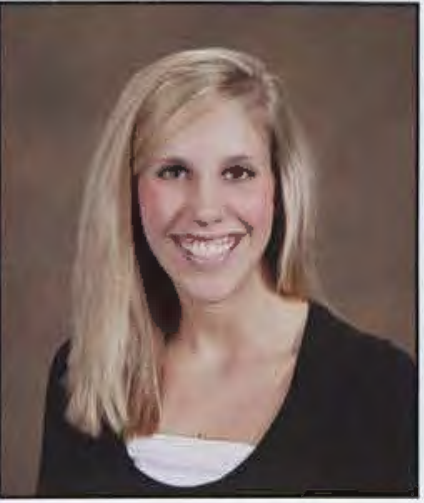

Lynn Verlander Nursing

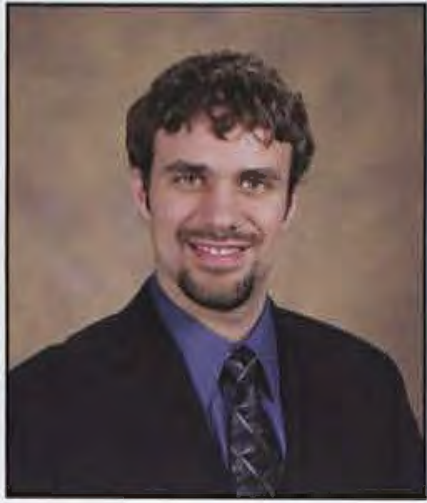

Timothy Vincent Mechanical Engineering

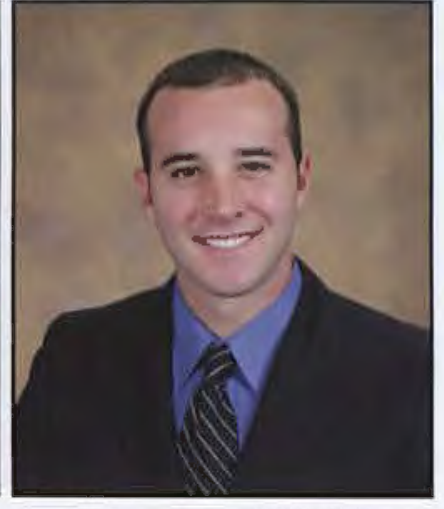

Philip Vinson Public Administration

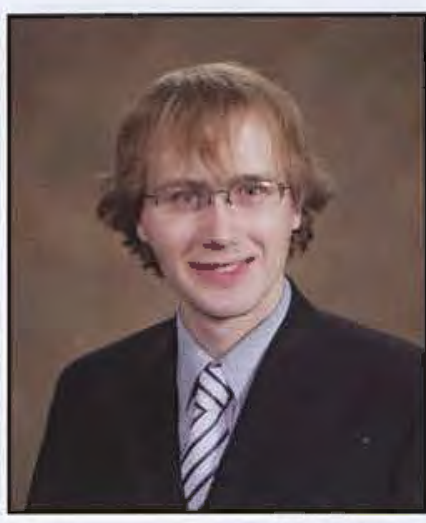

Ryan Voris History and Politcal Science

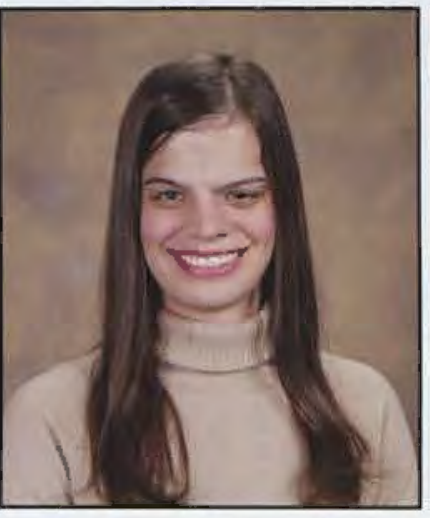

Grace Wagner Nursing

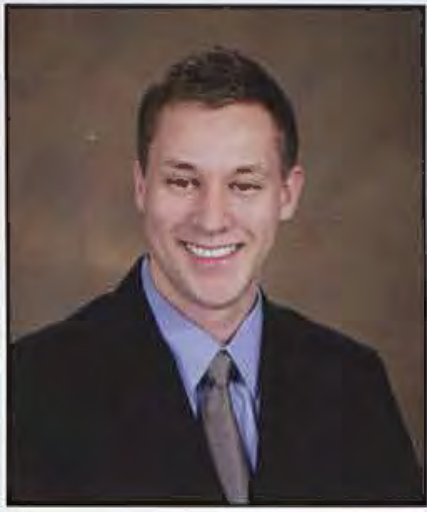

Micah Wagner History

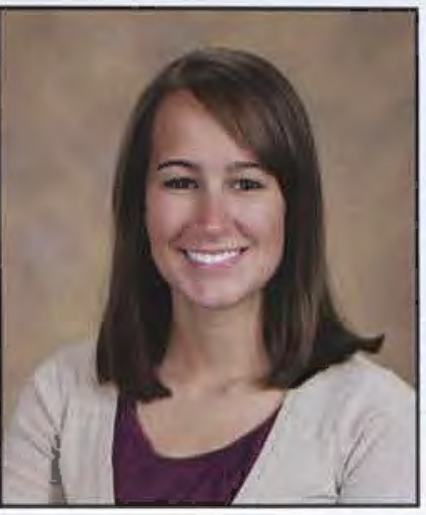

Brittany Walker Nursing

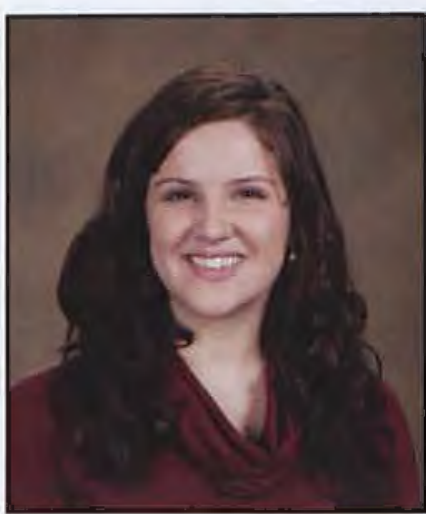

Elizabeth Walkley Nursing

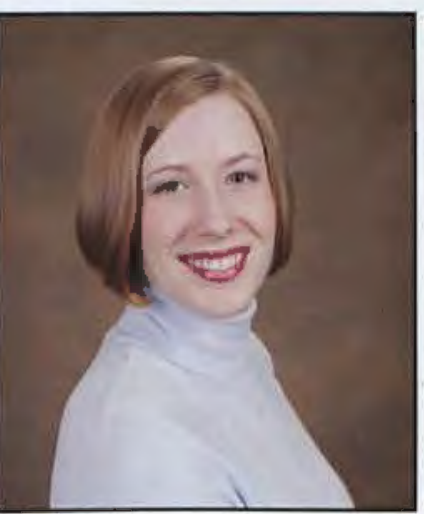

Sharon Waltz English

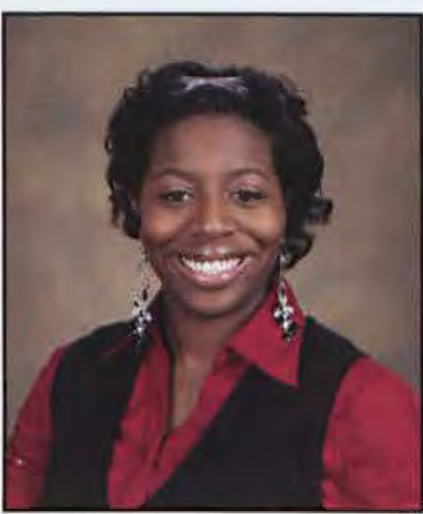

Jerica Ward International Relations/Social

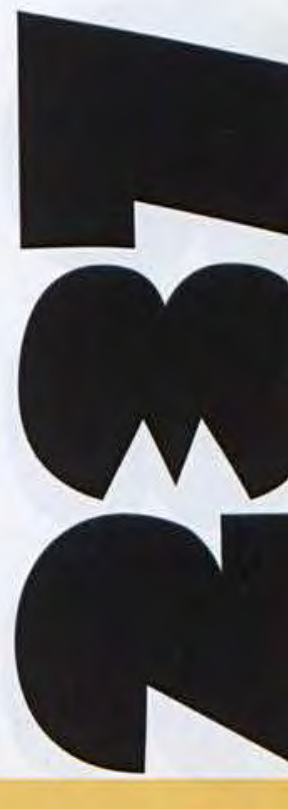




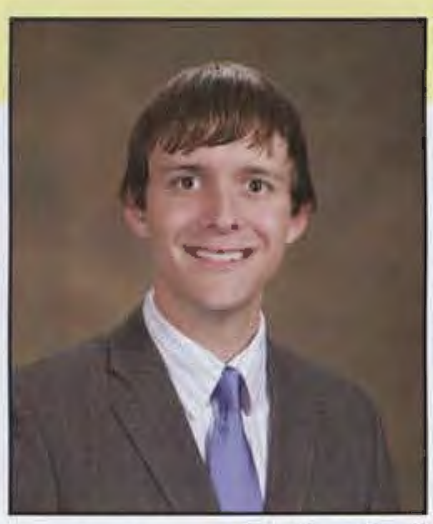

Stephen Ward Finance

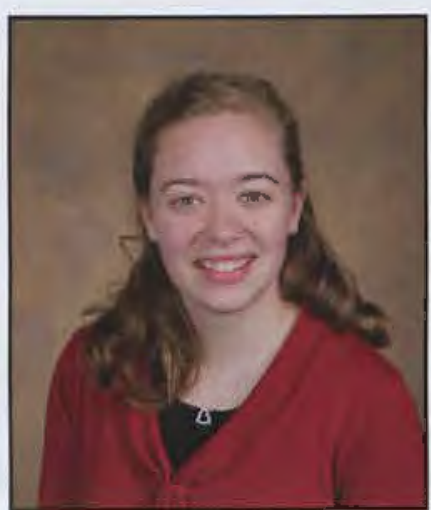

Amy Whitacre

Middle Childhood Education

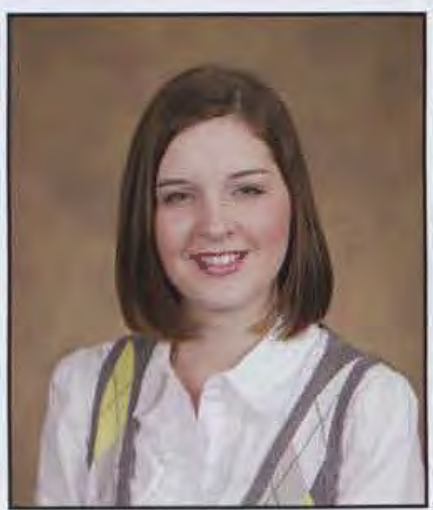

Abigal Wight Electronic Media

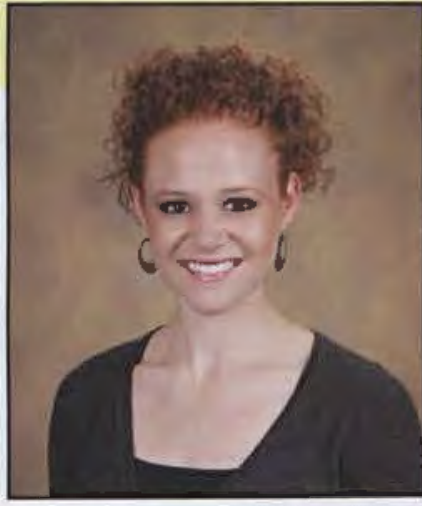

Brittany West Nursing

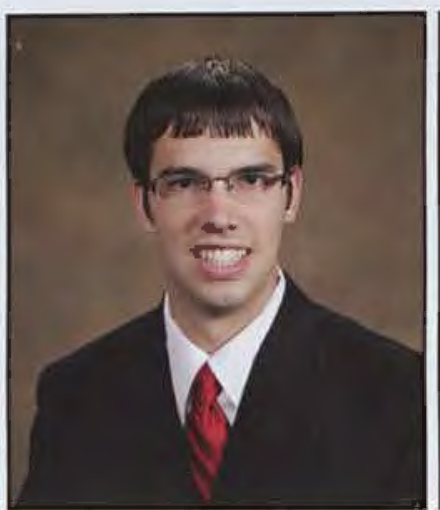

Jeffrey Whitacre Biology

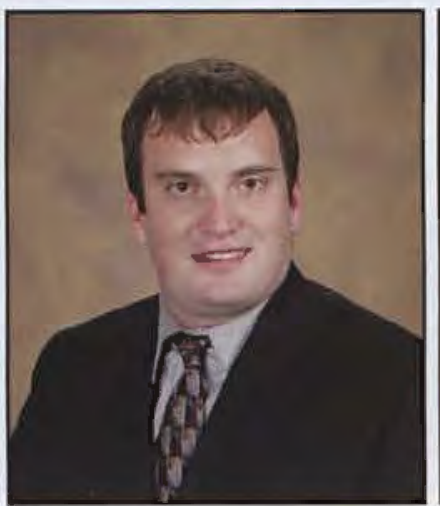

Tyler Whightman Electrical Engineering

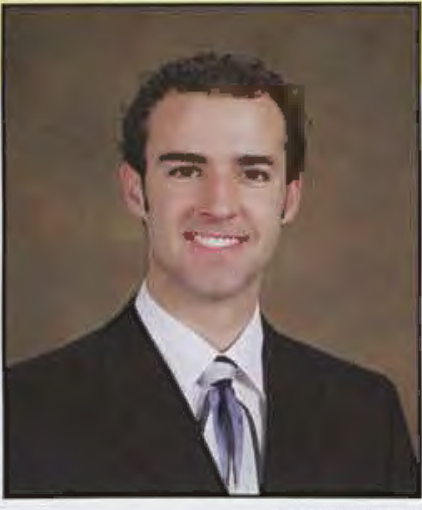

Justin West Biology

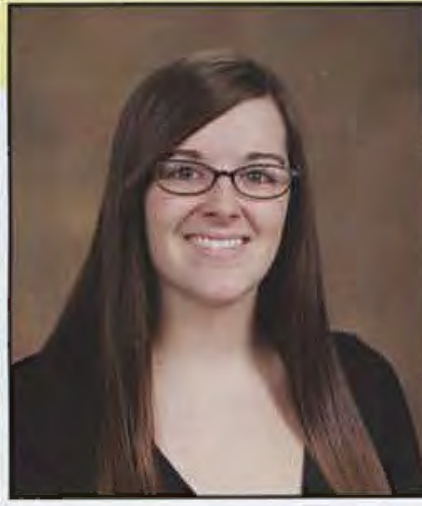

Rebecca Westerhof Business Management

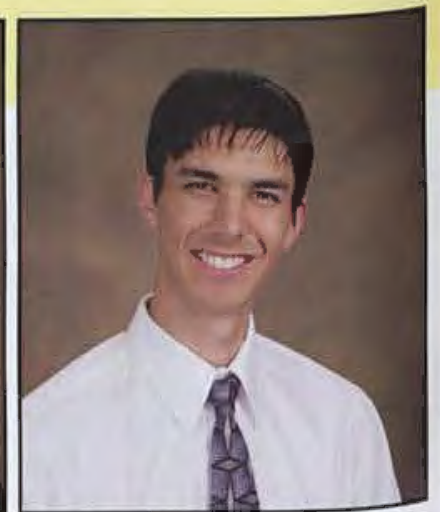

Nathan Wexler Graphic Design

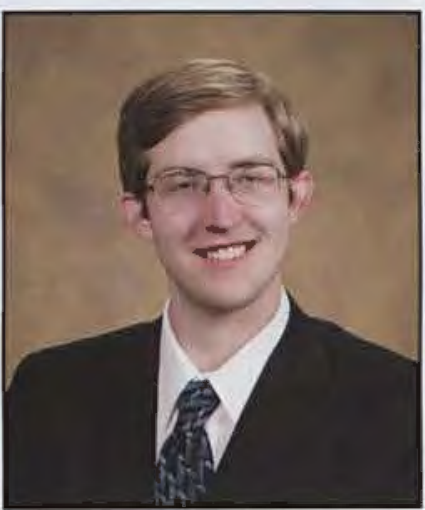

Ethan White

Computer Engineering

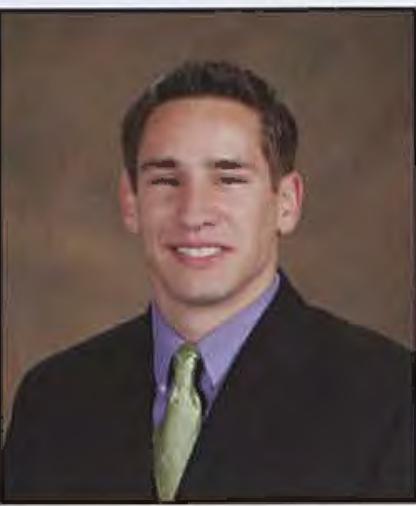

John Wildman

Biology

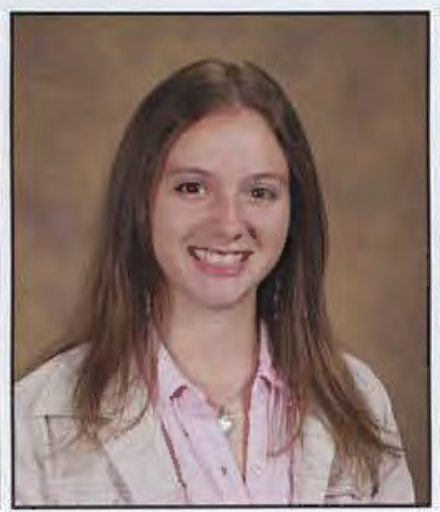

Sara White Social Work

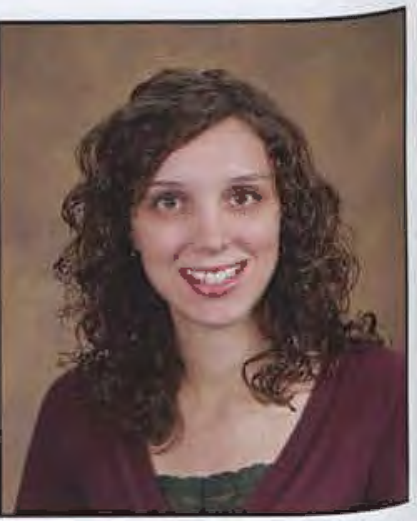

Lynette Whited Graphic Design

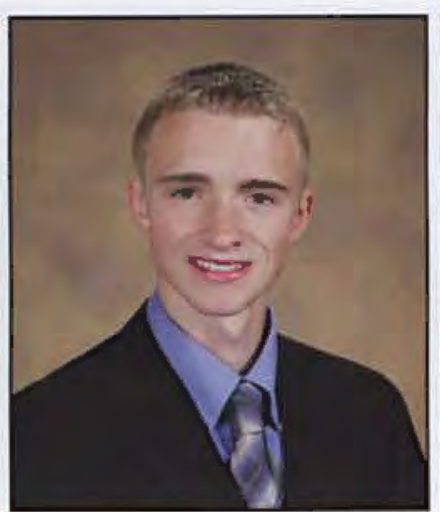

Benjamin Wiley Computer Science

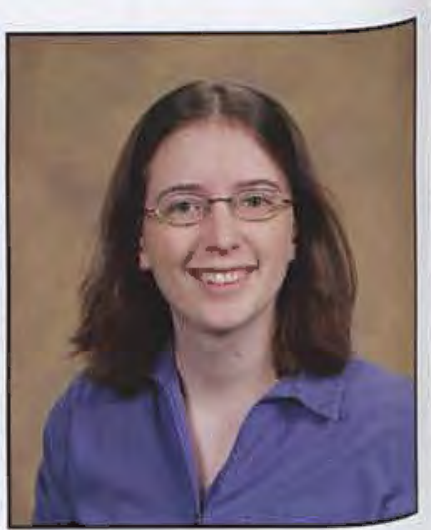

Valerie Williams Music

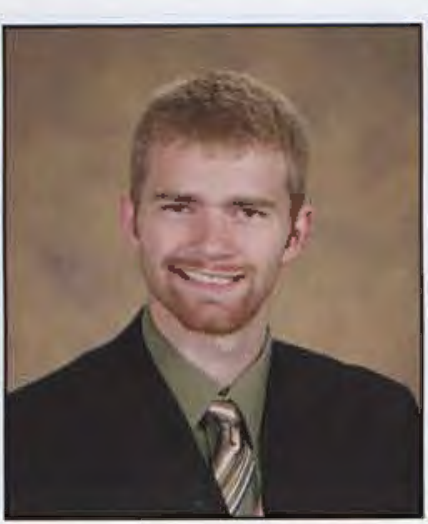

Micah Wilson Integrated Social Studies Education
Edward Winter

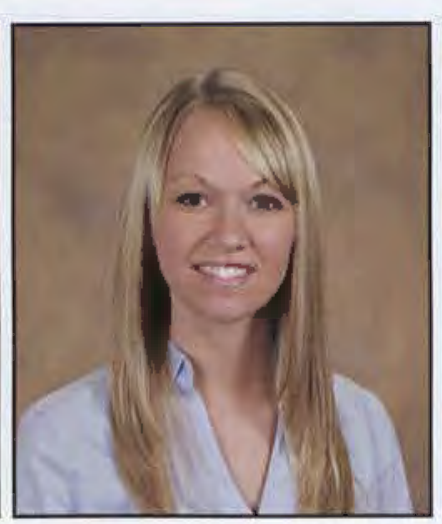

Lisa Wirt Mechanical Engineering

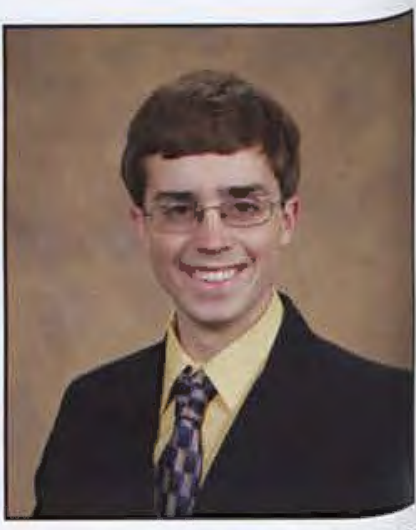

Jonathan Wolvertor International Studies Social Science

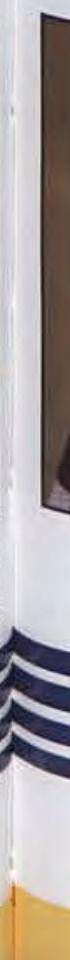




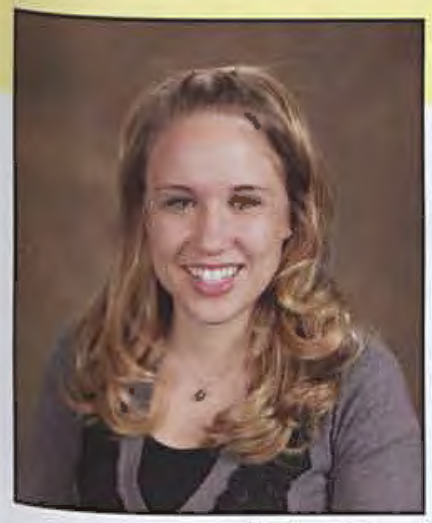

Whitny Wood

Nursing Organizational Communication

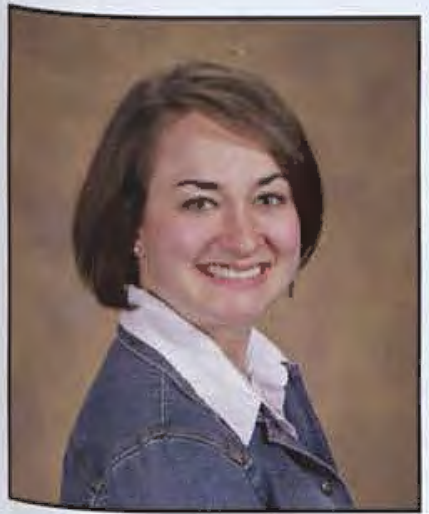

Katie Yahara Exercise Science

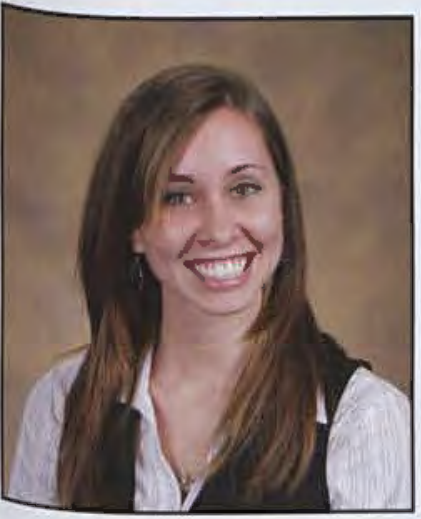

Bethany Young

Social Work

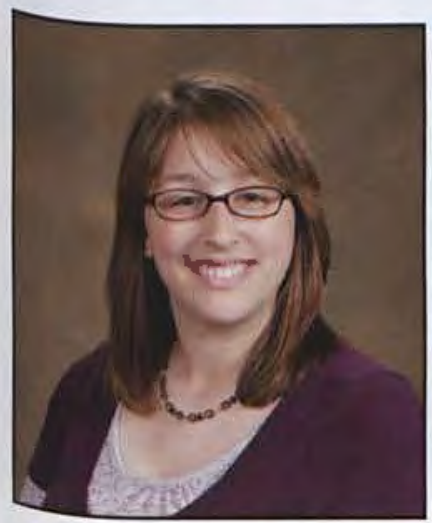

Bethany Ziemblicki

Biology

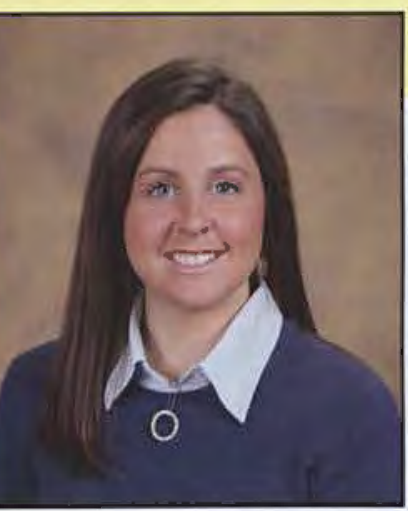

Clare Woods

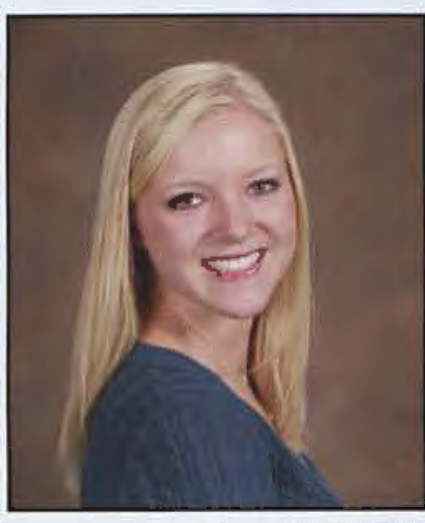

Jo Lyn Yoder Early Childhood Education

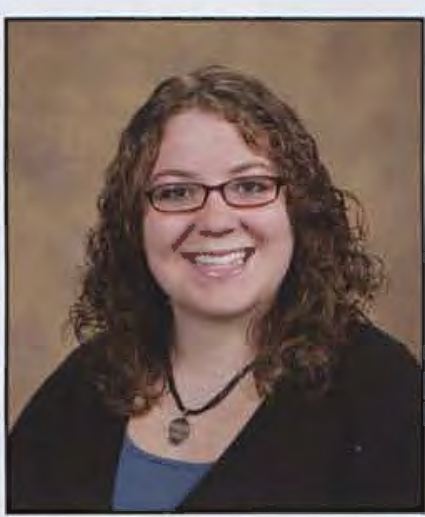

Shannon Young

Marketing

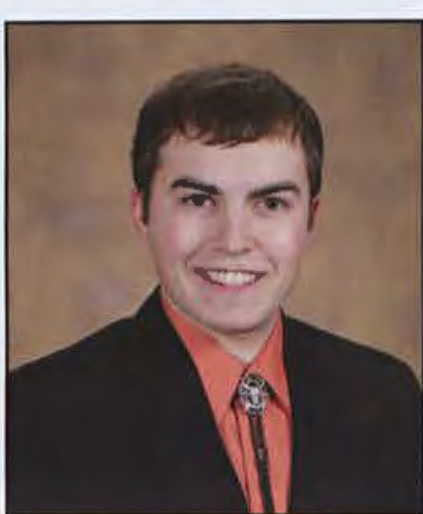

Daniel Zimmerman

Criminal Justice

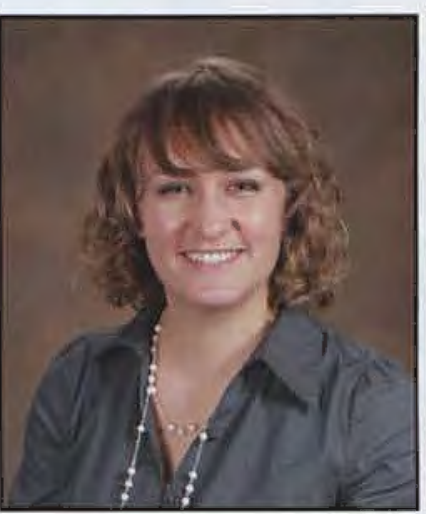

Rachel Yoder Social Work

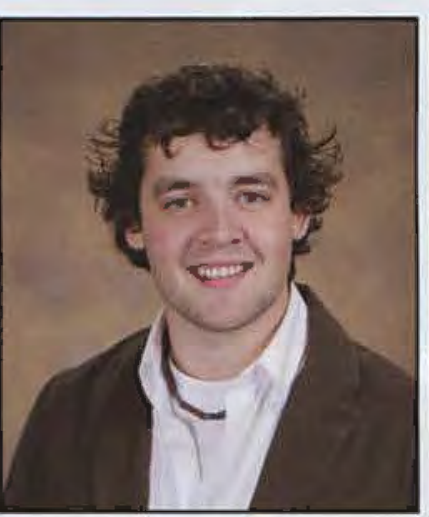

Lemuel Yutzy Social Work

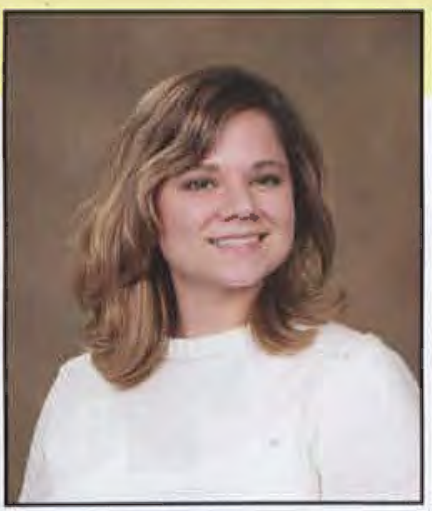

Leslie Wuenstel Biology

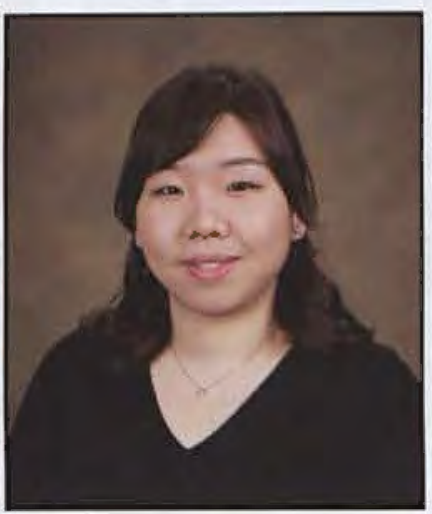

Jiyoung Yoo Electronic Media

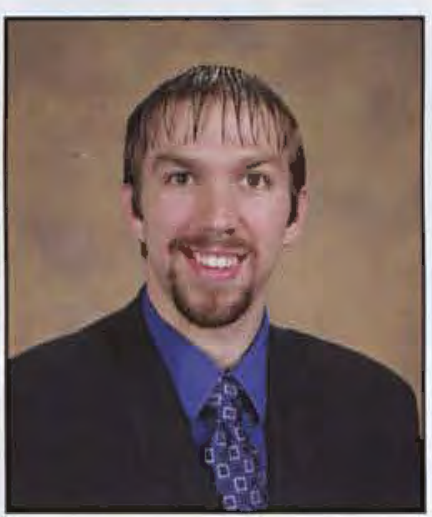

Matthew Zellner International Studies/ Missiology

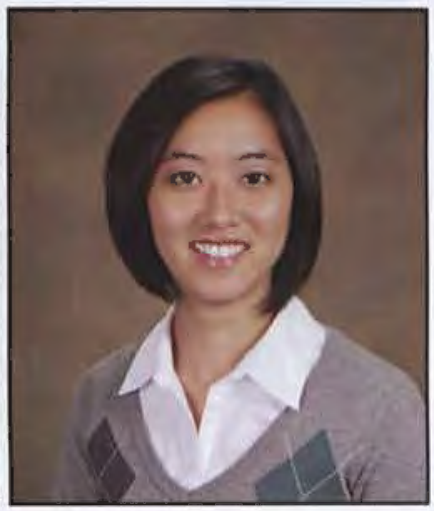

Bethany Young Nursing

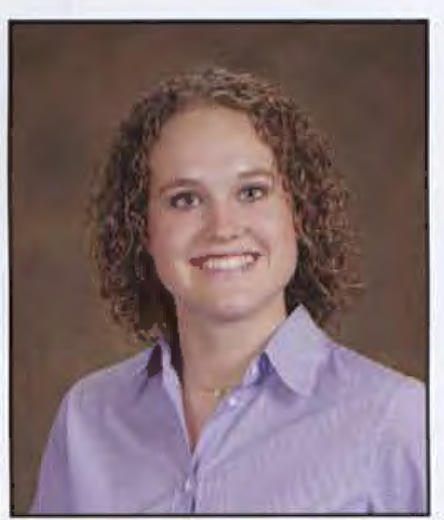

Michelle Zerminski Biology

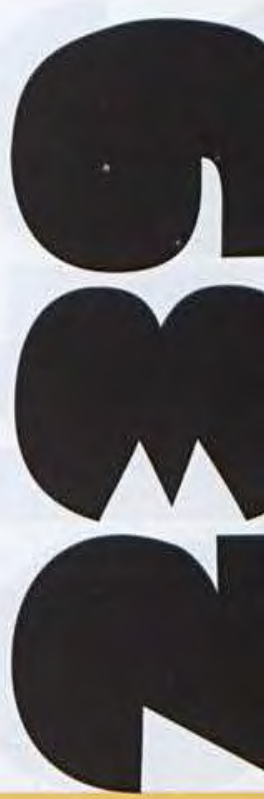




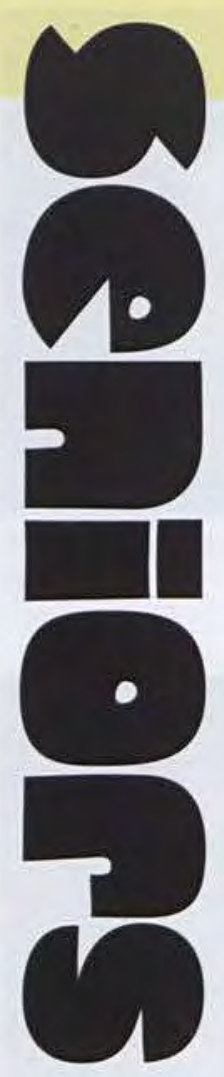

A bright group of biology girls take a moment to socialize without the lab goggles.

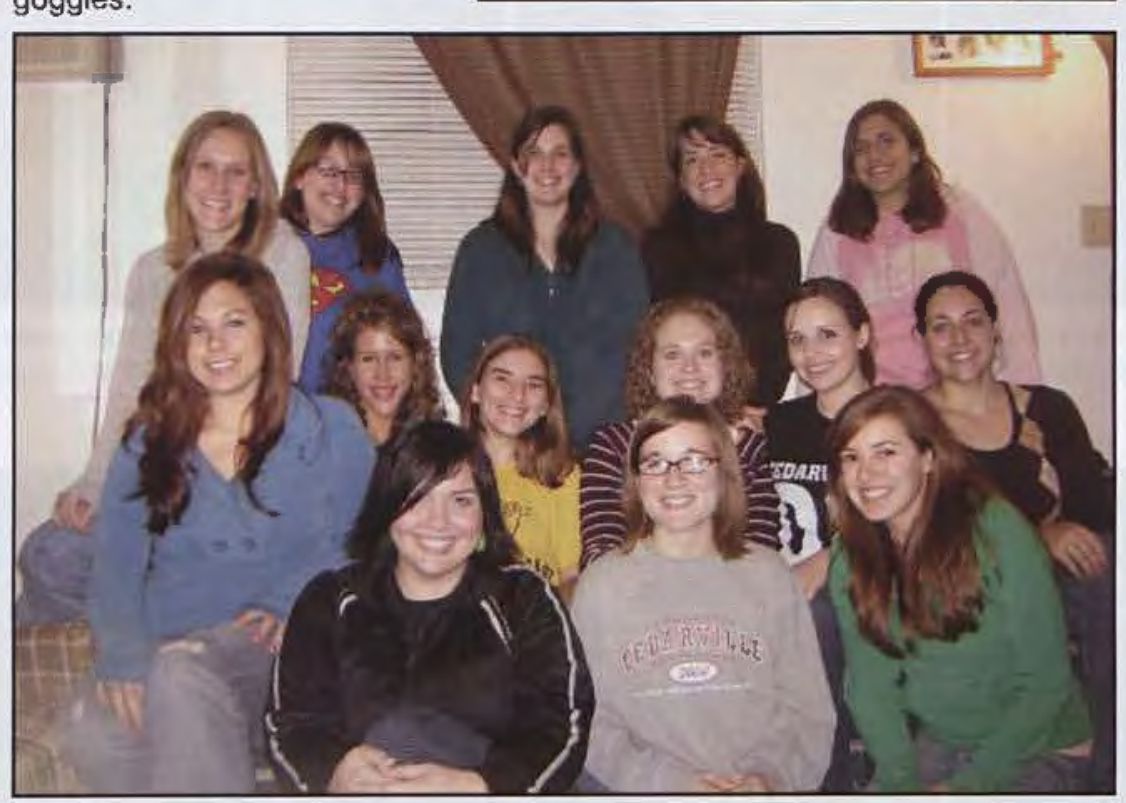

Who says seniors have to be concerned with the future? These two girls prove that everyone needs time to just act like a kid.

Nathan Sheppard, Andrew Arthur and Karie Pepe throw up some smiles as they celebrate at the seniors' 100 days party.

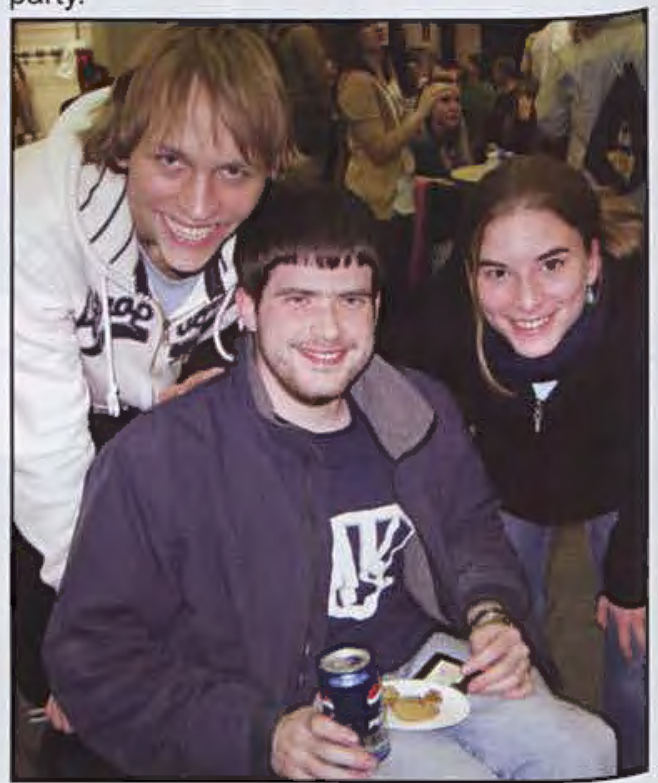

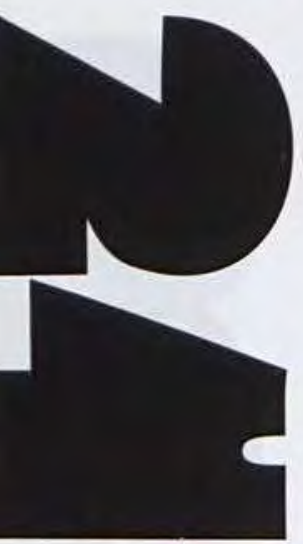

lan Casper and Josh Griffith go a little sweet on us at the annual Prism of Praise Concert during homecoming weekend.

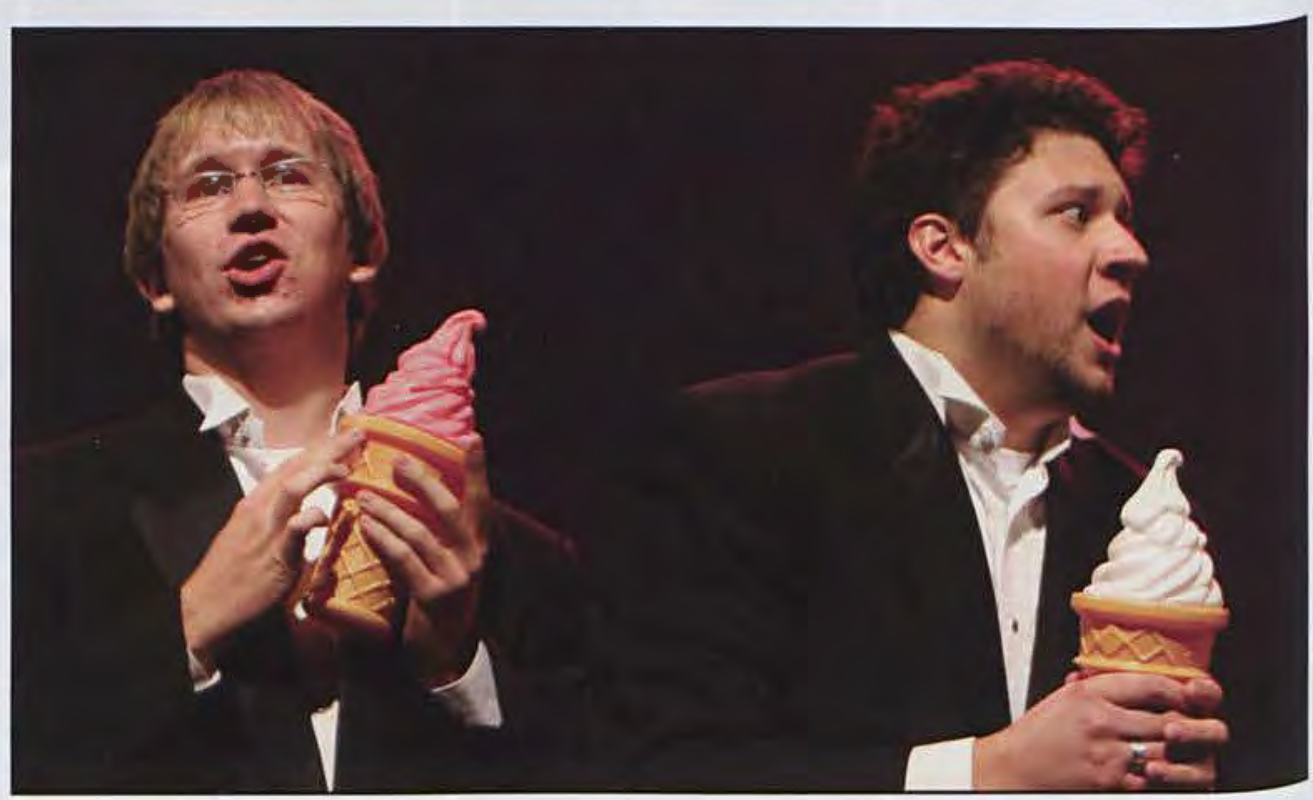



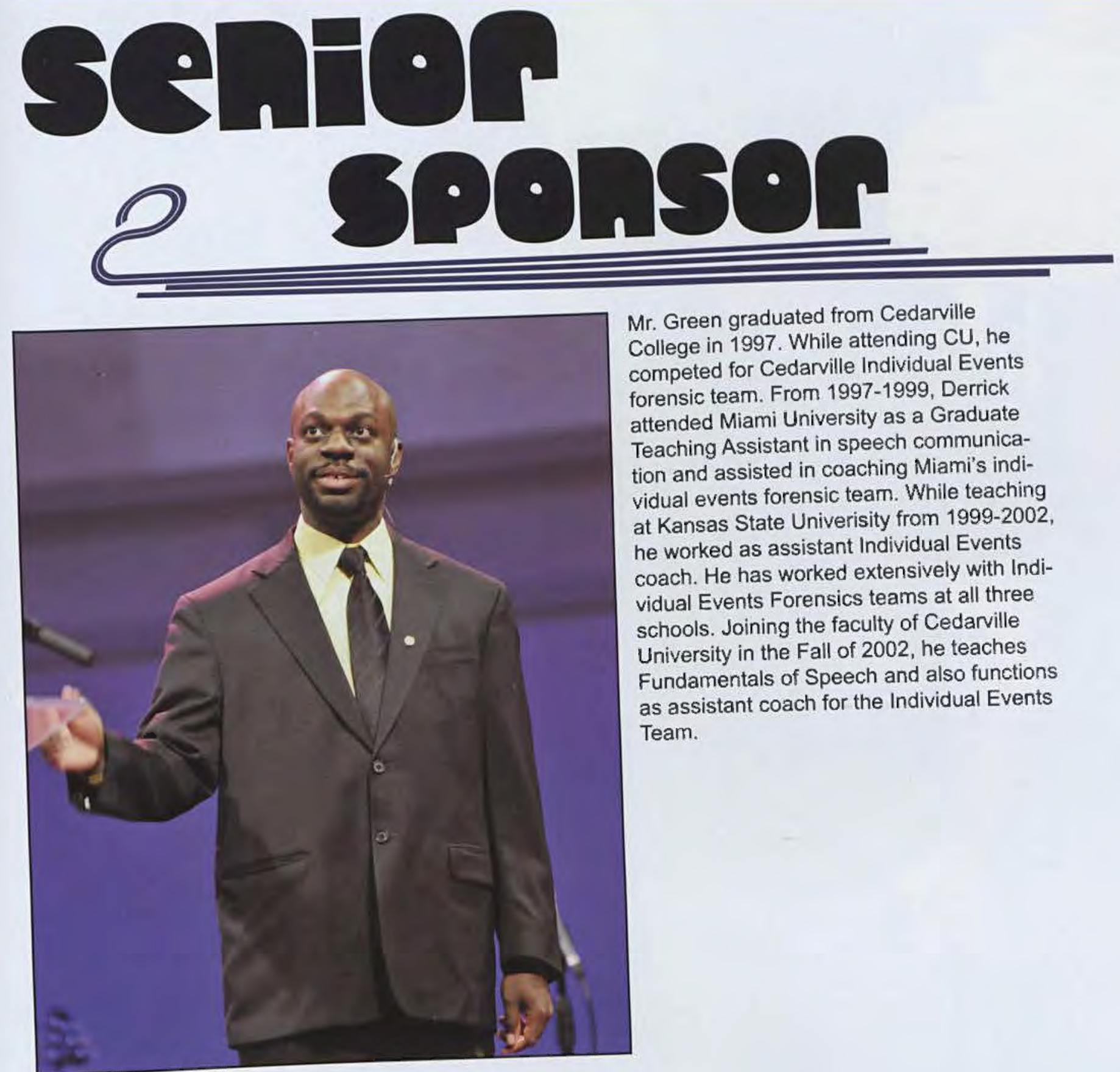

Mr. Green graduated from Cedarville College in 1997. While attending CU, he competed for Cedarville Individual Events forensic team. From 1997-1999, Derrick attended Miami University as a Graduate Teaching Assistant in speech communication and assisted in coaching Miami's individual events forensic team. While teaching at Kansas State Univerisity from 1999-2002, he worked as assistant Individual Events coach. He has worked extensively with Individual Events Forensics teams at all three schools. Joining the faculty of Cedarville University in the Fall of 2002, he teaches Fundamentals of Speech and also functions as assistant coach for the Individual Events Team.

"We had an incredible group of officers who worked diligently to serve their class, whether it was giving up a weekend to help make a float or staying up late making a plinko board, they did an exceptional job. As we as the Senior class head out into the turbulent job market, knowing only the immediate next step and no more, we're reminded of our God's great faithfulness. It is that knowledge that gives us hope and reminds us to leave here with a joy of the great things to come." -Andrew DaVis, senior crass prestdent 

"The shirt," she nods to me, eyes focused on the shirt of one of

the actors as her hands adjust the mic clip on the collar. We are backstage, making last minute adjustments on costuming. I nod in return, though the nod is unnecessary. She doesn't need to know that I understand exactly what she means: she expects it. The shirt. Of course. The fabric is slippery, thin, and loose. Not ideal for a mic. From here, only two options are available: make a new shirt or find a way to keep the mic in place. But I'm not thinking about that at this moment. I'm thinking about the other costumes, the necessity of the shirt for the portrayal of the character, the amount of time between now and opening night that we have to fix this dilemma, the hundreds of other aspects of the show that need to be addressed. This is what I do. This is what I love.

Perhaps the science of drama and the art of science will never meet face to face, but in my life they overlap in ways I have never expected. You may not see me, but I certainly see you. I am not blinded by the stage lights but rather in control of them. I am not at the front, but I'm everywhere else. You may never see me, but I exist so that you may have the full picture, the full effect of the director's vision that will allow you to escape not into the world of the actual but the world that I have helped to create-a world where your eyes drink their fill of a magical wonderland with eccentric mad hatters, or your spine tingles while trapped in a snowed-in lodge with a killer.

While Getting started weekend constitutes a lot of meeting and greeting, I postponed any chats with my soon-to-be science professors in order to make my way to the theatre section. Starting as the sound head, and even gracing the stage in the chorus of You're a Good Man, Charlie Brown, I eventually found my place as Stage Manager once again. The pairing of a chemistry major and a theatre minor may seem strange to some, but not to me. Theatre has its own periodic table of elements, all engineered to produce the vision of the director. Opening night allows all of these elements to merge, and the high that comes is barely describable: I cast off my role of serious stage manager and everything within me screams, "Yes! We did it!"

Going to the theatre is watching life unfold. The Bible itself acts almost as an epic play, and God has created spoken word to move the human soul toward Himself. Theatre is just a facet of God's design. Whether it is allowing a child to escape from a world where he can barely think beyond his next meal, partnering with counseling and collection centers with the money earned by the production, or promoting a message of hope and restoration, theatre is, and always will be, a way to bring glory to God. My passion is to maintain the vision and passion of the show and let it change you in whatever way it may.

"Here we go," she says, this time smiling into my eyes. Lights. Curtain. Action. 


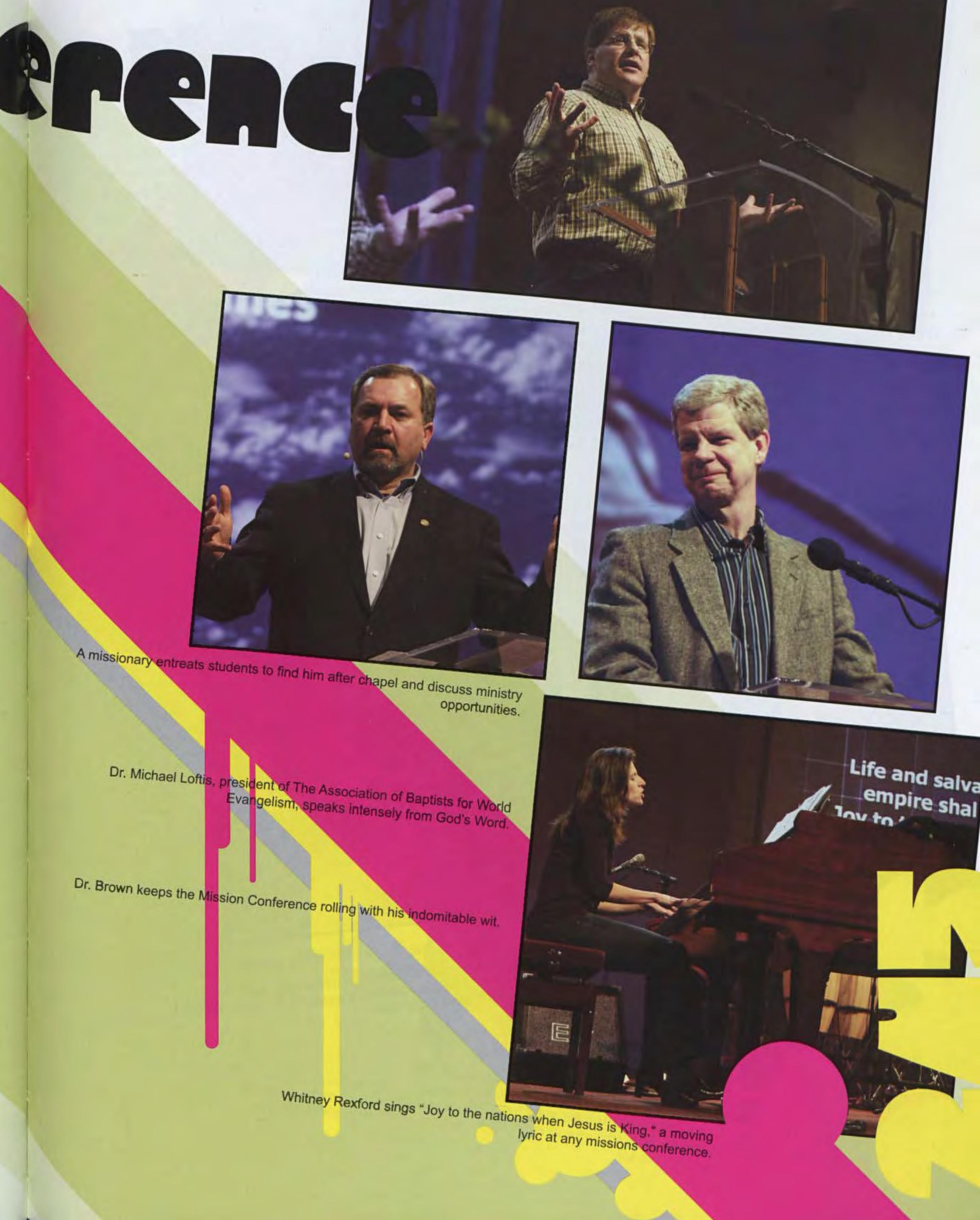




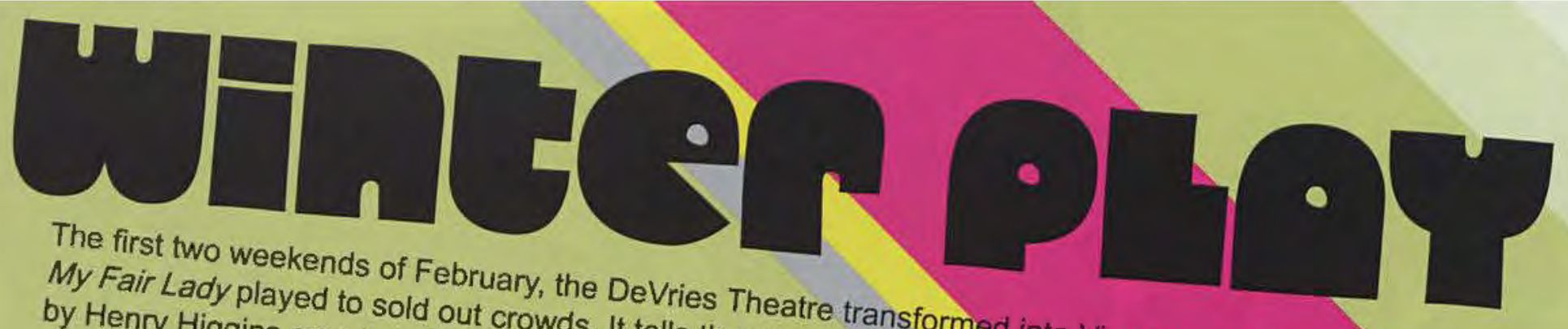

by Feir Lady played to sold out crowds. It tells the story of a guttersnipe. Eictorian Era England. The production of all people, regardless of . But, it's more than just a transformation. "My Fair Lady is transformed into a princess imagination, but for the first time in his life they dress. Whether Henry Higgins falls in clates the value of peoplecollect items for the imper. In order to emphasize the value of bachelor' realizes he needs solith Eliza is left to our people, Alpha Psi Omega partnered with else," says was a great were involved in the production and worked har and majors in the pursuit of an excellent production," said Christa Funke, Assistant Stage Manterent backgrounds
down to the extras, each performer played it up on stage, embodying the
of the crew did a fabulous job bringing all the office to the street adous job bringing all the technical aspects toging their characters. Not to bager. From the leads "There is nothing like scene changes. roaring cheer and like the feling you get when you played Mrs. Higgins. The audience fed energy to the actors who sent it right back. Not a mossica Hickling, who
magic of the theatre was not displayed.

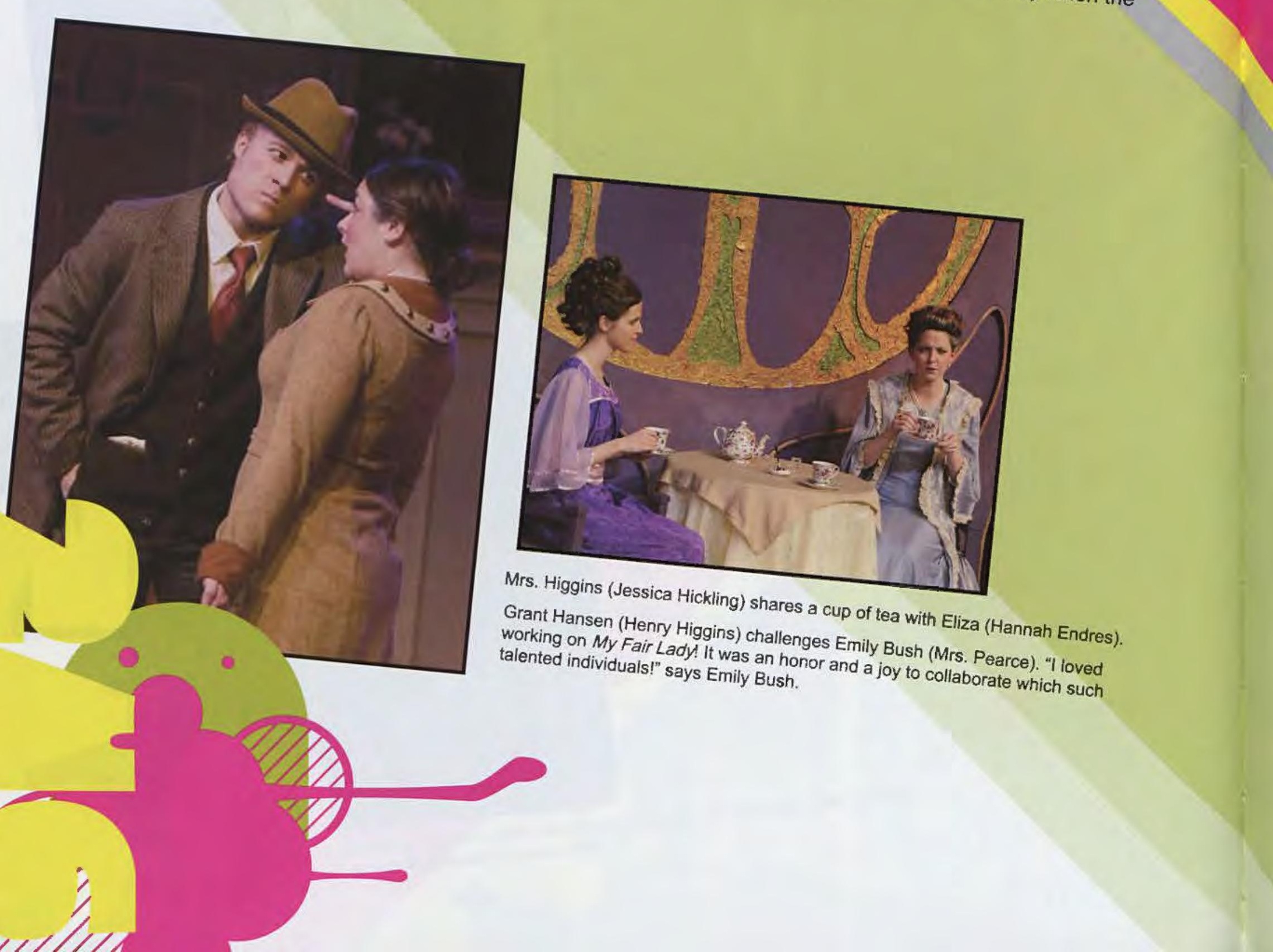



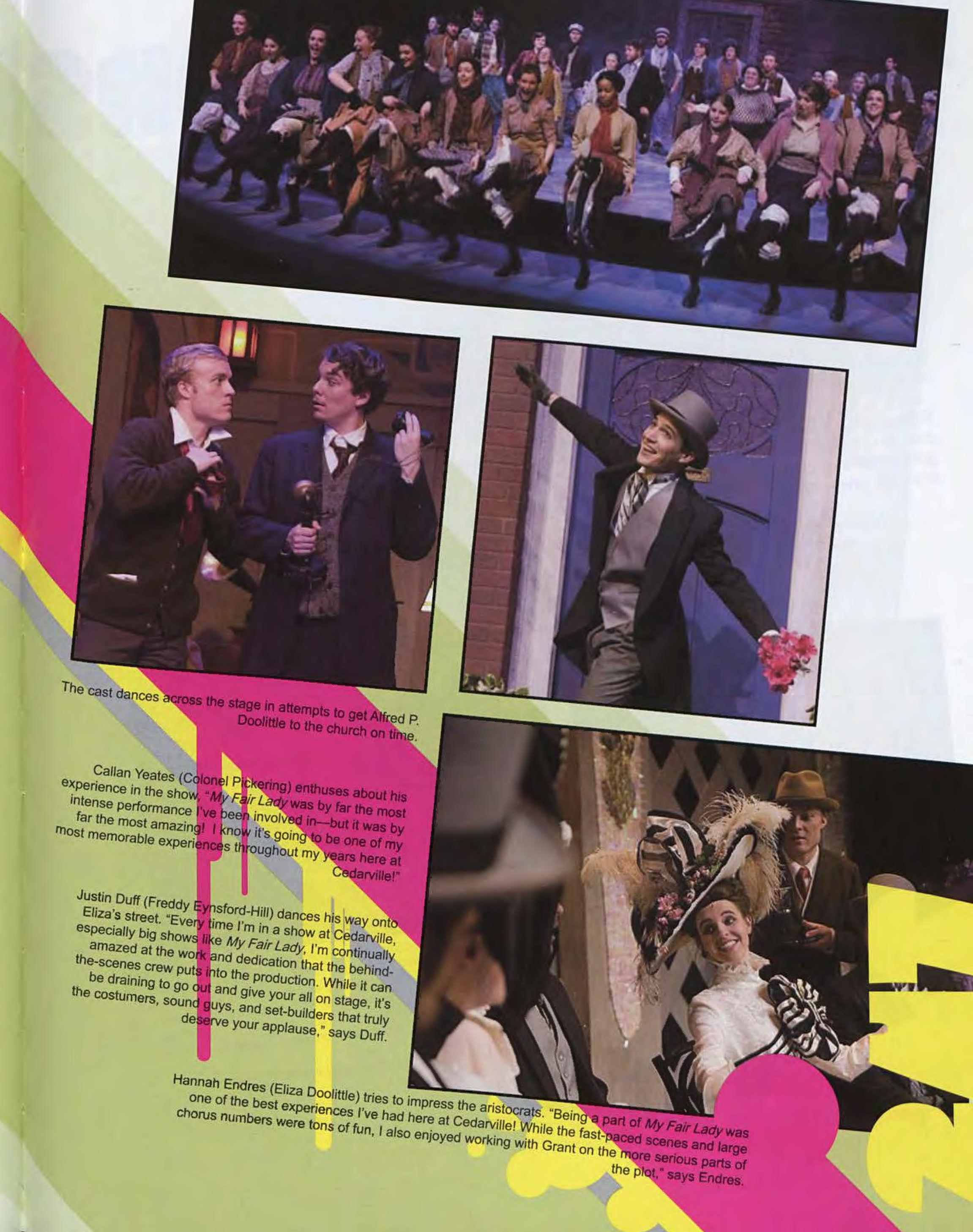

After strumming and singing along with her guitar, Alle Pennell declares,
"Playing for Interruptions

Cedarville experience. Food, friends, music-it doesn't get bets of my

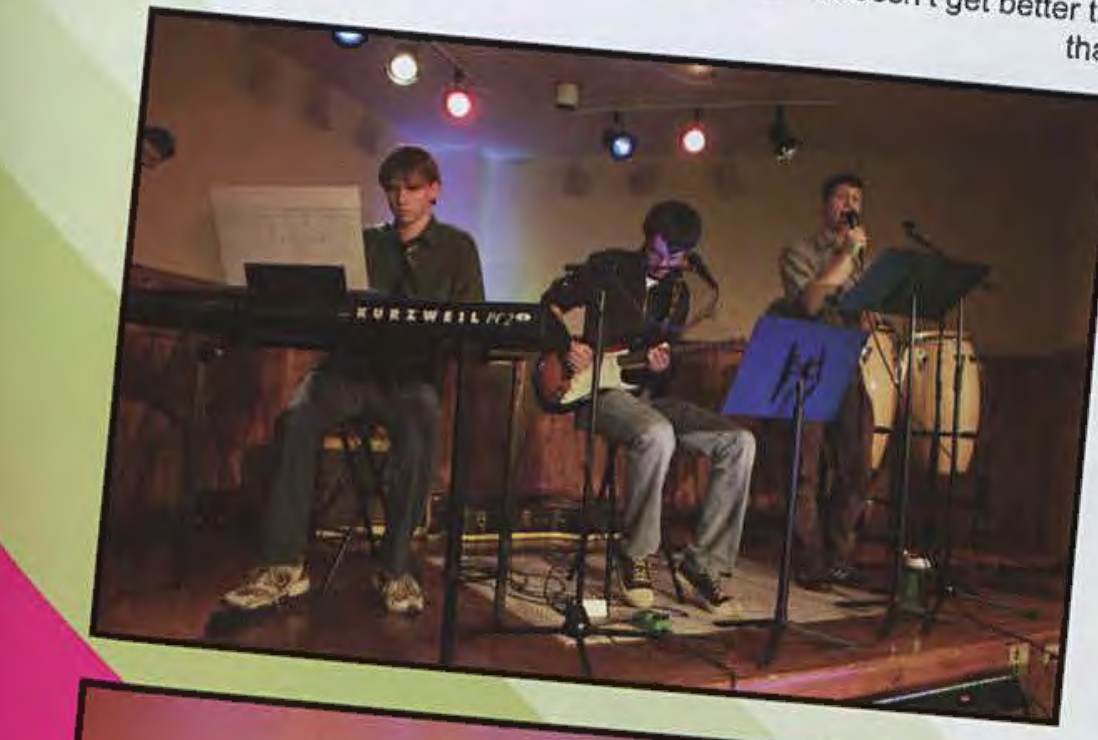
that!"
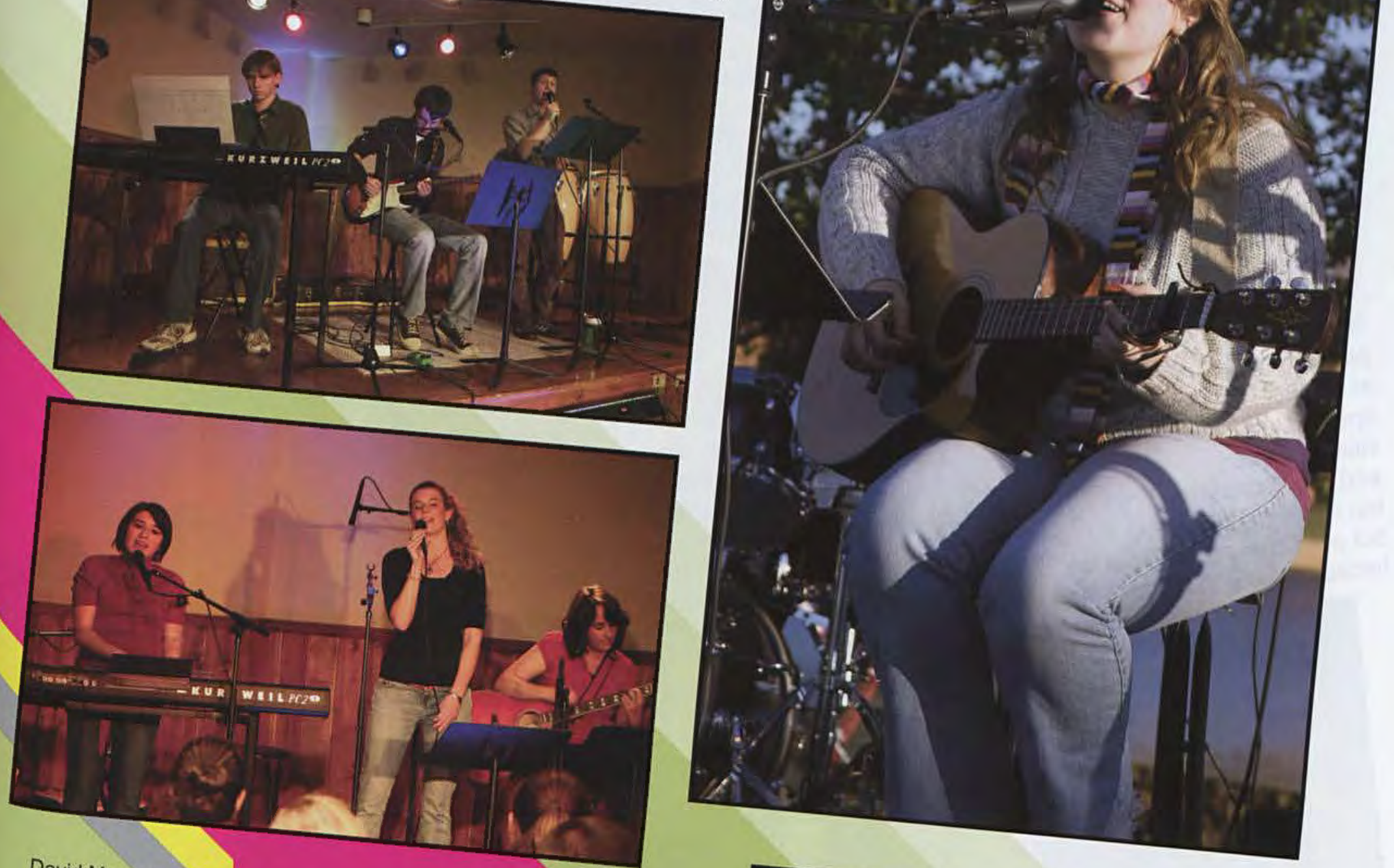

David Moser knows

Danielle Scarpone

turn into powerful me and Tiffany Hendricks sho

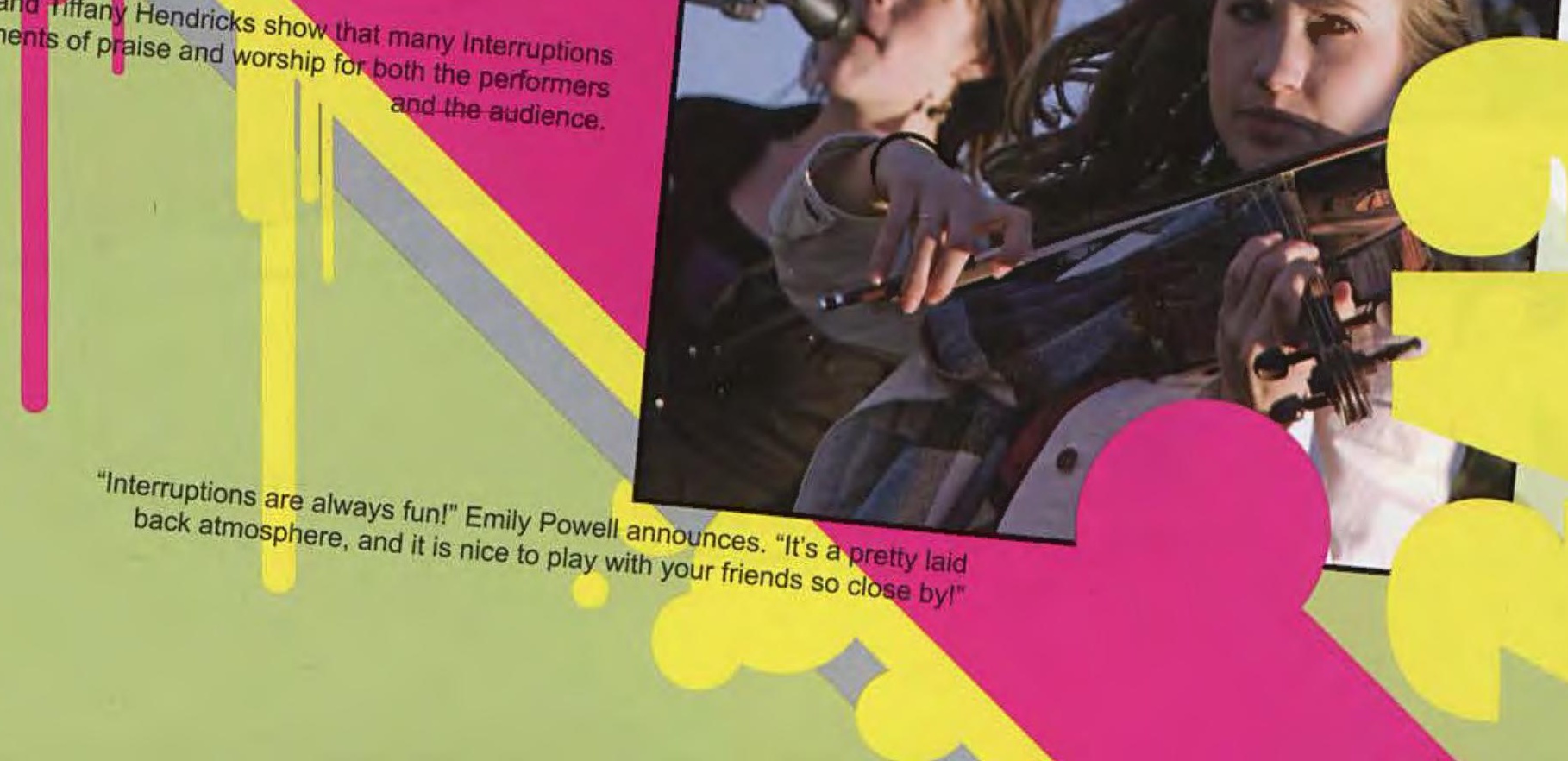




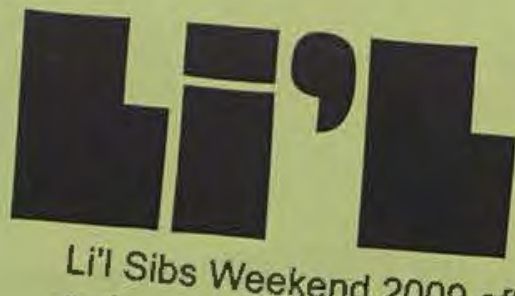

Li' Sibs Weekend 2009 offered an assortment of activived for the fos brother's or sister's and meaningful weekend! Friday mivies for all of the siblings were either classes, listening to SGA chay morning started early with little family. Over four hundred siblings to attend the Leeland concert or Ceating lunch in Chucks. Friday night, sting their older the day. the fun did not stop there. Saturday in the Steven's Play, My Fair Lady. of Vecinos. Meanwhile, clusters of siblings excions and financial aid Center was bustling with activity for most of team during the day. Siblings, students, and families participated in the various activities put on by Cedarville's cups $10 / 24$ other tone from its usual fast-paced and busy academic atmosphere. When asked what her favorite part of Lil shs Weeknic atmosphere. sister Hannah, said, "I like all the games and Weekend was, eleven year old Joy Miller, who was visiting her older
and I really wanted to come back."
Upstairs in the SSC event rooms, one cluster just really fun. This is my second year, and my mom, dad playing Mario Cart. Caleb (15), Heather (14) of four siblings seemed to be having a rowdy and adventurous time ville students gathered in one place. Harley, while reciated the community atmos promptly responded that the chapel top of the middle wall! I also really The oldest, Caleb, piped inpy to pause his Mario Kart race, said all of the Cedarbut after coming to Cedarville this we going to classes with Ashley. I ck climbing wall was my favorite. I mat game room

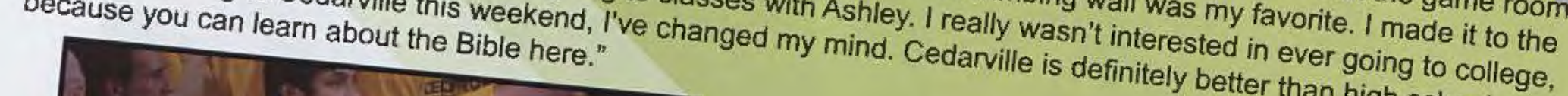

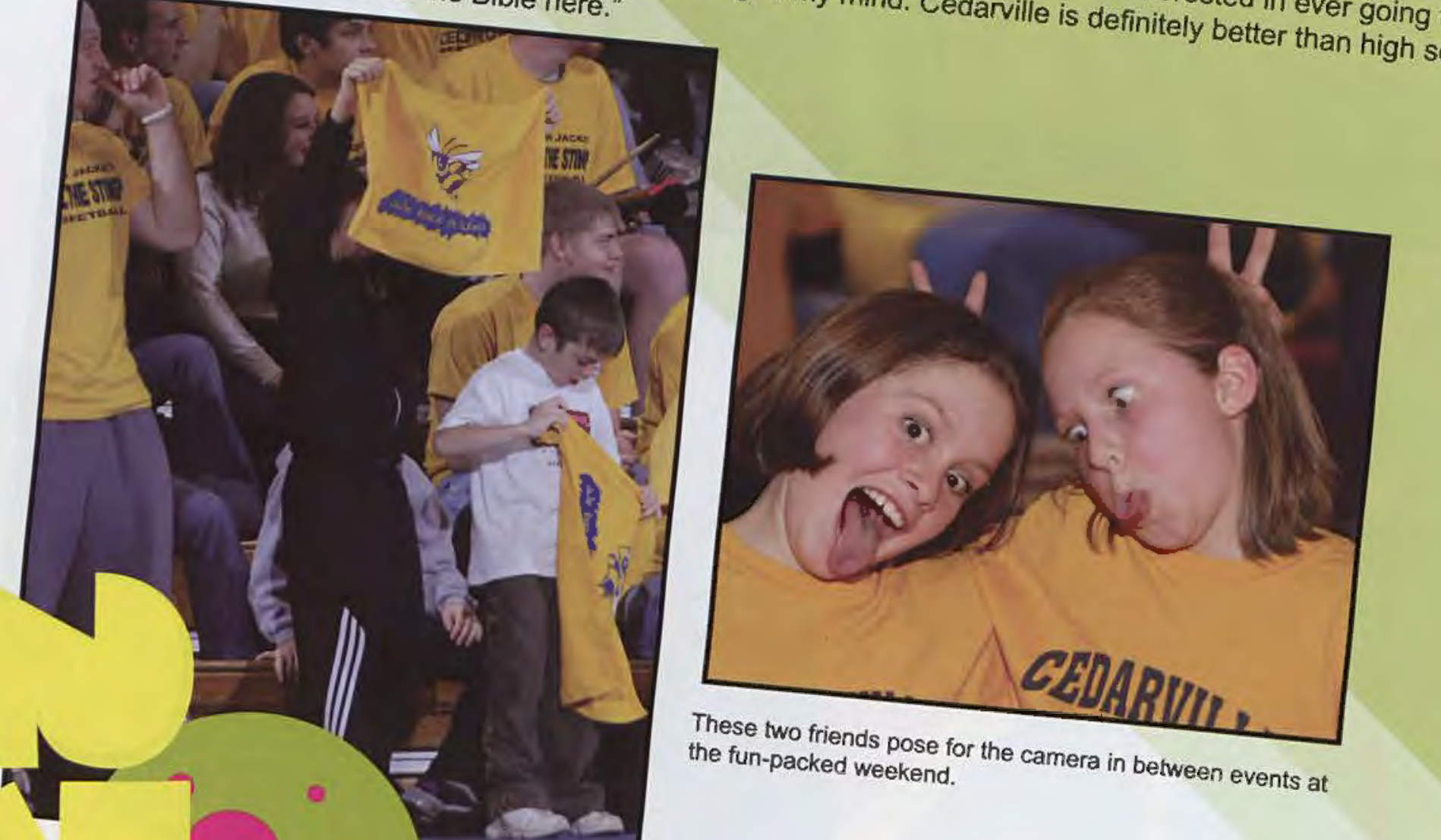




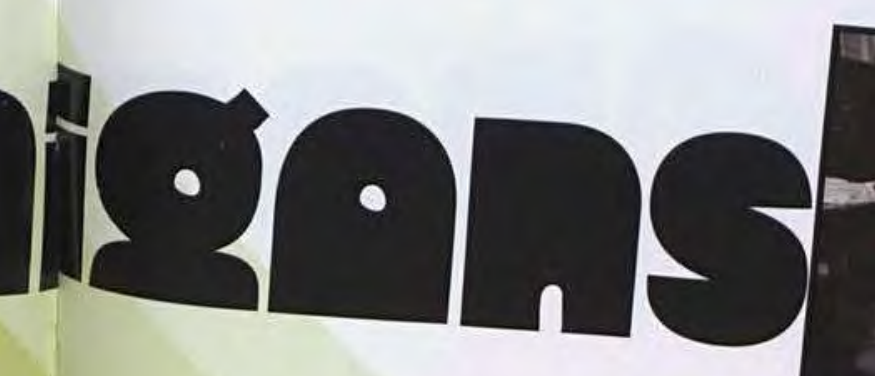

The Civil Rights bus tour makes a stop to get some much

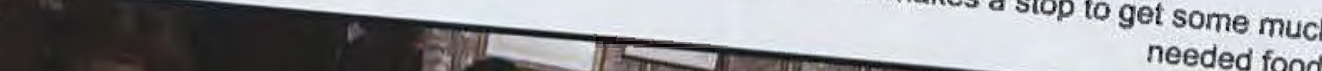
needed food.
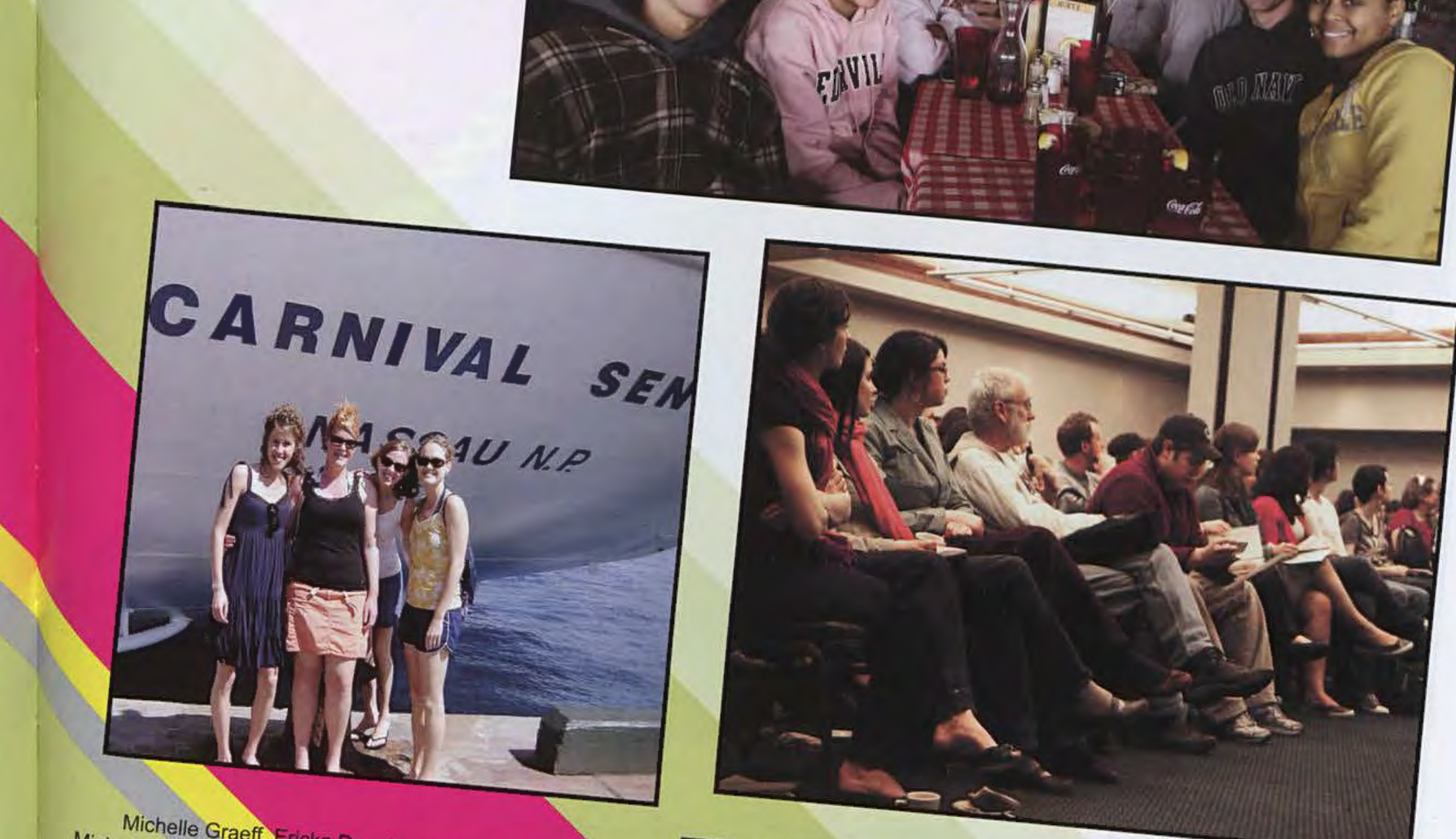

Michelle Zerminski spend spring breat, Jessica Huber, and
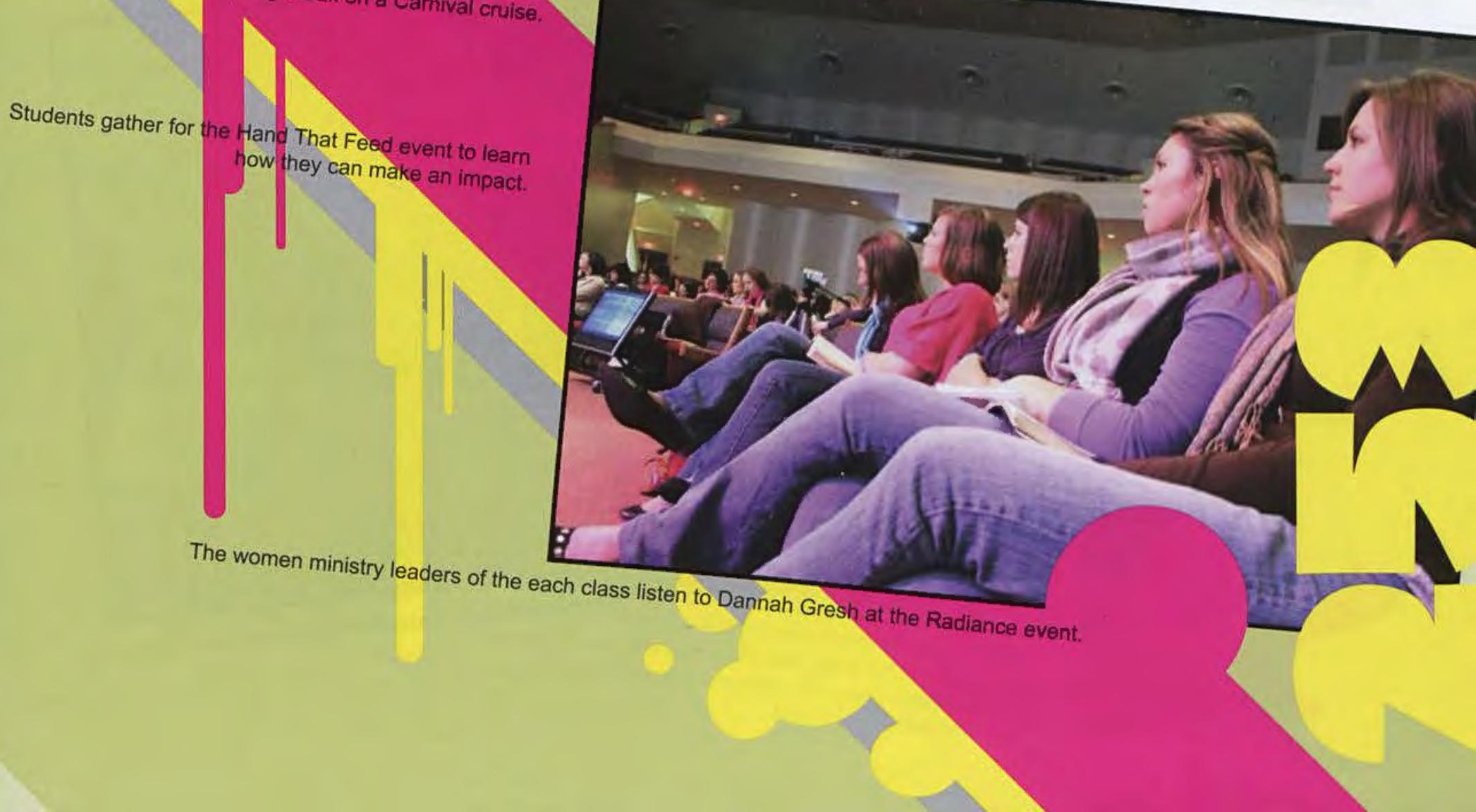


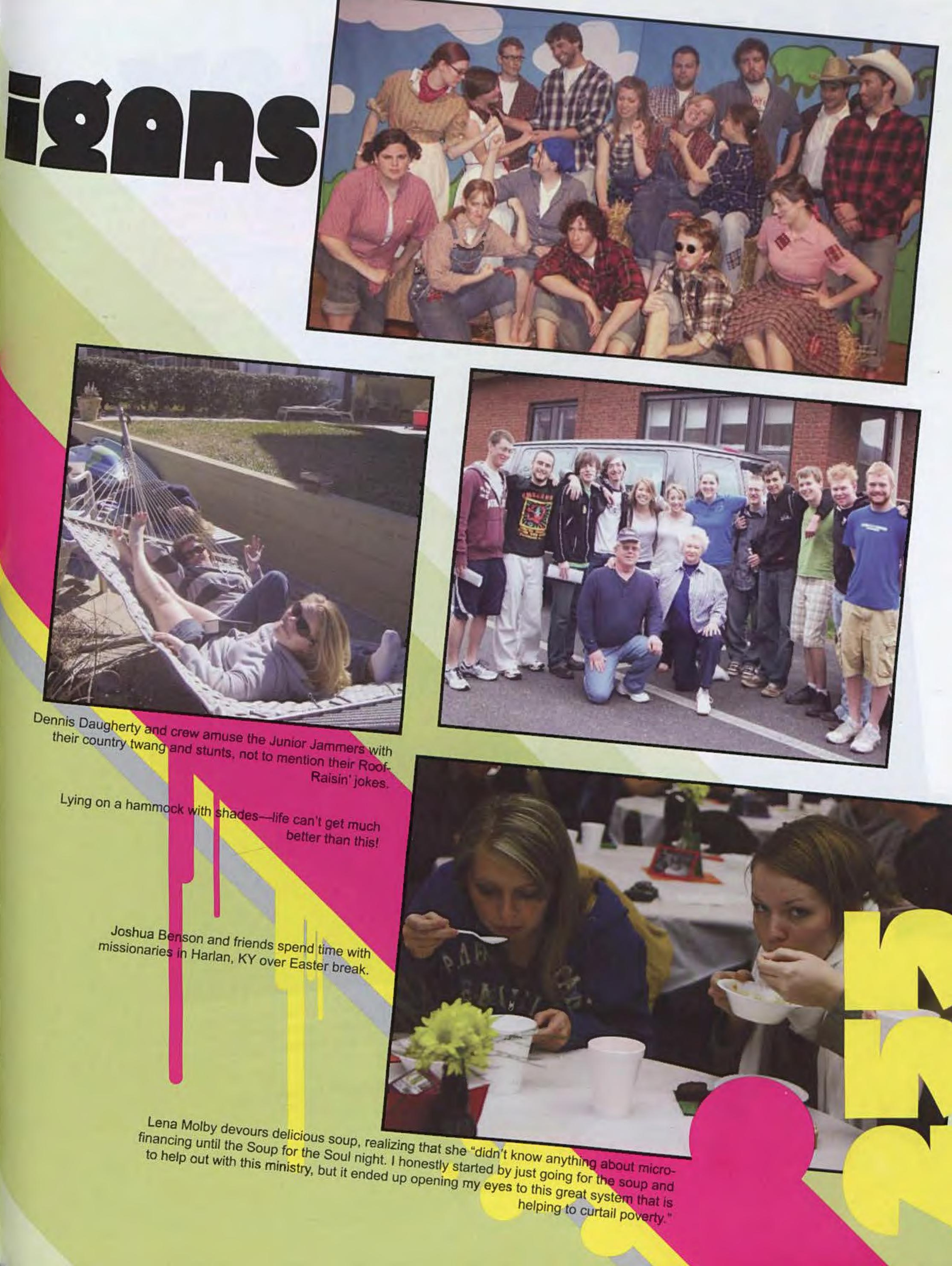




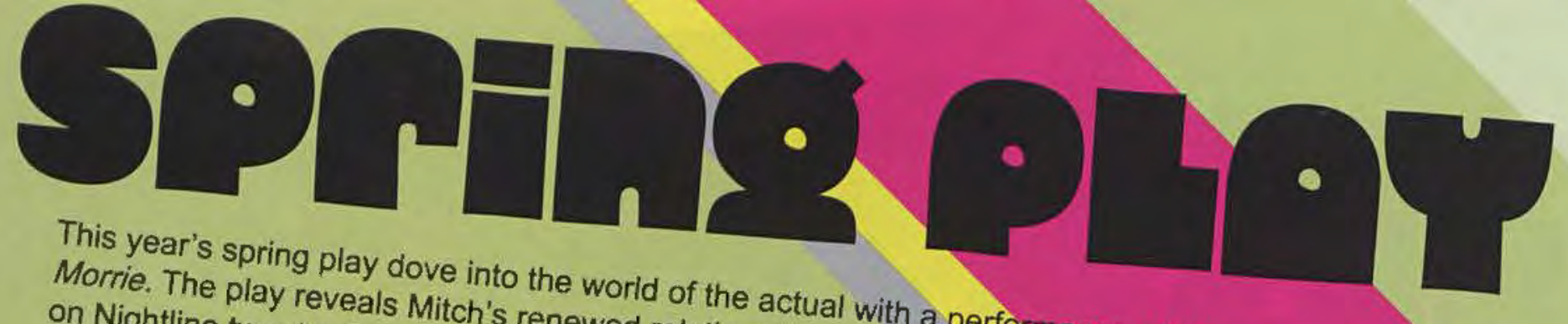
old professor a visit, which eventually turns interview reveals that Morrie is dying, Morrie, after Mitch sees Morrie the impoch the frivolity of society's obsession with youth aly meeting on Tuesdays. Mo ALS. Mitch decides to pay his a life you can be proud of in the end. me to audition fore having the opportunity to play Morrie Schwartz, reveals different experience. I have always played fairly exable." Derek Stockwell admits this wom and quirkiness. These emotion that has to go into every production was quite taxly chacters, but Mitch is somewhing Mitch "was a really The show was a great taxing, but the response from the people who saw it was tear jerker in a good success, and, as audience member sarah Murphy or mentors greatly impact your life."

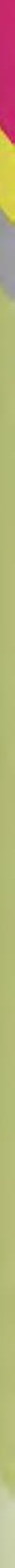


Morrie's love for dance is an expression of him living life to the

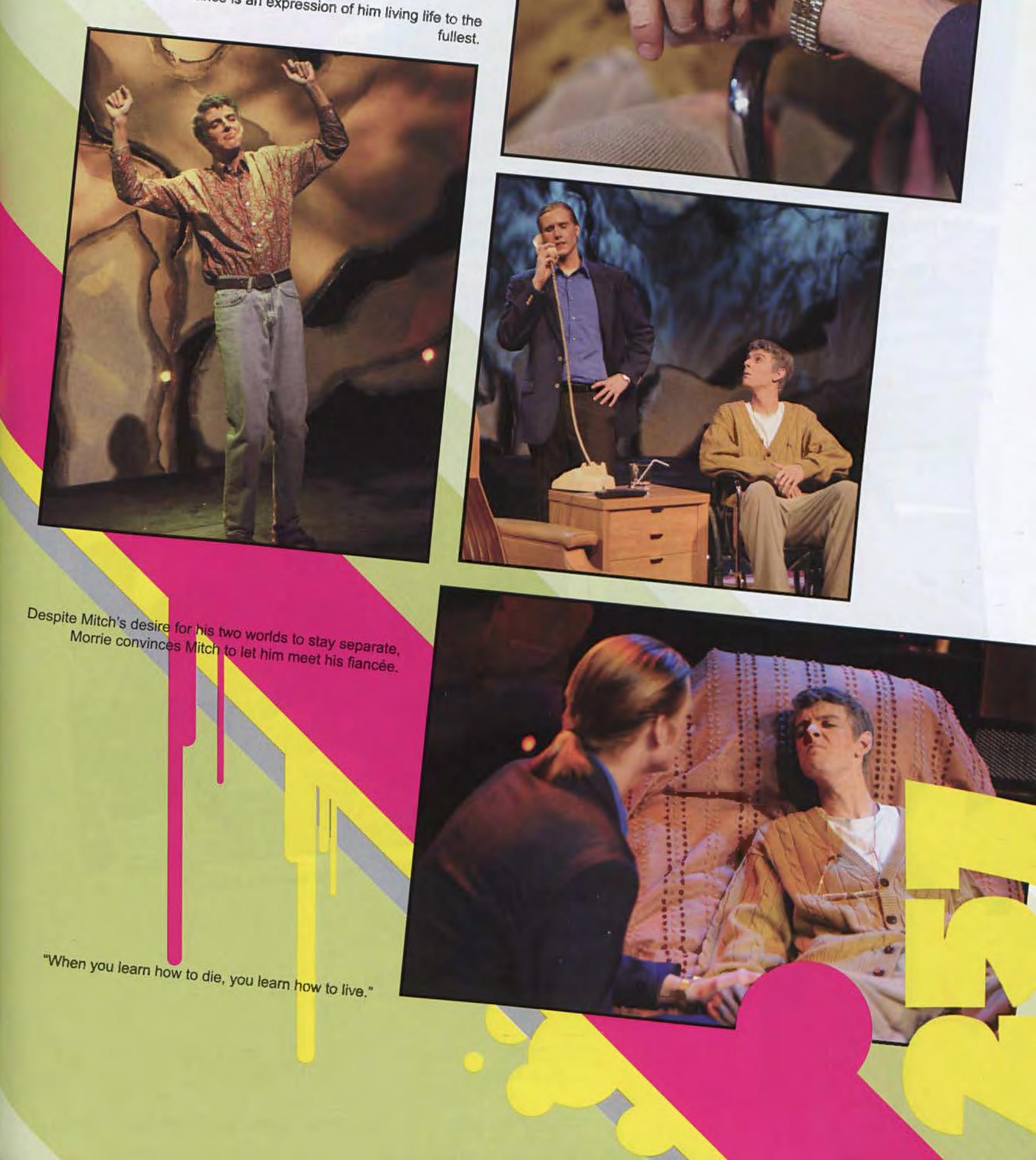


Hosts Justin Duff and Alex Clements try to dea us? Where did come? "Why is he looking at

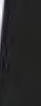
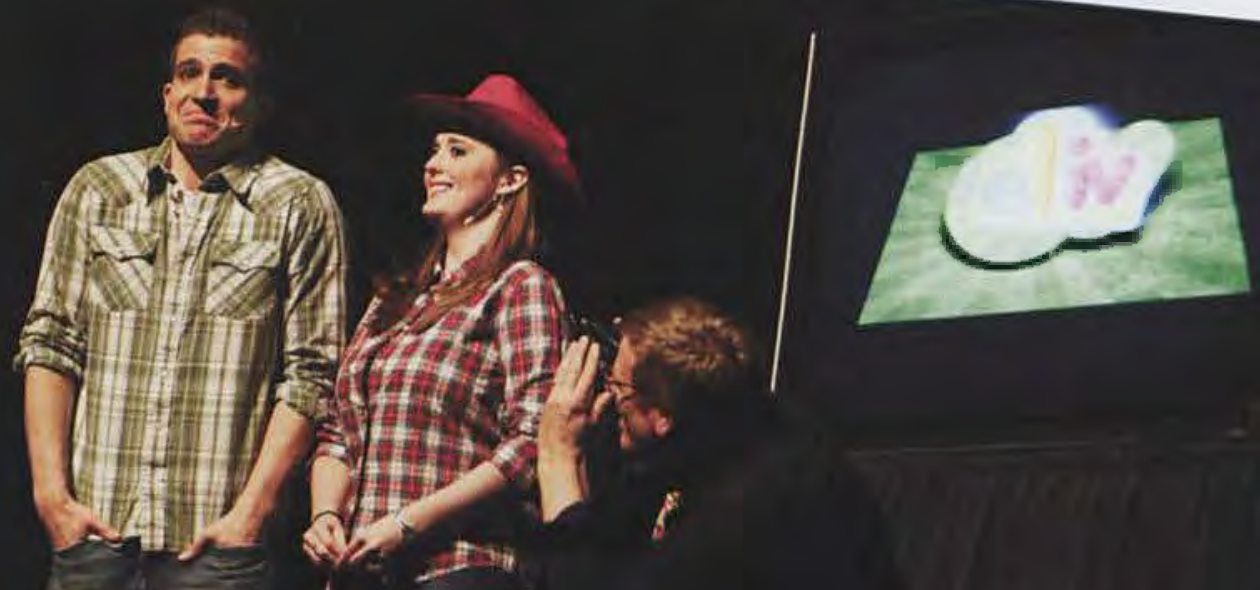

They just fit so well together! The Tetris blocks
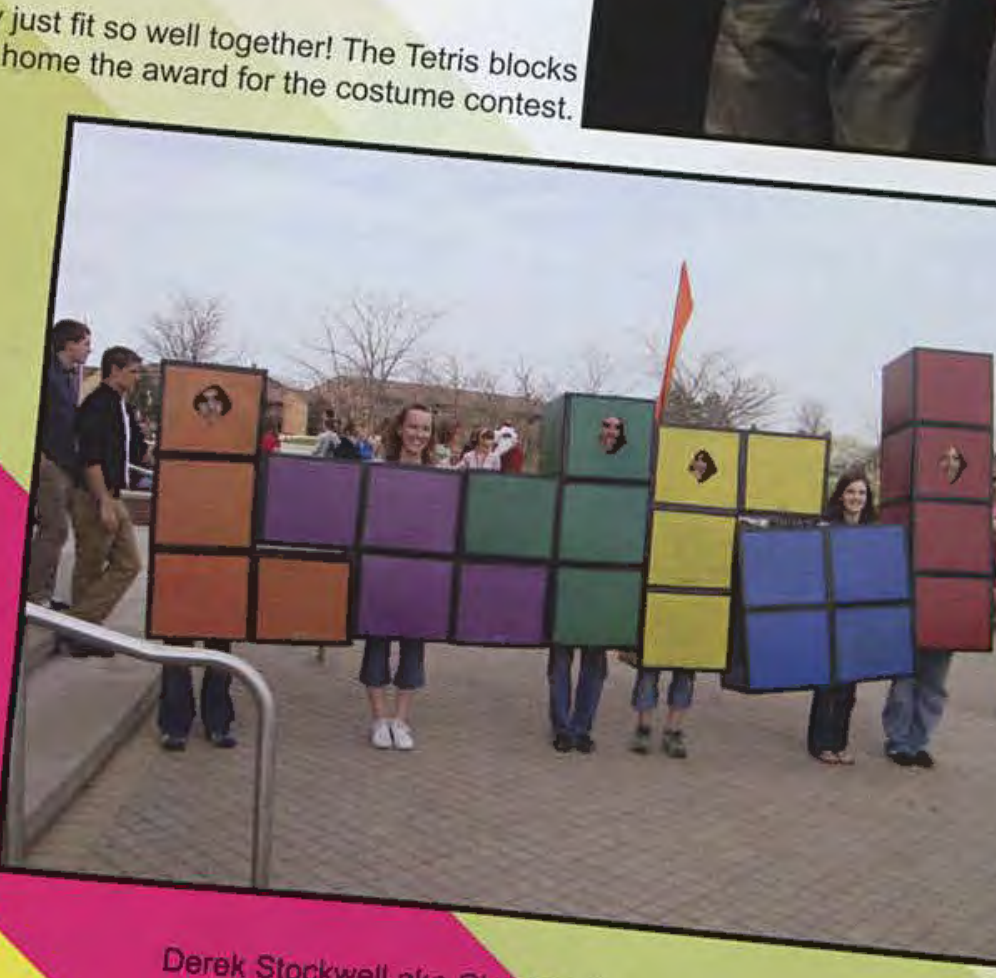

aka Tommy Pickles escape the playpen to Jason Erdmann
preshow.

preshow.

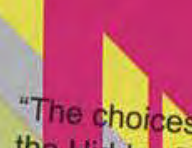

the Hidden Temple crew and your alonel" The Legends of parrots and green monkeys back memories of the purple guards or you'll lose your pendant watch out for the temple
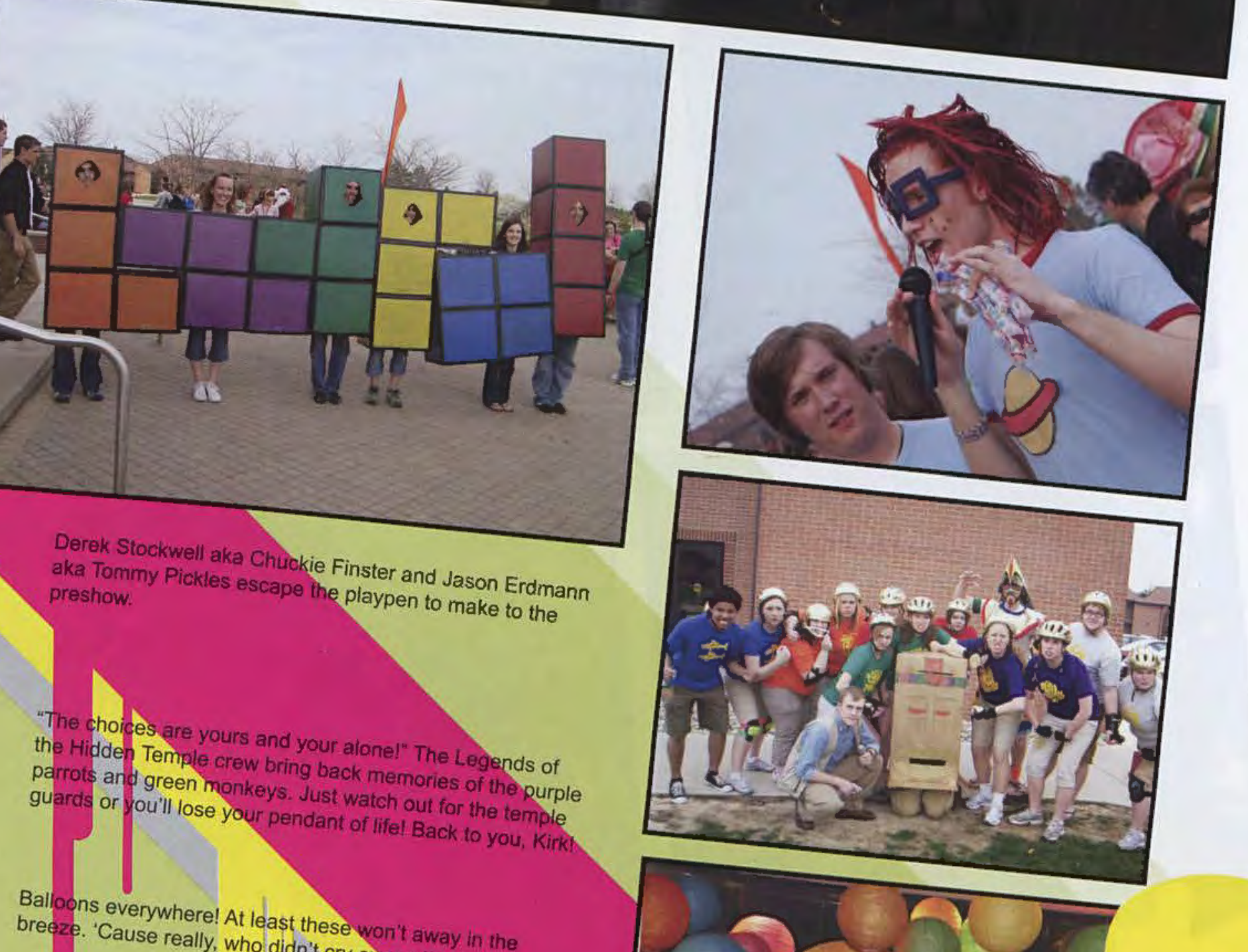

breeze. 'Cause really, who least these won't away in the 

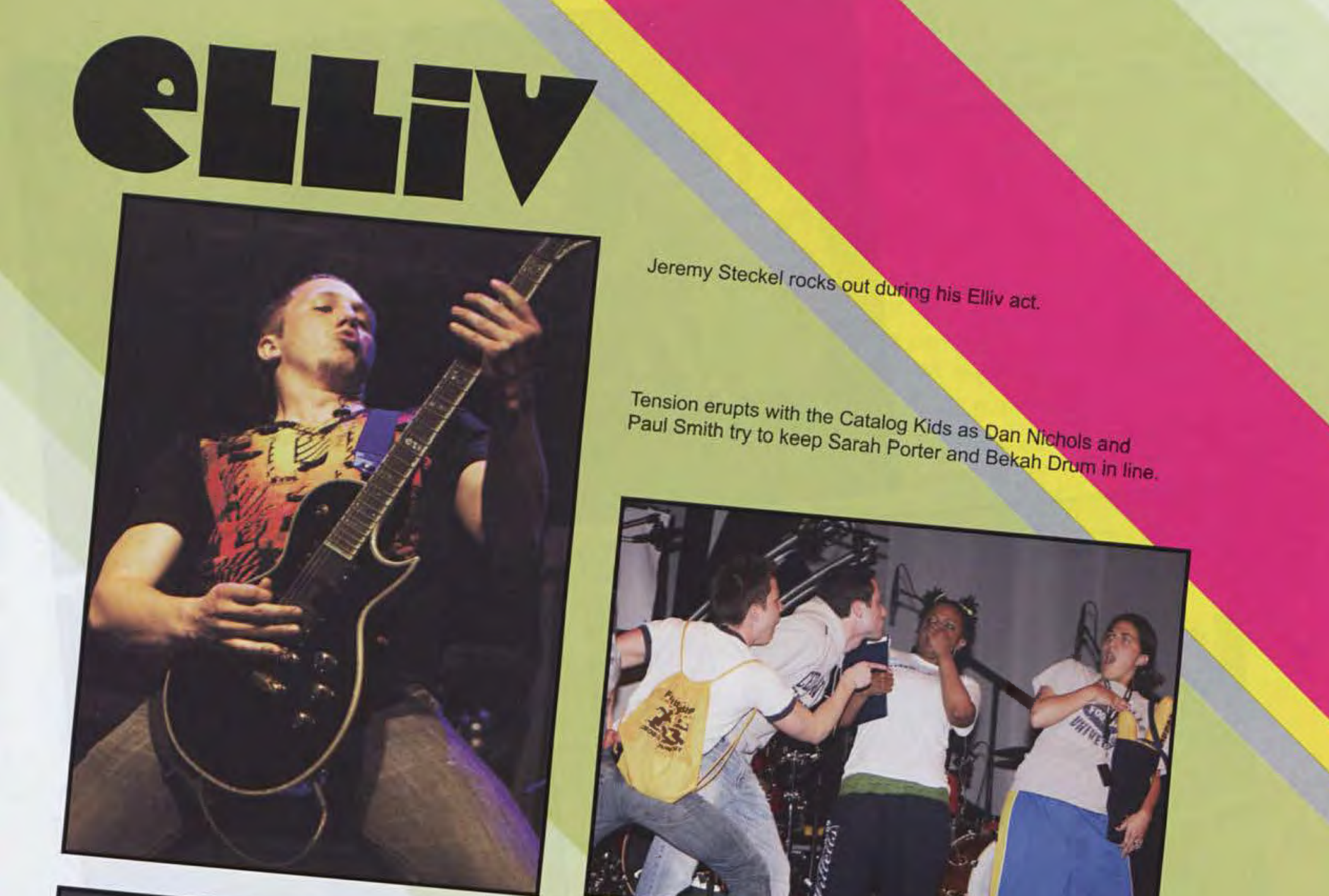

Paul Smith try to keep Sarah Pids as Dan Nichols and
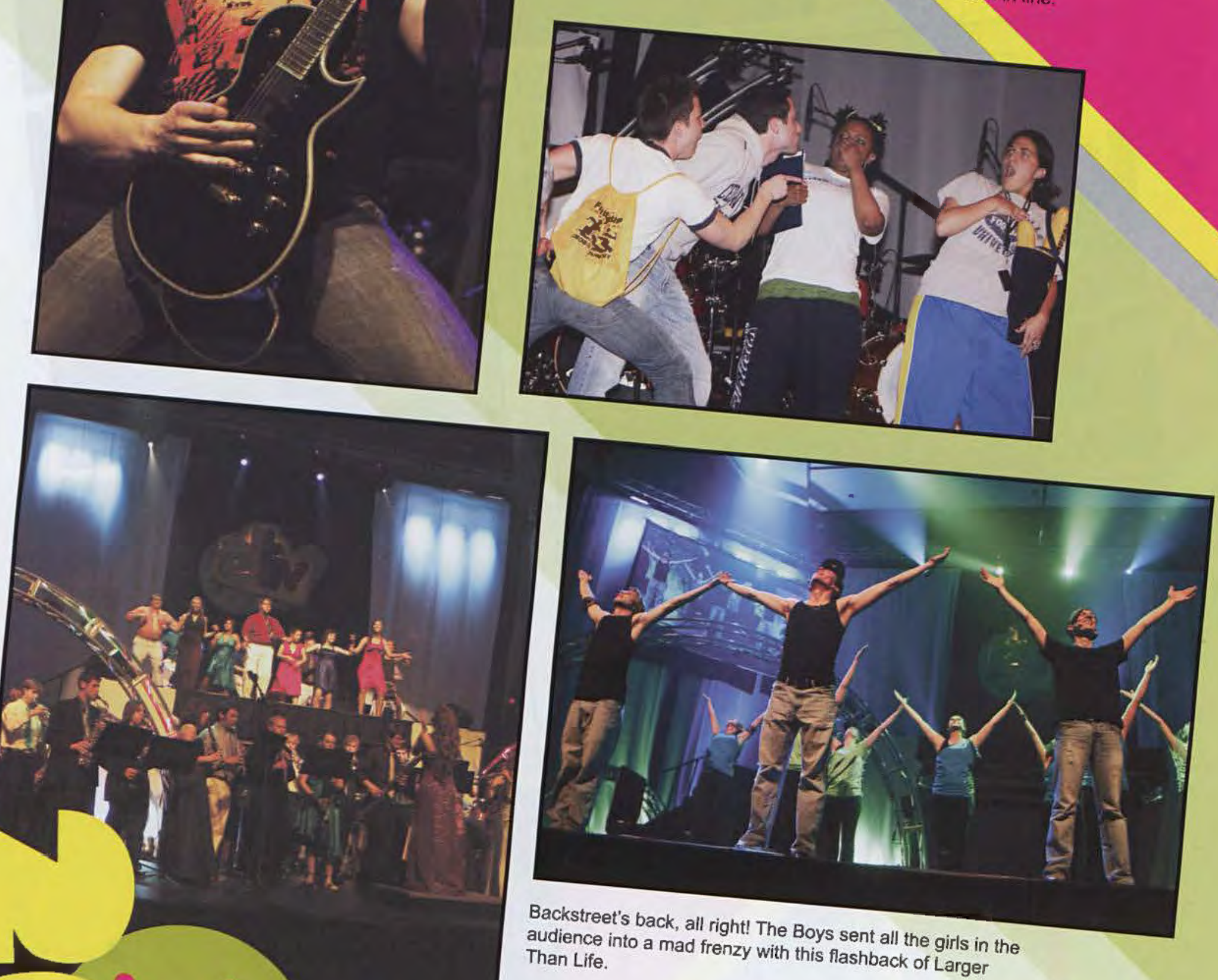
audience into a mad frenzy with this flashback of Larger
Than Life.

The Disney medley took us all back to those days of magical romance
and pretty princesses! 


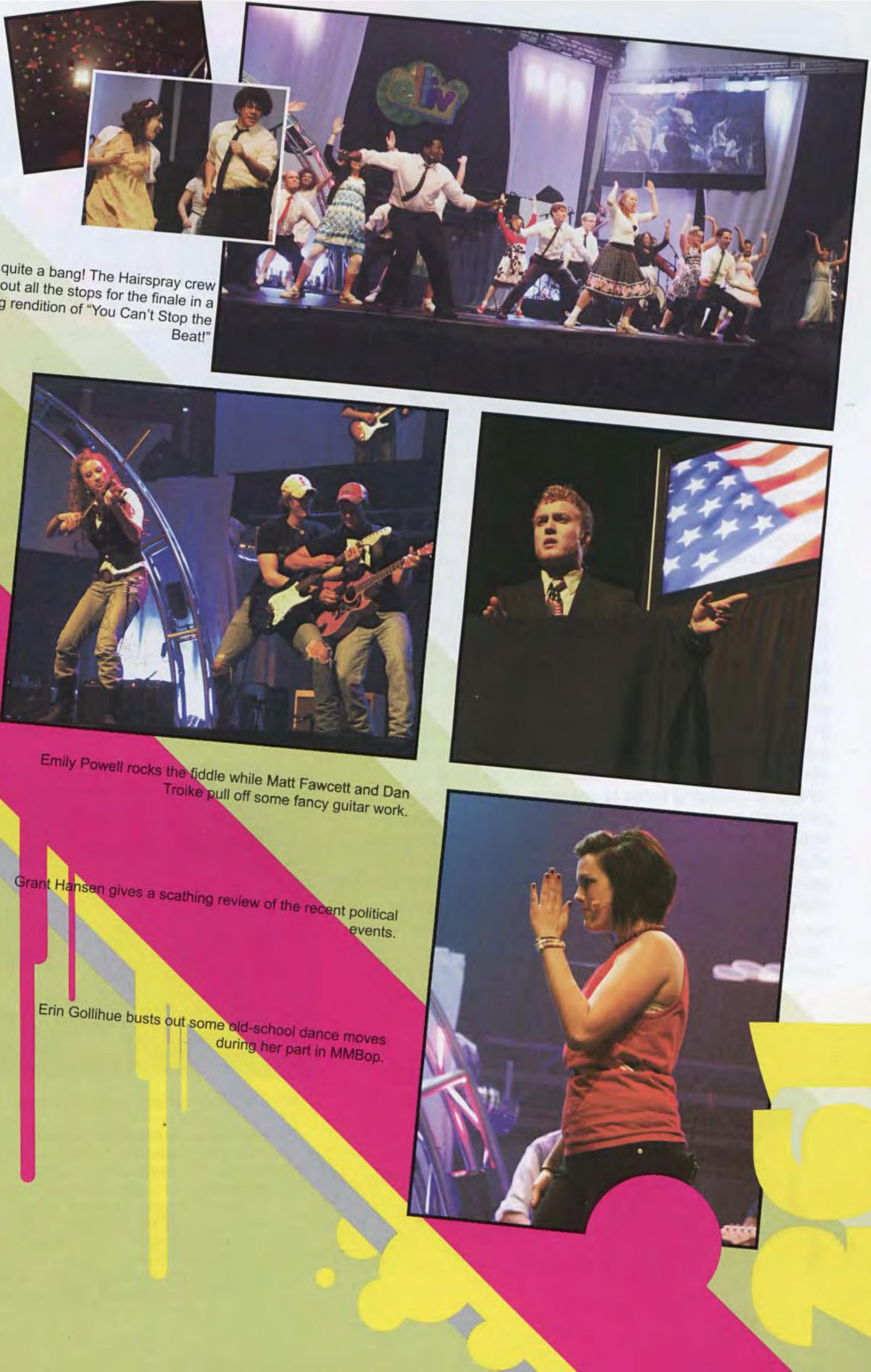




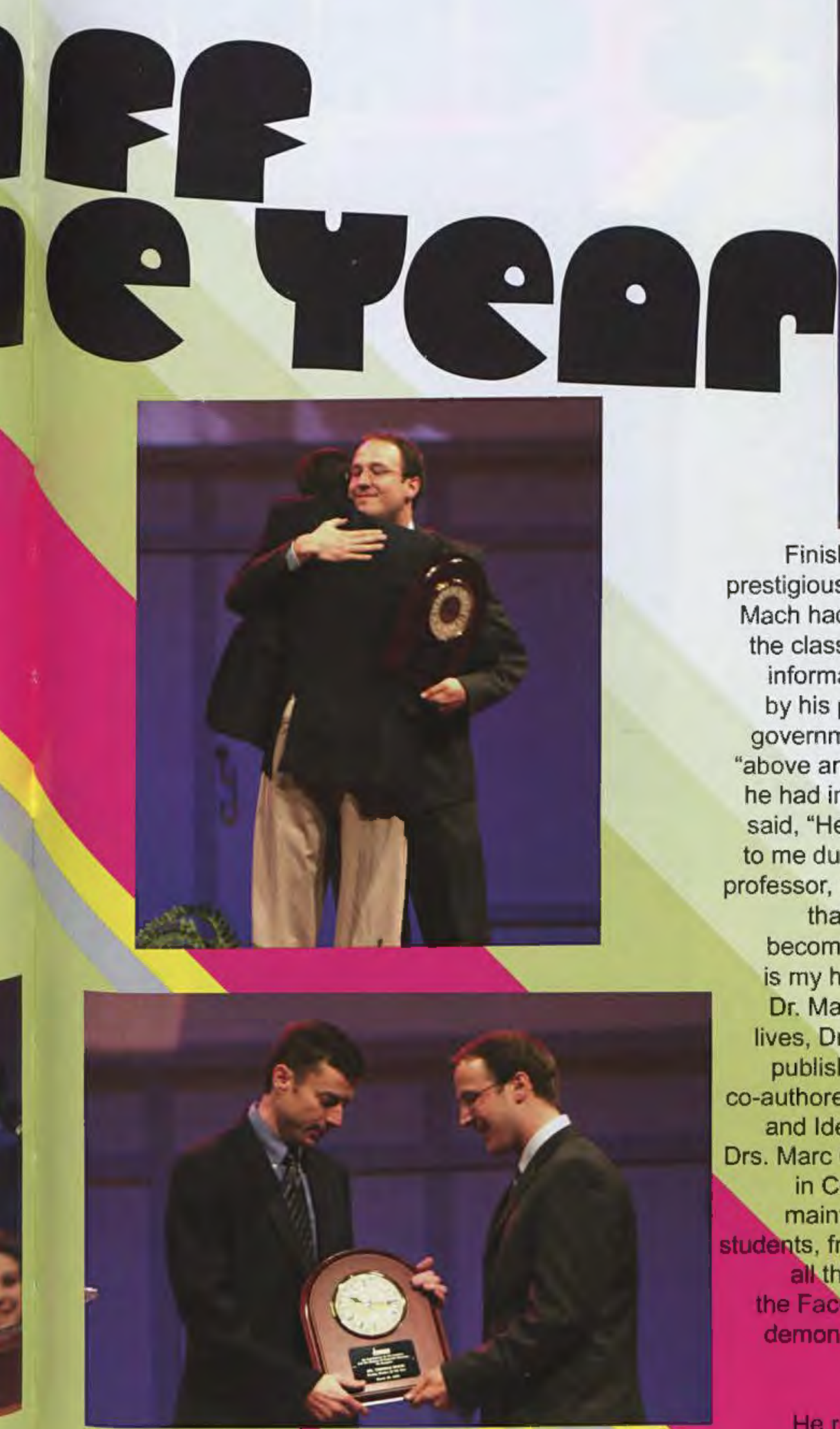

Mark Miller hugs the stunned Dr. Mach, who later reflected, "I was humbled and honored by the award. It was especially meaningful to me because it comes trom the students. It is a great privilege to work with the students here and the joy I have in doing so is its own reward. This recognition was another manifestation of the generous and gracious nature of our student body."

Dr. Mach recollects, "I did not know I had been nominated. My department made sure that I would be there in chapel that day by telling me that a colleague of mine was winning the award, and it was my job to get him there. So, I made sure he was there. Then, about halfway through the introduction, Mark Miller mentioned the SelfStudy, and I knew I had been duped."

Finishing his ninth at Cedarville, Dr. Tom Mach earned the prestigious faculty of the year award. As a history professor, Dr. Mach had surmounted the challenge of incorporating faith into the classroom and challenging his many students to evaluate information through a Christian worldview. Highly respected by his peers, he would serve as the chair for the history and government department the following year. Renowned for his "above and beyond" involvement in his students and advisees, he had influenced more students than he knows. One student said, "He has been an incredible encouragement and support to me during my time at Cedarville. I could not ask for a better professor, mentor, or friend." Sophomore Geoff Beck expressed

that "Dr. Mach has inspired me by showing my dream of becoming a history teacher (or professor) can be a reality. It is my hope that when I reach that level, I will be viewed as a Dr. Mach to my students." With such an impact on students' lives, Dr. Mach continued to advance his academic career by publishing a journal article every few years, and recently he co-authored 'Gentleman George' Hunt Pendleton: Party Politics and Ideological Identity in Nineteenth Century America" with Drs. Marc Clauson and Mark Caleb Smith. He also participated

in Cedarville's mentoring and Fit-to-be-tied programs and maintained generous office hours to assist and encourage students, frequently staying late to care for and advise students,

all the while not neglecting his family. In 2006 he received the Faculty Teaching Effectiveness Award further demonstrating the high regard his peers have of

him.

He ranks as a "real historian" according to Dr. Rich, an authentic Christian, and an exceptional professor. 

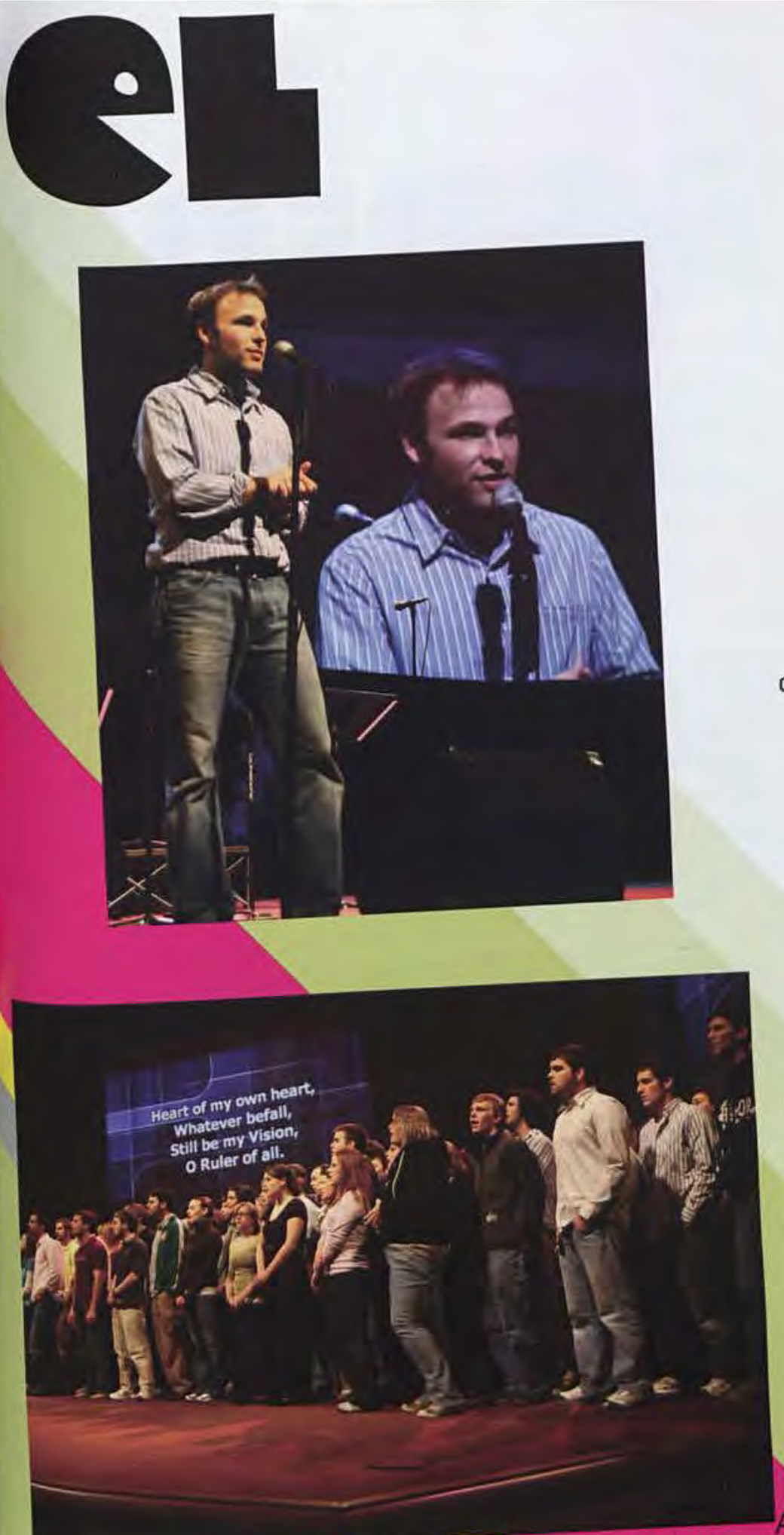

Several seniors commemorate the "Circle of Truth" from freshman year.

Senior Andrew Davis opens chapel with a word of prayer.

All the graduating seniors gather on the stage for one final farewell
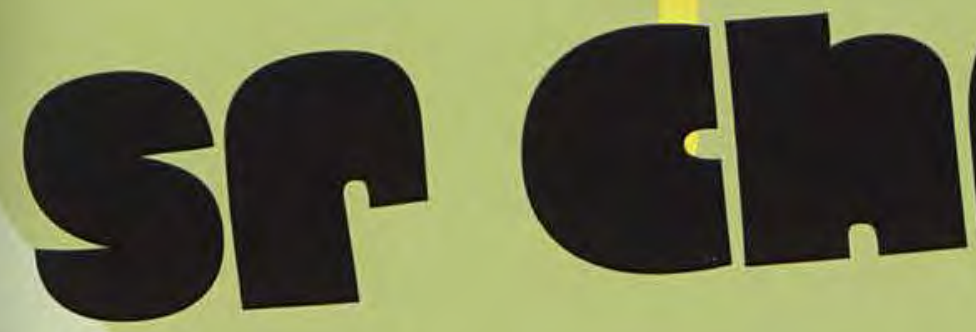

After four years at Cedarville, and six year for Chris "Cleveland" Harmon, the senior class took the opportunity to reflect back on their time here during Senior Chapel. The senior class officers organized the entire chapel, from the music and testimonies to the videos and skits.

Highlights from the past four years were presented in the form of hilarious skits. They even paid tribute to the infamous Early Arrivals Party from freshman year, where an unidentified man asked some fairly personal questions to the students during a "get-to-know-you" game. This event was commemorated with a song called "The Circle of Truth." Every senior who was at that freshman party knew exactly what they were talking about.

One student mentioned that her favorite lake story was about how Dr. Phipps got the Volkswagen Beetle at the bottom of Cedar

Lake. When asked what he learned from his roommate, senior Josh Saunders replied, "Well, my roommate is Peter Brandt, so I learned... perfection."

Four seniors also shared their testimonies during chapel, recapping their years at Cedarville and looking back on some of

the struggles as well as blessings they encountered along the way. Many of them offered words of wisdom and encouragement to the rest of the student body, telling of God's provision and faithfulness.

At the end of chapel, all the seniors made their way to the platform as a bagpipe sounded a verse of "Be Thou My Vision," and Andrew "Cos" Costerisan led everyone in singing. It was a fitting tribute to all the departing seniors. 
" 1 thirsty JS date +3 bowls of mystery punch $=$ 100 ways for a man to fail. Solution: Try "em all way through the punch selections

"IS means is that people dress up and cameras a photo Near the end, it became so much of models. Someone we clearly began to feel like our legs, which in the brilliant idea to model though un-matching, pair case revealed my classy, were a good choice though"

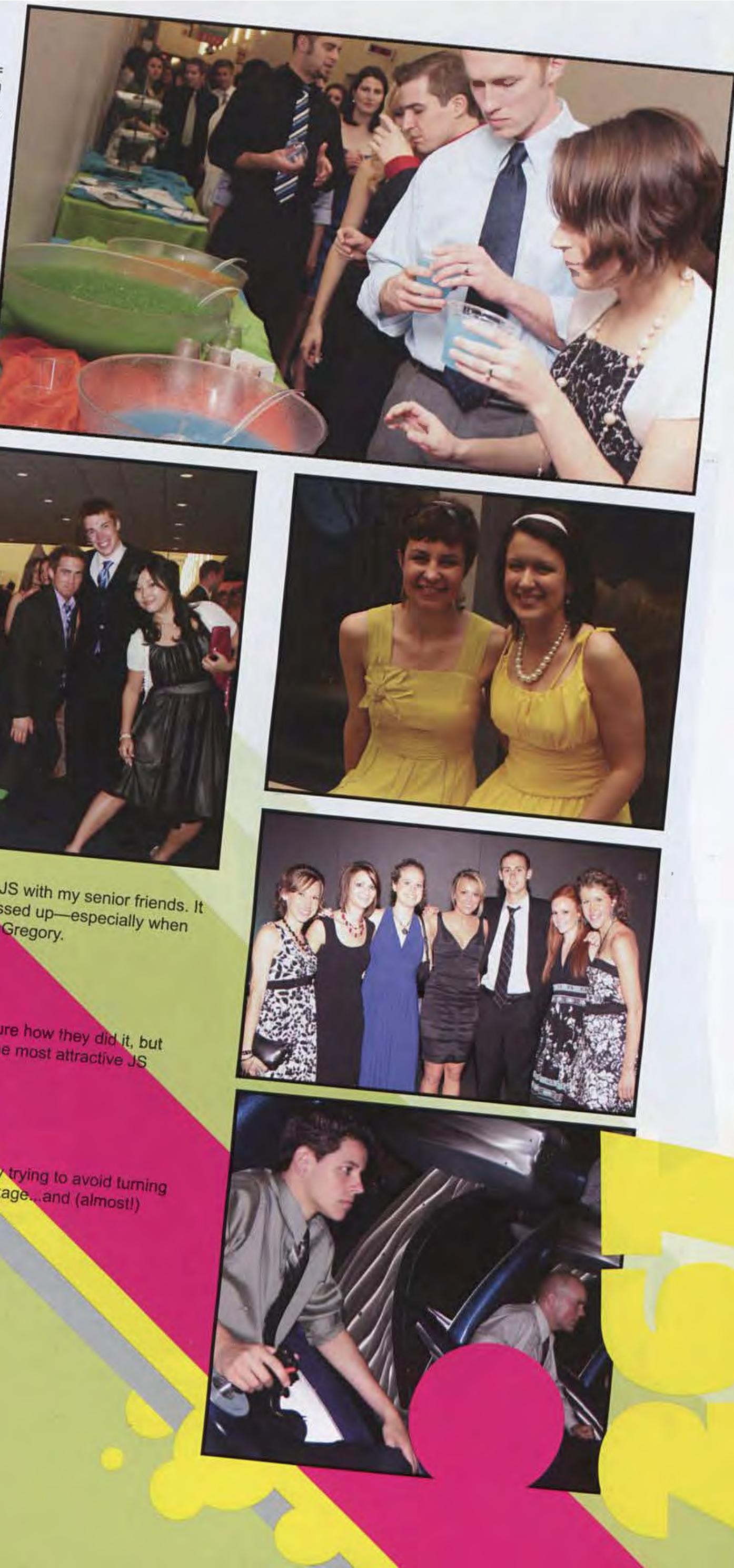




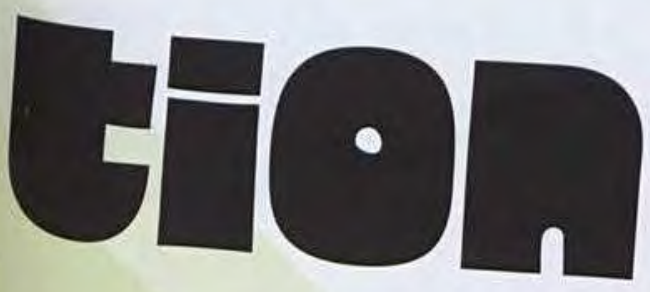

that marked the beginning of our last few daus a great event was a great time spent with close friends days at Cedarville. It Brown astunity to share some last thou and provided many of Brown as things began to come to thoughts with Dr and Mrs. and Mrs. Brown in a one-on-ome to a close. Speaking with Dr. experience. The setting is always a memorable

evident in several ways, but I could the student body is took time out of their busy schedul could not believe that they every graduating senior. I'm unaware to speak with each and president who cares so deeply for of any ether university's Even though Charissa Rowe home to the SSO due to rain Kimed from Dr. Brown's "they gave us some really yummy desserts, so l'm glad I went."

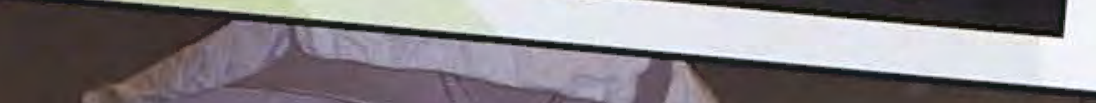

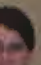
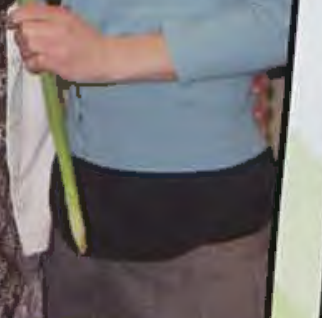

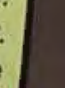




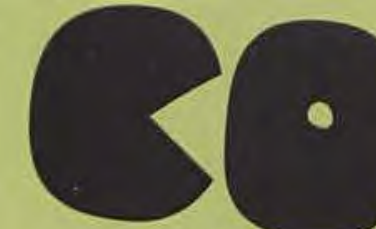

For Cedarville's 113 th

ating seniors s 113 th commencement, Dr. Mark Bailey, Presi

to cultivate in about standing strong in a culture thailey, President of Dallas Theologic

scholarship, which the university will The Class of 2009 donaty resists the light of Christ thinary spoke to the graduamount of ministry involvement.

(henior student who has "Light of the World" day experiences. ville, is what I will treasure. The laughterival at a destination point for me - the journ

Adam Evans during my time in college will tears, deep conversations, and mome journey, these past four years at Cedar-

declared. "I blinked and it was over. Now the only thing I have is like my four years at Cedar.
Rebekah Olsen discussed her emotion

led us in "Christ is All I Need." I sational graduation expering I have degree...and a wife! Thankille: a blur," Evans of my peers had to deal with this sang the familiar song one lence, saying, "Sitting there before you, Cedarville. perfect song for the perfect mom day, and I found great peace ime and thought of all the uncertainty and, Dr. Brown Perhaps all the gration, any place, all the time." been changed for the bes would agree with a final declaration

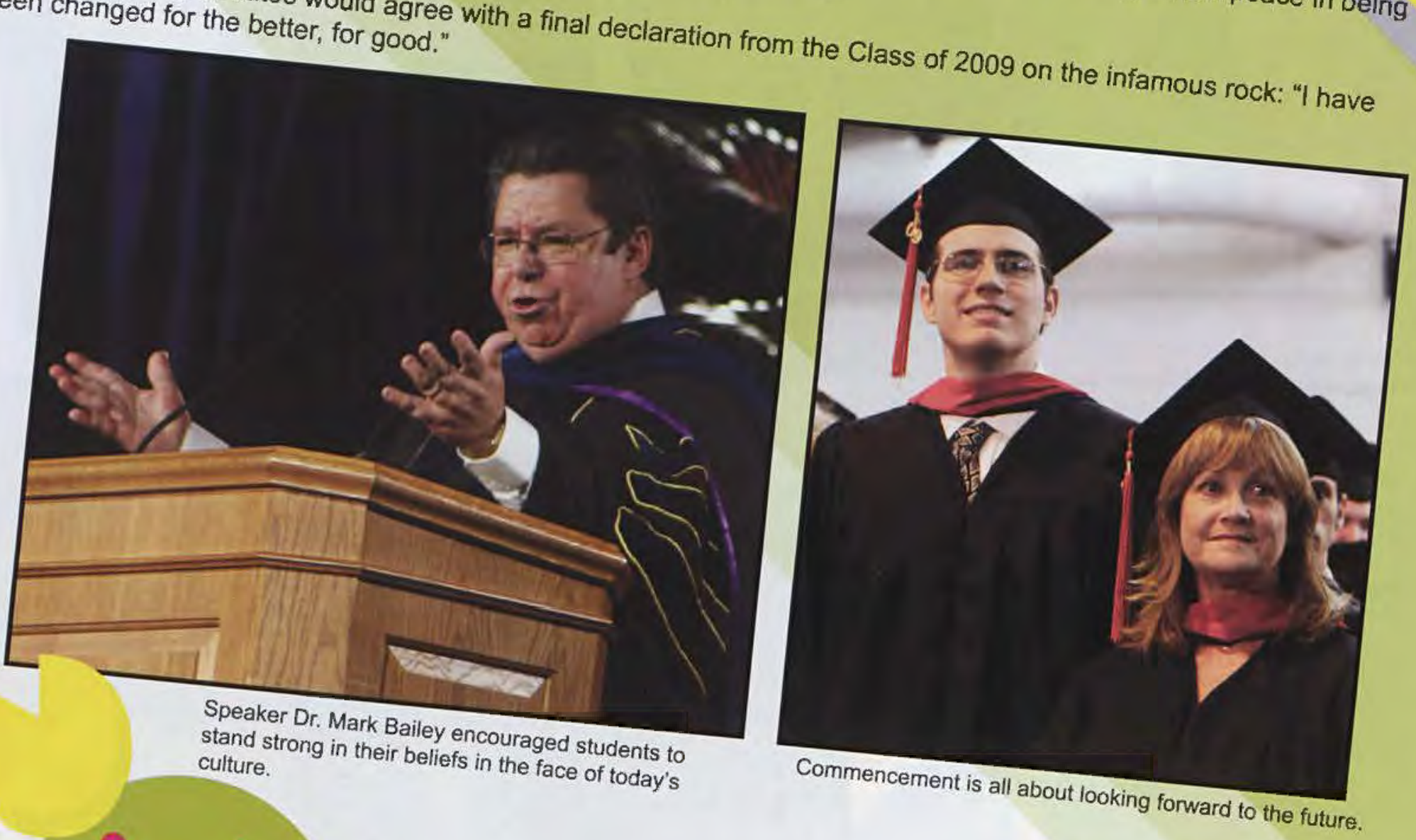




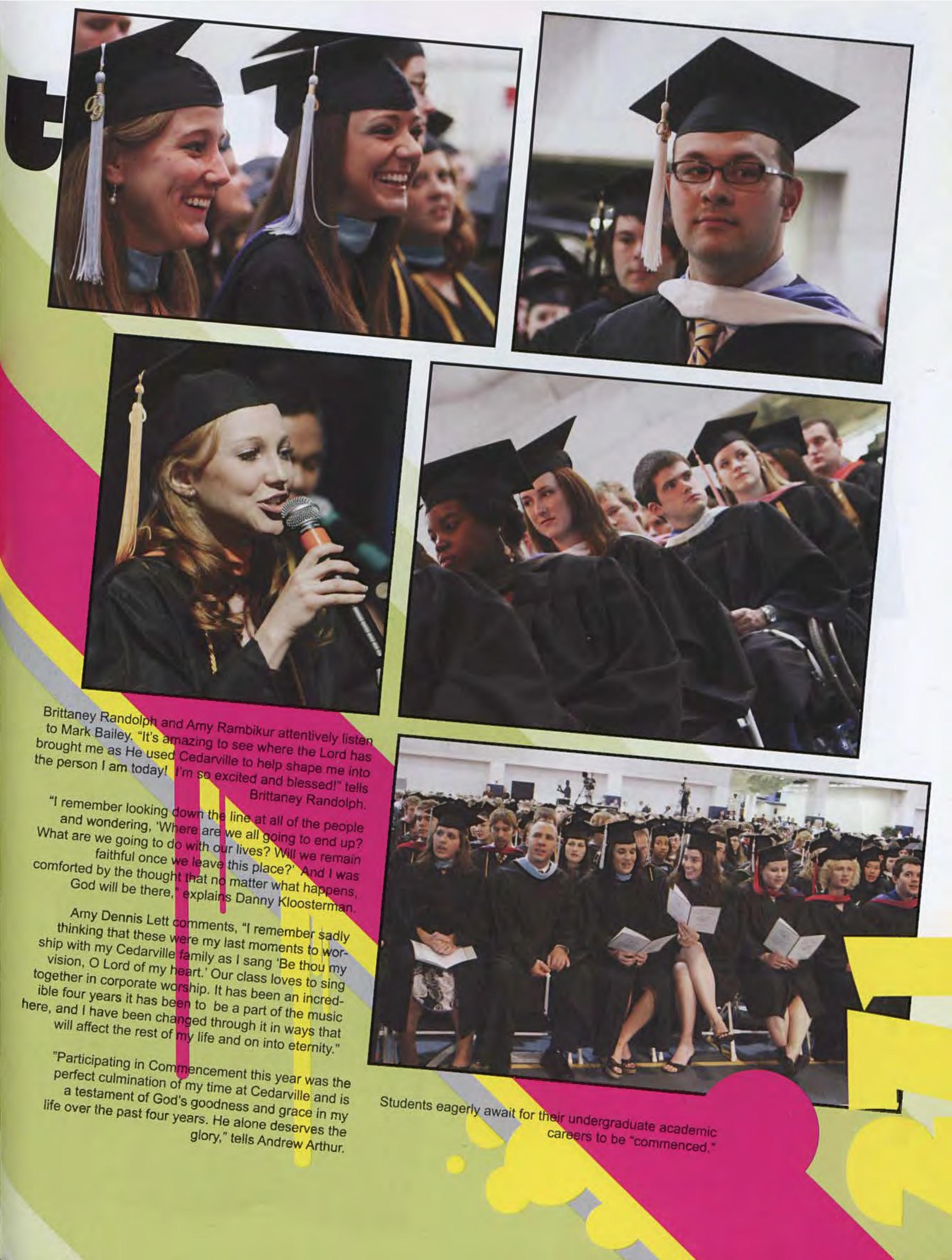




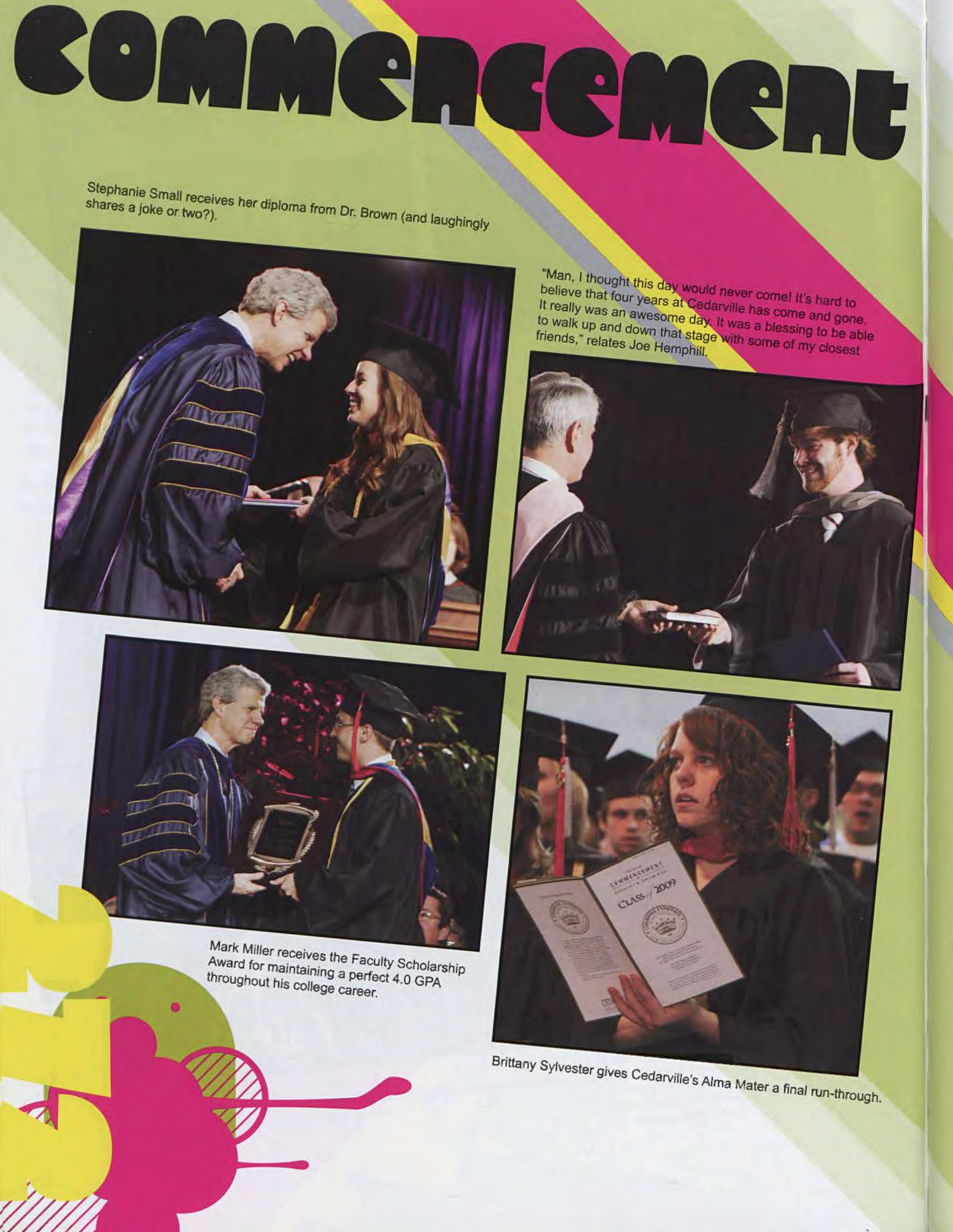



Stan Moran, Emily Vandett, and friends picture before heading off into the world

of music.

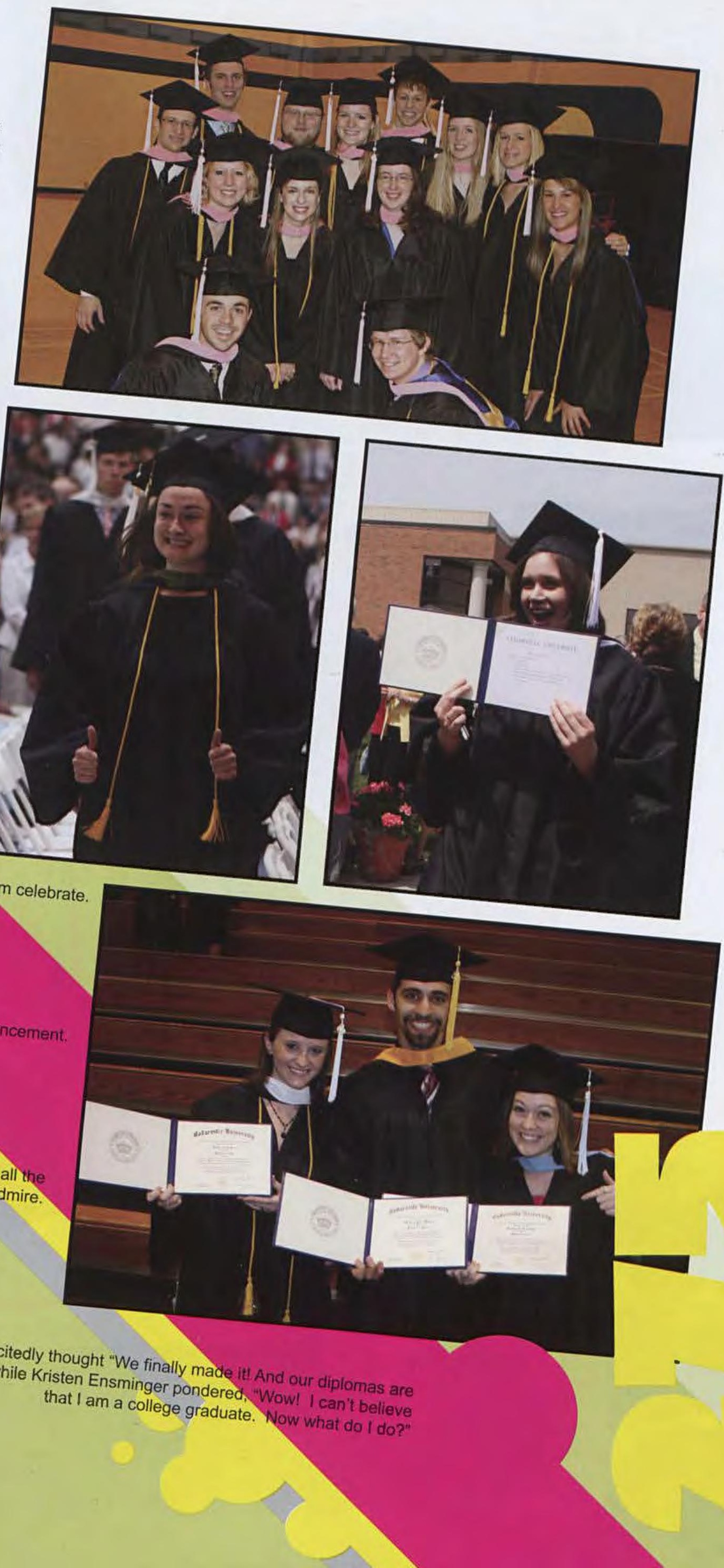




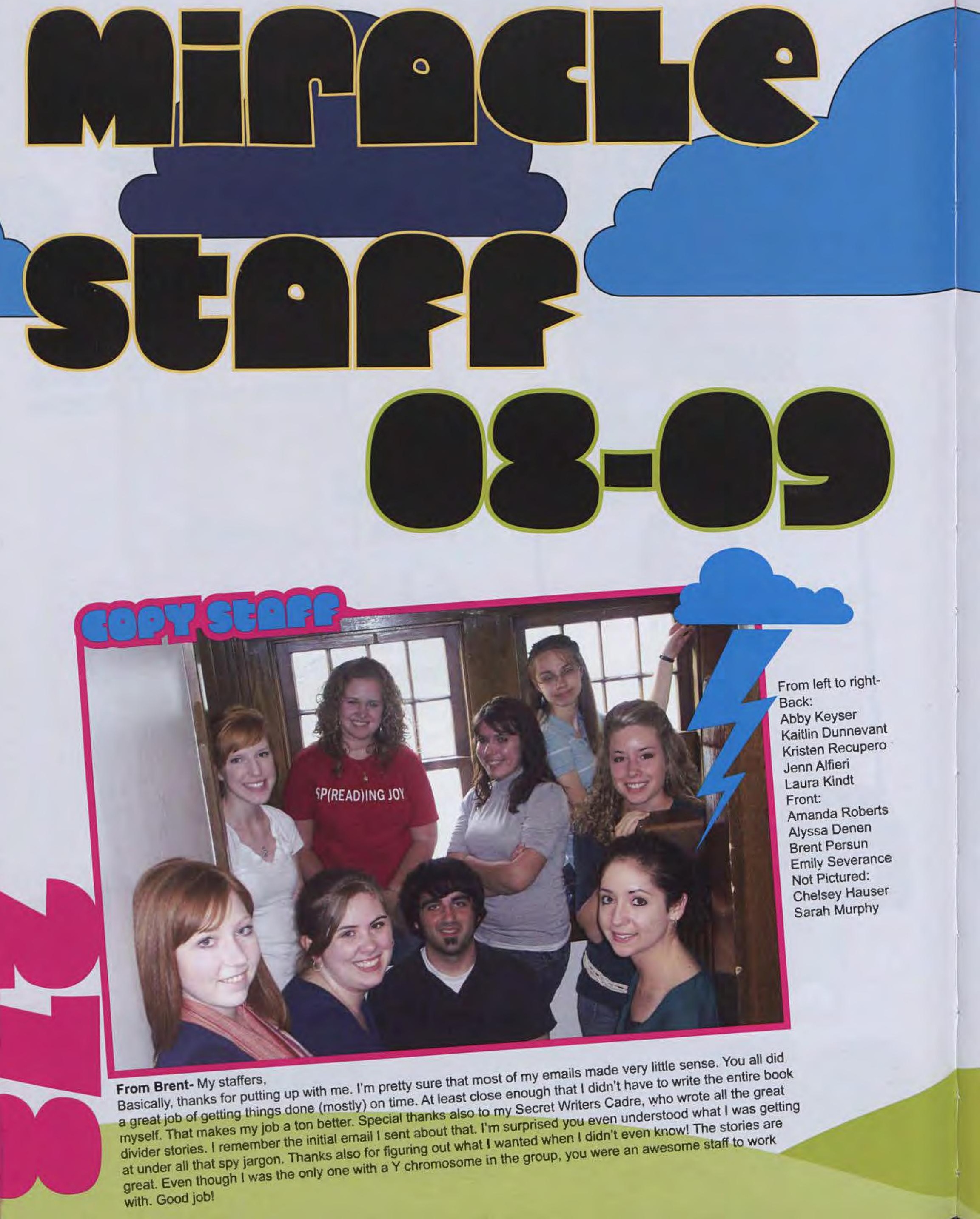





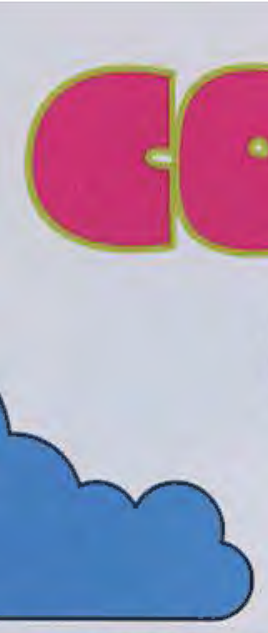

\section{PUbisther}

The 2009 Miracle was published by Jostens, Inc. It was printed at their plant located in State College, PA.

The Jostens representative was Eric Howard, and the plant coordinator was Crystal Gault.

\section{Gever}

The cover is a Custom Litho that was designed by David Elgena.

\section{Deper stedx}

The paper for this book is $80 \mathrm{lb}$ gloss finish. 294 all color pages were used.

\section{corer}

The 2009 Miracle was printed with four color process ink.

\section{cesign}

The 2009 Miracle yearbook design theme was created by David Elgena, and other design work is credited to Katy Russell, Trevor Plumley and Jana Linville.

\section{bhene}

The 2009 Miracle theme was a combined brainstorm of the editorial staff with input and commentary from the Miracle staffers.

The 2009 Miracle was produced on IBM computers. Layouts were created using Adobe InDesign CS3. Photos were cropped, resized, edited using Adobe Photoshop CS3. Copy was typed in Microsoft Word and then imported into InDesign.

\section{Evpogrepir}

The main fonts used in the book are Arial and Cooter. Stories are 11 point Arial. Captions are 9 point Arial. Titles and divider text are in Cooter and are of various sizes and colors. Page numbers are 120 point Cooter.

\section{Photegrephy}

The majority of pictures were taken by Josh Gigliotti and his photography staff. Student submissions were also accepted. Several photos were also taken by Scott Huck, photographer for Cedarville University. Jim Rainey acted as the DaVor representative for portraits.

The Miracle Yearbook office may be contacted for additional information about this book's production at:

\section{Miracle, Cedarville University}

251 N. Main St.

Cedarville, Ohio 45314 (937) 766-4995 miracle@cedarville.edu 



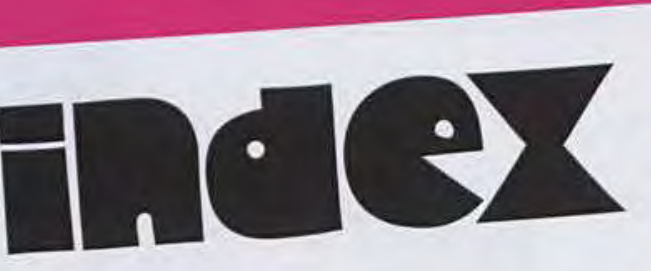

Anderson, Benjamin 210

Anderson, Frederick

$20,21,120$

Anderson, Haddon 186

Anderson, Meredith 88

Anderson, Stephanie

78

Andres, William

Andrews, Eric

Anfang, Laura

Anfang, Robert

Angle, Drake

Arch, Nicholas 210 .

269

Arthur, Andrew

240, 271

Arthur, Peter

120

Arthur, Rachel

142, 170, 200, 201

Askew, Luke 120

Atkeson, Daniel 193

Augenstein, Emily 120

Augustine, Bradley 120

Auyer, Jeremy 210

Ayers, Nannette 156

Ahrens, Hanna 120

Aker, Olivia $\quad 210$

Alfieri, Jennifer 156

264, 278

Allen, Amanda

116

Allen, Brietta $\quad 210$

Alliman, Ricardo 156 .

186

Allison, Sarah $\quad 156$

Amarante, Holly 210

Amerine, Levi $\quad 156$

Andersen, Eric $\quad 210$

Andersen, Sarah 99 , 200,201 , 210

Babbitt, Allison

210

Babcock, Devin

Backer, Laura

Bacon, Grant

194

Baden, John
Arbogast, Wesley 78

\section{Barkas, Elizabeth \\ 156 \\ Barker, Jacob \\ Barnard, Rachel \\ Barnes, Brianne \\ Barnes, Geoffrey \\ 122 \\ Barrett, Collin \\ 139 \\ Berron, Esther}

Barron, Hannah

102

Bartlett, Betty

Bertley, Erin

Bartling, Jason

Basford, Diana

Bash, Steven

120

Bashore, Jessica

Bates, Sarah

Battis, Christine

Baumer, Leanna

156, 171

Baylor, Rachel

277,279

Beachy, Rachel

118, Beals, Christian

Bearden, Molly

Beauchemin, Faith

Beck, Geoffrey

Becknell, Mark

Beelen, Alexander

203

Beesley, Amanda

Beight, Micheel

Bell, Beth

Bell, Eric

Bell, Katie

211

Bell, Nathan

Belote, Rebecca

Bender, Jason

Bender, Kevin

174

122

Bailey, Benjamin

Bailey, Julita

Baker, Elizabeth

Baker, Leah

204, 205

Baker, Stephanie 204

Baldwin, Chase 149 ,

156

Baldwin, Dane

Balint, Jennifer

Banz, Geneva

147

Banz, Jared

Baranowski, Brittany

210

Baranski, Jacob 211

Barclay, Samantha 78

Bardakjy, Scott 156

Barfell, Andrew 120

Barham, Nathanael 211

193

120, Benson, David

Benson, Heidi

Benson, Joshua

255

Benz, Erin

Beres, Stacie

Bernard, Kathleen

78,254

Bernecker, Mary

206

Bertagnoli, Kathleen

78

Bertsche, Eva

274

Bertsche, John

96,156

Berwager, Sarah

Besaw, Laura

Betori, Stefanie

92
Bell, Lauren

Bewley, Molly

Bidwell, Lauren

Bierer, Jesse

78 Bigg, Jenne

211 Bigler, Brett

176211

120, Bigler, Nicholas 193

120, Bingeman, Zachary Black, Dylan

156 Black, John

78, Blackburn, Erin 149

211 Blackburn, Kelly

78 Blacklidge, Sara

120166,252

156 Blackstone, Michelle

17. 78

Blackstone, Valerie 156

211 Blagg, Ethan

$78180,181,194$

78 Blake, John

72. Blatherwick, Alexandra 127

78. Bliss, Katie 157

78 Blocher, Brian

186 Blocker, Hugh

78 Bochman, Heidi

120 Bogan, Robert

263 Boice, Stephanie

211

Boice, Stephanie

202. Bollenbacher, Karissa

212

211 Bolyard, Ethan

194 Boward Gabrielle

211 Bowers, Rachel

211157

110, Bowes, Hanna

Bowlsby, Sarah

78 Bowman, Trevor

258193,212

120 Boyd, Heather

174 Bracy, Jordan

120. Bradley, Roderick Brady, Bonnie

Benjamin, Marcus 194 Brady, Patrick

Benner, Daniel 211 Brammer, Tacie

Bennington, Christopher 134

2

Bertsche, Benjamin 96

Betzold, Haeli

Brand, Amy

211 Brandes, Michael

Brandt, Adam

279

20, Brandt Julie

211188

211 Brandt, Katie

120 Brandt, Peter

4. 265

Brandt, Sarah

78. 166

Brannon, William

Braun, Nathanael

Breeden, Emma

Brenneman, Kyle

11. Brewer, Bethany

158

54. Brewer, Kaitlin

157

211 121,122

78, Bright, John 202

120
78 Brittan,

211 Brittin David

120 Brock, Peter

156 Brockmeyer Kevin

193, 157

Brodzinski, Dennis 79

121. Broesder, Kimberly 213 Brooker, Judson 180 ,

194, 212

121 Brooks, Anna

211 Brooks, Janelle

121, Brooks, Natalie 206

212 Brooks, Nevin

156. Brown, Alexis

Brown, Alisha

Brown, Andrea

Brown, Andrew

Brown, Brandon

170

Brown, Breanna

212 Brown, Brian

Brown, Courtney

206, 212

14. Brown, Danielle

Brown, Douglas

15779,80

212 Brown, Jacob

21279

157 Brown Jessica

121 Brown, Joshua

Brown, Lauren

Brown, Megan

140

78 Brown, Nathan 202

57 Brown, Nathaniel 12

12, Brown, Rachel 177

Brown, Rebekah 212

121 Brownfield, Rachel 79,

$79 \quad 176$

192, Brownfield, Sarah 176

Browning, Hayden 148

79 Bryan, Clayton 202

121 Bryan, Hannah 79

212 Bryan, Laura 79,

21285

121 Bryce, Alexandria 79

121. Bubacz, Christin 213

212 Buck, Hali

120 Briggs, Lydia

Buckholz Lori

Buesgens, Micaela 114

157,121

Buhr, Kristin

121 Bundy, Jonathan

258, Bunez, Bridget

Burch, Whitney

157. Burgman, Lisa 213

79 Burkhardt, Jessica 98

79 Burkholder, Brittany 79

121 Burkholder, Megan 121

121 Burris, Emily 157

157. Busenilz, Justin 157

Bush, Christopher 68

141, 121

Bush, Emily

109, 145,246

Butterfieid, Karen 
Chisholm, Eric $\quad 186$

Chiu, Emily 28

63, 121

Cho, So Mang 213

Christiaanse, Justine

$157,178,179$

Christiaanse, Paige 79

Christian, Angela 17.

$136,157,166,279$

Christiansen, Brenna

Christiansen, Heidi 107

213

Christiansen, Ruth 80

Chrystal, Stephanie 80

Ciarrone, Lauren 145

Ciolek, Nathan 214

Clark, Charles $\quad 80$

Clark, Courtney 80

Clark, Heather 80

Clark, Jordan $\quad 174$

Clark, Todd 158,

171

Clason, Nicholas 80

Clawson, Melissa 214

Clelland, Lucas 80

Clore, Luke $\quad 180$

Clouse, Adam 158

186, 187, 206

Clouse, Michael 121

Clymer, Kaydrie 214

Cobucci, Stephen 214

Cochran, Timothy 214 .

273

Cocks, Aaron 202

Cody, Andrew 158

Coffill, Samantha 158

Coffindaffer, Kari 214

Cole, Kraig 214

Coleman, Sarah 214

Colman, Kristin 158

163

Colton, James 214

Combs, Cortni 204

Comers, Andrew 27,

80

Comers, Luke 158

Compitello, Aubrie 122

Compitello, Everett 214

Condon, Lacie 176

188

Connelly, Ryan $\quad 174$

Conner, Amy 122

138

Convertini, David 202 ,

203

Conway, Jillanne 122

Cook, Felicia 158

Cook, Jazmine 158

Cook, Kaitlyn $\quad 158$

Cookson, Shaun 64

214

Cooper, Julianne 80

Coover, Matthew 80

Coppola, Joshua 80

Corbin, Kristin 81

196, 197

Cornell, Laura $\quad 214$

Cornish, Lindsay 81

Costerisan, Andrew 112,

214
Costilow, Christina 81

Cottings, Bethany 214

Couser, Jaklyn 81,

85

Covel, Janemarie 81

Cover, Laura 214

Cowden, Hannah 81 .

88

Cox, David 214

Cox, Michael 159

Cox, Rachel 159

Cox, Timothy 214

Craig. Sara 214

Crane, Miriam 81

Crawford, Rebekah 81

Crawford, Scott 174 ,

215

Crichlow, Sarah

215

Crickard, Charles 122

Croft, Jennifer 122

Cronin, Jennifer 122

Crowder, Lisa 215

Cuenin, Renee 215

Cuffman, Timothy 122

Culver, Jenna 215

Cummings, Elizabeth

122

Cummings, Lisbeth 159

Cummings, Sara 122

Cunliffe, Jennifer $\quad 81$

Cunningham, Jason 174

Curby, Erin $\quad 122$

Currie, Rachael 81

Curtis, Benjamin 215

Cvetich, Rebekah 147

Cyrus, Amanda $\quad 215$

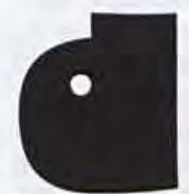

D'Anna, Mark $\quad 81$

Daly, Samantha 81

Dammer, Timothy 159

Daransky, Sara 123

Darling, Marie 123 ,

134

Dasuqi, Ashley

279

Daugherty, Dennis 255

Davenport, Nathan 202

David, Priscilla 94.

159

Davidson, Nathaniel81

Davidson, Robert 64 ,

215,273

Davies, Bethany

196,215

Davies, Cynthia

Davies, Jordan

181, 194

Davis, Andrea

91

Davis, Andrew

241, 265

Davis, Courtney

264

159,
Davis, Jewell

215

Davis, Virginia

Davison, Danny

Dayton, Katherine 81

DeBoer, Kyle

198

DeCook, Felicia

103

DeGraff, Hayley

DeGroft, Jennifer

159

DuPree, Briana

159 Duarte, David

Long, Leslie

215

DeLong, Samuel

DeRusha, Emily

196,215

Duff, Justin 111

4. Duarle, Rachel Duda, Ashley

215 Duerrwaechter, David

83. 190

DeVault, Katherine 159

$159,247,258,259,2$

Dulin, Joel

Deakin, Diana 158 ,

Dulis, Ezra

Dunger, Kellie

Dunger, Kellie

Deakin, Nathan 105 Dunn, John

Dean, Megan 81 Dunn, Nicholas

Dearden, Matthew 112, Dunnevant, Kaitlin 216,

113,258

Dearden, Rachel 215 Dunning, John 216

Decker, Jonathan 215 Durbin, Alexander 216

Deemer, Carolyn 159 Dybwad, Taylor 27

Deeter, Sarah

Dehart, Joshua 81

Dehnke, Emily 123

Dellicarpini, Leah 123

Demers, Jonathan 66 ,

123

Demeusy, David 81 ,

123

Denen, Alyssa

278

Dennis, Elizabeth 215 Eachus, Thomas 123

Earls, Christopher 216

Develbiss, Jenna 159 Early, Danielle 123

159 Eaves, Dorianne 159

123

Devine, Stephanie 72

Eberle, Matthew 216

Edens, Kathryn 123

215

Edwards, Heidi 81

123

92

Dickhoner, Barbara 159

Didik, Yekaterina 159

264, 281

Diebold, Alayna

206

Dion, David

Dixson, Jennifer 81

Dobutovich, Cassandra

26. 216

Dodson, Rebekah 81

Doenges, Timothy 148 ,

159

Dolan, Erin

\section{1}

215, Donaldson, Benjamin

216

Don

253

Donohue, Kelly

Dorbritz, Kristina

Dorsey, Arielle

Douglas, Abby

216

Ege, Malachi 123

Egenreider, Sarah 159

Eggleston, Stephen 159

Eisenlohr, Abby

135

159

Endres, Hannah

246,247

Engel, Aaron

Engel, Austin

68,217

England, Jessica

217

Engler, Amanda

Ennis, Curtis

Ensminger, Kristen 14

217, 275

6 Ensslen, Johanna 1

16 Enterline Kara 123

Erdmann, Jason 123 259

16 Erlandson, Jenna 217

59 Erlandson, Sarah 217

Eslick, Stephen 256

Eustace, Abigail 72 , 123

Evans, Adam

$208,217,270$

16 Evans, Jeffrey

180

Evans, Matthew 123

Everswick, Julianne 123

Ewers, Lacy 217

Ewing, Sean

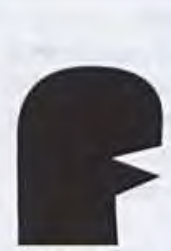

Fallin, Dea

217

Fanster, Megan 217

Fawcett, Matthew 153 261

Feczer, Justin

Fehl, Sarah

Feiler, Katherine

Felmet, Evan

Ferguson, Kristen

Ferkaluk, David

Ferrell, Andrew

Ferrell, Talitha

Fideler, Joseph

Fields, Jonathan

Edwards, Meredith 112, Fillion, Molly

171

Finkbeiner, Rachel 123

Firmin, Ruth 109

124

159 Fisher, Susan

Flack, Nathaniel

160

127. Flamm, Seth

127

122

Fleetwood, Jonathan 


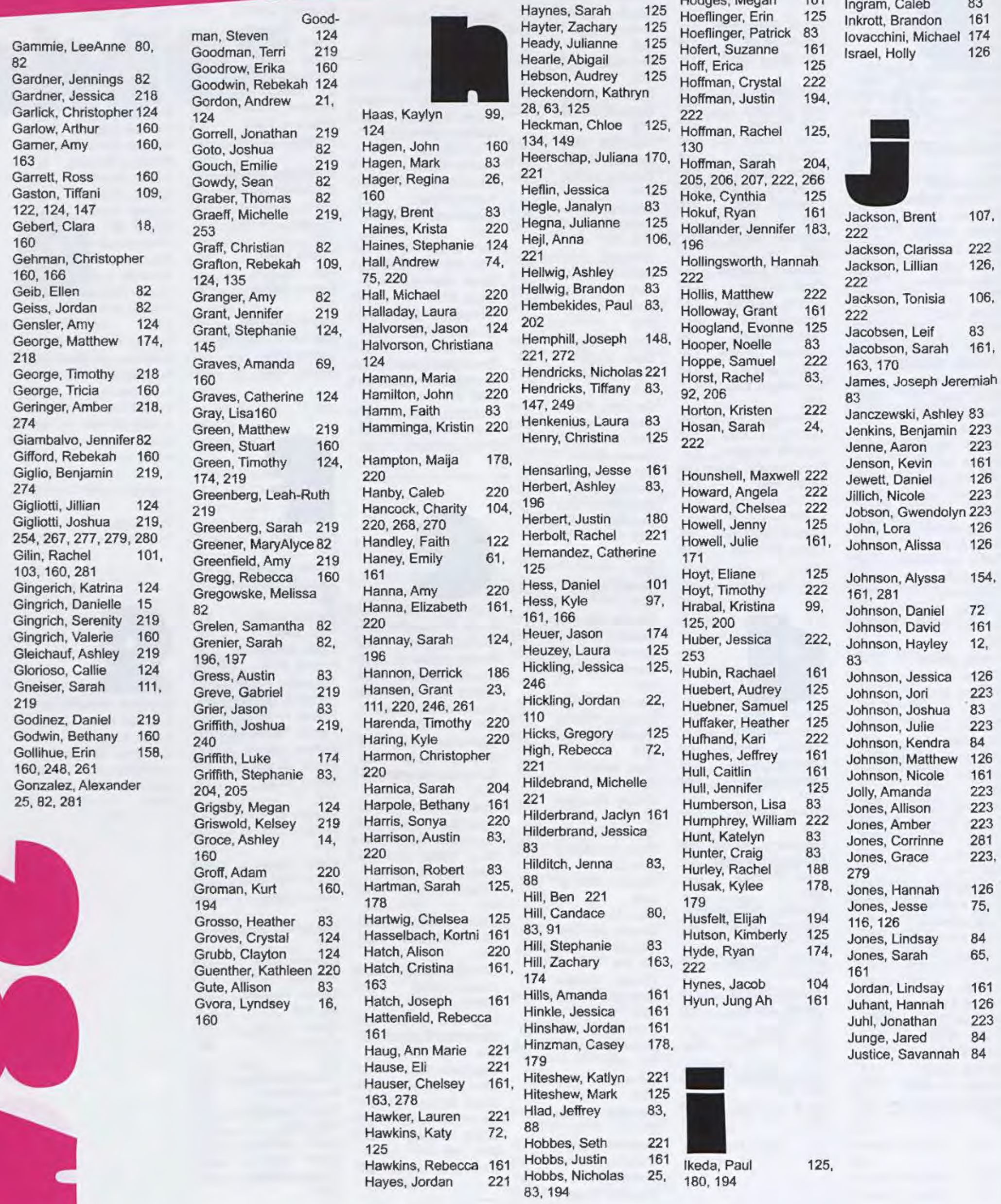


Kolody, Timothy 224 Komar, Grace 281 Komarek, Lauren 162 Koranek, Matthew 84 Korthals, Jessica 162 281

Kowalchuk, Rebekah

126

Kallstrom, Sarah 84

Kane, Erik 20

Kapp, Amanda 223

Kauffman, Elyse 18

Kearney, Alison 162

Keeports, Christine 162.

171

Keller, Stacey

183.

196,223

Kellogg, Joy

200, 201

Kelly, Amy

162 ,

Kelly, William

84

Kempf, Jacob 12

Kempton, Eric 194

Kepiro, Lindsey 126

Kerner, Nicholas 84

Kessler, Jessica 126

Keyser, Abby 278

Kidd, David $\quad 162$

Kilian, Benjamin 126

Kim, Jinsil 223

Kim, Seung

Kinch, Christina 84

Kindt, Laura 16,

$223,268,278$

King, Shannen 223

Kirby, Abigail $\quad 224$

Kirby, Kyle

Kirby, Laura

196

Kirby, Marie

Kiser, Andrea

224

Kitchen, Jeremiah 162

Kittle, Douglas 194

Kjellman, Andrew 162

Kleis, Benjamin 224

Kline, Courtney 224

Klink, Zachary 84 ,

180, 194

Klint, Charles

166

Rebecca 224

KIoosterman, Daniel

224, 271

Knepel, Ryan 84

Knight, Jonathan 22

162

Knoedler, Brittany 224

Knoedler, Jordan 75 ,

126

Knorr, Elizabeth 162

Knott, Christine 224

Knott, Rebeccah 84

Kobza, Carl $\quad 17$

Koch, Andrew 224

Koch, Kathryn 176

224

Koepke, Sara 204

Kohavi, Daniel 186 ,

187

Kohl, Grace

126

Kolody, Jessica
Kowalczyk, Valerie 224

Kowatch, Krista 224

Kraft, Emily

224

Kramer, Devin 162

Kramer, Laura 126

Kramer, Stephanie 84

Kranenburg, Julia 162

Kremer, Katherine 126

Krizo, Kathryn 162

Krogstad, Matthew 162

193

Kroll, Michelle 224

Kroninger, Krista 126

Krop, Caitlin 126

Kropf, Michelle 25 .

224

Kuhn, Kevin

194, 195, 224

Kuiken, Jesse

Kurtz, Lauren

Kushnir, Michelle 126

Kusky, Sarah

180 ,

84

225

126

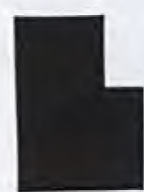

Labrado, Timothy 138

$225,274,281$

Lacey, Michael

180,194

Lagan, Daniel

Lagar, Natalie

84,

225

225

Laing, Amber

225

Lamb, Juliann

162

Lambright, Rhoda 225

Landers, Erin 178,

177

Lane, Lauren

84.

Lanphier, Katherine 84 ,

196

Lanphier, Renee 162

Lansford, Megan 225

Lantz, Noah

Larsen, Brooke

225

Larsen, Sandra

225

Laswell, Alyssa

Laswell, Ashleigh

Laswell, Hannah

Latario, Brian

Latario, Rachel

162

176.

61.
La Croix, Daniel 126
Latham, Stephanie 162, Lusk, Joshua

188

Lavaliee, Emilie

Lavoie, Evan

198

Law, Weslea

Lawrence, Joel

Lawson, Jedidiah

Lawton, Sarah

Leach, Jordan

Leaman, Tara

162,196

Lear, Jesse

Lear, Larissa

Lease, Alicia

Ledger, Peter

225

Lee, Alisha

225

Lee, Rachel

Leffel, Erin

145

Leffel, Jordan

Lehmann, Gail

Lehr, Sharon

Leitch, Hannah

Leman, Jordan

Lemon, Alison

189

Lempa, Dale

Lentz, Jason

Lenz, Jordan

Leonard, Caryne

Leonard, Christina

Leppke, Lindsay

Lester, Jennifer

Lethbridge, Tiffany

Lett, Amy 226

Lewis, Daniel

84,226

Lewis, Mary

Lightner, Joe

Lightner, Melody

Lindemann, Kara

Linden, Bethanne

139

Linden, Kyle

Linder, Nathan

Linebaugh, Patricia 14

162

Link, Jordan

Linville, Jana

279, 280

Liu, Annette

126 Lulz, Kathryn

4. Lyman, Daniel Lynch, Ernilie

225

178

225 Lynch, Jeffrey

26 Lyons, Whitney

174

114.

225
127

225

65 ,

188, Maat, Christian 195, 226

24 Mable, Kourtney 226

127. MacKenzie, Allison 85

MacKenzie, Katherine

225127,178

127 MacKenzie, Matthew

162226

225 Mace, Laura

162 Mack, Kyle

188, Mage, Peter

Maithel, Sarah

162 Manchester, Marcus

162226

198 Manning, Travis

4 Mansker, Kristen

26 Manzer, Stefanie

62 Marburger, Katie

162141,163

62 Marchena, Jason

Marks, Alison

20. Marot, Luke

Marriott, Richard

127 Marshall, Joseph

162 Marshburn, Justin 227

84 Martelli, Kevin 74

226 Martin, Amanda $\quad 227$

127. Martin, Andrew

Martin, Brent

19

29 Martin, Christie

Martin, Jessica

LoCicero, $S$

$162,166,185,266$

Locke, Paul

166, 190

Locke, Sarah

Lockridge, Heidi

Long, Dustin

Long, Kenneth

Looyengoed, Elyse 9 163

Loper, Brittany

Losch, Jessica

145,226

Losch, Nathan

Lounds, Courtney

Lowry, Kyle

Lowry, Sarah

Ludlow, Kyler

Lukasiewicz, Timothy

127

McClure, Georgiann 164,

200,201

58 McClure, John 227

226 McConnell, Lucas 164

226 McCoskey, Karen 128

176,206

127 McCown, Casey 227

. McCoy, Jonathon 85

McCoy, Michael 227

275

McCray, Anna

McCue, Jamin

227

McDonald, Jennifer 227

McDonald, Matthew 16 85

McDuffie, Jeremy

164

McEllhenney, Sarah 85

McFarland, Bryan 128

4, McGee, Katie 275

McGee, Miles 164

McGill, Jonathan 112

$113,227,250,264$

McGuire, Erin

McGunnigal, Kristine

227

McHugh, Courtney 227

27 Mclnturf, Callie 106 
Memec, Elyse 129

Muckley, Karen $\quad 86$

Mueller, Joseph 174

Muhlenkamp, Mitchell

128

Muhlenkamp, Whitney

164

Mukes, Jennifer 164

Muller, Joshua 86

Mullican, Gerhard 164

279

Mullikin, Laura 229

Mumme, Courtney 128 ,

206

Murphy, Sarah 164

256, 278

Murray, Erin $\quad 128$

Murschel, Cristin 164

Muschott, Bradley 229

Muschott, Brittany 86

Musselman, Brittany

164, 206

Musselman, Cherice 85

Musser, Jonathan 109

Myers, Janelle 164

Myers, Sarah $\quad 86$

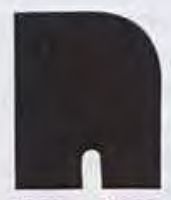

Napp, Kevin

164

Naramore, Randi 86

Nearhoof, Joshua 229

Nelson, Annalise 229

274

Nelson, Elizabeth 129

Nelson, Jennifer 163

164

Nelson, Katherine 129 ,

229

Nelson, Megan 229

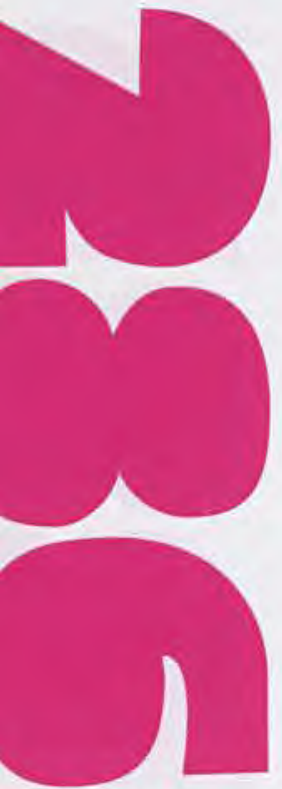

Neubecker, Daniel 86

Neuhart, Micaela 158

229

New-Day, Danielle 229

New-Day, Holly 164

Newman, Christopher

164

Newman, Justin 164

Ney, Sara 229

Nicholls, Jordan 86

Nichols, Daniel 164

260

Nick, Josef 86

Niday, Hannah 165

Niemiec, Matthew 174

175

Nikkel, Lucas

Nine, Jerilynn

Nine, Juliana

Noble, Emily

Noble, Garrett

Norris, Sarah

86

North, Brittany

Nosal, Bayley

188, 189

Nowak, Lauryn

142

Nowels, Kelly

Nygren, Ashley 86

Nyhuis, Julia

86

165

165

229

229

20 ,

230

67 ,

29

230

86

129

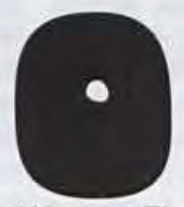

O'Connor, Thomas 129

O'Neal, Christine 230

O'Neal, Erica 230

O'Neal, Renee 165

O'Prandy, Justin 129

Oakes, Jordan 87

Oakley, Adam 87

Oana, Asheritah $\quad 165$

Obielodan, Mary 87

Ohlin, Bethany 165

Ojala, Brendan 192

193

Olley, Stuart 87

Olmstead, Hannah 165

Olney, Lucy 230

Olsen, Erin 165.

230

Olson, Karl

109

Olson, Toryn 230

Ommundsen, Corey 87

Ormsby, Daniel 129 ,

198

Ortiz, Melody

264

Osborn, Joseph

207

Ouellette, James
Ouellette, Amy

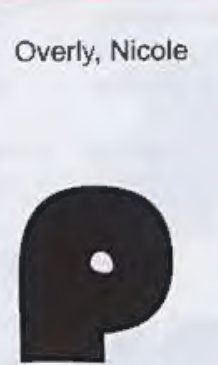

Pack, Helen

Pagano, Morgan

Page, Paul

Palmer, David

Paimer, Hallee

Palmer, Steven

143,165

Paney, Megan

129

Pankratz, Kelly

$146,158,163,165$

Paoloni, Krista

Parker, Laura

Parmerlee, Carissa 17

200

Parrott, Rachel

93

Pasma, Alyssa

Patrick, Charity

Patrick, Diana

Patrick, Melvin

atton, William

Payne, Jillian

Peak, Sarah

Peck, Jordan

Peden, Stacie

230

Peletis, Cassand

Pennell, Allison

249

Pepe, Karie

240

Pepper, Torrie

176, 177

Perhai, Brian

Pernicano, Leah

Perrel, Kelly

Perrien, Tanner

Perry, Rebecca

Persun, Brent

$276,278,281$

Petek, Elizabeth

Peters, Daniel

Peters, Trisha

Peterson, Ethan

Peterson, Kayla

Petke, Daniel

202

Phillips, Andrea

Phillips, Stacy

145

Phillis, Samantha

Philyaw, Drake

Philyaw, Erin

Piasecki, Andrew

Pickens, Julie

Pierce, Jillian

Plante, Kyle

Plasterer, Anna

129, 207

Plasterer, Ruth
165 P

Plumley, Trevor

Pollock, Lisa

Polston, Laura

Poore, Thomas

Port, Katherine

Porter, Charmaine

$258,273,281$

Porter, Sarah

$152,260,281$

Potter, Joseph

129 Powell, Emily

249,261

194 Powell, Jennifer

129 Presley, Jessica

16587,191

105

Pressly, Alexander

Preston, Heather

28. Price, Andrew

Price, April

Price, Christen 231

30 Price, Clinton

30 Price, Danielle

Price, Elisabeth

Price, Krista

87. Price, Leah

231

87 Prijatel, Kimberly

129 Prince, Amber

$8767,138,144$

258 Proctor, Pamela

87 Pruzanis

87 Pruzaniec, Danielle 1

165 Puffer, Christina

165

129 Putinski, Adam

69, 166

Putt, Brittany

230 Pyle, Gabriel

165. Pyles, Anna 231

230, Pyles, Joanna

165

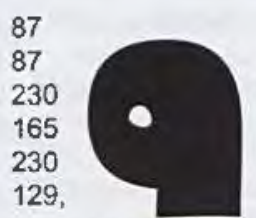

87

Oueeney, Vanessa 87

230

129

87.

230

67.

Rabenstein, Robert 87

87

230 Rader, Sarah 87

Rafoss, Jonn 231

129

Ragle, Zachary

Ralph, Taylor

Rambikur, Amy

153271

165 Ramsey Aaron

230

99.

Randolph, Brittaney 231

271

165

Rarick, Jessica

Rathburn, Jessica 87

Ratliff, Mark

Ray, Meagan

75,129

65 Raybuck, Lindsay

176, 206

Rayder, Kelley

130

43. Raymond, Jessica

Rea, Brittany

174 Reategui, Claudia 87

29. Rector, Ethan 130

, 264,278

Reed, Nathan

29 139, 165

65 Reed, Rachel

7 Reeder, Corey

31 Reeder, Jillian

2. Reep, Ryan 231

202 Reese, Morgan

87 Reilmann, Krista

231 Reke, David

Renke, Andrew

232

Reuther, Katelyn

166, 206

7. Rice, Heather

Rice, Marla

232

Richard, Meredith 165

129 Richardson, Lindsey

129. 165

Richardson, Noah 165

Rieches, Veselin 13

12996,130

11 Riehle, Adam 
Schellin, Bradley 233 Scherner, Rachel 111, 258

Schindehette, Joshua 131

Schindler, Paul 89

Schiabach, Grant 167

Schlegel, Kylie 279

Schlener, Jessica 233

Schlindwein, Ann Marie 167

Schlueter, Tiffany 233

Schmidt, Katherine 89 , 140

Schmidt, Stacie 131

Schoen, David 108 ,

233

Schultz, Erin

89

Scott, Cayla

Scott, Jessica

Scott, Kathryn

Scott, Megan

Scott, Seth

Scott, Tyler

174,233

Scull, Peter

Segebart, Kyle

Seibert, Jordan

233

Seifert, Samantha 167

Self, Christina 131

Seligman, Justin 131

Sergent, Andrea $\quad 167$

Serna, Denise 111 .

281

Servi, Bethany 89

Severance, Emily $\quad 89$,

278

Seybold, Susanne 89

Shackelford, Kelly 167

Shaffer, Daniel 131

Shanahan, Emily 131

Shanks, Catherine 69 ,

233

Shaughnessy, Joy 166

Shaw, Kelly 131

Sheers, Sarah $\quad 178$

233

Shenk, Brianna 167

Shephard, Juliana 58

Shepherd, Juliana 67 . 233

Sheppard, Nathan 233 240

Sherman, Amanda 131 Sherrell, Stephanie 167 Sherry, Mychaela 167 Sherwood, Rachel 233 Shetler, Ami $\quad 131$

Shifrin, Katherine 167

Shimeall, Peter 131

Shinabarger, Rachel

233

Shinabarger, Rebekah

167

Shinn, Brianna 89

Shirzadian, Michael 72

Shomper, Matthew 233

Shorey, Benjamin 72

Shorts, Mikal 234

Shriber, Andrew 234
Shum, Heman 89 Smith, Timothy

Smith, Travis

Sides, Justin

166, 167

Snead, Andrew

188. Sniffen, Apryl

189

Sierawski, Bethany 65 ,

234

Silveira, Matthew 180

Simmons, Emily 143

Simon, Trenton 234

Simpson, Brittany 182 .

183, 196, 234

Simpson, Jared

167

Simpson, Laura

89

Simpson, William

Sims, Grace $\quad 234$

Sims, Kelly 234

Sincock, Benjamin 234

Sisson, Joshua 202

Sjoquist, Ryan

Skelton, Julia

Skinner, Jessica

Slagh, Rachel

Slavish, Michael

Slifka, Mary

Sloan, Anna

113, 131

Stampfli, Catherine 131

Smidt Ty

Smith, Amanda

196, 234

Smith, Amie

Smith, Amy

Smith, Andrew

Smith, Brandon

Smith, Brennan

Smith, Clifford

Smith, Eric

Smith, Erika

Smith, Ethan

Smith, Jacob

Smith, Jenna

Smith, Jessica

196

Smith, Jonathan

Smith, Jordan

Smith, Kendra

93, 206

Smith, Kyle

Smith, Lydia

Smith, Matthew

Smith, Meagan

Smith, Nathanael

234

Smith, Patrick

Smith, Paul

$104,111,168,260$

Smith, Peter

Smith, Rebecca

Smith, Ryan

Smith, Sara

206

Smith, Sarah

206

Smith, Stephen

187, 267

Solomon, Amber

Sosey, Lauren

198

Spencer, Brianne

163, 168,174

Spring, Megan

Spurlock, Christopher

89

Sray, Camila

Stacy, David

Stacy, Melissa

Stafford, Ally

Stahl, Stephanie

Staley, Joshua

194

Start, Kaitlin

Staudt, Daniel

Stauffer, Aaron

Stauffer, Kelsey

Stearns, Grace

Steckel, Jennifer

Steckel, Jeremy

260

Steckel, Joshua

Steele, Meghan

Steele, Michael

Steffel, Charis

Steinbach, Mary

Steiner, Heather

Steinmetz, Jarrod

235

131 Stephens, David

235

Stephens, Emily

Stephens, Scott

234 Stern, Jennifer

131 Stevens, Daniel

89 Stevens, Sarah

196

Stevenson, John

167

Stewart, Ashleigh

Stikeleather, Erica

Still, Cheryl

Stock, Amanda

259

168, Stoltzfus, Colby

Storch, Jonathan

Storhaug, Sonja

Stout, Heather
Starr, Samuel 168

Strayhorn, Anna

168, 201, 236 Streitmatter, Amy 168

235 Strejc, Stephanie

89 Strejc, Stephanie 89

131168

168 Strode, Elizabeth 168

89 Strohm, Deanna 168

131 Studebaker, Elizabeth

$186,146,236,268$

Studebaker, Nathaniel

131168

168 Studebaker, Timothy

Sugg, Amanda 168

168 Sumner, Meredith 132

63. Surant, Zachary 168

Sutton, Amanda

89. Swaney, Abby 168

Swango, Jessica 90

Swanson, Timothy 90

131. Swayze, Jessica 236

Swett, Alanna

Swick, David

Swick, Stacie

89 Swieringa, Julia

168 Swisher, Mark 90

168 Swoveland, Jonathan

$\begin{array}{ll}89 & 132\end{array}$

235 Sylvester, Brittany 236,

89. 272

31

168

Stockwell, Derek

Strait, Daniel

Straton, Macy

Straw, Kevin

Strayhorn, Ali

168 ,

132

132

235

132

132

168

235,

Taylor, Joanna

132, 202

Taylor, Stephanie

Torr

158 ,

Townsend, Lauren 132

Veldt, Hadassah 90

Veldt, Mordecai 237

Veres, Joseph 142.

Trantina, Abigail $132 \quad 1$

Trautman, Seth 90 Verlander, Lynn 237

Travis, Christopher 236 Vincent, Timothy 237

Travis, Jeffrey 90

Travis, Mallory 236

Trego, Justine 236

Tremper, Heather 90

Trennepohl, Robert 180,

$194,195,236$

Tress, Brandon 168,

194. 195

Tribbett, Natalie

206

Trindal, Vienna

Troike, Danie

261

Trowbridge, Brent 236

Troxel, Hollis 132

180

Troyer, Kristin

Vinson, Philip 237

Vivas, Abraham 90

Volenec, Aaron 169

Voltz, Christopher 90 .

281

Voltz, Timothy

$67,138,237$

Voris, Ryan

60 ,

237 


\begin{tabular}{|c|c|c|c|c|c|c|c|}
\hline & & & & Woodruff, Elizabeth & 133 & Zimmerman, Jordan & \\
\hline & & Whitacre, Amy & 238 & Woods, Clare & 239 & Zion, Nathan & 134 \\
\hline Waiton, Joel & 104, & Whitacre, Jeffrey & 238 & Workman, Brady & 202 , & Zirkle, Tia 91 & \\
\hline 170 & & Whitaker, Christophe & & 239 & & orn, Christina & 2 \\
\hline Wands, Amanda & 90 & 91,96 & & Workman, Kimberly & 104 & & \\
\hline Ward, Jerica & 237 & Whitcomb, Jeffery & 133, & 133,244 & & & \\
\hline $\begin{array}{l}\text { Ward, Jernelle } \\
206\end{array}$ & 132, & 198 & & Worosher, Tyler & 16 & 236 & \\
\hline $\begin{array}{l}206 \\
\text { Ward, Laura }\end{array}$ & 90 & $\begin{array}{l}\text { White, Ethan } \\
\text { White, Mallory }\end{array}$ & 238 & 133 & & van der Wal, John & 236 \\
\hline Ward, Stephen & 238 & $\begin{array}{l}\text { White, Mallory } \\
204\end{array}$ & 109, & $\begin{array}{l}\text { Woyak, Joshua } \\
\text { Wuenstel, Leslie }\end{array}$ & 239 & & \\
\hline Ware, Matthew & 169 & Whitecavage, Katrin & & $\begin{array}{l}\text { Wuenstel, Leslie } \\
\text { Wuobio, Heather }\end{array}$ & 163 & & \\
\hline Waring, Amanda & 133 & 133 & & 196,239 & & & \\
\hline Warwick, Stephen 1 & 169 & Whited, Lynette & 238 & Wurz, Darren & 152 , & & \\
\hline Wasem, Rachael & 127 & Whitfield, Sarah & 133 & 169 & & & \\
\hline 133 & & Whitmore, Sarah & 133 & Wynalda, Jessica & 22, & & \\
\hline Washatka, Naomi & 62, & Wichael, Samuel & 133 & $98,133,135$ & & & \\
\hline 169 & & Wicker, Devin & 91 & Wynaida, Robert & 24, & & \\
\hline Watkins, Jaimie & 176 & Widman, Jamie & 133 & 163,169 & & & \\
\hline Watkins, Kelsey & 90 & 176 & & & & & \\
\hline $\begin{array}{l}\text { 176, } 196,197 \\
\text { Watkins, Sarah }\end{array}$ & 133, & $\begin{array}{l}\text { Wight, Abigal } \\
75,238\end{array}$ & 74 & & & & \\
\hline 176 & & $\begin{array}{l}75,238 \\
\text { Wilcox, Brett }\end{array}$ & 169 & & & & \\
\hline Watson, Katherine 5 & 57 , & Wildman, Jane & 91 & & & & \\
\hline 133 & & Wildman, John & 238 & & & & \\
\hline Waugh, Dakotah & 169 & Wiles, Krista & 91 & & & & \\
\hline Weaver, Cameron 1 & 133 & Wiley, Benjamin & 238 & & & & \\
\hline Weaver, Diana & 90 & Wiley, James & 238 & & & & \\
\hline Webster, Jennifer & 133 & Wilkins, Jeremiah & 91 & Yahara, Katie & 239 & & \\
\hline Weeks, Matthew & 169 & Wilkins, Sarah & 133 & Yeates, Callan & 247 & & \\
\hline Weidman, Amanda 1 & 169 & Wilkins, Veronica & 139 & Yeh, Benjamin & 91 & & \\
\hline Weilein, Janna & 169 & Wilkinson, Andrew & 133 & Yeiter, Matthew & 133 & & \\
\hline Wein, Cynthia & 169 & Willett, Matthew & 202 & 193 & & & \\
\hline Weirich, Kirsten & 206 & 203 & & Yoakam, Andrew & 169 & & \\
\hline Weiser, Elizabeth & 85, & Williams, Alexander & 91 & Yoder, Caitlin & 133 & & \\
\hline 90 & & Williams, Christophe & & Yoder, Janelle & 133 & & \\
\hline Weix, Elizabeth & 169 & 64,169 & & Yoder, Lance & 133 & & \\
\hline Welborn, Megan & 133 & Williams, Daniel & 91 & Yoder, Rachel & 239 & & \\
\hline & & Williams, Lauren & 200 & Yoo, Jiyoung & 239 & & \\
\hline Wells, Tyson & 194 & Williams, Mark & 169 & Yorgey, Jennifer & 133 & & \\
\hline Wemple, Lucas & 91 & 194 & & York, Andrew & 202 & & \\
\hline Wengerd, Jessica & 91 & Williams, Michael & 133. & York, Rachel & 26. & & \\
\hline West, Brittany & 238 & 174 & & 102,169 & & & \\
\hline West, Justin & 238 & Williams, Valerie & 238 & Young, Bethany & 239, & & \\
\hline West, Krista & 133 & Wilson, Lindsay & 184 & 266 & & & \\
\hline $\begin{array}{l}\text { West, Matthew } \\
91\end{array}$ & 27. & $\begin{array}{l}\text { Wilson, Micah } \\
238\end{array}$ & 202 & $\begin{array}{l}\text { Young, Brandon } \\
202\end{array}$ & 169. & & \\
\hline Westerhof, Rebecca & & Winik, Alexa & 91. & Young, Emily & 91, & & \\
\hline 277 & & 196 & & 204 & & & \\
\hline Wexler, Nathan & 238 & Winkle, Melissa & 133 & Young, Lauren & 88. & & \\
\hline Wheeler, Amanda 1 & 122 & Winter, Edward & 238 & 91,190 & & & \\
\hline & & Winter, Kenneth & 174 & Young, Linnea & 169 & & \\
\hline & & Winters, Daryl & 169 & Young, Shannon & 239 & & \\
\hline & & Wirt, Lisa 238 & & Yutzy, Lemuel & 239 & & \\
\hline & & $\begin{array}{l}\text { Wise, Kelly } \\
169,170,176\end{array}$ & 19, & & & & \\
\hline & & $\begin{array}{l}\text { Wiseman, Joshua } \\
194\end{array}$ & 180. & & & & \\
\hline & & Witry, Joshua & 169 & & & & \\
\hline & & Witt, Emily & 72, & & & & \\
\hline & & Wolf, Chelsea & 91. & & & & \\
\hline & & 93 & & & & & \\
\hline & & Wolf, Cherish & 133 & & & & \\
\hline & & 281 & & Zboran, Jessica & 133 & & \\
\hline & & $\begin{array}{l}\text { Wolverton, Jonathan } \\
238\end{array}$ & & $\begin{array}{l}\text { Zeiset, Ryan } \\
170\end{array}$ & 169 , & & \\
\hline & & Womack, Aubrey & 148 & Zellner, Matthew & 239 & & \\
\hline & $\because$ & 169 & & Zellner, Nicole & 133 & & \\
\hline & & Wong, Lydia & 183 & Zerminski, Michelle & 68 & & \\
\hline & & Wong, Rachel & 183, & 239,253 & & & \\
\hline & & 196 & & Ziemblicki, Bethany & 16 & & \\
\hline & & $\begin{array}{l}\text { Wood, Anna } \\
\text { Wood, Jordan }\end{array}$ & $\begin{array}{l}169 \\
72\end{array}$ & 239 & & & \\
\hline & & $\begin{array}{l}\text { Wood, Jordan } \\
\text { Wood, Whitny }\end{array}$ & 239 & Zimmerm & & & \\
\hline
\end{tabular}



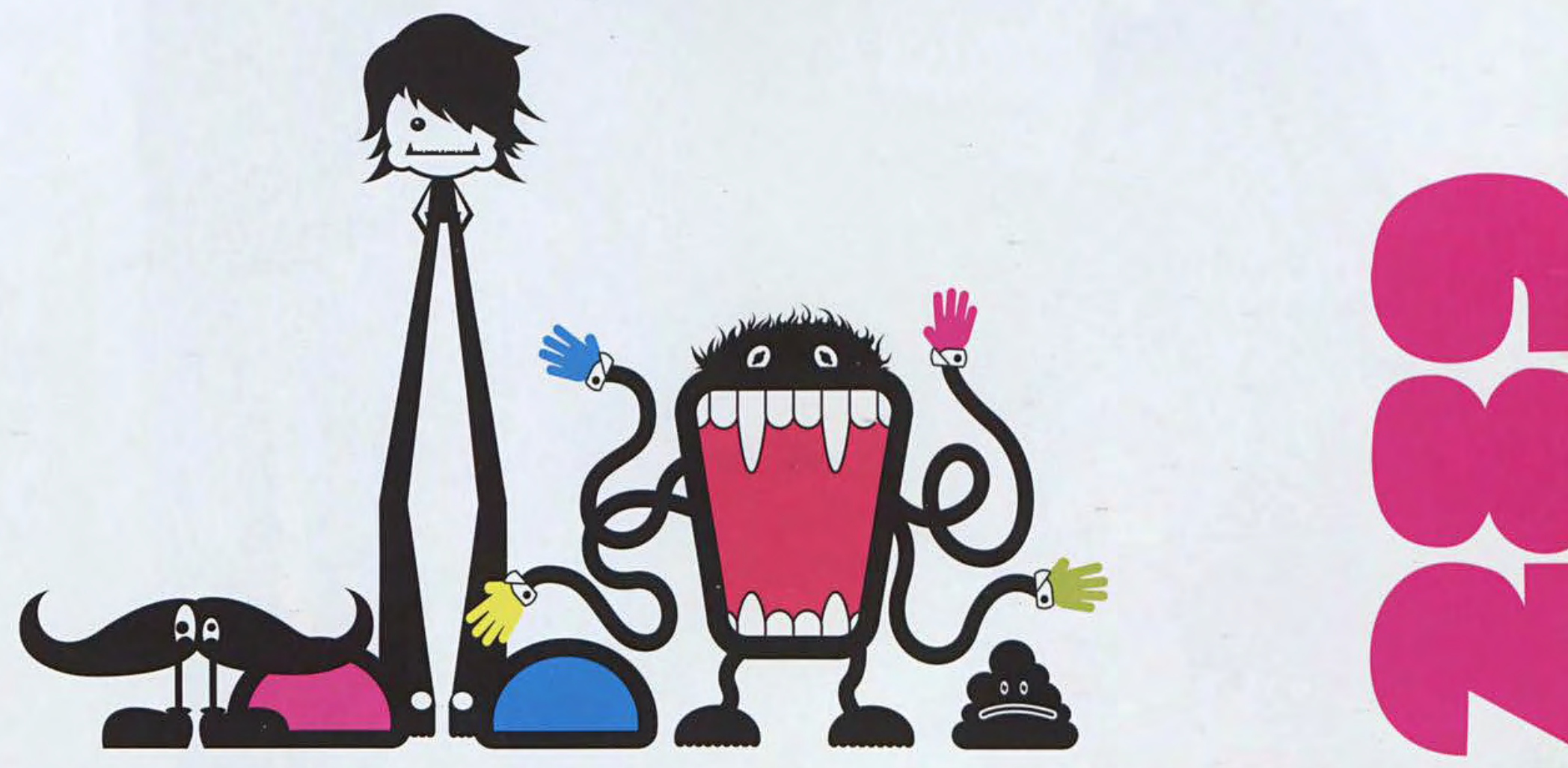


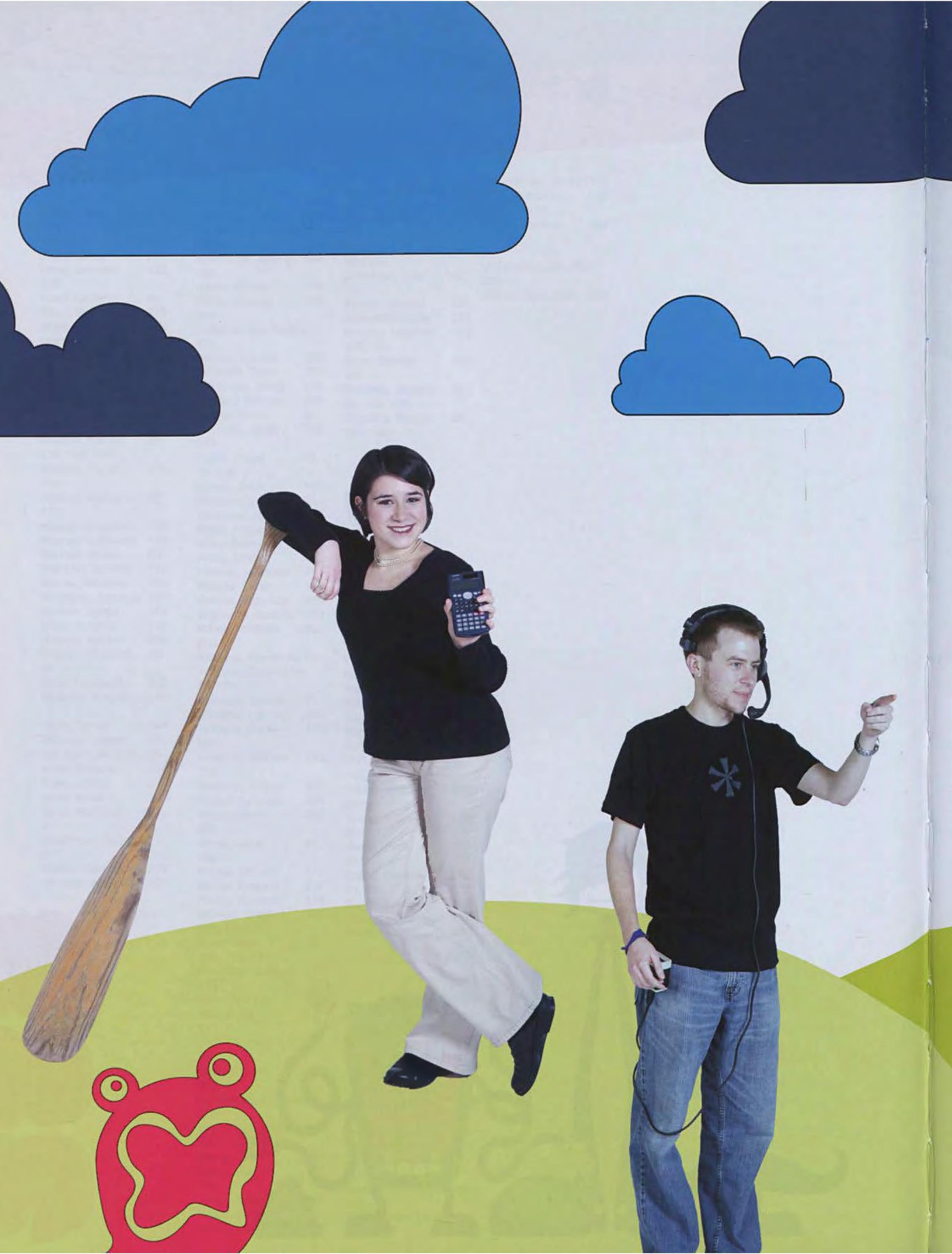




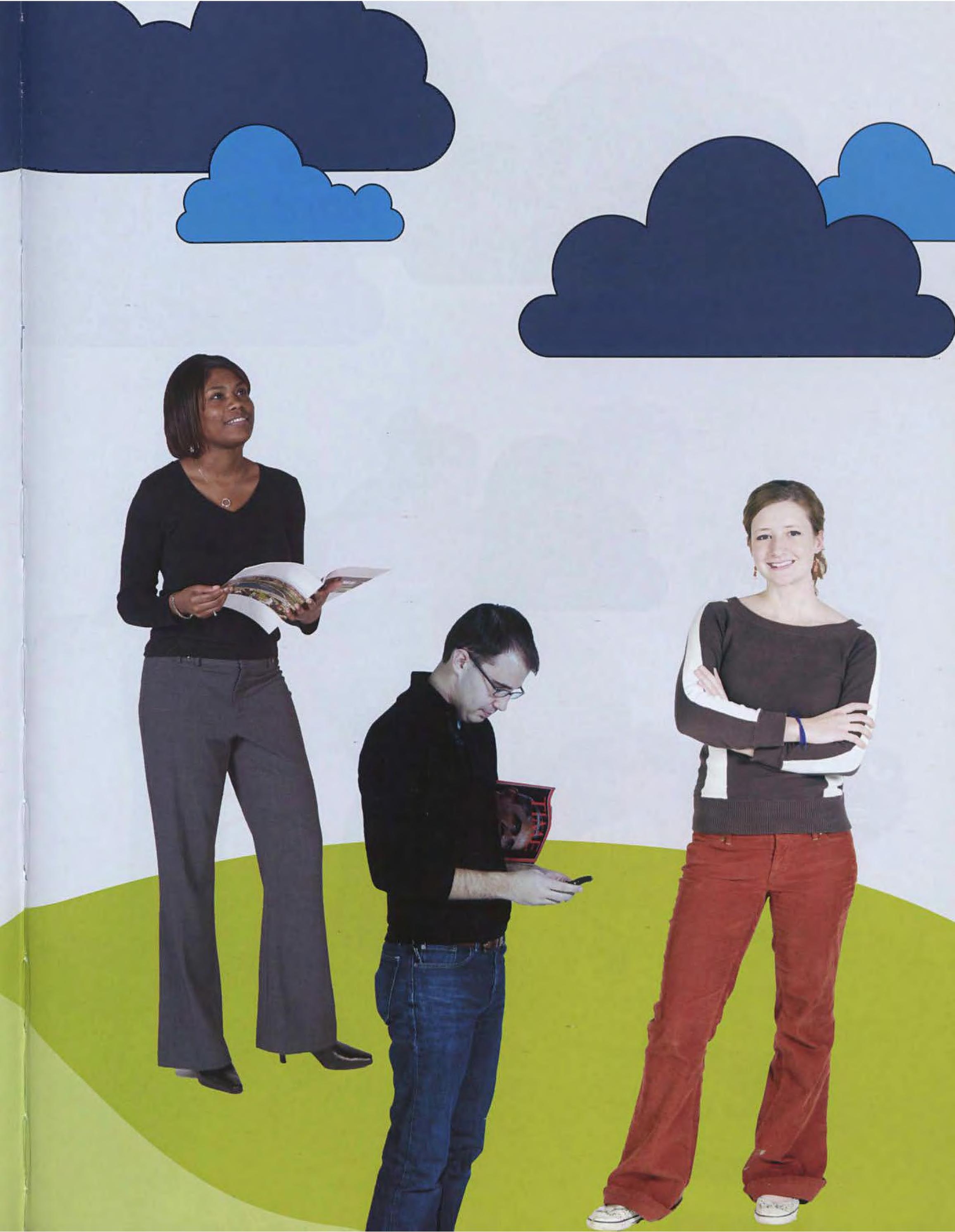



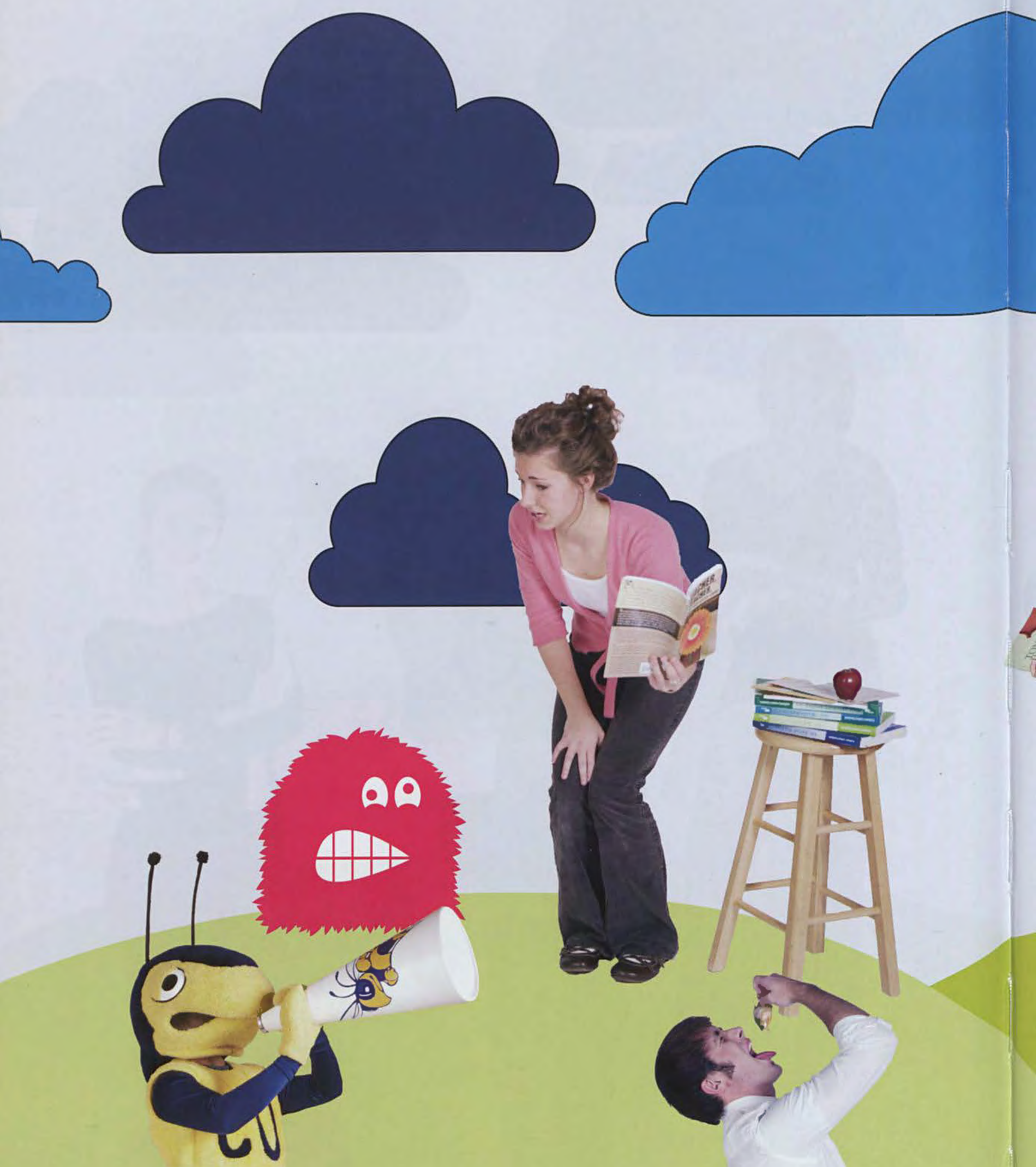


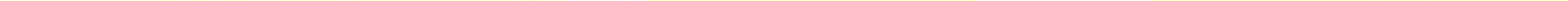








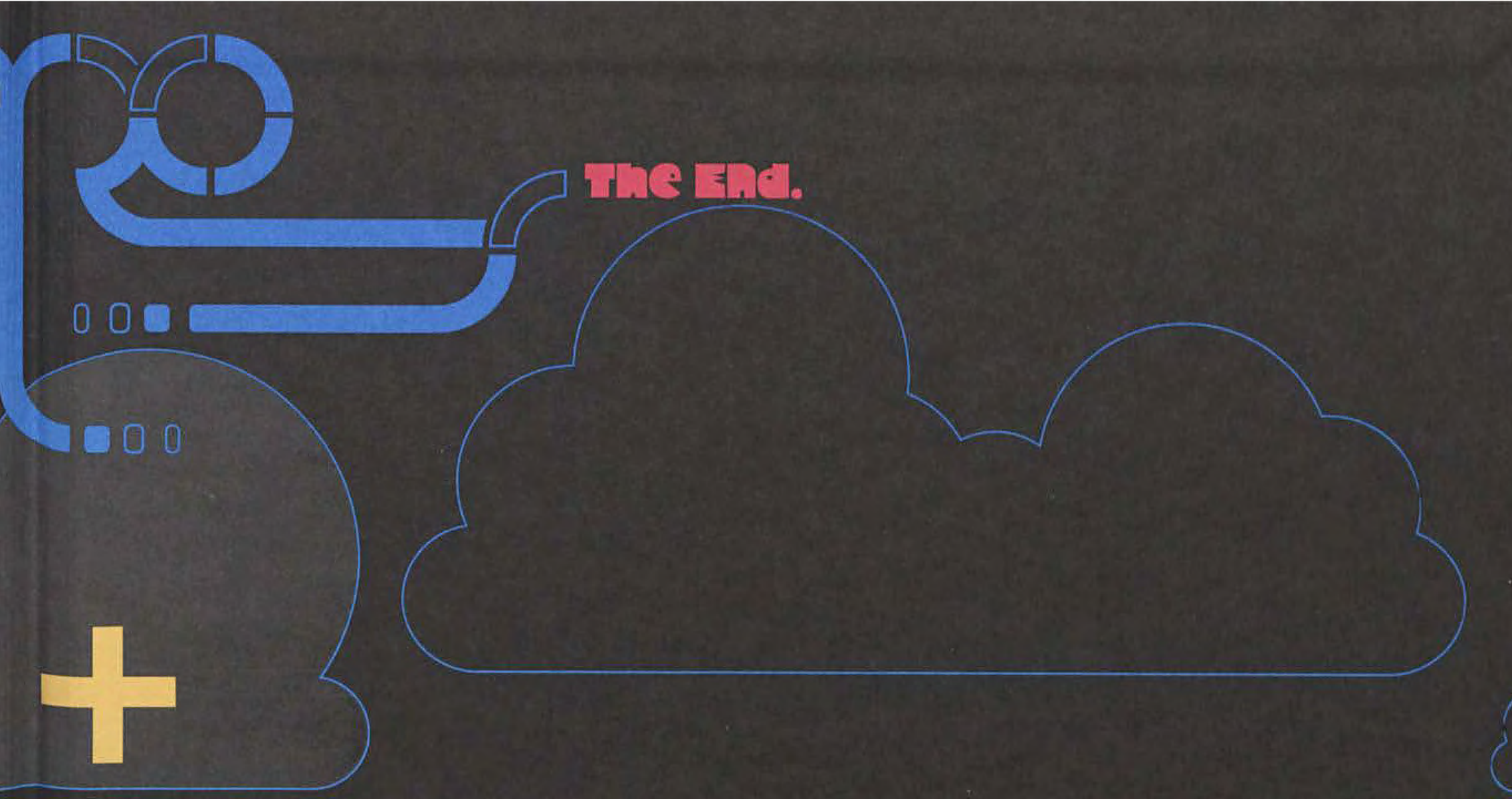



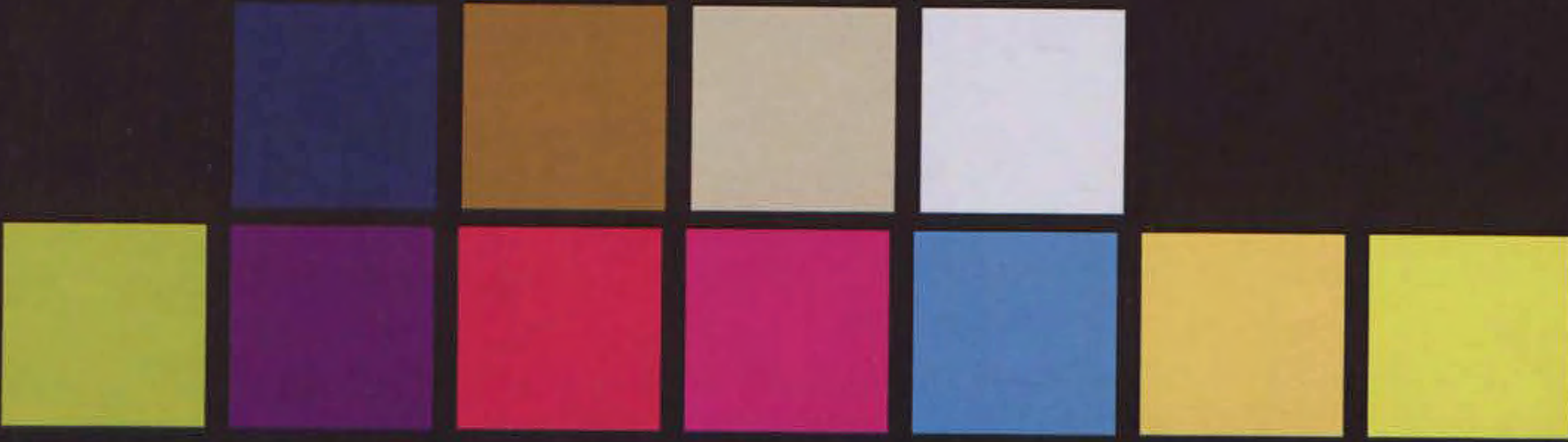